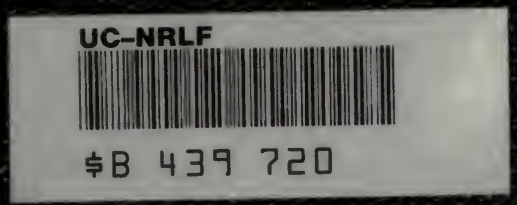




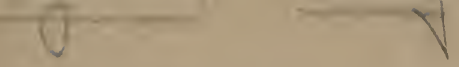

\section{DEPARTMENT OF PHARMACOLOG}

University of California Medical Schod

SAN FRANCISCO, CALIF.

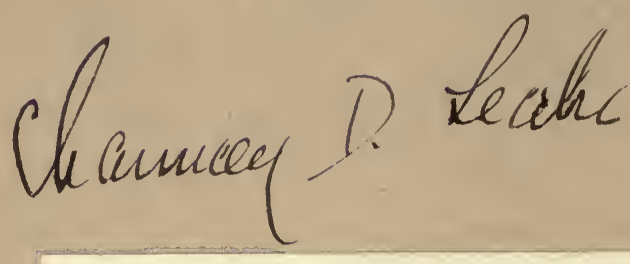

MEDICAZ SCMOOL LIBRARY

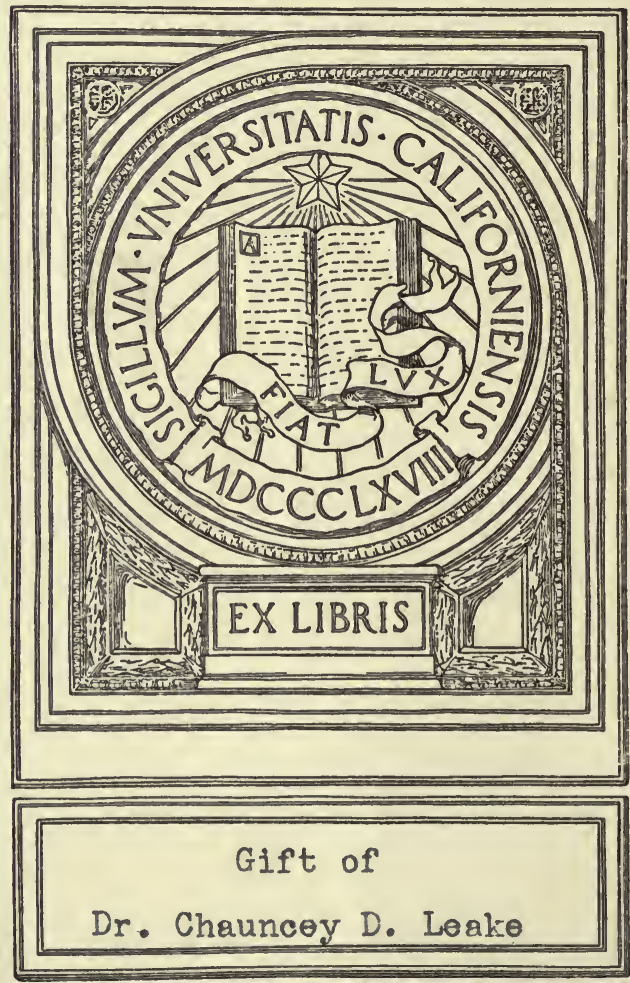


r.TB

3. 9 E. C.

6. Chramic L, L,

7. Pen mi is $\mathrm{i}$ Zins then 
Digitized by the Internet Archive in 2007 with funding from Microsoft Corporation 


\section{A TEXT-BOOK OF H I S T O L O G Y}
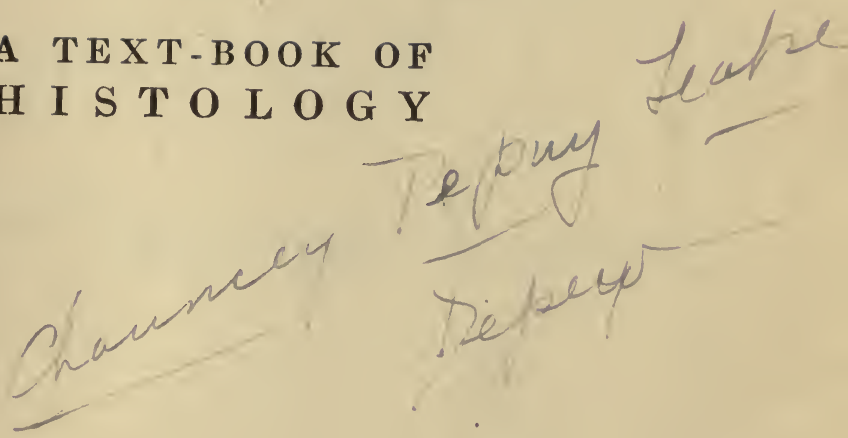

A TEXT-BOOK OF

H I S T O L O G Y

BY

HARVEY ERNEST JORDAN, A.M., Рh.D.

PROFESSOR OF HISTOLOGY AND EMBRYOLOGY, UNIVERSITY OF VIRGINIA

WITH FIVE HUNDRED AND NINETY-FOUR ILLUSTRATIONS IN THE TEXT, AND FOUR PLATES

NEW YORK AND LONDON D. A P PLETON A D COMPAN $\begin{array}{llll}1 & 9 & 2 & 0\end{array}$ 
COPYRIGHT, 1916, 1917, 1920, BY

D. APPLETON AND COMPANY 


\section{PREFACE}

In the preparation of this text-book of Histology we have kept foremost in mind the needs of medical students as we have come to understand them in our experience as teachers of the subject. These needs have been suggested by the common difficulties we have discovered on the part of the average student. It has been our effort to lessen these difficulties in the most practical way. Our experience has demonstrated to us the efficacy of the methods here pursued.

The bulk of the subject matter of Histology is now relatively stable. With respect to this nucleus our problem has largely been one of the best means of presentation. The secret of success in the acquisition of bistologic data is of course fundamentally an interest in the subject. Interest can best be stimulated by a revelation of relationships to future more clearly conceived practical ends; and it can best be sustained by the possession of principles and generalizations that serve at least provisionally to coördinate the mass of seemingly unrelated facts. These considerations force an approach to the subject of Histology largely from the viewpoint of function. The known or believed function dependent upon the structure described is therefore briefly indicated whenever this seems desirable. With the same end in view comparative anatomic and embryologic facts are frequently presented.

Since Embryology usually constitutes a separate course of the medical curriculum, the development of tissues and organs is discussed only to the extent deemed absolutely essential for a proper appreciation of structure. Since the anatomy of the Nervous System likewise properly constitutes a separate course, only the nervous tissues are here described, including the microscopic structure of the spinal cord, the cerebral cortex and the cerebellar cortex. A description of the structure of the brain stem is not included. An effort has been made to include the results of the latest investigations especially as regards cytologic data, particularly in relation to the genital glands. The organs of internal secretion are treated under a single head, and rather more fully, in view of their now evident importance, than has hitherto been the case.

The illustrations have been taken from various sources, duly cred- 
ited-including more than 300 originals-our one object having been to best elucidate the structures described. Photomicrographs of actual sections, combined with interpretive drawings and diagrams, appear to us the ideal illustrative procedure.

The majority of the photomicrographs are taken from Ferguson's "Normal Histology and Microscopical Anatomy." For a number we are indebted to our friend and colleague, Professor Albert H. Tuttle of the University of Virginia.

An attempt is made to adapt the book somewhat to prevailing nonuniform demands, by putting the more essential and what we regard as additionally desirable in different type.

References to the recent literature are inserted for the student who may wish to consult the more important original works upon which the later developments of Histology have advanced.

We gratefully acknowledge our indebtedness for illustrations and data taken from the recent and earlier literature, and our obligations to the publishers for their kindly help and courtesy.

Harvey ERnest Jordan Jeremiah S. Ferguson. 


\section{PREFACE TO THE SECOND EDITION}

IN the preparation of the second edition I have been guided by suggestions and criticisms made by teachers who have used the first edition. There appears to be very general agreement that the plan and scope of the book meet the desires of teachers and students of Histology in Medical Schools. The chief point of disagreement among teachers concerns the teaching value of the photomicrographs. I have tried to compromise the conflict of opinion on this point by substituting drawings for the less satisfactory photographs of the first edition. A number of new illustrations have also been added. To meet the demand for a laboratory guide, in general conformity with the text, a chapter has been added on "Directions for Laboratory Work."

In the first edition I used freely of the illustrations and certain portions of the text in Ferguson's "Normal. Histology and Microscopical Anatomy." My indebtedness for this material was recognized by publication under joint authorship. The present revision has made the work more exclusively my own, and I now assume sole responsibility for the book as it stands. Certain portions, however, still remain in essentials as originally published in Ferguson's excellent book, and I am under deep obligation to my friend and former colleague for this aid to my efforts to produce an acceptable textbook of Histology.

To all those who have in any way helped, especially by constructive criticism and by the loan of drawings for new illustrations, to improve the book in a second edition, I desire to express my gratitude. I am also again greatly indebted to my publishers for very generous cooperation in the work of revising the text and of changing many illustrations.

LABORATORY OF Histology and EMbRYology,

HARTEY ERNEST JoRdan. UNIVERSity OF VIRGINIA. 



\section{CONTENTS}

CHAPTER

I.-INTRODUCTION-PROTOPLASM-CELL _ . . . . . 1

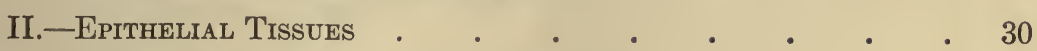

III.-Connective Tissue-Cartilage-Bone $\quad$ • $\quad$ • 49

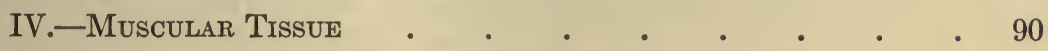

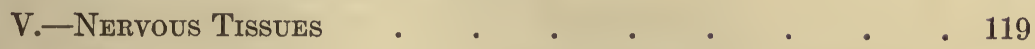

VI.-Peripheral Nerve Terminations-End Organs . 159

VII.-The Blood Vascular System • . . . . . . 176

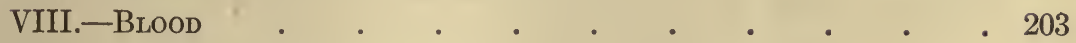

IX.-The Lymphatic SYstem • • . • • • . 225

X.-Mucous Membranes-Glands . . • • . 251

XI.-The Skin . • • • • • • • • • 262

XII.-The Respiratory System . . • • • . . 292

XIII.-The Digestive System • • • • • • . 320

XIV.-The Urinary System _ . • • • • . . 423

XV.-The Reproductive System . . . • . . . 455

XVI.-The Ductless Glands: Endocrin Glands • • • . 548

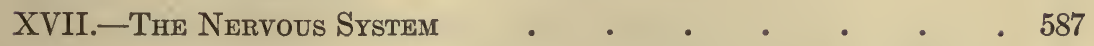

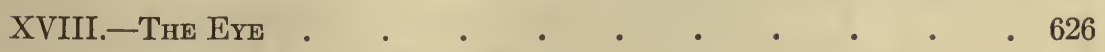

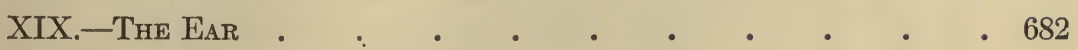

XX.-Histologic Technic . . 



\section{LIST OF ILLUSTRATIONS}

FIGURE

PAGE

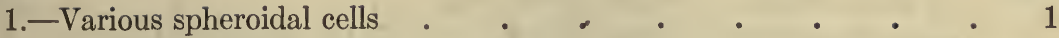

2.-Ameba proteus in motion . . . . . . . . 3

3.-Paramecium caudatum . . . . . . . . . 4

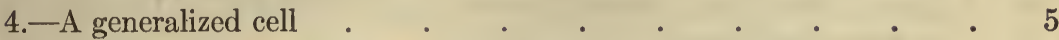

5.-A unicellular flaggellate animal (Tetramitus chịlomonas) . . 6

6.-Egg of a clam (Cumingia tellinoides) $\quad$. $\quad . \quad$. $\quad$. $\quad$. 7

7.-Primary spermatocyte of a turtle (Cistudo carolina) . $\quad$. 8

8.-Spermatid of opossum in early stage of metamorphosis into a spermium .

9.-Cells from the newly-hatched rainbow trout, treated according to Meves' technic for the demonstration of mitochondria (plastosomes)

10. - Two cells from the mesenchyma of the newly-hatched rainbow trout

11. - Intracellular network, or 'trophospongium' within a Purkinje cell of the cerebellum of Strix flammea

12.-Diagram illustrating the various theories of protoplasmic structure

13. - Egg of the blood starfish (Henricia sanguinolenta) in later growth period

14.-Pancreas cell of turtle, filled with zymogenic granules . .

15. - Motor nerve cell from the ventral horn of the spinal cord of the ox

16. - Interstitial cell from the testis of a twenty-one year old man. Showing granular and filamentous mitochondria

17.-A neuron (giant pyramidal cell, or cell of Betz) from the cerebral cortex of man, showing the neurofibrils . . . . . 13

18.-Developing fat cells

19.-Columnar ciliated epithelial cells. Showing canalicular apparatus

20.- Successive stages in the movement of an ameba

21. - A leukocyte from human blood in active ameboid motion . $\quad 16$

22. - Three cells from the epididymis of the rabbit _ . . $\quad$ • 16

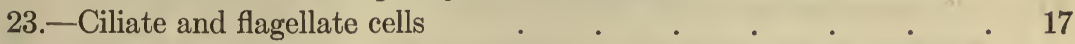

24.- Successive steps in amitotic division in tendon cell of new-born mouse

25.-Successive stages in the amitotic division of the ciliated cells lining the vasa efferentia of the epididymis of the mouse . 
FIGURE

26. - Multinucleated giant cell, from the yolk-sac of a $10 \mathrm{~mm}$. pig embryo

27.-Spermatocyte of Pyeris crategi, a butterfly, showing a cilium attached to the centrosome

28. -Diagrams illustrating successive stages of mitosis _ . $\quad$ - 21

29.-Cells from epidermis of the salamander . . . . . 22

30.-Successive stages of mitosis in the root tip of the dogtooth violet (Erythronium americanum)

31.-Successive stages in the maturation, fertilization and segmentation of the star-fish (Asterias forbesii) egg

32.-Transverse section of a frog embryo, showing the three germ layers

33.-Group of epithelial cells from the Malpighian layer of the skin

34. - A villus of the human placenta, showing a peripheral syncytium of irregular thickness

35.-Cells from the pancreas of Necturus, containing secretory granules and basal ergastoplasmic filaments

36.-Various forms of cells

37.-Polyhedral epithelium, from a section of the human liver .

38.-Goblet and columnar cells from the large intestine of the cat

39.-Columnar epithelium from the pyloric region of the human stomach

40.- 'Terminal bars' of cement substance as seen between the epithelial cells of a tubular secreting gland in the pyloric region of the human stomach

41. - Semidiagrammatic illustration of endothelium lining a large artery

44.-Tip of a villus of the synovial membrane from the knee-joint of an old man

45. - Columnar ciliated epithelium from the epididymis of a rabbit

46. - A group of cells from a transection of an acinus of the human pancreas; glandular epithelium

47. - Goblet cells as seen in a transection of a crypt of the large intestine in man

48.-Diagram showing the arrangement of the columnar and goblet cells of figure 47

49.-Stratified epithelium from the human esophagus

50.-Epidermis of the skin of the finger tip, showing extreme keratization of the epithelium .

51.-Pseudostratified columnar ciliated epithelium from a bronchial tube of man

52.-Diagram showing the manner in which all the epithelial cells of pseudostratified ciliated epithelium reach the basement membrane.

53.- Transitional epithelium from a transection of the ureter of an infant 
54.-Isolated cells -which may appear in human urine

55.-Embryonal connective tissue, early stage

56.-Embryonal connective tissue at a later stage than is represented in figure 55

57.-Subcutaneous areolar connective tissue of guinea pig _ _ _ 50

58. - Plasma cells of connective tissue from the human breast _. . 51

59.- Spindle-shaped connective tissue cells from the stroma of the human ovary .

60.-Pigmented cells from the choroid coat of the ox's eye .

61.-Granule cells from the fibrous connective tissue of the human mammary gland .

62.-Gelatinous connective tissue from the umbilical cord of a new-born infant .

63.-Reticulum of a cervical lymph node of man, from a thin section from which the lymphatic corpuscles had been partially washed out

64.-Dense fibrous tissue from the tendon of one of the ocular muscles of a child

65.-Longitudinal section of tendon of human finger .

66.-Portion of tendon from a cow

67.- Transverse section of portion of tendon of human finger

68.-Piece of tendon from tail of white mouse

69.-Isolated tendon cells

70.-Coarse elastic fibers from the ligamentum nuchae of the ox

71.-Transection of a fasciculus of the ligamentum nuchae of the ox, showing the very large elastic fibers embedded in a very delicate network of collagenous fibers .

72.- Portion of ligamentum nuchae of ox .

73.- Portion of a fat lobule from the areolar connective tissue surrounding the esophagus of a cat

74.-A group of fat cells from the subcutaneous tissue of a young rabbit

75.-Fat cells from a teased preparation of adipose tissue of man

76.--Adipose tissue

77. - Developing adipose tissue from the subcutaneous tissue of an infant

78. - Reticulum from the mucosa of the fundus region of the dog's stomach

79.- Section through a small lymph gland of a dog

80.-From a section through the medulla of a cervical lymph node of man

81.-Transection of a plate of hyaline cartilage, from the trachea of a child

82.-Cells and matrix of hyaline cartilage from the wall of a large bronchus of man

83.- Elastic cartilage from the human epiglottis, showing the large ovoid cartilage cells and the very delicate reticulum of elastic fibers 
FIGURE

85.- Notochordal tissue

86.-Transection through the compact bony wall of a human metacarpal bone

87.-Longitudinal section of ground bone from the shaft of the human femur .

88.- - Isolated bone cell shrunk away from wall of its lacuna

89.-An Haversian system, including the central canal, several lamellae, lacunae and canaliculi .

90.-Transverse section of Haversian canal, with contents .

91.-The primary changes in intracartilaginous bone formation

92.-A longitudinal section of the two distal phalanges from the finger of a five-months' human fetus

93.-Reconstruction of cartilage into bone

94. - Trabecula of primary enchondral bone, showing a central deepstaining core of calcified cartilage and a peripheral layer of osteoblasts .

95.-Trabecula of primary bone from the finger of a human fetus

96.-Intramembranous bone formation in the lower jaw of a sheep fetus

97. - Smooth muscle cells

98. - Smooth muscle cells from the pig's stomach

99.- Smooth muscle cells from the wall of the human intestine

100. - Smooth muscle cells from the wall of the human intestine

101.-Two stages in the histogenesis of smooth muscle, from the wall of the esophagus of a pig embryo

102.-A group of myoblasts from the heart muscle syncytium of a 48-hour chick embryo

103.-Cardiac muscle of guinea-pig, showing several branches, cross striations (ground membranes) and a number of intercalated disks

104.-Cardiac muscle cells from the pig's heart, isolated in equal parts of alcohol, glycerin, and water

105.-Cardiac muscle of the human heart; the abundant branches are plainly shown

106.-The central portion of the preceding figure, more highly magnified .

107.- Transection of a group of cardiac muscle fibers from a papillary muscle of the human heart

108.-Developing muscle fibers from the heart of a human fetus at seven months

109.-Cardiac muscle fibers

110. - Diagram of a striped muscle fiber, according to Heidenhain

111.-Six-lobed nucleus from the heart muscle of Limulus, showing the continuity of the nuciear wall with the telophragmata

112.-Seven nuclei, the product of amitotic division, lying in an undifferentiated mass of sarcoplasm, from the heart of Limulus 
113.-Longitudinal section of a trabecula of Limulus (King crab) heart muscle, showing an intercalated disk separating a contracted from an uncontracted portion

114.- Semidiagrammatic illustrations of various types of intercalated disks 104

115. - Successive stages of skeletal muscle histogenesis in mammals 105

116. - Transverse section of a striped muscle fiber of a newly-hatched rainbow trout, showing the process of myofibril increase by radial longitudinal splitting

117.- Striated muscle fibers ruptured by teasing, showing the sarcolemma 118. - Isolated fragments of striated muscle fibers, unstained . . 119. Striated muscle fibers of the dog, seen in transection . . . 107 120.-A portion of a striated muscle fiber seen in longitudinal section . 108 121. - A small portion of a muscle fiber of a crab showing beginning separation into fibrils

122.-Fibrils from the wing muscles of a wasp

123.-Longitudinal section of a portion of a striped muscle trabecula of Limulus, showing a nucleus of serrated contour with the telophragmata attached to the serrations

124. - Striated fiber from a leg muscle of the sea spider (Anoplodactylus lentus), showing the complexly striped condition characteristic of insect muscle

125.-Semidiagrammatic drawing, representing the appearance of the same fiber from the leg muscle of a beetle in ordinary and polarized light

126. - Lateral contractive wave of Cassida equestris

127. - Striated muscle fibers of the dog

128. - Striated muscle of a cat seen in transection

129. - Motor end-plate on an intercostal muscle fiber of a young rabbit

130.-Portion of a transection of a large tendon .

131.- Transverse section of tendon of tail of adult mouse . . .

132. - Portion of a muscle fiber from the tail of a $5 \mathrm{~cm}$. frog tadpole .

133.-Diagram of a neuron

134.-A unipolar ganglion cell of a frog

135.-Multipolar ganglion cell from the ventral horn of the gray matter of the spinal cord of the ox

136.-Pyramidal multipolar nerve cell from the cerebral cortex of a mouse

137. - Isolated nerve cells from the spinal cord of man

138.-Various types of nerve cells of the cerebellar cortex

139.-Three types of nerve cells supplying respectively cardiac (1), smooth (2) and (3) striated muscle

140. - A nerve cell from the trapezoid nucleus in the midbrain of a rabbit

141.-A neuron (giant pyramidal cell, or cell of Betz) from the cerebral cortex of man, showing the neurofibrils 
142.-Intracellular network (trophospongium) within a Purkinje cell of the cerebellum of Strix flammea

143.-Golgi cell, type I

144.-Golgi nerve cell, type II . . . . . . . . . . 130

145.-Isolated nerve fibers from a frog . . . . . . . . 133

146.-A small portion of a transection of the sciatic nerve of a dog . . 134

147. - A group of large medullated fibers from a nerve in the peritracheal areolar tissue of the cat

148. - Nerve fibers

149.-Cross and longitudinal sections of the same funiculus of nonmedullated nerve fibers (turned up at the left), showing the perineurium and the relationship of the neurolemma nuclei to the axis cylinder bundles of neurofibrils

150.-Cross-section of the trunk of the human vagus nerve, some distance below the nodose ganglion, showing medullated and non-medullated fibers .

151.-Successive stages in the degeneration process exhibited by the distal stump of a medullated axon (from sciatic nerve of adult dog) following section

152.-Regenerative stages in the proximal stump of the cut sciatic nerve of the dog, several millimeters above the level of section

153.-Transection of the spinal cord of an embryo chick

154. - Transection of the spinal cord of a child, fifth lumbar segment

155.-Portion of gray substance from the anterior horn of the spinal cord of man, showing nerve cell bodies, dendrons, medullated and nonmedullated portions of axons, and neuroglia

156.-Transverse section through the white substance of the human spinal cord

157.-Neuroglia from the spinal cord of a fetal pig

158.-A long-rayed astrocyte

143

144

159.-A short-rayed astrocyte, or mossy cell

144

160. -Neuroglia cell with adjacent fibers, from the pineal body of a yearling sheep

161.-Neuroglia cells and fibers from the spinal cord of an elephant

162.- Transection of the sciatic nerve of a dog

163.-Diagram of the origin and relations of the peripheral motor and sensory neurons

164.-Bipolar cell from a spinal ganglion of a fish

165.-Transformation of bipolar cells into unipolar cells in the Gasserian ganglion of the pig

166.-Section through the dorsal root ganglion of the first thoracic nerve - of a cat

167.-A nerve cell from a section of a human Gasserian ganglion 
168. - Schematic representation of the relations of the structures composing a spinal ganglion

169.-Common atypical, though probably perfectly normal, nerve cells from the spinal ganglion of the dog

170.-Sympathetic neurons

171.- The sprouting of an axon by a neuroblast from the spinal cord of a frog embryo

172.-The sprouting of an axon by a neuroblast from the spinal cord of a frog embryo

173.-Nerve endings in the epithelium of the larynx . . . . 160

174.-Tactile cells in the epithelium of the groin of a guinea-pig . $\quad 160$

175. - Schematic representation of a taste bud . . . . . . 161

176.-Taste bud from the human tongue . . . . . . . 162

177.- Tactile corpuscle of Meissner from the skin of the human toe $\quad 163$

178.-Tactile corpuscle of Meissner . . . . . . . 164

179.-Tactile corpuscle of Meissner . . . . . . . . . 164

180.-Ruffini's end organ . . . . . . . . . . . . 165

181.-End bulb of Krause from the margin of the ocular conjunctiva $\quad .165$

182.-Genital corpuscles from the clitoris of a rabbit . . . . 166

183. - A lamellar corpuscle from the mesentery of a cat _ . . . 166

184.-A lamellar corpuscle from the pleura of a child . . . . 167

185.-Lamellar corpuscle from the mesentery of a kitten _ . . 167

186.-A lamellar corpuscle in longitudinal section, showing a network of spiral elastic fibers . $\quad . \quad$. . . . . . 168

187.-Axial section of a corpuscle of Herbst from a duck's tongue . 168

188.-A papilla of the duck's tongue, containing a corpuscle of Grandry . 169

189.-Golgi-Mazzoni corpuscles from the subcutaneous tissue of the tip of the finger

190.-Motor nerve endings in striated muscle . . . . . . 171

191.-A muscle spindle from the psoas magnus of man $\quad$. $\quad . \quad 172$

192.-Middle third of a terminal plaque in the muscle spindle of an adult cat 173

193.-Neurotendinous end organ or tendon spindle of Golgi . _ . 174

194.-Nerve endings in cardiac muscle, from the heart of a cat . $\quad 175$

195,--Nerve endings in smooth muscle, from the intestine of a cat . $\quad 175$

196:-A small artery from the connective tissue of the anterior cervical region of man

197. - The external carotid artery of a child

198.-Transection of the wall of the aorta of a child $\quad . \quad+\quad . \quad 179$

199.-Part of a cross-section of the femoral artery of a dog . . $\quad 180$

200.-Transection of the celiac axis of man . . . . 181

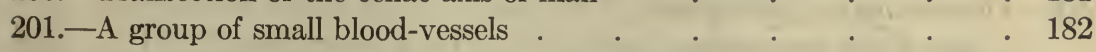

202.-Semi-diagrammatic illustration of small branch of pulmonary artery of ox 
FIGURE

203.-Semi-diagrammatic illustration of dividing small branch of pulmonary artery of guinea-pig .

204.-The capillary network connecting an arteriole and venule of the omentum of a young rabbit . . . . . . . . 185

205.-Capillary vessel of the frog's mesentery _ . . . . . 185

206.-Two sinusoidal vessels from the medulla of the human adrenal $\quad 186$

207.-Precapillary venule and arteriole _ . . . . . . . 187

208. - Transection of an arteriole and venule . . . . . . . 188

209.-Transection of the wall of the human vena cava $\quad$. $\quad$. $\quad$. 189

210.-A $13 \mathrm{~mm}$. human embryo . . . . . . . 192

211. - 'Vasoformative' cells from the mesentery of a rabbit seven days old 193

212.-The parietal layer of the pericardium of a child . _ . . . 196

213.-The endocardium . . . . . . . . . . 197

214.-Radial sections of the mitral valve, from the heart of a man . $\quad$. 198

215.-Human heart, opened from the right to show the atrioventricular bundle of His

216.-Reconstruction of the sinoventricular system (bundle of His) of the calf's heart

217. - Oxalated plasma of human blood clotted with thrombin, showing fibrin needles

218.-From a freshly prepared, unstained specimen of human blood . 205

219.-Blood cells from a specimen of freshly drawn unstained human blood 206

220.- Showing the action of water upon the red blood corpuscle . 206

221. - Five nucleated red cells (erythrocytes) from the blood of a frog . 207

222. - Three nucleated red blood cells (erythrocytes) from the marrow of a human rib . . . . . . . . 207

223.-A group of cells from normal human blood _ . . . 208

224.-A group of blood platelets, from the human blood . . . 209

225.-Outline drawings of living polymorphonuclear leukocytes of rabbit, from a drop of blood mixed with Ringer's solution to which a small amount of hirudin had been added to prevent coagulation .

226. Section of bone marrow from skull of $25 \mathrm{~mm}$. turtle embryo (Chelydra serpentina), showing three main stages in the hemopoiesis

227.- - Hemoglobin crystals

228. - Crystals of chlorid of hematin or hemin . . . . . . 213

229. -Wall of yolk sac of a $13 \mathrm{~mm}$. human embryo (Fig. 210), showing a small blood island and several small blood vessels containing erythrocytes . . . . . . . . . 215

230.-Large blood island from yolk sac of a $13 \mathrm{~mm}$. human embryo . 216

231. - Diagrammatic illustrations of successive stages in the transformation of the mammalian erythrocyte to form the erythroplastid

232.-Successive stages in the elimination of the erythroblast nucleus, from homoplastic cultures of blood of a $32 \mathrm{~mm}$. pig embryo 
233. - From a section of red marrow of a human bone .

234.-Types of cells from a smear preparation of the marrow of a human rib

235.- Subcutaneous lymphatic vessel of a fetal pig

236. - The growing end of a developing lymphatic vessel in the subcutaneous tissue of a fetal pig

237. - Lymphatic and blood vessels in the hilum of a human lymph node .

238.-Lymphatic capillary from the spermatic cord of a dog, showing nerve endings

239. - Transection of the pericardium of a child

240.-Section of a vascular synovial villus from the knee joint of a child

241.-A lymph nodule, solitary follicle, from the large intestine of man

242.-Diagrammatic illustration of a lymph node

243.-Transection of a cervical lymph node of a dog . . . . 237

244.- Transection of a mesenteric lymph node of a man . . . 237

245.-Diagram of the blood vessels of a lymph node . . . . 238

246.-Section of human hemplymph node ("splenolymph gland") . . 240

247. - Horizontal section through the faucial tonsil of a child . . . 241

248.-From a crypt of a dog's tonsil . . . . . . . 243

249.-The lingual tonsil of man . . . . . . . . 244

250.-Portion of spleen of cat, showing capsule (above and at left) and five splenic nodules

251.-Diagram of a lobule of the spleen . . . . . . . 247

252.-The origin of a vein in the splenic pulp . . . . . 248

253.-Types of cells from a smear preparation of the pulp of the human spleen

254.-Diagram of a mucous membrane having simple tubular glands $\quad$. 252

255.-Diagrams of the principal types of glands .

256.-Transection of three secreting tubules of the submaxillary gland of man

257.-Model of a reconstruction of the lacrimal gland of man

258.- -Reconstruction of a mucous gland from the respiratory region of the nasal mucosa of a child

259.-Reconstruction of an intralobular duct dividing into its terminal intercalary ducts and acini

260.- Epidermis of the foot

261.- Section of thin skin from abdomen of negro, showing the distribution of the pigment granules in dermal and epidermal cells

262.- Section of thin skin from abdomen of light brown mulatto .

263.-Skin from sole of human foot, showing spiral ducts of two sweat glands opening through the epidermis 
FIGURE

265.-Three early stages in the histogenesis of the skin

266. - From a section of the abdominal integument of an infant

267. - Several coils of a sudoriparous gland of the human finger

268.- Terminal phalanx of finger of human fetus

269.- Transection through the margin of a finger nail

270.-Longitudinal vertical section of the young nail and nail-bed of an infant

271.-Five stages in the development of a human hair

272.-From a section of the skin of an infant's arm, showing small immature hair follicles in transection

273.-From a section of the human scalp

274. - Transection of a hair near the middle of the root sheath

275.- -Regeneration of a hair

276. - Sebaceous glands in the scalp of a child

288

277. - Section of a sebaceous gland from the human scalp, through point of opening into a hair follicle (obliquely cut)

278.-Cells from the central portion of figure 277, showing two successive stages in sebum formation by process of fatty metamorphosis of the cytoplasm

279.-Reconstruction of the cutaneous blood vessels

280.--Photograph of Azoux model, showing nostril, pharynx, larynx and related structures

281.-From a section of the mucous membrane of the respiratory region of the human nose

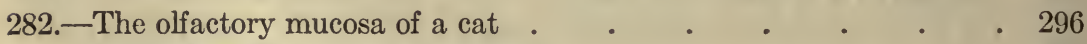

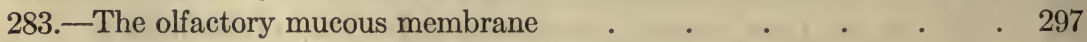

284.- -Vertical section of the olfactory mucosa of a kitten _ . . . 298

285.-Diagram of the relations of the neurons of the olfactory nerve and olfactory bulb

286.-A vertical section through the lateral wall of the human larynx . 301

287. - Transection of the wall of a child's trachea

302

288.-Mucus-secreting, tubulo-alveolar gland of the human tracheal mucosa

289.-A bronchus from the human lung

290.-Diagram of primary lobule of lung (lung unit)

303

291. - From a section of a child's lung

292.-From a section of a child's lung

293.-From a section of a child's lung

305

307

308

309

310

294.-Diagram of the three pulmonary lobules connected with a terminal bronchiole

295. - Two alveoli of a child's lung

296. - Transection of the pleura of an infant

297. - From a section of the pleura of man . 
299.-From the lung of a dog whose blood vessels had been injected with a gelatinous mass, and appear black

303.-Diagram of an axial ground section of tooth, showing the several stripes of the dentin and the enamel

304.-From a longitudinal section of the neck of a child's tooth and the adjacent alveolus

305.-From a section of a human tooth which had been ground to extreme thinness

306. - Section of fang parallel to the dentinal tubules, human canine

307.-Dentin from a ground section of a human molar, showing the dentinal tubules cut across

308.-Enamel prisms in transection

309. - A group of enamel prisms cut longitudinally, from the incisor tooth of the rat, showing their irregularly beaded character and the cross striations

310.-From a section of a human tooth which had been ground to extreme thinness

311.-Developing tooth from a human embryo $17 \mathrm{~mm}$. long _ . 331

312.-Dental anlages from a human fetus $40 \mathrm{~mm}$. long

313.-Two stages in the early development of the teeth, from a $25 \mathrm{~mm}$. pig embryo

314.-Developing tooth from a human fetus $30 \mathrm{~cm}$. long

318.-View of dorsum of tongue, showing the various papillæ, the tonsils and the fauces

319.- One lateral half of a coronal section of a dog's tongue .

321.-A filiform and a fungiform papilla, from an injected specimen of tongue of cat

323.- Two foliate papillæ from a rabbit's tongue, showing numerous taste buds along their lateral margins

325.-Surface view of Auerbach's intramuscular nerve plexus, from the esophagus of a cat

326.-Photomicrograph of a transverse section through upper third of cat's esophagus 
FIGURE

327. - Transverse section of human esophagus through lower third .

328.-From a section of the human esophagus

329.- Section through the stomach wall of man (pyloric region)

330.- The mucosa of the fundus region of the dog's stomach

331.-Longitudinal section of the fundus glands of man . . . .

332. - Transections of three glands of the fundus region of the human stomach

333.-A pyloric gland, from section of the dog's stomach . . . . 357

334.- - Portion of gastric gland from the fundus region of the stomach $\quad 357$

335.-Secretory capillaries of the fundus glands of the dog's stomach . 358

336.- The mucosa of the pyloric region of the human stomach _ . 359

337.-Blood vessels and lymphatics of stomach . . . . . . 361

338.-Termination of sympathetic nerve fibers . . . . . 362

339.-Schematic diagram illustrating probable relationship of sympathetic neurons in myenteric and submucous plexuses

340. - Section through the commencement of the duodenum at the pylorus

341.-From a longitudinal section through the duodenum of a cat

342.-The central portion of a Peyer's patch in the ileum of a dog's intestine

343.-Diagram of small intestine, showing the topographical relationship of the intestinal glands (crypts of Lieberkühn) to the villi .

344.- - Longitudinal section of villus

345.- Several villi from the small intestine of the $d$ og, in longitudinal section

346.-Reconstruction model of a Brunner's gland, from the human duodenum

347.- The blood-vessels of the small intestine of a dog, drawn after an injected preparation

348.- Intestinal mucosa of a frog during the absorption of fat . .

349.-Apex of an intestinal villus of a rabbit which had been fed with milk

350.-Section of large intestine of dog, showing intestinal glands (crypts of Lieberkühn) cut longitudinally .

351.- Section of portion of large intestine of $\mathrm{dog}$, showing the intestinal glands (crypts of Lieberkühn) cut across, their lining including columnar and goblet cells

352. - Transection of the vermiform appendix of man

353.-Semidiagrammatic representation of a small mucous gland from the oral mucosa of a rabbit .

354.-Corrosion model of an interlobular duct and its branches, from the human submaxillary gland

355.--Intercalary ducts and acini of the human submaxillary gland, corrosion model 
FIGURE

356.-A group of mucous acini, from the human submaxillary gland

PAGE

357.-From the sublingual gland of man

386

358.- Mucous acini of the retrolingual gland of the rat .

387

359.-Diagram of the arrangement of the cells in a mixed salivary gland

388

360.-Diagrams of A, parotid gland; B, submaxillary gland; C, sublingual gland; and $\mathrm{D}$, pancreas

389

361.-From a section of the human parotid gland

389

362.-From a section of the human submaxillary gland

390

363.-Reconstruction model of the sublingual gland of man . . .

364.- Nerve endings in a salivary gland

391

392

365.-Early stages in the development of the pancreas, illustrating conditions in the 5 and 7 weeks old human embryos

393

366.-From a section of the human pancreas, showing several lobules and the broad interlobular bands of connective tissue

367.-Drawing of an intercalary duct with three branches ending in acini to form centro-acinial cell groups . . . . . .

368.-Reconstruction model of the human pancreas

396

396

369.-Acini of the human pancreas

397

370.-Pancreatic acinus of cat cut transversely near fundus, showing the basal (prozymogen) filaments of the cells . . . .

371.-Cells from pancreas of Necturus

398

399

372.-Two adjacent acini from the guinea-pig's pancreas

399

373.- Section of an acinus from the guinea-pig's pancreas, showing the basal mitochondrial content and the central zymogen granules .

374.-Intercalary duct with branches, from pancreas of guinea-pig, showing highly branched tubules connected with the duct and with the islet

375.-Pancreatic islet

400

376.-From the human pancreas

377. - Section of a pancreatic islet from injected specimen of cat's pancreas to show the profuse blood supply

401

402

404

378. - A lobule of the pig's liver; the central vein lies in the middle of the figure .

379.-Diagram of liver lobules, the upper two cut transversely, the lower longitudinally

380.-From a section of the turtle's liver, showing the tubular arrangement of the parenchyma

381. - The reticulum of the dog's liver

408

382.- Stellate cells of von Kupfer in the liver of a dog

409

383.-A lobule of the pig's liver in longitudinal section, showing the relation of the central and sublobular veins and the arrangement of the hepatic cells

384.-A lobule of the human liver, seen in transection . $\quad . \quad \therefore \quad .411$ 
FIGURE

385.- Section of liver tissue showing the liver cell-cords, and the sinusoids lined with endothelium

386.-Diagram of four adjacent liver cells . . . . . . . . 413

387.-Bile capillaries of the hepatic lobule, from the liver of a cat . . 413

388. - Showing the connection between the intralobular and interlobular bile ducts in the cat's liver . . . . . . . 414

389. - Types of cells from a section of the normal human liver _ . 414

390.- Human liver cells, showing enlarged intracellular canaliculi, a condition characteristic of jaundice

391.-Diagram of a portal canal, including a branch of the portal vein, hepatic artery, hepatic (bile) duct, lymphatic and non-medullated nerve trunks

392.-From a section of the rabbit's liver whose blood vessels had been injected with a carmin stained gelatin mass; somewhat more than a single lobule is represented

393.-A sublobular vein of the pig's liver

394. - Intralobular nerve fibers in a rabbit's liver

395.-From a section through the wall of a dog's gall-bladder

396.-Reconstruction of the wall of a dog's gall-bladder

397.-Longitudinal section of kidney .

398. - Diagram of the structure of the kidney

399.-Reconstruction of a uriniferous tubule of an infant cortical ray in the lower left-hand corner

404.-From a longitudinal section of a convoluted tubule of the guineapig's kidney

405. - Cross section of a proximal convoluted tubule from the kidney of a mouse, showing basal filaments breaking up into granules centrally, and the central striated border of the cells

406.-A group of tubules from a transection of a renal pyramid of the human kidney; the section passes through the boundary zone . 407.-The distribution of the left renal artery . . . . . 408.-From the cortex of the human kidney . . . . . 442 409.- Nerve endings in a convoluted tubule of the frog's kidney _ . . 444 410.-Cast of the pelvis, infundibula and calices of the kidneys of a man . 445 411.-Transection of human ureter . . . . . . . . 446 412.-Transitional epithelium of dog's ureter $\quad$. . . . . . . 447 413.-Transection of the wall of a child's bladder _ . . . . 449 414. - The mucosa of a child's bladder in the contracted state of the organ 450 415. - Transitional epithelium of dog's bladder 
420.-Diagrams illustrating the metamorphoses of the indifferent urogenital system into the male and female systems

421.-Diagrams illustrating the process of maturation in the male and female gametes

422.-Primary spermatocyte of a grasshopper, Hippiscus tuberculatus, showing the compact accessory chromosome among the paler mossy prophase euchromosomes, and the idiosome . . . 423.-Chromosome groups of Schistocerca damnifica 424.-Diagram illustrating the behavior of the chromosomes during the first and second maturation divisions . . . . . 425.-The testicle with its system of efferent passages . . . . 426. - Seminal tubule of a man in transection

428.-Portion of a transection of a seminiferous tubule from the human testis, illustrating the various stages in spermatogenesis

429.-Successive stages in the metamorphosis of the spermatid into the spermatozoon cretions

443.-Prostatic genital corpuscles 
450.-From the ovarian cortex of an infant, showing many ova in the primary follicular stage

451.-Ovum, containing a yolk nucleus ('Dotterkern') at the left and above the nucleus .

452.-From a section of the ovarian cortex of a new-born kitten . $\quad$. 513

453.-A primary ovarian follicle of the human ovary . . . . . 514

454.-A vesicular (Graafian) follicle of the human ovary, somewhat more advanced than the preceding . . . . . . 515

455.-A nearly ripe Graafian follicle from the ovary of a dog _ . $\quad 516$

456.-Photomicrograph of a section of cat's ovary, showing two primary follicles and one vesicular

457. - Section through the peripheral portion of a corpus luteum, showing lutein cells.

458.- Portion of corpus luteum of rabbit

459. - A corpus albicans, from a section of the human ovary

521

522

460.-From a thick section of the ovary of a woman

523

461. - Transections of the human oviduct

524

462.-From a transection of the ampulla of the oviduct, showing the structure of the mucosa

463.- Transection of the uterus of an ape

527

464.- Transection through the body of the human uterus

529

465.-From a transection of the uterine mucosa

530

466.-From the cervix uteri of a girl of sixteen years, showing the cervical glands in section.

531

467.-A gland of the human cervix uteri in longitudinal section

468.-From a section of the human uterine mucosa at the first day of menstruation

469. - A group of decidual cells from the human uterus during the early stages of pregnancy

470.-Chorionic villi from the human placenta at full term . . . 536

471.-Chorionic villus at various stages of development _ . . . 537

472.-Vaginal mucosa . . . . . . . . . . 538

473.- Transection of a labium minus of an infant _ . . . 540

474.-From the actively secreting mammary gland of a woman _ . 542

475.- Model of a reconstruction of an intralobular duct and its acini from the active mammary gland of a woman

476.-Portions of several adjacent lobules, and a lactiferous duct, of the active mammary gland

477.-From a section of the human mammary gland in the resting condition 
481.- Reconstruction of a dog's adrenal

482.- - Section of part of an accessory suprarenal (chromophil body), newborn child

483.-From a section of the human thyroid gland

484.-Diagram of pharynx of human embryo showing the origins of the anlages of the thymus, thyroid and parathyroids (epithelial bodies) -560 485.-From the border of a mass of aberrant thyroid tissue of man, occurring in the region of the parathyroid glands . . . . 562 486.-Human parathyroid tissue, moderately magnified _ . . . 564 487.-A section through several lobules of the thymus of an infant . . 566 488.-A thymic corpuscle from the thymus of an infant _ . . 567

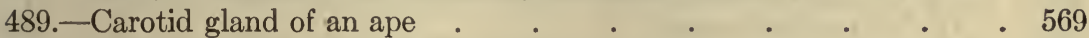
490.-From a section of the coccygeal gland of man _ . . . $\quad .570$ 491.-Median section through the anlages of the hypophysis cerebri of a 10 mm. cat embryo

492.- Sagittal view of a wax reconstruction of the hypophysis cerebri of the adult cat

493.- - Pars tuberalis, hypophysis of cat

494.- - Pars infundibularis, hypophysis of cat

495.- Pars distalis, hypophysis of cat

496.-Section of hypophysis cerebri of dog, showing portions of pars distalis of anterior or buccal lobe, residual lumen, pars tuberalis (pars intermedia), pars neuralis, and capsule

497.-Field from the center of a normal canine (puppy) pars anterior

498. - Semidiagrammatic representation of a median longitudinal section through the epiphysis of a $17 \mathrm{~cm}$. sheep fetus .

500.-Photomicrograph of a peripheral portion of the pineal body of a 21 cm. sheep fetus, showing several cysts and vascular trabeculæ, and an enormous number of intracellular melanic granules

501.-Photomicrograph of peripheral region of pineal body of a yearling sheep, to show the character of the parenchyma, the neuroglia cells and fibers, and the interneuroglia cells

502.-Two neuroglia and three interneuroglia cells from the pineal body of a lamb

504. Section of pineal body of an old sheep, showing 'brain sand' (acervulus) in the parenchyma

505.-Human embryo 2 millimeters long

506.-Transection through the Graf Spee embryo shown in figure 505 . (neural) groove to form the medullary (neural) canal and neural (ganglionic) crests 
FIGURE

508.-Cell lining the neural canal of the newly-hatched rainbow trout, showing mitochondria in an embryonic nerve cell $\quad$. $\quad$. $\quad 589$

509.-Section through medullary plate of a rabbit embryo _ . . . 589

510.-Section through medullary plate of closing neural groove of rabbit embryo

511.-Section through wall of later neural tube of rabbit embryo, showing a stage in the differentiation of the ependyma cells and the formation of a myelospongium

512.- Section of wall of forebrain of four-day chick embryo

513.-Diagram of a transection of the spinal cord of an early embryo, showing the migration of neuroblasts toward the marginal veil, and the ventral nerve root

514. - Transection of the spinal cord of a human embryo of four weeks 591

515.-Transection of the spinal cord of an embryo chick

516.-Reconstruction of the anterior portion of the body of a chick, the head distinctly differentiated, seen from the surface

593

517. - Transection of the spinal cord of a child, seventh cervical segment 593 518.-Diagram of the fiber paths of the spinal cord 595 519. - Transection of the spinal cord of a child, third sacral segment 518 520.- Transection of the spinal cord of a child, fifth lumbar segment 600 521. - Transection of the spinal cord of a child, eighth thoracic segment . 600 522.-Transection of the spinal cord of a child, fourth cervical segment 601 523.-Median sagittal section through the brain . 604 524.-From a section of the cerebellar cortex of man 605 525.-A Purkinje cell from the human cerebellar cortex 526. - A Purkinje cell from the cerebellar cortex of the rabbit 606 527.-Diagram of the cerebellar cortex 607 528. - Left lateral surface view of cerebral cortex in man, showing the lobes, main sulci, and the larger functional areas

529. - Large pyramidal cell of the cortex

530.- Scheme of the motor area of the cerebral cortex, showing the effect of various staining methods

531.-Disposition of the nerve fibers in the cerebral cortex of man

532.- Human cortex cerebri, motor area

533.-Human cortex cerebri, parietal lobe

534. - Human cortex cerebri, olfactory region

535. - Section of the spinal cord and its membranes, from the upper thoracic region .

536.-Dissection of eyelids and lacrimal apparatus

537. - Horizontal section of the right eyeball

538. - The anterior segment of a child's eye; meridional section 
541.-Corneal cells, isolated

542.-From a meridional section of the choroid coat . . . . 637

543. - The ciliary body and the adjacent structures; meridional sêction - 639

544.-The developing eye in meridional section; diagrammatic _ . 645

545.-Schematic reconstruction of the developing eye . _ . . 646

546.-The retina of a child's eye; meridional section _ . . _ . 647

547.--Pigmented epithelium of the retina, viewed in transection . . 647

548.- - Isolated rod and cone visual cells of the pig

648

549.-Diagram of the rod and cone visual cells, and their respective bipolar neurons

550.-A rod and cone visual cell from the fundus of the human retina, outside the macula lutea

551 - Two cones from the human retina . . . . . . 650

552.-From the human retina . . . . . . . . 65

553.-Diagrams of the human retina, showing the relationships to each other of the retinal neurons, and their disposition in the different layers

554.-From a meridional section of a child's eye, showing the layers of the retina at a point midway between the macula lutea and the ora serrata

555.-Horizontal cell from the retina of a calf

556.- Two amacrine cells from a transection of the retina of a calf

557.-A nerve cell of the large ganglion cell layer; from the retina of a cat .

558.-A fiber cell of Müller, or sustentacular cell, from the dog's retina .

560.-Developing rod and cone visual cells, from the retina of a $345 \mathrm{~mm}$. (6 mos.) human fetus

561.-Two early stages in the development of the rod and cone visual cells in the chick

562.-Diagram illustrating Balfour's theory to account for the inversion of the visual cells of the vertebrate retina

563.-Entrance of the optic nerve

565. - The nuclear zone at the margin of the crystalline lens of a child's eye, showing the transition of the lens epithelium to the lens fibers and the attachment of the suspensory ligament

566.- Schematic representation of the intrinsic blood vessels of the eye .

570.-Portions of two adjacent lobules of the lacrimal gland of the rabbit, showing two stages in secretory activity of the tubules 
572.-From the external acoustic meatus of man

573. - Transection of the tympanic membrane of a child $\quad$ • . $\quad$ • 687

574.- Section through the margin of the tympanic membrane of a child . 688

575.-The auditory ossicles _ . . . . . . . . . . 689

576.-The cavity of the tympanum, viewed from above $\quad . \quad$. 690

577.-Transection of the Eustachian tube; diagrammatic _ . . . 692

578.-The bony labyrinth . . . . . . . . 694

579.-Diagram of the membranous labyrinth in lateral view _ . 695

580.-Diagram of the right membranous labyrinth _ . . . 696

581. - The isolated membranous labyrinth . $\quad$. $\quad$. . . 696

582.-Transection of the margin of the macula acustica sacculi of a guineapig . . . . . . . . . 697

583.-Nerve endings in the macula acustica of a guinea-pig . . . 698

584.-Transection of a human semicircular canal . . . . 700

585.-Axial section through the cochlea of a fetal calf . . . . 701

586.-Axial section through a turn of the cochlea of a guinea-pig . $\quad 702$

587. - A radial section through Corti's organ in the first turn of the human cochlea . . . . . . . . 707

588.-Diagram of the organ of Corti . . . . . . . 711

589.-Axial section through Corti's organ of the guinea-pig, showing the terminal nerve fibrils . . . . . . . . 713

590. - Scheme of the vascular supply of the internal ear . . . 714

591. - Scheme of the vascular terminations in the wall of the cochlear canals

592.- Semidiagrammatic illustrations of successive stages in the development of the internal ear of the chick

593.-Wax reconstructions of three early stages in the development of the internal ear (membranous labyrinth) of man . . . . 718

594.-A method of preparing a paper box for paraffin embedding . $\quad$. 737

PLATES

A.- Successive stages in the spermatogenesis of Schistocerca damnifica .

B.-Successive stages in the spermatogenesis of Schistocerca damnifica (continued)

C.-Successive stages in the spermatogenesis of Schistocerca damnifica (continued)

D.-Successive stages in the growth, maturation, and fertilization of the egg of the starfish, Asterias forbesii . 


\section{A TEXT-BOOK OF \\ H I S T O L O G Y}





\title{
A TEXT-BOOK OF HISTOLOGY
}

\author{
CHAPTER I \\ INTRODUCTION-PROTOPLASM-CELL
}

\section{INTRODUCTION}

Definition of Histology.-Histology is the science of tissue structure, plant or animal. It concerns itself, therefore, chiefly with the structural characteristics and interrelationships of the component elements of tissues. These elements are the cells, and the material connecting or separating the cells, the intercellular substances. A tissue consists of cells associated in the performance of a specific function. A cell may be defined in a preliminary way as the unit of organic structure and function. The minuter details of histology involre also cell anatomy or cytology. Here we meet with the essential substance of the cells, the protoplasm, or bioplasm, the 'material basis of life.' We also meet with the chief 'organ' of cells, the nucleus. A completer definition of a cell may accordingly be given as a circumscribed mass of protoplasm containing a nucleus (Fig. 1). A complete histologic description embraces, therefore, details of the relationships of the component cells of a tissue, and of the protoplasmic structure and nuclear characteristics of the types of cells involved. Histology includes further the data of tissue origin and development, or histogenesis, and 1.

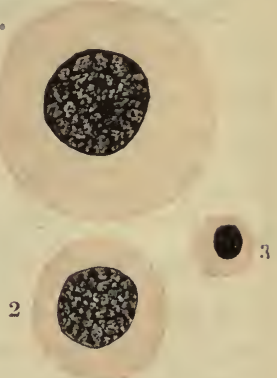

Fig. 1.-Various Spheroidal Cells.

1 , ovum from ovary of a child; 2 , spermatocyte; and 3 , spermatid, from the testicle of a rabbit. Hematein and $\operatorname{cosin} . \quad X$ 750 . of cell origin and development, or cytogenesis.

Cells are the 'building stones' of tissues; tissues combine to form organs; organs are associated into systems. Histology is accordingly a part of general anatomy; it is tissue anatomy; that part of histology which considers the relationships between tissues in organs is sometimes spoken of as microscopic anatomy. 
Historical Development of Histology.-Modern human histology had its origin in the work of Bichat (1771-1801). He did not employ the microscope; but his careful and extensive studies of the minute anatomy of tissues gave the impulse and general outline for later studies fy means of the microscope through which mammalian histology has grown to a relatively complete science. Great impetus was given also by the announcement of the 'cell theory' by Schleiden and Schwann in 1839, namely, the statement that all tissues are composed of structural units, or cells. Other epochal steps in histologic science were the recognition of the nucleus by Robert Brown in 1831, and of protoplasm by v. Mohl in 1846. Cytology arose almost as an incident to embryology. It traces its origin to the work of 0 . Hertwig on the fertilization of the sea urchin's. egg (1875). It is the infant anatomic science, its late development being due, largely, to its dependence upon the optical and mechanical refinements of the microscope. It deals with fundamental structures within the limits of visibility, and is destined to grow to vast proportions, as the already voluminous literature on 'mitochondria' ('plastosomes') in part foreshadows.

Relation of Histology to Other Biologic Sciences.-Histology aims to complete anatomic knowledge. It is thus the complement of gross anatomy. It furnishes also essential preliminary data for the understanding of pathology; abnormal structure and function become fully intelligible only in the light of normal histology. It is fundamental also to physiology, the science of normal function.

A certain function demands a specific structure; structure and function sustain reciprocal relationships. Normal function depends upon the normal structure of the cells involved in the function; abnormal function, or diseàse, is associated with altered cellular structure. Histology gains enormously in interest and value to the student who will always keep well in mind the function that a certain structure under consideration is called upon to perform. Embryology also to a considerable extent builds upon histologic and cytologic data.

\section{PROTOPLASM}

Chemical Constitution.-The unit of both structure and function is the cell. The essential constituent of cells is protoplasm. Protoplasm may be thought of as a physicochemical mechanism. Chemically, it is a very complex aqueous mixture of substances, containing the elements, 
carbon, oxygen, hydrogen, nitrogen, and small quantities of sulphur, phosphorus, calcium, sodium, chlorin, magnesium, potassium and iron.

The principal compounds of protoplasm are proteins, which furnish the main source of energy expended in function; carbohydrates; fats; and water, which constitutes about three-quarters of its weight. It is believed by one school of biologists (mechanists) that if we had the

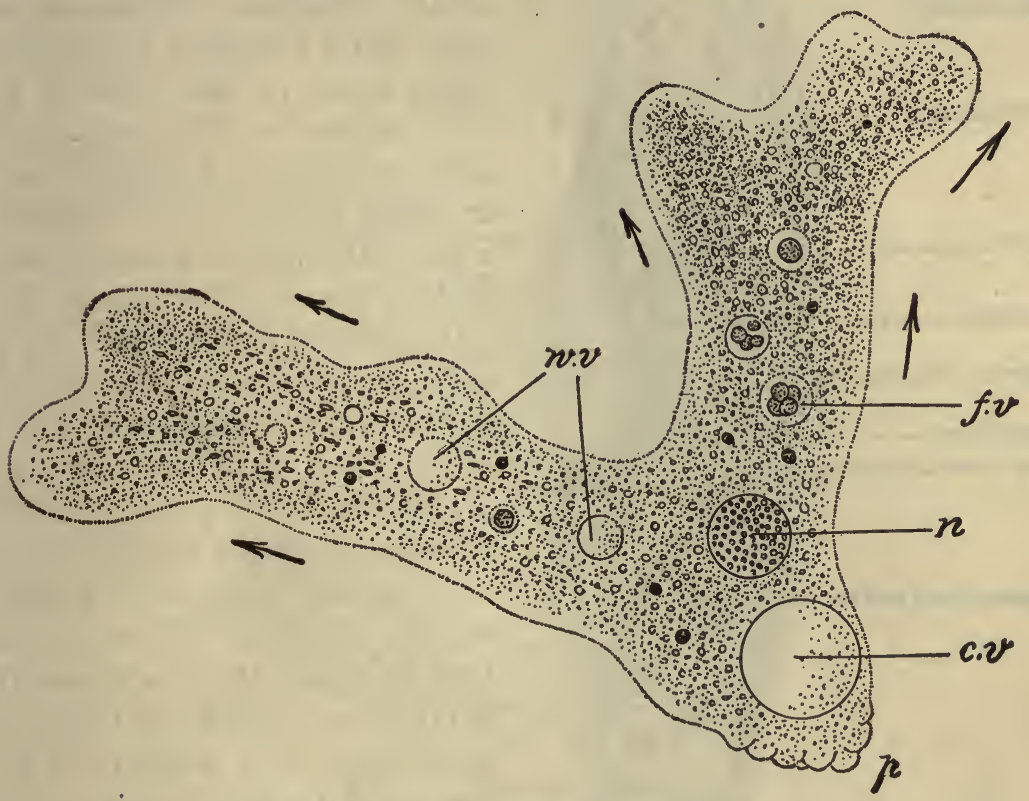

Fig. 2.-Ameba Proteus in Motion.

$c . v$., contractile vacuole; $f . v$., food vacuole; $n$., nucleus; $w . v$., water vacuoles. The arrows indicate the direction of the protoplasmic flow. Note the peripheral nongranular ectoplasm, and the granular endoplasm. (From Calkins' "Biology," H. Holt \& Co., after Sedgwick and Wilson.)

formula for the proper stereo-isomeric association of the elements and compounds of protoplasm, life could be artificially produced; another school of biologists (vitalists) assume an additional 'vital principle' as a prerequisite for life.

Physical Constitution.-Physically, protoplasm is a granular semifluid or gelatinous substance. It possesses properties characteristic of both solids and liquids. It is an aggregate of colloids and crystalloids. The physicochemical laws which govern the crystalloids and colloids underlie the properties of living matter. An organism is essentially 
an aqueous solution, holding in suspension colloidal substances of great complexity. Crystalloids are divisible into two groups: electrolytes and

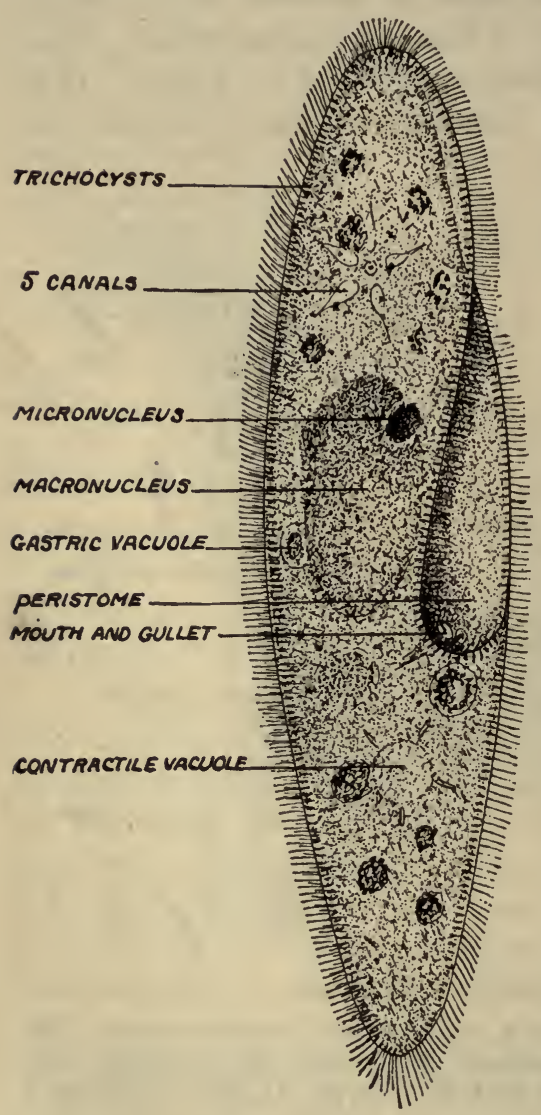

Fig. 3.-Paramecium caudatum.

Note the peripheral cilia and the granulo-alveolar character of the protoplasm. (From Calkins' "Biology," H. Holt \& Co.) non-electrolytes. The one (salts, acids, bases) in solution conduct the electric current, the others (urea, sugar) do not. Colloids exist in two states, a liquid or sol state, and a semi-solid or gel state. There exists no sharp line of division between colloids and crystalloids; these terms designate phases or states rather than substances; between them lie all kinds of intermediate grades. Protoplasm is a sol; and since its fluidity is due to water, it is commonly classed as a hydrosol. It passes readily into a gel condition, thus becoming a $h y$ drogel. In living protoplasm this metamorphosis is a reversible process. Agents which effect an irreversible gelation of protoplasm tend to bring life to a standstill. Fixation, or killing, of tissue for microscopic study consists in a separation of the more solid part of colloidal protoplasm from a more liquid part. Death is histologically such a process of coagulation. Living protoplasm may be studied to good advantage in the one-cell animal forms, Ameba (Fig. 2) or Paramecium (Fig. 3). These and other equally favorable protozoan forms are readily available from hay infusion cultures, and can be profitably employed for the demonstration also of the simpler modes of protoplasmic activity, and of the changes suffered by protoplasm in passing from the living to the dead condition. Since protoplasm is commonly organized into cells, the next step demands a knowledge of a typical or generalized cell. 


\section{THE CELL}

General Statements.-A generalized cell is of spheroidal shape (unmodified by pressure) and contains certain 'organs' and a variety of fundamental and secondary elements (Fig. 4). A cell (or protoplast; Hanstein) is a mass of protoplasm endowed with vital properties. The confines of such a cellular mass of protoplasm exist in a cell membrane.

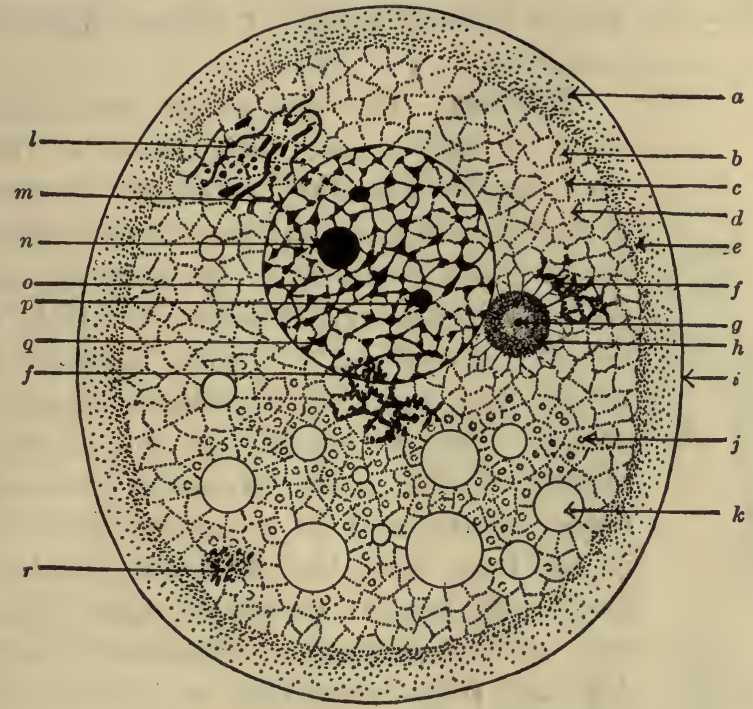

Fig. 4.-A Generalized Cell.

$a$, exoplasm; $b$, endoplasm; $c$, spongioplasm; $d$, hyaloplasm; $e$, microsomes; $f$, chromidia; $g$, centrosome (centriole); $h$, centrosphere, surrounded by astrosphere; $i$, cell membrane; $j$, deutoplasmic granule; $k$, fluid vacuole, or oil drop; $l$, mitochondria or plastosomes; $m$, nuclear membrane; $n$, nucleolus; $o$, linin; $p$, karyosome; $q$, chromatin (net knot); $r$, foreign inclusions, pigment, etc. (metaplasm).

This represents a differentiation product of protoplasm; when robust as in plant cells, it forms a cell wall. In certain cells, e.g., white bloodcells, it is apparently lacking; however, in these so-called naked cells the peripheral layer of protoplasm is more condensed and most probably subserves the osmotic function of a distinct membrane. In fact, the surfaces of protoplasm possess the properties of semipermeable membranes, probably lipoid in nature. An essential organ of the cell is the nucleus. It is trophic in function, the center of oxidation processes. In certain protozoa this is represented by scattered nuclear materials 
or granules (Fig. 5). The shape of the nucleus is spherical; typically it has a central location, but it frequently assumes eccentric positions. It is physically denser and more elastic than the extranuclear protoplasm. Its periphery simulates, or perhaps consists of, a membrane, the nuclear wall. Whether as a membrane it be complete or reticulated, whether of nuclear, cytoplasmic or composite origin, are undecided points. Recent

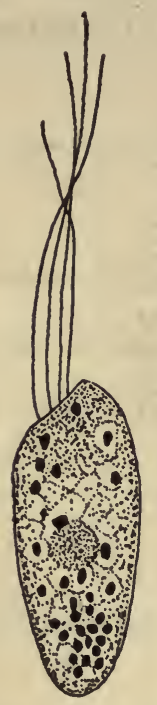

Fig. 5.-A UNIcellular Flagellate ANimal (TETRA MITUS CHILOMONAS).

Showing the nuclear material distributed as granules throughout the cell. (Redrawn from Calkins.) investigations on the nuclear membrane indicate that it is fenestrated; such conditions would permit of an easy escape of nuclear material into the cytoplasm.

Nucleus.-The protoplasm composing the nucleus is known as nucleoplasm or karyoplasm; that constituting the remainder of the cell, the cytoplasm. The nuclear constituents include a more fluid ground substance or nuclear sap (karyolymph: paralinin), throughout which extends a delicate reticulum of linin threads (Fig. 4). Upon these linin or achromatin threads are supported, more abundantly at the points of intersection of the mesh, granules (chromioles) and masses (net-kinots) of a substance staining deeply in the basic dyes, the chromatin. Spheroidal netknots are known as karyosomes. The linin is said to be achromatic. Whether it is chemically different from chromatin or simply more attenuated chromatin is disputed. The 'chromatic' granules themselves undergo changes in stainability: on the basis of reaction to acid and basic dyes, this substance is divided into oxychromatin (lantanin) and basichromatin. Linin and chromatin are regarded by some as different phases in the elaboration of the same substance. The nucleus includes, furthermore, usually one, frequently more, nucleoli. These do not grade into the nuclear sap, like the nuclear network, but are limited by a sharp line of demarcation. They may be achromatic, when they are known as plasmosomes, or they may take on chromatin, becoming chromatin nucleoli. It is uncertain whether the latter are identical in all cases with the karyosomes. The difference among nucleoli is more probably one of degree of abundance of chromatin. The nucleus is the metabolic organ of the cell; without a nucleus a cell may 
continue to live for a time, but it can neither grow nor undergo progressive differentiation. All changes in enucleated protoplasm are regressive, leading to death. The nucleus is also largely the reproductive center, as will be described below. The nucleolus plays the rôle, among other possible functions, of a center of storage, perhaps also elaboration, of chromatin. Nuclear protoplasm, more especially the chromatin, is relatively rich in phosphorus.

Astral System.-Another organ of a typical cell is the aster, astral system or attraction sphere. Its substance is collectively known as archoplasm. It usually lies outside of, but close to, the nucleus; in certain cells it is intranuclear, e.g., spermatocytes of Ascaris. It consists centrally of a granule, the centrosome; in this, in certain instances, may be differentiated centrally a smaller granule, the centriole; when the latter appears, the more outlying portion of the centrosome is designated the centroplasm. The centrosome may divide into two, becoming a diplosome, or in some instances it may become multiple, when it is known as a pluricorpuscular centrosome. Surrounding the centrosome is a clearer, minutely granular sphere, the centrosphere;

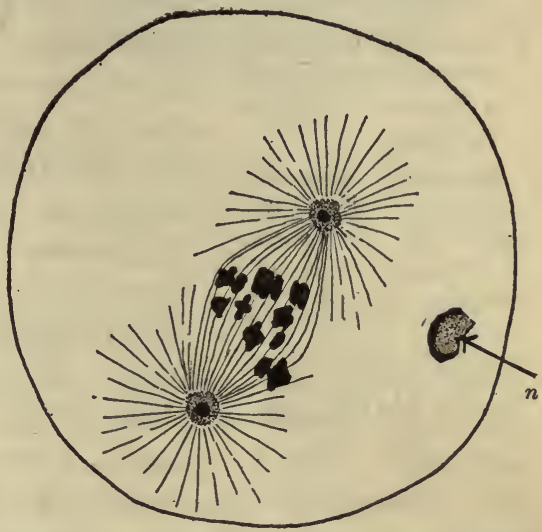

Fig. 6.-Egg of a Clam (Cumingia TELLINOIDES).

Showing the first maturation spindle with centrosomes and chromosomes at metaphase, and the disappearing nucleolus $(n)$ at the right. $\times 1000$.

radiating from this peripherally are delicate astral rays, collectively known as the astrosphere (Fig. 6). Structurally the aster is subject to considerable variations in different cells. On account of its relation to cell division, it is regarded as the dynamic center of the cell; viewed thus its substance is known as kinoplasm. The attraction sphere may or may not be visibly present; in all living cells it, or its analogue, is generally believed to be potentially present.

Cytoplasm.-The cytoplasm may be divided into a thin peripheral or cortical layer of less granular protoplasm, the exoplasm (ectoplasm), and the main central mass, the endoplasm. In certain highly differentiated cells the exoplasm is not discernible. In others, at certain stages in the development it contains the products of differentiations, when it is 
known as 'deuteroplasm' (Studnicka). The endoplasm is commonly described as consisting of a more fluid, finely granular ground substance,

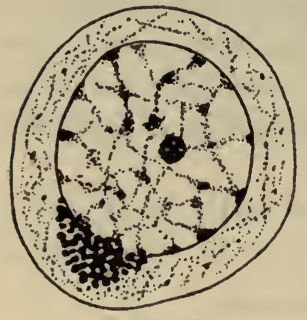

FI G. 7.-PR IMAR Y SPERMatocyte OF a Turtle (Cistudo CAROLINA).

Showing chromatic spherules ('chromidia') apparently in process of extrusion from the nucleus. $\times 1500$.

the hyaloplasm (paraplasm, interfilar mass, paramitome, enchylema, cytolymph), containing a delicate denser reticulum, or cytoreticulum, with polygonal or spheroidal meshes. The substance of the reticulum is called spongioplasm (mitome; filar mass). It is held by some to be continuous with the linin mesh of the nucleus. The granules of the ground substance, both free of and attached to the spongioplasm, are called microsomes. According to one interpretation the spongioplasm arises by coalescence of microsomes. A more recent interpretation regards both network and granule as simply more condensed portions of the hyaloplasm. The cytoplasm may contain, besides the aforementioned fundamental constituents, nutritive materials including yolk granules or globules (deutoplasm) ; vacuoles, foreign enclosures, e.g., bacteria, etc., and pigment (metaplasm) ; plastids (in plant cells); chromidia (Fig. 7), masses of chromatic granules, presumably of nuclear origin, and probably the raw material for certain differentiation products; and mitochondria or plastosomes.

Mitochondria.-Mitochondria are cytoplasmic elements of very variable form and of almost universal distribution. These are destined to bulk very large in immediate cytological investigations. They may prove to be very important elements of the more fundamental protoplasmic structure and function. In the germ cells of vertebrates, as in undifferentiated cells generally, they are for the most part, granular (chondriosomes) (Fig. 8); in the somatic differentiated cells filamentous or rod-shaped (chondriomites; chondrioconts; pseudochromosomes) (Figs. 9 and 10). Both chromidia and trophospongium (a canalicular network of the cytoplasm, prob-

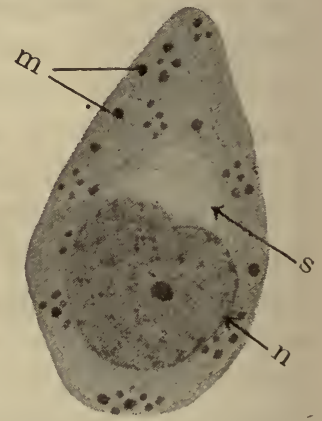

Fig. 8.-Spermatid of Opossum in EARLY Stage of MetamorPHOSIS INTO A SPERMIUM.

Showing granular mitochondria, $m$, in the cytoplasm. $n$, nucleus, $s$, archoplasmic sphere. $\times 2000$. ably concerned with circulation of nutritive material or secretion products) (Fig. 11) have been identified with mitochondria. Trophospon- 
gium at least is a distinct structure, and chromidia more probably also, though by some regarded as the elements from which the filamentous mitochondria are formed. Mitochondria have been credited with most

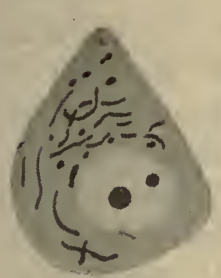

Fig. 9. $\stackrel{a}{-}$ Cells from the Newly Hatched Rainbow Trout, Treated According

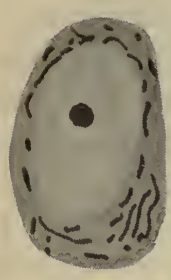

$b$

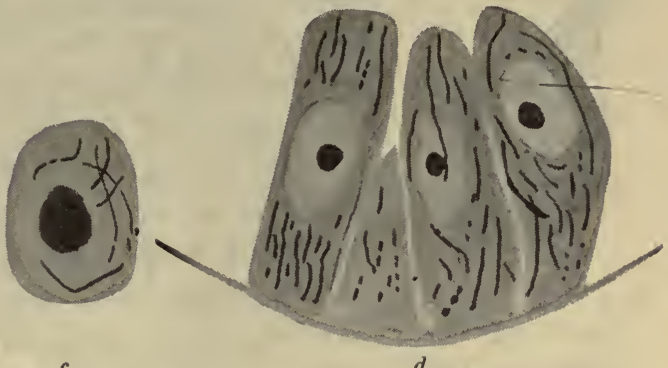
to Meves' Technic for the Demonstration of Mitochondria (Plastosomes).

$a$ and $b$, cartilage cells; $c$, young blood cell; $d$, epithelial cells from the intestine. $\times 2000$.

various functions, e.g., formation of presecretion and excretion granules, and the formation of various kinds of fibrils. M. Heidenhain regards the chondriosomes as vegetative organs of the cells subserving metabolism.
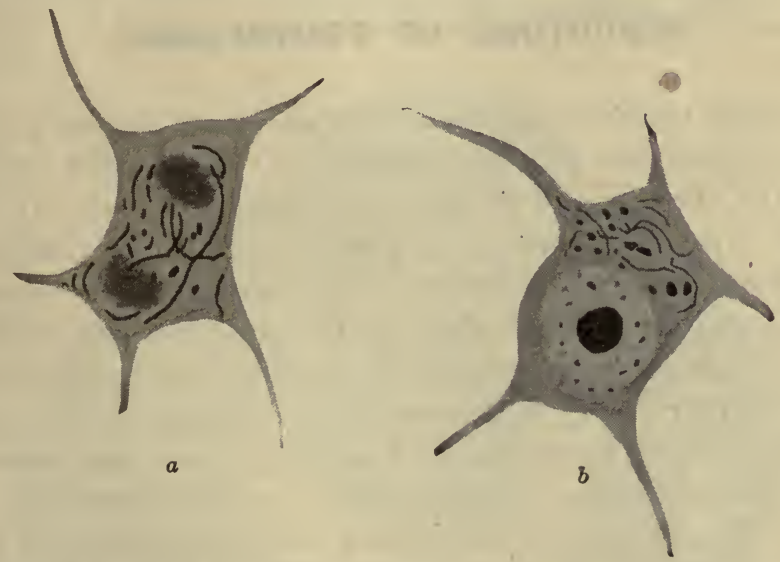

Fug. 10.-Two Cells from the Mesenchyma of the Newly Hatched Rainbow Trout.

The one to the left $(a)$ at late anaphase of mitosis, showing mitochondria (plastosomes). Meves' technic. $\times 2000$.

Our knowledge is as yet too limited to speak with assurance either as to their origin, complete function, or fate. One thing only is certain, namely, that they are actual constituents of the cytoplasm of practically 
every type of cells, at certain, perhaps all, stages of development and active function. They have been seen and studied even in living plant

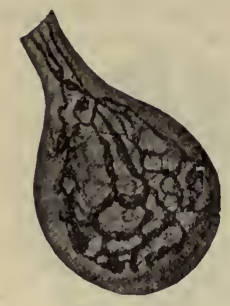

FIG. 11. - I N TR Acellular NetWORK, OR 'TROPHOSPONGIUM,' WITHIN A P URKINJE CELL OF THE CEREBELLUM OF STRIX FLAMMEA.

Golgi's stain. (After Golgi.) cells (Maximow), and in animal cells grown in artificial media they were observed by M. R. and W. H. Lewis (Amer. Jour. Anat., 17, 3, 1915) to move, to change shape, to divide into granules and again to reunite into filaments-facts which render inadmissible their interpretation in fixed material as lipoid precipitation products (FaurierFremier), and strongly suggest their connection with metabolic activity. They have the chemical composition of a lipoid (probably a phosphatid) united to an albuminoid base. It has been suggested that they are a support to, and the region of, oxidation. Certain investigators (Benda, Meves, Duesberg) regard them as the cytoplasmic basis of heredity, and ascribe to them an important rôle in histogenesis.

Schreiner's findings in developing fat cells suggest a nutritive significance (vide, p. 64).

\section{STRUCTURE OF PROTOPLASM}

Four main types of protoplasmic structure are generally recognized: (1) the homogeneous; (2) the granular; (3) the alveolar or foam type; and (4) the fibrillar, (a) reticular or sponge type and (b) filar (Fig. 12). The type of protoplasm of a particular cell may vary with the stage of development and function. In successive stages of development and differentiation the protoplasm of the same cell may pass from the apparently homogeneous, through the granular and granulo-alveolar, to a granulofibrillar type. Homogeneous protoplasm, as for example the ectoplasmic layer of ameba, is more probably to be interpreted as composed of minute, perhaps ultramicroscopic, colloidal granules ('colloidal biogens'). Young and undifferentiated cells commonly have a granular cytoplasm. In general it may be said that the actual fundamental type of protoplasm is the granular. This changes into the alveolar type by the appearance of spherules among the granules. The contents of the alveoli constitute the alveolar substance; the inter-alveolar sap, the hyaloplasm; the granules, the microsomes; and the walls of the alveoli may be identified with the spongioplasm of the reticular type of protoplasm. Another interpretation of alveolar protoplasm regards the content of the 
alveoli as hyaloplasm. The microsomes may in part be closely associated with the alveolar walls, perhaps forming them and the spongioplasm by

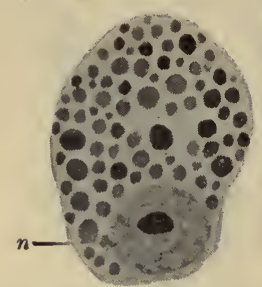

Fig. 14.-Pancreas

Cell of Turtle, Filled with ZyMOGENIC GRANULES.

$n$, nucleus, with nucleolus. $\times 2000$. the process of coalescence. The so-called alveolar protoplasm is in reality of the granulo-alveolar type. The spherules probably arise, at least in part, by a process of liquefaction of some of the granules. The alveologranular is probably, the commonest type of protoplasm. The process of transformation of the granular into the alveolar type can best be demonstrated in young growing eggs of invertebrates. Fig. 13 shows an egg in which the perinuclear protoplasm is predominantly alveolar, the more peripheral portion granular. The metamorphosis is apparently under the control of the nucleus.

Other commonly described types of protoplasmic structure may be interpreted in terms of mechanical (extraneous; artificial) alterations in the alveolar type. Thus a reticular type may be derived from the alveolar through modification (by pressure,

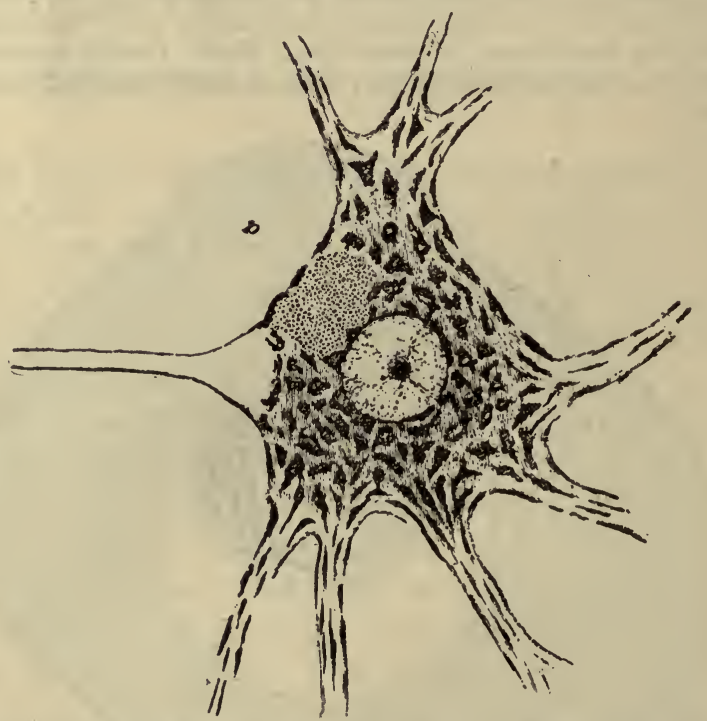

Fig. 15.-Motor Nerve Cell from the Ventral Horn of the Spinal Cord OF THE OX.

Showing Nissl granules in the cell body and its dendritic processes. The nongranular process at the left is the axon. $p$, pigment. (From Barker's "The Nervous System," after von Lenhossék.) 
or distortion) of the spherical alveoli into polyhedral or irregular compartments. Likewise the fibrillar or filar types may be interpreted as similar more extensive modifications resulting in ruptures of the alveoli and consequent finer or coarser indiscriminate aggregations of spongio-

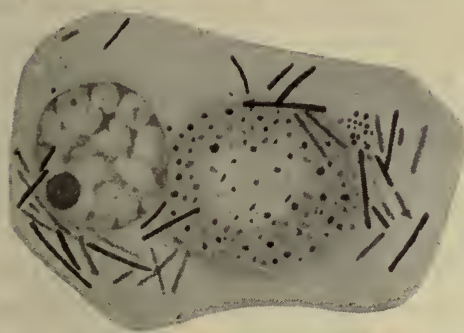

Fig. 16. - Interstitial Cell from the Testis of a Twenty-one Year OLD Man, Showing Granular and FilaMENTOUS MitochondRIA.

After Winiwarter.

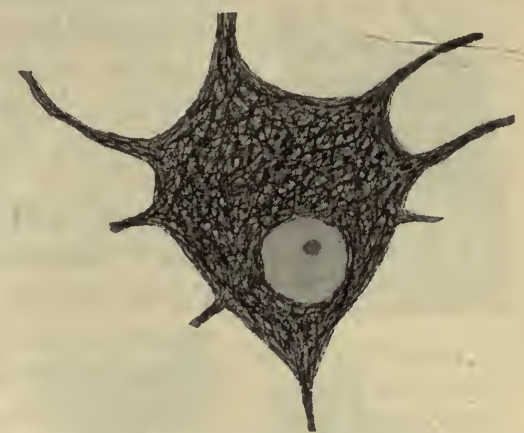

Fig. 17.-A Neuron (Giant Pyramidal Cell, or Cell of Betz) from the Cerebral Cortex of Man, ShowiNG THE NeUROFIBRiLS.

Bielschowsky technic. $\times 500$.

plasmic fibrils, or as the result of the coalescence of granules to form fibrils. The distinction between fundamentally granular and alveolar protoplasm, and secondarily derived types of granular and reticular (fibrillar) protoplasm must be emphasized. In the performance of specific functions, certain cells elaborate secretory granules (gland cells, Fig. 14; nerve cells, Fig. 15; cells with crystalloids, Fig. 16); others produce various types of fibrils (e.g., nerve cell, Fig. 17; connective tissue cells, and muscle cells) ; others elaborate fat spherules (e.g., Fig. 18) ; and still others a canalicular (trophospongium) apparatus (Fig. 19).

The foregoing description of protoplasmic structure pertains largely to the 'fixed' (dead) condition. In this connection the terminology employed will continue useful. But recent more refined physiochemical studies of living protoplasm have aroused considerable skepti-

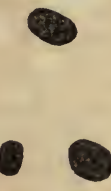

Fig. 18.-Developing Fat Cells.

The fat droplets, after extraction with alcohol and ether, appearing as vacuoles. Hematein and eosin. $\times 550$. cism respecting the verity of actual specific structures corresponding to the designations applied, more especially the spongioplasmic and linin network. Perhaps the most that can be said with certainty regarding the fundamental structure of protoplasm is to describe it as a 'granular gel.' Kite's studies 
(Amer. Jour. Phys., 32, 2, 1913) of the physical properties and molar structure of protoplasm in various cells, by combined methods of microdissection and vital staining, have led to clearer conceptions in this field. Kite accepts the interpretation of protoplasm as an emulsoid, the real structural

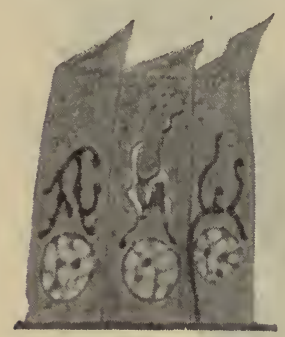

Fig. 19.-Columnar Ciliated Epithehial Celds, Showing Canalicular Apparatus.

After Holmgren. units of which are the colloidal particles; and he conceives of the optical image as the result of the combination of the physical phenomena of reflection, refraction, diffraction, absorption, dispersion, interference, and a scattering action on light. Living protoplasm is an apparently homogeneous and viscous hydrogel, holding in suspension in the form of granules ('microsomes') minute masses of denser gels, and liquid globules ('alveoli') which show many of the optical properties of an oil drop. The 'network' and 'granules' of the nucleus Kite regards as optical phenomena, areas of greater concentration in the nuclear gel, not separated from, but grading into the surrounding diluter gel of the 'nuclear sap.' Spindle fibers were successfully dissected out of the protoplasm as distinct, relatively rigid threads. These conclusions are in the main in accord with those deduced from the earlier physiochemical studies of protoplasm and colloids by Hardy and others. M. R. and W. H. Lewis (op. cit.) also find no sign of a reticular or of an alveolar structure in either cytoplasm or nucleus in cells studied in tissue cultures. They describe both cytoplasm and nucleus as 'finely granular almost homogeneous in appearance.'

\section{VITAL PROPERTIES OF CELLS}

Living protoplasm is capable of certain specific reactions (physiologica! processes) or functions. These reactions are spoken of as vital properties or attributes of protoplasm. They are general properties of living matter. They include primarily (1) metabolism; (2) irritability; (3) contractility; (4) reproduction.

(1) Metabolism.-Metabolism is that property of living protoplasm by virtue of which it can elaborate from raw food material the complex chemical compounds of protoplasm (anabolic phase, constructive metabolism, assimilation), and convert the same into kinetic energy for the performance of specific functions (katabolic phase, destructive metabolism, dissimilation), e.g., secretion and excretion. Metabolism generally involves growth and differentiation. Development also is 
fundamentally a metabolic process, and in essence consists of 'a progressive differentiation of complex and specialized structures and functions from relatively simple and generalized beginnings' (Conklin).

(2) Irritability.-Irritability, or sensitivity, is a fundamental or general property of protoplasm. It is characterized by a capacity to receive and make response to stimuli, by changes of vital processes. Its prerequisite is the protoplasmic property of conductivity, and its expression in many instances depends upon the property of contractility. In a comprehensive sense, stimulus is every alteration in the external vital condition (Verworn). 'The reaction to stimuli may exhibit itself in one of three modes: functional, nutritive, and formative (Verworn).
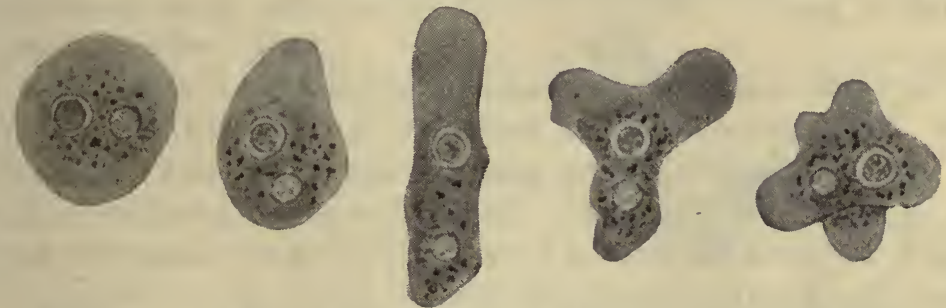

Fig. 20.-Successive Stages in the Movement of an Ameba.

The cells contain a nucleus, a contractile vacuole and protoplasmic granules. (After Verworn.)

Stimuli are of various sorts, e.g., thermal, mechanical, chemical, photic, solar, galvanic (or electric), fluid, current, gravity. The simplest resulting reactions, expressed in unicellular forms in terms of orientation (tropism) or contact (taxis)-represented by automatic responses or reflexes in higher forms-are respectively thermotropism, stereotropism (barotaxis; thigmotaxis), chemotropism, phototropism, heliotropism, galvanotropism, hydrotropism, rheotropism, and geotropism. Response may be either toward or away from the source of stimulus. In the case of the electric current or water currents, for example, simple organisms may orient themselves, or protoplasm may move, in line with or opposite to the current; these opposite reactions are called positive and negative tropisms respectively. Responses involve fundamentally metabolic changes.

(3) Contractility.-Motion results from response to certain stimuli, that is, by reason of irritability; and it is dependent upon the vital phenomenon of contractility. Motion is of various types, predominant among which are (a) ameboid, (b) ciliary, (c) molecular, (d) circulatory (streaming; protoplasmic), and (e) muscular. 
(a) Ameboid motility is exemplified in the movements of an ameba; hence the name. This consists essentially in the formation of a protoplasmic process or pseudopodium, into which the main mass of proto-

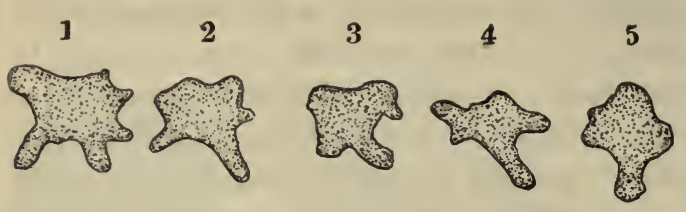

Fig. 21.-A Leukocyte from Human Blood in Active Ameboid Motion.

The figures indicate the successive forms assumed by the cell. Drawings were made at intervals of one minute. $\times 500$. plasm flows, thus producing progression (Fig. 20). The movement of the white blood-corpuscles is of this sort (Fig. 21).

\section{(b) Ciliary motility} is characteristic of hairlike processes of certain cells; such processes or cilia represent essentially permanently differentiated delicate pseudopodia. The method of cilium formation is illustrated in its simplest form in the transient vibratory processes that arise under certain conditions on leukocytes (Fig. 225). In metazoa generally ciliated cells are attached, motion being limited to the cilia, which are located on the free border. The function of cilia is to propel secretions toward the surface. The motion is wavelike and always in one direction. The cilia are generally attached to a double row of granules, the 'basal bodies' (Fig. 22), perhaps partition products of the centrosome. In Protozoa, e.g., Paramecium (Fig. 3), the entire surface of the cell may be ciliated; the function of the cilia here being progression, and the direction of stroke is reversible. Certain cilia are non-motile, e.g., in the epididymis, where they are closely clumped into brushlike masses (Fig. 25). The function of such cilia is, in part at least, to

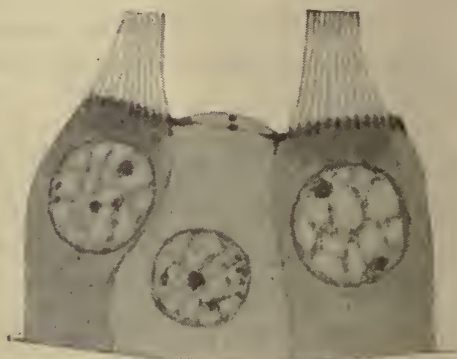

Fig. 22.-Three Cells from the Epididymis of the RabBit.

The non-ciliated cell has a diplosome at its free border. The adjacent ciliated cells have in place a double row of 'basal granules' to which the cilia are attached. (After v. Lenhossék.) furnish a means for the elimination of secretions. Flagellate motion is to be regarded as a variety of ciliary motion. A flagellum is commonly regarded as a more robust cilium. The purpose of flagella is to propel the cells to which they are attached. Usually in higher animals the number of flagella is limited to one to a cell. The best examples of flagella are furnished by spermatozoa (Fig. 23). 
(c) Molecular motility is a dancing or oscillatory movement of the granules in living protoplasm. Such granules may be non-living matter, pigment, etc. This type of motion is also called brownian movement. It is probably purely a physical phenomenon. It may be simulated by mixing finely divided carmin with glycerin.

(d) Circulatory or streaming movement is present in various-degrees in probably all living protoplasm. It is only when it is rapid that it becomes easily discernible. It is readily demonstrable in certain cells, e.g., chara and nitella; also less readily in certain protozoa (Paramecium). It must most probably be interpreted as a form of respiration. It is characterized by a flowing or streaming of the protoplasmic granules in a definite direction.

(e) The reason for listing muscular as a separate type of motility is mainly its predominance in animals and the fact that it does not apparently fully conform to any of the above types. It is characterized by a reversible process of contraction of specially differentiated muscle fibrils. It perhaps most closely resembles streaming motility. It leads to least confusion, in view of our present lack of definite knowl-
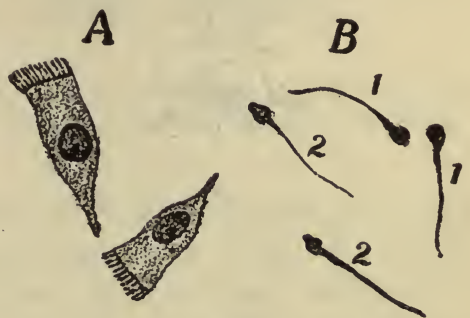

Fig. 23.-Ciliate and Flagellate Cells.

$A$, ciliated cells isolated from the trachea of a cat; $B$, human spermatozoa-1, in surface view; 2 , in profile. Examined fresh in normal saline solution. $\times 550$. edge regarding the physical and chemical phenomena underlying muscular motion, to speak of it as a distinct type. It will be further discussed under Muscle.

(4) Reproduction.-The essence of reproduction is cell multiplication. A living cell has the power of producing other cells like itself. Viewed philosophically, cells may conceivably arise in two different ways: (1) from non-living material, spontaneous generation (abiogenesis); (2) from preëxisting cells by division. Science has quite generally accepted the aphorism 'omnis cellula e cellula' (Virchow) as an expression of the whole truth. However, full acceptance of the doctrine of evolution logically compels belief in spontaneous generation: this not in any such crude form as that frogs may arise from the mud of rivers, or insects from dew or dung, but that given the conditions (conceivably possible somewhere in the universe to-day) prevalent when life first appeared as the original' mass of living protoplasm, the 'cytode' or 'cytoblastema', the inorganic may continually be passing into the primarily organic, e.g., 
monera (Haeckel), but not perceptible under our present means of search and observation. This is the position urged by one of the leading physiologists and histologists (E. A. Schaefer) of our day, following Spencer of the preceding generation.

However, in histology we need be concerned only with the derivation of cells from preëxisting cells. 'This proceeds in one of two ways: (a) direct, amitotic, or akaryokinetic; and (b) indirect, mitotic, or karyokinetic. The difference between the two inheres in a difference in behavior on the part of the nucleus (or karyon). Comparative studies of the

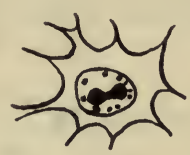

$\boldsymbol{a}$

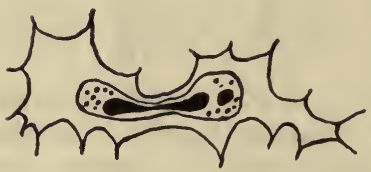

c

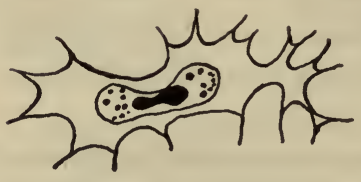

$b$

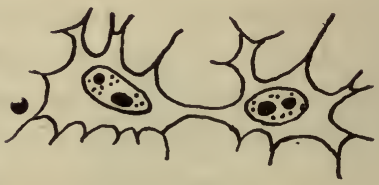

$d$

Fig. 24.-Successive Steps in Amitotic Division in Tendon Cell of New-born Mouse.

(After Nowikoff. $\times 800$.)

lower groups of animals and plants have revealed a fairly complete series of intermediate stages. On the basis of these facts it is believed by some (e.g., Strasburger) that amitosis is the primitive method of cell multiplication, mitosis the derived or more highly specialized type. Others regard amitosis as the derived, not the primitive form of division.

Cell division is presumably due to the fact that the area of the surface increases as the square, the volume as the cube, of the diameter. In consequence, the periphery becomes more favorably placed with respect to the nutritive medium than the more central portions. The time then arrives when the center must suffer nutritive want or when the nucleus becomes unable to exert its trophic functions at the distance of the ad- 
vancing periphery. Division of such an enlarged cell into two smaller cells reëstablishes the original and more favorable nucleo-cytoplasmic dimensional relationship.

(a) Amrtosis. - In typical amitosis the nucleolus first becomes bilobed and then divides (Fig. 24). This is followed by nuclear division,

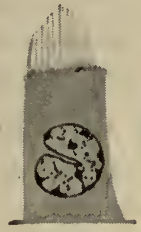

$a$

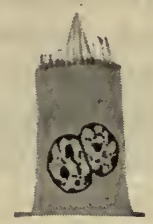

$b$

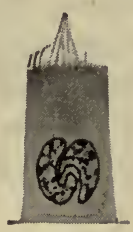

$c$

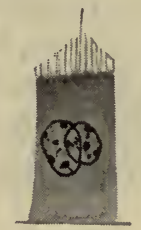

b

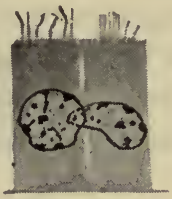

$e$

Fig. 25.-Successive Stages in the Amitotic Division of the Ciliated Cells Lining the Ductuli Efferentes of the Epididymis of the Mouse. $\quad \times 1500$.

each resulting nucleus enclosing one of the nucleoli. Nuclear division is followed by cytoplasmic division. A centrosome is generally neither active nor even visible during this process. This typical condition is rarely realized. It was first described by Remak (1841) for blood-cells. Usually nuclear division is independent of nucleolar fission, which may be lacking (Fig. $25)$. The nuclear fission proceeds variously by a medial or submedial annular constriction, or by progressive linear indentation of some portion of the surface. In certain instances the division takes place inside of the original nuclear membrane. The nuclear products may be of unequal size, and multiple (Fig. 26). Generally cytoplasmic division lags far behind nuclear division, or may even fail to appear, thus

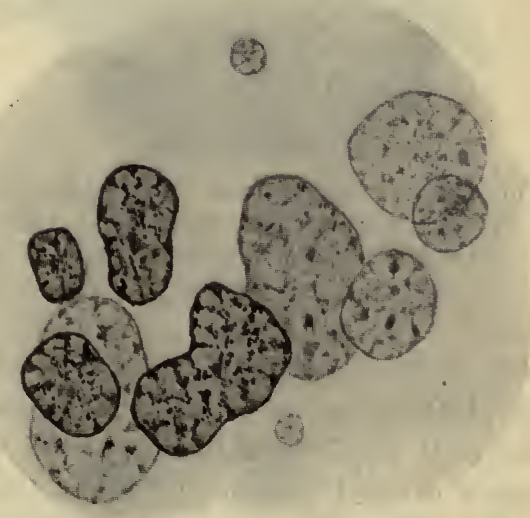

Fig. 26.-Multinucleated Giant Cell, From the Yolk-SaC of a 10 mm. Pig Embryo. $\times 2000$.

producing bi- or multinucleate cells. Amitosis effects a mass division of the nucleus; neither spireme nor chromosome nor achromatic spindle, so conspicuous in mitosis, appear. 'Until quite recently amitosis was generally regarded as a relatively rare and unimportant process. It was 
supposed to be associated only with highly specialized and pathological conditions leading inevitably to death. Cells once having suffered amitotic division were believed not to be capable of thereafter dividing mitotically. The work of Child (Biol. Bull., 1907) has shown, however, that it is probably of very wide occurrence. Instances have been described in most of the animal groups, including the vertebrates. Child has shown its occurrence in regions of rapid growth, as in various embryonic tissues, e.g., blastoderm of chick (Patterson), and where a secretion is elaborated or in places of reserve formation. These facts may be harmonized with its occurrence in starving, degenerating tissues on the basis of a common

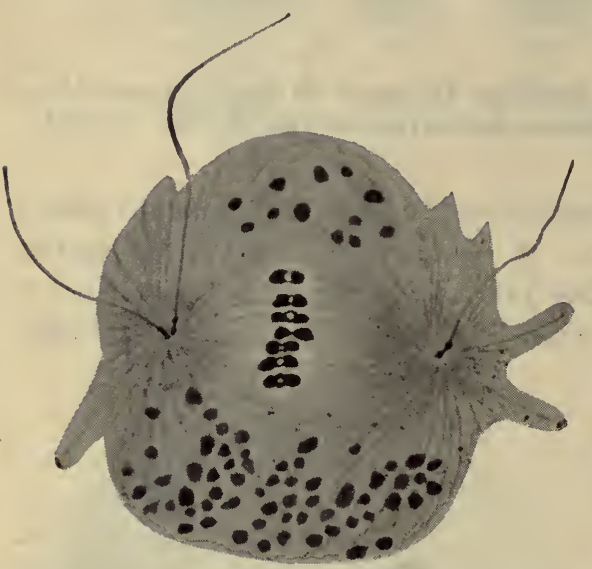

Fig. 27. - Spermatocyte of Pyeris CRategi, a Butterfly, Showing a Cilium Attached to the Centrosome. (After Meves.) : underlying condition, namely, relative scarcity of nutritive material. Wieman conceives of amitosis as due to scarcity of oxygen supply.

Where mitosis and amitosis are simultaneously present, it is more frequently the cells with the large nuclei, surrounded by a considerable amoun't of undifferentiated cytoplasm, that divide by mitosis. The factors underlying amitosis most probably exert their final effect indirectly through initial influence upon the centrosome. The best experimental evidence in favor of this view is supplied by Nathansohn who grew Spirogyra, normally dividing by mitosis, in a 1 per cent. solution of ether in water, when the cells divided amitotically. On transference to pure water, the cells again divided mitotically. The ether seems to have exerted a 'stupefying' effect upon the kinoplasm (centrosome material), compelling division by amitosis. Amitosis is now generally conceded to be of wide occurrence under certain conditions and in certain cells, but it is still quite unanimously disbelieved to occur in germ cells. In the latter it has perhaps not yet been certainly demonstrated to occur in cells actually in the germ cycle. In Mammalia amitosis can be demonstrated in the intermediate layers of stratified squamous (skin), transitional (bladder) and certain ciliated (epididymis, Fig. 25) epithelia; in the 

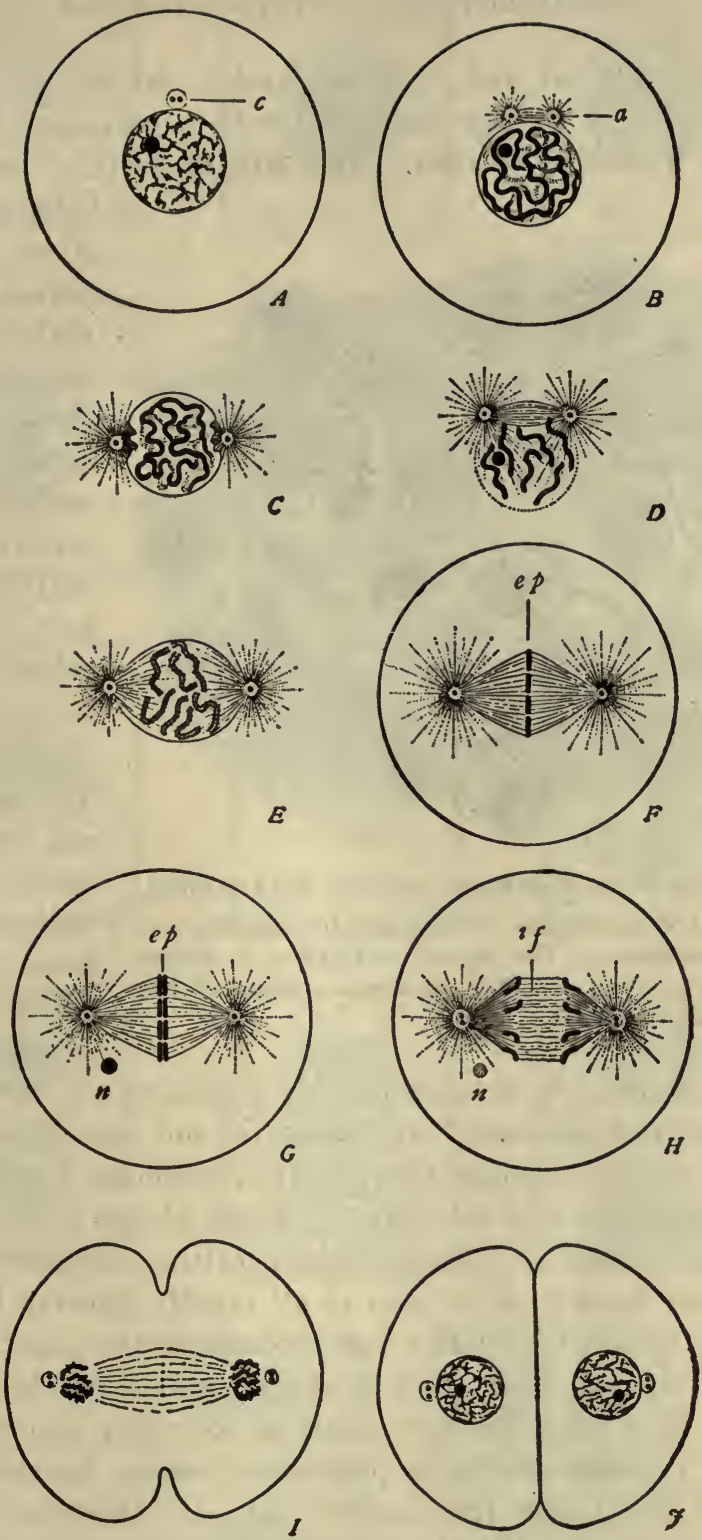

Fig. 28.-Diagrams Iludgstrating Successive Stages of Mitosis.

$A-F$, prophase; $G$, metaphase; $H$, anaphase; $I-J$, telophase. $a$, achromatic spindle; $c$, centrosome; $e p$, equatorial plate of chromosomes; if, interzonal fibers; $n$, nucleolus. (After Wilson.) 
medulla of the adrenal, and in decidual cells. In ciliated epithelia this mode of division is perhaps associated with a partition of the centrosome in the formation of cilia. This will be further discussed under

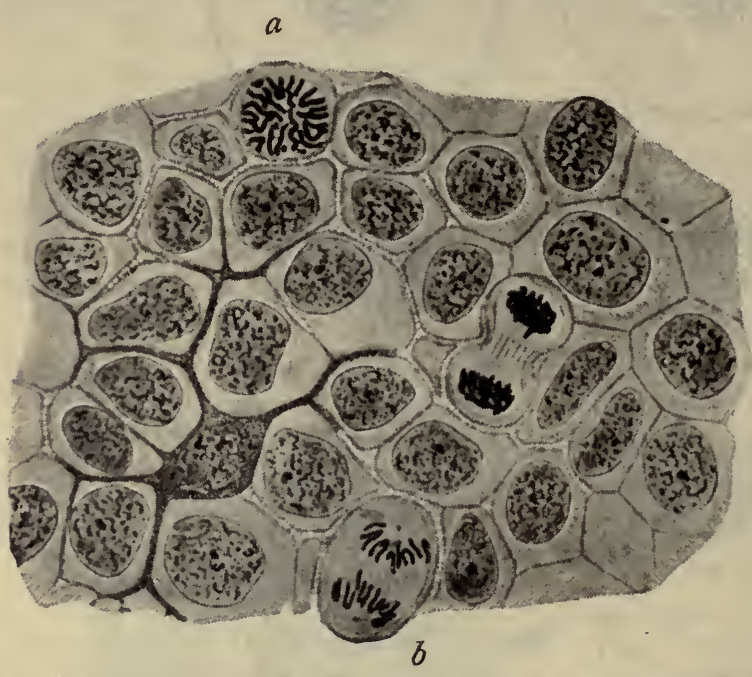

Fig. 29.-Cells from Epidermis of the Salamander.

Three cells are in process of division by mitosis. $a$, prophase; $b$, anaphase. The second cell above $b$, whose cell body is in process of fission, presents a stage of the telophase. (After Wilson.)

of as continuous, may be divided into (1) prophase; (2) metaphase; (3) anaphase; and (4) telophase. An alternative and preferable terminology employs the words anaphase (prophase), mesophase (metaphase) and kataphase (anaphase and telophase). These phases involve coincident changes in the nucleus and the archoplasm (attraction sphere, Fig. 28, A to J). Mitotic figures can be seen in all rapidly growing tissues. The process is an essentially similar one throughout the plant and animal kingdoms; variations relate only to details associated chiefly with the archoplasm. The most favorable locations for study of mitosis are the growing tips of roots of certain plants, e.g., onion, hyacinth, dogtooth violet; amphibian tissue (particularly skin and blood-cells), and the testes of grasshoppers. Mitosis in germ cells involves certain specialized features, and calls for additional theoretical consideration; hence the description of these maturation mitoses will be reserved for the chapter dealing with the ovaries and testes, where a complete account will follow. Among the simplest types of mitosis, and those best adapted for labora- 


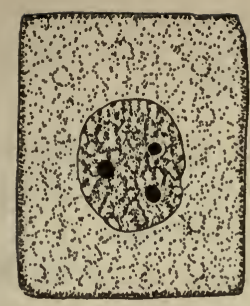

a

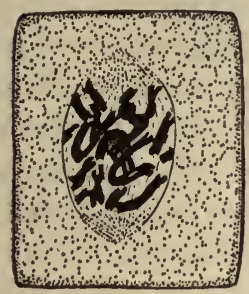

$d$

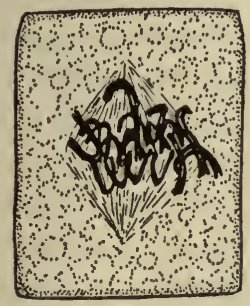

$g$

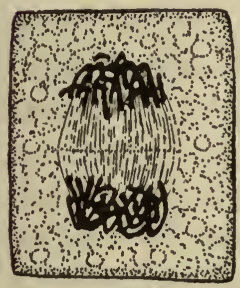

j

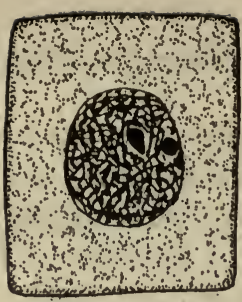

b

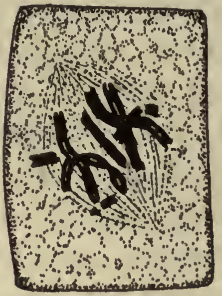

e

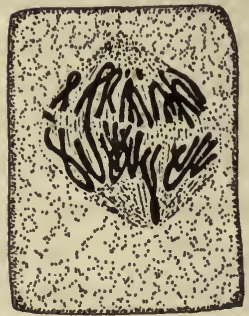

$h$

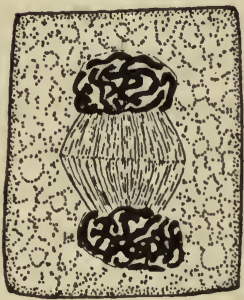

$k$

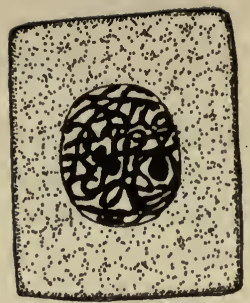

c

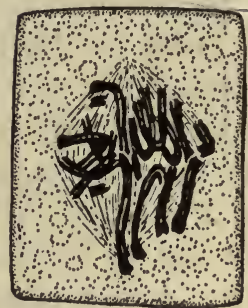

$f$

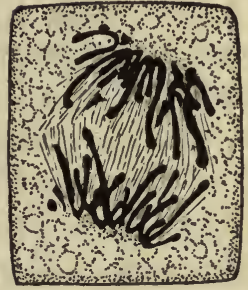

i

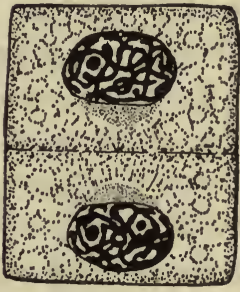

$l$

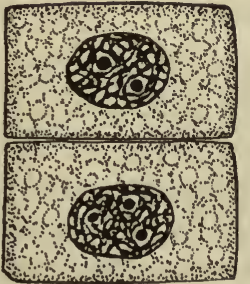

$m$

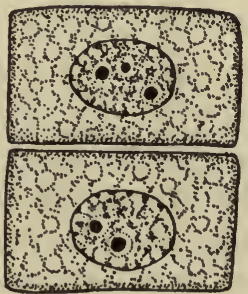

$n$

Fig. 30.-Successive Stages of Mitosis in the Root Tip of the Dogtooth Violet (ERYTHRONIUM AMERICANUM).

$a$, resting nucleus; $b$, close spireme; $c$, loose spireme; $d$, segmented spireme; $e$, late prophase; $f$ and $g$, metaphase; $h$ and $i$, anaphase; $j-l$, telophase (showing mid-body or cell plate); $m$ and $n$, daughter cells, $n$, with resting nuclei. $\times 1500$. 
tory study, at least as an approach to the subject, is that shown in the root tip of the dogtooth violet (Fig. 30, $a$ to $n$ ). The cells and the mitotic figures are here so large that all the major details can be easily recognized by use of the usual dry high power lenses of the microscope.

(1) Prophase.-This stage can again be subdivided into that (a) of the resting nucleus; (b) of the nucleus with close spireme; (c) of the nucleus with the loose spireme; and (d) of the segmented spireme. Coincident with these nuclear changes, the centrosome in animal cells divides into two (diplosome) ; these moieties move apart toward opposite poles of the nucleus and build a spindle (amphiaster) between themselves. Meanwhile the nuclear membrane begins to disappear, only a remnant distant to the achromatic spindle persisting at the end of the prophase. In the root tip cell of the dogtooth violet and in plant cells generally, the spindle appears in less conspicuous fashion than in animal cells. A centrosome is apparently lacking. The first indications of the spindle are the polar caps of faint radiations which grow medially to build the spindle. The resting nucleus (Fig. 30, a) is characterized by a random, granular, nuclear reticulum with net-knots and one or several nucleoli. This reticulum becomes changed into a delicate, deeply chromatic, probably continuous, close spireme (Fig. 30, b). By process of shortening and thickening, this changes into the loose spireme stage (Fig. 30, c). The nucleoli have meanwhile contributed chromatic substance to the spireme, but may persist for some time longer as achromatic, ultimately fragmenting or dissolving, bodies. On closer inspection the close spireme is seen to consist of a series of granules (chromomeres); during the loose spireme stage these become split, thus giving rise to a double row of granules. The loose spireme passes into the succeeding stage (segmented spireme, Fig. 30, d) by transverse segmentation into a number of rods or chromosomes. At this stage all indication of chromomeres is generally again lost, the chromosomes appearing as compact, deeply staining rods of various shapes.

The number of chromosomes is believed to be constant for all cells of a species. This belief rests upon data of actual counts in various insects and other lower animals and certain plant forms. Here the number is relatively small, and the individual chromosomes are large and can in consequence be readily counted. (Certain qualifying statements must be made in the chapter which includes a discussion of sex determination.) Attempts have recently been made to throw doubt upon the matter of a specific chromosome constancy, but it is only fair to note that these attempts have dealt with relatively unfavorable material, where exact chromosome counts 
are extremely difficult if not at present actually impossible. It may be noted in passing also, that the chromosomes are believed by many to be the bearers of the determiners of hereditary characters-a point to be further discussed below.

(2) Metaphase.-This is a relatively brief stage in mitosis. - It includes the period when the chromosomes are arranged upon the spindle in the equatorial plate. Seen in polar view this is called the monaster stage (Fig. 30, e). In this stage the chromosomes split longitudinally. In dogtooth violet the number of chromosomes is twenty-four. A common form of chromosome is the U-shaped type. The point of attachment to the spindle is the apex of the bent chromosome (Fig. 30, f). In the more rapidly growing cells the double or split condition of the chromosomes has remained discernible since the preceding telophase, a true resting stage having been omitted. At metaphase the already longitudinally split chromosomes are completely divided, and the separated moieties (daughter chromosomes) drawn toward opposite poles (Fig. 30, g).

(3) Anaphase.-The limits of this phase are indefinite (Fig. 30, $h$ to $j)$. It may be said to include all stages between that when the separation of the daughter chromosomes, resulting from the longitudinally splitting of the mother chromosomes, is severally consummated, and that when the groups of daughter chromosomes drawn to either pole are still distinct. Seen in side or oblique view the later stages of this phase present a double star arrangement of chromosomes-hence diaster stages (Fig. 30, i). The daughter chromosomes, an equal number at either pole, were drawn apart by activity of the outermost of the spindle fibers (called mantle fibers) presumably by process of contraction. The inner or 'interzonal fibers' constitute the ceniral spindle.

(4) Telophase.-Meanwhile a plate of granules (cell plate; mid$b o d y)$ has appeared in the equatorial region of the spindle. This marks the plane of the future division (Fig. 30, $\mathrm{j}$ and $\mathrm{k}$ ). In animal cells, an annular constriction appears peripherally in the cell membrane. This proceeds centrally throughout telophase until ultimately the mother-cell is divided into two daughter-cells. The constriction of the cells in division is generally interpreted as a phenomenon of alteration in surface tension. Coincidently with the steps of this process, the chromosomes and centrosomes (archoplasm material in plants) pass through the stages of the prophase, but in inverse order: segmented spireme, loose spireme, 

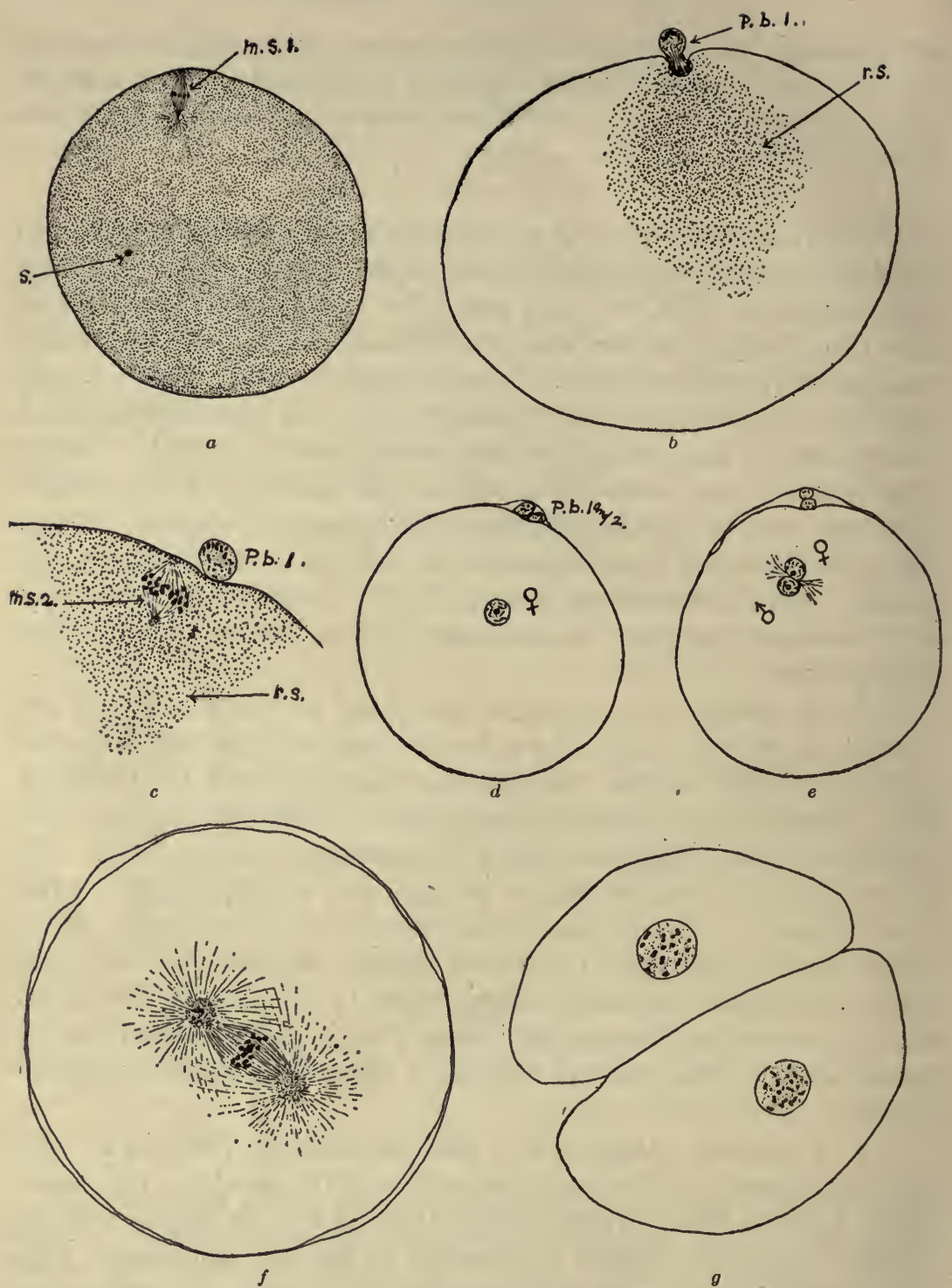

Fig. 31.-Successive Stages in the Maturation, Fertilization and Segmentation of the Starfish (Asterias forbesii) EgG.

$a$, first maturation spindle (m.s.1) and the spermatozoön $(s)$; $b$, formation of the first polar body $(p . b .1)$ and the residual substance of the nucleus $(r . s) ;$.$c , second$ maturation spindle (m.s.2); $d$, female pronucleus ( $q$ ); $e$, union of male $(\delta)$ and female pronuclei (the male pronucleus is derived from the spermatozoön); $f$, first segmentation spindle; $g$, two-cell stage of division. $d$ and $e$ are less highly magnified. 
close spireme, resting daughter nucleus with its daughter centrosome and ultimately a nucleolus (Figs. 30, k, l, m, n). Where a cell plate appears, division is consummated without constriction. In certain pathological tissues, e.g., cancers, the cells divide in various atypical ways, involving the formation of tri- and multipolar spindles.

\section{HISTOGENESIS}

Every higher organism begins as a fertilized egg or zygote; this involves the fusion of a male (spermatozoön) and a female (egg) germ cell (Fig. 31, a and e). 'The result of the fusion is a mingling of approximately equal parts of paternal and maternal chromatin (presumably the basis of specific heredity), a large mass of maternal cytoplasm and nutritive substance with a small, but perhaps important mass of male cytoplasm; and a coincident stimulus to development. The fertilized egg divides by mitosis into two spheroidal cells-the typical embryonic form-or blastomeres (Fig. 31, $f$ and $g$ ), and each of these again into two, the segmentation process

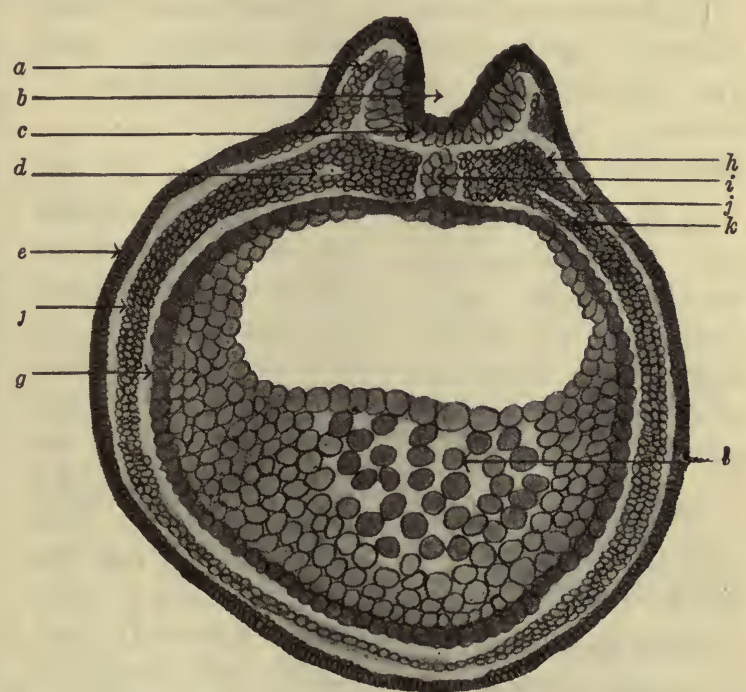

Fig. 32.-Transverse Section of a Frog Embryo, Showing the Three Germ Layers.

$a$, neural crest; $b$, neural groove; $c$, neural plate; $d$, cœlom; $e$, ectoderm; $f$; mesoderm; $g$, entoderm; $h$, somite; $i$, notochord; $j$, parietal mesoderm; $k$, visceral mesoderm; $l$, yolk; central opening, the primitive intestine. (Drawing by G. A. Pagenstecker.)

continuing until the adult organism results as an aggregation of innumerable cells. This process of growth through cell multiplication is accompanied by cell differentiation, which constitutes histogenesis. The first outstanding stage in the differentiation is that when the three funda- 
mental germ layers: ectoderm (ectoblast; epiblast), mesoderm (mesoblast), entoderm (endoblast; hypoblast) have appeared (Fig. 32). Back of this of course must lie a more fundamental differentiation, perhaps already present in the unfertilized egg, a predelineation of adult structure destined to develop from localized egg materials. By the process of histogenesis all the adult tissues arise from the several germ layers. This matter is summarized in the following table:

\section{ADULT TISSUE DERIVATIVES FROM}

ECTODERM

Epidermis and its derivatives: hair, nails, and epithelium of sebaceous, sweat and mammary glands.

Epithelium of mouth and its derivatives: enamel, taste buds, epithelium of salivary and other" buccal glands, and anterior portion of hypophysis.

Epithelium of anus, and distal portion of the male urethra.

Epithelium of nostrils and communicating glands and cranial sinuses.

Epithelium of conjunctiva and associated ducts and lacrimal glands. The lens, and the epithelium of the pars nervosa, ciliaris and iridica retinæ.

Epithelium of mem-
MESODERM

Epithelium of uriniferous tubules, renal pelves and ureters.

Epithelium of the seminiferous tubules and the associated excretory ducts of the testis; epithelium of oviduct and uterus; probábly also the sex cells. The cortex of the suprarenal gland. All muscular tissue; connective tissue; vascular tissue (blood and lymph vessels and cells), and lymphoid organs in general.

Epithelium (mesothelium) of pleuræ, pericardium and peritoneum; of the tendon sheaths, joint cavities and bursæ; and of the chambers of the eye, and the perily $\mathrm{mph}$ spaces of the internal

\section{ENTODERM}

Epithelium of digestive tract (including ph a ry $\mathrm{nx}$; excluding mouth and anus) and associated $\mathrm{glands}$ : pharyngeal, esophageal, gastric, intestinal, pancreas and liver, with gall-bladder.

Epithelium of middle ear (tympanum) and auditory (Eustachian) tube. Epithelium of respiratory system, beyond nostril. Epithelium of thyroid, parathyroids, and the thymic reticulum and corpuscles.

Epithelium of female urethra, proximal part of male urethra, and of the urinary bladder.

Epithelium of prostatic and Cowper's glands in the male, and of the glands of 
branous labyrinth of internal ear, and lining of external ear.

Epithelium lin ing the central canal of the spinal cord, and the ventricles of the brain.

All neurons and neuroglia of the nervous system.

Certain ductless glands : pineal, posterior (nervous) portion of hypophysis, medulla of suprarenal, and the chromaffin system or paraganglia.

Possibly smooth muscle associated with sweat glands, and in iris of eye. ear (scalæ tympani and vestibuli). 


\section{CHAPTER II}

\section{EPITHELIAL TISSUES}

\section{TISSUES}

A tissue in the histologic sense is a collection of similarly specialized cells united in the performance of a particular function, e.g., liver tissue. In certain tissues the cells are joined together by an intercellular cement substance, which is a secretion product of the cells themselves. Through this cement may extend the so-called 'intercellular bridges' or cytodesmata (Fig. 33), the minute intervening spaces forming delicate

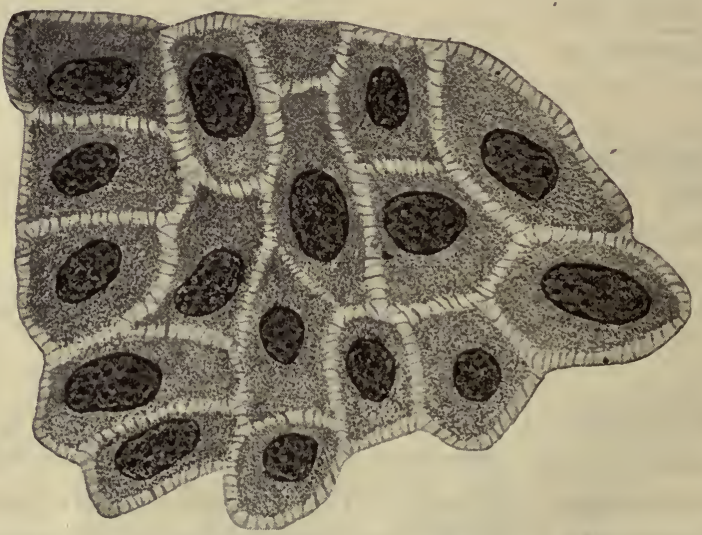

Fig. 33.-Group of Epithelial Cells from the Malpighian Layer of THE SKin.

The intercellular bridges are very distinct. Hematein and eosin. $\times 1,000$.

canaliculi, presumably for mediating the transfer of nutritive matterial from cells more favorably placed with respect to the source of supply to those less favorably located, e.g., epidermis; these bridges arise through process of vacuolization in the exoplasm of adjoining cells, the walls of the original vacuoles persisting as 'bridges.' Through such bridges, fibrils may extend from cell to cell. Practically every tissue contains 
also connective tissue elements for unification and support; also vascular and nervous constituents. Tissues in which the cell boundaries are absent are known as syncytia (Fig. 34). A syncytium may obviously arise

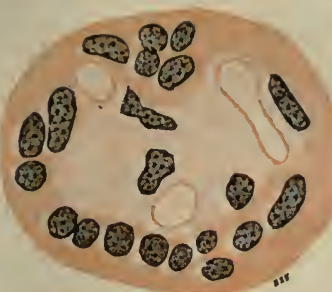

Fig. 34.-A Villus of the Human

Placenta, Showing a Peripheral Syncytium of Irregular Thickness.

The connective tissue inclosed by the syncytium contains three capillary vessels. Hematein and eosin. $\times 500$. through nuclear proliferation in the absence of cytoplasmic division, or as the result of the disappearance of original cell boundaries. We may distinguish

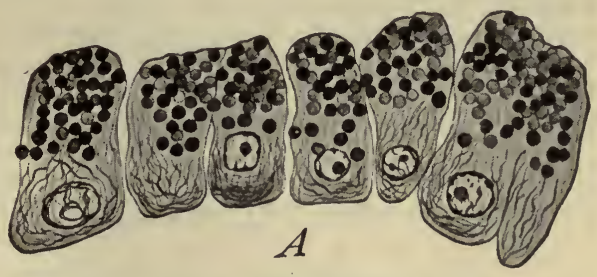

Fig. 35.-Cells from the Pancreas of Decturus, Containing Secretory Granules and Basal Ergastoplasmic FilaMENTS. (After Matthews.)

the following fundamental tissues: (a) epithelial; (b) connective; (c) muscular; (d) nervous; and (e) vascular.

Lymphoid tissue may be regarded as still another fundamental tissue;

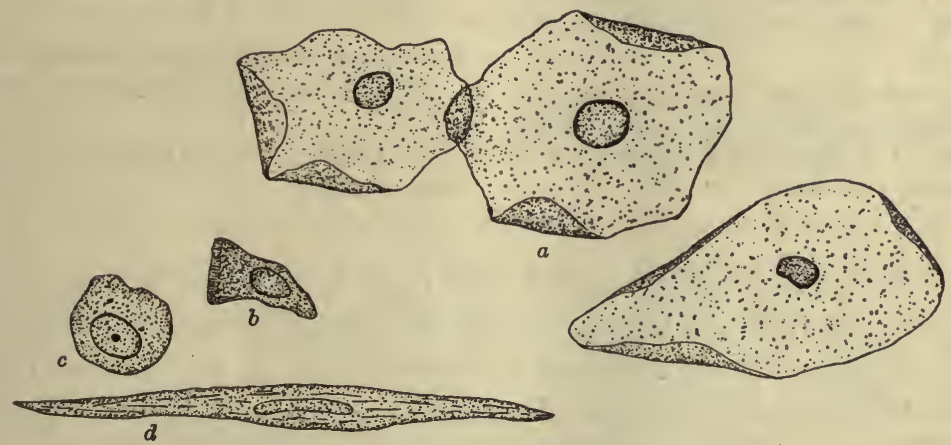

Fig. 36.-Various Forms of Cells.

$a$, squamous epithelium from the tongue; $b$, a columnar cell from the small intestine; $c$, a polyhedral or spheroidal cell from the liver; $d$, a smooth muscle cell from the muscular coat of the stomach. $\times 550$.

or it may be included under vascular tissue. In fact from the genetic viewpoint, vascular may be included under connective tissue, since both arise from the mesenchyma. 
Representatives of all of the fundamental tissues are generally found in all histologic preparations, or tissues in a general sense. Cells vary greatly both from the standpoints of shape and. contents in the various tissues-both depending upon the types and phases of function. The more usual form variations include: (a) spheroidal, spherical (e.g., embryonic cells and egg cells, Fig. 1, chap. I), polyhedral (spherical cells modified by pressure from adjacent cells, e.g., liver cells, Fig. 37) ; (b) scalelike or squamous (e.g., superficial cells of mucous membrane of mouth, Fig. 36, a) ; (c) columnar, prismatic or cylindrical (e.g., cells lining intestine, Fig. 38, b). Columnar cells, when very short, are

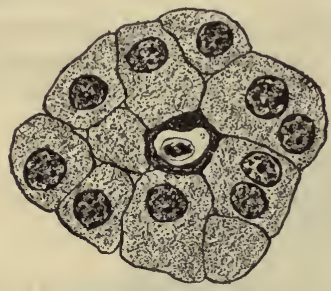

Fig. 37. - Polyhedral Epithelium, From a Section of the Human Liver.

The central blood capillary contains one leukocyte, and its wall contains the nucleus of a flattened endothelial cell. Hematein and eosin. $\times 550$.
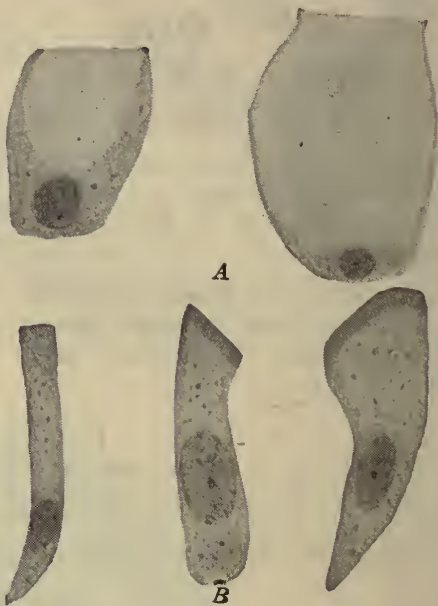

Fig. 33.-Goblet and Columarar Celle FROM THE LARGE INTESTINE OF THE CAt.

A, Goblet cells; $\mathrm{B}$, isolated columnar cells. $\times 900$.

usually designated cubical or cuboidal (e.g., bronchioles and rete testis, Fig. 43) ; intermediate lengths may be designated either tall cuboidal or short columnar; when modified by confinement in an alveolus into a pyramidal shape as in glands, they may be called pyramidal or 'glandular' (Fig. 46). Glandular cells, moreover, are characterized also by an internal differentiation commonly expressed in the form of granules or filaments. Columnar cells may be further modified by the appearance of cilia into ciliated epithelium (e.g., trachea, bronchial tube, Fig. 52), or of mucus into goblet cells or 'unicellular glands' (e.g., intestine, Fig. 38, a) ; or as specialized receptors for stimuli of special sense they may become modified. as neuro-epithelium (e.g., certain cells of eye, ear, nose and tongue). 


\section{EPITHELIAL TISSUES}

Epithelia are cellular membranes covering the surfaces and lining the internal cavities of the body. They serve for protection, secretion, excretion, and the reception of stimuli. The constituent cells may be-of-any of the above enumerated forms. The spheroidal types, however, are found only in embryonal membranes. The term spheroidal epithelium is sometimes employed to designate masses or solid columns of spheroidal cells, such as appear in the sex cords of the developing testis and ovary, and in the early stages of glands. They are in general, outgrowths or evagination from embryonic or undifferentiated epithelia.

An epithelium may consist of a single layer of cells, when it is called non-stratified or simple epithelium. A complete description, however, must include the name of the preponderating type of cell, e.g., simple columnar epithelium, or simple squamous epithelium, as the case may be. Moreover, an epithelium may consist of several or many layers, when it becomes a complex or stratified epithelium. The uppermost type of cells gives the name to

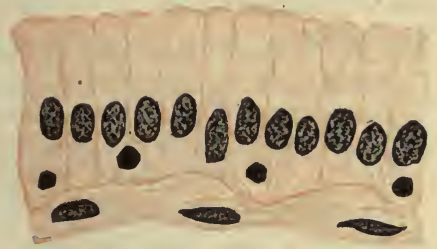

Fig. 39.-Columnar EpitheLIUM From the Pyloric REgion of the Human Stomach. (Profile view.)

Hematein and eosin. $\times 550$. stratified epithelium; for example, in the epidermis the outermost cell is of the squamous type, though the middle cells are polyhedral, and the innermost columnar; hence called stratified squamous epithelium (Fig. 49).

In the stratified epithel:a the superficial cells arise through cell division in the deeper layers, and if they become detached by abrasion, disintegration, or by other physiological or pathological processes, they may be replaced by cell reproduction occurring in the lower layers. When but a single layer of cells is present, as in the simple epithelia, loss of cells over large areas will obviously become more difficult of replacement by cell division. Hence it is that repair of extensively destructive pathological conditions involving such epithelial tissues becomes exceedingly difficult and often impossible, as, for example, in the alveoli of the lung.

Each epithelial cell is to some extent a secreting cell. Sometimes secretion is its chief function, as is the case with goblet cells, which might well be called 'unicellular glands,' and which secrete abundant mucus. The same is true of those cells which form the parenchyma of 
secreting glands-salivary glanrls, kidney, and liver. In many epithelia secretion is a subsidiary function, protection being the primary purpose.

In all epithelia a cement substance is present between the cells. This becomes especially abundant and dense between the distal ends of

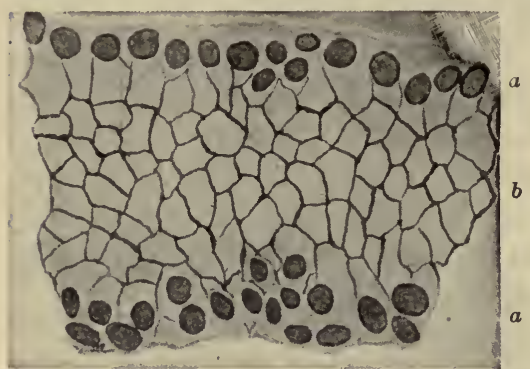

Fig. 40.- 'Terminal Bars' of Cement Substance as Seen between the Epithelial Cells of a Tubular Secreting Gland in the Pyloric Region of the Human Stomach.

The columnar epithelium is seen in profile at $a$; at $b$, the free ends of the cells are seen. Hematein. $\times 550$. the cells of columnar epithelium, and is here known as terminal bars (Fig. 40). Cement substance has the peculiar property of precipitating silver nitrate from solutions, which turns black on exposure to sunlight. This furnishes an especially favorable technic for demonstrating cell boundaries. All epithelia, simple or stratified, rest upon a homogeneous basement membrane or membrana propria, frequently a product of the cells themselves but occasionally of connective tissue origin, and a subjacent connective tissue supporting membrane or tunica propria (or corium). The latter only contains blood and lymph vessels from which the epithelial cell must draw nourishment by process of absorption, and transfer through 'intercellular bridges.' It furnishes support also for the nerve supply. We may now consider briefly the usual types of simple and stratified epithelia. The main facts are summarized in the appended outline:

\section{CLASSIFICATION OF EPITHELIA}

I. SIMPLE (NON-STRATIFIEI)) FPITHELIA-those which compose a membrane but one cell in thickness. Epithelial cells, usually spherical or polyhedral in shape, occur also en masse in the form of cords or clusters.

1. Squamous, composed of flattened, scale-like cells. (a) Lining closed cavities. Pavement epithelium

or (1) endothelium; heart, arteries, capillaries, veins, and lymphatic vessels.

(2) mesothelium; serous membranes.

(3) mesenchymal epithelium; synovial membranes, bursæ, and tendon sheaths, lining of the anterior 
1. Squamous, chamber of the eye, and of the perilymph spaces composed of flattened, scale-like cells. of the internal ear.

(b) Lining the alveoli of the lungs, some tubules of the kidney, the middle ear, and the membranous labyrinth of the internal ear.

(c) As the superficial cells of stratified epithelium (vide infra).
2 Columnar.
(A) Plain
(B) Modified
(1) Ciliated vary, lacrimal, and mammary glands, testicle, prostate, kidney, etc.
(c) The deepest layer of cells in stratified epithelium is composed of columnar-shaped cells, which, however, differ in structure from the true columnar type.
(a) Lining the uterus and ovi- ducts.
(b) Lining portions of the ventri- cles of the brain and central spinal canal of the embryo and infant. (In later life these cells lose their cilia.)
(2) Pyramidal or 'glandular'
(3) Goblet*
The secreting cells of all tubular glands-kidney, pancreas, sali- vary. glands, intestinal glands,
etc.
(a) Respiratory $t$ ract-n a s a l, pharyngeal, tracheal, and bron- chial mucous membranes.
(b) Alimentary tract-stomach, small and large intestines.

(a) Lining the mucous membrane of the alimentary tract-stomach, small intestines, large intestines, gall-bladder.

(b) Lining the ducts of all secreting glands-liver, pancreas, sali-

* Cells whose protoplasm has been converted into mucinogen. They may be considered unicellular, mucus-secreting glands. 

Columnar.

$\left\{\begin{array}{l}\text { (4) Neuro-epi- } \\ \text { thelium. }\end{array}\right.$
(a) Eye-the rod and cone cells of the retina.
(b) Ear-in the cristæ and mac- ulæ of the labyrinth and in Cor- ti's organ.
(c) Nose-in the olfactory mucous membrane (true neuron).
(d) Tongue - in the taste buds.

II. COMPLEX (STRATIFIED) EPITHELIA-those whose cells form several superimposed layers.
1. Squamous. $\{$ deeper, polyhe- dral; the deep- est, columnar in shape.

Forms the epidermis of the skin, and covers the free surface of those mucous membranes which clothe all orifices in direct connection therewith-viz., the conjunctiva and cornea; the external auditory canal; part of the nasal mucous membrane; mouth, pharynx, and esophagus; epiglottis and vocal cords; anus, as high as the internal sphincter; vagina and external portion of the urethra.

(a) Part of ductus deferens.

(b) Respiratory tract; nasal mucolumnar; deeper cells, $\left.\begin{array}{l}\text { (Pseudo- } \\ \text { stratified } \\ \text { columnar). }\end{array} \begin{array}{c}\text { polyhedral or } \\ \text { spindle-shaped. } \\ \text { (a) Non-ciliated } \\ \text { (rare) } \\ \text { (b) Ciliated. }\end{array}\right\}$ $\left.\begin{array}{l}\text { (Pseudo- } \\ \text { stratified } \\ \text { columnar). }\end{array} \begin{array}{c}\text { polyhedral or } \\ \text { spindle-shaped. } \\ \text { (a) Non-ciliated } \\ \text { (rare) } \\ \text { (b) Ciliated. }\end{array}\right\}$ $\left.\begin{array}{l}\text { (Pseudo- } \\ \text { stratified } \\ \text { columnar). }\end{array} \begin{array}{c}\text { polyhedral or } \\ \text { spindle-shaped. } \\ \text { (a) Non-ciliated } \\ \text { (rare) } \\ \text { (b) Ciliated. }\end{array}\right\}$ $\left.\begin{array}{l}\text { (Pseudo- } \\ \text { stratified } \\ \text { columnar). }\end{array} \begin{array}{c}\text { polyhedral or } \\ \text { spindle-shaped. } \\ \text { (a) Non-ciliated } \\ \text { (rare) } \\ \text { (b) Ciliated. }\end{array}\right\}$ $\left.\begin{array}{l}\text { (Pseudo- } \\ \text { stratified } \\ \text { columnar). }\end{array} \begin{array}{c}\text { polyhedral or } \\ \text { spindle-shaped. } \\ \text { (a) Non-ciliated } \\ \text { (rare) } \\ \text { (b) Ciliated. }\end{array}\right\}$ cous membrane and passages connected therewith, tear-ducts, auditory tube, etc., larynx, trachea, and bronchi.

Genital tract; epididymis and vas deferens.
Found only in the urinary system -viz., pelvis of the kidney, ureter, bladder, and first portion of the urethra. 


\section{NON-STRATIFIED EPITHELIA}

\section{Simple Squamous Epithelium}

\section{(Pavement Epithelium)}

This variety of epithelium comprehends two main groups: (1) the endothelia, lining the vascular system, and (2) the mesothelia of the serous membranes lining the large internal closed cavities-pleuræ, pericardium and peritoneum. This distinction is somewhat arbitrary but nevertheless useful, and derives justification in that endothelia arise in the first instance from syncytial mesoderm (mesenchyme) and mesothelium from epithelial mesoderm.

But according to Bremer (Amer. Jour. Anat., $16,4,1914)$, at least some of the earliest bloodvessels in man also arise from true mesothelial cells. Mesothelium lines the extra-embryonic body cavity and is reflected over the yolk-sac and body-stalk. In the latter location Bremer describes ingrowths of mesothelium into the mesenchyme, from which endothelium and blood-cells develop.

This classification should include also another group of closed cavities, namely, the tendon sheaths, bursæ, joint or synovial cavities, chambers of the eye, and the scalæ tympani and vestibuli of the internal ear. These cavities arise as splits, or by the union of isolated spaces, in the mesenchyma, the mesenchymal lining cells taking on epithelioid characters and arranging themselves in the form of a membrane. In their method of derivation these cells resemble more closely the earliest endothelial anlages. The most satisfac-

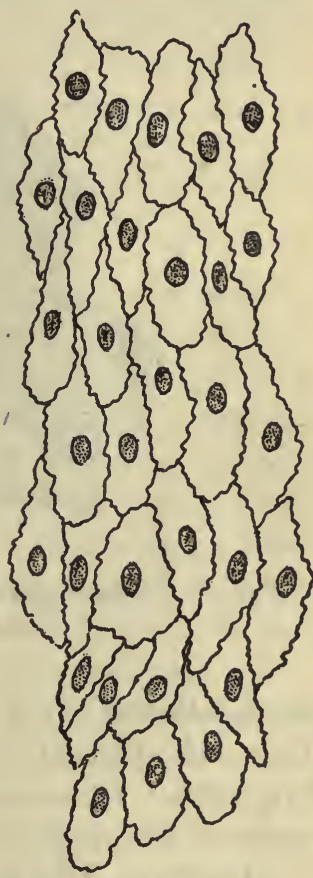

Fig. 41.-SemidiagramMatic ILlustration of ENdothelium LiNING a LARge ARTERY. tory disposition of this group of epithelia seems to be to classify them as 'false' or 'mesenchymal' epithelia, as proposed by F. T. Lewis.

Such epithelia have been experimentally produced by the introduction of small sheets of celloidin and masses of paraffin into the subcuta- 
neous tissue and cornea respectively, of laboratory animals : the connective tissue cells became changed into large flat cells, disposed in the manner of a mesothelium. These results suggest the conclusion that the mesothelial cells of pleura, pericardium and peritoneum may be regenerated in the event of destruction from exposed connective tissue cells of the subepithelial stroma (W. C. Clarke, Anat. Rec., 8, 2, 1914).

The individual squamous cells are flat plates bulging at the center where the oval nucleus is located, with serrated borders. In surface view

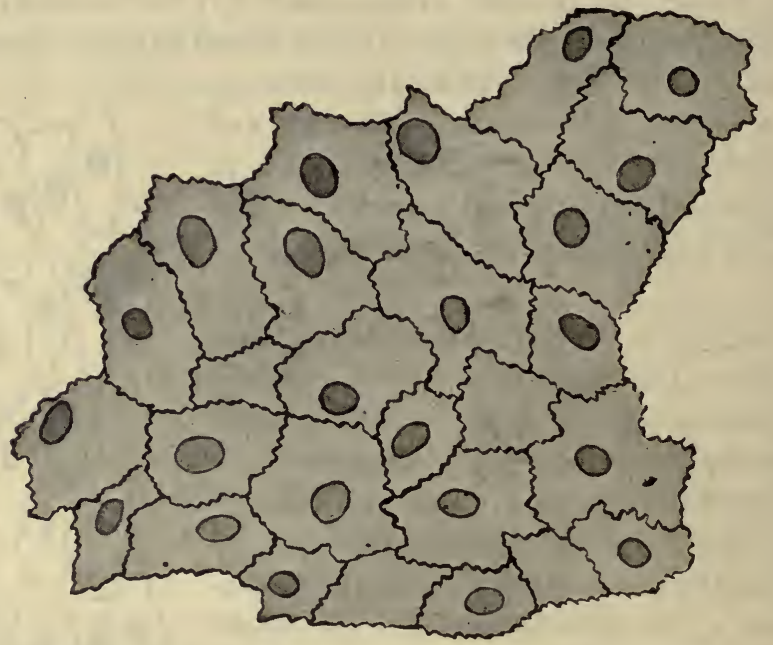

Fig. 42.-Mesothelium (surface view), from the Mesentery of a Rat. Silver nitrate and hematein. $\times 550$.

the endothelial cell is oblong, the long axis parallel with the long axis of the vessel (Fig. 41), while the mesothelial cell is polygonal in outline (Fig. 42). In sections through the nucleus, these cells in side view present a flat spindle-shaped appearance.

Mesothelia exhibit small intercellular spaces, the stigmata. They have been regarded as openings between the body cavities and lymph spaces and vessels; but are more probably transient structures, perhaps artifacts. Abdominal mesothelia of lower forms, e.g., frog, contain also permanent openings, or stomata, surrounded by specialized guard cells.

The mesenchymal epithelial cells of synovial membranes vary greatly in shape according to the degree of pressure to which they are subjected. They may thus be of the cuboidal or the squamous type; and they may even become pressed apart so as to expose the underlying connective tissue. (Compare Figs. 44 and 240.) 


\section{Simple Columnar Epithelium}

\section{(a) Plain}

This type of epithelium consists of columnar or cylindrical elements (Fig. 39), in transverse section presenting polygonal, frequently hexagonal, outlines (Fig. 40). It may be tall, medium or low columnar epithelium, depending upon the height of the individual cell of the particular membrane. The lower types may be designated cuboidal epithelia (Fig. 43). The phenomenon of polarity is partic-

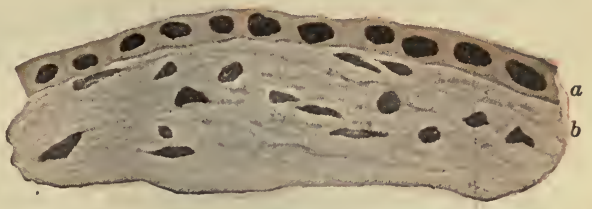

Fig. 43.-Cuboidal Epithelium from the Rete Testis of the Rabits.

$a$, epithelium; $b$, connective tissue. Hematein and eosin. $\times 550$. ularly well exhibited by a tall columnar cell, a condition inhering in a structural and functional differ-

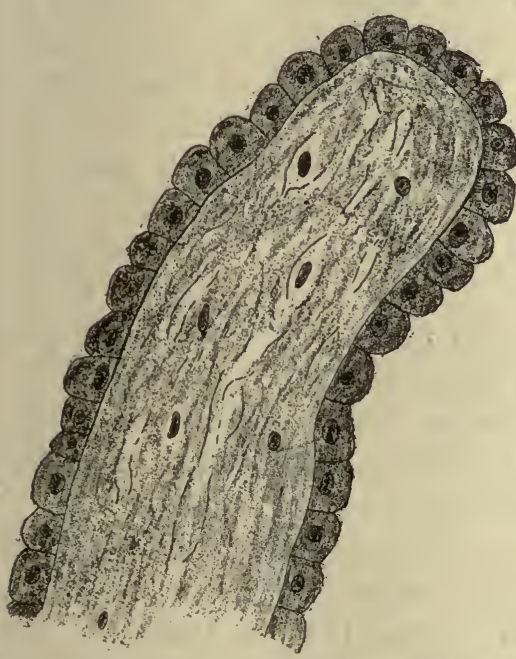

Fig. 44.-Tip of a Villus of the Synovial Membrane from the KneE-Joint of an Old Man.

The core contains capillaries embedded in a compact, delicately fibrillar stroma. A distinct basement membrane appears in certain regions. The epithelium is of the low columnar or cuboidal type. entiation between the attached, or proximal, and the free, or distal, end of the cell, dependent in a final analysis in larger measure upon distance from source of the nutritive and oxygenative stream in the blood. The nucleus is generally located nearer the proximal end; this end, moreover, tapers to a point and is occasionally bifid; and it contains the presecretion (ergastoplasm, prozymogen, etc.) granules, rodlets and fibrils in secreting epithelia (Fig. $35)$. The distal border is frequently striated (cuticular margin, striated border, Fig. 38, b), an appearance due to the presence of minute canals, or more frequently, short pseudopodia, mediating absorption or the elimination of secretion. Striated borders are particularly prominent in the columnar cells of the intestine. In the secreting cells of the 
kidney the marginal processes are more prominent and divergent, forming 'brush borders.' Where the cell membrane becomes greatly thickened on the free surface of a columnar cell, it is termed a crusta. Also, in neuroepithelial cells, the free border mediates reception, the attached pole transmission, of stimuli.

\section{(b) Modified Columnar Epithelium}

(1) Ciliated Epithelium.--Here the columnar cells carry upon their free surface a group of delicate hairlike processes called cilia, or a single flagellum (flagellate cells of lower forms), which during life are

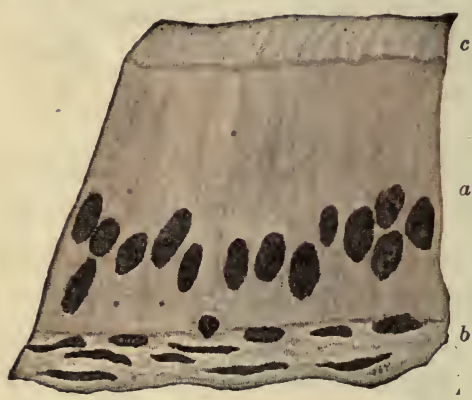

Fig. 45.-Columnar Ciliated EpiTHELIUM FROM THE EPIDIDYMis OF A RABBIT.

$a$, epithelium; $b$, connective tissue; $c$, cilia. A leukocyte is seen between the bases of the columnar cells. Hematein and eosin. $\times 550$. capable of a rapid vibratory or undulatory motion, a further expression of cell polarity. The direction of this ciliary motion is constant and is such as to produce a definite current within the fluids which bathe the surface of these cells whose direction is invariably from within toward the external surface of the body. In the human body the cilia occur almost exclusively upon the free extremities of columnar cells (Figs. 45, 51 and 52). In some of the lower animals, as for example in the mouth of the frog, cilia are found also upon polyhedral and pear-shaped cells. In simplest form cilia are pseudopodlike extensions of the cytoplasm of the cell body, and may be regarded as modifications of its exoplasm.

The ciliated border is separated from the protoplasm of the cell body by a fine chromatic line, which on higher magnification resolves into a number of knob-like segments, the basal granules (Fig. 22, Chap. I). The cytoplasm and nucleus of ciliated epithelium, except for the peculiarities dependent upon the formation of cilia, is similar to that of the simple non-ciliated columnar cells. Their cytoplasm as in other types, may contain vacuoles, pigment granules, metaplasm, and even secretory granules, e.g., epididymis.

(2) Glandular Epithelium.-This type derives its name from the fact of the predominance of the glandular function. 'This condition is 
structurally expressed in segregation of the presecretion bodies (granules, rods, threads) in the basal end of the cell, and of the secretion products (granules, spherules, and mucous or serous fluid) in the distal end (Fig. 35). Morphologically it represents simply a modified columnar cell, its pyramidal shape resulting from mechanical factors due to its disposition in saccular or spherical acini, the periphery of the central lumen of which is much less than the periphery of the acinus, necessitating the modification in shape of the individual cells (Fig. 46). The cells of glandular epithelium usually lack cuticular borders. Pyramidal or glandular epithelium is found in tubules of the kidney, salivary glands, the pancreas, in the secreting glands of the gastric and intestinal mucous membrane, in the mucous glands of the esophagus, pharynx, bronchial tubes and oral and nasal cavities, and in the secreting glands of the skin.

(3) Goblet Cell Epithelium.-A further important and very widespread modification of columnar cells in epithelia concerns the elaboration and storage of mucous secretion, giving to the loaded cells a goblet form (Figs. 47 and 48). Goblet cells may occur among

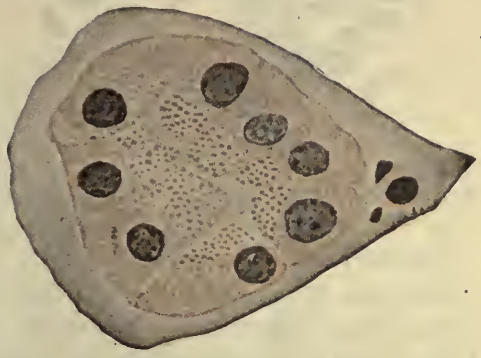

Fig. 46.-A Group of Cells from a Transection of an Acinus of the Human Pancreas; Glandular Epithelium.

Hematein and eosin. $\times 550$. either the plain or ciliated columnar cells. They are most abundant in the intestinal tract but are also to be found in the stomach, bronchial tubes, trachea, nasal mucous membrane, and in the ducts and tubules of mucus secreting glands. In such epithelial membranes certain columnar cells, if not indeed all of these cells, are destined to secrete mucus. The cytoplasm of such cells is converted into a glairy mass of a peculiar vitreous appearance, which occupies an increasing proportion of the free extremity of the cell. 'This 'mucinogen,' when acted upon by alcohol, is precipitated within the cell, and then forms fine basophilic fibrils or granules which stain deeply with the muchematein and mucicarmin solutions of P. Mayer. At the base of the goblet cell, its nucleus is embedded in a minute mass of unaltered granular cytoplasm.

The accumulation of mucus (mucinogen) within the cytoplasm expands the cell, finally. ruptures its wall in the direction of least resistance, and thus permits its mucous content to exude upon the free surface, leaving behind the small granular protoplasmic cell remnant attached to 
the basement membrane. The further history of these cell remnants is somewhat doubtful. They are possibly resorbed or removed, and finally replaced through mitctic division of adjacent cells. There is, however, some evidence to show that after function they are still capable of further

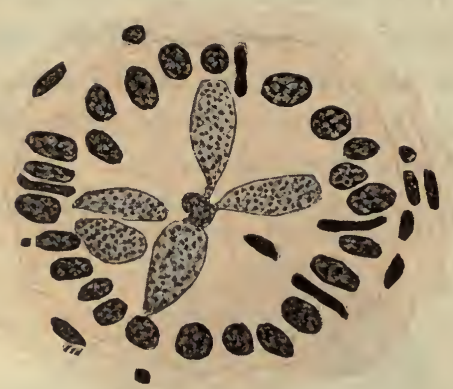

Fig. 47.-Goblet Cells as Seen in a Transection of a Crypt of the LARge Intestine of Man.

Sections of five goblet cells are seen among the columnar cells which line the tubule. Muchematein and eosin. $\times 550$.

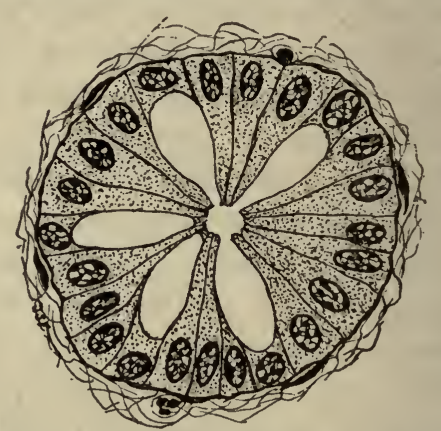

Fig. 48.-Diagram Showing the ARRANGement of THE Columar and Goblet Cells of the Preceding Figure.

The goblet cells are represented as being empty; their unaltered basal portions containing the nucleus are distinctly seen.

growth, whereby they may regain their original form and become again able to pass through the same stages of secretory activity.

(4) Neuro-epithelium.-The cells of neuro-epithelium are columnar elements specially differentiated to form nerve end-organs. They are usually elongated cells having a bulging nucleated center, their free extremity either projecting beyond the epithelial surface as a bundle of fine cilia or as a slender non-ciliated process which terminates within a pore-like opening directly connected with the free surface. Their attached extremity, tapering to a fine process, is in relation with the terminal arborization of the axis cylinder of a nerve fiber. Neuro-epithelium is found only in the several organs of special sense, and will be moro fully described as a part of these several organs. (See chapters of the Eye, the Ear, the Olfactory Organ, the Tongue, and on the Nerve EndOrgans.) 


\section{STRATIFIED EPITHELIUM}

\section{Stratified Squamous Epithelium}

This variety of epithelium occurs as a membrane of varying thickness but always comprising several cell layers. A straight line perpendicular to its free surface would penetrate from five to thirty or more epithelial cells. But while there is a wide diversity in the thickness of the epithelial layers, the character of the cells at any given level is very nearly con-

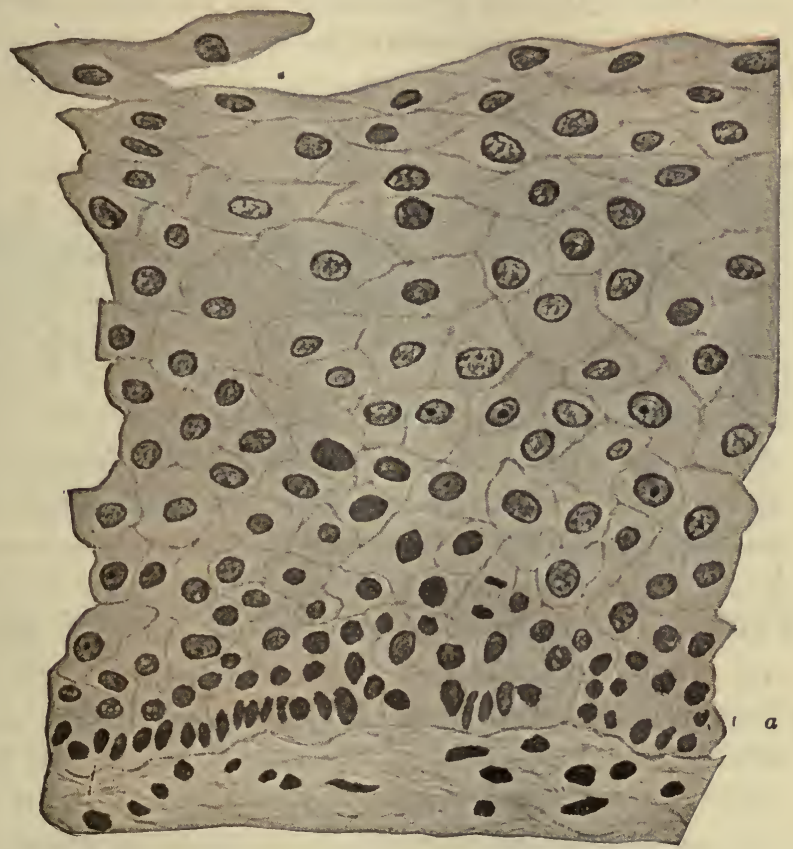

Fig. 49.-Stratified Epithelium from the Human Esophagus.

$a$, basement membrane; $b$, connective tissue. Hematein and eosin. $\quad \times 410$.

stant. Thus the deeper cells, those nearest the basement membrane, are nucleated, of soft consistence and may contain mitotic figures, indicating that it is at this level that cell reproduction is most active. Toward the surface of the membrane the cells become progressively of firmer consistence, so that the most superficial ones present a horny appearance as a result of the gradual keratization of the cytoplasm during the progress 
of the cell toward the surface. The keratization is apparently dependent upon surrounding physical conditions, for it is much more marked in the skin, which from constant and rapid evaporation is comparatively dry, than in the mouth, esophagus, or conjunctiva, where the epithelium is constantly moistened by glandular secretions; the margins of the lips, eyelids, etc., present an intermediate state of keratization.

With these chemical changes in the composition of the cytoplasm there are corresponding changes in its nucleus. In the deeper cells, the nucleus is oval or spherical and highly chromatic. 'Toward the surface, the nucleus becomes more and more flattened and more and more obscured by the cornification of the cell protoplasm. In the most superficial cells it is usually impossible to demonstrate the nuclei, except by acting upon their protoplasm with strong reagents such as caustic alkalies, soda or potassa.

But the most characteristic change in the cells of stratified epithelium is the progressive transition in shape undergone during their passage from the deeper layers to the free surface. . New cells, resulting from indirect division of the cells in the deeper layers, are by continued reproduction gradually pushed toward the surface, whence they are constantly being resquamated in small scaly masses. The pressure exerted in this process tends to gradually flatten these cells, so that their vertical diameter, that perpendicular to the surface, becomes progressively shorter the nearer they approach the free surface; on the other hand, their transverse diameter, that parallel to the surface of the epithelial membrane, is correspondingly increased. The deepest cells of the stratified epithelium-those which rest upon the basement membrane-are elongated in their vertical diameter and possess an irregularly columnar shape. Their nuclei are likewise elongated, oval or elliptical in shape. In the skin of brunettes and the dark-skinned races, and in the epithelium of the skin of the scrotum, perianal region, and areolæ of the breasts, these cells contain small granules of the pigment to which the color of the cuticle is largely due. This columnar cell layer is then described as the layer of pigment epithelium. Superficial to these, but still in the deeper layers, are polyhedral cells with spherical nuclei, which are known as prickle cells because of their prominent intercellular bridges. Superficial to the prickle cells, the epithelial cells become progressively more flattened, until at the surface, they are mere scales. This gradual transition from columnar and polyhedral cells below, to thin flat scales on the surface is characteristic of all stratified epithelium.

The thin superficial scales resemble very closely in shape and appear- 
ance the squamous epithelium previously described. The deeper cells have a finely granular cytoplasm and distinct nuclei except when obscured by the appearance of keratin within their protoplasm. Many of these cells contain coarse granules of eleidin and keratohyalin-substances chemically intermediate between the unaltered and keratized protoplasm.

As stated, the formation of keratin within these cells is more active in those membranes which are comparatively dry from exposure to the air. Consequently, it is most active in the epidermis of the skin. If stratified epithelium is at all times well moistened, as, for example, in the

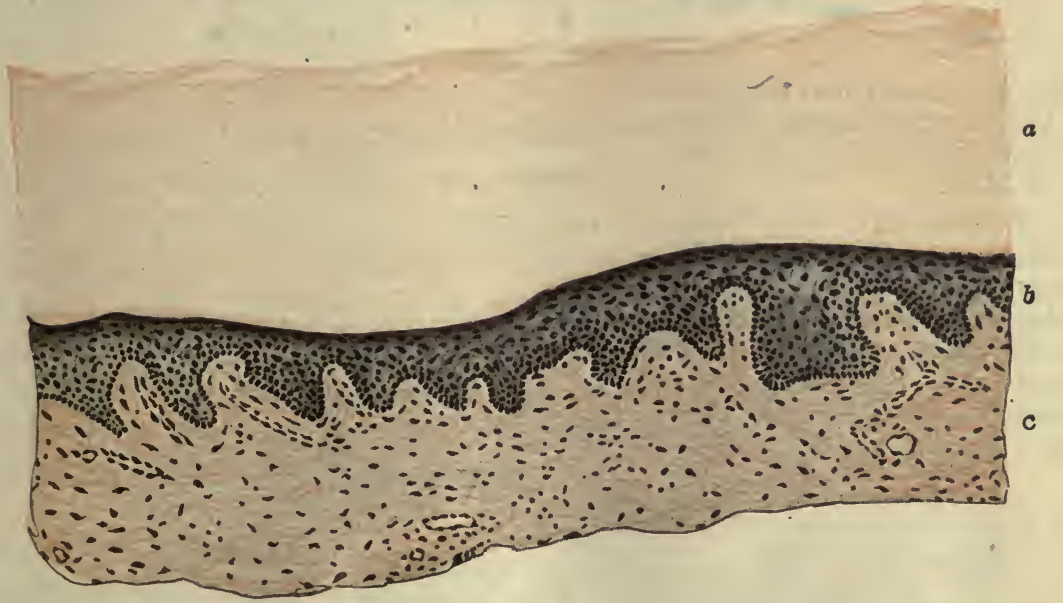

Fig. 50.-Eidermis of the Skin of the Finger Tip, Showing Extreme Keratization of the EPITHELIUM.

$a$, keratized epithelium; $b$, Malpighian or germinal layer; $c$, connective tissue. Hematein and eosin. $\times 50$.

mouth and esophagus, the formation of keratin is slight, and the soft polýhedral cells compose the major portion of the epithelial membrane which then has only a thin superficial covering of flattened scaly cells. In the comparatively dry epidermis, on the other hand, the flattened horny cells frequently occupy more than half the thickness of the epithelial layer (Fig. 50). In the superficial squamous cells of moist membranes the nucleus can always be readily demonstrated, even in the keratized cells of the extreme surface. Cells of the intermediate layers, especially those just above the prickle cell layer, frequently show nuclei in process of amitotic division. This condition is presumably associated with an early stage of degeneration dependent upon a scarcity of nutriment due to the relatively greater distance of these cells from the source of supply. 
It will assist in the understanding of the structure and morphological characteristics of the several layers of cells to think of the superficial

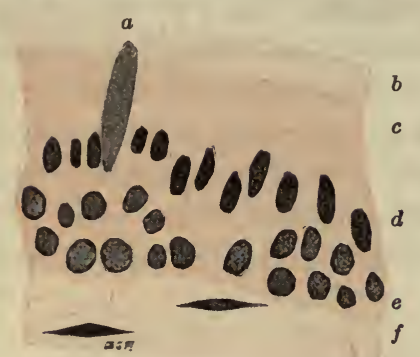

Fig. 51.-P s e U D o- Stratified

Columnar Ciliated EpitheLiUm from a Bronchial TUbe of Man.

$a$, a goblet cell; $b$, cilia; $c$, superficial cytoplasmic layer; $d$, deeper nucleated layer, the nuclei of the columnar cells are somewhat more deeply stained than those of the basal cells; $e$, basement membrane; $f$, connective tissue. Hematein and eosin. $\times 550$. squamous cells in terms of the innermost columnar cells, modified during the passage to the surface by mechanical (pressure), physical (desiccation), and chemical (keratization) factors.

\section{Pseudo-stratified Columnar - Epithelium}

The superficial cells only of this variety of epithelium are columnar in shape, and except in one or two unimportant places are always ciliated. The deeper extremities of these columnar cells taper to a point, and extend all the way to the basement membrane. Between the tapering ends of these cells small spindle-shaped and spheroidal cells are closely packed. The several varieties of cells thus appear to be superimposed, though all actually rest upon the basement membrane. The 'superficial' cells of this variety extend throughout the entire thickness of the membrane. Hence this form of epithelium may in one sense be called 'simple' rather than 'stratified.' The distribution of this variety of the epithelium is practically identical with that of ciliated cells. The deeper extremities of the columnar cells are occasionally bifid or even somewhat varicose in order the more closely to fit between the spindle-shaped and spheroidal cells of the deeper portion. 'The nucleus of these latter cells is usually situated a little below the middle of the columnar cell, so that all the nuclei of the epithelial membrane lie within its deeper half, thus giving to this portion a more deeply

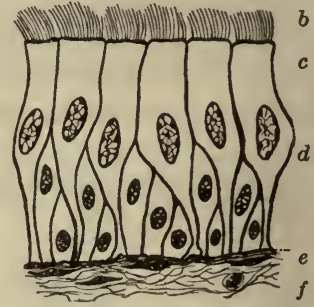

FIG. 52. - D I A G R A M SHowing THE MaNNER IN WHICH ALL THE EPITHELIA L Cells of Pseudostratified Ciliated Epithelium R E A H the Basement MemBRANE.

Letters as in the preceding figure. chromatic appearance when observed in stained sections under low magnification. The superficial half of the epithelial layer contains only 
the cytoplasmic portion of the columnar cells with their ciliated borders.

This type of epithelium is frequently designated simply 'stratified columnar'; and in fact in certain instances under conditions of further modification involving a separation of the taller cells from the basement membrane, it passes, over more or less extensive areas in the respiratory and male genital tracts, into actual stratified columnar epithelium. Toward the proximal end of the male urethra the epithelium is of the true stratified columnar (non-ciliated) type.

\section{Transitional Epithelium}

This variety resembles somewhat stratified squamous epithelium in that it is composed of several cell layers, the deeper cells of which are more nearly polyhedral but are somewhat flattened upon the free surface, but differs in having a smaller number of cell layers-in which respect it is 'transitional' between simple and stratified squamous epithelium-and in the character of the superficial cells. Transitional epithelium is not usually more than from three to ten cells deep, four to six being the rule. The number of cell layers and the consequent actual thickness of epithelial membranes is to a certain extent dependent upon their state of tension during life; thus the

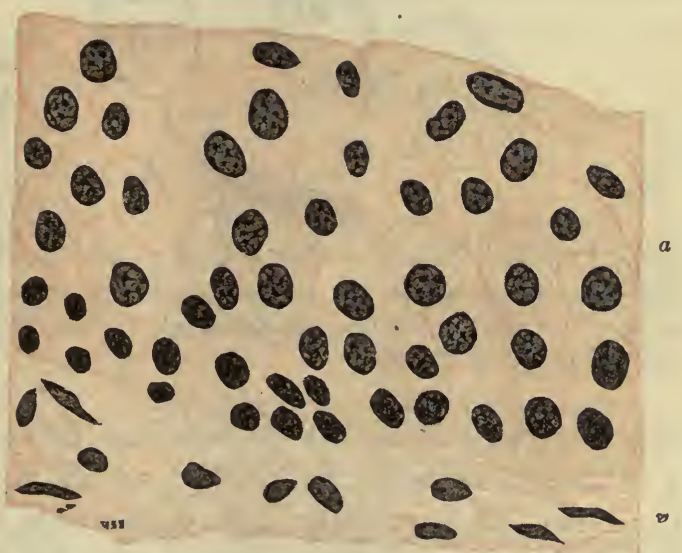

Fig. 53.-Transitional Epithelium from a TranSECTION OF THE URETER OF AN INFANT.

$a$, epithelium; $b$, connective tissue. Hematein and eosin. $\times 550$. transitional epithelium of the urinary bladder is much thicker when the organ is collapsed than during distension.

The deepest cells are polyhedral, and these form the greater portion of the membrane. Only the more superficial layers differ therefrom. Those polyhedral cells which lie in the midregion of the epithelial layer possess a peculiar flask or pear shape, with well-rounded bodies and a 
broad tapering process which is embedded between the adjacent cells of the deeper layers. The rounded extremities of the pear-shaped cells fit into peculiar indentations in the deeper surface of the superficial layer of epithelial cells, producing peculiar concave facets, which are specially characteristic of the detached superficial cells of transitional epithelium.

'The superficial cells, while somewhat flattened, usually have a thickness equal to one-sixth to one-third their transverse diameter. In this respect.they differ markedly from the superficial scaly cells of stratified squamous epithelium and are easily distinguished therefrom, even in the
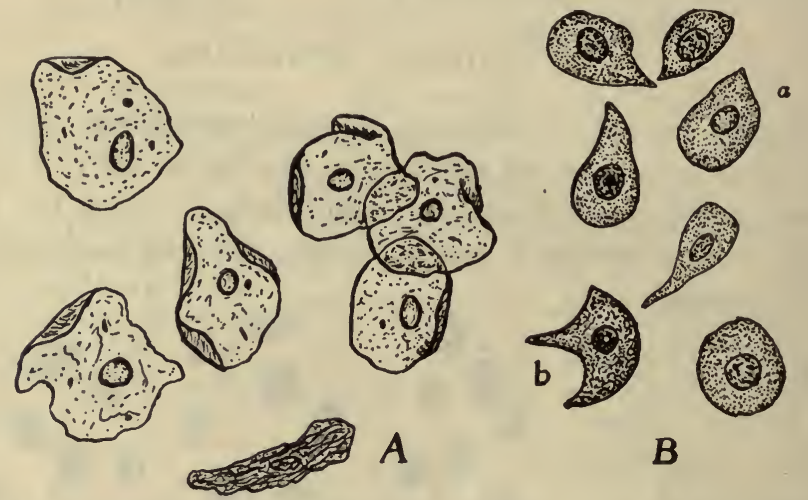

Fig. 54.-Isolated Cells Which May Appear in Human Urine.

$A$, from the vagina of a woman (stratified squamous epithelium); $B$, from the ureter of a child (transitional epithelium); $a$, cells from the deep layers; $b$, superficial cell. Moderately magnified.

isolated condition in which they are frequently found in the urine. The concave facets on their under surface, as well as the peculiar pyriform shape and small size of the deeper cells, are sufficient to distinguish the transitional cells from those of stratified epithelium.

There is little, if any, formation of keratin in transitional epithelium. This is possibly explained by the fact that, as it occurs only in the urinary system, this form of epithelium is always well moistened. Differentiation of this variety of epithelial tissue, though neglected by some authors, becomes most important in the clinical examination of urine where it is necessary to determine the origin of individual cells. Transitional cells from the bladder are easily distinguished from the stratified squamous cells of the vagina, urethra, or epidermis. 


\section{CHAPTER III}

\section{CONNECTIVE TISSUE-CARTILAGE-BONE}

\section{CONNECTIVE TISSUE}

General Statements.-Whilc in the epithelial tissues the cells are developed chiefly at the expense of the intercellular elements, in the connective or supporting tissues the conditions are the reverse. The intercellular elements are here developed out of all proportion to the connective tissue cells. The cells of these tissues therefore are scanty, the ground substance considerable, and within the latter a new element, the connective tissue fiber, makes its appearance. The fibers are of three

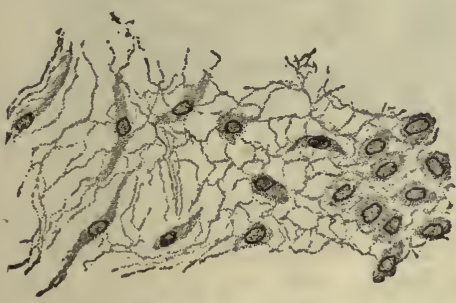

Fig. 55.--Embryonal Connective Tissue, Early Stage.

Highly magnified. (After Mall.)

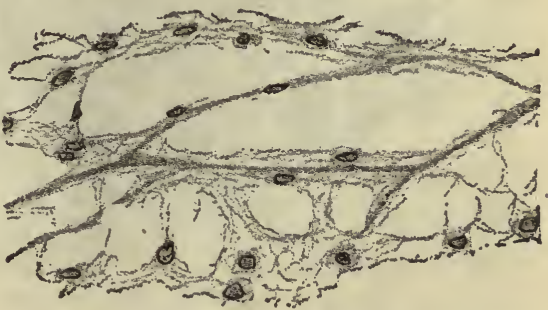

Fig. 56.--Embryonal Connective Tissue at a Later Stage than Is RepRESENTEd in Fig. 55. (After Mall.)

varieties: white or collagenous fibers, elastic fibers, and reticulum. In any given location either of these varieties may predominate to such an extent as to determine the character of the mature tissue, while in the immature forms of connective tissue it is the cellular elements which attain the greatest prominence.

The minute structure of connective tissue is subject to great and important changes during its development. Beginning as it does with the primitive mesoderm, connective tissue is originally a cellular structure. The cells of primitive connective tissue, the fibroblasts, not only increase in number by cell division but also secrete an intercellular ground substance of semifluid consistence. The fibroblasts fuse with each 
other and finally form a syncytial tissue, the mesenchyma, in which there promptly occurs a differentiation of the cytoplasm with the formation of an endoplasm and an exoplasm; and within the latter the fine fibrils soon make their appearance, according to Meves, by processes of fusion and chemical alteration of mitochondria (chondrioconta). This process continues, new ground substance and fibers being con-

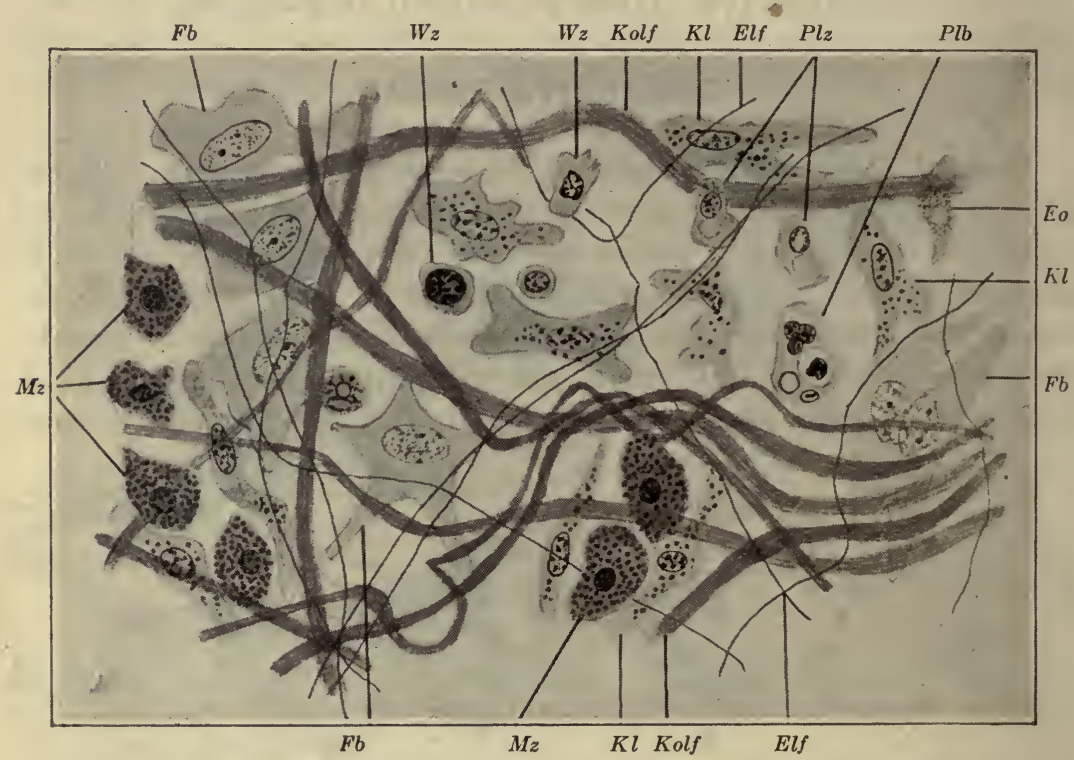

Fig. 57.-Subcutaneous Areolar Connective Tissue of Guinea Pig. (Maximow.)

$E l f$, elastic fiber; Kolf, collagenous (white) fiber bundles; $F b$, fibroblast (lamellar cell); $M z$, mast cell; $W z$, resting wandering cell (clasmatocyte); $P l z$, plasma cell; $K l$, clasmatocyte ('macrophage'); Eos, eosinophil. $\times 1750$.

stantly formed at the expense of the endoplasm, until finally the remnant of the latter again forms isolated cells. The culmination of these changes results in the mature fibrillar connective tissue in which the cells are shrunken and scarce, though still apparently capable of assuming renewed activity on demand of altered conditions. The definitive fibrils result in part from a longitudinal splitting of the coarser primitive fibers, collagenous (Mall), elastic and reticular.

Embryonic connective tissue is therefore typically cellular as compared with the mature type; its ground substance is abundant but the fibers, whose development is as yet incomplete, are scanty. Such embry- 
onic connective tissue is found not only in the fetus but also in early childhood and in the adult, especially during regeneration of destroyed areas of connective tissue, and in other more or less pathological conditions.

\section{Connective Tissue Cells}

Connective tissue cells not only vary in number as they approach maturity, but in their structure and appearance as well. The cells of embryonic connective tissue are comparatively large, are frequently stellate from the presence of numerous interlacing and sometimes anastomosing branches, and their cytoplasm has a typical reticular or granular appearance. In the later stages of their development ameboid motion has been observed in such cells, and within the limits of the tissue in which they are developed, they are presumably endowed with the power of locomotion.

In the neighborhood of developing blood-vessels plasma cells of large size and irregular shape are frequently seen. The cytoplasm of these cells

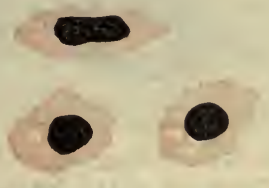

Fig, 58.-PLASMA Cells of ConnecTIVE Tissue From the Human Breast. Hematein and eosin. $\times 750$. is of considerable volume, is finely granular, stains readily in most dyes, especially the basic varieties, and is prolonged into broad protoplasmic branches of considerable length. Both in the cell body and in the processes vacuoles are so numerous as to give the cell a typically reticular

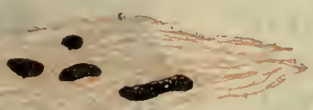

Fig. 59.-SPINDLE-SHAPED Connective Tissue Cells from the Stroma of the Human Ovary. Hematein and eosin. $\times 550$. appearance, a peculiarity which is emphasized by the removal of the contents of the vacuoles, as frequently happens in the preparation of microscopical specimens. Plasma cells are found in considerable numbers in the mucous membrane of the intestinal tract and in the subcutaneous tissue, where they are frequently of spheroidal form.

In the denser forms of mature connective tissue, where the cells are apparently subjected to more or less compression between the firm bundles of fibers, the connective tissue cells lose their typical embryonal stellate form and become somewhat fusiform; they are then known as the spindle cells of connective tissue. Such cells occur in great abundance in the stroma of the ovary and the mucosa of the uterus and oviduct.

In the mature tissue of the adult many of the cells become more or 
less flattened and are often closely applied to, or even wrapped around, the fiber bundles. These lamellar cells have a small nucleus, a consider-

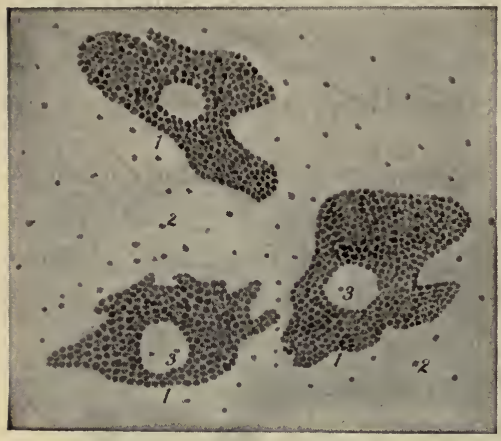

Fig. 60.-Pigmen'ted Cells from the

Choroid Coat of the Ox's Eye.

Unstained; hence, only the pigment granules appear in the figure. 1 , granules contained within the cytoplasm; 2 , free granules which have escaped from cells injured during the process of teasing; 3 , the non-pigmented nuclei.

The cytoplasm of certain cells found in connective tissue contains coarse basophil granules, which stain with dahlia and similar basic dyes. This type is known as basophil granule cells, or mast cells (Mastzellen of the German authors). The granules of other granulocytes are readily stained with acid dyes, such as eosin (eosinophil, acidophil or oxyphil granulocytes). According to the observations of H. B. Shaw (Jour. Anat. and Physiol., 1901), certain of the granule cells abound in those locations where fat is deposited, and have a special relation to the development of the fat cells of adipose tissue. These granulocytes of fibro-elastic connective tissue are apparently identical with those of the blood. Lymphocytes and phagocytic leukocytes are also present in connective tissue.

It is a disputed point whether the granulocytes of connective tissue differentiate from fibroblasts or from lymphocytes; the weight of evidence seems to incline to the latter position. Plasma cells seem more próbably altered fibroblasts but have also been regarded by some as lymphocyte derivatives. The so-called 
'resting-wandering' cells, or 'clasmatocytes,' are perhaps to be regarded as varieties of basophilic granulocytes characterized principally by the presence of irregular protoplasmic processes. According to Kite (Jour. Infec. Dis., 15, 2, 1914) the 'clasmatocytes' described for the frog by Ranvier in 1891 are lymphocytes which have protruded pseudopods. Evans classifies them with the 'macrophages' of Metschnikoff.

\section{Types of Connective Tissue}

The proportions and character of the cells and fibers present in any given connective tissue, to a certain extent determine its character. If the collagenous fibers of connective tissue-are closely packed in dense parallel bundles, the elastic fibers being comparatively insignificant in number, the type of connective tissue may then be said to be dense fibrous or white fibrous tissue.

In elastic tissue on the other hand, the yellow elastic fibers are highly developed, the white fibers forming only insignificant and very delicate sheaths which inclose the coarser elastic fibers.

Again, it is the variety of delicate connective tissue fiber known as reticulum which preponderates in reticular tissue, and if the meshes of this reticular network become infiltrated by lymphocytes, which then multiply by division until they exceed the other tissue elements, the connective tissue is then said to be of the lymphoid or adenoid variety. Large numbers of the fixed cells of areolar connective tissue may change into fat cells, the tissue as a whole then forming adipose tissue. In all we distinguish the following varieties of connective tissue: (1) Embryonal; (2) mucous; (3) reticular; (4) loose fibro-elastic or areolar; (5) dense fibrous; dense elastic; (7) adipose; (8) adenoid; (9) cartilage; (10) bone.

Embryonal Connective Tissue.-Embryonal connective tissue (Figs. 55 and 56) occurs not only in fetal and infantile life, but also during the regeneration of destroyed connective tissue areas and in pathological neoplasins. It is distinctly cellular in character. Its cells are spindleshaped and stellate, are much branched, and through their larger processes they frequently anastomose.

The fibers are extremely fine; they are not usually arranged in bundles, but form a delicate network which permeates the ground substance in every direction. In the very immature types the fibers are all of the collagenous variety; delicate elastic fibers appear later. The fluid ground substance forms an abundant mass of tissue juice which occupies the meshes of the fibrous net. 'The earliest developmental stages are identi- 
cal with mesenchyma; from the viewpoint of progressive differentiation it properly heads the list of connective tissues.

Mucous Connective Tissue.-Mucous, gelatinous or mucoid connective tissue occurs only in the umbilical cord, where it forms the 'jelly of Wharton,' and in the vitreous humor of the eye. Its semifluid ground substance is of a gelatinous consistence and forms the greater portion of the tissue; in the vitreous humor there is little else.

The cells are mostly of the branched lamellar variety, are few in num-

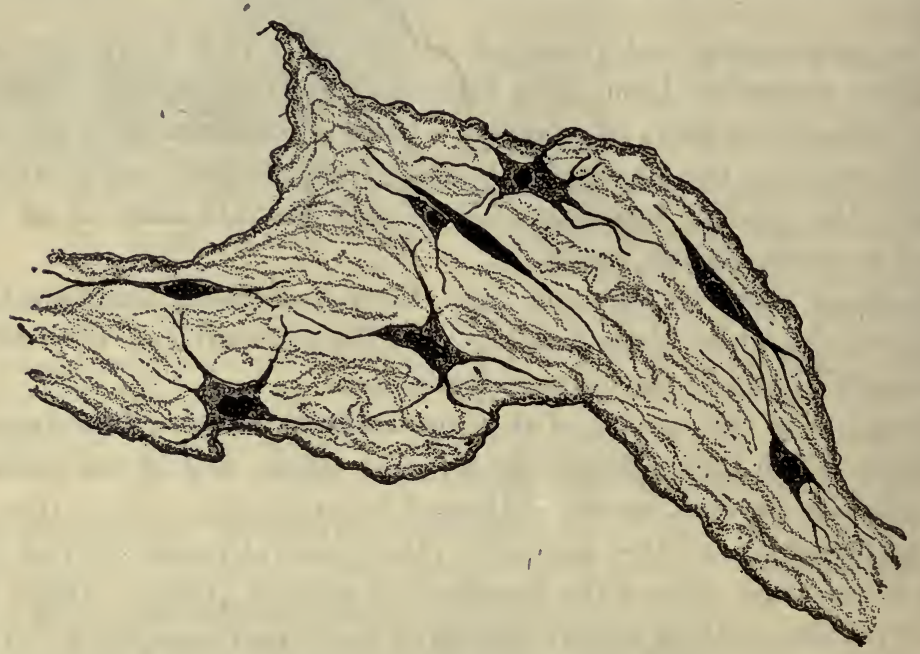

Fig. 62.-Gelatinous Connective Tissue from the Umbilical Cord of a NewBORN INFANT.

Safranin and water blue. $\times 410$.

ber in the vitreous, but more abundant in the umbilical cord. In the vitreous humor also, there are very few fibers; those which are present are very fine and form a delicate reticulum. In the umbilical cord the fibers are more abundant, and possess a tendency to form bundles which are disposed in parallel cylindrical layers around the large bloodvessels. Elastic fibers are wanting. This type lacks also nerves, bloodvessels and lymph-vessels; the two large umbilical arteries and the umbilical vein have no vascular connection with the mucous tissue of the cord.

The essential chemical body in mucus is mucin, a glycoproteid. The most typical mucous substance is the secretory content of goblet cells. The mucus of embryonic and gelatinous connective tissue is closely 
similar. Less closely similar 'mucous' substances of the more compact connective tissues are properly designated, mucoids.

Reticular Tissue (Reticulum).-Reticular tissue occurs as the stroma of adenoid tissue in the lymphatic glands and other lymphoid organs, and according to Mall (Johns Hopkins Hosp. Rep., 1896), is found also in the membrana propria of the secreting tubules of the stomach, intestine, kidney, testis, and thyroid, and in the marrow of bone and the walls of the pulmonary air sacs.

Like the other connective tissues, reticular tissue consists of cells, fibers, and ground substance; the latter, however, is no more than a fluid tissue juice which, at least in the lymphoid organs, is identical with the lymph. The fibers are extremely fine and are arranged in slender bundles, which freely anastomose to form a delicate close-meshed reticulum. Individual fibers can be readily demonstrated in these bundles only after the action of alkalies, digestion by artificial gastric juice, or wy other methods of dissociation, yet on careful examination indications of fibrillar structure can be zeen in the reticulum of fresh tis-

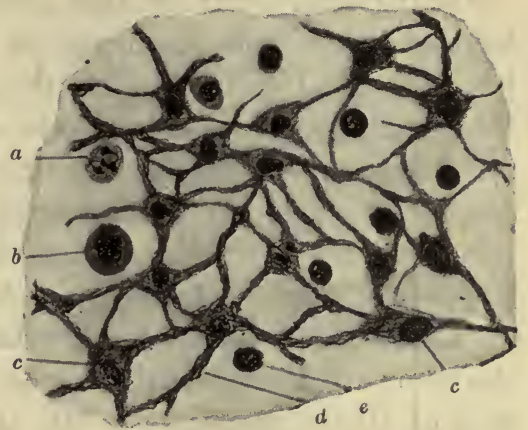

Fig. 63.-Reticulừ of a Cervical Lymph Node of Man, from a Thin SeCtion From Which the Lymphatic Corpuscles Had Been Partially WASHED OUT.

$a$, polynuclear lymphatic corpuscle; $b$, large mononuclear cell; $c$, connective tissue cells of the reticular tissue; $d$, fibrous bundle of the reticulum; $e$, small mononuclear lymphocyte. Hematein and eosin. $\times 500$. sue and in ordinary microscopical preparations. The chemical reactions of the reticular fibers are similar to those of collagenous fibers except that the former are much less readily digested by artificial gastric juice.

Flattened connective tissue cells clasp the bundles of reticular fibers; they are mostly found at the intersections of the anastomosing bundles. This fact was accountable for the former theory, which regarded reticular tissue as formed by the anastomosing branches of stellate cells. The careful investigations of Carlier (Jour. Anat. and Physiol., 1895) and others have shown the true nature of the lamellar cells and their underlying fiber bundles.

The fibers of reticular tissue very closely resemble the collagenous fibers of areolar tissue, but differ from them in having a clearer, more 
highly refractive appearance. Their digestion in pepsin begins only after an interval of two hours, while white fibers are digested in a few minutes; they also stain less readily than white fibers and yield reticulin, which differs somewhat from the gelatin of white fibrous tissue. The intimate histologic relation between the reticular and white fibrous tissue is shown . by the fact that the two tissues are frequently continuous and exhibit similar staining reactions.

Mall (Amer. Jour. of Anat., 1902) has attempted to show that reticular tissue should be considered as that form of connective tissue which has been least differentiated from the embryonic mesenchymal type. $\mathrm{He}$ accordingly considers the cells of the reticulum as formed by the undifferentiated endoplasm, and the reticular fibers as representing the specialized exoplasm of this most primitive type of true connective tissue. In the liver, the reticulum arises from the endothelial cells of von Kuppfer instead of from mesenchyme (Mall).

Loose Fibro-elastic or Areolar Connective Tissue. - Loose fibroelastic or areolar connective tissue (Fig. 5\%) is the most widely distributed of all the varieties; it fills all otherwise unoccupied spaces within the body, and in all microscopical sections areolar tissue is almost invariably to be found. It is also known as loose connective tissue in contradistinction to the more compact or dense varieties. This tissue connects the skin with the underlying structures, maintains the position and relation of adjoining muscles, surrounds the heart and its great vessels, envelops the abdominal viscera as submucous and subserous sheets, occupies the spaces of the mediastinum, and fills similar intervals between the various organs in all parts of the body. Areolar tissue of course varies in the degree of its laxity or density.

The ground substance of areolar tissue is a coagulable fluid, the tissue juice. Solutions of silver nitrate injected into the interstices of areolar tissue coagulate its tissue juice or ground substance and darken it slightly. It is then seen to be permeated by broad lymphatic channels, which are lined by delicate endothelioid mesenchymal cells (W. G. MacCallum, Arch. f. Anat., 1902 ; also Bull. Johns Hopkins Hosp., 1903).

Both collagenous and elastic fibers occur in areolar tissue, the former being far in excess of the latter. The comparatively loose reticular arrangement of the fibers of fibro-elastic tissue affords a most favorable opportunity for the study of these connective tissue elements.

The collagenous or white fibers in mature tissues are invariably arranged in bundles which interlace with one another to form an open network. Each bundle consists of a number of very fine fibers whose 
course is characteristically wavy or undulating. Though the individual fibers rarely branch, the fiber bundles frequently anastomose with one another. 'The white fibers are readily stained with most 'acid' dyes, and possess a special affinity for acid fuchsin. Chemically they consist of the albuminoid collagen, which on boiling in water yields gelatin, and is readily dissolved by boiling in dilute acids or alkalies. Collagen fibers are digested by artificial gastric juice in five or ten minutes but are

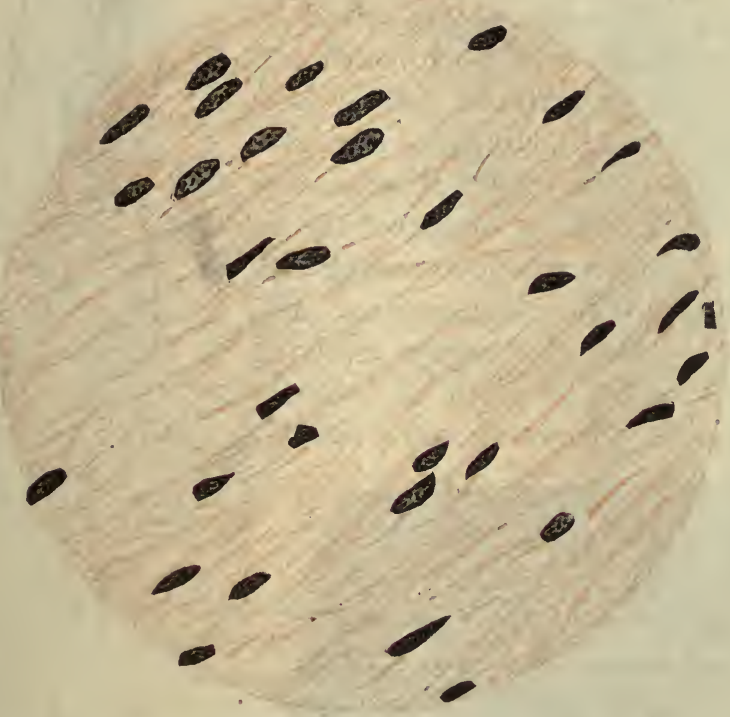

Fig. 64.-Dense Fibrous Tissue from the Tendon of One of the Ocjuar Muscles of a Child.

Hematein and eosin. $\times 550$.

scarcely altered after several hours when acted upon by solutions of pancreatin. After boiling, however, white fibers are readily digested by pancreatin. In dilute acids they swell and become transparent.

The elastic fibers of areolar tissue, in comparison with the collagenous fibers, are few in number. They occur as isolated fibers-never in bundles - which frequently branch and anastomose, forming in this way a very fine net with wide meshes, within which are the interlacing bundles of white fibers. The elastic fibers exist under a certain tension during life, so that their course under favorable conditions is invariably straight. When areolar tissue is removed from the body this tension is frequently relieved and the elastic fibers then curl up, especially at their free ends. 
Under these conditions they are no longer straight, but present a gracefully curved contour. The elastic fibers also possess a glassy, shining, or highly refractive appearance, the collagenous fibers by comparison looking dull and opaque.

Elastic fibers stain but slightly with most dyes; they are readily colored by orcein and by Weigert's elastic tissue stain (resorcin-fuchsin), both of which serve as specific dyes for these fibers, coloring the fibers dark brown or black. Elastic fibers are not dissolved by dilute acids or alkalies even when boiled, and are only digested by artificial gastric juice after a lapse of several hours; they are, however, readily digested in faintly alkaline solutions of pancreatin. They consist of the albuminoid body, elastin, which on boiling does not yield gelatin. Both collagenous and elastic fibers arise by a similar process involving transformation of the exoplasm of their respective fibroblast progenitors into a fibrillar

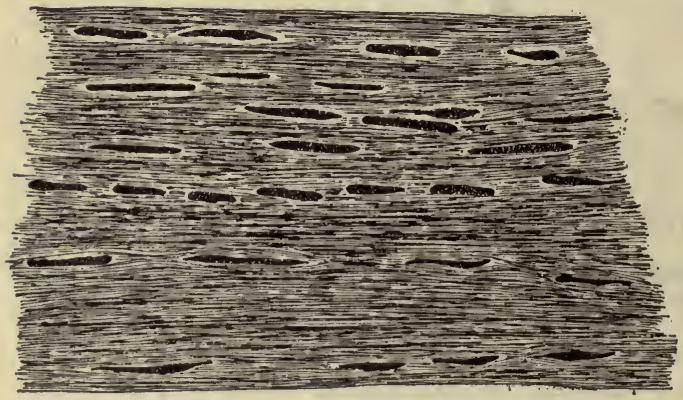

Fig. 65.-Longitudinal Section of Tendon of Human Finger.

Only the nuclei of the tendon cells are conspicuous, scattered in rows among the collagenous fibrils. The rows of nuclei mark the boundaries of the primary bundles. $\times 750$.

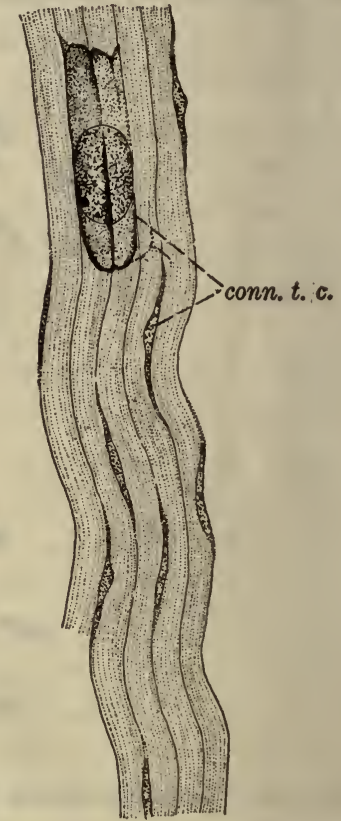

Fig. 66. - Portion of Tendon From a Cow.

conn. $t$. c., connective tissue cells (tendon cells) seen from the side and, in one case, from the surface. (From Dahlgren and Kepner's "Animal Histology," Macmillan Co.)

structure. Whether the fibers are deposited as such or arise by coalescence of more fundamental granular elements is a disputed point.

The cells of areolar tissue are few in number, but may include any of the several varieties, though lamellar and spindle cells together with leukocytes form the more common types. Many of the lamellar cells are 
closely applied to, or even wrapped around the bundles of white fibers. Fat cells occur in considerable numbers in all areolar tissue and in some places are aggregated into large groups which form lobules of fatty tissue.

Dense Fibrous Tissue.-In dense fibrous tissue the ground substance is comparatively deficient. Large bundles of collagenous fibers are arranged in approximately parallel rows, and are so closely packed as to

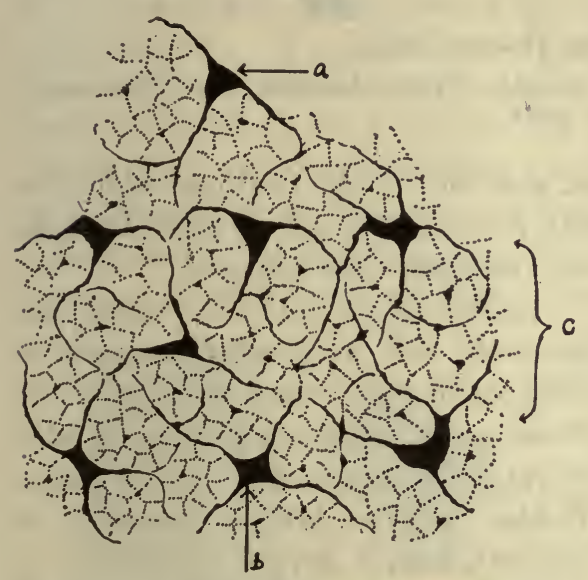

Fig. 67.-Transverse Section of Portion of Tendon of Human Finger.

$a$, three-winged cell; $b$, four-winged cell; $c$, primary bundle, completely ensheathed by the wings of tendon cells, and divisible into still smaller bundles of collagenous fibers outlined by finer processes of the wings. The individual fibers are not shown. Gold chlorid. $\times 1000$.

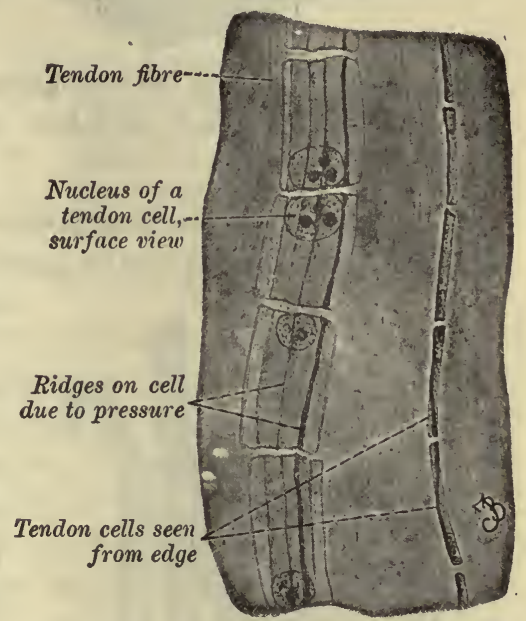

Fig. 68.-Piece of Tendon from TaIL of White Mouse.

Between the bundles of connective-tissue fibrils are cells arranged in rows. Some are seen in surface view, and others in optical section. $\times$ 400. (From Szymonowicz-MacCallum, "Histology and Microscopic Anatomy.")

form a dense, firm, highly resistant tissue. Its scanty connective tissue cells are of the lamellar variety, and are usually arranged in rows which occupy the interstices between the parallel fiber bundles.

Dense fibrous tissue occurs typically in tendons; in these the connective tissue cells often have a peculiar quadrate shape and are arranged in rows of exceptional regularity (Figs. 64-67). These should be studied in dissociated tendinous tissue. It also forms the ligaments, the fasciæ, the muscular sheaths (aponeuroses), and the enveloping capsules of many of the viscera. Thus it surrounds the liver, kidney, lymphatic nodes, and other organs; it also forms the valves of the heart, the tendinous rings 

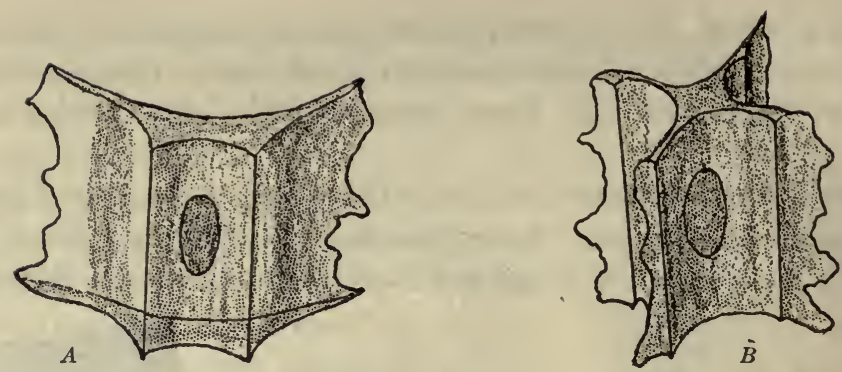

Fig. 69.-Isolated Tendon Cells.

$A$, with two 'wings'; $B$, with four 'wings.' (From Maximow, after Tourneau.) $\times 1000$.

which surround the cardiac orifices, and the chordæ tendineæ which are attached to its valves; and in general, it is found wherever great firmness

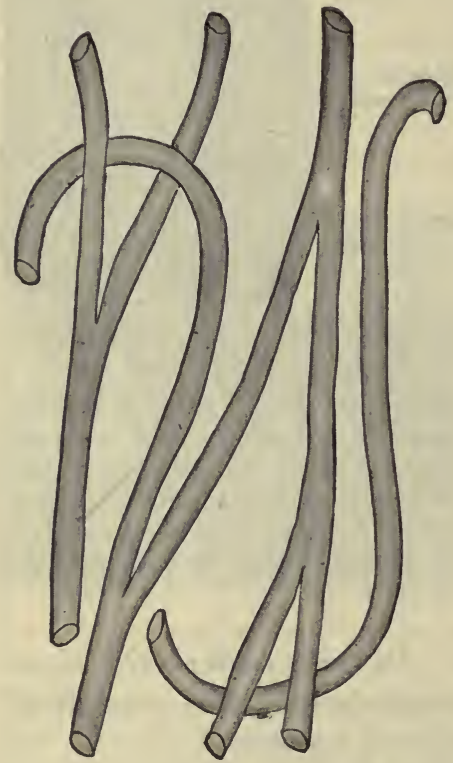

Fig. 70.-Coarse Elastic Fibers From the Ligamentum Nuche OF THE OX.

Isolated by teasing. Partly diagrammatic. $X$ about 250 . and resistance are required.

Elastic fibers in this tissue are relatively few in number and are so obscured by the dense bundles of white fibers as to be scarcely demonstrable except by means of the specific stains. Tendon will be further discussed in connection with striped muscle.

Dense Elastic Tissue.-I $n \quad t h$ i s form of tissue the elastic fibers are developed at the expense of the collagenous fibers. The ground substance is insignificant in amount, and the connective tissue cells are scanty and are confined to the white fibrous sheaths in which the elastic fibers are enveloped. The elastic fibers are of very large size (10 to $15 \mu$ ) as compared with those of other forms of connective tissue. But except for their larger size, these fibers have the same peculiar characteristics as the elastic fibers of areolar tissue. In their straight course, frequent branches, and their glisténing, highly refractive appearance, as also in their characte-istic reactions to specific dyes and other reagents, these fibers are identical 
with the elastic fibers of the other types of connective tissue.

The elastic fibers are bound together by delicate sheaths of very fine collagenous fibers, and are united into bundles by coarser bands of fibrous tissue. Elastic tissue is found in the ligamenta flava, the stylohyoid ligament and in the ligamentum nuchæ ('whitleather') of quadrupeds. In these locations it occurs in considerable quantity and has a peculiar yellowish color; it is for this reason that it is frequently described as yellow elastic tissue. It occurs also as fen-

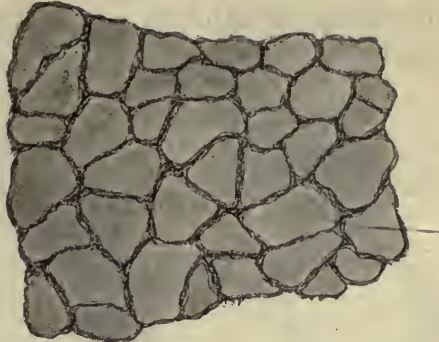

Fig. 71.-Transection of a FascicUlus of the Ligamentum Nuche of the Ox, Showing the Very Large Elastic Fibers Embedded in a Very Delicate Network of Collagenous Fibers.

Picro-fuchsin. $\times 550$. estrated membranes in arteries. These are formed by a coalescence of

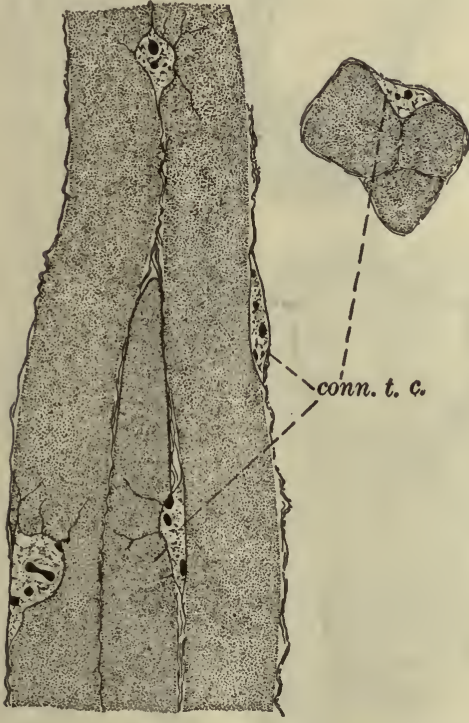

Fig. 72.-Portion of Ligamentum NuCHa OF Ox.

conn. t. c., connective tissue cells. (From Dahlgren and Kepner.) neighboring fibers. In the process of occlusion of the postfetal ductus arteriosus of the pig by increase in the amount of the elastic tissue in the wall of the artery, the new elastic fibers arise both from latent fibroblasts and by delamination of fibers from preformed elastic tissue (J. $P$. Schaeffer, Jour. Exp. Med., vol. 19, 1914).

\section{Adipose Tissue (Fat Tissue).-} Wherever areolar tissue occurs, adipose tissue may also be found; its distribution is therefore identical with that of areolar tissue. It forms a considerable mass, panniculus adiposus, beneath the skin of many parts. In it are embedded the kidneys, adrenals, and many lymphatic nodes. The mesentery and omentum are freely supplied with fat. The same tissue is found in the grooves of the heart wall and it also occupies the spaces of the mediastinum.

Adipose tissue is composed of lobules or groups of fat cells which are 
supported by fibrous bands and septa and are abundantly supplied with small blood-vessels.

The fat cells arise from the connective tissue cells by a deposit of fat droplets within the cytoplasm of the latter. These droplets continue to increase in number and fuse with each other to form globules

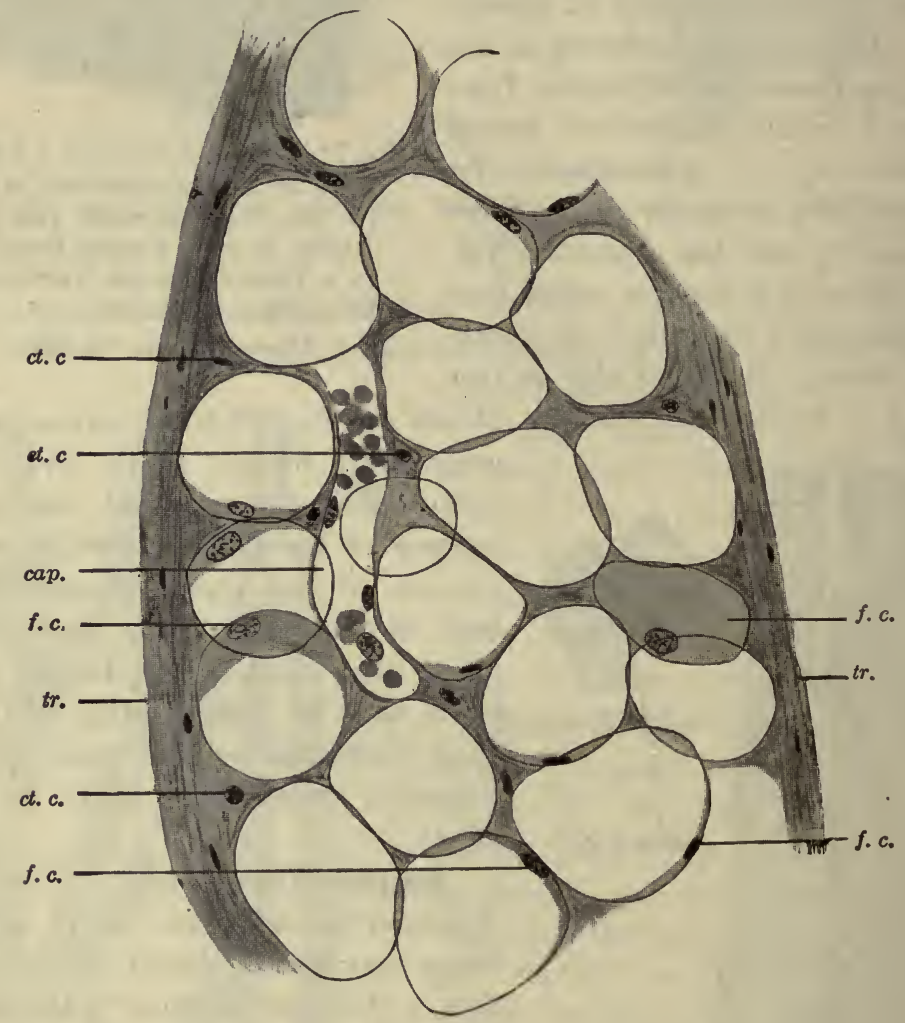

Fig. 73.-Portion of a Fat Lobule from the Areolar Connective Tissue Surrounding the Esophagus of a Cat.

cap., capillary; ct. c., nucleus of a connective tissue cell; $f$. $c$., fat cell showing nucleus; $t r$., trabecula of fibro-elastic connective tissue. $\times 500$.

of increasing size, until the cytoplasm finally becomes so excavated as to form a mere limiting membrane or cell wall (Fig. 74). The nucleus is pushed to one side in this process and is flattened against the cell membrane; it is usually embedded in a remnant of granular cytoplasm. Being thus distended with fluid fat, the cell acquires a spheroidal shape.

The routine specific stains for fat are osmic acid, which colors the 
fat globules black; sudan III, which gives a red reaction; and scharlack $R$ (fettponceau), which also stains fat red. For the successful application of these stains it is required that the tissue has not been previously subjected to treatment involving the use of alcohol or ether, since these reagents extract fat from the cells. Fat, in cooling, solidifies and precipitates delicate threads, the margarin crystals.

During periods of starvation or malnutrition, at which time fat decreases greatly in volume, many of the fat cells return to a condition which approximates their former state. As the fat is removed the cytoplasm of the cell increases in amount, but assumes a peculiar fluid appearance and is not readily colored by the usual dyes. These cells, which still contain a number of fat droplets, are known as 'serous' fat cells.

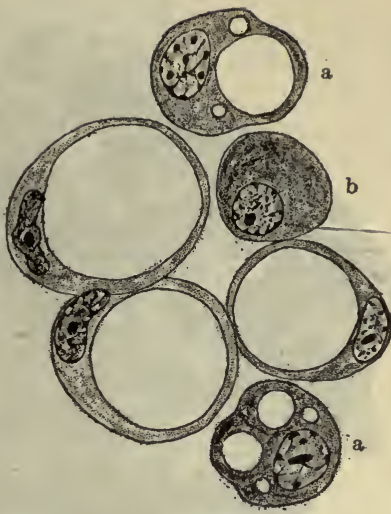

Fig. 74.-A Group of Fat Cells from the Subcutaneous Tissue OF A Young RABBIT.

Cells $a$ show stages in development; cell $b$ is cut tangentially through the nucleated pole. $\times 1000$.

The origin of the fat cell is still somewhat in doubt. It was for-

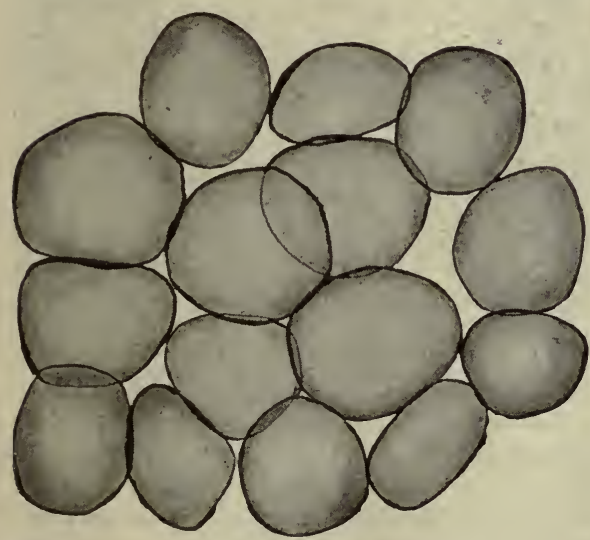

Fig. 75.-Fat Cells from a Teased Preparation of Adipose Tissue of Man. $\times 110$. merly thought that it might result from a deposit of fat within any of the connective tissue cells. A second theory maintains that it arises only from a special fat-forming connective tissue cell. The demonstration of large numbers of peculiar ovoid granular cells within areas where fat cells were undoubtedly forming in fetal and young subjects, and the demonstration of similar cells in areas showing fat formation in adult tissues, has lent support to the hypothesis that these granular cells are the only progenitors of the fat cells (Shaw, Jour. Anat. and Physiol., 1901). According to Weiskotten and Steensland (Anat. Rec., 


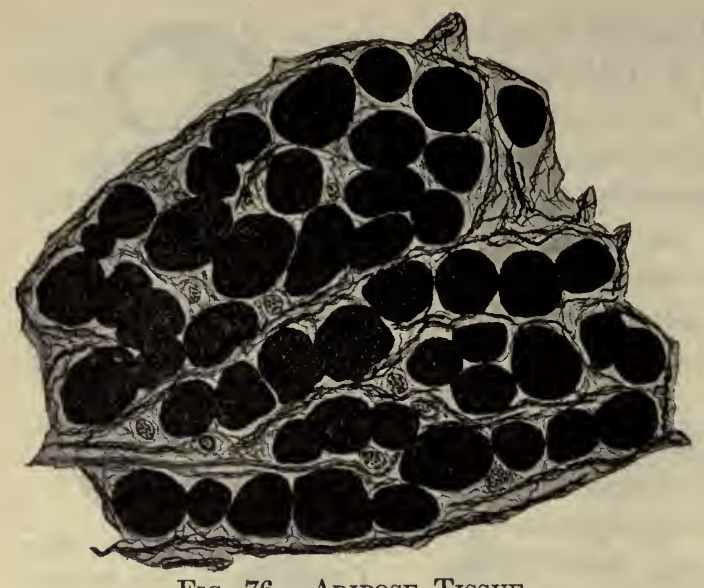

Fig. 76.-Adipose Tissue.

8, 2, 1914) fat cells can arise also by a process involving the enclosure of free fat spherules by endothelial cells. They suggest that fat cells may be modified endothelial cells rather than modified fibroblasts.

The forerunners of the original smallest fat droplets are granules (Altmann, 1890). - In the subcutaneous The fat cells have been blackened by osmium tissue of Myxine (Hag-
tetroxid. $\times 110$. fish) embryos, Schreiner (Anat. Anz. 48, $\%$, 1915) has described the process of fat elaboration in minute detail. The pre-fat granules originate from rod-like chromidia ('mitochondria') by process of segmentation. The chromidia arise as nucleolar buds which wander through the nucleus and traverse the nuclear membrane as spherical granules. These 'primary granules' elongate into rods, and subsequently segment into 'secondary granules,' which liquefy and coalesce to form the definitive fat spherules. This important investigation suggests a functional rôle for mitochondria in terms of a nutritive material upon which cell metabolism and differentiation may depend.

\section{Lymphoid Tissue (Ade-}

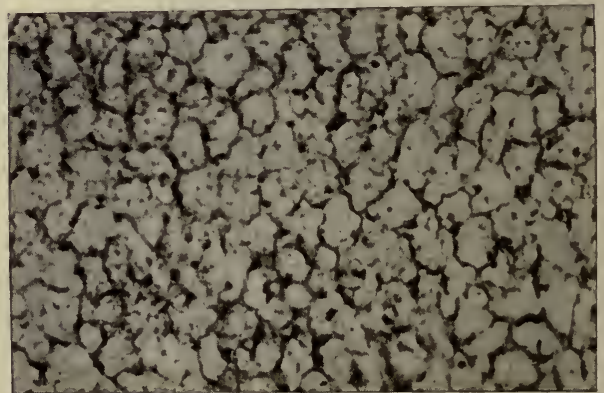

Fig. 77.-Developing Adipose Tissue from the Subcutaneous Tissue of an Infant.

The fat has been removed by immersion in alcohol and ether. The polygonal outlines of the fat cells are well shown. Within many of them is seen the finer cytoplasmic network by which the inclosed droplets of fat were invested; this network had not been completely replaced by the accumulation of fat. Hematein and eosin. Photo. $\times 325$. noid Tissue).-Lymphoid tissue is a reticular tissue the meshes of whose metwork are occupied by a closely packed mass of lymphocytes, cells with 


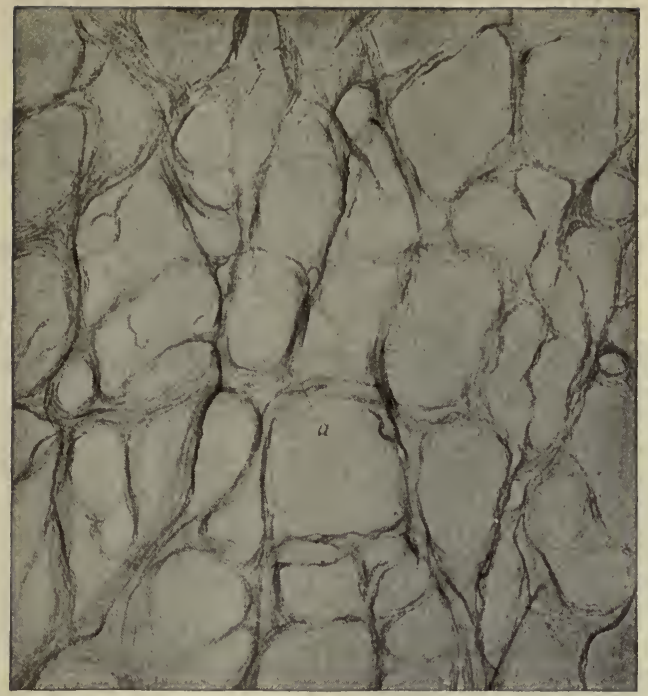

Fig. 78.-Reticulum from the Mucosa of the Fundus Region of the Dog's STOMACH.

The section was made parallel to the surface and the glandular tissue removed by shaking in water. Picro-carmin. $\times 125$. (After Mall.)

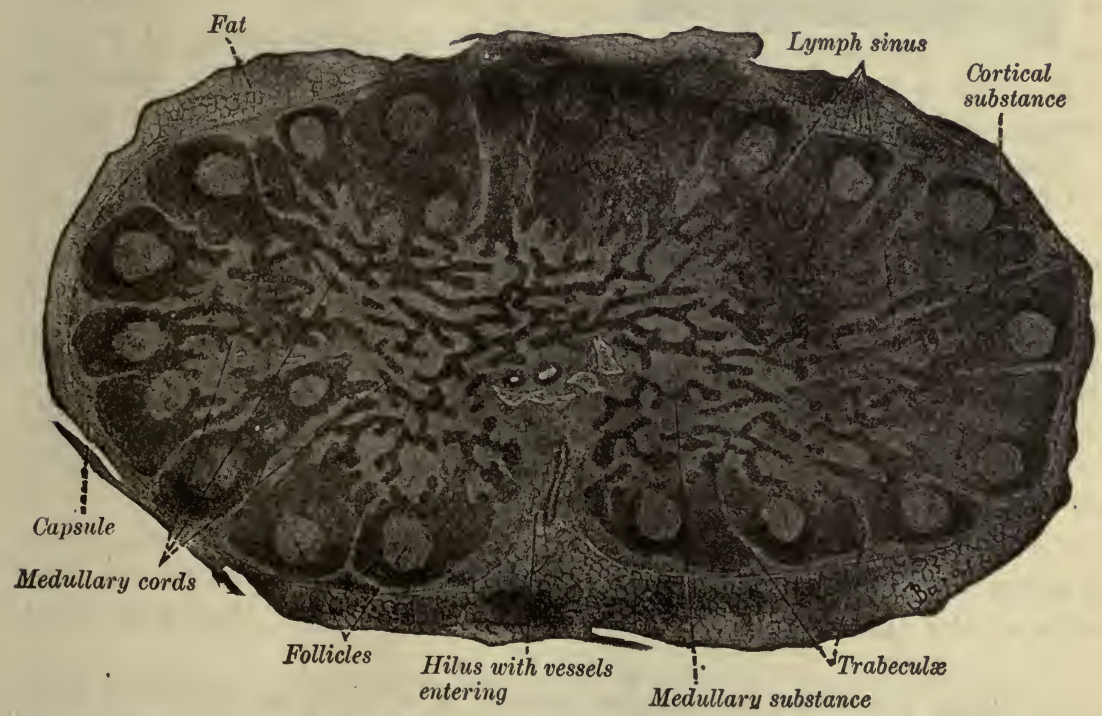

Fig. 79.-Section through a Small Lymph Node of a Dog. $\times 20$.

(From Szymonowicz-MacCallum, "Histology and Microscopic Anatomy.") 
a deeply staining nucleus enveloped by a narrow shell of homogeneous slightly basophilic cytoplasm. The lymph cells (lymphocytes) are so closely packed that it is almost impossible to distinguish the fine threads of the reticular stroma, except in those portions where some of the lymphatic cells have been washed out or displaced in the preparation of

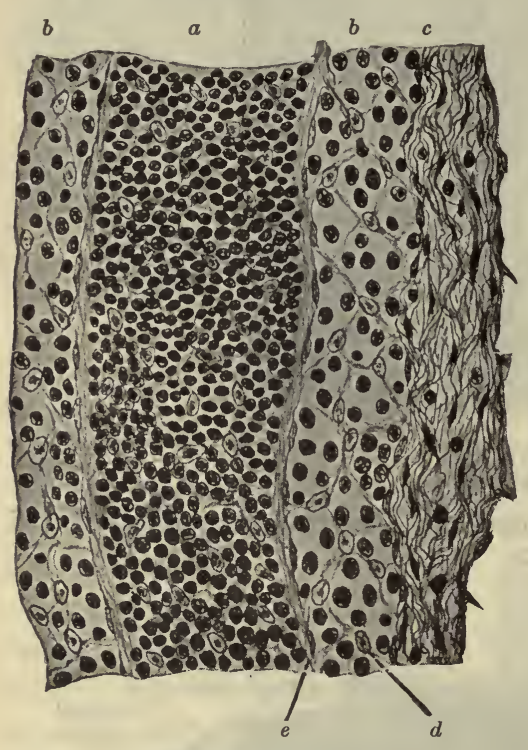

Fig. 80.-From a Section through the Medulla of a Cervical Lymph Node of Man.

$a$, a 'cord' of dense lymphoid tissue; $b$, looser lymphoid' tissue of the medullary sinuses; $c$, the margin of a fibrous trabecula; $d$, nucleus of the connective tissue reticulum; $e$, endothelial lining of the lymphatic sinus. Hematein and eosin. $\times 475$. the specimen.*

The density of the lymphoid tissue varies much, however, in different organs and even in different portions of the same organ. The denser accumulations of lymphoid corpuscles may form either ovoid lymph nodules or follicles, or long dense trabeculæ, the lymphatic cords, which are surrounded by looser portions of lymphoid tissue.

Lymphatic corpuscles are frequently infiltrated into the connective tissue of the mucous membranes, where they form irregular collections, which may be termed diffuse lymphoid tissue, in contradistinction to compact lymphoid tissue, which occurs in the lymph nodes, tonsils, thymus, and spleen, and in the aggregate and solitary nodules of the intestinal canal. Diffuse lymphoid tissue is found in the mucous membranes of (A) the respiratory tract-nose, nasopharynx, larynx, trachea, and bronchi; and (B) the alimentary tractmouth, tongue, pharynx, esophagus, stomach, and intestines.

In the basement membranes of certain tubular glands-e.g., sweat, kidney, tear and mammary-and in the peripheral portion of the large cells of the umbilical cord, Mallory (Jour. Med. Res., 1903 and 1905)

* Mall's technic for this purpose consists in injecting gelatin into a fresh lymph organ (e. g., spleen), freezing the tissue, and placing thin sections into warm water when the lymphocytes are largely carried away by the dissolving gelatin leaving the reticulum free. 
has discovered robust fibers extending also from cell to cell, resembling somewhat white fibers, but unrelated by transition elements to, and differing microchemically from, collagenous fiber's. They are said to be similar to the fibrils of neuroglia cells of nervous tissue and to the border or myoglia fibrils of plain muscle cells.

\section{Blood and Nerve Supply of the Connective Tissues}

The connective tissues, but especially the areolar variety, form a supporting substance through which the various blood and lymphatic vessels and nerve trunks are distributed to all portions of the body. Within the connective tissues these vessels are everywhere present, and from them the connective tissue itself receives its supply of capillary vessels and terminal nerve fibrils.

The vascular supply of the comnective tissties is very abundant. Small arteries, which are derived from the main trunks, form a capillary plexus throughout the tissue, the capillaries finally reuniting to form the venules.

It is in this capillary plexus that the fluid portions of the blood exude into the surrounding perivascular lymphatic or tissue spaces of the connective tissue. The tissue juices which arise in this manner are most active agents in the physiological processes of assimilation. From the tissue juice spaces, lymph reënters the abundant capillary lymphatic vessels to be finally returned to the venous blood. This transfer is mediated by process of filtration and osmosis, the tissue spaces being generally regarded as closed spaces making no direct connection with the lymphatic terminals. Of the several varieties of connective tissue, the adipose possesses the most abundant blood supply; the lymphoid, on the other hand, is most richly supplied with lymph.

Abundant nerves are distributed to the connective tissues, some of which, the sympathetic nerves, supply its blood-vessels while others, medullated, terminate in special forms of. sensory nerve end-organs.

\section{CARTILAGE}

Cartilage is a dense, firm, but elastic substance, resembling connective tissue in that it is developed from similar mesodermal cells. It contains a ground substance, the cartilage matrix, and at times, fibers which may be either collagenous fibers or elastic. The presence, absence, or 
character of these fibers determines the variety of cartilage. Three varieties are thus distinguished: hyaline cartilage, in which no specific fibers can ordinarily be demonstrated within the matrix ; elastic cartilage, whose matrix is permeated by elastic fibers; and fibrocartilage, whose matrix contains collagenous fibers.

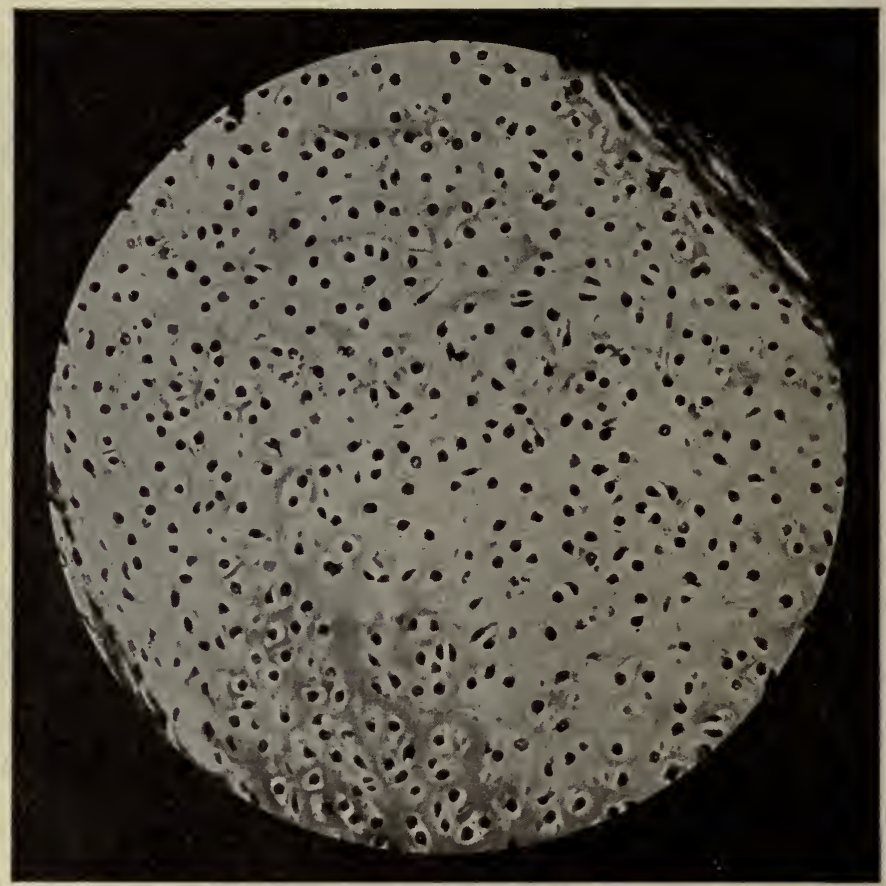

Fig. 81. - Transection of a Plate of Hyaline Cartilage, from the Trachea of A Child.

The margin of the fibrous perichondrium can be seen on either side of the plate of cartilage, in the upper right and lower left hand corners of the figure. Hematein and eosin. Photo. $\times 400$. The dark bodies are shrunken cells within lacunæ.

Hyaline Cartilage. - This is the most abundant of the three varieties, commonly known as gristle. It is found in the respiratory system, forming the cartilages of the nose, larynx, trachea, and bronchial tubes; in the costal cartilages of the ribs; as articular cartilages covering the ends of long bones; and in the fetus, where in the course of development of the bones, the entire skeleton, excepting only the flat bones of the skull and face, at first consist of hyaline cartilage. In most of these locations the cartilage occurs as platelike masses, which are invested by a vas- 
cular membrane of dense fibro-elastic tissue. This membrane is the perichondrium. The inner portion of this membrane is richly supplied with small cells, and it is from this cell layer, the chondrogenetic layer, that the cartilage is presumably developed.

The cartilage blastema is essentially mesenchyma. The chondrogenetic cells of this precartilage multiply, and deposit about themselves the structureless mass which first forms merely a capsule to the cell, but which as it increases in amount, separates the various cells by wider areas and becomes the cartilage matrix. The cells, which in the perichondrium are small and decidedly flattened, likewise increase in size during this process, and become more nearly spherical, so that those cartilage cells which lie near the center of the cartilaginous plates are spheroidal in shape, while those toward the surface are more and more flattened or elongated, their long axes gradually revolving from a perpendicular position in the center of the plate to one parallel with the perichondrium at the surface. Each cartilage cell is inclosed within a small space or lacuna, which during life it entirely fills.

Cell multiplication in cartilage is peculiar in that cell di-

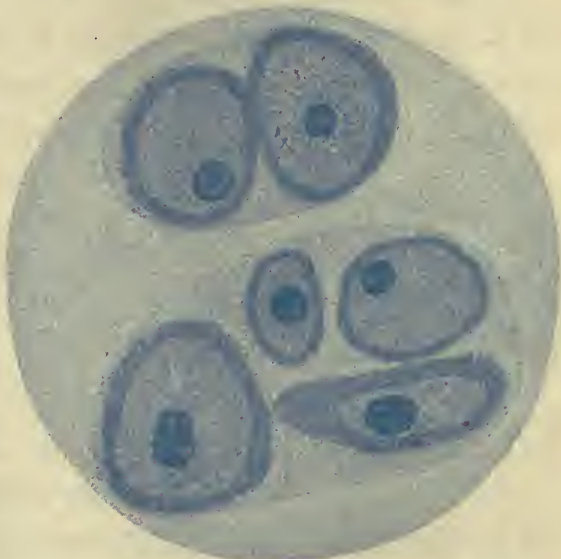

Fig. 82.-Cells and Matrix of Hyaline Cartilage from the Wall of a large Bronchus of MaN.

The grouping in pairs and fours, and the tendency to produce a so-called 'capsule,' are especially noticeable. Hematein. $\times 550$.

vision occurs within a firm capsule and results in the formation of two daughter-cells, which at first lie within the same encapsuled space. These two cells may each again undergo division within the same space with formation of four new cells. As a result of this peculiar method of cell division the cartilage cells are arranged in groups of two, four, or even eight cells. Each of the cells in the group deposits its capsule, and thus forms a matrix about itself, so that the increasing space thus produced between the cells of a group may separate them until they become completely isolated cartilage cells each within its own lacuna. In this way the matrix of the cartilage is produced. Enlargement of a cartilage plate occurs through a combination of interstitial and perichondrial growth. 
The matrix of hyaline cartilage is devoid of fibrous or cellular structure. Chemically it consists of collagen, chondromucoid and albuminoid substances. Von Korff (1914) interprets hyaline matrix as being composed of matrical fibrils masked by a homogeneous cementing substance.

During life, or if the tissue is examined in the fresh state, the cartilage cell entirely fills the lacuna in which it lies. But shortly after death shrinkage of these cells begins, so that after some hours a considerable space intervenes between the cell and the wall of its lacuna. It has been supposed that this space was occupied during life by lymph. It would,

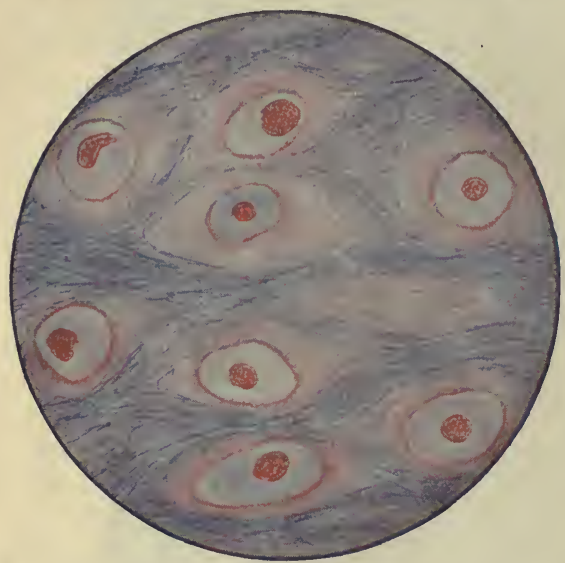

Fig. 83.-Eilastic Cartilage from the Human Epiglottis, Showing the Large Ovoid Cartilage Celis and the Very Delicate Reticulum of Elastic Fibers.

Ehrlich's triacid stain. $\times 550$. however, seem more probable that it is partially the result of post-mortem shrinkage of the cell.

Frequently, and especially in developing cartilage, concentric lines may be seen surrounding each lacuna. These lines have been described as the 'cell capsule.' They appear only to indicate the successive layers of material which have been deposited by the cell, and which have fused together to form its surrounding matrix.

Cartilage arises from a mesenchymal syncytium in which the matrix is formed from the exoplasm of the syncytial tissue, the cartilage cell representing its endoplasm. The so-called capsule of the cartilage cell would accordingly represent the partially modified border line between the original endo- and exoplasm, and would thus correspond to similar conditions which are observed in other forms of developing connective tissue.

Cartilage cells frequently contain small droplets of fat, and these may coalesce until the cell is completely transformed into a fat cell. Isolated masses of adipose tissue, resulting from the transformed groups of cartilage cells, thus make their appearance within the cartilaginous plates. This fatty metamorphosis is most marked in the elastic variety of cartilage.

By coloration with iodin, glycogen granules may also be demonstrated in the cartilage cells. 
Elastic Cartilage.-Elastic cartilage occurs in the external ear, in the auditory tube, in the epiglottis and in the cuneiform and corniculate cartilages and the vocal processes of the arytenoid cartilages of the larynx. It is essentially hyaline cartilage the matrix of which has become permeated with delicate elastic fibers forming a dense interlacing network. The large spheroidal cartilage cell lies in a lacuna bounded by a capsule and surrounded by a layer of hyaline matrix free of elastic fibers. The plates of elastic cartilage, like the hyaline variety, are surrounded by a dense fibrous perichondrium. Neither blood-vessels, nerves, nor lymphatics are distributed within the matrix of elastic cartilage.

Fibrocartilage. - This tissue forms the interarticular cartilages of the lower jaw, the clavicle, and the knee; composes the intervertebral disks and the other cartilaginous symphyses of the body; lines the tendon grooves of the bones, and forms the glenoid ligament of the shoulder and the cotyloid ligament of the hip. Fibrocartilage is intermediate in structure between hyaline cartilage and such very dense fibrous tissue as occurs in the tendons of muscles. At the attached margins of the

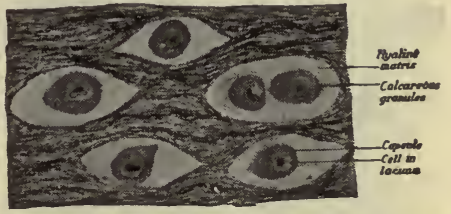

Fig. 84.-Fibrocartilage, From INTERVERTEBRAL Disk OF Ox. cartilaginous plates its tissue is continued by imperceptible gradations into the surrounding fibrous connective tissues. Like the other forms of cartilage, this variety is also non-vascular and devoid of nerves. .

Microscopically, fibrocartilage differs from such dense white fibrous tissue as is found in the ligaments and tendons, in that the meshes of the dense fibrous tissue of fibrocartilage are everywhere permeated by a hyaline matrix in which here and there are small groups of ovoid cartilage cells. Each cartilage cell is occasionally surrounded by a characteristic, concentric, lamellar appearance of the adjacent matrix, the socalled 'capsule.' Plates of fibrocartilage, unlike the other varieties, are not surrounded by a perichondrium.

A peculiar sort of connective tissue of entodermal origin is found in the nuclei pulposi of the invertebral disks. It is the sole adult vestige of the embryonic axis, the notochord. According to Williams (Amer. Jour. Anat., 8, 3, 1908), who carefully studied its cytomorphosis in the pig, "It is primarily cellular and epithelial; later it becomes a 
syncytial network with a mucin-like substance in its vacuoles; and finally it becomes cellular and closely resembles cartilage."

The Perichondrium.-The perichondrium is a dense fibrous membrane which surrounds each individual plate of cartilage. It is continuous with the surrounding connective tissue, and is well supplied with bloodvessels and lymphatics; it may also contain terminal nerve fibrils.

The cartilage itself is an absolutely bloodless and nerveless tissue. Neither are lymphatic channels demonstrable within the cartilage matrix.

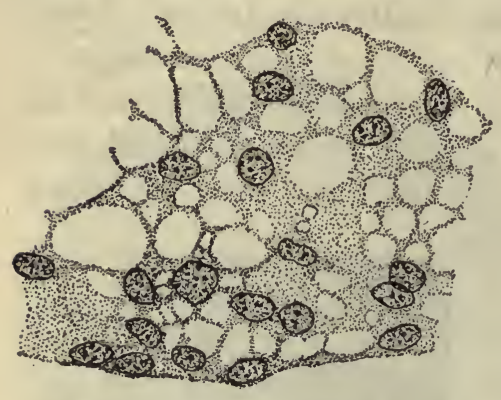

$A$

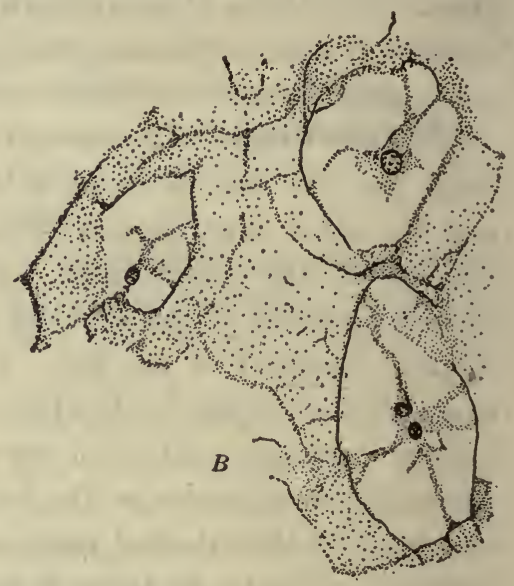

Fig. 85.-Notochordal Tissue.

$A$, from pig embryo of $150 \mathrm{~mm}$.; the syncytium contains many mucin-filled spaces. $\times 800$. $B$, from nucleus pulposus of an adult pig; the three cells shown are greatly vacuolated. $\times$ 452. (After L. W. Williams, Amer. Jour. Anat., 8, 3, 1908.)

After long maceration or artificial digestion the matrix assumes a granular or fibrous appearance, and small channels have been demonstrated within it, which have been said to connect the various lacunæ; but it is evident that these appearances were possibly the result of artificial destructive processes and could not therefore be considered as evidences of the presence of such structure in living cartilage.

\section{BONE}

General.-Bone is a firm calcareous tissue which is found only in the skeletal system. In the flat bones it forms a double layer of dense osseous tissue between which is a narrow space, bridged across at frequent intervals and thus subdivided into a number of compartments, the 
marrow cavities. This central stratum presents a spongy appearance as compared with the denser periphery; it is therefore said to contain spongy or cancellous bone, while the more superficial lamellæ contain compact bone.

In the long bones a similar condition exists in the epiphyses, which consist of a wall of compact bone within which the marrow cavity is subdivided by bony partitions into numerous compartments. The epiphysis consists, therefore, of spongy bone. The shaft or diaphysis of the bone, however, contains a single large marrow cavity whose walls, except for a thin layer at either end, consist entirely of compact bone. A little spongy structure is present for some distance at either end of the shaft, in that portion which adjoins the marrow cavity.

The ends and facets of the bones are covered by a disk of hyaline cartilage, which forms the articulating surfaces of those bones which enter into the formation of the movable joints. These articular cartilages are peculiar in that they are not covered by a perichondrium, and their deeper cells, which adjoin the bone, are so arranged that their long axes are perpendicular to the free surface, as is the case in the central portion of free cartilaginous plates. Toward the free surface of the cartilage the long axis of the cell lies more nearly parallel to the surface, as is likewise the case at the surface of cartilaginous plates

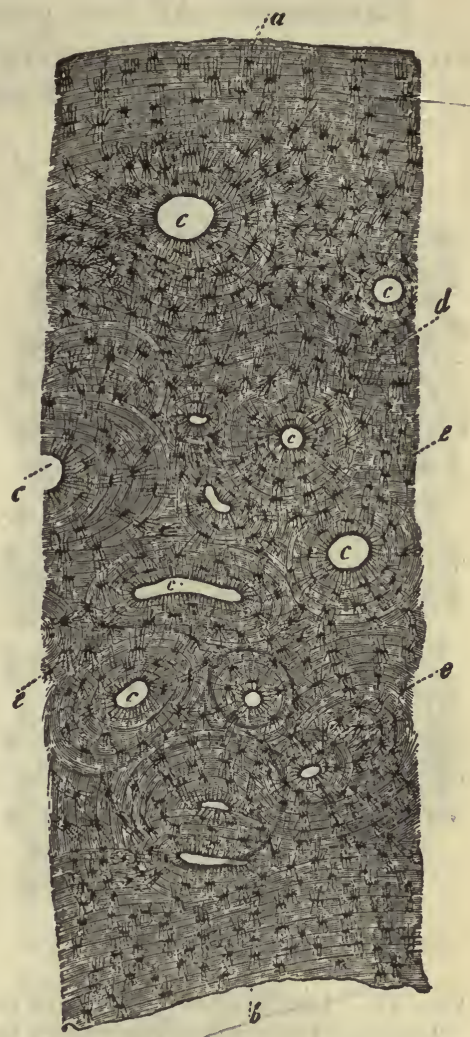

Fid. 86. - Transection throdgh the Compact Bony Wall of a Human Metacarpal Bone.

$a$, outer circumferential lamellæ; $b$, inner circumferential lamellæ; $c$, Haversian canals; $d$, interstitial lamellæ; $e$, lacunæ, with delicate radiating canaliculi. From a thin section of ground bone. $\times 90$. (After Kölliker.) elsewhere. In the long bones of younger individuals a plate of hyaline cartilage is found also at the epiphyseal lines between the epiphysis and the diaphysis. This plate, which extends through the entire axis of the 
bone, becomes ossified later in life. It represents the line of growth, and is the last portion of fetal eartilage to be transformed into adult bony tissue.

Periosteum.-All those portions of the bone which are not covered by an articular cartilage are supplied with a membranous coat of fibrous tissue, the periosteum. The outermost layer of this membrane consists

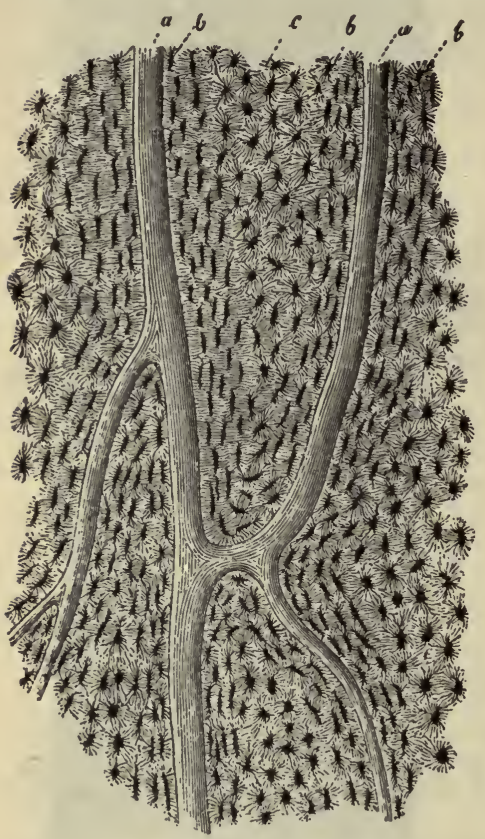

Fig. 87.-Longitudinal Section of Ground Bone From the Shaft of the Human Femur.

$a$, Haversian canals; $b$, lacunæ; $c$, canaliculi. $\times 100$. (After Kölliker.) of interlacing bundles of dense fibrous tissue in which are the larger bloodvessels, whose branches are distributed to the underlying bone. The inner portion of this layer forms a firm fibro-elastic stratum, which in older individuals is closely attached to the surface of the bone. The periosteum of developing and growing bone, however, contains a third or innermost areolar layer, in which are small blood-vessels, fine connective tissue fibrils, and numerous small osteogenic cells, the osteoblasts. After growth of the bone has ceased, the deepest layer of the periosteum contains few small blood-vessels and only occasional osteoblasts. These cells, however, are present in sufficient numbers to accomplish the regeneration of the bone after destruction of its osseous tissue.

The medullary surface of the bone is likewise supplied with an osteogenic membrane of fibrocellular tissue, similar to the innermost layer of the periosteum; it is known as the periosteum internum, endosteum, or membrana medullaris.

Compact Bone.-Compact bone, such as that composing the shafts of the long bones, consists of concentric lamellae of calcified fibrous tissue which constitute the Haversian systems, together with groups of parallel laminæ, which are interposed between adjacent Haversian systems and are known as the interstitial or ground lamella. Many of the interstitial lamellæ are the remains of Haversian systems which have been partially 
absorbed during the development of the bone. In a section through the shaft of a long bone the Haversian systems are found in the middle of the wall, while superficial to them and just within the periosteum are a number of lamellæ which may be traced much or all of the way around the circumference of the cylindrical shaft, and which are known as the external circumferential or periosteal lamella. On the inner surface of the compact bony wall is a similar group of parallel laminæ which adjoin the marrow cavity, and are known as the internal circumferential or endosteal lamella. In their finer structure the circumferential lamellæ are exactly similar to the cylindrical bony lamellæ of the Haversian systems.

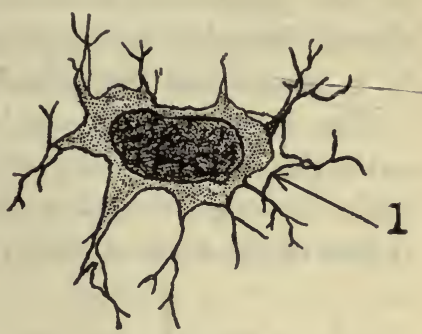

Fig. 88.-Isolated Bone Cell, Shrunk Away from Wall of Lacuna at $l$.

(Schäfer, after Joseph.)

Haversian System.-A Haversian system contains a small central canal (0.05-0.1 mm. dia.), which is occupied by connective tissue, marrow cells derived from the marrow cavity during the process of development,

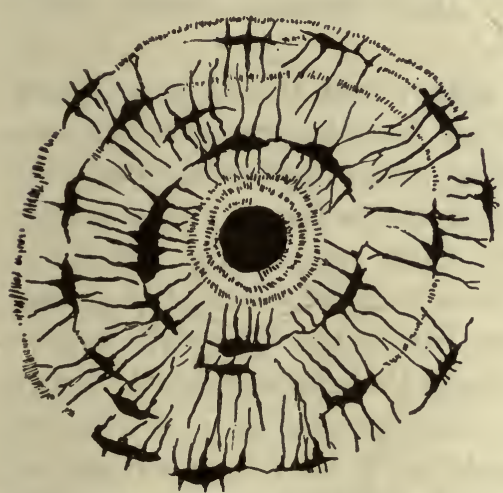

Fig. 89.-An Haversian Sxstem, Including the Central Canal, Several Lamellat, Lacuna aND Canaliculi. small blood-vessels, nerve fibers, and perivascular lymphatics. Concentrically arranged around the Haversian canal are parallel layers of dense fibrous tissue, the Haversian lamellax. The fiber bundles of this tissue form an interlacing network whose bundles frequently cross each other at right angles and whose interstices are occupied by a solid calcareous mass, consisting chiefly of the phosphates (about 80 per cent.) and carbonates of calcium. From four to twenty such calcareous lamellæ are found in each Haversian system. The organic substance of bone consists chemically of collagen, osseomucoid and small amounts of other albuminoid bodies.

Both in and between the lamellæ are many small ovoid spaces which are partially filled by small flattened cells, the bone cells; these spaces are known as the lacunce. From each lacuna minute canals, the canaliculi, 
radiate in all directions, thus placing the lacuna in open communicatio: with its neighbors, and eventually with the lymph spaces of the cen tral Haversian canal. The branching processes of the bone cells fre quently project for a short distance into the canaliculi. These cytoplasmic branches are more numerous in newly formed bone, later they are retracted and the cells become more or less shriveled in appearance.

The Haversian system, being developed about a centrål canal which marks the course of a blood-vessel, necessarily acquires a slender columnar shape, its long axis being usually disposed in a direction nearly parallel to that of the bone of which it forms a part. The Haversian canals fre-

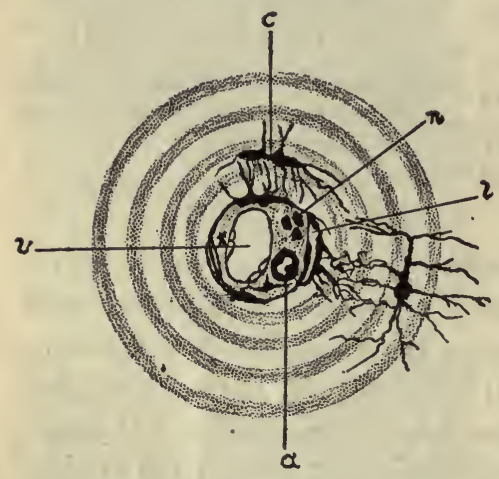

Fig. 90.-Transverse Section of Haversian Canal, with Contents.

$a$, arteriole; $v$, venule; $l$, lymphatic; $n$, non-medullated nerve fibers; $c$, bone cell. (After Schäfer.) quently branch to permit a corresponding division of their blood-vessels, and all of the Haversian canals are connected either directly or indirectly with the periosteum, the nutrient foramina, or the marrow cavity -thus forming a complete connected system between marrow cavity and surface-from the blood-vessels of which their vascular supply is derived.

INTERstitiaL LAMELLA.-The interstitial lamellæ are likewise composed of dense interlacing bundles of calcified fibrous tissue, within and between which are lacunæ, canaliculi, and bone cells, all disposed in a manner exactly similar to their arrangement within the concentric lamellæ of the Haversian systems. Coursing through the interstitial and circumferential lamellæ are Volkmann's canals, which are similar in origin, contents, and function to the Haversian canals but which are not surrounded by concentric lamellæ. Volkmann's canals frequently arise as branches of the Haversian canals which wander out, as it were, into the interstitial lamellæ.

Circumferential Lamelle.-The circumferential lamellæ do not differ in structure from the other osseous lamellæ. They possess the same arrangement of laminated calcareous connective tissue, with lacunæ, canaliculi, and bone cells, as in the concentric and interstitial lamellæ. Even more than elsewhere, however, the outer circumferential lamellæ are firmly bound together by collagenous and elastic fibers which pass from 
the periosteum into and through the superficial lamellæ; these are known as the perforating fibers of Sharpey. Similar fibers connect together the concentric and interstitial lamellæ. The perforating elastic "fibers are frequently surrounded by an envelope of fibrous connective tissue.

Bone Marrow.-Bone marrow consists of a variety of connective tissue, largely reticular, which is rich in fat cells and blood-vessets and which also contains osteogenic and hemogenic elements, the marrow cells or myelocytes. According to the relative proportion of these elements marrow is said to present two types, the yellow and the red marrow. The yellow marrow consists almost entirely of fat, with only occasional bands of true marrow tissue. The red marrow contains very little fat, but is so abundantly supplied with blood and marrow cells as to closely resemble a very vascular lymphoid tissue. The embryonic medulla of all bones contains fetal red marrow, but in later life the larger masses in the medulla of the shafts of the long bones are, in man, changed to the yellow variety. The red marrow, however, persists in the epiphyses of the long bones and in cancellous bone generally; it is especially characteristic of the marrow cavities of the ribs, vertebræ, base of the skull, and sternum. It is the source of supply of blood-cells in the adult.

RED MARRow.- - Red marrow consists of fibrous and reticular tissues which are infiltrated by marrow cells and richly supplied with small blood-vessels. The smaller veins possess exceedingly thin walls, readily pervious to the blood-cells. The walls are so delicate that it becomes very difficult to determine with certainty whether or not their endothelium, as also that of the capillaries, may be occasionally absent, thus placing the blood-stream in direct communication with the pulp of the bone marrow.

The hemogenic elements of marrow will be described under the subject of blood development, where red marrow must again be considered. At this point it is only necessary to describe the osteogenic elements. These are (1) the osteoblasts, or bone builders, and (2) the osteoclasts, or bone destroyers. The osteogenic process as a whole is of course dependent upon the blood, with all its hemal elements.

Osteoblasts.-These are cells which may assume various shapes depending upon their spatial relationship to the bony substance. When free they are of round or slightly oval shape; lining the marrow cavity or covering the bone as portions of the periosteum or applied to spicules of cancellous bone they may become considerably flattened. The nucleus is generally round or oval, deeply chromatic and granular. As spheroidal cells they have an average diameter of about 8 microns. They are with 
difficulty distinguished from lymphocytes except when characteristically arranged as a membranous coat upon the surface of bony walls or spicules. They become the bone cells of compact bone. Osteoblasts and lymphocytes are genetically closely related, both being relatively slightly differentiated mesenchymal cells. In the bone marrow of the turtle osteoblasts may differentiate into leukocytes. It seems probable that persistent fetal osteoblasts of adult red marrow may function as parent blood-cells.

OstEoclasts.-These are giant multinuclear cells, often containing as many as ten to twenty or more nuclei. They are the cells by whose agency bone is destroyed during the processes of development and growth. They are similar to, but not identical with, the polykaryocytes of hemogenic foci which are concerned with the process of blood-cell formation. The osteoclasts originate by a process of fusion of reticular cells of the marrow; the hemogenic polykaryocytes originate from lymphocytes (hemoblasts) by repeated amitotic division of the nucleus.

Blood Supply.-Marrow, and especially the red variety, is richly supplied with blood. The nutrient or medullary artery penetrates obliquely through the nutrient foramen to the marrow cavity of a long bone where it divides into an ascending and descending branch and supplies an abundance of small arteries to all portions of the medulla. The terminal arteries end in broad capillary vessels whose wide lumen and delicate endothelial walls determine their character as sinusoids. It was formerly thought that the endothelial walls of these vessels were here and there deficient, and although recent investigations discredit the former observations, the all-important fact remains that the endothelial walls are pervious to both red and white blood-cells. Certain of the terminal arteries anastomose with those of the cancellous epiphyses, and with the arteries which enter the Haversian canals of the compact bone from the periosteum.

Efferent veins return the blood from the sinusoidal capillaries of the marrow. These veins, passing as companion veins to the medullary artery through the nutrient foramen, or independently through separate foramina, as also those of the bony tissue, are not supplied with valves. Outside of the bones, however, these same veins contain abundant valves.

The Lymphatics.-The lymphatics of bone occur in great abundance in the periosteum, and as perivascular spaces penetrate the canals of Havers and Volkmann and thus reach the medullary cavity. The existence of lymphatics within the marrow, other than in the sheaths of the blood-vessels, is doubtful.

The Nerves.-The nerves accompany the blood-vessels in all portions 
of the bone and marrow and form a rich perivascular plexus which is distributed to the walls of the vessels; occasional side fibrils are also distributed to the marrow. Nerve endings have not been demonstrated in compact bone nor in the articular cartilages. In the periosteum terminal nerve fibrils are supplied to the musculature of the blood-vessels, and other sensory fibrils end in lamellar corpuscles.

Development of Bone-Bone tissue makes its appearance relatively late in fetal life. The long bones are first mapped out by masses of hyaline cartilage. The entire skeleton, with the exception of the flat bones of the face and those of the vault of the skull, is thus primarily formed by plates of fetal cartilage. The process by which these cartilaginous plates are transformed into bone is known as intracartilaginous or enchondral ossification. The process is essentially one of replacement of cartilage by bone, not one of change of cartilage into bone. The resulting bones are known as substitution bones.

The flat bones of the face and skull (including the interparietals, parietals, frontals, squamosals, tympanics, median pterygoid plate of the sphenoid, nasals, lacrimals, malars, palatine, vomer, maxilla, and a portion of the mandible) are formed directly from the mesenchymal blastema without the intervention of cartilage. This method of bone formation differs somewhat from the above and is known as intramemtranous ossification.

INTRACARTILAgINous Ossification.-This process begins with the formation of plates of hyaline cartilage whose shape corresponds more or less closely with that of the future'bone. 'This type of fetal cartilage differs from the hyaline cartilage of the adult only in the irregular form and distribution and greater abundance of its cartilage cells.

Each plate of fetal cartilage is enveloped by a layer of embryonal fibrous tissue, the fetal perichondrium. The outer portion of the fibrocellular layer is destined to become the periosteum of the future bone; its innermost portion contains many small round cells, which from their intimate relation to bone production, are known as osteoblasts. The inner portion of the perichondrium forms the osteogenic layer of the future periosteum.

Centers of Ossification.-Ossification of the cartilage begins at one or more points which are called centers of ossification. In the long bones, in which the process of bone formation can bè most readily traced, there are usually three such centers, one near the middle of the cartilaginous plate, from which the diaphysis is formed, and one epiphysial center at each extremity. The centers for the epiphyses make their appearance 
much later than that for the shaft of the bone, for the most part not until some months after birth, and from an extension of marrow from the primary center.

Enlargement of the Cartilage Cells.-The first indication of beginning bone formation is evidenced by an enlargement of the cartilage cells which promptly arrange themselves in rows or columns that radiate from the center of ossification (calcification). This process is accompanied by absorption of the adjacent cartilage matrix, so that the enlarged car-

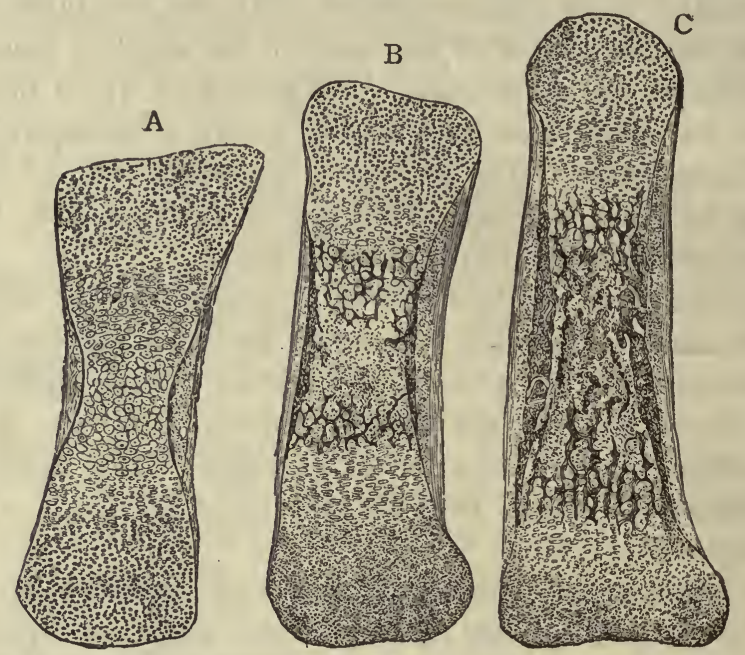

Fig. 91.-The Primary Changes in Intracartilaginous Bone Formation.

$A$, metatarsus; $B$ and $C$, phalanges of human fetus. In $A$, the earliest enlargement of cartilage cells at the center of ossification is shown. $B$ and $C$ are successively later stages. The bones are cut in longitudinal section. Carmin hematoxylin stain. $\times 27$. (After Toldt.)

tilage cells are contained within broad spaces or areola. The cartilage cells now appear to undergo a gradual but progressive absorption; their cytoplasm becomes shrunken and granular and finally disappears, even the nucleus at last succumbs to the process.

Calcified Cartilage.-The absorption of the cartilaginous matrix proceeds more rapidly in those portions which separate the individual cells in the columns than in those other portions which intervene between. the adjacent rows of cartilage cells. While the former portions are entirely absorbed, remnants of the latter remain, and in them calcium salts are deposited in an irregular manner. Calcified cartilage, the most primitive of the calcareous tissues, is thus formed. 


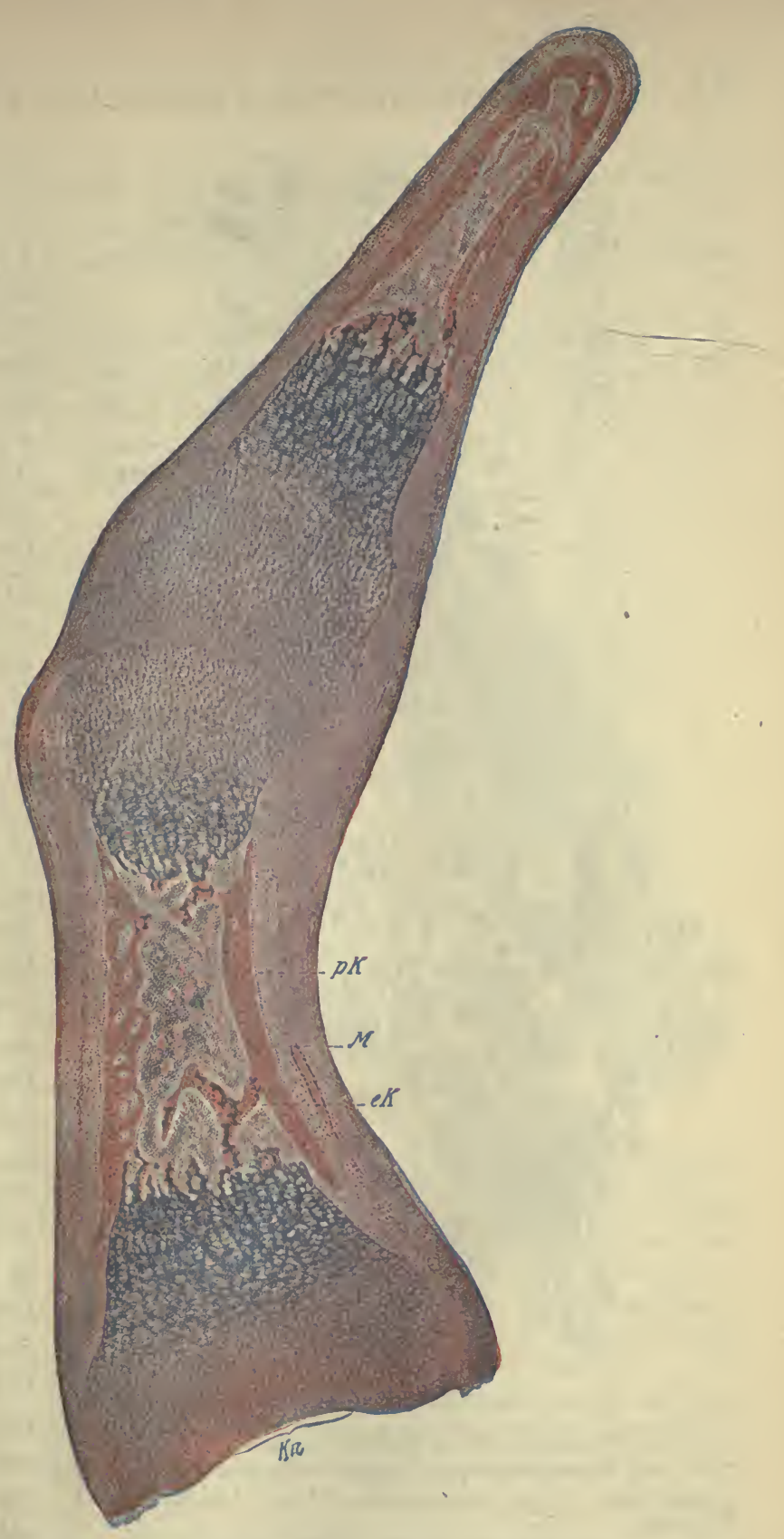

Fig. 92.-A Longitudinal Section of the Two Distal Phalanges from the Finger of a Five-months' Human Fetus.

$K n$, cartilage showing calcification and resorption; $e k$, enchondral bone; $M$, marrow cavity; $p k$, periosteal bone. $\quad \times 15$. (From Sabotta's "Histology.") 


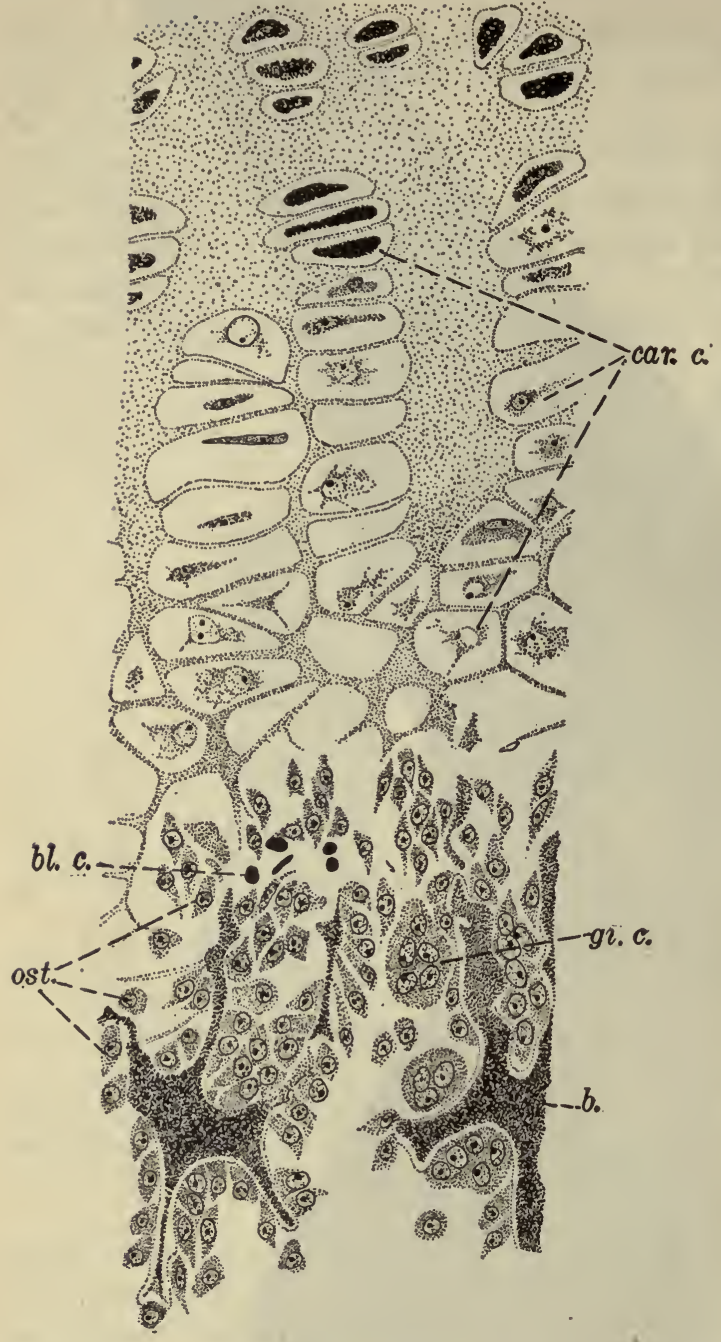

Fig. 93.-Reconstruction of Cartilage into Bone.

car. c., cartilage cells in successive stages of degeneration; ost, osteoblasts; gi. c., giant cells (osteoclasts); $b$, young bone; bl. c., blood cells. (From Dahlgren and Kepner.)
Primordial Marrow Cavities.-The absorption of the cartilage matrix results in the formation of broad spaces into which osteogenic buds of primitive marrow tissue push their way from the perichondrium. Thus the primordial marrow cavities are formed. The fetal marrow which now occupies these cavities is derived from the osteogenic layer of the primitive periosteum. The osteogenous tissue of this layer, containing osteoblasts, osteoclasts, a $\mathrm{n} \mathrm{d}$ developing blood-vessels, grows into the cartilage in the form of budlike cords which are preceded by absorption of the adjacent cartilage matrix. This so-called 'eruptive tissue, promptly reaches the center of ossification and burrows its way into the enlarged cartilage lacunæ whose cells are now replaced by primary osteogenic marrow. The destruction of cartilage is initiated and maintained by agency of the osteogenic tissue, presumably through specific cells, the so-called chon- 
droclasts, the morphological marks of identification of which are not yet known. According to some investigators (e.g., Retterer, 1900) the cartilage cells do not disintegrate but pass into the marrow cavity where they become osteoblasts.

Primary Bone.-The osteoblasts which thus gain access to the primary marrow cavities, now arrange themselves.along the surface of the remnants of calcified cartilage and begin the deposit at their proximal surface of the fibrous tissue and calcareous salts which compose the primary bone. The osseous matrix is commonly assumed to be the product of a transformation of the exoplasm of the osteoblasts. Many of the osteoblasts apparently become entangled in this newly formed tissue and form the bone cells. The fetal cartilage is thus transformed into a spongy mass of primary osseous tissue whose spicules are formed by a core of calcified cartilage upon which are deposited successive layers of bony tissue with their included lacunæ and bone cells. In sections stained with hematoxylin and eosin, the central strand of calcified cartilage is colored blue, the primary bone, red.

Axial sections of long bones at this stage of ossification show all the above changes in regular succession from the fetal hyaline car-

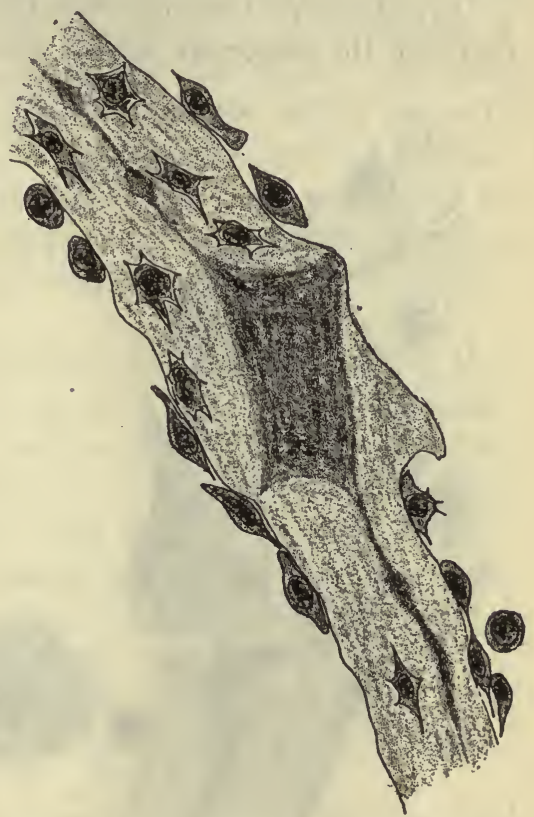

Fig. 94.-Trabecula of Primary Enchondral Bone, Showing a Central Deep-staining Core of Calcified Cartilage and a Peripheral LAYER of Osteoblasts.

Osteoblasts have become incorporated within the bone as bone cells. From the finger of a human fetus. tilage at the extremities to the primary bone with its marrow cavities in the center. The process of ossification steadily progresses toward the ends of the bone, the line of enlarged cartilage cells constantly advancing farther and farther from the original center of ossification.

Absorption of the Newly Formed Bone.-It is at this stage, however, that the giant cell osteoclasts become most active and the absorption of the newly formed bone progresses rapidly. The osteoclasts collect along 
the surface of the spicules of primary bone in considerable numbers and appear to sink into little recesses which they form within the bony tissue. The little bays which are thus formed in the primary bone are the lacunce of Howship. The continued absorption soon breaks down and removes the trabeculæ and partitions of spongy bone and forms a central medullary cavity. of constantly increasing size.

Perichondrial Ossification.-Coincident with these changes within the cartilage the osteogenic tissue which forms the inner layer of the peri-

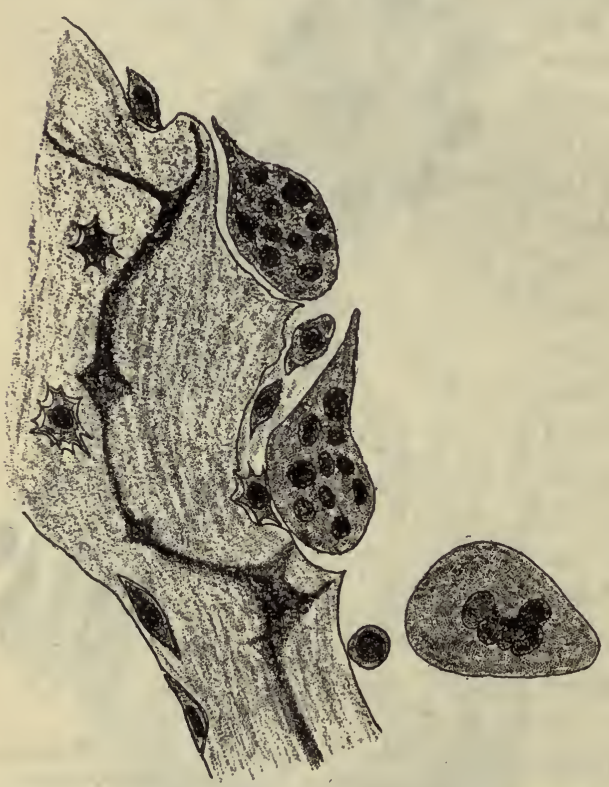

Fig. 95.-Trabecula of Primary Bone from the Finger of a Human Fetus.

Three giant cells (osteoclasts) are shown at the right, two resting in Howship's lacunæ. chondrium produces successive layers of bony tissue upon the surface of the fetal cartilage. This process of perichondrial (periosteal) ossification proceeds in a manner similar to that by which bone is formed in membrane which is not closely applied to cartilage.

Perichondrial bone formation is essentially of the intramembranous type. In essence there is no valid distinction between endochondral, perichondrial and membrane bone development, since each involves calcification of a fibrillar matrix by agency of the same cell, the osteoblast. At irregular intervals the osteoclasts collect and the primary perichondrial bone is absorbed. Into these cavities buds of vascular osteogenic tissue push their way to form canals of considerable length. Upon the surface of the canals which are thus hollowed out of the perichondrial bone, the Haversian spaces, the osteoblasts deposit successive concentric layers of bony tissue and the Haversian systems make their appearance. Finally, upon the surface of the periosteal bone successive layers of newly formed bony tissue compose the external circumferential lamella, while upon the wall of the medullary cavity a similar endosteal layer of bone-forming 
cells deposits the internal circumferential lamella. The Haversian canals are actually continuations of the marrow cavity, and the larger are even lined by endosteum.

With the formation of the perichondrial bone the lateral expansion of the organ by endochondral bone formation necessarily ceases. Henceforth increase in diameter of the bone is only produced by continued absorption internally of the compact bony wall and the formation of new bone beneath the periosteum by frequent repetitions of the processes of periosteal (perichondrial) ossification as already described. The remnants of those Haversian and circumferential lamellæ which are only partially absorbed in this process form the interstitial lamella of the mature bone. In the long bones and in flat cartilage bones ossification at first proceeds in the perichondrium, endochondral ossification appearing only later; in the short bones ossification is endochondral until the cartilage is entirely replaced by bone.

Epiphysial Ossification.-During the processes of endochondral and perichondrial ossification within the shaft of the bone, the epiphysial cartilages continue to grow. Finally, however, ossification begins in the epiphysis, osteogenic tissue having pushed in from the primary center of the diaphysis, and proceeding in the same manner as in the shaft, results in the formation of primary spongy bone, some of which is absorbed and replaced by more compact bony tissue, as occurs in the wall of the diaphysis. In its central portions the tissue retains its spongy arrangement and but few Haversian systems are formed. It is thus that the cancellous bone of this part, as also of the ends of the diaphysis, is formed.

At the point where the expanding centers of ossification of the shaft and epiphysis are about to meet, a line of unossified cartilage, the epiphysial line, persists until growth of the bone is complete. It is by growth of this cartilaginous disk, with continued formation of cartilage mainly on its inner surface, and its concomitant replacement by bone, that the bone increases its length. After ossification of this epiphysial synchondrosis at about the twenty-first year, growth in length must cease. Meanwhile the perichondrium has become periosteum.

The following is a résumé of the various stages of endochondral ossification:

1. Formation of the fetal hyaline cartilages from precartilage mesenchyme blastema.

2. Enlargement of the cartilage cells with a rearrangement into radiating cell rows at the center of ossification. 
3. Absorption of the cartilage matrix between the cells of the rows and finally also of the cells themselves. Calcification of persistent remnants of cartilage matrix between the rows of cells.

4. Eruption of the subperiosteal osteogenic tissue, invasion to center of cartilage plate, and the formation of primary marrow cavities at the center of ossification.

5. Gradual extension of the above processes followed by a deposit of primary bone by the osteoblasts upon the calcified cartilage spicules. Coincident osteoblastic deposit of perichondrial bone beneath and within the perichondrium of the cartilage plate.

6. Absorption of portions of the primary bone by the osteoclasts to form the large central marrow cavity or medulla. The absorption involves both the endochondral and the perichondrial bone and is accompanied by a further deposit of new bone at the periphery. In the perichondrial bone cylindrical axial channels are formed, in which the deposit of new bone produces the Haversian systems of the compact bony tissue.

Intramembranous Ossification.- This is the simpler and more direct method of bone formation. In principle it is identical with perichondrial ossification. Endochondral bone development differs from it only in respect of the additional processes involved in the removal and replacement of the hyaline cartilage.

Membrane bones, including the flat bones of the face and the vault of the cranium, arise directly in the mesenchyma. The first indication of ossification is the enlargement and rounding up of a group (or groups) of mesenchyme cells, and their association in the form of an irregular membrane. Among the cells appear bundles of delicate collagenous fibrils, the osteogenic fibers (Sharpey), radiating beyond the limits of the cell group. The cells of this initial ossific group begin to function as osteoblasts and deposit osseous matrix among the fiber bundles. This original osseous trabecula marks approximately the center of the future bone. The surrounding loose mesenchyma has meanwhile become increasingly vascular. Vaguely outlining the peripheral limits of the definitive bone appears a relatively thick layer of denser, more cellular mesenchyma, the cells in general maintaining a fusiform shape. This represents the primitive periosteum of the forming bone. The bone takes shape internally by the appearance of numerous trabeculæ, which arise in the manner described for the initial spicules and then unite into a 
bony sponge-like structure enclosing vascular mesenchyma, the primary marrow. The spicules of the cancellous bone contain numerous bone cells - the representatives of original osteoblasts which have become enmeshed in their own product of osseous matrix-and are covered with an epithelioid membrane of a single or double layer of osteoblasts, which contribute

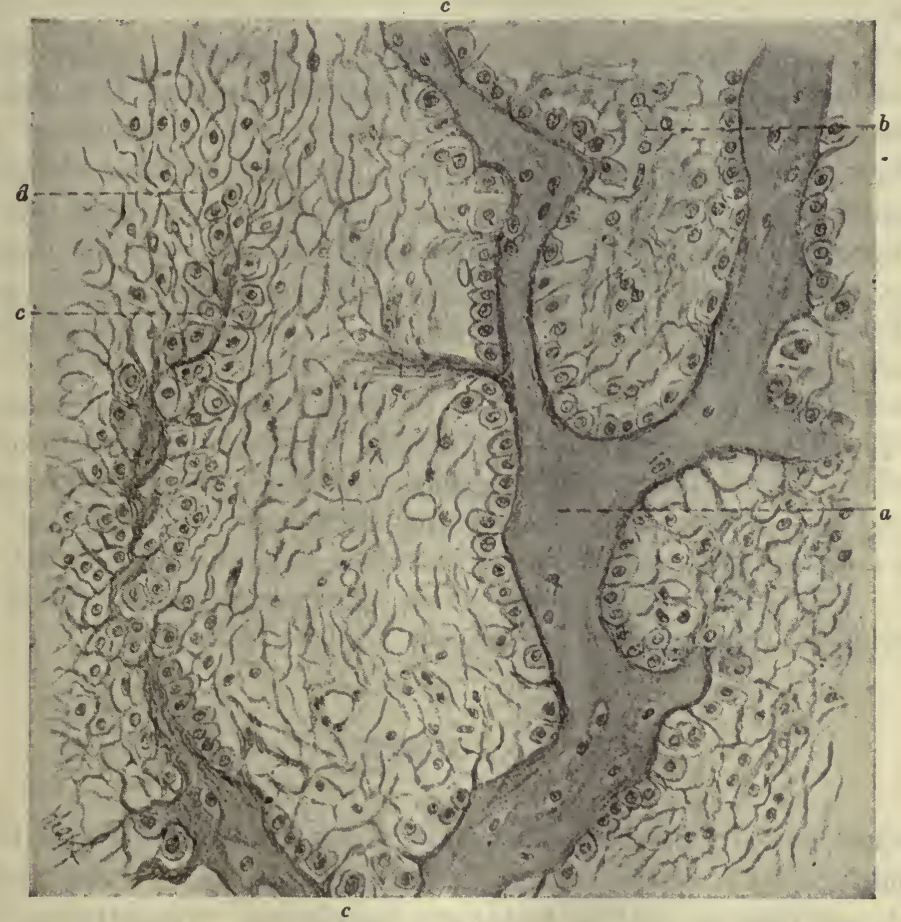

Fig. 96.-Intramembranous Bone Formation in the Lower Jaw of a Sheep Fetus.

$a$, bone; $b$, primary marrow cavity; $c$, osteoblasts; $d$, growing point of the primitive bone, beyond which primary marrow is developing in the connective tissue of the mesoblast. $\times 300$. (After Böhm and von Davidoff.)

to the further growth of the bony trabeculæ. The marrow includes besides osteoblasts and the specific marrow cells-somewhat less numerous than in the primary marrow of endochondral bone-numerous osteoclasts under whose absorptive agency, assisted by the productive activity of the osteoblasts, the inner conformation of the growing bone continually alters its details. Peripheral osteoblasts, arising from the inner layer of the periosteum, produce the more compact external plates of the bone. 
In the flat bones of the skull, the central cancellous bone is designated diploe, the peripheral compact bone, tables. Membrane bones may contain typical Haversian systems (Arey, Anat. Rec., 17, 1, 1919).

The conditions which determine that certain bones may arise directly in mesenchyma while others must pass through a cartilaginous stage are obscure.

It is commonly believed that periosteum is essential for bone regeneration, and its preservation is aimed at where new growth is desired after osteotomy. But according to W. Macewen ("The Growth of Bone," Maclehose \& Sons, Glasgow, 1912), who has made a comprehensive experimental study of osteogenesis in regenerating bone in dogs, the periosteum functions simply as a confining, nutritive, and protective membrane, but has no osteogenic significance. His observations lead him to conclude also that in the long bones the osteoblasts are derived from proliferating cartilage nuclei freed from the disappearing matrix. Under more favorable conditions regeneration is said to occur through direct osteoblastic activity, under less favorable conditions a cartilaginous transition stage intervenes. He deduces from his 'experiments that "diaphyseal bone is reproduced by proliferation of osteoblasts derived from preëxisting osseous tissue, and that its regeneration takes place independently of the periosteum." The periosteum is conceived as being an important factor in determining the conformation and growth limit of bone.

\section{JOINTS}

Joints are divisible into two main types, the movable and the 'immovable,' or (1) diarthroses and (2) synarthroses. These and their several modifications call for histologic description. Synarthroses include (a) syndesmoses, or joints in which the connecting substance is a dense fibro-elastic tissue joining the bones immovably as in the articulation of the skull (sutura), or where it consists of ligamentous tissue permitting slight movement as between the lower ends of the tibia and fibula; and (b) synchondroses, in which the connection is effected by cartilage, either hyaline (e.g., between the epiphyses and diaphysis of young bones) or fibrous (e.g., the intervertebral disks of the vertebral column).

In relation with diarthroses are in several instances (mandibular, lower radio-ulnar, costosternal, sternoclavicular, acromioclavicular) intra-articular menisci of fibrocartilage; here the articular cartilages of the bones concerned are also of the fibrous variety. The semilunar cartilages of the knee and the glenoid cartilage of the shoulder joint are also 
of the fibrous type. These cartilages serve to deepen the sockets in which the respective ends of the femur and humerus move and are known as adaptation cartilages or labra glenoidalia.

The joint cavity of a diarthrosis is enveloped in a capsule consisting of two layers, an outer fibro-elastic continuous with the periosteum and an inner cellular layer, the synovial membrane, consisting of epithelioid cells forming a mesenchymal epithelium. The function of the synovial membrane is to secrete a lubricating fluid, the synovia, consisting of about 94 per cent. water with small amounts of mucoid substances and oil. In the large joints the synovial (serous) membrane is thrown into villuslike folds (Fig. 240). The covering cellular membrane is occasionally imperfect; the cells vary from the flattened, typical mesothelial cells, to the cubic variety (Fig. 44), and rest directly upon a vascular, frequently fatty, fibrous stroma. 


\section{CHAPTER IV}

\section{MUSCULAR TISSUE}

\section{GENERAL CONSIDERATIONS}

Muscular tissue consists essentially of protoplasm in which the general vital property of contractility has become predominant. However, the path of contraction is practically limited to one direction, the long axis of the cell. This phenomenon of contractility results from the differentiation of specially contractile fibrils, the myofibrils, from the protoplasm of embryonic muscle elements, the myoblasts. The protoplasm of the muscular tissue is called sarcoplasm. Adult muscular tissue may be divided into three classes: smooth, cardiac, and striped. All three types arise from mesoderm, with the exception of the dilator and sphincter muscles of the iris of the eye, and the muscle of the secretory portion of the sweat gland-both of the smooth variety-which are generally believed to be of ectodermal origin. In lower forms, muscle tissue may be largely derived from the ectoderm and even from the entoderm.

The smooth muscle is in general limited to the viscera; it is not under the control of the will, hence also called involuntary muscle. The cardiac type is limited to the heart, and to the middle layer of the roots of the aorta, pulmonary artery, and pulmonary veins. It is striped, but like smooth muscle, controlled by the sympathetic nervous system; therefore independent of the will, hence also of involuntary type. So-called striped or skeletal muscle is practically limited to the skeleton, and subserves the function of skeletal movement. This group includes also the muscles of the eyeball, the ear, the upper third of the esophagus, diaphragm, and tongue. It is under the control of the will, hence designated voluntary. The striped muscle of the diaphragm and the esophagus is apparently only partially voluntary.

It is obvious from the above that there is demanded a more specific terminology: involuntary smooth (unstriped); involuntary striped (cardiac); and voluntary striped (skeletal). The three types pass through very similar, perhaps identical, earlier stages of histogenesis. The essen- 
tial difference seems to be one of degree differentiation. In general, skeletal muscle is most highly differentiated, cardiac muscle being intermediate between smooth and the voluntary striped type.

For a proper understanding of the structure of these three types it is necessary that we now consider the process of muscle histogenesis. The student should gather the several criteria by which he may distinguish between smooth, cardiac, and skeletal muscle, both in transverse and longitudinal sections.

\section{HISTOGENESIS AND STRUCTURE}

Smooth Muscle.-As stated above, the germ layer involved in muscle histogenesis is the mesoderm. Smooth muscle is derived chiefly (exception: arrectores pilorum) from the visceral or splanchnic layer. This is at first an epithelial structure of a single layer of cells, the primitive mesothelium. The cells subsequently proliferate and change their shape in general to a fusiform type. Intercellular connections (cytodesmata) are either maintained or established, and the tissue is permanently more or less in a syncytial condition. These so-called intercellular bridges are particularly pronounced and can be readily demonstrated in the tunica media of the blood-vessels of the umbilical cord. It must be emphasized, however, that the outlines of the genetic units in smooth muscle are always distinct, whereas in striped, especially the cardiac type, the outlines of the original myoblasts are lost.

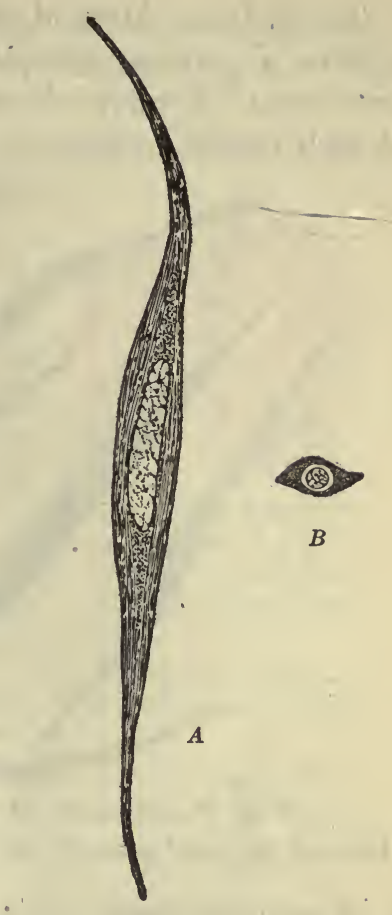

Fig. 97.-Siooth Muscle
Cells.

$A$, an isolated cell from the cat's intestine. The nucleus is surrounded by coarsely granular sarcoplasm, continuous peripherally with the finely granular interfibrillar sarcoplasm. The innermost myofibrils may erroneously suggest a cell membrane. The fusiform element is invested by a true cell membrane, or sarcolemma. $\quad \times 750$. $B$, oblique transverse section of a cell from the muscularis mucosæ of the cat's esophagus. The perinuclear sarcoplasm has contracted away from the nucleus leaving a clear space limited by a sharp line, external to which lies the perinuclear granular sarcoplasm. Hematoxylin and ensin. $\times 750$. 
The early myoblast, of short spindle shape with central oval nucleus, contains a granular cytoplasm, limited by a delicate membrane, the sarcolemma. The granules may be called myochondria; whether identical with cytomicrosomes or with mitochondria, whether of cytoplasmic

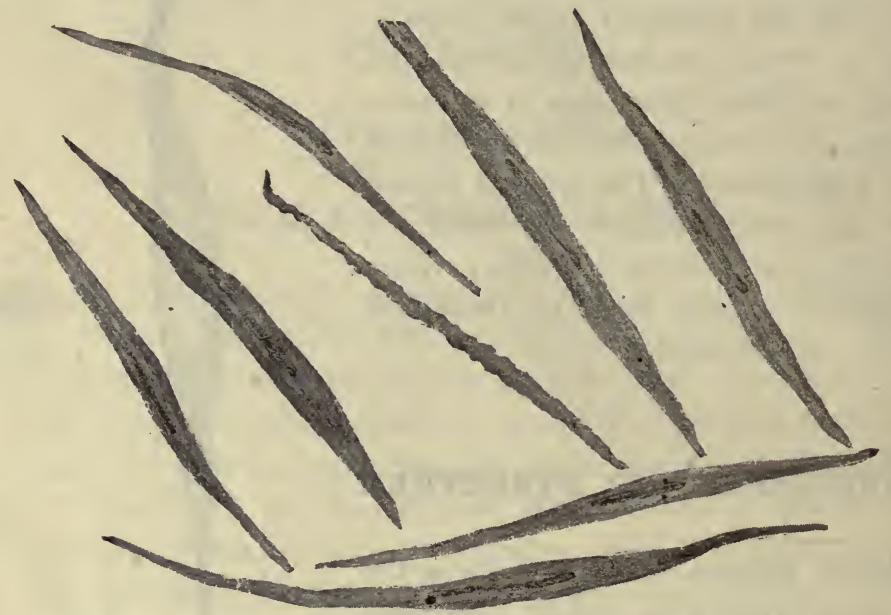

Fig. 98. - Smooth Muscle Cells from the Pig's Stomach.

Isolated in equal parts of alcohol, glycerin, and water. Unstained. $\times 410$.

or of nuclear origin, are disputed points. No evidence of a distinct spongioplasm is discernible. This observation tends to invalidate the teaching of certain histologists, that the contractile fibrils (myofibrils) represent modified spongioplasmic threads arranged in rectilinear meshes.

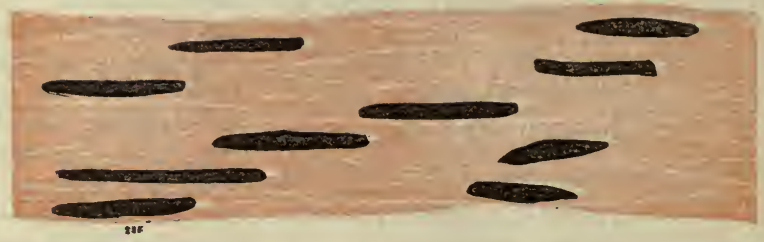

Fig. 99.-Smooth Muscle Cells from the Wall of the Human Intestine. Longitudinal section. Hematein and eosin. $\times 665$.

Moreover, it has been established by direct observation (McGill, et al.) that the myofibrils arise through process of alignment and subsequent fusion of the myochondria. MeGill (Internat. Monatschr. Anat. u. Phys., Bd. 24, 190\%) recognizes two types of myofibrils, namely, stouter peripheral border fibrils (myoglia) which may pass beyond the limits of a cell and form intercellular bridges; and the more central, or myofibrils 
proper, which are limited to the cells proper and are considerably more delicate. It is believed that border fibrils may subsequently arise by fusion of the more delicate fibrils.

The function of the border fibrils is disputed, some claiming that they serve to straighten the cell following contraction produced by the central fibrils, others claiming that they have a contractile rôle similar to the central myofibrils. Whatever their complete function may be, they certainly seem to bind, together with the connective tissue, the individual cells into a compact tissue in which coördinated movement, as in peristalsis of the intestine, becomes possible-an obviously important condition.

The oval or rod-shaped nucleus retains its central location, and is surrounded by a mass of granular, relatively undifferentiated sarcoplasm, containing mitochondria, lipoid, and glycogen gran-

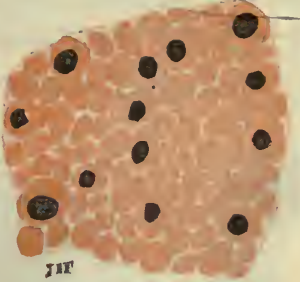

Fig. 100.- -умоотн M USCLE CeLLS FROM THE WALL OF the Human IntesTINE.

Transection. Hematein and eosin. $\times 750$. ules. It changes its shape with the phase and degree of contraction, occasionally even assuming a short, spiral form. It has been shown (McGill, Amer. Jour. Anat., 9, 4, 1909) that during contraction the nucleus decreases markedly in length and increases in thickness; and that the uniformly distributed chromatin granules stream toward the poles, where they collect in coarse strands. This structural intranuclear change is apparently unaccompanied by any change in volume.

Smooth muscle cells vary greatly in size-from the shortest of about 50 microns, to some of 500 microns in length in the pregnant uterus. When in the contracted condition, they show a number of broad, more deeply staining contraction bands, very conspicuous in the smooth muscle of the lower portion of the esophagus. As seen in transverse section these fibers vary in size from a mere point up to their maximum diameter, according as the section happens to pass through the end or through the middle of a fiber. Because of its central location, the nucleus is only found in the larger transections.

Smooth muscle fibers may be joined together in interlacing groups as in the wall of the uterus or bladder; or they may form broad membranous layers as in the wall of the alimentary tract; or again, they may form small isolated bundles, as in the skin. In any case, the muscle bundles are united by a delicate network of connective tissue. 
Smooth muscular tissue occurs chiefly in the walls of the hollow or tubular viscera. Its distribution may be classified as follows:

(1) In the alimentary tract: lower portion of the esophagus, stomach, small and large intestines.

(2) In the respiratory system: trachea and bronchial tubes.

(3) In the genito-urinary system: ureter, bladder, urethra, penis, prostate, vagina, uterus, oviduct, and ovary.

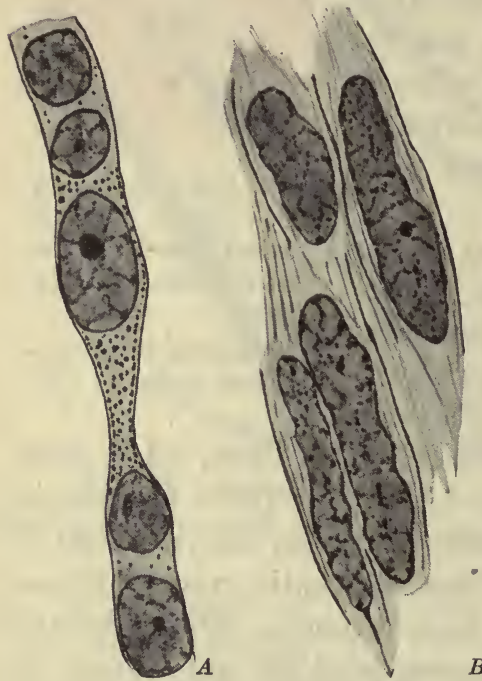

Fig. 101.-Two Stages in the Histogenesis of SMOOTH MUSCle, From the Wall of the Esophagus of a Pig Embryo.

$A, 10 \mathrm{~mm}$. stage of development. The central nucleus of the mesenchymal syncytium has become enlarged and is enveloped by a greater mass of cytoplasm. It represents a myoblast; theperipheral myochondria have become aligned preparatory to fusion to form a muscle fibril. $B, 21 \mathrm{~mm}$. stage of development. Four adjacent myoblasts, with peripheral stouter myoglia fibrils and central more delicate myofibrils. $\times 1500$.

quently acquire a cross striation. cle fibers or trabeculæ joined into an intricate close meshwork, by means of less robust branches. The nuclei retain their axial position in the 
fibers and are surrounded by an oval area of undifferentiated granular sarcoplasm. The cardiac fibers and their branches contain peripheral myofibrils, which during growth of the muscle arise by longitudinal splitting of the original fibrils and take position progressively toward the center. Cardiac muscle thus consists of a slender axial core of undifferentiated sarcoplasm swelling to an oval, more expansive mass where the

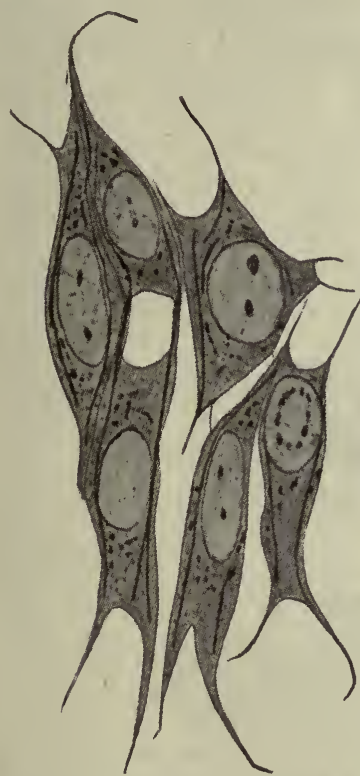

Fig. 102.-A Grodp of MroBLASTS FROM THE HEART Muscle Syncytium of A 48 Hour. Chick Embryo. Showing myofibrils, myochondria and mitochondria. The latter are the deeper staining granules. Meves' technic. $\times 2000$.

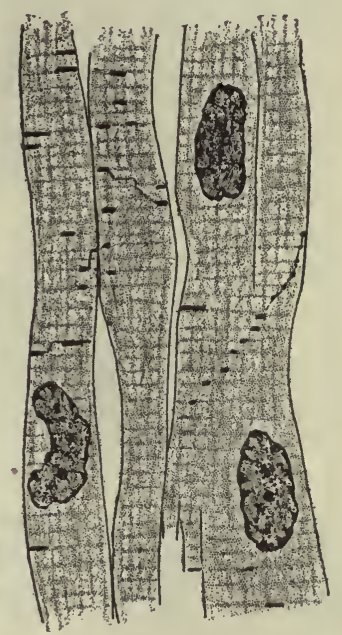

Fig. 103.-CardiaC Muscle of Guinea Pig, Showing SevERAL BRANCHES, Cross Striations ( G R O U N D M E M BRANES) AND A NUMBER OF INTERCALATED Disks.

Zimmermann's technic. $\times 1000$.

nuclei are located; this core is surrounded by successive rows of myofibrils arranged in groups representing Cohnheim's fields in transverse section; and the whole is invested by a delicate sarcolemma. The striations of the fiber result from the fact of a transverse alignment of identical areas in adjacent fibrill - a correspondence which indicates a definite functional stimulus to a structural modification (Fig. 103). The sarcoplasm contains mitochondria (Fig. 102), lipoid, albuminoid (interstitial gran- 
ules of Kölliker), and glycogen granules. Fat granules, Tiposomes' (Bell) of probably nutritive significance, and varying greatly in amount according to the functional condition of the individual, are normally present in cardiac muscle (Bullard, Amer. Jour. Anat., 14, 1, 1912). This fatty content can be demonstrated by the several microchemical technics for lipoids. According to Meves, Duesberg, and others,-the myofibrils of striped muscle differentiate from the mitochondria of the myoblasts; but since mitochondria can be demonstrated in highly developed

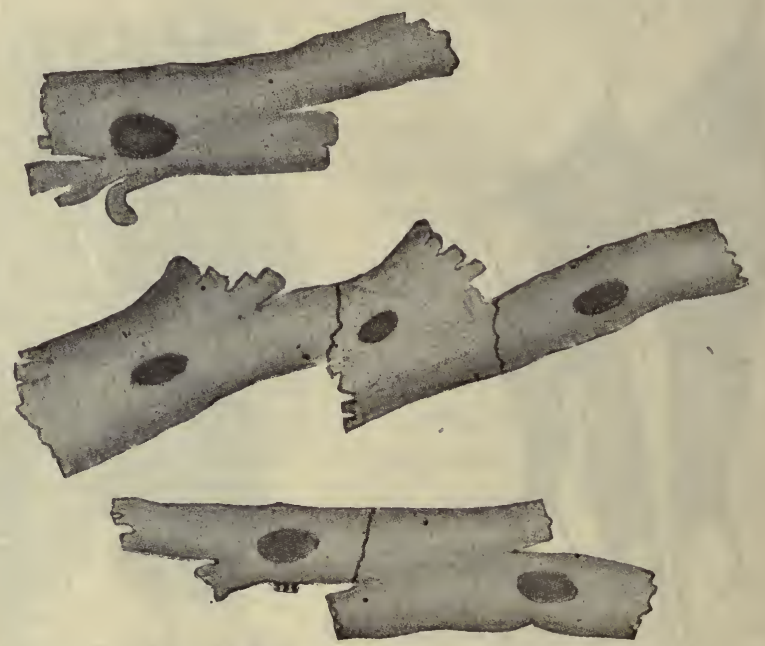

Fig. 104.-Cardiac Muscle 'Cells' from the Pig's Heart, Isolated in Equal Parts of Alcohol, Glycerin, and Water.

Unstained. (The nuclei are somewhat darker than they actually appear.) $\times 410$.

fibers (Fig. 116) it seems improbable that mitochondria have anything directly to do with the development of muscle fibrils.

It has been claimed that heart muscle and striped muscle generally can be interpreted in terms of muscle cells, and intercellular myofibrillæ, in analogy with connective tissue (Baldwin). But the presence of a continuous axial core of undifferentiated sarcoplasm, lack of a definite cell membrane separating this sarcoplasm from the outlying myofibrillæ, inability to separate such 'cells' by dissociation methods, and the extension of the telophragma to the nuclear wall, seem to render this view untenable.

The myofibrils must be further considered. No distinction between border fibrils and central fibrils, as in smooth muscle, is possible in cardiac muscle. But the myofibrils undergo greater differentiation. This 
expresses itself in an alternation of light and dark disks ('bands,' 'segments,' 'stripes'), said to consist of isotropic and anisotropic substances respectively. While the disks are conspicuous both in fresh and stained tissue, the demonstration of their physical properties under the polariscope is a matter of difficulty. Indeed with crossed Nichols the entire

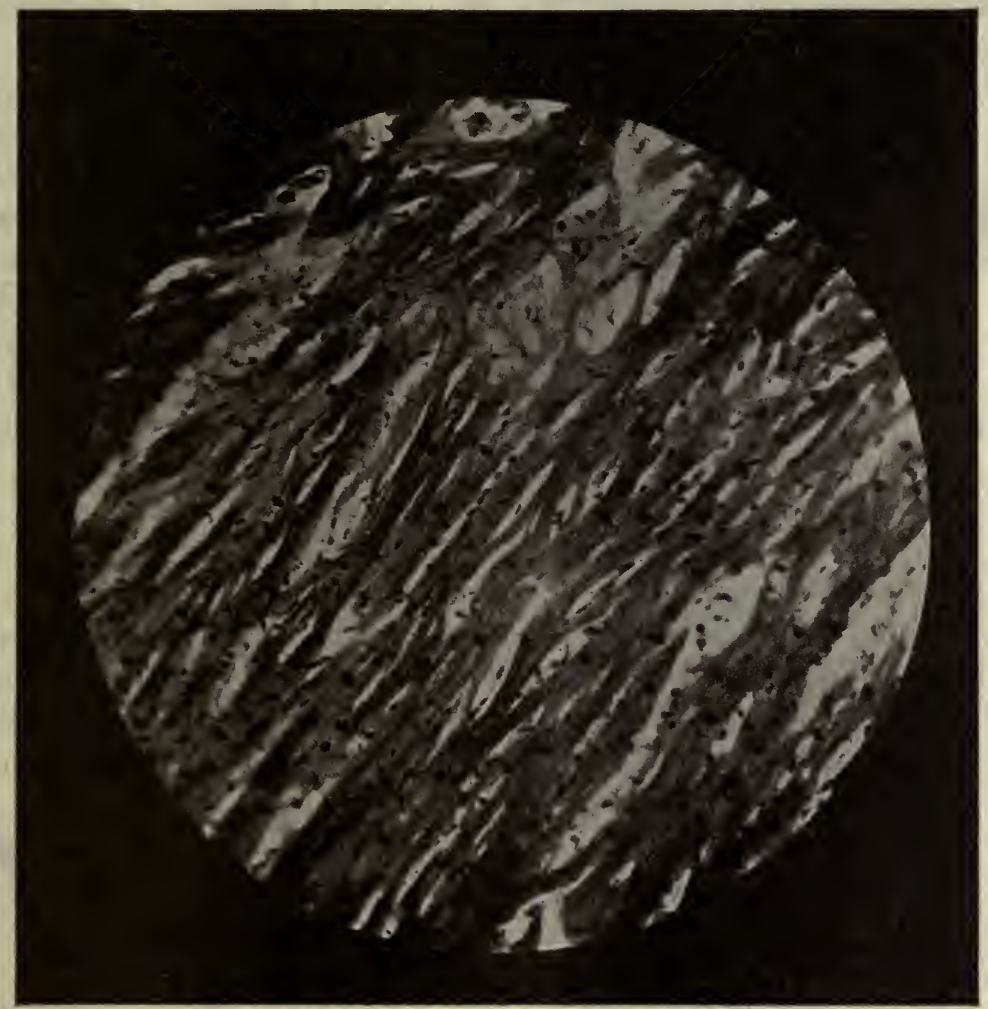

Fig. 105.-Cardiac Muscle of the Human Heart; the Abundant Branches Are Plainly Shown.

Longitudinal section. Hematein and eosin. Photo. $\times 120$.

fiber appears lighter than the field, showing the presence of anisotropic materials (granules) scattered throughout the fiber, but a definite banding corresponding to the light and dark disks of fresh muscle is not apparent in all striped muscle. It seems more probable that, though anisotropic granules are more abundant in the dark disk, they are not absent in the lighter disk; moreover, they are more or less definitely aggregated in the dark disk according to the phase of contraction. The 
lighter disk, or intermediate disk of Krause, is commonly designated by the letter $J$ (Isotrope streife); the dark disk, or transverse disk of Brïcke, by the letter Q (Querscheibe). On closer inspection the $J$

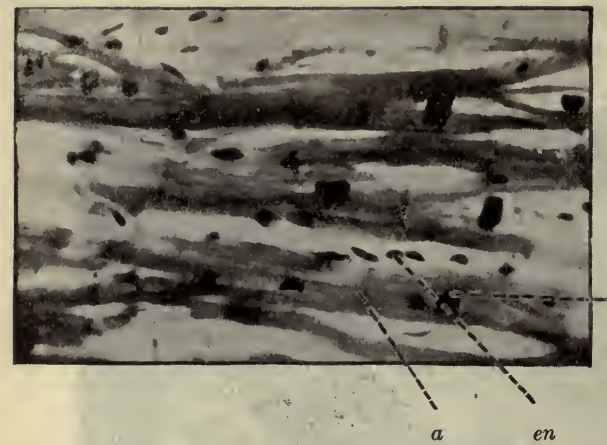

Fig. 106.-The Central Portion of the Preceding Figure, More Highly MagNIFIED.

$a$, intercalated disk; en, endothelial nuclei of a capillary; $N u c$, nucleus of 'muscle syncytium. Hematein and eosin. $\times 500$. disk is seen to be bisected by a dark disk or membrane, the ground membrane of Krause, designated by the letter Z (Zwischenscheibe). I n d e e d this is the most conspicuous stripe, and gives to the muscle, as seen under ordinary low magnification, its banded appearance in uncontracted fibers. The term telophragma has recently been employed by Heidenhain for this membrane. The myofibrils are intimately connected with it. Similarly, the Q disk is bisected by a narrow light disk, the median disk of Hensen $(H)$, which in turn is said to be bisected by the intermediate membrane of Heidenhain, or mesophragma (M, Mittelscheibe). Both telo- and mesophragmata (inophragmata) are supposed to unite with the sarcolemma peripherally, and to be structurally similar. The telophragma is in intimate connection both with the sarcolemma and the nuclear membrane. But the mesophragma, at least in striped muscle of certain forms, e.g., Limulus, is not a true membrane to which the fibrils are attached in the manner of the telophragma. Indeed it remains an open question whether heart muscle actually possesses a mesophragma.

The portions of a fibril, or sarcostyle, included - between successive telophragmata, constitute structural units, the sar-

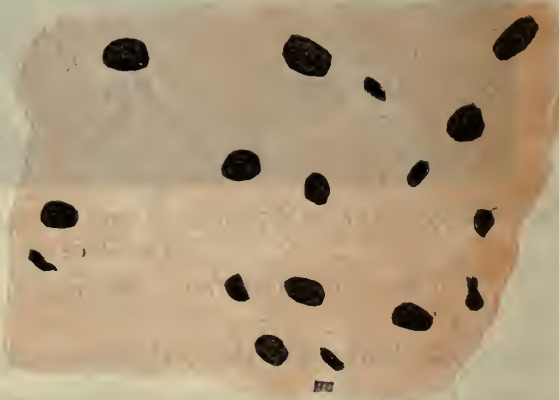

Fig. 107.-Transection of a Group of Cardiac Muscle Fibers from a Papillary Muscle of the Human Heart.

Hematein and eosin. $\times 550$. comeres, or inokommata (Heidenhain, Fig. 110). In inacerating fluids 
fractures occur at the $\mathrm{Z}$ lines. These membranes extend completely across the fiber, through the axial strand of sarcoplasm-a significant fact controverting the cellular idea of cardiac muscle originally advanced by Apathy. The interstitial granules of Kölliker (sarcosomes of Retzius) scattered throughout the $\mathrm{Q}$ and $\mathrm{J}$ disks in striped muscle, both cardiac and skeletal, are designated the $Q$ and $J$ granules respectively (Holmgren).

Intercalated Disks.-A unique characteristic of cardiac muscle pertains to the presence of the intercalated disks, 'junctional lines,' or bands of Eberth. These are barely visible in ordinary histologic preparations, but can be rendered conspicnous by the special technics of Heidenhain and of Zimmermann. In gross appearance they are of several sorts: straight bands, step-like forms, and serrated forms. The bands (disks) may extend completely across a fiber, or only the width of a single fibril (granule type) ; the step form may

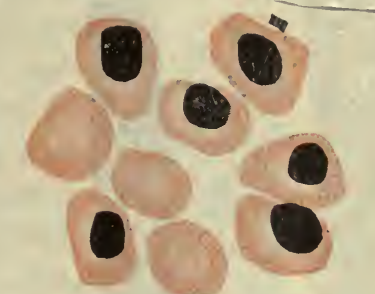

Fig. 108.-Developing Muscle Fibers from the Heart of a Human Fetus at Seven Months.

Fibrillæ are well developed at the periphery; the undifferentiated cytoplasm in the center presents a clear appearance and in some cases is partially occupied by the nucleus. Hematein and eosin. $\times 750$. consist of one or many groups of steps and risers, the 'risers' being the height of one or, occasionally, several inokommata; the saw-tooth type also may be of small or greater extent, and of the height of one or several inokommata. All three types may be arranged in rings or even longer or shorter spirals. The intercalated disks are peripheral in position, extending for varying depths, but never completely through a fiber, and never central to the axial sarcoplasm. They are occasionally on the same level with the nucleus. They have been found in the heart of representatives of all the animal groups to, and including, teleost fishes (Jordan and Steele). They are present sparsely and in simple form also in the heart of Limulus. They are probably a morphologic incident of the rhythmic contraction of cardiac muscle. They appear only late in fetal life, toward the end of the last week of gestation in the guinea pig.

The earliest disks are all of the coarsely granular band type. Subsequently they increase in number and complexity, the older stages being characterized by occasional saw-tooth forms. Once formed, they are evidently for the most part permanent structures, undergoing modification largely through mechanical factors. On closer inspection, under

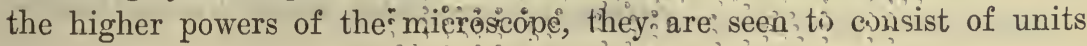




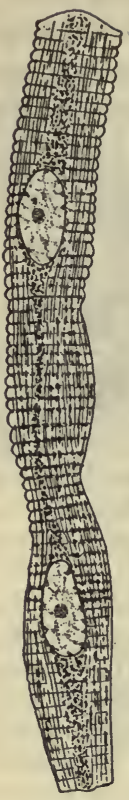

A

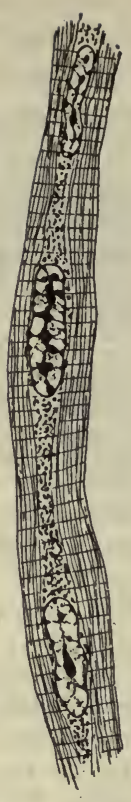

B

Fig. 109.-Cardiac Muscle Fibers.

$A$, portion of a fiber from a macerated preparation of a cat's heart, drawn according to its appearance in the optical longitudinal plane. Two nuclei are shown, connected by a continuous axial strand of coarsely granular sarcoplasm. The sarcolemma appears festooned between successive ground membranes. There is no evidence of a cell membrane separating the central granular from the peripheral non- or finely-granular sarcoplasm. $\times 1000 . \quad B$, median longitudinal section of a fiber from the ventricle of an adult white mouse. Note the continuity of the axial sarcoplasm. Hematoxylin and eosin. $\times 1000$.

corresponding to portions of a single fibril. These units may be granular or compact. The units are bisected or bounded on one side by the $\mathrm{Z}$ membrane. Association of the units in transverse lines gives rise to the band forms; they may be drawn into spirals by longitudinal traction of the fibers involved; unequal transverse and oblique tractions probably produce the step forms; the sawtooth form arises by process of longitudinal splitting of fibrils, enlargement of fibers, and the various tensions characteristic of hypertrophying fibers. The exclusive type of hypertrophied heart muscle is the more or less complex saw-tooth type. The practically exclusive type of atrophied heart muscle is the 'comb type,' a type produced from a band type by a modification involving longitudinal tension (Fig. 114, d). In brief, the unit of structure is a modified focus on a myofibril, in essence involving an accumulation of granules about the $\mathrm{Z}$ membrane. Such foci associated in various ways produce the various types of bands and steps, the latter in part due also to external mechanical factors, the extreme condition of such effect being sawtooth forms.

A significant point concerns the similarity between the phylogenetic and ontogenetic development of intercalated disks : that is, below birds, as in all fetal hearts; only simple bands appear; in birds as in young hearts, step forms are present; only in mammals and in old hearts do the more complex types appear. What then is the meaning of these disks? Any interpretation must be more or less tentative at present. It is easier to say what they probably are not, than what they probably are. They were originally interpreted as cell boundaries, or intercélular cement lines (Schweig- 
ger-Seidel); this interpretation has recently been again supported by Zimmermann. This interpretation would mean that from a syncytium a cellular tissue has secondarily arisen by the appearance of cement lines, secondary cells having been formed in a syncytium, irrespective of the original genetic units. A number of facts render this interpretation inadmissible, chief among which are their occasional supernuclear position, and their peripheral location. A more recent interpretation conceives of them as places where the muscle fiber grows, that is, as sarcomeres in the making (Heidenhain). Among the countervailing facts to such interpretation are chiefly the absence of transition stages, their relative scarcity at the period of greatest growth of the heart, and their continued abun-

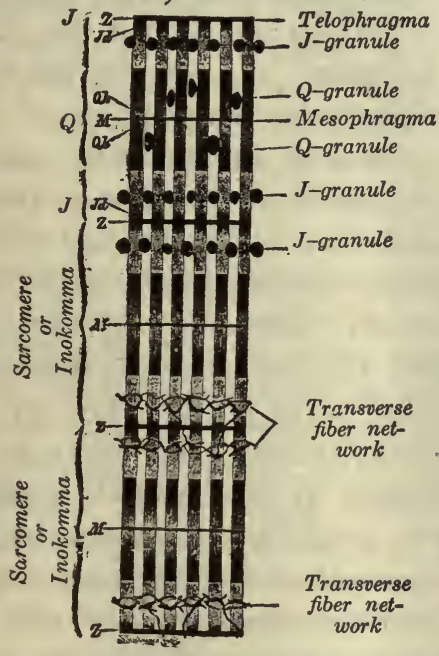

Fig. 110. - Diagram of a

Striped Muscle Fiber, ACCORDing to Heidenhain.

The transverse fiber network may be a trophospongium.

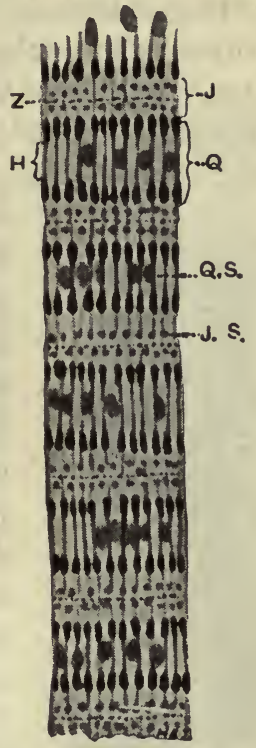

FIG. 111.-LongITUDINAL SECTION OF PORTION OF WING MUSCLE Fiber of Mantis, at Mid-phase of Contraction.

$z$, telophragma; $h$, Hensen's disk; $j$, isotropic disk; $q$, anisotropic disk;

q. s., $q$-sarcosome;

$j$. s., j-sarcosome. $\times 1600$.

dance in full-grown, even aged, hearts. The suggestion has been made that they are related to a phase of contraction. This seems more likely. Since, once formed, disks are largely permanent, and undergo subsequent modification; they must represent an irreversible condition of the contraction phase. The interpretation of the disks as irreversible contraction bands rests upon the similarity of the simplest types and the contraction bands of Rollet, both characterized by accumulation of dark stain- 
ing granules about the $\mathrm{Z}$ membrane. In the older hearts, where they are mechanically modified, and in diseased hearts, as in hypertrophies, where probably a chemical modification is also suffered, they represent lines of weakness. These are the locations of fracture in fragmented and.segmented pathological hearts - a significant point in relation to 'heart failure.'

Heart muscle is syncytial in structure, and the myofibrillæ pass uninterruptedly through the intercalated disks. These facts are of special importance because of their bearing on the opposed theories of the origin and conduction of the stimulus to the heart beat, the myogenic and the neurogenic. A complete cellular structure with actual cement lines, combined

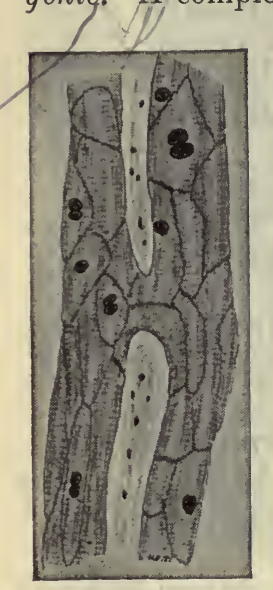

FIG. 112.--LoNGITUdinal Section OF PORTION OF ATRIo - VENTRICULAR BUNDLE OF HEART' OF BeEF. MANY OF THE Cells are BinuCLEATED. $\times 160$. with the fact that the atria are apparently completely separated from the ventricles by intervening connective tissue, was once urged as a strong argument against the validity of the myogenic theory of heart beat-the theory which proclaimed the adequacy of heart muscle to initiate and conduct the stimulus to contraction without the intervention of nerve elements, that is, to beat automatically and independently of the nervous system. The neurogenic theory, which holds that nerve elements are essential for the conduction of stimuli for contraction, on the other hand, seemed contradicted by the observation that in the chick the heart beat rhythmically before the appearance of nerve fibers. However, there remained the possibility that nerve fibers were present but uncemonstrable by the method employed; also that while nerves might be unnecessary for maintaining rhythmic contraction during embryonic life, they nevertheless became necessary in fetal and adult life.

The discovery of the atrioventricular bundle of $H$ is (1893) at first added apparently the strongest evidence to the support of the application of the myogenic theory of heart beat in the mammalian. This is a muscular bundle which effects an intimate connection between the atria and the ventricles. See heart, p. 199.

An important matter is the observation that the final ramifications of the bundle of His are identical with the so-called Purkinje fibers. These have long been known, especially in the sheep's heart, where they are unusually large and abundant. They are limited to a region directly under the ventricular endocardium. They are coarser, less branched, with fewer intercalated disks, almost exclusively of the band type, than are the fibers of the myocardium proper. They would 
seem to represent a younger or less highly differentiated stage of muscle fiber. In cross section they are of greater diameter, with fewer peripheral myofibrils and a far greater amount of central undifferentiated sarcoplasm, rich in glycogen. According to Lange (Arch. mikr. Anat., Bd. $84,1914)$ the Purkinje fibers cannot, however, be regarded as remains of embryonic muscle cells, since they are clearly distinguishable already in very young mammalian embryos; they constitute the non-nervous apparatus for conducting stimuli to heart beat.

The myogenic theory accordingly seemed well established. It was apparently strongly supported by the experiments of Erlanger (1906), who clamped the bundle in the dog's heart and produced a condition of 'heart block'-a disturbance of the coördinated rhythmicity of the atria and ventricles-without, however, interfering with the conduction of impulse, since there resulted no stoppage of contraction in the ventricles. But the subsequent discovery of abundant nerve fibers and ganglion cells (Tawara, Wilson, McGill) in the bundle, intimately related to the cardiac fibers, robbed this experiment of its specific applicability and seemed for a time to force an interpretation favoring the neurogenic theory. Carlson, moreover, demonstrated its validity for the Limulus heart, in which, after the removal of its ganglion, it could not be made to beat. But more recently Burrows (1911) has shown that single cells of a 14- to 18-day embryo chick heart, grown in artificial culture media, may begin to beat automatically and rhythmically-an observation which would seem to settle the point that heart muscle may beat rhythmically in the absence of nerve supply or even nerve stimulus.

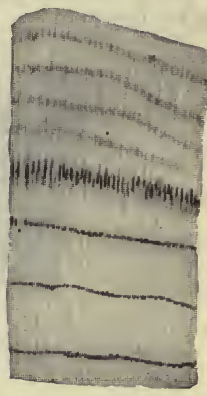

Fig. 113.-LongITUdinal SeCtion of a Trabecula OF LIMULUS (KING $\mathrm{Cr}_{\mathrm{RAB}}$ ) Heart Muscle, Showing AN INTERCA L A TED Disk SeparatING A ContractED FROM AN UNCONTRACTED PORTION.

Nitric acid-alcohol fixation (Zimmermann's technic), iron-hematoxylin stain. $\times 1300$.

Furthermore, Hooker (Jour. Exp. Zool., 11, 2, 1911) showed that in the frog larvæ in which the nervous system was entirely removed, the cardiac muscle will differentiate and function normally, independently of nervous control. The myogenic theory is further supported by the fact that it has been possible to revive the excised heart of man to rhythmic activity 20 hours after death (Flack, "Further Advance in Physiology," Ed., Hill, 1909), whereas the longest time that a nerve cell is known to survive (in the intestine) is $3 \frac{1}{2}$ hours (Cannon and Burkett, Amer. Jour. Phys., vol. 32, p. 347, 1913). In the superior cervical ganglia, nerve cells may survive 1 hour, while in the brain the maximum time of survival is said to be 15 minutes. 
Any interpretation of the intercalated disks as actual intercellular structures (cement substance) would seem inconsistent with the myogenic theory of the heart beat, which now seems largely to prevail. The present,status of the matter seems to be that the origin of stimulus to heart beat in vertebrates is myogenic, in invertebrates probably neurogenic. The difference may inhere in the absence in the hearts of invertebrates of a muscular coördinating structure analogous to the atrioventricular bundle of vertebrate hearts.

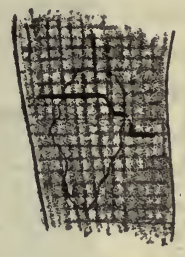

$a$

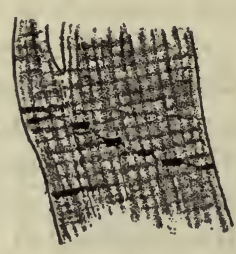

$b$

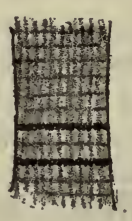

$c$

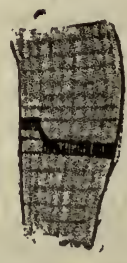

$d$

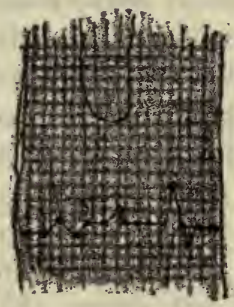

e

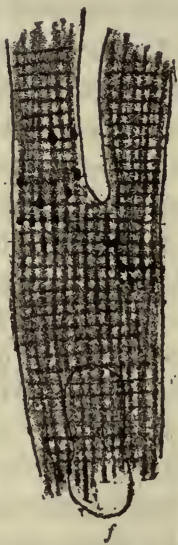

Fig. 114.-Semidiagrammatic Illustrations of Various Types of Intercalated Disks.

$a$, from guinea pig's heart; $b$, from chipmunk's heart; $c$, from monkey's heart; $d$, from monkey's heart; $e$, from guinea pig's heart; $f$, from chipmunk's heart. $\times 1200$.

Voluntary Striped or Skeletal Muscle.-The unit of structure of skeletal muscle is essentially the same as that described for cardiac muscle, namely, a myofibril or sarcostyle. The fiber is likewise divisible into successive sarcomeres or inokommata. A difference in detail inheres in a greater definiteness of striation, and a greater complexity, due to the presence generally of an additional disk in the $J$ stripe. This stripe or accessory disk of Engelmann ( $N$ line; nebenscheibe) bisects the 
portion of the $J$ disk between the $\mathrm{Z}$ line and the succeeding $\mathrm{Q}$ disk. It is interpreted more or less tentatively by Heidenhain as due to a linear arrangement of $\mathrm{J}$ granules. The median segment of the $\mathrm{J}$ disk, that is,

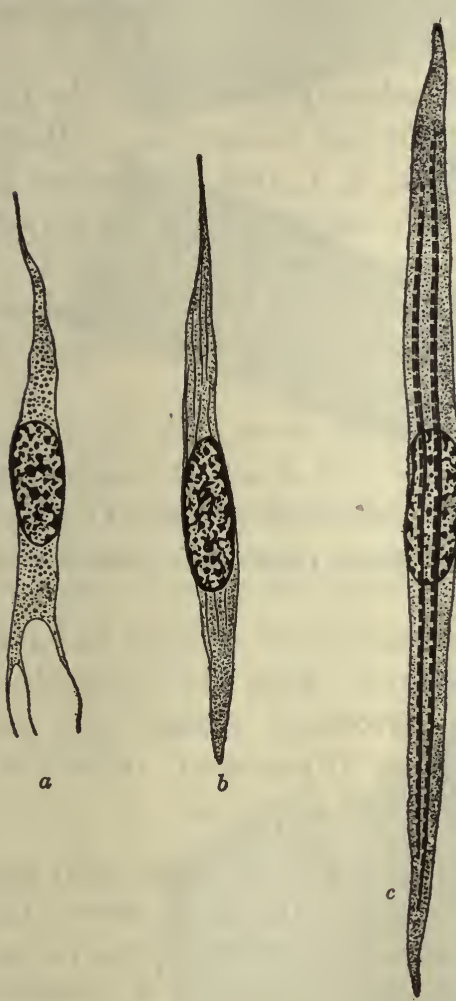

Fig. 115.-Successive Stages of Skeletal Muscle Histogenesis in Mammals.

$a$, myoblast with fine cytoplasmic granules (myochondria), from a 13 $\mathrm{mm}$. sheep embryo; $b$, myoblast with homogeneous myofibrils, from a $10 \mathrm{~mm}$. guinea-pig embryo; $c$, myoblast with cross striped fibrils, from a $8.5 \mathrm{~mm}$. rabbit embryo. (From Heidenhain, after Godlewski.) the portion between the $\mathrm{N}$ line and the $\mathrm{Z}$ line, is called the terminal disk of Merkel (E disk; endscheibe). This complex condition of striping is conspicuous only in certain insect muscles (e.g., leg and wing muscles of wasp, etc.) where powerful activity or great rapidity of beat is required. In general, complexity of striation of conspicuous character corresponds with a relatively greater irritability and a capacity for more powerful or relatively more rapid function.

Another difference between skeletal and cardiac muscle pertains to the diameter of the fiber and the position of the nucleus. In skeletal mus-

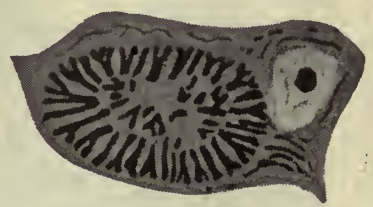

Fig. 116. - Transverse Section of a Striped Muscle Fiber of a Newly-hatched RaINBow Trout, Showing the Process of MroFIBRIL INCREASE BY RADIAL LONGITUDINAL SPLitTing.

Mitochondria are seen in the peripheral sarcoplasm and around the nucleus at the right. Meves' technic. $\times 2000$.

cle the fiber has a greater diameter, is more nearly circular in cross section, the myofibrils are scattered throughout its diameter, and the nuclei are peripheral, lying just beneath the sarcolemma. The nuclei are envel- 


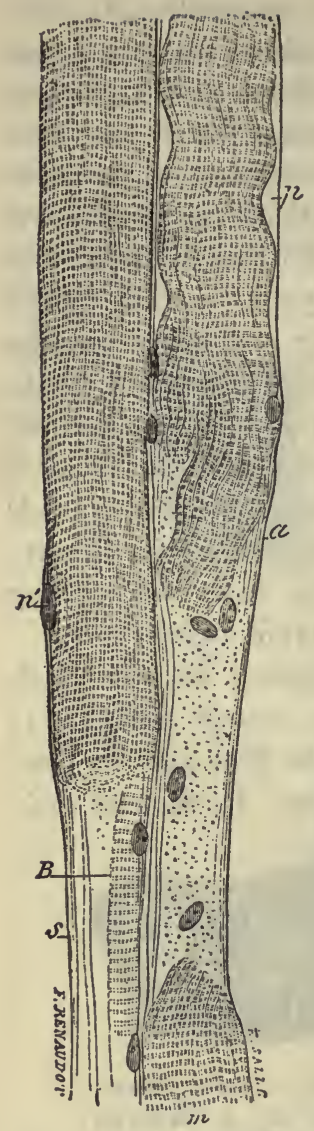

Fig. 117.-Striated Muscle Fibers Ruptured by Teasing, Showing the SarcoLEMMA.

$a$, ruptured end of the muscle fiber; $B$, a bundle of fibrils projecting from the torn end; $m$, a muscle fiber; $n^{\prime}$, a nucleus of the muscle cell; at $p$, the muscle substance has shrunken away from the sarcolemma; $s$, sarcolemma. Moderately magnified. (After Ranvier.) oped in a small amount of granular sarcoplasm. The sarcolemma is a homogeneous, plastic, nonnucleated membrane, the representative and product of the cell membrane of the original myoblasts.

In routine laboratory preparations only the $\mathrm{Z}, \mathrm{Q}$, and $\mathrm{J}$ stripes are conspicuous. Meigs (Zeitschr. allg. Phys., 8, 1, 1908) recognizes at most only three different substances in striped muscle sarcostyles (fresh wing muscle of fly) : Q, Z, and M. He regards $J$ and $H$ as optical effects dependent upon the reflection of light by the $\mathrm{Z}$ and $\mathrm{M}$ membranes.

Thulin (Arch. mikr. Anat., 86, 3, 1915) records the absence of both $\mathrm{Z}$ and $\mathrm{M}$ membranes in the wing muscles of certain insects (Coleoptera) and the analogous (pectoral) muscles of birds and bats.

The perinuclear sarcoplasm contains filar and granular mitochondria. Both the perinuclear and interfibrillar sarcoplasm contain also fat granules and globules (liposomes), interstitial granules of Kölliker and glycogen.

Heidenhain (Anat. Anz., 44, 11-12, 1913) has conclusively shown that in the trout embryo the progenitor (the manner of whose origin is unknown) of the definitive myofibrillæ is a single, stout, deeply staining column, close to the nuclear wall externally, which undergoes a succession of radial and concentric longitudinal divisions. This observation would seem to dispose of the assumption of a direct mitochondrial origin of myofibril$1 æ$ in this form at least, in the manner of the current descriptions. And this conclusion is strengthened by the demonstration of mitochondria throughout the earlier development (Fig. 116).

Skeletal muscles develop from a portion of the mesodermic segments or primitive somites, called the myotomes. The myoblasts pass through 


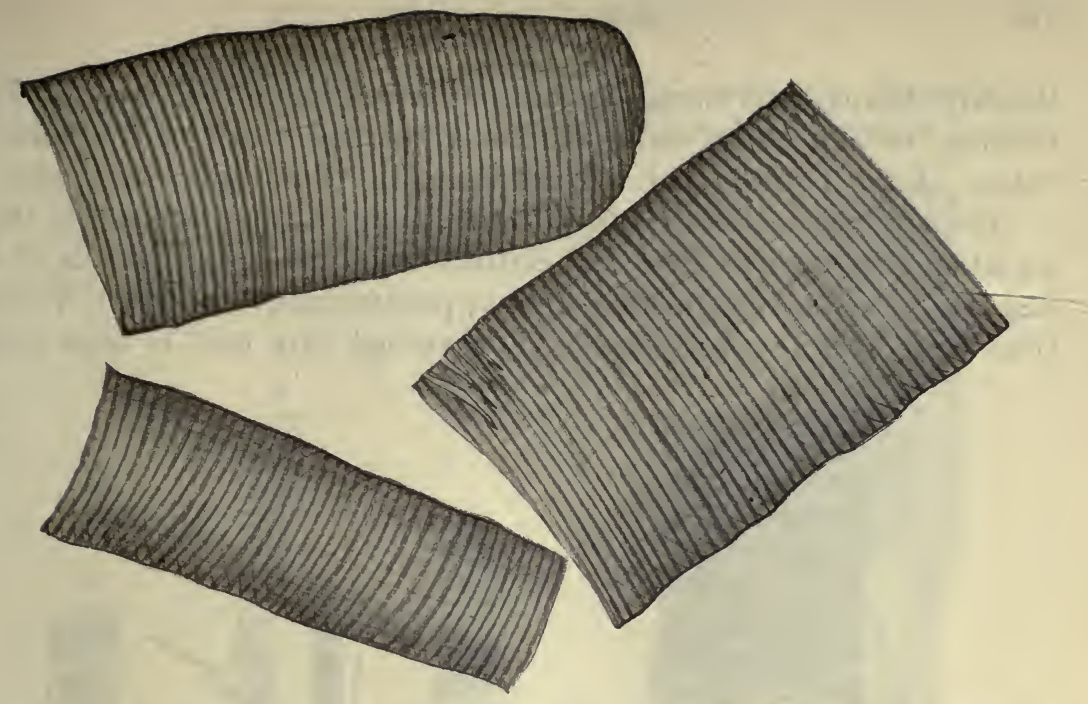

Fig. 118.-Isolated Fragments of Striated Muscle Fibers, Unstained.

The one above is from the end of a fiber; that on the right shows at one end a tendency to cleavage into transverse disks. $\times 360$.

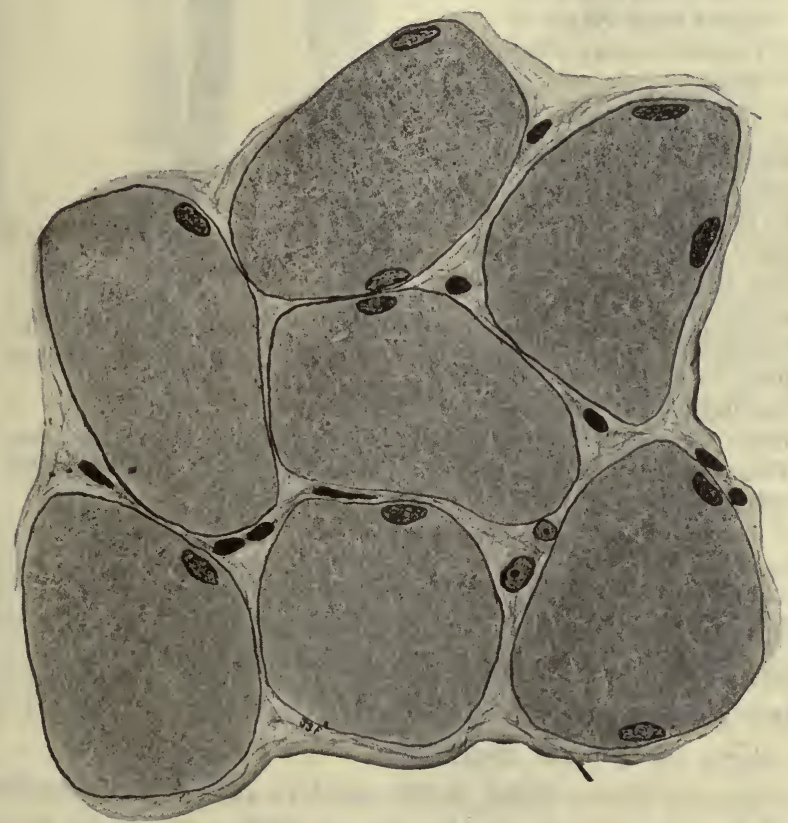

Fig. 119. - Striated Muscle Fibers of the Dog, Seen in Transection.

The areas of Cohnheim are indistinctly outlined. Hematein and eosin. $\times 490$. 107 
the early histogenetic changes already described for cardiac muscle. In the frog, irritability was shown by Hooker to follow closely upon differentiation of the fibrillæ, and the establishment of nervous connections.

The adult skeletal muscle fiber is a multinucleated structure. Is this condition the result of fusion of distinct myoblasts, or of growth of a single myoblast accompanied by nuclear proliferation? Both interpretations have been advanced; many observational data tend to show that

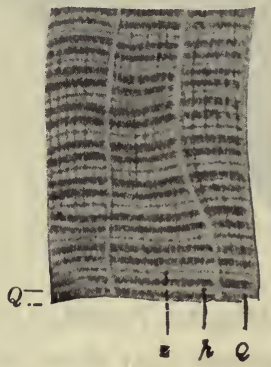

Fig. 120.-A Portion of a Striated MusCle Fiber Seen in Longitudinal SeCTION.

The alternate light and dark cross striations are well shown. $h$, light line, Hensen's line, in the middle of the dark disk $Q$. $z$, dark line, Krause's membrane or Dobie's line, in the middle of the light disk. Hematein. $\times 1200$. (After Böhm and von Davidoff.)

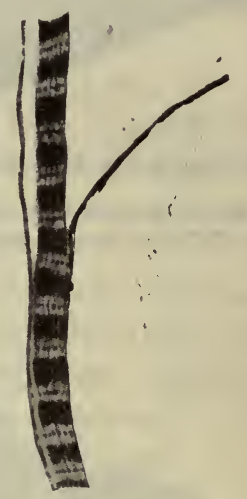

Fig. 121.-A Small Portion of a MusCLE FibER OF A Crab Showing BeginNing Separation inTo FibRILS.

Drawn from a photograph. $\times 600$. (After Schäfer.)

a skeletal muscle fiber represents a myoblast which has elongated and multiplied its nuclei. In the trout embryo, however, considerable fusion of myoblasts occurs. The mode of nuclear division appears to be at first mitotic, and subsequently amitotic. In the tongue a small number of branched fibers have been described.

Striped muscle fibers differ in the relative amounts of myofibrillar and sarcoplasmic content. When the myofibrillæ are relatively preponderant and the interstitial granules sparse, the fiber is known as 'light'; 
when the sarcoplasm, with its interstitial granules, is relatively abundant, 'dark.' In the latter fibers many of the nuclei may be centrally located.

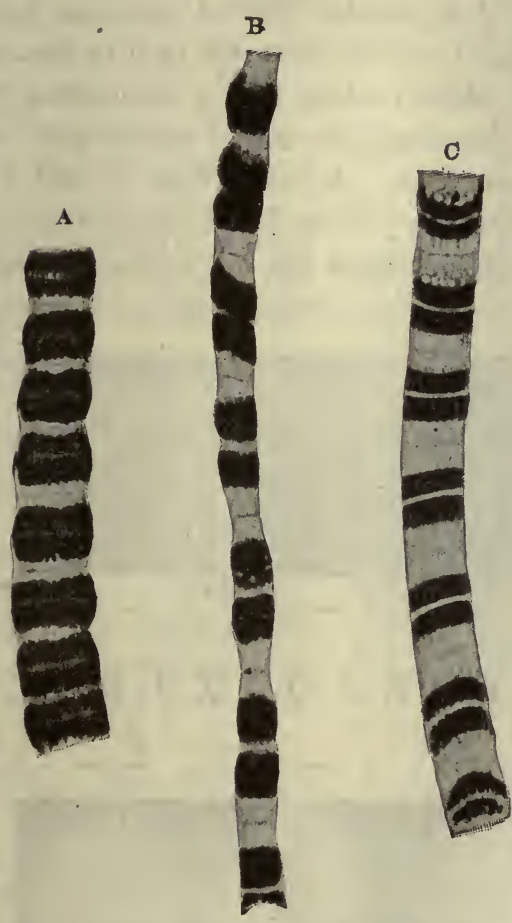

Fig. 122.-Fibrils From the Wing Muscles of a Wasp.

$A$, compressed; $B$, stretched; $C$, uncontracted. The alternate dark and light disks are prominent; the membrane of Krause in the light disk, and the line of Hensen in the dark disk are well shown. Very highly magnified. (After Schäfer.)

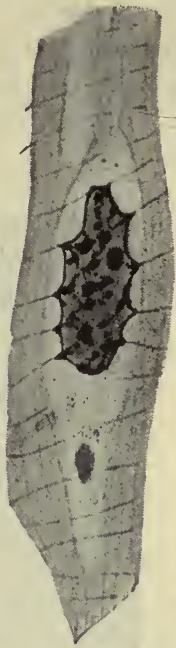

FIG. 123.-LongiTUDinal SeCtion of a Portion of a Striped Muscle Trabecula OF LIMULUS, Showing a NuCLEUS OF SERRATED CONTOUR WITH THE TELOPHRAGMATA ATTACHED TO THE Serrations.

The nucleus lies in an undifferentiated mass of sarcoplasm containing below a deutoplasmic granule. The adjacent myofibrils simulate a cell membrane. Fleming's fluid, iron-hematoxylin. $\times 2000$.

Most mammalian muscles contain both types of fibers, but with the 'light' greatly in excess. When a muscle consists chiefly of 'light' fibers it is a 'white' muscle; when the 'dark' fibers are abundant it may be 
called 'red' muscle. The former variety, for example the biceps muscle, acts more energetically but is more easily fatigued; the latter, like the

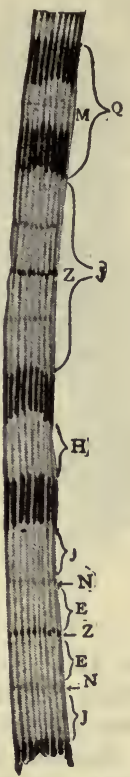

FIG. 124.-S T R I A T E D Fiber FROM a LEg Muscle of the Sea SPIDER (ANOPLODACTYLUS LENTUS), Showing the Complexly Striped ConDition Characteristic of Insect MusCLE.

$\mathrm{Q}$, anisotropic disk; $\mathrm{J}$, isotropic disk; M, membrane of Heidenhain (mesophragma); Z, membrane of Krause (telophragma); $\mathrm{H}$, median disk of Hensen; N, accessory disk of Engelmann; E, terminal disk of Merkel. $\times 1000$. muscles of mastication, respiration, the eyeball, and cardiac muscle, are functionally characterized by slower activity but less ready, fatigue. The interstitial granules accordingly seem to be of nutritive significance. They are generally more abundant in the $\mathrm{J}$ than in the $\mathrm{Q}$ segments. The $\mathrm{J}$ granules are of spherical form and smaller than the oval $\dot{Q}$ granules. Striped muscle fibers contain also a trophospongium (Holmgren). Adult muscle cannot regenerate, but is replaced by scar tissue. A young fiber is said to be able to regenerate, the process involving movement of proliferating nuclei toward the cut surface. Muscle growth, as with exercise, depends upon enlargement
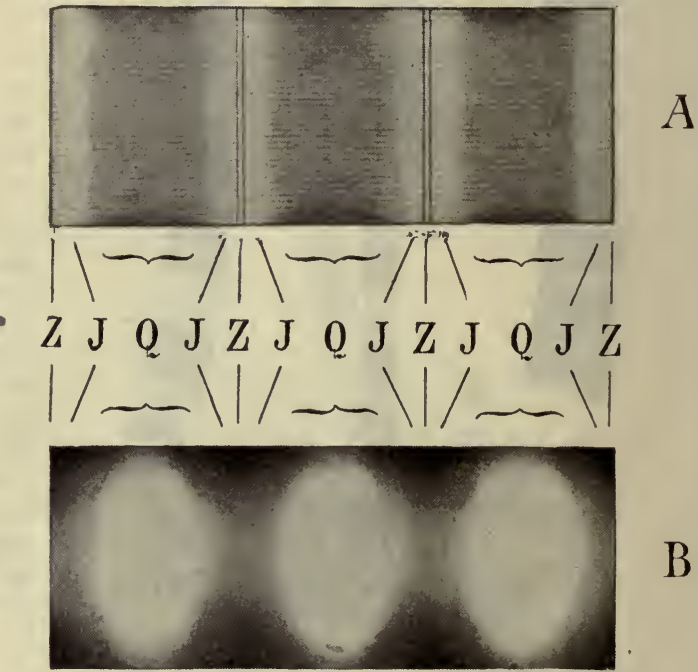

B

Fig. 125.-Semidiagrammatic Drawing, Representing the Appearance of the Same Fiber from the Leg Muscle of a Beetle in Ordinary and Polarized Light.

A, appearance in ordinary light; B, appearance in polarized light. (After Meigs.)

of the muscle fiber, consequent upon a multiplication of fibrillæ by process of longitudinal splitting, and their individual enlargement. 


\section{MUSCULAR CONTRACTION}

The physics and chemistry of muscle constituents are too inadequately known to permit anything like a confident description of the mechanism of contraction. It has been suggested that muscular contraction is essentially a reversible coagulation process. The rapidity of the process seems a fatal objection to this explanation. A plausible interpretation follows the analogy afforded by the action of catgut suspended in water, the temperature of which is suddenly raised by passage of an electric current, namely, a swelling and consequent shortening of the fiber (Engelmann). In muscle, the myofibrillæ may be conceived of as corresponding to the catgut of the experiment, the semifluid sarcoplasm to the water, and the nerve impulse to the electric current. The rapidity of muscular activity, however, again seems a difficulty. However, the optical changes undergone by a contracting muscle give evidence in favor of such interpretation. The fibrillæ shorten and thicken in con-

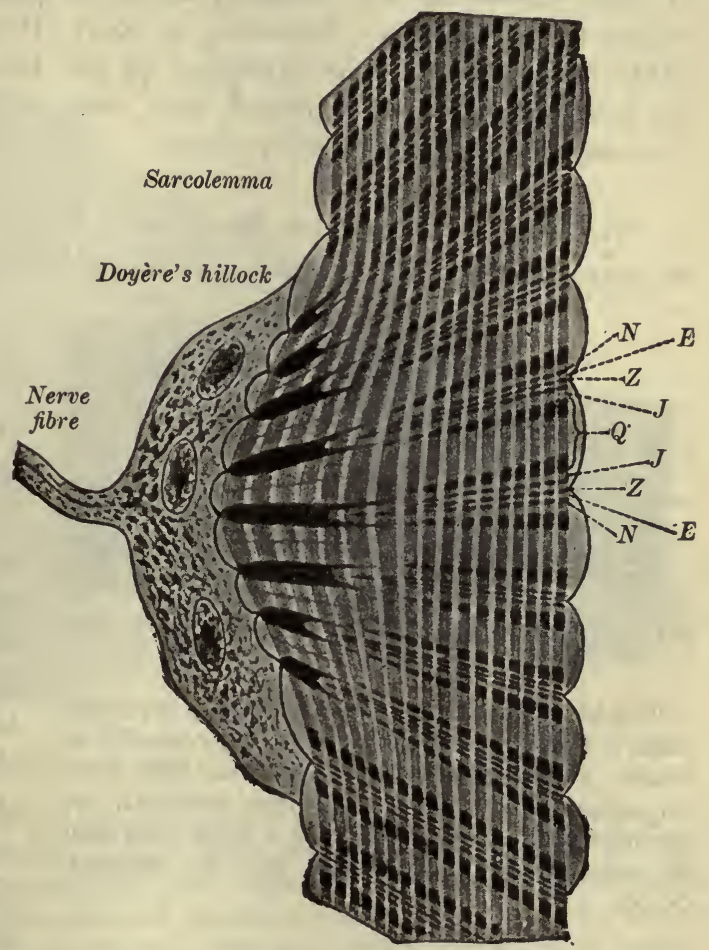

Fig. 126.--Lateral Contractive Wave of Cassida EQUESTRIS. (After Rollet.)

The formation of the contraction band is well seen, at the left, as thick black lines. (From SzymonowiczMacCallum, "Histology and Microscopic Anatomy.") traction, and there is a rearrangement of the optically different substances of the fiber; the dark granules aggregate about the $\mathrm{Z}$ line, so that it seems to have disappeareda change which involves also the disappearance of the original $\mathrm{Q}$ stripe. We may be fairly certain that the explanation of muscular contraction must be sought for in physical and chemical changes in the myofibril, it being 
the essential contractile element, the sarcoplasm serving largely as a nutritive substance. However, it should also be mentioned that it has been held that the sarcoplasm may be the essential contractile substance.

Meigs questions the pertinency of Engelmann's 'artificial muscle' in his catgut experiment, and the validity of the suggested hypothesis of contraction as a thermodynamic phenomenon. He regards contraction as the result of a rise in osmotic pressure and the consequent imbibition of fluid caused by the breaking down of larger into smaller molecules within the sarcostyles. According to Roaf (Proc. Roy. Soc., Series B. 88, 1914) contraction can be explained on the hypothesis that lactic acid is set free, and that this combines with certain proteins to form salts, with a consequent rise of osmotic pressure.

We must now consider the muscle as a whole. For this purpose we may select any well-known muscle, for example, the biceps. A muscle

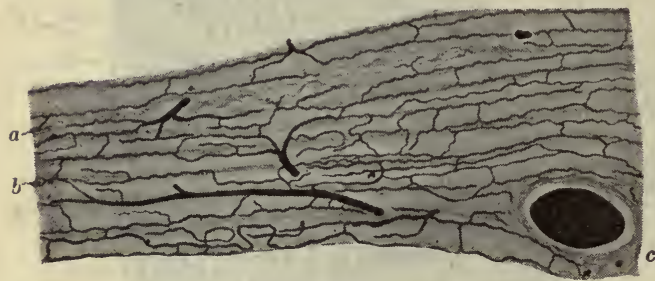

Fig. 127.-Striated Muscle Fibers of the Dog.

The blood-vessels have been filled by injection with a gelatinous mass and are represented in black. One whole fasciculus and one fiber from an adjacent fasciculus have been included. $a$, perimysium; $b$, endomysium; $c$, a large vein seen in transection. The section was not stained. $\times 53$. into larger and smaller collections of muscle fibers, each bundle imperfectly separated from its fellows by septa from the perimysium, the endomysium. The ultimate subdivisions of the endomysium completely envelop each fiber and blend with the sarcolemma. This brings us to an individual fiber. Each fiber is enclosed in a sarcolemma. The myofibrillæ are collected into larger and smaller bundles, Kölliker's columns, separated from each other by semifluid, granular sarcoplasm; these collections in cross section are known as the areas of Cohnheim. They represent the definitive division products of the original group of fibrils (Heidenhain). The ultimate histologic units are the myofibrillæ or sarcostyles. 
But these may consist of still more delicate fibrils; in Limulus muscle, for example, the myofibrils may be ressolved into successively finer iibrils to the limits of visibility.

Heart muscle can be similarly, but less precisely divided, the endocardium and epicardium corresponding to the epimysium. Fasciculi and perimysium are not so readily distinguished, but the endomysium is re-

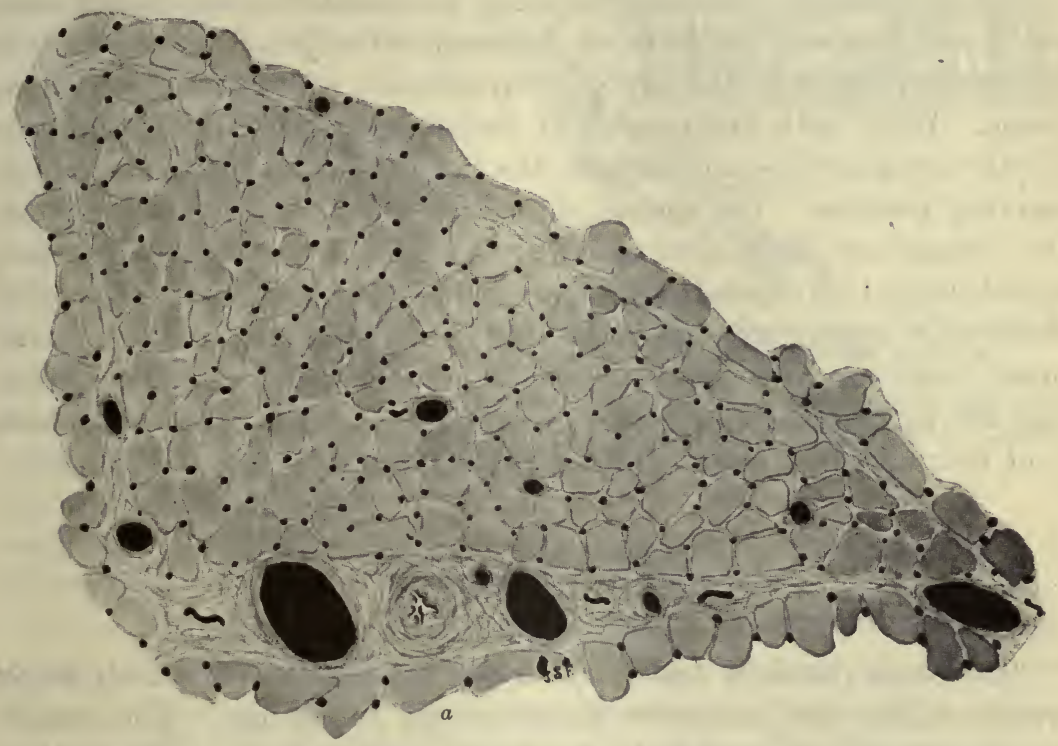

Fig. 128.-Striated Muscle of a Cat Seen in Transection.

The blood-vessels have been injected and are black in the figure. At $a$ an artery is contracted and empty. The heavy black vessels are veins and arterioles; the small black dots are capillaries in transection. One whole fasciculus is represented and is surrounded by a delicate perimysium of connective tissue. Between the muscle fibers is the still more delicate endomysium. The larger vessels are almost exclusively found in the perimysium. The section was not stained. $\times 80$.

lated to cardiac muscle in a manner essentially similar to that described for skeletal muscle.

The student should have well in hand the several criteria for the differentiation of the three types of muscle, both in transverse and longitudinal sections; and of smooth muscle from the dense white fibrous connective tissue. In brief, cross sections of skeletal striped muscle can be readily distinguished on the basis of their greater diameter and the peripheral position of the nucleus. Both cardiac and smooth muscle 
have a central nucleus and peripheral fibrillæ; but the fibers of the cardiac muscle are more or less polygonal in outline and more constant in size, excepting occasional branches, while the smooth cells are circular in outline and of very diverse diameters, depending upon the different levels at which the section passes through adjacent fusiform cells. In longitudinal sections the cardiac muscle can be easily recognized by its branching character and the presence of intercalated disks; smooth muscle by the fusiform character of its associated cells. Smooth muscle is frequently difficult to distinguish from compact, white fibrous connective tissue. When both are present in the same section, stained with the routine hematoxylin-eosin technic, the two exhibit a slight difference in staining reaction. The smooth muscle commonly stains a deeper red; the collagenous fibers have a'lighter orange tinge. Moreover, from a morphological standpoint, while portions of the white fibrous connective tissue may appear spindle-shaped, thus simulating the unit of smooth muscle structure, the associated nuclei of enveloping connective tissue cells are peripheral to the bundle, whereas the nucleus of smooth muscle is of course in the center of the analogous structure, the muscle cell.

\section{BLOOD SUPPLY}

The blood-vessels of voluntary striped muscle distribute their larger trunks within the connective tissue of the epimysium. The smaller branches penetrate the endomysium and supply a rich capillary plexus with long rectangular meshes. This network of capillaries surrounds the muscle fibers so completely that each fiber is placed in relation with four or five capillary vessels which run parallel with the long axis of the fiber. The blood supply of cardiac muscles is in general similar, but even more abundant and intimate, with respect to its terminal meshes. The blood supply of smooth muscle is relatively meager.

Numerous lymphatics occur in the perivascular connective tissue. These lymphatic vessels are especially abundant in cardiac muscle.

\section{NERVE SUPPLY}

Skeletal muscle is innervated both by cerebrospinal and sympathetic nerves, supported in the connective tissue enrelopes and septa. The former include both sensory and motor fibers ending in muscle spindles 
and motor end-organs respectively. . These endings will be further described under Peripheral Nerve Terminations. The sympathetic or 'accessory fibers' (Fig. 129) - relatively sparse and delicate, and in close relationship to the motor fibers and endings - terminate in special 'endplates,' close-meshed networks of generally oval outline. Boeke (Anat. Anz., 44, 15-16, 1913) suggests that they may mediate the maintenance of muscle tone.

Cardiac muscle is supplied only with sympathetic motor fibers. These terminate on the muscle fibers in brushes of fibrils, but without highly specialized endings. Sensory fibrils from the vagus are distributed to the cardiac endomysium. Each smooth

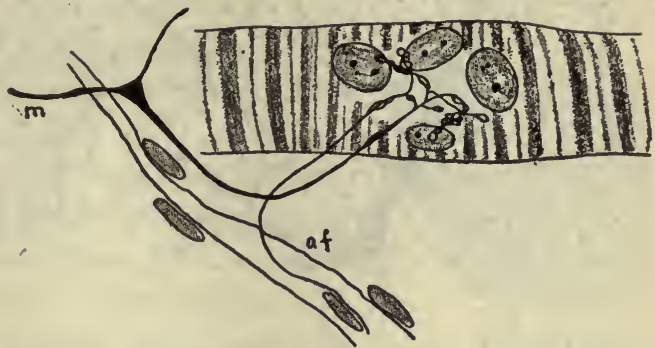

Fig. 129.-Motor End-Plate on an Intercostal Muscle Fiber of a Young Rabbit.

The motor nerve fiber $m$ is accompanied by an accessory (sympathetic) fiber, af. (After Boeke, Anat. Anz., 44, 15, 1913.) muscle cell, likewise, is supplied with a sympathetic fibril, ending in minute knobs or plates.

According to Malone (Amer. Jour. Anat., 15, 1, 1913), the three types of muscle are innervated by three histologically distinct types of nerve cells, representing specific functional differences. The cells supplying heart muscle are from the standpoint of size and granular (chromophilic) content, intermediate between those supplying smooth and those supplying skeletal striped muscle. (See Fig. 139 below.)

\section{TENDONS}

A tendon, taken as a whole, is invested by a dense fibro-elastic membrane, the epitendineum,.or vagina fibrosa. Where tendons play in bony grooves this may be modified into a tendon sheath, the epitendineum acquiring a mucous cavity, when it becomes known as a vagina mucosa. Septa from the epiter dineum penetrate the mass and divide the tendon imperfectly into irregular columns, the tertiary bundles. These are further divisible into more regular aggregations of fibrils, completely enveloped by a peritendineum, and are the tendon fasciculi. These correspond to the muscle fasciculi enveloped by 


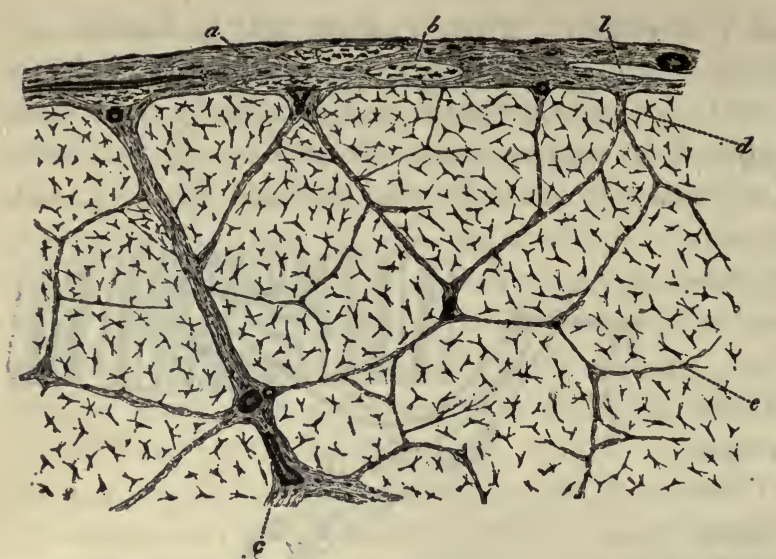

Fig. 130.-Portion of a Transection of a Large Tendon.

$a$, fibrous capsule with circular, and at $b$, longitudinal bundles of connective tissue; $c, d$, and $e$, fibrous septa between the fasciculi of the tendon; $l$, lymphatic cleft. Moderately magnified. (After Schäfer.) perimysium. In the mouse, tendons consist of from 1 to 11 fasciculi; in the chick from 2 to 5 (Loevy, Anat. Anz., 45, 10-11, 1913). Each fasciculus consists of elementary bundles of collagenous fibrils, enveloped by a complete sheath formed by the anastomosing processes of the tendon cells (cells of Ranvier). The cell bodies lie between these primary bun-

dles; they are connected to each other by their processes, forming an 'endothelial' tube (Ranvier), the cells of which have a characteristic mesothelial appearance in silver nitrate preparations.

In the tendons of the tail of the mouse, Loevy describes the cells as flat, rectangular, and rhomboidal; they are parallel to the long axis of the tendon, two of their surfaces extended into flat plates or wings which effect a union with 'wings' of adjacent cells. A cell may have from 2 to 4 wings. The wings or plates have been interpreted as elastic in nature, but do not react to specific stains for elastic tissue. Each cell contains a spherical or oval deeply staining nucleus; the nuclei

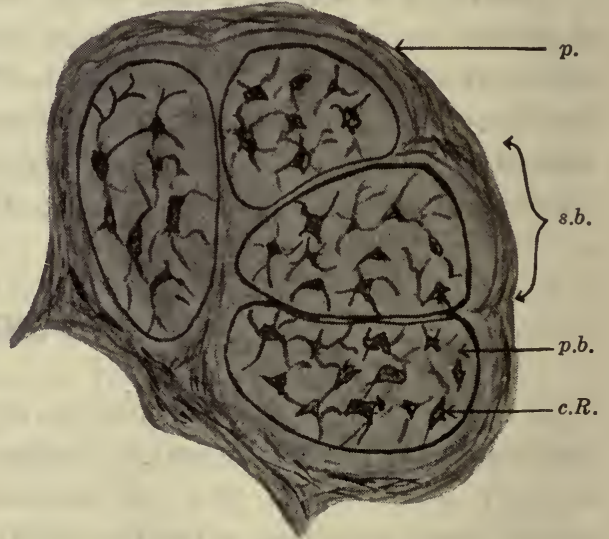

Fig. 131.-Transverse Section of Tendon of Tail of Adult Mouse.

It consists of four secondary bundles or fasciculi, s.b.; $p . b$. , primary bundle; $p$., epitendineum; c.R., tendon cell (cell of Ranvier). (After Loevy, Anat. Anz., 45, 10, 1913.) 
of successive cells are often so placed as to be immediately adjacent. According to Loevy the fibrils are developed from fibroblasts; the definitive tendon cells, which form the primary bundles, arise from cells of Ranvier. Both come from mesenchyme cells, but the fibroblasts entirely disappear, while the cells of Ranvier persist as the characteristic winged tendon cells.

Ligaments, fascia, and aponeuroses are very similar to tendon, but are less compact and contain more elastic tissue.

Bursæ are mesothelium-lined sacs in connection with the large diarthroses and certain locations where tendons are subject to friction.

Tendons are supplied with blood-vessels and sensory nerve endings, in a manner very similar to skeletal muscle.

The exact manner of the attachment of striped muscle to tendon is still disputed. According to certain investigators (O. Schultze, Arch. f. mikr. Anat., Bd. 79, 1912), the myofibrils and tendon fibrils are directly continuous through the sarcolemma. Others (Baldwin, Morph. Jahrb., Bd. 45, 1912) hold that the muscle ends sharply, remaining striped to its termination, and that the rounded or pointed end is completely enveloped by the sarcolemma (Fig. 132). The muscle fibers are described as being dovetailed into the tendon, the tendon fibrils being attached to the sarcolemma. This is the more commonly accepted interpretation; but it seems probable that both types of muscle-tendon connections occur in different muscles, for in certain muscles the cross striations become gradually more vague toward the tendon, and the point of

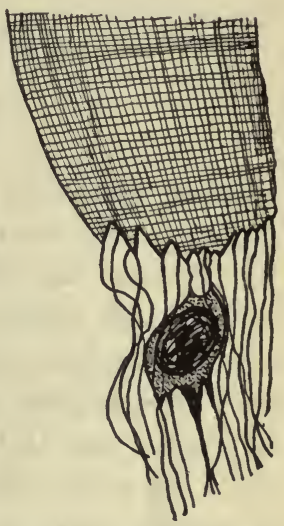

Fig. 132.-Portion of a MUSCle FIBER FROM THE TAIL of A 5 CM. Frog TADPOLE.

Each cone-shaped sarcolemma process has attached to it a tendon fibril. Two of the processes derive fibrillæ from a large fibroblastic cell situated among the tendon fibrillæ. (After Baldwin.) $\times 1000$. transition from muscle to tendon is by no means sharply marked. Moreover, the fact that certain ligaments and aponeuroses arise normally by transformation of muscle adds support to the idea of muscle-tendon continuity.

Baldwin distinguishes two general types of muscle termination with respect to tendon: one in which the long axes of tendon and muscle fiber coincide; a second in which they meet at an angle. In neither type does he recognize a direct continuity between muscle and tendon fibrils. 
In the first type the sarcolemma presents pointed ends, to which the tendon fibrils are attached; in the second, the sarcolemma presents a flat surface which rests directly against the attached structure, whether fascia, periosteum, or ligaments. Jymphatics are abundant in tendons. 


\section{CHAPTER V}

\section{NERVOUS TISSUES}

\section{GENERAL CONSIDERATIONS}

Nervous tissue comprehends those tissue elements which are peculiar to the nervous system. In the protoplasm of nervous tissue proper (neuroplasm) the fundamental properties of irritability and conductivity have become predominant. The nervous system includes the cerebrospinal-comprising the central (brain and spinal cord) and the peripheral (cerebral and spinal nerves) portions-and the sympathetic divisions. For convenience we may speak also of the central and peripheral nervous systems, the latter including the sympathetic division. The essential unit of structure, comparable to the cell of other tissues, is here the neuron, or neurocyte. A neuron is a nerve cell in the broadest sense of the term. It consists of the cell body (nerve cell of the older writers, cyton), together with all of its processes. These latter are divisible into two varieties, the axon and the dendrons (dendrites).

The neurons are among the largest cells of the body. Their cell body is of variable size, in some cases extremely minute, at other times sufficiently large to be readily observed with the unaided eye. Their processes, usually of considerable number, vary in length from a millimeter or less, up to half the height of man. It is therefore obviously impossible to study microscopically at one time the entire course of these longer processes. This circumstance renders it advisable to retain the term nerve fiber of the older writers to designate, not as was the former conception, a histological entity, but rather that portion of those long processes of the nerve cell which pursues its course, as a rule, outside of the gray matter of the central portion of the cerebrospinal division.

On this basis we may divide the neuron into the nerve cell and the nerve fiber. The former term includes the cell body, or cyton, with its dendrons and the proximal portion of its axon; the distal portion of the axon forming the essential part of a long nerve fiber. The nerve cells 


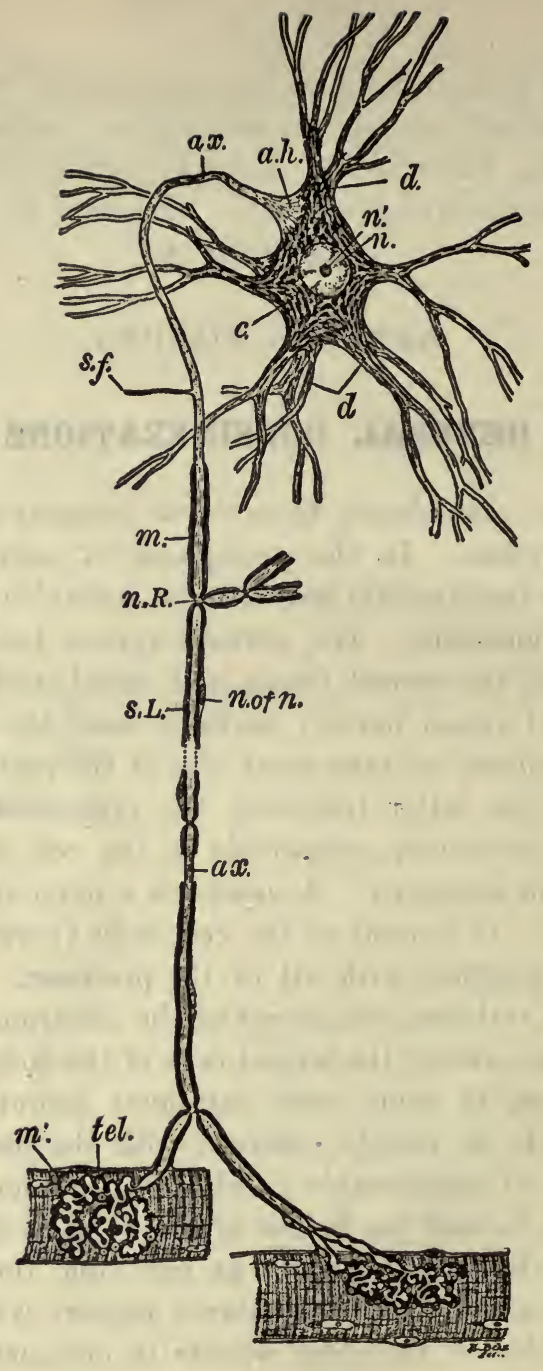

Fig. 133.-Diagram of a Neuron.

$a h$, axon hillock; $a x$, axon; $c$, cytoplasm, the Nissl granules have been stained; $d$, dendrons; $m$, myelin sheath of the nerve fiber; $m^{\prime}$, muscle fiber; $n$, nucleus; $n^{\prime}$, nucleolus; $n$ of $n$, nucleus of the neurolemma of the nerve fiber; $n R$, node of Ranvier; $s f$, collateral; $s L$, segment of Lantermann; tel, telodendrion or terminal arborization which here forms a motor end-plate. (After Barker.) 
are found throughout the gray matter of the central portion and in the peripheral ganglia of the cerebrospinal division and in the sympathetic ganglia. Nerve fibers occur in the white matter of the central portion and in the nerve trunks and ganglia of the peripheral portions of the nervous system.

In the peripheral nervous system the nervous tissues are chiefly supported by the connective tissues, but in the central portion a special form of supporting tissue, the neuroglia, is also found. This is described below.

\section{THE NERVE CELL}

\section{(Cyton, Cell Body, Perikaryon, Ganglion Cell)}

This term, as already stated, includes the cell body with its dendrons and the proximal portion of its long axon. The cell bodies vary in size from $4 \mu$ to $200 \mu$ in diameter. Their shape is chiefly dependent upon the number of their dendritic processes. Unipolar nerve cells, with but a single process, are flask-shaped or pyriform; bipolar cells, whose processes are usually derived from opposite extremities, are most frequently fusiform; multipolar nerve cells, from the considerable number of their processes, are irregularly stellate.

Nucleus.-The cytoplasm of the cell is finely granular and contains a large vesicular nucleus which, as a rule, is excentrically situated. The appearance of this large nucleus is quite characteristic of the nerve cell as distinguished from the cells of other tissue. The nuclear membrane is distinct and highly chromatic. The contents of the nucleus, however, except for the large spherical nucleolus which is quite constantly present, is noticeably deficient in chromatin. Those few small karyosomes which are present are mostly adherent to the inner surface of the nuclear membrane. The achromatic nucleoplasm forms the greater portion of the nucleus. Occasionally the chromatin forms still finer granules, and is more equally distributed throughout the nucleus. A large, chromatic, centrally situated nucleolus is nearly always present.

Cytoplasm. - The finer structure of the cytoplasm of the nerve cell is the subject of considerable difference of opinion. The studies of Nissl have shown that it is divisible into a substance which is readily stained by methylene blue, thionin, etc. (the stainable substance of Nissl, tigroid of von Lenhossék), and an apparently homogeneous substance which is not so readily stained (the unstainable substance of Nissl). 


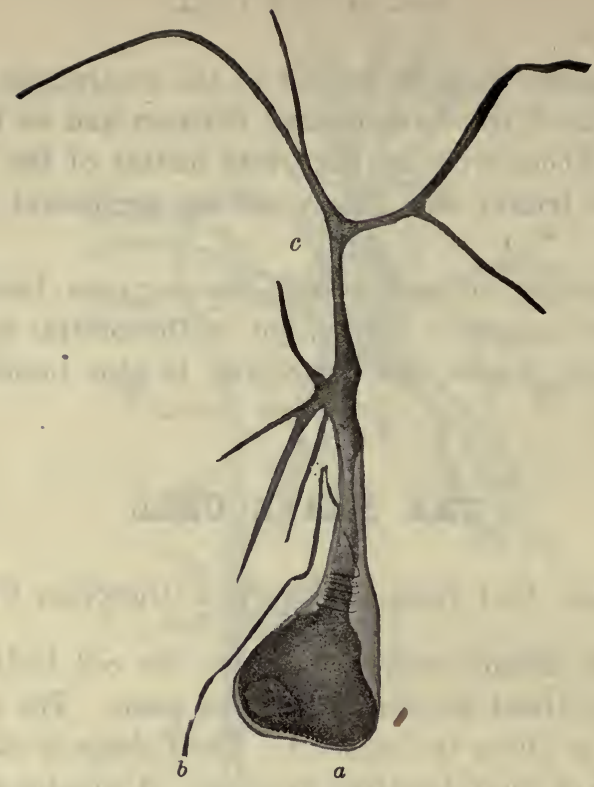

Fig. 134.-A Unipolar Ganglion Cell of a Frog.

$a$, cell body; $b$, axon; $c$, dendron. Methylene blue. Highly magnified. (After von Smirnow.)

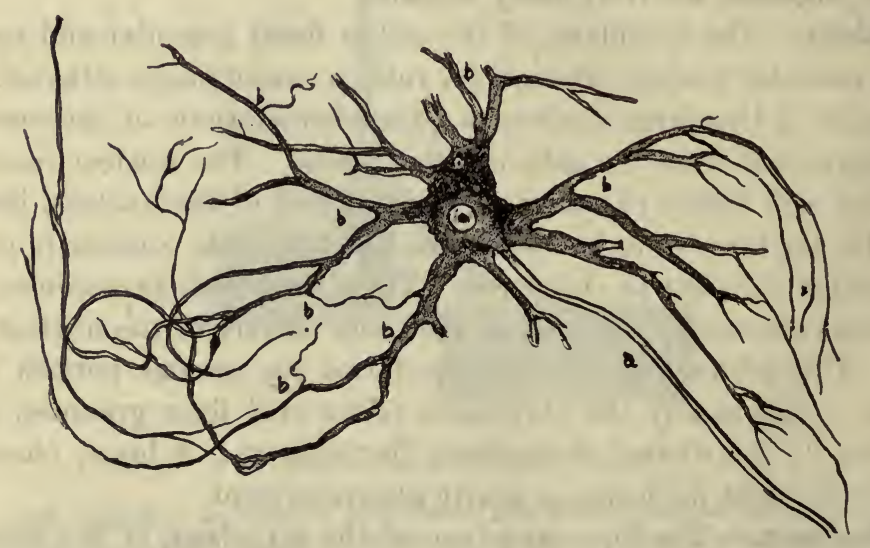

Fig. 135.-Multipolar Ganglion Cell from the Ventral Horn of the Gray Matter of the Spinal Cord of the Ox.

$a$, axon; $b$, dendrons. (From Barker, after Deiters.) 
Nissl's substance, chromophilic or tigroid substance, occurs in the form of flake-like granules of varying size and irregular shape. Their disposition within the cytoplasm is subject to considerable variations in different nerve cells, but according to Nissl it is fairly constant in cells of the same location for any given species. The amount also of the chromophilic substance is subject to variation depending upon the functional condition of the individual. It has been shown that the substance is greatly diminished by fatigue (Dolly) and after surgical shock (Crile). In general also, the longer the axon the greater the amount of chromophilic substance. Chemically, it is a nucleoproteid. There is considerable histologic evidence to indicate that it has a nuclear origin, appearing first in the form of 'chromidia,' and it is accordingly sometimes designated as cytochromatin. Mühlman has shown, however, that tigroid nuclein is soluble in weak soda solutions while nucleus nuclein is not. It has been suggested (Heidenhain) that it may perhaps have an accessory nuclear function. According to Held it is present as a diffuse continuous substance, coagulated in the form of flakes and granules in fixed tissues.

Those nerve cells in which the Nissl substance is abundant are said to be in a pyknomorphous, those in which it is scanty in an

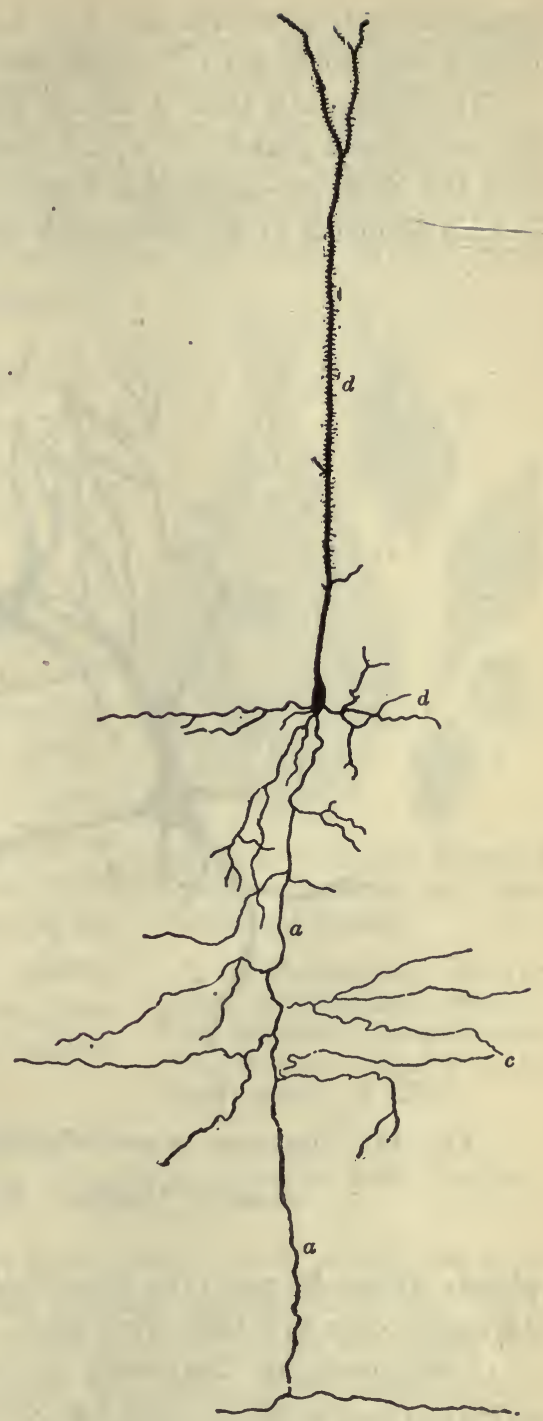

Fig. 136. - Pyramidal Multipolar Nerve Cell from the Cerebral Cortex of a Mouse.

$a$, axon; $d$, dendrons; $c$, collaterals. Golgi teohnic. (Barker, after Ramón y Cajal.) 
apyknomorphous condition. The Nissl granules are apparently used up during functional activity of the nerve cell.

The brain-cells show a strong affinity for adrenalin, the secretion of the suprarenal glands; this fact leads Crile (1914) to strongly suspect that the Nissl substance is a volatile, extremely unstable combination of certain elements of the brain-cells and adrenalin because the suprarenal

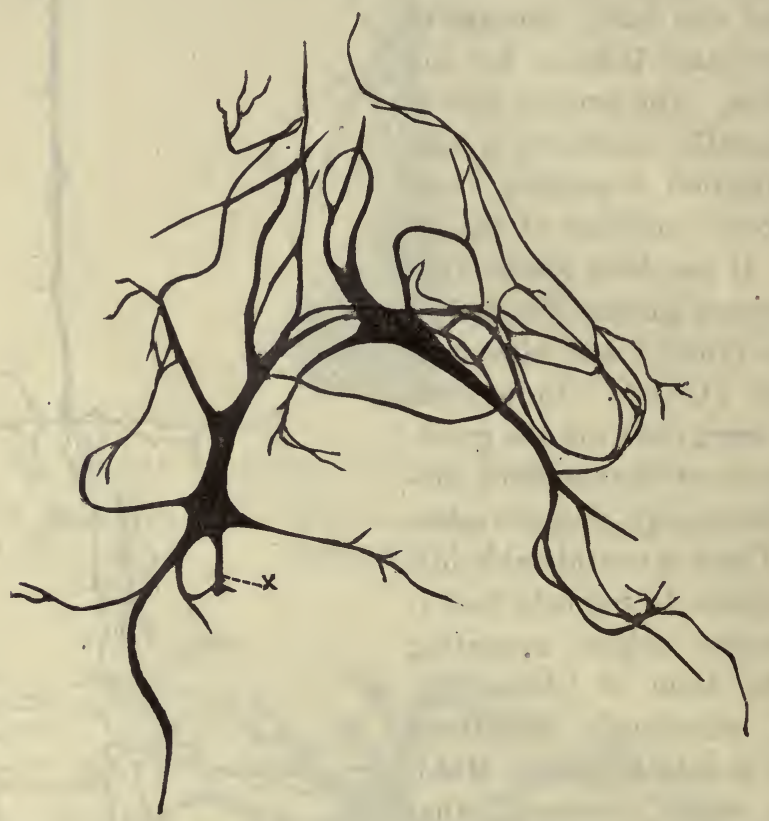

Fig. 137.-Isolated Nerve Cells from the Spinal Cord of Man.

$x$, axon. Carmin. $\times 160$. (After Sobotta.)

glands alone do not take the Nissl stain, and the brain deprived of adrenalin does not take Nissl stain.

Nissl substance disappears in case of lesion of the neuron, but reappears in abundance after temporary injury and recovery of the cell. Such disappearance after section of the axon (axonal reaction) is accompanied by a swelling of the neuroplasm and the peripheral migration of the nucleus, after from ten to fifteen days.

Concerning the finer structure of the unstainable substance of Nissl, comparatively little is known. With varying methods of fixation this portion of the cytoplasm has been found to show very fine fibrils (neuro- 
fibrils, Fig. 141) (Schultze, Flemming, Apáthy, Bethe) and fine acidophil granules (neurosomes of Held; probably mitochondria). Besides these structures there remains a homogeneous ground substance or hyaloplasm, which, though of extreme physiological importance, in the usual histological preparations presents no structure. Centrosomes and attraction spheres have been frequently observed in the nerve cells of the lower vertebrates, and occasionally in those of mammals.

The cytoplasm of many nerve cells contains a characteristic brownish-yellow pigment, whose fine granules have a tendency to accumulate in the vicinity of the nucleus.

Mitochondria also have been reported in ganglion cells of the rabbit (Schirokogoroff, Anat. Anz., 43, 19 and 20, 1913). Cowdry (Amer. Jour. Anat., 17, 1, 1914) describes granular and rod-like mitochondria in the spinal ganglion cells of a number of vertebrates, including man. They are said to occur throughout the entire neuron, axon as well as dendrons. They are regarded as fundamental constituents of the neuroplasm. It is suggested that they are concerned with the metabolism of the neurocyte.

Neurons are incapable of division; de-

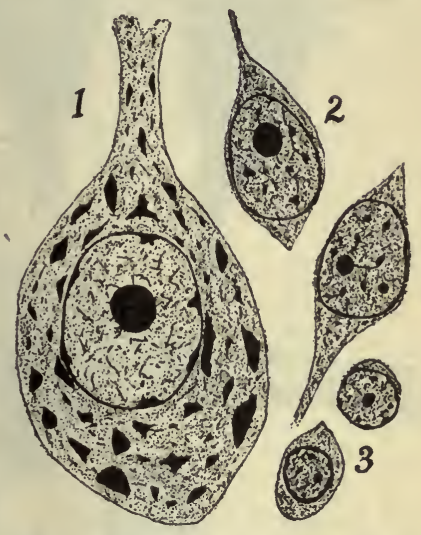

Fig. 138.-VARious TyPes OF Nerve Cells of the Cerebellar Cortex.

1, cell of Purkinje; the cytoplasm contains large flakes of Nissl substance; 2 and 3 , smaller nerve cells, 'granule cells.' Nissl's stain. $\times 1200$. stroyed neurons cannot be replaced; the axon, however, may regenerate.

End fibrils of other neurons have been demonstrated within the cytoplasm of the nerve cell. Apáthy has likewise demonstrated that fibrils occasionally pass from one neuron to another, so that we no longer consider that a neuron, though a structural unit, is in all cases anatomically independent of all other neurons. The present status of this much discussed question seems to be comparable to that of the cell, as a histological unit of structure, which though formerly. thought to exist independently of other cell units, has since been found to be frequently connected, as by the intercellular bridges of epithelium and of smooth muscle. The neurons of the nervous system therefore, while being usually related to one another by contiguity or by contact only, may occasionally be more 


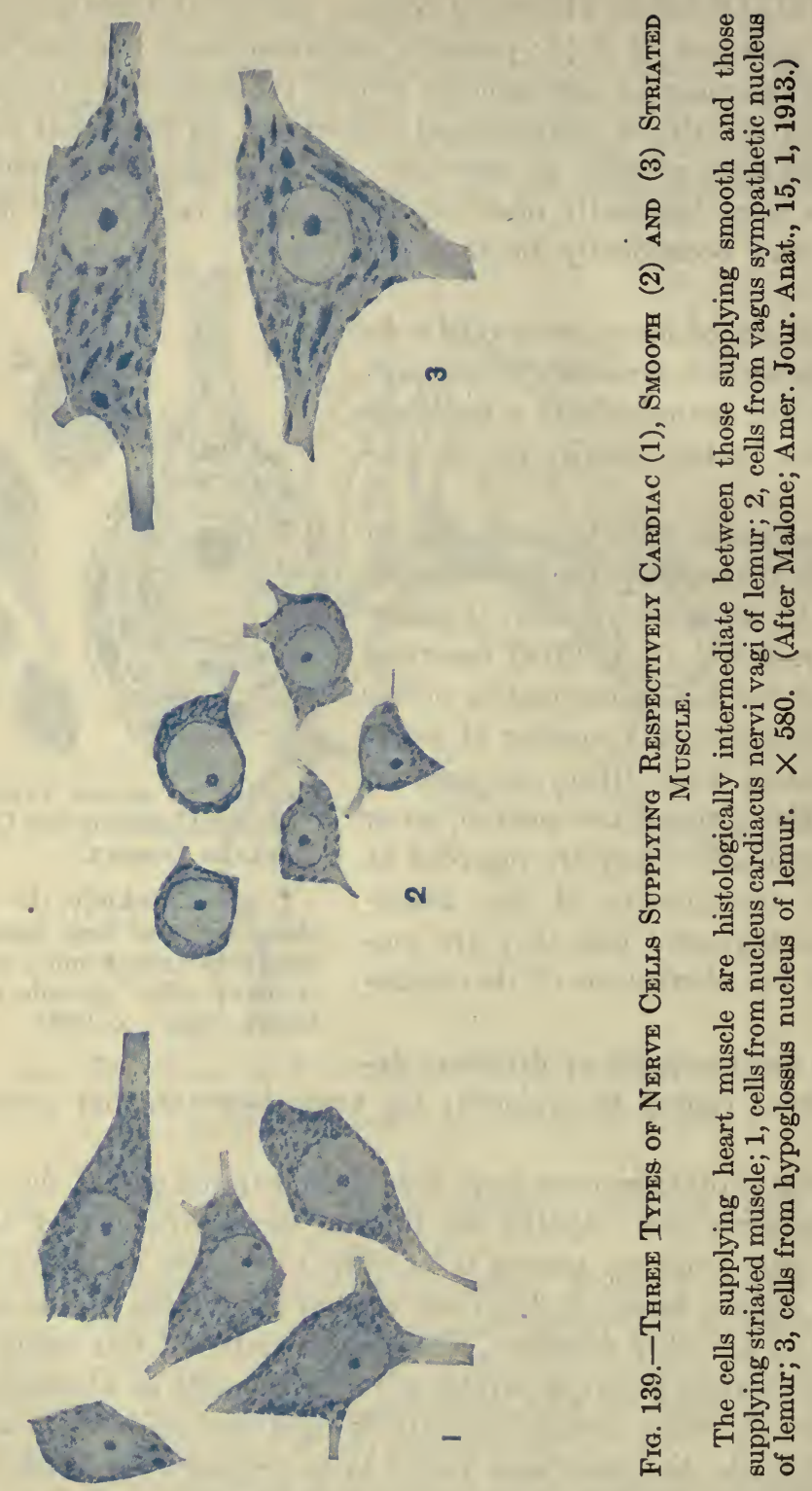


directly connected by fibrillæ, which pass from the processes of one neuron to the cell body or processes of a second neuron (Apáthy, Bethe), or by 'concrescence,' as described by Held.

The nerve cells are surrounded by a narrow interval which separates them from the surrounding tissue. This is presumably a lymphatic or tissue fluid space. Holmgren has demonstrated also the presence, within the cytoplasm of the nerve cell, of minute canaliculi which form an intra-

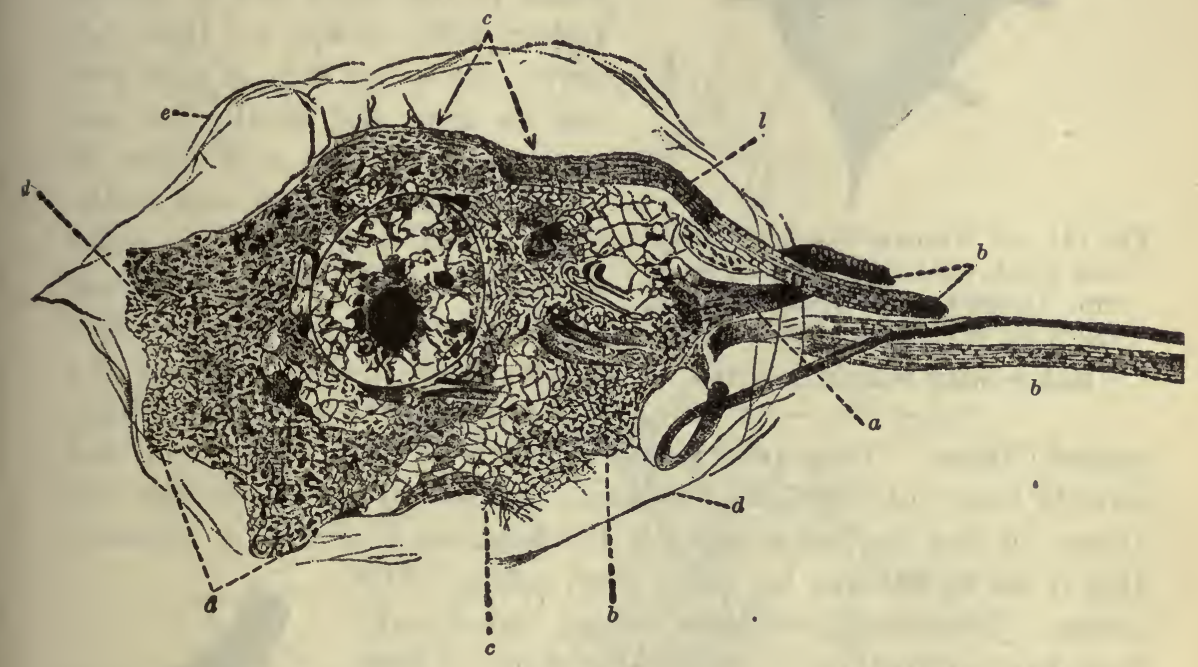

Fig. 140.-A Nerve Cell from the Trapezoid Nucleus in the Midbrain of a RABBIT.

$a$, axon; $b$, axons of other nerve cells which terminate in relation and apparently fuse with the cytoplasm of the cell body; $c$, points of fusion or zones of concrescence; $d$, dendrons which have been cut off close to the cell body; $e$, neuroglia. The cytoplasm shoẃs a neurofibrillar network and Nissl granules. Iron hematoxylin. Very highly magnified. (After Held.)

cellular network, more abundant near the surface of the cell, and which he has termed juice canaliculi, or trophospongium. These canaliculi may possibly account for the peculiar intracellular network which Golgi has demonstrated in the periphery of the nerve cell, by a modification of his rapid silver impregnation method.

The processes of the nerve cell are of two varieties: the one, broad, granular, and rapidly dividing in the vicinity of the cell body into a number of branching subdivisions, is the dendron; the other, long, slender, and finely but distinctly fibrillar, arises from the cell body direct, or 
from the base of a dendron, and passing for a considerable distance from the cell body, finally enters the nerve fiber as its axis cylinder, or termi-

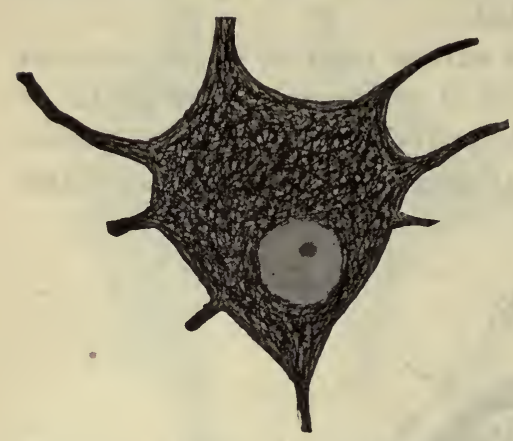

Fig. 141.-A Neuron (Giant Pyramidal Cell, or Cell of Betz) from the Cerebral Cortex of Man, Showing the Neurofibrils.

Bielschowsky technic. $\times 500$. nates in relation to some distant nerve cell. This latter process is the axon. Each cell body usually possesses a single axon and several dendrons. Cells without an axon are found in the retina and in the olfactory bulb; except for these, all nerve cells in the body of man possess an axon and usually but one such process. The subdivision of nerve cells into uni-, bi-, and multipolar cells is, therefore, chiefly based upon the number of their dendrons.

Dendrons (Dendrites, Protoplasmic Processes).-The dendrons of a nerve cell vary from one to a considerable number.' They arise from the cell body by a broad stem, and quickly' break into branches which can be traced for a considerable distance-in fact, the arborization of the dendrons is usually so extensive that it can be followed for only a short portion of its course. Occasionally dendrons do not branch until they have arrived at a considerable distance from their parent cell-body.

The structure of the dendron is, to all appearances, similar to that of the cell-body. The chromophilic substance is continued for some distance into the arborizing dendrons, which often possess a finely fibrillar appearance. In Golgi-stained preparations the dendrons frequently present a thorny appearance, due to the clustering along their borders of minute lateral projections, the gemmules.

The terminal filaments of the dendronic arborization are frequently in relation with the cell bodies or axons of other neurons, less frequently with the dendrons of other neurons. Such contact relationship is known as synapsis.

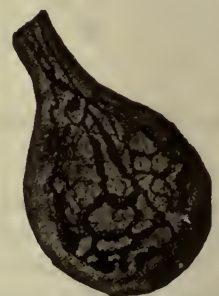

FIG. 142. - INTRACELlular NeTWORK (TrophoS P O N G I U M ) WITHIN A PURKINJE Cell OF the Cerebellum oF Strix flammea.

Golgi's stain. (After Golgi.)

Dendrons are cellulipetal processes, transmitting impulses to the cyton. The Axon (Neuraxis, Neuraxon, Neurite, Axis Cylinder Process).- 
This process in contradistinction to the dendron, is long and slender, as a rule does not arborize near its parent cell-body, is of smooth and regular contour in Golgi preparations, and contains no chromophilic substance. It arises from the cell body, or less frequently from the base of a dendron, by a conical, clear area, the axon hillock or implantation cone, which, like the process itself, is devoid of chromophilic granules. It consists of

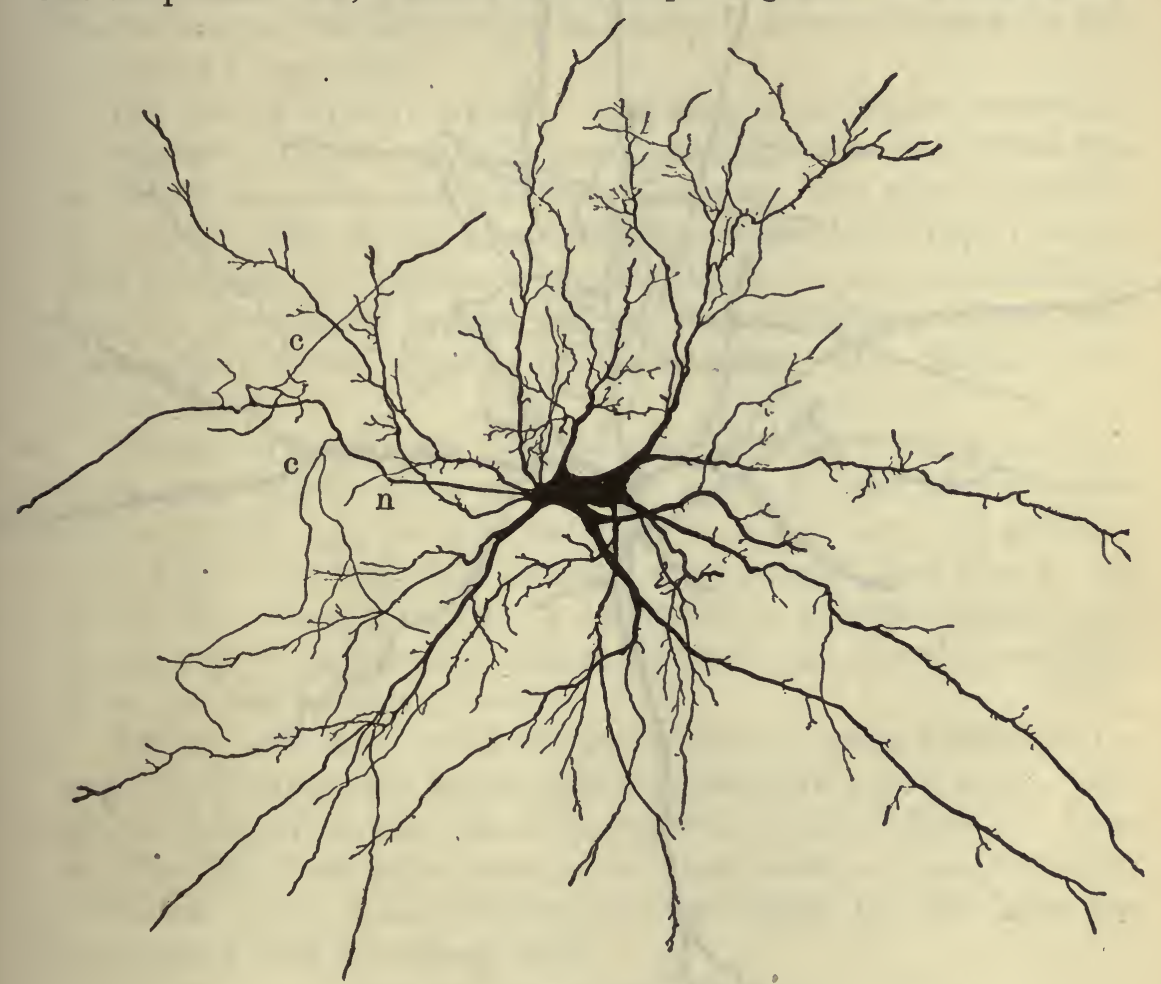

Fig. 143.-Golgi Celi, Type I.

$c$, collaterals; $n$, axon. Golgi's stain. (After Kölliker.)

a bundle of delicate neurofibrils (axon fibrils) embedded in axoplasm. During early developmental stages the fibrils increase in number by a splitting of preëxisting fibrils.

At some little distance from the parent cell-body the axon gives off very fine lateral branches, the collaterals, which leave the parent stem at the nodes of Ranvier at nearly right angles. These delicate branches, as also the axon proper, finally terminate by a sudden end arborization, or telodendrion, by which each axon is placed in relation with a large 
number of neurons, or a considerable area of surface. The telodendrion may terminate in minute knobs or plates, the neuropodia. The telodendrion is structurally apparently an efficient mechanism for mediating

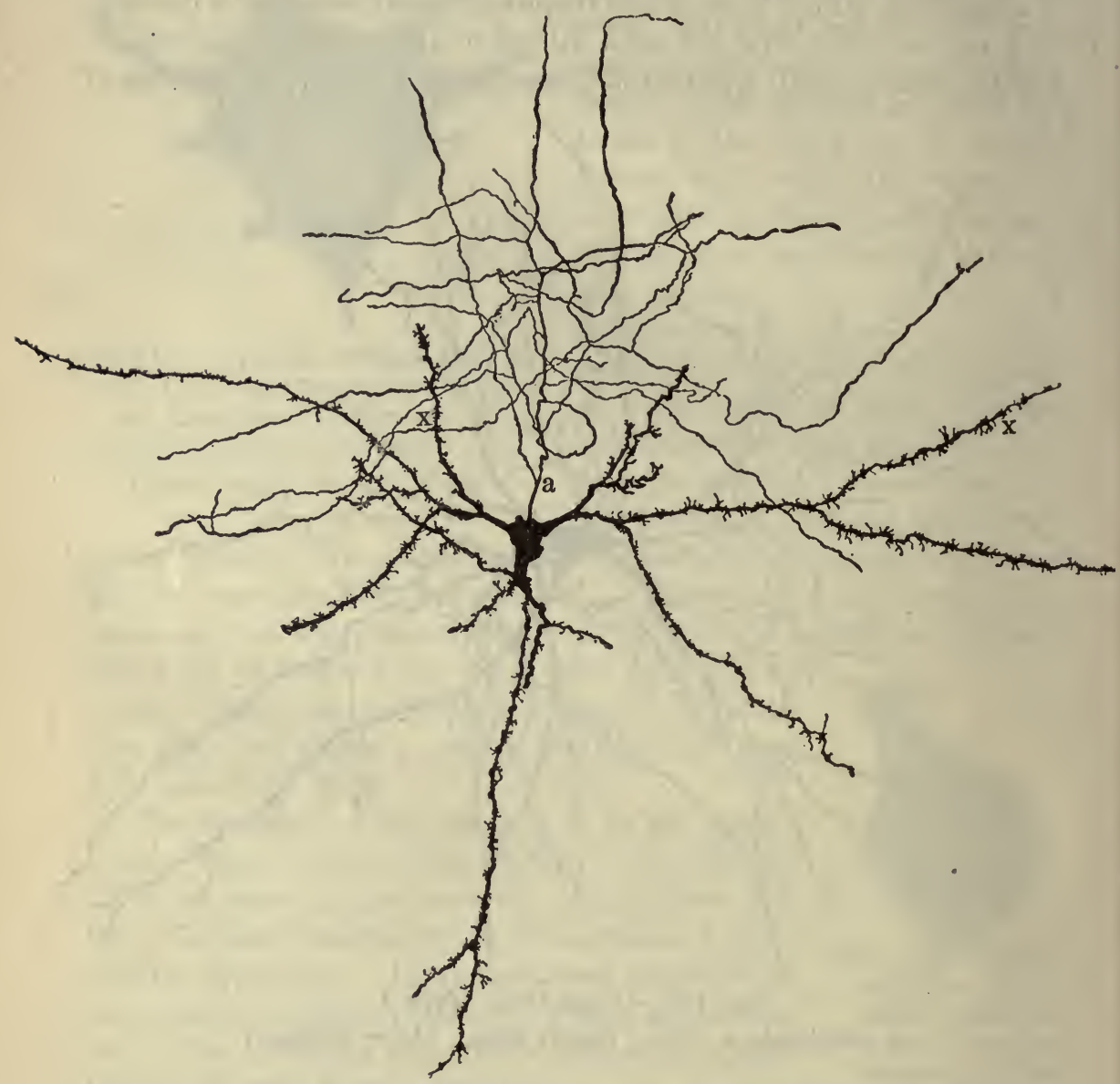

Fig. 144.-Golgi Nerve Cell, Type II.

$a$, axon; $x$, dendron. (After Kölliker.)

the phenomenon of 'axon reflex' (Langley). The parent stem of the axon may be finally exhausted in its collaterals, or it may in turn end in a terminal arborization. Collaterals are said to be more frequent in the proximal than in the distal portion of the axon. The axon transmits impulses away from the cell-body; it is a cellulifugal process. 
According to the length of their axons, neurons are divided by Golgi into two types.

1. Golgi cells; Type I (Deiters' cells).

2. Golgi cells, Type II (Golgi's cells).

The cells of Type I possess a long axon which passes beyond the confines of the gray matter in which it arises and usually becomes the axis cylinder of a nerve fiber.

The cells of Type II possess a short axon which forms its terminal arborization in the vicinity of its parent cell-body. The cells of this type are usually association and commissural neurons; they place in conduction relation other not very remote neurons. The cells of Type I, on the other hand, are more frequently projection neurons; they are distributed from the nerve centers to other and perhaps very different tissues, their courses lying in the long projection tracts and nerve trunks of the nervous system.

The cells of Type II are therefore most frequently intrinsic or endogenous neurons, their whole course lying in one division of the central nervous system, e.g., the gray matter of the spinal cord. The cells of Type I are more frequently extrinsic or exogenous; they arise in one part of the nervous system to be distributed to a distant portion, e.g., they arise in the peripheral ganglia and enter the spinal cord to terminate in its gray matter, or vice versa.

The size of a nerve cell is thought to bear a general relation to the length of its axon, the larger cells possessing the longer axons. The cells of Golgi's Type I are therefore larger than those of Type II. Likewise the cells of the motor tracts, whose axons are as a rule much longer than those of the sensory tracts, are characterized by their large size as compared with the sensory cells.

\section{THE NERVE FIBER}

The origin of the nerve fiber and its relation to the other portions of the neuron will be appreciated by tracing the course of the axon of a motor nerve cell of the ventral horn of gray matter in the spinal cord. This process, arising in the central gray matter, is at first a naked axon. It soon leaves the gray matter to traverse the white matter and makes its exit from the spinal cord as the axis cylinder of one of the fibers of a ventral nerve root. On leaving the gray matter the axon acquires a 
cylindrical sheath of myelin substance, the medullary sheath, myelin sheath, or white substance of Schwann.

On entering the ventral nerve root, which lies outside of the white matter of the spinal cord, the axon receives an epithelioid membranous sheath, the neurolemma or nucleated sheath of Schwann. The axon retains these two sheaths until near its termination, when the sheaths suddenly stop, the axon becoming again naked as it breaks into terminal fibrils.

Not all nerve fibers are medullated, nor do they all possess a neurolemma. The axons of the central nervous system are not supplied with a neurolemma until they pierce the meninges to enter the nerve roots. Those of the gray matter also have no appreciable medullary sheath. The axons of the peripheral nerve trunks and ganglia are all supplied with a neurolemma except at their terminals, as already explained. Yet some of the peripheral axons have a medullary sheath, while others have none. An axon with its enveloping sheaths constitutes a nerve fiber, and upon the presence or absence of these sheaths nerve fibers may be classified as follows:

A. Medullated nerve fibers......... $\begin{cases}1 . & \text { With a neurolemma } \\ 2 . & \text { Without a neurolemma. }\end{cases}$

B. Non-medullated nerve $\{3$. With a neurolemma fibers. \{4. Without a neurolemma-alemmal.

1. Medullated Nerve Fibers with a Neurolemma.-Nearly all the nerve fibers of the cerebrospinal nerve trunks and ganglia and some of those of the sympathetic nerves are of this type. These nerve fibers consist essentially of three cylindrical structures: the axis cylinder, which is the continuation of the axon of a nerve cell, and which forms the central portion or core of the nerve fiber; the medullary sheath, which forms a hollow cylinder inclosing the axis cylinder, and which suffers frequent interruptions, as will be described; and the neurolemma, which is an extremely thin investing sheath forming an uninterrupted envelope from the point where the nerve fiber leaves the central nervous system to a point near the end of the fiber where the axis cylinder breaks into its terminal fibrils. To these structures an investing sheath of connective tissue, the sheath of Henle, is sometimes added. It is derived from the connective tissue endoneurium in which the nerve fibers are embedded. It serves to support the capillary blood-vessels destined for the supply of the nerve fibers.

The Axis CyLinder.-The axis cylinder presents a finely fibrillar 
structure. The nature of these fibrils is not well understood. They are apparently continuous with the neurofibrillar network of the cell body.

In certain nerve fibers of the lower animals these fibrils have a tendency to collect into the center of the axis cylinder, leaving a peripheral clear zone; this distribution is especially characteristic of those fibers

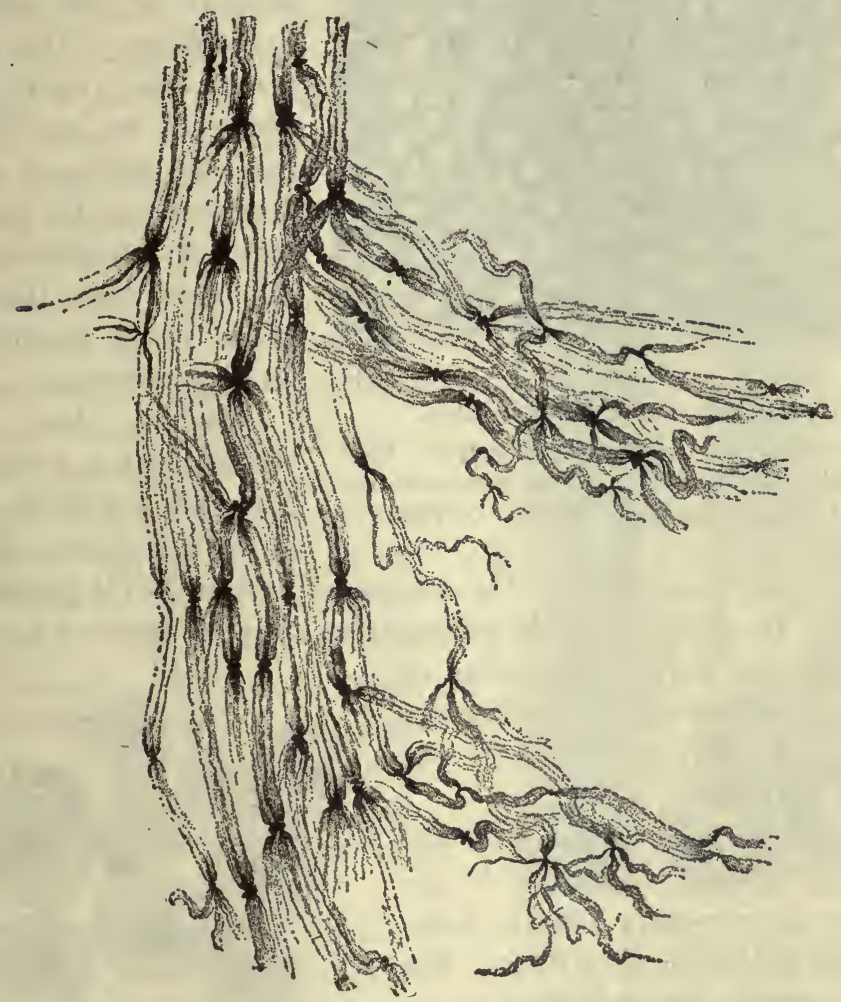

Fig. 145.-Isolated Nerve Fibers from a Frog.

The axis cylinders, the enveloping myelin sheaths, and the nodes of Ranvier are clearly shown. Intra-vitam methylene blue stain. (Barker, after von Kölliker.)

which are not supplied with a medullary sheath. In mammals, however, the fibrillæ occupy a larger portion of the axis cylinder, the clear peripheral area being correspondingly diminished until in man it can scarcely be recognized. The fibrils of the lower animals are also coarser.

Apáthy, studying chiefly the lower animals, has considered these 'ultimate fibrilles' to be the conducting element of the nerve fiber. Others, however, lay greater stress upon the intervening clear portion, the 
neuroplasm of Schiefferdecker or axoplasm, as containing the active conducting substance of the fiber.

According to Verworn, Lenhossék and R. Goldschmidt, these elementary fibrillæ (axon fibrils) in the axis cylinder are nothing else than

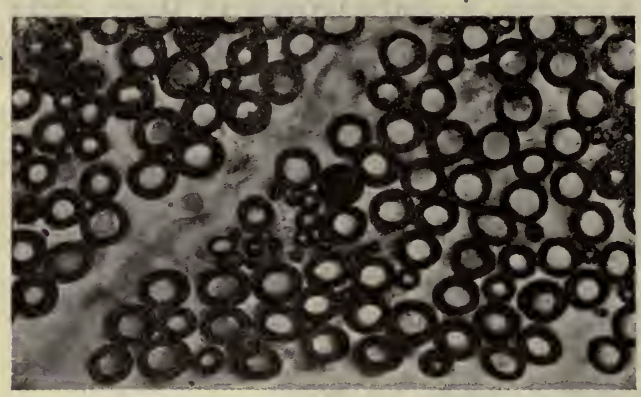

Fig. 146.-A Small Portion of a Transection of the Sciatic' Nerve of a Dog.

Nerve fibers are seen in transection; their myelin sheaths are black, their neuraxes unstained. Osmium tetroxid. Photo. $\times 700$. skeletal substance for the support of the semi-fluid neuroplasm. The circumstance that many of the fibrils of an axis cylinder may be sectioned without diminution of the maximum effect of stimulation favors the view that the neuroplasm is the essential conducting substance.

Tashiro has demonstrated that a living nerve gives off a definite amount of carbon dioxid, and that when the nerve is stimulated the amount of carbon dioxid production is increased. He conceives of the propagation of nerve impulses as a chemical change, the propagation being in essence a restoration of equilibrium in the nerve fiber disturbed at the point of contact.

The axis cylinder is, under certain conditions at least, found to be inclosed by an extremely delicate membrane, the axolemma of Kühne. The existence of this membrane as an integral part of a living axis cylinder has been denied by others. It may be simply a fixation artifact.

The Medullary Sheath (White Substance of Schwann, Myelin Sheath). - The medullary sheath forms a cylindrical investment for the

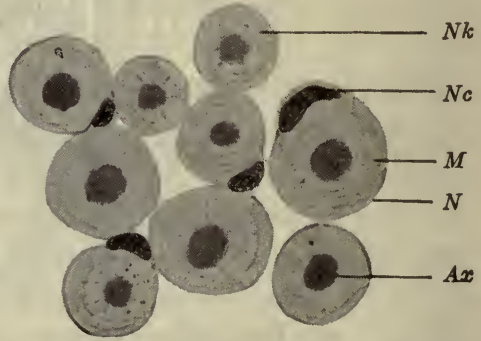

Fig. 147.-A Group of Large MedULLATEd Fibers from a Nerve in the Peritracheal Areolar TisSUE of the Cat.

$A x$, axis cylinder; $N k$, neurokeratin framework; $N c$, neurolemma cell and nucleus; $M$, medullary sheath; $N$, neurolemma. $\times 1000$. axis cylinder. Medullated fibers vary greatly in diameter according to the amount of myelin present., It appears to be retained in position by the neurolemma, for when the 
latter is ruptured the myelin exudes in the form of 'myelin drops.' The myelin thus obtained possesses the physical properties of a fat. It is also capable of being blackened by osmium tetroxid. By extraction with ether the myelin can be removed, leaving behind a supporting framework of neurokeratin. The function of myelin is probably nutritive, though it has been regarded as an insulating substance. It is thought to be present in small amounts even in so-called non-medullated fibers. It seems reasonable to suppose that it may have a double function, that is, nutritive and in part insulating.

At frequent intervals in the course of the nerve fiber its myelin sheath suffers complete interruption, thus forming the annular constrictions or nodes of Ranvier. At these points the neurolemma dips in until it is in contact with the axis cylinder. Both axis cylinder and neurolemma are continued past the node without interruption.

The successive nodes of Ranvier divide the nerve fiber into internodal segments. Within each internodal segment the medullary sheath, on blackening with osmium tetroxid, presents clear intervals which penetrate the myelin sheath in such manner as to give the appearance of obliquely disposed clear lines or incisions. These incisures of Schmidt (Schmidt-Lantermann lines) have not been satisfactorily explained and can not be demonstrated in the living fiber, yet they present a constant form and are always present in

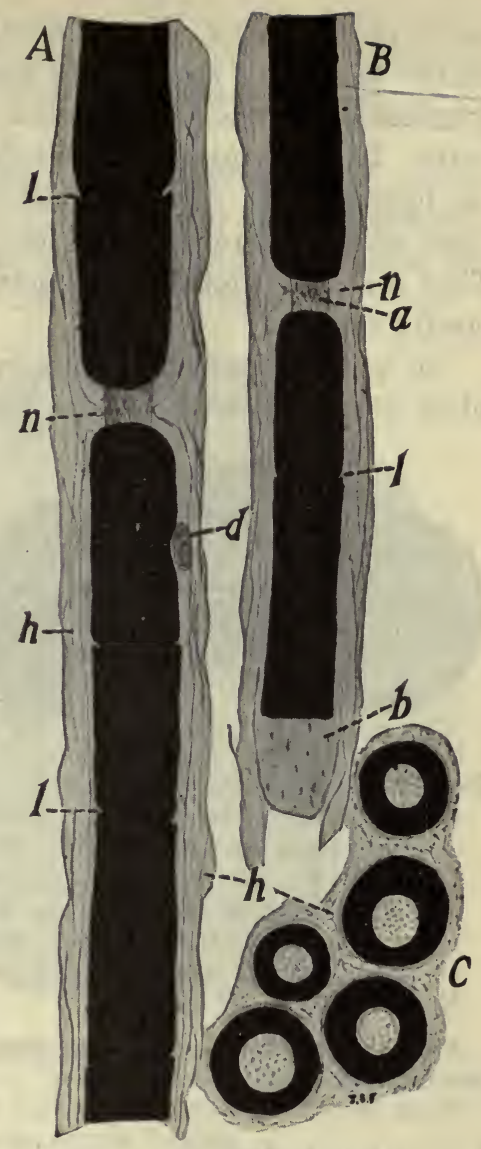

$A$ and $B$, from the sciatic nerve of a rabbit, isolated by teasing, and viewed in profile; $C$, a group of nerve fibers in transection, from the sciatic nerve of a dog. $a$, axon; $b$, neurolemma projecting beyond the torn end of the fiber; $d$, nucleus; $h$, endoneurium or fibrous sheath of Henle; $l$, SchmidtLantermann lines; $n$, nodes of Ranvier. Osmium tetroxid. $A$ and $B$, $\times 670 ; C, \times 900$.
Fig. 148.-Nerve Fibers. 
osmic preparations. These incisures subdivide the interannular segments of the medullary sheath into medullary segments. Schmidt originally considered them to be the optical expression of folds in the outer fibrous coats. Lantermann and others claim to have shown that they are within the neurolemma. They are believed by others to represent the limits of cones of neurokeratin. The incisures may point in different directions. They are more probably artifacts, representing fractures in the delicate myelin sheaths.

In preparations of fresh nerve fibers which have been treated with silver nitrate according to the method of Ranvier, the solution is found

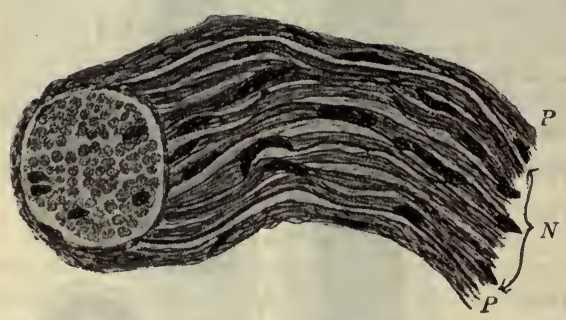

Pig. 149.-Cross and Longitudinal Sections of the Same Funiculús $(N)$ of Non-medullated Nerve Fibers (TuRned Up at the LefT), Showing the Perineurium $(P)$ and the RelaTIONSHiP OF THE NeUrolemma NUClei to the Axis Cylinder Bundles of NeUrofibrils.

From the peritracheal areolar tissue of the cat. $\times 1500$. to enter the fiber most readily at the nodes of Ranvier, so that if blackened by exposure to the sunlight, minute +-like appearances are seen at each node. By prolonged maceration in weak solutions of silver nitrate the solution penetrates still farther and the blackened axis cylinder is found to possess spiral transverse markings which are quite characteristic. The true meaning of these appearances has not been satisfactorily explained. Because of the apparent greater permeability of the fiber at these points, these peculiarities have been taken to indicate a certain relation of the annular constrictions to the nutrition of the fiber.

The Neurolemma (Nucleated Sheath of Schwann).-The neurolemma is the outermost of the nerve fiber sheaths. It is of ectodermal origin and makes its appearance prior to the medullary sheath. It forms a very delicate membrane, which incloses the myelin substance, and at each node of Ranvier comes into contact with the axis cylinder.

Attached to the inner surface of the neurolemma in each internode, and usually but one for each internodal segment, is an oval nucleus. The nucleus is surrounded by a minute amount of finely granular cytoplasm. This structure is taken to indicate that the embryonal neurolemma is formed by cells which became spread out over the surface of the primitive fiber, one cell, as a rule, supplying each internodal segment; 
and its nucleus with a minute amount of undifferentiated protoplasm is, according to this hypothesis, considered to remain as a permanent part of the neurolemma.

2. Medullated Nerve Fibers without a Neurolemma.-This type of nerve fiber composes the white matter of the central nervous system. The axis cylinder does not, of course, differ in the least from those of the previous variety and will need no further description.

The medullary sheath also is similar in its finer structure to that of the previous type, but since no neurolemma is present, these fibers possess no nodes of Ranvier. The medullary sheath of the fibers found in the white matter of the brain and spinal cord, is therefore uninterrupted. Its surface is in direct contact with the neuroglia network, which forms the supporting tissue of these organs, the innermost layer being condensed into a membrane which simulates a neurolemma. These fibers are accompanied by sheath cells

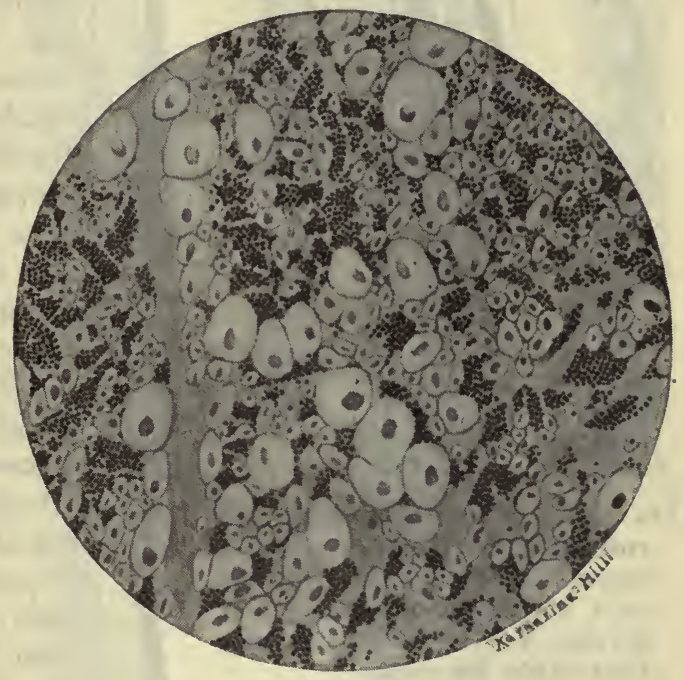

Fig. 150.-Cross-section of the Trunk of the Human Vagus Nerve, Some Distance Below the Nodose Ganglion, Showing Medullated and Non-medullated Fibers.

Pyridin-silver. $\times 680$. (After Ranson.) (Hardesty), homologues of the neurolemma cells of other fibers, which aid in the formation and maintenance of the myelin.

3. Non-medullated Nerve Fibers with a Neurolemma (Sympathetic Nerve Fibers, Remak's Fibers).-The most of the fibers of the sympathetic division are of this type. The axis cylinder does not differ from that of the previous types. The medullary sheath is entirely absent or, at most, only slightly developed in these fibers. The neurolemma is perhaps incomplete at times, but exhibits frequent nuclei along the course of the fiber. The neurolemma nuclei appear to be embedded in the superficial portion of the axis cylinder. Fibers of this type are of quite frequent occurrence also in the cerebral (cephalic; 
cranial) nerves of the cerebrospinal division. Other cerebrospinal nerve fibers lose their medullary sheath and finally also their neurolemma prior to their termination.

The recent work of Ranson has shown that even in the typical medullated spinal and cerebral nerves non-medullated fibers are very abundant.

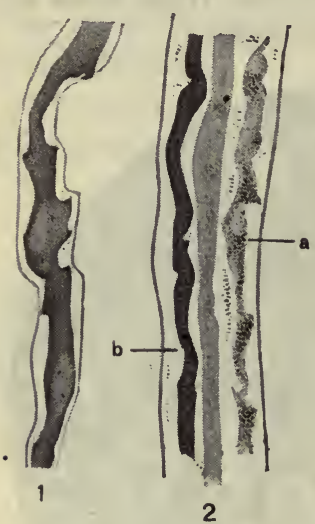

2

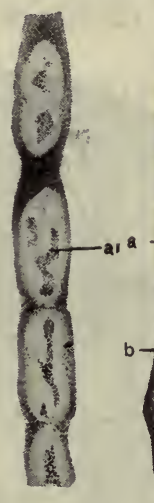

3

In the vagus of the dog, for example, the non-medullated fibers actually preponderate below the diaphragm. Ranson states that of these no considerable portion can be of sympathetic origin and that only a few represent medullated fibers which have lost their myelin distally. The non-medullated fibers of the vagus are said to comprise both afferent and efferent fibers, the latter arising from cells in the ganglia (jugular and nodose) connected with the vagus nerve (Anat. Rec., 24, 1, 1914). The spinal nerves also are shown to contain more non-medullated than medullated fibers (Amer. Jour. Anat., 12, 1, 1911).

\section{Non-medullated Nerve} Fibers without a Neurolemma. are at different stages of degeneration, the neurolemma can be seen bounding the unstained myelin sheath. (3) on the eighth day, the fragmented axon is surrounded by an elliptical segment of myelin (a). (4) on the nineteenth day, $a$, nucleus, $b$, droplet of myelin containing fragments of axon. (Ranson, Jour. Comp. Neur., 22, 6, 1912.)

-These fibers are naked axis cylinders and as such are found at the cytoproximal end of the axon in the gray matter of the central nervous system, and at

4 the cytodistal end prior to the termination of the axon in its arborization of terminal fibrils.

In man nerve fibers are of this type throughout their entire course only in the olfactory nerves.

All portions of the neuron, its axon and collaterals as well as its dendrons, are dependent upon the cell body for nutrition; hence each nerve cell becomes the so-called trophic center for all of its processes. 
The entire nervous system may be considered as an enormous tangle, formed by the interlacing processes of an innumerable number of neurons whose complex fiber paths place all portions of the body in communication with all other portions.

Nerve cells are unequally distributed throughout the central division of the nervous system; they therefore occur in more or less distinct groups or nuclei, from each cell of which an axon is frequently distributed along the same path. The larger bundles thus formed are called funiculi,fasciculi, or fiber bundles; the smaller ones tracts.

Since each fiber of such a tract is dependent for nutrition upon the nerve cell from which it arises, the tract as a whole must depend upon its nucleus of origin for its nutrition. Each nucleus therefore becomes the trophic center for the fiber tract to which it gives origin.

It may be readily demonstrated that if any such group of axons be cut or otherwise separated from its trophic center, that tract will promptly
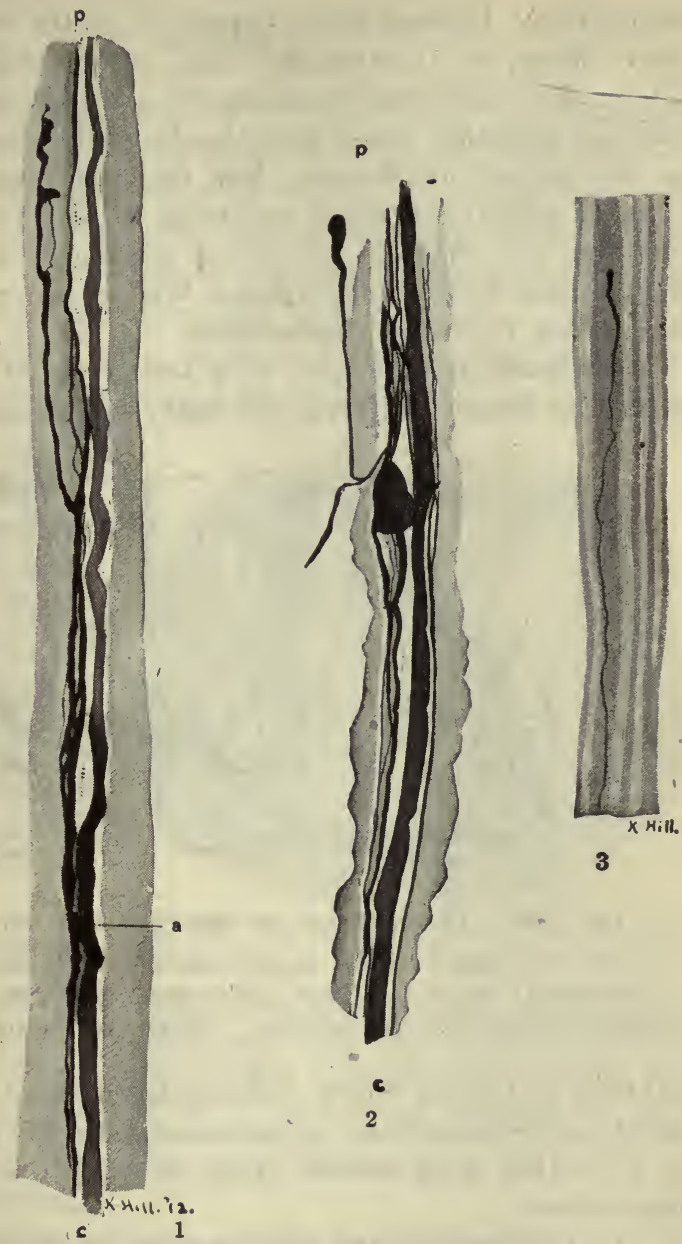

3

Fig. 152.-Regenerative Stages in the Proximal Stump of the Cut Sciatic Nerve of the Dog, Several Millimeters above the Level of SeCtion.

$p$, toward the periphery; $c$, toward the center. (1) on the nineteenth day after section; $a$, point in the old medullated axon from which arises an extremely short branch which at once divides into two. (2) on the twenty-fifth day. (3) five protoplasmic strands down which a new axon is growing. (Ranson.) (Pyridin-silver preparations.) 
degenerate. If these axons happen to be the axis cylinders of medullated nerve fibers, as is often the case, their myelin sheaths become rapidly altered in composition and acquire a tendency to disintegrate into small globular granules, which stain deeply with osmic acid when used according to the method of Marchi. For the experimental demonstration of this form of partial cell death occurring in that portion of the neuron which has been cut off from its cell of origin, we were originally indebted to the eminent English physiologist Waller; the resulting changes are therefore called Wallerian degeneration.

Obviously that portion of a neuron or of a fiber tract which, after injury or disease involving its path, still retains its connection with its

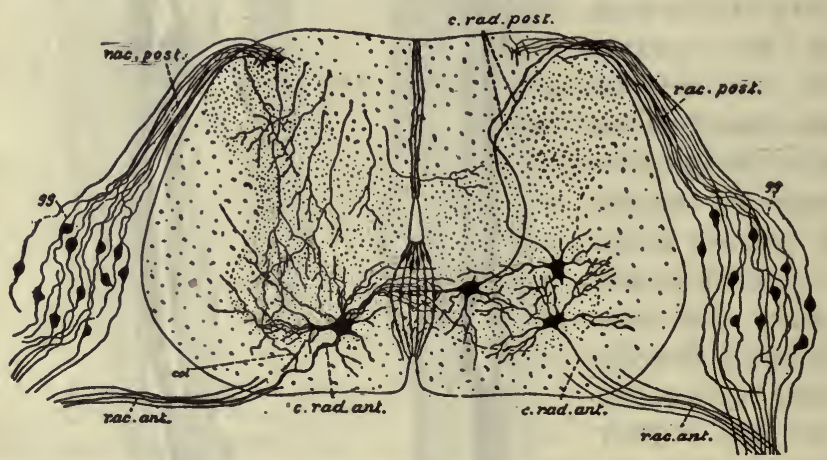

Fig. 153.-Transection of the Spinal Cord of an Embryo Chick.

c. rad. ant., axons to the ventral roots; c. rad. post., axons to the dorsal roots; $\mathrm{col}$, collateral from an axon back to the gray matter; gg, dorsal root ganglion; rac. ant., ventral root; rac. post., dorsal root. (After van Gehuchten.)

cell body or trophic center, will not degenerate. This part of the neuron is called its central portion, in contradistinction to its distal portion, the latter of which has been severed from its trophic center and is consequently degenerated.

To the study of the various types of Wallerian degeneration we are indebted for many of the facts by means of which the intricate tangles of axons composing the various fiber tracts of the central nervous system have been partially unraveled.

The contiguous relationship of different neurons within the nervous system occurs in any one of several ways. The terminal arborizations or telodendrions of one neuron may interlace with:

a. the telodendrions of axons belonging to other neurons,

b. the telodendrions of collaterals of other neurons,

c. the dendrons of other neurons, or

d. the terminal arborization may surround, basket-like, the cell body of other neurons. 


\section{NEUROGLIA}

Both the gray and the white matter of the central nervous system contain a peculiar supporting tissue, the neuroglia, which consists of two elements, the glia cells and the glia fibers. The latter are very probably produced by the glia cells, of which they were formerly considered to be processes. They consist of a substance similar to, perhaps identical with, the neurokeratin framework of myelin.

The Glia Cells.-The glia cells, as seen in Golgi preparations, are divisible into two distinct types, the ependyma cells and the astrocytes.

The EPENDYMA CeLLS may be considered as undifferentiated relics of the embryonal cells, from which both glia and true nerve or ganglion cells were developed. These cells line the central canal of the spinal cord and the ventricles of the brain, in which latter organ they also form the covering or outer coat of the telæ choroideæ.

The ependyma consists of long nucleated columnar cells whose free ends, in fetal and early life, carry a tuft of cilia; in adult life they are usually non-ciliated. The attached ends

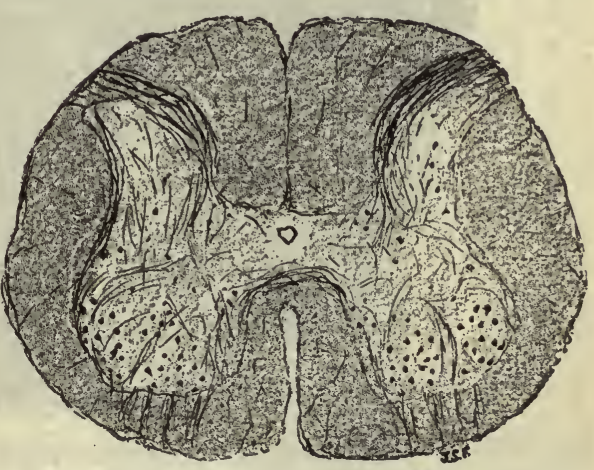

Fig. 154.- Transection of the Spinal Cord of a Child, Fifth lumbar Segment.

The central H-shaped gray substance consists of nerve cell bodies, dendrons, non-medullated portions of axons, and neurogliar supporting tissue. The enveloping white substance consists of medullated axons supported by neuroglia. Weigert stain. $\times 7$. of these cells are embedded in the surrounding gelatinous tissue, and are frequently prolonged for some distance as a fine branched process. In this way the ependyma of the spinal cord enters into the formation of the central gelatinous substance, in which the branched processes of its cells ramify in a glia-like manner. In the fetus the filamentous processes extend from the central canal all the way to the periphery of the spinal cord. In the adult the ependyma cells are prone to so multiply as to almost occlude the central canal; their processes have apparently become shorter, and now reach the surface of the spinal cord only at its dorsal median sulcus.

The Astrocytes, when stained by the Golgi method, apparently consist of a small cell body and an innumerable number of long slender 


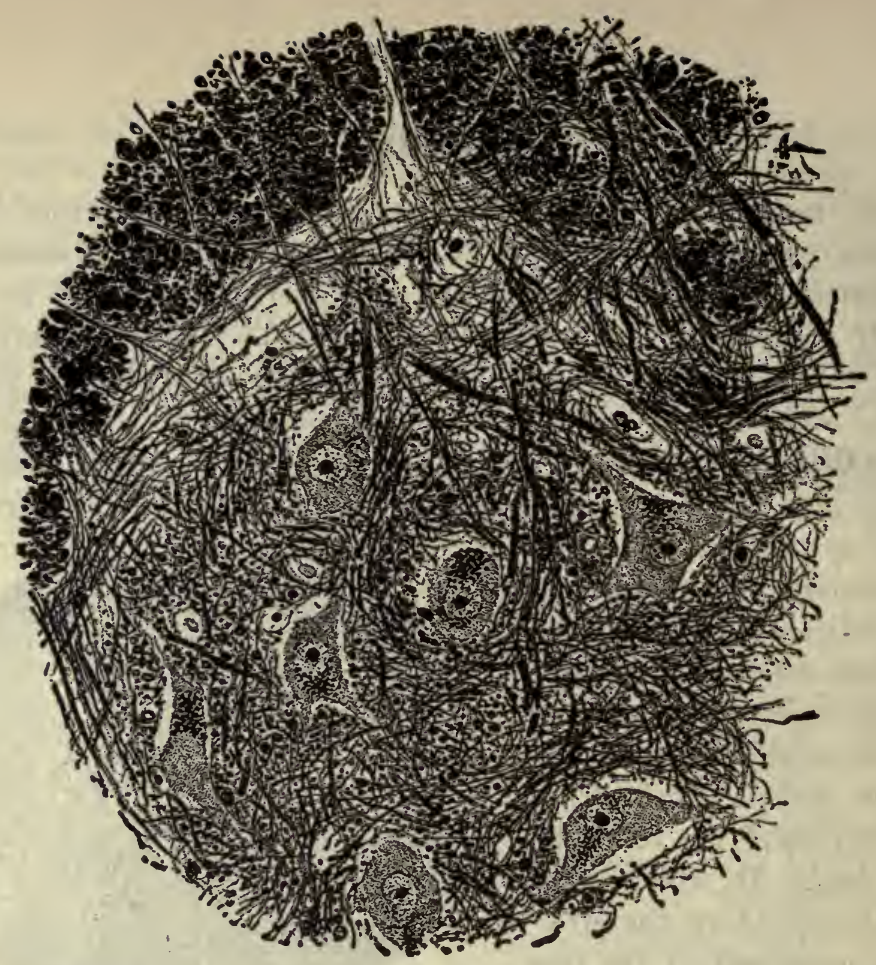

Fig. 155.-Portion of Gray Substance from the Anterior Horn of the Spinal Cord of Man, Showing Nerve Cell Bodies, Dendrons, Medullated and Non-medullated Portions of Axons, and Neuroglia.

(From Salinger, after Kölliker.)

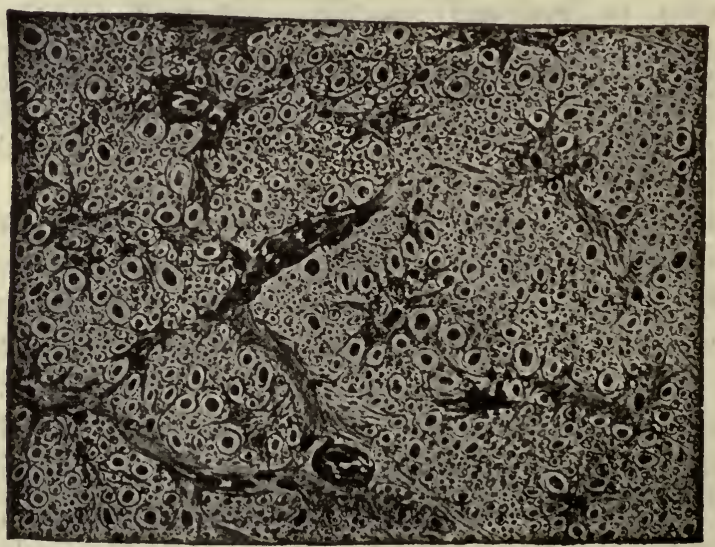

Fig. 156.-Transverse Section through the White Substance of the Human Spinal Cord.

The dark oval bodies are cross-cut axis cylinders; the surrounding light halos represent the myelin sheath. The coarser trabeculæ are connective tissue, continuous with the finer neuroglia framework. (After Salinger.) 
processes. Two varieties of these cells are recognized: the spider cell or long-rayed astrocyte, with a small cell body and very many exceptionally long and slender processes; and the mossy cells or short-rayed
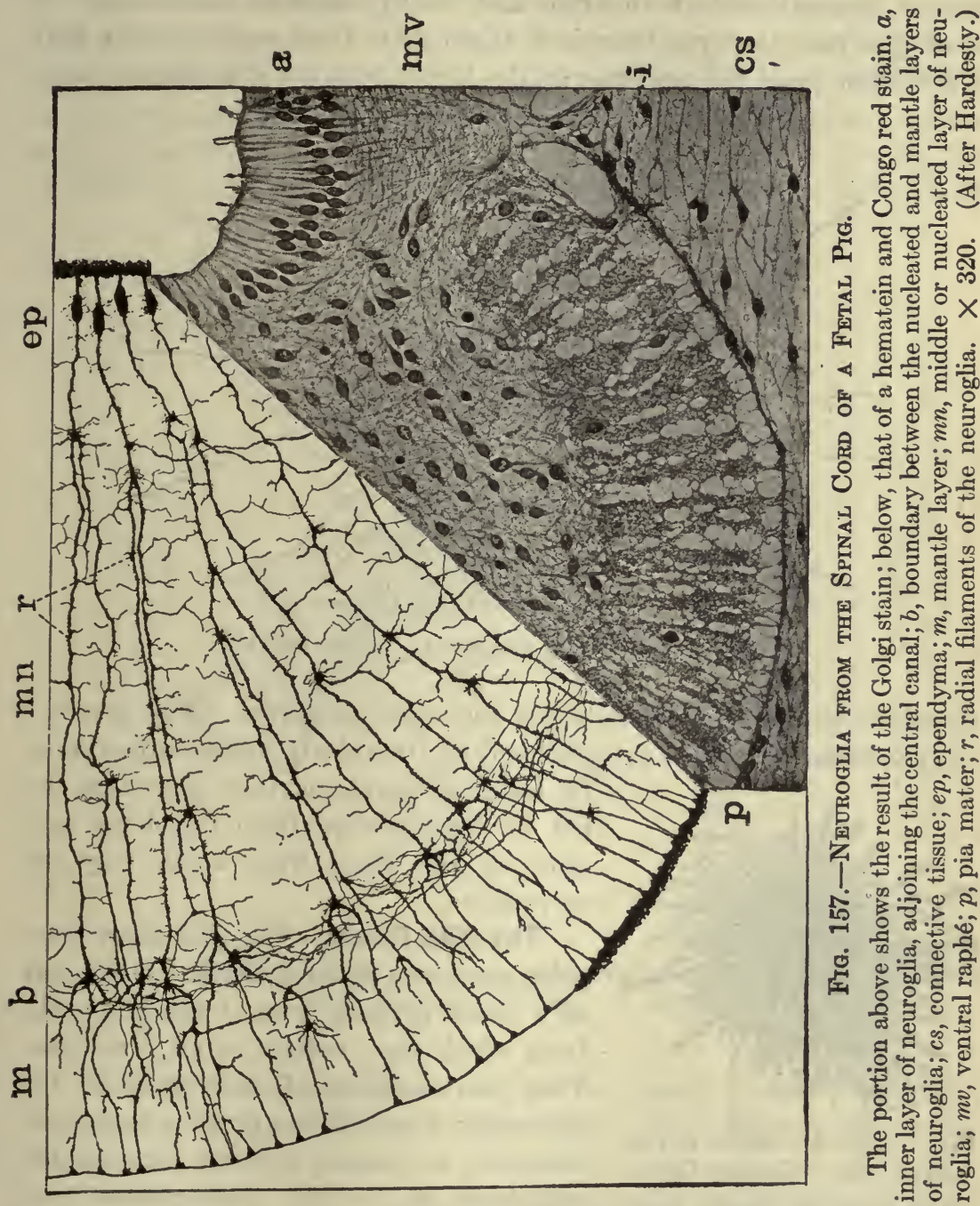

astrocytes, whose processes are shorter and somewhat thicker but decidedly more varicose than those of the long-rayed type.

Recent investigations by means of the staining methods of Weigert, Mallory, and Benda, have demonstrated that the astrocytes, as seen in 
the Golgi preparations, probably include two distinct structures, the glia cells and the glia fibers.

Glia cells, as seen in sections prepared according to these methods, appear as small cells with large and deeply staining nuclei. In the small glia cells the cytoplasm is so slight as to form scarcely more than a mere rim about the nucleus; in the larger cells the cytoplasm is more

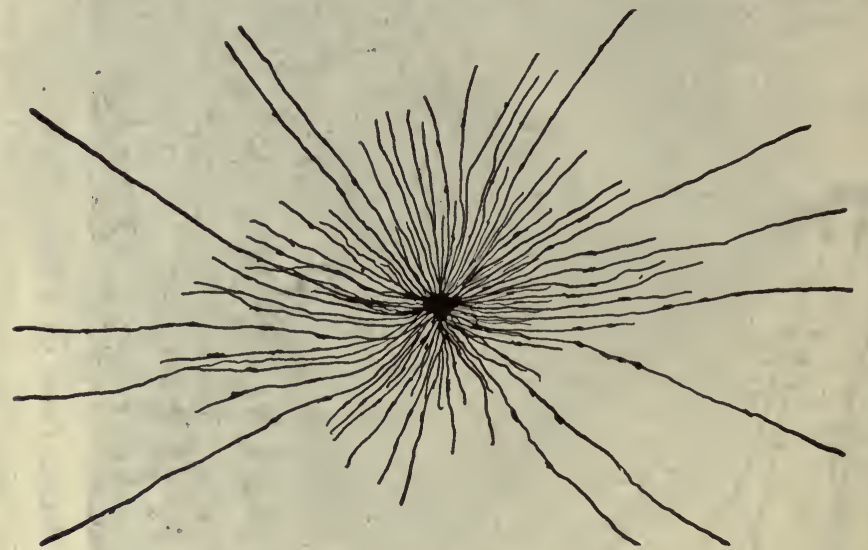

Fig. 158.-A Long-Rayed Astrocyte.

Golgi's stain. Highly magnified. (After Berkley.)

abundant and the processes larger and more numerous. The presence of cytoplasmic processes gives the cell an irregularly stellate appearance.

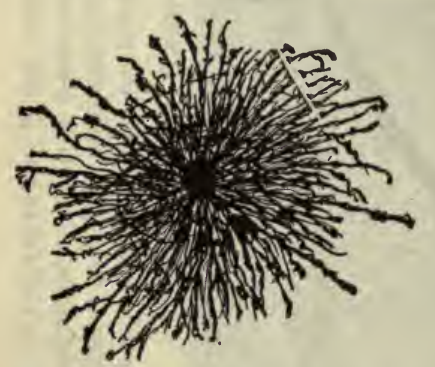

Fig. 159.-A SHORT-RAYED Astrocyte, or Mossy Cell.

Golgi's stain. Highly magnified. (After Berkley.)
In Golgi preparations these processes can not be distinguished from the dense network of glia fibers with which they are surrounded.

The Glia Fibers.-The glia fibers comprise numerous filiform fibrils which occur as a dense network around the glia cells, from which they radiate in all directions. They pass alongside of, over, or under the glia cells; their filaments have even been described as passing entirely through the cytoplasm of the cell. Nevertheless they appear at all points to be anatomically distinct from the cell body.

The relation of the glia cells to the fibers of neuroglia is perhaps comparable to the arrangement in fibrous or reticular tissue. The fibers 
of each of these tissues appear to be ontogenetically derived either directly or indirectly from its cells, yet when fully formed they often exist as anatomically distinct elements.

Occurrence of Neuroglia.-Neuroglia cells and fibers occur in both gray and white matter of the central nervous system, though perhaps more abundant in the latter. The fibers radiate for considerable distances from their glia cells; and thus form a supporting tissue for the nerve elements. They are frequently in intimate relation with the blood-vessels, on the walls of which many of the glia fibers, particularly

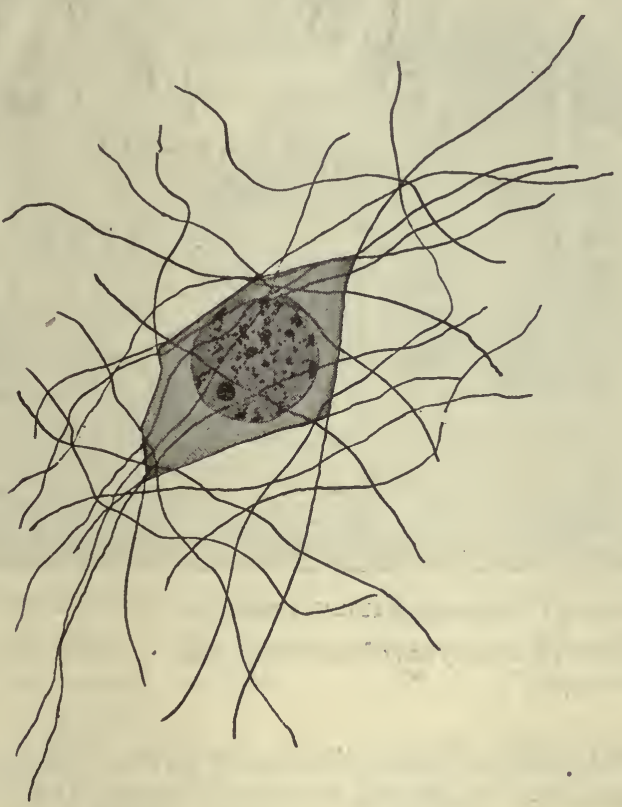

Fic. 160.-Nendoglia Cell with Adjacent Fibers from the Pineal Bodr of a Yearling Sheep. $\times 1500$.

the thicker or mossy variety, terminate in expanded plates, which, in some parts, form an almost complete outer membranous coat of the vessel.

The astrocytes are ontogenetic derivatives of the embryonic ependyma cells. From their point of origin around the neural canal they wander to all portions of the central nervous system, and even into the optic and olfactory tracts, which are embryonic outgrowths from the fetal cerebral vesicles. Thus neuroglia occurs throughout the brain 
and spinal cord, and also in the olfactory nerves, the optic chiasm, and the retina of the adult.

The supporting tissues of the central nervous system include, besides the neuroglia, numerous bands or trabeculæ of fibrous connective tisŝu,

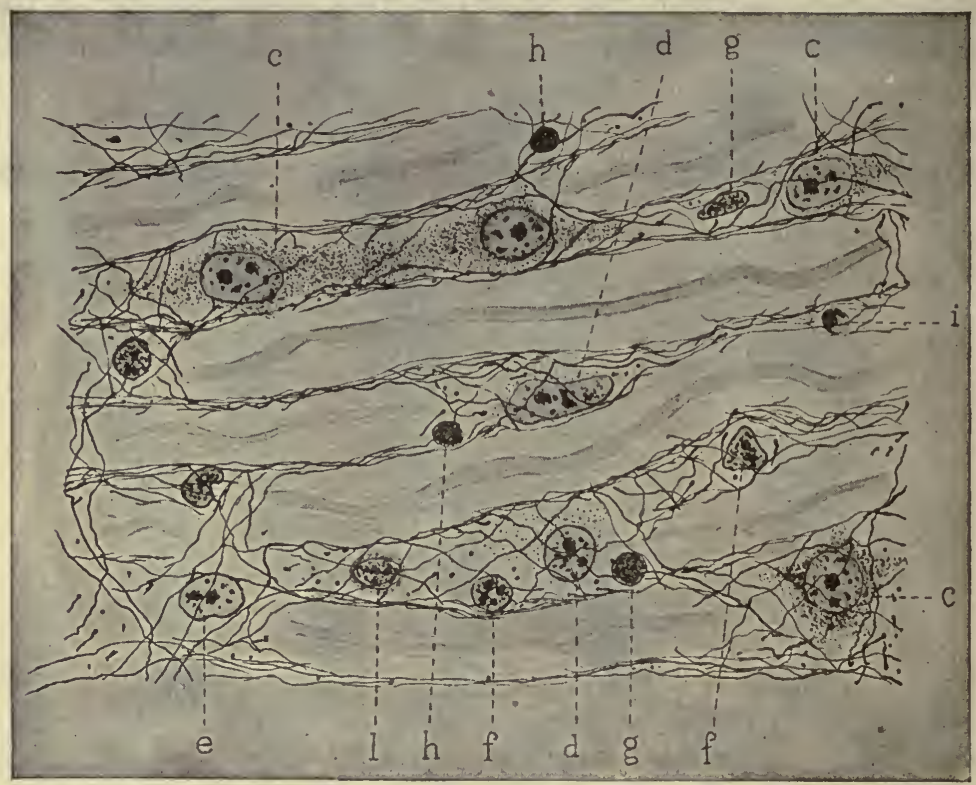

Fig. 161.-Neuroglia Cells and Fibers from the Spinai Cord of an Elephant.

The letters indicate various types of neuroglia cells. $\cdot l$, a leukocyte. Benda's stain. $\times$ 940. (After Hardesty.)

which push inward from the pia mater, carrying with them the vascular branches for the supply of the nervous tissues, and which penetrate deeply into the substances of the spinal cord and brain.

\section{NERVE TRUNKS}

Structure.-The nerve fibers of the peripheral nervous system are united into bundles to form the nerve trunks or nerves. Each nerve is surrounded by a heavy connective tissue sheath, the epineurium, which sends trabecula-like septa into the nerve. These septa subdivide the nerve trunk into smaller bundles of nerve fibers, the funiculi. The funiculus forms a compact bundle of nerve fibers, and is in turn invested with a sheath of dense connective tissue, the perineurium. Hence the 
perineurium stands in the same relation to the funiculus as does the epineurium to the whole nerve trunk.

From the inner surface of the perineurium, septa pass into the funiculus and break up to form a fine connective tissue framework, the endoneurium, for the support of the individual nerve fibers. On separating the fibers of a funiculus with needles a portion of this fibrous endoneurium remains adherent to the surface of the nerve fiber and gives the appearance of an outermost fibrous sheath, the so-called connective tissue sheath of Henle.

Nerve trunks frequently branch, the branches being formed. either by an individual funiculus or by groups of funiculi. In the smaller nerve trunks the funiculi are further subdivided. It is by anastomosis of the funiculi that most of the nerve plexuses are formed. Individual nerve fibers of the medullated ${ }^{\circ}$ type do not generally branch except in those portions which are naked

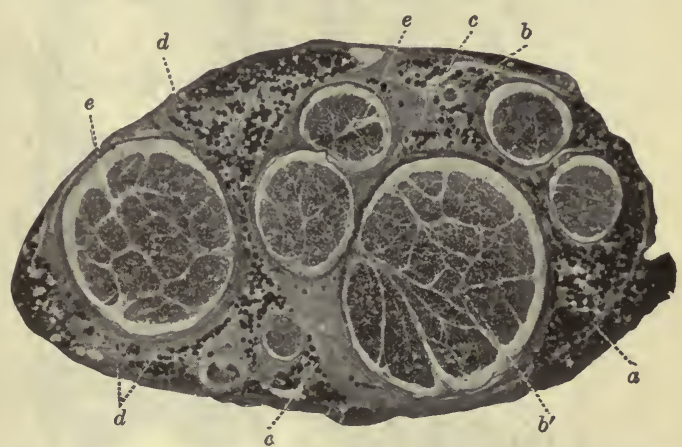

Fig. 162.-Transection of the Sciatic Nerve of A Dog.

The fat cells and the myelin sheaths of the nerve fibers have been blackened by osmium tetroxid. $a$, fat cells; $b, b^{\prime}$, bloou vessels, that at $b^{\prime}$ lies within a funiculus; $c$, epineurium; $d$, perineurium; $e$, coarser bands of the endoneurium. Osmium tetroxid. Photo. $\times 30$. axis cylinders, viz., at the cytoproximal portion of the axon by means of collaterals, and at the cytodistal portion by means of end arborizations. Occasionally also collaterals arise at a node of Ranvier.

Vascular Supply.-The nerve trunks are well supplied with bloodvessels. The larger of these are found in the epineurium, and from them branches of considerable size enter the septa to be distributed throughout the perineurium to the funiculi. The coarser septa of the endoneurium contain minute arterioles and venules. A capillary network with elongated meshes occupies the finer divisions of the endoneurium, its vessels being thus brought into contact with the nerve fibers.

Perivascular lymphatic vessels abound in the epineurium and its septa, and lymphatic tissue spaces are found throughout the connective tissue of the nerve trunk. Where the cerebrospinal nerve trunks pene- 


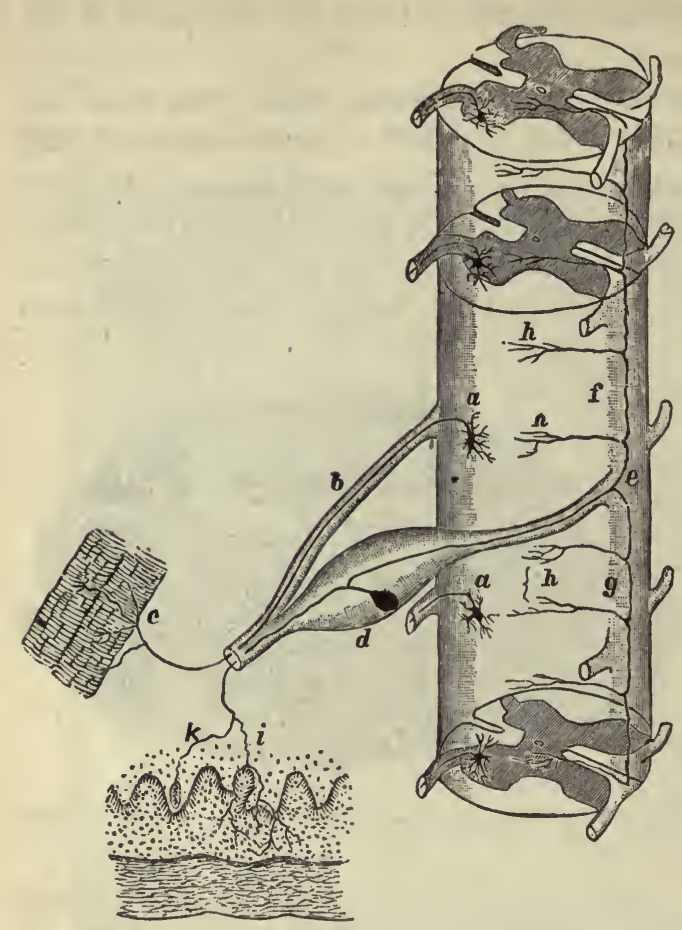

Fig. 163.-Diagram of the Origin and Relations of the Peripheral Motor and Sensory NeuRONS.

A cylindrical section of the spinal cord, with its ventral and dorsal nerve roots, is shown. $a$, nerve cell of the ventral horn whose axon passes through the ventral nerve root, $b$, to its peripheral termination, $c$; at $d$ is a unipolar sensory nerve cell in the dorsal root ganglion; its process immediately divides into a peripheral and a central branch. The central branch enters the spinal cord and at $e$ divides into an ascending, $f$, and a descending, $g$, branch from both of which numerous collaterals, $h$, enter the gray matter and terminate in fine end brushes. The peripheral branch of the spinal ganglion cell enters a spinal nerve and finds its way to its termination which is here represented in the skin; it terminates partly by free endings among the epithelial cells, $i$, and partly in connection with a sensory end organ, $k$, in this case a tactile corpuscle of Meissner. (After von Lenhossék.) trate the meninges these lymphatic vessels are said to be continuous with the similar vessels of the. dura mater.

Minute nerve fiber bundles, nervi nervorum, are also found in the epineurium; their fibers are mostly, if not entirely, distributed to the bloodvessels.

\section{GANGLIA}

A ganglion may be described as a group of nerve cells occurring in the course of a peripheral nerve trunk. The largest of the ganglia form - fusiform swellings in the course of the nerve, which are visible to the naked eye. The smallest, on the other hand, contain not more than half a dozen nerve cells, and these must be sought with the aid of the microscope and can only be found by the most careful observation.

Whatever may be their size, all ganglia appear to have a similar structure, except for those differences which characterize the sympathetic as 
distinguished from the cerebrospinal type. The essential elements of structure are the nerve cells, nerve fibers, and a supporting framework of rather dense fibro-elastic connective tissue.

Many of the nerve cells of the adult mammal are unipolar in the cerebrospinal ganglia, but are usually multipolar in the sympathetic. The spinal ganglia of the lower vertebrates and of the embryo mammal,

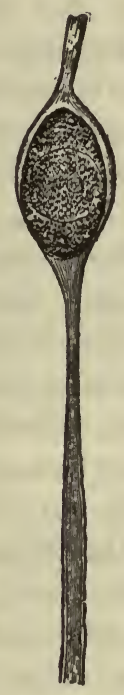

Fig. 164.-BipoluR

CELL From a Spinal GangLion of a Fish. Corti.)

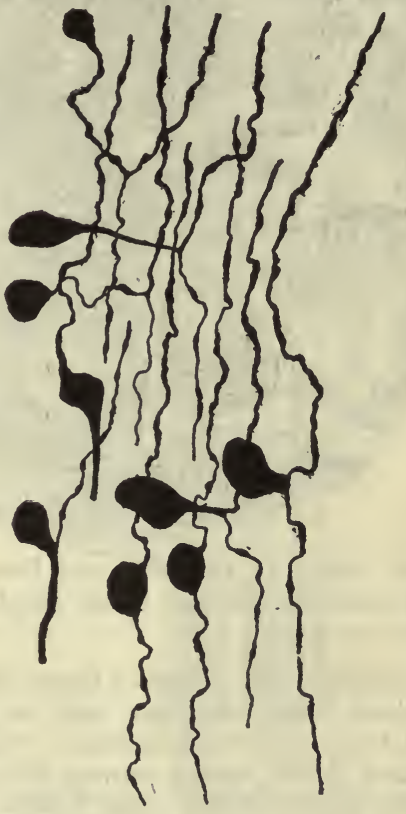

Fig. 165.-Transformation of Bipolar Cells into Unipolar Cells in the Gasserian Ganglion of the Pig. ten.)

(Barker, after van' Gehuch-

however, contain bipolar ganglion cells. In mammals the two processes of the embryonal neuron fuse to form a single one which branches in a $\mathrm{Y}$ - or T-like manner soon after leaving the parent cell body.

In the ganglia of the acoustic nerve the primitive bipolar condition of the neuron is retained; and the cell body is not surrounded by a capsule.

The nerve cells of all other ganglia are surrounded by a capsule of flat epithelioid cells which form a complete investment for the nerve 


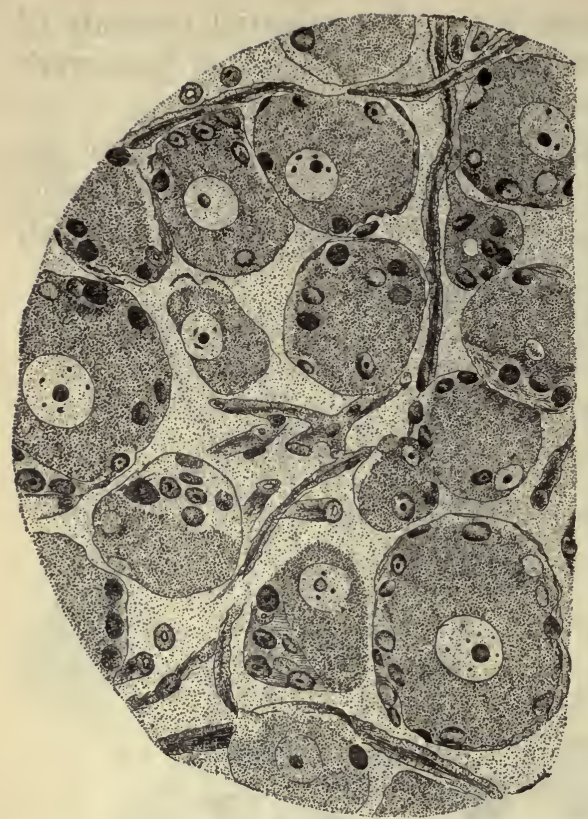

Fig. 166.-Section throdgh the Dorsal Root Ganglion of the First Thoracic Nerve of a CAt.

The ganglion cells contain a large vesicular nucleus, with nucleolus, and are enveloped by a nucleated capsule. Several medullated fibers appear among the ganglion cells. (From Barker, after Hodge.)

frequently proximally convoluted and, after branching in T-shape fashion, passes out of the ganglion to become the axis cylinder of a medullated nerve fiber, and (2) cells with a slender axon which breaks up within the ganglion and whose terminal arborizations form a pericapsular plexus around the cell capsule;-from this plexus fine end branches penetrate the capsule to form a pericellular arborization about the nerve cell itself. The cells of this latter type suggest association neurons cell and its processes, being continuous with the neurolemma. The capsule is not, however, as a rule, elosely applied to the cell, but leaves a narrow interval which is occupied by lymph or 'tissue juice.'

In their structure the ganglionic neurons do not appear to differ in any way from other neurons. The large vesicular nucleus with its distinct nucleolus readily distinguishes these cells from those of neighboring tissues.

The studies of the ganglion cells by Dogiel, Ranvier, and Cajal have done much to explain the relations of these cells to each other, especially in the sympathetic system, where they were formerly but little understood. In the spinal ganglia Dogiel (Anat. Anz., 1896) described two types of ganglion cells: (1) a unipolar cell in which the axon is thick and

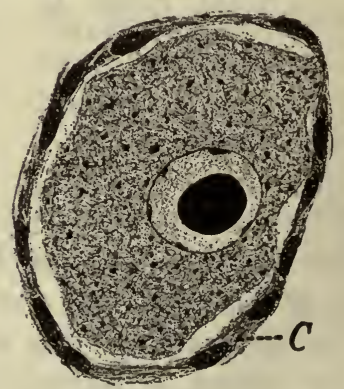

Fig. 167.-A Nerve Cell from a Section of a Human GasSE Ria n Ganglion.

$C$, capsule. Nissl's stain. $\times 500$. 
within the ganglion. Nerve fibers from the sympathetic ganglia also enter the spinal ganglia and form pericellular arborizations about the cells of the second type. Dogiel found also that multipolar ganglion cells occur in the spinal ganglia of the adult as well as of the embryo.

The more recent work of Cajal (1905), Dogiel (1908), and Ranson (1912) has revealed a third distinct type of cell formerly apparently included under Dogiel's Type I: smaller, pyriform, unipolar. cells with non-medullated axon, rarely convoluted, dividing into a central and a peripheral branch, the exact terminations of which are unknown; but having according to Ranson apparently the same distribution as the coarser medullated fibers of Type I, and probably afferent in nature (Jour. Comp. Neur., vol. 22, 1911). In cat and rat Ranson estimates the number of these cells at two-thirds that of the total number. These are the cells which contribute the bulk of the very numerous non-medullated fibers of the spinal nerves, only a small portion of which are believed to arise in sympathetic ganglia.

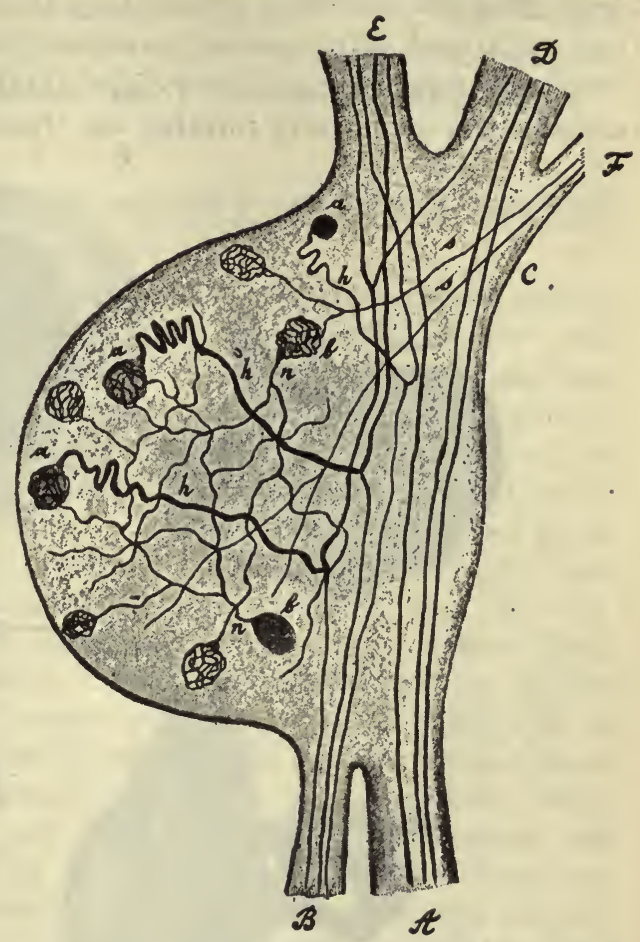

Fig. 168.-Schematic Representation of the Relations of the Structures Composing a Spinal Ganglion.

$A$ and $B$, ventral and dorsal spinal nerve roots; $C$, a spinal nerve; $D$ and $E$, its ventral and dorsal divisions; $F$, its ramus communicans. $a$, nerve cells of the first type, whose neuraxes divide and form the axis cylinder of a peripheral and a central nerve fiber; $b$, nerve cells of the second type, whose neuraxes, $n$, end in a felt work about the cells of the first type; $s$, sympathetic nerve fibers which terminate in a basket work about the cell bodies of the second type of ganglion cells. (After Dogiel.)

With the exception of relatively few cells of bi- and multipolar form, all of the nerve cells of the spinal ganglia are unipolar in the adult condition. In the case of the larger cells, the medullated ason before it 
leaving the capsule is more or less extensively convoluted over the cell body forming in the extreme condition a so-called 'glomerulus.' These same cells are variously modified by the presence of short, coarse and fine intra- and extracapsular processes (both dendrons and collaterals) frequently terminating in 'end disks' (Huber). Such processes may fuse more or less extensively forming the 'fenestrated' variety of cells. The

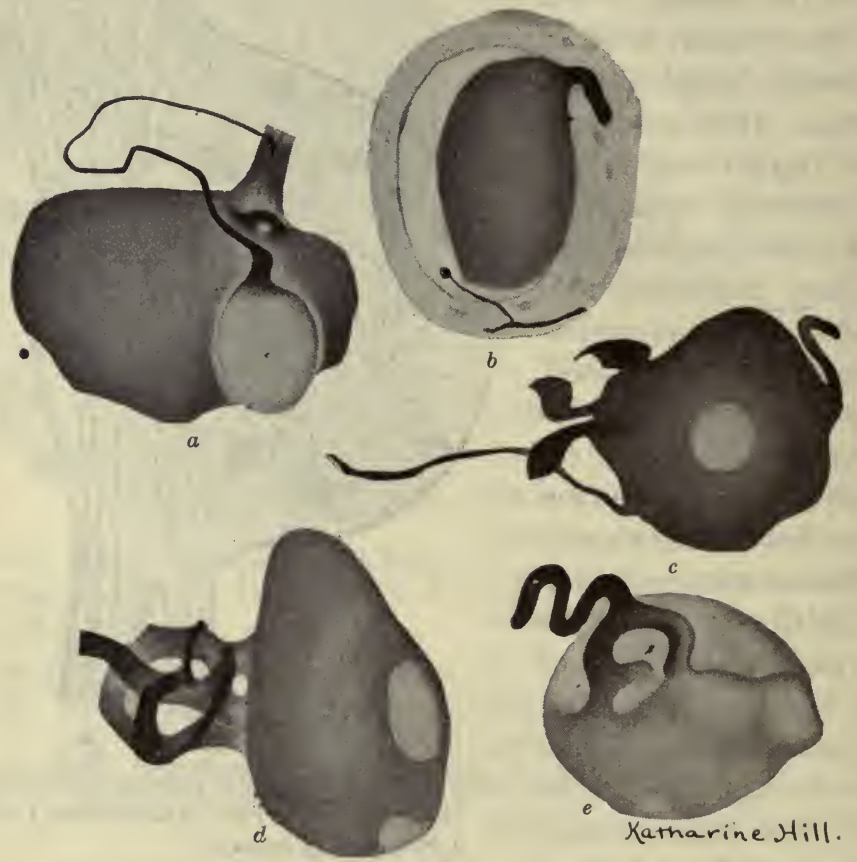

Fig. 169.-Common Atrpical, thodgh Probably Perfectly Normal, Nerve Cells from the Spinal Ganglion of the Dog.

$a$ and $b$, cells with collaterals ending in 'end bulbs'; $c$, a multipolar cell; $d$ and $e$, 'fenestrated' cells. (Ranson, Jour. Comp. Neur., 22, 2, 1912.)

axon, prior to its division, may split at one or several points, for longer or shorter distances, into two or many portions, and reunite again into a single fiber; rarely also the axon may have two or more points of origin, probably the result of fusions of collaterals with the cell body. These more complex atypical forms are said to predominate in man (Ranson, Jour. Comp. Neur., 24, 6, 1914). Ranson regards them to some extent at least as transient modifications, which may return to the simpler unipolar condition. Nageotte (190\%) has suggested that 
the phenomena of end disks and fenestrations signify regenerative activity. They are relatively more abundant in regenerating transplanted ganglia. But they are abundant also in pathological ganglia (Nageotte, 1906), and in fetal ganglia (Huber, 1913). No conclusive evidence has yet been presented that these modified forms signify functional derangement.

\section{THE SYMPATHETIC DIVISION OF THE NERVOUS SYSTEM}

The sympathetic division of the nervous system (autonomic system) consists essentially of three sets of ganglia: (1) the ganglionated cords (sympathetic trunks; vertebral ganglia); (2) the prevertebral plexuses; and (3) the visceral or terminal plexuses, including chiefly the myenteric and submucous plexuses of the alimentary canal. The ganglia of the ganglionated cords are segmentally arranged, and interconnected transversely (caudally) and longitudinally by plexuses of non-medullated fibers. They are connected also with the spinal nerves by the white and gray rami communicantes. Homologous ganglia in the head region, less definitely related to the cerebral nerves, are the ciliary, sphenopalatine, submaxillary, sublingual, parotid and otic ganglia. The prevertebral plexuses develop from non-segmentally arranged anlages which originate as outgrowths at certain levels of the early ganglionated cord. These plexuses contain fewer and smaller cells, with a preponderance of fibers, e.g., cardiac, celiac (semilunar; solar), hypogastric and pelvic plexuses. The myenteric and submucous plexuses are located in the muscle and submucous layers respectively of the esophagus, stomach and intestine. Here the cells are still smaller and less numerous than in the prevertebral plexuses, and the fibernetwork is less dense. A plexus is a network of nerve fibers with few cells; where the nerve cells are relatively abundant, the plexus is known as a ganglion. The embryonal cells (neuroblasts) which develop into sympathetic neurons have migrated from the neural crest, possibly in part also from the wall, of the primitive spinal cord.

Langley employs the term 'autonomic nervous system' for all that portion of the peripheral nervous system not included among the cerebrospinal nerves, commonly designated as the 'sympathetic system.' This comprises four components: (1) the sympathetic proper, including the autonomic fibers arising from the thoracicolumbar regions of the spinal cord, together with the associated vertebral ganglia and their postganglionic neurons; (2) sacral autonomic, preganglionic fibers included in the roots of the second, third and fourth sacral nerves, together with the associated postganglionic neurons; (3) cranial autonomic, a group of fibers arising from the midbrain and the medulla (this component is separated from the sympathetic proper by the whole extent of the cervical region 
of the spinal cord, which region lacks white rami communicantes); (4) enteric, including the myenteric and submucous plexuses of the digestive tube. Langley proposes also the term 'parasympathetic' to designate the sacral and cranial autonomic fibers, since in many parts of the body they overlap the distribution of the sympathetic proper.

In the sympathetic (or autonomic) ganglia Dogiel (Anat. Anz., 1896) likewise recognized two cell types, in general smaller than those of the

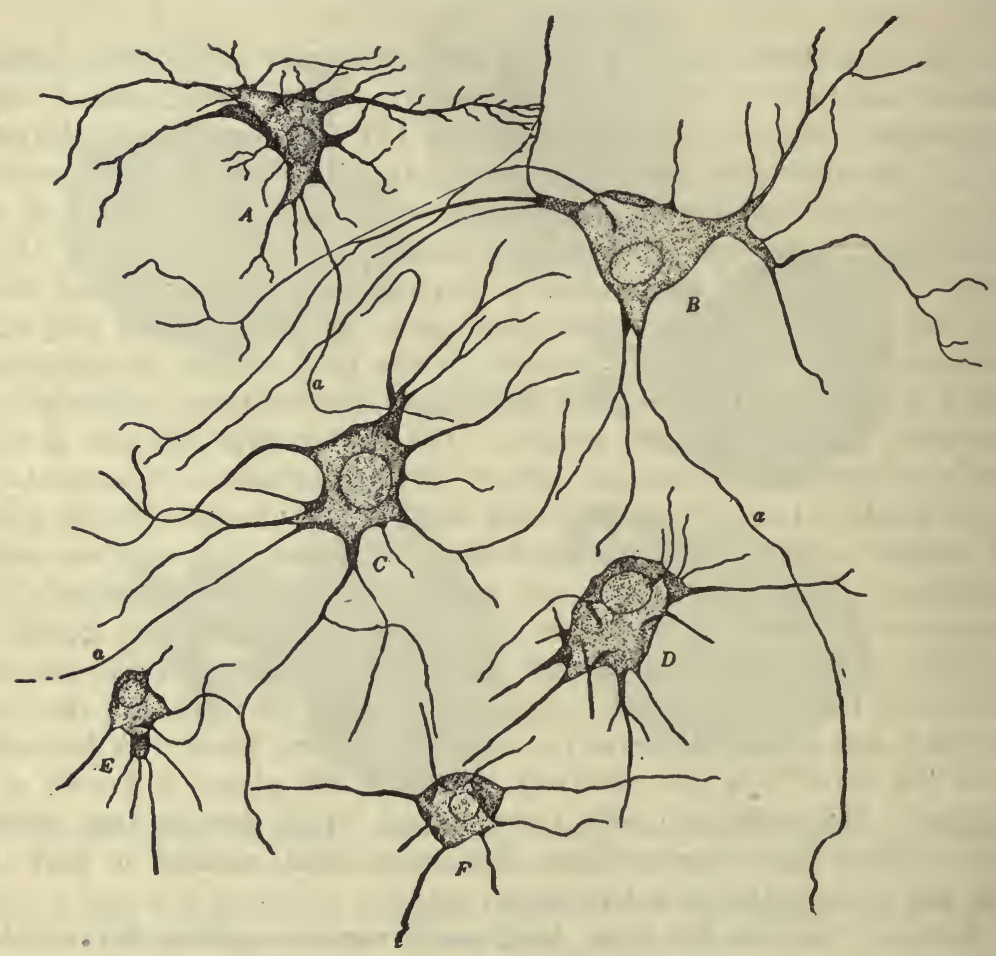

Fig. 170.-Sympathetic Neurons.

$A$, in myenteric plexus, ileum of eat; $B$ and $C$, in myenteric plexus, ileum of dog; $D, E, F$, in submucous plexus, ileum of dog; $a$, axon. $A$ corresponds to Dogiel's Type I, a motor neuron; $B$ and $C$ correspond to Dogiel's Type II, probably sensory neurons. (After Kuntz, Jour. Comp. Neur., 23, 3, 1913.)

spinal ganglia: (1) small multipolar fusiform or stellate nerve cells with 5 to 20 dendrons and an axon which enters the nerve trunks as a nonmedullated fiber, but may later acquire a thin medullary sheath-motor neurons; (2) larger spheroidal nerve cells with 1 to 16 dendrons and a 
single axon which also enters the nerve trunk as a non-medullated nerve fiber, but may later acquire a very thin medullary sheath, perhaps sensory neurons. The dendrons of the second type are distinguished from those of the first by being very long and slender and also by entering the nerve trunks, to pass, presumably, to neighboring ganglia. The dendrons of the first cell type on the other hand, are shorter, thicker, and end in relation with other cells within the same ganglion. Carpenter and Conel report also intermediate types in the cat.

In certain rodents (rabbit and guinea pig) many of the neurons of the vertebral and prevertebral autonomic ganglia are bi-nucleate (Carpenter and Conel, Jour. Comp. Neur., 24, 3, 1914).

The ganglionic cell group is excentrically placed as regards the axis of the nerve trunk, some funiculi apparently passing the ganglion without being in any way connected with its nerve cells.

The sympathetic differ from the cerebrospinal ganglia chiefly in the relative preponderance of non-medullated nerve fibers in the former and of the medullated type in the latter. Just as the cerebrospinal ganglia receive a few non-medullated sympathetic fibers, so also the sympathetic ganglia receive, through the medium of the white rami communicantes, a certain number of medullated nerve fibers from the cerebrospinal system. Moreover, with the intense staining method of Weigert, very thin medullary sheaths may now be demonstrated where formerly they were not suspected.

The sensory and motor neurons of the cerebrospinal division show characteristic differences in their chromophilic substance. In the cerebral and spinal ganglia the cell bodies of the sensory neurons contain fine Nissl granules evenly distributed throughout the cytoplasm. The motor cell bodies from the spinal cord contain fewer and much larger chromophilic flakes. The sympathetic neurons likewise present a characteristic and constant appearance: the chromophilic granules are intermediate in size and generally massed toward the periphery (Malone; Carpenter and Conel).

The ganglia are supplied with blood vessels and lymphatic vessels in a manner similar to the nerve trunks in whose course they occur.

The earlier conception of the nervous system interpreted the nerve fiber (axon) as the fusion product of a chain of cells extending from its proximal to its distal end. The axis cylinder fibrils were regarded as differentiation products of the cytoplasm (Schwann; Apáthy; et al.). The view which now prevails interprets the axon as the outgrowth of the cell body to which it is attached (His; Cajal; et al.). The tissue culture 


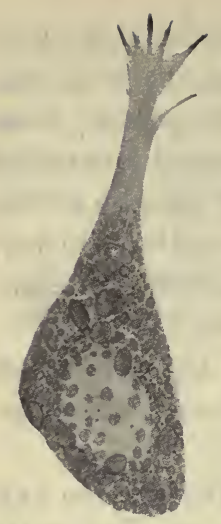

Fig. 171.-THe Sprouting OF AN AXon By a NeuROBLAST FROM THE SPINA L Cord of a Frog Embryo.

From a live specimen grown in lymph; the cell body is filled with yolk granules; the protoplasmic process (axon) is of hyaline appearance and undergoes ameboid movements. (Harrison.)

work of Harrison and others has established the outgrowth view upon a firm basis of observational data. By growing small pieces of the embryonic spinal cord of frogs in lymph, Harrison could observe the cells sprouting an axon process (Figs. 171 and 172). $\mathrm{He}$ describes the beginning of a nerve fiber as an outflow of hyaline protoplasm from cells which were situated within the central nervous system. The experiments of Harrison upon frog larvæ demonstrate further that the sheath cells of the neurolemma of motor and sensory fibers have their origin in the ganglionic crest, therefore ectodermal, and that they are unessential to the formation of the fibrils of the axis cylinder. He excised the dorsal half of the cord, including the neural crest, and observed that in such larvæ the fibers of the motor roots did not acquire sheath cells. On the contrary, when he excised the ventral half of the cord, dorsal root fibers developed normally with a neurolemma, but the sheath cells which migrated to the location where the ventral fibers normally appear were unable to produce these fibers in the absence of neuroblasts in the ventral half of the cord. (Anat.Rec., 2, 9, 1908.)

The influence which guides the nerve along its proper path is apparently exerted by the tissue which is to be innervated. The essential factors comprising this influence are obscure; they may be of a chemotropic nature. It must be emphasized, however, that the connection between a particular nerve and its tissue terminal is made relatively early, that is, while the two elements are still spatially relatively closely associated. Probably mechanical stimuli, inducing thigmotropic reactions, also play an important rôle in determining the path of a nerve

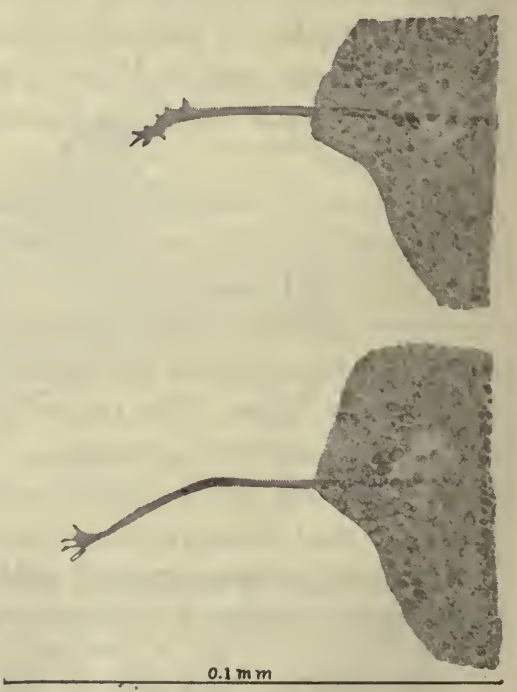

Fig. 172.-The Sprouting of AN Axon by a Neuroblast from the Spinal Cord of a Frog Embryo.

Two views of the same nerve fiber, grown in lymph, taken twenty-five minutes apart. (Harrison.) 
fiber. The earlier relations are of course modified during growth; the definitive relation between nerve and end-organ are acquired by mutual adjustment. Recently Harrison has contributed further experimental-evidence in support of the view that the growing axon is guided through a stereotropic response (Jour. Exp. Zool., 17, 4, 1914).

By cultivating sympathetic neurons from pieces of the intestine of the embryo chick in saline solutions, W. H. and Margaret R. Lewis (Anat. Rec., $6,1,1912$ ) have been able to demonstrate that here also the fibers arise as outgrowths of nerve cells.

\section{NEURONE THEORY}

The work of Harrison, the Lewises and many others, including both experimental and morphological investigations, leave scarcely any further doubt of the accuracy of the Neurone Theory of Waldeyer (1891), which simply applies the Cell Theory of Schleiden and Schwann (1838$39)$ to the nervous system. It holds that the unit of structure is the neuron (neurocyte), consisting of cell-body (cyton) and processes, including one axon, and one or several dendrons. The nervous system consists therefore of innumerable associated neurons. Neurons arise each from a single embryonic cell, the neuroblast, retain their independence throughout life, and make connection with each other in general only by contact, which, however, is sufficiently intimate to insure functional continuity. A neuron exhibits a structural and functional polarity; and constitutes a trophic unit for the maintenance of whose metabolic activity a nucleus is necessary.

Further confirmation of the outgrowth interpretation as opposed to that of autogenesis of the axon has recently been furnished by the experiments of Clark (Jour. Comp. Neur., 24, 1, 1914) on the domestic fowl. By prolonged exclusive feeding of polished rice he induced degeneration in the peripheral medullated nerves. On return to an adequately nutritive diet regeneration, accompanied by a return to normal locomotion and function, followed. The material thus gave opportunity for a microscopic study of the steps in the nerve degeneration and regeneration. When the degenerative process had not been excessively prolonged a new axis cylinder grew down the old medullary sheath, which returned to normal; when greatly prolonged the myelin disappeared and the nuclei of the neurolemma multiplied, giving an appearance very similar to that of embryonic nerve fibers ('bandfasern' stage). Clark concludes that the function of these excessive sheath cells is the removal of the degenerating myelin, a new medullary 
sheath being supplied probably by joint influence of the new axis cylinder and the neurolemma cells.

Mitochondria of granular and rod forms are abundant in the neuroblasts. Meves, Duesberg and others have claimed that these differentiate into neurofibrils. The recent work of Cowdry (Amer. Jour. Anat., 15, 4, 1914) on chick embryos proves the untenability of this view. Cowdry shows that the neurofibrils arise as a differentiation of the ground substance of the neuroblast; and that mitochondria persist in undiminished numbers throughout the period of neurofibril-development. Moreover, it is now known that mitochondria are present also in adult neurons. They are apparently fundamental cytoplasmic constituents of a metabolically active cell.

Spinal ganglion cells of certain adult mammals (cat and rabbit) have been kept alive in tissue cultures for as long as twenty days (Minea, Anat. Anz., 46, 20, 1914). The cells remain apparently normal, augment their amount of chromophilic substance, produce new neurofibrils, develop short processes with end-plates (neuropodia) and become fenestrated, but do not proliferate. 


\section{CHAPTER VI}

\section{PERIPHERAL NERVE TERMINATIONS: END ORGANS}

All peripheral nerve fibers end either as terminal fibrils or in relation to a highly specialized end organ. The function of these latter bodies is apparently included in the changing of ordinary stimulimechanical, thermal, chemical, etc.-into a nerve impulse, or, vice versa, the changing of a nerve impulse to a cell stimulus which results in motion, secretion, etc., according to the nature of the tissue cells which are thus stimulated. Some of the nerve end organs are connected with efferent (motor) fibers, others with afferent (sensory) fibers. Nerve endings are found in nearly all the tissues of the body with the exception of cartilage and the calcareous tissue of the bones.

\section{NERVE ENDINGS IN EPITHELIUM}

Intra-epithelial nerve fibrils are derived from the nerve fiber plexuses in the subjacent connective tissue; the epithelium usually receives a very abundant nerve supply. The following types of intra-epithelial nerve endings have to be considered.

1. End Fibrils:- This form of nerve termination has been demonstrated in all the varieties of epithelium. Terminal nerve fibers enter the epithelial tissue as naked fibrils, often somewhat varicose, which form a delicate plexus between the epithelial cells. The terminal fibrils of this plexus frequently end in minute knoblike enlargements which are in contact with the surface, but rarely, if ever, penetrate the interior of the epithelial cells. The 'trefoil plates' of Bethe represent unusually large end knobs.

2. Tactile Cells (Merkel).-These are modified epithelial cells, with clear cytoplasm and a slightly vesicular nucleus, which are found in the deeper layers of the stratified epithelium of the epidermis and in the root sheaths of hairs. These cells are recognized by their vesicu- 
lar character and by the fact that they occur most abundantly in the interpapillary portions of the epidermis. The deeper surface of the

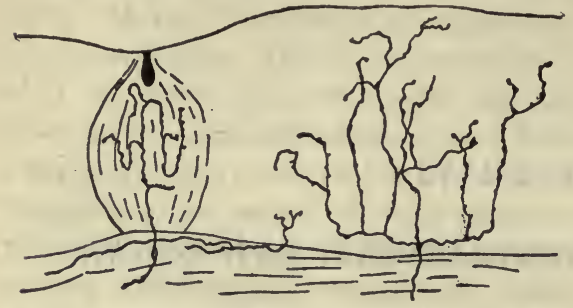

Fig. 173.-Nerve Endings in the EpiTHELIUM OF THE LARYNX.

On the left a taste bud; on the right, nerve endings in the stratified epithelium are represented. (After Retzius.) tactile cell rests in a cuplike expansion of a terminal nerve fibril which is known as the tactile meniscus.

3. Neuro-epithelium.-The cells of some types of neuroepithelium, e.g., the olfactory cells, are true nerve cells; others are modified epithelial cells, in relation to which the nerves terminate by intercellular end fibrils. The neuro-epithelium of the eye and the ear will be described in the chapters devoted to these organs, that of the gustatory organ forms typical nerve end organs, the taste buds.

Taste Buds (Gustatory Organ).-These end organs appear to be concerned with the special sense of taste. They occur in the stratified epithelium of the base of the tongue, uvula, soft palate, and epiglottis. Disse has also found similar structures in the nasal mucous membrane. They are most abundant on the lateral surfaces of the circumvallate papillæ of the tongue and on the walls of the sulci in the foliate papillæ which are most highly developed in the rabbit. They are occasionally found on the fungiform papillæ of the tongue, where they occur in considerable numbers in fetal life but mostly disappear be-

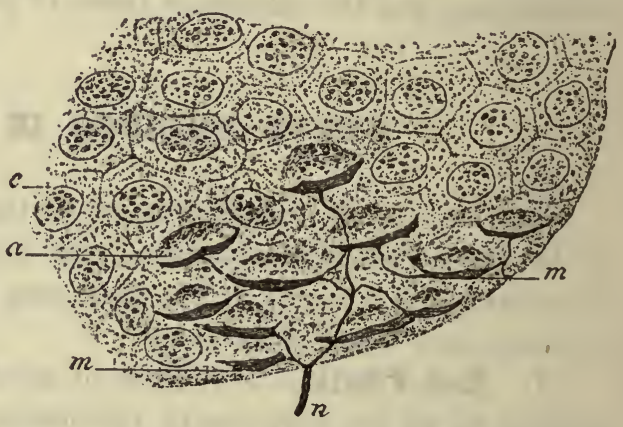

Fig. 174.-Tactile Cells in the Eithelium of the Groin of a Guinea-pig.

$a$, tactile cell; $c$, epithelial cell; $m$, tactile meniscus, at the end of a nerve fibril; $n$, nerve fiber. Chlorid of gold. Highly magnified. (After Ranvier.)

fore birth, and in the lateral walls of the sulci about the circumvallate papillæ.

Taste buds are ovoid, ellipsoidal, or spheroidal masses which occupy almost the entire depth of the epithelial layer. Their broad base rests 
upon the basement membrane, their narrower apex extends nearly to the surface of the epithelium. The apex of the bud is thus-covered by the superficial squamous epithelial cells except for a narrow tubular opening which overlies the superficial pole of the end organ. This canal presents an external and an internal ostium, respectively designated the outer and inner taste pore. The inner taste pore leads into a goblet-shaped depression in the apex of the taste bud, into which the cuticular processes of the gustatory cells project. Composite buds with two and three pores are common in the foliate papillæ of the rabbit; Heidenhain

(Anat. Anz., 45, 16, 1914) reports also buds with four, five and six pores.

The taste buds consist essentially of two varieties of cells, the gustatory and the sustentacular. The latter include the broad outer sustentacular or tegmental cells at the surface of the bud, the inner sustentacular cells within, and the basal cells which lie near the basement membrane.

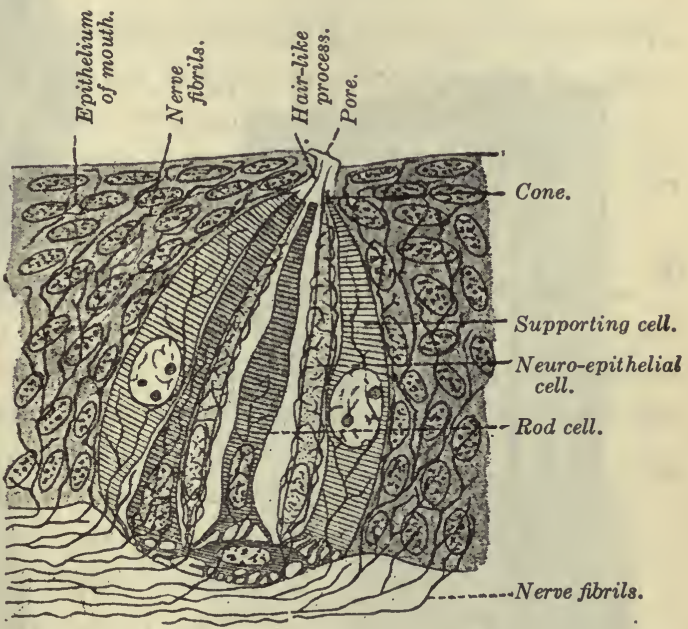

Fig. 175. - Schematic Representation of a Taste Bũ.

(After Hermann, from Böhm and von Davidoff.)

The Gustatory Cells.-The gustatory cells are slender neuro-epithelial structures whose nucleus causes a fusiform enlargement near their center or toward the basal end. Their cytoplasm is finely granular; their nucleus stains deeply and is ovoid or rod-shaped. The distal end of the cell carries a delicate, highly refractive cuticular process which projects beyond the apices of the sustentacular cells and as far as the inner taste pore. Their proximal end is often bifid, forked, or so flattened as to form a footlike extremity which is connected with the basal cells by fine processes. Sapid substances in solution enter the pore and stimulate the taste cells through the hair processes.

Sustentacular Cells.- The outer and inner sustentacular cells are elongated epithelioid cells, having an ovoid or spheroidal vesicular 
nucleus which causes no bulging of the protoplasm, and a coarsely reticular and frequently vacuolated cytoplasm. The distal ends of the cells taper to blunt points which collectively form the lateral wall of a goblet-shaped cavity at the apex of the taste bud. The proximal end
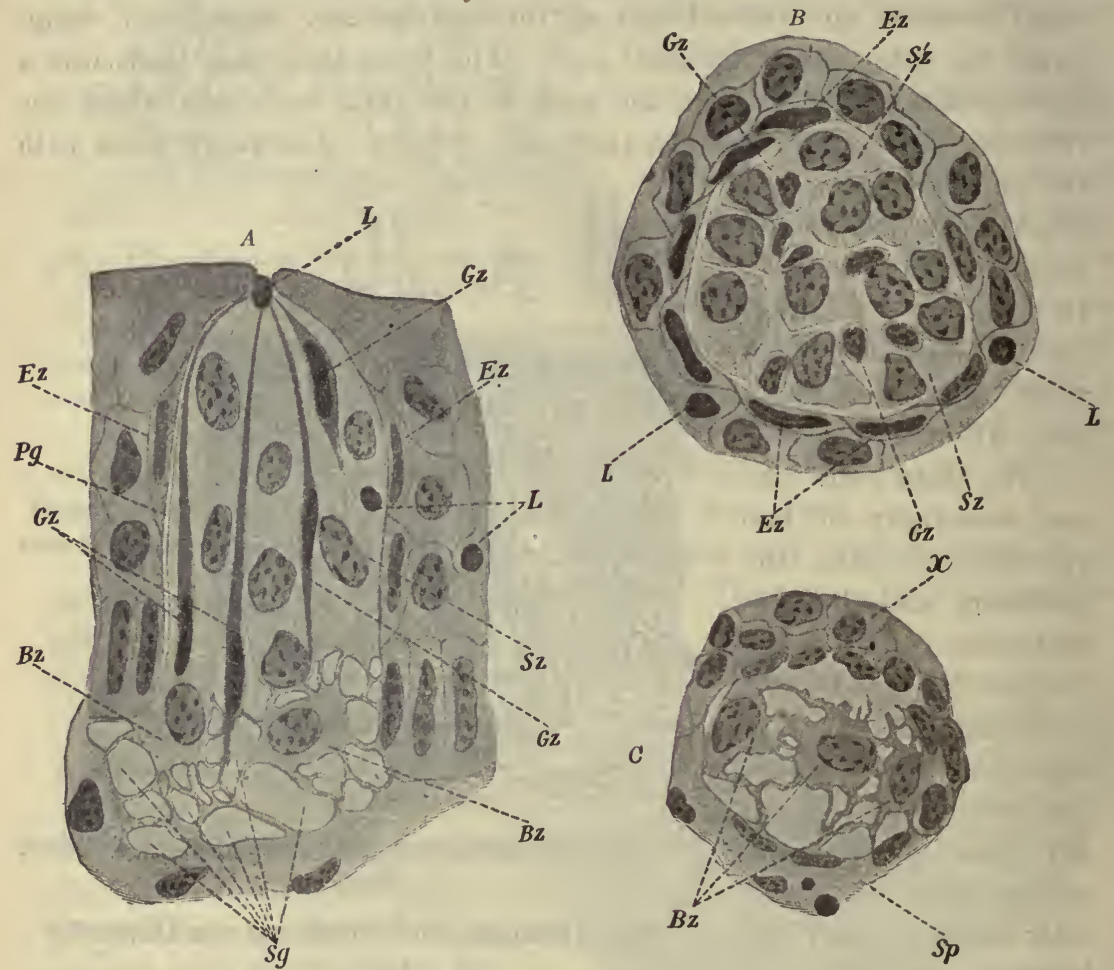

Fig. 176.-Taste Bud from the Human Tongue.

$A$, in longitudinal section; $B$, transection through the deeper third; $C$, transection through the base. $B z$, basal cells; $E z$, extra-bulbar cells; $G z$, gustatory cell; $L$, leukocytes, in $A$ one of these is seen in the pore; $P g$, perigemmal space; $S g$, subgemmal spaces; $S p$, connective tissue of the tunica propria; $S z$, sustentacular cells; $x$, cells of the adjacent epithelium. (After Graberg.)

is broad, often blunt or serrated, and, like the gustatory cells, it is connected with the basal cells by protoplasmic processes.

The Basal Cells.-The basal cells are flattened bodies with small ovoid vesicular nuclei and a relatively small amount of cytoplasm which is prolonged into numerous processes that appear to be continuous with the sustentacular and gustatory cells. These cells have been considered as having a similar function to the sustentacular cells. 
The Fibers. - The nerve fibrils of the taste buds are derived from a sub-epithelial plexus which distributes terminal fibrils to the gustatory and sustentacular cells,-intragemmal fibers,-and to the intervening portions of the stratified epithelium,-intergemmal fibers,-where they terminate in end fibrils. Von Lenhossek (Anat. Anz., 1892) states that the intragemmal and intergemmal fibers are never derived from the same nerve fiber. Circumgemmal fibers, distributed as varicose fibrils to the surface of the taste bud, may, however, arise from the same nerve fiber as the intragemmal branches.

Those nerve fibers which enter the taste buds form fine varicose fibrils which are closely applied to, but are not continuous with, the gustatory and the sustentacular cells. The terminal twigs of these fibrils end by minute end knobs which are scarcely distinguishable from the varicosities (Fig. 175).

\section{NERVE ENDINGS IN CONNECTIVE TISSUE}

The nerve fibers form extensive plexuses in the connective tissues from which terminal branches are distributed to the epithelium (free sensory endings), the walls of the blood and lymphatic vessels (sympathetic vasomotor endings), and to the numerous sensory end organs (encapsulated endings) which occur in abundance in most of the connective tissues. Nerves also terminate in connective tissue by free end fibrils some of which, as in the epithelial tissues, possess minute end knobs. Free nerve endings of this nature occur in the tendons, the lungs, the stomachal and intestinal mucous membranes, the meninges, and in the superficial layer of the corium of the skin and the hair follicles.

The following types of nerve end organs are found in connective tissue:

1. Tactile Corpuscles (Touch Corpuscles of Meissner).-These organs are formed by the terminal expansion of a

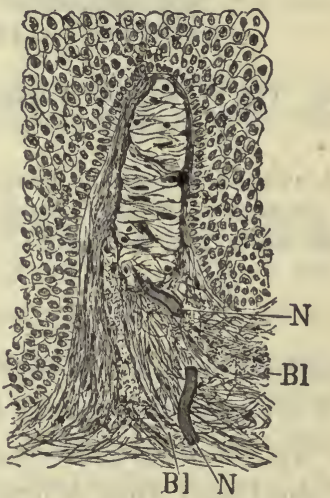

Fig. 177.-Tactile Corpuscle of Meissner from the Skin of the Human Toe.

$B l$, blood-vessel; $N$, medullated nerve fiber. Highly magnified. (After Schiefferdecker.) nerve fiber, which forms a varicose plexus inclosed within a delicate connective tissue sheath. The nerve fiber, or its primary branches, prior to 
its ultimate division makes several spiral turns about the corpuscle. The course of the nerve fiber gives the corpuscle a peculiar spirally striated appearance. Within the corpuscle the nerve fiber breaks into a plexus of varicose fibrils, many of which end in knobbed extremities. The cor-

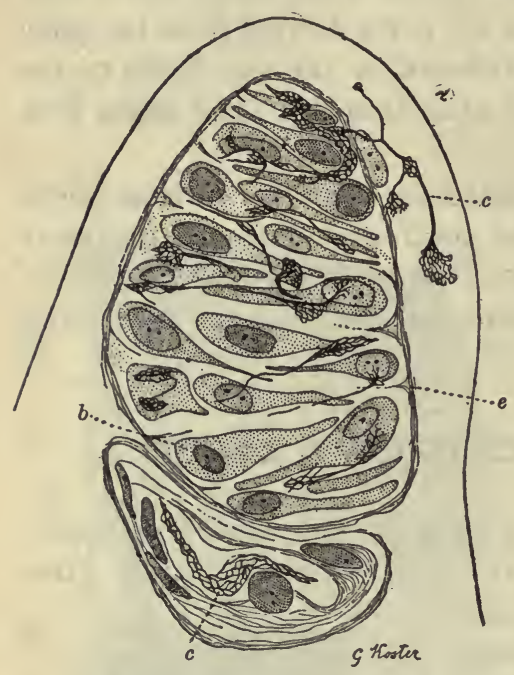

Fig. 178.-Tactile Corpuscle of MeIsSNer.

$b$, epithelio cells; $c$, nerve endings; $e$, connective tissue capsule. (Maximow, after Van de Velde.)

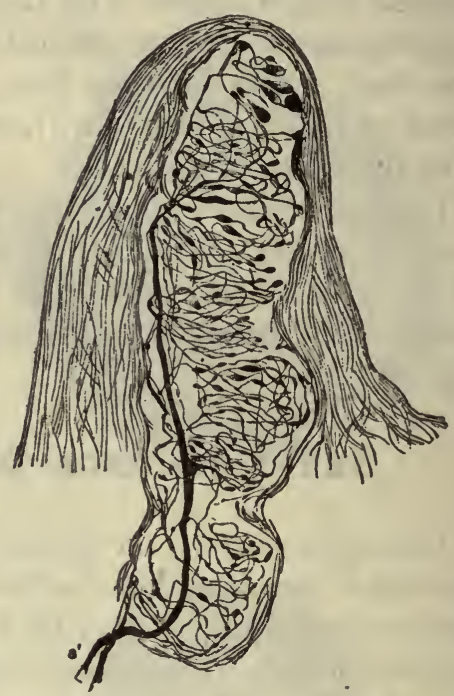

Fig. 179.-Tactile CoRpuscle of MEISSNER.

$a$, nerve fibrils which enter the corpuscle and supply its nerve skein. Methylene blue. Very highly magnified. (After Dogiel.)

puscles also contain many flattened or cuneiform epithelioid cells which are interspersed among the nerve fibrils.

Tactile corpuscles occur in largest numbers in the cutaneous papilla of the finger tips, where there may be as many as twenty to the square millimeter. They are found in considerable abundance also in other highly sensitive regions, including especially the corium of the toe tips, the lips, nipple, conjunctiva, glans penis and clitoris. The cutaneous senses comprehend four different qualities of sensation: pressure, warmth, cold and pain. These are mediated by two distinct groups of sensory fibers ending in the skin: the one conveys the impulses for pain and extremes of temperature (protopathic sensibility), the other for light pressure and small changes of temperature (epicritic sensibility). The 


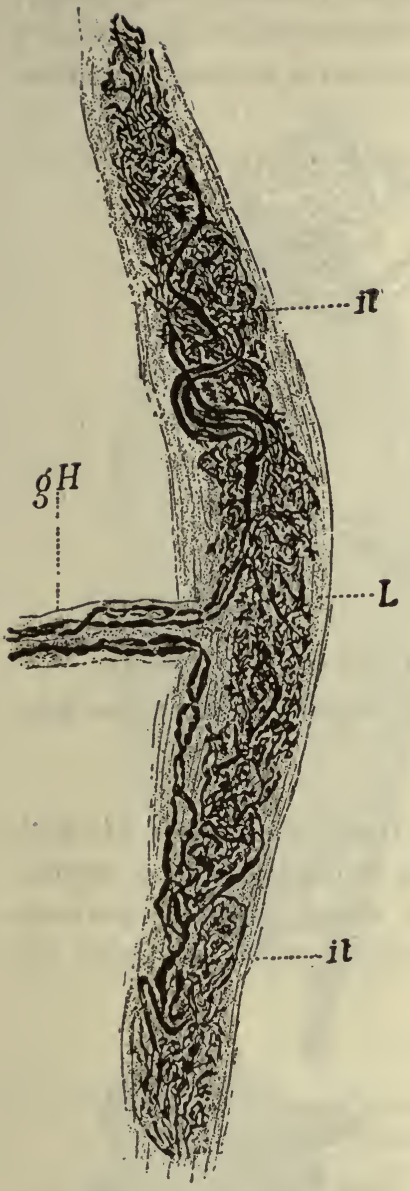

Fig. 180.-RUFFini's END ORGAN.

A single nerve fiber breaks up to form the tangle of nerve fibrils within the organ. $g H$, medullary sheath; $i l$, terminal fibrils of the axis cylinder; $L$, connective tissue capsule. (After Ruffini.) ally at its end. Now and then a single branching nerve fiber is distributed to several of these end organs. various subcutaneous endings mediate subcutaneous sensibility to pressure and movement.

2. Ruffini's End Organs.-These bodies, also known as terminal cylinders, resemble the tactile corpuscles in structure but possess a definite, though thin, connective tissue sheath within which the terminal arborization of the nerve, fiber is embedded in a granular core. They occur

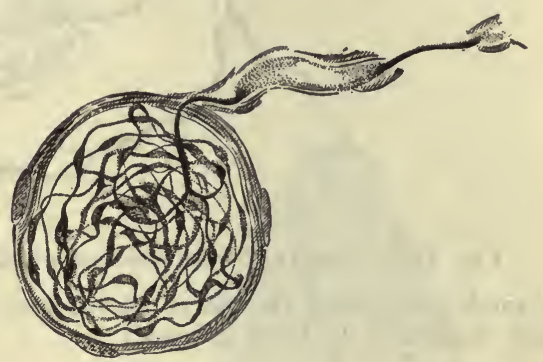

Fig. 181.-End Bulb of Krause from the Margin of the Ocular Conjunctiva.

The axon forms a dense skein within the encapsulated bulb. Methylene blue. Highly magnified. (After Dogiel.)

in the deeper part of the true skin near its junction with the subcutaneous tissue and in the connective tissue septa of the latter, whereas the tactile corpuscles are found in the papillary layer of the skin. Ruffini (Arch. ital. de biol., 1894) states that they occur in large numbers in the skin of the finger tips, where they rival in number the rather more deeply placed Pacinian corpuscles.

The Ruffini organs are cylindrical in shape and their nerve fibers usually enter at the side of the organ, though occasion- 
3. Fnd Bulbs (Krause).-These structures (bulbous corpuscles), together with those which follow, form the true so-called encapsulated nerve end organs. In the end bulbs the nerve forms a terminal arboriza-

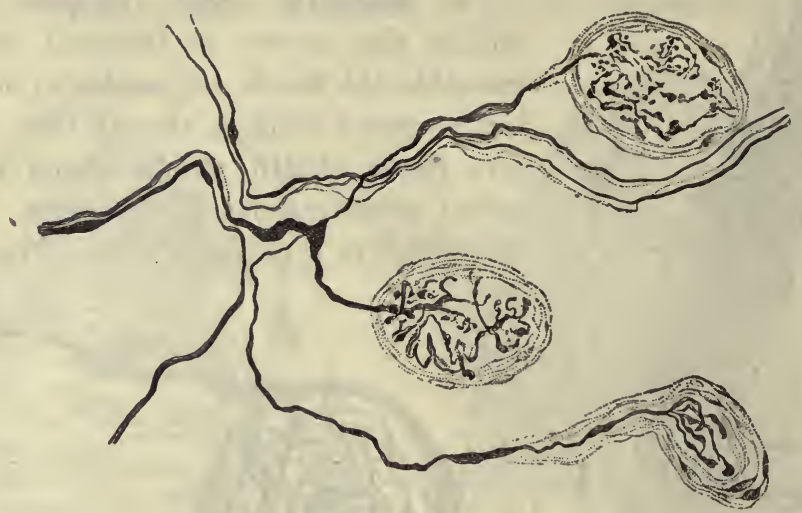

Fig. 182.-Genttal Corpuscles from the Cuitoris of a Rabitt.

A single axon from the nerve plexus enters each corpuscle. Methylene blue. Highly magnified. - (After Retzius.)

tion of the varicose and knobbed fibrils which freely anastomose (Dogiel, Ruffini). The bulb is invested with a distinct connective tissue capsule. On entering the bulb the nerve fiber loses its sheaths and the perineurium, now represented by Henle's sheath, becomes continuous with the
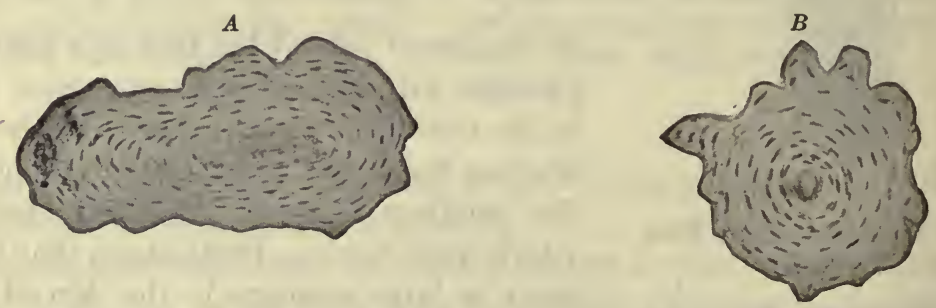

Fig. 183. - A Lamellar Corpuscle from the Mesentery of a Cat. $A$, a nearly axial section; $B$, a transection. Hematein and orange $\mathrm{G} . \quad \times 410$.

capsule of the bulb. Within the capsule the nerve fibrils are embedded in a granular inner bulb.

The end bulbs vary much in both size and shape. They may be either spheroidal, ovoid, twisted or convoluted, branched or compound, or cylindroid. They are abundantly found in the conjunctiva, but also 
occur in the corium of the skin. Similar, though more highly developed, end bulbs form the so-called genital corpuscles which are found in considerable numbers in the connective tissue of the glans penis, prepuce, and clitoris. In some of the smaller (cylindrical) end bulbs found in the tendons, the mucous membranes, and in certain portions of the skin,

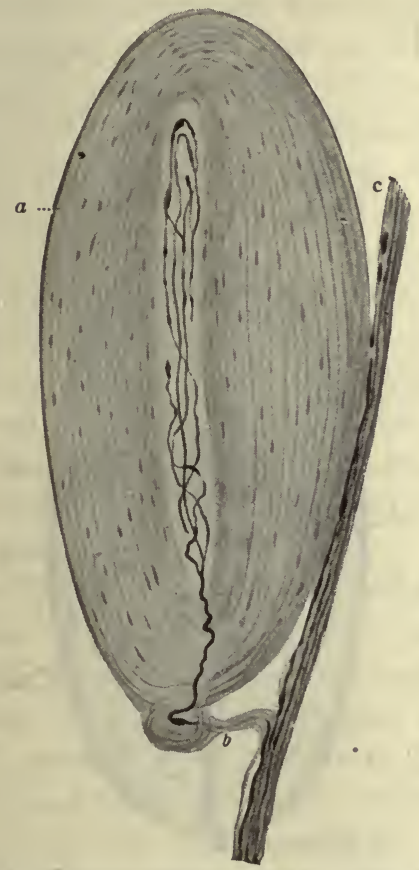

Fig. 184.-A Lamellar Corpuscle from the Pleura of a Child.

$a$, lamellæ; $b$, nerve fiber; $c$, nerve. Methylene blue. Moderately magnified. (After Dogiel.)

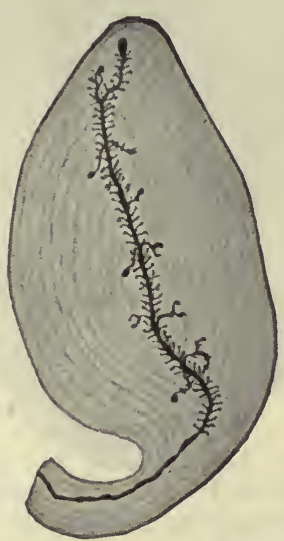

Fig. 185. - Lameliar Corpuscle from the Mesentery of a Kitten.

The nerve fiber shows lateral processes, many of which are knobbed. Methylene blue. Moderately magnified. (After Sala.)

the nerve fiber fails to divide but ends near the distal extremity of the bulb in a small fusiform end knob.

4. Lamellar Corpuscles (Pacinian Corpuscles, Vater's Corpuscles, Vater-Pacinian Corpuscles).- These are among the largest of the nerve end organs. In the mesentery of the cat they are of macroscopic size, varying in length from two to three millimeters. They assume the form of a large ovoid thickening, placed upon the end of a nerve fiber. The 
Pacinian corpuscle consists of a thick lamellated connective tissue coat, and a central granular protoplasmic core which is pierced by the nerve fiber. The medullated nerve fiber enters the axis of the corpuscle, its Henle's sheath becoming continuous with the superficial capsule of con-

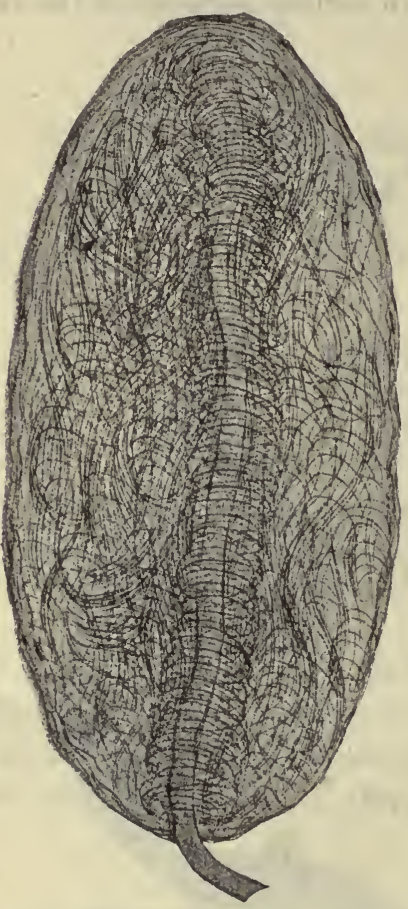

Fig. 186.-A Lameluar Corpuscle in Longitudinal Section, Showing a Network of Spiral Elastic Fibers.

Weigert's elastic tissue stain. Highly magnified. (After Sala.)

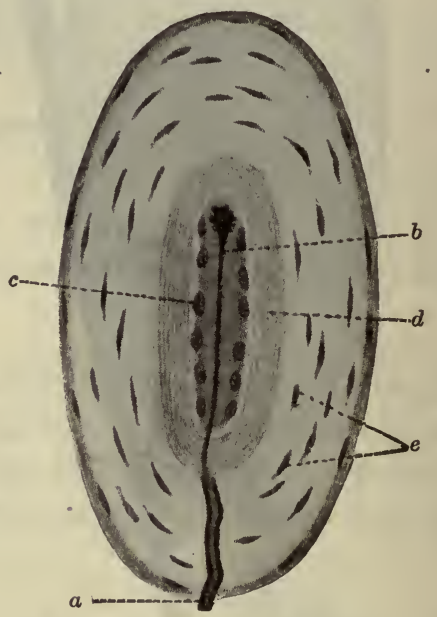

Fig. 187.-Axial Section of a CorPUSCle of Herbst From a DUCK's Tongue.

$a$, medullated nerve fiber; $b$, naked axial nerve fiber with a bulbous end; $c$, nuclei of the core; $d$, inner concentric capsule; $e$, nuclei of the outer lamellated capsule. $\times 380$. (After Sobotta.)

nective tissue. The nerve fiber on entering the core loses its medullary sheath, and after traversing a greater or less portion of the core divides into two to five branches which end near the distal pole in a disk-like expansion. In its course through the core, the nerve fiber gives off fine lateral twigs (Sala, Retzius).

The connective tissue sheath consists of a granular protoplasm which 
is permeated by densely felted spiral fibers (Sala) and is divided into ten to fifty concentric lamellæ by lines of flattened connective tissue cells and fibers. According to Schwalbe, however, these cells form an endothelioid coat on either surface of each lamella. Lamellar corpuscles are occasionally compound, two or more adjacent corpuscles being supplied by branches of the same nerve fiber.

Lamellar corpuscles are found in large numbers in the subcutaneous tissue of the finger tips and of the penis, as well as in the skin of other parts, in the mesentery and the connective tissue of neighboring organs, e.g., the pancreas, in the prevertebral connective tissue of the abdomen and mediastinum, near the walls of the large blood-vessels, in the serous membranes,-pleura, pericardium, peritoneum-in the periarticular connective tissue and periosteum, in the sheaths of the larger nerve trunks, and in the connective tissue of the thyroid gland and of the skeletal muscles.

5. The Corpuscles of Herbst (KeyRetzius Corpuscles).-The corpuscles of Herbst are similar in structure to the lamellar corpuscles except that the core which surrounds the axial nerve fiber contains cuboidal tactile cells. They occur only in the cere of aquatic birds.

\section{The Corpuscles of Grandry} (Merkel's Corpuscles).-The corpuscles of Grandry, also found only in aquatic birds, contain several tactile cells of ectoblastic origin similar to those found in

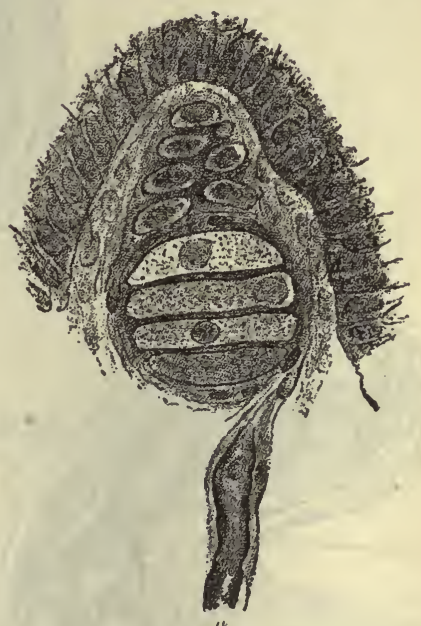

Fig. 188.-A Papilta of THE Duck's Tongue, Containing a Corpuscle of Grandry.

The corpuscle contains four large cells, between which are the tactile menisci of the nerve ending. $n$, nerve. Highly magnified. (After Merkel, from Kölliker.) the epidermis. Each cell is in relation with a ring or meniscus formed by the expanded end of a nerve fiber. The whole is included within a thin connective tissue capsule and may be regarded as a compound tactile cell occurring in connective tissue.

7. The Golgi-Mazzoni Corpuscles.-The Golgi-Mazzoni corpuscles described by Ruffini (Arch. ital. de biol., 1894) somewhat resemble the Pacinian corpuscles in that they possess a lamellar, though relatively very thin, connective tissue sheath and a central granular core. The core, however, is relatively excessive in size, and the entering nerve fiber 
breaks into a number of branches with discoid terminal expansions similar to those found in the nerve endings of Golgi in the tendons. They

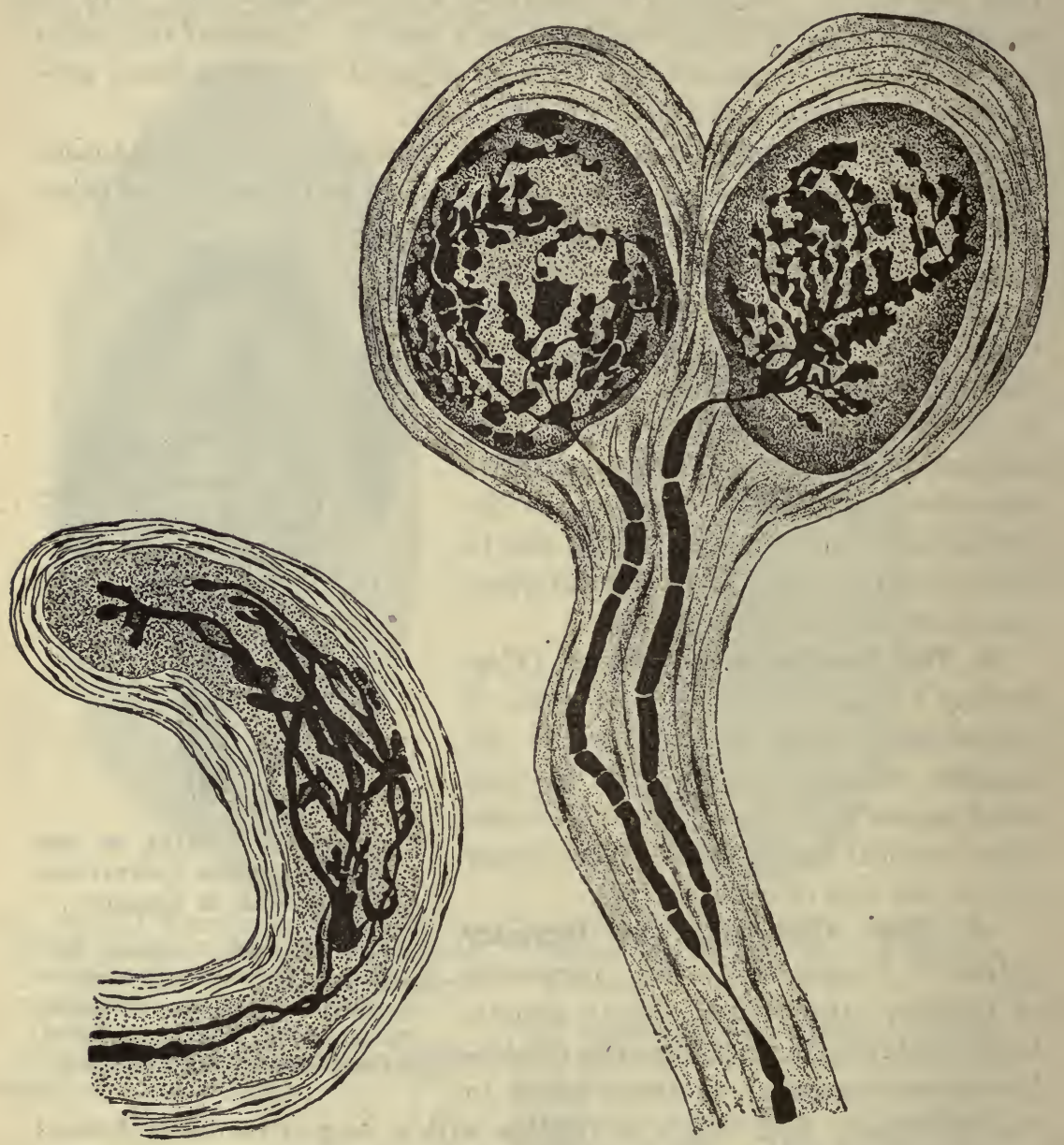

Fig. 189.-Golgi-Mazzoni Corpuscles from the Subcutaneous Tissue of the Tip of the Finger. (After Ruffini.)

occur also in the corium of the skin in certain regions (e.g., the external genitalia, finger tips), and in the conjunctiva and the periosteum. 


\section{NERVE ENDINGS IN MUSCLE AND TENDON}

\section{A. Voluntary Striped Muscle}

1. Motor End Plates.-These organs form the intramuscular endings of motor axons whose cell bodies are found in the ventral horns of the gray matter of the spinal cord. The efferent fibers reach the muscle through the many cerebrospinal nerve trunks. On entering the muscle
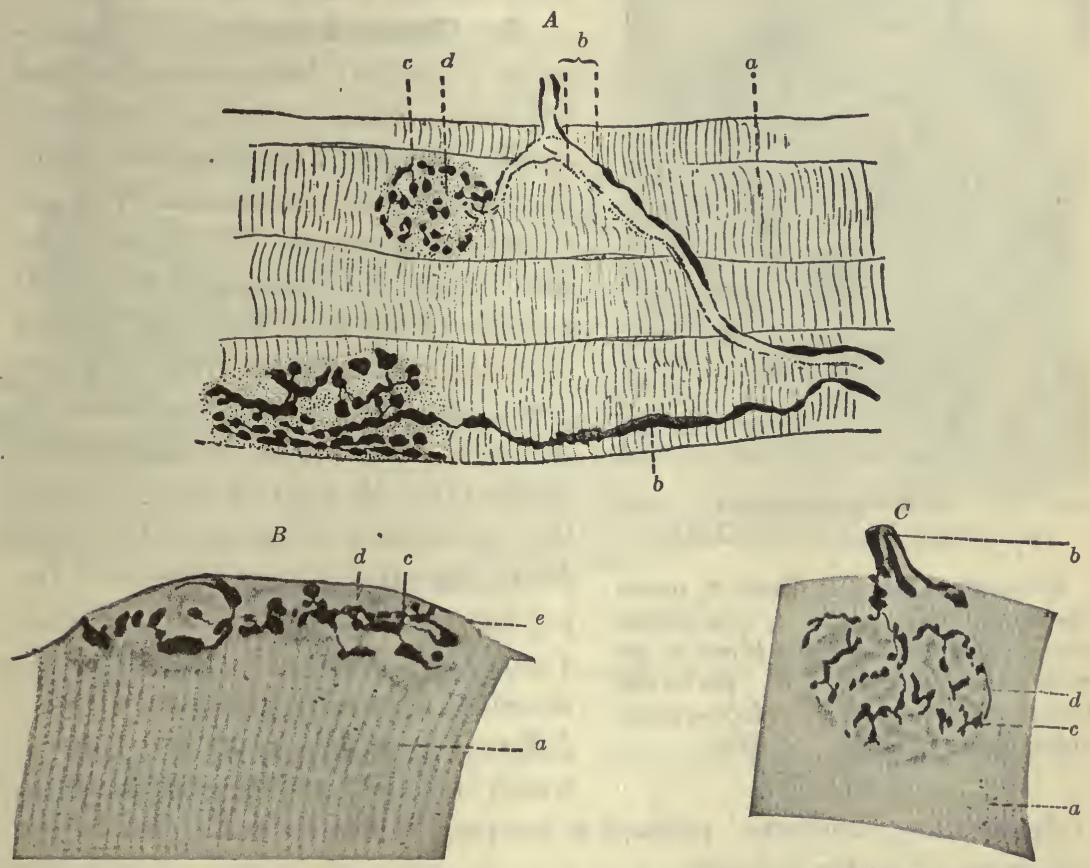

Fig. 190.-Motor Nerve Endings in Striated Muscle.

$A$, from a lizard; $B$, from the guinea-pig; $C$, from the hedgehog. $A$ and $C$ are surface views; in $B$ the end plate is seen in profile. $a$, muscle fiber; $b$, nerve fiber; $c$, nerve ending in the form of a 'brush'; $d$, the sole plate; $e$, sarcolemma. $A, \times 160 ; B, \times 700$; $C, \times 1200$. (After Böhm and von Davidoff.)

these nerves form a plexus in the perimysium from which nerve fibers are distributed within the muscle bundles. Here they form an abundant plexus of branching nerve fibers within the endomysium, the ultimate branches being of sufficient number to supply one or more terminal nerve fibers to each muscle fiber. 
At the surface of the muscle fiber the nerve fiber loses its medullary sheath, its neurolemma becomes continuous with the sarcolemma of the muscle cell, and its naked axis cylinder divides into two to five branches; which end, often after repeated subdivision, in flattened terminal disks, distributed in mammals over a limited, in amphibians over a broad area, but which never completely encircle the cylindrical muscle cell.

The terminal expansions of the axon rest upon a granular, slightly

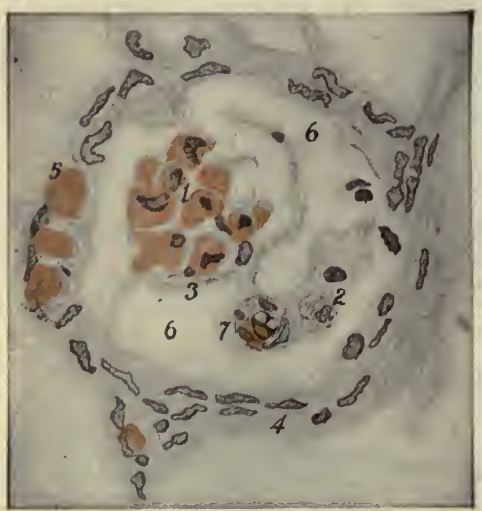

Fig. 191.-A Muscle Spindle from the Psoas Magnus of Man.

1 , intrafusal muscle fibers; 2 , nerve fibers; 3 , axial sheath; 4 , connective tissue capsule; 5 , muscle fibers of an adjacent fasciculus; 6 , peri-axial lymphatic spaces; 7 , blood-vessel. Hematein and eosin. $\times 470$. raised sole plate which contains many ovoid muscle nuclei, the sole nuclei.

2. Muscle Spindles (Neuromuscular Spindles, Neuromuscular End Organs).-These are sensory nerve endings which are concerned with the so-called muscle sense. They are especially numerous in the extrinsic muscles of the tongue, in the small muscles of the hand and foot, and in the intercostal muscles (Huber, Amer. Jour. of Anat., 1902). They have not been found in the muscles of the diaphragm. A detailed description of the developing neuromuscular spindle in the extrinsic eye muscles of the pig has recently been given by Sutton (Am. Jour. Anat., 18, 1, 1915). He describes a coarsely granular 'plaque,' different from both muscle and nerve, which he inclines to regard as an 'intermediary structure,' perhaps a receptor substance analogous to the sole plate of motor endings.

A muscle spindle contains from five to twenty striated muscle fibers of small size, and an almost equal number of nerve fibers. The whole is inclosed within a connective tissue capsule of considerable thickness. The bundle of intrafusal muscle fibers is again surrounded by a delicate axial sheath of connective tissue which is united to the capsule by bands of fine fibrous tissue which span the broad periaxial lymphatic space. The larger of these fibrous bands support the nerve fibers, on their way to the intrafusal muscle cells, together with several small blood-vessels.

The muscle spindles form long fusiform bodies (from 1 to 5 millimeters in length) whose muscle fibers at the pole of the spindle may be 
commected with the teudon, or they may join other muscle fiber bundles. The muscle spindles are usually found in the fibrous septa of the perimysium. Compared with the adjacent muscle fibers, the intrafusal fibers have a smaller diameter, are less distinctly but more coarsely striped, and contain some centrally located nuclei.

Either one or several nerre trunks enter the spindle, usually near its equator rather than at its poles. The nerve fibers branch repeatedly

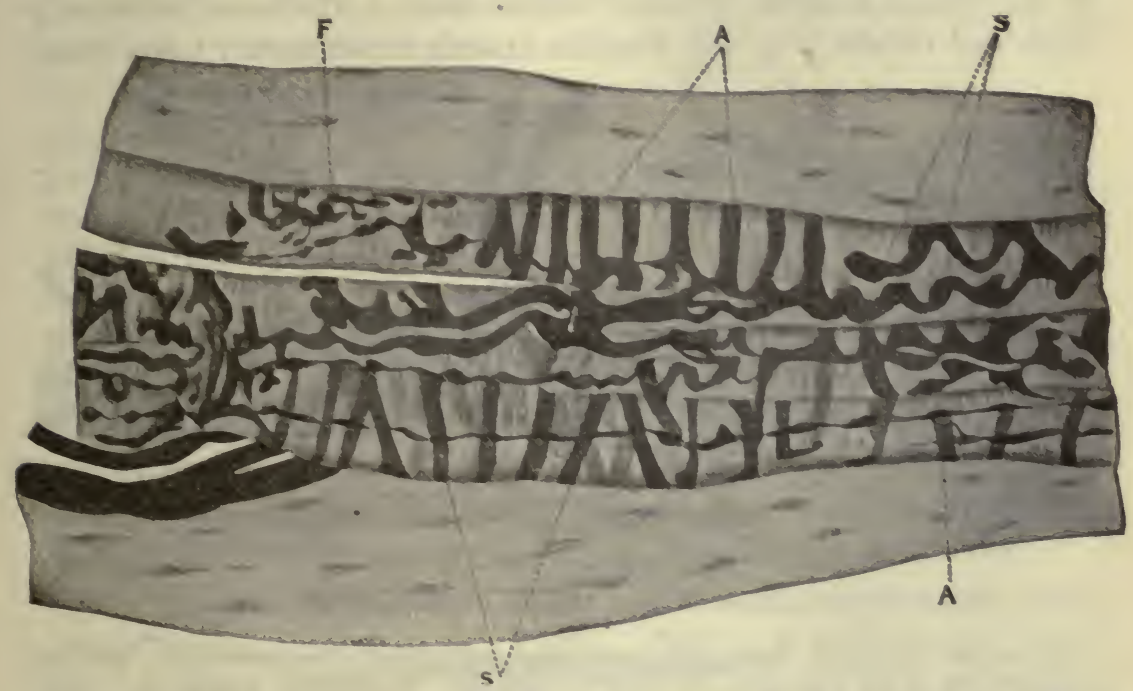

Fig. 192.-Middle Third of a Tervinal Plaque in the Muscle Spindee of an Adult Cat.

$A$, rings; $F$, dendritic branchings; $S$, spirals. Chlorid of gold preparation. Highly magnified. (After Ruftini.)

in the intracapsular connective tissue, and finally pierce the axial sheath as naked processes which form a rich arborization of terminal fibrils about the intrafusal muscle fibers. Ruttini distinguishes three types of terminal nerve fibrils: (1) annular, which form rings around the muscle fibers; (?) spiral, which are spirally twisted about the intrafusal fibers; and (3) dendritic branchings, in which the axons break into numerous irregular processes with laminate expansions.

Motor end-plates for the muscle fibers of the spindle as well as sympathetic vasomotor nerves for its blood-ressels have also been demonstrated within the muscle spindles.

That the muscle spindles are sensory and not motor organs has been 
demonstrated by Sherington (Jour. Physiol., 1894), who found that they were not affected by the muscular atrophy following section of the peripheral motor neurones, and by Horsley ("Brain," 1897) and others who have found that the muscle spindles are unaffected in cases of extreme muscular atrophy in man.

3. Neurotendinous End Organs. (Golgi End Organs, Tendon Spindles).-These organs occur in the tendons of muscles near the junction of the tendon bundles with the muscle fibers. They are fusiform in shape and consist of a thin lamellar capsule of connective tissue which

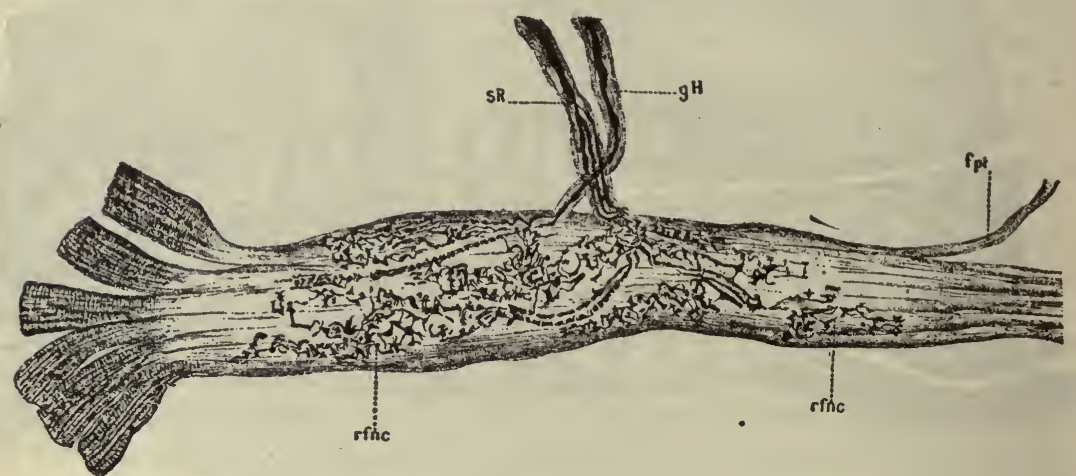

Fig. 193.-Neurotendinous End Organ or Tendon .Spindle of Golgi.

$f p t$, bundle of tendon fibers; $g H$, medullated nerve fiber; $r f n c$, ribbon-like terminal ramifications of the neuraxis; $S R$, node of Ranvier. Moderately magnified. (After Ciacio.)

incloses several intrafusal tendon bundles of dense fibrous tissue. A narrow lymphatic space intervenes between the capsule and the intrafusal tendon bundles.

Nerve fibers enter the spindle and give off several medullated branches which run between the tendon bundles near the axis of the spindle. These finally form naked end fibrils with branching end plates, which surround the tendon bundles in an annular or spiral manner (Ciacio, Arch. ital. de biol., 1891). Since the structure of the Golgi tendon spindles closely resembles that of the muscle spindles, they are probably of similar function.

4. Pacinian Corpuscles and End Bulbs of Krause.-In addition to the special motor and sensory end organs described above, Pacinian corpuscles and end bulbs of Krause are also found in the connective tissue of striated muscles. 
End plates of 'accessory' non-medullated, probably sympathetic, fibers have also been described in striped muscle (Perroncito, Huber and De Witt, and Boecke). Muscle tonus is believed to depend upon this innervation.

\section{B. Cardiac and Syooth Muscle}

The nerves (sympathetic) of the heart are distributed to the cardiac ganglia, whence non-medullated fibers pass to all portions of the organ and form a very rich plexus in the intermuscular connective tissue. Fine terminal fibrils are distributed from this plexus to the muscle fibers, upon whose surface they end in varicose swellings and end knobs. While most of these fibrils are probably motor in function, others which end in the intermuscular connective tissue are

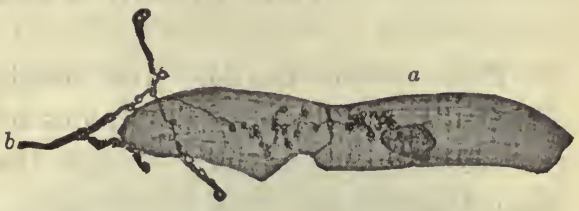

Fig. 194.-Nerve Endings in Cardiac Muscle from the Heart of a Cat.

$a$, muscle cells; $b$, nerve fiber. Methylene blue. Highly magnified. (After Huber and De Witt.) more probably afferent (sensory). Occasional endings in cardiac muscle resemble the simpler motor end organs of skeletal muscle.

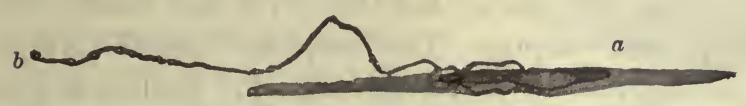

Fig. 195.-Nerve Endings in Smooth Muscle, from the Intestine of a $\mathrm{CA}_{\mathrm{A}}$.

$a$, muscle cell; $b$, nerve fiber. Methylene blue. Highly magnified. (After Huber and De Witt.)

In smooth muscle, plexuses of sympathetic nerve fibers occur in the intervals between the bundles of muscle cells. Secondary plexuses of $\mathrm{n}$ a k e d fibrils are found among the muscle cells, and from this plexus fine lateral fibrils are distributed to the muscle cells, upon whose surface they end in small terminal granules or end knobs. Many of the nerve fibers in smooth muscle are undoubtedly of sensory function.

The nerve endings and the distribution of the peripheral nerve fibers in the various organs of the body are more fully described in the several chapters devoted to those organs. 


\section{CHAPTER VII \\ THE BLOOD VASCULAR SYSTEM}

This system includes the heart, arteries, capillaries, and veins. These structures form a continuous set of branching tubes, which convey the blood from the heart, through the arteries and capillaries, and back again through the veins to the heart. In the capillaries a portion of the blood plasma transudes into the tissue spaces, where it forms the tissue juices, and from which it is returned to the blood by the lymphatic vessels, the terminal branches of which empty into the subclavian veins.

This entire vascular system is completely lined by a single layer of flattened epithelial cells, the endothelium. The cells are united edge to edge by an intercellular cement substance, to form a continuous membrane throughout the entire system. The blood-vessels include the arteries, capillaries, and veins, and these, together with the heart, will form the subject of the present chapter. The lymphatic vessels (lymph vascular system) will be described in connection with the lymphatic system. The blood and lymph vessels together with their contents comprise the vascular tissue.

\section{ARTERIES}

The arteries convey the blood from the heart to all the tissues of the body. They are therefore almost universally present, but vary in size from the aorta down to minute unnamed vessels of microscopic caliber. They are divisible, according to size, into the large, mediumsized, and small arteries, the arterioles, and what may be termed the arterial capillaries, or precapillary arteries. The large arteries include only the aorta and the largest of its immediate branches (innominate, common carotids, subclavians and common iliacs), and the pulmonary artery,--the conducting arteries; the medium sized (distributing) arteries comprise nearly all the remaining named arteries of the body; small arteries, arterioles, and precapillary arteries include those un- 
named arteries which are to be found in nearly all of the organs and tissues of the body.

Medium-sized Arteries.-A medium-sized artery will be first described, as presenting the typical arterial structure. Such a vessel consists of three coats:

1. The internal coat-tunica intima, or interna.

2. The middle coat-tunica media.

3. The external coat-tunica adventitia, or externa.

The internal coat, tunica intima, presents three layers, the innermost being the layer of endothelial cells, the outermost a layer of elastic tissue, the fenestrated coat of Henle, or internal elastic membrane; between these is a delicate fibrous membrane or tunica propria, which constitutes the middle layer. This layer is regarded by some as the product of the endothelium.

The endothelium comprises only a single layer of flattened or squamous cells, placed edge to edge to form a continuous membrane of simple pavementepithelium. These cells are irregularly polygonal in outline, with serrated margins, and are somewhat elongated in the direction of the axis of the vessel. They are loosely

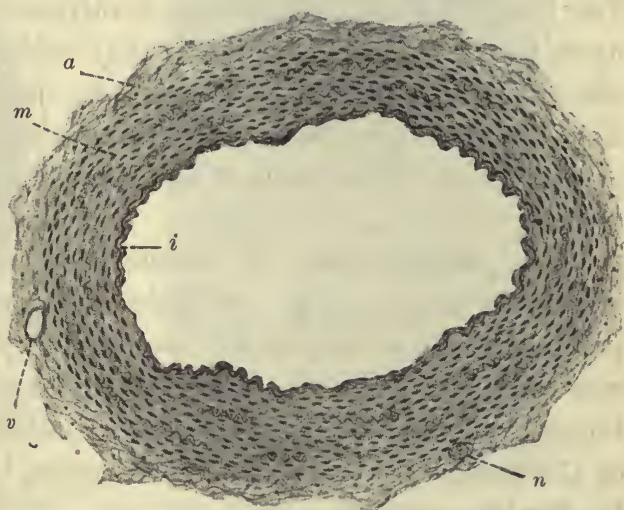

Fig. 196.-A Small Artery from the CoNnective Tissue of the Anterior Cervical REgION OF MAN.

$a$, tunica adventitia; $i$, tunica intima; $m$, tunica media; $n$, a small non-medullated nerve trunk; $v$, a minute venule. Hematein and eosin. $\times 370$. attached to the elastic membrane by the middle layer of fine fibrillar connective tissue, in whose ground substance small branching connective tissue cells are found. The thickness of this connective tissue layer varies proportionately to the size of the vessel. In the largest arteries it increases in amount also with age, becoming especially well developed in the aorta. In the smaller arteries and in certain of the larger, e.g., external iliacs, and the main branches of the abdominal aorta, it is so scant as to be essentially lacking. The thickening of the intima in the aorta coincident with increasing age is commonly interpreted as a compensatory mechanism necessitated by the increasing diameter of the vessel 
due to loss of elasticity resulting from a transformation of elastin into elacin.

The internal elastic membrane is a layer of elastic tissue, consisting of an intimately united fibrous mass, which completely encircles the artery. In the smaller vessels the elastic fibers of this layer form only a reticulated structure, but in the larger arteries they are so abundant and so closely interwoven as to form a complete membrane, which can be readily stripped from the subjacent tissue. If the membrane thus prepared is examined microscopically, it will be found to present numerous small openings at points where the elastic tissue is deficient. It is this appearance which led to its description as a 'fenestrated membrane.' The internal elastic membrane is intimately united to the tunica media, upon which it rests; in fact, it may perhaps be better considered as the innermost layer of this tunic, for, in the larger arteries, e.g., the aorta, it can only with difficulty be distinguished from the adjacent layers of elastic tissue which form a large portion of the tunica media of these vessels.

The tunica media, or middle coat, contains smooth muscle, sheets of elastic tissue, and a very delicate fibrous connective tissue. The proportion of these elements present in any given artery varies with the size of the vessel. Muscular tissue usually predominates, but in the larger arteries elastic tissue is so abundant as to appear quite in excess of the muscular; in the smaller arteries, however, the muscular tissue is by far the more abundant.

The smooth muscle fibers are circularly disposed in the wall of the vessel; they are short, of irregularly serrated outline, and are intimately united with one another. Quite frequently the muscle fibers possess short branches which interdigitate with those of neighboring fibers. In the larger vessels they are arranged in layers which alternate with the sheets of elastic tissue. Small bundles of longitudinal smooth muscle fibers are occasionally found in the outer portion of the tunica media.

The elastic tissue of the middle coat is disposed in membranous sheets which, in the larger vessels, are embedded in a fine fibrillar connective tissue. In these vessels, also, the fibro-elastic membranes thus formed alternate with the layers of smooth muscle, throughout the entire thickness of the tunica media. In consequence of the relaxation of the normal arterial tone and the contraction of the muscular wall in rigor mortis, as seen in the usual preparations, these elastic layers, as well as the internal elastic membrane, are thrown into wavy folds.

The external coat, tunica adventitia, consists chiefly of fibrous con- 
nective tissue. Relatively few elastic fibers occur in this coat, and these for the most part lie in its inner portion, adjoining the tunica media. In the larger arteries, when especially abundant, the elastic fibers form an incomplete layer, which may be termed the external elastic membrane. Like the internal elastic membrane, this layer might well be considered as

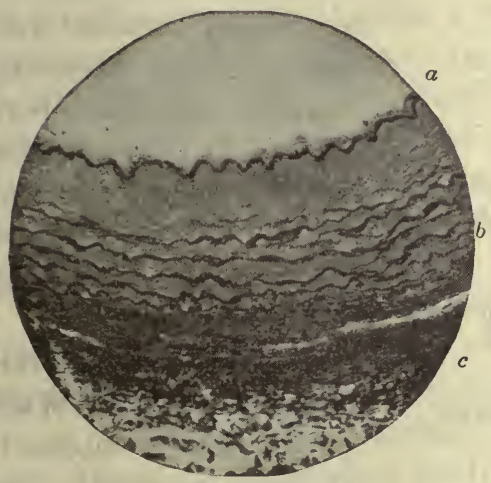

Fig. 197.-The External Carotid Artery of a Child.

$a$, tunica intima, the internal elastic membrane is prominent; $b$, tunica media, containing smooth muscle and several wavy layers of elastic tissue; $c$, tunica adventitia, containing many transversely and obliquely cut elastic fibers and much wavy connective tissue. Photo. (After Magrath.)

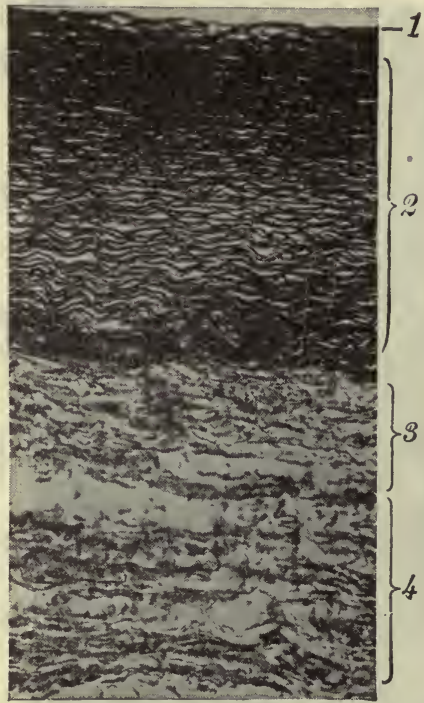

Fig. 198.-Transection of the Wall of the Aorta of a Child.

The elastic tissue is deeply stained. 1, tunica intima; 2, tunica media, 3 , tunica adventitia; 4, areolar connective tissue. Weigert's elastic tissue stain and picro-fuchsin. Photo. $\times 64$.

belonging to the tunica media, of which coat it would then form the outermost stratum.

The collagenous fibers of the tunica adventitia are disposed in dense interlacing bundles, to form a firm, unyielding coat. At the periphery of the artery the connective tissue bundles of the adventitia intermingle with those of the adjacent areolar connective tissue, in which the bloodvessels are nearly always embedded, hence the outer boundary of this coat is usually more or less ill defined.

The fibrou's bundles of the adventitia are disposed somewhat obliquely or diagonally about the artery, thus forming a closely felted connective 
tissue network. Small nutrient blood-vessels, both arteries and veins (vasa vasorum), and minute nerve trunks with occasional ganglia, occur in this coat. From these vasa et nervi vasorum capillaries and fine nerve fibers, both sensory and autonomic vasomotor, are distributed to the mus-

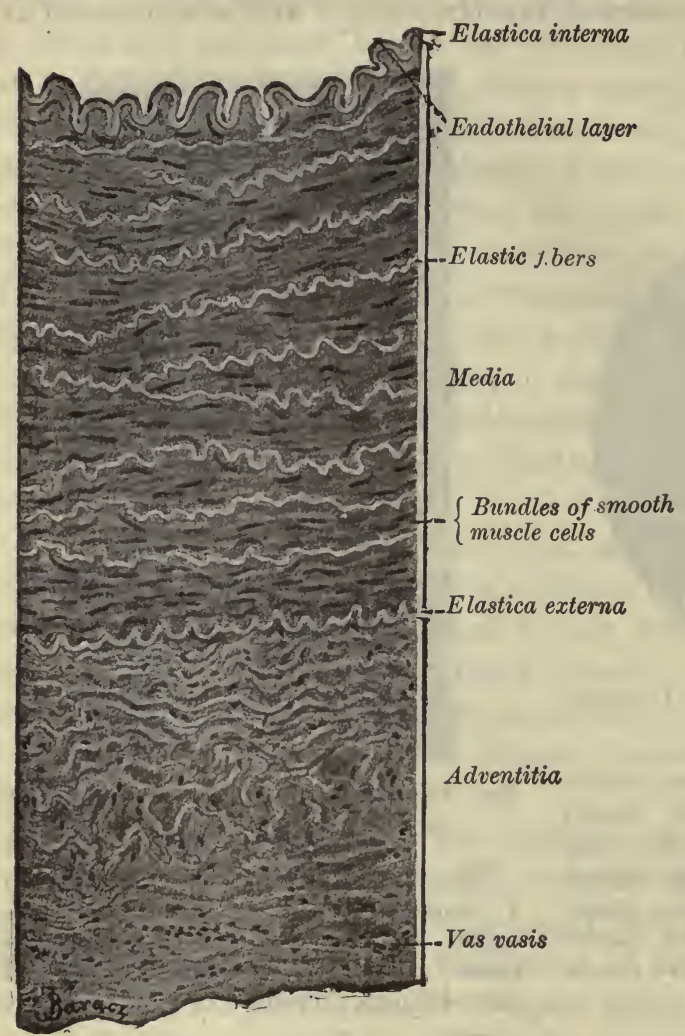

Fig. 199.-Part of a Cross-section of the Femoral Artery of a Dog. $\times 150$. (From Szymonowicz-MacCallum, "Histology and Microscopic Anatomy.") cular coat. No bloodvessels are found in the tunica intima. In the larger vessels the adventitia may contain also an occasional lamellar corpuscle. The adventitia contains abundant perivascular lymphatics. Nervi vasorum are said to be lacking in the blood-vessels of the brain and spinal cord.

\section{General Characteris- tics of the Arterial Wall.} -The tunica media is almost invariably the thickest of the arterial coats. In the mediumsized vessels, e.g., the iliac arteries, the adventitia is often of nearly equal thickness, but in the smaller vessels it is much thinner. The arterial wall as a whole, also, is very thick as compared with the lumen of the vessel, and is much

thicker than that of a vein of corresponding size.

The wall of the larger arteries is relatively thinner as compared with the lumen than is the case with the arterioles; in the latter vessels the thickness of the arterial wall often exceeds the diameter of their lumen. In certain small arteries, e.g., those of the liver, even this ratio may be exceeded.

The arterial wall contracts firmly in rigor mortis, hence the arteries 
after death contain but little blood, and because of the density of the tissues which compose their wall, these vessels retain, as a rule, their cylindrical form.

Large Arteries.-The largest arteries differ from the medium-sized type in the excess of elastic tissue and relative deficiency of muscle in their media, the extreme thinness of their adventitia, and the relative thinness of their wall, as a whole, when compared with their lumen. Elastic tissue is especially abundant in all of these vessels; in the media it equals-in volume the muscular tissue, in the adventitia it forms a dense network of elastic fibers. In the aorta and the pulmonary artery the elastic tissue surpasses the muscular in the media. These vessels lack a distinct internal and external elastic membrane.

The adventitia of the largest arteries is extremely thin, that of the thoracic aorta being not much thicker than its fibrous tunica intima; this coat, therefore, forms but a small portion of the vascular wall in vessels of this type.

Small Arteries.-In the small arteries the elastic tissue is relatively decreased and the smooth muscle noticeably increased. The tunica intima

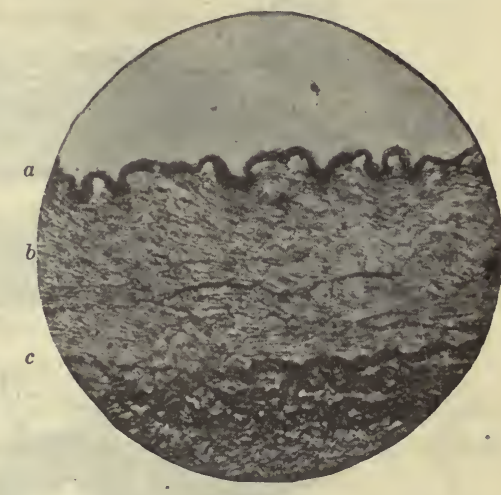

Fig. 200.-Transection of the Celiac Axis of Man.

$a$, tunica intima with a prominent internal elastic membrane; $b$, tunica media, consisting chiefly of smooth muscle; $c$, external elastic membrane in the inner portion of the tunica adventitia: Photo. (After Magrath.) of these vessels is thin, and is limited externally by an internal elastic membrane, which stands out prominently because of the relative deficiency of elastic tissue in the tunica media.

In the tunica media of these vessels the plates of elastic tissue which characterize the larger arteries are scarcely to be found. This coat in the small arteries contains very little tissue other than smooth muscle.

The external elastic membrane is indistinct, and the adventitia is not more than one-half to two-thirds as thick as the tunica media.

Arterioles.-The arterioles possess a relatively thicker wall than any other vessel of the arterial system. Their tunica intima is thin, but little fibrous tissue being contained within it, and the internal elastic membrane is represented only by a very incomplete layer of elastic fibers. 
The tunica media of the arteriole forms two-thirds to three-fourths of its wall, and consists almost entirèly of firmly united smooth muscle fibers. The adventitia, much thinner than the media, contains bundles of white fibers and delicate interlacing elastic fibrils.

Precapillary Arteries.-The smallest arterioles pass into what may be termed the precapillary arteries. In these minute vessels the wall consists of scarcely more than the endothelial lining, about which is an incomplete layer of circular muscle fibers, interspersed with occasional

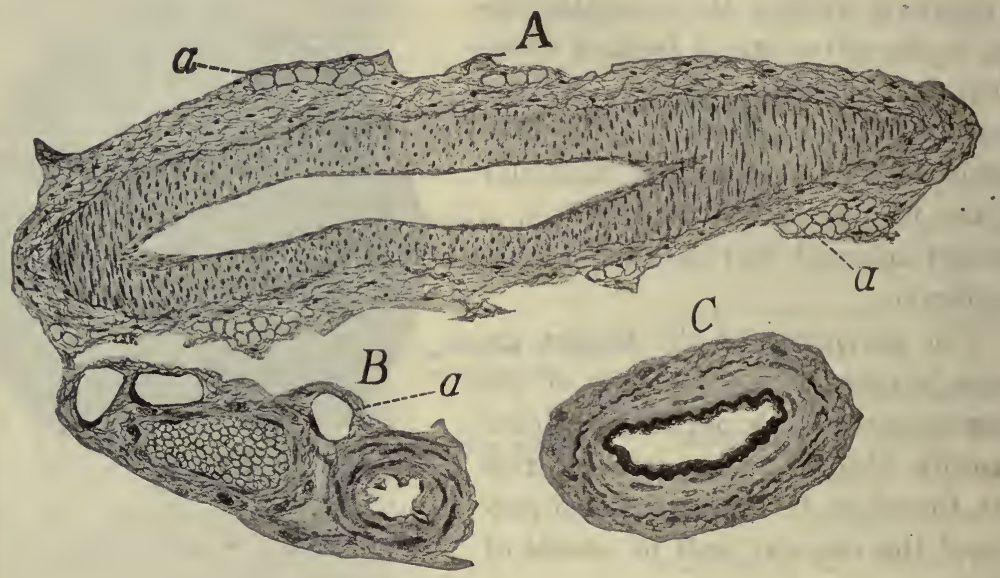

Fig. 201.-A Group of Simall Blood-vessels.

$A$, small artery obliquely cut; $B$, arteriole and venule, the latter filled with blood; $a$, fat cells. $A$ and $B$ are from the connective tissue of the anterior cervical region. Hematein and eosin. $A, \times 110 ; B, \times 550$. $C$, a small artery near the descending aorta of man; the internal and external elastic membranes are rendered distinct by the stain. Hematein, Weigert's elastic tissue stain, and picro-fuchsin. $\times 550$.

collagenous and elastic fibers. On approaching the capillaries the endothelial tube is gradually laid bare. It is the smooth muscle which is the last of the tissues to disappear from the arterial wall, whereas beyond the capillaries it is the fibrous tissues which are first added to the endothelial tube to form the wall of the smallest venules (Fig. 20\%).

Atypical Arteries.-Certain atypical arteries differ markedly from the typical structure above described.

The umbilical arteries are almost exclusively muscular, and practically lack elastic tissue. The muscle is arranged in two distinct layers: an inner longitudinal, and a wide outer circular; external to these is usually a 
more or less complete third layer of scattered bundles of longitudinally arranged smooth muscle cells. The umbilical vein is very similar but eontains more elastic fibers, and a distinct internal elastic membrane.

The cerebral and meningeal arteries have very thin walls and, exclusive of a relatively very well developed internal elastic membrane, contain but little elastic tissue.

The iliac, splenic, renal, superior mesenteric and dorsalis penis contain scattered longitudinal bundles of muscle in the media next the intima.

In the pulmonary arteries the media is exceptionally well developed. This is the case to an extreme degree in the pulmonary arterioles of the cat. The pulmonary arteries and veins are very similar in structure. In the guinea pig and opossum the media of the arterioles consists throughout of thick oval segments of circularly disposed smooth muscle

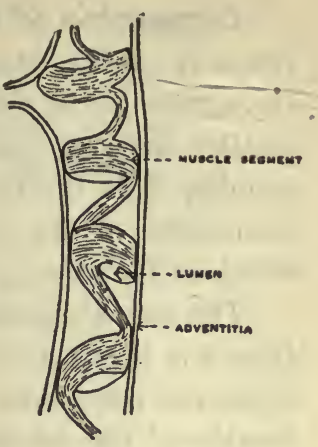

Fig. 202.-SEMI-DiAGRAMMatic IllusTRATION OF SMALL Branch of PUlMoNARY ARTERY OF Ox.

(After Piana.) $\times 70$. alternating with narrow intervals where the muscle layer is relatively thin. In ox, sheep and pig such segmented condition of the media is modified in that the segmentation is spirally disposed.

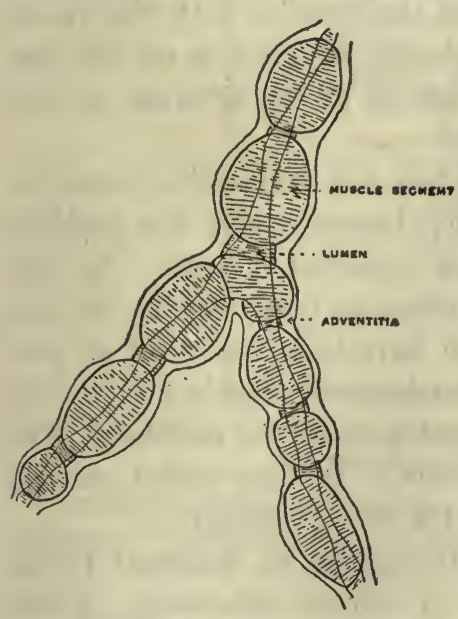

Fig. 203.-Semi-diagrammatic

IlLUSTRATION OF Dividing Small Branch of Pulmonary Artery of GUinea-PIG.

Pulmonary arterioles of opossum are almost identical. $\times 50$.

The media of the roots of the aorta and the pulmonary artery consists largely of cardiac muscle.

In the subclavian artery the longitudinal surpasses the circular muscle in the media. In the arch of the aorta and in the upper portion of the descending aorta longitudinal -muscle bundles are found in the intima, media and adventitia (von Bardeleben). The common carotid, common iliac and common femoral (cruralis) contain both longitudinally and spirally arranged muscle fibers in the media. In general, where large arteries are subjected to bendings the circular muscle fibers are reinforced by oblique (spiral) and longitudinal bundles in the media. This is conspicuously the case in the common iliacs, the popliteal, and the brachial arteries (MacCordick, Anat. Anz., 44, 11, 1913). 
Comparison of Large and Small Arteries.-The larger arteries are typically elastic, the smaller typically muscular. In the larger vessels the elastic tissue forms about one-half of the entire wall; toward the smaller arteries this tissue progressively diminishes until, in the arterioles, it is limited to an incomplete internal elastic membrane, the homologue of the complete elastic coat or fenestrated coat of Henle, which is found only in larger vessels.

The smooth muscle, on the other hand, increases in relative amount from the larger to the smaller arteries. While in the largest vessels it forms not more than one-third, in the arterioles it represents about threefourths of the arterial wall.

In the largest arteries the adventitia is relatively very thin. That of the medium-sized vessels is much thicker, and the ratio of connective tissue as found in the wall of these vessels remains fairly constant down to the arterioles. In the wall of the precapillary arteries connective tissue is very scanty.

\section{CAPILLARIES}

The capillaries are minute tubes, $\dot{5}$ to $13 \mu$ in diameter, which, in nearly all the tissues of the body, connect the arteries with the veins. Their wall is formed by a layer of endothelial cells which on the one hand is continuous with the endothelial lining of the arteries, on the other hand with that of the veins.

As a rule there are neither muscle fibers nor connective tissue in the wall of the true capillaries; occasionally, however, very fine isolated circumferential elastic fibers encircle the endothelial tube. In the minute arterioles and venules, which are about to terminate in or take origin from the true capillaries and which have been described as precapillary arterioles and venules, a very thin layer of muscle fibers or of connective tissue is added to the endothelial wall of the capillary. On the arterial side the muscle is the first tissue to be thus added, on the venous side the fibrous connective tissue is the first to appear.

The endothelium of the capillary wall consists of flattened platelike cells which are joined edge to edge by cement substance. These cells are somewhat elongated in the axis of the vessel, the shape of the cell, as in the arteries and veins, depending upon the size of the vessel, - the smaller the vessel the more elongated its endothelial cells. The margins of these cells are extremely irregular, hence they present a wavy or serrated outline. 


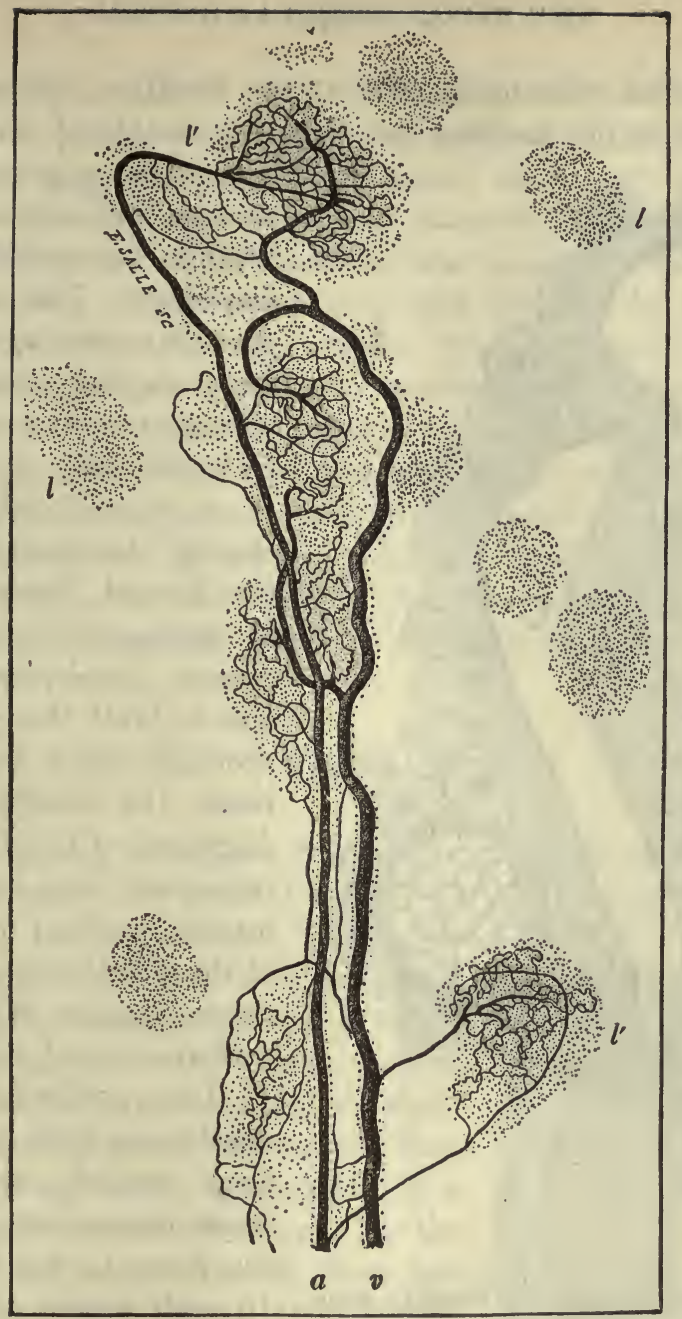

Fig. 204.-The Capillary Network Connecting an Arteriole and Venule of the Omentum of a Young Rabbit.

The blood-vessels have been injected. The discolorations at $l$ and $l$ are due to the presence of lacteals beneath the endothelium; at $l^{\prime}$ and $l^{\prime}$ these are surrounded by the capillary network. $a$, arteriole; $v$, venule. Considerably magnified. (After Ranvier.)

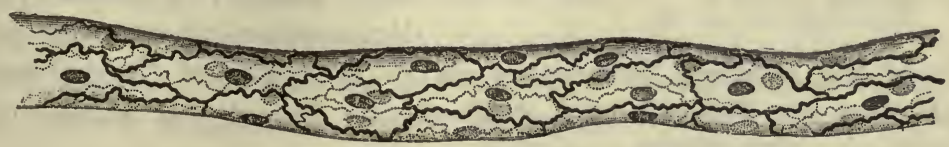

Fig. 205.-Capillary Vessel of the Frog's Mesentery.

Treated with nitrate of silver to show the outlines of the endothelial cells. Highly magnified. (After Ranvier.) 
Although the endothelial cells of the capillary wall appear to be firmly united to one another, yet they are capable of being separated

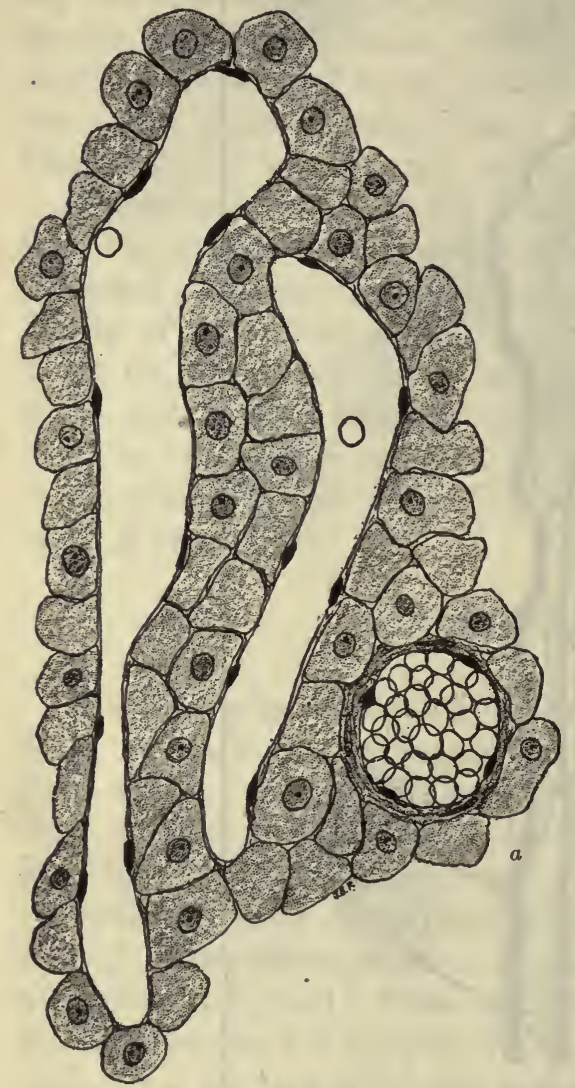

Fig. 206.-Two Sindsoidal Vessels from the Medulla of the Human Adrenal.

Each contains the outline of a single red blood corpuscle for comparison of size. At $a$, a small vein is shown; it is filled with blood and possesses a much thicker wall than that of the sinusoids. Hematein and eosin. $\times 410$. sufficiently to permit the ready passage of white blood-cells through the capillary wall, by diapedesis. The capillary wall does not appear to be an inactive factor in this process, for inert pigment granules may also penetrate the wall of these vessels, the endothelial cells immediately closing the aperture which is thus formed. Nevertheless, purely mechanical means, e.g., increased blood-pressure, appear also to favor this process. The openings which are formed between the endothelial cells by diapedesis of blood-cells are very transitory; they are almost immediately closed by the activity of the endothelium. Such transitory breeches of the capillary wall are termed stigmata.

The capillaries branch and anastomose with one another to form networks, the outlines of whose meshes vary according to the tissue in which they occur. In such tissues as muscle and $\mathrm{nerve}$ they form elongated meshes whose long axes are parallel to those of the muscle or nerve fibers; in the looser, more areolar tissues they form large meshes of irregular form; while in the capillary membranes, as in the walls of the pulmonary alveoli, they are disposed in a close net, the diameter of whose meshes scarcely exceeds that of the capillaries.

With but few exceptions capillaries occur in all the tissues of the 
body. In epithelium and in cartilage there are no blood-vessels of any kind, and in the splenic pulp it is doubtful if true capillaries occur. In certain tissues large vascular spaces occur, which are comparable to the capillaries in that their wall consists of scarcely more than the endothelial tube, but which differ from the true capillaries in the extreme size of their lumen. These vessels have been described by Minot (Jour. Bost. Soc. of Med. Sc., 1900) as sinusoids. They are found in the erectile tissues, adrenals; coccygeal gland, parathyroids, in the maternal placenta, and in the fetal liver, heart, pronephros, and mesonephros. They differ from capillaries also in that they generally do not connect arteries and veins, but are either exclusively arterial or venous. In the adult only venous sinusoids occur. Retia mirabilia are capillary plexuses on arterioles or venules; the best example of a rete mirabile in the human body is the arterial capillary plexus on the efferent glomerular arteriole of the kidney.

\section{VEINS}

The blood having passed the capillaries, enters the smallest radicals of the venous system, the precapillary venules, and passes thence through the venules to the larger veins. The progressive increase in the caliber of these successive vessels is accompanied by a corresponding increase in the thickness of their wall. Thus, while the endothelial tube alone composes the capillary wall, the endothelium of the precapillary venule is encircled by a delicate connective tissue membrane. In the venule occasional smooth muscle fibers are added to the wall of the smaller vessel, and in the vessels of this caliber the fibrous tissues have been so increased that the vascular wall, as in the artery, can be said to possess three coats.

Precapillary Venules.-The wall of the precapillary venule consists of the endothelial lining, which is surrounded by a very delicate connective tissue membrane in which are very few elastic and white fibers.
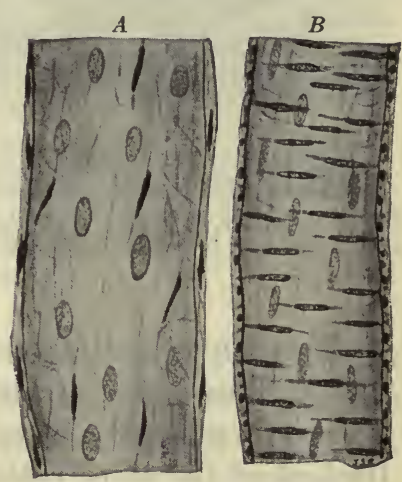

Fig. 207. - Precapillary VenUle AND Arteriole.

The lighter nuclei are those of the endothelium. The darker nuclei in the venule are in connective tissue cells; in the arteriole they are in the muscle cells. $A$, venule; $B$, arteriole. Partly diagrammatic. Highly magnified. 
Venules.-In the venule the tunica intima consists of little more than the endothelial lining. Its media and adventitia are not as yet distinctly differentiated, the former being distinguished only by the incomplete layer of circularly disposed smooth muscular fibers. The extremely thin adventitia is composed almost wholly of white fibers, the greater part of which are circularly disposed. Very few elastic fibers occur even in vessels of this size.

Small Veins.-In the small veins the three coats are fairly distinct, the vascular wall being, however, much thinner than in the artery of corresponding size.

The endothelium of the tunica intima is supported by a very delicate connective tissue membrane which as yet contains but few elastic fibers.

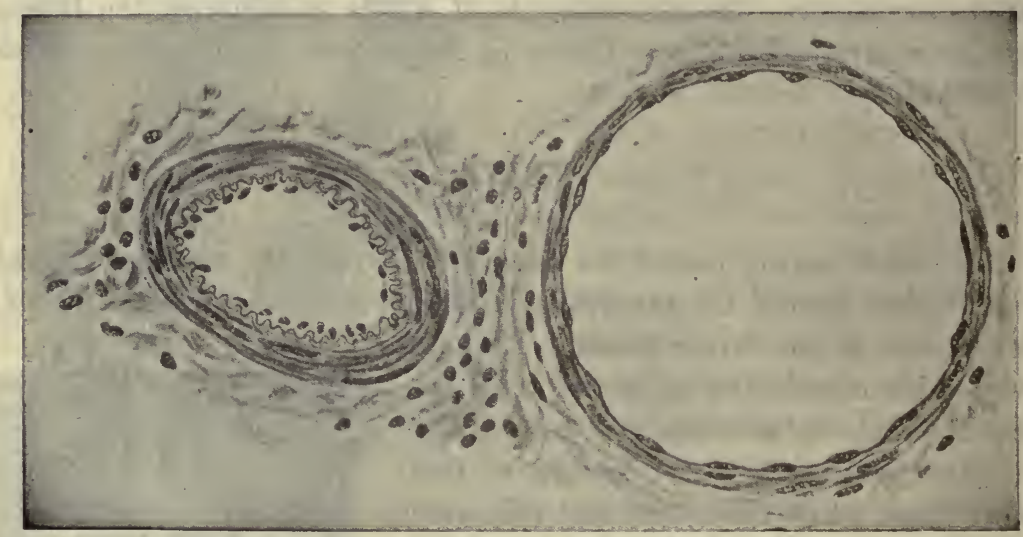

Fig. 208.-Transection of an Arteriore and Venule. $\times 250$. (After Schäfer.)

The tunica media consists of a thin layer of circularly arranged smooth muscle fibers intermingled with a delicate fibrous tissue; elastic fibers are relatively scarce. The adventitia, though considerably the thickest of the three coats, is as yet a thin membrane. It consists of fibrous connective tissue, elastic fibers being scarcely demonstrable except by means of the specific stains for this tissue.

Larger Veins.-The wall of the larger veins closely resembles that of the corresponding artery, except that the venous wall is much thinner and contains far less elastic tissue. The tunica intima of the medium and $\operatorname{lar}_{\check{C}}$ e veins presents a lining endothelium, a thin layer of delicate connective tissue fibers, and an incomplete internal elastic membrane. The last named is never so prominent as in the artery. 
The tunica media contains smooth muscle fibers, the most of which are circularly arranged. A somewhat smaller proportion of delicate connective tissue completes this coat.

The media is best developed in veins of the lower extremities; it forms a thinner layer in veins of the upper extremities, and is relatively scant in the large veins of the abdominal cavity.

The adventitia of the larger veins consists of interlacing bundles of dense white fibers, among which is a network of fine elastic fibers. Occa-

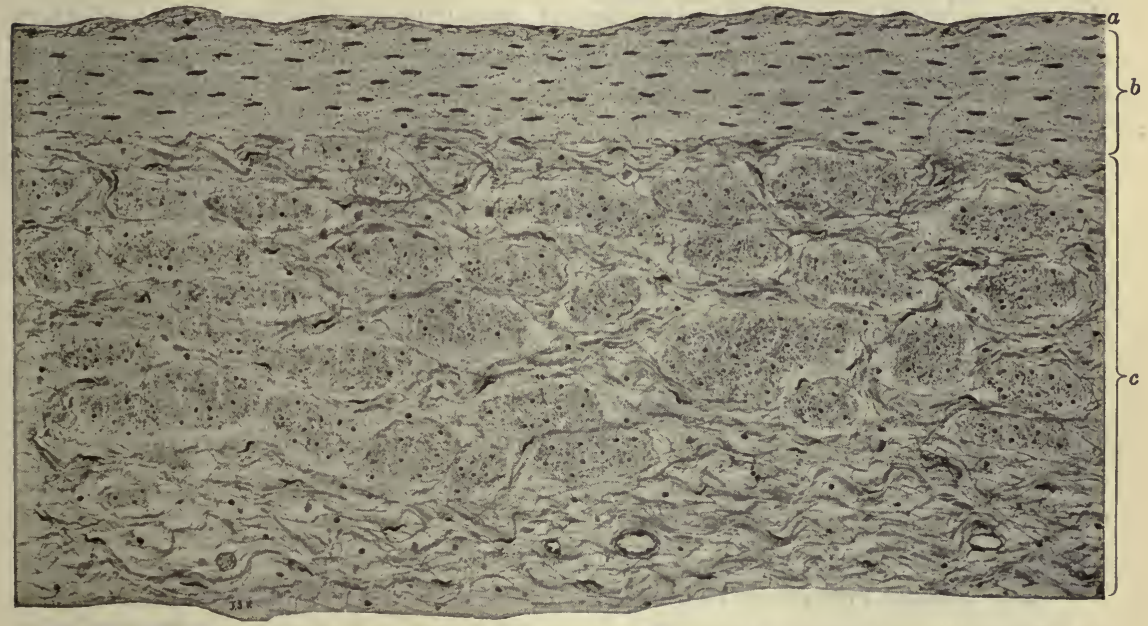

Fig. 209.- Transection of the Wall of the Human Vena Cava.

$a$, tunica intima; $b$, tunica media; $c$, tunica adventitia. The inner portion of the tunica adventitia contains numerous bundles of longitudinal smooth muscle fibers which have been cut across. Hematein and eosin. $\times 90$.

sional small bundles of longitudinal smooth muscle fibers occur in the adventitia of the largest veins. In these vessels also, a very incomplete external elastic membrane may be demonstrated by the specific stains for elastic tissue.

Nerve fibers and minute blood-vessels, vasa vasorum, occur in this coat and distribute their terminal branches to the two outer coats of the vessel. The intima of the vein, as in the artery, is non-vascular. The venæ vasorum of veins empty directly into the lumen.

Atypical Veins. - In certain tissues the veins present noticeable departures from the typical structure. Longitudinal muscle fibers are found in many of the larger veins of the abdominal and thoracic cavities. 
The cephalic, basilic, mesenteric, iliac, femoral, saphenous, uterine and the dorsalis penis veins contain small longitudinal bundles in the intima. Certain veins, e.g., saphenous, femoral, and popliteal, contain a layer of longitudinal muscle in the intimal portion of the media.

The adrenal veins contain, almost exclusively, longitudinal muscle fibers, and in the renal, suprarenal, portal, splenic and phrenic veins and the inferior vena cava these fibers form the greater portion of the tunica adventitia.

In the pulmonary veins the circular muscle fibers are highly developed, the tunica media of these veins almost equaling in thickness that of the corresponding pulmonary artery. As in other large veins, however, elastic tissue is notably deficient in the tunica media of the pulmonary veins. The muscle of the roots is partially of the cardiac type.

The tunica media of the largest veins, e.g., the venæ cavæ, jugular, innominate and subclavian, contain much fibrous and considerable elastic tissue, the latter often forming incomplete membranous layers, which alternate with the muscle, as in the arteries. Such structure is, however, limited to the very largest of the veins. In the superior vena cava and the hepatic vein the media is practically replaced by adventitia.

The cranial veins (cerebral and meningeal) are conspicuous for the almost entire absence of muscle from their walls, the large meningeal sinuses being surrounded by a dense fibrous coat derived from the dura mater, and lined by the usual endothelium. In the veins of the retina also, and those of bones, a media is essentially lacking.

The venous spaces of the erectile tissues have already been mentioned as presenting to some extent the sinusoidal type of structure, these large venous cavities possessing an extremely thin wall, in structure scarcely more than endothelial lining. The afferent artery projects into the broad vascular lumen, from which the efferent vein makes its exit.

\section{Comparison of the Larger and the Smaller Veins.-Comparing} the larger with the smaller veins, the excess of elastic and muscular tissue in the former is most noticeable. In the absence of specific stains, elastic tissue can scarcely be recognized in the venules and smaller veins. In the medium-sized vessels it is scanty, but is present in considerable quantity in the largest vessels.

The precapillary veins and venules contain scarcely any smooth muscle. This tissue becomes more distinct in the small veins and steadily increases. proportionately to the size of the vessel; in the largest veins it is again relatively deficient.

Comparison of the Vein with the Artery of Corresponding Size. -The lumen of any given artery is always much smaller than the total 
lumen of its venæ comites (usually two in the case of the smaller arteries, one vena comes in the case of the medium-sized), the ratio being about one to three. Hence, of any two vessels in close proximity to each other, the vein would more likely possess the larger caliber; the artery, on the other hand, would have the thicker wall.

As compared with the arteries, the veins are notably deficient in elastic and muscular tissue. In the wall of most veins the white fibrous is in excess of all other tissues. For this reason the adventitia is almost invariably the thickest of the three coats of the vein, whereas in the artery the media is always the thickest coat.

The internal elastic membrane, which can be readily recognized even in the smaller arteries, is limited to the large veins. Alternating layers of elastic and muscular tissues are to be seen even in the medium-sized arteries, but this arrangement is likewise confined to the largest of the veins.

The wall of the vein as a whole is much thinner in proportion to its lumen than that of the corresponding artery; it is also less rigid. For this reason the wall of the vein is much more likely to collapse after death than is the thicker and more rigid arterial wall. Because of the preponderance of muscle in the wall of the artery its contraction in rigor mortis is more powerful than that of the vein; the vein therefore is apt to be distended with blood while the artery contains but little. A certain number of blood-cells can usually be found in almost any type of blood-vessel.

Valves occur at intervals of considerable length along the course of the larger veins. These are not found in the arteries. Each valve consists of one, usually two, and occasionally more crescentic folds or reduplications of the tunica intima between which is a slightly increased amount of connective tissue, the elastic fibers of which are more abundant on the side next the lumen. The valves therefore are suspended free in the lumen of the vessel and are covered on either side with a layer of endothelium which is continuous with that lining the vein.

The valves open with and close against the blood current. They occur generally distal to the point of entrance of venous tributaries. They are more abundant in the veins of the extremities and are lacking in the superior and inferior venæ cavæ, in the hepatic, portal, renal, uterine, pulmonary, umbilical, cerebral and meningeal veins, in the veins of bones, and in veins of less than 2 millimeters diameter. They obviously assist the flow of blood to the heart against the influence of gravity. The general absence of valves in the veins of the abdomen and thorax, and their 
abundance in the veins of the extremities, especially the lower, is probably to be interpreted in terms of a quadrupedal ancestral condition.

The fact should be borne in mind that it is because of their relative infrequency that valves are not often met with in those transections of the smaller veins which are seen in nearly all microscopical preparations.

\section{THE DEVELOPMENT OF BLOOD-VESSELS}

The earliest anlage of the blood vascular system is a mesenchymalike layer, the angioblast, which appears between the entoderm and mesoderm at the distal pole of the yolk-sac (Fig. 210) at a very early stage of the embryonic development (1 millimeter, Minot). In this layer appear accu-

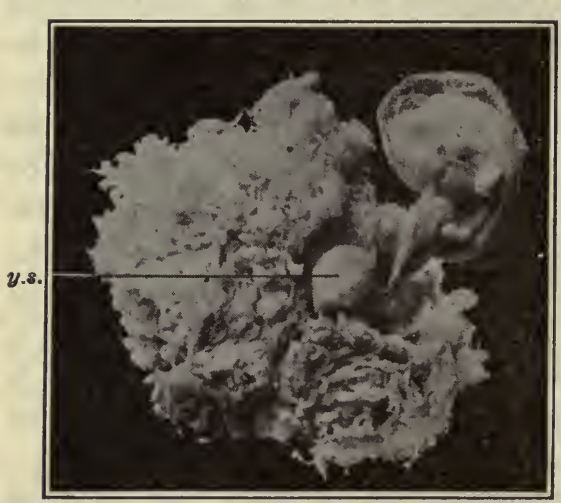

Fig. 210.-A 13 mm. Human Embryo.

The chorionic vesicle is cut open, revealing the embryo enveloped in the amnion, and the yolk-sac (y.s.). $\times 1 \frac{1}{2}$. mulations of rounded cells in the form of anastomosing irregular solid cords. The peripheral cells become flattened to form an endothelial tube; the central cells separate and scatter in the vessels as primordial blood-cells floating in a plasma, probably a secretion product of the cells. This network of primitive blood-vessels grows toward the embryo in the shape of tubes and solid nucleated sprouts (angioblast cords, Bremer) and, converging to form two large vessels, invades the tissue of the embryo in the region of the developing heart as the vitelline veins. Subsequently other vessels, both arteries and veins, appear in the embryo. Such vessels are preceded by capillary plexuses, as demonstrated by Evans (Anat. Rec., 3, 9, 1909), in which the definitive vessels arise as paths in the original network selected, enlarged, and modified under the influence of mechanical factors incident to the flow of the main stream of the blood. There can be no doubt that the original anlages of the blood-vessels arise by a confluence of separate spaces (angiocysts),--possibly always connected by angioblast cords, - and tubes formed in the angioblast; likewise there is no doubt that the embryonic blood-vessels sprout as tubes and solid cords and thus grow into adjacent regions (Fig. 211). But the features of vasculogenesis concerning which there remain decided differences of opinion are (1) the nature and origin of the angioblast, that is whether of mesodermal, ento- 
dermal or of dual origin; (2) the manner of origin of the primary vascular stems in the embryo, whether by invasion through growth from the extraembryonic primitive vascular area, or by a process in the body mesenchyma similar to that through which the primitive vessels arose in the yolk-sac (umbilical vesicle). The evidence seems to favor the mesodermal origin of the angioblast. The advocates of vasculogenesis by invasion (Evans, Minot, Bremer and others) regard the original angioblast, very early differentiated from mesenchyma, as the sole future source of endothelium, to which is ascribed a strict specificity throughout development. The advocates of the in situ method of origin (Maximow, Huntington, Schultze, Miller and others), on the contrary, conceive early vasculogenesis as a process of progressive fusion of tissue spaces and mesenchymal cells involving a continued differentiation of endothelium from mesenchyma.

The total evidence seems to favor the view that in earliest stages blood-vessels may arise in the mesenchyma of the embryo and that these

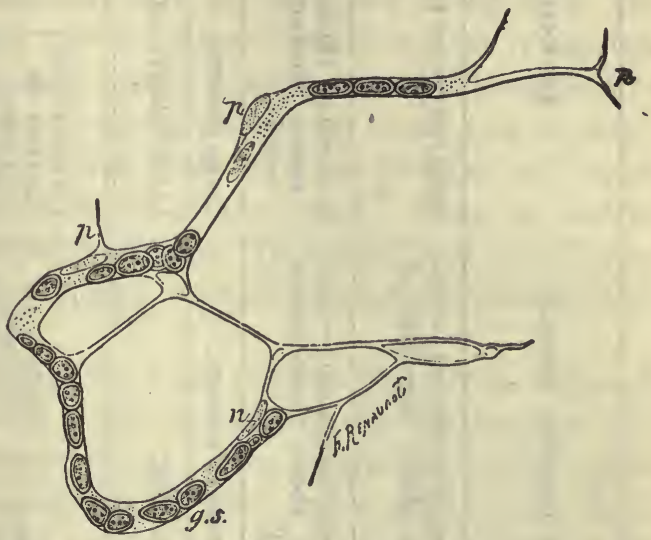

Fig. 211.- 'Vasoformative' Cells from the Mesentery of a Rabbit Seven Days Otd.

g.s., red blood cells; $n$, nucleus of the vascular endothelium; $p$, points of growth, at which extension occurs. Highly magnified. (After Ranvier.)

primitive stems may be added to by discrete anlages all of which may fuse to form the vascular net out of which develop the future main vessels.

The vessels of later embryonic and fetal stages probably arise solely as sprouts from these earlier stems.

The chief point of uncertainty concerns the point in time when vasculogenesis passes from a process including sprouting and fusion of separate anlages, to one where extension is exclusively by terminal growth. Both arteries and veins have a like origin in capillary plexuses.

The final anastomosing sprouts of endothelium represent the definitive capillaries. The development of the definitive wall of arteries and veins involves the formation of extra-endothelial layers of muscular and connective tissue elements from the surrounding mesenchyme, and their association into the several tunics of the various subdivisions of these vessels. 
The essential matters in the foregoing chapter may be summarized in the following schemes.

\begin{tabular}{|c|c|c|c|c|c|c|c|}
\hline 迹 & 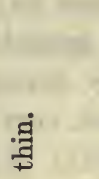 & 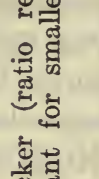 & 1 & 章 & 密 & 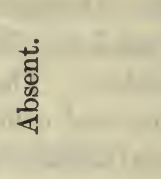 & 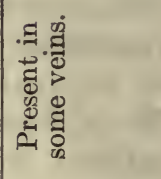 \\
\hline 量 & 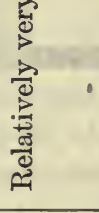 & 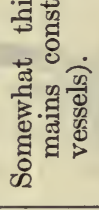 & 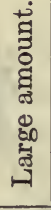 & 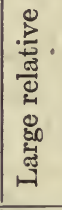 & 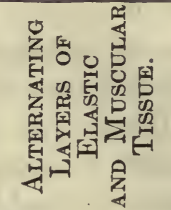 & 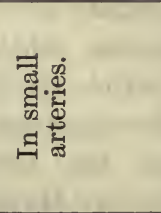 & 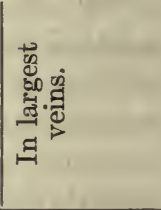 \\
\hline \multirow{2}{*}{ 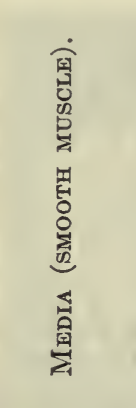 } & \multirow{2}{*}{ 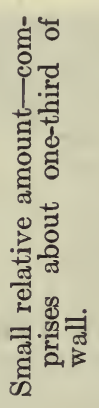 } & \multirow{2}{*}{ 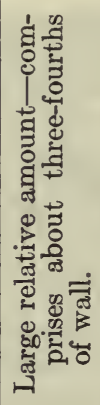 } & \multirow{2}{*}{ 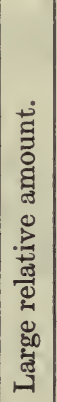 } & \multirow[b]{2}{*}{ 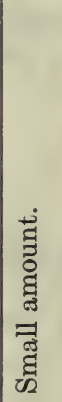 } & 起星 & 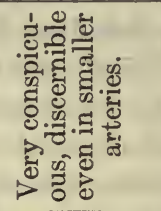 & 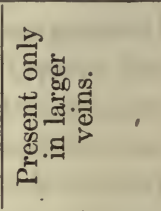 \\
\hline & & & & & 曷 & . & 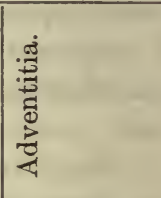 \\
\hline \multirow{2}{*}{ 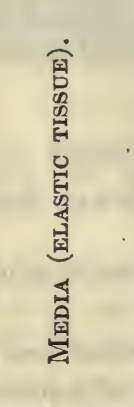 } & \multirow{2}{*}{ 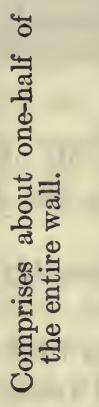 } & \multirow{2}{*}{ 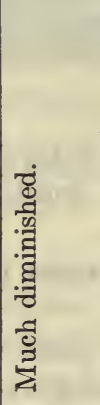 } & \multirow[b]{2}{*}{ 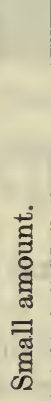 } & \multirow[b]{2}{*}{ 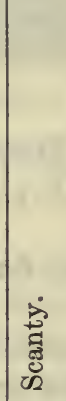 } & 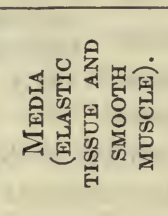 & 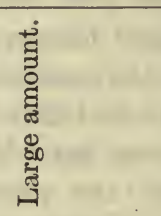 & 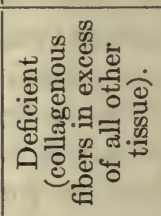 \\
\hline & & & & & $\stackrel{3}{3}$ & 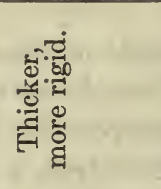 & 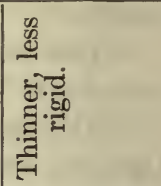 \\
\hline 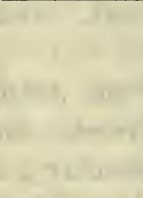 & 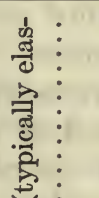 & 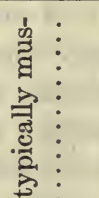 & ( & $\vdots$ & 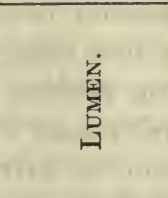 & 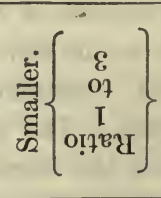 & 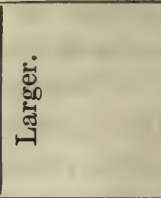 \\
\hline mis & 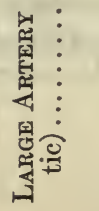 & 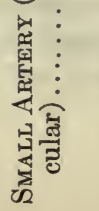 & 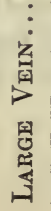 & 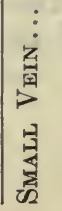 & & 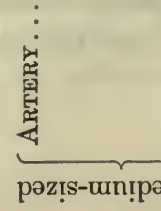 & $\begin{array}{c}\vdots \\
\vdots \\
\text { 畜 } \\
p^{2}\end{array}$ \\
\hline
\end{tabular}




\section{HEART}

The wall of the heart consists of interlacing bundles of cardiac muscle fibers, the myocardium, which are covered externally by the epicardium, a serous membrane which forms the visceral layer of the pericardium. Internally the muscular wall of the heart is lined by the endocardium, which resembles the serous membranes in that it consists of pavement epithelium supported upon a layer of fibro-elastic connective tissue. The endocardium lines all the cavities of the heart, and its endothelium is directly continuous with that of those arteries and veins which are connected with the cavity of the heart. - Thus the entire vascular system-heart, arteries, capillaries, lymphatics, and veins-may be said to be lined by an uninterrupted sheet of pavement epithelial cells, the endothelium.

Myocardium.-The muscle cells of the myocardium are so disposed as to form long fibrous bundles which by their figure-of- 8 arrangement are interwoven with one another to form a dense interlacing mass of muscle bundles. (For detailed description see Mall, Amer. Jour. Anat., 11, 3, 1911.) The structure of these cardiac muscle fibers has already been described. Because of the irregularity of their disposition, transections of the cardiac wall present sections of muscle fibers which have been cut in every conceivable direction.

Between the muscle fibers is a very delicate framework of fibrous connective tissue, the endomysium, which surrounds the muscle fibers and supports the abundant capillaries, arterioles, and venules, with which they are supplied. The proportion of connective tissue in the normal myocardium as compared with the muscle is, nevertheless, very small.

In certain portions of the myocardium connective tissue is more abundant. Thus it is slightly increased in the vicinity of the endocardium, in the papillary muscles, and near the bases of the cardiac valves. At the surface of the heart, beneath the epicardium, especially in the various grooves on the surface of the heart, the connective tissue is still more abundant, and may contain groups of fat cells. It is through these accumulations of connective tissue that the larger blood-vessels are distributed to the myocardium.

Epicardium.-The epicardium, like the other serous membranes, consists of a layer of pavement cells, so joined edge to edge as to form a complete mesothelial coat. Here and there the mesothelium presents 
small openings at the angles between its cells; these stomata are surrounded by minute, finely granular cells and are perhaps connected with the lymphatic vessels.

The mesothelium of the epicardium is supported upon a thin layer of dense areolar tissue in which are many small blood-vessels and lymphatics. Fibers from the deeper surface of this layer are prolonged into the myocardium to become continuous with its endomysial connective tissue. The larger of these connective tissue trabeculæ accompany the branches of the larger arteries and veins which are distributed to the muscular wall of the heart.

Endocardium.-The endocardium consists of a lining membrane of polygonal endothelial cells supported upon a thin layer of delicate

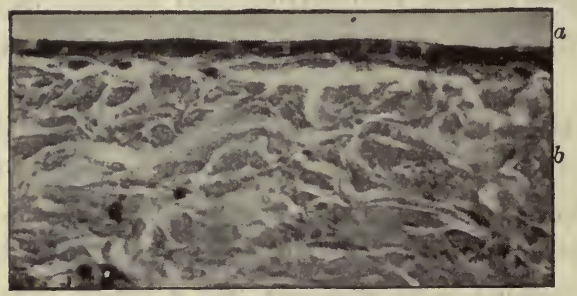

Fig. 212. - The Parietal Layer of the Pericardium of a Child.

$a$, mesothelium; $b$, connective tissue. Hematein and eosin. Photo. $\times 500$. fibrous connective tissue, of endothelial origin (Mall). In this membrane is a network of elastic fibers, and a small amount of smooth muscle. The endothelium of this membrane is continuous with that of those blood-vessels which open from the cavities of the heart. Its connective tissue also forms a continuous layer with that of the tunica intima of these ves-
diac wall-endocardium, myocarsels; in fact, the three coats of the cardiac wall-endocardium, myocar-
dium, and epicardium-might well be compared with the corresponding three coats of the arterial and venous walls-the intima, media, and adventitia. In either organ, the inner coat consists of a lining membrane of endothelium, and a supporting membrane of connective tissue; muscle in large part composes the middle coat, while the outer coat is typically a connective tissue layer.

Valves. - At the cardiac orifices the entire thickness of the endocardium is folded upon itself to form a double layer, between the folds of which an intervening stratum of dense fibro-elastic tissue is inserted. These endocardial folds form the cardiac valves. The number and shape of their cusps are dependent upon the location. The semilunar valves of the aortic and pulmonary orifices consist of three crescentic endocardial folds; at the auriculoventricular orifices the tricuspid valve consists of three, the bicuspid or mitral of two, folds.

The margin of the valvular cusp or fold is extremely thin; just within 
the margin, however, the central mass of dense fibrous tissue is somewhat thickened to form, in each cusp, a dense rim which during-valvular closure secures the firm and accurate approximation of the free margins of adjacent cusps. At the apex of the valvular cusp, where the adjacent fibrous margins of the valve meet, the dense connective tissue, particularly in the semilunar valves, is considerably thickened to form a nodule, the corpus arantii. These corpora or noduli, in the aged, are frequently subject to calcareous infiltration.

Muscular fibers are continued from the adjacent cardiac wall into the dense fibrous tissue at the base of the valve, except in the case of the semilunar valves of the pulmonary and systemic aortæ. This muscle

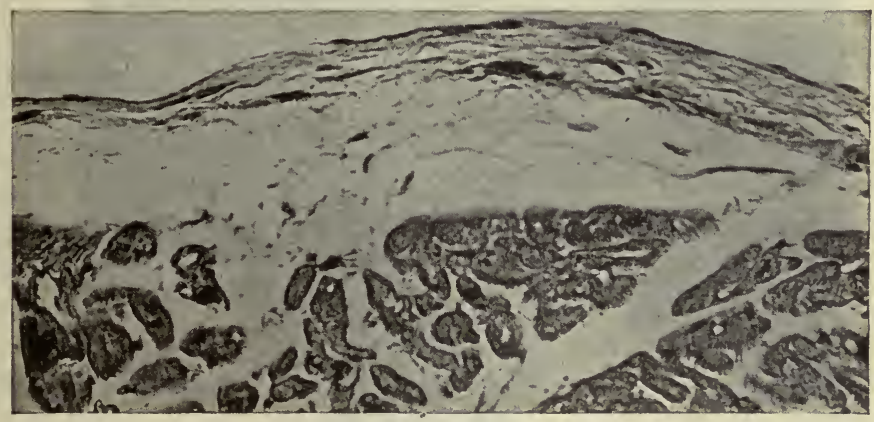

Fìg. 213.-The Endocardium.

From the ventricular wall of the heart of man. Hematein and eosin. Photo. $\times 469$.

is generally non-striped, and probably functions as a sphincter. The base of the valve is also surrounded by a ring of fibrous tissue, the annulus fibrosus, whose interlacing bundles are so closely packed as to give them an almost cartilaginous feel. At the auriculoventricular orifices, these fibrous rings are continuous with the auriculoventricular septum, from which the muscle bands of the myocardium take their origin.

Chordæ Tendineæ. - These are firm, unyielding cords, composed of parallel bundles of dense collagenous fibers, with a few elastic fibers, and covered with a very thin endocardium continuous with that of the ventricular wall and cardiac valve. These fibrous bands unite the apices of the papillary muscles to the ventricular surfaces of the mitral and tricuspid valves. At the apex of the papillary muscle the fibrous bundles of the chordæ intermingle with the muscle fibers, and are continued into the endomysial connective tissue, which is especially abundant in those 
portions of the myocardium. At their valvular attachment the fibrous bundles of the chordæ tendineæ turn almost at right angles, and spread out, in a somewhat radial manner, to become continuous with the dense fibrous tissue which forms the interior of the valve.
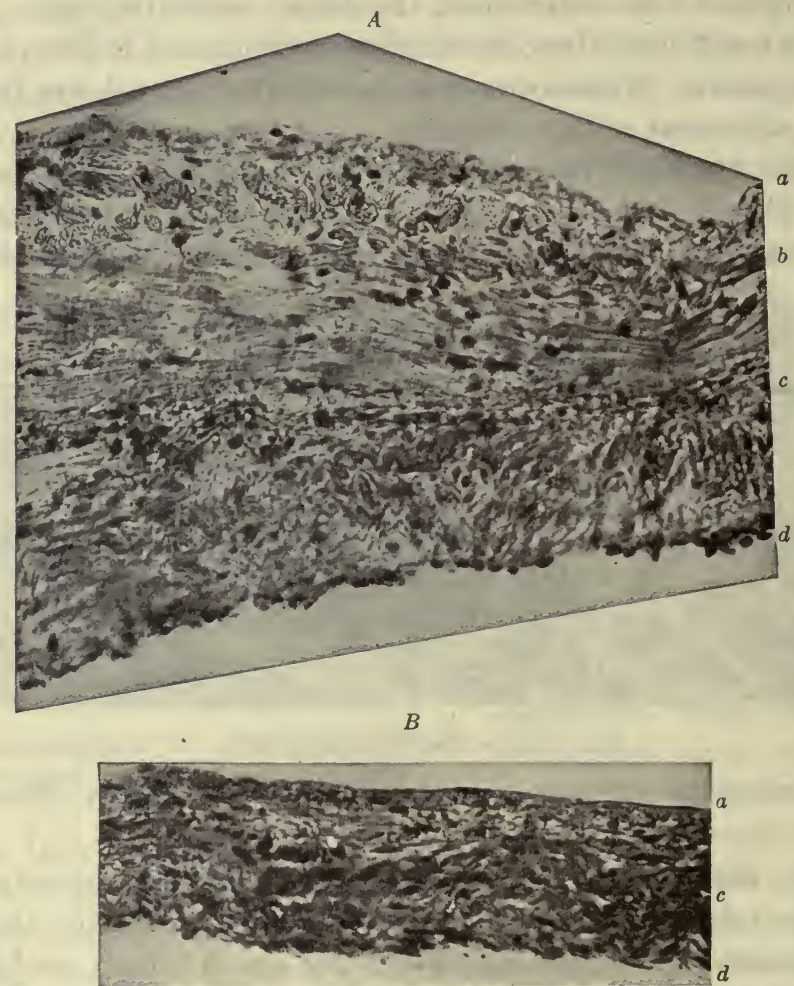

Fig. 214.-Radial Sections of the Mitral Valve, from the Heart of a Man.

$A$, from the base of the valve showing the extension into it of cardiac muscle fibers from the wall of the heart; $B$, from the mid-region of the valve. $a$, auricular endocardium; $b$, muscle fibers; $c$, dense fibrous tissue; $d$, ventricular endocardium. Hematein and eosin. Photo. $\times 800$.

Columnæ Carneæ.-The columnæ carneæ are columelliform projections of the myocardium into the ventricular cavity. They consist of cardiac muscle fibers, largely of the Purkinje fiber variety, which are disposed in their long axis, and are covered by reflections and reduplications of the endocardium. The irregular contour of the ventricular cavities appears to be entirely due to the projecting columnæ carneæ. 
These muscular columns may present any one of three modes of attachment to the myocardium: (1) they may be attached along their entire extent; (2) they may be attached only at their two ends, the midportion being free; (3) they may be attached to the myocardium at one end only, the other end projecting into the ventricular cavity as a papillary muscle, from whose apex chordæ tendineæ pass to the auriculoven-

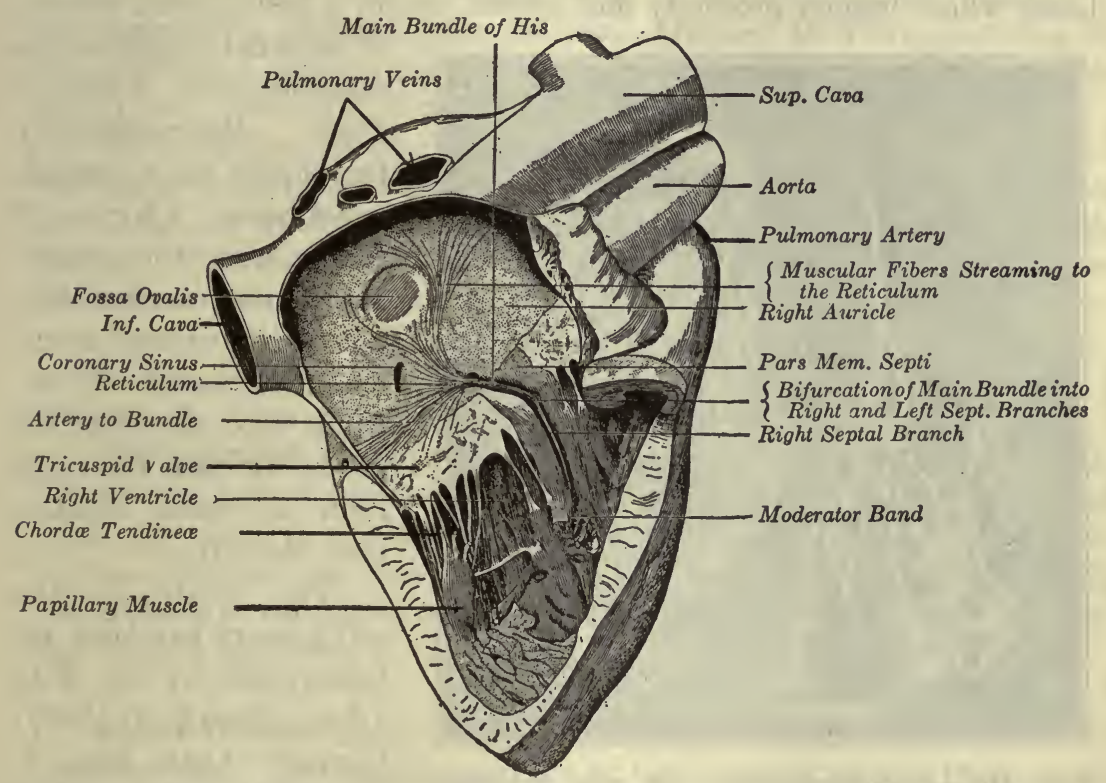

Fig. 215.-Human Heart Opened from the Right to Show the AtriovenTRICULAR BUNDLE OF His.

The illustration shows also a heart valve, the chordæ tendineæ, and the papillary muscles. (After Curran, Anat. Rec., 3, 12, 1909.)

tricular valves. Either of the last two forms may, in transections of the ventricles, appear as isolated islands of muscular tissue surrounded by endocardium and lying apparently free within the cavity of the ventricle. Columnæ carneæ which span the ventricular cavity constitute moderator bands. One such band is frequently present in the right ventricle near the apex, and occasionally one appears in the left ventricle.

Atrioventricular Bundle.-The atrioventricular bundle of His was discovered in the human heart by His, Jr., in 1893. Previously in the same year it had been noted by Kent in the heart of a number of mammals. It has since been seen in every species of mammal investigated. 
It consists of a dense meshwork of cardiac muscle fibers rich in sarcoplasm, in the form of a band taking origin in scattered fibrils in the posterior wall of the right atrium near the septum in the atrioventricular groove (sinus region; hence, sinoventricular conducting system, Retzer, 1908), and coursing forward in the interatrial septum into the upper anterior portion of the interventricular septum, where it divides into two limbs which branch profusely and spread out in a complicated system

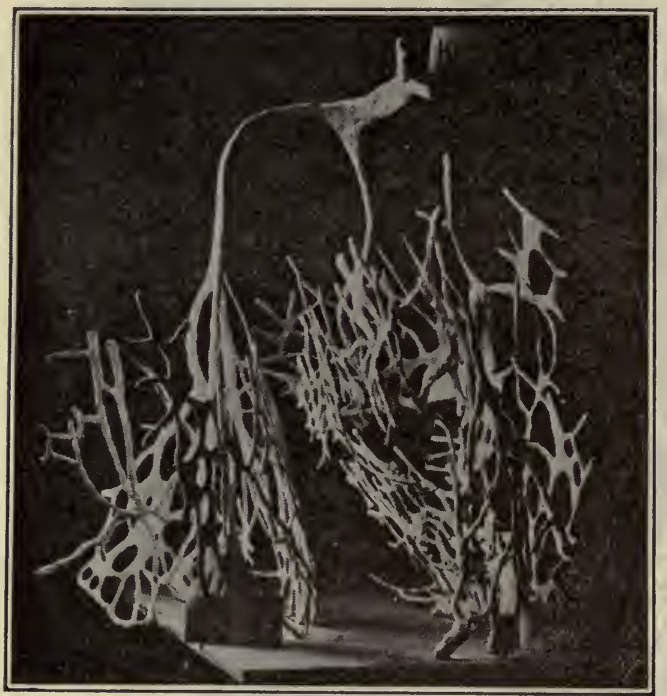

Fig. 216.-Reconstruction of the Sinoventricular System (Bundle of His) of THE Calf's Heart.

(De Witt, Anat. Rec., 3, 9, 1909.) of terminal branches, the subendocardial Purkinje fibers. Macroscopically the bundle has a grayish appearance; where it passes from the interatrial to the interventricular septum (pars membranacea septi) it expands into the so-called node. In man the right limb is much smaller than the left.

The bundle of the calf's heart has been reconstructed by De Witt (Anat. Rec., 3, 9, 1909). Curran (Anat. Rec., 3, 12, 1909) has described a constant bursa or lubricating mechanism in relation with the bundle, furnishing protection against friction during contraction of the heart. He describes "its connection with all parts of both auricles through three large trunks and a number of smaller twigs, and not, as was once thought, merely arising in the right auricle only."

Tawara (1906) first carefully described the histology of this bundle in several mammals, including man. De Witt more recently (1909) has extended the study in this same field. "In the sheep and calf, where the fibers are most typical and most clearly differentiated from the myocardial fibers, the fibers are much larger than the myocardial fibers, with fewer fibrils and much more sarcoplasm." She describes the bundle as a muscular syncytium. "Connective tissue and especially elastic fibers 
are much more abundant than in the myocardium." The bundle contains abundant ganglion cells and nerve fibers. It is also very rich in glycogen. In the ox it is distinctly cellular (Fig. 112).

It would seem, on the basis of its constancy of presence and structure, and its probably independent blood and nerve supply, that the atrioventricular bundle has a function independent of the myocardium, probably of the nature of a neuromuscular end-organ (Retzer; DeWitt), providing for the conduction of the impulse to contraction, and the coördination of the atrial and ventricular rhythm.

A muscle bundle of closely similar structure intimately related with the vagus and sympathetic nerves, the 'sino-atrial node,' has been described by Keith and Flack (1907) at the juncture of the sinus venosus and the atrium. It is believed to be the place of origin of the impulse to the heart beat, from which it is transmitted to the atrioventricular bundle.

Laurens (Anat. Rec., 2, 8, 1913) has described an analogous muscular connection between auricles and ventricles in certain reptiles, where it assumes the form of an inverted funnel-shaped tube.

Development of Heart.-The anlage of the heart arises from the fusion of a pair of parallel endothelial tubes in the paramedial angioblast, each surrounded by primitive mesenchyma. The endothelium of the resulting sac differentiates into the endocardium of the definitive heart, while the connective tissue and muscle develop from the mesenchyma in a manner essentially similar to that described for the blood-vessels.

Blood-Vessels.-The heart is supplied with blood through the coronary arteries. The larger branches of these vessels pursue their course beneath the epicardium in the superficial grooves of the cardiac wall. From these large arteries, smaller branches are distributed to the epicardium and to the muscular wall, the latter vessels penetrating as far as the endocardium, in whose connective tissue they form a meager capillary plexus.

The capillaries of the myocardium are extremely abundant. They form elongated meshes between the muscle fibers, the circumference of each muscle fiber being in relation with several capillary vessels. The veins return the blood from these rich capillary plexuses and pursue a course similar to that of the arteries, the larger veins being always found in the broader connective tissue septa. In the right atrium certain small veins, the vence minima, empty directly into the cavity of the heart.

The lymph supply is very abundant and intimate. The lymph vessels form two superficial plexuses, the endocardial and the epicardial, both draining into the larger lymph vessels at the base of the heart. 
Nerve Supply.-The nerve supply of the vascular system is by means of fine branches from the cerebrospinal and sympathetic systems. In the heart these minute nerve trunks end in the various cardiac ganglia, most of which are found in the connective tissue of the heart, e.g., the coronal plexuses about the orifices of the aorta and pulmonary artery. From these ganglia sensory nerve fibers are distributed to the endocardium and epicardium, and motor fibers to the myocardium. The most of the former are connected with the vagus, the latter with the sympathetic trunks. Through both the vagus and the sympathetic trunks are distributed also efferent cerebrospinal fibers: the accessory nerve through the vagus contributes 'inhibitory' fibers, the cervical spinal nerves through the inferior cervical ganglia contribute 'acceleratory' fibers.

From the cardiac ganglia branches pass to form a coarse plexus in the connective tissue between the muscle bundles, the perimysial plexus, from the branches of which a fine plexus is distributed to the endomysium. The terminal branches end in relation with the surface of the muscle fibers.

The pericardium contains numerous encapsulated nerve endings (corpuscles of Golgi and Mazzoni). According to Martynoff (Arch. mikr. Anat., vol. 84, 1914) unencapsulated endings also are present, of three types: coils, dendriform terminal ramifications, and modified dendriform endings. He describes also naked terminal filaments ending on the bases of the mesothelial covering cells.

The blood-vessels are similarly supplied, minute ganglia occurring here and there in the adventitia or adjacent connective tissue. From these ganglia sensory branches are distributed to the adventitia and intima and motor branches to the tunica media. Naked nerve fibrils can be traced to the smallest blood-vessels, and even in the capillaries terminal fibrillæ are found in relation with the endothelial wall. 


\section{CHAPTER VIII}

\section{BLOOD}

Blood may be regarded as a tissue in which the intercellular material is a fluid, the plasma. The plasma contains fibrin in solution (fibrinogen) ; on exposure to air, as in case of cuts, the fibrin is precipitated in the form of delicate needle-like crystals (Fig. 21\%), leaving a clear strawcolored liquid, the serum. The active element in causing this precipitation is thrombin (or prothrombin), probably liberated by the bloodplatelets on disintegration under the influence of air or the stimulus of a roughened area on the wall of the blood-vessels. The blood-cells become entangled in this fibrin net forming a clot or thrombus. Plasma is very similar to, but not identical with, the lymph of the lymph vessels.

The formed elements, or blood-cells, are of two main classes, red and white, or erythroplastids (erythrocytes) and leukocytes. The red blood corpuscles or plastids owe their characteristic color to the presence of hemoglobin. Single corpuscles have a light yellowish-green color, in masses they appear red. The function of the hemoglobin is to carry oxygen in weak combination as oxyhemoglobin for transportation through the blood-vessels from the lungs to the tissues, where it is employed in the oxidation processes upon which life depends.

\section{THE RED BLOOD CELL}

The erythroplastid is a circular biconcave disk, of 7.5 microns (a micron is $1-1000$ of a millimeter) diameter. They are present in practically all histologic preparations, hence they serve well as a ready scale for approximate determination of size of cellular elements in the same microscopic field. Every section; blood preparation, as of a drop under a cover-slip; and even the blood-vessels of living mammals, contain also a larger or smaller number of cells of saucer shape (cup shape; 
bell shape, etc.). These are believed by some (Weidenreich, Lewis, Minot, and others) to be the more normal in shape; however, there remains

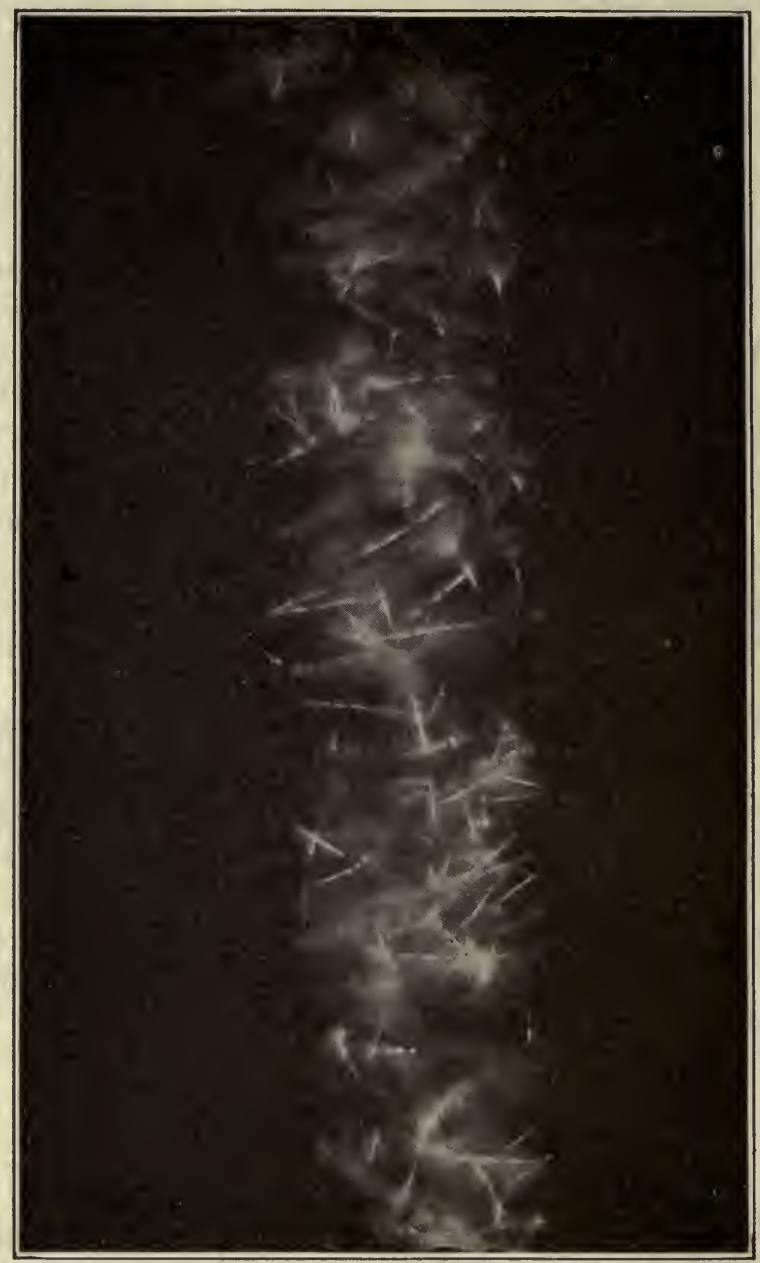

Fig. 217. - Oxalated Plasma of Human Blood Clotted with Thrombin, Showing Fibrin Needles. $\times 512$.

(After Howell, Amer. Jour. Physiol., 35, 1, 1914.)

little doubt that the circular biconcave disk shape is the normal (original), the saucer or cup shapes, the derived forms, the result of modifi- 
cation by extrinsic factors. 'The erythroplastid is of course a very delicate structure, and slight tractions or tensions incident to passage through exiguous confines, or osmotic currents set up in fixing fluids, or the coagulation of its protoplasm, would produce modifications and distortions. Almost any conceivable mechanical modification would of

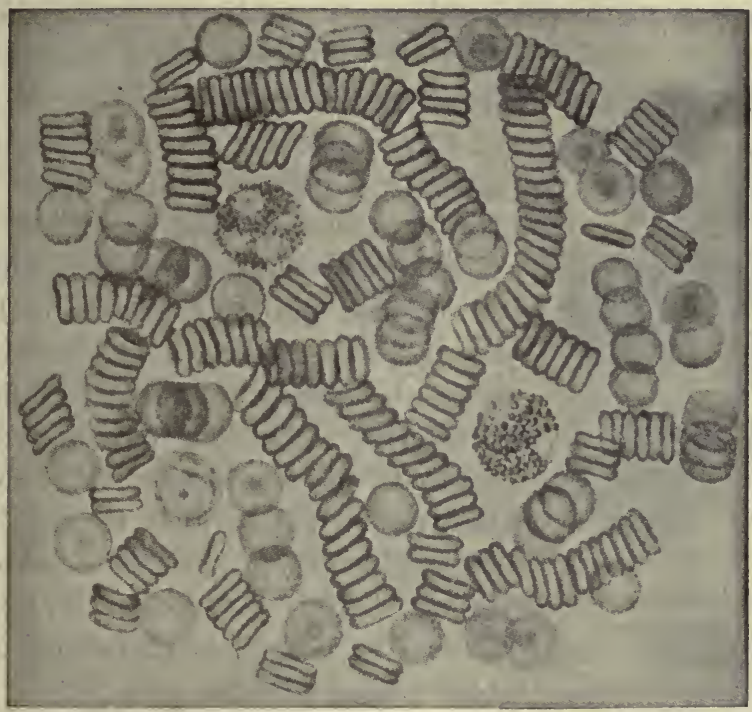

Fig. 218.-From a Freshly Prepared, Unstained Specimen of Human Blood.

Three leukocytes, an eosinophil, a polynuclear, and a lymphocyte, are represented. Many red blood corpuscles (erythroplastids), some on the flat, some in rouleaux and in profile, are also shown. $\times 1200$, but reduced somewhat in reproduction. (After Schäfer.)

necessity change a circular biconcave disk into some sort of cup-shaped element.

The red blood element lacks a nucleus, hence the propriety of insistence on the term 'plastid' or 'corpuscle' in preference to cell (cyte). The red elements of all mammals are non-nucleated. The lower forms have nucleated elements (erythrocytes), frequently of ellipsoidal form. Mammalian red elements of greatly divergent sizes (2.5 $\mu$ in musk ox; $9.4 \mu$ in elephant; $\operatorname{dog}, 7.5 \mu$ ) are all of circular outline, except those of the camelidæ (llama, camel, etc.) which are elliptical. The erythroplastids are generally believed to be enclosed by a delicate membrane, and 
to contain a very delicate stroma (spongioplasm) and fluid matrix (hyaloplasm, with hemoglobin). The red corpuscles have a tendency when exposed, as in a drop mounted under a cover-slip, to arrange themselves in rows, like coins in a pile, concave surfaces apposed, forming rouleaux. Drop preparations show after a short time also an increasing number of plastids with puckered or spiny surfaces, crenated corpuscles. This condition results from evaporation producing a medium of greater density than
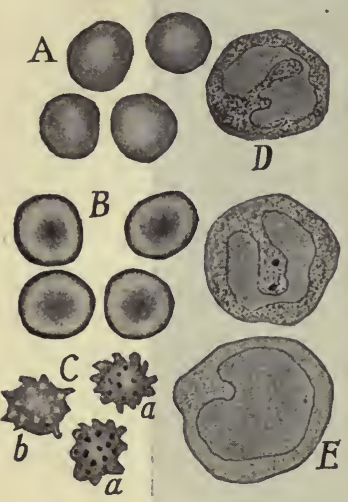

Fig. 219. - Blood Cells From a SPECIMEN OF Freshly Drawn UNstained Human Blood.

$A$, red blood corpuscles, deep focus, showing a light center and dim margin; $B$, the same with a higher focus; the center, being slightly out of focus, is dim while the margin is light; $C$, crenated red corpuscles from the margin of the preparation; $a$, deep focus; $b$, higher focus; $D$, two polymorphonuclear leukocytes; $E$, large mononuclear leukocyte. $\times 750$. that of the normal blood plasma. Any medium of density equal to that of the plasma of any particular blood is spoken of as an isotonic solution for that blood. The solution in most common use for human blood is a 0.9 per cent. solution of sodium chlorid in distilled water. Solutions of higher density are hypertonic; these produce exosmotic currents causing destruction leading through crenation. Solutions of lower density, for example, water, are hypotonic; they produce destruction (hemolysis) through endosmosis causing swelling, a stage of which shows a saucer-shaped corpuscle. This is accompanied by laking, or extraction of hemoglobin, giving rise to blood shadows; and final bursting, leaving a débris called hemokonia.

The number of red corpuscles in the blood is subject to constant variation between wide limits. Many Schäfer.) physiologic condi-

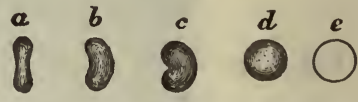

IG. 220. - Showing THE ACTION OF WATER UPON THE RED BLOOD CoRPUSCLE.

$a$, the corpuscle in profile; $b-e$, various stages in the transformation which leaves only a 'shadow' $e$; diagrammatic. (After

tions influence their total number, as well as the relative proportion of red elements to the white. The average number of red corpuscles in the adult male is about 5,000,000 per cubic millimeter. In young robust persons the number may be considerably higher. The number may also be much reduced by considerable hemorrhages or by the imbibition of large quantities of fluid. Profuse perspiration tends to produce 
by great increase of nuclear and cytoplasmic constituents, gives rise to the so-called GIANT CELLS. These are of two sorts, depending upon whether the nucleus is single or multiple, the megakaryocyte and the polykaryocyte. The latter represents a modification of the former, and must not be confused with the osteoclasts of developing bone.

Megakaryocytes are practically limited to bone-marrow. They frequently show long and numerous pseudopodia. These, as also the cellbody proper, show a differentiation of the cytoplasm into a superficial hyaline layer and a central basophilic granular core. According to Wright, constriction and segmentation of these pseudopods give rise to the BLOOD-PLATELETS (plates; plaques). These commonly hold positions at the center of masses of converging fibrin fibrils in blood clots, in consequence of which they are supposed to be the essential elements, probably liberating 'thrombin', in clotting, hence their synonym, thrombocyte. This term, however, is ill-

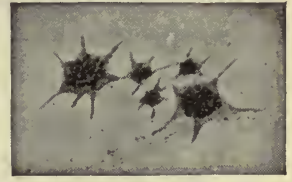

Fig. 224.-GRoUP OF Three SMall aND Two LARge Blood Platelets. $\times 2000$. chosen, for these elements contain no nucleus. What simulates a nucleus is the central spheroidal mass of basophilic granules. Blood-platelets are capable of ameboid motility. They vary in diameter from 2 to 4 microns, and in number per cubic millimeter from 200,000 to 800,000. Analogous elements of avian, reptilian and ichthyoid bloods are nucleated spindle cells, or true thrombocytes. Wright's observations on mammalian megakaryocytes furnish at present the best data for the genetic interpretation of blood-platelets. However, almost every conceivable mode of origin, notably from extruded nuclei of erythrocytes, and fragmenting leukocytes have had, and still claim, prominent supporters.

Granulocytes.--The granulocytes comprise three varieties distinguished on the basis of their cytoplasmic granules: (1) neutrophils; (2) eosinophils (oxyphils) and (3) basophils, or mast leukocytes. The nucleus is of the polymorph type, perhaps occasionally, polynuclear. This nuclear condition consists in a commonly crescentic chain of nuclear masses connected by frequently extremely delicate nuclear strands.

The NEUTROPHILs are characterized by their fine cytoplasmic granules, having a neutrophil staining reaction (lilac color) in mixtures of acid (eosin) and basic (methylene blue) dyes. They range in size from $7.5 \mu$ to $10 \mu$ in diameter. They are predominantly phagocytic. They constitute in normal adult life about 70 per cent. of the total whitecell blood content. 
The EOsinopHILs contain larger spheroidal eytoplasmic granules which show a special affinity for eosin. They are slightly larger than the neutrophilic granulocytes, and comprise about 4 per cent. of the leukocytes of normal blood.

The BASOPHILs are characterized by an extremely variable polymorphous nucleus, but especially by their spheroidal and irregular basophilic granules of greatly varying sizes. They occur to the extent of only about 0.5 per cent. in the circulating blood. They are of approximately the same size as the eosinophils. Their significance and genetic relationship is uncertain, but they are usually interpreted as degenerating granulocytes, the granules being variously regarded as products of nuclear fragmentation, and as cytoplasmic products of mucoid degeneration. Maximow (Arch. mikr. Anat., 83, 1, 1913), however, regards the 'mast cells' of the blood as specialized kinds of granulocytes, distinct from similar cells of the tissues, and without sign of degeneration.

Cowdry has demonstrated mitochondria, by vital staining with janus green, in all types of leukocytes, except mast leukocytes, including platelets. The lymphocytes and polymorph neutrophils contain them abundantly. They are onily sparsely present in eosinophils. Mitochondria are said to be totally absent in the erythroplastids of normal adult human blood (Intern. Monatschr. Anat. u. Physiol., 31, 4, 1914).

P. Ehrlich in a series of communications announced that by coloring the leukocytes with various stains he was able to distinguish by their reaction, several types of granules. These he called $(\alpha)$ oxyphil or acidophil, which were deeply stained by eosin, acid fuchsin, etc.; $(\beta)$ amphophil, which were stained both by eosin, and by dahlia and like dyes; $(\gamma)$ basophil, which were stained deeply by dahlia, thionin, etc.; $(\delta)$ certain cells whicb neither after staining with eosin, etc., nor with dahlia, etc., could be made to show any granules other than the nodes of cytoreticulum; (द) neutrophil, which can be stained only by a due admixture of acid and basic dyes, as of fuchsin and methylene blue, or the so-called 'triacid mixture' of Ehrlich.

The demonstration of these characteristics presupposed a division of dyes 'nto three primary classes:

1. Acid-e.g., eosin, orange-G, acid fuchsin, aurantia, erythrosin.

2. Basic-e.g., methylene blue, dahlia, thionin, hematin.

3. Neutral-which are only formed by the interreaction of examples of each of the two preceding classes; the neutral dye is supposed to arise de novo in such mixtures, as a result of chemical reaction. 
The application of such a classification of stains to other tissues than the blood has, however, been found to present considerable difficulties.

According to Kite the cytoplasm of the polymorphonuclear leukocytes has nothing of the nature of a cell membrane, but they are completely naked, nor do they contain a spongioplasm and hyaloplasm. "The cytoplasm is a jelly in which are embedded large numbers of globules." The structures usually termed cytoplasmic granules are of the nature of separation products; they do not grade into the surrounding cytoplasm. All leukocytes undergo also certain definite structural transformations, characterized by the appearance of pseudopods changing into vibratile cilia. Kite suggests that the protoplasmic processes may be prominently concerned in phagocytosis. Under certain conditions erythroplastids may be made to protrude similar processes (Jour. Infect. Dis., 15, 2, 1914).

\section{HEMOGLOBIN}

Hemoglobin is a very complex chemical compound of iron with a globulin; it gives the characteristic color
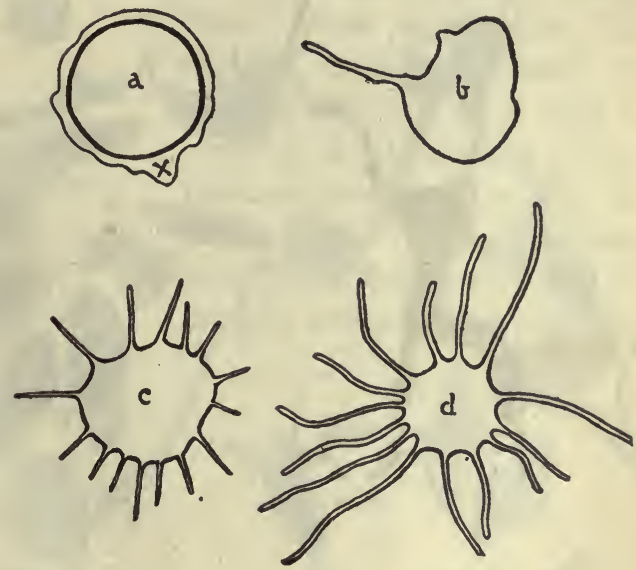

Fig. 225.-Outuine Drawings of Living PolyMORPHONUCLEAR LEUKOCYTES OF RABBIT, From a Drop of Blood Mixed with Ringer's Solution to Which a Small Amount of Hirudin Had Been Added to Prevent CoagULATION.

In the course of half an hour the cells develop retractile undulatory processes. $a$, hyaline-surface phase; $x$, hyaline layer; $b$ and $c$, ciliated phase; $d$, flagellated phase. Leukocytes of all classes of vertebrates undergo similar changes. (After Kite, Jour. Infectious Dis., 15, 2, 1914.) to the blood. It combines readily with oxygen to form oxyhemoglobin, a loose chemical combination by which the oxygen is carried from the lungs to the tissues, and which gives the brighter red color to the arterial as compared with the venous blood. The hemoglobin is held either in solution or in unstable chemical union by the cytoplasm of the erythroplastids. It escapes from these corpuscles after rupture, or it may be extracted by ether, and is 
then prone to crystallize on evaporation in the form of minute brownishyellow prisms. The crystals of the various species of any genus belong to a crystallographic group (Reichert) but generic differences are frequently striking; thus, in human blood they are long rhombohedra (Fig. 227 ), in guinea pig, tetrahedra, in squirrel, hexagonal plates, in rat,

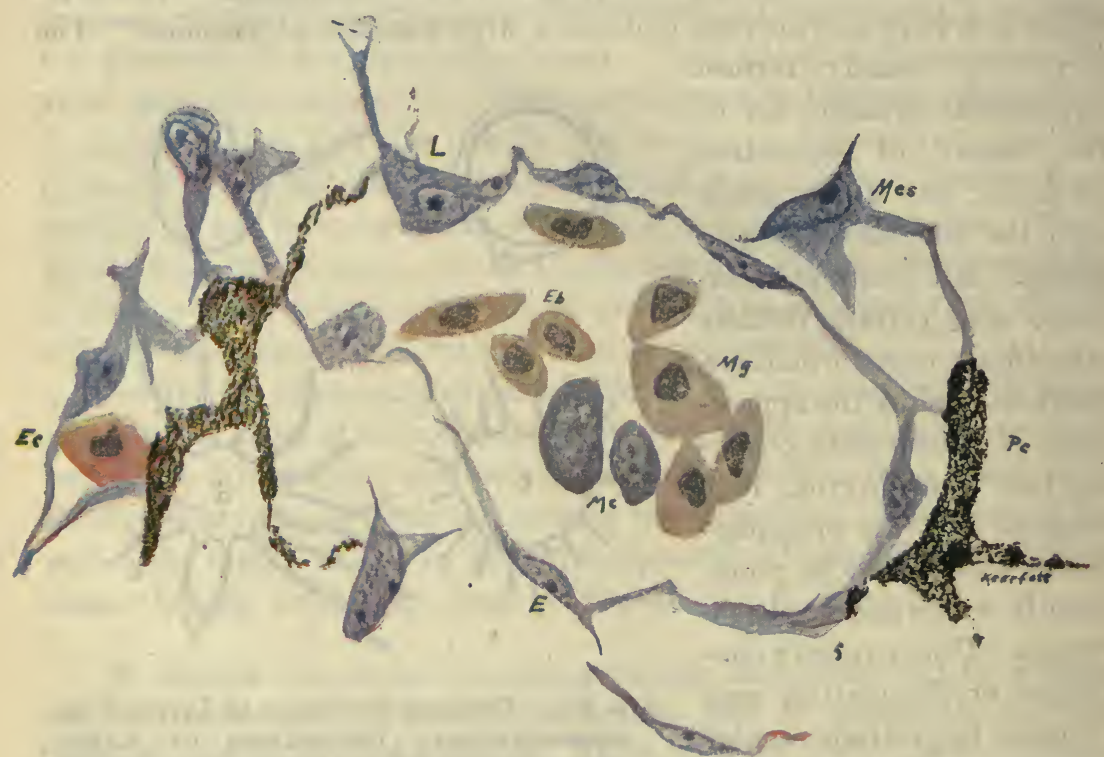

Fig. 226.-Section of Bone Marrow from Skull of 25 mm. Turtle Embryo (Chelydra serpentina), Showing Three Main Stages in the Hemopoiesis.

$L$, hemoblast (lymphocyte), differentiating in the mesenchyma and passing into a blood-vessel; $M c$, blood mother cell (hemoblast); $M g$, megaloblast; $E b$, erythroblast; $E c$, erythrocyte; $E$, endothelial cell; $M e s$, mesenchymal cell; $P c$, pigmented cell. Helly fixation; Giemsa stain. $\times 700$.

elongated six-sided plates, in.hamster (a rodent), rhombohedra, and in dog they appear as rhombic prisms which are diamond shaped in crosssection.

Various other crystalline, and also amorphous hemoglobin derivatives may occur as decomposition products. The iron of the coloring matter of the hemoglobin may be thus obtained in the form of hematin, a soluble, amorphous protein compound of a brownish-red color. If hematin is combined with hydrochloric acid the chlorid of hematin, hemin, is produced. Hemin occurs in deep brownish-red crystals, known also as Teichmann's crystals, which differ somewhat according to the animal 
species from which they are obtained; those of human blood take the form of triclinic plates (Fig. 228). Hemin crystals derive a certain importance as a forensic test for the presence of blood, and they may be obtained from old and dried-up specimens as readily as from fresh blood. The procedure for testing a suspected stain consists in heating

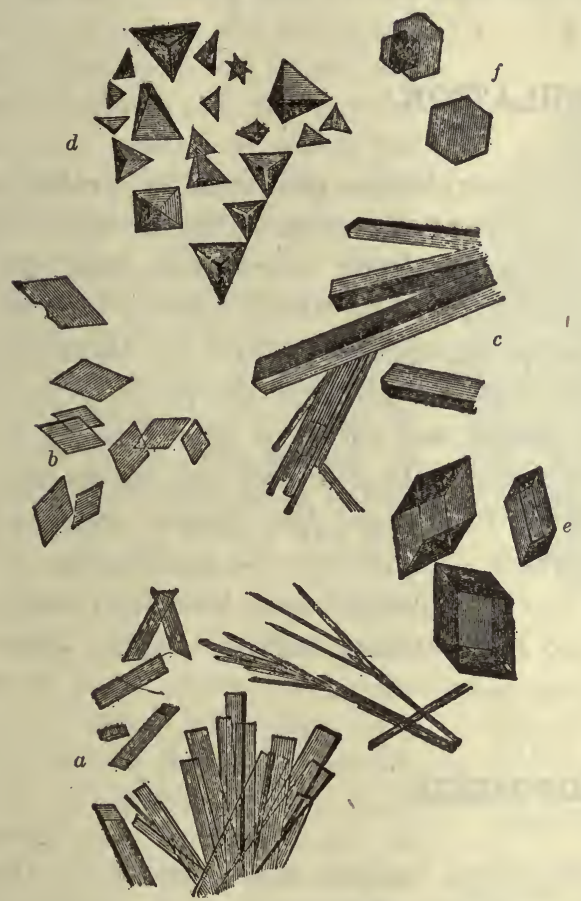

Fig. 227.-Hemoglobin Crystals.

$a$ and $b$, from human blood; $c$, from the cat; $d$, from the guinea-pig; $e$, from the hamster; $f$, from the squirrel. (After Ranvier.) to the boiling point in a drop of acetic acid, a drop of a normal salt solution of the specimen. From fresh blood the crystals may be produced by heating together on a slide a drop of blood, a grain of sodium chlorid, and a drop of acetic acid. Hemin crystals prove only that hemoglobin is present, but give no precise in-

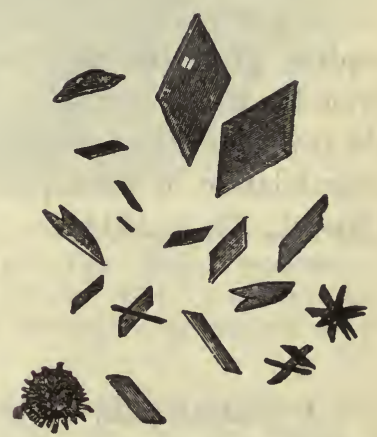

Fig. 228. - Crystals of Chlorid of Hematin or HeMin.

(After Ranvier.)

formation as to the species from which the blood in question came, for the crystals obtained from many mammals are apparently identical.

Hematin may decompose also into hemosiderin, an iron-containing hemoglobin derivative, in the form of light-brown granules, frequently found in phagocytic lenkocytes as the product of cellular digestion of effete erythroplastids. Hemosiderin granules thus appear in the spleen and bone-marrow. Iron in the tissues, and ferruginous pigments generally, can be recognized by application of the ferrocyanid test, which stains the iron blue. 
When extravasations of blood occur within the tissues of the body, as, for example, in the corpus hemorrhagicum of the ovary, the hematin is frequently deposited as hematoidin, an iron-free derivative of hemoglobin which forms stellate groups of yellowish needle-like crystals.- Hematoidin is apparently identical with bilirubin, a bile pigment.

\section{COAGULATION}

According to Howell's theory of coagulation, prothrombin, probably produced at least in part by disintegrating platelets, in the presence of calcium is converted into thrombin. The latter precipitates from the fibrinogen of the blood plasma the fibrin needles which mass to produce a mesh in which the corpuscles become aggregated to form the thrombus or clot. Howell has recently studied with the ultramicroscope the process of coagulation in oxalated blood plasma and solutions of fibrinogen to which a solution of thrombin was added. He describes clotting as proceeding after the manner of crystal formation; the crystals begin as granules which adhere to form threads. Clotting is 'an aggregation of the invisible particles (amicrons) to visible particles and then the further consolidation of these particles into rigid looking needles' (Amer. Jour. Physiol., 35, 1, 1914).

\section{HEMOPOIESIS}

A consideration of blood development involves the questions of the sources of primary origin and the genetic relationship of the different varieties of cells. The blood-forming or hemopoietic organs comprise (1) the yolk-sac; (2) body mesenchyme and endothelium of primitive bloodvessels of the embryo; (3) liver and spleen; assisted by lymph organs in the production of lymphocytes, and finally (4) marrow of fetal and adult bone. These several sources function in development in the order enumerated, though the successive stages overlap to some extent. With the assumption of function on the part of bone-marrow-the earliest foci being the scapular, pelvic, vertebral and costal elements-the other blood-forming organs cease activity. However, in certain diseases the spleen may possibly be stimulated to renewed function; and lymph organs function throughout life in the production of lymphocytes. From the standpoint of the germ layers hemapoiesis is more probably limited to the mesoderm (mesenchyme). This is certainly true largely at least, probably completely, of sources outside of the yolk-sac. 
The yolk-sac wall is the earliest locus of blood-cell origin. This structure is a vesicle lined by columnar entodermal cells, invested by mesenchyme, the surface cells of which become arranged in the form of a $\mathrm{mcm}$ brane, the mesothelium. The first anlage of blood appears in the shape of irregular masses and cords of cells next the entoderm. These cords anastomose at the same time that they acquire lumina through a process which involves the peripheral differentiation of cells into endothelium and the appearance of plasma in which the central cells are suspended. The very intimate spatial relationship of these bloodislands to the entoderm has led many to incline to an entodermal origin of the earliest blood anlages. The layer in which blood first appears in the yolk-sac has been named angioblast, a term which does not commit one to any view as to primitive germ layer origin. However, the importance of the matter of origin seems great only when one believes in the strict specificity of germ layers. Such position is hardly tenable in view of the fact that originally there is only one germ layer, the ectoderm, and the origin of the mesoderm from

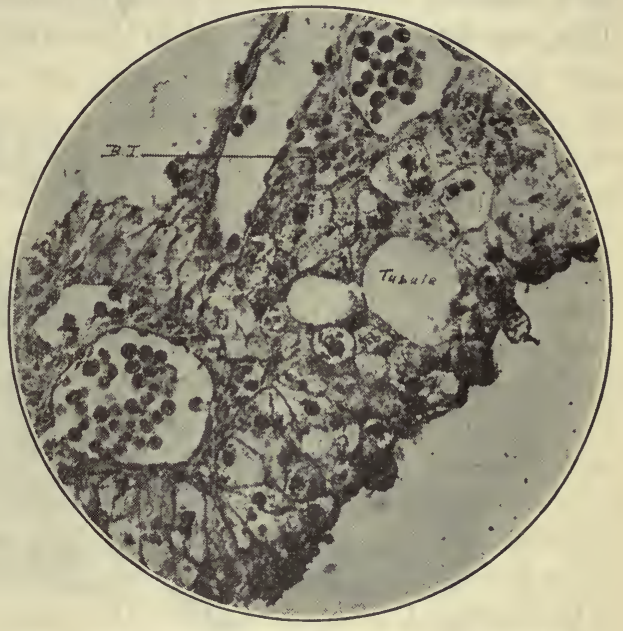

Fig. 229.-WALl of YolK-SaC of a 13 MM. Human Embryo (Fig. 210), Showing a Small Blood Island (B. I.) and Several Small Blood Vessels Containing EryTHROCYTES.

The wall consists of stratified columnar and cuboidal entodermal cells (to the right), a middle mesenchymal layer (angioblast), and a covering of mesothelium. $\times 240$. the primitive streak, a phase of fusion of ectoderm and entoderm. Moreover, in certain forms, e.g., Tarsius, a primate, it is claimed by Hubrecht that mesoderm has a double origin from ectoderm and entoderm, as well as from primitive streak. Again in subsequent early embryonic stages it is believed by many that blood-cells arise by process of differentiation of body mesenchyme. In the further discussion of hemopoiesis we may take for granted the origin of blood exclusively from mesenchymal elements.

Regarding the question of the original blood mother-cell two sharply contrasting views are held, expressed in the monophyletic (unitarian) and polyphyletic (dualistic) theories of blood-cell origin. The former holds that all types of blood elements, red and white, have their origin directly in one and the same primordial blood cell ('hemagonium,' hemoblast); the 
several types are conceived to arise through process of differentiation along distinct lines. The polyphyletic theory holds to a dual origin, from genetically distinct mother-cells, for red and white cells; or to a multiple origin, tracing erythrocytes and the several varieties of leukocytes each along different lines of differentiation to different mother-cells. The later investigations into this problem of blood-cell origin-done with an admittedly superior technic - tend to show that the monophyletic view is correct. This was first well supported by the work of Saxer, who gave the name

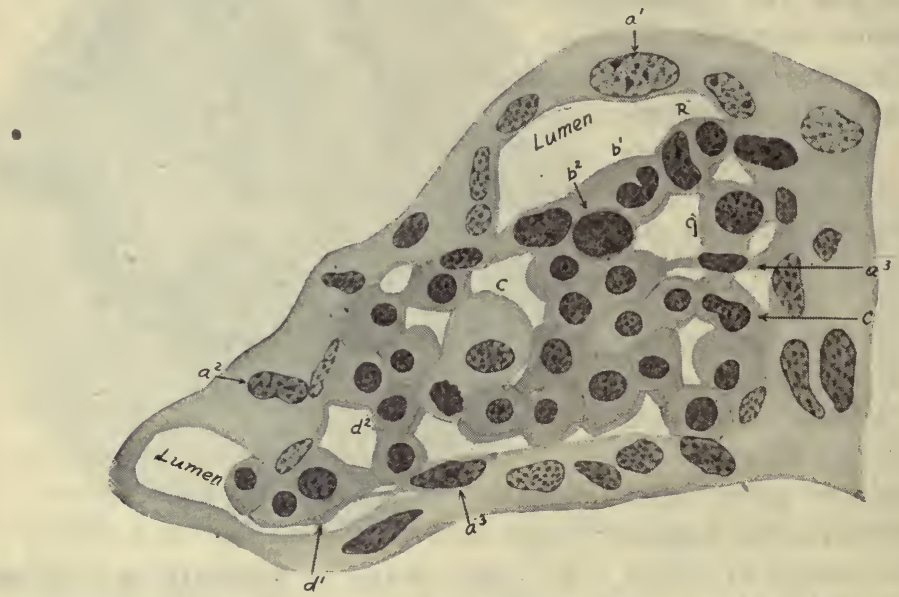

Fig. 230.-Large Blood Island from (Fig. 210), a 13 mm. Human Embryo.

$a^{1}$ and $a^{2}$, mesenchymal cells; $a^{1}$ is differentiating into an endothelial cell; $a^{3}$, endothelial cells becoming separated from wall to form hemoblasts ('lymphocytes'); $b^{1}$ and $b^{2}$, hemoblasts (mesameboids); $c$, megaloblasts; $d^{1}$ and $d^{2}$, normoblasts; $R$, binucleated giant cell. $\times 750$.

'primary wandering cell' to the common blood-mother cell; more recently by the work of Maximow, who applies the name 'lymphocyte' (primary lymphocyte) to this cell, on the ground of its resemblance to the large lymphocyte of adult blood. This lymphocyte (hemoblast) is according to Maximow originally a cell of the general mesenchyma, the earliest differentiation stages of which are characterized by increase of basophily of the cytoplasm, separation from the general syncytium, and assumption of ameboid properties. These steps involve blood-island stages.

We may now trace the steps through which the primitive blood-cell passes in the process of attaining the adult condition of the various types of cells. Of two daughter-cells one differentiates into an erythroblast; the other may remain as a primitive blood-cell to further function as a mothercell; at the same time primitive blood-cells may continually differentiate in adult life from the mesenchyme of bone-marrow. An alternative view 
accepted by some, e.g., Minot,-and still in accord with the monophyletic teaching-regards the later embryonic, fetal and adult hemopoietic organs as simply foci of proliferation and differentiation of primordial blood-cells (hemoblasts) originally derived from the angioblast and subsequently carried by the blood stream to these locations (red marrow, etc.) where they persist throughout life.

We will follow first the several steps in the developmental process of the red element, to which taken together the name 'erythrocyte' may be appropriately applied. According to Maximow, in the forms studied by him, there are two lines of erythrocytes, a primitive and a definitive line; the primitive line dies out after a brief span, and, since the definitive line differs only in the matter of smaller size of cells, we may confine our attention to that line.

The several stages in the differentiation process are: (1) megaloblast; (2) normoblast; (3) erythroblast and (4) erythroplastid ('erythrocyte'). All except the last may multiply by mitosis. The megaloblast differs from its progenitor, the hemoblast, in having somewhat more cytoplasm, and a small amount of hemoglobin, which gives it a light reddish-brown color. Its nucleus is relatively large and vesicular and contains a delicate granular network; this stage corresponds to the ichthyoid stage of Minot; a seemingly appropriate terminology, since it calls attention to the similarity between the adult (phylogenetic) stage of a lower form, and the embryonic (ontogenetic) stage of a higher form. The normoblast is a daughter-cell of the megaloblast and is of somewhat smaller size, containing more hemoglobin, with a somewhat denser nucleus, with larger net-knots; this corresponds to the sauroid stage of Minot, the significance of which term is the same as explained above for ichthyoid. The erythroblast differs from the normoblast in having an increased amount of hemoglobin, but mainly in its smaller, more compact nucleus. This cell becomes an erythroplastid through loss of nucleus. (It conduces to simplicity and clearness to regard the megaloblast and normoblast as two developmental phases of the erythroblasts.) The manner by which the nucleus disappears has been much disputed. It has been held to be absorbed (karyolysis); Howell has described its extrusion either as a single or fragmented body (karyorrhexis) in the cat; Emmel (Amer. Jour. Anat., 16, 2, 1914) has recently described for pig a process by which the red corpuscle arises through budding, leaving a nucleated remnant.

Certain erythroblasts may be smaller than the average, others larger; these dimentionally atypical forms are termed respectively microcytes and megalocytes.

The large lymphocyte of the blood is believed to be derived from the primitive blood-cells by slight differentiation; perhaps it represents a potential hemoblast. The small lymphocyte is simply a daughter-cell of a large; a large, a grown small lymphocyte. 
The primitive blood-cell may by slight differentiation become a leukoblast; according to whether the cytoplasm elaborates neutrophilic, acidophilic, or basophilic granules, it becomes a polymorphonuclear neutrophil, eosinophil, or basophil ('mast-cell') leukocyte. The transition stage, from
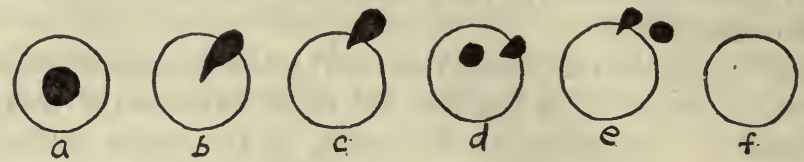

Fig. 231.-Diagrammatic Illustrations of Successive Stages in the Transformation of the Mammalian Erythrocyte (a) to Form the ErythroPLASTID $(f)$.

$b$ and $c$, by extrusion of the nucleus as a whole; $d$ and $e$, by extrusion of the fragmented nucleus.

the standpoint of the nucleus, between the leucoblast with spherical nucleus and the granulocyte with polymorphous nucleus, is the large mononuclear leukocyte (transitional leukocyte). According to Kyes (1915), certain large mononuclear leukocytes are derived from the reticulum of lymph nodes. Both the reticular cells and their leukocyte derivatives are said to be phagocytic.

Giant cells are derived from the leukoblast, or perhaps primitive bloodcell, along a separate line of differentiation, characterized by absence of cy-
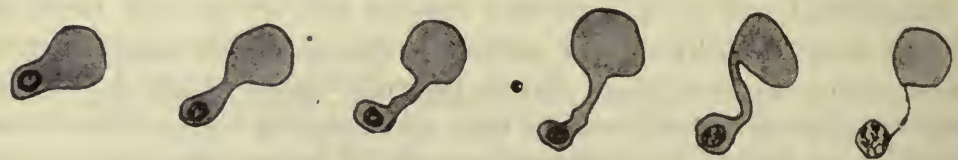

Fig. 232.-Successive Stages in the Elimination of the Erythroblast Nucleus, from Homoplastic Cultures of Blood of a 32 m. Pig Embryo.

This is regarded by Emmel as a 'somewhat imperfect case of constriction,' but it illustrates the fundamental similarity between erythroplastid formation by extrusion of nucleus (Howell) and by cytoplasmic constriction. (After Emmel, Amer. Jour. Anat., 16, 2, 1914.)

toplasmic division, excessive growth, and giant or multiple nucleus. The multinucleate condition is attained apparently by both mitotic and amitotic division of the nucleus. From the megakaryocyte pseudopodia are derived the blood-platelets as explained above. Blood development in marrow passes through the same phases, the hemoblast stage here being generally known as the myeloblast, and characterized by a considerable finely granular neutrophilic cytoplasmic content. The above is given in outline in the following scheme, adapted largely from the work of Maximow on hemopoiesis in the rabbit embryo. (Arch. mikr. Anat., Bd. 73, 1909.) 


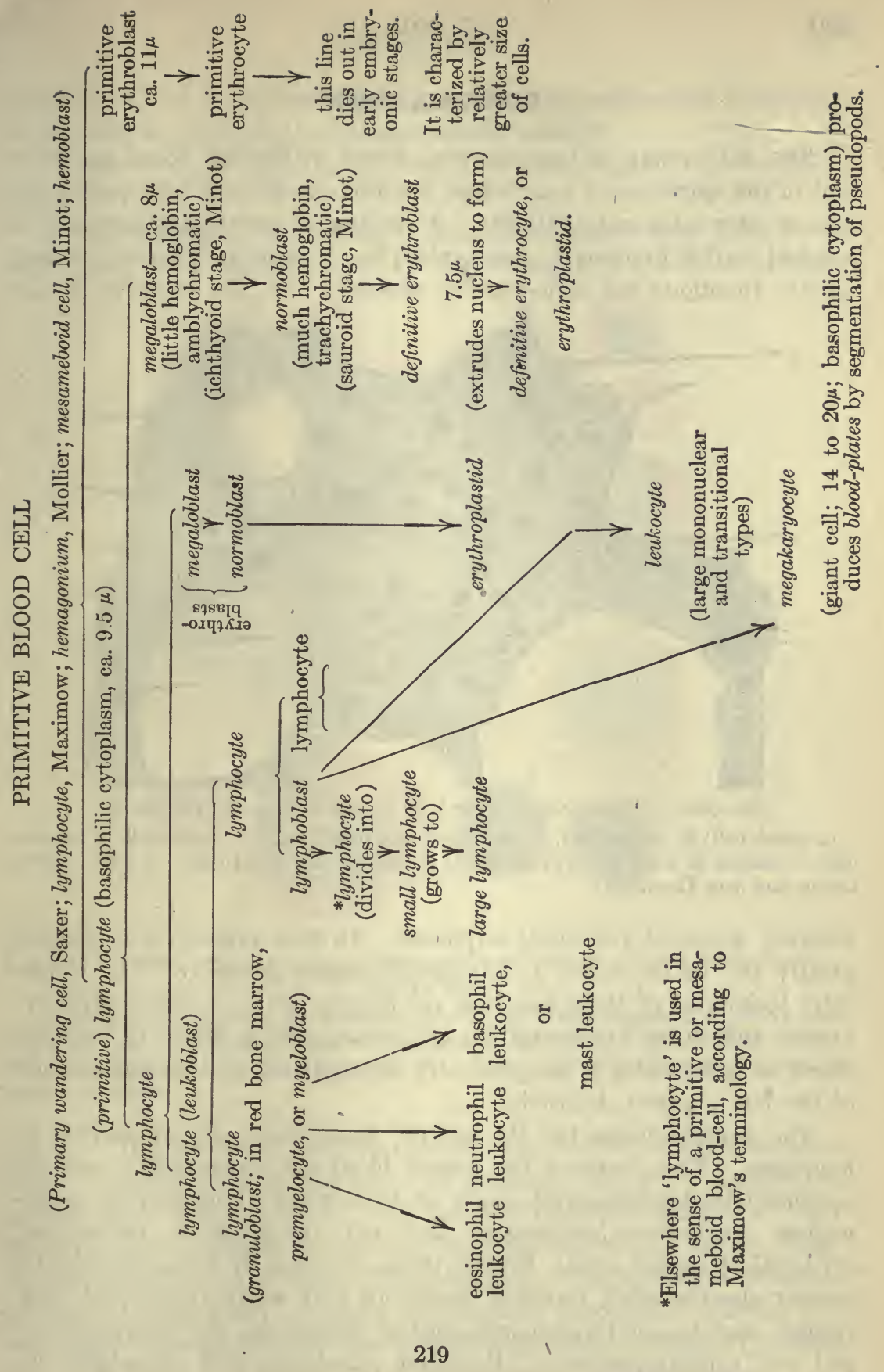




\section{BONE-MARROW}

The red variety of bone-marrow, found in the flat bones generally and in the epiphyses of long bones, functions as the sole hemopoietic organ of later fetal and adult life. According to certain authorities it is assisted in this function to some extent by the spleen. Besides a hemopoietic function, red bone-marrow possesses also the capacity of de-

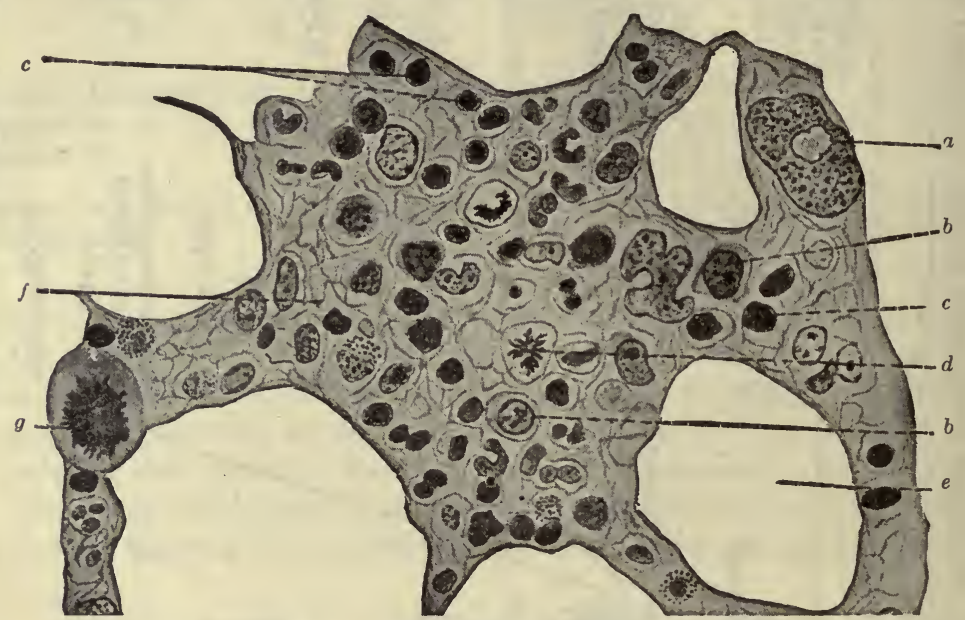

Fig. 233.-From a Section of Red Marrow of a Human Bone.

$a$, giant cell; $b$, leukocytes; $c$, nucleated red blood cells; $d$, mitosis in a marrow cell; $e$, outline of a fat cell; $f$, reticulum; $g$, mitosis in a giant cell. $\times 680$. (After Böhm and von Davidoff.)

stroying worn-out red blood-corpuscles. In this process it is assisted greatly also by the spleen and lymphoid organs generally. The phagocytic leukocytes of these locations are largely active in this destructive process and in the transportation of the hemoglobin débris to the liver where as hematoidin it is apparently appropriated in the manufacture of the bile pigment, bilirubin.

The generic name for the specific hemogenic cells of marrow is myelocyte. This includes the parent blood-cell (hemoblast) and the intermediate developmental stages of both white (lymphocytes, mononuclear leukocytes-leukoblasts) and red (megaloblast, normoblast, erythroblast stages) cells. Besides these, there are of course abundantly present also the adult forms of blood-cells both white (eosinophil, neutrophil, and basophil polymorphonuclear leukocytes, and lymphocytes) and red (erythroplastids). Potential osteoblasts and osteoclasts are 
also present, but indistinguishable from the lymphocytes and hemogenic polykaryocytes respectively unless specially stained.

A description of the cells in their order of hemogenic cytomorphosis follows :

1. Myeloblast (Premyelocyte; Hemoblast, Mesameboid Cell; Primitive Blood Cell; 'Lymphocyte'):-This is the parent blood-cell of bone-
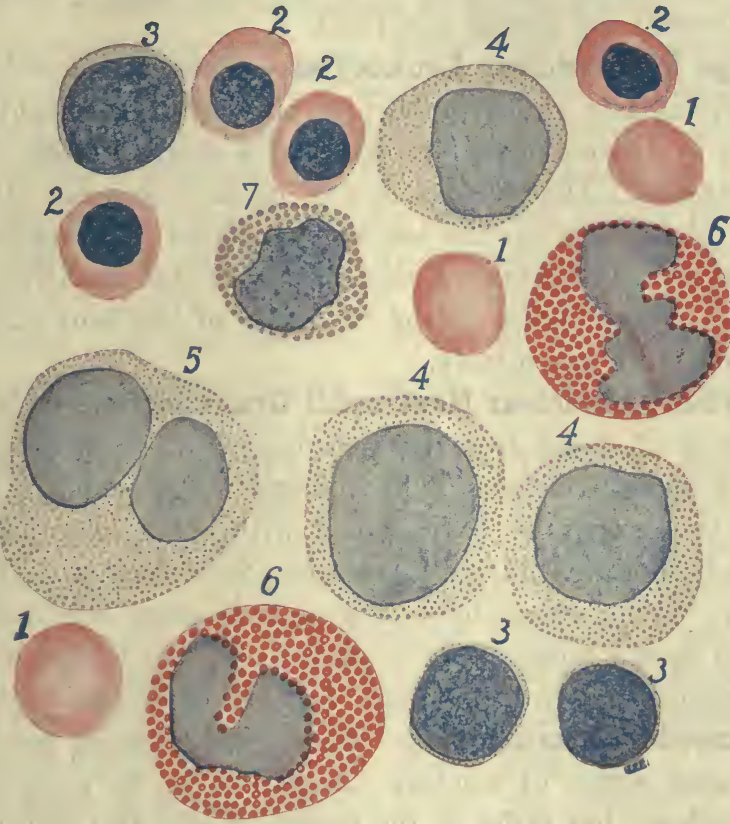

Fig. 234.-Types of Cells from a Smear Preparation of the Marrow of a Human Rib.

1 , red blood corpuscles; 2 , nucleated red blood cells, erythroblasts; 3 , small lymphocytes; 4, large mononuclear cells with neutrophil granules; 5 , polynuclear neutrophil; 6, eosinophil cells; 7, a basophil cell. Eosin and methylene blue. Nocht's stain. $\times 1200$.

marrow. It produces by proliferation and slight differentiation the more direct ancestors of both the white (leukoblast) and red (megaloblasterythroblast) cells. Mother- and daughter-cells are similar in their relatively large granular nuclei, well developed cell-body, ameboid capacity, and proliferative activity. The cytoplasm of the myeloblast is homogeneous or very finely granular and slightly basophilic; likewise the leukoblast. The megaloblast may be distinguished by its slightly oxyphil character due to the presence of a small amount of hemoglobin. 
2. Lymphocytes.-These include larger and smaller varieties. They are similar to, and in part identical with, the above-described cells, the myeloblasts. They include both cells differentiated from marrow and cells transported by the blood stream. They are indistinguishable from the osteoblast. In short, these several cells of similar characteristics may be identical in their capacity to function as progenitors of blood-cells, and may represent the parent blood-cell.

3. Large Mononuclear Leukocytes.-These cells are distinguished from the myeloblasts and lymphocytes only by their slightly modified nucleus, and by the occasional presence of a small number of fine neutrophilic granules. The nucleus is oval or bean-shaped. It represents a transition phase between the less differentiated lymphocyte stage and the granulocytes. It contains a centrosome located usually in that portion of the cytoplasm lying in the concavity of the bent nucleus. This cell may proliferate extensively.

4. Polymorphonuclear Neutrophil Granulocytes.-These include a graded series of stages from the standpoint of shape of nucleus, and amount of granular content. The younger stages contain a less complicated nucleus, generally bean-shaped with centrosome and relatively fewer (some basophilic, 'unripe') granules; these have a slight proliferative capacity. Older stages have progressively more complicated nuclei, characterized by a lobulated chain (with from two to five segments) of dense chromatin, and are no longer capable of mitotic division.

5. Eosinophil Granulocytes.-These cells resemble the neutrophils in their graded series of nuclear forms, ending in a lobulated polymorphous nucleus; but differ in the matter of size and staining capacity of the cytoplasmic granules. These granules are of fairly uniform size and of spherical shape, but much larger than the neutrophilic granules, and are strongly acidophilic, showing special affinity for the cytoplasmic stain, eosin. In the younger forms the granules are basophilic. The older types are identical with those of the blood. Again, only the less differentiated, that is, those with oval nucleus and a centrosome, may divide mitotically.

The commonly accepted view of the origin of neutrophilic and oxyphilic cytoplasmic granules is that they arise intracellularly under nuclear influence, perhaps from less differentiated nuclear extrusions or chromidia. Originally these granules are basophilic ('unripe'). However, Weidenreich regards the eosinophil granules as the ingested hemoglobin particles of degenerating and fragmenting erythroplastids. Their considerable presence in red marrow and spleen where blood-cell disintegra- 
tion is extensive, lends weight to this hypothesis. Likewise Badertscher (Amer. Jour. Anat., 15, 1, 1913) finds that eosinophil leukocytes and free eosinophilic granules are very abundant in the vicinity of degenerating muscles in salamanders during the time when the gills atrophy, and believes that the eosinophilic granules are ingested fragments of degenerated muscles and red blood-corpuscles. Eosinophils do undoubtedly increase greatly in number during certain infectious diseases characterized by extensive tissue disintegration, e.g., trichiniasis; but disproof of intracellular origin-a teaching more in accord with our knowledge of cytoplasmic granule origin through protoplasmic activity-demands direct evidence of extensive granular ingestion, which is lacking. Moreover, the microchemical nature of eosinophil granules differs from that of hemoglobin; also, they differentiate from basophilic granules and in hemogenesis in the turtle, for example, eosinophils appear before hemoglobin-containing cells are present. The free eosinophil granules in degenerating tissues are more likely derived from disintegrating eosinophils, abundant in such regions.

6. Basophil Granulocytes (Mast cells).-These are identical with the mast leukocytes of the blood, and possibly also with the cells of this name in connective tissue, the latter perhaps representing degenerating phases of the former. They are characterized by a variable polymorphous nucleus, apparent lack of centrosome, extremely slight proliferative capacity, and presence of spheroidal non-uniform basophilic cytoplasmic granules. They are numerically increased in marrow and the circulating blood and in the spleen in certain diseases.

7. Giant Cells or Myeloplaxes.-These are relatively enormous cells (of 30 to 100 microns diameter). They may have either a single large, frequently lobulated annular, nucleus (megakaryocyte) or several, even many, nuclei (polykaryocyte). The megakaryocyte is a derivative of the lymphocyte (myeloblast). The polykaryocyte represents a later modification of the megakaryocyte, the lobulated nucleus having become broken up into separate nuclei. These polykaryocytes ('hemogenic giantcells') have been erroneously regarded as identical with the multinucleated osteoclasts. They are in fact potential erythroblasts, comparable to 'blood-islands,' with only slight or no phagocytic capacity. After Wright's technic the cytoplasm of these cells presents fine purple granules. Osteoclasts do not contain such 'metachromatic' granules. The polykaryocyte of the yolk-sac of the 10-millimeter pig embryo can be seen to differentiate into erythrocytes, a hemoglobin-containing area developing about the several nuclei, the whole finally breaking up into an equal num- 
ber of red cells. Giant cells are characteristic elements of all hemopoietic organs. The megakaryocytes protrude long pseudopodia which segment into blood-platelets (Wright), abundantly present in red marrow.

8. Erythrocytes.-These cells include the several developmental forms of red cells: (a) megaloblast, (b) normoblast, (c) erythroblast and (d) erythroplastid. The megaloblast is very similar to the leukoblast except that the cytoplasm contains a slight amount of hemoglobin, and therefore gives an oxyphilic staining reaction. The nucleus is granular, with a delicate chromatic network. This is the so-called ichthyoid stage of Minot. Normoblast and erythroblast are closely similar stages (sauroid stage of Minot), characterized by the relatively smaller and denser more chromatic nucleus, and a relatively more extensive shell of cytoplasm with increasingly more hemoglobin. The erythroplastid develops from the erythroblast through loss of nucleus, generally by extrusion.

Evans (Amer. Jour. Physiol., 37, 2,1915) has directed attention anew to the phagocytic large mononuclear leukocytes, the 'macrophages' of Metschnikoff (1892). On the basis of a specific response to vital azo dyes he identifies them as a group distinct from the large mononuclear elements and lymphocytes of the blood, and includes among them certain endothelial and reticular cells and the 'clasmatocytes' (Ranvier) or 'resting wandering cells' (Maximow) of connective tissue, all of which may become free macrophages. These cells in mammals, of round or elongated shape, range in diameter from about 10 to 30 microns; they contain a stoutly cresentic nucleus of irregular contour and excentric position; the cytoplasm is weakly basophilic, covered with delicate pseudopods of various sizes, and frequently filled with large vacuoles. As endothelial cells they may line the capillaries and venules of the liver ('von Kuppfer cells'), spleen, red bone-marrow, hemal glands, and the lymphatic sinuses of lymph nodes. They include also reticular cells of lymph nodes, spleen pulp. and bone-marrow, and the clasmatocytes of connective tissue. As free cells they occur in the serous cavities, lymph sinuses of lymph nodes, spleen and hepatic capillaries. They are abundant in transudates and exudates in serous cavities. Only under pathological conditions do they appear in the peripheral blood stream. Weidenreich identified them with the large mononuclear leukocytes of the blood and lymph. Evans' experiments, on the contrary, give no indication that leukocytes are converted into these cells. They originate chiefly from endothelia. They are phagocytes and are active also in the normal physiological processes. They handle the blood and bile pigments, and fats and lipoids. The protoplasm of the macrophages is characterized especially by its response to finely particulate matter. Macrophages share the function of phagocytosis with the polymorphonuclear elements of the blood. 


\section{CHAPTER IX}

\section{THE LYMPHATIC SYSTEM}

The lymphatic series includes a system of lymphatic channels which collect the lymph from the various tissues of the body and return it to the large veins of the neck, where it mixes with the blood. In the course of this lymph vascular system are various aggregations of lymphoid or adenoid tissue which occur in the form of lymph nodules or follicles, lymph glands or nodes, and the lymphoid organs. These organs are the tonsils, thymus, and spleen. The lymphatic vessels also stand in intimate relation if not in direct communication with the serous and synovial membranes and the bursæ.

\section{LYMPH}

Like the blood, the lymph may be considered as a primary tissue whose intercellular elements are entirely of a fluid nature. In most portions of the body, lymph is a colorless fluid which is scantily provided with corpuscular elements, the lymphatic corpuscles. The lymphatic corpuscles are identical with the leukocytes of the blood. In the lymph most of these cells are of the mononuclear form, the small mononuclears or lymphocytes being the most abundant. Lymph also contains a small proportion of polymorphonuclear cells, which not only are derived from the lymphoid tissues, but as wandering cells find their way into the 7ymphatic vessels from the tissues generally. Blood-platelets are not present in lymph (Howell); prothrombin is liberated by the lymphocytes.

The cells of lymph, predominantly of the small lymphocyte type, are derived from the numerous lymphoid masses (nodes and nodules) through which the lymph passes on its way from the tissues to the subclavian veins. According to Davis and Carlson (Amer. Jour. Physiol., rol. 25, 1909) the number of lymphocytes contributed to the blood daily may be more than the total permanently present in the blood. Since the number in a cubic millimeter remains fairly constant, a number must 
be daily consumed in the body equal to the number added to the blood. Many of course actually suffer destruction, but it seems probable that a considerable number also first undergo differentiation into granulocytes, and perhaps as potential hemoblasts may function as parent cells of erythrocytes.

In addition to the leukocytes lymph contains fat globules and glycogen. These are mostly the products of absorption from the intestinal tract, in which process the lymphatic vessels play an important rôle. In the lymphatic vessels of the intestine during absorption fat globules are so abundant as to impart to the lymph a milky white color; this variety of lymph is termed the chyle. These fat globules are rapidly removed by the lymphoid organs, since even in the presence of abundant chyle only comparatively few fat globules escape into the general blood current. The lymph of other portions of the body than the abdominal region, therefore, contains relatively little fat.

The lymph, unlike the blood, circulates in but one direction, viz., toward the heart. It must therefore be formed in the tissues generally. The blood plasma constantly escapes through the walls of the capillary vessels into the surrounding lymphatic spaces of the tissues. It is these tissue spaces which have been considered as forming the beginning of the lymphatic system. Recent evidence, however, goes to show that the tissue spaces are not directly connected with the lymphatic vessels, but that just as the plasma exudes into the tissue spaces by processes of secretion, osmosis, and filtration, so the tissue juices, as the predecessors of lymph, enter the lymphatic vessels by similar processes of secretion, osmosis, and filtration. Lymph is also formed by absorption, which occurs chiefly in the alimentary tract.

Under favorable conditions the lymph will coagulate, though more slowly than blood, the fibrin forming a firm, colorless clot in which the leukocytes are entangled. Because of their tendency to adhere to the sides of the vessel-thus circulating at the periphery of the currentthe lymph cells are most likely to be found at the periphery in those: post-mortem clots which occur within the lymphatic vessels.

\section{LYMPHATIC VESSELS}

\section{(Lymphatics)}

The lymphatic vessels vary in size from that of the smallest capillary vessels up to that of the thoracic duct. The smaller vessels, lymphatic 
capillaries, form anastomosing meshes in all tissues where blood capillaries are found. They are most abundant in the perivascular connective tissues, where they form a dense plexus about the wall of the blood-vessels.

The wall of the lymphatic capillary, like that of the blood capillary,

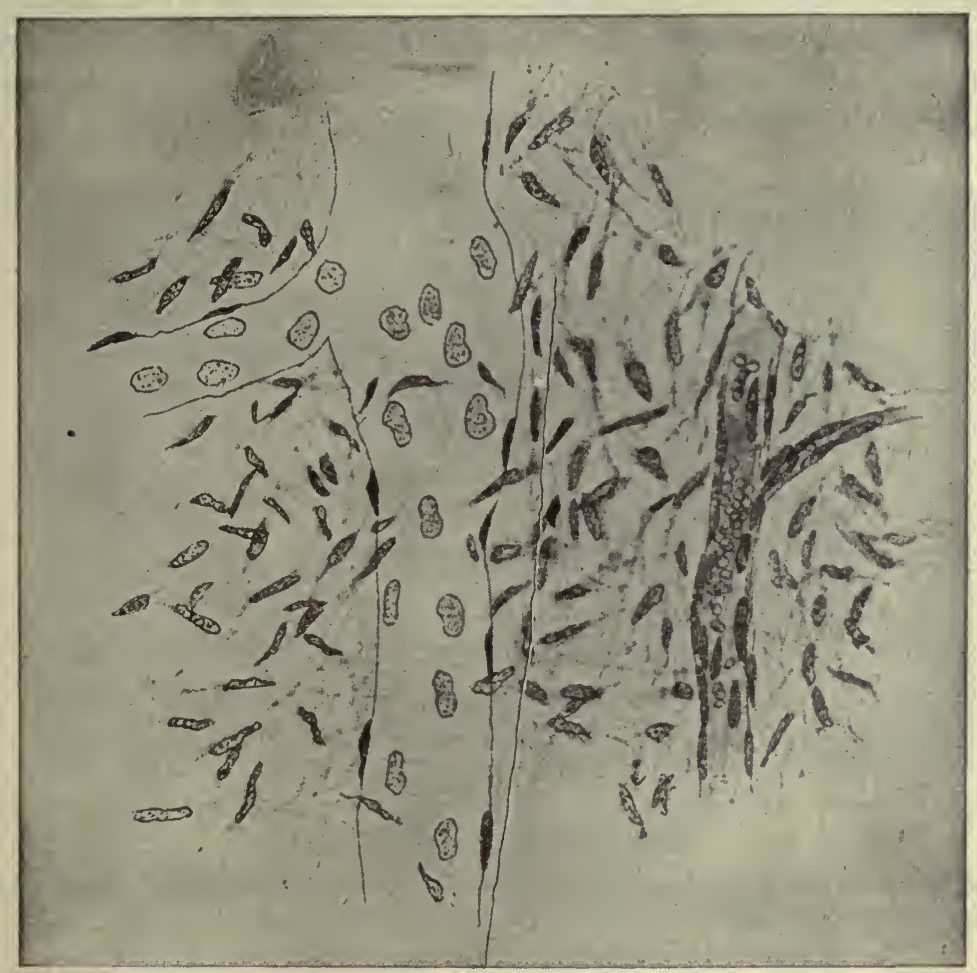

Fig. 235.-Subcutaneods Lymphatic Vessel of a Fetal Pig.

At the right is a small blood-vessel. Hematein and eosin. Highly magnified. (After MacCallum.)

consists of a single layer of endothelium. This endothelium probably forms a complete lining for the lymphatic capillary and is continuous through larger and larger vessels with that of the veins, from which, according to Sabin (Amer. Jour. Anat., 1902), the lymphatics are originally developed.

The relation of the lymphatic capillaries to the tissue spaces is not as yet definitely settled. It was formerly thought that these spaces were 
continuous with the lymphatic capillaries, but the more recent observations, represented by those of MacCallum (Johns Hop. Hosp. Bull., 1903), seem to show that the capillaries of the lymphatic system, like those of the blood vascular system, form a series of branching channels which are open only toward the veins. According to this conception, therefore, the tissue juices, formerly also considered as lymph, are contained within a separate series of channels, the tissue spaces and lymphatic canaliculi, and they enter the true lymphatics only by processes of osmosis and the secretory activity of the lymphatic endothelia.

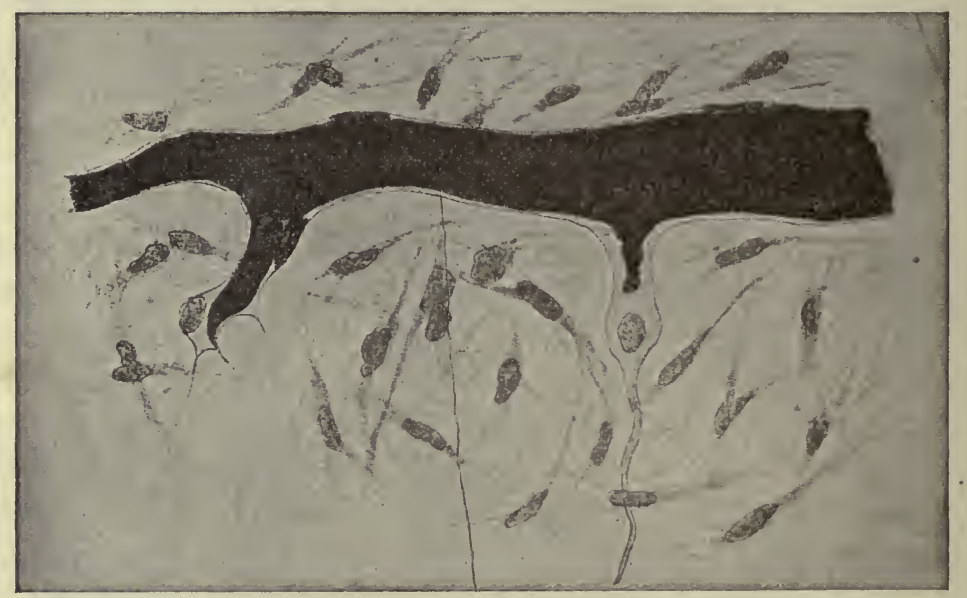

Fig. 236.-The Growing End of a Developing Lymphatic Vessel in the Subcutaneous Tissue of a Fetal Pig.

The lumen of the vessel has been filled with a dark injection mass. Highly magnified. (After MacCallum.)

The lymphatic capillaries are of rather irregular caliber, generally greater than that of blood capillaries, and possess frequent sinus-like dilatations, which peculiarity is also characteristic of the larger lymphatic vessels.

The lymphatic capillaries soon acquire an adventitial sheath of fibroelastic tissue and pass into the smaller lymphatic vessels. On attaining a size of from 0.2 to 0.8 millimeter their wall is differentiated into the same three coats which are found in the veins. Except for the fact that they contain lymph instead of blood, these vessels closely resemble the small veins, and like some of the latter vessels they possess frequent valves. 
The tunica intima of the lymph vessel consists of an endothelial lining with a thin delicate fibro-elastic membrane. The tunica media is thin and contains circular smooth muscle fibers. The adventitia is the thickest coat of the lymph vessel. It consists of fibro-elastic connective tissue and longitudinally disposed bundles of smooth muscle fibers.

The wall of the lymph vessels is supplied with small blood-vessels and nerves, in the same manner as the veins. The nerves form a plexus in

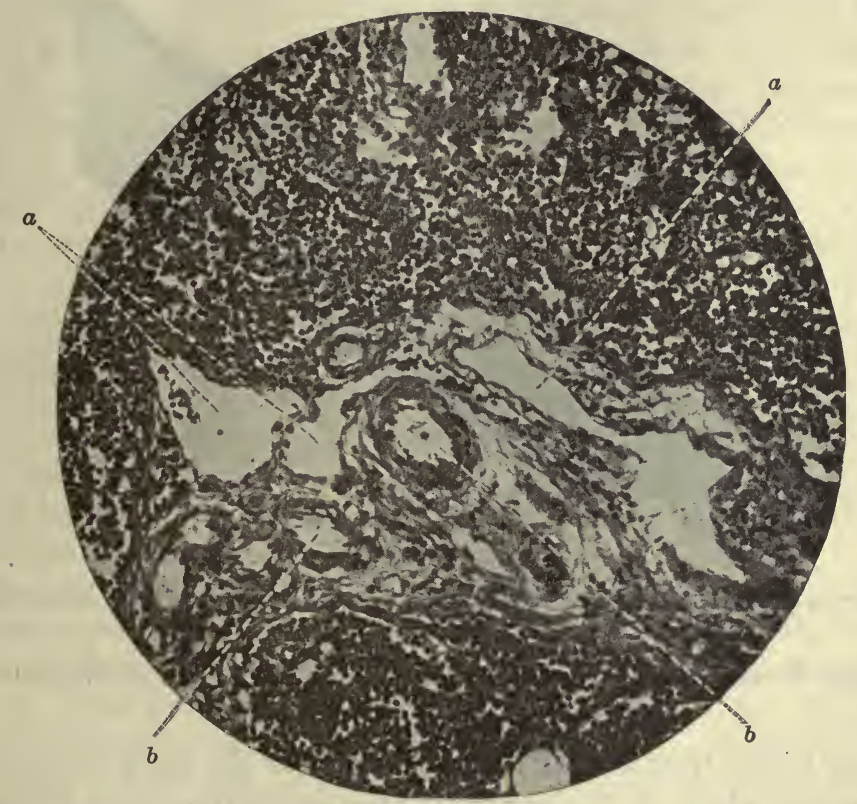

Fig. 237.-Lymphatic and Blood Vesseld in the Hilum of a Human Lymph-node.

$a$, lymph vessels; $b$, blood-vessels. Hematein and eosin. Photo. $\times 160$.

the adventitia from which branches are distributed to the media and intima. Kytmanof (Anat. Anz., 1901) has traced the fine nerve fibrils to the smallest lymphatic capillaries, where, as in the intima of the larger vessels, they end in close relation to the endothelial cells.

To summarize: the lymphatic capillaries arise by one of three methods :

1. As lymphatic plexuses in all connective tissues; the most abundant of these are the perivascular lymphatics.

2. As dilated pouches having, blind extremities, as in the villi of the small intestine, where they are known as lacteals. 
3. By direct communication with the stomata of the serous membranes. The presence of true stomata in the serous membranes of man with the exception of possibly certain portions of the peritoneum is disputed.

The lymph is derived from the tissue juices and by absorption from the alimentary tract, and is conveyed by the lymphatic capillaries to

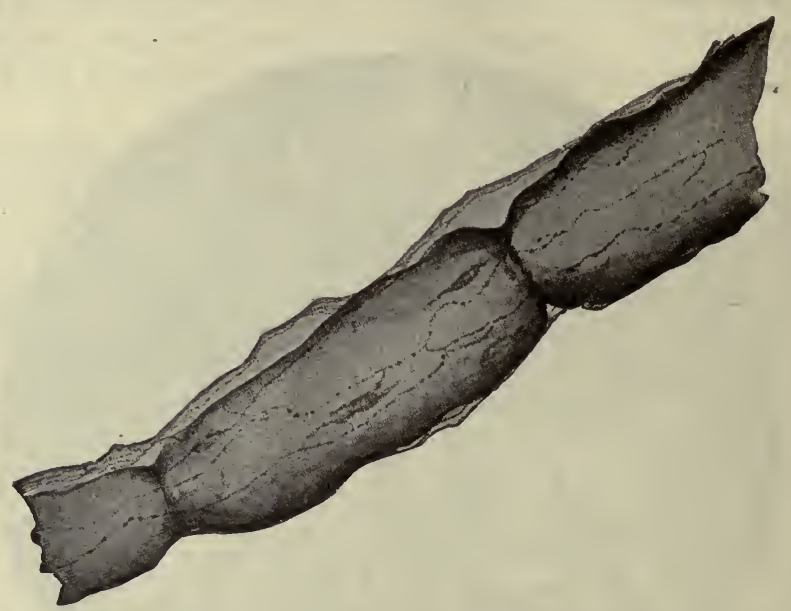

Fig. 238. - Lymphatic Capillary from the Spermatic Cord of a Dog, Showing Nerve Endings.

a, nerve fibers. Methylene blue. Highly magnified. (After Kytmanof.)

larger and larger lymph vessels, which resemble the small veins in their structure, and which finally empty into the subclavian veins of the neck, at their junction with the internal jugulars.

The main lymph channels are the thoracic duct on the left, and the right lymphatic duct. Only the thoracic duct drains the abdominal lymphatics and is thus much the larger vessel. Toward its distal end it expands into a receptacle for the absorbed chyle, the cisterna chyli.

\section{THE DEVELOPMENT OF LYMPH VESSELS}

According to Sabin the unammalian lymphatic system has its primary origin in two paired and one unpaired venous sprouts: the jugular, inguinal (sciatic), and mesenteric (retroperitoneal) lymph sacs. Certain investigators (Huntington, Mem. Wistar Inst., 1911; McClure, Anat. Rec., 6, 6, 
1912, and others) interpret these sacs as the products of fusions of still more primitive discrete lymphatic anlages which arose as mesenchymal spaces; and their connection with the subclavian, sciatic and renal veins as secondary unions. Sabin 'and others regard the lymphatic endothelium once having sprouted froni the venous endothelium as strictly specific, and the entire lymphatic system as a derivative by sprouting and fusion of these three sets of anlages.

Huntington and McClure derive the definitive lymphatic system by a progressive fusion of isolated mesenchymal spaces (mainly in the extraintimal portion of disappearing veins) and cells in the paths of the future lymphatic trunks. According to the one school lymphatic endothelium can arise only by proliferation of preëxisting endothelium; according to the other, endothelium can continually differentiate from young mesenchyma.

The recent observations of Clark (Anat. Rec., 3, 4, 1909) on the growing lymphatics in the tail of living frog tadpoles where the process of sprouting could be clearly followed, leaves no room for doubt that lymphatics spread through sprouting, but the material and data give no information as to the manner of origin of the initial anlages, which is the real question at issue. It is perhaps as yet too early to decide the matter on the basis of available evidence, but the extensive histologic data of Huntington and McClure strongly support their claim of primary lymphatic origin by confluence of isolated mesenchymal spaces.

The beautiful injections of Miss Sabin which show a progressively enlarging continuous system in pig embryos apparently flatly contradict this hypothesis; but the objection cannot be fairly ignored that the advocates of lymphatic origin through fusion of isolated spaces base their claims on appearances before the establishment of a continuous system or even the several sets of lymphatic sacs, and the further fact that the injection method is unsuitable for revealing lymphatic anlages existing as isolated spaces. As concerns endothelium in general, Huntington (Amer. Jour. Anat., 16, 3, 1914) regards an endothelial cell as simply an adaptive form of a mesenchymal cell, 'modified in accordance with definite hydrostatic and other purely mechanical factors,' resulting from the presence of blood or lymph. On drainage of the fluid and consequent release of pressure, the endothelial cell is believed to be capable of again reverting to 'the type of the indifferent mesenchymal cell.' Kampmeier (Amer. Jour. Anat., 17, 2, 1915) presents evidence from a study of sections of the young toad embryo apparently demonstrating the primary origin of lymphatic endothelium only from venous endothelium; but the primary sacs and ducts arise by a confluence of these earlier discrete venous buds. A concise discussion and summary of this subject is given by McClure (Anat. Rec., 9, 7, 1915.) 


\section{THE SEROUS MEMBRANES}

The serous membranes form closed sacs which line the great cavities of the body and are reflected over the viscera to form a double covering, the two layers of which are freely movable over one another. Of these two layers the one, the parietal layer, is attached to the wall of the body cavity, the other, the visceral layer, covers the surface of the inclosed organ.

The serous membranes consist of a mesothelial lining and a supporting membrane of areolar connective tissue which is richly supplied with

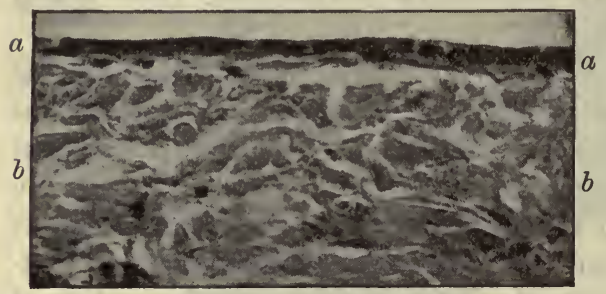

Fig. 239.-Transection of the PericarDIUM of a Child.

$a-a$, mesothelium; $b-b$, submesothelial connective tissue. Hematein and eosin. Photo. $\times 500$. capillary blood-vessels and lymphatics. The mesothelium consists of large flat cells, pavement epithelium, whose serrated margins are firmly united by an intercellular cement substance. Here and there minute openings are seen which are surrounded by very small mesothelial cells; these stomata have been found to be in certain instances directly connected with the lymph vessels.

Some regard them as transient fenestra, others as artifacts.

Tunica Propria.-The mesothelium rests upon a layer of areolar tissue which is richly supplied with small blood-vessels and lymphatics, forming an abundant vascular plexus beneath the mesothelium. The serous membrane is either directly united to the wall of the cavity and the surface of the organ which it envelops, or it may be attached by a loose layer of submesothelial connective tissue.

The thickness of the mesothelial cells varies in different portions of the serous membranes and is somewhat dependent upon the age of the individual. In most portions it is no more than a pavement epithelium, but over the surface of the functionally active ovary these cells are much thickened and acquire a cuboidal shape; thus it forms the 'germinal epithelium' of the ovary. In young individuals, viz., in fetal life and early childhood, the cuboidal cell type is found in many portions of the peritoneum, pleura, and pericardium.

The synovial membranes resemble the serous in their structure. They 
are clothed by a single layer of pavement cells which is said to be incomplete in places. This epithelium (mesenchymal epithelium) is supported upon a layer of firm fibrous tis s u e richly supplied with both lymph and blood capillaries. In the recesses of the joints the synovial membranes are frequently thrown into $\mathrm{s} \mathrm{m}$ a $1 \mathrm{l}$ villous folds, which are chiefly formed by the inner portion of the fibrous coat and are covered w i t h epithelium; these are the synovial villi.

The bursa and the synovial sheaths of the tendons are of similar structure.

Both the serous and the synovial mem-

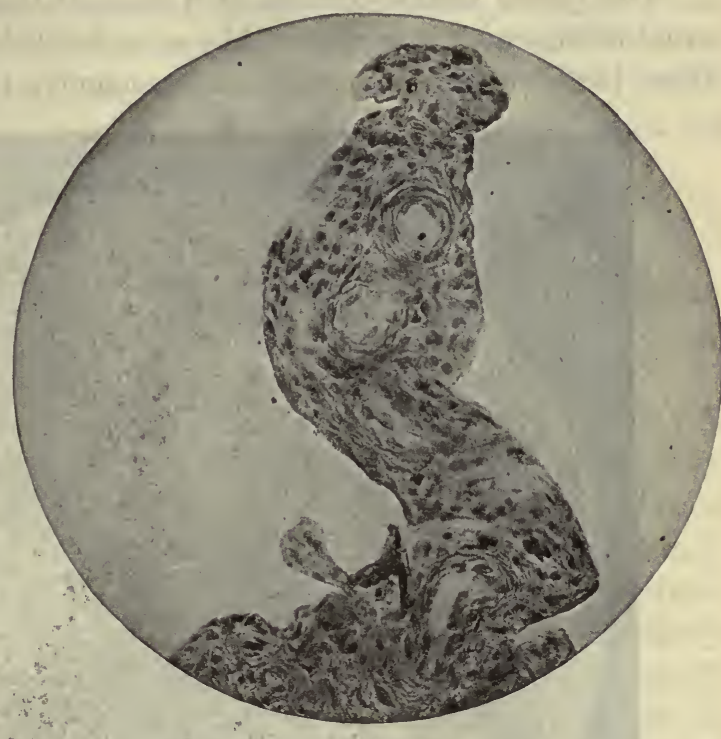

Fig. 240.-Section of a Vascular Synovial Villus from the Knee Joint of a Child.

Hematein and eosin. Photo. $\times 200$. branes are moistened by fluid which contains leukocytes in small numbers, and closely resembles the lymph and tissue juice in its composition.

\section{LYMPH NODULES}

\section{(Lymph Follicles)}

The lymph nodule is a structural unit of lymphoid tissue which may exist independently, as in the solitary nodules of the intestinal tract, or may form groups or accumulations consisting of a greater or less number of nodular units. In this latter condition they occur in the mucous membrane of the small intestine as Peyer's patches, in the tongue as the lingual tonsil, in the fauces as the faucial tonsils, in the pharynx as the pharyngeal tonsil, in the wall of the laryngeal cavity, in the spleen as the Malpighian (splenic) corpuscles, in the lymph nodes as the peripheral 
lymph nodules, and in the thymus, where we may consider the lobule of the organ as being the structural equivalent of a lymph nodule.

The lymph nodule consists of a mass of lymphoid tissue, usually of ovoid form, which is surrounded by or embedded in connective tissue. In those locations where it exists independently the nodule is completely

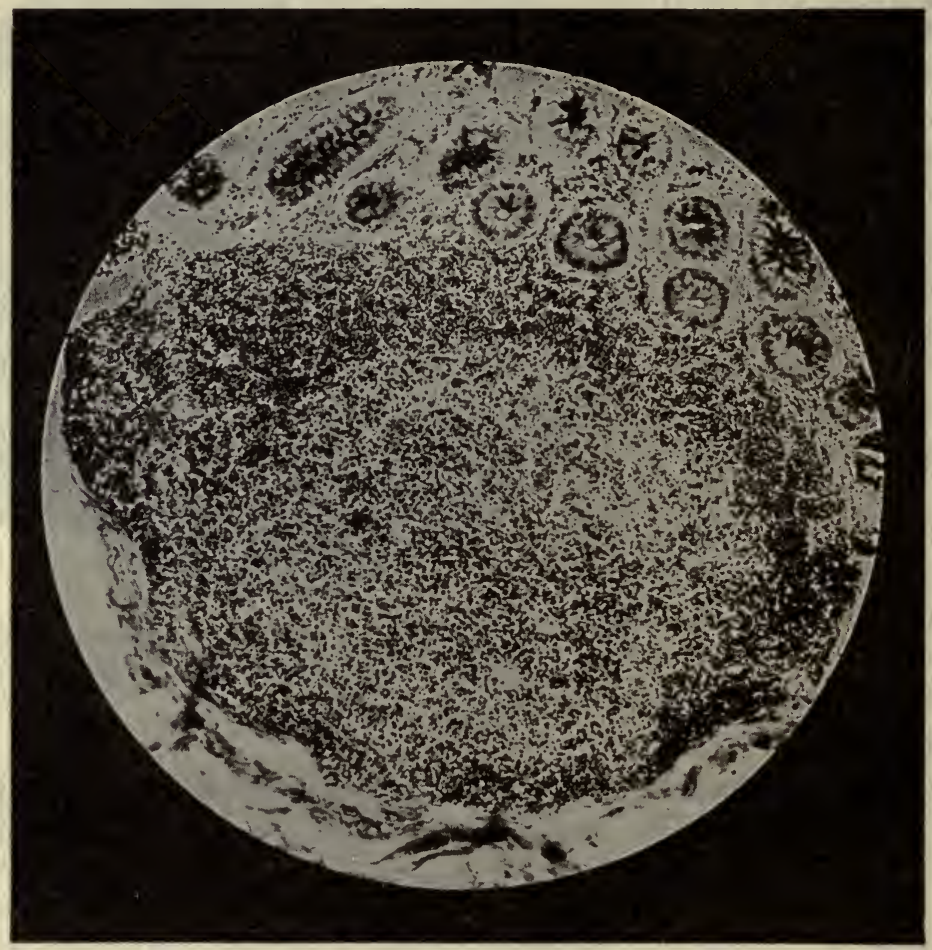

Fig. 241.-A Lymph Nodule, Solitary Follicle, from the Large Intestine of MaN.

In the upper part of the figure the edge of the intestinal mucosa is shown; it contains many secreting tubules which have been cut in transverse or oblique section and are lined by columnar epithelium and goblet cells. Photo. $\times 80$.

surrounded by the connective tissue in which it lies. In other places, as in the lymph nodes, the nodule is only partially surrounded by the connective tissue trabeculæ of the organ. Not only do fine branches from the surrounding connective tissue bundles penetrate the periphery of the nodule, but the reticulum of the nodule is continuous with these trabeculæ, thus forming a supporting stroma in which the lymphocytes are embedded. 
The lymphocytes are loosely packed in the center of the nodule, and in this portion cell division by mitosis is most active. This central portion is the germinal center of Flemming. The germinal center is surrounded by a denser circumferential layer of lymphoid tissue in which cell division is less active. Between this denser portion and the surrounding connective tissue the lymphocytes are again more loosely packed, and over a greater portion of the nodule are separated from the trabeculæ by a lacuna-like space, the peripheral lymph sinus.

The nodule is usually supplied with a thin-walled artery, occasionally two, which penetrates to the middle of the nodule to form a wide meshed capillary plexus. The capillaries, at the periphery of the nodule, unite to form two or more veins, which are contained in the adjacent connective tissue.

The lymph cells are mostly of the mononuclear type of leukocyte, the small mononuclear or lymphocyte type being the most abundant. Polymorphonuclear and eosinophil leukocytes are also found in the lymph nodules, though in much smaller numbers. Mitosis is most frequently observed in the large mononuclear type. Because of the nomadic tendencies of the leukocytes the boundaries of the lobule are not always sharp, the lymph cells frequently infiltrating the surrounding connective tissue so as to render it most difficult to distinguish the latter from the true lymphoid tissue of the nodule.

\section{THE LYMPH NODES}

\section{(Lymph Glands)}

These structures occur in the course of the lymph circulation in various parts of the body. They are found in the neighborhood of the large joints, as in the axilla, the groin, the popliteal space, in the prevertebral and mediastinal connective tissue of the abdominal and thoracic cavities, and in the mesentery. They are frequently in relation with the large arteries, e.g., the renal, internal and external carotids, etc.

Each lymph node consists of a mass of nodular lymphoid tissue inclosed within a fibro-elastic connective tissue capsule. The capsule also contains a little smooth muscle tissue, but this is never so abundant as to form any considerable portion of the fibrous membrane; in fact, as compared with the somewhat similar capsule of the spleen, that of the lymph node is notably deficient in smooth muscle. 
An afferent lymph vessel, pursuing its course within the capsule, enters the lymph node by a number of subdivisions which penetrate the deeper layers of the capsule and open into a peripheral lacunar space, the lymph sinus, which separates the inner surface of the capsule from the adjacent lymphoid tissue, but which is bridged across at frequent intervals by the fine strands of lymph reticulum.

The lymphoid tissue, which forms the substance of the node, consists of a dense peripheral

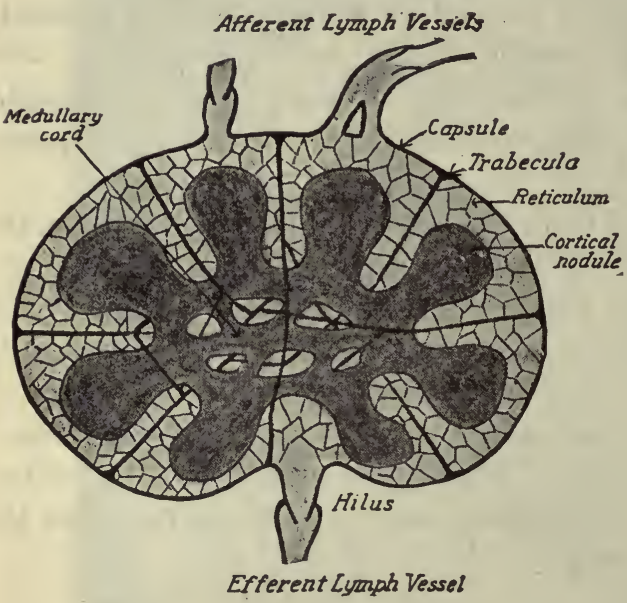

Fig. 242.-Diagrammatic Illudstration of a LympH NoDe. portion, the cortex, formed by closely packed lymph nodules, and a, looser medulla in which are columnar accumulations of dense lymphoid tissue, the lymph cords.

Cortex.-The nodules of the cortex are partially se parated from each other by septum - like trabeculæ which extend inward from the fibrous capsule, and along which the peripheral lymph sinuses are continued into the substance of the node to partially surround its lymph nodules.

Each lymph nodule is thus surrounded, except at its central pole, by a peripheral lymph sinus, into which the afferent lymphatic vessels pour their contents. The lymph on entering the gland is thus permitted to enter the spaces of the reticulum and percolate through the lymph nodules of the cortex before it can reach the looser portions of the medulla. Each of the nodules of the cortex contains a germinal center in which lymphocytes are actively formed by mitosis, and from which the lymphocytes readily escape along the lymph channels of the reticulum into the more open meshes of the medulla.

Medulla.-The medulla occupies the center of the gland, and at one point, the hilum, it reaches the surface. At this point a considerable mass of fibrous trabeculæ enters the medulla, carrying with it the larger blood-vessels to be distributed to all portions of the gland. The finer 
ramifications of these medullary trabeculæ are continuous with those of the cortex.

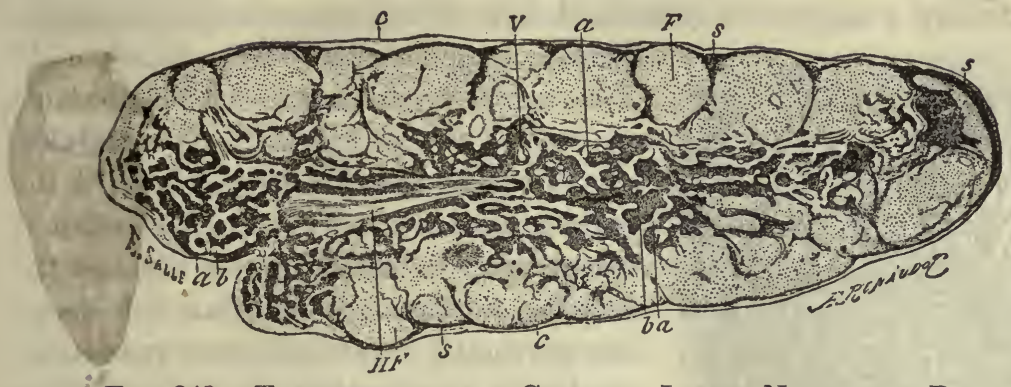

Fig. 243.-Transection of a Cervical Lymph Node of a Dog.

The denser portions of lymphoid tissue are light in the figure. $a$, medullary cord of dense lymphoid tissue; $b$, looser lymphoid tissue of the cavernous medulla; $c$, capsule; $F$, dense lymph nodule of the cortex; $H F$, fibrous tissue containing the large vessels of the hilum; $s$, peripheral lymphatic sinus; $V$, blood-vessel. Magnified several diameters. (After Ranvier.)

The lymphoid tissue of the medulla is divisible into the denser branching lymph cords, in which the lymphocytes are closely packed, and the

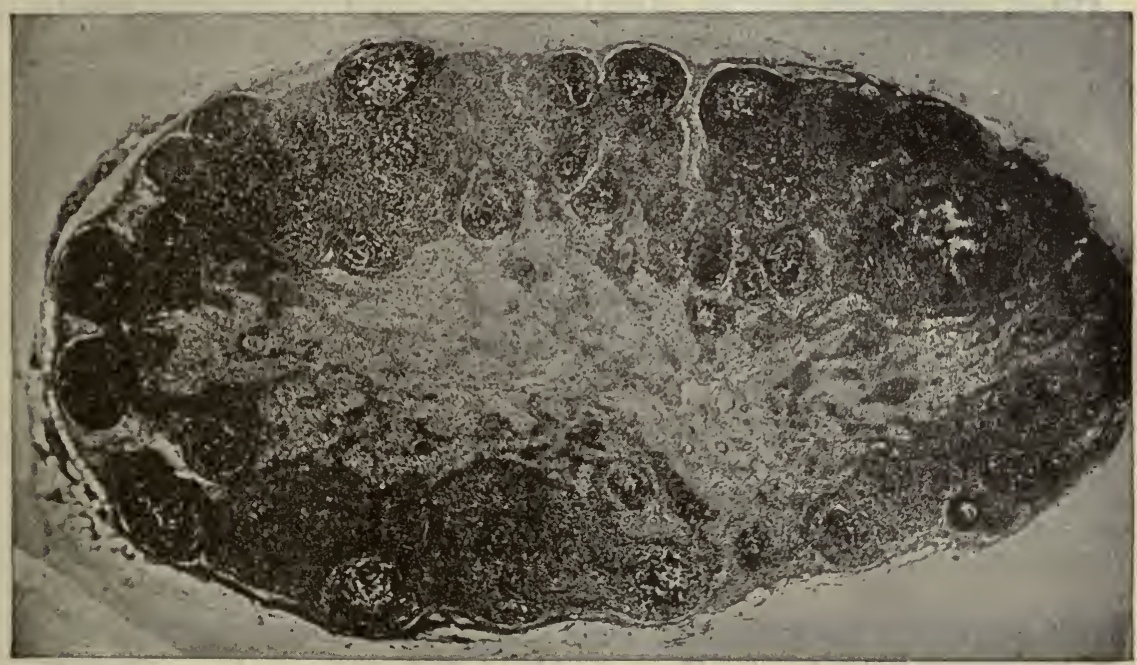

Fig. 244.-Transection of a Mesenteric Lymph Node of a Man.

Hematein and eosin. Photo. $\times 38$.

intervening pulp spaces, in which lymphocytes are less numerous, and the reticulum of which is continuous with that of the cortical nodules. 
The pulp spaces are broad channels, which are occupied by a reticulum whose meshes are partially filled with lymphocytes. They are bounded by a layer of endothelioid cells which everywhere incloses the

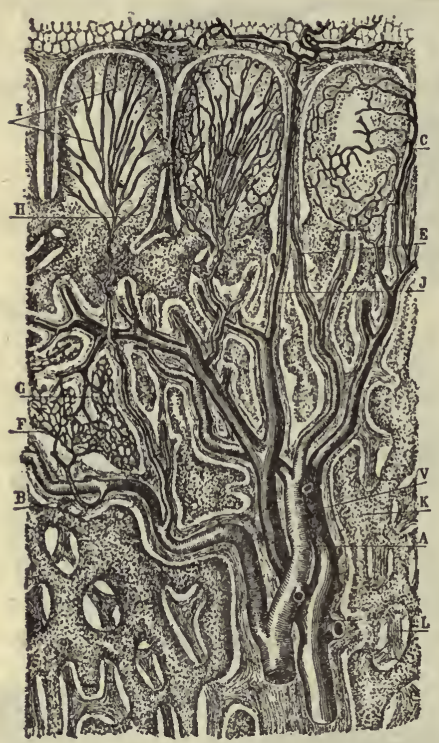

Fig. 245.-Diagram of the Blood-Vessels of a LyMPH NoDE.

A composite section of three follicles and the medullary cords of a mesenteric lymphatic node of the dog. $A$, artery; $B$, medullary artery; $C$, follicular vein; $E$, artery going to the capsule; $F$, capillaries in the periphery of a cord; $G$, medullary vein; $H$, follicular artery; $I$, arterial capillaries in a follicle; $J$, vein from capsule; $K$, cord; $L$, trabecula; $V$, vein. $\times 501$. (After Calvert.) denser lymph cords. The function of these cords would seem to be comparable to that of the peripheral lymph nodules.

The pulp spaces are open toward the cortex, whence they receive the afferent lymph after it has percolated through the nodules, but toward the hilum the spaces are continued into the efferent radicles of the lymph vessels which, in the connective tissue of this part, unite into larger trunks, and finally form several efferent lymph vessels of considerable size.

The reticulum of the lymph gland is a close-meshed network of interlacing fibrillar bundles, which are here and there clasped by flattened endothelioid connective tissue cells. Reticulum is but poorly stained with either acid or basic dyes, is destroyed by acids and bases, but is not digested by pancreatin. After prolonged action of Weigert's specific stain for elastic tissue it is but slightly colored.

Lymph Cells.-The great majority of these cells are of the small mononuclear or lymphocyte type. Large mononuclear cells with a considerable cytoplasmic body are also very numerous. Polymorphonuclear neutrophil leukocytes, though of frequent occurrence, are less abundant than the previous varieties. Eosinophil cells are present in small numbers, and large basophilic mast-cells are occasionally seen, though according to Carlier (Jour. Anat. and Physiol., 1893) they are mostly confined to the connective tissue. Drummond (Jour. Anat. and Physiol., 1900) also found large multinuclear giant cells, megakaryocytes, similar to those of the bone-marrow; these were, however, very rare.

Many of these cells, after proper fixation, show mitotic figures. This 
mitosis has been most frequently observed in the large mononuclear type, and is most abundant in the germinal centers of the nodules. The small mononuclear and polymorphonuclear types have also been shown to be capable of cell reproduction by indirect division. Reproduction by direct division of leukocytes appears to be rare, if indeed it ever actually occurs.

The mononuclear as well as the polymorphonuclear forms appear to be phagocytic. Among the inclusions which have been found within these cells are fat globules, pigment granules, red blood corpuscles in partial disintegration, insoluble pigments, such as carbon granules, etc., and bacteria. The cells of the reticulum are also believed to be phagocytic.

Blood-vessels.-The arteries enter the lymph node at its hilum, and, following the trabeculæ within which they lie, are distributed to all portions of the organ. In the medulla branches are distributed to the lymph cords, in which they form a wide-meshed capillary plexus.

The terminal branches of the primary divisions of the afferent artery are distributed to the nodules of the cortex. A single nodular branch (Calvert, Anat. Anz., 189\%) enters the nodule and passes straight toward its center, where it breaks into a plexus of divergent capillaries which unite at the surface of the nodule to form small venous radicals.

The veins follow the internodular trabeculæ in their course toward the medulla, where they enter the medullary trabeculæ, are augmented by venous radicals from the capillary plexuses of this portion of the gland, and thence follow the trabeculæ to the hilum, where they unite to form the efferent vein.

Certain of the arteries also pass from the medulla through the internodular trabeculæ to the capsule of the gland, to which they supply a capillary plexus. The blood is returned through veins which retrace the course of the arteries and enter the large veins of the medullary trabeculæ. The spleen contains no lymphatics beyond the capsule.

\section{HEMOLYMPH NODES}

\section{(Hemal Nodes)}

These structures, which closely resemble the lymph nodes, were first described by H. Gibbes (Quart. Jour. Mic. Sc.), in 1884. He found them in the connective tissue between the renal artery and vein, in the human subject. They have since been found in the prevertebral connective tissue, and in the mediastinum and mesentery. They are larger and 
more numerous in the ruminants, ox, sheep, etc., than in man. Their size varies from that of a millet seed to that of a pea. In color they closely resemble a minute extravasation of blood.

These organs are essentially lymphatic structures in which the lymphoid tissue is arranged in the form of cords rather than in nodules. The

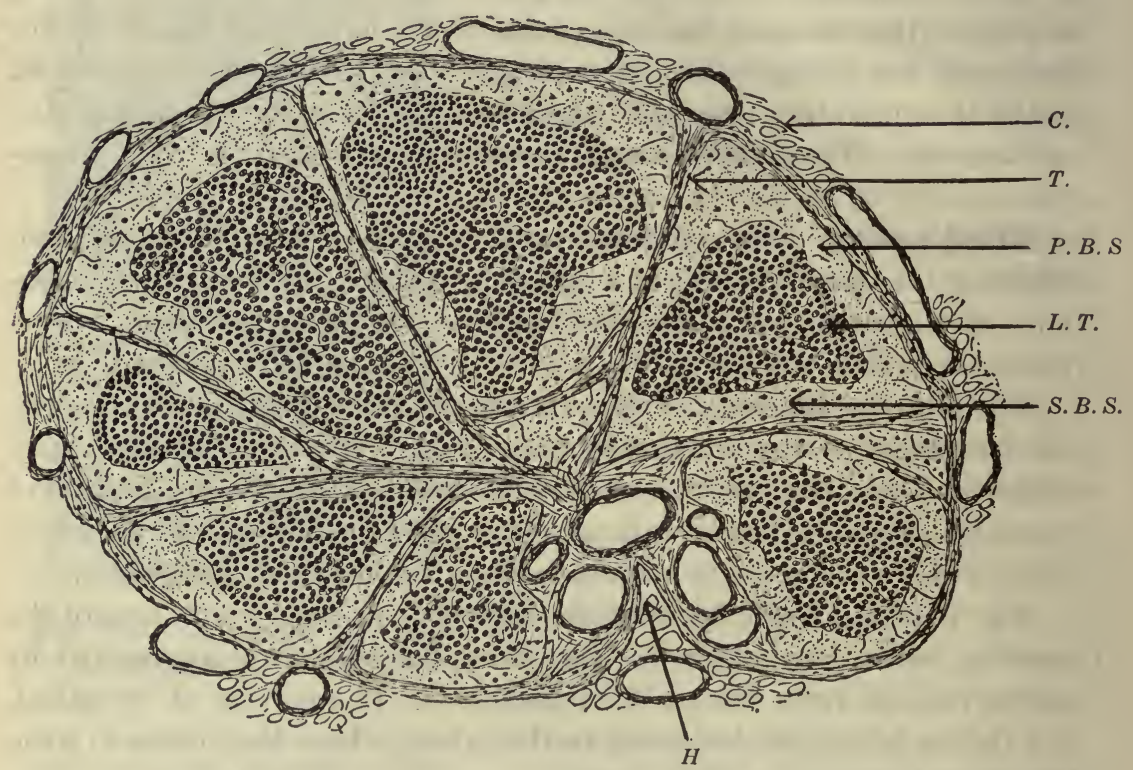

Fig, 246.-Section of Human Hemolympi Node ("Splenolymph Gland").

C., capsule; T., trabecula; P.B.S., pẹipheral blood sinus; L. T., lymphoid tissue, largely in the form of cords; S. B. S., secondary blood sinus; H, hilus. (After Warthin.)

node is inclosed by a fibrous capsule, beneath which is a broad sinus filled with blood. In this fact lies the chief distinguishing feature of these glands.

The peripheral blood sinus, which is analogous to the peripheral lymph sinus of a lymph node, sends into the interior of the organ a greater or less number of secondary sinuses. Based largely upon the abundance of these secondary sinuses, the hemolymph nodes have been divided into two varieties, named by Warthin (Jour. Bost. Soc. Med. Sc., 1901) the 'splenolymph glands' and the 'marrowlymph glands.' 
In the splenolymph type, which is the more abundant, the node is of small size and is well filled with secondary blood sinuses. The lymphoid tissue is supported by a similar reticulum, and contains the same varieties of lymph cells as in the lymph nodes.

In the marrowlymph nodes a somewhat similar structure is found. The blood sinuses are less numerous and lymph nodules do not occur (Vincent, Warthin). The eosinophil leukocytes are more numerous than in the splenolymph type, and the marrowlymph nodes as a rule are the larger.

Huntington (Amer. Jour. Anat., 16, 3, 1914) has suggested that

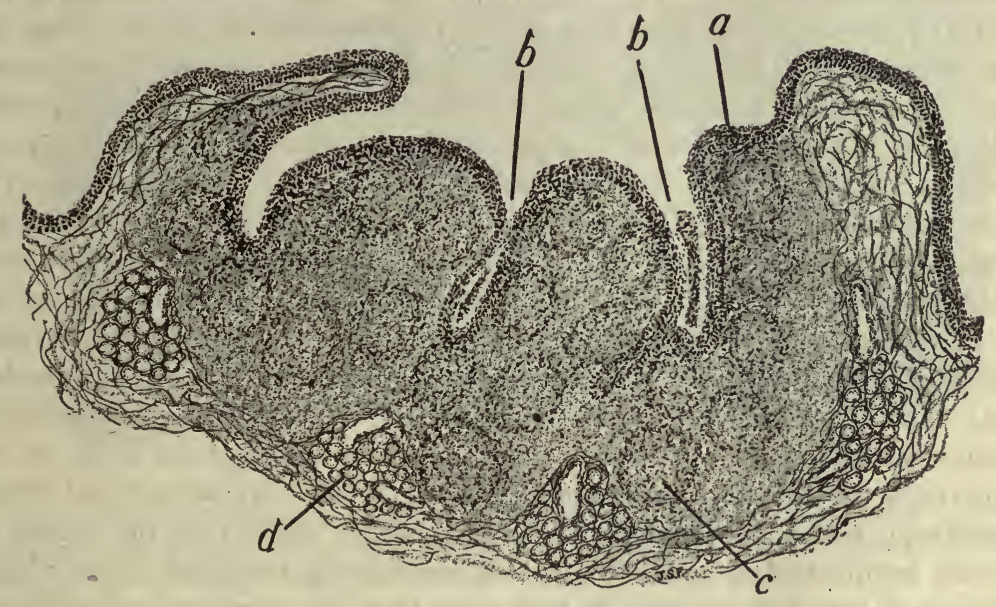

Fig. 247.-Horizontal Section throdgh the Faucial Tonsil of a Child.

Semi-diagrammatic. $a$, stratified epithelium; $b$, crypts; $c$, lymph nodule; $d$, mucussecreting gland. Hematein and eosin. $X$ about 20.

some of the structures described as hemolymph nodes may be post-natal hemopoietic foci, in which erythrocytes develop from the endothelium of the lymph channels. They probably function as accessory spleens having a combined lymphopoietic and phagocytic activity.

Intermediate types between the lymph nodes and the splenolymph type (Vincent, Jour. Anat. and Physiol., 189\%) on the one hand, and between the splenolymph node and the spleen and marrowlymph type on the other hand, are of frequent occurrence.

Blood Supply.-The afferent artery, according to Drummond (Jour. Anat. and Physiol., 1900), enters the hilum with the connective tissue, and through the trabeculæ reaches all parts of the node. In the lymphoid 
tissue its branches form a capillary plexus whose vessels open into the blood sinuses. All the sinuses, peripheral and secondary, communicate with each other, and from them the blood is ultimately collected into two or more thin-walled veins. In the center of the gland these vessels unite to form an efferent vein which passes out at the hilum.

\section{DEVELOPIENT OF LYMPH NODES}

Lymph nodes arise through the invasion of primary lymphatic capillary plexuses by lymphocytes. The first lymph nodes arise in the regions of the axilla and groin during the third month of development. Such areas become circumscribed by the development of a capsule from the surrounding mesenchyma. The capsular tissue is continued into the developing node in the form of trabeculæ, terminating in a dense network of delicate reticular fibers. Hydrostatic conditions probably determine the formation of a peripheral lymph sinus. The retention of certain channels (internodular and medullary sinuses) between the peripheral sinus and the efferent lymphatics at the hilum is likewise probably determined mainly by the operation of like factors, brought into play through the appearance of cortical nodules. These nodules arise as regions of proliferative activity of lymphocytes. The node has meanwhile early become invaded at a point which becomes the hilum by a vascular and nerve supply. Nodules arise as accumulations of proliferating lymphocytes about the cortical arterial twigs. Hemolymph nodes apparently arise in a manner similar to the origin of ordinary lymph nodes, and become only secondarily modified. The reticular tissue of lymph nodes may in part arise from the capillary endothelium.

The function of lymph nodes is the production of lymphocytes, which become phagocytic leukocytes. Besides having a leukopoietic rôle, lymph nodes probably function also as centers for the dissolution of worn-out blood elements, in which process phagocytosis predominates, the lymphocytes being in part assisted by the endothelial cells of the capillaries. Lymphoid aggregations also serve as 'ymph filters,' the phagocytes removing from the lymph bacteria and other noxious products.

\section{THE TONSILS}

The Faucial Tonsils (Palatine Tonsils; Amygdalce).-The tonsils consist of a mass of lymphoid tissue which projects slightly from either side into the cavity of the fauces, and is covered by a layer of stratified epithelium continuous with that which lines the oral and pharyngeal 
cavities. The lymph nodules which compose the tonsil immediately underlie the epithelial coat, and are embedded in areolar connective tissue.

The epithelial coat here and there penetrates the substance of the organ in the form of invaginated funnel-shaped depressions, the crypts ('follicles' of the tonsils). The direction of the crypts in the upper third of the tonsil is downward and outward (C. P. Johnson). The ducts of many mucous glands open into the recesses of these branching crypts. The mucus-secreting glands lie in the loose connective tissue which sur-

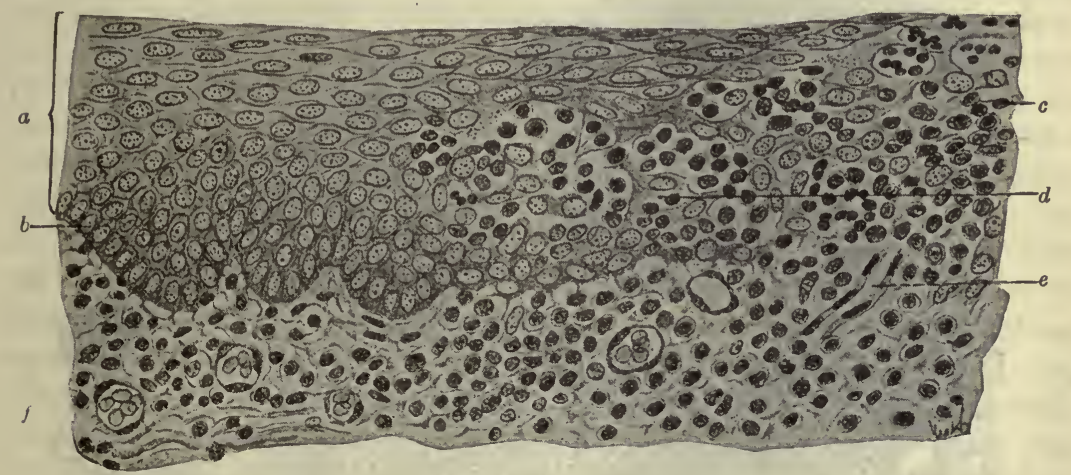

Fig. 248.-From a Crypt of a Dog's Tonsil.

$a$, stratified epithelium; $b$, basal margin of the epithelium; $c$, infiltration of the epithelium by leukocytes; $d$, spaces in the epithelium filled with leukocytes and epithelial cells; $e$, blood-vessel; $f$, lymphoid tissue.' $\times 150$. (After Böhm and von Davidoff.)

rounds the tonsil on all but its faucial surface. The crypts are lined throughout by a layer of stratified epithelium, which is continuous with that on the free surface of the tonsil, but which becomes progressively thinner as it recedes into the deeper recesses of the crypts.

Many of the lymph cells migrate into the intercellular spaces of the epithelial layer, and even penetrate to the free surface; thus they find their way into the oral cavity, where they are found in large numbers in the saliva, as 'salivary corpuscles.' If such salivary corpuscles are examined in a drop of saliva, freshly prepared, the fine intracellular granules of the polymorphonuclear leukocytes will be seen to undergo an active dancing movement, Brownian motion. The salivary corpuscles are derived not only from the faucial tonsils but from the other lymphoid tissue which is in relation with the oral mucous membrane, e.g., the lingual and pharyngeal tonsils.

The passage of leukocytes through the epithelial surface of the faucial 
tonsil is so very active that at times the epithelium becomes completely filled with these cells, and it is then difficult to distinguish it from the adenoid tissue beneath. The normal tonsils atrophy after puberty.

The Lingual Tonsil.-A collection of lymph nodules is also found at the base of the tongue in the median line, between the circumvallate papillæ and the epiglottis. This, because of its similarity in appearance and in structure to the faucial tonsil, is called the lingual tonsil.

In the lingual tonsil, however, the nodules are grouped about a

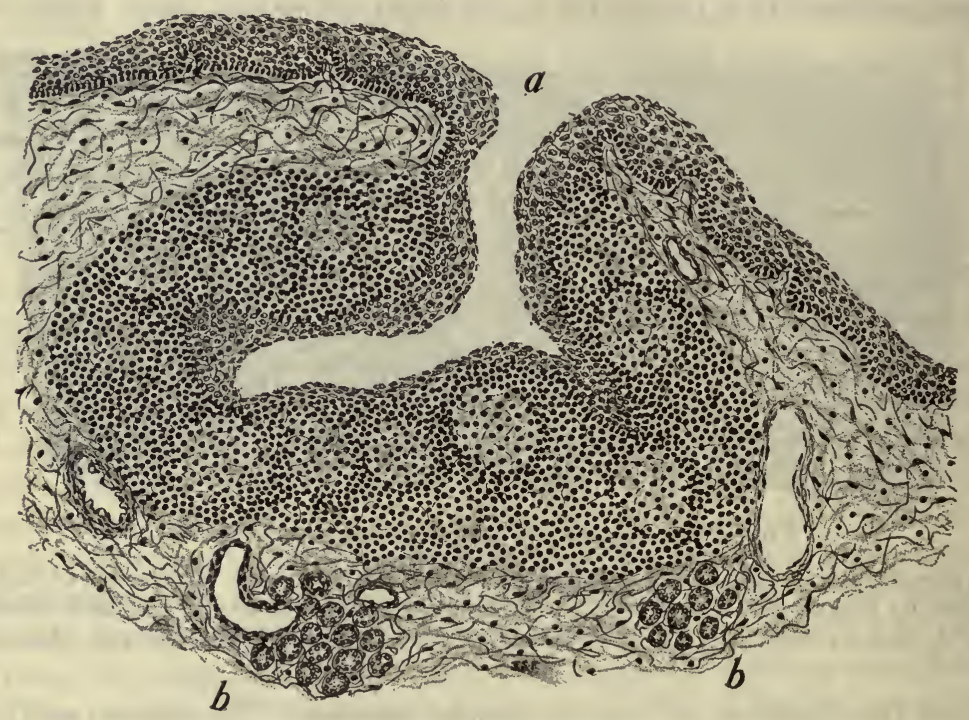

Fig. 249.-The Lingual Tonsil of Man.

$a$, a crypt; $b$, von Ebner's glands. Hematein and eosin. $\times 45$.

single wide-mouthed crypt, the foramen cacum lingui. This crypt is frequently branched, and into it the many mucous glands of the neighboring lingual mucosa pour their secretion.

The Pharyngeal Tonsil.- The posterior wall of the nasopharynx is supplied with a similar accumulation of lymph nodules, the pharyngeal tonsil. It lies in the median line and extends downward from between the orifices of the auditory (Eustachian) tubes for a distance of three centimeters (Klein). It contains a considerable number of lymph nodules and several small crypts. The lateral extensions in the vicinity of the tubal orifices are sometimes known as the tubal tonsils.

The pharyngeal tonsil is prone to hypertrophy in youth, in which 
case it forms the adenoid growths which are so common in strumous children.

Viewing the several tonsils and the associated lymphoid tissue as a whole, it will be perceived that they constitute a lymphoid ring at the gateway to the alimentary and respiratory tracts. The function of the lymphoid tissue is to produce phagocytic leukocytes for the protection of the body against bacteria and other noxious products. The tonsillar crypts offer favorable foci for the lodgment, invasion and attack of such harmful elements. The location of this annular mass of lymphoid tissue is significant; it is placed where it can apparently best perform a necessary function. When called upon to increase its functional activity lymphoid tissue responds by hypertrophy; this of itself may cause inconvenience by obstructing the channels employed in respiration and phonation. But when unable to respond adequately and thus successfully cope with the infecting material, the tonsils become diseased. This is commonly considered to call for removal of the involved lymphoid masses. But it would seem that the excision of the tonsils would result in handicapping the organism in its perpetual combat with bacteria, by depriving it of a means of defense; moreover in the case of removal of the faucial tonsils, proper phonation may also be interfered with. However, excised tonsillar tissue is probably largely compensated for by regeneration and hypertrophy of other noninvolved lymphoid tissue. Nevertheless it has been suggested that much could be gained through prophylactic means consisting largely perhaps in the promotion of nose breathing and the prevention of chronic nasal cold in infants.

\section{THE SPLEEN}

Structure.-The spleen is the largest lymphoid organ of the body. It is located to the left and dorsally between the stomach and diaphragm, has an irregular oval outline, measures about five inches in length and three inches in thickness, and weighs about seven ounces. It is subject to great variations in size and shape. It is enveloped by a thick fibroelastic capsule, or tunica albuginea, containing smooth muscle in its inner portion. External to this is also a peritoneal investment, or tunica serosa. The capsule of the spleen of the ox is especially robust, and rich in smooth muscle. At one point, the hilum, the capsule projects into the spleen as a large mass of trabecular tissue. Over the entire surface also other trabeculæ project from the capsule into the parenchyma of the organ. These trabeculæ are of similar structure to the capsule. The supporting tissue of the parenchyma is a delicate reticu- 
lum. This is continuous with the fibro-elastic terminal processes of the fibromuscular trabeculæ.

The primary trabeculæ divide the parenchyma imperfectly into roughly pyramidal compartments about one millimeter in diameter, with three trabeculæ for each lobule. This lobulation is faintly indicated by surface markings. According to Mall this unit of structure, the splenic lobule, is further subdivided into about ten smaller compartments by

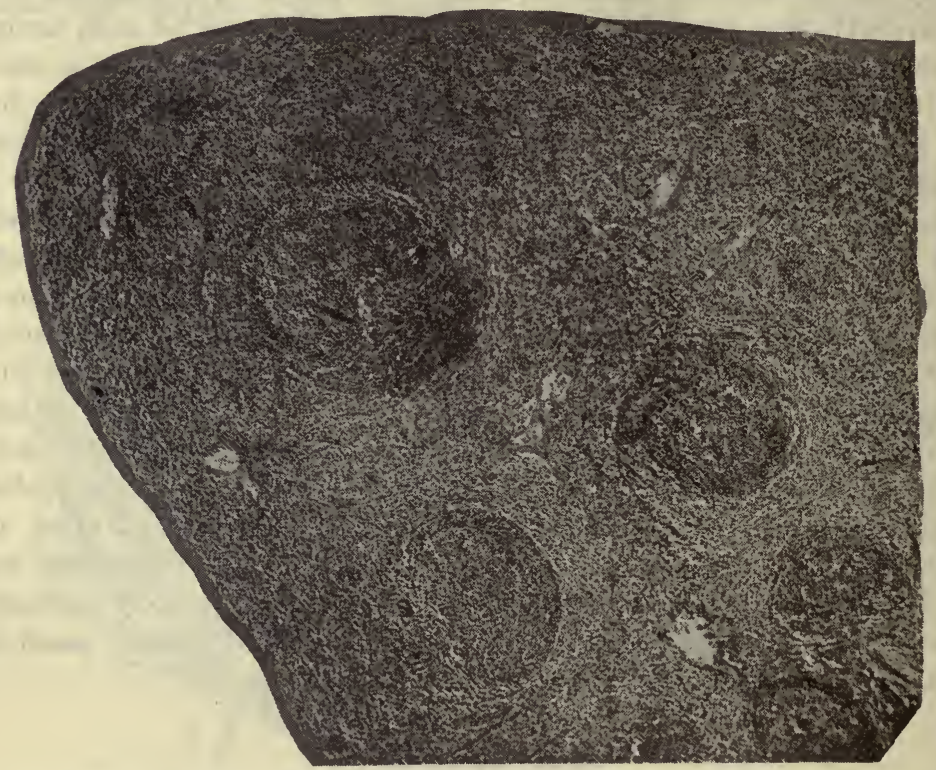

Fig. 250.-Portion of Spleen of Cat, Showing Capsule (Above and at Left) and Five Splenic Nodules.

Between the nodules can be seen vascular trabeculæ continuous with the capsule. $\times 60$.

anastomosing septa, continuous with the primary trabeculæ. The division of the spleen into lobules, and their subdivision into lobular compartments has structural significance also from the viewpoint of the blood supply. A knowledge of the microscopic structure of the spleen is dependent upon an understanding of the distribution of the bloodvessels.

Blood-vessels.--The splenic artery enters at the hilum, associated with the splenic vein. The larger arterial branches are located within the coarser trabeculæ continuous with the connective tissue of the hilum and still accompanied by the larger tributaries of the splenic vein. The 
smaller arteries part company with the veins when they leave the trabeculæ and pass into the apices of the lobules. At the point of entrance into the lobule the adventitia of the intralobular artery becomes infiltrated with lymphocytes, forming thus a spherical or fusiform lymphoid mass, the splenic nodule (Malpighian corpuscle) characteristic of the spleen. This arterial vessel gives off numerous branches to the splenic nodule, s o m e of which pass beyond the confines of the nodule into the splenic pulp. Some of these nodules contain germ centers. In infancy all of the nodules are said to contain germ centers.

The artery usually passes excentrically through the nodule. The nodules a $\mathrm{r}$ e fre-

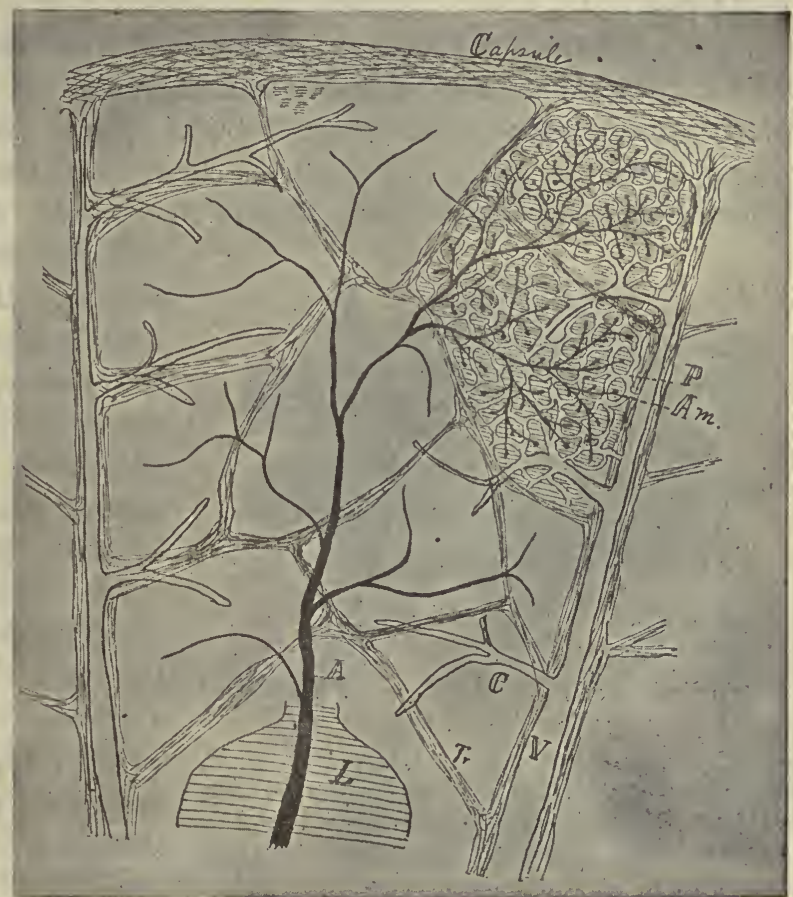

Fig. 251.-Diagram of a Lobule of the Spleen.

$A$, artery lying in the center of the lobule; $A m$, a terminal ampulla of the artery; $C$, intralobular vein; $L$, a splenic corpuscle; $P$, venous plexus within the pulp of the spleen; $T r$, fibromuscular trabecula within the lobule; $V$, interlobular vein, lying in a large trabecula. (After Mall.) quently situated

at the point where the artery branches and in consequence contain two arterial vessels. Beyond the splenic nodules, the intralobular artery breaks into a number of twigs, one for each lobular compartment. Within each compartment, the arteriole divides into a brush of delicate precapillary arterioles, the penicilli of Ruysch. On these appear an ellipsoidal condensation of reticular tissue forming the so-called splenic ellipsoids. These arterioles" (six to ten microns in diameter) are known as sheathed arteries. The splenic pulp of the lobules can be divided into somewhat 
denser cords of uncertain outline, the splenic pulp cords, and slightly looser intercordal pulp, the venous sinuses, corresponding to the sinuses of the medulla of lymph nodes. 'The penicilli are located in the pulp cords. Terminally these penicilli expand into dilatations, the ampulloe of Thoma.

The exact method of passage of the blood from the terminal arterioles to the initial venules is uncertain and disputed. It is certain only that blood passes freely at this point into the splenic pulp. This gives the adenoid tissue a deeply red or purple color, in contrast to the light pink color of ordinary lymph nodes, due to the presence of innumerable ery-

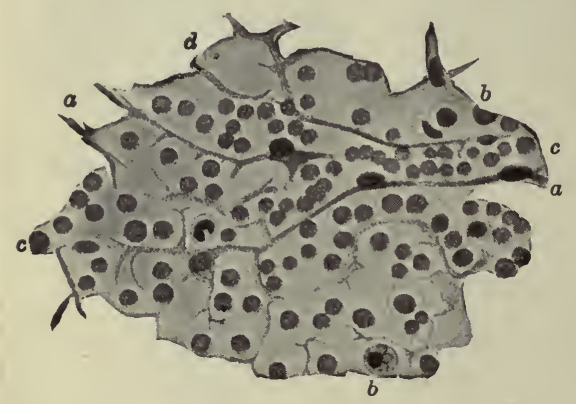

Fig. 252.-The Origin of a Vein in the Splenic Pulp.

$a$, venous endothelium; $b$, leukocytes; $c$, red blood corpuscles (appearing rather too dark in the reproduction); $d$, a mesh of the splenic pulp. Highly magnified. (After Bannwarth.) throplastids. It seems probable that the walls of the connecting capillaries and the initial venules (cavernous veins, venous sinuses) are fenestrated, permitting the free passage of blood from these vessels into the spleen pulp.

According to some authorities the blood passes by two routes from the arterioles to the venules; (1) through the arterial ampullæ directly into the venules (venous ampullæ); and (2) from other terminal arterial twigs into the spleen pulp, from where it is collected by the venules.

The veins thus begin as wide sinusoidal channels ( pulp veins) within the splenic pulp. At first, and for a considerable distance, they follow an independent course through the pulp, receiving at the same time frequent accessions of blood from other venous radicals. Finally, however, the veins enter the larger trabeculæ, but are still devoid of more complete coats than the thin membrane of fibro-elastic tissue which surrounds the endothelial tube, but which is now ensheathed by the trabecular tissue. Henceforth the path of the veins lies within the trabeculæ (interlobular veins), and is directed toward the hilum. On approaching the hilum the larger veins acquire the usual venous coats. Having arrived at the hilum, they form several efferent vessels which, in the outlying connective tissue, form by their union the splenic vein. 
Differentiation of Spleen from Lymph Node.-The spleen in section can be readily differentiated from a lymph node: it lacks a definite subdivision into cortex and medulla characteristic of the lymph node, its pulp contains a preponderating number of red blood corpuscles, and its capsule is relatively very robust and contains a greater amount of smooth muscle. The spleen may be thought of as a congested lymph node, consisting wholly of medulla, throughout which are scattered lymph nodules, the splenic nodules. It might quite properly be described as a huge hemolymph node.

Splenic Cells.-Besides erythroplastids and occasional erythrocytes, the pulp contains also the several varieties of leukocytes: lymphocytes, granulocytes, a few megakaryocytes, and blood-platelets. The leukocytes of the spleen are largely of the large mononuclear type. These are notably phagocytic, frequently containing erythroplastids, fragments of cells, pigment and other granular débris. They are in a sense specific for the spleen, hence called splenic cells. Megakaryocytes are abundant in the fetal spleen during its period of erythropoietic function, but rare in the adult spleen.

Lymph Supply. - The

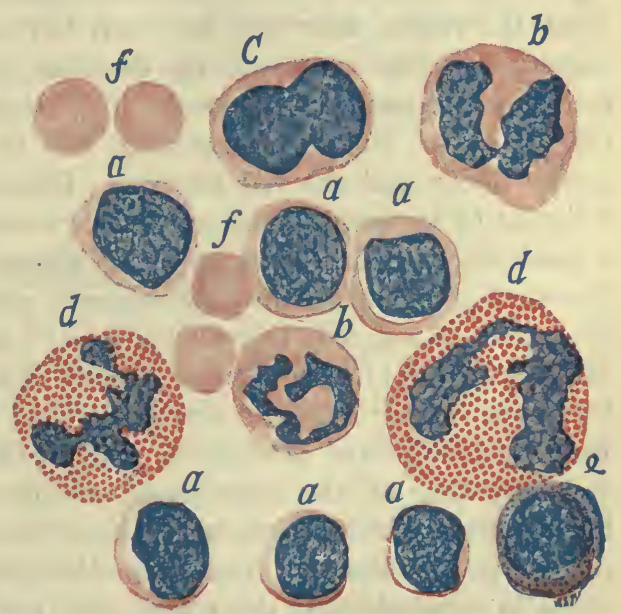

Fia. 253.-Types of Cells from a Smear Preparation of the Pulp of the Human Spleen.

$a$, lymphocytes; $b$, polymorphonuclear neutrophil leukocytes whose granules are not stained by the method used; $c$, large mononuclear leukocyte; $d$, eosinophil cells; $e$, basophil cell; $f$, red blood corpuscles. Hematein and eosin. $\times 1200$. lymph supply of the spleen is relatively scanty. The capsular is independent of the parenchymal system, which latter consists. chiefly of perivascular lymph spaces and vessels draining toward the hilum.

Nerve Supply.-The innervation includes both medullated and nonmedullated fibers. The latter predominate, and are distributed to the smooth muscle of the capsule, trabeculæ, and blood-vessels.

Functions.-The spleen functions as a leukopoietic organ and as a blood filter. This is indicated both by the direct evidence of lymphocyte 
proliferation and the ingestion of erythroplastid débris by the splenic and endothelial cells, and by the fact that the proportion of lymphocytes to erythroplastids in the splenic vein is very much greater than in the splenic artery. After severe hemorrhage or certain anemias the spleen may resume its fetal erythropoietic function. Numerous small supernumerary spleens, of varying size but usually about the size of a pea, are frequently found in the vicinity of the spleen.

Besides the production of lymphocytes and the destruction of senile red corpuscles, the normal adult spleen has been credited also with an erythropoietic rôle, and with a function concerned with the metabolism of iron. That the spleen does not, however, have any specific function absolutely essential to life is proved by the fact that it may be removed without serious consequence. Obviously its function may be taken over by some other organs. Such compensatory rôle is usually attributed to the hemolymph nodes and the red marrow. However, splenectomy in dogs is not followed by increase in the number or size of the hemolymph nodes, nor by a production of accessory spleens (Meyer, Jour. Exp. Zool., 16, 2, 1914). Removal of the spleen in dogs seems to exert a stimulating effect upon the formation of red cells in bone-marrow (Krumbhaar and Musser, Jour. Exp. Med., 20, 2, 1914). Pearse and Pepper (Jour. Exp. Med., 20, 1, 1914) found that splenectomy caused a transformation of yellow into red marrow. The result is interpreted as showing that in the absence of the spleen the marrow may take on the function of storing and elaborating the iron of the blood pigment for future utilization by new red cells. The experiments of Austin and Pearse (Jour. Exp. Med., 20, 2, 1914) on the contrary lead them to conclude that the spleen does not exert a constant and important function on iron metabolism. The complete function of the spleen apparently remains largely unknown. Like the thymus it is sometimes classified among the organs of internal secretion.

Development.-The anlage of the spleen appears at the beginning of the second month as a condensation and swelling in the mesenchyma on the left border of the dorsal mesogastrium. The overlying mesothelium proliferates extensively and its cells invade the mesenchyma obliterating the line of demarcation. The early histogenesis is obscure. The mesenchyma is potentially capable of producing all the definitive elements of the spleen: connective tissue capsule and framework, and lymphocytes. The mesothelium, genetically closely related to mesenchyma, probably aids in the general process. Probably also the bulk of the later lymphocytes invade the spleen from without. 


\section{CHAPTER X \\ MUCOUS MEMBRANES-GLANDS}

The histologic structures which are necessary for the formation of a secretion include an epithelial surface, and a tunica propria of connective tissue which supports the requisite blood and lymphatic vessels and the controlling nerve supply. These structures may either form smooth membranous surfaces or apparent epithelial invaginations. The former are found on the surface of the mucous membranes, the latter are the secreting glands.

\section{MUCOUS MEMBRANES}

The mucous membranes may be said to include all those secreting surfaces which are directly or indirectly connected with the surface of the body, hence their epithelial clothing is continuous with that of the skin. The mucous membranes form the lining coat of the respiratory and alimentary systems, together with the ducts of their secreting glands; in the nose this membrane is continuous through the tear ducts with the conjunctiva of the eye and through the auditory (Eustachian) tubes with the lining membrane of the middle ear. The broad expanse thus formed is known as the gastropneumonic mucous membrane. A second membranous sheet, the genito-urinary mucous membrane, clothes the organs of the genital and urinary systems; it thus forms the lining membrane of the vagina, uterus, and Fallopian tubes, of the urethra, bladder, ureters and pelvis of the kidney, of the ducts and tubules of the prostate gland, the testis, and the smaller secreting glands which are connected with the genital system.

A mucous membrane consists of a superficial layer of epithelium of varying type, which rests upon a basement membrane (membrana propria) and is in turn supported by an investment of connective tissue, the tunica propria, or corium. The tunica propria is richly supplied with small blood-vessels and lymphatics; its nerve fibrils are not only distributed to the walls of the blood-vessels but in many cases send ter- 
minal filaments which enter the epithelial layer and terminate in contact with the secreting cells. The mucous membranes are mostly contained within hollow organs which are subject to alternate collapse and distention; hence the membranes are frequently much folded. The deeper portion of the mucous membrane usually contains a more or less well-defined layer of smooth muscle fibers, the muscularis mu$\cos \infty$.

The mucous membranes, as their name indicates, are nearly all moist-

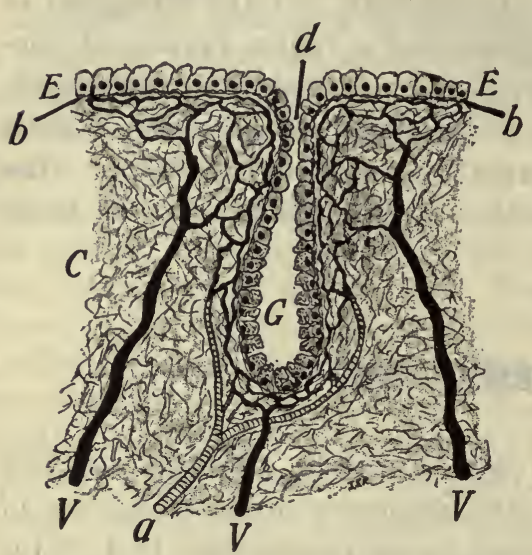

Fig. 254.-Diagram of a Mucous Membrane Having Simple Tubular GLANDS.

$a$, artery; $b-b$, basement membrane; $\iota$, connective tissue; $d$, duct of the gland, lined by cuboidal cells; $E$, epithelium of the free surface with clear, columnar cells; $G$, lumen of the fundus of the gland, lined by granular, serous secreting, columnar cells surrounded by secretory capillaries; $V$, veins. The arteries are striped, the capillaries and veins, black. Nerves are not represented. ened by a mucus-containing secretion. The relative amount of mucus which its secretion contains, and consequently the viscidity of the secretion, bears a close physiologic relation to the intensity of the mechanical irritation to which the membrane is subjected. Thus the mucus-secreting goblet cells of the gastropneumonic membrane are here and there reënforced by numerous mucus-secreting glands of considerable size; these are especially abundant in the oral cavity, pharynx, and esophagus, and in the nose, trachea, and bronchi; in the urinary system even the goblet cells are absent.

The basement membranes, upon which the epithelium of the mucous membranes and the secreting glands is supported, are connective tissue structures. They are sometimes formed by interlacing bundles of delicate collagenous fibers intermingled with numerous elastic fibers. Frequently, however, they consist of reticular tissue. Basement membranes of this nature have been demonstrated by Mall and his pupils in the mucous membranes and glandular tubules of the stomach, intestine, liver, salivary glands, kidney, testis, and thyroid. Occasionally basement membranes are homogeneous or hyaline in structure and present a more or less clear or glassy appearance. 


\section{GLANDS}

The secreting glands may be quite properly considered as invaginations of the epithelial surfaces of the mucous membranes. They appear as such in the embryo. Their earliest anlage is formed by a solid or funnel-shaped process of epithelium, in which a distinct lumen soon appears, and which grows into the surrounding mesoblast, carrying with it its embryonal tunica propria.

\section{Classification of Glands}

The form of the glandular invaginations is subject to great variation. They may be straight and simple, more or less branched and compound, convoluted or coiled, or the tubules may terminate in minute ampullary
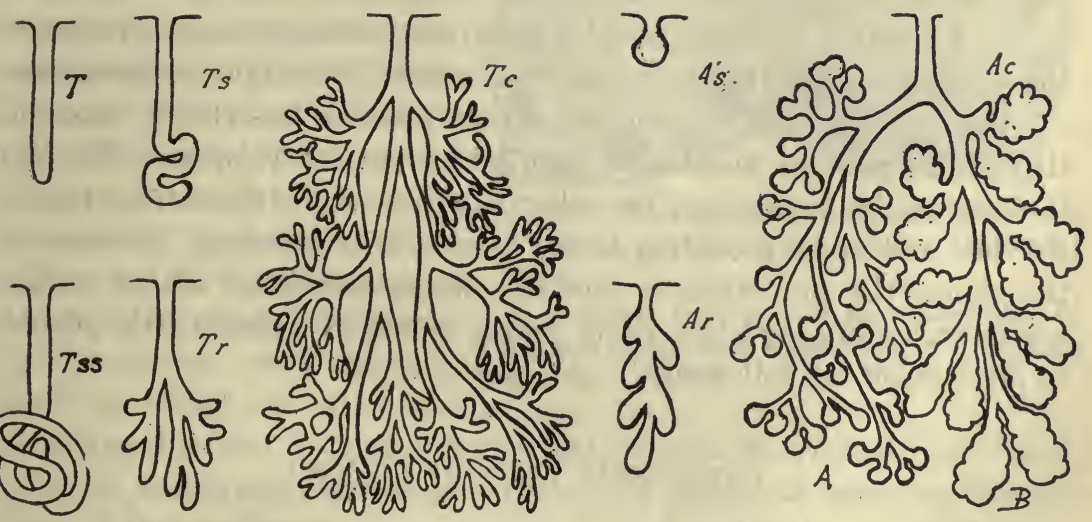

Fig. 255.-Diagrams of the Principal Types of Glands.

$T$, simple tubular (intestinal glands); $T s$, slightly coiled tubular (intestinal glands and small sweat glands); $T$ ss, more extensively coiled tubular (larger sweat glands); $T r$, branched tubular (gastric glands); $T c$, compound tubular (kidney, lacrimal glands); $A s$, simple alveolar or saccular (small sebaceous glands); $A r$, branched alveolar (larger sebaceous glands, tarsal glands of eyelid); Ac, compound tubuloalveolar or racemose (A) (salivary glands); compound saccular (B) (mammary gland). In major part after Prenant, Bouin and Maillard.

enlargements, the acini or alveoli. The invaginations may also be distinctly tubular and of approximately equal diameter throughout, or they may form pouch-like saccules. According to the form of the organ, it is thus possible to distinguish the following 


\section{HISTOLOGIC TYPES OF GLANDS:}
I. Tubular
II. Saccular
(alveolar)
1. Simple.
2. Convoluted.
3. Branched.
4. Compound.
5. Compound tubulo-acinar (alveolar); racemose.
1. Simple.
2. Branched.
3. Compound.

III. Ductless glands; endocrine glands.

Glands of the tubular and saccular types contain an actively secreting portion or fundus and a duct. Such externally secreting glands are also known as 'exocrine' glands. In the ductless ('endocrine') glands the duct is absent. The duct, though its epithelium may take some part in the formation of the glandular secretion, primarily serves to convey the secretion of the fundus to the free surface of the mucous membrane.

The epithelium of the duct, as a rule, more or less closely resembles that of the mucous membrane upon whose surface it opens. The epithelium of the fundus, on the other hand, usually differs from that of the duct and varies according to the nature of its secretion. In many of the glands the epithelium is typically mucus secreting; others produce a clearer, watery, and less viscid serous secretion. Hence it is possible to distinguish the following

\section{PHYSIOLOGIC TYPES OF GLANDS:}

I. Serous glands.

II. Mucous glands.

III. Glands which are both mucous and serous (mixed glands).

IV. Glands which are neither mucous nor serous.

This physiologic classification is not in any way the equivalent of the histologic gland types mentioned above. Thus both serous and mucous glands, in different locations, form almost every variety of tubular gland.

\section{Description of Physiologid Types}

The glands of the fourth type are too varied in their structure to be considered collectively to advantage. The reader is referred to 
the several chapters in which they are described in detail. This type includes the testis, the prostate, the ceruminous glands, many of the ductless glands, and also some authors describe the ovary and the lungs as comforming to the glandular type of structure.

The mixed glands include some tubules which are characteristically mucous, while others are typical serous secreting. Occasionally both types of secreting cells are contained within the same tubule.

Mucus-secreting cells possess the general characteristics which have been previously recited under the head of goblet cells (Chapter II). When void of secretion the cytoplasm of mucous cells is granular, their nucleus centrally situated, and their shape more or less columnar. The pre-secretion accumulates in the central portion of the cell and occupies an area, adjacent to the glandular lumen, which steadily increases in size until the greater part of the cytoplasm has been replaced; the nucleus is pushed to the proximal or attached end of the cell ; :and the whole cell often be-

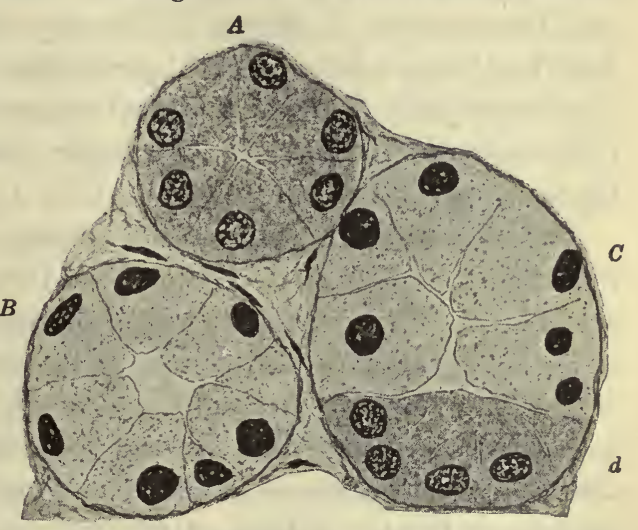

Fig. 256.-Transection of Three Secreting Tubules of the Submaxillary Gland of MaN.

$A$, a serous tubule; $B$, a mucous tubule; $C$, a mucous tubule with a demilune, $d$. Hematein and eosin. $\times 665$. comes swollen and distended to more than double its original size. Finally the cell membrane ruptures and the mucus pours out upon the free surface of the membrane.

At the base of the mucus-secreting cells, and between them and their basement membrane, are groups of epithelial cells having a finely granular cytoplasm, which form crescentic cell masses, the demilunes of Heidenhain (crescents of Gianuzzi). In the tubules of some glands these demilunes are extremely minute, in others they occupy a conisiderable portion of the epithelial coat and encroach upon the glandular lumen. Their significance is not definitely understood. They have been considered as representing either secreting cells which are in a state of rest following the discharge of their secretion, or as primordial cells which by reproduction give origin to true mucus-secreting cells. It is quite possible that both of these functions are assumed by the several 
cells which compose the demilunes. Many of these cells contain an independent, intracellular, secretory, canalicular system, which indicates a specific and independent functional rôle.

Mucus, the product of the mucus-secreting cells, possesses peculiar properties. In the fresh condition it has a clear, glairy appearance and a pearly white color. Acted upon by alcohol or acids it gives a heavy precipitate of stringy white flocculi. Within the tissues these delicate flocculi stain slightly with basic dyes and readily with the muchematin and mucicarmin of Mayer. The very clear glairy appearance of the fluid. and the slightly basophil properties of the precipitated flocculi are so characteristic that when typical mucus-containing cells are once carefully observed they can be thereafter readily distinguished from other types of epithelium.

Serous-secreting cells differ greatly in appearance with the varying character of their secretions, yet they present certain general characteristics. These cells are unquestionably capable of alternate phases of secretory activity and comparative rest. At the end of a period of activity they appear shrunken and small, and the lumen of their tubule is consequently increased in size. Their nucleus is centrally located, and their cytoplasm is relatively devoid of secretion and frequently presents a faintly rodded or striated appearance.

During rest secretion accumulates within the cell, and the cytoplasm consequently becomes either clearer or more granular, according as the nature of the secretion is watery, or is granular and zymotic in character; thus the secreting cells of the sweat glands become clearer as their secretion accumulates, whereas those of the pancreas become more granular.

As a rule the pre-secretion accumulates at the central end of the cell, the nucleus is thus crowded toward the basement membrane and is surrounded by the least altered cytoplasm. The whole cell becomes swollen and distended by the accumulated secretion and the tubular lumen is consequently diminished in size or even occluded.

Finally the period of secretory activity arrives, and the secretion is poured into the glandular lumen; the cells become shrunken and the lumen of the tubule correspondingly dilated. The cytoplasm returns to its former condition; if the secretion is of a granular character the cell becomes clearer, but if watery the cytoplasm acquires a finely granular appearance. The nucleus resumes its former central location and the cell enters upon a second period of constructive and accumulative activity. 
Many of the serous-secreting cells contain minute intracellular canals which connect with a network of intercellular passages about the cell. The intercellular canaliculi may, on the one hand, open into the glandular lumen, or they may communicate with the tissue spaces of the tunica propria. This system of intracellular and intercellular canaliculi may thus serve either as a system of nutrient channels or as a network of secretory capillaries by which the secretion is conveyed from the interior of the secreting cells to the lumen of the gland or even to the duct system. Nutrient and secretory canaliculi of this nature have been demonstrated in the secreting cells of the liver, cardiac glands of the stomach, salivary glands, pancreas, adrenal, and epididymis, but they are not by any means confined to the actively secreting cells, for they have been found in the cells of bladder epithelium (Holmgren) and are highly developed in the nerve cells (Holmgren, Golgi, et al.).

\section{Description of Histologic Types}

Simple Tubular Glands.-Simple tubular glands occur in the: mucous membrane of the small and large intestine as the crypts of Lieberkühn or intestinal glands. In shape these glands resemble a test-tube. They form straight tubules which open on the free surface of the membrane, are of approximately equal caliber throughout, and at their deeper end terminate in a blind extremity. The tubules are lined with epithelium and are embedded in a thin vascular tunica propria. Their epithelium includes the usual columnar and goblet cell types, the latter being more abundant near the mouth of the gland. Near the blind extremity are certain granular cells, the granules of some of which are slightly basophilic: other cells possess coarse granules which are highly acidophil, as demonstrated by Kultschitsky (Arch. f. mik. Anat., 1897) in the intestinal glands of the dog, an observation which is easily corroborated for the simple tubular glands in the small intestine of man.

Convoluted Tubular Glands.-Convoluted tubular glands occur as the sweat glands of the skin, the ceruminous glands of the ear, and the glands of Moll in the eyelids. The above are typical simple coiled glands. Certain other glands, which are less typically coiled but are more or less convoluted near their blind extremities and are frequently branched, are also to be included under this type. Such glands are the pyloric glands of the stomach, and the small mucous glands of the oral and nasal cavities, pharynx, larynx, trachea, bronchi, and esophagus. Some of these glands, and especially those of the pyloric end of the 
stomach, present terminal acinar dilatations, hence they also resemble to some extent a small tubulo-acinar type of gland.

The typical coil glands consist of a duct whose epithelium resembles an attenuated layer of the stratified epithelium upon which they open, and a fundus or secreting portion which is lined by columnar epithelium of the glandular type. They also possess a connective tissue basement membrane and a vascular tunica propria.

Branched Tubular Glands.-Branched tubular glands include the cardiac and pyloric glands of the stomach and the glands of the uterine

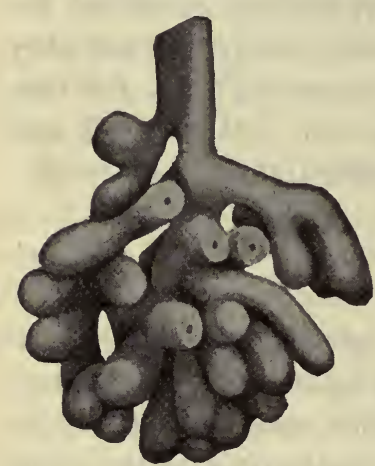

Fig. 257.-Model of A RECONSTRUCTION OF THE LACrimal GLand of Man.

The tubular duct divides into the terminal secreting tubules, forming a compound tubular gland. $\times 170$. (After Maziarski.) mucous membrane. These glands possess a duct whose epithelium corresponds in type with that of the surface upon which they open. Several secreting tubules open into this duct by means of a short constricted portion, the neck. The fundus or secreting portion, after a typically spiral course, ends with a blind extremity which is often curved or hooked. This portion of the gland is clothed with columnar or glandular epithelium and invested with a thin basement membrane and tunica propria.

Compound Tubular Glands.-Compound tubular glands include the kidney, testis, lacrimal gland, and liver. The finer structure of the glands of this type is so peculiar that the reader must be referred to the several chapters in which they are more fully described.

Compound Tubulo-alveolar Glands (Tubulo-acinar or Racemose Glands).-This is the most widely distributed of all the types of secreting glands. It includes the parotid, the submaxillary, the larger mucous and serous glands of the oral cavity, and of the nose, pharynx, trachea, bronchi, and esophagus, the duodenal (Brunner's) glands, the pancreas, bulbo-urethral (Cowper's) glands, urethral (Littrés) glands, and the large mucous glands of the cervix uteri.

The form of these glands may be likened to a much branched tree, whose stem as the main excretory duct opens upon the free surface of a mucous membrane, and the branches and twigs as the larger and smaller interlobular ducts reach out in all directions to finally end in minute alveolar dilatations, the secreting acini. 
Except for the ducts of certain mucous glands whose epithelial coat resembles that of the mucous membrane to which they are attached, the ducts of this type of secreting gland are lined by columnar cells whose cytoplasm frequently presents a rodded appearance at the deeper end of the cell. The acini contain typical serous or mucous, secreting epithelium. Occasionally the secreting cells are also found for some distance beyond the acinus in the lining membrane of the smallest ducts.

The tubules and acini of these glands are

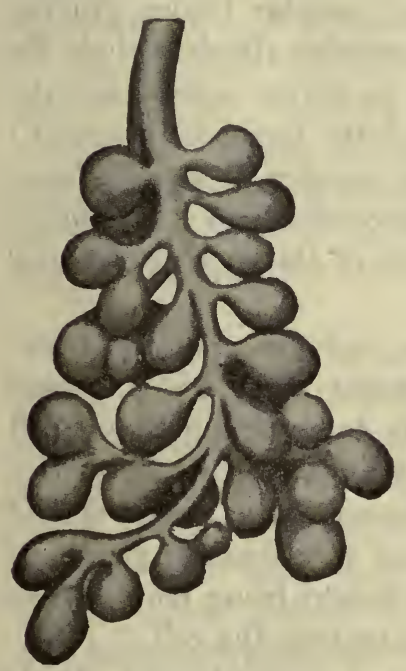

Fig. 259.-Reconstruction OF AN INTRALOBULAR DUCT Dividing into Its TerMiNAL INTERCALARY DUCtS AND ACINI.

The terminal divisions of a large compound tubulo-acinar gland. The model was made from serial sections of the human pancreas. $\times 344$. (After Maziarski.) invested with a basement membrane and a delicate tunica propria. The acini are united by the connective tissue into small groups which inclose a

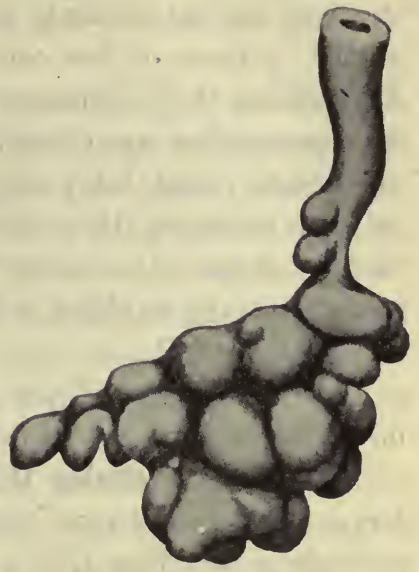

Fig. 258.-Reconstruction of a Mucous Gland from the Respiratory Region of the Nasal Mucosa of a Child.

The duct passes directly into the secreting alveoli. A typical small tubulo-alveolar gland. $\times$ 200. (After Maziarski.) central duct of the smallest type, the intercalary (or intermediate) duct. These acinar groups are again united into the lobules of the gland by fine bands of connective tissue, and broader bands of loose connective tissue cement the many lobules into one glandular mass. The intercalary ducts by union within the lobule form numerous small intralobular ducts which approach the periphery of the lobule and at its margin open into the interlobular ducts; the latter are found in the broader septa of connective tissue between the lobules. The interlobular ducts by union with one another result in progressively larger branches which finally form the main excretory duct of the gland.

Simple Saccular Glands.-Simple saccular glands occur as the 
smallest sebaceous glands of the skin. These are small glandular pouches with a short duct, a constricted neck, and a dilated fundus which, instead of having a single coat of epithelium as in most of the tubular glands, is more or less completely filled with a mass of epithelial cells. The cells as they approach the duct of the gland show progressive stages of degeneration and disintegration which culminate in the formation of a thick, viscid, fatty secretion. Since these cells form their secretion by disintegration they are obviously capable of passing through the various stages of secretory activity but once, and hence they must be renewed by the repeated mitotic cell division which occurs at the periphery of the saccule.

The epithelium of the secreting saccule rests upon a distinct basement membrane and is invested with a very vascular tunica propria.

Branched Saccular Glands.-Branched saccular glands include the larger of the sebaceous glands of the skin, in which several saccules pour their secretion into a common duct, and the tarsal (Meibomian) glands of the eyelids in which a considerable number of saccules open into an axial canal by which the secretion is conveyed to the terminal duct. The structure of each glandular saccule of this type is identical with that of a simple saccular gland.

Compound Saccular Glands.-This type includes only the mammary gland. It consists of a system of tubular ducts which possess ampullary dilatations and many branches. Its ducts terminate in small saccular alveoli which have a thin epithelial lining. During the period of their inactivity the lining epithelial cells are much flattened and the acini appear shrunken. The epithelium of the lactating gland, on the other hand, is cuboidal or columnar, the height being more or less dependent upon the accumulation of secretion within the cell.

The secretion is formed in the same manner as in the tubular glands with an additional process of fatty infiltration by which fat droplets are formed within the cytoplasm. These droplets collect in the central portion of the cell and are finally discharged into the lumen of the acinus with apparent rupture of the cell membrane and the escape of a portion of its superficial cytoplasm. The epithelium is thus capable of repeated secretion.

The mammary glands may be considered as offering an intermediate type between the branched saccular and the tubulo-acinar types.

Ductless Glands (Endocrine Glands).--Under the head of glands it is necessary to consider certain structures which apparently contain 
secreting epithelium and which present a more or less distinct tubular arrangement. These bodies are the adrenals, thyroids, parathyroids, carotid glands, coccygeal gland, paraganglia, hypophysis cerebri, and epiphysis cerebri (pineal gland). These are also known as organs of internal secretion.

While these glands do not possess an excretory duct, nevertheless some of them certainly, and the others probably, form certain products which find their way into the blood or lymph as so-called 'internal secretions.' The epithelium of the glands may form either alveoli, tubules, or solid cell columns, which are supported by very delicate connective tissue tunics. Many blood-vessels, often of the thin walled sinusoidal type, are found within these tunics and are thus brought into intimate relation with the epithelial parenchyma. In some instances lymphatics are distributed in a similar manner within the gland.

The property of internal secretion is not peculiar to the ductless glands. It has long been ascribed to the liver cells in connection with their influence upon nitrogenous and carbohydrate metabolism, and, in fact, many secreting glands, even though not of vital importance, are nevertheless found to influence the economy in certain ways which can not be accounted for by the properties of their external secretions.

Finally, it must be emphatically stated that the types of secreting glands, as above described, are not bound by hard and fast lines, but many forms will be found which might well be placed under either of two or more types. Hence any classification of secreting glands becomes more or less arbitrary; nevertheless such a classification is of extreme importance as serving to establish in the mind. of the student certain typical pictures with which individual glands may be compared, and important structural details will thus be noticed which might otherwise escape observation. 


\section{CHAPTER XI}

\section{THE SKIN}

The skin, or integument, consists of an outermost. ectodermal layer of stratified squamous epithelium, the epidermis (cuticle), and a subjacent mesodermal layer of dense connective tissue, the corium or derma (derma vera, cutis vera), homologous with the tunica propria of the mucous membranes. The corium contains the nerves and the nerve end organs of special sense, and rests upon a subcutaneous layer of areolar and adipose connective tissue which, blending with fascia or periosteum, unites the skin to the underlying muscles and bones.

The skin serves a composite function: protection, regulation of body temperature, tactile sensation, and excretion. Its excretory rôle is in fact accessory to the kidney.

The skin is typically a stratified organ, and for convenience of description may be divided into the following layers:

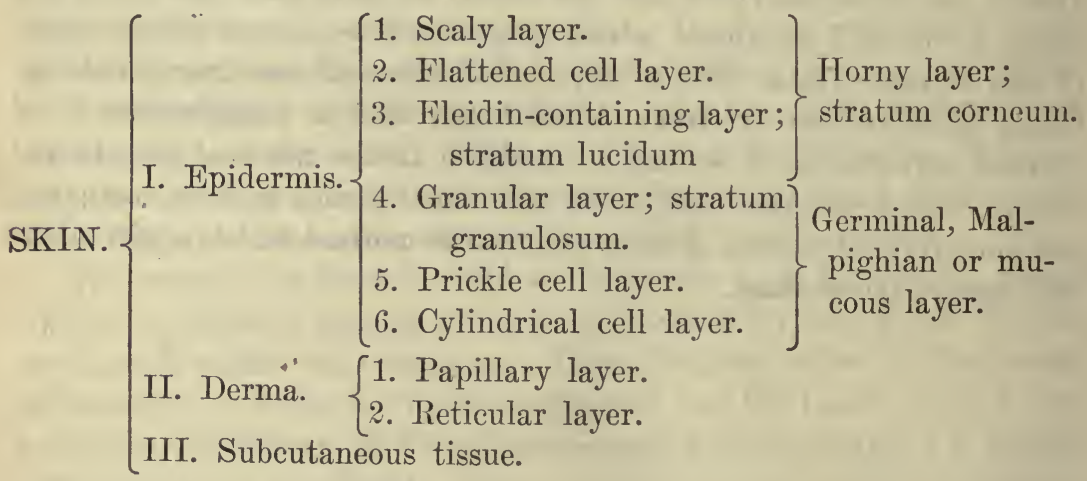

\section{THE EPIDERIMIS}

The epidermis (cuticle) or scarf skin serves for the protection of the more sensitive corium or 'true skin.' It is formed by a dense layer of stratified epithelium and varies in thickness in different portions 
of the body, being thickest upon those surfaces which are exposed to the: greatest mechanical violence, e.g., the palms of the hands and soles of: the feet; and thinnest in the least exposed portions, e.g., inner sides: of the arms and the back.

The layer of stratified squamous epithelium composing the epidermis differs from that of the mucous membranes in that its superficial cells contain an abundance of keratin, a peculiar horny material. The production of keratin in the cells of stratified epithelium appears to be more or less dependent upon the desiccation which occurs in those cells which form the comparatively dry cutaneous surface. The cornification can scarcely be demonstrated in the stratified squamous epithelium of the moistened mucous membrane of the mouth, esophagus, etc.; it is present though not pronounced in the partially moistened margins of the eyelids, lips, labia minora, glans penis, etc. In the epidermis, however, cornification is pronounced and characteristic in all portions of the body.

The thickness of the cornified layets appears to be in proportion to, if not entirely dependent upon, the amount of mechanical violence to which the cutaneous surface is subjected. Accordingly the increased thickness of the epidermis covering the palms and soles is found to be due almost entirely to an increase in the superficial horny portion of the epidermis, the germinal

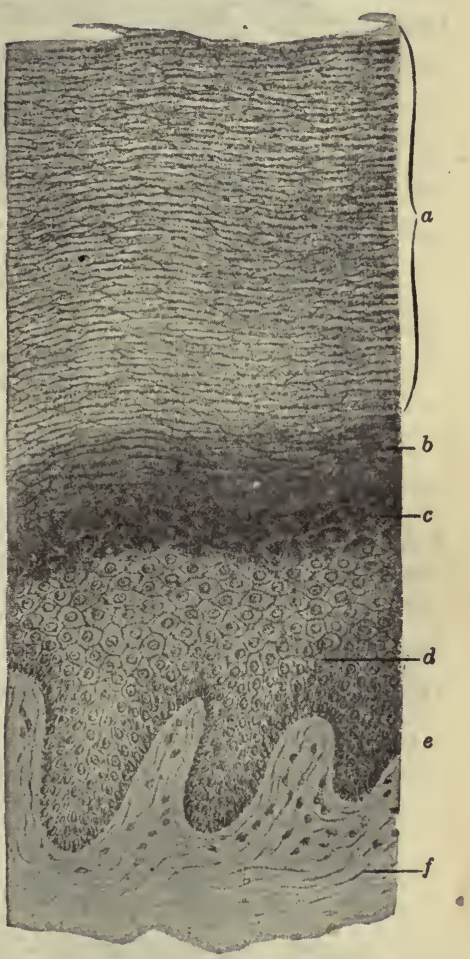

Fig. 260.-Epidermis of the Foot.

$a$, flattened cells; $b$, stratum lucidum; $c$, granular layer; $d$, germinal layer; $e$, cylindrical cell layer; $f$, derma. Picrocarmin. Moderately magnified. (After Ranvier.) layers being no more pronounced than in other portions of the body.

The epidermal tissue is divisible into a superficial horny portion consisting of flattened, desiccated, cornified cells-the stratum corneum or horny layer-and a deeper protoplasmic, so-called 'mucous' portion, which consists of polyhedral and cylindrical cells-stratum germinativum, or mucosum, rete mucosum, rete Malpighii. 
Cylindrical Cell Layer (Stratum Cylindricum).-The deepest cells of the stratum mucosum are elongated in a direction nearly perpendicu-

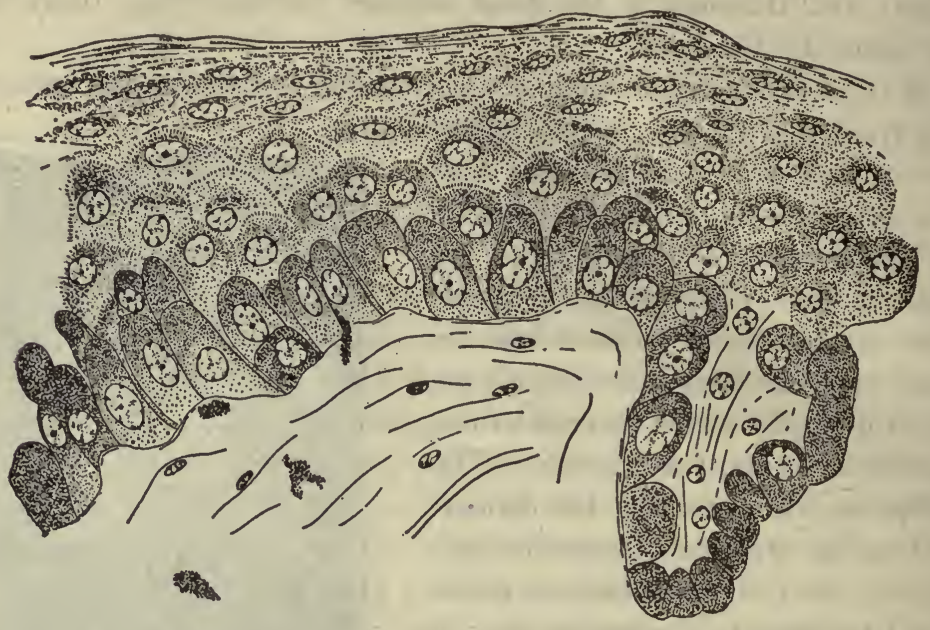

Fig. 261.-Section of Thin Skin from Abdomen of Negro, Showing the Distribution of the Pigment Granules in Dermal and Epidermal Cells. $\times 750$.

lar to the basement membrane upon which they rest; they are thus irregularly cylindrical in shape. It is these cells which in the pig-

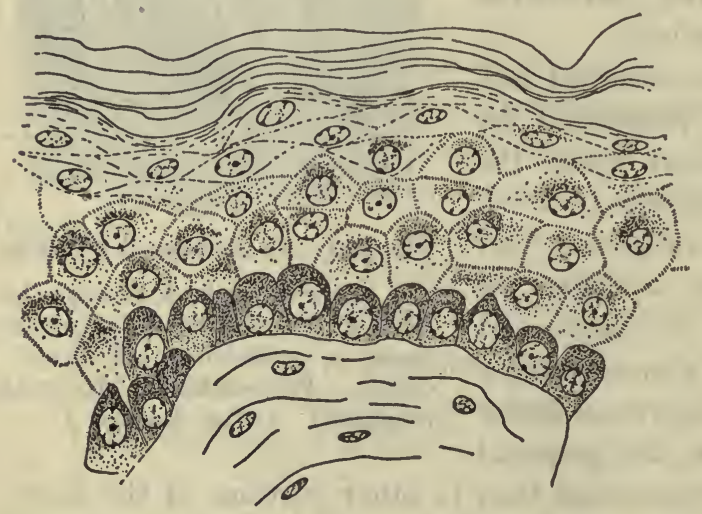

Fig. 262.- Section of Thin Skin from Abdomen of Light Brown Mulatto. $\times 750$. mented portions of the body, i.e., areolæ of the nipples, $\mathrm{scr}$ ot u m, circumanal region, etc., and in the skin of brunettes and the colored races contain the pigment which gives rise to the darkened color of the skin.

In the skin of the negro, the pigment ( $\mathrm{mel}$ a n i c) granules, while most abundant in the basal cylindrical cells, are nevertheless present, but in progressively smaller number, in all of the outer cell layers. In the 
various shades of mulatto skin, there is a close correspondence between the degree of color and the number of pigmented layers of epidermal cells. Certain connective tissue cells of the corium of pigmented skins :also contain abundant melanin granules; in the darker skins such cells are numerous. However, the dermal pigmented cells are not correctly regarded as the sources of supply of melanic granules for the epidermal cells, as has been maintained. The latter can produce their own granules; both dermal and epidermal pigmented cells owe their condition to the same underlying cause (Jordan; Amer. Nat., vol. 45, 1911). "The pigment granules are said to arise as a differentially staining nuclear ssubstance ('pyrenoid substance') which passes through the nuclear membrane into the cytoplasm, where it gradually acquires the character of pigment granules (Meirowsky: "On the Origin of the Melanotic Pigment in the Skin and the Eye," Leipzig, 1908). The nuclear origin of the pre-pigment granules is, however, disputed by some. The more recent investigations indicate that the physiology of melanic pigment formation involves the interaction of a nuclear oxidase (tyrosinase) with an extranuclear, perhaps cytoplasmic, chromogen (tyrosin).

The processes of mitotic cell division are very active in these columnar cells, and they, with the adjacent portion of the prickle cell layer, form the stratum germinativum of Flemming, in which the regeneration of the epidermis occurs. The cylindrical cells are firmly united to the basement membrane by delicate cytoplasmic fibrils, the intercellular bridges. Their nuclei are ovoid in shape, and vesicular in appearance.

Prickle Cell Layer (Stratum Spinosum).-Superficial to the cylindrical cells is a stratum of polyhedral epithelium which extends inward between the adjacent papillæ of the corium (interpapillary region of the epidermis), and is therefore thick in these portions, but is relatively much thinner over the apices of the dermal papillæ (suprapapillary portion of the epidermis).

The polyhedral cells of this layer contain a soft granular cytoplașm and a very chromatic, though vesicular, spheroidal nucleus. They are. separated from one another by narrow intercellular spaces which are bridged across by innumerable delicate cytoplasmic fibrils. These fibrils. connect adjacent cells and are frequently continued without interruption through one, two, or even three or four neighboring cells. Their course is characteristically curved, the convexity being directed toward the nucleus. Those portions of the numerous cytoplasmic fibrillæ which span the intercellular. spaces form the so-called intercellular bridges. 
It is because of the resulting spinous appearance that the polyhedral cells have been termed prickle cells (Schultze).

In the thinner portions of the epidermis the prickle cells are immediately covered by several layers of hard flattened cells whose nuclei have partially or wholly disappeared, and whose cytoplasm has been changed into a horny, keratin-containing mass. The flattening and desiccation of these cells becomes more pronounced as they approach

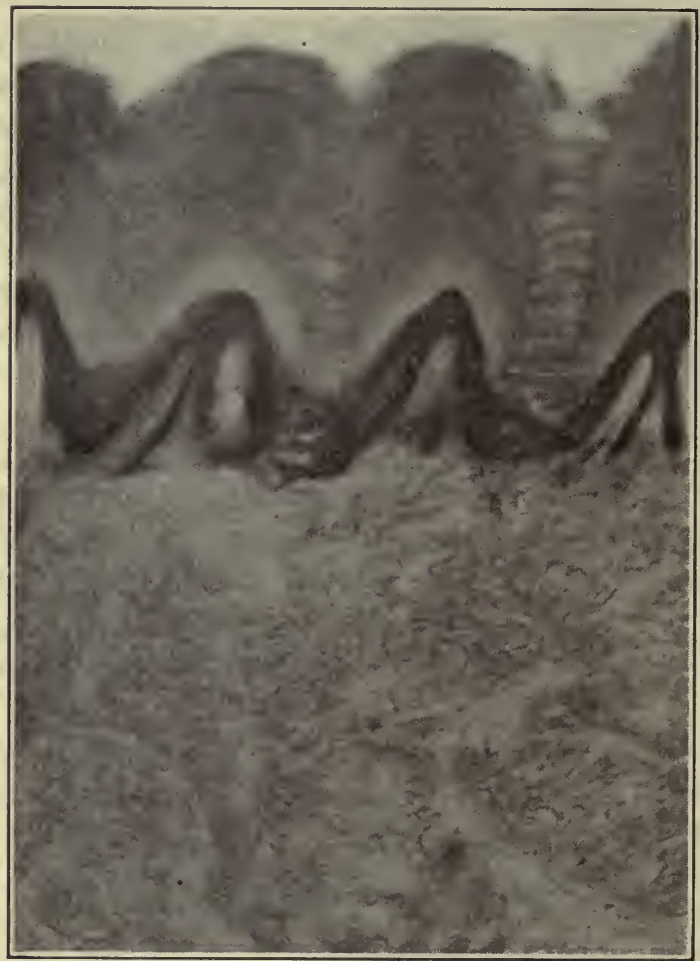

Fig. 263.-Skin from Sole of Human Foot, Showing Spiral Ducts of Two Sweat Glands Opening THROUGH THE EPIDERMis.

The stratum germinativum is represented by the dark, wavy line; below this is the stratum papillare of the derma, above the stratum lucidum. the surface. In the thin portions of the epidermis the change from the prickle cell layer to the horny layer is abrupt.

In the thicker portions of the epidermis, as in the palms of the hands, the change is more gradual, and results in the appearance of two additional cell layers, in the cytoplasm of whose cells are intermediate products of chemical metamorphosis, keratohyalin and eleidin, which may be considered as the predecessors of the keratin or pareleidin which is peculiar to the cells of the horny portion.

Granular Layer ( $S$ tratum Granulosum).-In the thicker parts of the cuticle the most superficial prickle cells become slightly flattened, and coarse granules appear within their cytoplasm. These cells form the granular layer (stratum granulosum), a double cell layer which occupies the superficial portion of the rete mucosum. 
The cells of the granular layer are flattened and angular. They possess an indistinct, apparently degenerating nucleus, and their eytoplasm contains large plate-like granules of keratohyalin (eleidin of Ranvier), which are strongly basophil and stain readily with most nuclear dyes.

Eleidin-containing Layer (Stratum Lucidum).-The granule cells are abruptly transformed into the shiny cells of the stratum lucidum, which is the deepest layer of the horny portion of the epidermis. The cells of this layer possess an indistinct nucleus, are irregularly flattened and angular in shape, are more or less fused together at their adjacent margins, and contain a smooth, highly refractive, glassy cytoplasm which reacts feebly to most staining reagents, but is deeply colored by safranin.

The stratum lucidum is so named because of its highly refractive appearance; it is usually about two cells thick. Its cytoplasm contains eleidin, a substance which is probably intermediate in chemical composition between the keratohyalin of the stratum granulosum and the keratoid pareleidin of the horny cells.

Flattened Cell Layer and Scaly Layer (Stratum Corneum and Stratum Disjunctum of Ranvier).-Above the stratum lucidum the horny layer consists of flattened cornified cells which are closely

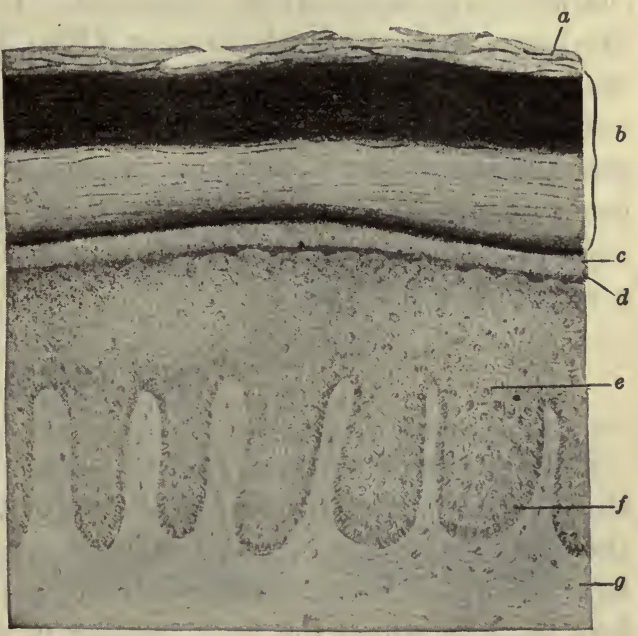

Fig. 264.-Transection of the Epidermis of the Foot.

$a$, superficial scaly layer; $b$, layer of flattened cells, the inner and outer portions of which have been characteristically blackened by osmium tetroxid; $c$, stratum lucidum; $d$, granular layer; $e$, prickle cells; $f$, cylindrical cells; $g$, papillary layer of the derma. Osmium tetroxid, carmin. Moderately magnified. (After Ranvier.) packed and somewhat fused and blended with each other at their faintly serrated margins. Intercellular bridges and spaces have almost entirely disappeared. The nuclei of the cells are no longer demonstrable, and their cytoplasm has been changed into a dry, shiny, highly refractive mass of 'keratin' (pareleidin) which responds but slightly to ordinary 
stains. Its superficial layers stain deeply in osmic acid, indicating a considerable fatty alteration. If, however, these cells are acted upon by solutions of strong alkalies, soda, potassa, etc., the outlines of the degenerated nuclei reappear. As the cells are pushed nearer the free surface, by the process of cell division in the deeper layers and the coincident desquamation of cells from the free surface, they become more and more flattened and desiccated and more completely and firmly fused together until at the surface they form the partially detached cell masses or scales-scaly layer, stratum squamosum-which are eventually removed by continued desquamation.

It is the thicker portions of the epidermis only, which possess all the characteristic layers above described. In other portions of the body the horny layer is much thinner (Fig. 262). In these thinner parts the cuticle of the epidermis consists of a prominent rete mucosum which is covered by a relatively very thin layer of horny cells. The stratum granulosum, in such portions, is not usually demonstrable, the stratum lucidum is absent or indistinct, and the entire horny layer consists only of flattened cornified cells, the more superficial of which form a very thin scaly layer.

\section{THE DERMA}

The derma or corium (derma vera, cutis vera) forms a connective tissue bed or matrix upon which the epidermis lies. It is divisible into two strata, a deeper reticular layer in which coarse fiber bundles interlace to form a loose connective tissue network, and a superficial papillary layer in which the finer bundles of connective tissue form a more closely meshed network.

The Papillary Layer (Stratum Papillare).-The surface of the papillary layer presents numerous conical elevations, the papilla of the corium, which project into corresponding cup-shaped cavities in the under surface of the epidermis. Many of the connective tissue papillæ contain tactile end-organs (touch corpuscles of Meissner), and terminal filaments of the nerve fibers. They may therefore be regarded as the special organ of tactile sensation. Other papillæ contain no touch corpuscles but are richly supplied with capillary blood-vessels. Two types are thus distinguished, the tactile papilla and the vascular papilla.

Papillæ are most abundant in the palms of the hands and the soles of the feet, where they are mostly arranged in rows which are responsible for the fine lines and ridges visible to the naked eye. In other portions 
of the body they are less numerous and are often less regularly disposed.

The papillary layer consists entirely of white fibrous and elastic-connective tissues which form a supporting membrane for the finer branches of the cutaneous blood-vessels and nerves. The elastic tissue supplies a rich network of fine fibrils to all portions of the papillary layer, and just beneath the epidermis it forms a delicate elastic membrane whose fibers intermingle with the hyaline cuticular deposit of the columnar epidermal cells to form a firm resistant basement membrane. Many of the elastic fibers of the papillæ, especially the more superficial ones, pursue a peculiar archiform course from the base to the apex of the conical papillæ. In this way they surround and inclose the centrally situated capillaries and the tactile corpuscles of the papillæ.

The Reticular Layer (Stratum Reticulare).-The deeper portion of the corium consists of interlacing bundles of connective tissue fibers which form a dense meshwork. These bundles are much coarser than those of the papillary layer with which they are imperceptibly blended. The reticular layer contains the larger blood-vessels of the corium, many small nerve trunks, the ducts and parts of the secreting portions of the sweat glands, the more superficial sebaceous glands, and many of the smaller hair follicles. Lamellar corpuscles and nerve end-organs of Ruffini are also found in this layer.

The skin of the face contains many striated muscle fibers which are derived from the insertions of the mimetic muscles. The corium of the scrotum (where it forms the tunica dartos), of the penis, perineum, and areola of the nipple contain much smooth muscle, intermingled with which is a considerable amount of elastic tissue.

\section{SUBCUTANEOUS TISSUE}

The subcutaneous tissue (tela subcutanea, subcutis) consists of bands and septa of fibrous connective tissue which extend from the deeper margin of the derma to the underlying fasciæ of the muscles, the periosteum of the bones, etc. The direction of these fibrous bundles is very variable. The more nearly parallel to the cutaneous surface the fiber bundles are, and the looser the meshes which they form, the greater is the mobility of the skin.

The meshes of the subcutaneous network are occupied by lobules of adipose tissue. When abundant the subcutis is termed panniculus adiposus. The subcutaneous tissue contains the main nerve trunks and 
larger blood-vessels of the skin, the larger sudoriparous and sebaceous glands, and the coarser hair follicles. It also, together with the deeper part of the derma, contains the nerve end-organs of Pacini, Ruffini, and the Golgi-Mazzoni corpuscles (see Chapter VI).

Small bundles of smooth muscle fibers which form the arrectores pilorum muscles take origin from the deeper surface of the corium and are inserted into that portion of the hair follicle which is embedded in the subcutaneous tissue. These fusiform or columnar muscle bundles are found in connection with all the hairs, but in the scalp they are most highly developed and lie most deeply in the subcutaneous tissue.
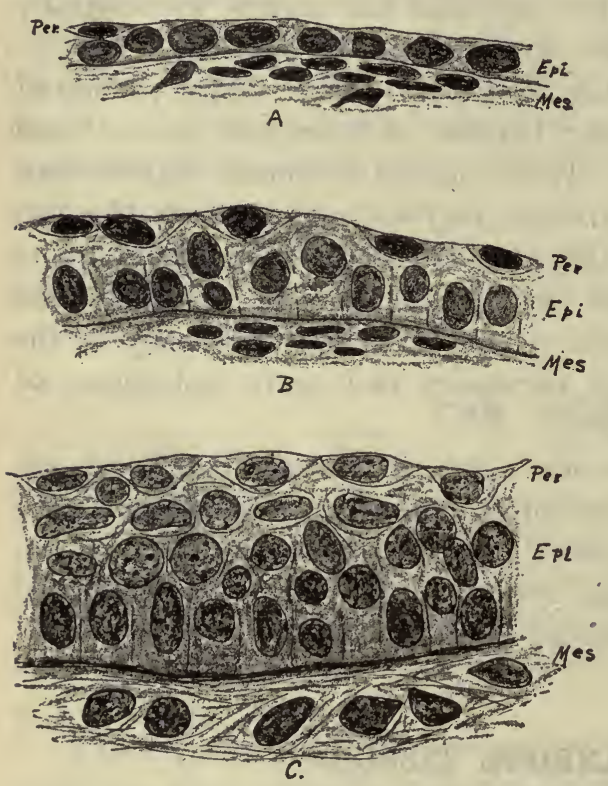

Fig. 265.-Three Early Stages in the Histogenesis of the Skin.

$A$, single-layered epidermis from dorsal body wall of a $5 \mathrm{~mm}$. human embryo; $B$, twolayered epidermis, from dorsal body wall of $13 \mathrm{~mm}$. human embryo; $C$, multiple-layered epidermis from nose of $21 \mathrm{~mm}$. pig embryo. Per, periderm (epitrichium); Epi, epidermis; Mes, mesenchyma differentiating into the derma. $\times 750$.

two layers, a superficial periderm (epitrichium), and a deeper germinal layer.

At the level of the vascular plexuses between the corium and the tela subcutanea, Häggquist (Anat. Anz., 45, 2, 1913) has described a thick bundle of smooth muscle not previously recognized. It lies directly beneath a cold spot, and is not found in skin lacking cold spots. The muscle is believed to contract reflexly when a cold object is placed on the skin and constrict the local blood supply.

\section{DEVELOPMENT AND GROWTH OF THE SKIN}

The skin may be said to arise with the first differentiation of the embryo into its three germ layers. The ectoblast, which is at first a single cell layer, becomes a double layer by the end of the first month. It continues to increase in thickness until by the end of the second month it can be differentiated into
chium), and a deeper germinal 
The periderm forms a layer of peculiar dome-shaped cells with flattened margins and a vesicular center. It continues to form the superficial layer of the epidermis until about the sixth month, when it is lost by desquamation. The germinal layer consists of a deep stratum of cylindrical cells and one or two superficial strata of spheroidal vesicular cells. The latter are known as the stratum intermedium. By the fifth or sixth month cell differentiation has advanced in the intermediate portion until cornification can be distinguished in its superficial cells.

Further development is analogous to the growth of the mature epidermis; new cells are rapidly formed in the deeper portion, stratum germinativum, and are steadily pushed toward the surface, their migration being either accompanied by slight, or later by more pronounced cornification, which in the latter case gives rise to the stratum granulosum, stratum lucidum, and horny layer, but in the former produces only relatively slight flattening of the superficial cells without the appearance of keratin or the disappearance of the nucleus.

The derma arises from the superficial layers of the mesoblast as ordinary connective tissue, in which the appendages of the skin make their appearance as ingrowths from the epidermis. Certain mesenchymal cells form the smooth muscle fibers of the arrectores pilorum muscles and of the derma of those locations where muscle is present in the mature skin. Other mesenchymal cells produce the fat lobules of the subcutaneous tissue. Papillæ appear during the fourth or fifth month but do not attain their completed development until much later.

\section{CUTANEOUS APPENDAGES}

The cutaneous appendages include the sudoriparous glands, the nails, the hairs, and the sebaceous glands.

\section{Sudoriparous Glands \\ (Glandula Sudoriparce, Sweat Glands)}

The sudoriparous glands occur in all portions of the skin, but more abundantly in certain locations, e.g., palms of the hands and soles of the feet,-where their number has been estimated at between two and three thousand to the square inch-axillæ, groin, and circumanal region. Over the back, where they are least numerous, their number is said to be less than five hundred to the square inch. They are long, coiled or convoluted, tubular glands whose secreting portions lie in the 
subcutaneous tissue and in the deeper part of the corium; their ducts extend through the corium to the under surface of the epidermis where the lining epithelium of the duct becomes continuous with the cells of the interpapillary portion of the stratum germinativum. In its further course through the epidermis the duct of the gland forms only a

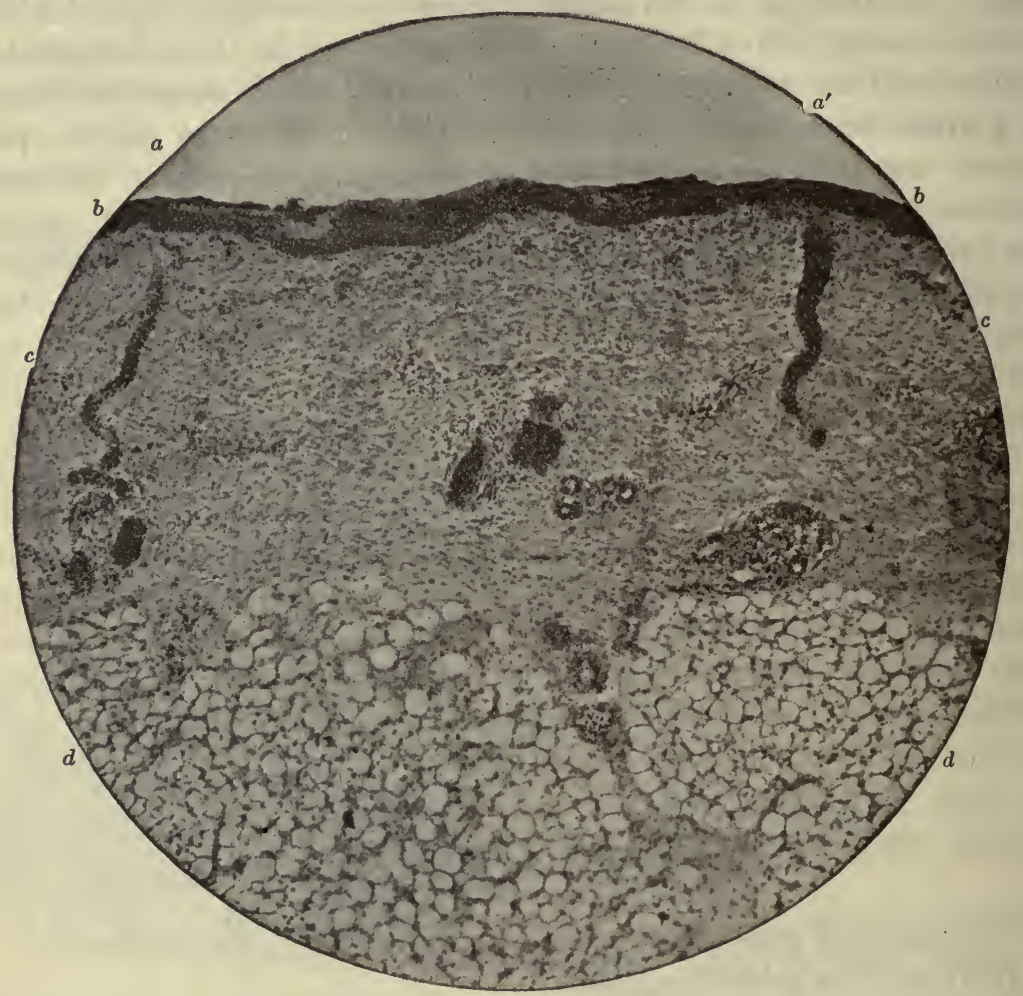

Fig. 266.-From a Section of the Abdominal Integument of an Infant.

Beneath $a$, and $a^{\prime}$, sweat glands are seen; the secreting portion of $a^{\prime}$ is detached from its duct; $b, b$, epidermis; $c, c$, derma; $d, d$, panniculus adiposus. Hematein and eosin. Photo. $\times 65$.

tortuous spiral cleft or passage whose wall is formed only by the concentrically placed cells of the various epidermal layers through which it passes. The glands of the axilla and circumanal region are branched.

The secreting or coiled portion of the gland (fundus) consists of a secretory epithelium resting upon a delicate hyaline membrana propria in whose outer portion are concentrically disposed connective tissue fibers. The inner portion of this membrane contains many longitudinal 
fusiform fibers whose nature is somewhat doubtful, though they have been most frequently considered to be smooth muscle fibers. These fibers are frequently branched, their processes often extending between the cells of the secreting epithelium nearly to the lumen of the gland.

The secreting epithelium of the fundus consists of tall columnar cells which possess a large spheroidal chromatic nucleus and a finely granular cytoplasm. The basal portion of their cytoplasm is often slightly rodded and the cells are so closely pressed together that it is frequently impossible to distinguish their outlines. The secreting cells are disposed in a single layer and, except after active secretion, are so

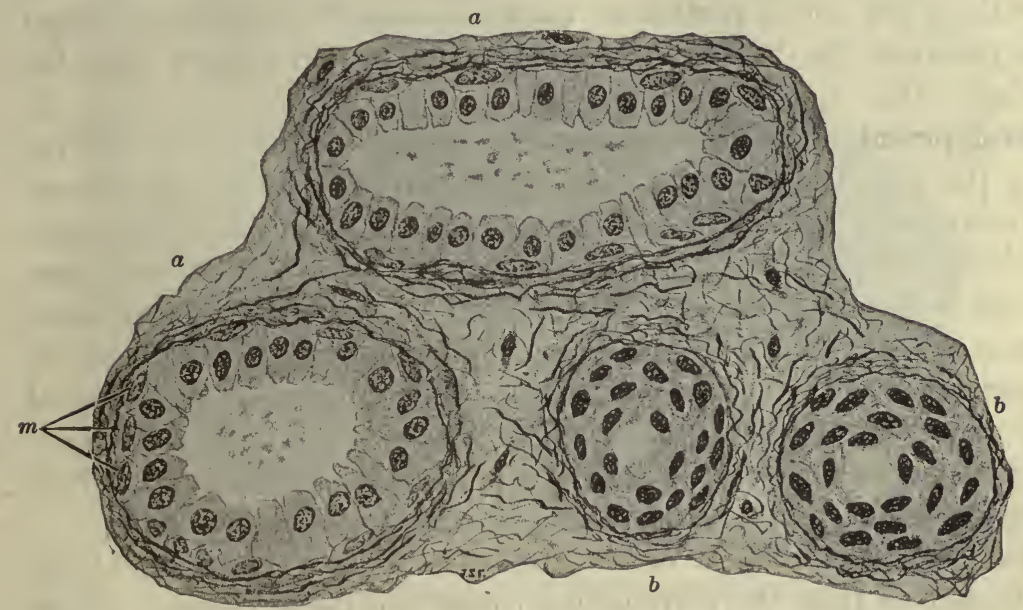

Fig. 267. - Several Coils of a Sudoriparous Gland of the Human Finder.

$a$, secreting portions, their lumen containing traces of secretion; $b$, ducts; $m$, muscle cells. Hematein and picrofuchsin. $\times 550$.

tall as to leave only a very narrow, central, glandular lumen. During secretory activity the cells become shrunken and their cytoplasm more granular. After a period of rest the cytoplasm again becomes clear and vesicular in appearance and the cells are much distended. The secretion reaches the lumen through intra- and intercellular canaliculi.

The ducts are lined by a double, occasionally triple, layer of somewhat flattened epithelial cells, which rest upon a delicate membrana propria continuous with that of the secreting portion. The gross diameter of the duct is much less than that of the secreting portion of the gland, yet the lumen of the duct may be larger. That portion of the duct which is lined by the thin stratified epithelial layer pursues a spiral course through the subcutaneous tissue and the derma. It finally 
reaches the epidermis, which it enters in the interval between the dermal papillæ (interpapillary portion of the epidermis). Its lining epithelium is continuous with that of the stratum germinativum, and in its course through the epidermis the wall of the duct consists solely of the surrounding epidermal cells. The stratum granulosum and adjacent portion of the horny layer in the immediate neighborhood of the duct is invaginated into the stratum mucosum, which is thus considerably thinned by the passage of the duct.

The sweat glands are abundantly supplied with capillary bloodvessels and small non-medullated nerves, which form plexuses about the walls of the coiled portion of the gland, and from which terminal fibrils penetrate the basement membrane and end in contact with the secreting cells.

Development.-The sudoriparous glands first appear in the embryo during the fifth month as solid columnar ingrowths from the stratum germinativum of the epidermis. These processes grow inward through the primitive corium to its junction with the looser subcutaneous tissue. Here the cell columns become thickened and convoluted, and at about the same period their lumen appears. The glandular lumen is not at first connected with the free surface, but as the cells of the germinal layers of the epidermis gradually replace those which are more superficial the epidermal portion of the duct is formed. At about the seventh month the lumen of the duct opens upon the epidermal surface.

The membrana propria of the fundus and dermal portion of the duct is derived from the surrounding connective tissue elements of the mesenchyma.

\section{The NAILS}

The nails are produced by a peculiar modification of the epidermis by which the stratum lucidum becomes greatly thickened (Bowen, 1899) while the horny layer (eponychium of the embryonic nail) is at the same time wanting. The nail is divisible into the nail body and nail root; the former comprising the exposed, the latter the hidden portion of the organ. The root of the nail is overhung by a fold of the skin, the thickened horny layer at the margin of which forms an adherent border, the eponychium of the adult nail.

The nail groove or sulcus is included between the overhanging skin and the root of the nail. It is deep at its proximal end but is shallow at the lateral margins of the nail. The distal or free border of the nail projects over the skin at the tip of the finger and the thickening of 
the horny layer of the subjacent epidermis forms the so-called hyponychium.

Finer Structure.-The nail consists of two layers, the superficial stratum lucidum and the deeper germinal layer. These are continuous at the border of the nail with the corresponding layers of the epidermis which lines the nail groove. At the distal border, however, the nail

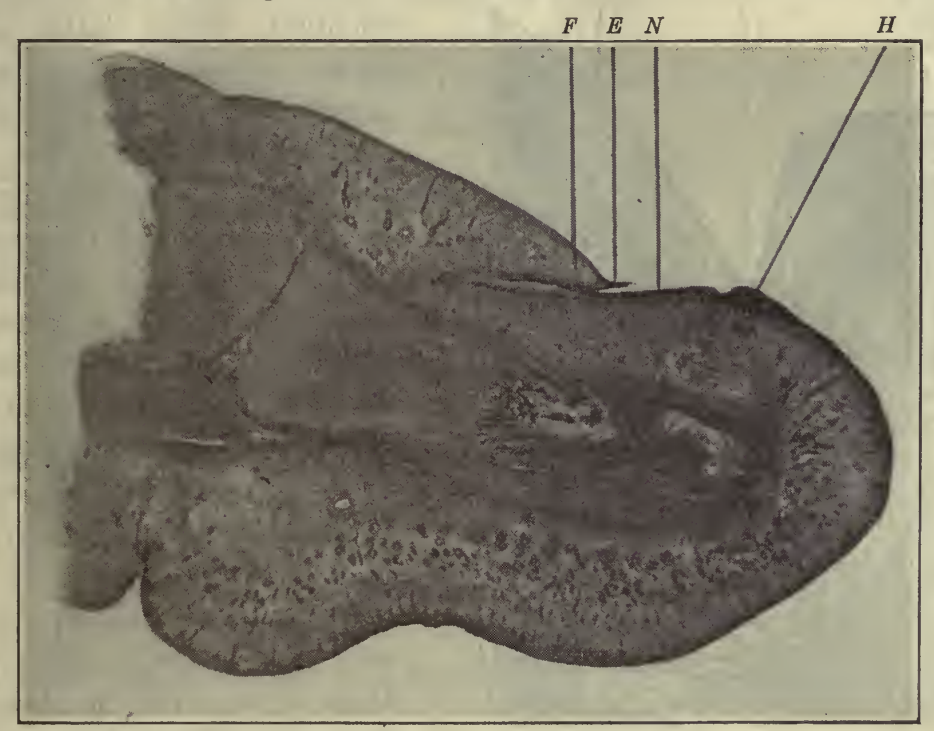

268.-Terminal Phalanx of Finger of Human Fetus.

Showing $N$, nail; $F$, nail fold; $H$, hyponychium; $E$, eponychium; developing sudoriparous glands, and developing bone.

proper or thickened stratum lucidum ends in a free margin. The finer structure of these two layers does not essentially differ from that of the corresponding layers of the epidermis.

The stratum lucidum in the body of the nail is very thick and its cells are so completely blended with each other through the excessive eleidin production that it is impossible to distinguish their outlines. By maceration in alkaline solutions, however, the outlines of both cells and nuclei may be caused to reappear. In the nail root the stratum lucidum increases rapidly in thickness as it grows distalward; in the body of the nail this layer is not very materially thickened as it approaches the distal or free margin.

The stratum germinativum is of nearly equal thickness in all portions of the nail body. In the nail root it is somewhat thicker and 
forms the nail matrix of Ranvier. In this portion also is a distinct stratum granulosum, a layer which is absent or rudimentary beneath the body of the nail. It is the presence within this layer of numerous keratohyalin granules which renders the root of the nail opaque and thus forms the dull white lunula which contrasts with the transparent,

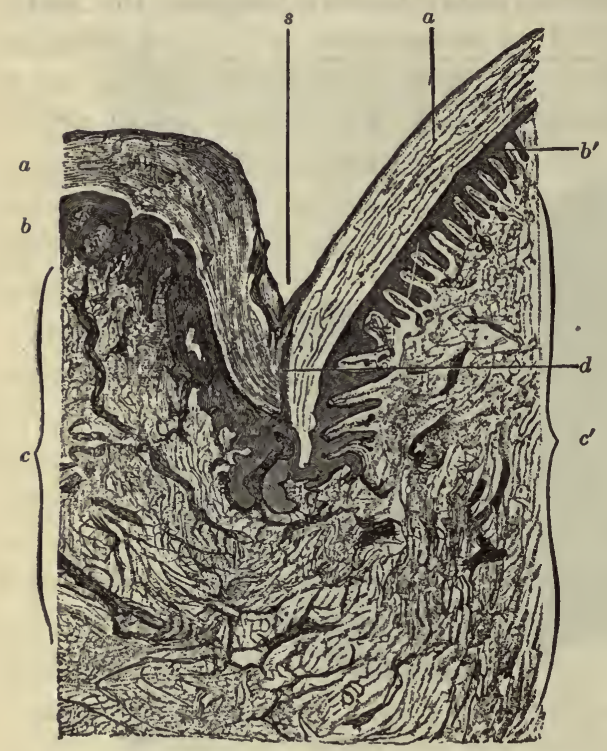

Fig. 269.-Transection throdgh the Margin of a Finger NaIL.

On the left is the skin, on the right the nail. $a, a^{\prime}$, horny layer; $b, b^{\prime}$, germinal layer; $c, c^{\prime}$, corium; $d$, margin of the nail; $s$, nail sulcus. Moderately magnified. (After von Brunn.) eleidin-containing, st $\mathrm{r}$ a $\mathrm{tu} \mathrm{m}$. lucidum, which latter layer alone covers the germinal layer of the nail body (Unna).

The Nail Bed.-The nail rests upon a very vascular corium or nail bed (matrix of authors) which is continuous: with the corium or derma of the skin. The nail bed is sometimes regarded as active in nail formation, but this process is now generally believed to be limited to the matrix of the root. The nail bed at the margins of the nail is provided with papillæ as in other portions of the skin, but beneath the body of the nail its surface is raised into longitudinal ridges which possess only very minute secondary papillæ.

Nail Growth.-The growth of the nail occurs in the matrix of the nail root. The cells of the stratum germinativum of this portion, having been once formed by active mitosis push obliquely forward and outward toward the nail body. It is thus that the more advanced are constantly carried onward toward the free border. The growth of the nail occurs at the rate of about one thirty-second of an inch per week (Schäfer).

Development.-In the fetus the nail appears as a direct formation of the epidermis, which is very early evidenced by a thickening of the stratum lucidum in the nail area. The nail is therefore at first. covered by the superficial peridermal cells of the cuticle. The nail groove is rapidly formed by an invasion of the mesoblast by the epidermal 
cells which become piled up at the margin of the groove to form an excessive horny layer, the definitive representative of the embryonic eponychium. At the distal extremity of the nail the superficial cells are also accumulated into a considerable mass which forms a prominent hyponychium. Further growth of the nail pushes its distal margin forward over the hyponychium so that the border becomes free shortly prior to birth. The peridermal cells are then shed and the nail body finally presents, at about the time of birth, its naked stratum lucidum.

\section{THE HAIR}

Development.-The structure of the hair will be most readily appreciated if preceded by a brief introductory sketch of its development.

THE HAIR GERM.-The hairs ${ }^{\circ}$ arise at any time after the third month of fetal life, their earliest anlage appearing as a slightly increased proliferation of the cells of the germinal layer of the epidermis. The further multiplication of the cylindrical cells produces a solid columnar ingrowth of the epidermis which penetrates into, and sometimes through, the primitive derma. The spheroidal cells of the intermediate layer of the epidermis increase in size, assume a vesicular character, and finally by fatty degeneration form the epidermal hair canal through which the future hair reaches the surface.

The Hair Column.-The columnar epidermal ingrowths, hair columns or hair pegs, come into early relation with the anlage of the hair papilla which is formed by a proliferation of the mesenchymal cells at the tip of the hair column. Further development of the papilla

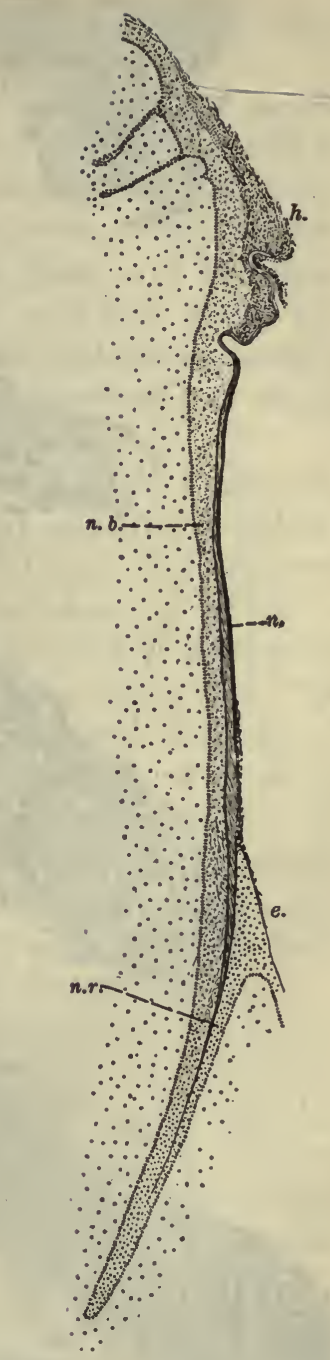

Fig. 270.-Longitudinal

Vertical Section of THE Young NaIl AND NAIL-BED OF AN INFANT.

$n$. $r$, nail root or lunula; $n$. , nail; $n$. $b$., nail bed; $e_{\text {., }}$ eponychium; $h$., hyponychium. (From Dahlgren and Kepner.) 


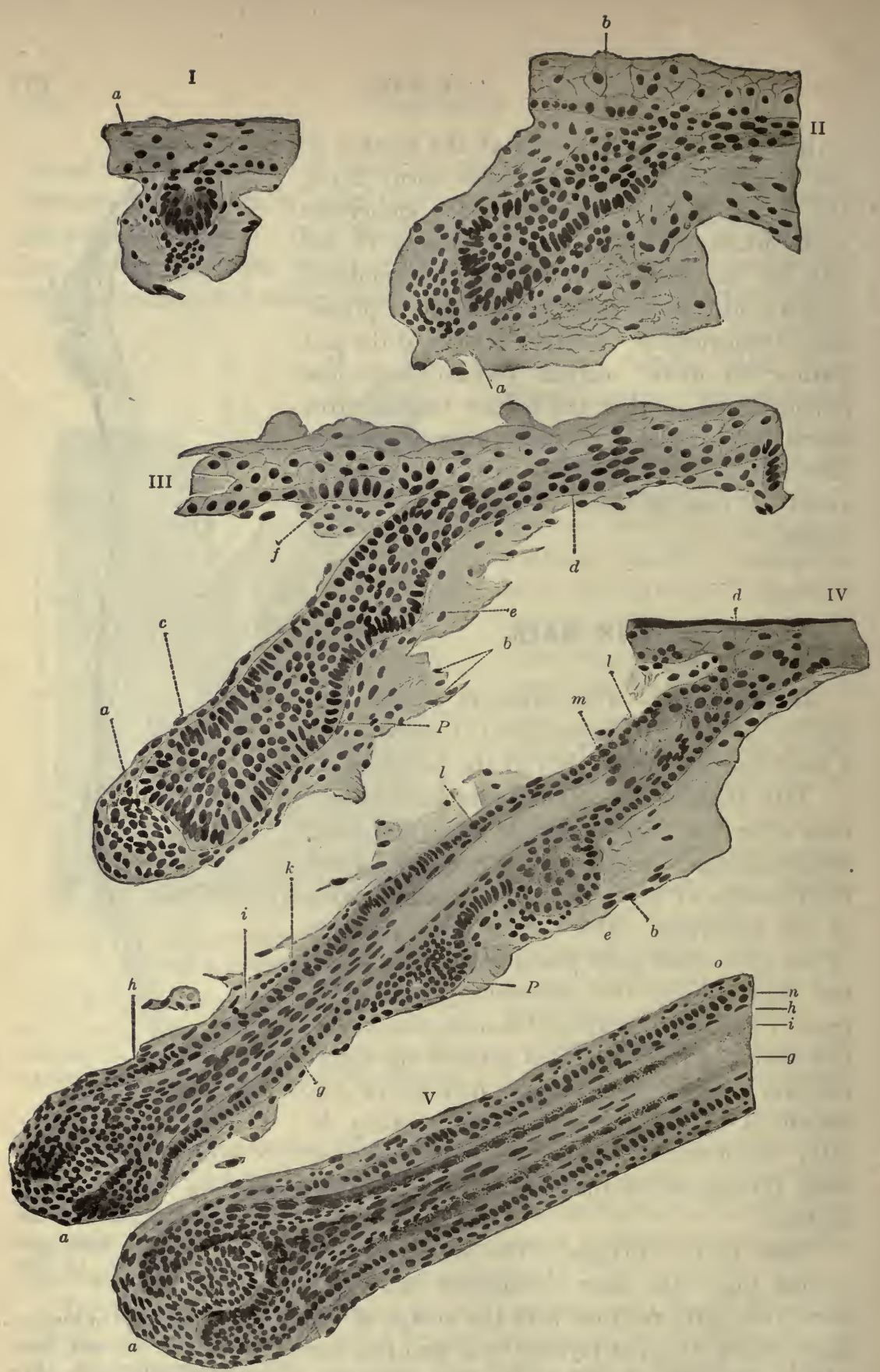

Fig. 271.-Five Stages in the Development of a Human Hair.

$a$, papilla; $b$, arrector pili; $c$, the line is directed toward the primordial shaft; $d$, cells which form the hair canal; $e$, sebaceous gland; $f$, hair germ in epidermis; g, hair shaft; $h$, Henle's layer; $i$, Huxley's layer; $k$, cuticle of the root sheath, ? inner root sheath; $m$, outer root sheath in tangential section; $n$, outer root sheath in longitudinal section; $o$, dermal root sheath; $P$, epithelial bed. $\times 460$. (After 
produces an indentation of the advancing hair column and gives rise to a true dermal papilla of considerable size.

The HaIr Bulb.-Coincident with the formation of the papilla there is an increased proliferation of the cells of the hair column by which it is surrounded, and which therefore represents the future hair bulb. Two other swellings appear in the hair column; one, the more superficial, forming the anlage of the sebaceous gland, and the other, the deeper, forming the so-called epithelial bed or matrix of the hair which stands in close relation with the growth and. future regeneration of the hair. This second swelling is sometimes interpreted as simply offering a point of attachment for the arrector pili muscle.

The development of the hair papilla produces a slight evagination of the epithelium of the hair bulb, which is just sufficient to redirect the growth of central cells of the hair column toward the cutaneous surface. It is thus that the younger cells which arise by mitosis in the germinal layers of the hair bulb are pushed outward along the axis of the hair column where they form the shaft of the future hair. The growth of the hair from the germinal cells of the hair bulb is accompanied by beginning cornification of the newly formed cells of the primitive hair shaft and of the intermediate cells of. the hair column. The growth of the shaft is, however, preceded by enlargement, vesiculation, and fatty degeneration of the central cells of the hair column, thus producing a central canal through which the hair may grow, and which later becomes continuous with the hair canal of the epidermis.

The Hair Follicle.-At this stage the hair column has become differentiated into a peripheral follicle, the primitive root sheath, and a central hair. Continued multiplication of the cells in the germinal layer of the bulb pushes the advancing tip of the hair nearer and nearer the surface until it forces its way into the epidermal hair canal. Finally the thin cuticular covering is ruptured and the eruption of the hair shaft occurs.

Further differentiation of the cells of the epidermal root sheath and the formation of a mesenchymal or dermal sheath of connective tissue completes the development of the hair follicle. This process is frequently repeated and results in the formation of new hairs not only during fetal life, but also, in constantly decreasing numbers, throughout childhood and adult life.

The Mature Hair.-Its development teaches that the hair follicle, being formed as it were by an invagination of the epidermis, contains a dermal and an epidermal sheath and that the outer portion of the 
latter, being identical with the deeper portion of the epidermis, must possess a close structural resemblance to the rete mucosum, while its inner portion, like the horny layer of the skin, is more or less cornified. There is thus an outer and an inner epidermal root sheath corre-

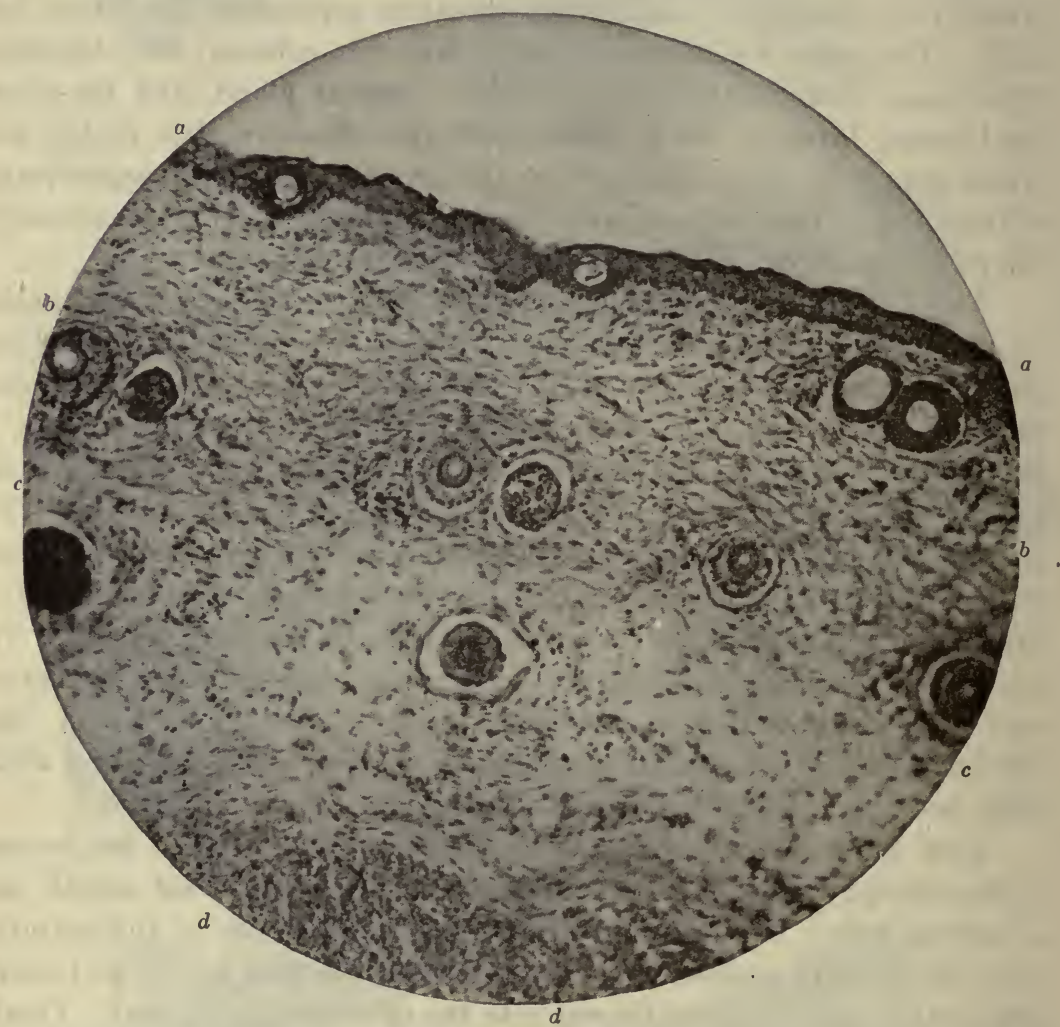

Fig. 272.-From a Section of the Skin of an Infant's Arm, Showing Small Immature Hair Follicles in Tr $\angle$ NSEction.

$a-a$, epidermis; $b-b$, derma; $c-c$, subcutaneous connective tissue; $d-d$, muscle. Hematein and eosin. Photo. $\times 95$.

sponding respectively to the mucous and horny layers of the epidermis; the cornified portion, inner root sheath, becomes progressively thinner toward the hair bulb. The hair, on the other hand, represents an excessively developed horny layer whose rete mucosum is found in the germinal layer of the hair bulb.

The mature hair is divisible into a hair shaft or free portion, and a hair root or concealed portion. The latter is inclosed within an epi- 
dermal and a dermal root sheath which together form the hair follicle. The Hair Shaft.-Sections of the hair shaft present a thin cuticle which consists of delicate horny scales whose free edges are imbricated upward, viz., toward the tip of the hair. Within the cuticle the hair may consist solely of a hair cortex formed by flattened and very much elongated horny epithelial cells, which frequently retain the remnant of a nucleus, and whose keratized cytoplasm is often much pigmented; or the axis of the coarser hair may contain enlarged angular cells in which eleidin granules and much pigment are found. In the latter case the hair is said to possess a medulla. The medulla is seldom if ever present throughout the entire length of the hair. When present it sometimes contains numerous air bubbles which, together with the paucity of pigment, produce the lighter shades of hair peculiar to certain individuals.

In the light of its development it is obvious that the several layers of the hair shaft are comparable to the homologous layers of the horny epidermis, the cuticle, cortex, and medulla of the hair being respectively homologous with the scaly layer, the flattened cell layer, and the eleidincontaining layer or stratum lucidum of the epidermis.

The HAIR Root.-The root of the hair, except for the fact that it is immediately invested with a hair follicle, does not in any way differ in structure from the hair shaft. It possesses the same three layers, the medulla, however, being very irregularly developed.

The imbricated cells of its cuticle interdigitate with the similar cells of the cuticle of the inner root sheath in the deeper half of the follicle; in its superficial half, viz., above the opening of the sebaceous gland, a narrow space intervenes between the cuticle of the hair and that of the root sheath.

The axis of the hair root is always inclined at an angle to the epidermis; it therefore makes with the epidermis an obtuse angle on one side and an acute angle on the other. The arrector pili muscle is always found on the side of the obtuse angle; it therefore, by drawing the hair follicle and its inclosed hair root nearer the perpendicular, causes the erection of the hair. The sebaceous gland is included in the angle between the arrector muscle and the hair follicle. Contraction of the muscle may aid also in the expulsion of the sebum. Extreme contraction of the arrectores pilorum muscles may result from fright, causing the hair to 'stand on end'; in a similar manner cold air may effect, through the pilomotor fibers, the erector muscles of the small hairs distributed over the body and cause the so-called 'goose-flesh.' 


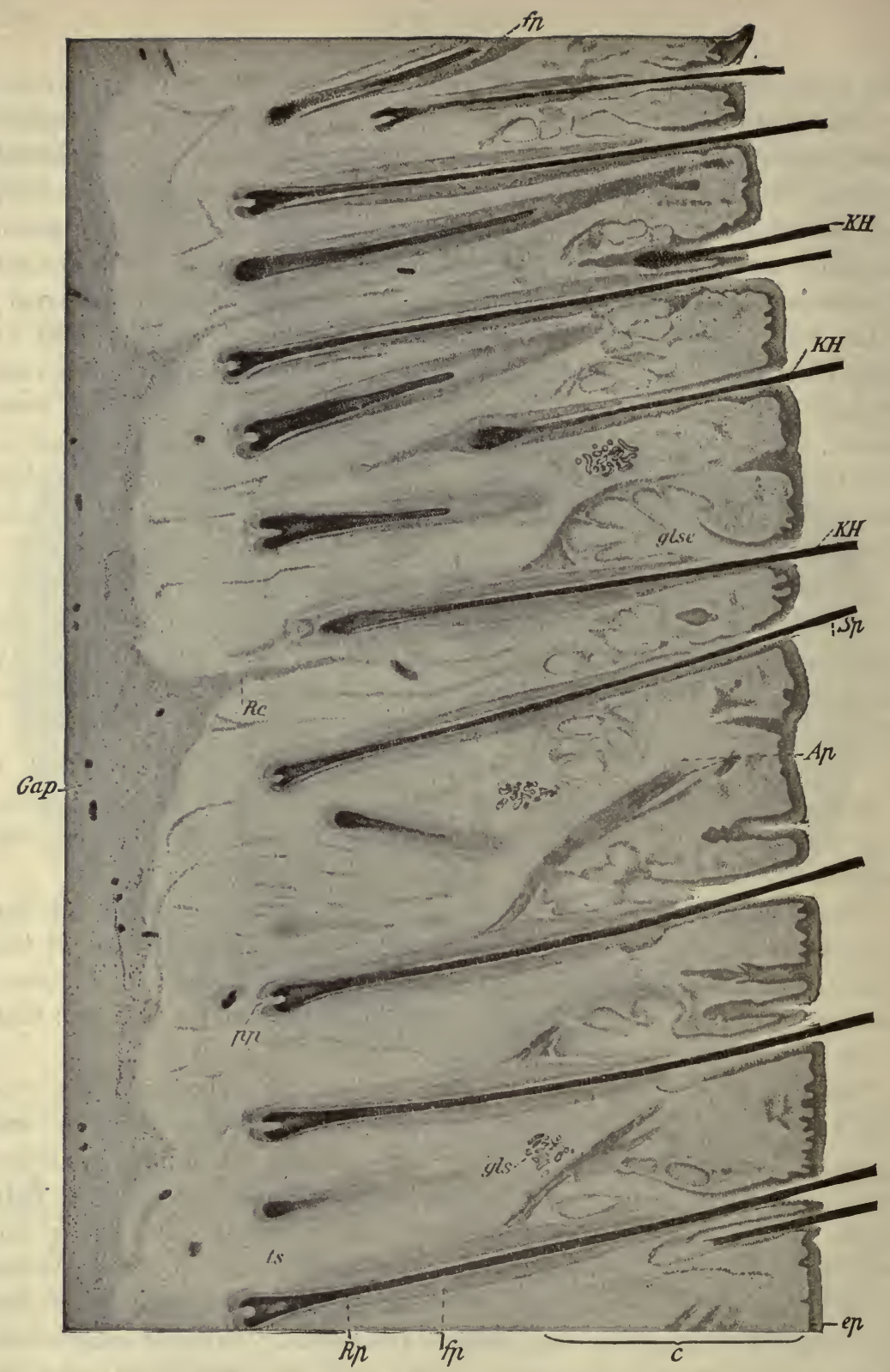

Fig. 273.-From a Section of the Human Scalp.

$A p$, arrector pili muscle; $c$, corium; $e p$, epidermis; $f p$, epidermal root sheath; Gap, muscular aponeurosis; gls, sudoriparous gland; glse, sebaceous gland; $K H$, so-called 'club-hairs' in various stages of molting and regeneration; $p p$, papilla of the hair; $R c$, fibrous band in the subcutaneous tissue; $R p$, hair root; $S p$, hair shaft; ts, subcutaneous adipose tissue. Hematoxylin and eosin. $\times 15$. (After Sobotta.) 
The Epidermal Root Sheath.-The epidermal root sheath consists of an inner and an outer portion, each of which at about the midlevel is divisible into three layers corresponding to the three similar layers of the horny and the mucous portions of the epidermis. In those portions of the follicle and in those individual hairs in which the process of cornification is less advanced these subdivisions cannot all be demonstrated, and it is only in the most highly developed hairs that they are typically found. This is in accordance with the structure of the epidermis, in which the subdivisions of its horny and mucous portions are typically found only in the more highly developed portions, e.g., the palms and soles.

Inner Root Sheath.-The cuticle of the inner root sheath consists of thin horny epithelial scales which are imbricated downward, viz., toward the hair bulb, and which interdigitate, in the deeper portion of the follicle, with the similar scales of the hair cuticle. The direction of the imbrication explains the removal of the epidermal root sheath when the hair is artificially extracted.

The mid-layer of the inner root sheath, layer of Huxley, one or two cells thick, consists of horny cells which are somewhat flattened, and in which the semblance of a nucleus is sometimes present. It corresponds to the flattened cell layer of the epidermis.

The outer layer of the inner root sheath, layer of Henle, is frequently wanting or imperceptibly blended with the preceding layer. Its cells are clear and highly refractive and their nuclei can but rarely be demonstrated in the usual microscopical preparations. The layer is seldom more than one cell deep. It is homologous with the stratum lucidum of the epidermis.

Outer Root Sheath.-The outer root sheath is continuous with the stratum mucosum of the epidermis and therefore contains similar cell types. The granular layer, as in the epidermis, is frequently absent or rudimentary, but can be readily demonstrated in hematein-stained sections of the more highly developed hair follicles. It rests upon a layer, several cells deep, of spheroidal prickle cells. The outermost layer of the outer root sheath is formed by a basal layer of cylindrical cells. It grows progressively thinner toward the hair bulb, where its cells become mingled with the germinal cells of the hair matrix.

Dermal Root Sifeath.-The dermal root sheath presents three layers, an innermost basement membrane or glassy layer, a layer of circular connective tissue fibers and a similar layer of longitudinal fibers. These layers are obviously homologous with the basement mem- 
brane, the papillary layer, and the reticular layer of the derma, respectively. The dermal root sheath is, however, devoid of papillæ.

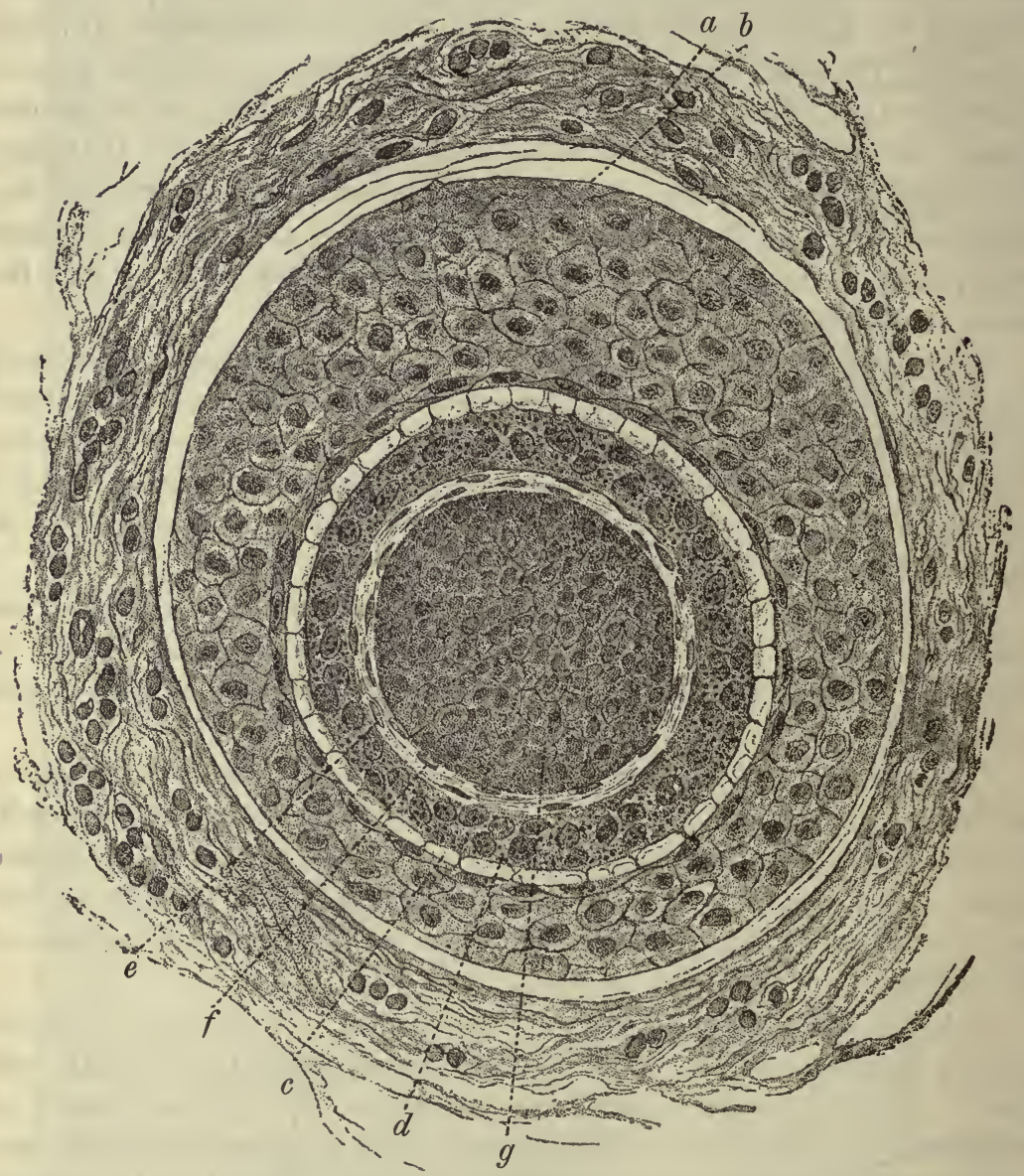

Fig. 274.-Transection of a Hair Near the Middle of the Root Sheath.

$a$, dermal root sheath; $b$, outer margin of the epidermal root sheath-the light space is the glassy membrane, the polyhedral cells form the outer root sheath; $c$, Henle's layer of the inner root sheath; $d$, Huxley's layer; $e$, cuticle of the root sheath; $f$, cuticle of the hair; $g$, cortex of the hair shaft. Highly magnified. (After Kölliker.)

The glassy membrane is a peculiarly thick homogeneous membrane which is chiefly mesoblastic in origin, but whose innermost portion is formed as an exoplasmic product of the adjacent epithelium. This 
membrane is highly refractive and contains very few connective tissue cells or fibers.

The circular fibers of the dermal root sheath contain interlacing. bundles of connective tissue fibers, which are mostly disposed in a ringlike manner. Elastic fibers are absent. Within this layer is a dense anastomosing plexus of capillary blood-vessels, together with a rich subepithelial plexus of non-medullated nerve fibers.

The longitudinal fibers of the connective tissue root sheath also form interlacing fiber bundles, most of which are somewhat obliquely disposed. The bundles are coarser than those of the preceding layer and contain a few elastic fibers. This portion of the root sheath contains many small blood-vessels and nerves which supply the plexuses of the circular layer.

The hair follicle in transverse section varies in different races from circular to elliptical form. In the Chinese race the diameter of the follicle and the hair is $100 \times 7 \%$ to 85 , in the European $100 \times 62$ to 72 , and in the Negro $100 \times 40$ to 60 . The more elliptical the form of the follicle the greater the curl of the hair.

Atypical Portions of the Hair Follicle.-As already indicated, the hair follicle presents some structural differences at various levels. The typical arrangement is found only in the mid-portion of the follicle.

In its superficial portion the hair lies free in the follicular lumen, the interval between it and the inner root sheath being only partially occupied by the fatty secretion of the sebaceous gland which enters the lumen of the follicle at the deeper portion of its middle segment. At this level also, the root sheaths of the hair offer a gradual transition from their typical structure to that of the dermal and epidermal layers with which they are continuous.

The hair bulb likewise differs prominently from the typical structure of the hair root. In this portion the germinal layers are very highly developed at the expense of the horny layers. It is, therefore, in this portion that growth is most active. The cells of this region are often deeply pigmented. The increased size of the germinal layer, moreover, produces a distinct bulging of the follicle, which incloses the hair papilla and results in the peculiar bulbous shape of the extremity of the hair follicle.

The Hair Papilla.-The structure of the hair papilla is identical with that of the vascular papillæ of the derma except that it is constructed upon a much larger scale. It consists of a conical or clubshaped elevation of connective tissue which indents the extremity of 
the hair bulb. It contains an abundant plexus of capillary blood-vessels and a rich supply of non-medullated nerves. It also contains an undue proportion of connective tissue cells.

Regeneration of the Hair.-Hairs are being continuously shed and regenerated, the average life of a hair of the scalp being stated as sixteen hundred days (Stöhr). The shedding of a hair is first heralded by an atrophy of its papilla and a cornification of its bulb. Growth ceases, and the hair, firmly adherent to its root sheath, is gradually carried, by the continued growth of the latter, nearer and nearer the surface of the skin.

Its excursion leaves behind a narrowed cell column which still unites the hair with its former papilla.

From this rudiment a new hair germ may form (Unna), a new papilla develop, and the resulting hair grow toward the surface in the path of the molting hair, its eruption being preceded by the falling of its predecessor. The formation of the new hair germ very probably occurs at a point nearly corresponding with the insertion of the arrector pili muscle, where there is a swelling of the root sheath which has been already mentioned as the matrix of the hair follicle. This matrix appears very early in the development of the hair, but remains quiescent until regeneration becomes necessary, when the cells are said to proliferate and grow downward filling the space between the atrophic hair and the new bulb.

In infancy and youth shed hairs are also compensated for by new formation from hair germs appearing at the germinal border of the epidermis, the process proceeding in the manner already described for the development of the hair.

\section{The Sebaceous Glands}

These are branched saccular glands which may be subdivided into two classes, (1) those whose ducts open into the hair follicles, and (2) those whose ducts open upon the free surface of the epidermis. The former are by far the more numerous; the latter occur in the skin of the face, red margins of the lips, labia minora, glans penis and prepuce, and the tarsal glands of the eyelids. With the above exceptions the distribution of the sebaceous glands is coextensive with that of the hairs. They are therefore absent from the palms of the hands and soles of the feet.

A sebaceous gland consists of a dilated saccular fundus, a constricted 
ressels. Branches from this plexus pass to the papillary layer, where they form a second (subpapillary) plexus from which terminal arteries are distributed to the capillaries of the papillæ.

The distribution of the veins is similar to that of the arteries. The primary plexus is found in the papillary layer; occasionally a second plexus immediately underlies the first, and from these, venules pass to the deeper part of the corium, whence after free anastomosis they proceed to the subcutaneous tissue, collecting on the way the venules returning from the hair follicles and secreting glands, and from the subcutaneous connective tissue. The very rich capillary network in the papilla of the hair bulb is worthy of special mention.

The lymphatic vessels of the skin begin as a terminal lymphatic plexus in the corium, which collects the lymph from the tissue spaces of both derma and epidermis. The vessels of this plexus communicate with a subcutaneous lymphatic plexus of larger vessels which follow the course of the blood-vessels on their way to reach the neighboring groups of superficial lymph glands.

\section{NERVE SUPPLY}

The skin is abundantly supplied with large nerve trunks, both sympathetic and cerebrospinal (sensory), which find their way along the subcutaneous fat and send branches directly to the larger blood-vessels, the hair follicles, the sebaceous and sudoriparous glands, to the corpuscles of Pacini, Ruffini, and Golgi-Mazzoni, and to the end bulbs of Krause, which lie in the connective tissue.

In the cutis vera the nerve trunks form a plexus of delicate fiber bundles in the reticular layer, with a secondary, more closely meshed plexus of finer nerve bundles in the papillary layer. From these plexuses fibrils are distributed to the smaller blood-vessels and to the papillæ, where many end in tactile corpuscles. Other fibrils penetrate the epidermis, terminating as naked fibrils or on tactile cells.

In the region of the hair follicle small branches form a network of fibrils in the dermal root sheath. Branches (pilomotor nerves) are also distributed to the arrectores pilorum muscles.

In the sudoriparous glands the nerves form a fine plexus about the membrana propria (epilamellar plexus), from which naked axis cylinders penetrate the basement membrane and terminate between the secreting cells. 


\section{CHAPTER XII}

\section{THE RESPIRATORY SYSTEM}

The respiratory system may be said to comprise a true respiratory organ, the pulmonary alveoli, in which the interchange of gases between the air and the blood occurs, and a system of duct-like passages leading

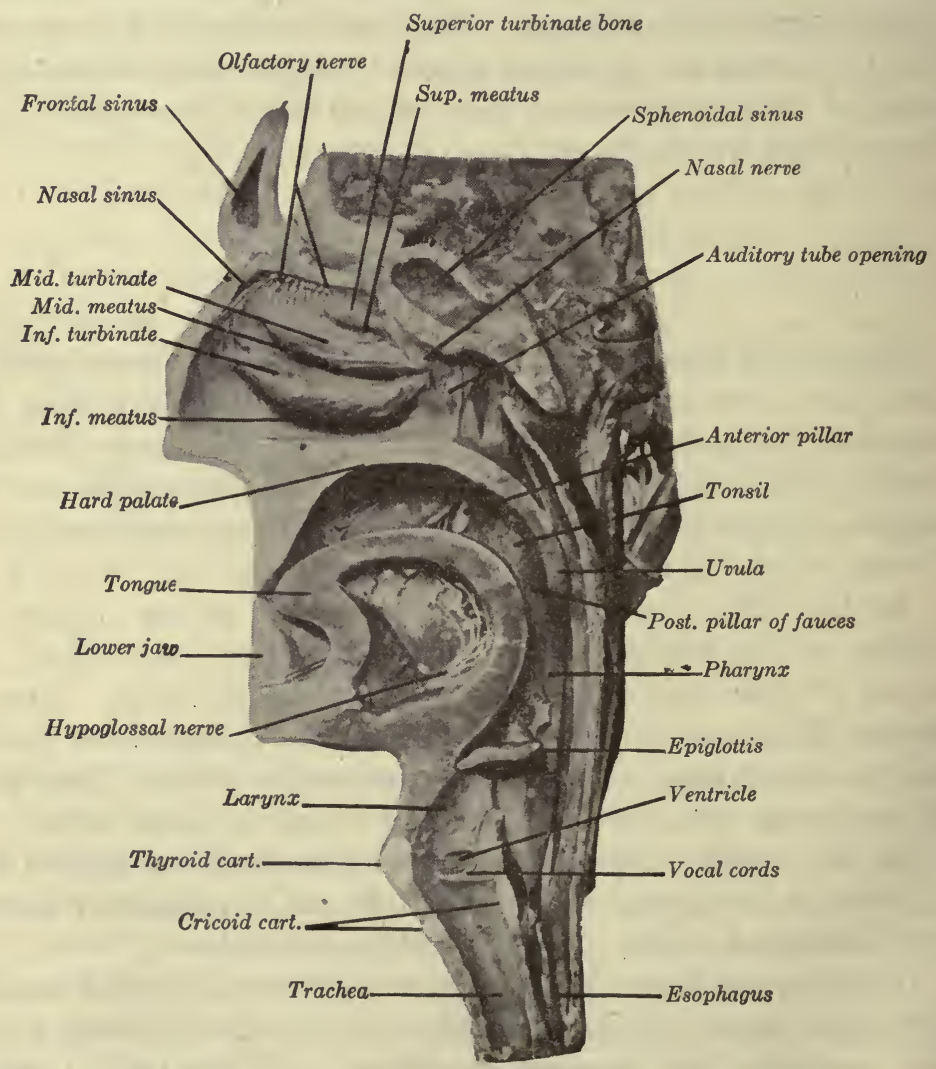

Fig. 280.-Photograph of Azoux Model, Showing Nostril, Pharynx, Larynx and Related Structures. 
thereto, which, beginning with the nasal cavity, successively includes the nasopharynx, larynx, trachea, and bronchi of gradually diminishing caliber, and which finally ends in the terminal bronchioles leading to the air sacs and pulmonary alveoli.

The arrangement of these several portions of the respiratory system has been frequently compared to the structure of the tubulo-acinar glands. From this point of view the larynx and trachea form the duct stem of the gland, the bronchi form the branching interlobar and interlobular ducts, and the terminal bronchioles (intercalary ducts) end in the numerous acinar air saccules of the lung.

Development.-The original anlage of the respiratory system, beginning with the larynx, is a short linear ventromedial evagination from the cephalic end of the primitive esophagus. This tracheal groove becomes separated, distally, thus forming a tube, which grows backward, meanwhile dividing distally into the bronchi which undergo further division to form the successively finer branches of the pulmonary system. The respiratory epithelium is thus of entodermal origin, and becomes enveloped in connective tissue of mesodermal origin.

\section{THE NASAL CAVITY}

This cavity is bounded by a cartilaginous and bony wall and is lined by a mucous membrane which, according to the nature of its epithelium, may be divided into three portions: (1) the vestibule, (2) the respiratory portion, and (3) the olfactory portion.

Its external and internal openings are the nares and choance respectively. Communicating with the nasal chambers are the sphenoidal, maxillary, frontal, and palatal accessory sinuses, and the ethmoidal air cells. Their lining membrane is continuous with, and histologically essentially like, though thinner than, that of the respiratory portion of the nostril.

\section{The Vestibule}

The vestibule of the nose corresponds very closely to the cartilaginous portion of the nasal wall. Its mucous membrane is continuous anteriorly with the skin and posteriorly with the mucous membrane of the respiratory portion. The vestibule is lined by stratified squamous epithelium, which offers a gradual transition from the moist respiratory epithelium to the dense horny epidermis of the skin. Near its external ori- 
fice are numerous coarse stiff hairs, vibrissa, connected with which are many sebaceous glands. The vibrissæ have no associated arrectores pilorum muscles. Some of the glands also open directly upon the surface of the mucous membrane.

The fibrous tunica propria of the vestibule is continuous with the corium of the skin, and in it are embedded the deeper portions of the vibrissæ and the secreting portions of the sebaceous glands. By its deeper surface the tunica propria is closely attached to the perichondrium of those plates of hyaline cartilage which form the septum and alæ of the nose.

\section{The Respiratory. Portion}

The respiratory portion of the nasal mucous membrane (Schneiderian membrane) clothes the middle and inferior meatus of the nose. It is continuous anteriorly with the mucous membrane of the vestibule,

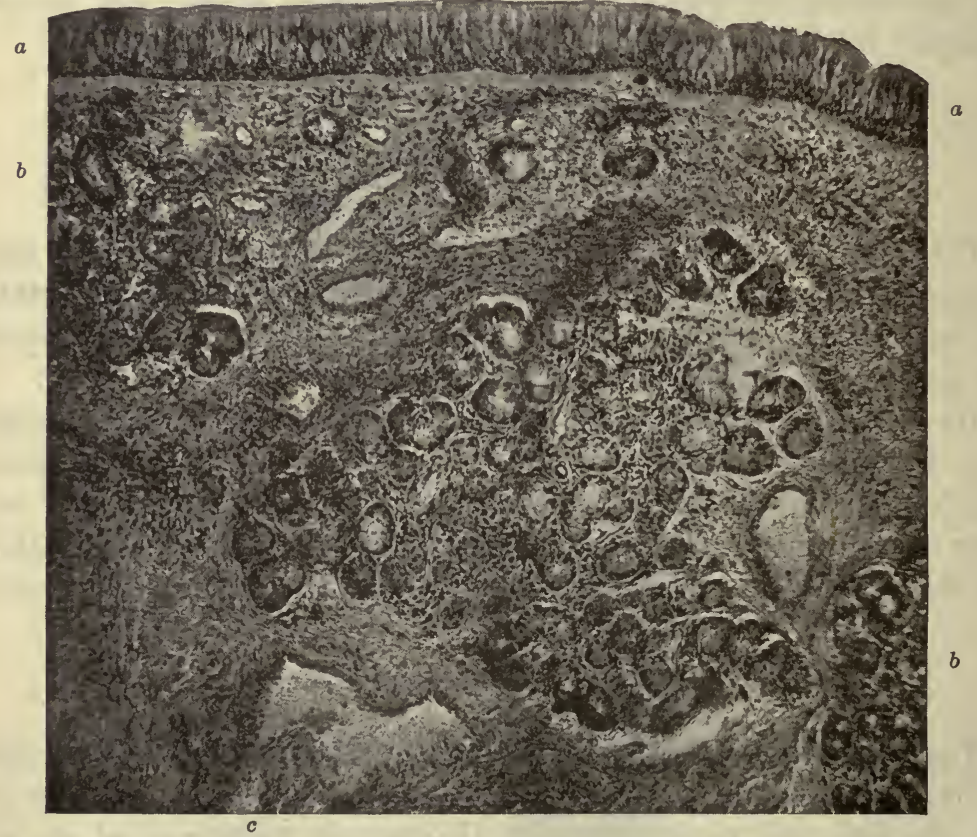

Fig. 281.-From a Section of the Mucous Membrane of the Respiratory Region of the Human Nose.

$a-a$, ciliated epithelium; $b, b$, mixed mucoserous glands; $c$, blood vessel. Hematein and Congo red. Photo. $\times 185$. 
above with the olfactory mucous membrane, and posteriorly with that of the nasopharynx. The respiratory region is lined by columnar ciliated epithelium of the pseudo-stratified type, which also contains many mucus-secreting, goblet-cells.

The epithelium rests upon a distinct basement membrane which reacts to the specific stains for elastic tissue. The tunica propria consists of a very vascular connective tissue; it varies much in thickness in different portions. It is thinnest in the accessory sinuses and is thickest where it covers the turbinal bones and the adjacent portions of the nasal septum. The tunica propria is richly supplied with both mucous and serous glands. The smaller ones, in the thinner portions of the mucous membrane, are somewhat convoluted; the larger and more numerous are tubulo-acinar glands. Many of the latter are mixed glands containing both mucous and serous acini. They produce an abundant secretion.

The Schneiderian membrane is in all portions extremely vascular, many of its vessels having very thin walls. The thicker portions over the turbinals and the septum are typically erectile. The dense connective tissue of these portions is permeated with broad venous channels which are surrounded by bands of smooth muscle. Other muscular bundles are longitudinally distributed. The small arteries are contained within the fibromuscular stroma.

The subepithelial portion of the tunica propria contains fine inter-' lacing bundles of connective tissue and many capillary blood-vessels. Here and there it is also infiltrated with lymphocytes and occasional very minute solitary nodules are found. The lymphatics of the Schnei- derian membrane lead posteriorly to the lymph nodules of the nasopharynx.

\section{The Organ of Jacobson}

Associated with the respiratory portion is the rudimentary vomeronasal organ (of Jacobson). In the embryo of one month it appears as a tubular extension, one on either side, into the corium of the median septum, opening anteriorly. In transverse section it has a semicircular outline, with its convexity mesial. In lower forms, e.g., Amphibia, it persists in the adult as a functionally important organ concerned with smell. It is innervated by fibers from the olfactory nerves, vomeronasal nerves, and by the nervus terminalis, which is present also in man (Johnston, Anat. Rec., 8, 4, 1914). In the cat the tall columnar ciliated epithelium (pseudo-stratified) includes true sensory cells, similar to the 
olfactory cells. The nervus terminalis contains some medullated fibers, and may contain both afferent and efferent components (Johnston). In the rabbit it innervates in addition to Jacobson's organ, also a wide area of the nasal septum (Huber and Guild).

\section{The Olfactory Portion}

The olfactory portion of the nasal mucous membrane, the olfactory organ, lines the superior meatus, a portion of the superior turbinate bone and a corresponding portion of the median septum, and its irregular border here and there invades the upper portion of the middle meatus. It consists of a fibrous tunica propria and a clothing of

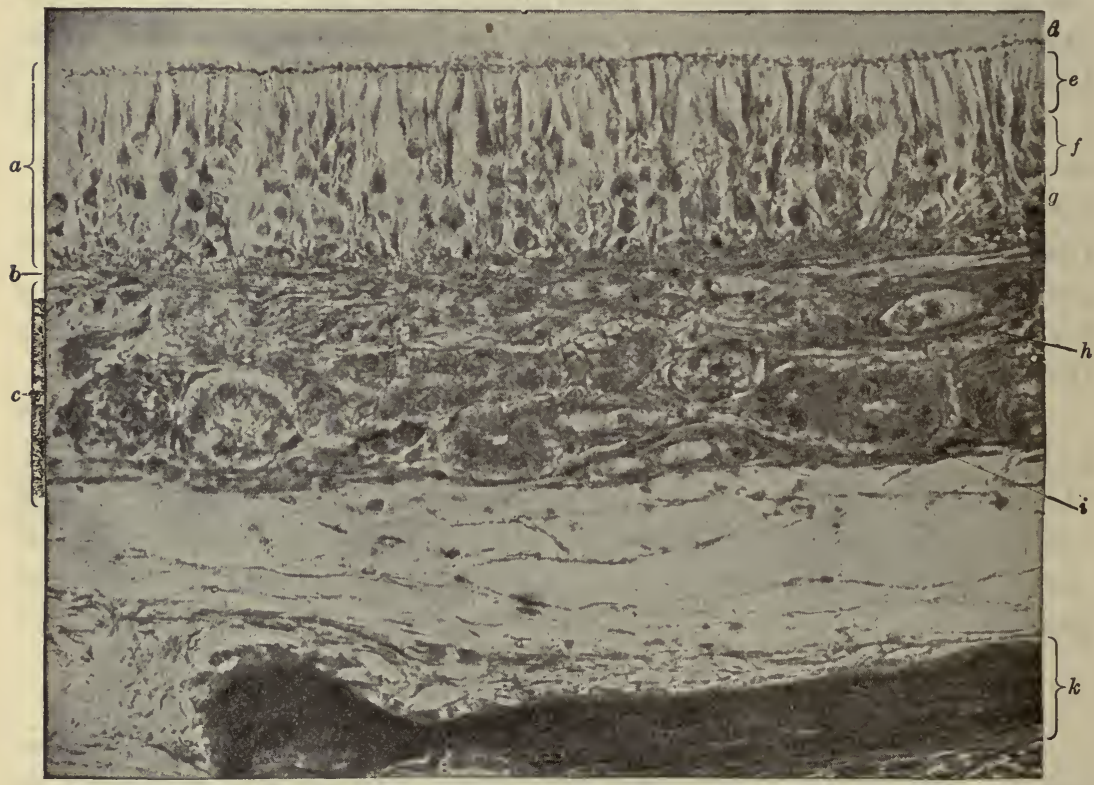

Fig. 282.-The Olfactory Mucosa of a Cat.

$a$, epithelium; $b$, basement membrane; $c$, corium; $d$, cuticle; $e$, sustentacular cell layer; $f$, olfactory cell layer; $g$, basal cells; $h$, blood-vessel; $i$, a tubule of Bowman's glands; $k$, bone. Hematein and picrofuchsin. Photo. $\times 270$.

neuro-epithelium. The tunica propria contains elastic as well as collagenous fibers, and many small tubulo-acinar serous glands, the olfactory glands of Bowman. Beneath the epithelium is an indistinct basement membrane. 
The neuro-epithelium contains three intermingled cell types, the sustentacular, olfactory, and basal cells.

The sUstentacular CELLS are columnar ciliated epithelial cells which possess a distinct cuticular margin. Their nuclei are ovoid, and, since they lie at the same level, they form a continuous superficial zone of oval nuclei. The deep ends of the cells are often branched; they interlace with one another and with the processes of the olfactory and

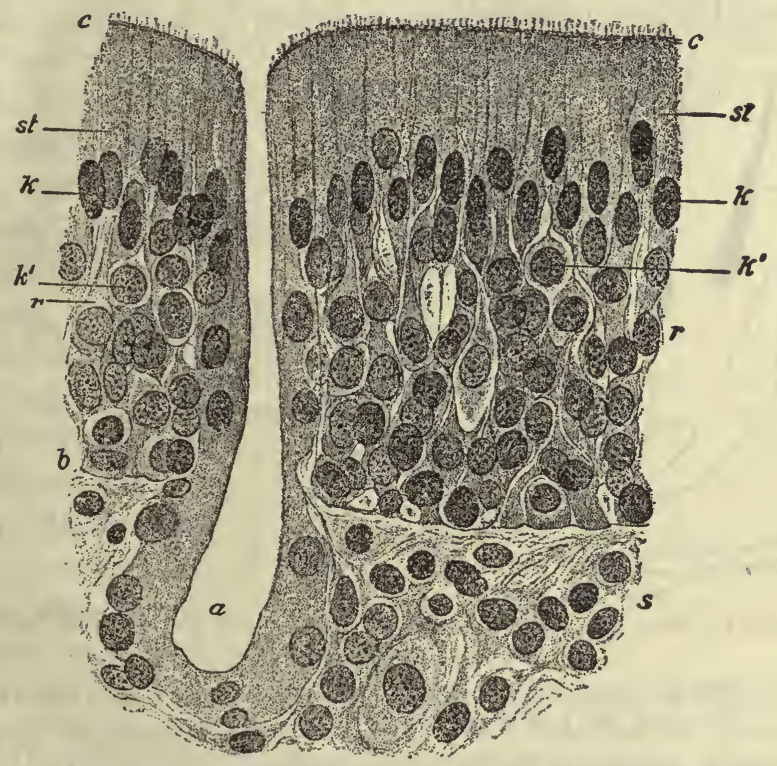

Fig. 283.-The Olfactory Mucous Membrane.

$a$, duct of gland; $b$, basal cells; $c$, cuticular border with olfactory cilia; $k$, nuclei of the sustentacular cells; $k^{\prime}$, nuclei of the olfactory cells; $r$, layer of olfactory cells; $s$, corium, containing connective tissue cells and nerve fibers; st, sustentacular cells. $\times 465$. (After Kölliker.)

basal cells. The cytoplasm of the sustentacular cells is finely granular and contains a yellow pigment.

The OLFACTORY CELLS occupy a unique position among neuro-epithelial cells in that they are true nerve or ganglion cells. They possess a small cytoplasmic body and two processes, a distal and a central. Their nuclei are spherical and are disposed in several rows beneath the nuclear zone of the sustentacular cells; thus they form a broad zone of spherical nuclei. The distal process of the olfactory cell projects as a slender filament whose free end, carrying several fine cilia, reaches the surface of 
the membrane through a pore-like opening in the cuticular membrane which is formed by the cuticle of adjacent sustentacular cells. The central process of the olfactory cell penetrates to the tunica propria and becomes a non-medullated nerve fiber of one of the many rami of the olfactory nerve; it passes to the olfactory bulb, where its terminal arborization with the dendrites of the mitral cells forms the olfactory glomeruli.

The BASAL CELLS are flattened cells which form the deepest nuclear zone of the olfactory neuro-epithelial layers. Their cytoplasm is finely

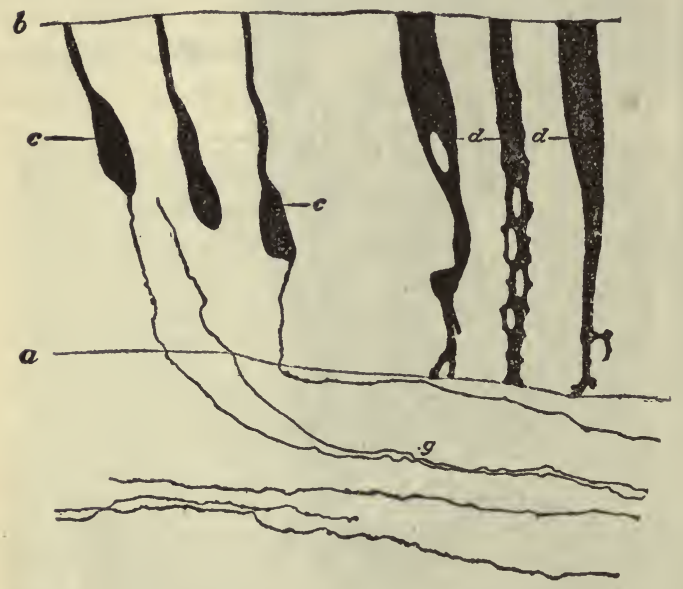

Fig. 284.-Vertical Section of the Olfactory Mucosa of a Kitten.

$a$, deep, and $b$, superficial border of the epithelium; $c$, olfactory cells; $d$, sustentacular cells; $g$, olfactory nerve fibers in the corium of the mucosa. Golgi stain. $\times 325$. (After Kölliker.) granular and their nuclei are ovoid or flattened. Frequently they send a short process between the branched ends of the sustentacular cells.

Many small nerve trunks occur in the tunica propria. The great majority of these are non-medullated and are formed by the central processes of the olfactory cells, which processes are true axons. Several of the smaller superficial fiber bundles unite in the deeper part of the tunica propria to form one of the small olfactory nerves. Taken together, about twenty in number on each side, these form two olfactory nerves, unique among cerebrospinal nerves in being collectively non-medullated. A few medullated fibers, derived from the trigeminus, are also found in the tunica propria; they distribute their terminal vasomotor branches to the blood-vessels, and, by fine sensory filaments which end between the epithelial cells, supply the neuro-epithelial layer. The trigeminus fibers supply a similar innervation to the respiratory mucosa.

The blood vessels of the olfactory mucous membrane are abundant. Their capillary plexuses form several layers in the tunica propria, and their veins mostly empty, through the ethmoidal veins, into the superior 
longitudinal sinus-a most significant fact. On the other hand the veins of the respiratory region return their blood to the internal maxillary vein, while some of those of the vestibule anastomose with the radicals of the facial vein which supply the adjacent skin.

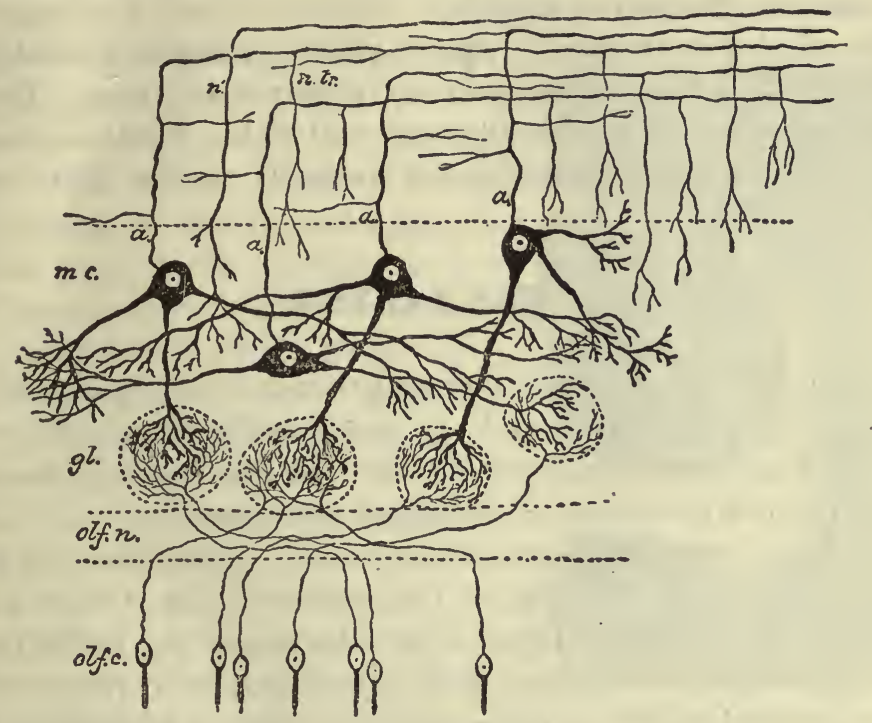

Fig. 285.-Diagram of the Relations of the Neurons of the Olfactory Nervi AND Olfactory Bulb.

olf. c., olfactory nerve cells, located in the olfactory region of the nasal mucosa, whose axons enter the olfactory nerve, olf. $n$., and terminate in relation with the dendrites of the mitral cells, $m c$., in the olfactory glomeruli, $g l$. The axons of the mitral cells, $a$., enter the olfactory tract, where they make a sharp bend and pass toward the cerebrum giving off frequent collaterals. At $n^{\prime}$ a nerve fiber appears to end by a free ramification among the mitral cells of the olfactory bulb. (After Schäfer.)

The lymphatics of the olfactory region can be readily injected from the sub-dural spaces of the meninges. They form a network in the connective tissue of the tunica propria.

\section{THE NASOPHARYNX}

This cavity, like that of the nose, is limited by a bony wall. Its mucous membrane is continuous anteriorly with that of the respiratory portion of the nose, and posteriorly with that of the oropharynx. The 
structure of its mucous membrane resembles that of the Schneiderian membrane, but its dorsal wall, in addition to the ciliated epithelium, the thin-walled blood-vessels, and the numerous secreting glands, contains many small nodules of lymphoid tissue. These nodules form a considerable mass, the pharyngeal tonsil.

The ciliated epithelium of the nasopharynx is also continuous with the lining epithelium of the auditory (Eustachian) tube. The tunica propria is firmly adherent to the bony wall of the dorsal surface, but is more loosely attached laterally and ventrally to the pharyngeal and palatine muscles.

\section{THE LARYNX}

The wall of the larynx is formed by several large plates of hyaline cartilage-thyroid, cricoid, and arytenoid cartilages-which are firmly united by ligamentous bands of fibrous tissue; the cartilaginous wall incloses a mucous membrane of considerable thickness.

The larger cartilages are of the hyaline variety and are prone to ossify in adult life. The tips of the arytenoids, the cornicula of Santorini, the cuneiform cartilages of Wrisberg, and the epiglottis are of the elastic variety of cartilage, and, though frequently much infiltrated with fat, are not, like the hyaline cartilages, subject to ossification. The median portion of the thyroid cartilage is also generally elastic in character; this portion does not generally ossify in women. In the lateral hyothyroid ligaments are the minute triticeous cartilages, generally hyaline but occasionally fibrous in character.

The intrinsic muscles of the larynx, taking origin from these cartilages, pursue their course beneath the mucous membrane.

The upper portion of the larynx, including the greater part of the epiglottis, as far as the false vocal cords is lined by stratified squamous epithelium which is continuous with that of the pharynx.

The epithelium of the vocal cords and that covering the anterior surface of the arytenoids is also of the stratified squamous variety. The remaining portions of the larynx, including the base of the epiglottis on its laryngeal surface, the ventricle, and the entire portion below the level of the true vocal cords, are lined by columnar ciliated epithelium of the pseudo-stratified type. The ciliary motion is directed toward the pharynx. The epithelium rests upon a basement membrane which is less highly developed than in other portions of the respiratory tract.

The tunica propria consists of fibro-elastic connective tissue in which 
are many small tubulo-acinar mucous glands. These are most abundant in the region of the ventricle and the false vocal cords. In this region also there is much diffuse lymphoid tissue, and the lateral and dorsal wall contains several solitary nodules which are so constant in their appearance as to warrant designating them 'laryngeal tonsil' (Fränkel). Occasionally, h ow e ver, lymph nodules are not present in the mucous membrane of the human larynx. The deeper portion of the tunica propria in certain parts, e.g., in the false vocal cords, contains a few striped muscle fibers in addition to those of the named muscles of the larynx. The false vocal cords and the aryteno-epiglottic folds contain loose fibrous tissue and frequently much fat.

The true vocal cords are formed by dense bands of elastic and a few collagenous fibers which are covered by a mucosa clothed with stratified squamous epithelium. Their free margin is sharply defined; at their attached margin, however, they blend indistinctly with the tunica propria. The free margin of the vocal cords has no connective tissue papillæ on the surface of the tunica propria, but toward the trachea superficial papillæ of connective tissue project into the deeper surface of the stratified epithelium.

The mucous membrane of the larynx

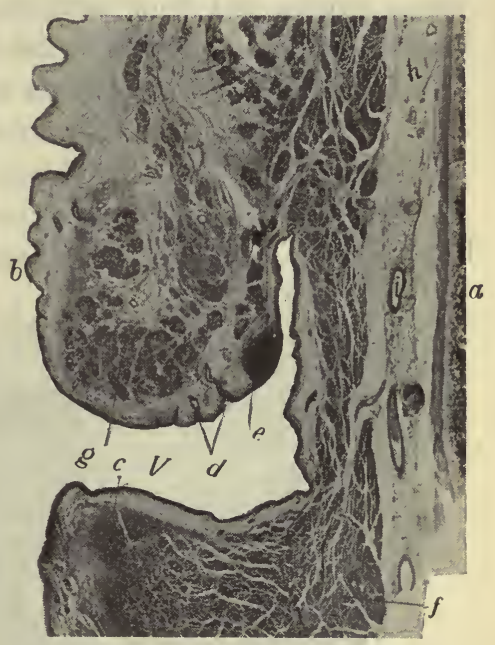

Fig. 286.-A Vertical Section THROUgh the Lateral Wall of the Human Larynx.

$a$, cartilage; $b$, laryngeal mucosa, clothed with ciliated epithelium; $c$, transection of the vocal cord, in this region the mucosa is clothed with stratified epithelium; $d$, ducts of mucous glands; $e$, lymphoid nodule; $f$, muscle; $g$, mucous glands; $h$, submucosa; $i$, bloodvessel; $V$, ventricle of the larynx. Hematein and eosin. Photo. $\times 8$. is freely supplied with blood-vessels and lymphatics. The latter terminate in the deep cervical lymph nodes. The nerve fibers form an abundant plexus in the laryngeal mucosa, from which motor fibers are distributed to the muscles and sensory fibers to the epithelium. The latter end in fine fibrils between the cells of the lining epithelium. In the stratified squamous epithelium, especially that of the epiglottis, small taste buds are also found; none, however, occur on the vocal cords. 


\section{THE TRACHEA}

The trachea proper extends from the lower border of the cricoid cartilage to the point where it bifurcates into the two primary bronchi, a distance of about four and one-half inches. The wall of the trachea somewhat resembles that of the larynx. It consists of three layers:

1. The mucous membrane, or mucosa.

2. The submucosa.

3. The fibrocartilaginous coat, or adventitia.

The mucous membrane presents slight longitudinal folds, and is lined by columnar ciliated epithelium, like that of the larynx, which

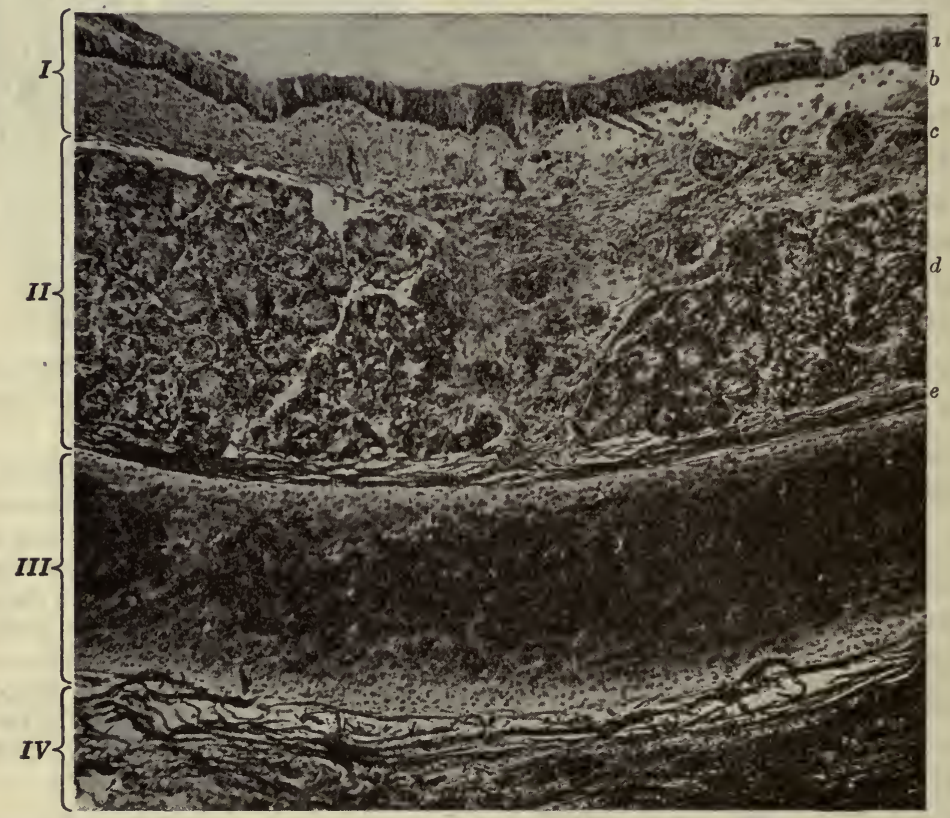

Fig. 287. -Transection of the Wall of a Child's Trachea.

$I$, mucosa; $I I$, submucosa; $I I I$, cartilage; $I V$, outer fibrous coat; $a$, columnar ciliated epithelium; $b$, tunica propria; $c$, layer of elastic fibers; $d$, mucous glands; $e$, perichondrium. Hematein and eosin. Photo. $\times 90$.

rests upon a delicate basement membrane. The tunica propria includes a thin inner layer of connective tissue which is richly supplied with small blood-vessels and infiltrated by many lymphocytes, and an outer 
layer of elastic tissue most of whose fibers are longitudinally disposed. The elastic layer begins in the region of the vocal cords in the larynx and is continuous below with the similar layer of the bronchial mucous membrane. Elastic fibers are more numerous in the trachea of the lower mammals than in that of man. A muscularis mucosa, a characteristic structure of the mucosa of the digestive tube, is lacking in the trachea. The elastic membrane occupies the position held by the muscularis mucosa in other organs.

The submucosa consists of loose areolar tissue which contains many small tubulo-acinar mucous glands. The ducts of these glands penetrate the mucosa and open upon the free surface of the trachea. They supply an abundant mucous secretion. This coat also contains the larger blood-vessels and nerves which are destined for the supply of the mucosa.

The fibrocartilaginous coat is formed by the C-shaped 'ring cartilages' of the trachea (from sixteen to twenty in number) which are firmly united to one another by ligamentous membranes of fibrous tissue continuous with the perichondrium of adjacent cartilage plates. The cartilages are of the hyaline variety and are subject to more or less ossification as age advances. They rarely overlap each other, so that but a single plate of cartilage forms the wall at any given point. Their borders are irregular, and horizontal sections near the

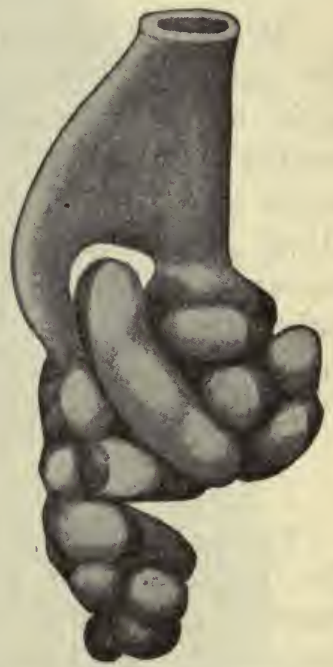

FIG. 288.-MUCUS-SeCRETING, TUBULO - ALVEOLAR GLAND OF THE Human Tracheal MuCosa.

The terminal dark areas are demilunes. Reconstruction. $\times 200$. (After Maziarski.) upper or lower margin of the cartilage frequently pass through several projections, which, unless properly interpreted, would lead one to infer that the cartilaginous plate was interrupted.

The interval between the ends of the C-shaped cartilage plates is occupied by a membrane of smooth muscle whose transverse fibers unite the adjacent ends of the cartilages. The muscle fibers are inserted into the perichondrium of the cartilages. Many of the fibers are obliquely, and a few of the outermost are longitudinally, disposed. This muscular portion of the tracheal wall forms the so-called trachealis muscle. The mucous membrane and submucosa of this portion of the trachea are unusually thick and their mucous glands are exceptionally large. The 
loose fibrous tissue which invests the outer surface of the cartilaginous coat contains many small sympathetic nerve trunks and ganglia.

\section{THE LUNG}

At the root of the lung the trachea divides into a primary bronchus for each lung. By repeated subdivisions-the earliest branches being given off at acute angles, the later ones at more obtuse angles-the smaller and smaller bronchi finally end in minute terminal bronchioles which lead through the alveolar ducts to the pulmonary air sacs and alveoli. One may thus distinguish between primary, secondary, and tertiary bronchi, and bronchioles. The mode of division for the main series of bronchi is monopodial, of the smaller bronchi a mixed dichotomy and monopody (Miller, Jour. Morph., 24, 4, 1913). According to Piersol the bronchioles are the branches of the fourth or fifth order.

\section{BRONCHI}

The wall of the primary bronchi is similar in structure to that of the trachea, but in bronchial tubes which are one or two divisions removed (secondary and tertiary bronchi) from the primary bronchi the plates of cartilage are no longer $\mathrm{C}$-shaped, and a complete muscularis mucosæ, within the cartilages, forms the outermost boundary of the mucous membrane. In such tubes-typical bronchi-the wall, as in the trachea, comprises:

1. A mucosa.

2. A submucosa.

3. A fibrocartilaginous coat.

The mucosa, a continuation of that of the trachea, is lined by tall, columnar, pseudo-stratified, ciliated epithelium which rests upon a distinct elastic basement membrane. The epithelium is thrown into wavy longitudinal folds. . The tunica propria is extremely vascular; it possesses an abundant supply of thin-walled veins of small caliber, together with many lymph vessels. Its connective tissue forms a delicate fibrous reticulum in the meshes of which are many lymphocytes. The outer portion of the tunica propria contains bundles of fine longitudinal elastic fibers, which form a complete layer about the tube. This elastic layer is thickest opposite the ridges and thinnest opposite the troughs of the epithelial waves.

The outer boundary of the mucous membrane contains a well- 


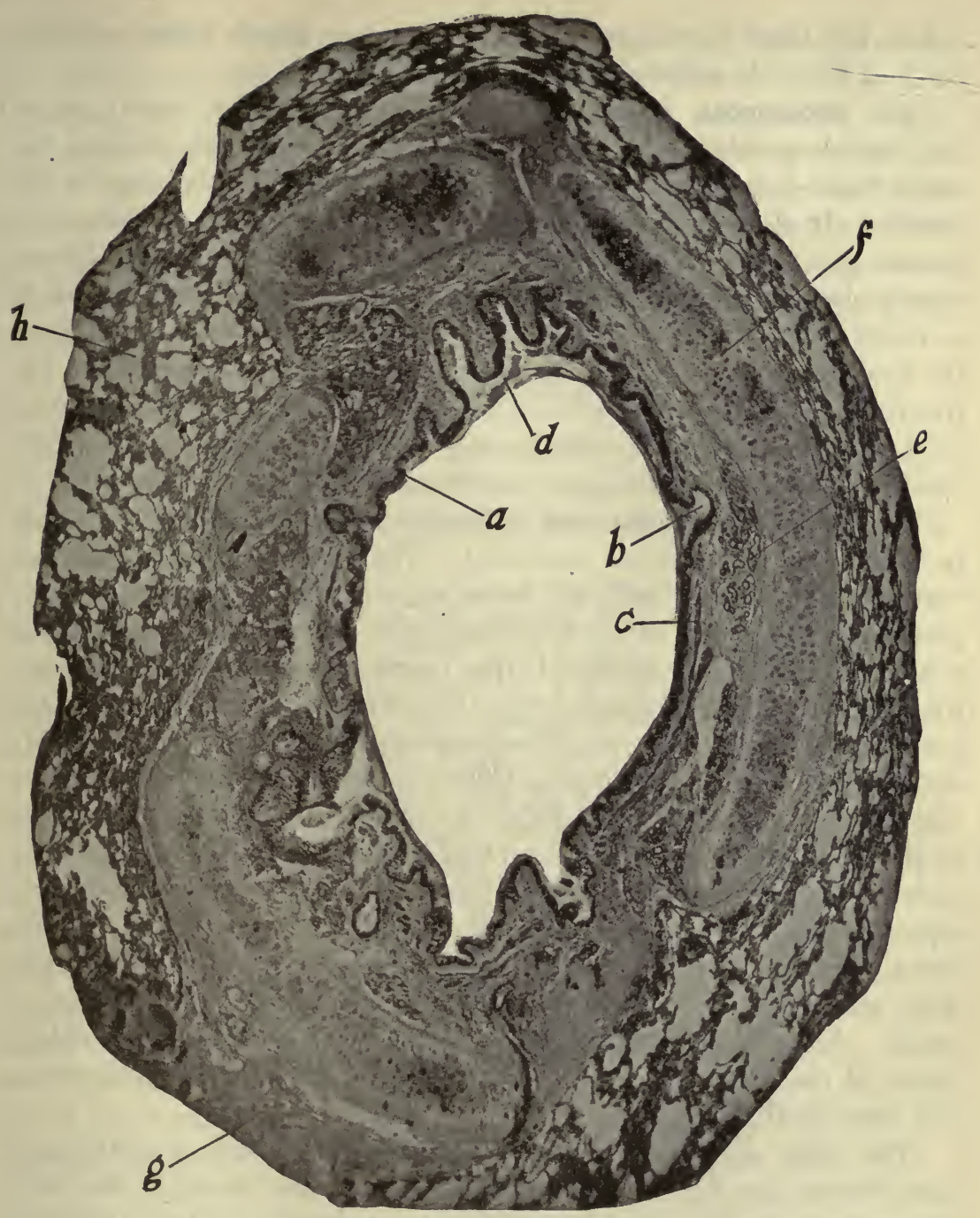

Fig. 289.-A Bronchus from the Human Lung.

$a$, lining epithelium; $b$, duct of a mucous gland; $c$, muscularis mucosæ; $d$, accumulated mucus, etc., bathing the surface of the epithelium; $e$, mucous glands; $f$, hyaline cartilage; $g$, outer fibrous coat; $h$, pulmonary alveoli. Hematein and eosin. Photo. $\times 34$.

developed muscularis mucosa composed of interlacing bundles of circular smooth muscle fibers. This layer forms a complete muscular coat which 
is here and there pierced by the ducts of mucous glands whose secreting portions lie in the submucosa.

The submucosa, by its broad-meshed areolar tissue, loosely unites the macous membrane to the cartilage plates. This coat contains the larger blood-vessels, nerves, and lymphatics which are distributed to the mucosa. It also contains the secreting portions of many tubulo-acinar mucous glands, which occur in groups that in the larger bronchi almost completely surround the tube. The number and size of these glands is in direct proportion to the size of the bronchus. The efferent ducts of the mucous glands penetrate the muscularis mucosæ and open upon the free surface in the interval between adjacent folds of the epithelial lining. In the tunica propria the ducts possess ampullary dilataiions which are lined by ciliated cells and contain portions of the mucous secretion.

The fibrocartilaginous coat is formed by a dense fibrous membrane in which the cartilages are embedded. The plates of hyaline cartilage vary much in number and size, being more or less highly developed in proportion to the size of the bronchial tube. They possess at all times a somewhat crescentic shape. In the larger bronchi three or four cartilage plates with overlapping edges encircle the entire tube. In the lower mammals, e.g., the pig, these overlapping cartilages are so highly developed that the plates often lie three or four deep; in man they are rarely more than one or two deep. As the bronchi diminish in size by division, the cartilage plates are no longer of sufficient size to completely encircle the wall but leave broad intervals in which this coat is only represented by fibrous tissue. In tubes of a diameter of 0.8 to 1 millimeter, bronchioles, the cartilages entirely disappear, and in these or somewhat smaller bronchioles the mucous glands are, likewise, no longer found. According to Cutore (Anat. Anz., 47, 13, 1914), the cartilage. plates of the intrapulmonary bronchi contain elastic fibers, and are in fact true elastic cartilages.

The outer surface of the cartilages is invested with a clothing of loose fibrous tissue of varying thickness-sometimes known as the outer fibrous coat-in which the branches of the pulmonary artery and veins and also many nerve trunks and ganglia are found. In the larger bronchi the two vessels, pulmonary artery and pulmonary vein, are found on opposite sides of the tube; in the bronchioles only one vessel, the artery, is in relation with the tube, the vein pursuing an independent course within the pulmonary tissue.

Near the root of the lung many small lymph nodes are found in the outer fibrous coat. In the smaller bronchi these are represented by 
solitary nodules which, it is important to note, are always found in the fibrous connective tissue which forms the outer portion of the bronchial wall. The bronchial lymph nodes and nodules are deeply pigmented, the volume of the pigment being dependent upon the age and occupation of the individual. It is apparently derived by absorption from the surface of the bronchi and is therefore absent in infancy, deficient in youth, abundant in adult life, and especially abundant in those individuals whose occupations have necessitated the inhalation of a dusty atmosphere.

\section{The Bronchioles}

The bronchioles possess neither cartilage, mucous glands, nor lymph nodules. Their epithelium, though still ciliated, is low-short columnar, or, in the smaller bronchioles, cuboidal. The tunica propria contains many lymphocytes and the elastic tissue forms an almost complete layer of longitudinal elastic fibers.

The muscularis mucosæ is relatively more highly developed than in the larger bronchi; it completely encircles the wall and is invested with an adventitious layer of fibrous tissue which contains the small arteries, n e r ves, lymphatics, a capillary plexus with elongated meshes, and occasional venules.

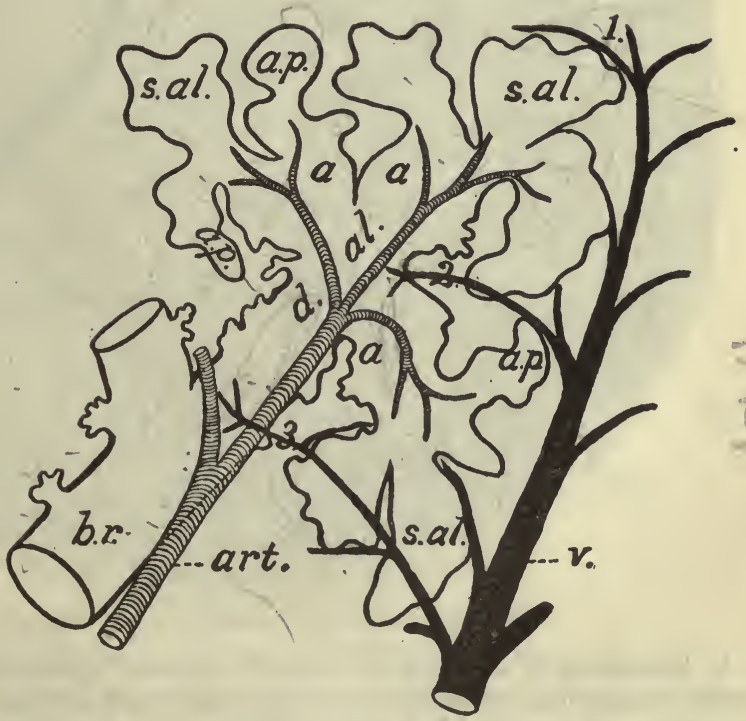

Fig. 290.-Diagram of Primary Lobule of Lung (LUNG UNIT).

b.r., respiratory (terminal) bronchiole; $d$. al., alveolar duct; $a$, atria; s. al., alveolar or air sac; a.p., pulmonary alveoli; art., pulmonary artery; $v$., pulmonary vein. (After Miller.)

The fibrous coat

of the bronchiole here and there blends with the fibrous bands of interlobular tissue and is in contact with the adjacent pulmonary alveoli. 
Each bronchiole enters the apex of a pulmonary lobule (secondary) and divides into terminal so-called respiratory bronchioles (terminal bronchioles).

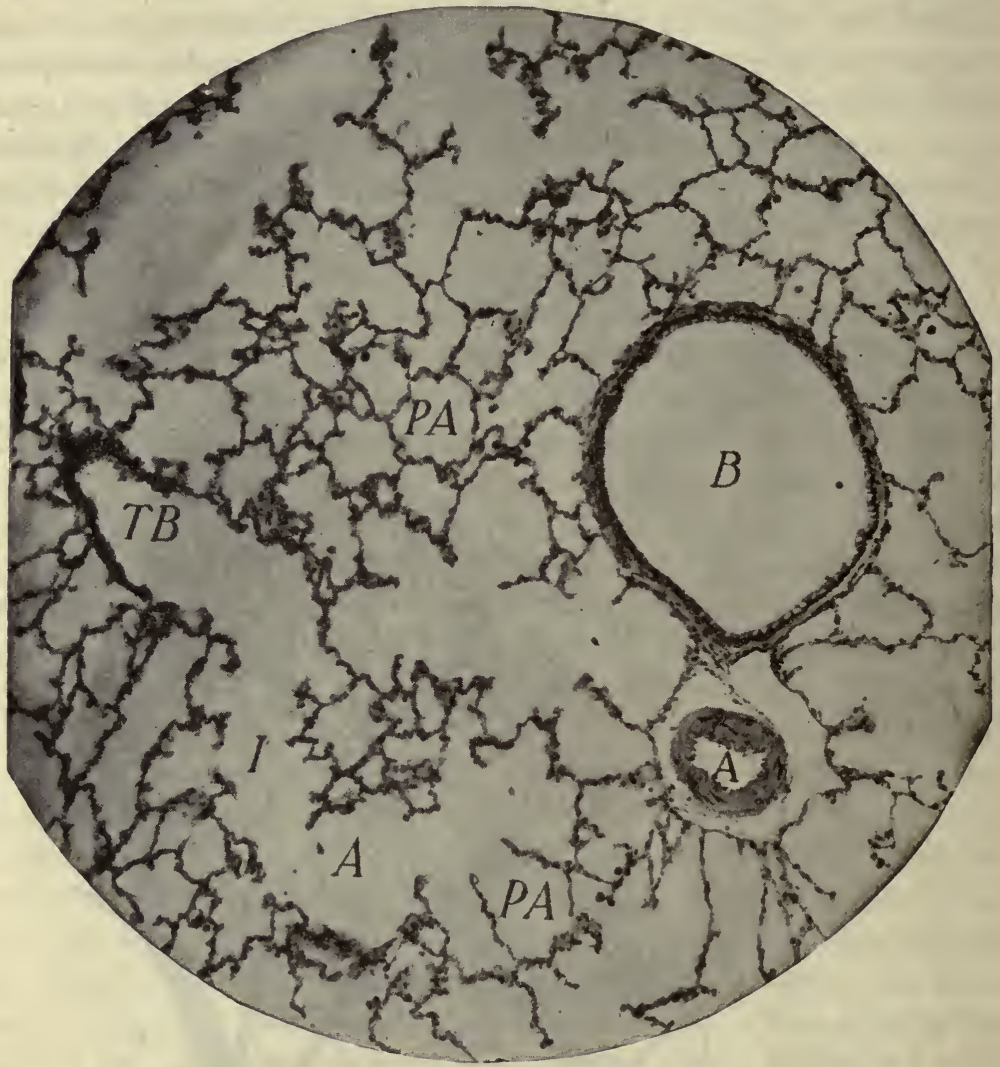

Fig. 291.-From a Section of a Child's Lung.

$B$, bronchiole in transection, with its adjacent pulmonary artery, $A ; T B$, bronchiole, ending in a terminal bronchiole, from which are derived the alveolar duct, $I$, the atrium, $A$, and the pulmonary alveoli, $P A$. In the center of the figure a pulmonary alveolus, $P A$, is seen in transection; many similar ones are shown. Hematein and eosin. Photo. $\times 45$.

The respiratory bronchiole bears alveoli which become more numerous toward its distal end. It thus contains a variable epithelial lining consisting proximally of a low columnar, sometimes ciliated, epithelium, with distally a more flattened non-ciliated type, and in the alveoli a typical greatly flattened respiratory epithelium including non-nucleated 
plates. It has a diameter of 0.5 millimeter or less. The epithelium rests upon a thin fibromuscular coat, the continuation of the mucous membrane of the bronchioles. The muscle still forms an almost complete though very thin investment of circular fibers; the muscle fibers,

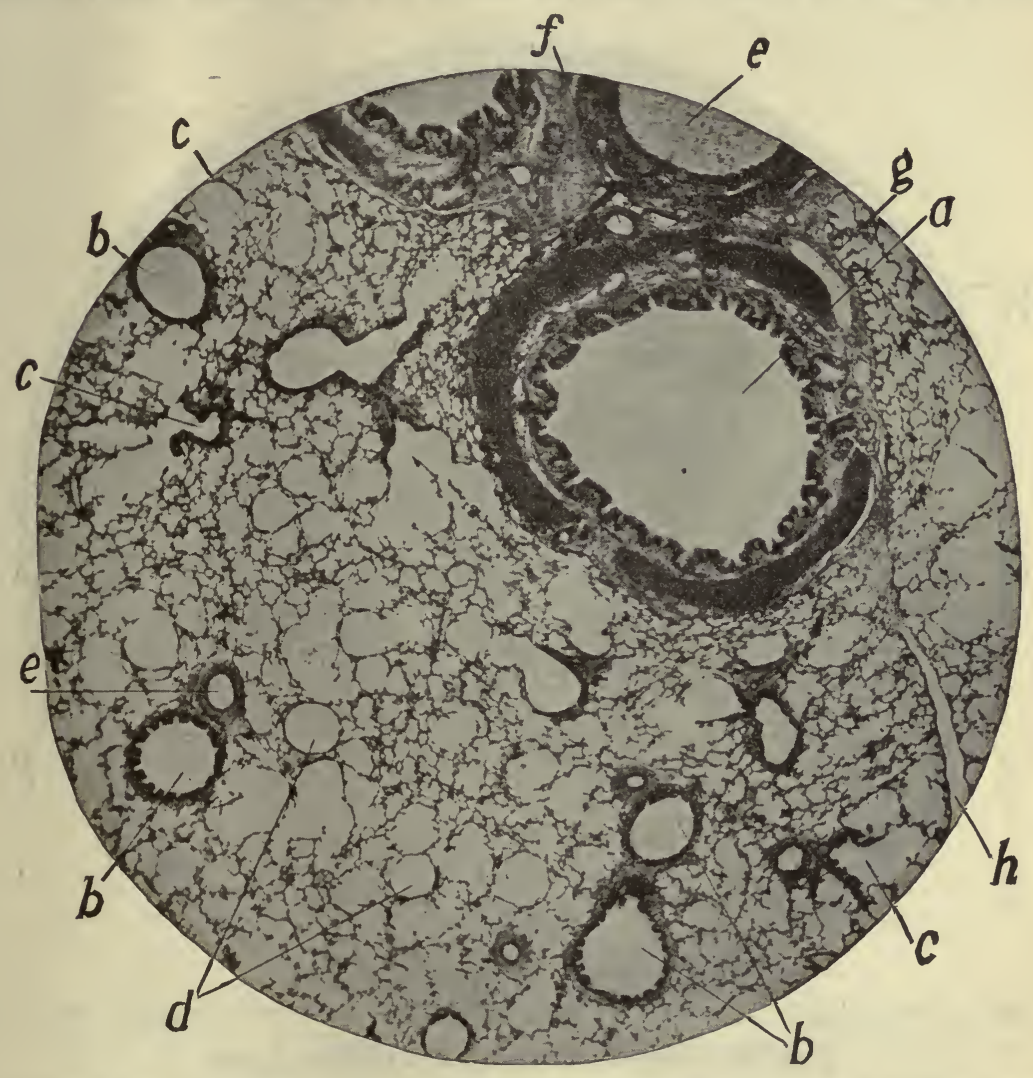

Fig. 292.-From a Section of a Child's Lung.

$a$, a small tertiary bronchus; $b$, bronchioles; $c$, bronchioles ending in terminal bronchioles, alveolar ducts, etc.; $d$, terminal bronchioles in transection, they have a more regular contour and thicker wall than the alveoli; $e$, pulmonary arteries; $f$, a bronchial artery; $g$, a bronchial vein; $h$, interlobular fibrous septum. Hematein and easin. Photo. $\times 62$.

however, are not continued into the wall of the pulmonary air sacs. The elastic fibers, derived from the elastic layer of the bronchioles, pass over to the alveolar walls in which they form a delicate network.

The respiratory bronchioles are short branching tubules leading to 
broader spaces, the alveolar ducts (infundibula of Schultze), which are surrounded by air saccules with pulmonary alveoli. According to Miller the alveolar ducts bear numerous alveoli, and are lined with flattened respiratory epithelium, and contain scattered bundles of smooth muscle which end in a delicate sphincter where the duct passes into the non-

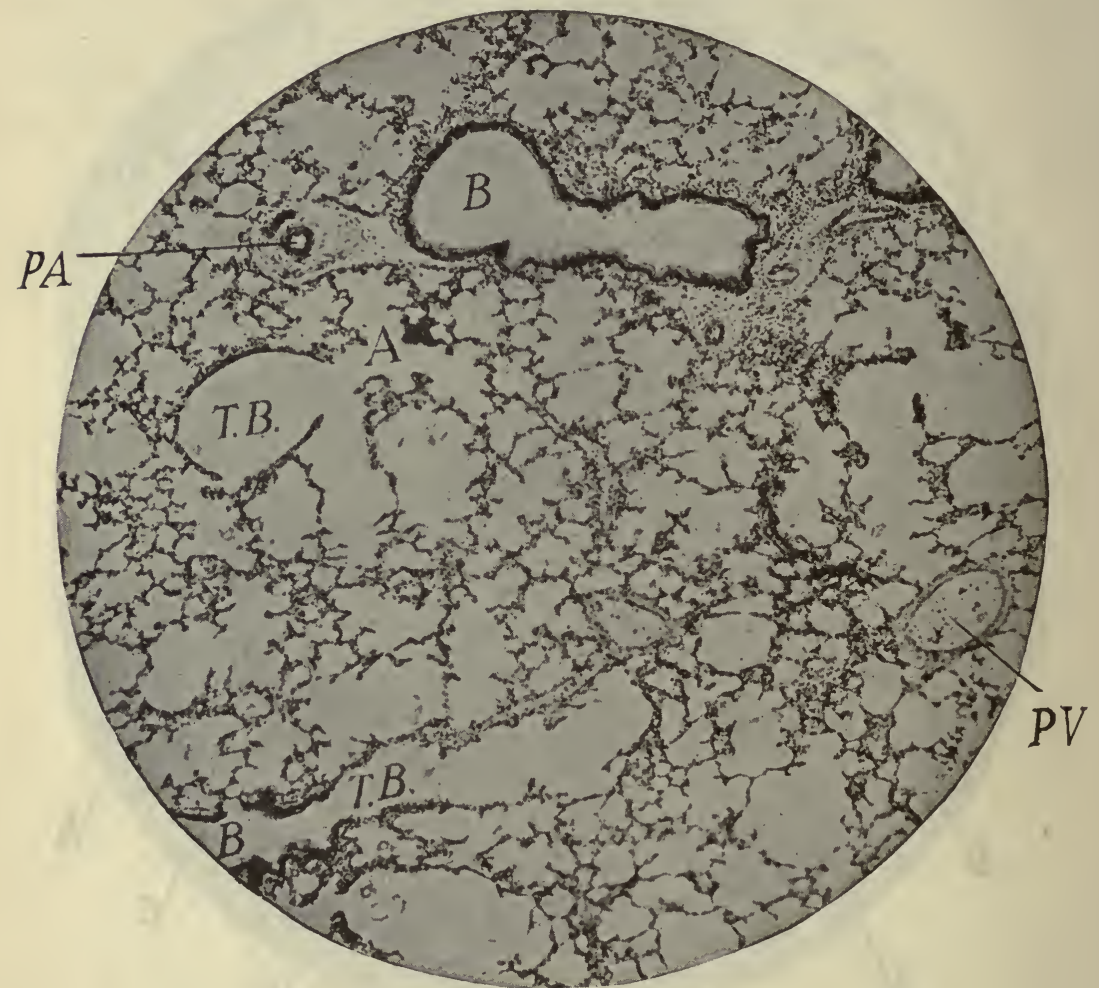

Fig. 293.-From a Section of a Child's Lung.

$A$, atrium; $B$, bronchioles ending in terminal respiratory bronchioles, $T B ; P A$, pulmonary artery; $P V$, pulmonary vein. Hematein and eosin. Photo. $\times 50$.

muscular atria, from three to six for each alveolar duct. Each atrium, of more or less circular outline, opens into a variable number (two to five) of irregular and variable alveolar saccules (air sacs). Each saccule bears on its periphery numerous pulmonary alveoli. The epithelium of the entire bronchial tree, including the nucleated respiratory cells, contains abundant mitochondria (Meves and Tsukaguchi, Anat. Anz., 46, 1, 1914). 


\section{The Pulmonary Alveoli}

The pulmonary alveoli are minute air cells, open toward the alveolar ducts, whose extremely thin wall consists of a capillary network, a deli-

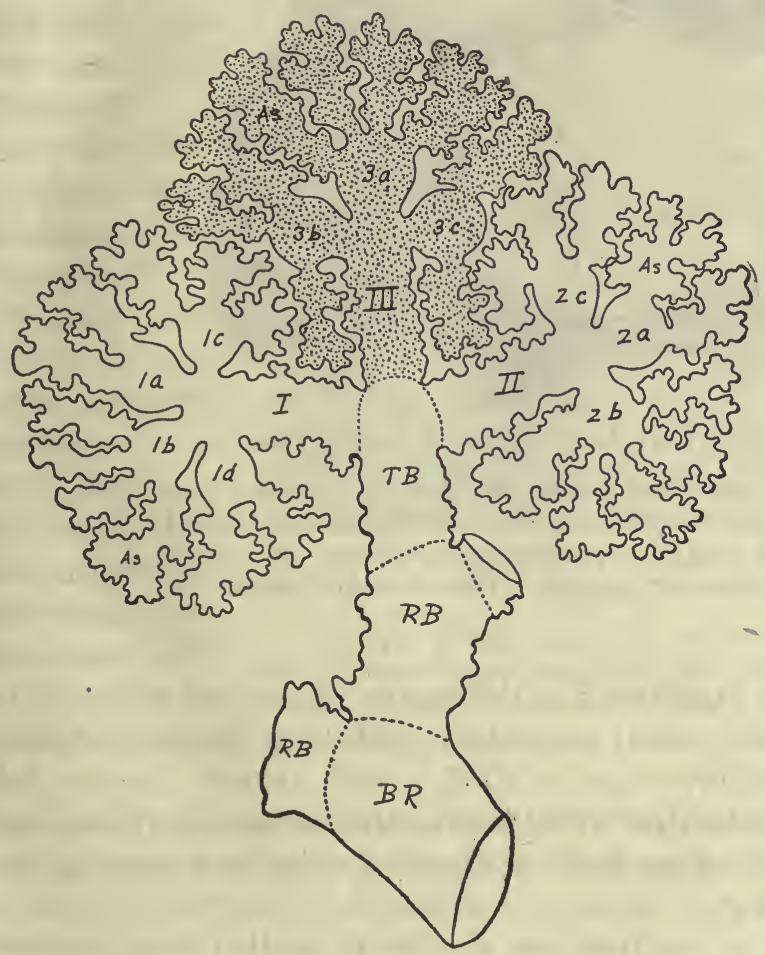

Fig. 294.-Diagram of the Three Pulmonary Lobules Connected with a Terminal Bronchiole (TB).

The middle lobule is stippled. $B R$, bronchiole; $R B$, respiratory bronchiole of the first order; $T B$, respiratory bronchiole of the second order (terminal bronchiole); $I, I I, I I I$, alveolar ducts; 1,2 , and $3 a, b, c$ and $d$, atria; $A s$, alveolar sacs with pulmonary alveoli or air cells. (Adapted from Miller, Jour. Morph., 20, 4, 1913.)

cate fibro-elastic reticulum, and a lining epithelium. The alveoli are so densely clustered about the alveolar duct that the capillary plexus, in the form of a reticulated membrane of wide capillary vessels, is exposed to the air of two adjacent alveoli, being separated therefrom only by its own endothelium and the epithelial lining of the alveolus. 
The lining epithelium of the alveoli, continuous through the alveolar ducts with that of the respiratory bronchioles, consists of flattened cells and broad protoplasmic non-nucleated plates. These cells are narrower

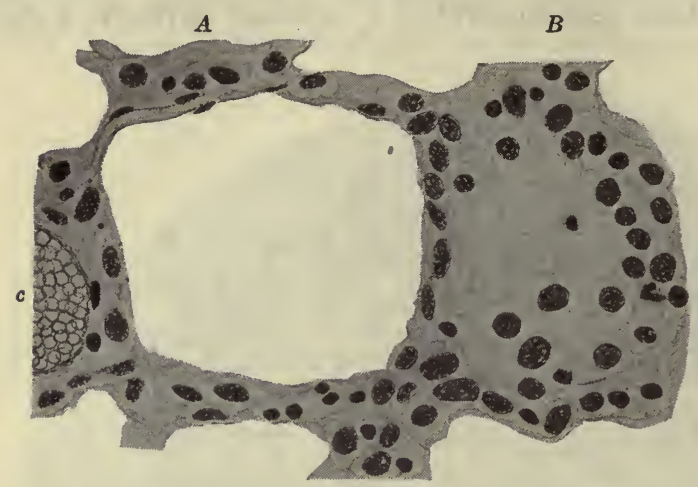

Fig. 295.-Two Alveoli of a Child's Lung.

In $A$, the wall is cut across and viewed in profile; $B$, a tangential section showing the cup-shaped bottom of the alveolus and the pulmonary epithelium in surface view; $c$, a pulmonary venule. Hematein and eosin. $\quad \times$ 425 .

among the capillaries; in the meshes of this net a few white fibers are found. The normal respiratory epithelium does not become phagocytic (Miller, 1911).

An alveolar duct with its atria, alveolar saccules, blood-vessels, lymphvessels and nerves forms a natural unit of structure, the primary pulmonary lobule.

Pores leading from one alveolus to another have been described, but Miller denies their presence in the lung of the cat (Jour. Morph., 24, 4, 1913).

\section{The Pleura}

The pleura is a serous membrane whose visceral layer (pleura pulmonalis) envelops the lung, and whose parietal layer (pleura costalis, diaphragmatis et mediastinalis) lines the thoracic cavity.

The surface of the pleura is clothed with a layer of mesothelium which rests upon a 'subserous' layer of connective tissue. The mesothelium contains frequent 'stomata' which in the costal pleura are only present over the intercostal spaces. 
These pleural pores have been the subject of considerable discussion. They have been regarded by some as giving direct vent to lymphatics; but W a l ters (Anat. Hefte, Bd. 46, 1913) has quite conclusively shown that they are artifacts, an interpretation maintained also by Miller.

The connective tissue contains an abundant network of elastic fibers. It is loosely attached to the chest wall but is more firmly united to the pulmonary tissue. Normally the pleura contains no lymph nodes (Miller).

The pleura contains many small blood-vessels and an abundant plexus of blood and lymphatic capillaries. Its innerva-

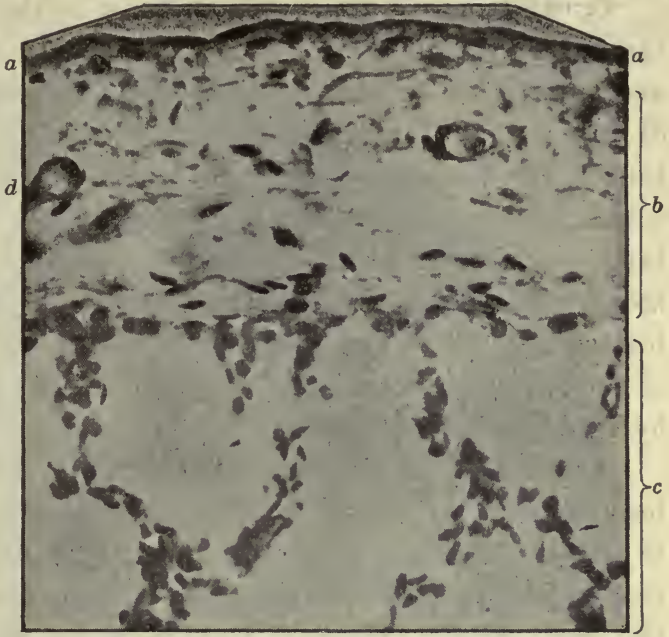

Fig. 296.-Transection of the Pleura of an INFANT.

$a-a$, layer of mesothelium; $b$, submesothelial connective tissue; $c$, pulmonary alveoli; $d$, a small bloodvessel. Hematein and eosin. Photo. $\times 470$. tion includes both sympathetic and cerebral (vagus) fibers. Both are apparently non-medullated. The sympathetic nerve fibers are supplied to the walls of the blood vessels; they are vasomotor in function. The vagus fibers are sensory in nature, and terminate in lamellar corpuscles, and as fine free sensory fibers.

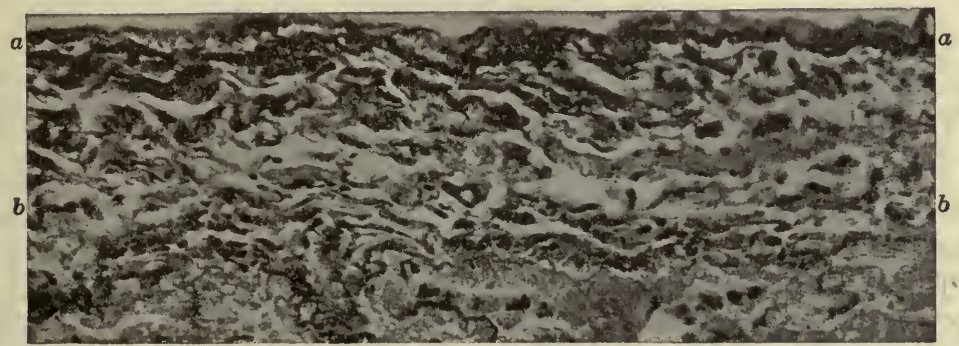

Fig. 297.-From a Section of the Pleura of Man.

The elastic tissue appears black. $a-a$, mesothelial surface; $b-b$, submesothelial connective tissue. Weigert's elastic tissue stain, hematein and picrofuchsin. $\times 110$. 


\section{The Lobule of the Lung}

If carefully examined, the surface of the pulmonary pleura presents minute polygonal areas (10 to $25 \mathrm{~mm}$. in diameter), the bases of the anatomical (or secondary) lobules, whose borders mark the attachment of fine bands of interlobular connective tissue, outlined by pigmented lines. In microscopical preparations still finer bands may be found, which traverse the pulmonary tissue in the direction of the root of the lung, and which partially outline minute conical areas, the true pulmonary (or primary) lobules, or lung units, whose bases are directed toward the pleura, and their apices toward the root of the lung. In many of the lower mammals, e.g., ox, these lobules are more distinctly outlined by interlobular connective tissue than is the case in man.

At the apex of the secondary lobule a small bronchiole (intralobular bronchiole) enters and divides into its respiratory bronchioles (from 30 to 100). This secondary pulmonary lobule consists of a collection of the smaller units or primary pulmonary lobules above described. At the same point a terminal branch of the pulmonary artery enters with the bronchiole and supplies the anastomosing capillary plexus in the alveolar walls. Branches of the bronchial artery do not supply any of the primary intralobular structures, and the pulmonary veins which return the blood from the alveolar capillaries arise at the periphery of the primary lobule and immediately enter the interlobular connective tissue.

The interlobular connective tissue contains the smaller branches of the pulmonary veins, the lymphatics returning from the pleura, and the non-medullated nerve trunks which are destined for the supply of the pleura and the intralobular pulmonary tissue.

\section{Blood Supply of the Lungs}

The blood supply of the lungs is derived from two distinct sources, the pulmonary arteries and the bronchial arteries. The former is destined chiefly for aëration in the capillaries of the alveolar walls, the latter for the nutrition of the bronchial walls.

The pulmonary artery enters at the hilum in company with the vein and the bronchus. It follows the bronchus throughout its course and gives an arterial branch to each of its subdivisions. The large arteries nearly equal in size the bronchus in relation to which they lie, but the smaller branches are not more than one-fourth to one-fifth the 
diameter of their bronchus. Throughout their course the branches of the pulmonary arteries lie on the wall of the bronchi, viz., in the outer fibrous coat or attached thereto by a broad band of fibrous tissue. More-

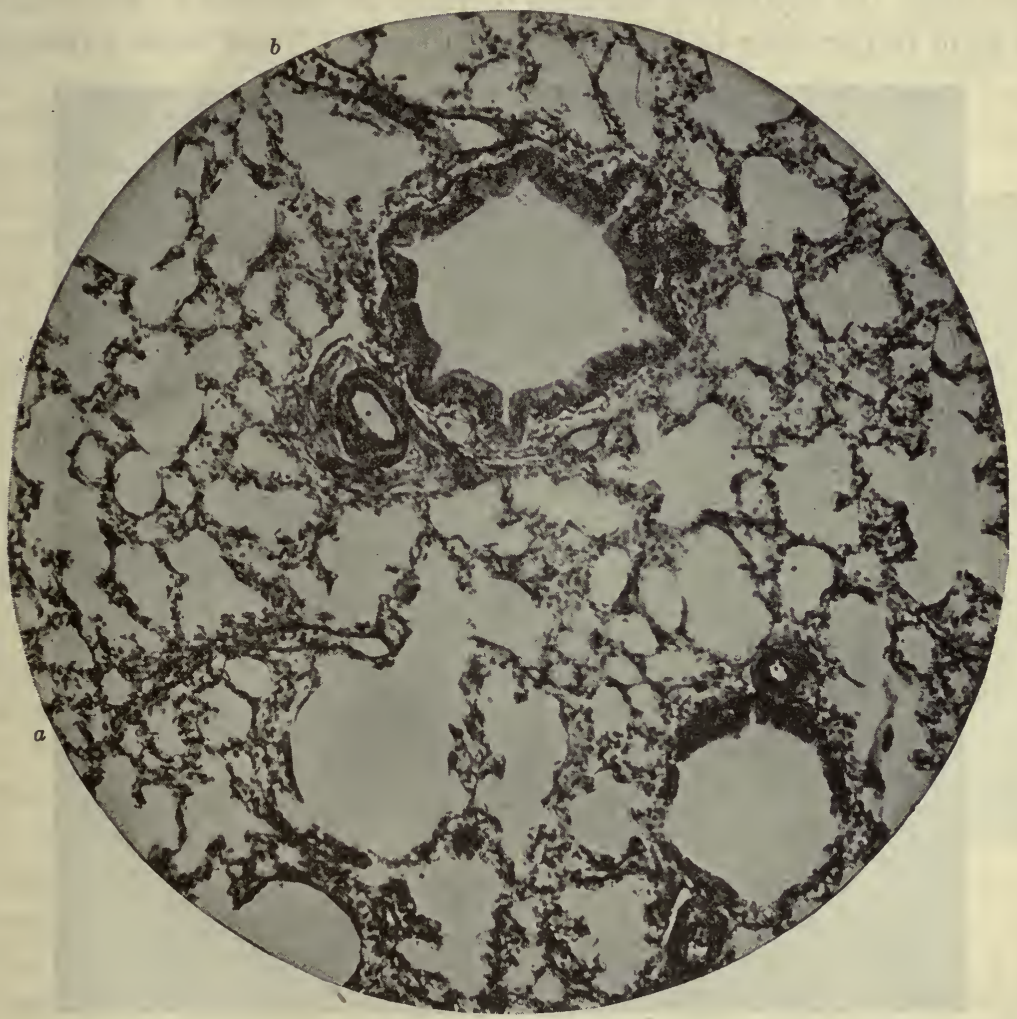

Fig. 298.-From the Lung of a Child.

At $a$, the origin of a pulmonary venule in the wall of a lobule is shown; at $b$, the pulmonary venule is just coming into relation with the bronchiole. Hematein, Weigert's elastic stain, and picrofuchsin. Photo. $\times 105$.

over each bronchus is accompanied by only one branch of the pulmonary artery and receives no capillaries from it.

At the apex of the secondary pulmonary lobule the pulmonary artery enters with the bronchiole; it resolves into smaller arterioles corresponding in number approximately to the number of respiratory bronchioles, each of which again breaks into several small twigs-one for each atrium, according to Miller-which supply the capillary networks in the walls of the alveolar ducts and alveoli. The pulmonary capillaries form an ex- 
ceedingly dense net of anastomosing vessels in the walls of the alveoli, the meshes of the capillary net being frequently, in the deeper portions of the lung, of less diameter than the vessel itself. At the periphery of the lobule the capillaries converge to form several venules which unite to form larger veins in the interlobular tissue. These veins pursue an

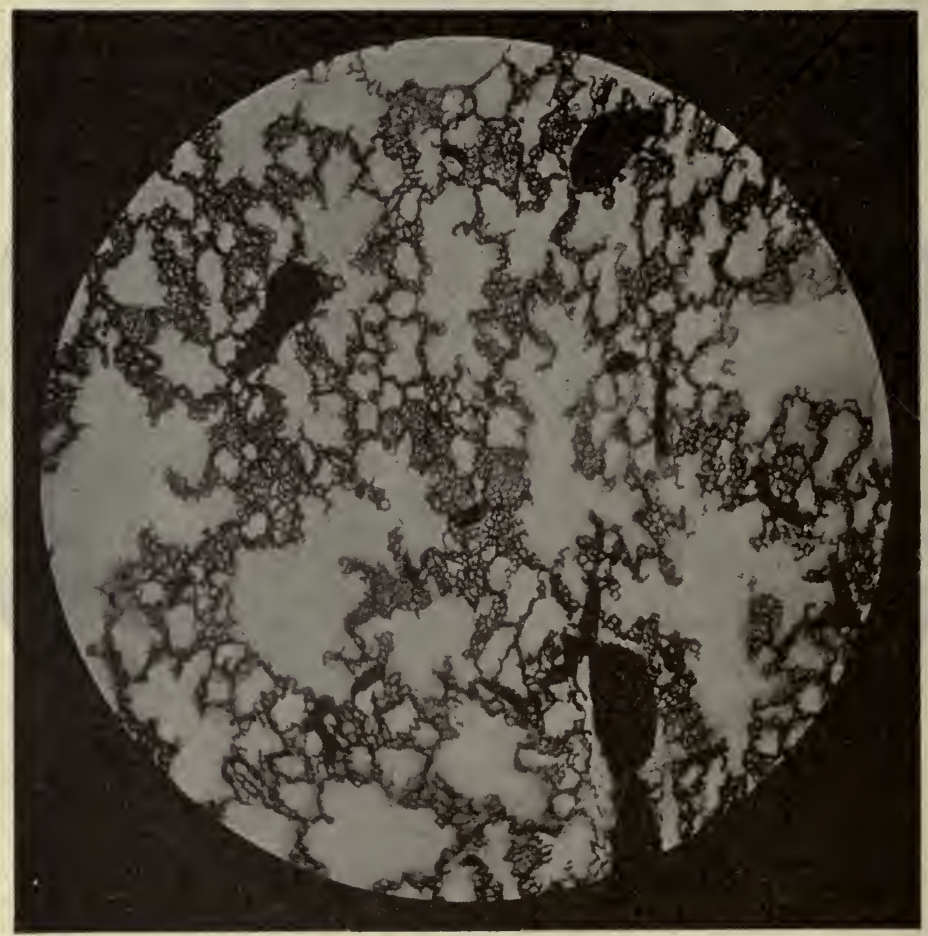

Fig. 299.-From the Lung of a Dog Whose Blood-vessels Had Been Injected with a Gelatinous Mass, and Appear Black.

The outlines of the pulmonary alveoli and atria are well shown. Many of the alveoli have been cut tangentially and present a surface view of the capillary network; in others the alveolar wall is cut across and is seen in profile. $\times 125$.

independent course and are always found at a considerable distance from the bronchioles and lobular branches of the pulmonary artery. (See Fig. 290.)

The smaller branches of the pulmonary artery near the surface of the lung give arterial twigs to the adjacent portions of the pleura. From the capillaries of the pleura minute venules enter the interlobular tissue and join the interlobular veins. 
The interlobular veins (pulmonary veins) follow the fibrous septa toward the hilum. They soon come into relation with the bronchi and are then found on that side of the bronchus opposite the pulmonary artery. The vein, like the artery, lies outside of the bronchial wall in the adjacent fibrous tissue. It is, as a rule, only those bronchi whose wall contains cartilage plates which are in relation with both pulmonary artery and vein; the smaller bronchioles are usually accompanied by the artery only. Those veins which accompany the bronchi receive smaller branches from the bronchial wall and by union with their fellows form larger and laxger vessels which finally make their exit as the pulmonary veins and pass to the left auricle of the heart.

The bronchial arteries also follow the bronchial tubes in all their ramifications. The larger branches are found in the outer fibrous coat near the cartilages, the smaller ones lie in the submucous and mucous coats. In contradistinction to the pulmonary vessels the bronchial arteries are found in the wall of the bronchi, not

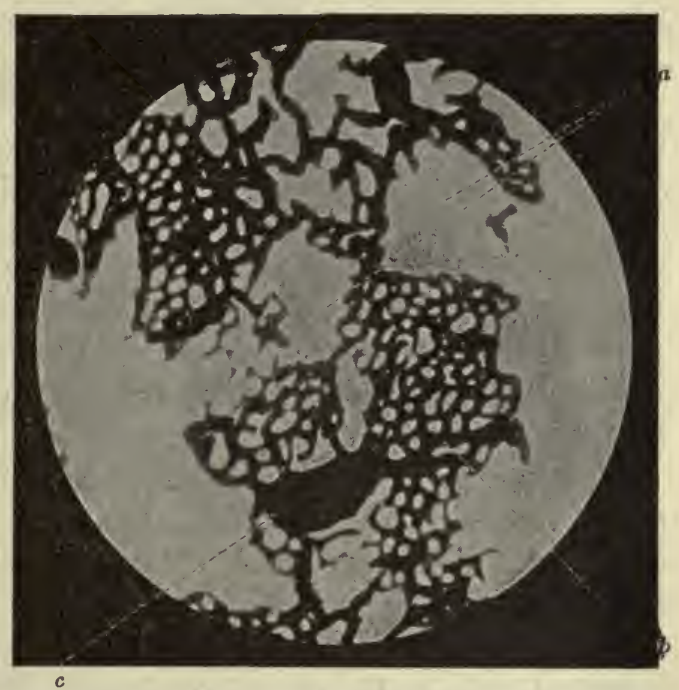

Fig. 300.-From the Central Portion of the Preceding Figure.

$a$, two pulmonary alveoli in transection; $b$, tangential section showing the bottom of an alveolus; $c$, a minute pulmonary venule. Photo. $\times 500$. outside of the bronchial wall. They supply capillaries to all of the tissues of the bronchi. The bronchial capillaries reunite to form small venules whose course differs with the size of the tube. In the terminal bronchioles these venules pass directly to the interlobular veins, and, according to Miller, the pulmonary veins receive a similar acquisition at each division of the bronchi. In the larger bronchi, however, the venules unite within the bronchial wall to form the radicals of the bronchial vein which, lying in the fibrous tissue of the bronchial walls, retrace the course of the bronchi to the hilum, where they make their exit as the bronchial veins and join the azygos veins. Thus, only the walls 
of the larger bronchial tubes are supplied with bronchial blood, and, according to Schaffer, a few branches at the root of the lung are also distributed to the adjacent pleura. Many of the bronchioles, the respiratory bronchioles, and also the alveolar ducts, pulmonary alveoli, and the pleura all receive their nutrition from the pulmonary arteries. Finally it may be said that there are no anastomoses between the pulmonary arteries and veins except among the capillaries of the alveolar walls.

\section{LYMPHATICS}

The pulmonary lymphatics form a plexus in the walls of the bronchi and bronchioles, penetrating to the mucous membrane. Branches from this plexus frequently anastomose with perivascular lymphatic vessels about the branches of the pulmonary artery and reins. A close network of lymphatic vessels is also found in the pleura, its efferent vessels passing into the interlobular tissue to join those vessels which accompany the veins. The pulmonary lymphatics are supplied with frequent valves and numerous anastomoses. At the atria the lymphatics pass to the interlobular septa, so that the alveolar walls lack lymph vessels.

The lymphatic vessels of the bronchi are connected with larger lymphatic vessels of the outer fibrous coat and with the lymph nodules in the walls of the larger tubes. Many of the larger vessels in the outer fibrous coat of the bronchi, and also those which accompany the pulmonary artery, enter those lymph nodes which are in relation with the bronchial walls at the root of the lungs. The pleural lymphatic plexus and the vessels accompanying the pulmonary veins, after pursuing much of their course through the interlobular connective tissue in company with the pulmonary veins, also open into the bronchial lymph nodes. Much pigment is conveyed through these vessels and is deposited in $(a)$ the interlobular connective tissue, $(b)$ the fibrous tissue about the pulmonary arteries, and most abundantly in $(c)$ the bronchial lymph nodules and nodes.

Lymphoid tissue forms an important constituent of the lungs, serving as filters in the lymph circulation and as centers to which the phagocytes carry their collected material. ' It occurs as nodes, nodules, or as smaller masses of more diffuse lymphoid tissue; it may be peribronchial, periarterial, perivenous or subpleural in position. Lymph nodes are associated with the larger divisions of the bronchi and located at the points of branching. The smaller masses are found in greatest 
numbers at the periphery of the primary and secondary lobules (Miller, Anat. Rec., 5, 3, 1911).

\section{NERVE SUPPLY}

The nerves of the lungs are derived from the anterior and posterior pulmonary plexuses of the sympathetic system. They are distributed to the walls of the blood-vessels, where they form a delicate plexus with terminal fibrils among the smooth muscle fibers, and to the walls of the bronchial tubes. Small nerve trunks, with which many minute ganglia are connected, occur in large numbers in the outer fibrous coat of the bronchi.

From these nerve trunks and ganglia fibrils are distributed to the bronchial mucous membrane, in which they supply the muscularis mucosæ, and form a terminal plexus beneath the epithelial coat. These fibers have been traced to the respiratory bronchioles, where they are said to form a delicate plexus within the lobule in the interalveolar walls (Wolff, Arch. f. Anat., 1902). Sensory fibrils are supplied by the vagus nerve. The vagus contributes to the bronchi and their subdivisions also motor fibers, for the most part probably indirectly through the sympathetic ganglia. 


\section{CHAPTER XIII}

\section{THE DIGESTIVE SYSTEM}

The digestive system includes the cavities of the mouth, pharynx, esophagus, stomach, and intestines, together with the accessory glandsthe salivary glands, pancreas, and liver. Associated with the mouth and coöperating in the function of the essential organs of digestion are also the teeth and the tongue.

\section{THE MOUTH}

The walls of the oral cavity comprise a mucous membrane, a submucous layer of connective tissue, and a muscular or bony paries.

The mucous membrane (mucosa) is clothed with a layer of stratified squamous epithelium which presents, at the margin of the lips, a gradual transition to the epidermis of the skin, and at the fauces is continuous with the lining epithelium of the faucial isthmus and the pharynx.

The tunica propria (corium, stratum proprium) upon which the epithelium rests, consists of dense areolar tissue, the superficial portion of which specially abounds in elastic fibers. This portion of the corium consists of rather delicate connective tissue bundles which at frequent intervals are prolonged into the epithelial coat in the form of minute conical papillæ, similar to those of the skin, whose height varies with the location. The tallest papillæ are found on the gums and at the margins of the lips, the lowest on the inner surface of the cheeks and the soft palate.

The papillary layer of the corium contains a plexus of capillary bloodvessels which is connected with a network of small arteries and veins in the deeper part of the tunica propria.

The submucosa consists of looser connective tissue which blends insensibly with that of the mucosa, and unites the mucous membrane to the subjacent muscles and bones forming the wall of the oral cavity. In most portions the buccal mucous membrane is but loosely connected 
with the underlying parts, but in the hard palate and the gums this union is very firm.

Lymphoid tissue occurs in considerable abundance in the oral mucous membrane. Areas of diffuse lymphoid tissue are of frequent occurrence and small lymph nodules are occasionally found. The lymphatic vessels form a plexus in the tunica propria, which empties into larger vessels in the submucosa.

\section{Secreting glands} occur in considerable sbundance in all portions of the buccal mucous membrane except that covering the gums. The glands are of the tubulo-acinar type and produce either a pure mucous secretion or, in the case of the larger ones, a mixed mucous and serous secretion. The ducts of the glands are lined by columnar cells which, near the mouth of the duct, of-

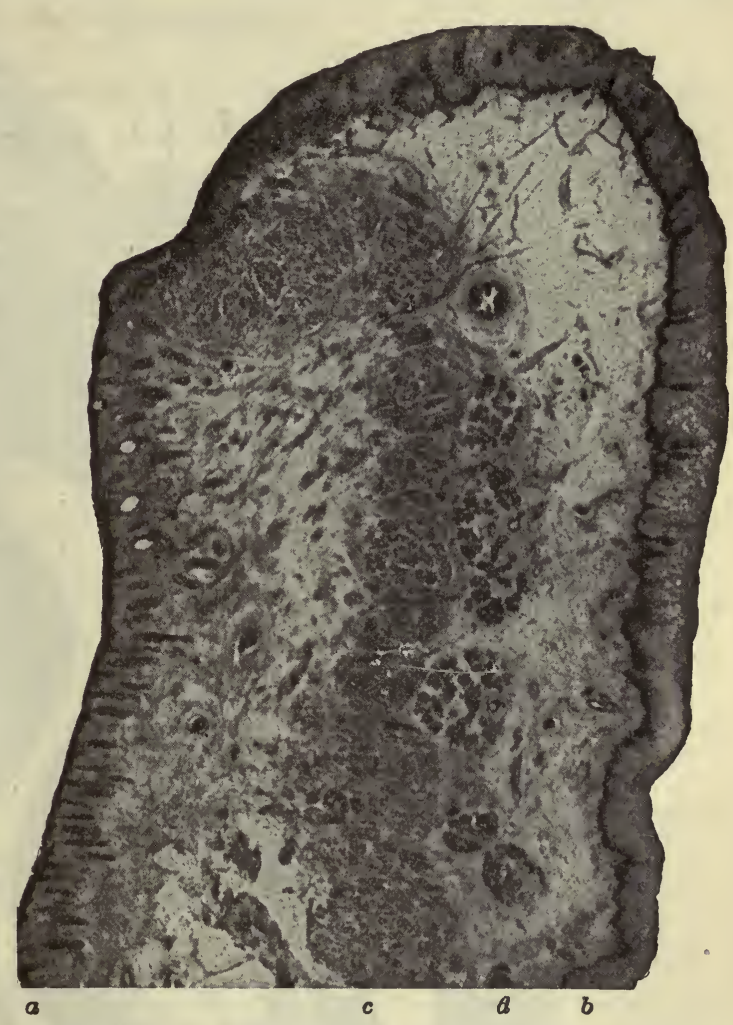

Fig. 301.-From a Section throdgh the Lip of AN INFANT.

$a$, cutaneous surface; $b$, epithelium of the oral mucosa; $c$, layer of striated muscle; $d$, layer of mucous glands. Hematein and eosin. Photo. $\times 10$. fer a gradual transition to the stratified squamous epithelium of the mucosa. The glandular epithelial cells of the secreting portions become swollen and clear after a period of rest, but are shrunken and present a faint cytoplasmic reticulum after activity. The different glands of the same region, and even different cells in the same gland, often exhibit various stages of secretory activity. The fundus of the secreting glands frequently extends into the loose connective tissue of the submucosa. At the margin of the lips and 
more rarely in the neighboring portions of the buccal mucous membrane are small sebaceous glands which open directly upon the free surface. The oral glands will be further described below under salivary glands.

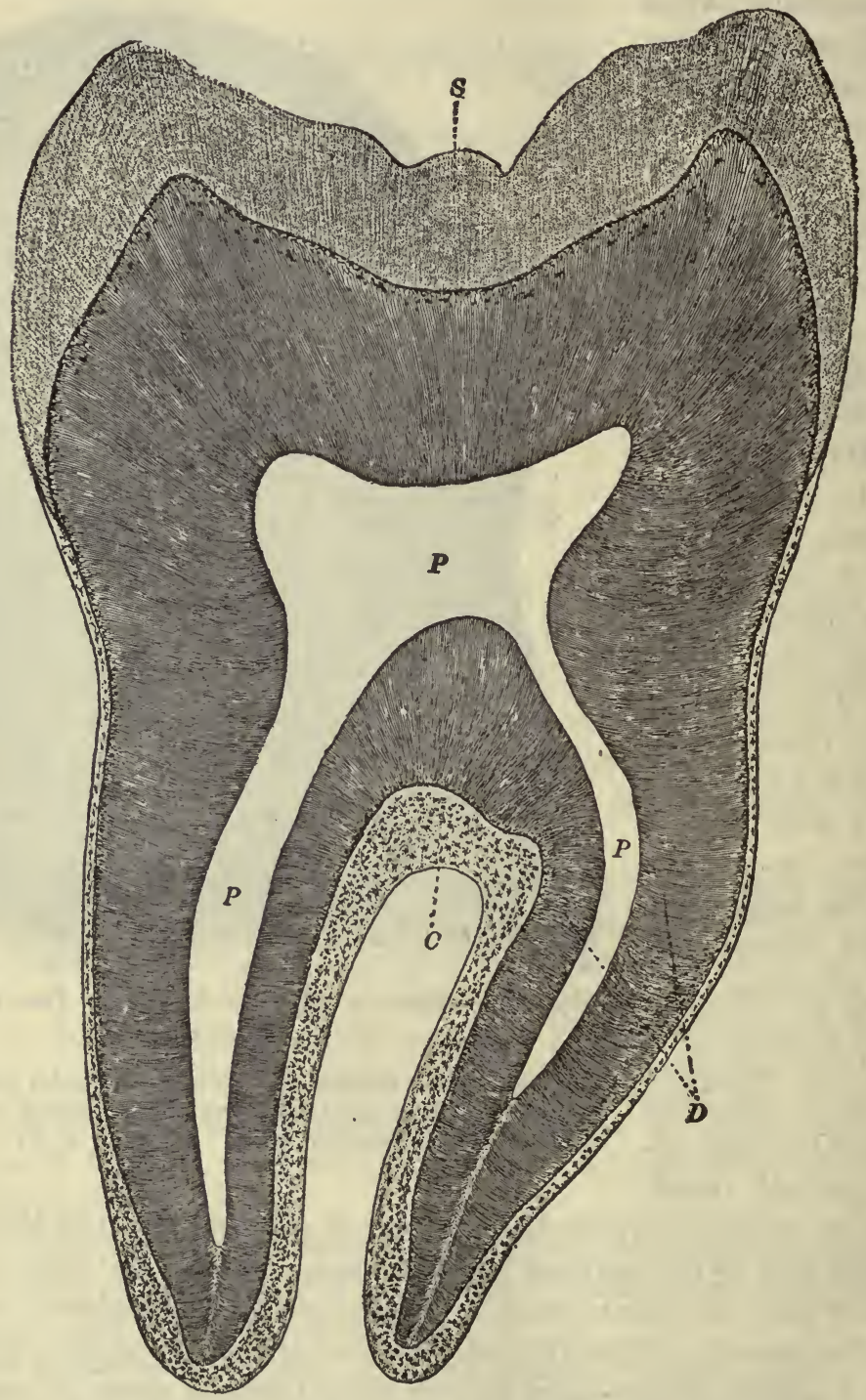

Fig. 302.-Axial Section of a Human Molar Tooth.

$C$, cementum; $D$, dentin; $P$, pulp cavity; $S$, enamel. $\times 8$. (After Sobotta.) 


\section{THE TEETH}

\section{Structure}

Each tooth rests in a bony socket in the alveolar process of the maxillary bone, and is also held in place by the periosteum of the alveolar sac and the adjacent portion of the gum. The tooth is divisible into a free portion or crown, and a concealed portion or root which usually consists of from one to three fangs. The slightly constricted border between the root and the crown, which is surrounded by the soft tissues of the gum, is known as the neck of the tooth.

The tooth consists of a superficial calcareous portion and a central medulla, the dental or pulp cavity, which occupies the axis of the tooth and which contains a peculiar embryonal type of connective tissue, very similar to reticular tissue, the dental pulp. At the tip of each fang is an opening, the foramen apicis dentis, leading to a narrow canal which penetrates the wall of the tooth and permits the entrance of the nerves and blood-vessels which supply the pulp.

The calcareous wall of the tooth is formed by three distinct tissues: (1) dentin or

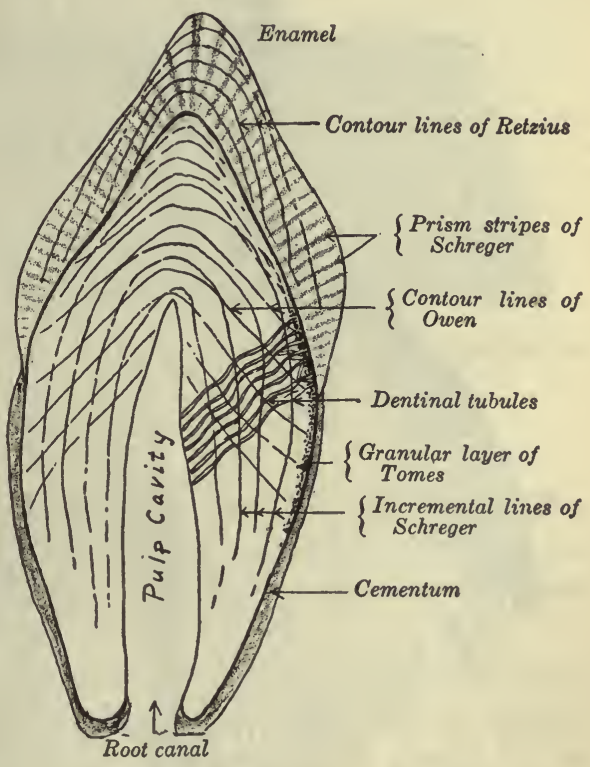

Fig. 303.-Diagram of an Axial Ground Section of Tooth, Showing the Several Stripes of the Dentin and the ENAMEL. ivory; (2) enamel; (3) cementum. The dentin incloses the entire pulp cavity and is in turn covered by the enamel and cementum, the enamel forming the superficial layer of the crown, the cementum that of the root of the tooth.

The Dental Pulp.-The dental pulp is an embryonal type of connective tissue which is rich in branching stellate cells and poor in fibers. It contains no elastic fibrils, and the delicate collagenous fibers 
instead of forming bundles are arranged in an interlacing network, the fine fibrils of which are in intimate relation with the connective tissue

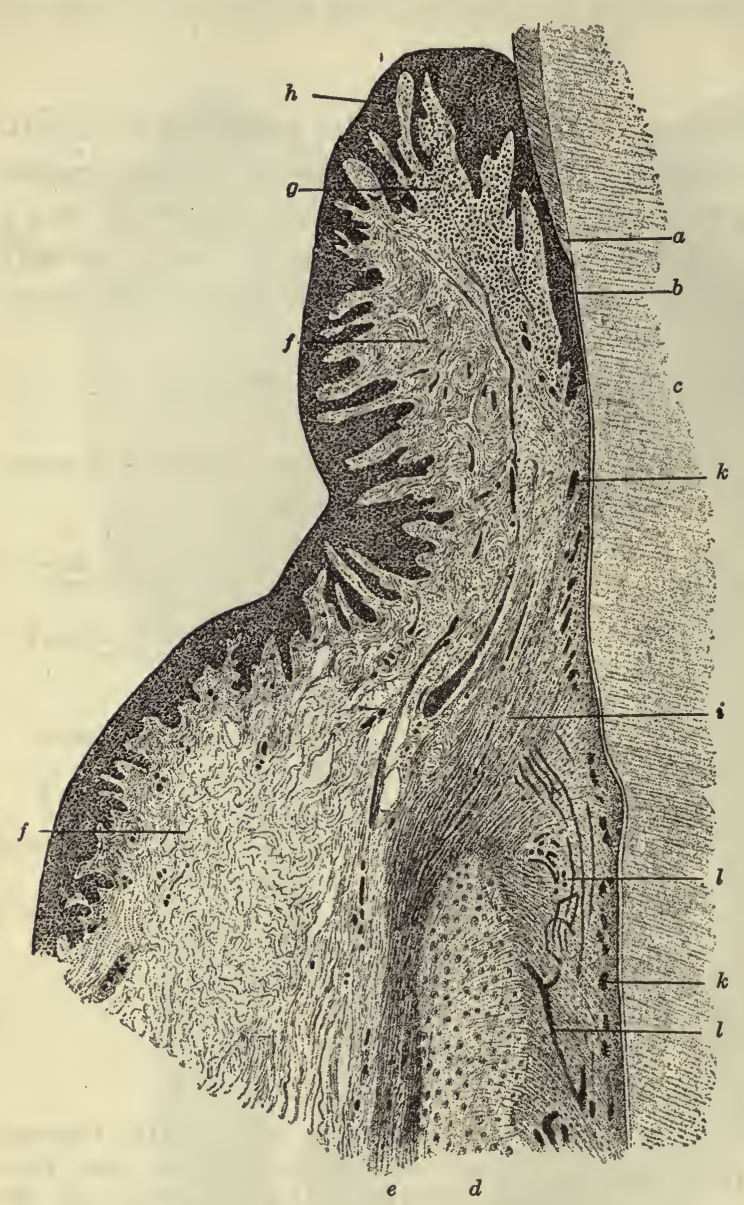

Fig. 304.-From a Longitudinal Section of the Neck of a Child's Tooth and the Adjacent Alveolus.

$a$, enamel; $b$, cementum; $c$, dentin; $d$, bone; $e$, periosteum; $f$, corium; $g$, lymphoid tissue; $h$, stratified epithelium of the gum; $i$, circular dental ligament; $k$, epithelial remnants; $l$, blood-vessels. $\times 25$. (After Kölliker.) cells. It accordingly closely resembles reticular connective tissue. The stellate connective tissue cells are scattered throughout the entire pulp, but at the periphery of the cavity a re closely crowded and are much en larged. These peripheral cells form a layer of odontoblasts which is in contact with the dentin.

$\mathrm{T} h$ e odontoblasts are cylindrical branched connective tissue cells whose long axis (about $40 \mu$ ) is perpendicular to the surface of the adjacent dentin. From their apex a delicate process is sent into the dentinal canals, in which they frequently extend all the way through the dentin. Lateral processes from the cell bodies of the odontoblasts interlace with each other and firmly unite the cells into a membranous layer. Other processes are given off irom the base of these cells and intermingle with the fibers of the pulp, 
so that if this tissue is forcibly separated from the dentin the odontoblasts remain adherent to the connective tissue of the pulp. The nuclei of the odontoblasts are found near their inner or basal extremity. 'Their cytoplasm is of considerable extent as compared with that of the other connective tissue cells of the pulp.

The dental pulp is richly supplied with blood-vessels, derived from a nutrient artery which enters through the root canal, its several branches forming a network of minute arterioles and capillary vessels in the center of the pulp cavity, and a peripheral close-meshed capil-

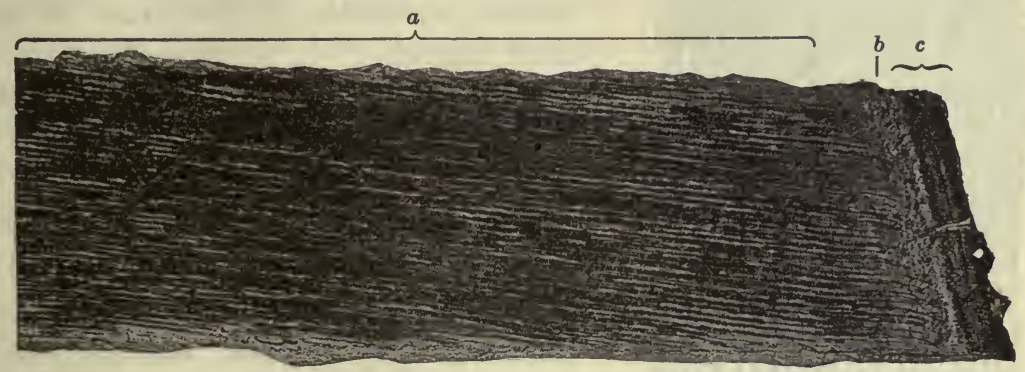

Fig. 305.-From a Section of a Human Tooth Which Had Been Grodnd to Extreme Thinness.

$a$, dentin; $b$, granular layer of Tomes; $c$, enamel. Photo. $\times 150$.

lary network which is in close relation with the layer of odontoblasts. Numerous delicate lymphatic vessels, uniting to leave the root as one or several large vessels, have also recently been demonstrated in the tissue of the dental pulp (Schweitzer).

A rich nerve supply is derived from fine branches which also enter by the root canal. Most of the nerve fibers lose their myelin sheaths soon after they enter the pulp. They form a primary plexus in the connective tissue from which fine fibers pass to the periphery and form a marginal plexus beneath the odontoblasts. From here delicate terminal sensory fibrils ramify over the odontoblasts and pass in great abundance into the dentinal tubules, usually two to each tubule (Mummery, Proc. R. Soc., Series B, 85, 1912). This peripheral distribution of nerve fibrils explains the extreme sensitiveness of the dentin. Sympathetic fibers supply the muscle cells of the pulp arterioles.

Dentin.-The dentin surrounds the entire pulp cavity except at the opening of the root canal. It is a fine calcareous substance which resembles bone in that it consists of a collagenous fibrous matrix and is infiltrated with lime salts. The matrix is a fine fibrous network of 
dense connective tissue, the majority of whose fibers are disposed in a longitudinal direction. The meshes of the matrix are almost completely

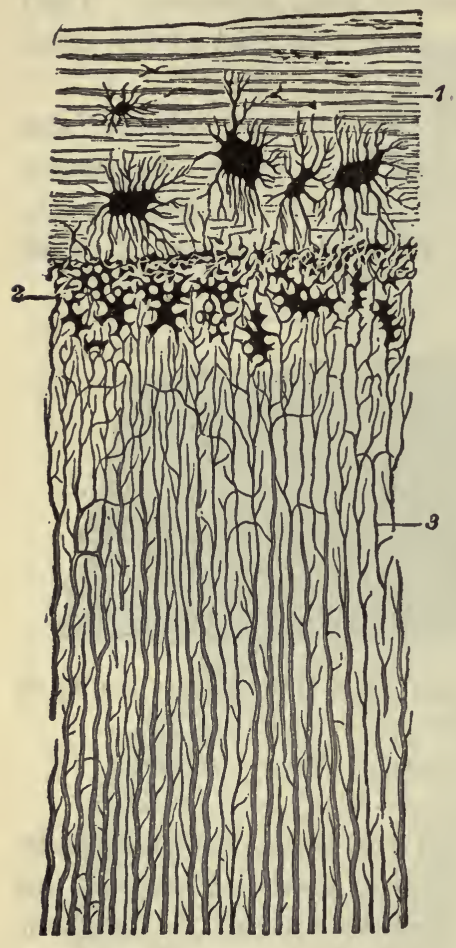

Fig. 306.-Section of Fang

Parallel to the Dentinal

Tubules, Human Canine. (Waldeyer).

1, cementum, with large lacunæ, canaliculi and indications of lamellæ; 2, granular layer of Tomes with large interglobular spaces; 3 , dentinal tubules. $\times 300$. (From Quain's "Anatomy.") filled by a deposit of calcareous salts which gives the dentin its bony consistence. Dentin consists of about 28 per cent. organic and 72 per cent. earthy matter. The latter includes calcium phosphate, about 67 per cent.; calcium carbonate, about 3 per cent.; and magnesium phosphate, with a trace of calcium fluorid.

Here and there, especially toward its peripheral border and near the apex of the tooth, the dentinal matrix fails to become calcified. Such uncalcified areas, interglobular spaces, are encroached upon by the rounded or globular margins of the adjacent calcified matrix which forms the so-called dentinal globules.

The dentin is everywhere permeated by a system of canaliculi, the dentinal tubules or canals, which extend in a radial manner from the pulp cavity outward to the cementum and enamel Their course is characteristically curved, resembling the letter $s$. The cavity of the dentinal tubules is partially occupied by the dentinal processes (fibers) of the odontoblasts, an arrangement which may be compared to that existing between the processes of the bone cells and the canaliculi of bone. At their inner extremity the dentinal tubules are 2 to $4 \mu$ in diameter, but they taper very gradually, especially in the outer portion of their course, where they finally reach a diameter of no more than 0.5 to $1 \mu$.

The dentinal tubules may divide dichotomously in the inner third of their course; beyond this point they give off very fine lateral twigs, which at first leave the parent tubule nearly at right angles, but later are slightly inclined outward. At their distal end most of the dentinal 
tubules divide into a group of terminal branches, some of the arborizations being very extensive, others consisting of but two or three subdivisions. The coarser branches are frequently looped, the distal end of the loop often anastomosing with adjacent tubules. In their course through the dentin those canaliculi which enter the larger interglobular spaces are continued through these spaces without interruption.

The walls of the dentinal tubules are formed by extremely dense calcareous dentinal sheaths (of Neumann) which are very resistant to the action of acids. The curvatures in the course of the dentinal tubules are of two types; the longer primary curves and the shorter spiral secondary curves. They occur with extreme regularity and as a result give rise to certain parallel lines in the substance of the dentin which follow the contour of the dentinal surface. These are known as the incremental lines of Schreger.

A second system of dentinal striæ, visible in ground sections of tooth under low magnification, are the contour lines of Owen (arched incremental lines of Salter). They run nearly parallel to the lines of Schreger in the crown and toward the tip of the root, but elsewhere cut these lines at wide angles (Fig. 303). They represent lines of defective calcification between suc-

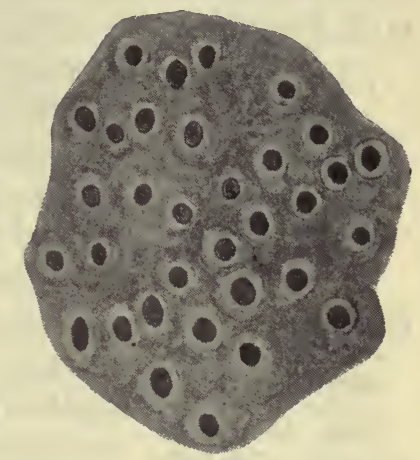

Fig. 307.-Dentin FRoM A Ground Section of a $\mathrm{Hu}-$ man Molar, Showing the Dentinal Tubules Cut Across.

The tubules appear as dark round or oval areas in the dentinal matrix. Each tubule is surrounded by a narrow lighter halo, corresponding to the sheath of the tubule, perhaps an optical effect. $\times 750$. cessively deposited layers of dentin.

The superficial portion of the dentin is formed by the granular layer of Tomes, in which there are no dentinal tubules, but instead there are in this layer numerous small interglobular spaces from which minute canaliculi radiate in various directions. Many of these canaliculi are connected, on the one hand with the dentinal tubules, and on the other with the canaliculi and bony lacunæ of the cementum. The canaliculi of the granular layer are readily distinguished from the adjacent dentinal tubules by the extreme irregularity of their course, which contrasts sharply with the straight or regularly curved course of the dentinal tubules.

The granular layer is relatively thick in the root of the tooth, but 
becomes much thinner toward the neck. Beneath the enamel it becomes so thin that toward the apex of the tooth it is scarcely demonstrable. At this point also, occasional dentinal tubules are continued for a short distance into the enamel, though this condition is more characteristically developed in some of the lower mammals (e.g., Rodentia) than in man.

Enamel.-The enamel, which covers the exposed crown of the tooth, is the hardest tissue of the body. About 90 per cent. of its earthy matter is calcium phosphate; about 4 per cent. is calcium carbonate; less than

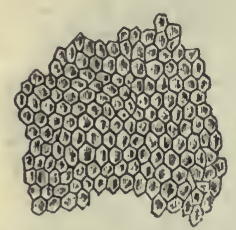

Fig. 308.-Enameu

Prisms in TranSECTION.

From the tooth of a calf. $\times 350$. (After Kölliker.)

5 per cent. of its substance consists of organic matter. It contains a slightly larger trace of calcium fluorid than dentin.

Its unit of structure is a calcareous cylinder, the enamel prism. 'These prisms or 'fibers' radiate outward from the dentin and are disposed after the manner of a mosaic. They are firmly united to each other by a very thin layer of calcified interprismatic cement substance. They represent calcified columnar ectodermal cells.

In transverse section the enamel prisms have a polygonal, frequently quite regular hexagonal, outline. In certain bundles, especially toward the periphery, the prisms have the shape in transverse outline of stout crescents. In longitudinal view the prisms present a slightly beaded appearance, the constricted portions being darker and delicately cross-striped. This peculiar structure and optical condition results from the manner of the formation of the prisms by the deposition of successive globules of preënamel substance, subsequently becoming calcified to form definitive enamel. The enamel cement exhibits a reciprocal beading. Since the external surface of the enamel is greater than the internal, and since the enamel prisms are of approximately uniform diameter throughout, additional shorter prisms, pointed at their inner end, are interpolated toward the surface. The striped and beaded character of the enamel prisms is especially conspicuous in the teeth of rodents.

The enamel prisms are grouped into bundles within which the constituent prisms are parallel. The course of the prism bundles, however, is variable, so that, though following a more or less radial course through the enamel, the prism bundles frequently cross one another at acute angles. In axial longitudinal sections of ground tooth this crossed arrangement of the prism bundles produces the appearance of radially disposed alternating dark and light bands. This banding is seen under 
low magnification, and is especially conspicuous in reflected light; it is due to the difference with which the groups of transverse and longitudinally cut enamel prisms reflect the rays of light. The dark bands are known as the radial lines, or the prism stripes of Schreger.

Ground sections of dried tooth show also brownish lines in the enamel having an arrangement approximately parallel with the surface of the tooth. These contour lines of Retzius are explained by von Ebner as the result of air-filled fissures in the dried enamel. They are also said to be the result of the wavy direction of the enamel prisms. They are most probably caused by imperfect calcification, marking growth stages in the development of the enamel.

Cementum.-The dental cement, or crusta petrosa, is a thin layer of bony tissue which invests the root of the tooth. It forms a very thin layer at the neck of the tooth, but gradually increases in thickness as it approaches the tip of the fang.

The cementum consists of parallel layers of bony lamellæ between which many lacunæ with their bone corpuscles are included. Bone canaliculi radiate from the lacunæ and frequently open into the interglobular spaces of the granular layer. There are no Haversian systems in the cementum, but the thicker portions are frequently penetrated by vascular canals which, like Volkmann's canals, are not accompanied by concentric lamellæ. The cementum is firmly united to the granular layer of the dentin, the matrix of the two tissues being continuous.

The cementum is invested with a periosteal coat, the periodontium, pericementum, alveolar periosteum,

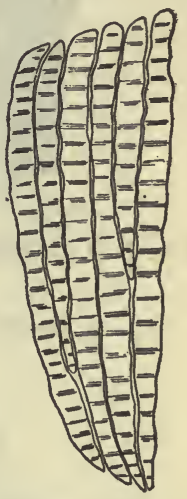

Fig.309.-A Group O F E N A M E L Prisms Cut LonGIT UDIN A L L Y FROM THE INCISOR TOOTH OF THE RAT, SHOWING TheIr IRR E G U L A R L Y Beaded CharacTER AND THE Cross StriaTIONS.

Shorter and slenderer prisms are interpolated peripherally. $\times 375$. or root (peridental) membrane, of dense fibrous tissue which, at the neck of the tooth, unites with the dense connective tissue of the gum to form an annular thickening of very dense fibrous tissue which encircles the tooth and is known as the circular dental ligament. The root membrane contains no elastic fibers, but sends considerable numbers of slender white fibrous bands (Sharpey's fibers) into the cementum. These bands effect a firm anchorage of the tooth to the alveolar wall. They are analogous to the perforating fibers of Sharpey which bind the periosteum to. the osseous lamellæ. 


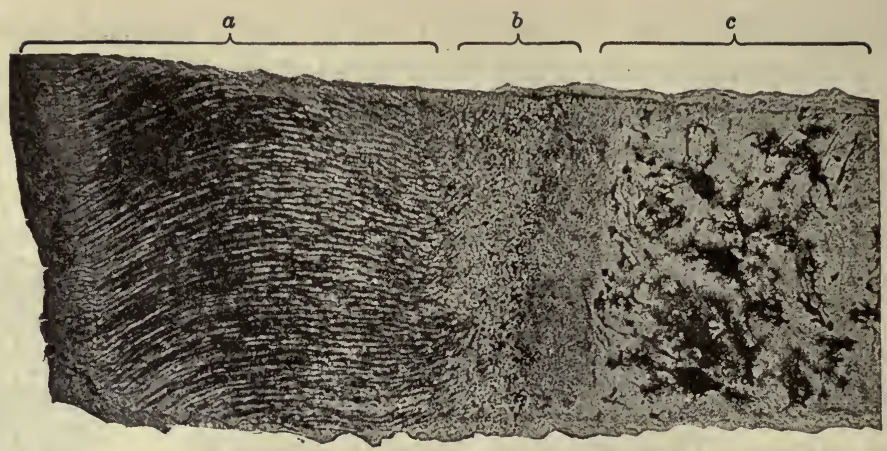

Fig. 310.-From a Section of a Human Tooth Which Had Been Grodnd to Extreme Thinness.

$a$, dentin; $b$, granular layer of Tomes; $c$, cementum. Photo. $\times 140$.

\section{Development of the Teeth}

The teeth arise in part from the ectodermal epithelium of the oral cavity and in part from the mesenchyma of the alveolar processes. In the seventh week of fetal life there appears upon the surface of the maxillary ridges a thickening of the epithelium which grows into the subjacent mesenchyma in the form of a longitudinal plate or shelf, the labiodental strand, whose position is indicated superficially by a dental groove which indents the epithelial surface of the primitive gum. The labiodental strand divides at its deeper border into a nearly vertical continuation, the labial lamina, which subsequently becomes hollow to form the labiogingival groove; and a horizontal inwardly directed shelf, the dental lamina, which forms the earliest anlage of the enamel, the enamel organ.

At the beginning of the third month the dental shelf shows upon its deep margin a series of shallow inverted cups, one for each of the temporary teeth, produced by an up-pushing of a corresponding series of cone-shaped areas of condensing mesenchyma at the site of each tooth germ. Each mesenchymal thickening forms the anlage of a dental papilla. That portion of the dental shelf which spreads out laterally to cover the dental papilla of each tooth forms its enamel germ, from which the dental enamel is eventually produced. Further development of the enamel germ and dental papilla causes the former to surround the papilla like a cap. Figures 311 and 312 show four stages in the early development of a tooth. 
During the third month of fetal life the anlages of all primitive (deciduous, milk, or temporary) teeth are formed in the above manner. At about the same time, also, a posterior growth upon the lingual side of the thin portion of the dental shelf which still connects the enamel germs with the oral epithelium, forms the anlages of twenty of the permanent teeth. The twelve additional permanent molars arise at a later period and in a similar manner by a dorsal extension of the dental lamina which grows backward through the connective tissue of the alveolar process as a solid cell column from which the enamel germs are formed and into which the dental papillæ grow.

Further development of the dental anlage includes the differentiation of the enamel germ on the one hand and of the dental papilla on the other. From the former the enamel and the cuticular epithelial membrane arise; the latter produces the dental pulp and the dentin.

The Enamel Germ.

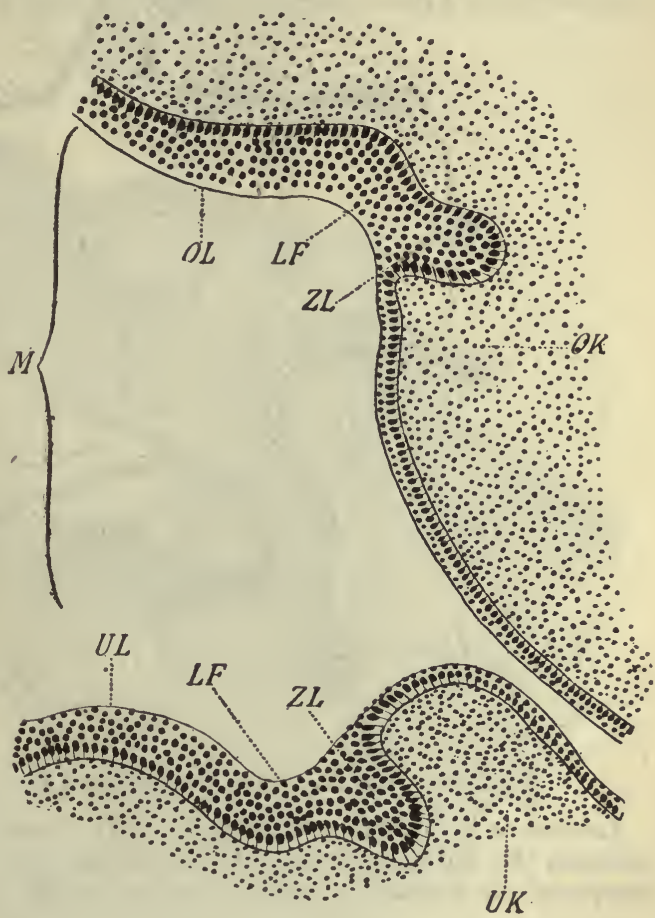

Fig. 311.-Developing Tooth from a Human ЕмвRYo 17 мм. LoNG.

$L F$, dental groove; $M$, oral cavity; $O K$, mesoblast of upper jaw; $U K$, anlage of lower jaw; $O L$, epithelium of the primitive upper lip, and $U L$, of the lower lip; $Z L$, dental lamina (labiodental strand). $\times 120$. (After C. Röse.)

-The enamel germ or enamel organ soon differentiates into three layers: (1) an inner enamel epithelium which forms the enamel prisms; (2) an outer enamel epithelium which lines the dental sac; and (3) an intervening enamel pulp.

The Inner Enamel Epithelium.-The innermost cells of the enamel organ, viz., those which rest directly upon the dental papilla, soon become elongated and attain a cylindrical form. The nucleus moves toward the distal pole, and the original basal end becomes modi- 


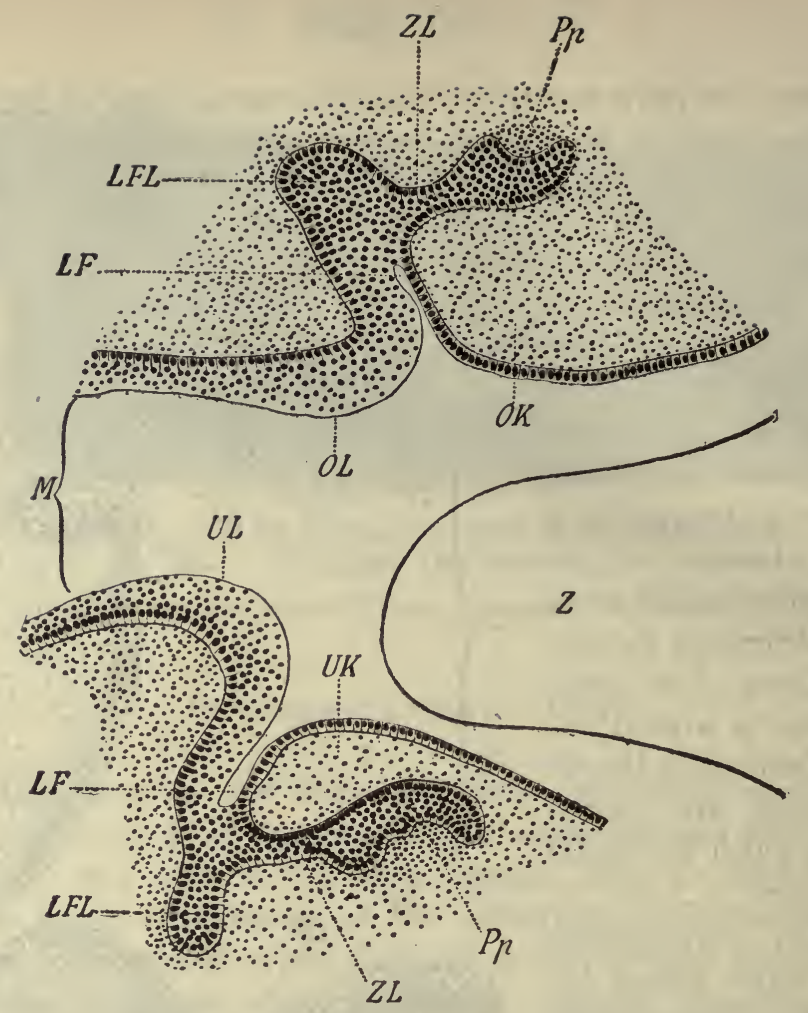

Fig. 312.-Dental Anlages from a Human Fetus 40 mm. Long.

Letters as in the preceding figure. $L F L$, labial lamina, or anlage of the groove between the lip and the mandibular process; $P p$, dental papilla; $Z$, outline of the margin of the tongue; $Z L$, dental lamina. $\times 60$. (After C. Röse.)

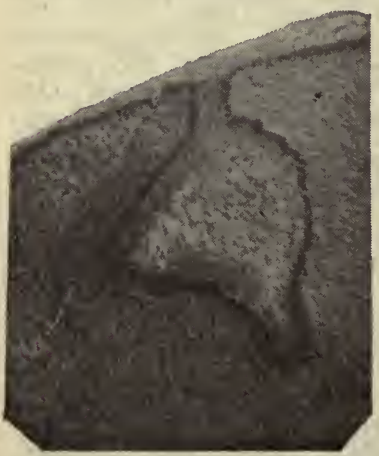

A

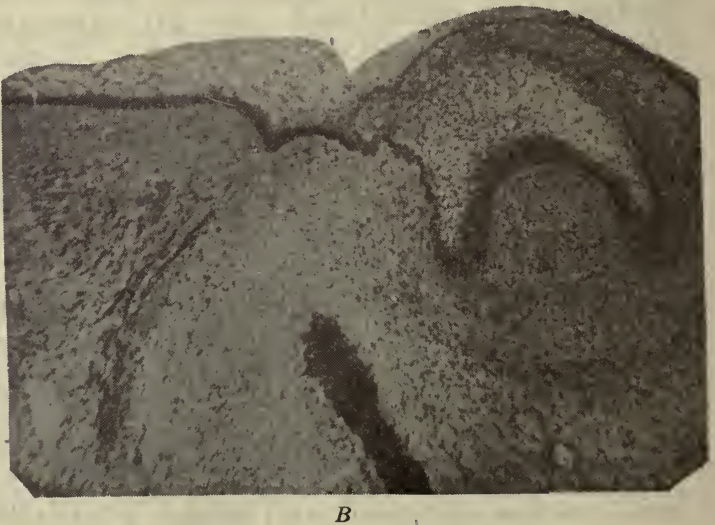

Fig. 313.-Two Stages in the Early Development of the Teeth, from a 25 mm. Pig Embryo.

In $B$ is seen the developing bone and striped muscle of the lower jaw. In both stages the dental papilla and the three layers of the enamel organ are clearly shown. 
fied to take on the characteristics of the distal end of a columnar cell. A cuticular border appears upon the inner (originally basal) extremities, and as the calcareous substance of the enamel begins to be deposited fine processes are seen extending inward from the extremities of the enamel cells, Tomes' processes. It is around these processes that the permanent

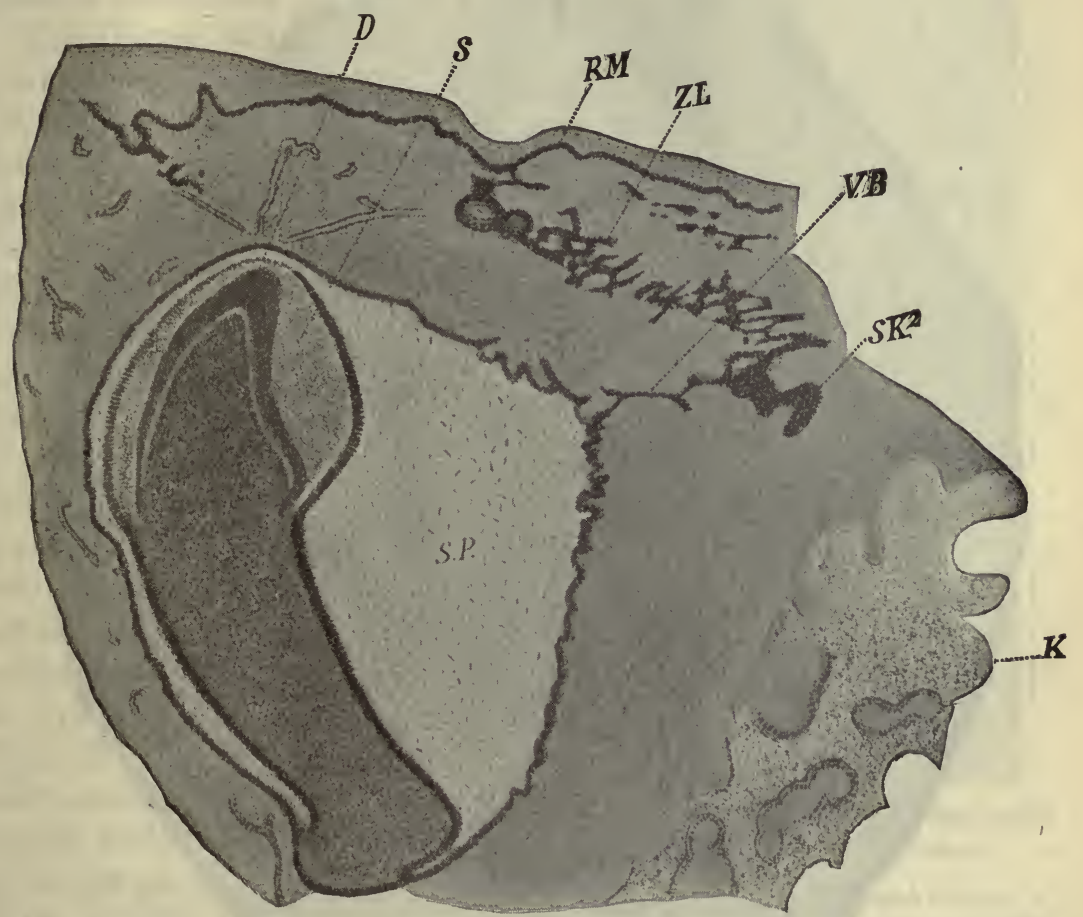

Fig. 314.-Developing Tooth from a Human Fetus 30 cm. Long.

$D$, dentin; $K$, bone of the jaw; $R M$, germinal layer of the oral mucosa; $S$, enamel organ; $S K^{2}$, enamel anlage of the permanent tooth; $V B$, epithelial bridge still uniting the anlages of the temporary and permanent teeth; $Z L$, disintegrating dental lamina; $S P$, enamel pulp. $\times 30$. (After C. Röse.)

enamel is deposited first in the form of globules, which become calcified and meanwhile fuse to form the enamel prisms.

The deposit of lime salts by the cylindrical cells of the inner enamel layer, ameloblasts (adamantoblasts), occurs earliest at the apex of the dental germ. Thus, it is the enamel of the face of the tooth crown which is first formed, and this is therefore its thickest portion. The enamel on the sides of the tooth crown appears later and hence it gradually 
tapers in thickness as it approaches the neck of the tooth, in which latter place the last formed enamel is found.

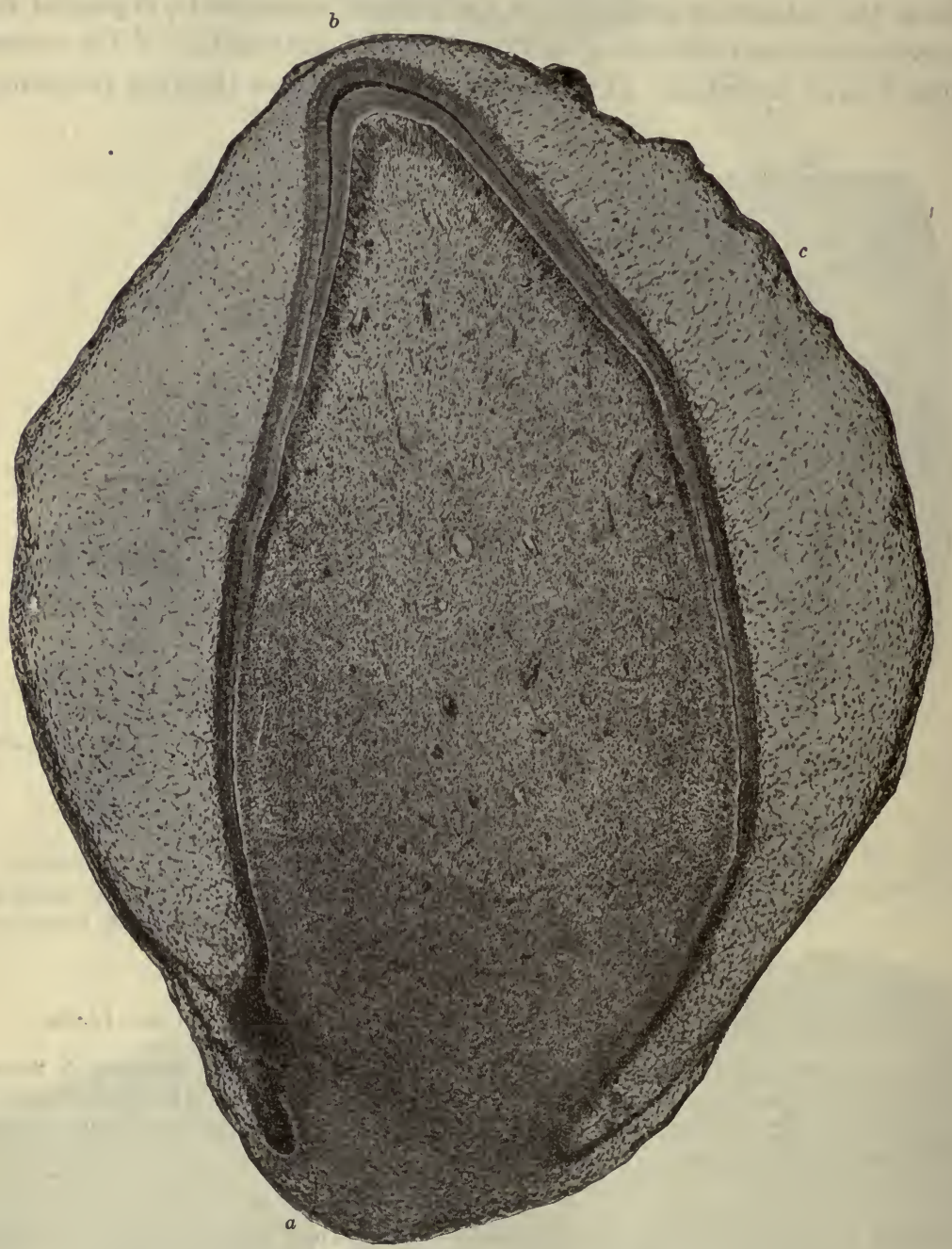

Fig. 315.-A Developing Tooth From an Infant's Jaw.

$a$, papilla; $b$, crown; $c$, outer enamel epithelium. Hematein and eosin. Photo. $\times 65$.

The nucleated bases (originally apices) of the cylindrical cells of the inner enamel epithelium are also marked by a sharp cuticular margin and rest upon the adjacent cells of the enamel pulp, the innermost cells 
of which retain a characteristic epithelial appearance. The thin layer formed by the uncalcified bases of the adamantoblasts, which still cover the free surface of the enamel at the eruption of the tooth, remains as the highly cornified dental cuticular membrane (of Nasmyth). This is soon lost over the crown by reason of mechanical wear.

The Outer Enamel EpITHELIUM.- $\mathrm{T}$ h e outermost cells of the enamel germ are immediately in contact with the mesenchyma of the primitive jaw. This connective tissue forms, toward the end of the third month, a vascular investing sheath or dental sac, which incloses the entire dental germ and finally, by gradually encroaching upon the narrow neck which still connects the enamel germ with the dental lamina, severs the connection of these organs so that the primitive tooth lies free within the dental sac. The outer enamel epithelium, which lines all portions of the dental sac except at the base of the dental papilla, forms several layers of flattened epithelial cells. Remnants of this cell layer frequently persist, in re-

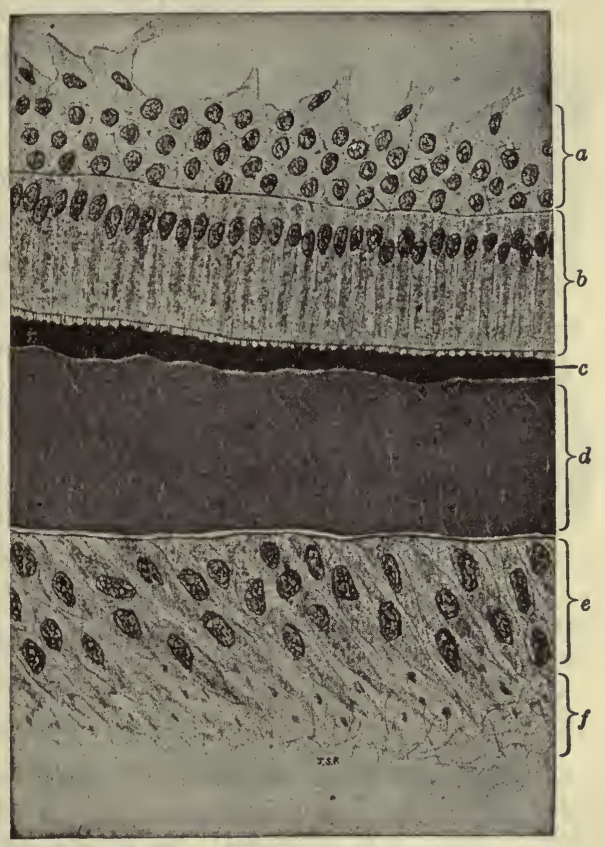

Fig. 316.-A Portion of Fig. 315, Near the Apex of the Developing Tooth.

$a$, enamel epithelium; $b$, ameloblasts; $c$, enamel; $d$, dentin; $e$, odontoblasts; $f$, border of the dental pulp. Between the formed enamel and the ameloblasts Tomes' processes can be seen. Hematein and eosin. $\times 550$.

lation to the inner margin of the bony alveolus whose wall is produced by intramembranous ossification in the connective tissue surrounding the embryonic dental sac.

The Enamel PulP.-This structure is produced by a remarkable differentiation which occurs within the mid-portion of the enamel organ. The epithelial cells of this region, which at first appear to form a delicate syncytium, become separated by wider and wider intercellular spaces, and are thus drawn out into stellate forms with long anastomosing processes. The resulting cells closely resemble in form the connective 
tissue cells of embryonal or gelatinous connective tissue. They are, however, inclosed on all sides by the epithelial cells of the inner and outer enamel epithelium and, like other epithelial tissues, are never penetrated by blood-vessels.

The enamel pulp appears to serve a purely mechanical function, it

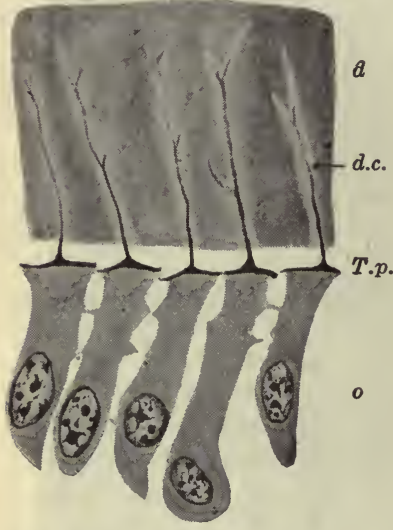

Fig. 317.-ODONTOBLASTS AND DENTIN OF THE Tоотн OF A NEW-BORN Cat.

$o$,odontoblasts; $T p$, Tomes' fibrils, prolongations of the odontoblasts; $d c$, dentinal canal in the dentin $(d)$. $\times$ 500. (After Prenant, Bouin and Maillard.) being a soft tissue through which the growing tooth readily pushes its way to the surface.

The Dental Papilla.-The dental papilla is a mesenchymal structure which is invested by and grows into the enamel organ. Coincident with the appearance of the ameloblasts in the enamel organ, the superficial cells of the dental papilla become enlarged, elongated, and so arranged as to form a continuous layer of odontoblasts on the surface. of the papilla. These cells apparently secrete a thin homogeneous layer, membrana preformativa (Raschkow), which serves as a basement membrane upon which the ameloblasts deposit the enamel prisms; it also forms the anlage of the granular layer of Tomes.

The odontoblasts now form the DENTIN in a manner entirely analogous to the deposit of bone by the osteoblasts, processes of the odontoblasts being included within the deposit of dentin to form the dentinal fibers. Irregular spaces, occurring in the dentin and granular layer, in which no calcification occurs produce the interglobular spaces.

The central mass of the dental papilla develops the reticular tissue of the dental pulp. The blood-vessels and nerves enter the pulp through the base of the papilla, which thus becomes the anlage of the root canal.

The CEMENTUM is formed by intramembranous ossification occurring in that portion of the dental sac which invests the base of the dental papilla and the primitive root of the tooth.

The process of cementogenesis is essentially similar to that of perichondrial ossification. The vascular mesenchyma of the dental sac breaks through the epithelial sheath (the root extension of the enamel organ), and through the agency of modified mesenchymal cells similar 
to the osteoblasts, the cementoblasts, deposits cementum upon the dentin of the root. The first deposit is made in the neck region, and-the deposition progresses from thence to the apex of the root, where the thickest layer is subsequently found. Cementoblasts become enclosed in lacunæ of the cementum and persist as cementum cells. The peripheral layer of the root portion of the sac differentiates into a dense fibroelastic lamina which serves both as a pericementum to the tooth, and as periosteum to the bone of the jaw. The pericementum includes besides cementoblasts also odontoclasts which become active as tooth destroyers at about the age of five years. They absorb the roots of the deciduous teeth, and thus open the way for the eruption of the permanent teeth.

\section{THE TONGUE}

The tongue consists essentially of a mass of voluntary muscle invested by a continuation of the mucous membrane of the mouth and pharynx. The fibers of this striated muscle are separated into two lateral halves by a median septum of dense connective tissue which extends from the base to the tip of the organ, and is known as the lingual septum.

The Muscle Fibers.-The muscle fibers include two groups: (1) the intrinsic, those of the lingualis or tongue muscle proper; (2) the extrinsic, those entering from without and serving to attach the tongue: the genioglossus, the hyoglossus, the styloglossus, the palatoglossus, and the chondroglossus. The fibers are disposed in three planes and are so arranged that the bundles cross one another at right angles. They thus form: (1) sagittal or vertical fiber bundles which are slightly inclined outward from the septum linguæ and are derived from the lingualis muscle, supplemented in the inferior median portion by fibers from the genioglossus and the hyoglossus; (2) longitudinal fibers peripherally distributed and running from the base to the apex of the tongue, derived chiefly from the lingualis but supplemented on the under surface by the styloglossus, and chondroglossus; (3) transverse or horizontal fibers extending laterally from the septum linguæ, which are also derived from the lingualis muscle; and include a few fibers from the palatoglossus.

The interlacing bundles of muscle fibers are embedded in loose areolar and adipose tissue. The muscle fibers are inserted into the corium of the lingual mucous membrane, their sarcolemma being firmly 
adherent to the connective tissue of the mucosa, which invests the rounded blunt extremity of the muscle cell. Many of these muscle fibers are branched.

The Mucous Membrane.-The mucous membrane of the tongue

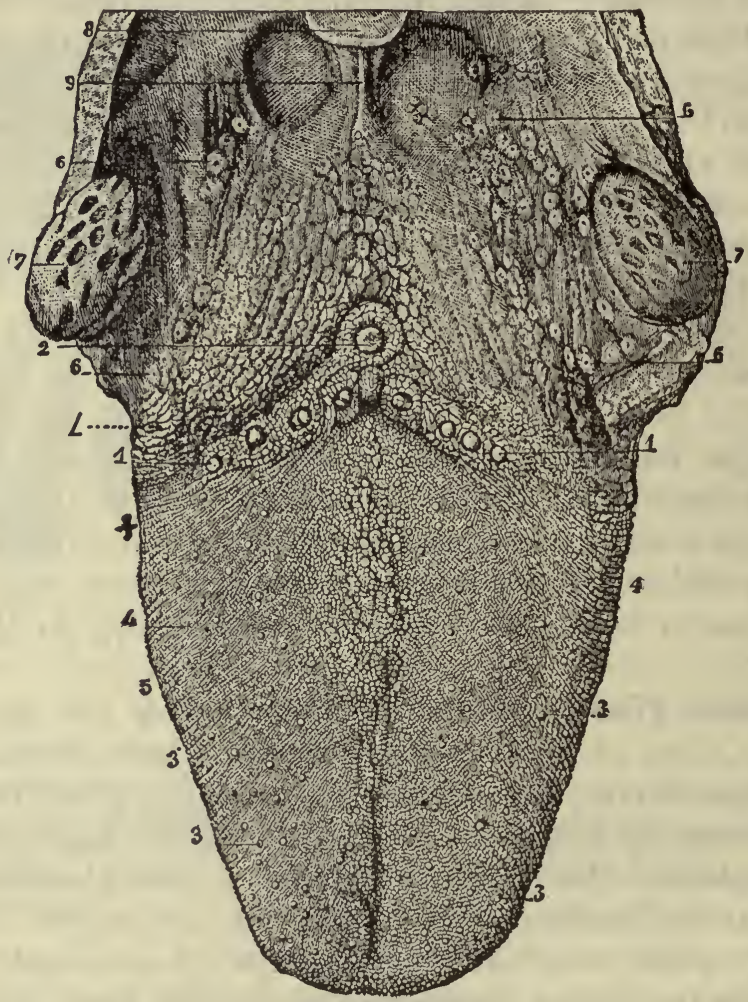

Fig. 318.-View of Dorsum of Tongue, Showing the Various Papille, the Tonsils and the Fauces.

1, circumvallate papillæ; 2 , foramen cecum; 3 , fungiform papillæ; 4, filiform papillæ; 5 , transverse and oblique rugæ; 6 , mucous glands; 7 , tonsils; 8 , tip. of epiglottis; 9 , median glosso-epiglottidean fold; $f$, foliate papillæ; $L$, lenticular papillæ. (After Sappey.)

consists of a thick corium, and an epithelial covering. The deeper part of the CORIUM, consisting of loose areolar tissue, is intimately connected with the muscle. The superficial portion of the corium, containing denser areolar tissue, carries upon its surface dorsally connective tissue papilla of unusually large size which project into the 
glands of Nuhn. The serous glands of von Ebner are confined to the region of the circumvallate papillæ at the base of the tongue. They pour their secretion into the trench which surrounds the base of the papilla or into the crypts of the lingual tonsil. Other lingual glands, of the small tubulo-acinar mucous type, occur at various portions of the dorsal surface of the tongue, being especially abundant in the lymphoid area.

The Lingual Tonsil.-The lingual tonsil (Fig. 249) is a considerable collection of lymphoid nodules which is found at the base of the tongue in and about the median line. These nodules aré grouped about a large funnel-shaped crypt, the foramen cecum, which opens at the apex of the V formed by the group of circumvallate papillæ and which in the embryo forms the lingual extremity of the so-called duct of the thyroid gland (thyroglos-

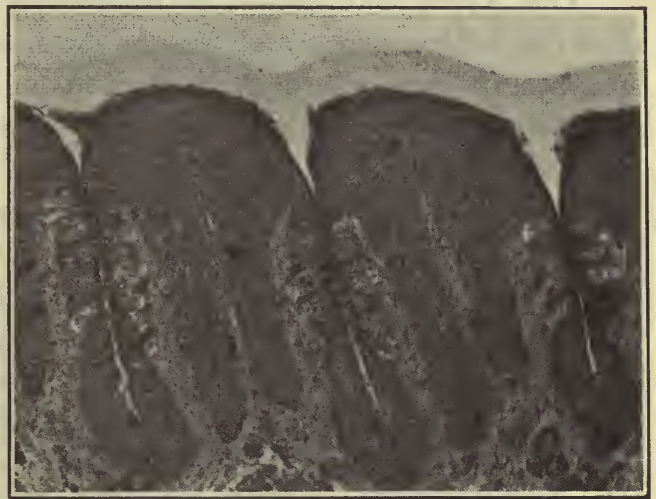

Fig. 323.-Two Foliate Papillat From a RaBbit's Tongue, Showing Numerous Taste Buds Along Their Lateral Margins. sal duct). Several smaller crypts are also included in the region of the lingual tonsil.

The lymphoid nodules are embedded in the mucosa or corium of the tongue and are surrounded by mucous glands, many of whose ducts penetrate between the nodules to open into the branching crypts. Lymphocytes, apparently derived from the nodules, infiltrate the surrounding connective tissue and epithelium and find their way into the lumen of the tonsillar crypts.

The Blood-vessels of the Tongue.-The blood-vessels of the tongue are supplied by large arteries (branches of the lingual artery) which, with the corresponding veins, are embedded in the muscular portion of the organ and supply capillary vessels to this tissue. From these arteries, also, small arterial branches enter the deeper portion of the corium and form a capillary plexus which supplies the connective tissue and whose terminal ramifications extend to the very apex of the connective tissue papillæ. The blood is returned by veins which pursue a similar course. 
The Lymphatics.-The lymphatics form a superficial set of small vessels and tissue spaces beneath the epithelial layer, which are especially abundant in the region of the lingual tonsil at the base of the tongue. The lymphatic vessels of this superficial plexus frequently encircle the lymphoid nodules. A deeper plexus of lymphatics in the loose connective tissue of the submucosa receives the lymph from the superficial plexus and conveys it by efferent lymphatic vessels to the deep cervical lymphatic glands.

The Nerve Supply.-The nerve supply of the tongue comprises cerebral, both sensory and motor fibers, and sympathetic fibers. The sympathetic elements supply the glands and blood-vessels; small ganglia occur along their course. The motor fibers supplying the striped muscle are derived from the hypoglossal nerve. These end in motor endpıates. The sensory fibers arise from the lingual branch of the trigeminal and the glossopharyngeal nerves. These mediate both general sensibility and the sensation of taste. The fibers which receive the stimuli of general sensibility end as naked varicose fibrils in the connective tissue of the body of the tongue, and in the submucosa of the papillæ. They are accompanied by small ganglia. Certain fibers also end in muscle spindles. The fibers of special gustatory sensation are also distributed to the papillæ; the chorda tympani (branch of the facial) component of the lingual branch of the trigeminal supplying the anterior two-thirds, the glossopharyngeal the posterior one-third of the tongue. At the base of the tongue small nerve bundles of taste fibers are distributed to the circumvallate papillæ, and form a subepithelial plexus from which fibrils are distributed: (1) to the interior of the taste buds where they end in relation with the gustatory cells, intragemmal fibers; (2) to the surface of the taste buds, perigemmal fibers; and (3) to the intervening portions of the epithelial layer, where they end between the epithelial cells as in other parts of the tongue, intergemmal fibers (Fig. 175, page 161). 'The taste buds of the soft palate are innervated through the palatine nerves (great superficial petrosal component) of the trigeminal.

\section{THE ALIMENTARY CANAL}

\section{General Characteristics of the Walls}

It is convenient to consider collectively under this head the pharynx, esophagus, stomach, and the small and large intestines. This tract 
forms a continuous tube whose wall has, throughout its entire extent, many common characteristics. Thus the wall in all portions consists of

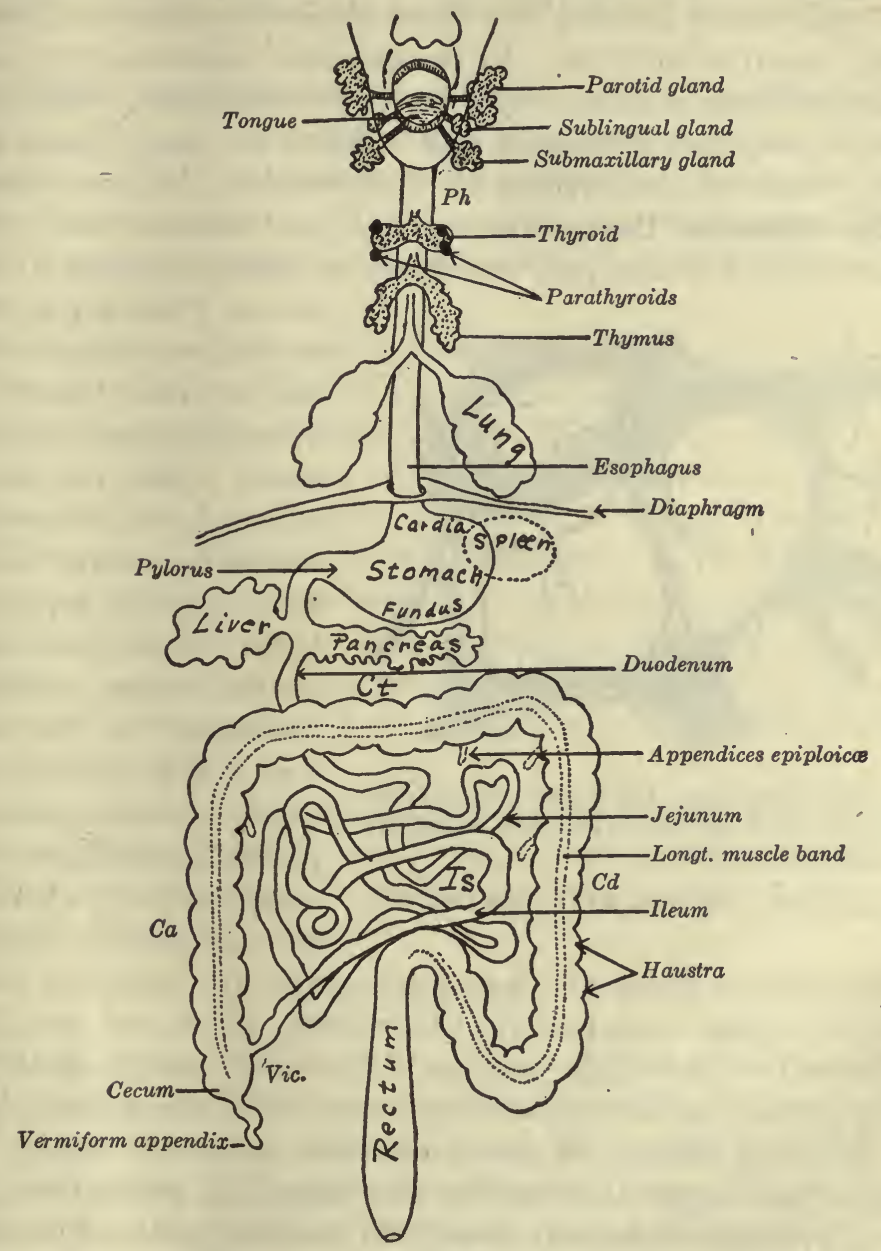

Anus

Fig. 324.-Diagram of the Alimentary Canal of Man.

$I s$, small intestine; $C a, C t$, and $C d$, ascending, transverse and descending portions of the colon; $P h$, pharynx; Vic, ileocolic valve. (Adapted from Wiedersheim.)

four coats which are respectively known, from within outward, as the mucous, submucous, muscular, and fibroserous. The three outermost coats are of very similar structure in all portions of the tract.

The Fibroserous Coat.-In the abdominal cavity the outermost 
coat is derived from the peritoneum, by which the stomach and intestines are invested. In the upper portion of the tract, pharynx and esophagus, the serous coat is replaced by a layer of areolar connective tissue which usually contains much fat. In the abdomen the homologous subserous connective tissue is covered by a layer of mesothelium. The connective tissue of the outer fibroserous coat contains the larger blood and lymphatic vessels whose branches are distributed to the three inner coats.

The Muscular Coat.-The muscular coat, situated next within the fibroserous, is divisible into two layers, an outer longitudinal the direc-

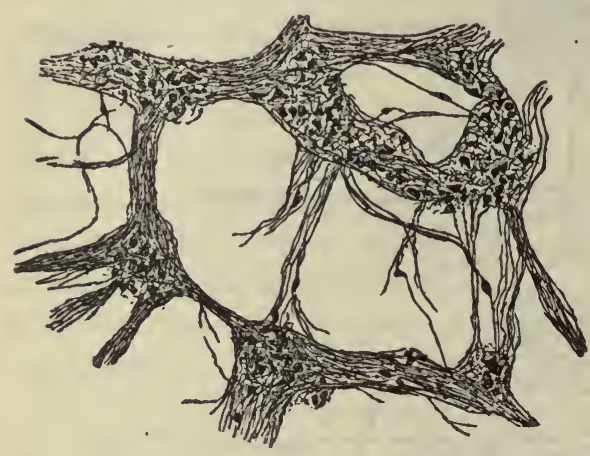

Fig. 325.-Surface View of Auerbach's Intramuscular Nerve Plexus, from the Esophagus of a Cat.

Methylene blue. $\times 40$ to 50. (After DeWitt.) tion of whose fibers is parallel to the long axis of the canal, and an inner transverse layer whose fibers are circularly disposed. The two layers are united by a thin septum of areolar connective tissue which serves for the support of the larger blood-vessels and lymphatics, whose capillaries are distributed to the muscular coat. This septum also contains a coarse-meshed nerve plexus, consisting of small anastomosing nerve trunks which are composed in large part of non-medullated fibers, at whose intersections are numerous small sympathetic ganglia, the myenteric (Auerbach's) plexus and ganglion.

Below the level of the junction of the middle and lower third of the esophagus, and including the musculature of the stomach and intestines, the muscle is entirely of the non-striated or smooth variety. In the pharynx and upper third of the esophagus, the striated or voluntary type of muscle is exclusively found. In the mid-portion of the esophagus both striated and non-striated muscle occur in varying proportions, occasional striated fibers being found even in the lower third of the organ.

The Submucous Coat.-The submucous coat consists of loose areolar tissue, and serves for the support of the larger blood and lymphatic vessels which supply this coat and the mucosa. A second plexus of nerve fibers, similar in structure to the intramuscular plexus, is found in the deeper layers of the submucosa, and is known as the 
submucous (Meissner's) plexus. Its nerve trunks and ganglia are somewhat smaller than those of the myenteric plexus. The submucous plexus supplies the muscular and glandular tissues of the mucous membrane.

The Mucous Membrane.-The mucous coat or mucosa of the gastro-intestinal canal contains four typical structures, (1) an internal lining epithelium; (2) the muscularis mucosæ which forms the outermost layer; between these is (3) a tunica propria or corium of diffuse lymphoid or areolar tissue, which serves chiefly for the support of (4) the secreting glands.

The muscularis mucosa usually consists of a double layer of smooth muscle, the outer being longitudinally, the inner circularly disposed. This layer is most highly developed in the esophagus.

The tunica propria consists of delicate fibro-elastic and reticular tissue whose volume is in inverse proportion to that of the secreting glands. It is most abundant in the esophagus. In the stomach and intestines it is considerably infiltrated by lymphocytes and often contains diffuse lymphoid tissue. Small lymph nodules are also found in the deeper part of this membrane; they progressively increase in size toward the lower portion of the tract, where they form the solitary nodules of the intestine.

The nature of the lining epithelium and the type of glands differs in each succeeding portion of the canal, and must, therefore, together with the other peculiarities of the several subdivisions of the tract, be separately considered.

\section{The Pharynx}

The pharynx may be subdivided, upon histological as well as physiological grounds, into (1) an upper respiratory portion, or nasopharynx, and (2) a lower portion, oropharynx and laryngopharynx; only the latter of these properly belongs to the alimentary tract. The soft palate and uvula form a thin partition between the naso- and oropharynx. The nasopharynx has already been described as a part of the respiratory system (see Chapter XII).

The mucous membrane of the lower portion of the pharynx is lined by stratified squamous epithelium which rests upon a thick corium of areolar tissue. The tunica propria is well supplied with thin-walled blood-vessels and lymphatics, and contains many mucus-secreting glands of the tubulo-acinar type whose secreting portions lie deeply embedded in the connective tissue of the muscular coat. 
There is no muscularis mucosæ in the mucous membrane of the pharynx; its place is taken by a layer of connective tissue which is exceedingly rich in longitudinal elastic fibers. This layer lies immediately upon the muscular coat, into which processes of fibro-elastic tissue extend between the muscular bundles; hence this fibro-elastic layer also serves as a submucosa.

The superficial layer of the corium contains diffuse collections of lymphoid tissue and occasional small lymph nodules.

The muscular coat of the pharynx is formed by its constrictor muscles. Their striated fibers mostly pursue an oblique course. Where these muscles are not immediately attached to the periosteum of the vertebræ, the pharynx is invested with an outer coat of areolar connective tissue by which it is loosely united to adjacent organs.

\section{Esophagus}

The esophagus or gullet is a short tube about 25 centimeters (10 inches) in length, connecting the pharynx with the cardia of the stomach. Its wall contains the usulal four coats: (1) the outer fibrous; (2) muscular; (3) submucous; and (4) mucous (fig. 327).

The Outer Fibrous Coat.-The outer fibrous coat envelops the wall of the esophagus and unites it to the adjacent organs. It consists of loose fibrous tissue, and contains the blood and lymphatic vessels and nerve trunks which supply the three inner coats. It is not invested by a serous layer.

The Muscular Coat.-The muscular coat contains an outer longitudinal and an inner circular layer of muscle fibers, which are separated by a narrow septum of loose fibrous tissue. In the upper third of the esophagus the muscle is of the striated variety, in the, middle third it is mixed, in the lower third it is-generally-smooth. The distribution of the muscle in the lower third is subject to great individual variation, and occasionally striated fibers are often found all the way down to the diaphragmatic opening.

The fibrous septum between the muscular layers contains the larger blood-vessels and the myenteric nerve plexus.

The Submucous Coat.-The submucous coat forms a layer of areolar connective tissue which firmly unites the muscular and the mucous coats. It contains those blood and lymphatic vessels, together with the submucous nerve plexus, whose branches supply the mucous membrane. It also contains a considerable number of tubulo-acinar 
serous connective tissue which is covered by mesothelium. The serous coat supports the larger blood and lymphatic vessels and nerve trunks which supply the organ.

The Muscular Coat.-The muscular coat of the stomach consists in general of two layers of smooth muscle fibers-a thin outer, longitudinal, and a much thicker inner circular and oblique layer. The regular circular arrangement of these fibers is much distorted 'by' the peculiar dilatation and partial rotation to which the stomach. is subjected in the course of its development, and as a result of this change obliquely placed fibers form a considerable portion of the muscular coat.

The oblique fibers are most numerous toward the cardiac end of the stomach, where they form a third muscular layer, the innermost portion of the muscular coat. The Tongitudinal fibers are most abundant toward the cardiac and pyloric orifices and along the lesser curvature; in the fundus and mid-region of the stomach they form only a very thin layer. The circular fibers form the thickest of the three muscular layers and are nearly equally distributed in all portions, except that at the cardiac and pyloric orifices they become much thickened to form the sphincter muscles. The pyloric sphincter is especially well developed.

The layers of the muscular coat of the stomach are united by thin septa of connective tissue; that between the longitudinal and circular layers contains the myenteric nerve plexus and the larger blood-vessels which supply this coat.

The Submucosa.-The submucosa consists of loose areolar tissue which supports the blood-vessels, lymphatics, and the submucous nerve plexus, all of which distribute their branches to the mucous membrane. In no portion of the stomach does this coat contain glands.

The Mucous Coat.-The muscularis mucosæ forms a thin but complete layer from one end of the stomach to the other, and marks the outer boundary of the mucous membrane. It usually consists of two thin layers, an inner circular and an outer longitudinal. Here and there muscle fibers extend from the muscularis mucosæ into the corium between the gastric glands.

The surface of the mucosa is clothed with tall columnar epithelium, and the whole membrane is thrown into wavy folds, an arrangement which is permitted by the very loose meshes of the submucous areolar coat. The corium of the mucosa is closely packed with tubular secreting glands, which open on the surface by wide-mouthed, crypt-like ducts or foveolæ, and are embedded in a fine fibroreticular tissue containing many lymphocytes. 
The character of the gastric glands differs somewhat in various portions of the stomach. The three varieties, according to their dis-

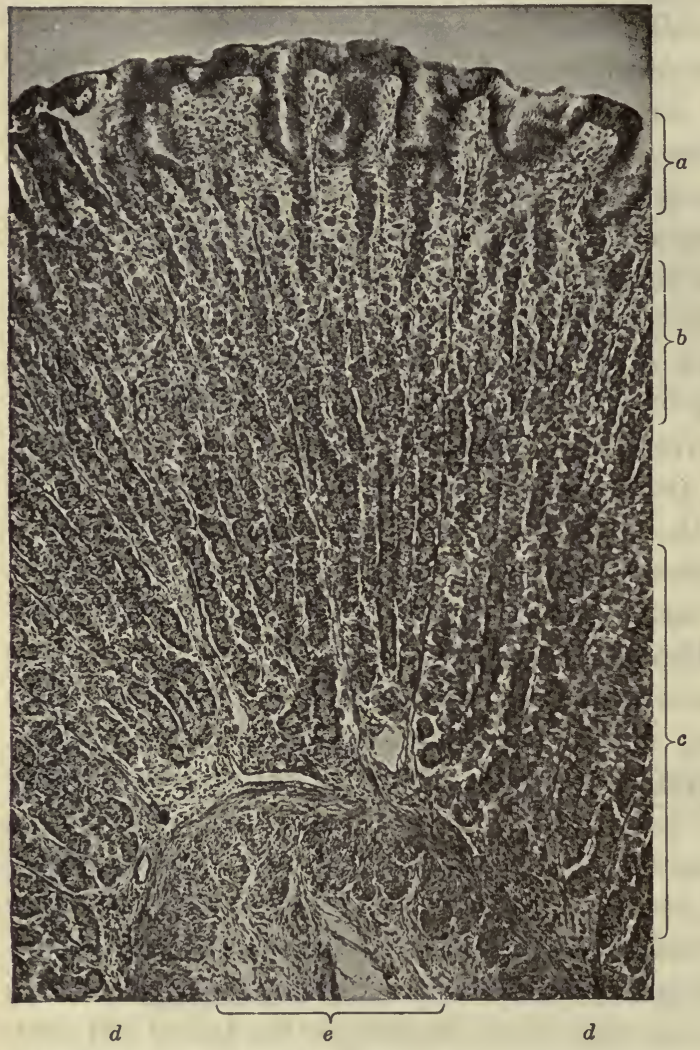

Fig. 330.-The Mucosa of the Fundus Region of the Dog's Stomach.

$a$, gastric crypts; $b$, neck region, and $c$, fundus portions of the gastric glands, the parietal cells being much more abundant in the former; $d$, muscularis mucosæ; e, submucosa. Hematein and eosin. Photo. $\times 80$. also continued over that portion of the corium which occupies the intervals between adjacent ducts, where it forms the true lining epithelium of the stomach. Its cells secrete a clear muco-albuminous fluid.

Between the distal ends of the cells terminal bars occur; they possess also indistinct cuticular borders.

The secreting portion, or fundus, of the gland is five to eight times as the fundus glands, the pyloric glands, and the cardiac glands.

THE FuNDUS GLA N D S ( $P$ e p t ic Glands).-These are somewhat branched tubular glands which possess short ducts, the crypts or foveola, and relatively long secreting portions, several of which open, by means of short constricted portions, the necks of the glands, into the bottom of each crypt.

The excretory ducts or crypts are lined with tall columnar cells which possess a remarkably clear cytoplasm distally, and whose nuclei lie at the proximal or attached. ends of the cells. This epithelium rests upon a distinct basement membrane of reticular tissue (Mall) ; it is

tribution, are known 
less removed from the lumen they possess an extensive system of pericellular secretory canals which invest the cell in a basket-like manner and convey its secretion to the glandular lumen, where it mixes with the

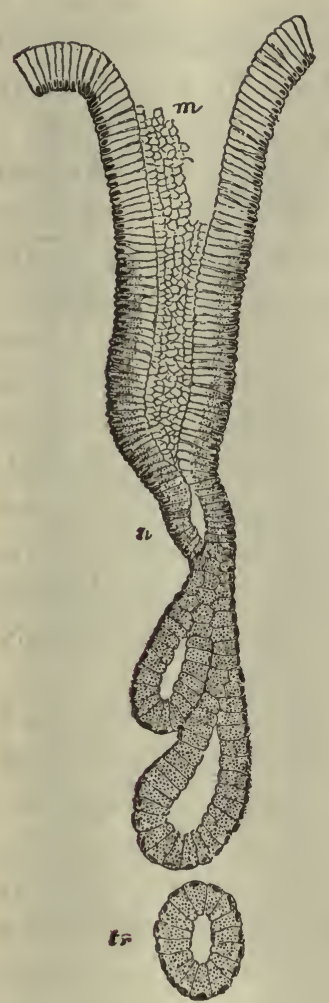

Fig. 333. - A Pyloric Gland, From Section of the Dog's Stomach. (Ebstein.)

$m$, mouth; $n$, neck; $t r$, a deep portion of a tubule cut transversely. (From "Quain's Anatomy.") secretion of the chief cells. The parietal cells also possess a system of intracellular canaliculi.

The parietal cells are commonly believed to secrete the $\mathrm{HCl}$ of the gastric juice. But Harvey and Bensley (Biol. Bull., 23, 4, 1912) claim to have shown that free $\mathrm{HCl}$ is not present in these cells; their content is said to be chemically neutral or alkaline, and to consist largely of chlorids. The results of experiments with rabbits and various other vertebrates indicate that chlorin is secreted by the parietal cells in the form of a chlorid of an organic base, and that the $\mathrm{HCl}$ is only set free after the secretion is poured out of the gland into the foveola. Hammett (Anat. Rec., 9, 1, 1915), however, presents further evidence tending to show the presence of acid in the parietal cells.

\section{Pyloric Glands.-} These are branched con-

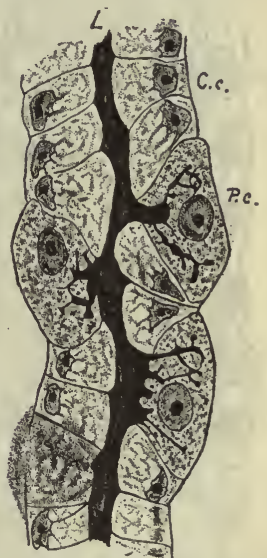

Fig. 334.-Portion of Gastric Gland FROM THE FUNDUS REgION OF THE Stomach.

$L$, lumen, ending in intracellular secretory canaliculi in the parietal cells (P.c.); C.c., chief cells. Prepared by the Golgi chromate of silver impregnation method; highly magnified. (After Zimmermann.)

voluted tubular glands with relatively long crypt-like ducts, into the bottom of which several secreting tubules open. According to Piersol, they occupy the pyloric fifth of the stomach.

The typical convolution is found only in the fundus of the gland, the course of the ducts being nearly straight. The branching, on the 
other hand, is chiefly confined to the ducts, which occupy the superficial two-thirds to three-fourths of the entire depth of the mucous membrane. In the pyloric mucosa, therefore, three zones may be distinguished: a superficial, middle, and deep.

The superficial zone is narrow and contains the wide-mouthed crypts

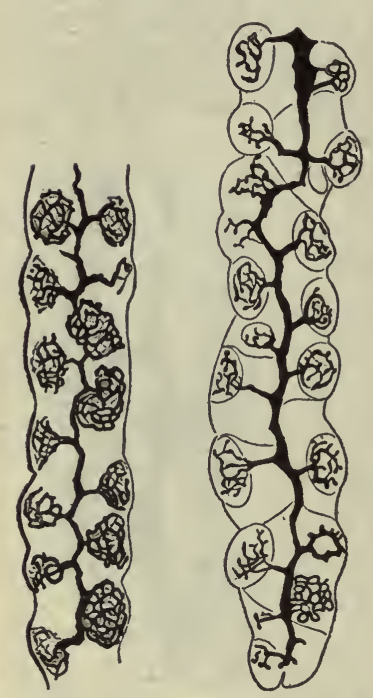

Fig. 335.-Secretory CapILLARIES OF THE FUNDUS Glands of the Dog's Stomach.

Golgi stain. (After Müller, from Oppel.) or foveolæ which are lined by tall columnar cells similar to those of the fundus crypts.

The middle zone contains the narrowed portion of the ducts and is the broadest of the three zones. Several of the narrow ducts open into each foveola and further branching of the secreting tubules occurs to a limited extent. The epithelium of the ducts is of the low columnar variety, whose deeply stained basal nuclei are spheroidal or ovoid, and are progressively flattened as the secreting portion is approached. The superficial cytoplasm of these cells stains readily with muchematein and often has a coarsely granular or reticular appearance.

The deepest zone contains the convoluted secreting portions and is sharply marked off from the adjacent ducts, since in a transection of the stomach wall its tubules, owing to their convolution, are nearly all cut across, while the ducts are in longitudinal section; the clear tall columnar epithelium and broad lumen of the fundus also, contrast strongly with the low finely granular epithelium and narrow lumen of the duct. It is this narrow zone of peculiar convoluted tubules, lying just within the muscularis mucosæ, by which the pyloric mucous membrane is most readily distinguished from all other regions of the alimentary canal.

The tall columnar cells of the fundus possess a remarkably clear cytoplasm which reacts distinctly, though feebly, to the specific stains for mucus. The nuclei are flattened against the base of the cell and thus contrast sharply with the spheroidal nuclei of the ducts and crypts.

During secretion the cells become shrunken and their nuclei approach the center of the cell and become more nearly ovoid or spheroidal in shape. 
There is no sharp line of demarcation between the fundus and pyloric regions, the glands offering a gradual transition from the one type to the other. Thus, in the human stomach, there is a broad transition

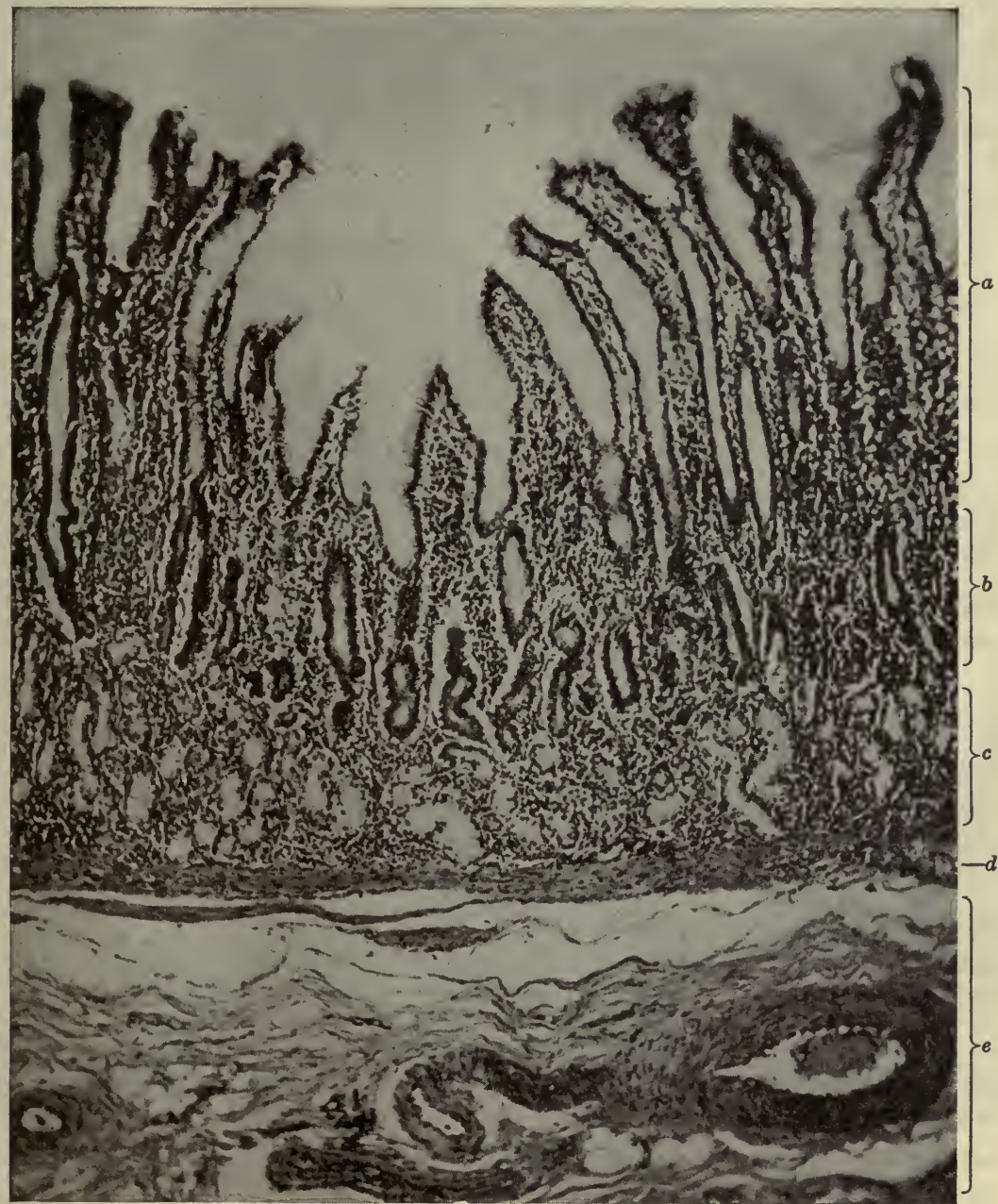

Fig. 336.-The Mucosa of the Pyloric Region of the Human Stomach.

$a, b$, and $c$, respectively the crypt, neck, and fundus zones of the glands; $d$, muscularis mucosæ; $e$, submucosa. Hematein and eosin. Photo. $\times 151$.

zone which contains both fundus and pyloric glands. Indeed, in many individuals, parietal cells may be distributed throughout almost the entire gastric mucosa. 
The Cardiac Glands.-A narrow region, about 5 millimeters in width, at the cardiac orifice of the human stomach contains glands whose form corresponds with that of the fundus glands, though they are slightly more branched and are rather more tortuous, but which are lined by relatively clear columnar epithelium. Only occasionally are the chief and the parietal cells, which are characteristic of the fundus glands, interspersed among the clear secreting cells of these tubules. The cardiac glands, therefore, appear to offer a transition from the esophageal to the more numerous fundus glands of the stomach. In certain mammals, e.g., the pig and the Marsupialia, the cardiac glands occupy a much larger area.

The Conium.-The corium of the mucosa consists of a delicate fibroreticular connective tissue which supports the blood and lymphatic vessels and is more or less infiltrated with lymphocytes. Hence in many portions it possesses the character of diffuse lymphoid tissue, though this tissue is characteristic of the interglandular rather than the interfoveolar portion of the tunica propria. In the latter situation, in sharp contrast to the intestinal villi with which the student may confound this region, the corium is decidedly fibrous and contains relatively few lymph corpusclés.

In the deeper part of the mucosa occasional small lymph nodules, homologues of the solitary follicles of the intestine, are seen. These nodules ('lenticular glands') lie just within the muscularis mucosæ and do not, as a rule, penetrate into the submucosa. In the cardiac region they may lie very near the free surface of the mucosa.

Blood Supply.-The large blood-vessels, derived from the branches of the celiac axis, enter through the subserous connective tissue of the omentum and form arches at the greater and lesser curvatures of the stomach.

From these arches, arteries lying in the subserous connective tissue are distributed to the ventral and dorsal surfaces of the gastric wall. These vessels supply branches which penetrate the muscular coat, giving off, on the way, arterioles to the intramuscular septum, and secondarily to the intramuscular capillary plexus, and spread out in the areolar tissue of the submucosa in which they form an extensive arterial plexus. Branches from this submucous plexus enter the mucous membrane and form a dense capillary plexus whose elongated meshes inclose the secreting glands.

Near the surface of the mucosa these vessels enter a plexus of small venules which, by union, form larger branches and convey the blood 
The Lymphatics.-The lymphatics arise by vascular loops or dilated extremities between the secreting glands of the mucosa. At the outer border of the mucous membrane they form a delicate anastomosing plexus from which-branches penetrate the muscularis mucosæ and enter a broad submucous plexus whose efferent vessels pierce the mus-

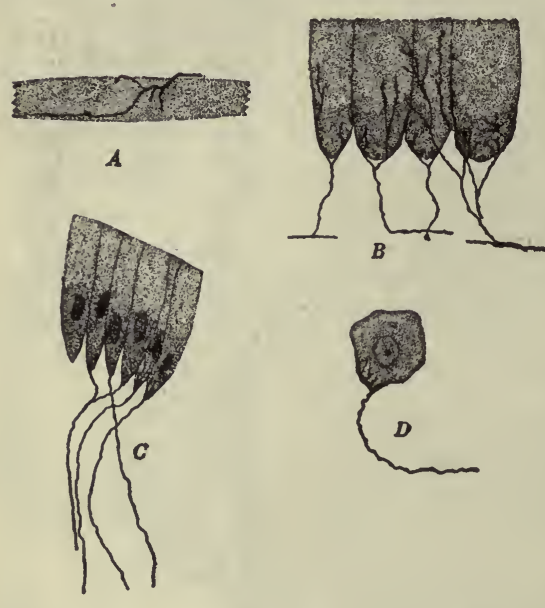

Fig. 338.-Termination of Sympathetic Nerve Fibers.

$A$, on smooth muscle cell; $B$, on cells of the digestive epithelium, ileum of cat; $C$, on cells of the digestive epithelium, stomach of cat; $D$, on parietal cell, stomach of cat. (Kuntz, Jour. Comp. Neur., $23,3,1913$. cular coat on their way to lymph nodes which are situated in the folds of the omentum at either curvature of the stomach.

The Nerves.-The nerves of the stomach are derived from sympathetic trunks, the splanchnic nerves, and from the vagi. The vagi are believed to be chiefly excitatory, the splanchnics inhibitory in function. The nerves enter with the blood-vessels and pierce the muscular coat. They connect with two plexuses of anastomosing nerve trunks: the myenteric (Auerbach's), in the intramuscular fibrous septum, which contains ganglionic enlargements at many of its intersections and distributes its fibrils to the smooth muscle; the submucous (Meissner's), lying in the deeper part of the submucosa, which also contains small ganglia at the intersections of its anastomosing branches. This latter plexus is much finer and contains smaller neurons than that of the muscular coat. Pericellular capsules are apparently lacking in these plexuses (Müller, Kuntz). The submucous plexus distributes its fibrils to the mucosa, where they terminate in and about the walls of the blood and lymphatic vessels, and to the epithelium of the secreting glands, where they end as varicose fibrils upon the cells. Kuntz (Jour. Comp. Neur., 23, 3, 1913) suggests that the ganglia of the myenteric and submucous plexus include both motor and sensory neurons, and that the fibers which terminate on cells of the digestive epithelium are the dendrons of sensory cells. 


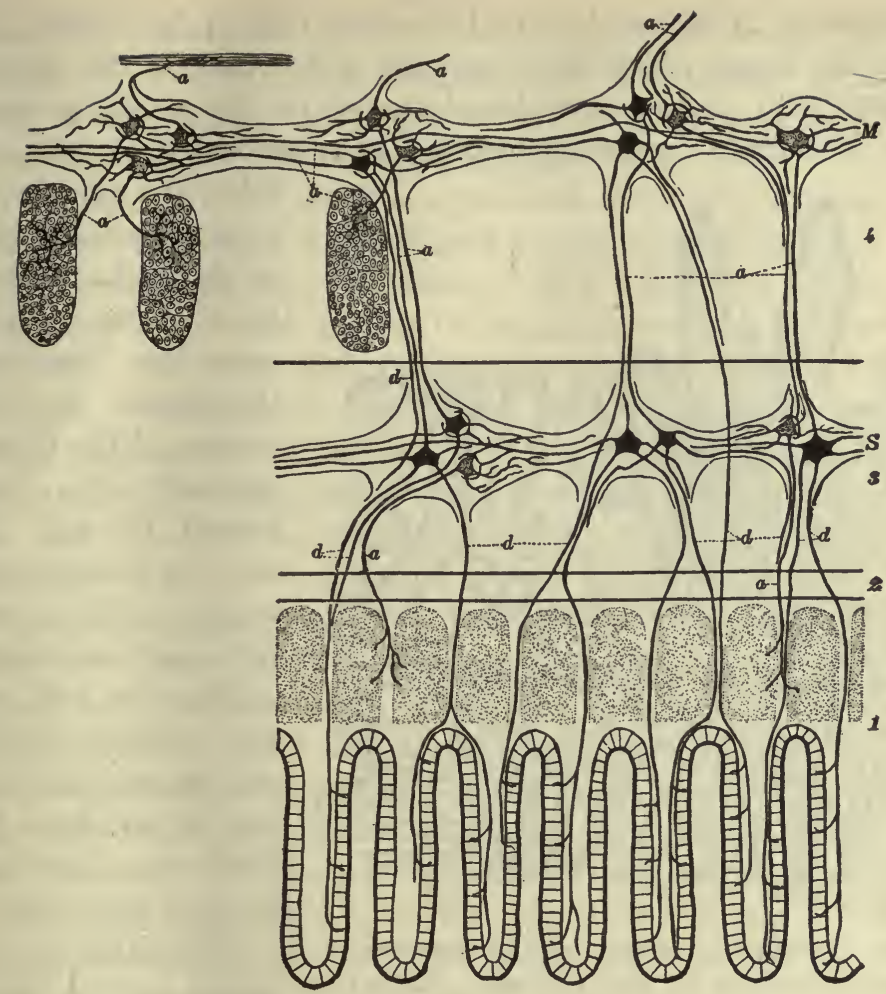

Fig. 339.-Schematic Diagram Illustrating Probable Relationship of Sympathetic Neurons in Myenteric and Submucous Plexuses.

Motor neurons, stippled; sensory neurons, solid. 1, tunica propria; 2 , muscularis mucosæ; 3 , submucosa; 4 , muscularis; $M$, myenteric plexus; $S$, submucous plexus; $a$, axons; $d$, dendrons. (Kuntz, Jour. Comp. Neur., 23, 3, 1913.)

\section{Small Intestine}

The small intestine constitutes the longest portion of the digestive tube. It connects the pylorus with the colon. It measures about $71 / 2$ meters (24 feet) in length. It may be divided into three segments: (1) the duodenum, about 11 inches in length; (2) the jejunum, including the upper two-fifth, about 9 feet, and (3) the ileum, including the lower three-fifth, about 14 feet, of the remainder. The duodenum lacks a mesentery, it is only partially enveloped by a serosa, and it has the greater diameter, about 47 millimeters (2 inches). Below the duodenum the caliber of the small intestine gradually decreases until 
a diameter of 27 millimeters (a little over $1 \mathrm{inch}$ ) is attained, at the end of the ileum. The three portions differ also in the shape and number of villi, and in other histologic details which will be described below. The inner surface is modeled by a succession of tall circular.

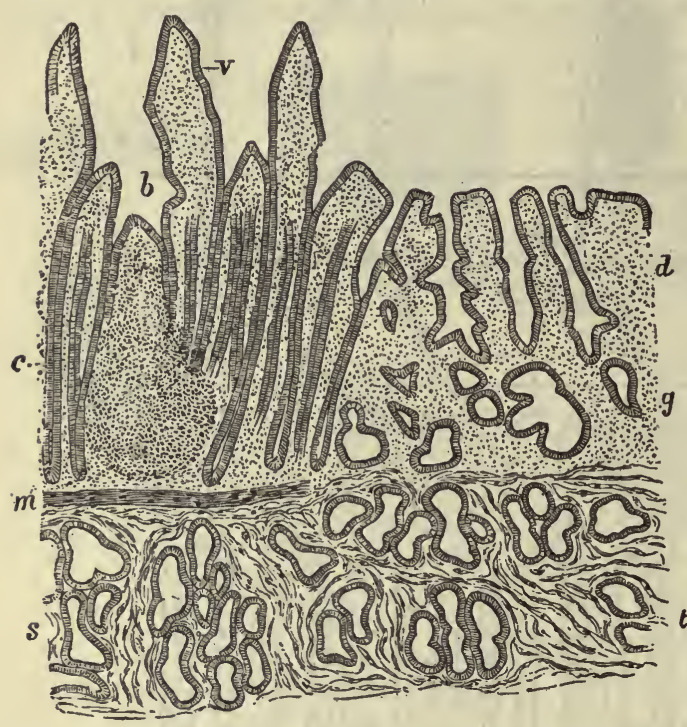

Fig. 340.-Section through the Commencement of the Duodenum at the Pylorus. (Klein.)

$v$, villi; $b$, apex of a lymphoid nodule; $c$, crypts of Lieberkühn; $m$, muscularis mucosæ; $s$, secreting tubes of Brunner's glands; $d$, ducts of pyloric glands of stomach; $g$, tubes of these glands cut across in mucous membrane; $t$, deep-lying tubes situated in submucous tissue, and corresponding with Brunner's glands of the intestine. (From Quain's "Anatomy.") folds, the valvula conniventes or plica circulares, involving the submucous layer. These plicæ become less closely-spaced throughout the lower portion of the ileum and generally disappear toward its end. They serve to increase the absorbent surface of the intestinal mucosa, and unlike the-villi, which are scattered over their surface, they are not subject to variations dependent upon altering degrees of distention.

The structure of the serous coat of the small intestine is identical with that of the stomach. The muscular coat consists of an inner and an outer layer of unstriped muscle fibers which are separated by a thin connective tissue septum. The inner circular layer is much thicker than the outer longitudinal.

The regular disposition of the muscle fibers as an outer longitudinal and an inner circular layer serves as a guide to the recognition of the direction in which a given microscopical section has been cut. In transections of the intestine the muscle fibers of the outer layer of the muscular coat are transversely cut; in longitudinal sections of the organ the same fibers are seen in longitudinal section.

The Submucosa.-The submucosa of areolar connective tissue is 
only a very indistinct cuticular border and such border is entirely lacking in the fundus cells of the glands. The epithelium of the glands appears to take no part in the process of absorption and therefore contains no fat globules. It secretes a mucous fluid.

At the neck of the gland the epithelium frequently contains mitotic figures which have been demonstrated in man (Schaffer, 1897) as well as in the lower mammals (Bizzozero, 188\%). Little or no mitosis has been demonstrated in the fundus of the gland or upon the free surface of the villi. On these facts the so-called wander theory of Bizzozero is founded. According to this theory there exist in the neck of the glands certain indifferent cells which are capable of reproduction by mitosis and whose daughter-cells move toward the free surface, being at the same time differentiated into either the goblet or the columnar cells of the villi.

Bizzozero originally considered that the granule cells of Paneth at the fundus of the glands were intermediate phases-in the formation of goblet cells, but as there is little or no mitosis in the region where these peculiar cells occur and as the granule cells are never displaced toward the surface, it seems more probable that, as also in the gastric glands, the indifferent genetic cells of the neck of the tubule develop on the one hand the superficial goblet and columnar cells which clothe the villi, and on the other hand, the true secreting cells in the fundus of the intestinal glands.

The granule cells of Paneth (Arch. f. mikr. Anat., 1888) are confined to the extreme tip or blind extremity of the fundus of the glands. They are pyramidal or low columnar cells whose spheroidal nuclei are situated close to the basement membrane. Their cytoplasm presents a delicate reticulum which is filled with coarse granules which in some cells are of a basophil nature (Klein, Amer. Jour. Anat., 1906). In others they contain still coarser granules which are strongly eosinophil. The exact function of these peculiar cells is unknown, but that they are true secreting cells seems highly probable.

Still other types of granular cells of unknown significance have recently been described by Kull (Arch. mikr. Anat., 81, 3, 1913) in the fundus of the intestinal glands, and among the epithelial cells clothing the villi: (1) 'acidophil cells,' with the basal oxyphilic granules finer than those of the Paneth cells; and (2) 'chromaffin cells' with yellowish basal granules, coarser than those of the acidophil cells and finer than those of the cells of Paneth. These three types of cells are present in man and certain vertebrates; they are said to have no genetic relation- 
ship. The 'chromaffin cells' of the intestinal epithelium were first recognized by Schmidt, who designated them as 'yellow cells' (Arch. mikr. Anat., Bd. 66, 1905). Ciacco (Arch. Anat. e Embri., 6, 3, 1907) reports similar cells also in the duodenal (Brunner's) glands. Champy

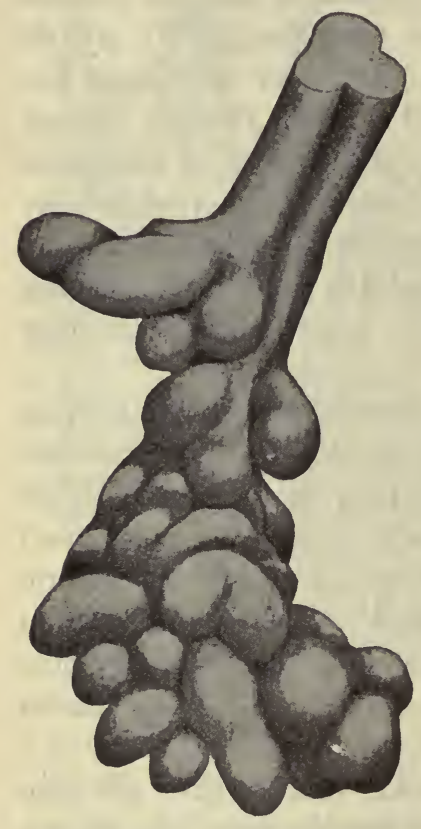

Fig. 346. - Reconstruction Model of a BRunNer's Gland, From the Human Duodenum.

Three partially blended ducts pass into the submucosa and end in expanded alveoli. $\times 344$. (After Maziarski.) (Compt. rend. Soc. Biol., T. 66, 1909) has described also mitochondria in the cells of the intestinal glands.

The intestinal glands are confined to the narrow deeper zone of the intestinal mucous membrane. Their lumen, after fixation, contains only the coarsely reticular mucous secretion.

The student should be warned to distinguish carefully between the transverse sections of the tubular glands which are confined to the deep zone of the mucous membrane and the similar sections of the villi which are only found in the superficial zone and whose epithelial coat, instead of inclosing a mere reticular mass of mucous secretion invests an organized body of diffuse lymphoid tissue.

'The Duodenal (Brunner's) Glands. - The duodenal glands of Brunner are tubulo-acinar glands which furnish a muco-albuminous secretion. They appear to represent the continuation into the intestine of the pyloric glands of the stomach, and they occur in decreasing proportion throughout the entire length of the duodenum; around the duodenal papillæ, however, they become locally more numerous. They are sharply distinguished from the pyloric glands by their larger size. Moreover, the secreting portion of the duodenal glands is only found in the submucosa and the deeper part of the mucous membrane, where the secreting acini form very numerous groups, the tubules of each of which are connected with the terminal subdivision of a duct.

The ducts of the duodenal glands open on the free surface between the villi by means of crypt-like tubules which are lined by tall columnar 
epithelium and can only with difficulty be distinguished from the adjacent intestinal glands. In the deeper part of the mucous membrane the $\mathrm{ducts}$ branch and pursue a somewhat tortuous course to the fundus of the gland, where the terminal acini of each subdivision of a duct are invested with a distinct fibrous capsule.

The secreting epithelium of the duodenal glands consists of tall columnar cells which surround a wide lumen. When loaded with secretion the cells are swollen and clear, but become shrunken and granular after a period of activity. Their cytoplasm reacts to the specific stains for mucin only when these are applied for a considerable time in concentrated solution (Bensley). The spheroidal nucleus is situated at the proximal or basal end, and as the cell fills with secretion the nucleus becomes progressively flattened.

Blood Supply.-The blood supply of the small intestine resembles that of the stomach. The branches of the mesenteric arteries pass around the intestinal wall in the subserous connective tissue. From this point they penetrate the muscular coat to form intramuscular and submucous plexuses. From the latter a few branches sup-

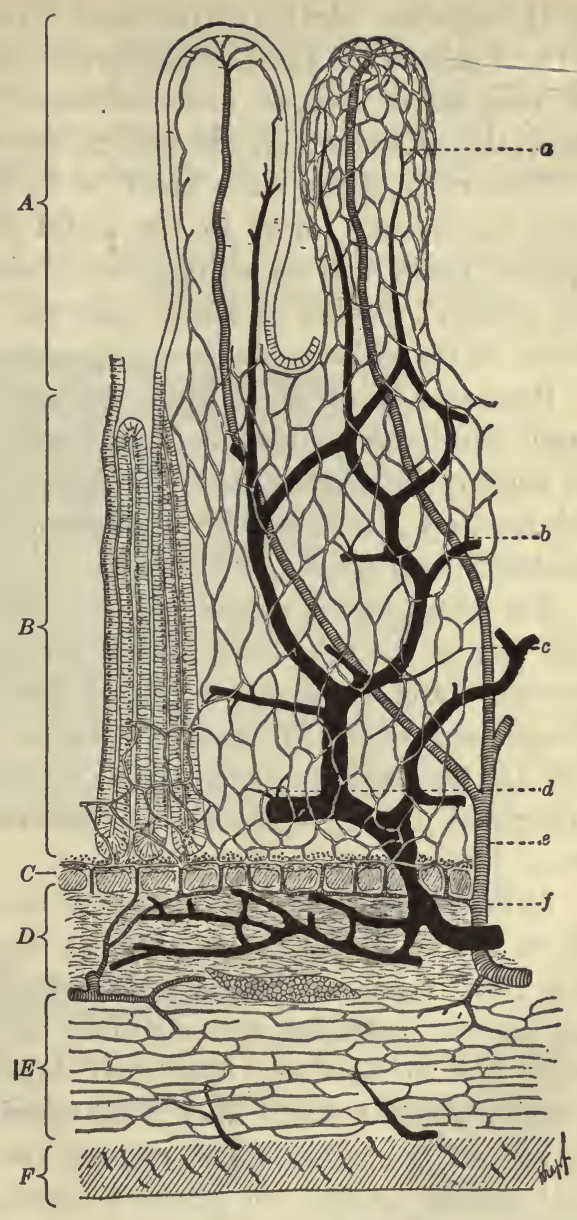

Fig. 347.-The Blood-vessels of The Small Intestine of a Dog, Drawn after an Injected Preparation.

The arteries are striped, the veins black, the capillaries open. $A$, villi; $B$, glands; $C$, muscularis mucosæ; $D$, submucosa; $E$, circular, and $F$, longitudinal layer of the muscular coat; $a$, venule beginning from the capillaries of the villus, and at $b$, from those among the glands; $c$, artery to the villus; $d$, venules in the deeper part of the mucosa; $e$, main arterial trunk to several adjacent villi; $f$, arterial branch to the glandular region. Highly magnified. (After Mall, from Oppel.) 
ply the adjacent portion of the inner layer of the muscular coat, but most of them pass to the mucous membrane, in which a plexus lies just within the muscularis mucosæ and distributes its branches to the capillaries about the intestinal glands and to the intestinal villi.

The artery of the villus enters at its base, and distributing capillaries along its course, forms in the distal part of the villus an abundant capillary network from which efferent venules return by a similar course. The artery, however, is found near the axis, the venules near the periphery of the villus.

Branches from the submucous and mucous arterial plexuses also supply capillaries to the duodenal glands in the duodenum as well as to the solitary and agminated lymph nodules. About each of the lymph nodules they form circular anastomoses, from which radial capillaries are distributed within the nodule.

The veins pursue a course exactly similar to that of the arteries. On their way to the mesenteric vessels they form mucous, submucous, intramuscular, and subserous plexuses, and drain into the portal system. The portal vein and its main tributaries lack valves. Valves are present only in the smaller tributaries, beginning in the tunica muscularis throughout the digestive tube and prevailing generally in the mesenteric veins.

Lymphatics.-The lymphatics or lacteals of the small intestine begin in the distal part of the villi as lymphatic capillaries, each having, as a rule, a pouched, blind extremity. During the digestion of fats they become distended with a whitish fatty lymph called chyle. At their origin the lacteals are frequently branched, or they may even form a scanty anastomosis. They finally unite to form a central lacteal in the axis of the villus, which empties into a rich plexus about the intestinal glands, or like the efferent vessels of this plexus, they may pass directly to the larger lymphatic vessels of the submucosa.

From the submucous plexus numerous efferent lymphatic vessels penetrate the muscular coat, receiving the lymph from the vessels of the intramuscular septum. They empty into the larger lacteal vessels of the mesentery which are intimately connected with numerous mesenteric lymph nodes. In the mucosa and submucosa the lacteals form sinuses which surround the bases of the solitary and agminated nodules. Thus, much of the chyle is permitted to come into relation with the parenchyma of these organs before leaving the intestinal mucosa.

Nerve Supply.-The nerve supply of the intestine is exactly similar to that of the stomach. The non-medullated fibers form an intra- 
muscular myenteric ganglionic plexus (Auerbach's) for the supply of the muscular coat, and a submucous plexus (Meissner's) which supplies branches to the blood-vessels and to the glands of the mucosa. The finer branches in the mucous membrane penetrate to the villi, forming a delicate plexus of naked fibrils about its blood-vessels and lacteals, and upon its epithelium.

Intestinal Absorption.-The absorption of fat consists essentially of three phases: (1) its absorption into the intestinal epithelium; (2)

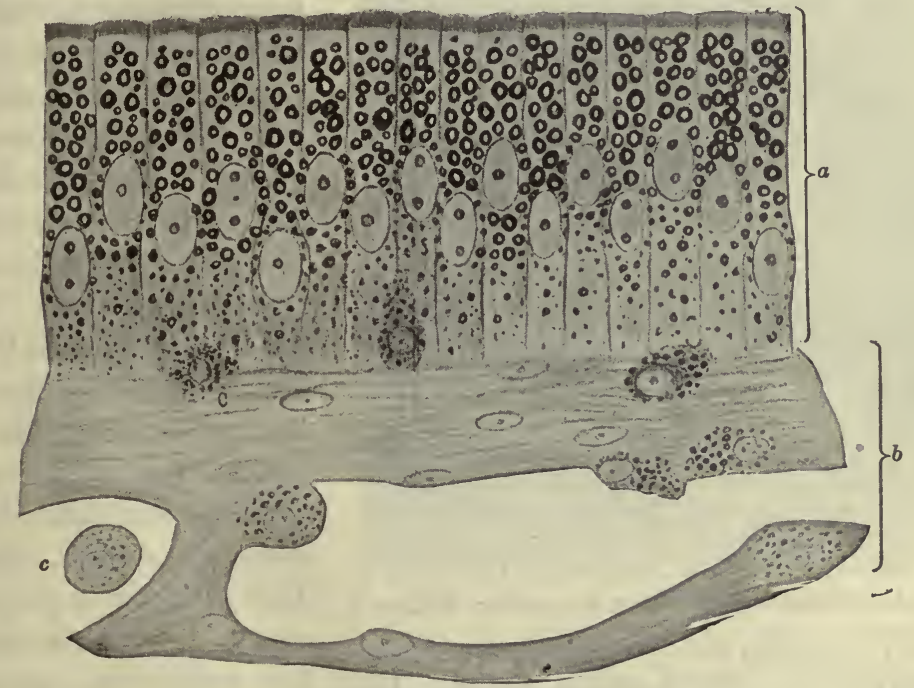

Fig. 348.-Intestinal Mucosa of a Frog During the Absorption of Fat.

$a$, epithelium; $b$, tunica propria; $c$, an ameboid leukocyte. Osmium tetroxid. Highly magnified. (After Schäfer.)

its secretion into the lymphoid tissue of the villus; and (3) its entrance into the lacteal vessels. In an animal killed during the absorption of fat, the intestinal villi, after fixation by solutions of osmium tetroxid, contain fat in (a) the epithelium, (b) the lymphoid tissue, and (c) the central lacteal.

In the epithelium, fat is contained in the form of fine droplets which are most numerous in the distal or free ends of the cells. 'They are also found in the intercellular spaces. During absorption the epithelial cells of the villi become much swollen and elongated. As the process subsides they return to their former size, and become relatively shrunken. When most distended the intracellular fat droplets are the 
most abundant; as the cells shrink the intercellular droplets increase relatively in number (Drago, Richerche d. lab. ant. norm. d. r. univ. d. Roma, 1900). The relative size of the epithelial cells and the abundance of intra-epithelial fat is apparently dependent upon the activity of the processes of absorption.

As to the manner in which the fat enters the epithelium there is some doubt. Schäfer (Internat. Monatsch. f. Anat. u. Physiol., 1885) suggested that the leukocytes by their ameboid activity inclose the

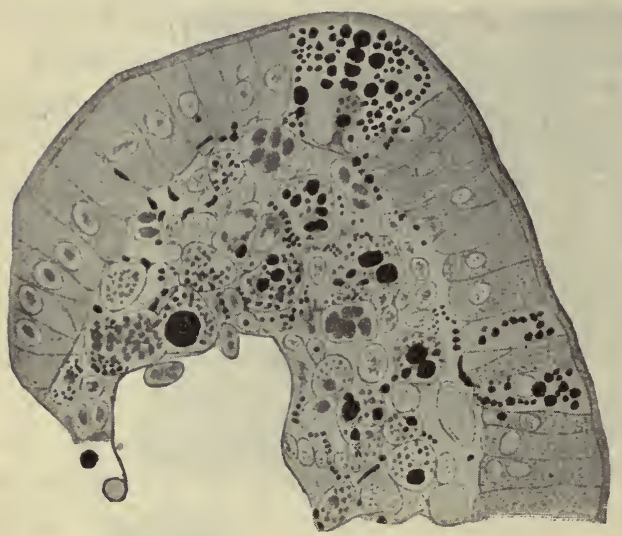

Fig. 349.-Apex of an Intestinal Villus of a Rabbit Which Had Been Fed with Milk.

The fat droplets have been blackened by fixation with picric acid and osmium tetroxid. The figure shows the distribution of fat during certain stages of absorption. .Alum carmin stain. Highly magnified. (After R. Heidenhain, from Oppel.) emulsified droplets in the intestinal lumen and convey them into the substance of the villi. It seems more probable that the fats are saponified in the intestinal tract, and enter the epithelium in solution (Whitehead, Amer. Jour. Physiol., 24, 2, 1909). Here they are again synthesized into neutral fat by the activity of the epithelium (Pflüger, Arch. f. d. ges. Physiol., 1900). Such a process accounts for the abundance of fat within the distal portions of the cells. The droplets are then secreted into the intercellular and subjacent tissue spaces.

The second phase of absorption includes the transference of the fat particles to the lacteal. This process appears to depend partially, at least, upon the activity of the leukocytes, as suggested by Schäfer, the particles of fat thus finding their way through the diffuse lymphoid tissue. According to Reuter (Anat. Hefte, 1902); fat droplets are found in the tissue spaces as well as in the lymph corpuscles of the diffuse lymphoid tissue, a fact which would seem to indicate that other agencies aid in the transit of the fat from the epithelium to the lacteal than are accounted for by the purely mechanical theory of Schäfer.

The third phase includes the secretion of the fat into the lumen of the lacteal; this is, at least partially, accomplished by the disintegra- 
rectum and in the vermiform appendix. In the latter the nodules are more or less confluent, a condition which is not found elsewhere in the large intestine. In the appendix the greater portion of the mucous membrane is invaded by lymphoid tissue, and the glands are much diminished in both number and size (Fig. 352).

The Vascular and Nerve Stuply,-The vascular and nerve supply of the large intestine is identical in its arrangement with that of the small intestine. The mucous membrane contains a capillary plexus of blood and lymphatic vessels in the corium a bout the glands. The nerves of the large intestine include both medullated and non-medullated fibers. The latter supply its muscular coats and blood-vessels. The former end in naked varicose or knobbed fibrils beneath and upon the epithelium of the glands. The us ual myenteric (Auerbach's) and submucous (Meissner's) plexuses ap-

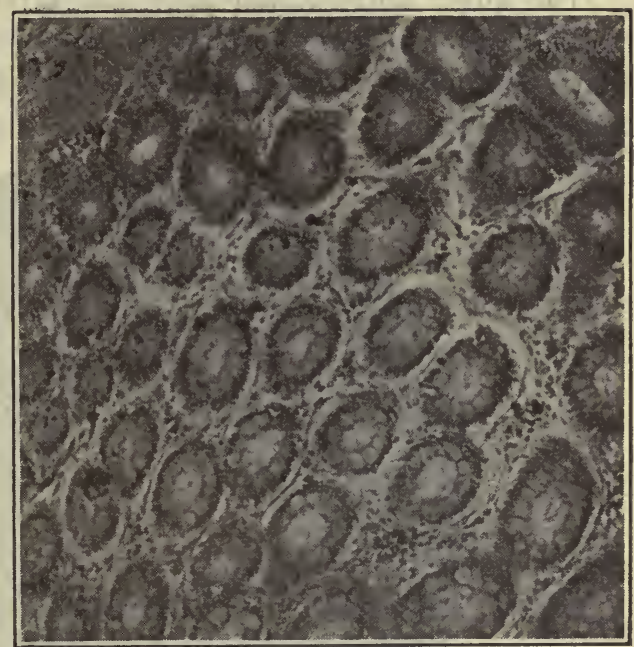

Fig. 351.-Section of Portion of Large Intestine of Dog, Showing the Intestinal Glands (Crypts of Lieberkühn) CUT Across, Their Lining Including Columnar and Goblet Cells. pear in the large intestine with the same structure and location as elsewhere in the alimentary canal.

In the rectum the lining epithelium is continuous at the anus with the stratified squamous epithelium of the skin. In this region also, the circular fibers of the inner layer of the muscular coat are much thickened to form the internal rectal sphincter. Lymphoid tissue abounds in the rectal mucous membrane. The glands are less numerous but larger than in the colon proper, and the mucosa is thicker. In the lower portion of the rectum the mucosa is thrown into a number of ${ }^{\circ}$ longitudinal folds, the rectal columns, at which level the columnar epithelium changes to stratified squamous type.

The Ileocecal (Colic) Valve.-The ileocecal valve, which guards the orifice by which the small intestine opens into the large, is formed 
by a reduplication of the mucous membrane, which is strengthened by a thickening and overlapping of the circular muscular layers of both small and large intestines. The valve itself consists of an upper and lower segment enfolding the slit-like orifice; laterally the two folds unite to form the frenulum, which encircles the colon and marks the boundary between it and the cecum.

The outer longitudinal muscular layer is continued directly from the wall of the ileum to that of the cecum, and therefore pursues a relatively shorter course than either the internal muscular layer or the mucous membrane. Section of only the outer layer of the muscular coat

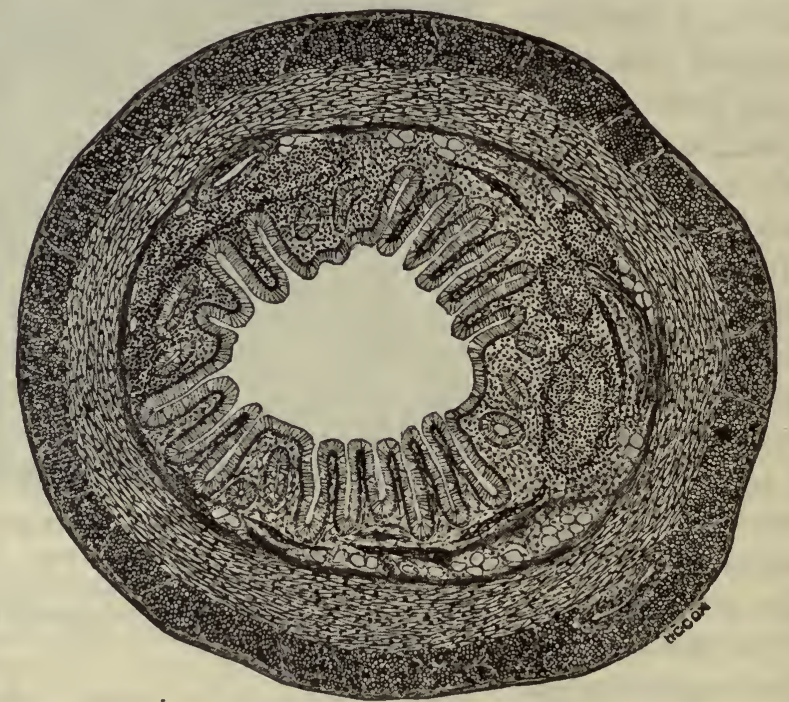

Frg. 352.-Transection of the Vermiform Appendix of Man.

The tela submucosa contains three lymph nodules (at the right), two of which have pushed beyond the scattered bundles of the lamina muscularis mucosæ into the lamina propria of the tunica mucosa, a mass of diffuse lymphoid tissue (at the left), and some adipose tissue. $\times 11$.

permits one to straighten the fold of the intestinal wall and thus obliterate the valve. In other words, the outer muscular layer is not included in the valvular reduplication.

The muscularis mucosce is slightly thickened at the margin of the valve. At this point also, the villi become shorter and at the margin of the cecal surface of the valve they entirely disappear.

The following tabular statement of the more important characteristics of the several portions of the alimentary tract may be of assistance to the student in the identification of microscopic sections of these organs. 


\begin{tabular}{|c|c|c|c|c|c|c|c|}
\hline 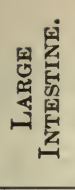 & 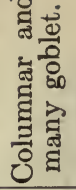 & 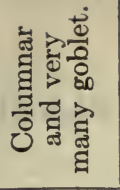 & 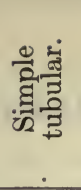 & 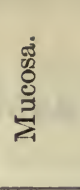 & 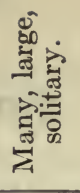 & 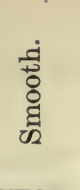 & 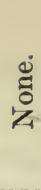 \\
\hline 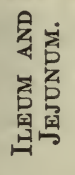 & 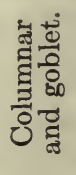 & 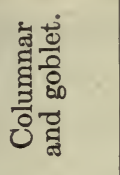 & 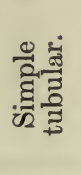 & 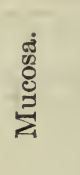 & 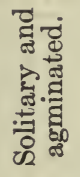 & 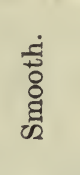 & 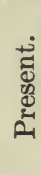 \\
\hline 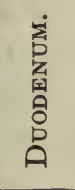 & 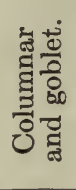 & 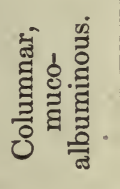 & 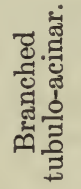 & 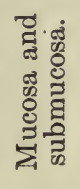 & 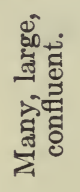 & 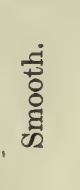 & 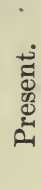 \\
\hline 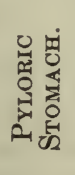 & 范 & 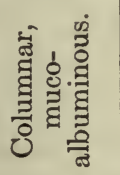 & 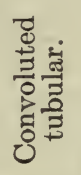 & 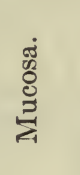 & $\begin{array}{l}\text { न]ं } \\
\text { हैं } \\
\text { है } \\
\text { हैं. }\end{array}$ & $\begin{array}{l}\text { क्ते } \\
\text { : } \\
\text { की }\end{array}$ & $\begin{array}{l}\text { ¿ } \\
\text { ż }\end{array}$ \\
\hline 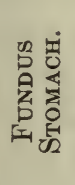 & 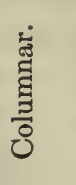 & 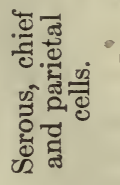 & 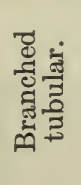 & 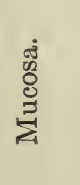 & 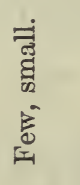 & 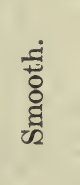 & $\begin{array}{l}\dot{g} \\
\text { Z } \\
z\end{array}$ \\
\hline 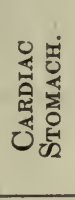 & 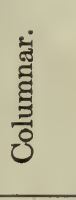 & 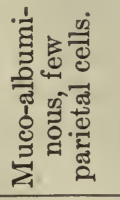 & 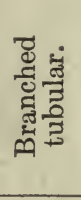 & 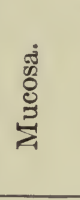 & 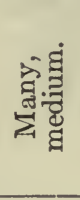 & $\begin{array}{l}\text { क्षं } \\
\text { ठ․ } \\
\text { \& }\end{array}$ & $\begin{array}{l}\text { ¿ } \\
\text { z } \\
\text { z }\end{array}$ \\
\hline 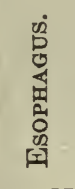 & 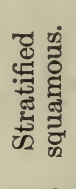 & 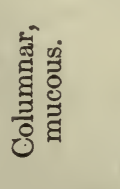 & 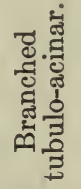 & 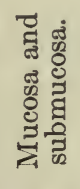 & 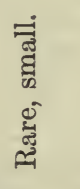 & 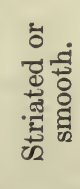 & $\begin{array}{l}\dot{0} \\
\text { ¿ } \\
\text { 乙 }\end{array}$ \\
\hline & 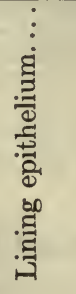 & 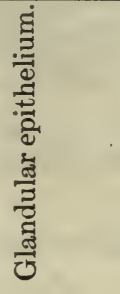 & है & 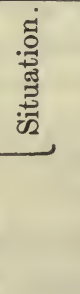 & 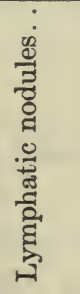 & 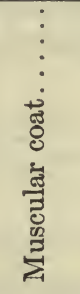 & $\stackrel{3}{3}$ \\
\hline
\end{tabular}




\section{THE SALIVARY GLANDS}

General Considerations.-The salivary or oral glands include the smaller glands of the oral cavity and three pairs of large compound tubulo-acinar glands, the parotid, submaxillary, and sublingual glands.

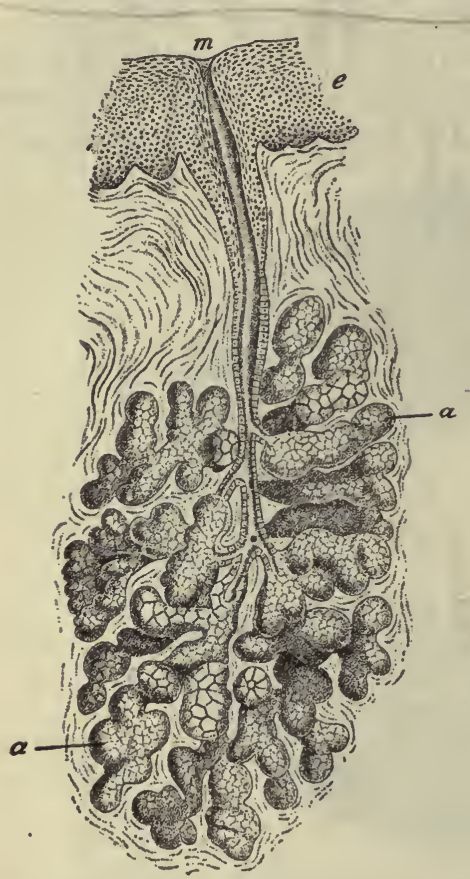

Fig. 353.-Semidiagrammatic RepRESEntation of a SMall Mucous Gland from the Oral Mucosa of a RaBBit.

$a$, mucous alveoli; $e$, epithelium of the oral mucosa; $m$, mouth of the glandular duct. $\times 70$. (After Kölliker.) The three large pairs are commonly designated as the salivary glands proper, the smaller as the accessory salivary glands. All these are of the tubulo-acinar type, but certain ones secrete a mucous fluid while others produce an albuminous secretion which contains no mucus. The former are known collectively as the mucous, the latter as the serous salivary glands. Still other salivary glands secrete-a fluid which is intermediate in composition, and as these glands contain certain alveoli which resemble those of the mucous, and others which are similar to those of the serous glands, this type is known as mixed salivary glands.

The salivary glands may therefore be subdivided into:

I. Mucous glands; glands on the anterior surface of the hard and soft palate (palatine glands) and the mucous glands of the margins and root of the tongue.

II. Nixed glands; submaxillary, sublingual, molar, buccal, labial and the anterior lingual glands (of Nuhn).

III. Serous glands; parotid, and von Ebner's glands at the base of the tongue.

The form of the salivary glands will be appreciated by examination of the accompanying diagram (Fig. 353) which represents one of the smaller glands of this type. The larger ones are constructed in the 
same manner, the larger number of their secreting alveoli or acini arising through a more complex duct system.

The larger ducts of the gland are lined by columnar cells, which,

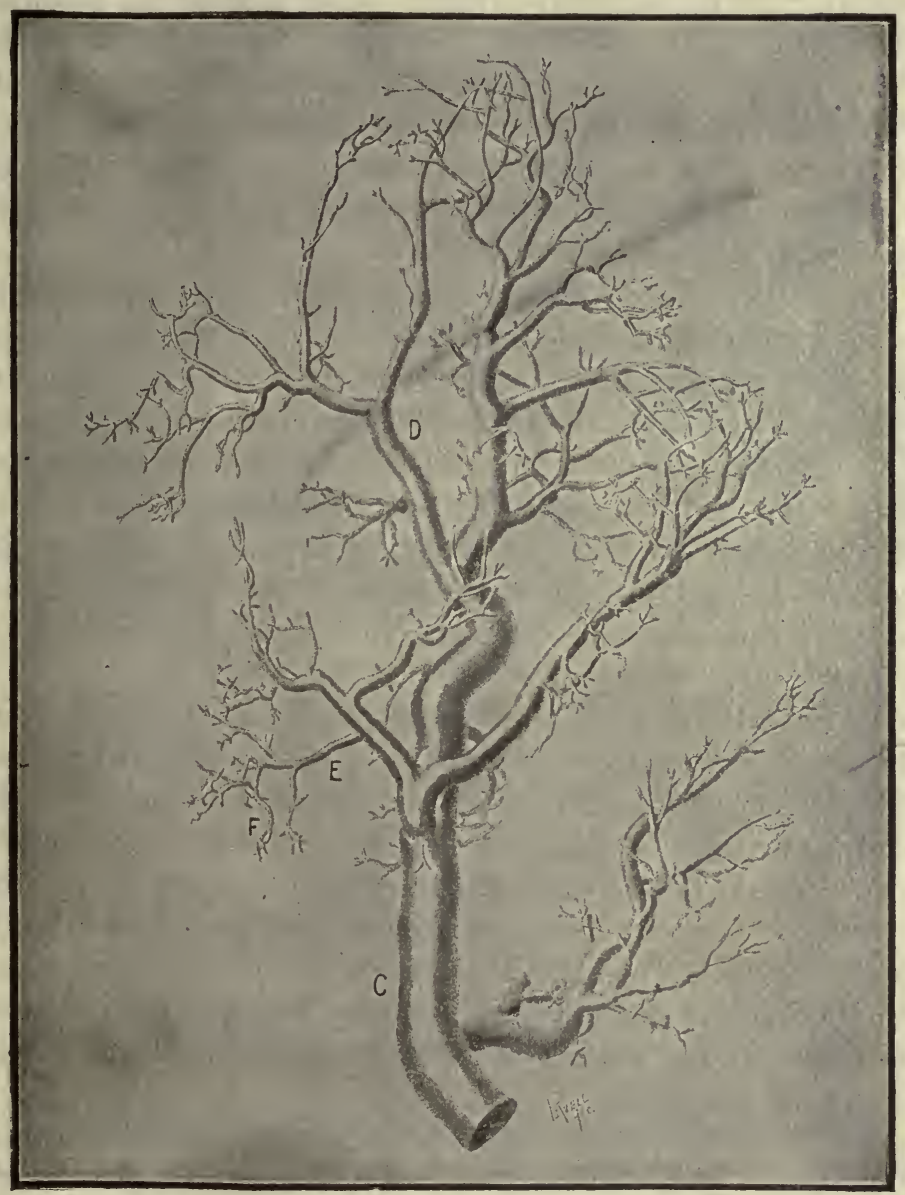

Fig. 354.-Corrosion Model of an Interlobular Duct and Its Branches, from the Human Submaxillary Gland.

$C$, interlobular duct; $D$, large intralobular duct; $E$, small intralobular duct; $F$, intercalary duct. $\times 12$. (After Flint.)

as they approach their termination, become superposed and thus offer a gradual transition to the stratified squamous epithelium upon whose surface they open. The epithelium rests upon a basement membrane 
which, in the larger ducts, is invested with a fibro-elastic coat containing a few longitudinal smooth muscle fibers.

The ducts divide and subdivide in an arborescent manner, the larger branches lying in the connective tissue which invests the lobules into which the gland is subdivided, while the smaller branches are found within the lobule. The duct system is thus divisible into interlobular and intralobular ducts; the latter include generally an excretory portion continuous with the interlobular duct, a modified 'salivary' secretory portion, and a constricted intercalary or intermediate portion connecting with the acinus.

In the smaller glands of the mouth the number of subdivisions of the duct system is relatively small, but in the larger salitary glands the small ducts are practically innumerable. Thus, in the submaxillary gland, Flint (Amer. Jour. of Anat., 1902) found that the interlobular, duct system formed 1,500 terminal branches, each of which entered a lobule and was further subdivided into intralobular and intercalary ducts before terminating in the secreting acini. The larger glands may therefore be said to bear to the smaller ones represented in Fig. 353 , a relation which is comparable with that of a full-grown tree to the youngest sapling.

The larger salivary glands are enveloped by ô fibro-elastic capsule continuous with the adjacent areolar tissue. From this capsule coarse trabeculæ enter to divide the gland into groups of lobules, the lobes. The lobules are invested by more delicate septa from the interlobar trabeculæ. The ducts of the interlobar connective tissue may be designated interlobar ducts to distinguish them from the interlobular ducts between the lobules. These are the excretory ducts of the system; the 'salivary' intralobular ducts have a secretory function. The glandular tissue is known as the parenchyma, the connective tissue as the interstitial tissue of the gland.

The smaller intralobular ('salivary') ducts are lined by columnar epithelium whose cells contain two zones, one on either side of the centrally situated nucleus. The distal zone or free extremity of the cell is finely granular, the proximal zone or base presents a characteristic striated appearance which is apparently due to a fibrillar structure of the cytoplasm in this portion of the cell. The basal fibrillæ are probably, in part at least, the mitochondria which are present in all functionally active cells. The epithelium is easily detached from its basement membrane by the artificial contraction of the tissues during fixation and hardening. 
The lumen of the ducts is of considerable diameter and contains the reticulated or granular particles of the secretion. The larger ducts lie in the connective tissue septa which invest the lobular groups of acini. Each of these groups is derived from the ramifications of the terminal branch of an interlobular duct which enters the lobule to divide into numerous intralobular ducts, and secondarily, through a short intermediate or intercalary portion, into the secreting alveoli or acini. The intercalary ducts are lined by low cuboidal epithelium and are the smallest tubules of the gland. As the duct passes into 'the acinus the tubule is increased in size, and its secretory epithelium becomes taller. The tubular acinus is more or less tortuous and possesses a sacculated or alveolar appearance.

The epithelium differs accordingly as it secretes a mucous or a serous fluid. Thus the acini are either mucous or serous secreting.

The Serous Acini.-The serous acini contain pyramidal epithelial cells of sufficient height to almost completely fill the tubule; hence the lumen is very narrow. The form of the secreting cells is somewhat irregulara fact which apparently depends upon their crowded condition within the acinus. The

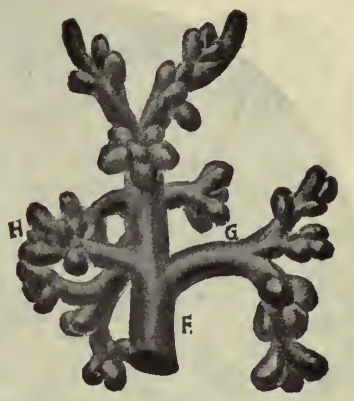

FIG. 355.-I N TER C A L A R Y DUCTS AND ACINI OF The Human SUbmaxilLARY GLaND, Corrosion MODEL.

$F$, small intralobular duct, $G$, intercalary duct; $H$; , acini. $\mathrm{H}$ igh ly magnified (After Flint.) nucleus is situated in the central portion or in the proximal end of the cell, and is spheroidal in shape. The cytoplasm is finely granular, the granules being more prominent in the distal portion of the cell.

The epithelium rests upon a basement membrane within which, beneath the bases of the secrefing epithelial cells, are certain flattened 'basket-cells' which here and there send short processes between the cells of the secretory epithelium and thus provide cup-like depressions which receive the bases of the secreting cells. The function and origin of these 'basket-cells' is not at present known. They are readily recognized by their deeply stained and flattened nuclei which are contained within the thin cytoplasmic cell body. They may be immature acinal cells, destined to replace worn-out secretory celis.

The appearance of the secreting epithelium varies with its ac- 
tivity. During rest the granular secretion accumulates within the cell, until the non-granular zone is reduced to a narrow rim at its basal extremity and the nucleus is obschred and pushed somewhat basalward. The cell becomes therefore much swollen and the alveolar lumen almost obliterated.' During activity the zymogen granules are discharged into the lumen; the cell shrinks and becomes clearer; the nucleus appears more distinct, and the granular zone becomes progressively narrower,

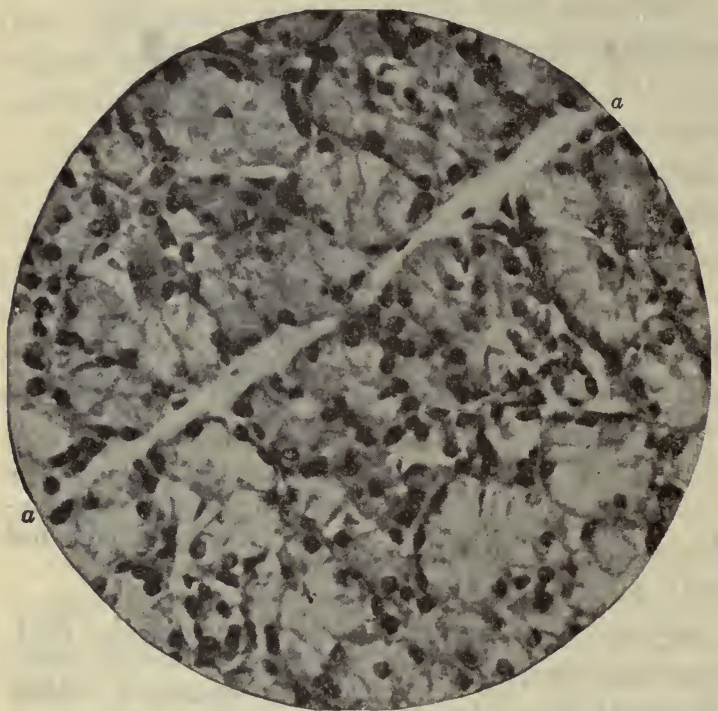

Fig. 356.-A Group of Mucous Acini, from the Human Submaxillary Gland.

$a-a$, interlobular connective tissue. Hematein and eosin. Photo. $\times 510$. the basal non-granular zone being correspondingly increased in breadth. In this basal zone elongated granules have been demonstrated, which in part are to be regarded as prozymogen ('b a s a l filaments' of Solger), in part (revealed by s pecia l technic) as mitochondria.

The serous cells are provided with systems of secretory canaliculi which, beginning at the glandular lumèn, invest the cell with a network of canals which lie in the intercellular substance

and may even send short offshoots or intracellular canaliculi into the body of the cell itself. These canaliculi are considered to be characteristic of the serous acini and are not found in relation with the cells of the mucous acini (Fig. 358).

The Mucous Acini.-The mucous acini may contain only mucussecreting epithelium, or they may also include certain finely granular acidophil cells which resemble the epithelium of the serous glands. The former variety of acinus is found in the mucous glands at the base of the tongue and in the soft palate; the latter in the sublingual gland, in the lingual glands of Nuhn, and in the mucous glands of the lips and cheeks. 
The serous appearing cells of the latter form of mucous acinus are frequently arranged as crescentic groups-bordering upon the adjacent mucous cells. Such groups are known as the demilunes (of Heidenhain) or crescents (of Gianuzzi; Fig. 35\%). They occur at the periphery of the acinus, their base being applied to the membrana propria, their inner margin sometimes reaching the glandular lumen, but more frequently separated therefrom by the overlapping of the adjacent mu-

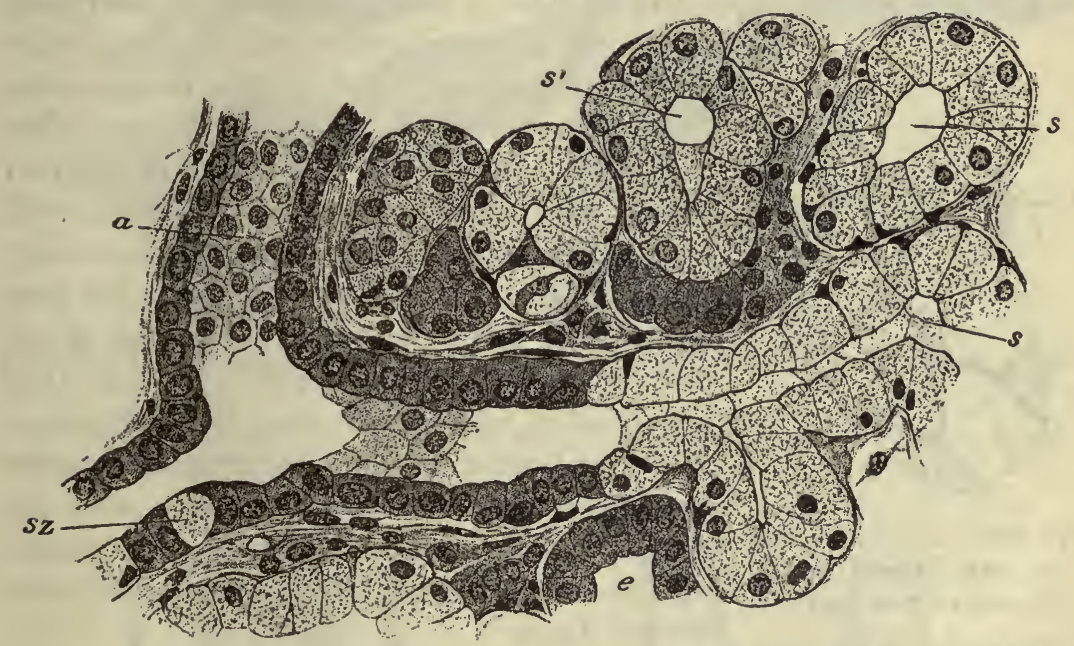

Fig. 357.-From the Sublingual Gland of Man.

$a$, salivary intralobular duct; $e$, acinus whose cells contain no mucus; $s$, mucous acini, at $s^{\prime}$ with a demilune; sz, mucous cells in the duct. $\times 500$. (After Kölliker.)

cous cells. The demilunes are frequently found at the blind extremity of the secreting acinus, but they may also occur along its sides.

The nature of the demilunes is the subject of considerable discussion. Heidenhain (Arch. mikr. Anat., 1869) first advanced the theory that the mucous cells were destroyed during secretion, and that the function of the demilunes was therefore to replace the disintegrated mucinous cells. This theory has been practically abandoned, for no one has yet demonstrated active cell division in the demilunes, a process which would necessarily be concomitant with the rapid development of mucus from demilune cells.

Hebold (1879) is responsible for the theory, strongly supported by 
Stöhr, that the demilunes represent an inactive, the mucous cells, an active phase of mucus secretion. The easy demonstration of intermediate stages in many mucus-secreting glands lends strong support to this theory, and in the present state of our knowledge it seems beyond doubt that such a process actually occurs in at least some of the mucussecreting glands.

A third theory, more recently advanced by Solger (Anat. Anz., 1894) and stoutly supported by Krause (Arch. mikr. Anat., 1895, 1897, and

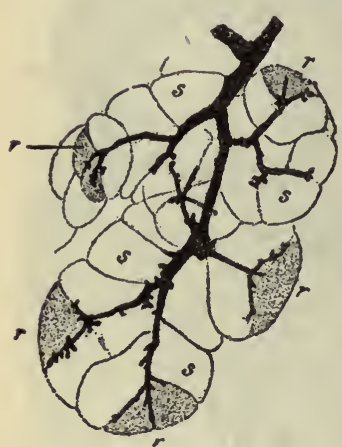

Fig. 358.-Mucous Acini of the Retrolingual GLand of the Rat.

The ducts and secretory capillaries havebeen blackened. $r$, demilunes with secretory capillaries; $s$, mucous cells. Golgi method. $\times 500$. (After Kölliker.)

lobule may contain only mucus-secreting eells.

After the customary preparation by fixation and staining, the mucous cells present a coarse basophilic reticulum which occupies the distal portion of the cell. Coarse granules, with proper fixation and in fresh tissue as well, can be demonstrated within the meshes of the reticulum. These granules are readily colored by the so-called specific mucus stains (Mayer's muchematein and mucicarmin, safranin, and thio$\operatorname{nin})$.

In the mucous cells the nucleus is crowded to the base or proximal end of the cell and flattened against the basement membrane. It is surrounded by a small remnant of finely granular cytoplasm, which, after 


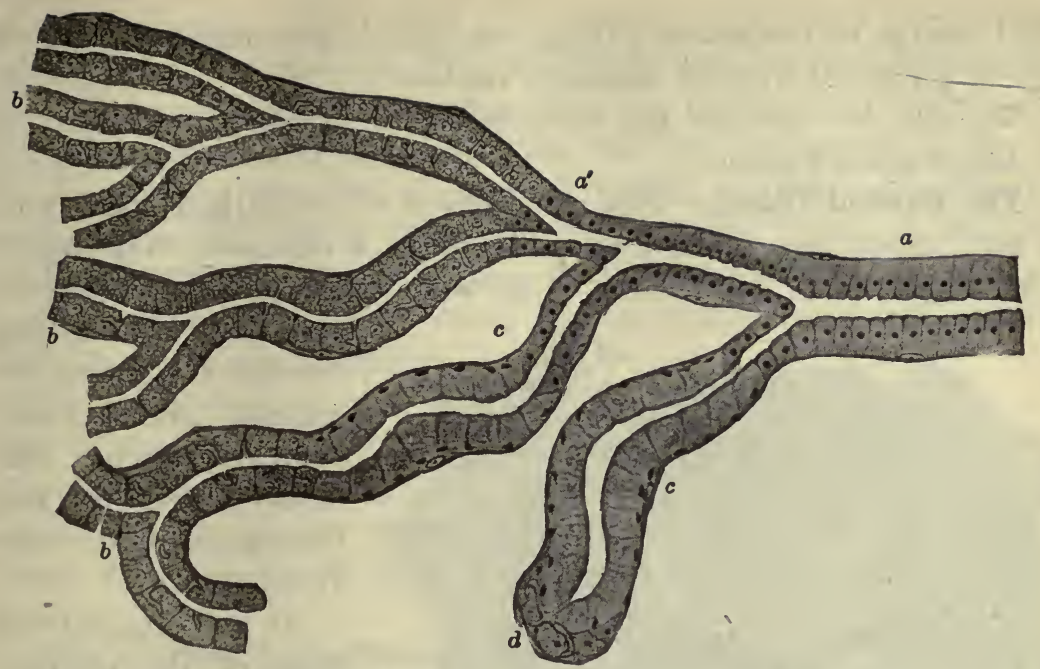

Fig. 359.-Diagram of the Arrangement of the Cells in a Mixed Salivary GLAND.

$a$, intralobular duct; $a^{\prime}$, intercalary duct; $b$, serous-secreting tubules; $c$, mucussecreting tubules; $d$, demilune. (After Krause.)

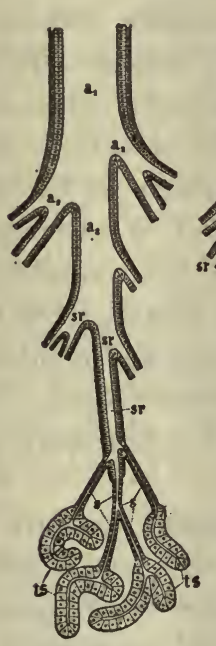

A

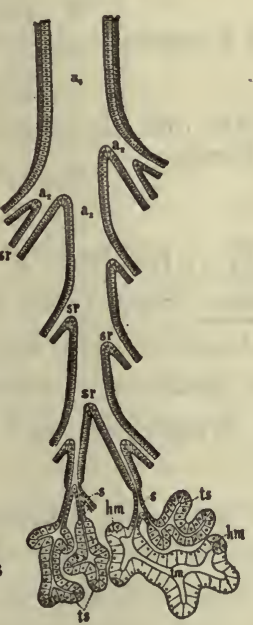

$B$

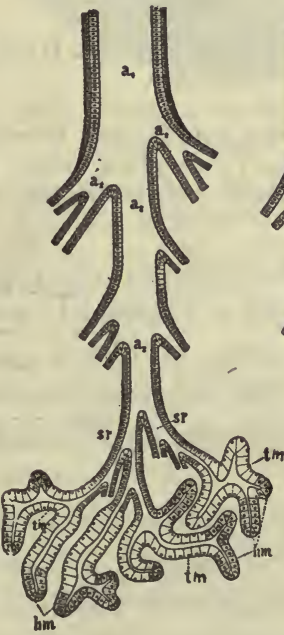

$C$

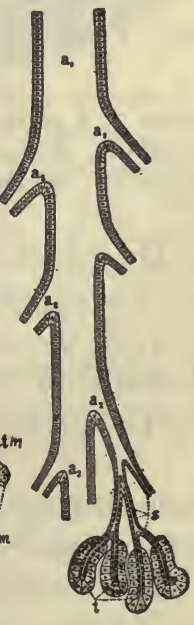

D

Fig. 360.-Diagrams of $A$, Parotid Gland; $B$, Submaxillary Gland; $C$, Sublingual Gland; and $D$, Pancreas. (After Sobotta.)

$a_{1}$, interlobular duct; $a_{2}$, intralobular duct; $s r$, salivary intralobular dinet; $s$, intercalary duct; $h m$, demilune; $t s$, serous alveolus; $t m$, mucous alveolus. 
the discharge of the mucus during secretion, is presumably capable of reloading the cell with its mucinous content.

We will now consider the more important peculiarities of each of the larger salivary glands.

The Parotid Gland.-This is the largest of the salivary glands and in man, sheep, dog, cat and rabbit is purely a serous-secreting organ. However, in sheep, dog and cat it contains also a variable number of

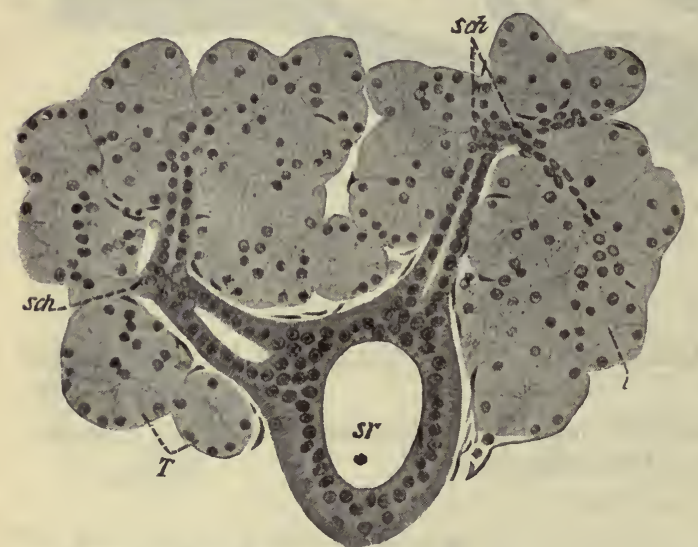

Fig. 361.-From a Section of the Humán Parotid GLaND.

$l$, lumen of a serous acinus; sch, intercalary duct; $s r$, intralobular duct; $T$, secreting acini. Hematoxylin and eosin. $\times 280$. (After Sobotta.) mucous alveoli during the first year; these degenerate and entirely disappear during the second year (Brock and Trautmann, Anat. Anz., $47,17,1914)$.

It is situated in front of the external ear, and overlaps inferiorly both faces of the upper portion of the ramus of the mandible. A variable number of small accessory lobules, including mucus-producing alveoli, lie along the course of the main parotid (Stenson's) duct. The latter opens into the mouth at the level of the second upper molar. The parotid is invested by a dense fibro-elastic sheath, septa from which divide the gland into lobes and lobules. The lobes and lobules are firmly united by the dense but narrow bands of connective tissue; these contain the larger ducts, b广ood-vessels, lymphatics, and a few small ganglia.

The secreting acini are relatively long and tortuous; they are frequently branched or forked. Because of the relatively low height of their serous-secreting cells the acini appear slender and their lumen is irregutar, indistinct, and rery narrow. The 'basket cells' upon which the secreting cells rest are highly developed in the parotid and often form a complete investment for the acinus.

The acini of the parotid are all of one type. The only other tubules within the lobules of this gland are the intercalary and the intralobular 
or salivary ducts. The former are characterized by their very narrow caliber and low epithelium. They are slender tubules which open on the one side from the acini and on the other into the branched terminals of the more spacious salivary ducts. 'The 'salivary' (secretory) portion of the intralobular duct has a somewhat greater diameter also than the excretory portion, which is histologically similar to the interlobular ducts. In the parotid the salivary ducts are relatively short as compared with the intermediate ducts, but are readily recognized by their striated columnar epithelium, which is deeply colored by acid dyes (eosin, etc.), and are thus sharply distinguished from the secreting cells of the acini, which stain poorly with these dyes.

The Submaxillary Gland. - In man and in most mam. mals this organ is a mixed salivary gland; that of the bear and dog contains the largest, that of man and the apes the smallest proportion of mucous acini (Krause, Arch. mikr. Anat., 189\%). In man the proportion of mucous to serous alveoli is about one to five (Piersol). The submaxillary is situated in the floor of the mouth under the posterior por-

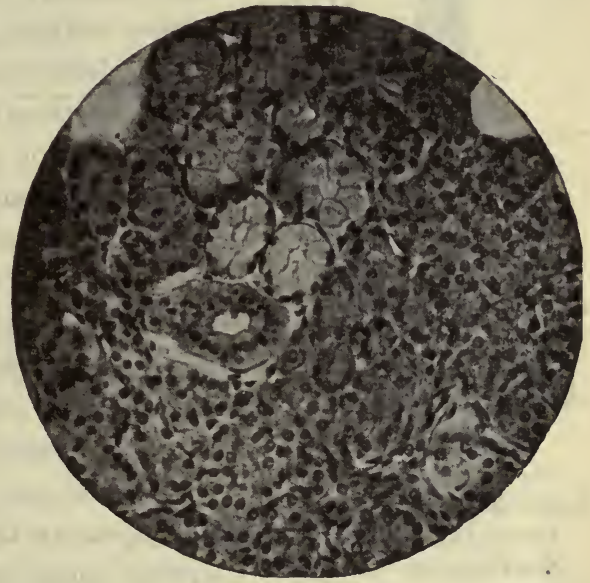

Fig. 362.-From a Section of the Human Submaxillary Gland.

In the center is a small salivary duct, just above which are three mucous acini, the uppermost one possessing a demilune. The other acini are serous. Hematein and eosin. Photo. $\times 370$. tion of the mandible. The main submaxillary (Wharton's) duct opens into the mouth one on either side of the frenulum of the tongue, sometimes joined by the duct of the sublingual gland.

The serous acini of the submaxillary are shorter and less typically tubular than those of the parotid, and they are lined by taller secreting cells. The diameter of the acinus is therefore slightly greater in this gland than in the parotid. Its mucous acini contain a relatively large proportion of demilunes.

The intercalary ducts are considerably shorter than in the parotid, while the salivary intralobular ducts are much longer in the submaxillary. The interlobular connective tissue is not quite so delicate as in 
the parotid. It contains many sympathetic ganglia of relatively large size. Small lamellar corpuscles of simple construction are occasionally found in the interlobular connective tissue (Krause).

The Sublingual Gland.-This is the smallest of the three pairs of large salivary glands. It is not invested by a distinct capsule. It lies under the floor of the mouth anteriorly, one on either side of the frenu-

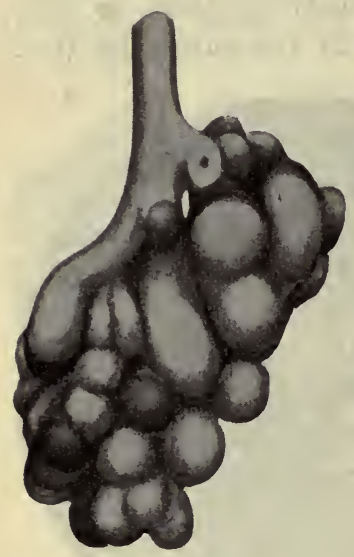

Fig. 363.-ReconstrucTION MODEL OF THE Sublingual Gland of MAN.

An intralobular duct terminating in salivary ducts and acini. $\times 285$. (After Maziarski.) lum. It is a mixed gland in man, dog, cat and rabbit. It differs from the submaxillary, however, in that all of its acini are mucous. It becomes 'mixed' by reason of the presence of very many demilunes (serous cells). Although isolated sections which pass through the larger collections of demilune cells may appear as sections of serous-secreting tubules, if examined in longitudinal section or by reconstruction the true mucous character of each lobule is apparent. Many of the terminal acini of the sublingual gland, however, although much branched, contain no demilunes.' The 'basket cells' are readily recognized in the acini of this gland though they are less highly developed. here than in the parotid. Its intralobular duct system lacks intermediate ducts, and is largely of the 'salivary' type.

Accompanying the sublingual gland proper, or larger component, are a variable number (5 to 20) of accessory sublingual glands of various sizes. They consist largely of mucous alveoli, each gland opening into the mouth by an independent duct. The duct of the sublingual proper (duct of Bartholin) opens at the side of the frenulum.

Blood Supply.-The salivary glands possess a rich blood supply. The arteries accompany the glandular ducts within the interlobar and interlobular connective tissue, and thus reach all the lobules of the gland. Small arterial twigs enter the lobule from all sides and form a rich capillary plexus in the delicate connective tissue coats of the acini. The capillaries are thus brought into intimate relation with the secreting cells, from which they are only separated by the basement membrane of the acinus. The veins return by a similar course, the smallest venules passing out of the lobule into the connective tissue septa in which they retrace the course of the arteries. 
Lymphatics.-Lymphatics are relatively few and are for the most part confined to the interlobular septa, where they form cleft-like spaces which lead to true lymphatic vessels and so on to the lymph nodes of the cervical region.

Nerve Supply.-The salivary glands are abundantly supplied with nerves, which are derived from both sympathetic and cerebral trunks. They are distributed to the walls of the blood-vessels and ducts, and to the secreting cells of the acini. The nerve trunks are found in the interlobular connective tissue where they are supplied with small ganglia which are most abundant in the submaxillary and least numerous in the parotid gland.

The sympathetic fibers which innervate the blood-vessels proceed from the superior cervical ganglion. They are believed to be vasodilator fibers. The cerebral nerves, which supply fibers to the gland cells, are the facial and the glossopharyngeal. The parotid gland is supplied by the glossopharyngeal; the fibers (secretory and yasoconstric-

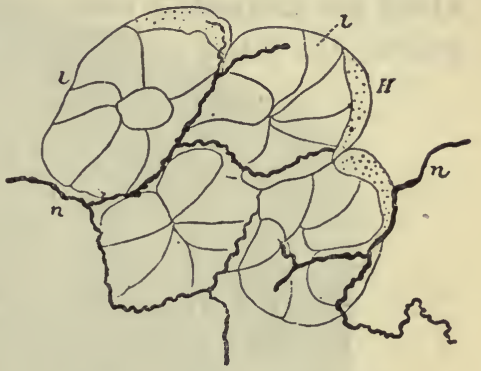

Fig. 364.-Nerve Endings in a Salivary Gland.

$H$, demilune; $l$, secreting acini; $n$, nerve fibrils. Highly magnified. (After Retzius, from Rauber.) tor) passing by way of the tympanic nerve (nerve of Jacobson), small superficial petrosal, otic (sympathetic) ganglion, and the auriculdtemporal branch of the inferior maxillary division of the trigeminal nerve. The submaxillary and sublingual glands receive their secretory fibers and vasoconstrictor fibers from the trigeminal nerve, by way of the chorda tympani nerve and the submaxillary (sympathetic) ganglion. The fibers beyond the otic and submaxillary ganglia are believed to be true postganglionic fibers; that is, axons of cell bodies situated in these ganglia. Sensory fibers are said to pass to the ducts.

Delicate fiber bundles from the interlobular nerve trunks enter the lobules and form a plexus of naked fibrils about the walls of the acini, known as the epilemmal plexus, from which terminal fibrils pierce the basement membrane and as hypolemmal fibers end in contact with and between the secreting cells. Small ferminal expansions, varicosities, or end knobs are found in the course of the hypolemmal fibers. 


\section{rumch like pusated \\ THE PANCREAS}

The pancreas is a flat elongate body lying behind the stomach between the loop of the duodenum and the spleen. It arises as two anlages: a smaller from the common bile duct (ventral pancreas), and a larger from the duodenum (dorsal pancreas). These subsequently fuse to form a single organ drained mainly by the duct of the ventral component, which has meanwhile made an anastomosis with that of the dorsal component, the latter proximal to the anastomosis nevertheless commonly
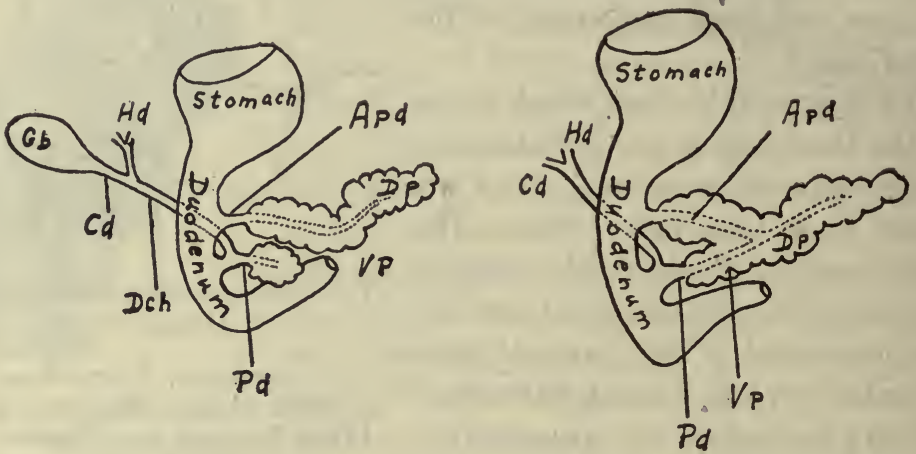

Fig. 365.-Early Stages in the Development of the Pancreas, Illustrating Conditions in the 5 and 7 Weeks Old Human Embryos.

$D p$, dorsal pancreas; $V p$, ventral pancreas; $P d$, pancreatic (Wirsung's) duct; $A p d$, accessory pancreatic (Santorini's) duct; $D c h$, ductus choledochus; $c d$, cystic duct; $H d$, hepatic duct. (Adapted from Kollman.)

remaining pervious and functional. The pancreas bears a close structural resemblance to the salivary glands, more especially the parotid. It is a compound tubulo-acinar gland which contains an immense number of small lobules associated into tobes and which pours its secretion into the lumen of the duodenum by means of the chief (Wirsung's) and accessory (Santorini's) pancreatic ducts. The tobules are united by a delicate and relatively very loose fibro-elastic conneetive-tissue.

The ducts of the pancreas branch and arborize in the same manner as those of the-salivary glands. The interlobular ducts are lined by a single layer of columnar cells; in the larger divisions (interlobar ducts) occasional goblet cells are found. The wall of the interlobular pancreatic ducts is much thicker than in those of the salivary glands, for 
they possess a much thicker connective tissue coat, in which are also many longitudinal smaoth muscle fibers.

On entering the lobule the duct is immediately transformed into

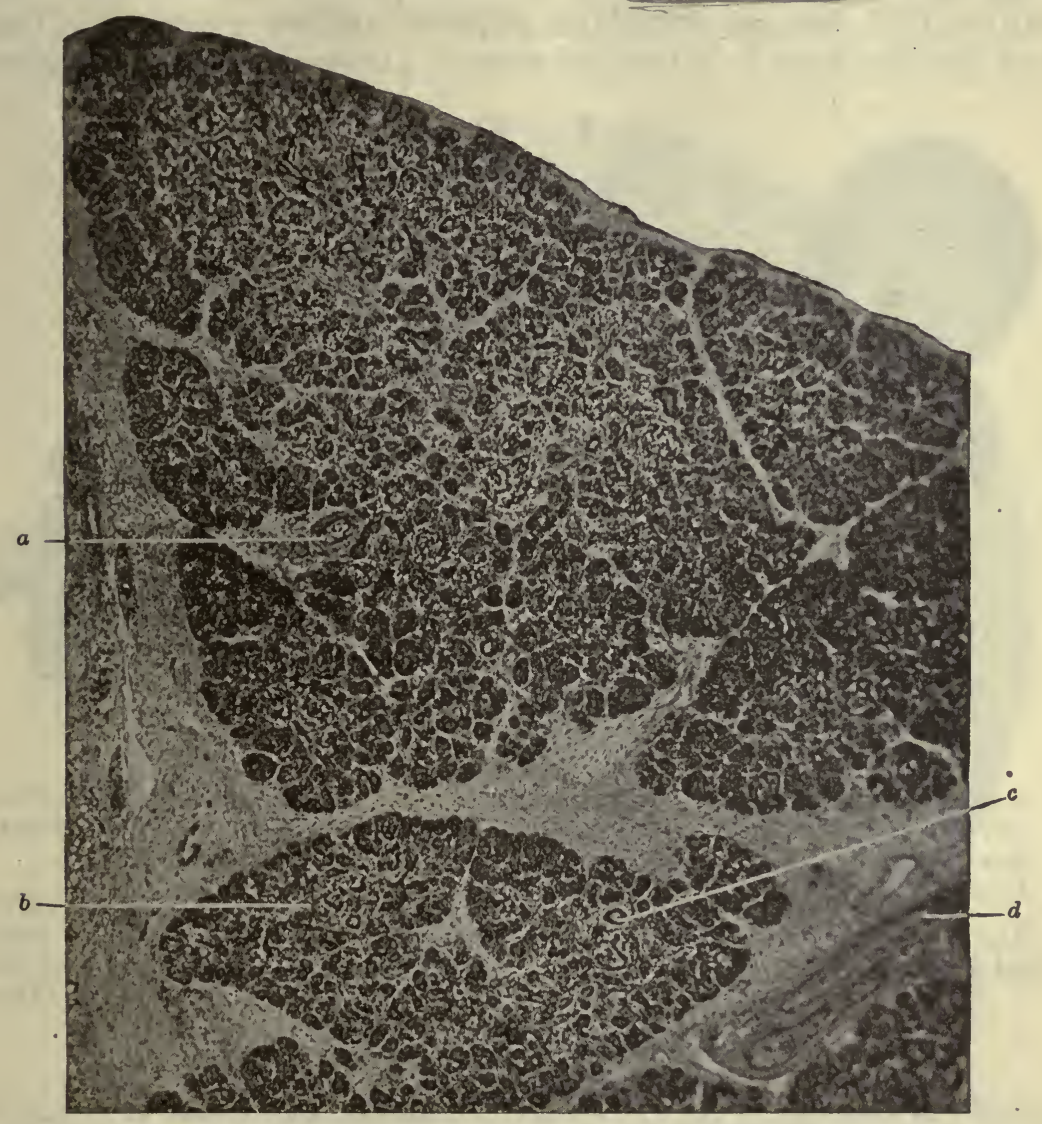

Fig. 366.-From a Section of the Human Pancreas, Showing Several Lobules and the Broad Interlobular Bands of Connective Tissue.

$a$, blood-vessel; $b$, pancreatic islet; $c$, intralobular duct; $d$, interlobular duct and accompanying artery. Hematein and eosin. Photo. $\times 45$.

the intercalary (intermediate; junctional) type; in the pancreas there. are no specialized intralobular ducts lined by columnar striated epithelium as in the salivary glands. The intercalary ducts are very slender tubules which are lined by low columnar or flattened epithelium. Because of the absence of larger intralobular ducts the intercalary portions. are relatively very long and much branched. 
On approaching its termination the lining cells of an intercalary duct are still more flattened and often acquire a considerable breadth. They are elongated in the direction of the long axis of the tubule, and in surface view have an irregularly polygonal outline (Bensley). They pass into the acini in a peculiar manner. Instead of offering a direct

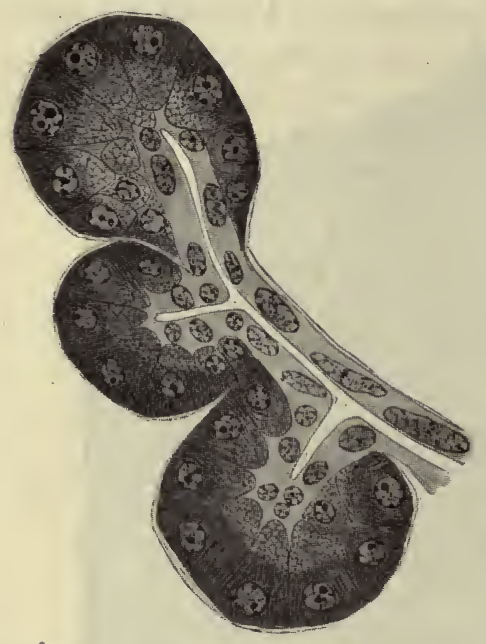

Fig. 367.-Drawing of an Intercalary Duct of Cat, with Three Branches Ending in Acini to Form Centro-acinal Cell Groups.

The acinar cells contain numerous basal filaments. $\times 750$.

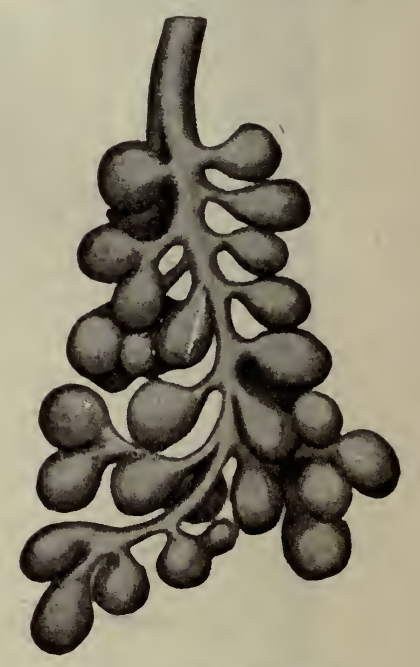

Fig. 368.-ReConstruction Model of the Human Pancreas.

The intralobular duct gives off long intercalary ducts, which, after branching, end in the acini. $\times 344$. (After Maziarski.)

transition from the duct epithelium to that of the acinus the cells of the former frequently appear as if telescopled into the lumen of the acinus. Thus the centro-acinal cells (of fangerhans) are produced, and consequently the centro-acinal cells correspond closely in appearance with 'those of the intercalated ducts. They seem to occupy the lumen of the acinus, and are only separated from the distal ends of the acinal cells by the secretory eapillaries which place the secreting cells in communication with the fumen of the duct. The centro-acinal cells are characteristic of the pancreatic acjni.

The Acini.-The acini of the parcreas p pssess an irregular tubular form with frequent alveolar dilatations. Their lining epithelium rests 
upon a reticular basement membrane within which are thin 'basket cells.' A delicate connective tissue stroma invests the-acini or alveoli.

The secreting cells are tall and irregularly columnar of pyramidal in shape. Their nucleus lies in the proximal third of the cell and is surrounded by reticular or very finely granular cytoplasm. The cyto-

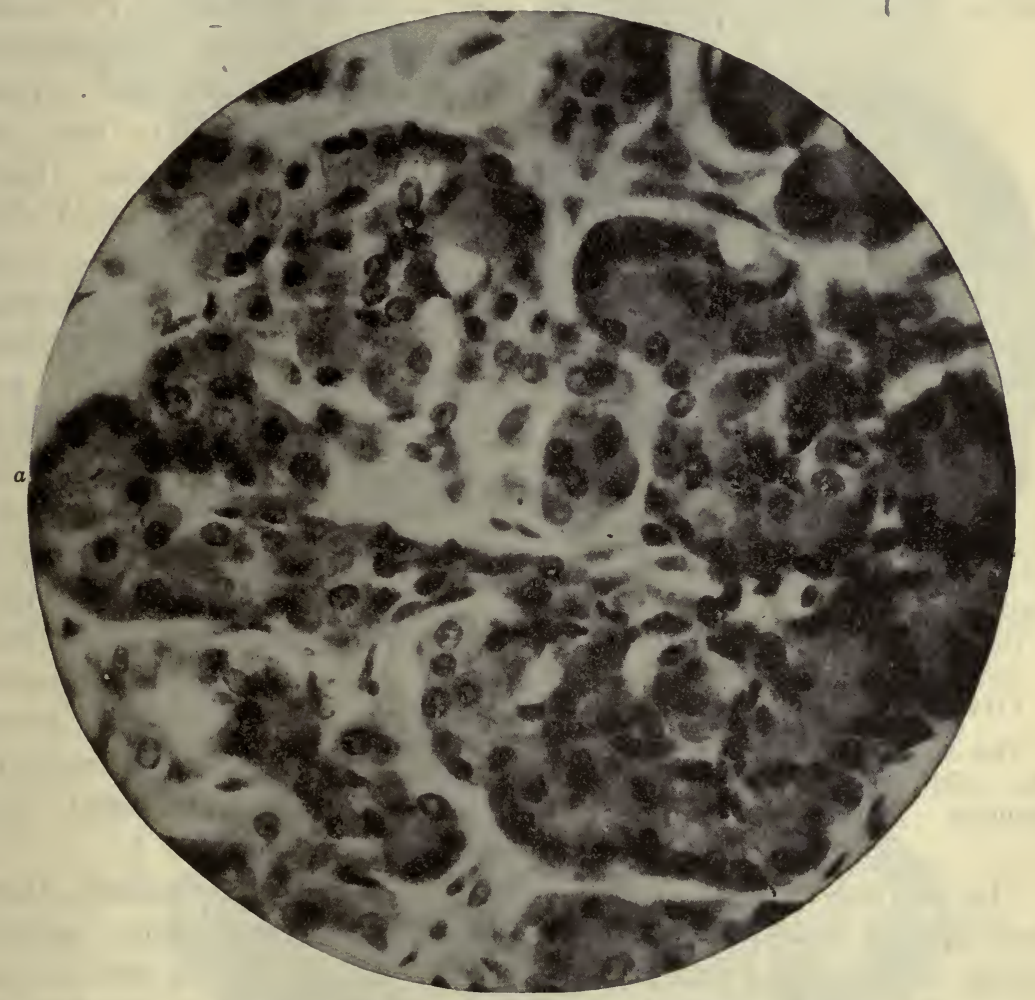

Fig. 369.-Acint of the Human Pancreas.

The acinus at $a$ is connected with an intercalary duct, cut in tangential section, and occupying the center of the figure. Hematein and eosin. Photo. $\times 950$.

plasm of the inner zone of the cell, on the other hand, is filled with coarse zymogen granules whose number is dependent upon the activity of the gland. During fasting the granules accumulate until eventually they almost completely fill the cell, but during digestion they disappear with the discharge of the secretion, the width of the granular zone gradually decreasing, that of the non-granular fibrillar basal zone being correspondingly enlarged (Figs. 3\%1, A and B). 
With the increased breadth of the basal zone during secretion, there appears in this portion of the cell a structure which/has been described by Nussbaum (Arch. mikr. Anat., 1885) as the Nebenkern, and which has been carefully studied by Mathews (Jour. of Morph., 1899). This is a spheroidal basophil body which lies near the nucleus and is frequently surrounded by a clear area of cytoplasm. Its origin

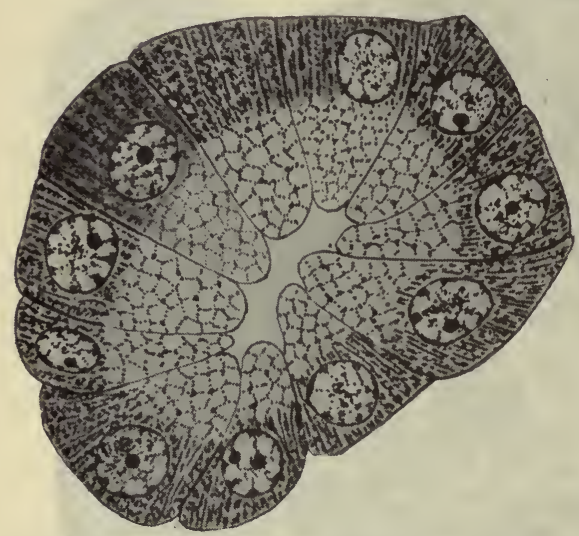

Fig. 370.-Pancreatic Acinds of Cat Cut Transversely near Fundus, Showing the Basal (Prozymogen) Fillaments of the Cells.

The alveoli of the central portions of the cells represent dissolved zymogen granules. $\times 1500$. and function are undetermined and it is possible that several distinct bodies have been included under the name. Ogata (Arch. f. Physiol., 1883) considers that it is derived from the nucleus by the extrusion of its plasmosome, an opinion which seems to be shared by von Ebner (Kölliker's Handbuch, 1902, Bd. iii, 5, 250). The studies of Mathews have shown that at least in certain instances it is distinctly fibrillar and suggest that it may be concerned with the mechanism of secretion. It most probably represents a post-secretion remnant of ergastoplasmic basal filaments.

In addition to this fibrillar complex of basophilous substance, there may be seen in appropriately fixed and stained preparations, another group of fibrils, the mitochondria. These can be seen in fresh acinar cells and in tissue preserved in fluids which lack acids (mitochondria are dissolved by acids), when they also give to the basal portion of these cells a striated appearance. These basal bodies have been extensively studied by Bensley (Amer. Jour. Anat., 12, 3, 1911) in the pancreas of the guinea pig, and their independence from the basal filaments of Solger established. Mislawsky also (Arch. mikr. Anat., 81, 4, 1913) has recently studied the mitochondria in the acinar cells of the rabbit's pancreas. He finds no evidence to indicate that they segment into the zymogenic granules. They are described as interstitial elements of the protoplasmic reticulum, more probably connected with the general cell metabolism. There is apparently no good evidence in support of the 

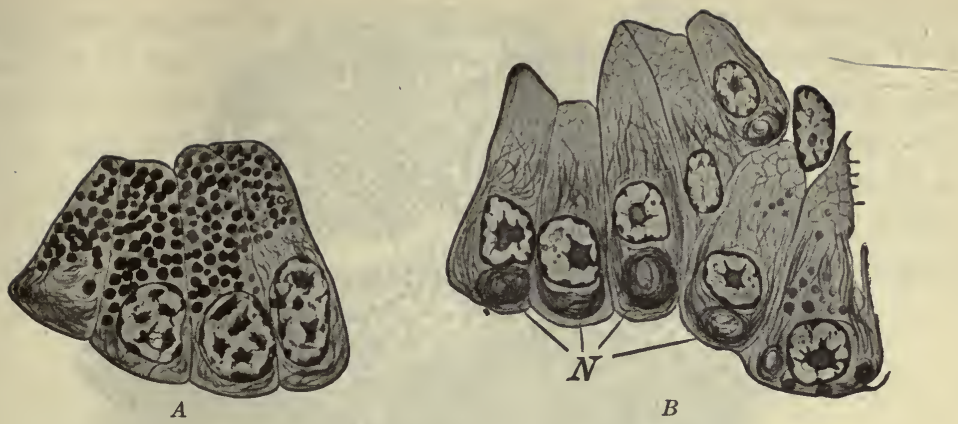

Fig. 371.-Cells from Pancreas of Necturus.

$A$, after rest, and filled with zymogen granules; $B$, after activity, showing the presecretion (basal; ergastoplasmic) filaments, and the so-called nebenkern $(N)$. (After Mathews.)

idea that either the basal filaments of Solger or the mitochondria give origin to secretory granules, by a process of segmentation.

Pancreatic Islets.-The lobules of the pancreas contain, in addition to the acini and ducts, certain larger and smaller spheroidal collections of polyhedral cells which lie in the inter-acinar connective tissue, the pancreatic islets (islands of Langerhans; intralobular or interalveolar

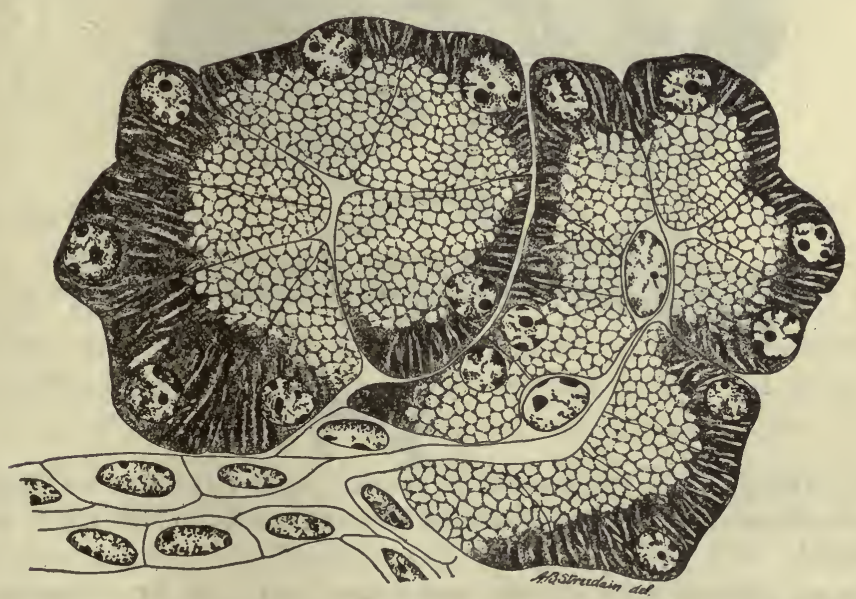

Fig. 372.-Two Adjacent Acini from the Guinea-pig's Pancreas.

,The one at the right shows an entering intercalary duct and two centro-acinal cells. The acinar cells are filled proximally with a basophilous substance (basal filaments), and contain distally numerous alveoli, the representatives of dissolved zymogen granules. $\times 1200$ (After Bensley, Am. Jour. Anat., 12, 3, 1911.) 


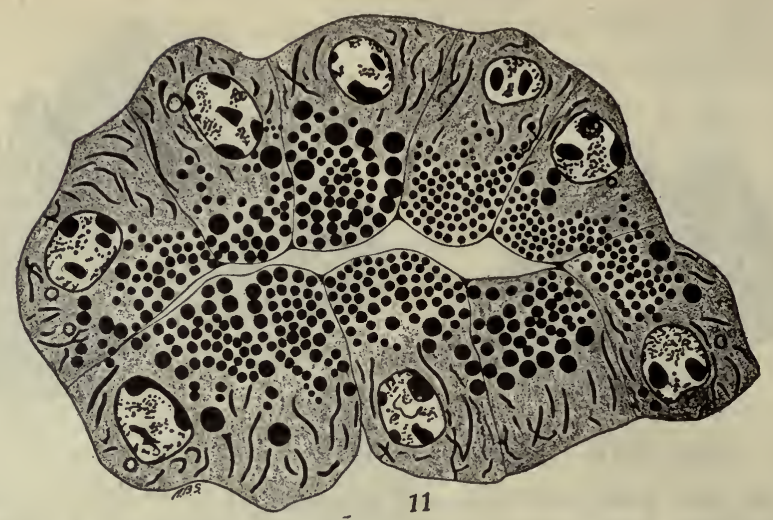

Fig. 373.-Section of an Acinus from the Guinea-pig's Pancreas, Showing the

Basal Mitochondrial Content and the Central Zymogen Granules.

Bensley's mitochondrial technic. $\times 1200$ (After Bensley, Am. Jour. Anat., 12, 3, 1911.)

cell groups). The islet cells are arranged in irregular cords, frequently only two cells deep, lying in the meshes of capilliform sinusoids, from which they appear to be separated by little more than the endothelial

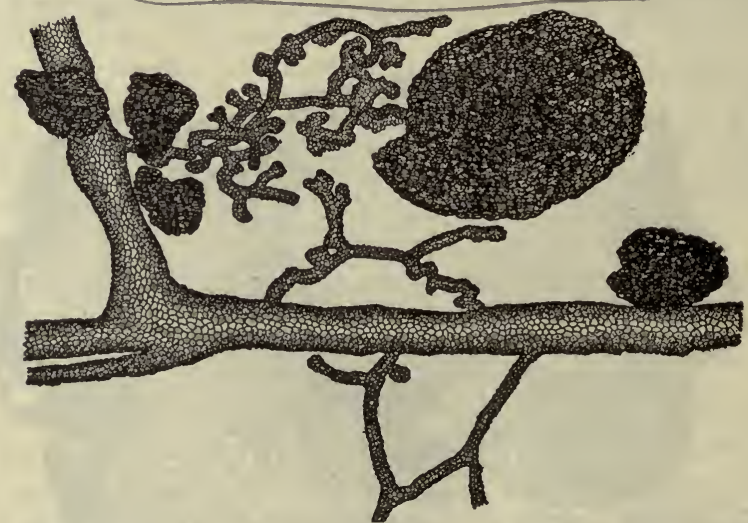

Fig. 374.-Intercalart Duct with Branches, from Pancreas of Guinea-pig, Showing Highly Branched Tubules Connected with the Duct and with AN IsLET.

Intravitam staining with pyronin and neutral red. $\times$ 50. (Bensley, Amer. Jour. Anat., 12, 3, 1911.)

wall. The cells are divisible into two distinct, apparently independent types, on the basis of their granular content (Lane, Amer. Jour. Anat., $7,3,190 \%)$. The 'A' and ' $\mathrm{B}$ ' granules differ morphologically and micro- 
chemically; it is suggested that they indicate a twofold secretion (Lane). The granules of the islet cells of both types differ markedly also from those of the acinar cells. The islets are of various sizes, ranging from those with only a few or even a single cell to those with many cells (3 millimeters in diameter). The number of islets also varies greatly in different individuals. Thus De Witt (Jour. Exp. Med., vol. 8, 1906) estimated the amount of islet tissue in three apparently normal subjects

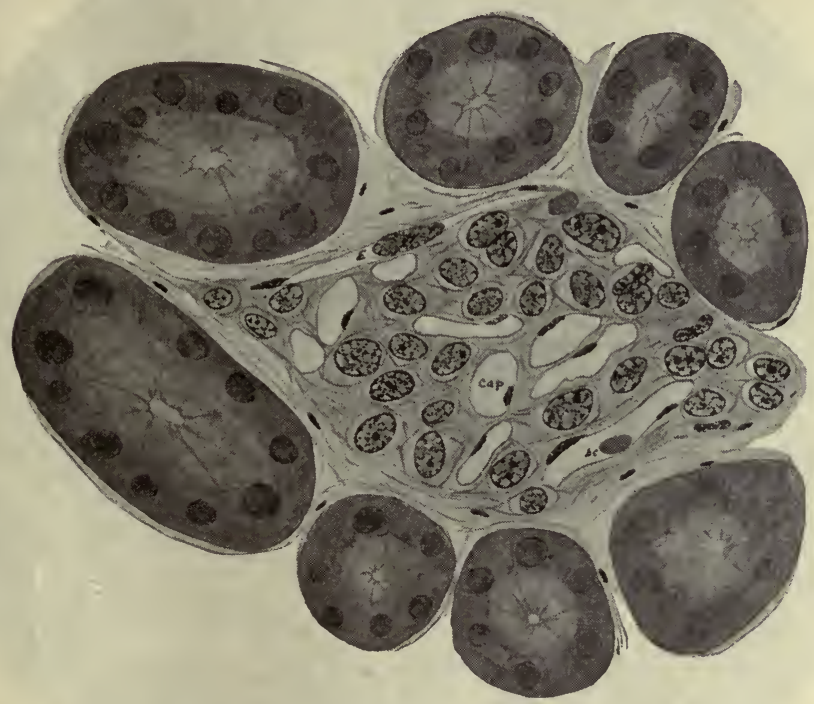

Fig. 375.-Pancreatic Islet of Cat.

$E$, endothelial cell; cap, capillary; $b c$, red blood corpuscle. $\times 750$.

at 1-25, 1-50 and 1-125 of the total volume of the pancreas. By means of intravitam stains (neutral red and janus green), Bensley succeeded in staining differentially the islets of the guinea pig's pancreas. $\mathrm{He}$ counted from 13,000 to 56,000 in different specimens. Clark (Anat. Anz., 43, 3, 1913) employed this method in a study of human pancreases secured shortly after death, and estimates the average number of islets at 12 per milligram. In one male subject of 24 years and 140 pounds weight, he estimated the total number of islets at $1,760,000$; in another of 29 years and 135 pounds weight, only 662,166. Opie (Johns Hopkins Hosp. Bull., 1900) first observed that the islets were more abundant in the tail and least abundant in the head of the pancreas; this observation is confirmed by both Clark and Bensley. Laguesse (Jour. de Physiol. et 
de Path., 13, 1, 1911) described the islets in continuity with the ducts of the acini. Such continuity is described also by Bensley for many of the islets in the guinea pig's pancreas. Bensley's studies have disclosed these further points of relationship between the islets and the zymo-

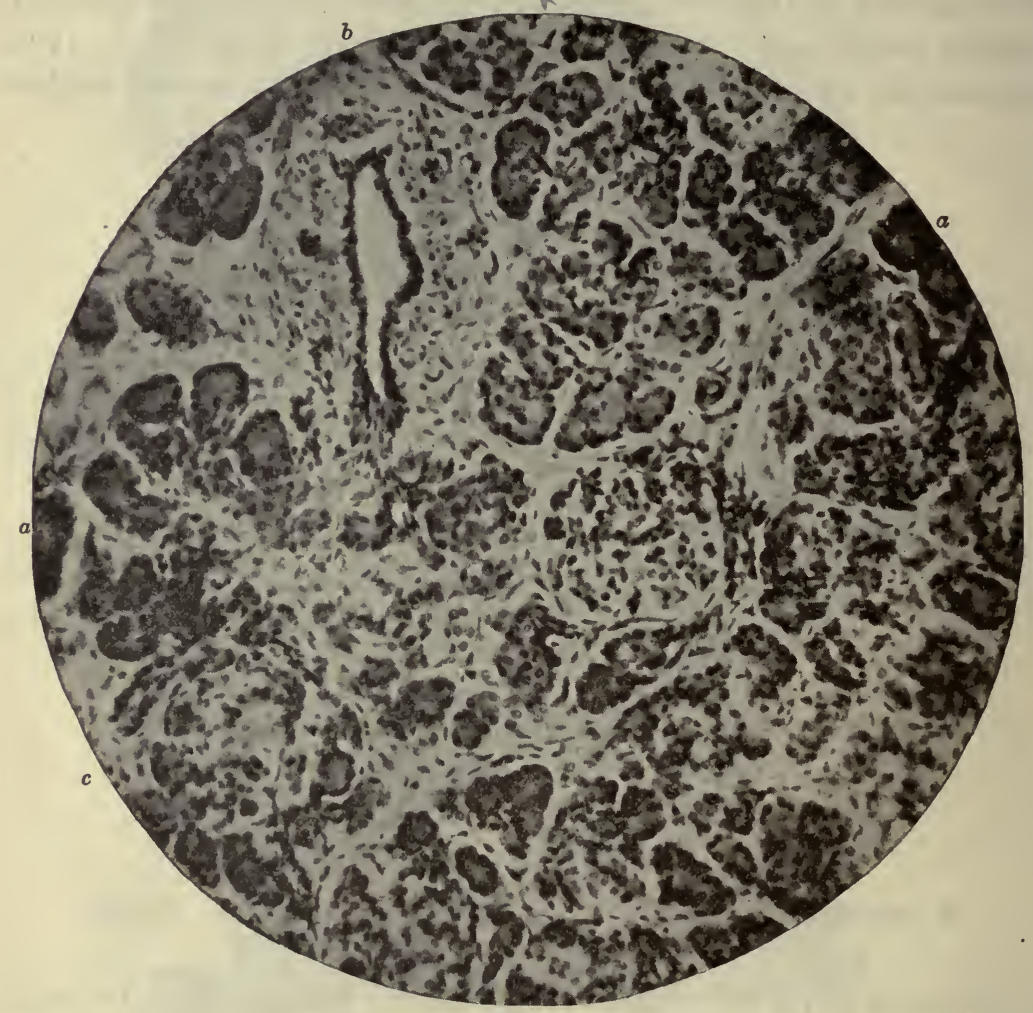

Fig. 376.-From the Human Pancreas.

$a$, acini; $b$, is placed above an interlobular duct; $c$, a pancreatic islet; a second islet, circular in outline, lies near the center of the figure. Hematein and eosin. Photo. $\times 330$.

genous parenchyma: Islets may be located (1) in the interlobular connective tissue, but connected with the duct system; (2) in the lobules, also unconnected with the acini (encapsulated), but directly connected with the interlobular duct system; (3) in the lobules-these include the great majority-and in connection with either acini or ducts or both; and (4) islets unconnected with either ducts or acipi, both in the interlobular connective tissue and in the acinar parenchyma. 
The tubules (intercalary ducts) are said to branch and anastomose freely and to be capable of differentiating either into acini, or islets, or both. They may produce also small mucous glands which open into the tubules; and single islet cells may be formed along the ducts. Notwithstanding their intimate developmental relationship the islets remain isolated from the general exocrin parenchyma, since the lumen of the connecting tubules does not penetrate its substance. There is no satisfactory evidence to show that islet tissue may be increased or diminished, or that acinus tissue may change into islet tissue, or vice versa, concomitant with experimentally induced alterations in nutritive and functional conditions, as has been repeatedly claimed; nor is there evidence of a transition between islet and acinar cells. Islets and acini have a common embryonic origin, but once differentiated they are not capable of transformation one into the other (Bensley). The islets appear in the human pancreas about the time the embryo attains a length of 50 millimeters (Lewis).

The islets are believed to constitute a group of internally secreting organs. The evidence for such conclusion is derived from a series of experiments by Opie and many others. When the pancreas is removed, a form of diabetes follows, characterized by the appearance of sugar in the urine. When the pancreatic duct is simply ligated, the flow of pancreatic juice is checked and atrophy of the acinar tissue ensues, but no disturbance in carbohydrate metabolism results, nor is any alteration produced in the islet tissue. Moreover, in cases of death following diabetes mellitus, Opie demonstrated gross degenerative changes. Homans' recent experiments (Jour. Med. Research, March, 1914) have extended the evidence in support of the conclusion that the islets are concerned in the metabolism of sugar. When more than three-fourths of the pancreas was removed in cats, the main duct being left intact, fatal diabetes occasionally followed. Microscopic examination of the islets revealed the following conditions: (1) ip those instances where diabetes did not follow the operation, the islet cells showed signs of over-activity indicated in part by a disappearance of secretory granules; (2) in the subjects which died from diabetes following the operation, the islet cells showed degenerative changes, the acinous tissue having remained unaltered.

Blood Supply. - The large blood-vessels of the pancreas accompany the interlobular ducts, but after repeated subdivision these vessels part company, and the smaller arteries pursue a separate course through the interlobular connective tissue. Thus they reach all portions of the 26 
gland and supply capillaries to the intralobular connective tissue about the acini. Certain arterial branches also enter the islets and form a specially rich plexus of broad capillaries (sinusoids) within these cell groups. The veins return the islet blood by a similar course.

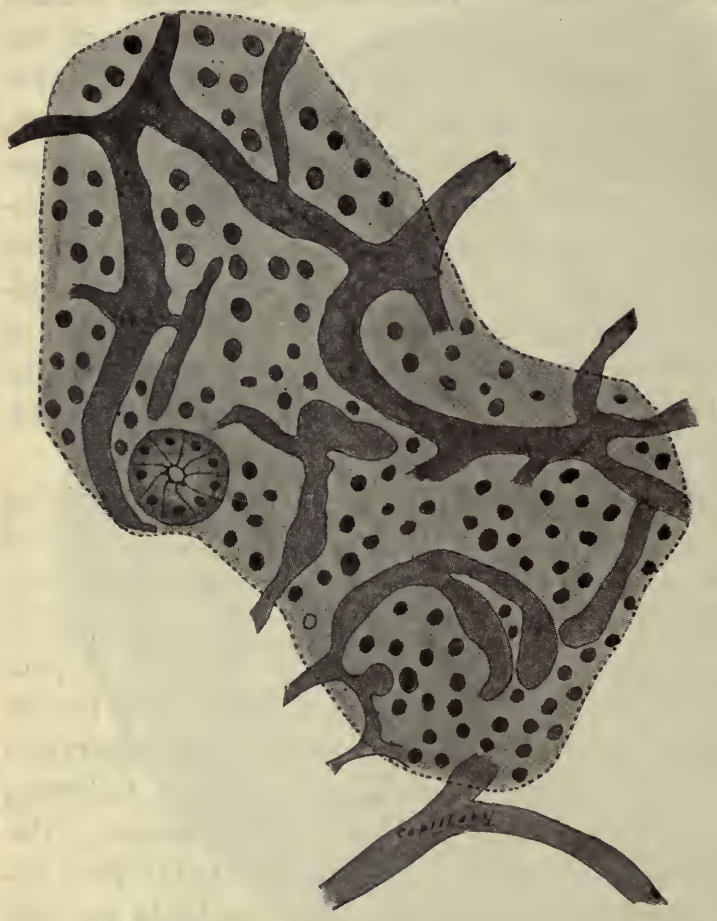

Fig. 377.- Section of a Pancreatic Islet from InJeCted Specimen of Cat's Pancreas to Show the Profuse Blood Supply.

The capillaries were accurately drawn by aid of a camera lucida. Only the nuclei of the islet cells are indicated; one acinus is shown at the left. $\times 500$.

The Lymphatics. -The lymphatics are. mostly confined to the interlobular tis s u e, where they are in relation with the bloodvessels.

The Nerves.-The nerves a re derived from the sympathetic system, and occur as small trunks within the interlobular connective tissue. $\mathrm{Nu}$ merous small ganglia occur in their course. As in the salivary glands the nerves supply the vascular walls. About the secreting acini they form a delicate network of naked fibrils, from which end-branches penetrate the basement membrane and terminate upon the secreting cells. Lamellar corpuscles are occasionally found in the interlobular connective tissue of the pancreas.

Résumé.-Finally the attention of the student should be specially directed to the presence of the pancreatic islets, the centro-acinar cells, the very distinct inner granular and outer fibrillar zones of the secreting cells, the thick walls of the interlobular ducts, the absence of intralobular ducts except of the intercalary type, and the loose character of the interlobular tissue as the distinguishing characteristics of the pancreas. 


\section{THE LIVER}

The liver is the largest gland of the body, and may be classed as a peculiar form of compound tubular gland whose cells resemble the serous-

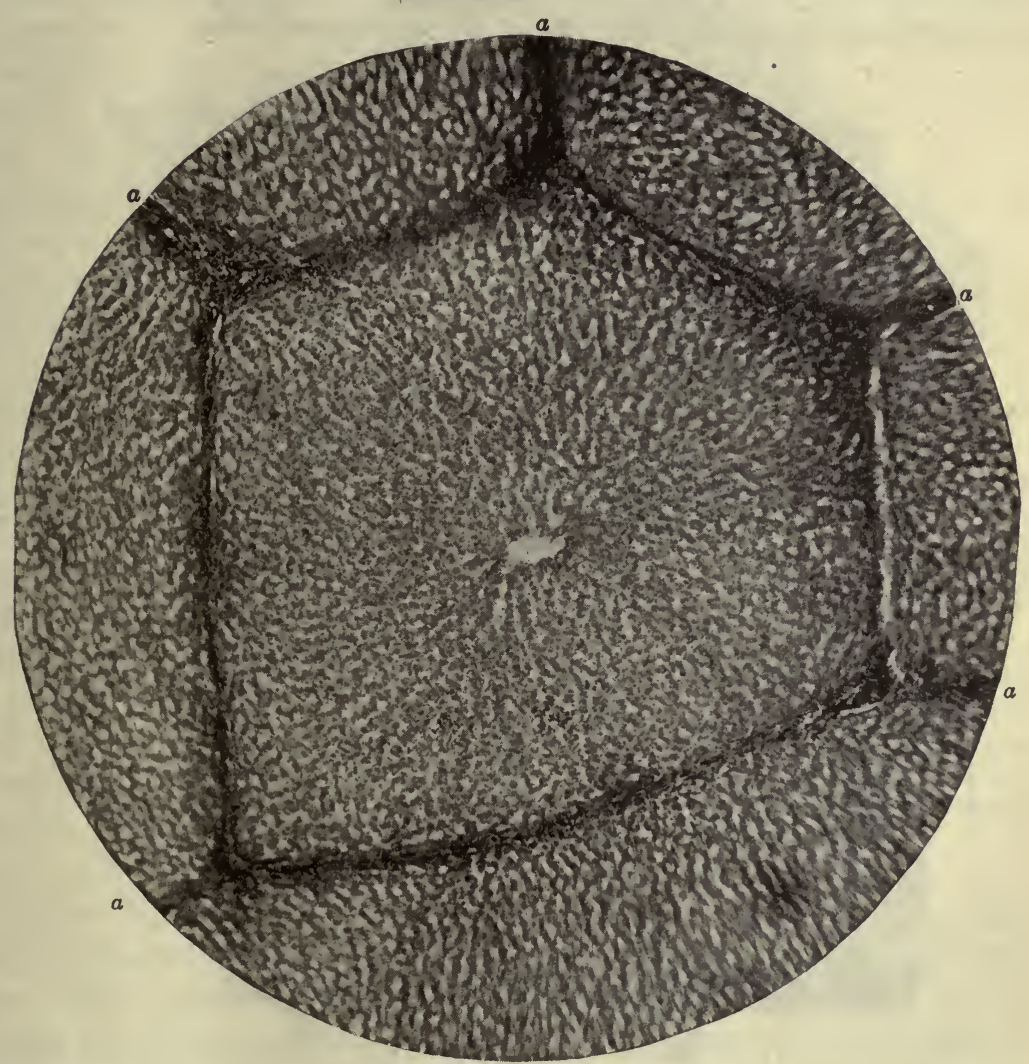

Fig. 378.-A Lobule of the Pig's Liver; the Central Vein Lies in the Middle of the Figure.

a, capsule of Glisson. Hematein and eosin. Photo. $\times 115$.

secreting type. The organ is invested with a connective tissue sheath, the greater portion of which is clothed with peritoneal epithelium. From this connective tissue capsule, fibrous bands or septa are continued into the substance of the organ and permeate to all its portions. These processes of connective tissue, collectively forming the capsule of Glisson, are most abundant at the transverse fissure where they contain the large 
blood-vessels and hepatic ducts-this fissure serving as a hilum for the organ. The liver is very irregular in outline and shape, and comprises five so-called lobes of very unequal size. In the adult its weight is

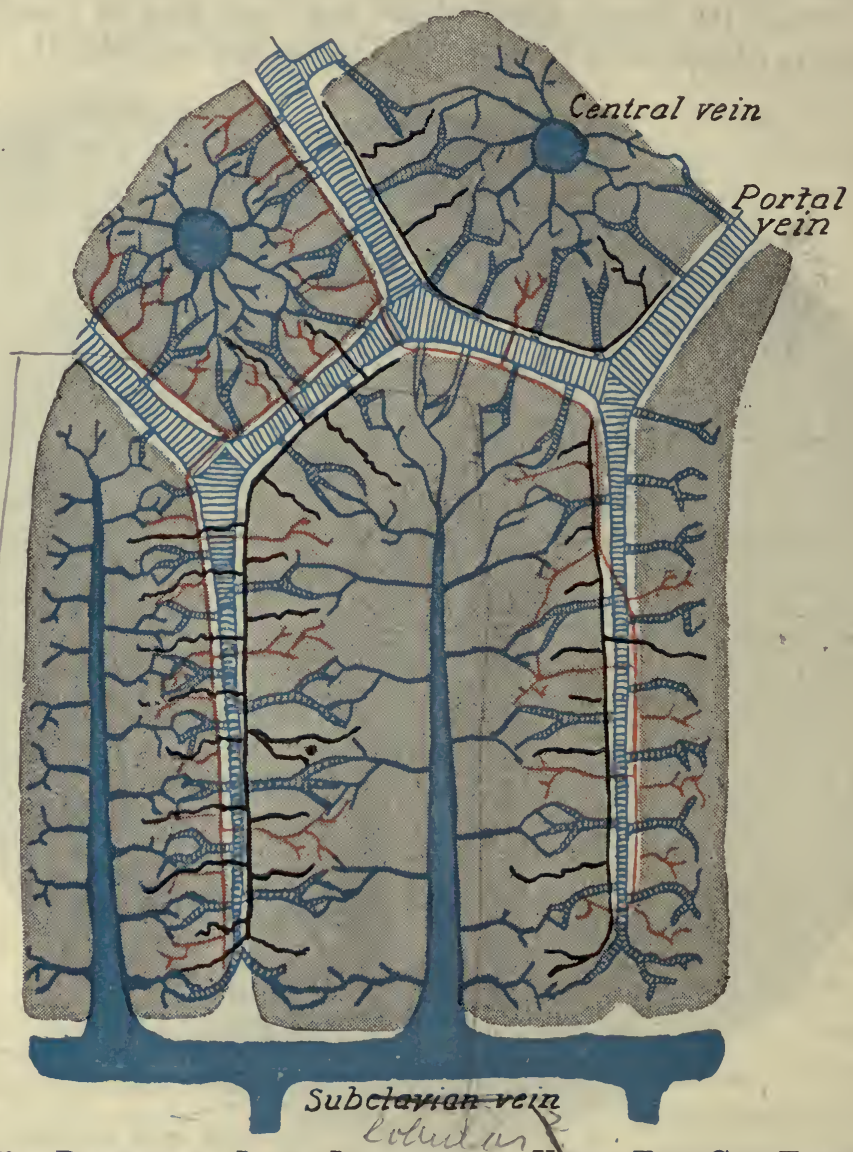

Fig. 379.-Diagram of Liver Lobules, the Upper Two Cut Transversely, THE LOWER LONGITUDINALLY.

The portal veins are blue striped; the hepatic veins (central with branches, and sublobular) are solid blue; the hepatic arteries are red; and the bile canals black. (After Merkel.) Cells not indicated.

about one-fortieth that of the body, about 3 to $33 / 4$ pounds. The parenchyma of the organ arises from a tubular outgrowth of the embryonic duodenum, hence entodermal; the interstitial tissue develops in part from the mesenchyma of the caudal layer of the primitive dia- 
phragm (septum transversum) and of the intervening ventral mesentery throughout which the entodermal tubules ramify, and in part (the-reticulum) from endothelium of the original venous sinusoids (Mall).

The liver is dependent for its structural characteristics upon the peculiar disposition of the connective tissue of Glisson's capsule, as alse of the blood-vessels whose-branehes it contains; for by these tissues the substance of the liver is extensively subdivided into minute collections of hepatic cells, each group forming an anatomie unit, the hepatic lobule, which in addition to the hepatic cells contains a connective tissue reticulum and the smaller blood-vessels and secretory capillaries (bile canaliculi).

The hepatic lobules are of cylindrical shape, about 2 millimeters in length and 1 millimeter in diameter (Báiley). In transverse section they present a polygonal (hexagonal or pentagonal) outline. In the dog they are

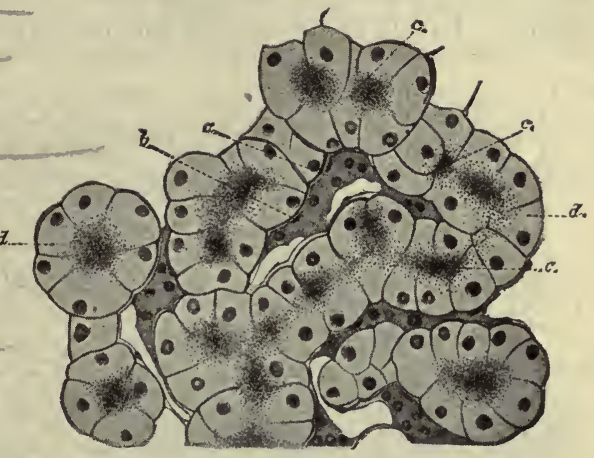

Fig. 380.-From a Section of the Turtle's Liver, Showing the Tubular Arrangement of the Parenchyma.

$a$, blood capillary, partially filled with clotted blood; $b$, vascular endothelium; $c$, darkened central portions of the hepatic cells; $d$, peripheral portion of the hepatic cells. Osmium tetroxid; carmin. $\times 400$. (After Shore and Jones.) short polyhedra about 0.7 millimeter high, and 0.7 millimeter in diameter; the entire liver containing 480,000 (Mall, Amer. Jour. Anat., 5, 3, 1906). They are analogous to the lobules of compound tubulo-acinar glands, inasmuch as they contain the secreting parenchyma of the organ, but are very different from the latter in the arrangement of the secreting cells which, in the human liver, do not present either a tubular or acinar structure, but form solid cell columns. Thus in the human liver the tubular character of the gland is scarcely apparent, yet in the liver of many of the lower animals, notably in that of the turtle and frog, the cells form typical tubules within the indistinct hepatic lobules.

The bile formed by the liver cells is conveyed to the duodenum by an excretory system, beginning with innumerable interlobular bile dućts which receive the intralobular secretory captlaries, and, leaving the lobule from all its sides, find their way through the interlobular con- 
nective tissue of the capsule of Glisson and unite with their fellows to form larger and larger bile ducts, which finally join to form the main excretory or hepatic duct; the latter unites with the cystic duct of the gall-bladder to form the common bile duct through which the bile reaches the intestine. The gall-bladder-which is in principle a diverticulum from the hepatic "duct-is simply a reservoir for the storage of bile; it is absent in some animals, for example, the horse and the elephant. In all their course the bile ducts are in close relation with the

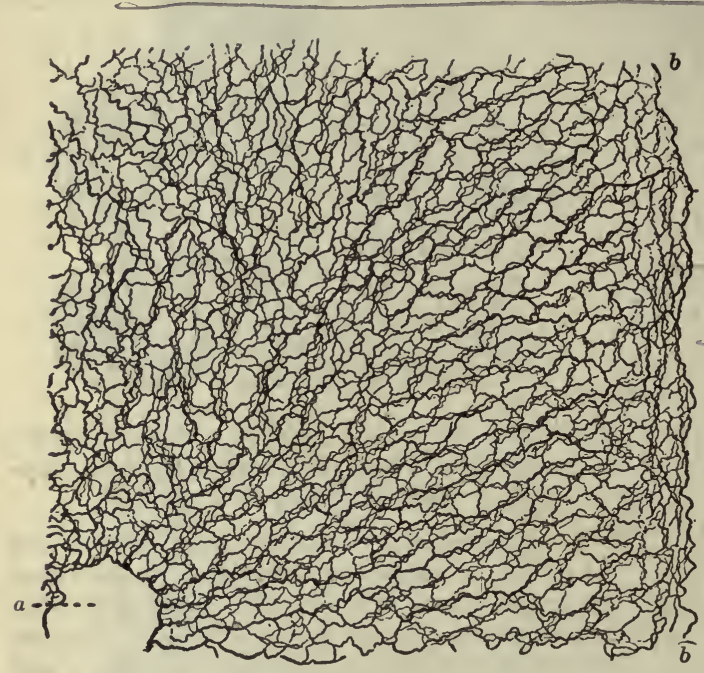

Fig. 381.-The Reticulum of the Dog's Liver.

$a$, central vein; $b$, capsule of Glisson at the margin of the lobule. Gold chlorid. $\times 120$. (After Böhm and von Davidoff.)

hexagonal lobules-together with the more delicate intralobular reticulum. These tissues convey the blood-vessels, lymphatics, nerves, and bile ducts.

The fibrous framework, which forms both the outer fibrous capsule of the liver and the capsule of Glisson, contains both collagenous and elastic tissue, the latter being fairly abundant-a fact which sharply contrasts with the complete absence of elastic fibers from the interior of the hepatic lobules.

The intralobular connective tissue is extremely delicate, and consists of very fine fibrils and stellate cells (of von Kupfer) which form a delicate reticulum, in which the capillary blood-vessels and columns of liver 
cells are suspended. The anastomosing strands of reticulum converge from the periphery toward the center of the lobule, thus following the course of the blood capillaries and cell columns. This reticular tissue (Mall) exists in so small a quantity and is so extremely delicate that although it can be readily studied after removal of the liver cells, as by artificial digestion, in ordinary preparations-except those of extreme thinness-it can-scarcely be discovered in the minute clefts between the cell columns and the blood capillaries. It differentiates, at least in part, from the endothelium of the original venous sinusoids; the stellate cells seen in Golgi preparations also represent endothelial elements. The endothelial cells of the intralobular capillaries are actively phagocytic.

The volume of the interlobular connective tissue which forms Glisson's capsule varies greatly in different animals. In the liver of the pig and the camel this tissue is very extensive, and forms a complete investment for each lobule. In man it is very limited in amount and is confined to minute areas between the adjacent angles of the lobules, with an occasional fragment separating the lateral sur-

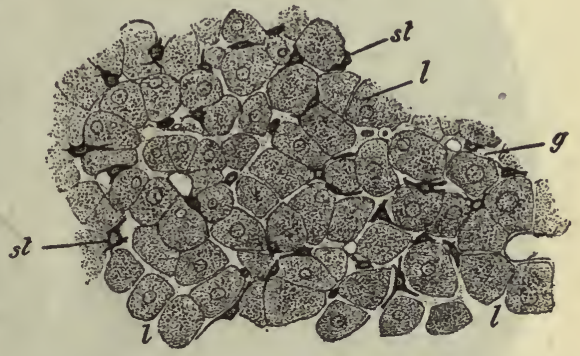

Fig. 382.-Stellate Cells of von KupFER IN. THE Liver of a Dog. faces of neighboring lobules. It is in the latter portions, viz., between the opposed surfaces of the lobules, that the branches of the hepatic veins (sublobular veins) are found. The interlobular veins, the subdivisions of the portal vein, together with the bile ducts and the branches of the hepatic artery are found at the angles of adjacent lobules; hence the portal canals, which contain these vessels, should always be sought in this location, while the sublobular veins, which run alone and form no part of the portal canals, will be found between the opposed surfaces of the lobules.

The capsule of Glisson also contains many lymphatic vessels and nonmedullated nerve fibers.

The Hepatic Lobule.-The lobule is the structural unit of the liver and consists chiefly of hepatic cells which are arranged in radiating cords. In shape the lobule is an irregularly hexagonal pyramid, the exact number of its faces being extremely variable. The periphery of the lobule is outlined by the connective tissue of Glisson's capsule which 
either completely invests each lobule, as in the pig's liver, or forms only a very incomplete investment, as in the liver of man.

Blood enters the lobule from the vessels of the portal canals and finds its way, through converging capillaries, from the periphery to the center of the lobule. Here it enters the intralobular or central vein,

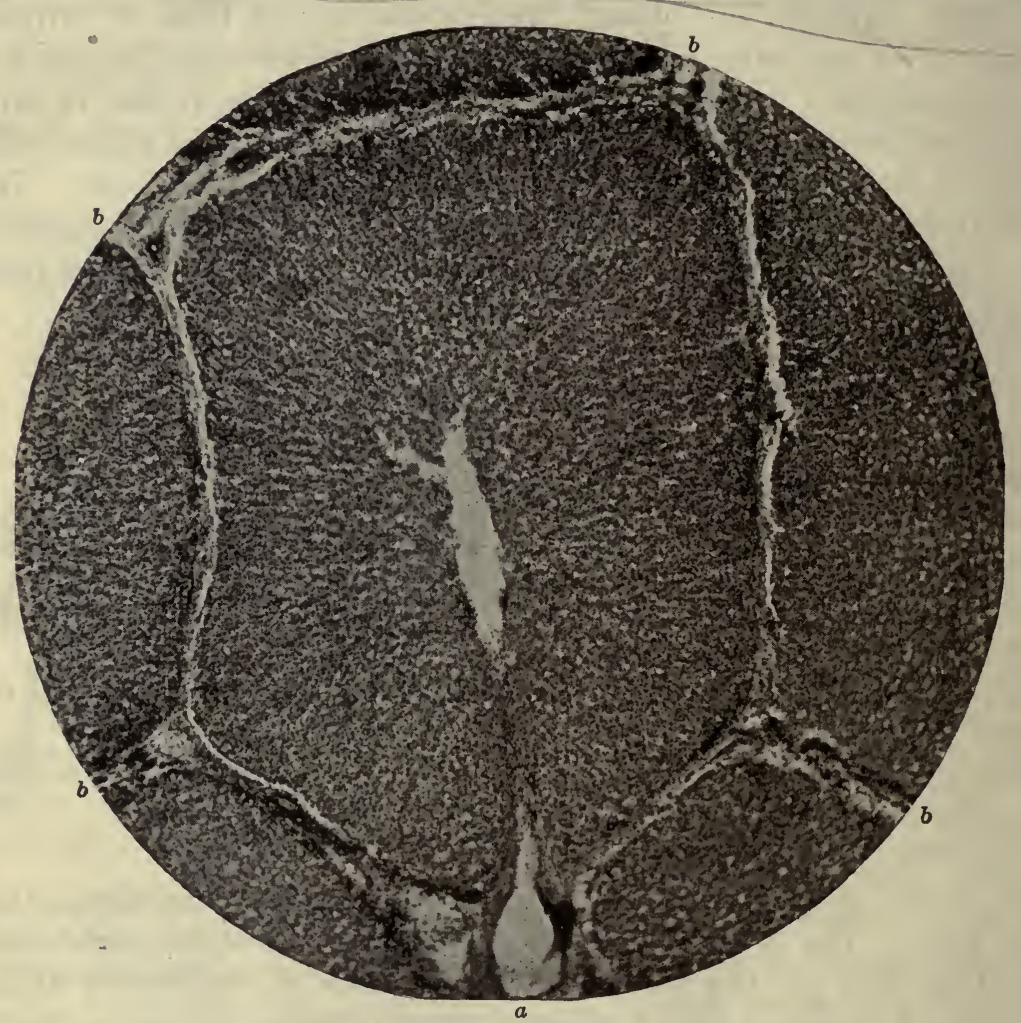

Fig. 383.-A Lobule of the Pig's Liver in Longitudinal Section, Showing the Relation of the Central and Sublobular Veins and the Arrangement of the Hepatic Cellos.

$a$, sublobular vein; $b$, capsule of Glisson. Hematein and eosin. Photo. $\times 68$.

which occupies the axis of the lobule and conveys the blood thence to the sublobular veins, which again lie in the interlobular connective tissue of Glisson's capsule.-

The hepatic cells accupy the meshes of the intralobular capillaries (sinusoids) and are arranged in cords which radiate from the central vein toward the periphery. The frequent anastomoses of the cappillaries 
as they approach the central vein produce great irregularities in the arrangement and length of the cell cords. Each cord, however, reaches the periphery of the lobule after a more or less tortuous course, and it is here-that the secretory bile capillaries, which are found within the cell cords, become continuous with-the minute bile ducts of the-portal canals.

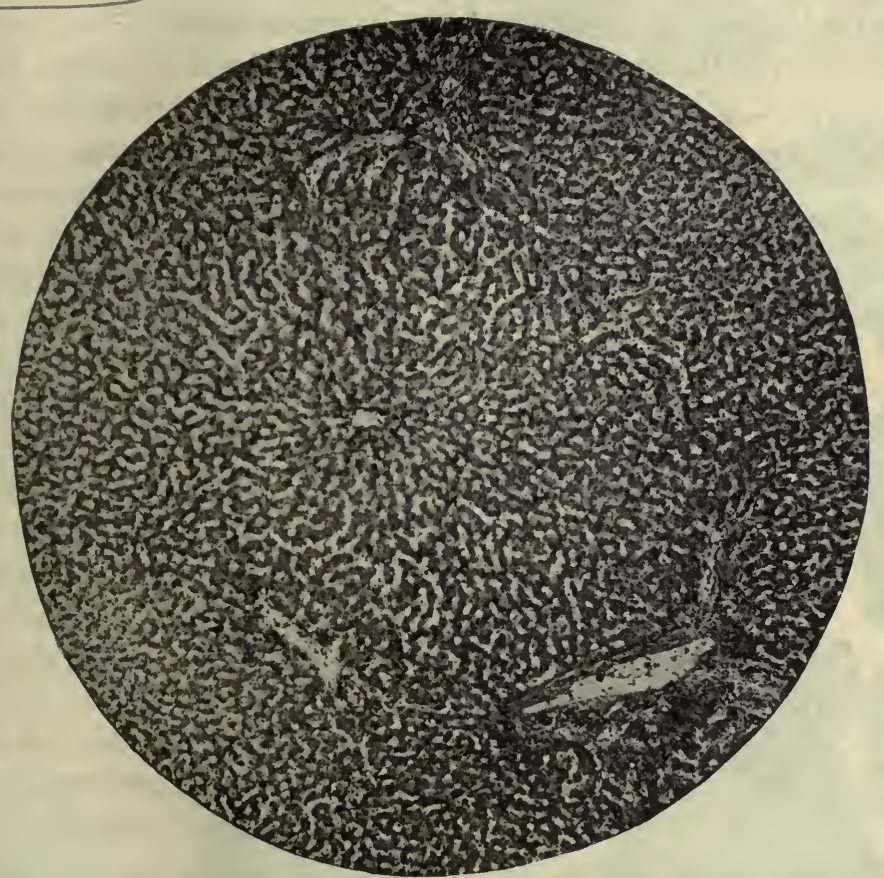

Fig. 384.-A Lobule of the Human Liver, Seen in Transection.

It is outlined by three small portal canals and contains a single central vein. Hematein and eosin. Photo. $\times 50$.

The Bile Capillaries.-The bile capillaries occur as secretory canaliculi between the opposed surfaces of the hepatic cells. They are thus found within the cell cords and stand in the same relation to the hepatic cells as though each cell cord formed a tubule-whose capiltary lumen, the bile canaliculus, was surrounded by only two secreting cells, whereas in other tubular glands a larger number of cells encircle the lumen of the secreting tubule. Hence the bile canaliculi and the blood capillaries are never in contact, but are always separated by at least one-third to one-half the diameter of a hepatic cell. The bile canaliculus occurs on that surface of the hepatic cell which is in contact with other 
cells within the cord; the blood capillary, on the other hand, is in relation with that surface of the hepatic cell which forms the periphery of the cell cord.

The blood capillaries are suspended in the fine meshes of the delicate reticulum which has already been described as the intralobular connective tissue, and which also invests the cords of hepatic cells. This reticular connective tissue is of relatively insignificant volume.

The bile capillaries are true secretory canaliculi by which the bile,

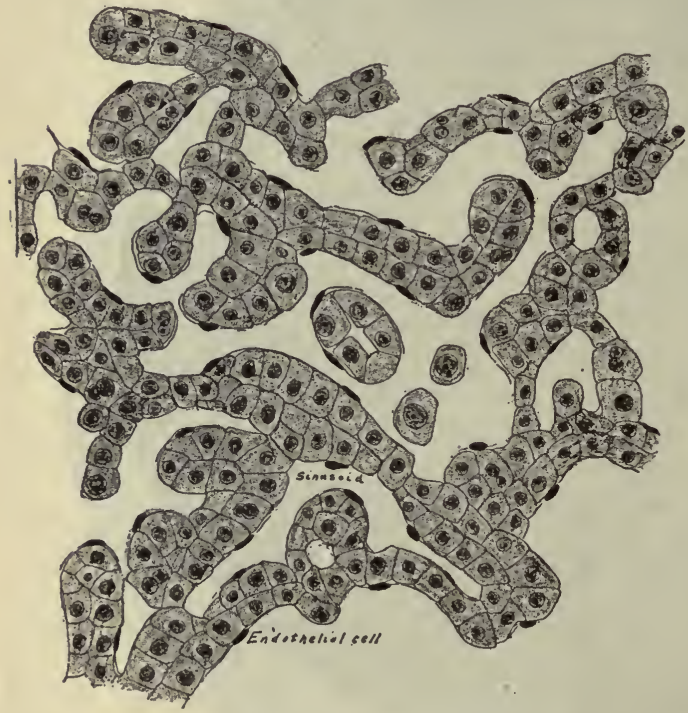

Fig. 385.-Section of Liver Tissue of Cat, Showing the Liver Cell-Cords, and the ArtiFicially Enlarged Sinusoids Lined with ENdoTHELIUM. $\times 375$. after secretion by the hepatic cells, finds its way along the anastomosing cell cords to some point at the periphery of the lobule, where the cell cord becomes continuous with a minute bile duct, the secreting cells within the lobule presenting a rapid transition to the very low columnar or flattened epithelium of the interlobular bile duct. The immediate lining of the intralobular bile canaliculus is a delicate cuticular membrane, probably the product of the hepatic cell.

\section{The Hepatic Cells.} -These are large polyhedral cells which possess one, or very frequently two, spherical nuclei and a coarsely granular cytoplasm. A true cell membrane may be regarded as being absent, yet there is often a sharply defined exoplasm which forms the surface of the cell and simulates a true membrane.-

The nuclei of the hepatic cells are rich in chromatin, and stain deeply. They are situated well within the cell, but usually in an eccentric position. Frequently they contain a distinct nucleolus.

The cytoplasm of the hepatic cells is finely reticular, the meshes being filled with coarse granules of irregular size. Many of these are undoubtedly glycogenic granules, and show a decided color reaction when 
treated with Lugol's solution of iodin after alcoholic fixation. The amount of glycogen present varies with the diet. After digestion and absorption of a carbohydrate meal it is greatly increased, but disappears during fasting. Even when glycogen is quite deficient, the hepatic cells still present a granular appearance from the presence of other substances, possibly zymogens.

Fat Globules.-Fat globules occur in hepatic cells in limited-numbers, and appear to be a normal constituent. The

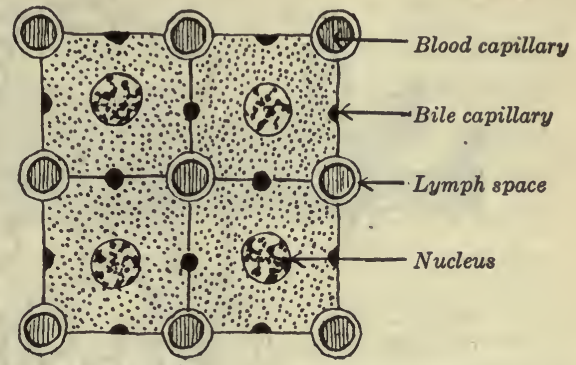
Fig. 386.-Diagram of Four Adjacent Liver Cells. globules vary much in size, but are all very small. Their number is also dependent upon diet and digestion. During absorption of a fatty meal, fat globules occur in considerable numbers, and are most numerous in those hepatic cells which are
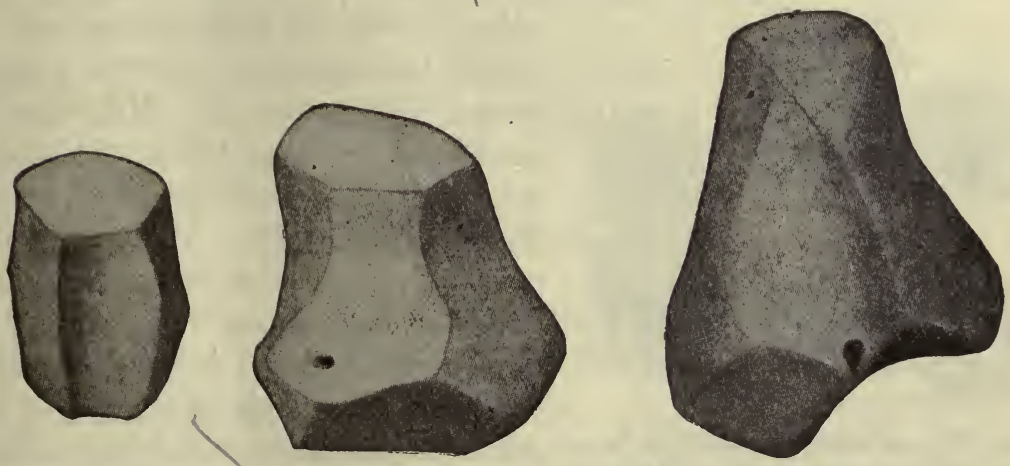

Fig. 387. Isolated Lobules of the Pig's Liver. X12.5 (After F. P. Johnson; Am. Jour. Anat., Vol. 23, 1918.)

at the periphery of the lobule. They are not normally found in the vicinity of the central vein.

The hepatic cells also frequently contain brown or yellowish-brown 


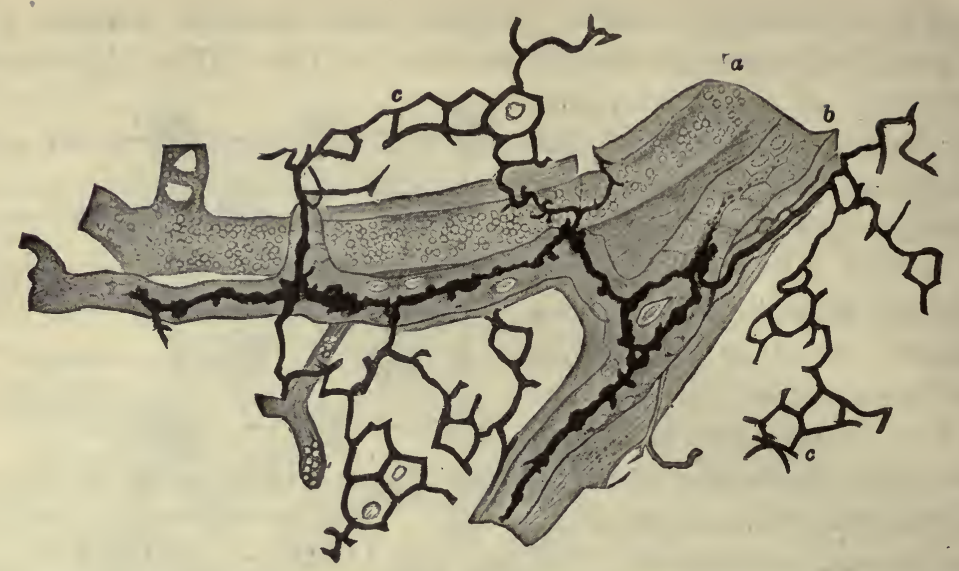

Fig. 388.-Showing the Connection Between the Intralobdlar and Interlobular Bile Ducts in the Cat's Liver.

$a$, interlobular vein; $b$, interlobular bile duct; $c$, intralobular bile capillaries. Golgi stain and hematein. Highly magnified. (After Geberg.)

granules of ferruginous PIGMENT, which are more prone to occur in the interior of the lobule near the central vein. When present in con-

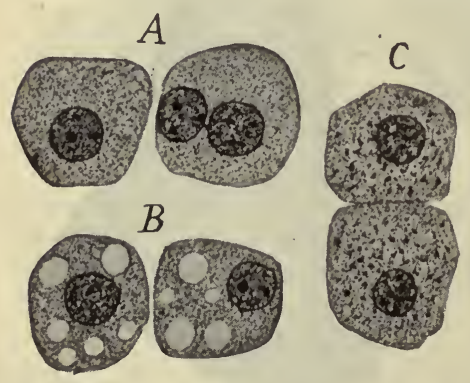

Frg. 389.-Types of Cells From a Section of the Normal Human LIVER.

$A$, the usual type of liver cell; $B$, fatty, and $C$, pigmented cells. Types $B$ and $C$ were very scarce. Hematein and eosin. $\times 900$. pear under certain functional conditions, but it is uncertain whether they are definite preformed channels, or simply transient secretory canals. longer be considered a normal constituent of the hepatic cell. Mitochondria have also been described in the hepatic cell (Policard, Compt. Rend. de Soc. et Biol., 1, 66, 1909). An intracellular canalicular apparatus can also be demonstrated within the cytoplasm. By some these channels have been interpreted as intracellular terminals of the intercellular (intralobular) bile canaliculi, by others as fixation artifacts, and still others regard them as a trophospongium. Schaffer (1902) interprets them in part as channels in relation with the blood sinusoids. Such intracellular canaliculi undoubtedly apsiderable amount this pigment can no (no 
longitudinal muscle fibers also appear in the walls of the excretory ducts, and so accumulate in the wall of the gall-bladder and common bile duct as often to form a distinct layer.

As has been pointed out by Mall (1906) the hepatic lobule cannot be regarded as the homologue of the lobule of other glands. For the portal canal with its bile duct, the excretory duct of the glandular unit, should be axial to the functional lobular unit. Moreover, an interlobular bile duct drains not the whole of a particular lobule, but portions of a number of adjacent lobules. The functional unit (portal lobule), in contradistinction to the structural unit (hepatic lobule), is accordingly that portion of liver tissue (portiens of a number of hepatic lobules) supplied by an nltimate branch of the vessels of a portal canal. Thus a hepatic lobule is drained by several bile ducts, and conversely, a single ultimate bile duct drains portions of several hepatic lobules.

Blood Supply. - The liver is supplied - with -blood from two independent sources, the hepatic artery and the portal vein. That supplied by the artery is of minor importance and is destined only for the nutrition of the connective tissue framework of the organ.

On entering the liver at the transverse fissure, the HEPATIC ARTERY gives off numerous capsular branches which ramify in the capsule of the liver and supply capillaries to its connective tissue Other branches, the direct continuation of the hepatic artery, enter the portal canals and by repeated division form the interlobular arteries, which ramify in the tissue of Glisson's capsule, and whose vaginal branches supply capillaries to this connective tissue. These capillaries, as well as those from the capsular branches, become continuous, at the periphery of the lobule, with the intralobular capillaries which are derived from the branches of the portal vein.

The Portal Vein.-The portal vein likewise enters at the transverse fissure, bringing to the liver the blood collected from the capillaries of the organs of digestion and absorption. It divides into numerous branches which follow the portal canals, in which they are known as the interlobular veins, and in this way reach all portions of the organ.

The interlobular veins throughout all their course give off small branches which at once enter the periphery of the hepatic lobules and immediately break into a brush of capillary vessels. These intralobular capillaries (capilliform sinusoids, Lewis) converge toward the center of the lobule and anastomose to form a capillary network, in the elongated meshes of which are the cords of hepatic cells. These capillaries approach the center of the lobule where they unite to form the intralobular or 
centrat veip. The central vein frequently begins in the form of a $\mathrm{Y}$, its two or more branches finally uniting to form a single vessel which pursues its course through the axis of the lobule. The central vein makes its exit at the periphery of the lobule and enters the interlobular connective tissue where it unites with its fellows to form larger $s u b$ lobular veins. The sublobular are easily distinguished from the interlobular veins by their thicker walls and by the fact that the former

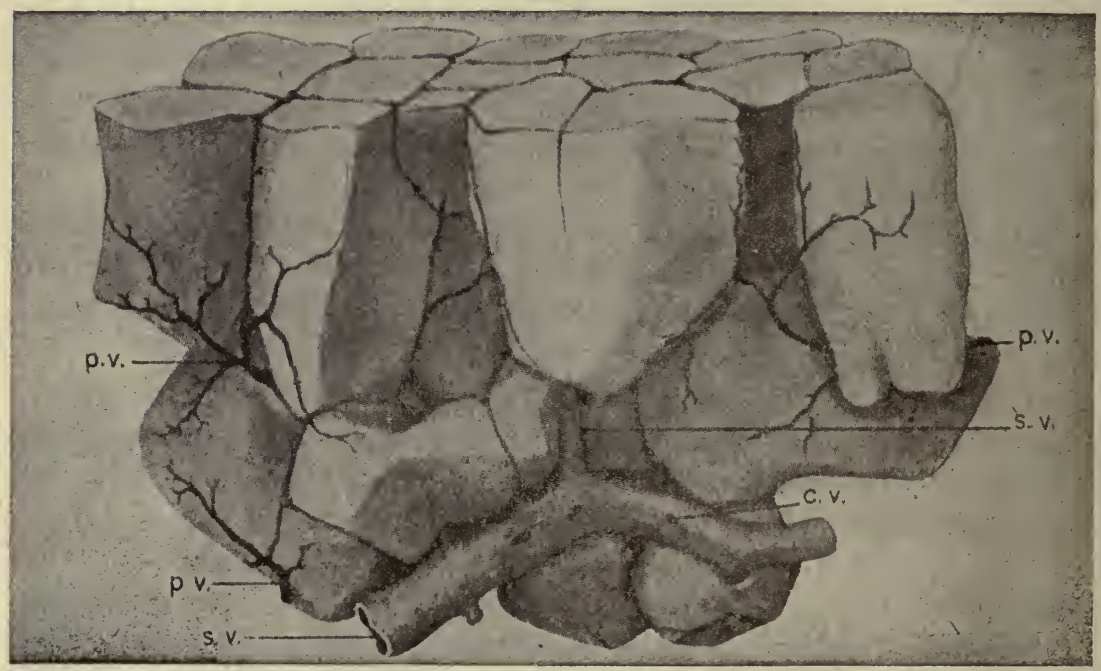

Fig. 393.-A Group of Surface Lobules of the Pig's Liver. p. v., portal vein; 8. v., sublobular (hepatic) vein; c. v., central (hepatic) vein. X10. (After F. P. Johnson.)

pursue an independent course through the tissue of Glisson's capsule, being nowhere in relation with either artery or duct.

The sublobular veins are, as a rule, vessels of considerable size, and by frequent union with their fellows become constantly larger. In their general direction they tend toward the dorsal surface of the liver and finally make their exit as four or five large hepatic veins which enter the inferior vena cava.

The blood supply of the liver, 30 per cent. of which can be accounted for in the hepatic artery, 60 per cent. in the portal vein (Mac- 
leod and Pearce, Amer. Jour. Physiol., vol. 35, 1914), is peculiar in that: (1) the greater portion of the blood has already passed through the capillaries of the digestive organs before entering the liver; (2) its arterial supply is relatively meager and supplies only the connective tissue framework, intermingling with the portal blood at the periphery of the lobule; (3) its intralobular capillaries are extremely abundant and are in intimate relation with the hepatic cells, each cell coming into contact with four to six capillary vessels.

The course of the blood through the vessels of the liver will be readily appreciated by reference, to the following table which indicates the succession of the hepatic blood-vessels:

1. Portal vein

2. Interlobular veins

3. Branches to lobule
1. Hepatic artery

2. Interlobular arteries

3. Vaginal branches and capillaries in Glisson's capsule

\section{Intralobular capillaries \\ 5. Central vein (intralobular) \\ 6. Sublobular veins \\ 7. Hepatic veins \\ 8. Vena cava inferior}

The three classes of veins, interlobular, central, and sublobular are readily differentiated by the fact that the two latter lie alone, while the interlobular veins are always in company with the ducts and arteries within the portal canals. Moreover the central vein has almost no connective tissue wall until near its exit from the lobule, where it passes into the sublobular branches; the sublobular veins, on the other hand, possess a relatively thick connective tissue wall and even some smooth muscle, except in the very smallest, which are to be regarded as mere interlobular continuations of central veins which soon unite to form the larger sublobular vessels.

Lymphatics. - The lymphatics of the liver may be considered as consisting of a superficial set which supplies the hepatic peritoneum and the capsule of the liver, and which is continuous with a deeper set in Glisson's capsule. The lymphatics of the deep set begin as perivascular spaces within the lobule, from which the lymph enters larger lymphatic vessels in the interlobular connective tissue, which follow the vessels of the portal canals to their exit from the liver; the larger lymphatics pass to the abdominal lymph nodes. Other lymphatics follow the sublobular and hepatic veins and pass to the mediastinal lymph nodes. 
Nerves.-The nerves of the liver are mostly of the non-medullated variety. They follow the portal canals and are distributed to the walls of the blood-vessels, the walls of the bile ducts, and to the capsule of the liver. Naked fibrils from these trunks also enter the lobules and form

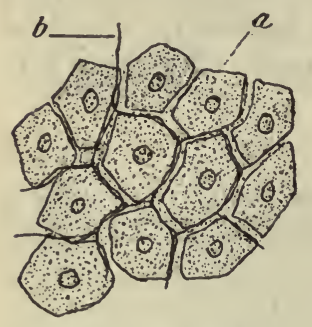

Fig. 394. - Intralob-

ular Nerve Fibers IN A RABBIT's LIVER.

$a$, hepatic cells; $b$, nerve fiber. Golgi stain. Highly magnified. (After Berkley.)

a plexus among the hepatic cells (Korolkow, Anat. Anz., 1893), in relation with which they form fine terminal brushes and varicose end knobs (Berkley, Johns Hop. Hosp. Rep., 1895).

The liver secretes the bile- a fluid which aids in the digestion and absorption of fats-and in fact probably serves also an excretory rôle. Bile has a greenish-yellow color, due in part to the presence of the pigment bilirubin, which is believed to be identical with the hematoidin elaborated from the hemoglobin of the erythroplastids disintegrating in the spleen, and carried to the liver by way of the splenic and portal veins. Under certain pathological conditions known as jaundice, bile finds its way within the liver directly into the blood stream where it produces hemolysis of the red corpuscles. In addition to its bile-producing activity, the liver functions also as an organ of internal secretion, in the conversion of glycogen into sugar and the elaboration of urea. Experimental evidence indicates that the liver is concerned greatly also with the production of fibrinogen (Whipple, Amer. Jour. Physiol., 1914).

\section{THE GALL-BLADDER}

The wall of the gall-bladder consists of three coats: (1) mucous; (2) muscular; (3) fibroserous. The mucous membrane is markedly folded or corrugated, the irregularly polygonal depressions being relatively broad at the fundus but becoming narrower toward the neck of the organ. The lining epithelium is of the tall columnar variety, with spheroidal or ovoid nuclei which lie near the base of the cell. The free extremity of the epithelial cells presents an indistinct cuticular border. The epithelium follows all the folds of the mucosa and lines the intervening depressions.

The corium of the mucosa consists of delicate connective tissue and contains a few smooth muscle fibers derived from the muscular coat. 


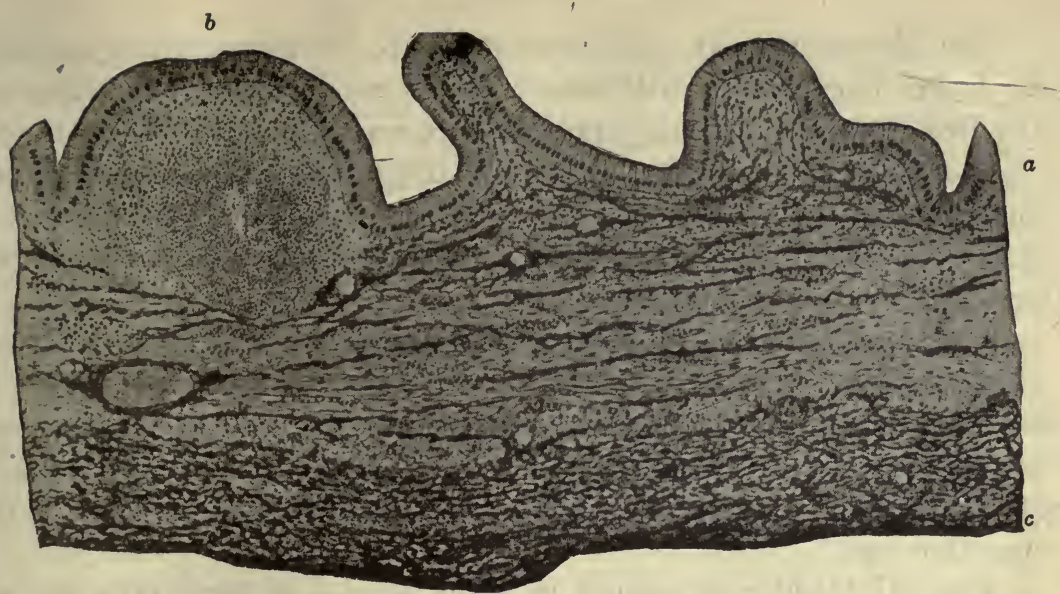

Fig. 395.-From a Section throdgh the Wall of a Dog's Gall-bladder. $a$, epithelium; $b$, lymph nodule; $c$, serous coat. $\times 80$. (After Sudler.)

It is connected with the muscularis by a thin layer of denser connective tissue which contains blood and lymphatic vessels and which simulates a submucosa.

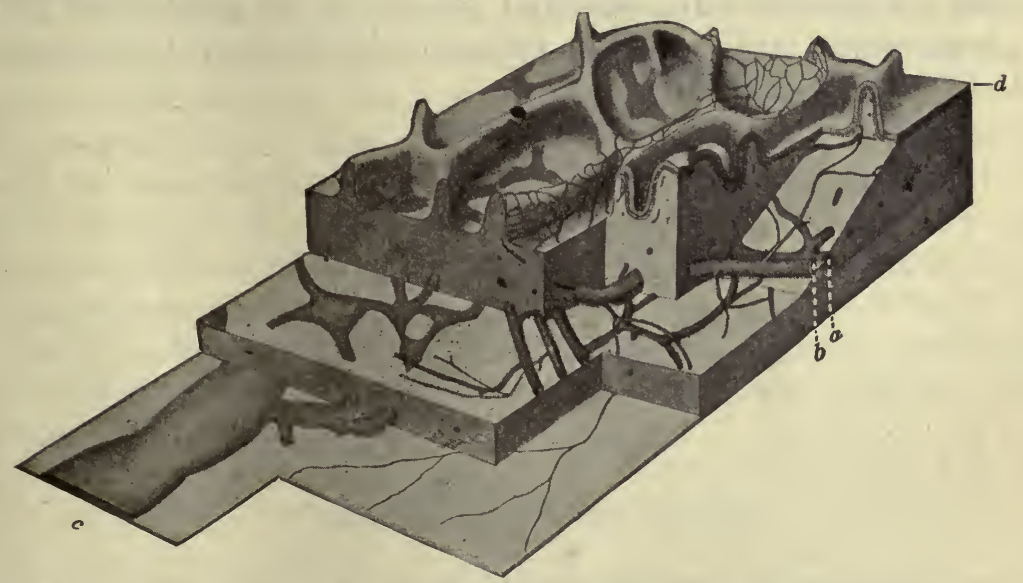

Fig. 396.-Reconstruction of the Wall of a Dog's Gall-bladder. $a$, vein; $b$, artery; $c$, lymphatic vessel; $d$, epithelium. $\times 60$. (After Sudler.)

The gall-bladder possesses a distinct muscular wall, consisting of numerous interlacing smooth muscle bundles the most of which are circularly disposed. Occasionally they form fairly distinet circular and longitudinal layers. 
The fibroserous coat consists of loose areolar tissue, which contains the larger blood-vessels with which the organ is abundantly supplied. The free surface of the gall-bladder also receives a peritoneal investment.

Occasional mucous glands occur in the mucosa of the gall-bladder. These are mostly of small size and widely separated, but toward the neck of the organ they increase in both number and size. 'They form short, branched, convoluted tubules.

The blood-vessels form a plexus just outside the muscular coat, from which branches are distributed to the peritoneal coat and to a plexus in the depth of the mucosa from which capillaries are supplied to the muscular layers and to a subepithelial plexus. The nerves are distributed to the blood-vessels and to the muscular wall. Minute ganglia occur in the muscular coat.

The gall-bladder is lacking in many species of vertebrates including the lamprey, pigeon, rat, horse, certain ruminants and the porpoise. In these instances a well-differentiated cystic anlage suffers regressive changes in later embryonic life and ultimately disappears. With the disappearance of the gall-bladder, the biliary system generally undergoes compensatory enlargement. Dilatation of the bile ducts is frequently associated with congenital absence of the gall-bladder in the human subject, and commonly follows cholecystectomy (see Scammon, Anat. Rec., 10, 8, 1916). 


\section{CHAPTER XIV}

\section{THE URINARY SYSTEM}

This system includes the kidneys, which are two large bean-shaped glands, together with their excretory passages, the ureters, which conduct the urine to the urinary bladder, whence it is voided through the urethra.

\section{THE KIDNEY}

Each kidney is a gland of the compound tubular type, measuring about four and one-half inches in length, two and one-half inches in width, and one and one-half inches in thickness. Its secretion, the urine, is produced by the uriniferous or renal tubules, which are long tortuous canals beginning near the surface of the kidney and finally ending at the hilum of the organ where they pour their secretion into the calyces of the renal pelvis. The uriniferous tubules are in intimate-relation with the renal blood-vessels which supply rich capillary plexuses to the entire extent of the tubules. Each uriniferous tubule consists of both tortuous and straight portions, and these are so regularly disposed as to produce macroscopical variations in the appearance of the different portions of the renal parenchyma according as the tortuous or the straight portions of the tubules predominate. These variations result in the following topographical subdivisions.

\section{TOPOGRaphy of the Kidney}

If the kidney be divided parallel to its long axis by an incision extending from its convex surface to the hilum, the cut surface shows that the parenchyma is divisible into a superficial cortex and a central medulla. The slit-like hilum of the organ opens into a deep excavation, the renal sinus, which is occupied by the renal pelvis and its subdivisions, the infundibula and calyces, into which the medulla projects in the form of several conical pyramids. The pelvis of the kidney, the expanded 
funnel-form beginning of the ureter, toward the renal parenchyma divides into two, sometimes three, infundibula, which in turn subdivide into several calyces, each of which incloses the conical apex of a projecting medullary or renal (Malpighian) pyramid.

The Medulla.-The medulla of the kidney consists of a number of these conical renal pyramids (usually twelve to fifteen) each of whose

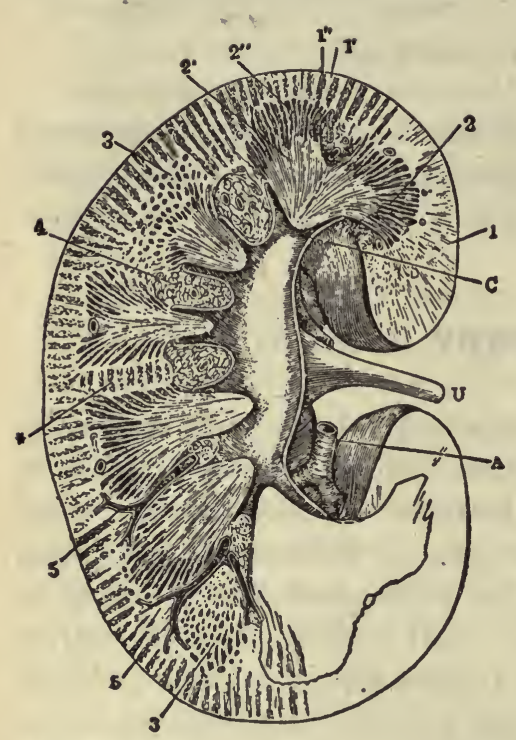

Fig. 397.-Longitudinal Section of KIDNEY.

1 , cortex; $1^{\prime}$, cortical rays; $1^{\prime \prime}$, labyrinth; 2 , medulla; $2^{\prime}$, papillary portion of medulla; $2^{\prime \prime}$, boundary layer of medulla; 3 , transverse section of tubules in the boundary layer; 4 , fat of renal sinus; 5 , artery; *, renal column of transverse medullary rays; $A$, branch of renal artery; $C$, renal calyx; $U$, ureter. (After Tyson and Henle, from Hill.) apices, as already stated, is received into the extremity of a renal calyx. Occasionally a calyx may receivé two papillæ. The base of each pyramid is embedded in the adjacent renal cortex, and that portion of the cortex which is interposed between the bases of adjacent pyramids, and thus brought into relation with the fibrous and adipose tissue which envelops the pelvis and calyces at the hilum of the organ, composes the cortical renal columns (of Bertini).

Each renal pyramid may be subdivided into a central free portion, the apical or papillary zone of the medulla, which is received into a calyx, and an outer or basal portion, which is embedded in the renal cortex and is known as the boundary zone of the medulla. These two portions of the medulla, the papillary and boundary zones, can be readily distinguished, since the latter contains only narrow tubules and is highly vascular, while the former, relatively deficient in blood-vessels, contains the broad terminations of the uriniferous tubules, the papillary ducts (of Bellini) which converge toward the apex of the pyramid where they open into the calyces.

The Cortex. - The cortex of the kidney, on careful observation, presents numerous dark lines or delicate columans which radiate from the base of the pyramids outward toward the surface of the organ. These radiating columns are the medullary rays (pyramids of Ferrein) or 
pars radiata of the cortex. They contain straight portions of the uriniferous tubules; these are continuous with the similar tubules in the boundary zone of the medulla. These columns lie within the cortex and not, as their name might be taken to indicate, in the medulla. They are termed 'medullary rays' because of their peculiar relation to the medulla, from which they extend outward in a radial direction. It would seem more proper to designate them 'cortical rays.'

That portion of the cortex which invests the cortical rays and which includes all the remaining cortical portions of the organ, consists of extremely tortuous tubules, and is characterized by the presence of small globular bodies, each of which contains a tuft of capillary vessels. These are the renal corpuscles (Malpighian bodies) which are characteristic of the kidney. The portion of the cortex in which they occur includes the entire cortical substance with the exception of the cortical rays, and is known as the renal labyrinth or pars convoluta. The labyrinth is divided into: (1) the renal columns, already mentioned; (2) the intercolumnar portions, or

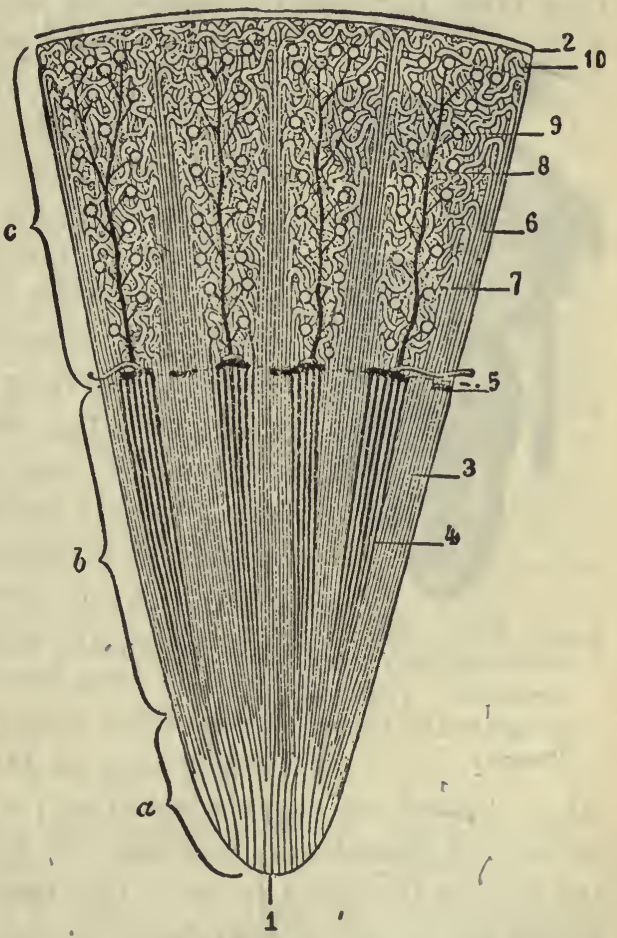

Fig. 398.-Diagram of the Structure of THE KIDNEY.

$a$, papillary zone, and $b$, boundary zone of the medulla; $c$, cortex; 1 , apex of a renal pyramid; 2 , capsule; 3 , tubules of the medulla; 4 , vasa rectæ; 5 , vascular arcades; 6 , a cortical ray; 7 , labyrinth; 8 , interlobular artery; 9 , renal corpuscle; 10 , 'cortex corticis.' (After Testut.) labyrinth proper, which includes that portion of the labyrinth which invests the cortical rays, and which, in sections cut parallel to these columns (longitudinal sections) appears as a portion of cortex inserted between the adjacent rays; (3) a narrow boundary zone of the cortex, 'cortex corticis' of Hyrtl, which is included between the fibrous capsule 
of the organ and the tips of the cortical rays, and in which the renal corpuscles, though present, are relatively few in number.

The Renal Lobule--In fetal and infantile/life the kidney is distinctly lobed. This condition is permanent in some animals-e.g., reptiles, birds, porpoise, ox, bear-each lobe consisting of a renal pyramid

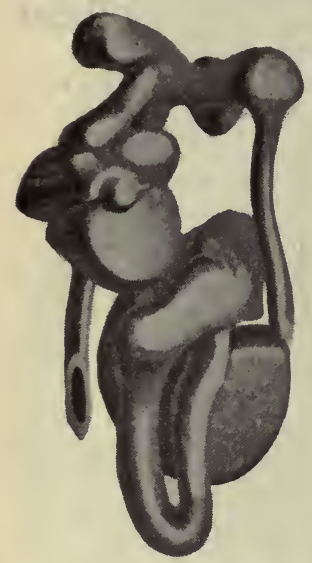

Fig. 399. - RECONSTRUCTION OF A URINIFEROUS TUBULE OF AN INFANT. (After Stoerk.) with its related portion of cortical substance. In man, after the first year, the renal lobes completely fuse and eventually leave scarcely a trace of the early lobed condition.

Internally this fetal lobed condition is in part indicated by the definitive renal pyramids, from eight to eighteen in number, each representing the product of fusion of from two to nine primitive lobes. In certain mamnals-e.g., mouse, rabbit, cat, guinea pig-neither a primitive nor definitive internal or external lobar arrangement appears. Such kidneys are designated unipyramidal or unipapillary, in contradistipction to the multipapillary type. In certain of the perissodactyls (elephant, horse) no distinct pyramids are present.

The term renal lobule or renculus, as applied to the adult human kidney, refers to a still smaller subdivision of the organ, one which includes a single cortical ray together with that portion of the cortical labyrinth by which it is immediately invested. Its peripheral boundary is marked by the interlobular blood-vessels. This lobule is the anatomical unit of the kidney and is thus comparable to the portal and to the pulmonary lobule, except that its arterial supply enters at the periphery. The tortuous secreting portions of its uriniferous tubules are contained in the labyrinth at the periphery of the lobule, while its straight conducting portions lie in the cortical ray in the axis of the lobule. The larger interlobular arteries and veins lie at the periphery, where they supply branches to several adjacent-lobules.

\section{The Renal Connective Tissues}

The kidney is enveloped by a fibrous capsule, the tunica fibrosa, which is loosely attached to the substance of the organ and contains the usual proportion of elastic fibers together with a little smooth muscle. A fatty layer, the tunica adiposa, invests the capsule. At the hilum of 
the organ the capsule is continuous with the connective tissue which envelops the renal pelvis, infundibula, and calyces, and which, in the intervals between adjacent calyces, comes into relation with the cortical substances of the renal columns.

This connective tissue of the hilum is of the areolar variety and contains much adipose tissue. It supports the large arteries and veins as they pass along the surface of the renal pelvis on their way to and from the renal columns, where they enter or leave the parenchyma. Sympathetic nerve fibers and a few small ganglia are also found in this region.

The connective tissue of the interior of the organ, interstitial tissue, is very scanty, and in most parts consists only of isolated fibrils which invest the blood-vessels and the renal tubules. It forms a very delicate reticulum by which the walls of the uriniferous tubules are loosely united. If the epithelium of these tubules is removed, a delicate fibrous network remains; this network incloses a homogeneous basement membrane upon which the lining epithelium ordinarily rests. Elastic fibers scarcely occur among the tubules of the kidney. The interstitial tissue is slightly increased in amount about the larger blood-vessels, the renal corpuscles of the cortex, and the small blood-vessels of the boundary zone of the medulla. At the apex of the renal pyramid it invests the large papillary ducts in considerable quantity.

\section{The Uriniferous or Renal Tubules}

The uriniferous tubules begin in the cortical labyrinth as the capsules of the renal corpuscles. Assuming a tubular form they then pursue a tortuous course through the pars convoluta and finally enter the boundary zone of the medulla, where, much reduced in size, they form the loop of Henle, which consists of a short, descending, thin limb, a U-shaped loop, and a long, ascending or thick limb. This last division, after recrossing the boundary zone of the medulla, enters a pars radiata and returns to the region of its origin, where it becomes again convoluted. A short arched tubule connects this convoluted portion with a straight collecting tubule of the cortical ray. The collecting tubules traverse the whole length of the ray, uniting with their fellows and receiving other arched tubules along their entire course. They then cross the-boundary zone of the medulla, and finally, in the papillary zone, having meanwhile received numerous accessions of straight collecting tubules, form the 
large terminal tubules, the papillary ducts, which pour the urinary secretion into the renal calyces.

Each uriniferous tubule may thus be subdivided into several portions

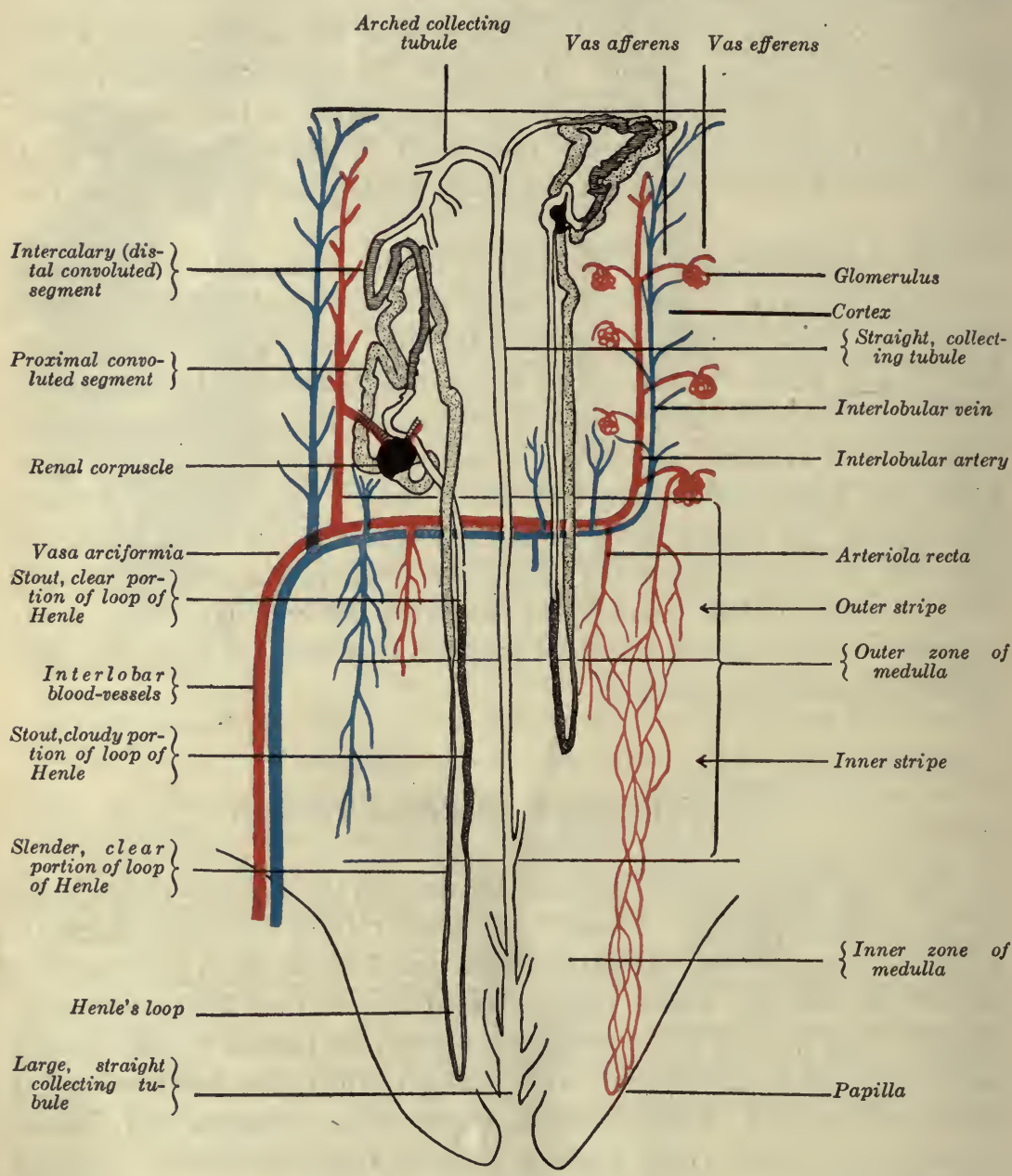

Fig. 400.-Diagram of Uriniferous Tubule of a Mammal. (Adapted from Peter and Merkel.)

which differ from each other, not only in their location, but also in the character of their lining epithelium. The successive portions which compose a single uriniferous tubule may be enumerated as follows: 
1. Capsule of the renal corpuscle.

2. Neck of the tubule.

3. Proximal convoluted portion.

4. Descending limb of Henle's loop.

5. Loop of Henle. Medullary loop.

6. Ascending limb of Henle's loop.

7. Distal convoluted portion.

8. Arched collecting tubule.

9. Straight collecting tubule.

10. Papillary duct.

It should be borne in mind that all of these several portions form only successive parts of a single uriniferous tubule. Those portions of the urine which are secreted into the capsule of the renal corpuscle must therefore find their way through each of these successive portions before it can reach the excretory passages of the renal calyces, pelvis, and ureter.

1. The Renal Corpuscle (Malpighian Body).-A renal corpuscle consists of a spherical tuft of capillary vessels, the glomerulus, which in the course of its development is invaginated into the end of the uriniferous tubule, and thus comes to be enveloped by a double layer of flattened epithelial cells known as the glomerular capsule (of Bowman).

The inner visceral layer of the capsule closely invests the entire surface of the glomerulus, except at that point wherethe afferent and efferent vessels. enter and leave the capillary tuft; at this point the visceral epithelium is reflected outward and becomes continuous with the parietal layer. The sur-

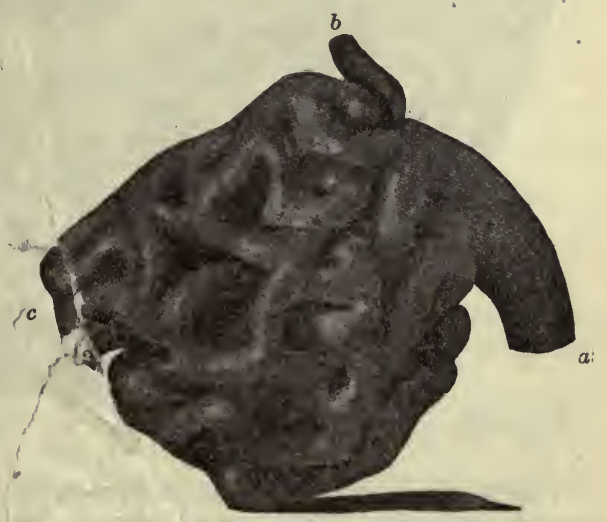

Fig. 401.-Feconstruction of a GlomerULUS OF THE HuMAN KidNEY.

$a$, afferent 'arteriole; $b$, efferent arteriole; c, capillaries. $\times 444$. (After Johnston.) faces of these two layers are almost in apposition; the narrow interval between them which results from the slightly eccentric position of the glomerulus forms the first portion of the lumen of the uriniferous tubule. At that pole of the renal corpuscle which is opposite the entrance of its 
blood-vessels the capsule opens, through a narrow neck, into the first or proximal convoluted portion of the uriniferous tubule.

The glomerulus is a true arterial rete mirabile, since it receives an afferent artery, which, after forming the capillaries of the glomerulus,

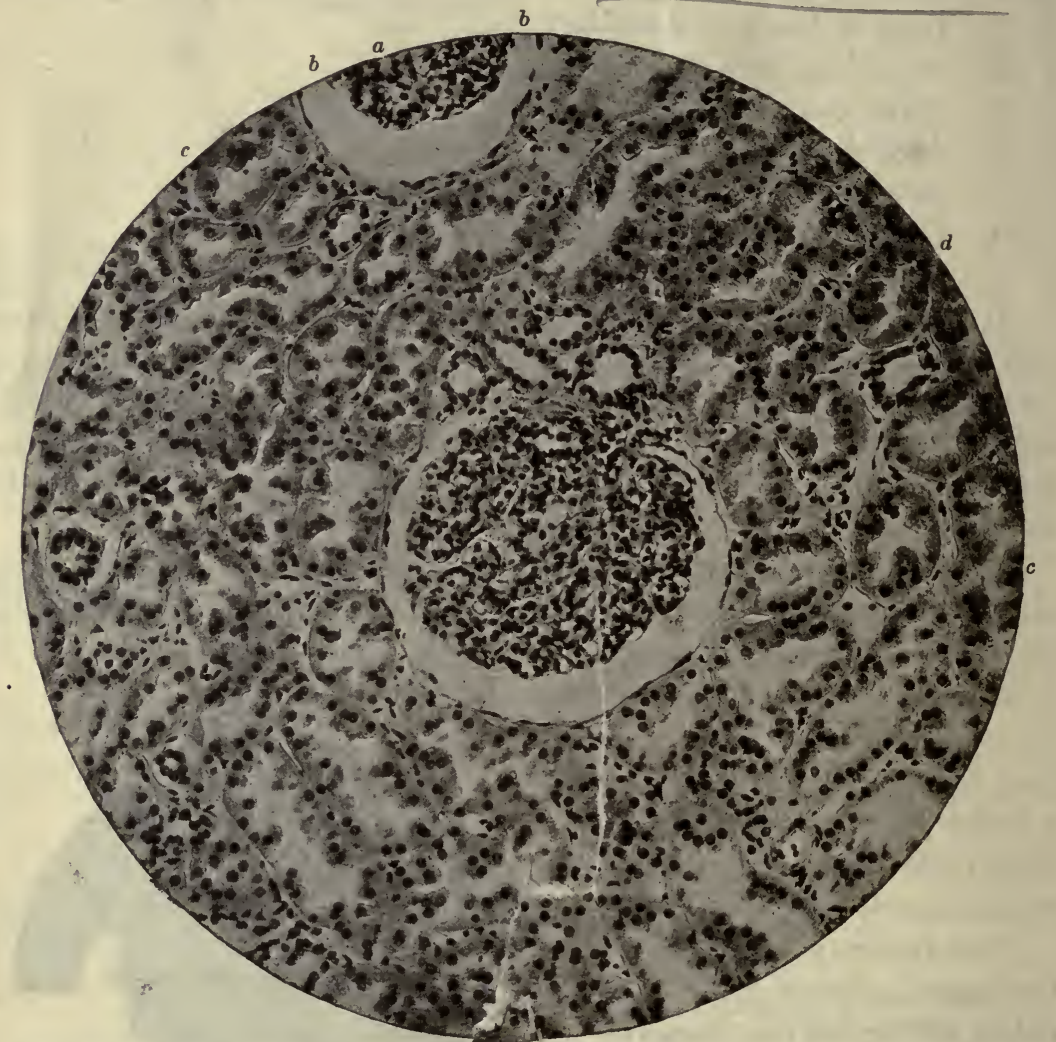

Fig. 402.-From the Cortical I Ibyrinth (Pars Convoluta) of the Human KIDNEY.

A large renal corpuscle is in the center of the figure. At its upper border are several sections of distal convol ated tubules. The great majority of the tubules shown are from the proximal ecnvoluted portions. $a$, a portion of a glomerulus; $b-b$, parietal layer of the capsule; $c$, proximal convoluted tubules; $d$, just within this point is a transection of . junctional tubule having relatively low and clear epithelium and a broad lumer. Hematein and eosin. Photo. $\times 135$.

passes out as an efférent artery to again enter a capillary plexus about the neighboring tubules of the renal cortex. The afferent vessel is of somewhat larger caliber than the efferent-a noteworthy fact because of its relation to the intraglomerular blood pressure. 
On entering the glomerulus the artery divides into two vessels which immediately subdivide with the formation of five branches (Johnston). Each of these branches forms a series of anastomosing capillary loups whose convexity is directed away from the entering artery. The capillary loops reunite, in a similar manner, to form the efferent vessel, which leaves the glomerulus in company with the afferent; but, once out, they soon part company, the efferent vessel breaking into a second capillary plexus about the neighboring tubules. Within the glomerulus the capillaries are united by a very delicate but scanty connective tissue containing no elastic fibers.

The visceral layer of the capsule is firmly adherent to the walls of the glomerular capillaries. It consists of a single layer of flat epithelial cells which are intimately blended with each other and with the endothelium of the capillaries. The epithelial cells possess a clear cytoplasm and a flattened ovoid nucleus, which, being thicker than the body of the cell, produces a considerable bulging. In fetal and infantile life the shape of the cells of this layer is cuboidal or even low columnar, but becomes more and more flattened as development progresses, until the epithelium finally simulates a layer of endothelial cells.

The epithelium of the parietal layer is also cuboidal in fetal life, but during development becomes nearly as much flattened as that of the visceral layer. Its single layer of finely granular cells forms a complete lining for the capsule. It rests upon a homogeneous basement membrane which is invested by a thin layer of connective tissue. This fibrous layer is rather more highly developed about those renal corpuscles which lie near the medulla than about those of the more peripheral portions of . the cortex.

2. The Neck of the Tubule.-In this portion of the tubule the flattened epithelium of the glomerular capsule rapidly changes to the low columnar type of the proximal convoluted portion. This section is extremely short; it forms a constricted portion which marks the beginning of the tortuous tubule. This constriction is more apparent than real, since the caliber of the tubule in the neck is as great as in the succeeding portion whose external diameter is, however, much increased by the increasing height of the epithelial cells. This portion of the tubule, being in relation with the renal corpuscle, is necessarily found in the cortical labyrinth.

3. The Proximal Convoluted Portion.-This is commonly the longest and broadest portion of the uriniferous tubule. Collectively the convoluted tubules form the greater part of the cortical labyrinth, in 
which region only they occur. This portion of the tubule is remarkable for the irregularity of its course, it being twisted and bent upon itself in a most tortuous manner. Arising at the renal corpuscle, it at first passes toward the surface of the organ, but soon turns about and runs toward

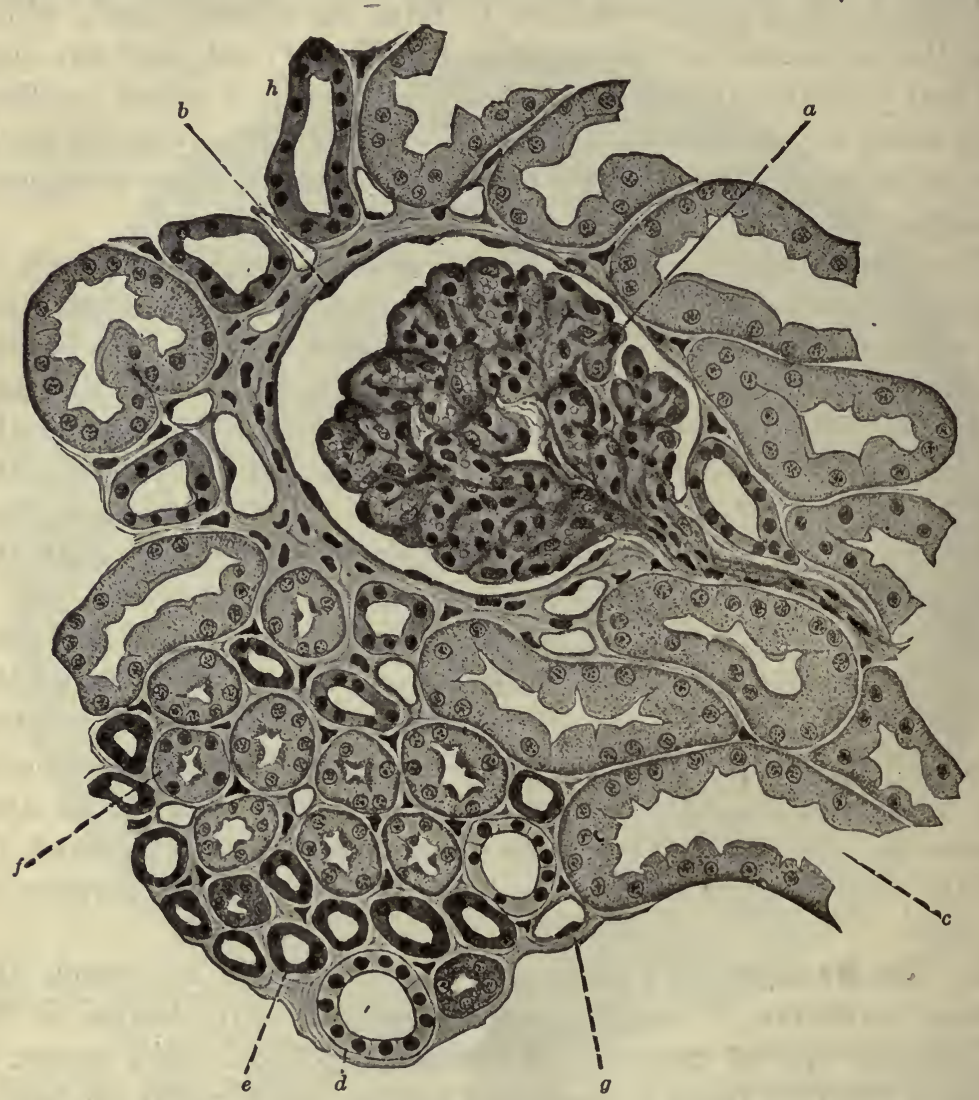

Fig. 403.-From the Cortex of the Human Kidnex, Showing a Transection of a Cortical Ray in the Lower Left-hand Corner.

$a$, glomerulus; $b$, glomerular capsule; $c$, proximal convoluted tubule; $d$, collecting tubule; $e$, ascending limb of Henle's tubule; $f$, spiral tubule; $g$, blood-vessel; $h$, distal convoluted tubule. Hematoxylin. $\times 200$. (After Schaper, from Stöhr.)

the medulla, at first with extreme convolutions, but later pursuing a rather spiral course (spiral tubule). On reaching the border of the medulla the tubule becomes sharply constricted and enters the medullary boundary zone at the thin descending limb of Henle's loop. 
The epithelium of the convoluted tubule is of the columnar or pyramidal type, its cells having broad, firmly united bases and conical free apices. The lateral tnargins of the cells are often so intimately blended at the base as to resemble a syncytium. When isolated, or if outlined by impregnation with silver salts, the borders of the epithelial cells are extremely irregular and are deeply fluted or serrated, the serrations of each cell interdigitating with those of its neighbors. The deep fluted ser-

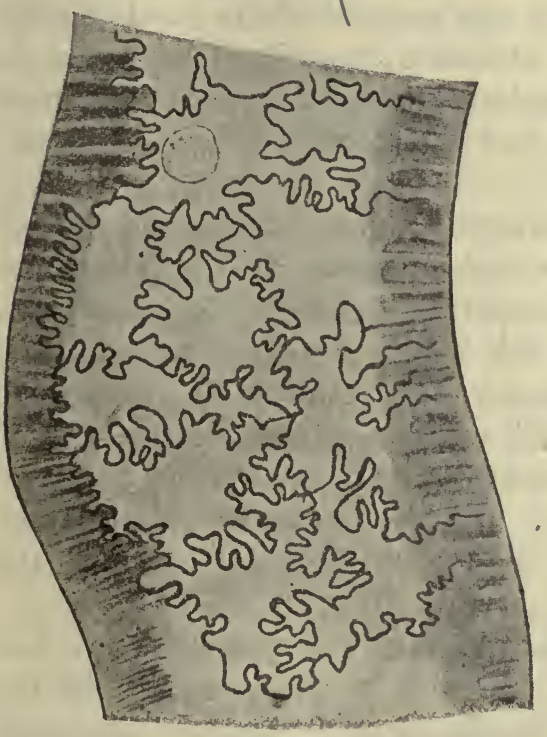

Fig. 404.-From a Longitudinal Section of a Convoluted Tubule of the 'Guinea-Pig's Kidney.

The cell outlines have been blackened by the Golgi method. Very highly magnified. (After Landauer.)

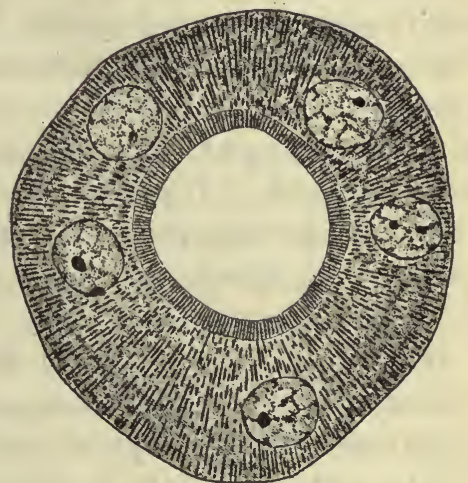

Fig. 405.-Cross Section of a Proximal Convoluted Tubule FROM THE KIDNEY OF A Mouse, Showing Basal Filaments (Probably Largely MitochonDRIal) Breaking UP INTO Granules Centrally, and the CenTRAL Striated Border of the Cells.

Meves' mitochondrial technic. $\times 1000$.

rations of the interlocked epithelium gives many of its cells a coarsely striated appearance, the striation being more prominent beneath the centrally situated nucleus than in the apical portion of the cell. Other longitudinal striations in the proximal or basal portion of the cell are the result of a linear arrangement of granules and filaments which occur in this part. These appearances often give the epithelium of the convoluted tubules a peculiar striated or 'rodded' character. The granules and filaments are probably largely mitochondrial in nature.

The apices of the epithelial cells are very easily destroyed, but when 
perfectly preserved often present a delicately striated, cuticular border ('brush border'). The remaining portions of the cytoplasm are finely granular.

The nuclei of the epithelial cells of the convoluted tubules are spherical in shape, and do not stain very deeply with nuclear dyes as compared with the more distinct and deeply staining nuclei of the collecting tubules. Thus they appear as if partially clouded by the granular cytoplasm, an appearance which is greatly exaggerated with the onset of acute inflammatory processes, which, on attacking the kidney, are prone to involve the convoluted tubules. The chromatin is quite evenly distributed throughout the nucleus and the nuclear membrane is not easily demonstrated.

The lumen of the convoluted portion of the uriniferous tubule is of variable caliber; it presents frequent slight dilatations. The caliber also depends, to some extent, upon the secretory activity of the epithelium, whose cells become shrunken, and the lumen correspondingly dilated, during active secretion. The diameter varies from 40 to 60 microns. The convoluted tubules are most actively engaged in the secretion of urine, but the further changes accompanying their secretion have not yet been satisfactorily demonstrated. It is generally believed that the water and salts of the urine are secreted in the glomerular capsule, the urea in the convoluted tubules.

4. Descending Limb of Henle's Tubule (The Thin or Narrow Tubule of Henle).- In this portion, which is, typically, located in the boundary zone of the medulla, the uriniferous tubule becomes very much narrowed (8-15 $\mu$ ), but the decreased diameter is the result of diminished height of the lining epithelium rather than of any change in the caliber of the tubule. The length of this portion of the tubule is very variable; typically it corresponds very nearly with the breadth of the medullary boundary zone.

The lining epithelium of the descending limb is of a peculiar flattened shape. Its cells possess an ovoid nucleus which, being thicker than the surrounding portions of the cell, projects slightly into the lumen of the tubule. The bulging nuclei of opposite sides of the tubule are not in apposition but interlock with one another, the nuclei of one side of the tubule being opposed to the cell margins of the opposite side. The lumen of longitudinal sections through the axis of the tubule thus acquires a sort of zigzag outline. The nuclei stain deeply but possess an evenly distributed chromatin. The cytoplasm of the epithelium is very finely granular, and although its cells are intimately adherent at their 
lateral margins they do not present the typical striations which are characteristic of the preceding portion.

5. The Loop of ffenle.-As the descending limb enters the loop of Henle, the tubule makes an abrupt turn and returns toward the cortex. The location of the loop, being dependent upon the variable location in the cortex of the renal corpuscle and the variable length of the thin segment, may be in any portion of the medulla except the extreme tip of the pyramids; its most frequent site, however, is near the junction of the boundary and papillary zones.

The structure of the loop may be that of either the descending or the ascending limb of the typical loop. It is also subject to great variations, since the change in structure from the narrow to the broad type, though it typically occurs just prior to the formation of the loop, is frequently delayed until well into the ascending limb. As a rule, the change in type occurs earlier when the loop lies in the boundary zone, and later when it occurs nearer the apex of the renal pyramid; the thick ascending limbs do not occur in the papillary zone of the medulla.

6. The Ascending Limb of Henle's Loop (The Broad or Thick Limb).-This portion of the tubule returns through the boundary zone of the medulla and enters a cortical ray, its course being parallel to that of the descending limb. It then passes toward the surface of the kidney, but finally leaves the ray and enters the labyrinth to reach that renal corpuscle (close to the vas efferens) from which the uriniferous tubule took origin, and in relation to which the tubule again acquires a tortuous course (distal convoluted portion). Within the boundary zone of the medulla this portion of the tubule is much broader than the preceding division, but it becomes somewhat reduced in size in its course through the cortical ray.

The epithelium of the ascending limb is of a short cuboidal form. Its cytoplasm resembles that of the lining epithelium of the convoluted portion, although the nuclei in the tubule of Henle are rather more distinct. Basal striations are also less distinct than in the convoluted tubule, the lateral serrations less deep, and the cell outlines sharper. The cells of this portion frequently possess a slightly imbricated arrangement.

The recent careful comparative studies of mammalian kidneys by Peter ("Untersuchungen über Bau and Entwickelung der Niere," Jena, 1909) have revealed certain important details. Peter divides the medullary portion of the renal pyramid into an outer and inver zone; these portions have no precise correspondence with the boundary and papillary zones above 
mentioned. In the outer zone Peter describes an outer and an inner stripe, the line of demarcation being the very definite level where the proximal convoluted tubule passes into the thin, clear, narrow limb of Henle's loop (Fig. 400). The point of transition from outer to inner zone is marked by a change in the character of the epithelium lining the distal limb of the long Henle's loops; the epithelium becomes cloudy (granular) and thicker. In view of the great variability in the character of the epithelium at the vari-

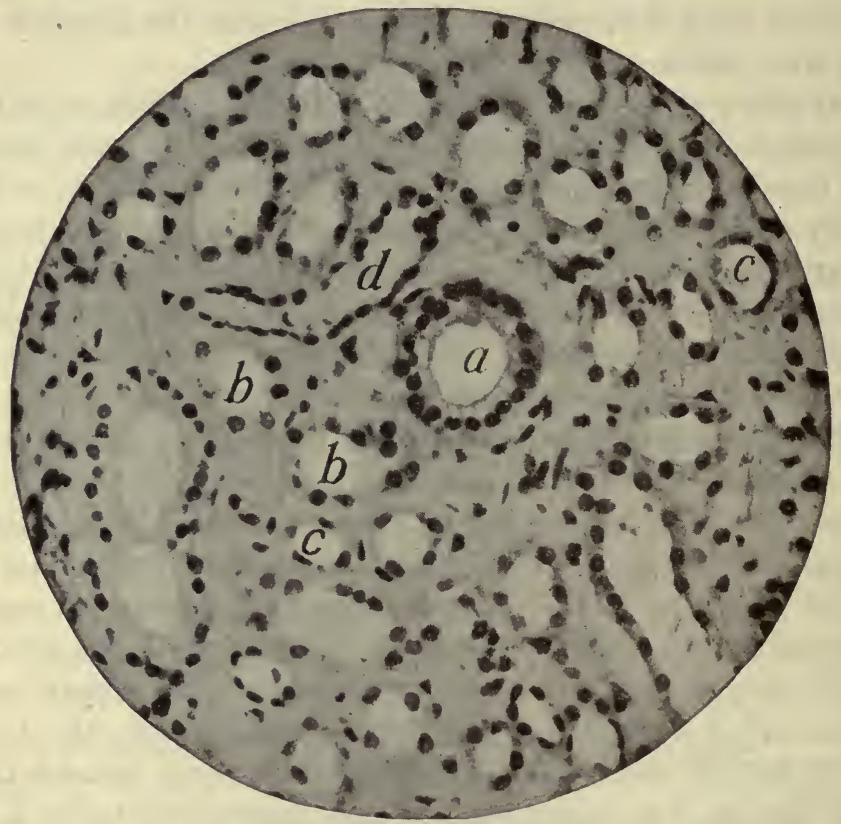

Fig. 406.-A Group of Tubules from a Transection of a Renal Pyramid of the Human Kidnex; the Section Passes through the Boundary Zone.

$a$, collecting tubule; $b$, ascending limb of Henle's loop; $c$, descending limb; $d$, loop of Henle. Hematein and eosin. Photo. $\times 275$.

ous levels in loops of different lengths, and the recognition by Peter of a thicker cloudy segment, it seems better to speak of thin clear, thick clear, and thick cloudy segments of the loop. In kidneys of the dog and cat there are no really short loops, none lying within the outer zone. In the kidney of the pig many loops lie in the cortex; such loops lack entirely the thin, clear narrow limb.

7. The Distal Convoluted Portion (Intercalary or Intermediate Portion).-This portion of the uriniferous tubule begins close to the vascular pole of the renal corpuscle, and, after several irregular contor- 
tions which are confined to the region of the cortical labyrinth, enters an arched collecting tubule. The distal is much shorter than the proximal convoluted portion. Its caliber is subject to great irregularities, so much so that its early turns have been characterized as the irregular or zigzag portion of the uriniferous tubule. The epithelium of this section resembles that of the proximal convoluted portion but is lower, more cuboidal, and striations-are indistinct.

This portion terminates the typically secretory portion of the uriniferous tubule. Beyond here the tubule possesses more the function of a duct, hence its epithelium shows a decided change in character. Hitherto it has possessed the peculiar character, the typically granular cytoplasm, of a secreting type of cell. Beyond this section the epithelium is no longer so granular but possesses a characteristically clear appearance. The secretory and excretory portions of the renal tubule have a separate embryonic origin, and only secondarily unite to form a continuous duct.

8. The Arched Collecting Tubule (Junctional Tubule).-This is a short portion of the uriniferous tubule which connects the distal convoluted portion in the cortical labyrinth with the straight collecting tubules of the cortical rays. Its course is characteristically arched.

The epithelium of the arched tubule consists of clear cuboidal cells with distinct outlines and deeply stained, sharply defined nuclei. The chromatin of the nucleus is irregularly distributed, forming numerous karyosomes, and the nuclear membrane is-distinct. The cytoplasm is relatively devoid of granules, and unlike that of the secreting epithelium does not possess a strong affinity for the acid dyes.

9. The Straight Collecting Tubules.-These portions of the tubules begin in the cortical rays, where they receive the arched tubules, and, proceeding to the medulla, become considerably increased in size. They penetrate the boundary zone of the medulla, all pursuing a parallel or slightly convergent course, and occasionally uniting with each other. On entering the papillary zone, by frequent union they become rapidly larger, and in the apex of each pyramid finally form about a score of large terminal papillary ducts. Though shorter than the convoluted tubules, the straight collecting portions, because of their direct course, traverse a broader area of the renal tissue; beginning near the peripheral end of the cortical rays, in these columns they cross nearly the whole breadth of the renal cortex and entering the medulla extend from base to apex of the renal pyramid.

Throughout their whole course they progressively increase in size and caliber. A corresponding progressive increase in the height of their epi- 
thelial cells likewise occurs, so that the lumen of the straight tubules of the medulla is not only actually greater than that of those of the cortical rays, but the walls of the former tubules are also considerably thicker. The extreme of this progression is found in the broad lumen and tall epithelium of the papillary ducts.

The epithelium of the straight tubules, like that of the arched, possesses a clear cytoplasm, distinct and deeply/staining chromatic nuclei, and well defined cell outlines. Beginning in the cortical rays with a low columnar type, it gradually increases in height in the course of the tubule until, in the papillary zone, the epithelium acquires a tall columnar form. The clear cytoplasm and distinct nuclear membranes of the epithelium of the collecting tubules stand out in sharp contrast to the granular cytoplasm and the evenly distributed chromatin in the nuclei of the lining cells in the secreting portions of the uriniferous tubules.

10. Papillary Ducts. (Ducts of Bellini).-These are the wide mouths of the uriniferous tubules which are formed by the dichotomous union of from ten to thirty collecting tubules, and which empty their secretion into the renal calyces at the apex of the renal pyramids. They attain a diameter of from two hundred to three hundred microns. They are lined by tall columnar cells with an exceptionally clear cytoplasm which has an affinity for the basic in preference to the acid class of dyes. The nuclei are spheroidal or ovoid in shape and lie in the basal portion of the cell. At their termination several papillary ducts frequently open into a common depression or foveola which is lined by an involution of the layer of transitional epithelium, derived from that of the renal calyx, by which the free papillary portion of the renal pyramid is clothed.

It is obvious that the entire renal tubule, from the glomerular capsule to the papillary duct, is a continuous canal whose epithelial wall, supported by a thin homogeneous basement membrane varies in character in each succeeding portion. Thus the proximal and distal convoluted portions and the typical ascending limbs of Henle's loops possess a granular, rodded or striated, acidophil, secreting epithelium; the capsule has thin cells of an endothelioid type; the typical descending limb and loop of Henle are lined by flattened finely granular and faintly acidophil epithelium; the curved and straight collecting tubules and papillary ducts possess a clear columnar epithelium. It should also be noticed that the several portions of the renal tubule occur in different topographical sub- 
divisions of the kidney and that, therefore, each of the subdivisions contains only certain characteristic portions of the renal tubule. Thus

are found in the-
CoRTICAL LABYRINTH.
(Pars Convoluta) $\left\{\begin{array}{l}\text { Renal corpuscle. } \\ \text { Neck of the tubule. } \\ \text { Proximal convoluted portion. } \\ \text { Distal convoluted portion. } \\ \text { Arched collecting portion. }\end{array}\right.$

Cortical Rays....... S Spiral portion of the convoluted tubule.

(Pars radiata)

Ascending limb of Henle's tubule.

Straight collecting portion.

Boundary Zone of the
Medulla ...........

Descending limb of Henle's tubule.

Papillary Zone of the Loop of Henle's tubule.

Medulla........ Straight collecting portion.

Papillary duct.

The following outline, based upon the investigations of Peter, gives a more detailed classification of the different portions of the renal tubule in the medulla :

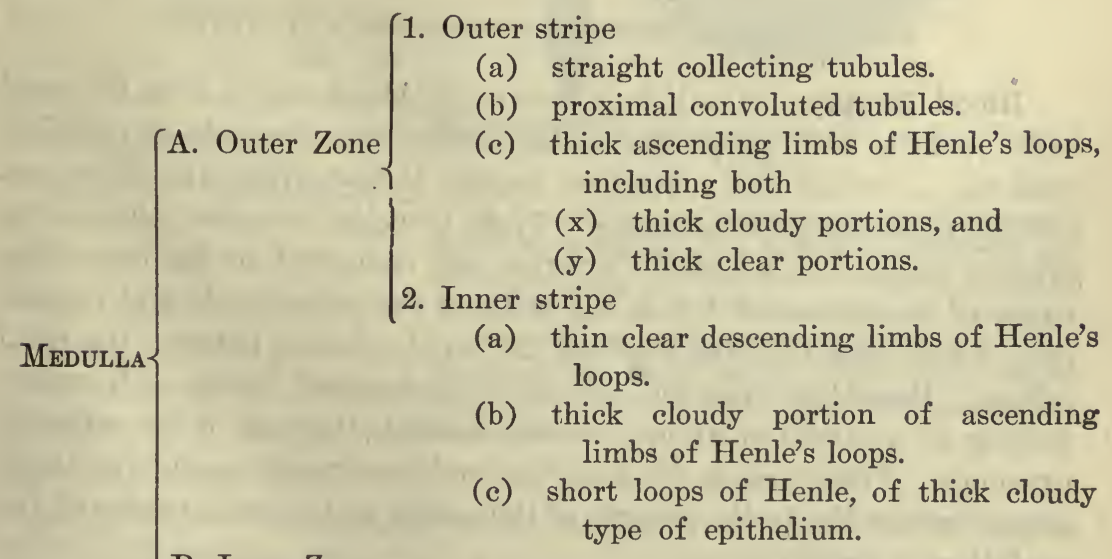

B. Inner Zone

(a) straight collecting tubules.

(b) long loops of Henle, entirely of thin clear type of epithelium. 
The following tabular résumé may be of service by emphasizing the more important peculiarities of the several portions of the uriniferous tubule.

\begin{tabular}{|c|c|c|}
\hline Portion of Tubule. & EPithelium. & Location. \\
\hline $\begin{array}{l}\text { Renal corpuscle. } \\
\text { Neck. }\end{array}$ & Flattened, endothelioid. & $\begin{array}{l}\text { Cortical labyrinth. } \\
\text { Cortical labyrinth. }\end{array}$ \\
\hline Proximal convoluted. & Low columnar, granular, & Cortical labyrinth. \\
\hline Spiral portion of above. & $\begin{array}{l}\text { Low columnar, granular, } \\
\text { and rodded. }\end{array}$ & Cortical rays. \\
\hline Descending limb. & $\begin{array}{l}\text { Low cuboidal or flat- } \\
\text { tened, granular. }\end{array}$ & $\begin{array}{l}\text { Medulla (boundary and } \\
\text { papillary zones). }\end{array}$ \\
\hline Loop. & $\begin{array}{l}\text { Varies; like either the } \\
\text { preceding or following. }\end{array}$ & $\begin{array}{l}\text { Medulla (boundary and } \\
\text { papillary zones). }\end{array}$ \\
\hline Ascending lim & $\begin{array}{l}\text { Cuboidal or low colum- } \\
\text { nar, granular, imbri- } \\
\text { cated. }\end{array}$ & $\begin{array}{l}\text { Boundary zone of me- } \\
\text { dulla and cortical rays. }\end{array}$ \\
\hline Distal convoluted. & $\begin{array}{l}\text { Low columnar or pyram- } \\
\text { idal, granular, and rod- } \\
\text { ded. }\end{array}$ & Cortical labyrinth. \\
\hline Arched collecting. & $\begin{array}{l}\text { Cuboidal, clear cyto- } \\
\text { plasm, dark nucleus. }\end{array}$ & Cortical labyrinth. \\
\hline Straight collecting. & $\begin{array}{l}\text { Cuboidal, changing to } \\
\text { columnar. }\end{array}$ & $\begin{array}{l}\text { Cortical rays and both } \\
\text { zones of medulla. }\end{array}$ \\
\hline Papillary duct. & Cuboidal, tall columnar. & Papillary zone of medulla. \\
\hline
\end{tabular}

\section{Renal Blood-vessels, Iymphatics and Nerves}

Blood Supply. - The kidney receives its blood supply from the renal artery, which, as it enters the hilum, divides into two sets of principal branches, of which the ventral set supply three-fourths, the dorsal set one-fourth of the renal substance.. These principal branches, the arterice proprice renales, or interlobar arteries, are embedded in the connective tissue of the sinus and follow the walls of the infundibula and calyces, upon which they lie, thus reaching the renal/columns between the renal calyces. Here they entèr the cortical substances and dividè, each branch passing in a curved or arched manner beneath the base of the adjacent, pyramids. These vessels form an incomplete arterial arcade (arciform artery) which lies in 'the'margin of the cortex at the outer border of the medullary boundary zone.

From the arterial arcade, branches are given to the medullary tissue of the pyramids on the one hand, and on the other to the cortical substance. Those branches which enter the medulla are slender vessels which 
pursue a characteristically straight course between the parallel tubules of this region and are known as the arteriolic recto. They branch freely at acute angles and form a rich capillary plexus in the boundary zone, the longest of the vessels reaching beyond the limits of this region to supply a less abundant plexus to the papillary zone of the medulla.

The cortical branches of the arterial arcades are vessels of considerable size which enter the labyrinth between the cortical rays and as interlobular arteries. (cortical arterioles) pass toward the surface of the organ, a few of the longest branches reaching the fibrous capsule with whose vessels they anastomose. 'Throughou't their whole course the interlobular arteries give off numerous short branches, w h i c h leave the parent stem at a wide angle, and pass directly to a renal corpuscle as the afferent artery (arteriole; vas afferens) to its glomerulus. Here it supplies the capillary plexus in the manner already described (page 431).

Certain of the afferent arterioles are peculiar in

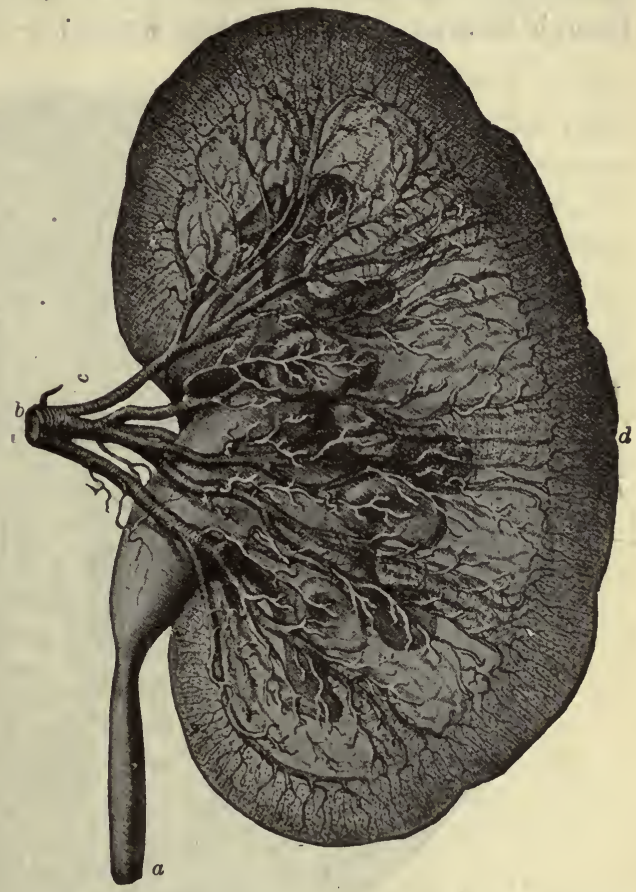

Fig. 407.-The Distribution of the Left Renal Artery.

Of the six arteriæ propriæ renales, five enter in front of the renal pelvis, and, lying upon the wall of the calyces, are distributed from the arterial arcade to both cortex and medulla. $a$, ureter; $b$, renal artery; $c$, arteriæ propriæ renales; $d$, the dark border is the cortex, within which is the lighter medulla. (After Brödel, from Szymonowicz and MacCallum.) that (1) they give off small branches which supply capillaries directly to the convoluted tubules of the cortical labyrinth; and (2) they occasionally form a small rete mirabile before they reach the glomerulus. By far the greater portion of the branches of the interlobular arteries, however, pass directly to the glomeruli. The capillaries of the glomerulus reunite to form an efferent 
vessel which, after leaving the corpuscle, promptly breaks into a second capillary plexus about the adjacent tubules.

The efferent vessels of those glomeruli which lie in that portion of the cortex adjoining the medulla, frequently pass to the boundary zone of the latter region where they form the arteriæ rectæ spuriæ which, though lacking a true arterial structure-they possess no circular mus-

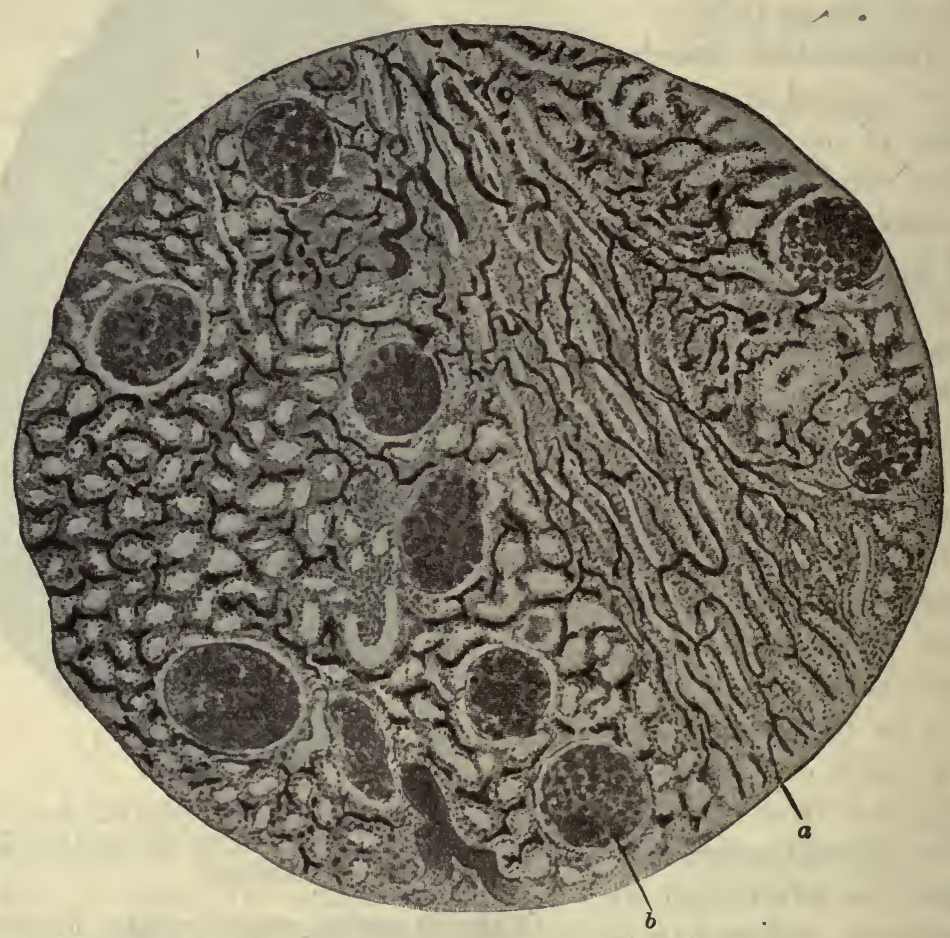

Fig. 408.-From the Cortex of the Human Kidney.

The blood-vessels have been injected and appear dark. A cortical ray and the adjacent labyrinth is included. $a$, cortical ray; $b$, a glomerulus in the labyrinth. Moderately magnified. (After Disse.)

cle fibers-pursue the same course as the true arteriolæ rectæ and assist the latter in supplying the capillary plexus of the medulla. The medullary capillaries, like those of the rays in the cortex, form a network with elongated meshes which surround the parallel tubules of this region. The capillaries of the cortical labyrinth, being distributed among irregular tortuous tubules, possess a more polygonal mesh:

The interlobular veins begin near the surface of the organ. where 
small venules, derived from the cortical capillaries and from occasional anastomoses with the capsular vessels, unite to form broad thin-walled venous spaces, the stellate veins of Verheyn, just beneath the capsule. From these subcapsular vessels the interlobular veins arise, and pass toward the medulla in company with the interlobular arteries, throughout their course collecting the minute venules which return the blood from the capillaries about the tubules of the renal cortex.

Arriving at the border of the medulla, but still embedded in the cortical substance, the interlobular veins turn sharply, at nearly right angles to their former course, and by free anastomoses form a venous arcade (arciform vein), which receives the venulce recta coming from the capillary plexuses of the medulla, and at the border of the renal pyramid enters a renal column to unite with similar vessels coming from the borders of the adjacent pyramids. The union of these vessels forms large venous trunks which leave the renal columns, in company with the arteries, as the vence proprice renales (interlobar veins). They enter the connective tissue of the hilum, traverse the wall of the calyces, infundibula, and renal pelvis, and finally unite to form the renal vein.

TABLE SHOWING THE COURSE OF THE RENAL CIRCULATION

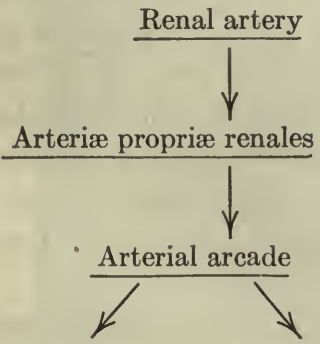

Interlobular arteries

Interlobular
$\downarrow$

Afferent artery to renal corpuscle

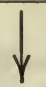

Capillaries of the glomeruli
Renal vein

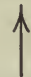

Venæ propriæ renales

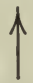

Venous arcade

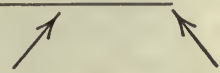

Venulæ rectæ Interlobular veins

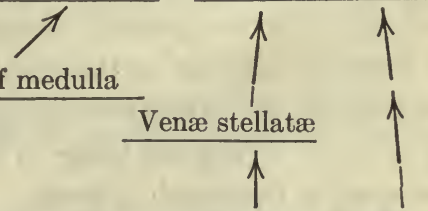

Capillaries of the cortical labyrinth
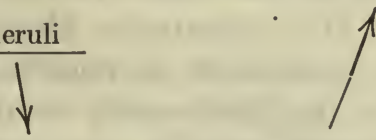

Efferent vessel from renal corpuscle 
The venulæ rectæ are peculiar in their typically straight course, and in the fact that the cells of their endothelium are extremely longso long indeed, as frequently to present a somewhat fibrous appearance, the elongated axis of the cell being parallel to the long axis of the vessel.

The capsule of the kidney is supplied by branches of the lumbar, phrenic, and suprarenal arteries, which form a rich, capillary plexus.

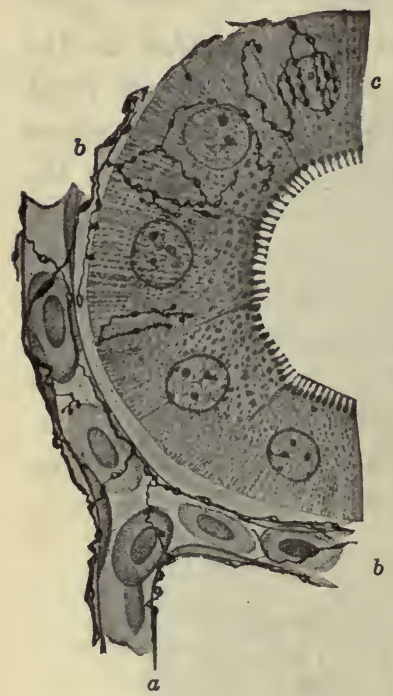

Fig. 409.-Nerve Endings in a Convoluted Tubule of the Frog's Kidney.

$a$, nerve fiber; $b$, blood-vessel; $c$, secreting epithelium. Methylene blue; cochineal. Very highly magnified. (After von Smirnow.)

These vessels anastomose with the terminal branches of the interlobular arteries of the kidney in the manner above described-a fact which acquires surgical importance from its relation to the establishment of a collateral circulation.

The table on page 443 may be useful as a résumé of the more important paths in the course of the renal circulation. In it the names of the several vessels are arranged in order and the arrows indicate the direction of the-blood current.

Lymphatics.-The renal lymphatics consist of a superficial set which forms plexuses in the perirenal fat and deeper layers of the capsule, and a deep set which supplies the parenchyma of the organ. These two sets of lymphatic vessels are in communication by frequent anastomoses. The vessels of the superficial set convey their lymph to the neighboring lymph glands of the lumbar region.

The deep renal lymphatics accompany the arteries and veins throughout their course. They form narrow cleftlike vessels of irregular caliber in the scanty interstitial tissue between the uriniferous tubules of both cortex and medulla. These vessels are relatively few in number. They converge to the hilum of the organ where they pass to the nearby lymphatic glands.

Nerves.-The nerves of the kidney include both medullated and non-medullated fibers. The sympathetic fibers form a ganglionated plexus in the connective tissue about the renal pelvis. From this plexus fibers are distributed to the blood-vessels of the capsule and to the parenchyma of the organ. The latter accompany the blood-vessels, dis- 
tributing their fibers to the walls of the arteries and veins and to the uriniferous tubules of both cortex and medulla.

The parenchymal branches form an 'epilemmal plexus' in the interstitial connective tissue about all portions of the uriniferous tubule. From this plexus fibrils pierce the membrana propria and anastomose to form a 'hypolemmal plexus' about the base of the epithelial cells. Terminal fibrils penetrate between the epithelial cells where they form minute end knobs.

\section{RENAL PELVIS AND URETERS}

The excretory passages of the kidney include the renal pelvis, the ureters, the urinary bladder and the urethra. All of these portions possess certain common characteristics. They have three coats, mucous, muscular, and fibrous, and are lined by a common type of epithelium,

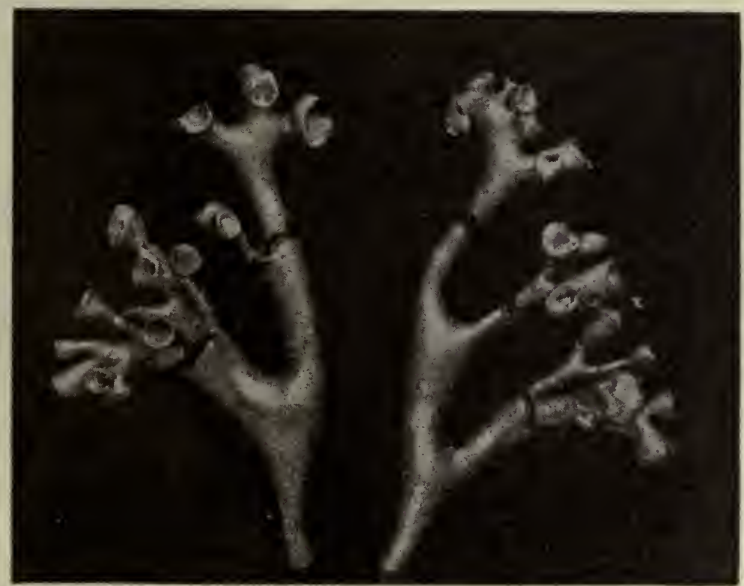

Fig. 410.-Cast of the Pelvis, Infundibula and Calices of the Kidneys OF a Man.

Showing the expansion of the ureter, the subdivision of the pelvis, and the concave facets by which the calices fit over the apices of the renal pyramids. (After Hauch.)

transitional, which extends from the renal pelvis to the prostatic portion of the urethra.

The mucosa of the renal pelvis and ureter is lined by transitional epithelium which rests upon a fibrous tunica propria. The epithelium consists of several cell layers, of which the superficial is formed by broad 
cuboidal cells, or thick flattened plates, whose form varies with the state of distention of the organ, and whose deep surfaces are indented by the rounded ends of the pear-shaped cells which form the deeper layers. The deepest cells are smaller and are irregularly spheroidal in shape. They

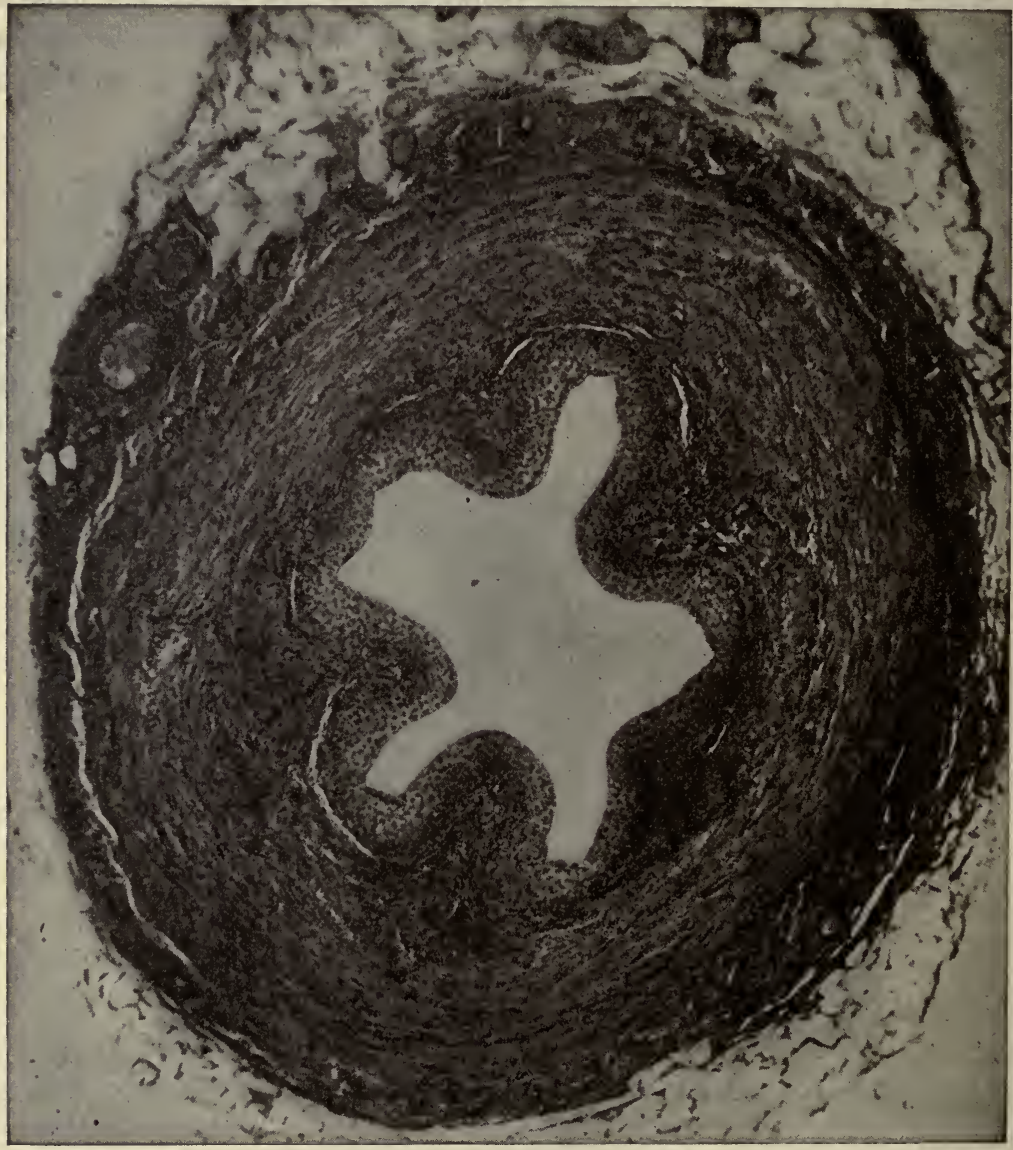

Fig. 411.-Transection of Human Ureter.

Hematein and eosin. Photo. $\times 113$.

are firmly attached to the underlying connective tissue which here and there projects into the epithelial layer carrying with it the most superficial capillary vessels. The cells of the deeper layers divide by karyokinesis and push toward the surface to replace the more superficial cells which are gradually desquamated. Direct cell division occurs in the 
large plate-like cells of the superficial layer. The thin transitional epithelium of the calyces is continuous with the columnar epithelium of the papillary ducts.

The tunica propria, continuous with the renal interstitial tissue, contains both collagenous fibers and elastic fibers. It is indistinctly divisible in to a superficial denser portion, and an open-meshed deep portion whose fibrous bands loosely attach the mucous membrane to the muscular coat. This deep layer is analogous to the submucosa of the alimentary tract. The mucous membrane is thrown in to numerous deep folds or rugæ which in the ureter have a longitudinal direction; $\mathrm{t} \mathrm{h}$ i s condition gives to the canal in transverse section a stellate appearance. Irregular folds or invaginations of $\mathrm{the}$ epithelium occur in the renal pelvis
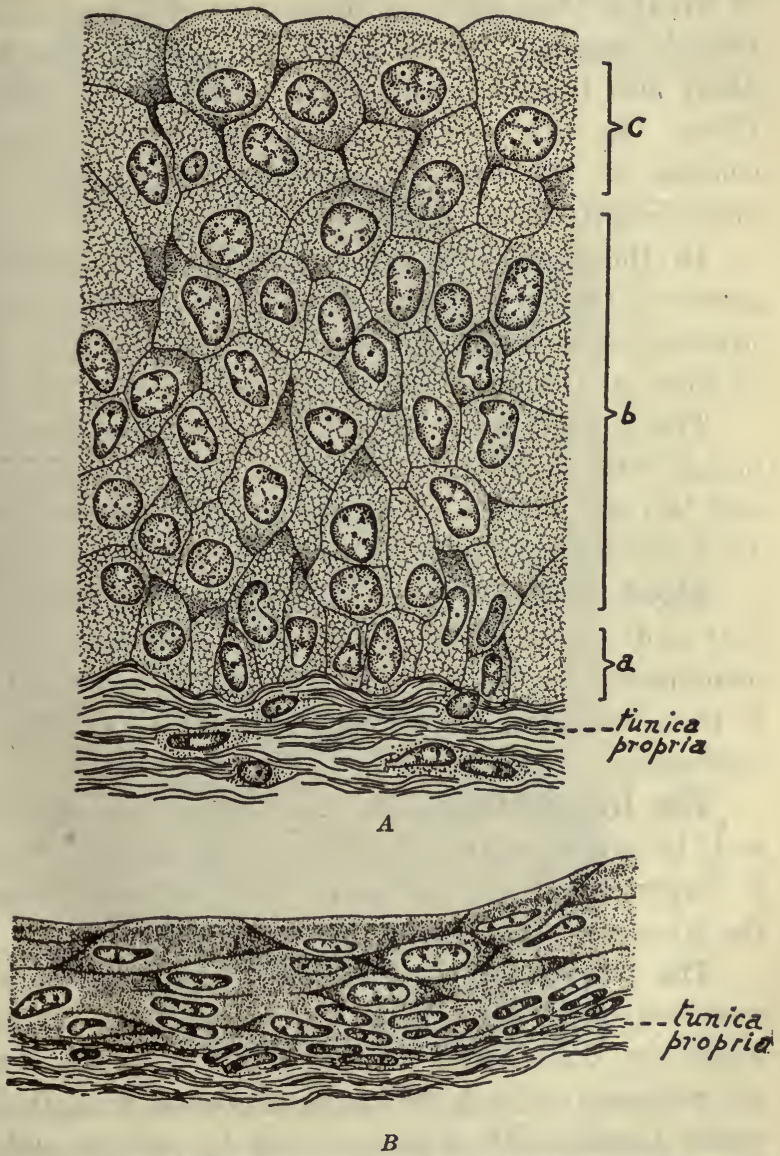
Fig. 412.-Transitional Epithelium of Dog's Ureter.

$A$, in the contracted condition; $B$, in the distended condition; $a$, basal layer of cubic cells: $b$, middle layer of polygonal cells; $c$, superficial layer of rectangular and ovoid cells. (R. W. Harvey, Anat. Rec., 3, 5, 1909.) $\times 750$.

and have been described as glands, but true secreting glands are not found. Occasional lymphocytes occur in the mucosa, and small lymph nodules have also been found but cannot be regarded as of constant 
occurrence. The mucosa becomes gradually thinner as it is traced through the infundibula and calyces and is reflected upon the surface of the renal pyramids.

The muscular coat of the ureter consists of a well-defined layer of circular fibers within which are many discrete bundles of longitudinal, smooth muscle. In the lower half of the ureter a third layer, whose fibers also have a longitudinal direction, is found outside the circular fibers. In this portion of the ureter, therefore, the muscular coat consists of three layers, an inner longitudinal, middle circular, and outer longitudinal.

In the renal pelvis and calyces the muscular coat becomes progressively thinner toward the renal substance, and at the apex of the pyramid consists chiefly of circular fibers which are slightly thickened to form a 'sphincter' about the papilla.

The outer fibrous coat of the ureter consists of areolar tissue which blends. with that of the surrounding parts. In the renal pelvis this coat becomes continuous with the connective tissue capsule of the kidney. (For development of excretory passages, see next Chapter.)

Blood Supply.-The larger blood-vessels lie in the outer fibrous coat and distribute branches to the muscular coat and to the mucous membrane. In the latter they form a superficial capillary plexus which is in unusually intimate relation with the deeper cells of the lining epithelium.

The Lymphatics.-The lymphatics begin in an intramuscular plexus and, by scanty vessels, in the deeper part of the mucosa. They pass to larger vessels in the outer coat, which possess valves, and convey the lymph to the neighboring lymph nodes.

The Nerves. - The nerves form a coarse plexus in the outer fibrous coat which contains many small ganglia. From this plexus motor fibers are distributed to the muscular layers, and sensory fibers to the mucosa. The latter form a plexus beneath the epithelium from which terminal fibers pass to end by minute end brushes in the connective tissue and by varicose fibrils between the deep cells of the epithelium.

\section{THE URINARY BLADDER}

The wall of the urinary bladder closely resembles that of the ureter. It consists of mucous, muscular, and fibro-serous coats. 'Its mucous membrane is lined by transitional epithelium like that of the ureter 
and renal pelvis. It is therefore impossible to determine from which of these portions detached epithelial cells are derived when found by microscopical examination of the urine. Epithelium may become de-

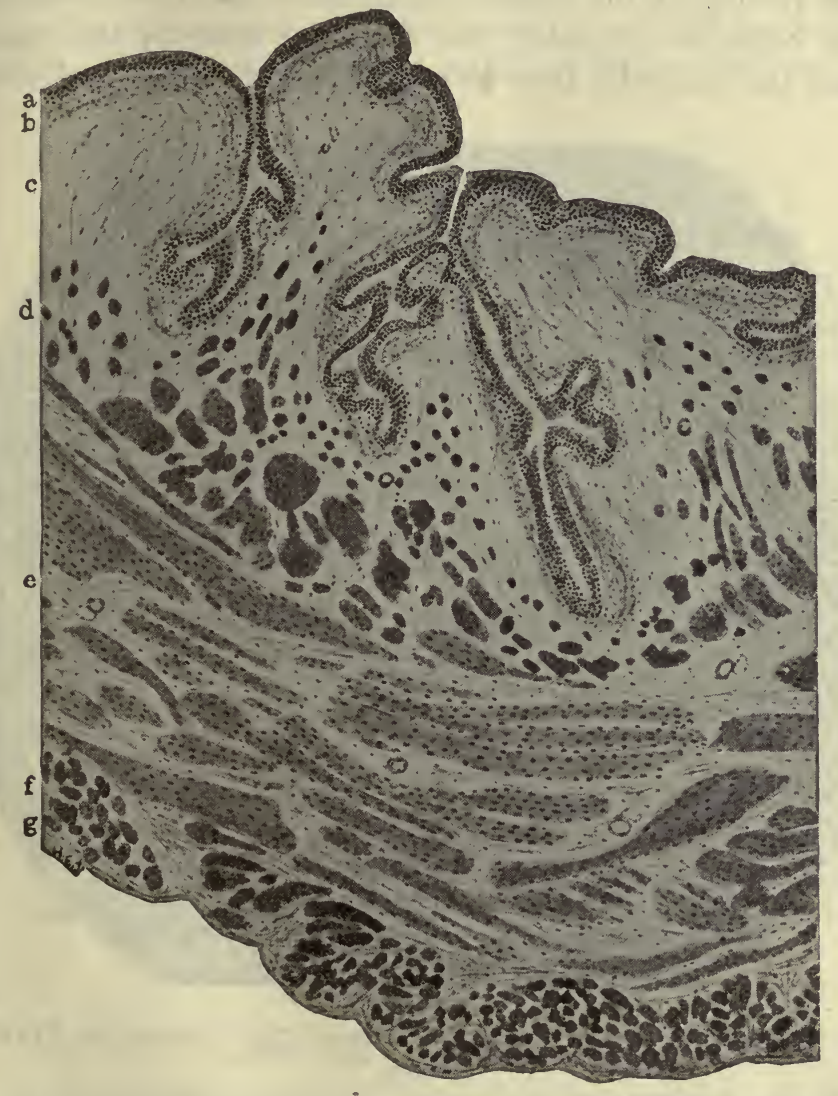

Fig. 413.-Transverse Section of Urinary Bladder of Dog.

$a, b$ and $c$, compose the tunica mucosa; $d, e$ and $f$, the tunica muscularis; $g$, tunica serosa; $a$, transitional epithelium; $b$, lamina propria mucosæ; $c$, deep portion of tunica mucosa, corresponding to a tela submucosa; $d$, internal longitudinal muscle layer; $e$, middle circular muscle layer; $f$, external longitudinal muscle layer. The tunica mucosa is thrown into larger and smaller folds. $\times 20$.

tached from any portion of the excretory passages, and as a result of inflammatory changes not only the superficial cells but also the deeper pear-shaped cells may be desquamated.

The character of the epithelium varies with the collapse and distention of the organ. When empty the mucous membrane is thrown 
into deep folds or rugæ, whose surface also presents secondary folds of irregular direction. The epithelial layer is relatively thick, and is thicker on the sides of the folds than upon either their apices or bases where the folding of the epithelium increases the tension of its cells. When the organ is distended, the folds are more or less completely obliterated, the epithelial layer is much thinned, often until it appears

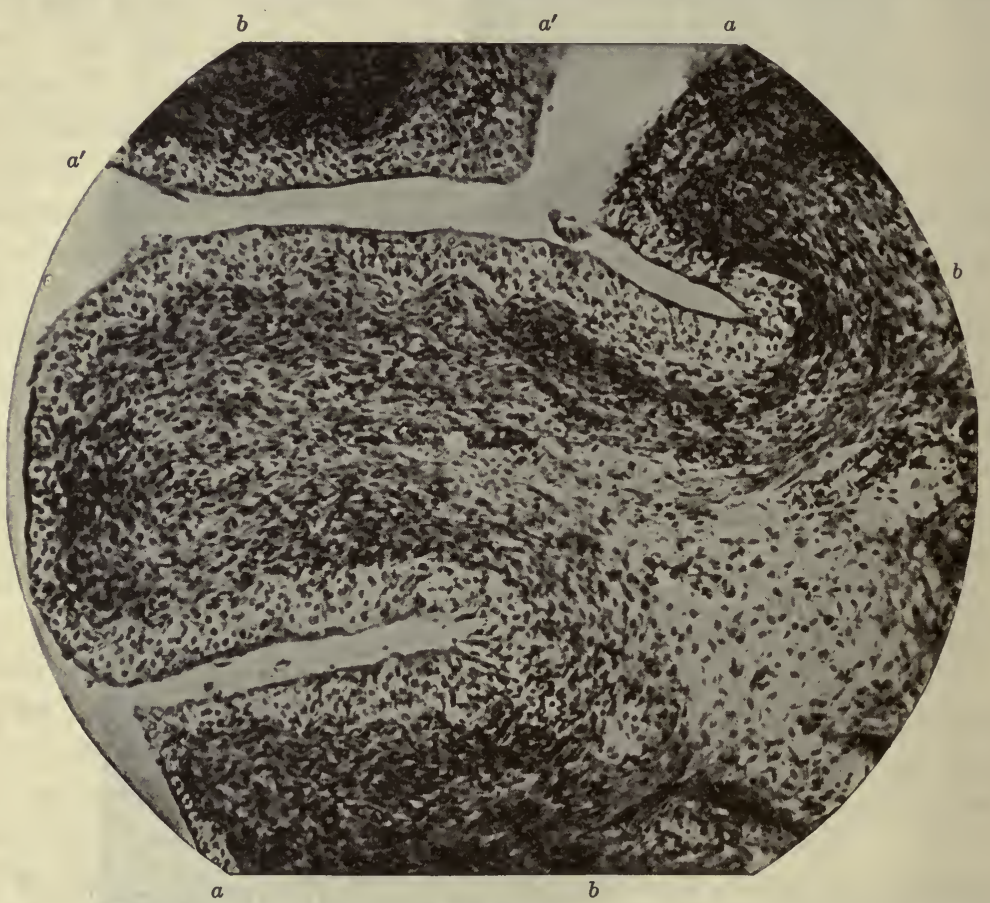

Fig. 414.-The Mucosa of a Child's Bladder in the Contracted State of the ORGAN.

$a-a$, and $a^{\prime}-a^{\prime}$, transitional epithelium; $b$, fibromuscular tissue of the mucosa. Hematein and eosin. Photo. $\times 216$.

to consist of no more than a double layer of cells, and its cells become much broader and relatively much thinner than in the collapsed or empty condition. Hence the transitional variety of epithelium, by which the organ is lined, may be said to be remarkable for the extreme elasticity of its cells.

The mucous membrane, except near the urethral orifice, contains no glands. In this location, however, the bladder of adult man contains a few small mucus-secreting glands, lined by columnar cells (Köl- 
liker). These are not present in infancy. They have been interpreted as vestigial prostatic tubules.

The tunica propria resembles that of the ureter and is loosely united to the muscular coat. This latter layer is formed by interlacing bundles of smooth muscle. It varies much in thickness according to the condition of the organ, being relatively thick when the viscus is

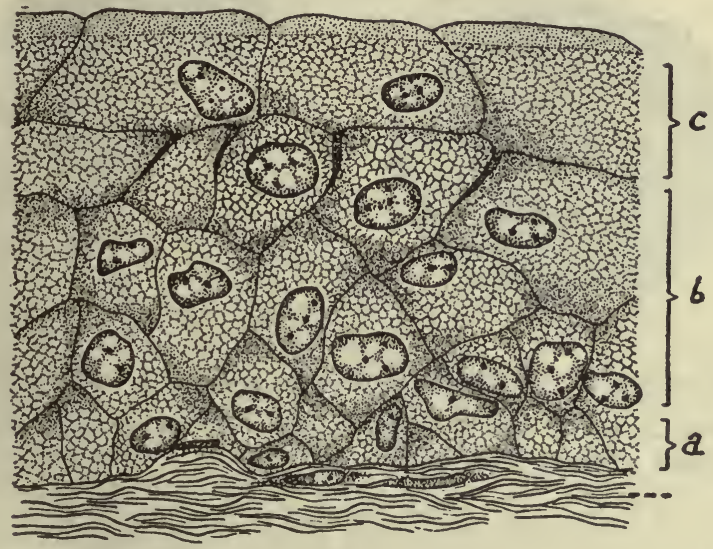

$A$

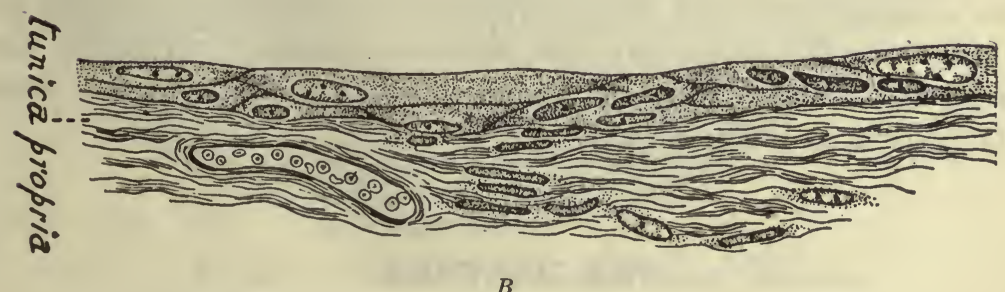

Fig. 415.-Transitional Epithelium of Dog's Bladder.

$A$, in the contracted condition; $B$, in the distended condition. (R. W. Harvey, Anat. Rec., 3, 5, 1909.)

empty and very thin when it is completely distended. In most portions three indistinct layers can be observed, a middle thick layer of circular fibers, and an inner and outer longitudinal layer. The outer longitudinal muscle is most distinct on the anterior and posterior surfaces of the organ.

The outermost coat of the bladder consists of areolar tissue, having very broad meshes; in the lower portion of the bladder this coat blends with the connective tissue of the adjacent organs. Over the fundus of 
the organ its outer fibrous coat is covered by the peritoneum, the connective tissue of which is indistinguishable from that of the outer coat of the bladder; it is clothed by the peritoneal mesothelium.

Vascular and Nerve Supply.-The vascular and nerve supply of the bladder is exactly similar to that of the ureter. The larger blood-

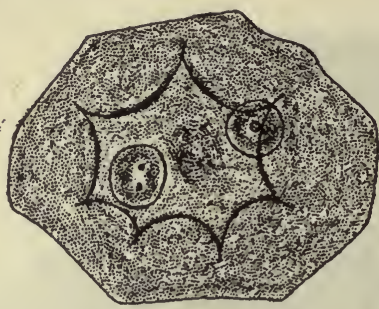

A

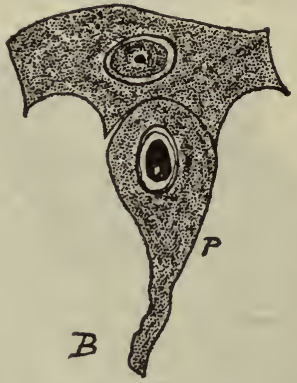

Fig. 416.-Epithelial Cells from the Bladder of the Rabbit.

$A$, as seen from the under surface, showing the depressions made by the underlying polygonal and pyriform cells $(P) ; B$, side view of similar cell. (After Klein, from Schäfer.)

vessels occur in the outer fibrous coat, whence they distribute branches to the muscular coat and mucous membrane. Many small ganglia are also found in the outer coat, and motor and sensory nerves are distributed to the musculature and to the epithelium and connective tissue of the mucosa, as in the ureter.

\section{THE URETHRA}

\section{The Female Urethra}

The mucosa of the female urethra is lined by a variable type of epithelium. Being continuous with the mucosa of the bladder, the epithelium of the urethra is at first of the transitional variety, but as it approaches the meatus this is changed to a stratified squamous type which is continuous with that of the vestibule. In certain individuals the superficial cells of the midportion of the urethra are much elongated and assume an irregular stratified columnar type.

The epithelium rests upon a tunica propria of dense areolar connective tissue whose outer portion blends with a looser connective 
tissue which contains many broad venous channels and forms a sort of spongy erectile tissue. This erectile coat is surrounded-by a thin muscular coat whose innermost fibers, longitudinal in direction, are continued outward to the meatus, and whose outer circular fibers, de-

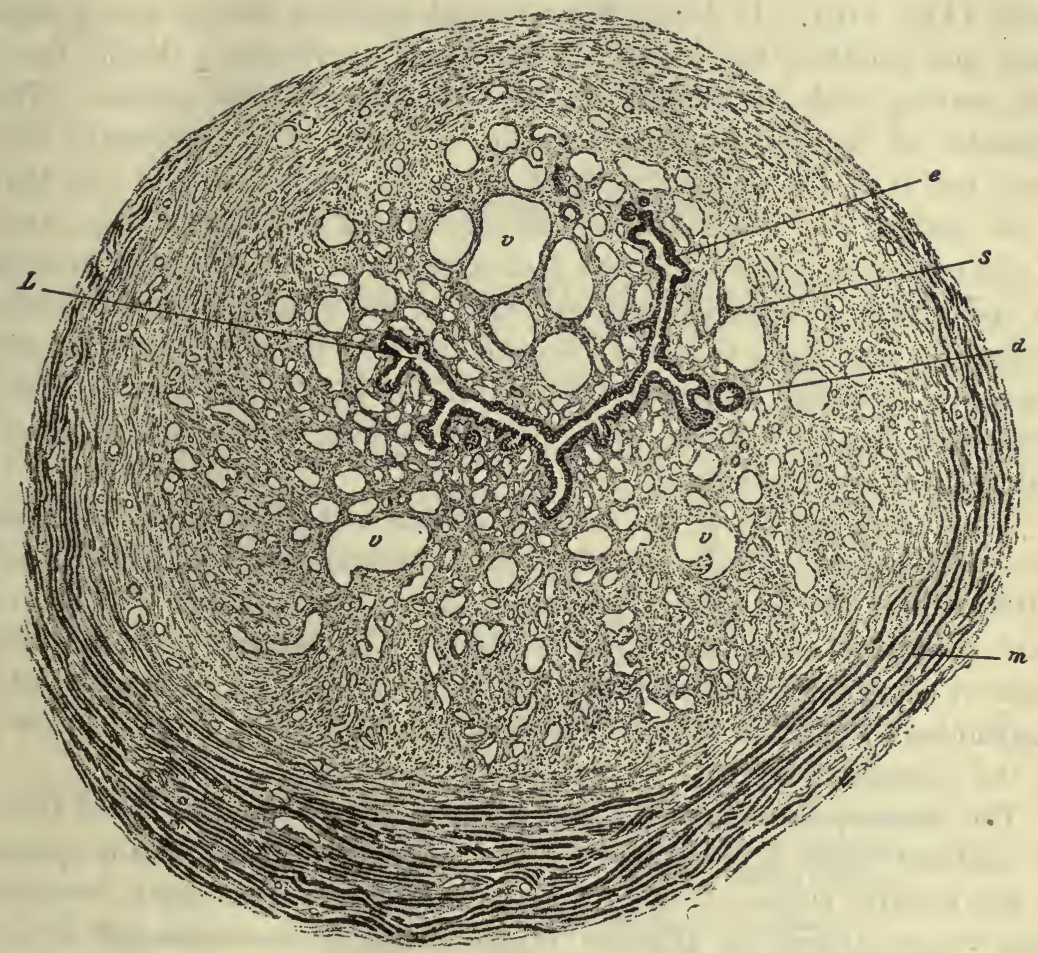

Fig. 417.-Transection of the Female Urethra.

$d$, gland-like diverticulum; $e$, urethral epithelium; $L$, urethral lumen; $m$, striated fibers of the urethral muscle; $s$, erectile tissue of the tunica propria, containing many venous spaces and smooth muscle fibers. $\times 10$. (After Kölliker.)

ficient toward the meatus, are often slightly thickened near the neck of the bladder to form an indistinct sphincter urethræ muscle. This coat contains occasional striated muscle fibers. The female urethra is scantily supplied with urethral' glands which open near the meatus and supply a meager mucoid secretion. 


\section{The Male Urethra}

The male urethra conducts the urine from the bladder to the surface, its course being through the axis of the corpus spongiosum of the penis (Fig. 446). It forms the terminal segment also of the genital canal and conducts the semen; it accordingly performs a double function, serving both the urinary and the male reproductive system. The character of its epithelium is variable, not only in its successive portions, but it is also subject to great individual variation, and like that of the bladder and ureter changes its appearance according as the canal is collapsed or distended. In the first or prostatic portion of the urethra the epithelium is of the transitional type; in the membranous and penile portion its superficial cells are elongated so that the epithelium usually acquires a stratified columnar form; near the meatus the type is again ex.changed for a stratified squamous epithelium which is continuous with that of the glans penis.

The tunica propria of the urethra consists of areolar tissue in which are embedded the small branched tubulo-acinar urethral glands (of Littré) lined by columnar, mucus-secreting cells. These glands are more abundant along the upper surface. The mucosa also contains frequent lacunar invaginations of the epithelium, and is thrown into longitudinal rugæ, its lumen being obliterated except when distended by the passage of urine.

The tunica propria, and especially that portion which is distant from the urethral canal, is permeated by the thin-walled broad venous spaces of the erectile tissue of the corpus spongiosum. The septa between these venous spaces, in addition to the dense areolar tissue of which they chiefly consist, contain many bundles of longitudinal smooth muscle. In the deeper portions of the urethra circular muscle fibers are also found in the outer part of this coat, and near the orifice of the bladder they are somewhat thickened to form the sphincter urethræ Moreover, the corpus spongiosum is invested with a thick sheath of dense fibrous connective tissue. At the apex of the prostate circularly disposed striped muscle fibers form a second urethral sphincter, the external vesical sphincter.

Nerve Supply.-The nerve supply of the urethra includes both sensory and motor spinal fibers, and sympathetic fibers for the smooth muscle and the blood-vessels. 


\section{CHAPTER XV}

\section{THE REPRODUCTIVE SYSTEM}

\section{GENERAL CONSIDERATIONS}

The reproductive system differs structurally, widely in the two sexes. The several organs involved, however, are in general strictly homologous in the male and female organism. The differences result from a divergent specialization concomitant with a division of labor in the reproductive act and process. The specialization involves both progressive and regressive differentiation, the latter producing certain

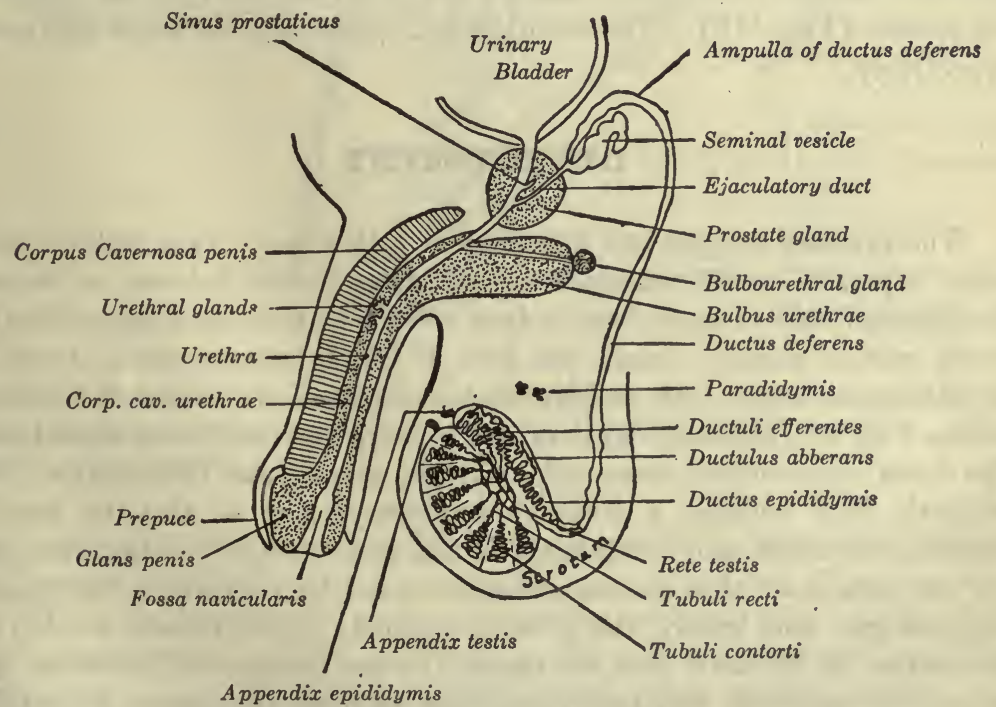

Fig. 418.-Diagram of Male Genitalia. (Adapted from Merkel.)

vestigial appendages, of different origin in the opposite sexes. The MALE ORGANS OF REPRODUCTION include a primarily and essentially internal group of genital organs, namely, the testis, and its associated 
ducts, glands and appendages; and an external genital organ, the penis (Fig. 418.) The FEMALE ORGANs of REPRODUCTION likewise include an internal group of genitalia, namely, the ovary, with its ducts and the

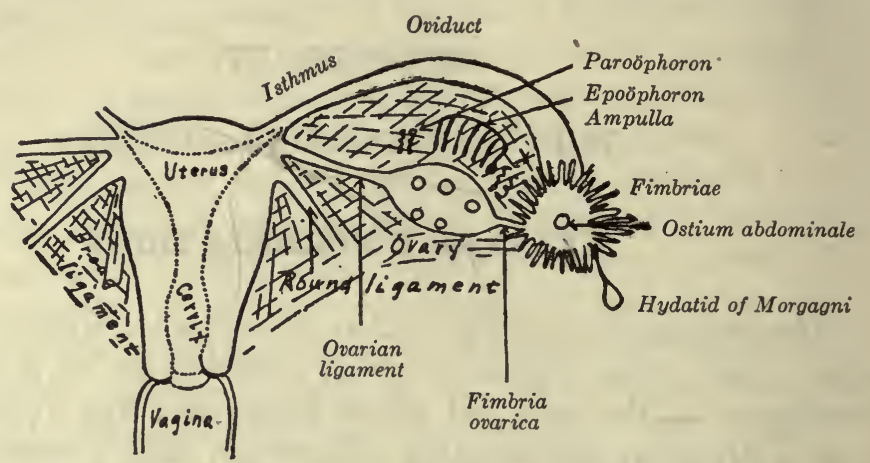

Fig. 419.-Diagram of Female Internal Ginitalia.

associated glands and appendages; and a group of external genitalia and glands (Fig. 419). The essential sex organs are the testis and ovary, respectively.

\section{DEVELOPMENT}

The primary anlages are the same in both sexes. They include essentially: a pair of undifferentiated sex-glands or gonads located on the midventromesial side of each fetal kidney (Wolffian body or mesonephros); a double pair of parallel canals, the fetal Wolffian (mesonephric) ducts and the Müllerian ducts. An embryo at this stage of development (13 millimeters, Fig. 210, about 40 days) is said to be in the indifferent sexual stage. The ducts communicate terminally with the gonads and the exterior. Subsequently they undergo a different development, as do also the terminal associates (gonads, and urogenital sinus, respectively) in the two sexes. For the details of this process reference must be made to a text-book of Embryology. But briefly, the primitive gonads in the female develop into the ovaries, in the male into the testes. In the female the Müllerian ducts become the oviducts, and fuse proximally to form the uterus and vagina; in the male, this duct suffers regressive changes and persists only as vestiges: the appendix testis, and the sinus pocularis. On the contrary the Wolffian duct becomes vestigial in the female, persisting as the appendix fimbria (hydatid of Morgagni) and the canal of Gärtner; while in the male, it develops into the main portion of the definitive duct system: ductus epididymis, and ductus deferens. The tubules of the fetal kidney (Wolffian 
body) also contribute to the excretory duct system. In the male, they persist in part (10 to 15$)$ to form the ductuli efferentes; several may become vestigial forming thus the ductuli aberrentes, the paradidymis and the appendix epididymis. In the female, these ducts early disappear for the

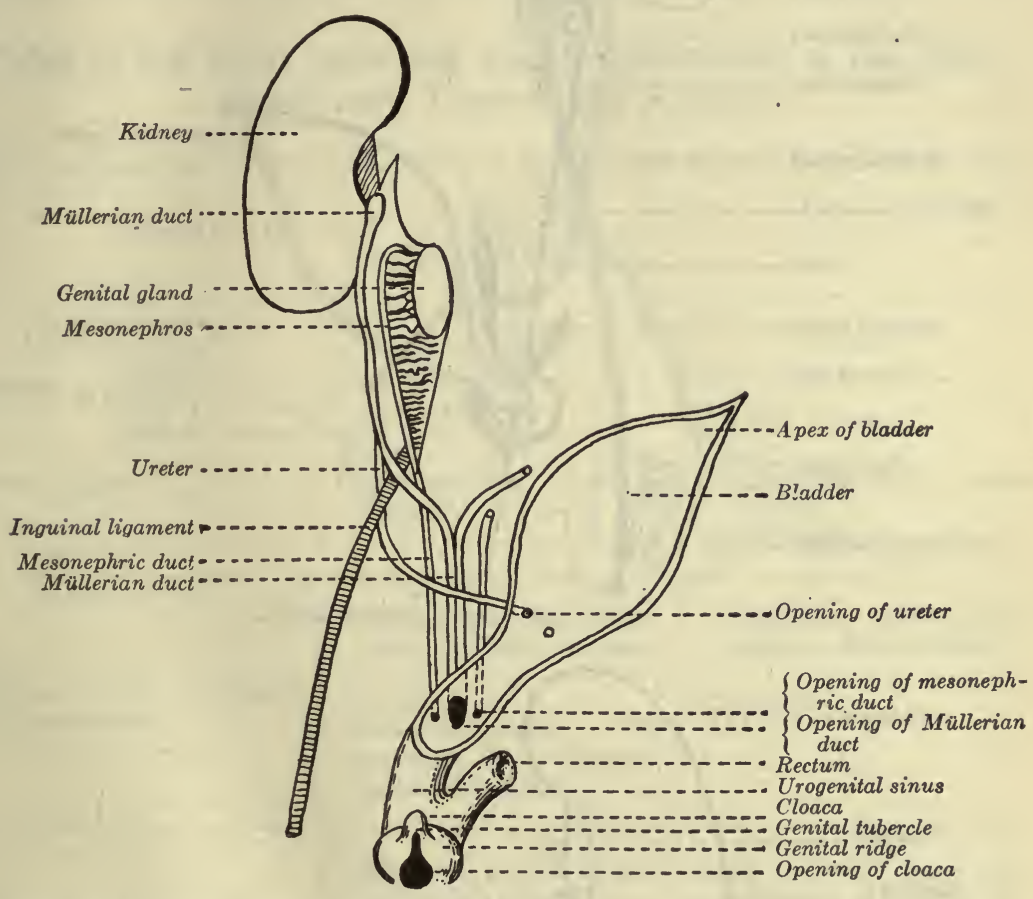

A

Fig. 420.-Diagrams Illustrating the Metamorprosses of the Indifferent (A) Urogenital System into the Male (B), and Female $(C)$ Systems. (From Polak, after Hertwig.)

most part-a variable number persisting as vestigial structures: the epoophoron and the paroöphoron.

The seminiferous tubules (including the recti and rete tubuli) of the testis arise as solid cords of cells continuous with the peritoneal epithelial covering of the gonad, and apparently as derivatives of this so-called 'germinal epithelium.' 'These cords subsequently acquire a lumen, and connect with the efferent tubules. In the ovary likewise such cell cords (sex-cords) appear, continuous with the peritoneal (germinal) epithelium; from these develop the ovarian follicles with their ova, as will be described below. The relation of the extra-regional 'primordial germ-cells' to the germinal epithelium and to sex-gland derivatives, discussed in the next section, 


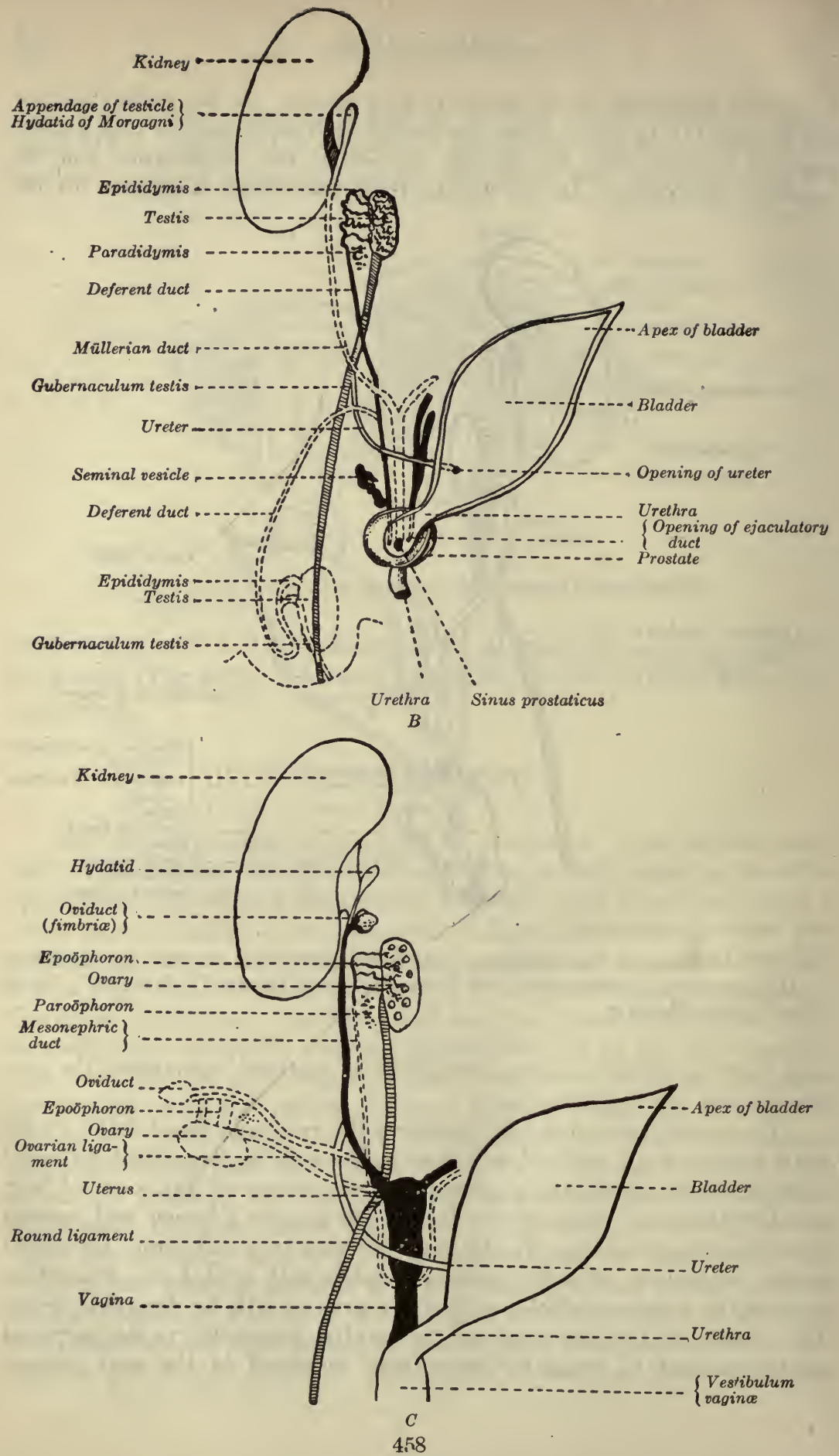


remains in doubt. Felix (Keibel and Mall's "Human Embryology," vol. 2) inclines to the view that in man the primordial germ-cells (primitive genital cells) disappear, and that they bear no genetic relationship to the primitive germ-cells (secondary genital cells) of the gonad.

\section{Table of the Adult Male and Female Derivatives of the Fetal (Indifferent) Reproductive System.}

(The vestigial structures are given in italics, synonyms in parentheses.)

Internal Genitals.

\begin{tabular}{|c|c|c|}
\hline Indifferent stage. & Male. & Female. \\
\hline Genitai ridge. & $\begin{array}{l}\text { Testis. } \\
\text { Gubernaculum (mesor- } \\
\text { chium). }\end{array}$ & $\begin{array}{l}\text { Fimbria ovarica. } \\
\text { Ovary } \\
\text { Ovarian ligament (meso- } \\
\text { varium). } \\
\text { Round ligament. }\end{array}$ \\
\hline $\begin{array}{l}\text { Wolffian body (tubules) } \\
\text { (Mesonephros). }\end{array}$ & $\begin{array}{l}\text { Globus major of epididy- } \\
\text { mis (ductuli efferen- } \\
\text { tes). } \\
\text { Paradidymis. } \\
\text { Ductuli aberrentes. } \\
\text { Appendix of epididymis } \\
\text { (stalked hydatid). }\end{array}$ & $\begin{array}{l}\text { Epoöphoron (Paronarium, } \\
\text { organ of Rosenmüller). } \\
\text { Paroöphoron. }\end{array}$ \\
\hline $\begin{array}{l}\text { Wolffian duct (Mesonephric } \\
\text { duct). }\end{array}$ & $\begin{array}{l}\text { Duct and globus minor } \\
\text { of epididymis. } \\
\text { Ductus deferens. } \\
\text { Seminal vesicle. } \\
\text { Ejaculatory duct. }\end{array}$ & $\begin{array}{l}\text { Chief vesicular appendage } \\
\text { (Hydatid of Morgagni). } \\
\text { Collecting tubule of epo- } \\
\text { ophoron. } \\
\text { Canal of Gärtner. }\end{array}$ \\
\hline Müllerian duct. & $\begin{array}{l}\text { Appendix testis (sessile } \\
\text { hydatid). } \\
\text { Sinus pocularis (Uterus or } \\
\text { vagina masculina, or } \\
\text { utriculus prostaticus). }\end{array}$ & $\begin{array}{l}\text { Oviducts (Uterine or Fal- } \\
\text { lopian tubes). } \\
\text { Uterus. } \\
\text { Vagina. }\end{array}$ \\
\hline
\end{tabular}

External Genitals.

Urogenital sinus.

Genital tubercle.

Genital folds.

Genital swellings.

Prostatic and membranous portions of male urethra.

Penis.

Scrotum.
Urethra.

Vestibule.

Clitoris.

Labia minora.

Labia majora. 
The outline on the preceding page summarizes in tabular form the main facts regarding the development of the reproductive system.

In both sexes the Wolffian duct proximally sprouts a duct which dilates and subdivides distally, meanwhile separating from the parent duct and making a secondary connection with the developing bladder. The subdivisions elongate to form the collecting portion of uriniferous tubules, and unite with ontogenetically distinct tubules (the secretory portions) to form the complete tubules. The dilatation and earlier subdivisions become the pelvis and calyces, and the proximal portion of the original anlage persists as the ureter.

\section{GAMETOGENESIS}

The study of the sex or genital glands, the ovary and the testis, is perhaps best approached by way of a consideration of the mechanism by which they perform their respective specific functions, namely the production of ova and spermatozoa ripe for union. The common process is known as gametogenesis. The end products are the male and female gametes, or sperm and ova. In the male the process is known as spermatogenesis, in the female, oögenesis. The act of subsequent union of the gametes is called fertilization, and the fertilized egg is the zygote.

The result of gametogenesis is the preparation of a primary germcell for union with a gamete from the opposite sex. The essence of the process is known as maturation, and involves prominently mitotic cell division. However, the method of division is not of the simple homeotypic type, where a chromosome simply divides longitudinally into two daughter chromosomes, but is of the type called heterotypic, the chief characteristic of which is the formation of tetrads. The latter are of various sorts, all, however, characterized by a four-lobed condition representing a quadripartite double or bivalent chromosome (Plate B, figs. 15-18, page 467).

Both sperm and egg trace their ancestry back to primordial germ cells, indistinguishable in the sexually undifferentiated organism, except for a difference in chromosome content, which difference is commonly indiscernible. In certain instances, e.g., Ascaris, the germ cell can be distinguished from the soma cell at the two-cell stage. In a number of vertebrates, e.g., dogfish, turtle, etc. (Allen), the primordial germ-cell has been traced from a position among the entoderm cells lining the gut of the young embryo through a migration into the differentiating gonad, ovary or testis. Swift (Amer. Jour. Anat., 15, 4, 
1914) has traced the primordial germ-cells in the chick from a crescentic area in the entoderm in front of the head end of the primitive streak, through their migration to the gonad by way of the developing bloodvessels. The earlier anatomists, on the other hand, derived the primordial germ-cells from the mesothelial covering (germinal epithelium) of the germ gland region of the primitive kidneys, the mesonephroi. This mode of origin, either exclusively or in part, is still supported by certain investigators.

Whatever the actual source of origin-whether entodermal or mesodermal, whether specific or otherwise-and whenever the time of differentiation, whether early or relatively late, the primordial germ-cells in the gonad undergo extensive proliferation, increasing greatly in numbers. The earlier generations may be inclusively designated primary spermatogonia or oögonia respectively, the final generation taking the term secondary gonia. The question of the origin of the primordial germ-cells bears upon the hypothesis of the continuity of germ-plasm.

Spermatogenesis.- Since gamete production is easier to follow, though apparently more specialized, in the male, spermatogenesis is advantageously first described. For the purpose we may employ the active testis of Schistocerca damnifica, a common grasshopper. Grasshopper material of many species is peculiarly favorable for a demonstration of the process of spermatogenesis. This scheme will apply with slight qualification to most grasshoppers. The reduced number of chromosomes is the same, namely, 12 ; moreover, this material shows peculiarly well the accessory or sex chromosome, and thus serves as a splendid basis for a discussion and comprehension of the salient facts and recent theories touching the problems of the determination, control, and inheritance of sex.

The grasshopper testis is a long tubular structure subdivided into a number of compartments, or cysts, each filled with cells of approximately the same stage of development, the successive stages in spermatogenesis being represented in successive cysts from distal to proximal pole. For purpose of readiest study there is required, then, a median longitudinal section of an entire testis in active condition. As is obvious, such material is useful also for illustrating mitosis but, with the exception of the spermatogonial and second maturation mitoses, cannot be regarded as typical; but for application and extension of a preliminary knowledge of simple mitosis it is perhaps unexcelled. It must be emphasized that this peculiar type of cell division, characterized chiefly by the phenomena of synapsis and tetrad formation, in- 


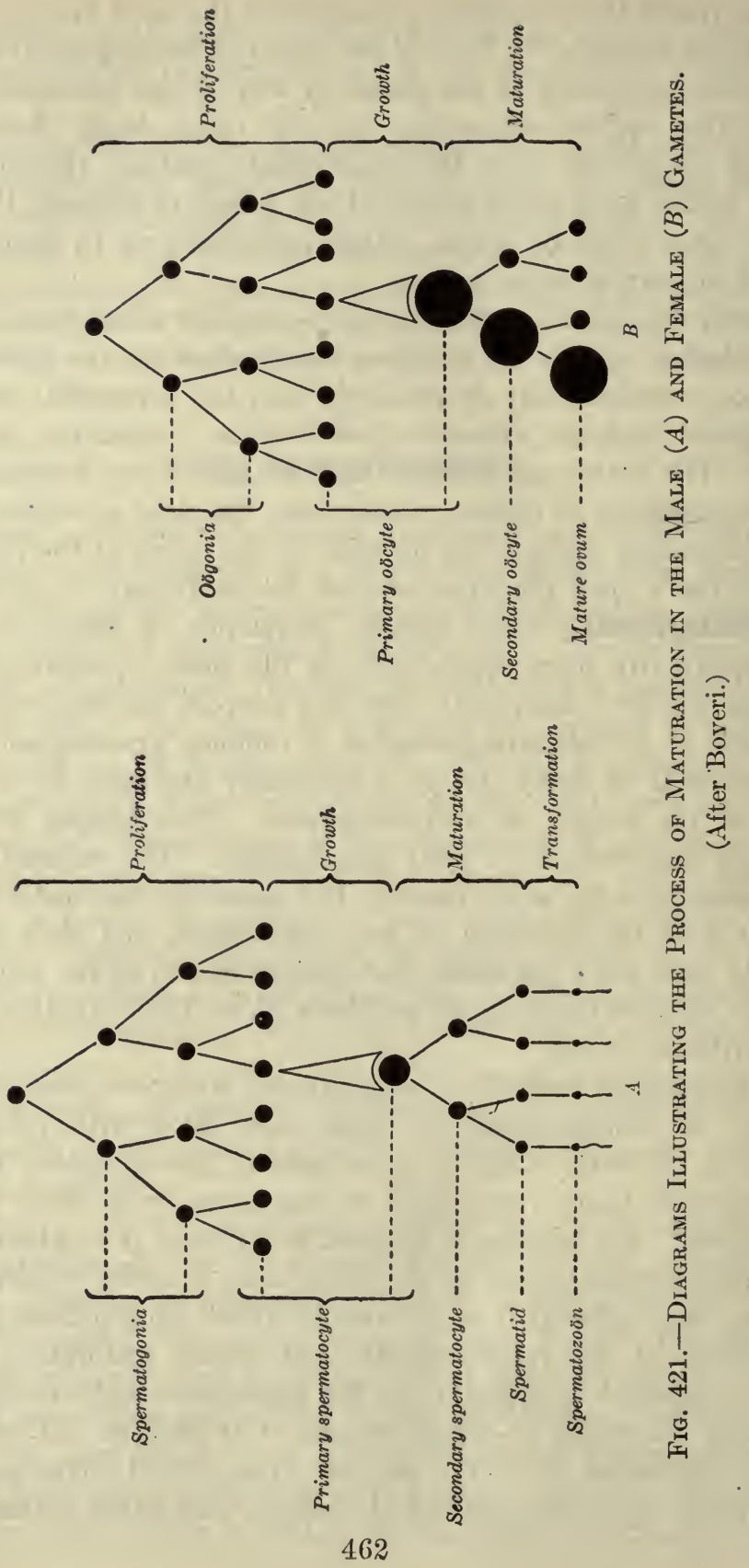


volving a reduction as well as an ordinary equation division, is limited to the maturation mitoses occurring in active testes and ovaries. The appended scheme will serve to show graphically the steps in spermatogenesis and oögenesis, and the correspondences and differences between the two.

We may begin the detailed description with the last generation of spermatogonia, the secondary spermatogonia. In the early resting stage the nucleus is poly-vesicular, each vesicle representing one or several chromosomes (Plate A, Fig. 1). Subsequently the separate vesicles fuse to form a greatly lobulated nucleus. In Fig. 2 one vesicle is shown still separated; this probably represents the accessory or sex chromosome; subsequently it also fuses with the main nucleus and remains generally indistinguishable from the other nuclear elements until early stages of the succeeding generation of cells, the primary spermatocytes (Fig. 8). Figs. 3, 4, 5, 6 and 7 represent successive stages in the karyokinetic division of the secondary spermatogonia. In the telophase of the spermatogonial division and the earliest prophase of the primary spermatocytes, as well as subsequently, the sex chromosome (allosome; heterochromosome) does not pass through the diffuse and spireme phases of the ordinary chromosomes (autosomes; euchromosomes) but remains chromatic and relatively compact. Subsequently it condenses considerably more (Fig. 8) and thereafter persists until late spermatid stages as a compact, deeply chromatic body, generally of oval or bilobed form, and usually close to or on the nuclear wall (Figs. 10; plate B, 14 and 20; and plate $\mathrm{C}, 27)$. Following the early growth stages, when the nucleus is in the resting or diffusive chromatic stage (Fig. 8), a series of changes occur known as synapsis. The most characteristic phase is the one in which the spireme becomes closely aggregated and polarized (synizesis) and subsequently segmented, the segments becoming looped and attached by their open ends to the nuclear wall over a constricted area, generally in the vicinity of the sex chromosome. The loops now free one attached end and unite in pairs by their free ends forming taller loops, oriented similarly to the smaller loops (bouquet figure, synaptenic nucleus). The number of loops is now half the original number, which represented the full number of chromosomes. This chromosome pairing is the essence of synapsis. An end to end union as here described is termed telosynapsis (metasyndesis); side by side union, described in certain forms, parasynapsis (parasyndesis). The important point is the reduction to half (plus one, the accessory) the original number (diploid) of chromosomes; the reduced number is 
termed the haploid group. The loops now lose their polarized condition (postsynaptic phase) and become scattered throughout the nucleus, which has meanwhile grown in size, and become more or less closely united into a more or less continuous thread. This soon shows evidences of a longitudinal split (Fig. 12), the diplotene phase; subsequently this undergoes transverse segmentation into half the original number

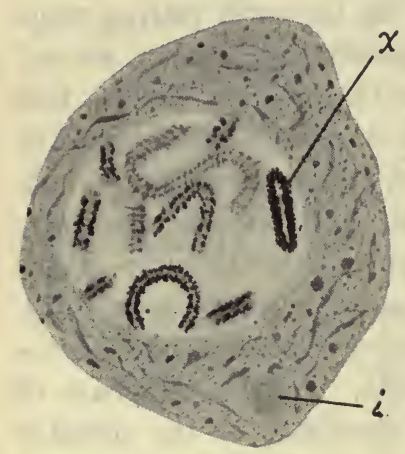

F1g. 422.-Primary SpermaTOCYTE OF A GRASSHOPPER, Hippiscus Tuberculatus, Showing the Compact Accessory Chromosome (x) Among the Paler Mossy Prophase EuchroMOSOMES, AND THE IDIOSOME (i).

The cytoplasm contains many granular and filamentous mitochondria. Flemming fixation; iron-hematoxylin stain. $\times 1800$.

father and mother when the egg was fertilized preceding development. At synapsis the chromosomes actually pair and fuse; and obviously in the manner indicated in $(A)$, for the chromosomes can again be arranged in a graded series, corresponding approximately in size and range to the pairs indicated. Since the accessory has no mate the reduced number of chromosomes is 12 .

It is now quite generally believed that the chromosomes of a pair represent paternal and maternal contributions; thus from the viewpoint of inheritance, assuming that the chromosomes are the vehicles of certain hereditary characters and qualities, the offspring of a pair of parents inherit 


\section{Plate A.}
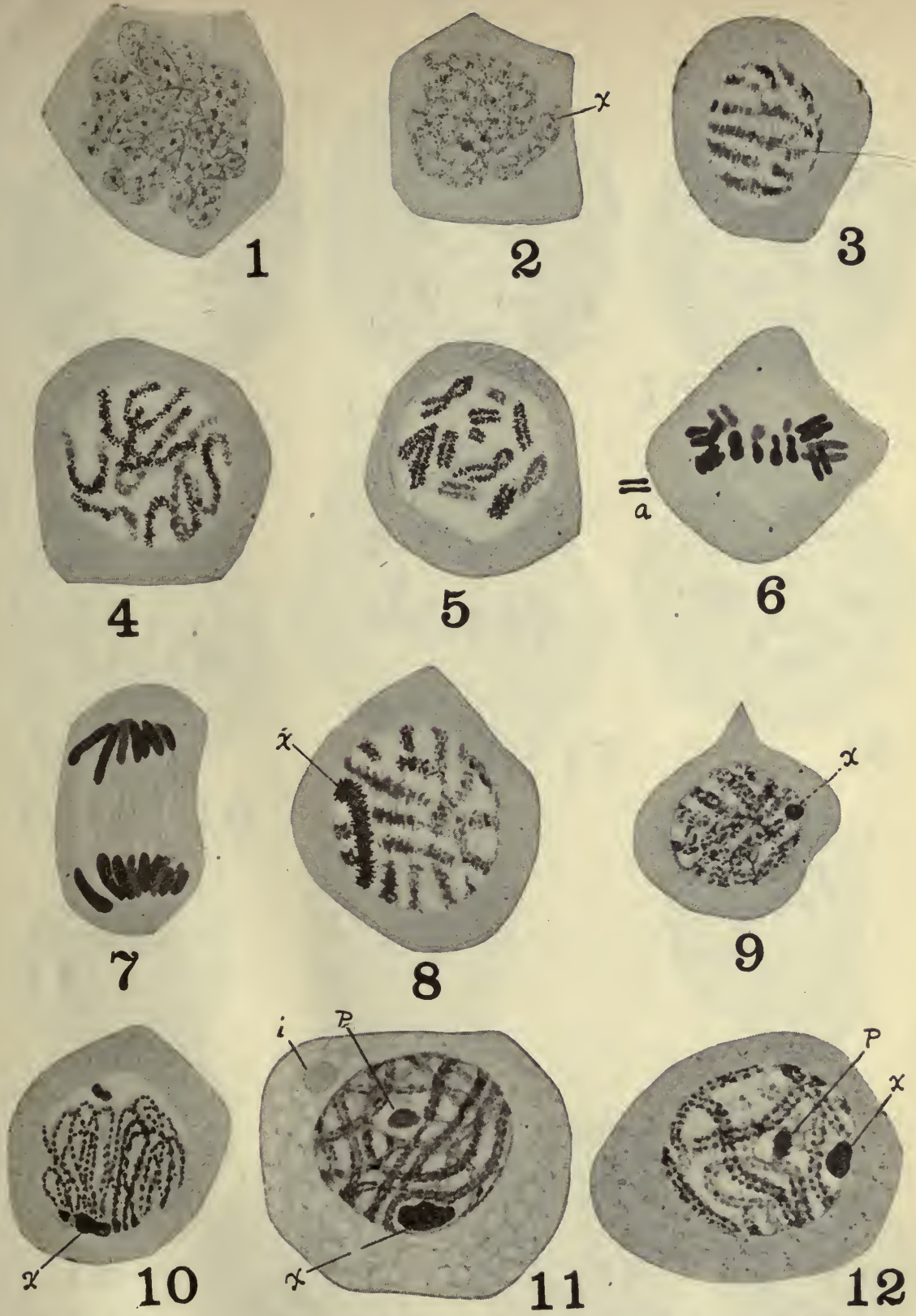

Piates A, B, and C.-Successive Stages in the Spermatogenesis of SchistoCERCA DAMNIFICA.

$x$, accessory or sex chromosome; $p$, plasmosome; $i$, idiosome. 1 to 7 , spermatogonia; 8 to 19 , primary spermatocytes; 20 to 26 , secondary spermatocytes; 27 to 30 , spermatids, differentiating (31 to 35) into the spermatozoön (36). 10, synapsis stage; 18 and 24 , metaphase plates of chromosomes; 6 a, diagram of a longitudinally split univalent chromosome from the metaphase plate of a spermatozonial mitosis; $16 \mathrm{~b}$, diagram of a bivalent chromosome, in tetrad condition, from the metaphase plate of the first maturation spindle, undergoing the reduction division; 23c, longitudinally split univalent chromosome entering the second maturation spindle for the equation division. (Flemming fixation; iron-hematoxylin stain.) $\times 1750$. 

Plate B.
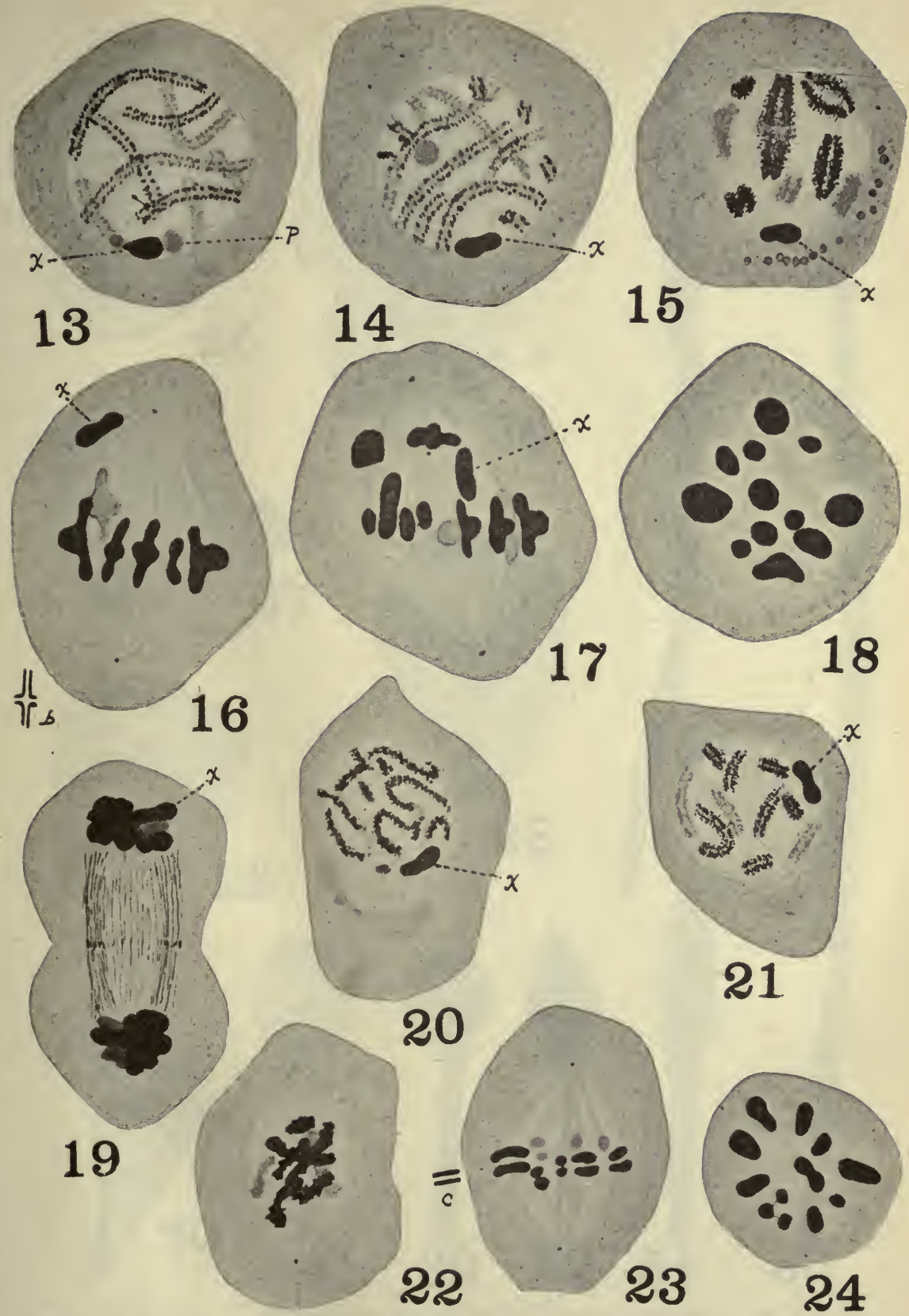

Plates A, B, and C.-Successive Stages in the Spermatogenesis of SchistoCERCA DAMNIFICA. 

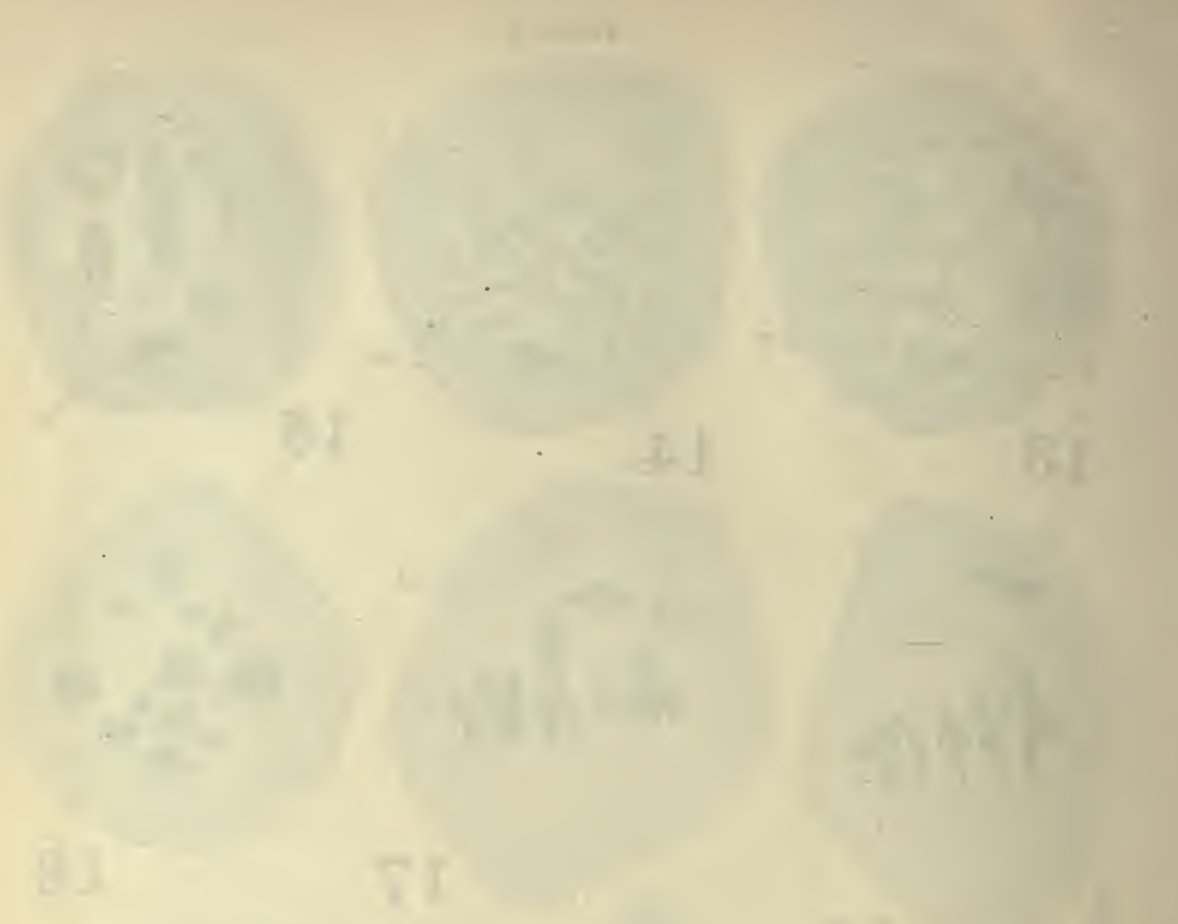
Plate C.
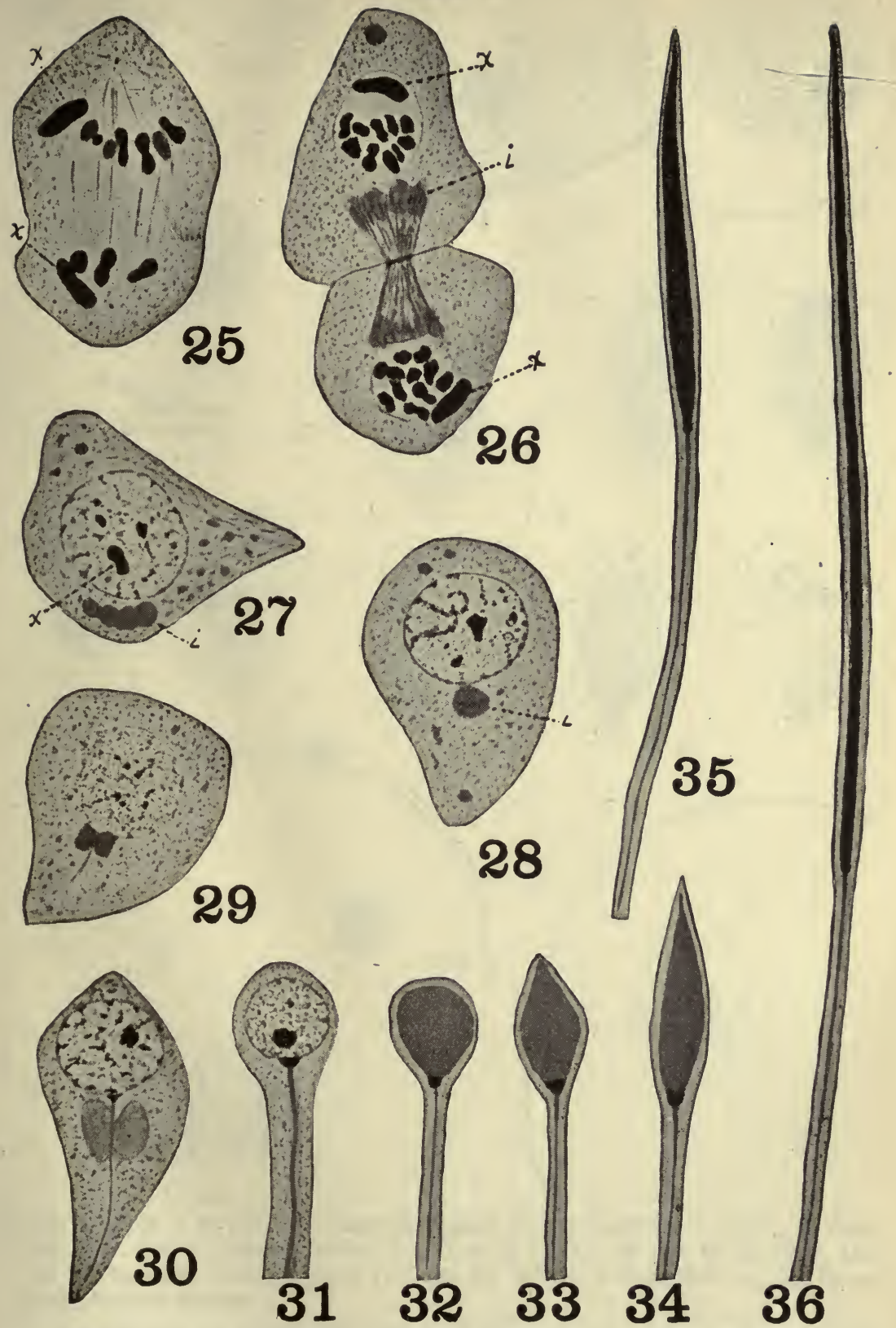

Plates A, B, and C.-Successive Stages in the Spermatogenesis of SchistoCERCA DAMNIFICA. 
$4 \sqrt{2}+12$

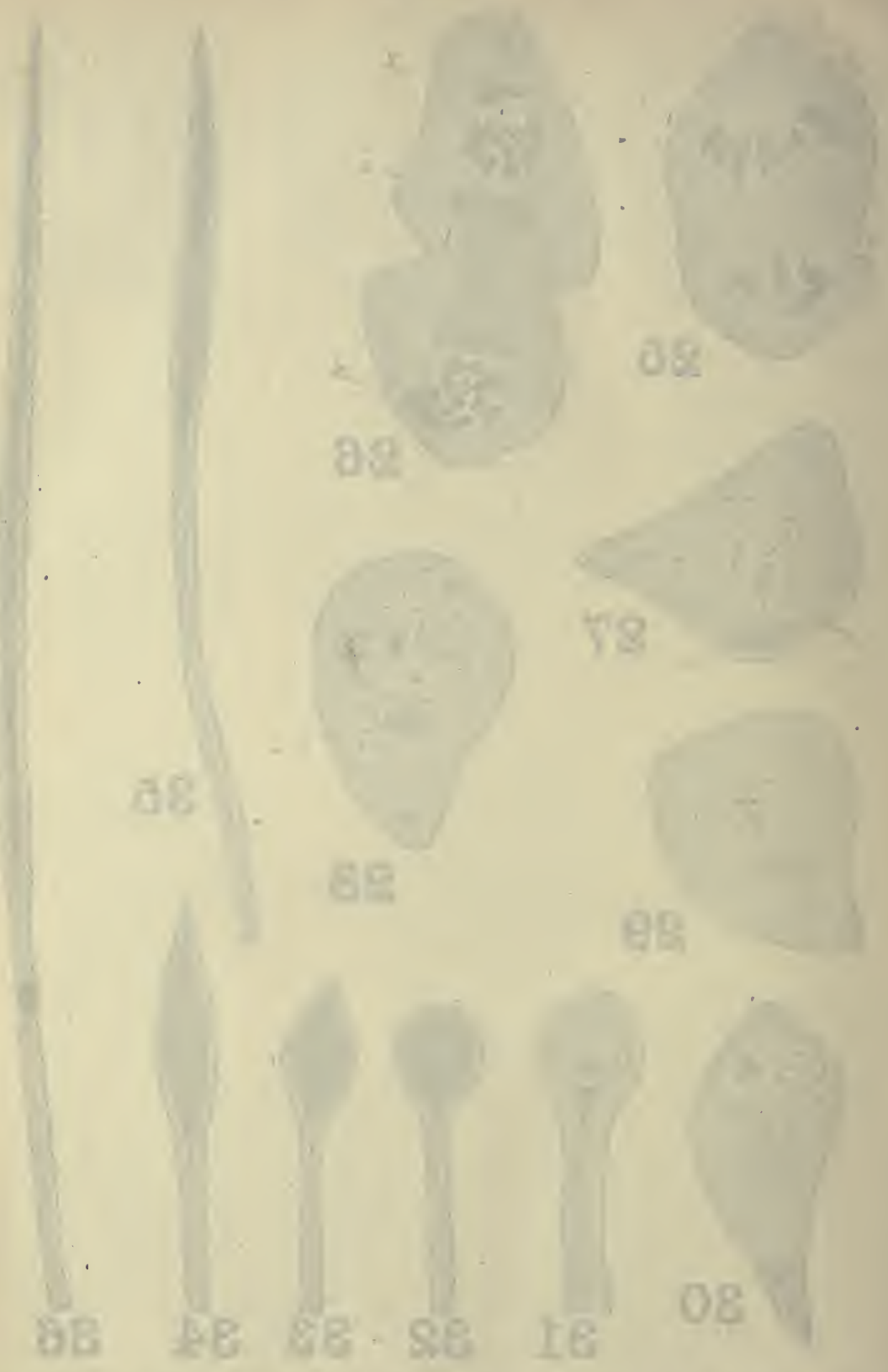

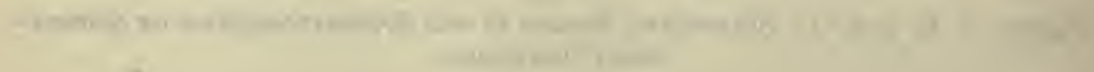
$+$ 


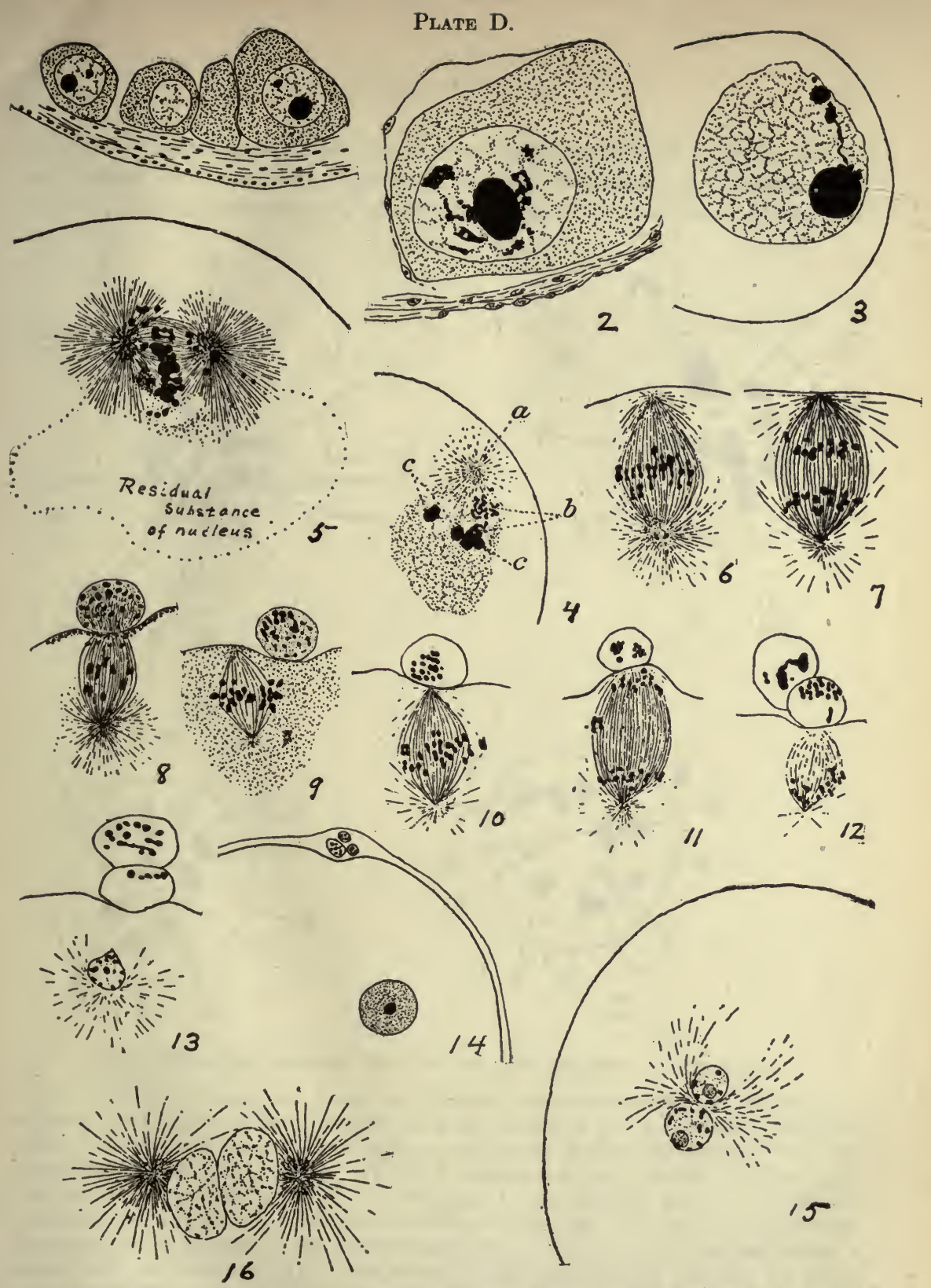

Plate D (Figs. 1 to 16 ). - Successive Stages in the Growth (1 and $2, \times 2100$, AND $3, \times 700)$, Maturation $(4,5,6,7,8,9,10,11,12$ and 13, X 2100, AND $14, \times 700)$, and Fertilization (15 and $16, \times 700)$ of the Egg of the StarFISH, Asterias forbesii.

In the ripe egg (3) the chromosomes are aggregated in a group connected with the nucleolus by a chromatic thread. In 4 the nucleolus is fragmenting $(c)$ and the chromosomes ( $a$ and $b$ ) are taken onto the first maturation spindle (5). In 13 two polar bodies are present, and the female pronucleus is forming. In 14, the mature egg, three polar bodies are present. In 15 and 16, the male and female pronuclei are fusing to contribute their chromosomes to the first segmentation spindle. 


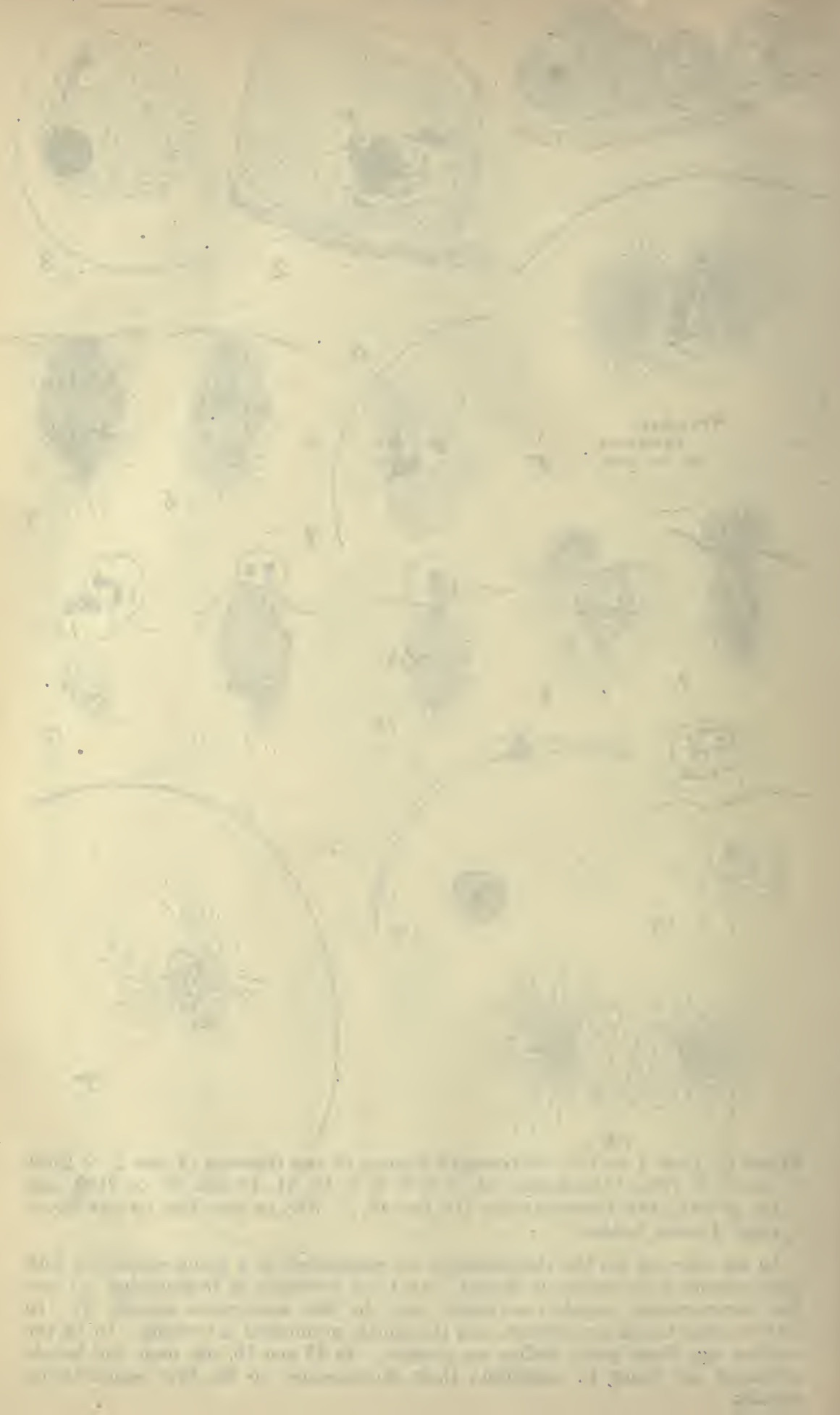


by way of chromosomes only from their grandparents. For their parents are simply, as it were, the 'guardians' of their grandparental chromosomes which, following the synapsis phase of growth (the final stage of the pre-
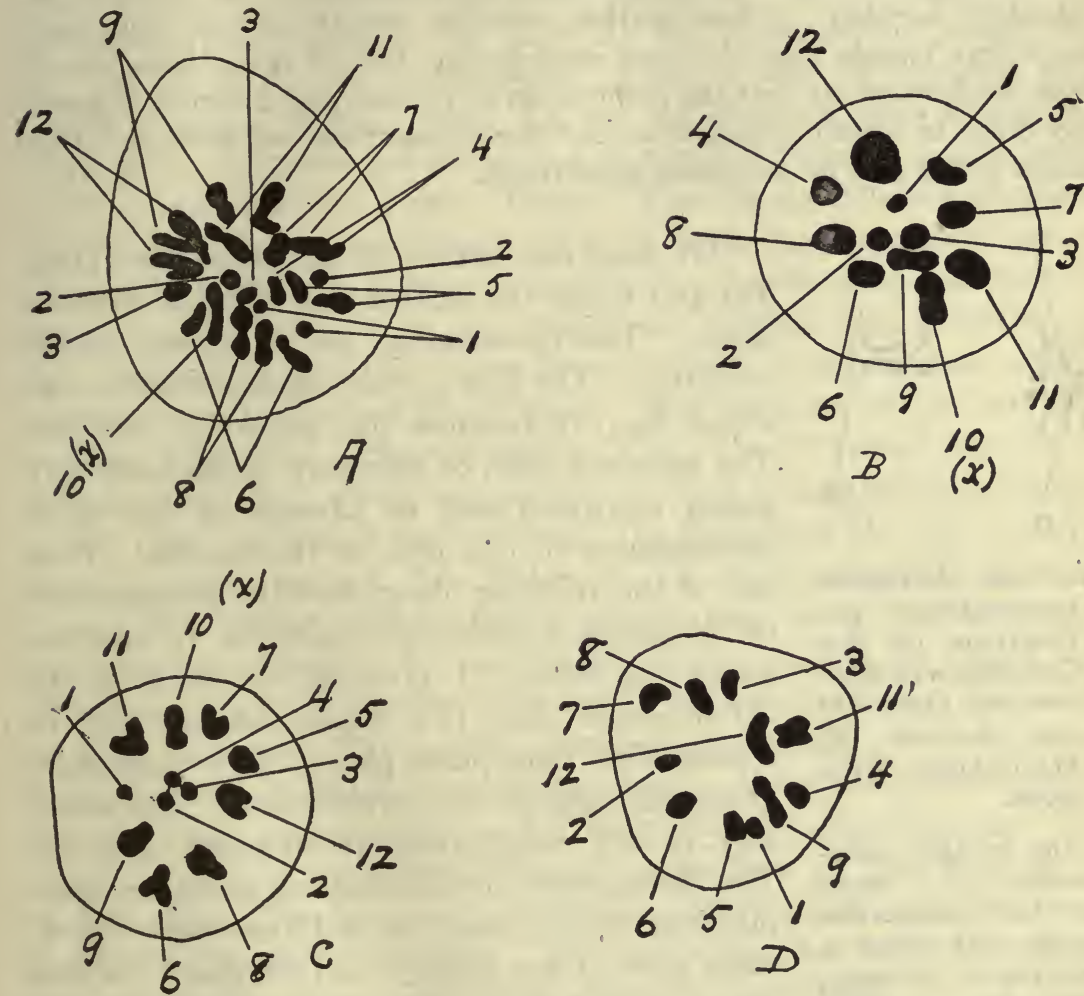

Fig. 423.-Chromosome Groups of Schistocerca damnifica.

(A) Diploid chromosome group of dividing spermatogonium of Schistocerca, showing the 11 pairs of chromosomes, and the unpaired (10-x) accessory chromosome. (B) Haploid group of primary spermatocyte, showing 11 bivalent chromosomes and the accessory $(10-\mathrm{x})$. The 11 bivalents fall into a series with the same size relationships as the pairs of chromosomes in the diploid group. $(C$ and $D)$ Chromosome groups of secondary spermatocytes, one $(D)$ with 11 univalent chromosomes, like those of the diploid pairs, the other $(C)$ with similar chromosomes, plus the accessory $(x)$. All mature eggs' presumably contain 12 chromosomes, homologous with $\mathbf{C}$.

vious fertilization which resulted in the parents) become redistributed in various combinations in the maturing germ cells from which the offspring develop, and determine their hereditary possibilities. In other words the germ-plasm is a continuous substance handed on from generation to gen- 
eration. Its 'elements' are the same, except in so far as the complement is halved during maturation and restored at fertilization by addition of an equal quota from another line of descent. This halving and doubling is the physical mechanism which underlies the phenomenon of variation involved in heredity. Offspring thus resemble parents because both have had origin largely from the same germ-plasm: lack of resemblance results from the fact mainly that the germ-plasm of parents and offspring of necessity differ in certain elements since different additions are made at fertilization in the case of successive generations.

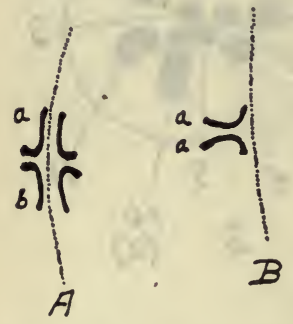

Fig. 424.-Dingrams ILLUSTrating THE Behavior OF THE Chromosomes DurING The First $(A)$ AND Second (B) Maturation DivisIONS.

The bivalent chromosome $a+b$ enters the first maturation spindle and suffers a reductional division, chromosome $a$ being separated from chromosome $b$. In the second maturation division, each chromosome undergoes an ordinary longitudinal division. This division is already foreshadowed during the first or heterotypic division, producing the tetrad condition of the bivalent chromosome.

We may now return to the metaphase (Fig. 16) and follow the further course of the chromosomes. The chromosomes are in typical tetrad condition. The first division most probably separates the chromosomes that paired at synapsis. The unpaired, odd, or accessory chromosome (x) passes undivided and in advance of the other chromosomes to one pole of the spindle. Thus one of the resulting daughter-cells, prespermatid or secondary spermatocyte, receives 11 chromosomes, the other, 11 plus the accessory, or 12 chromosomes. Fig. 17 is a side view and Fig. 18 a polar view (metaphase plate), of the metaphase group, showing all 12 chromosomes. The accessory is still recognizable at telophase, Fig. 19. The chromosomes of the resulting secondary spermatocytes do not pass into a diffuse stage but remain more or less compact and chromatic-a condition characteristic of the later segmented spireme stage of mitosis. In half the cells the accessory appears in its typical shape, staining reaction, and position, as shown in Fig. 20. This shows also a remnant of the original spindle. Attention should here be called to the fact that in many forms chromatic particles have been described passing from the nucleus into the cytoplasm, during the growth stages of the primary spermatocyte, particularly at the synapsis phase. Similar conditions have occasionally been described also for the spermatogonia, and in several instances even for the secondary spermatocytes. These extrusion products are called chromidia, 
and are regarded by some as the raw material for mitochondria, to which latter certain investigators have attached hereditary significance.

The further description of spermatogenesis will be confined to that half of the secondary spermatocytes containing the accessory or sex chromosome; conditions in the other half are closely similar except for the absence of the odd chromosome. Figs. 21 and 22 illustrate successive prophase stages. Figs. 23 and 24 illustrate side and polar views of the metaphase of the second maturation mitosis; in the latter all 12 chromosomes are shown. Plate C, Figs. 25 and 26 show anaphase and telophase stages respectively. Fig. 27 is the early spermatid stage, the accessory still conspicuous. A conspicuous cytoplasmic element of these stages is the idiozome (i), apparently derived from a portion of the disappearing spindle. Later stages of the metamorphosis of the spermatid are shown in Figs. 28 to 31 . The idiozome divides into two moieties (Fig. 29). These elongate (Fig. 30) and spread out over a filament (Fig. 31), which has grown out from a dark-staining granule on the outer surface of the nucleus. The cytoplasm also concurrently flows backward around this filament. In conformity with what is known of a similar granule with a similar function in various forms, this granule represents the centrosome of Fig. 23, but its origin and history are obscure. Further steps in the metamorphosis are illustrated in Figs. 32 to 36 . The mature spermatozoön consists of a long, relatively lightly chromatic, lance-shaped head, a deeply-staining thimbleshaped middle piece (body), and a long gradually tapering tail. The latter consists of the central filament ensheathed by the products of the idiozome. The entire spermatozoön is furthermore enveloped in a close-fitting sheath of cytoplasm. The head originated from the nucleus, and the middle piece presumably from the centrosome and a small contribution of cytoplasm.

Determination of Sex.-Anticipating somewhat, we may say that the process of oögenesis is essentially similar to that described for spermatogenesis. The cardinal difference relates to a numerical inequality between the functional descendants of a spermatogonium and an oögonium: in the former case the four resulting cells are all functional, and from the standpoint of their numerical chromosome content of two sorts; in the latter, three of the resulting cells are abortive (polocytes), only one remaining functional. Of the two classes of spermatozoa, one has an even number of chromosomes, the other an odd number. All mature eggs have the same number of chromosomes as that of the spermatozoa with the greater number. This description applies strictly only to those instances where an odd 
or accessory chromosome appears in the male, or where its representative is an odd group. Cases are known among insects, where the homologue of the accessory may have a mate of smaller size (or possibly even equal size, but of typical allosome behavior-idiochromosomes), or where the accessory representative may be an even group, in which instances the numerical relationships are different, but the principle of sexual relationship remains the same.

Confining our attention then to the simplest case, as illustrated in our specimen of the grasshopper, we may note that all the cells of the female, including the ooggonia, contain 24 chromosomes. Two of these correspond in size to the accessory of the male group, which group in all soma cells and all spermatogonia comprises 23 chromosomes. We recall that one-half of the spermatozoa received 11 chromosomes, the other half 11 plus the accessory, or 12 chromosomes. All mature eggs contain 12 chromosomes. When therefore a spermatozoön with 11 chromosomes fertilizes an egg with 12 chromosomes, the resulting organism will have 23 chromosomes, and become a male; when, on the contrary, a spermatozoön with 12 chromosomes fertilizes an egg with 12 chromosomes, the organism will have 24 chromosomes and become a female.

An essentially similar relationship is now known to obtain in many forms, chiefly insects, but including also various vertebrates, even mammals and man. According to Winiwarter (Arch. de Biol. T. 27, 1912), the white human male produces spermatozoa of two sorts, one with 23 chromosomes, the other with 24 ; the male cells containing 47 chromosomes, the female 48. There is some uncertain evidence to indicate that the negro has half the number of chromosomes possessed by the white man, the mulatto consequently possessing numerically intermediate groups.

With regard to the function of the accessory chromosome in relation to sex determination, two obvious possibilities present themselves: (1) it may qualitatively determine sex, that is, carry the determiner upon which sex depends; (2) it may act in a quantitative way, that is, sexual difference may depend upon differences in the amount of chromatin. On the contrary the presence of one accessory in the male, and two (homologues) in the female may be simply accompaniments of sex in a sense somewhat similar to secondary sexual characters (e. g., combs and spurs in poultry), these as well as other sexual differences being in common dependent upon some more recondite fundamental sex 'determiner.' Experimental evidence tends increasingly to show that all organisms carry the potentialities of both sexes, and that sex differences are largely differences in degree of differentiation; in other words, that femaleness represents an inhibited male. The greater amount of chromatin in the female, due to the presence of two sex chromosomes-as against one in the male-may be conceived to inhibit the higher grade of differentiation demanded by maleness.

Attempts have frequently been made recently somehow to bring sex 
determination in line with our knowledge of inheritance in general, and more particularly with the principles of Mendelian inheritance. This accordingly would seem to be the logical place for a brief presentation of the main facts of Mendelism, and the hypothesis that attributes to the chromosomes the function of vehicles, or determiners for hereditary qualities. Barring the sexual numerical differences above discussed, the specific number of chromosomes is constant; that is, all cells of the white human male have 47 , of the female 48 chromosomes. The more variable, superficial, or more recently acquired characters are believed by many to depend upon the presence in the developing egg-consequently the cells of the organism-of certain chromosomes, the so-called 'determiners.' The more fundamental characters are believed to inhere in the cytoplasm of the egg. For example, the vertebrate condition of a man is thus believed to result from the constitution of the egg; the color of his eyes from the presence or absence of some determiner or factor in a particular chromosome.

To elucidate the matter further, we must regard an organism as a congeries of almost numberless characters; of these some are like those of the mother and her ancestors, others like those of the father and his ancestors, others apparent blends. In Mendelian phraseology these characters are called unit characters, and their material basis is located in particular chromosomes. We may select a single pair of unit characters in order further to present the principles of Mendelian inheritance. Suppose we consider the coat color of gray and white guinea pigs. When individuals characterized by these coat colors are crossed, the resulting offspring, first generation, are all gray in color. The gray color is said to be dominant, the white recessive. However, the potentialities or determiners for white coat color are still present in the gray hybrid, for if such hybrids are inbred, there result gray and white offspring in the proportion, of 3 of the former to 1 of the latter. The latter if inbred produce only white offspring, hence called pure recessives, but of the 3 gray only one if bred with its own type (pure dominants) will produce gray; two out of the three if inbred produce again 3 gray to 1 white. The proportion of 1 pure dominant to 2 hybrid dominants to 1 pure recessive is the well-known Mendelian formula for one pair of unit characters in cross-breeding of specific varieties. The character of this formula depends upon the fact that no germ cell carries both determiners of a pair of unit characters, but only one or the other. It thus follows that from the standpoint of a single pair of unit characters (e. g., color of iris) there are two categories of eggs and likewise of sperm. According to the laws of chance there will then be one chance for the determiner of either character (e. g., gray and white coat) to meet its like, to two chances that it may meet its opposite, hence the formula $1: 2: 1$ :

The central concepts of Mendelian inheritance thus are (1) unit characters; (2) the phenomenon of dominance; and (3) the principle of seg- 
regation, producing a purity of the germ cells. The unit characters are external features, the hypothecated determiners for these are provisionally located in the chromosomes.

Sex is of course inherited in the sense that an individual resembles either its father or its mother in respect of sex. Moreover, considering a species as a group, the two sexes are approximately numerically equal. This is the ratio expected when a Mendelian pure recessive (homozygote) is crossed with a hybrid dominant (heterozygote) - for again by the laws of chance, the chances are equal that a determiner shall meet with its opposite or its like. On the basis of these considerations mainly, coupled with the data of sex-linked inheritance (see Morgan, "Heredity and Sex," 1915), sex is conceived by some to be inherited in Mendelian fashion, the individual with a single accessory (male) being regarded as the heterozygote, that is, digametic; that with two accessories, the homozygote, that is, the homogametic. However, such serious contradictions and difficulties arise when the matter is thus strictly interpreted that a simple Mendelian view of sex ratios must probably be abandoned. In any event the interpretation that regards the heterochromosome, when present in the duplex condition, as in the female, as an inhibitor to male sex development from the viewpoint of secondary sex characters, accords well with much of the experimental evidence from vertebrates. The bulk of the best evidence tends to show that sex is determined at fertilization, and that it cannot thereafter be altered by control of environmental conditions. Relatively much chromatin may be thought to underlie the anabolic condition characteristic of femaleness, relatively less chromatin the katabolic conditions which subserve the high differentiations of maleness.

Oögenesis. - We must now return to a detailed description of the oögenesis. Splendid material for the presentation of the facts concerning this process is the egg of Ascaris, or that of certain molluscs, or the more easily available egg of some echinoderm, e.g., starfish. This description will confine itself to the starfish egg. The essential points can be well illustrated with this material (Plate D, Figs. 1-16). In contrast to the spermatozoön, which is relatively minute, highly motile, and with a minimum amount of cytoplasm, the egg on the other hand grows enormously in size (ratio of sperm to egg 1:500,000 \pm ), is non-motile, and has a large amount of cytoplasm heavily laden with yolk material. This phase of the oögenesis is known as the growth period; during the early portions of this period synapsis occurs, and subsequently chromatic material (chromidia) appears in the cytoplasm, presumably extruded by the nucleus, and in part at least changed into yolk. This generation is the primary oöcyte, the preceding generation being 
oögonial, primary and secondary, characterized by extensive proliferation. The primary oöcyte divides in a manner similar to the primary spermatocyte, that is by heterotypic mitosis, but the daughter-cells are very unequal in size, each, however, with the same number of chromosomes (18); the small abortive cell is called the polar body, or better polocyte, while the large cell becomes a secondary oöcyte. Both polocyte and secondary oöcyte again divide, the end result being three similar polar bodies and one large oötid or mature ovum, now ready for fertilization. It must be recalled that the maturation process is in essence a process of eliminating one-half of the original quota of chromosomes, and a quantitative reduction of its original amount of chromatin to one-fourth. At fertilization, by fusion of egg and sperm, the specific number of chromosomes is reëstablished-differing in many cases in male and female of the same species-and the chromosomes by growth regain or even exceed their original size. With this brief consideration of the function of the germ glands in general, we may proceed to a description of the structure of the male organs of reproduction and subsequently of the female organs.

\section{MALE ORGANS OF REPRODUCTION}

These organs include the penis and the testes, together with their accessory glands, and the excretory ducts which connect the testes with the urethral canal. The excretory ducts include the epididymis, ductus (vas) deferens, seminal vesicles, and ejaculatory ducts, and with their termination in the urethra there are connected the ducts of the prostate gland and the bulbo-urethral glands (of Cowper), whose secretion mixes with that of the testes to form the semen. The male urethra serves the double function of a urogenital canal.

\section{Internal Genital Organs}

\section{Testis}

The testis is to be regarded as a gland with a double function; it produces cells (spermatozoa), hence a cytogenic gland; and an internal secretion, hence, in part an endocrin gland. In connection with the testis must be considered also its excretory duct system, and the various glands accessory to it. The relationship of these various structures is shown in diagram, Fig. 418. 
The testis is encased in a robust fibro-elastic capsule, the tunica albuginea, the innermost layer of which is of looser texture and very vascular, hence called the tunica vasculosa. External to the albuginea is a double-layered sac of peritoneum, the tunica vaginalis, its visceral layer closely adherent to the capsule. The human testis measures about one and one-half inches in length, one and one-quarter inches in width and one inch in thickness. Septa continuous with the capsule

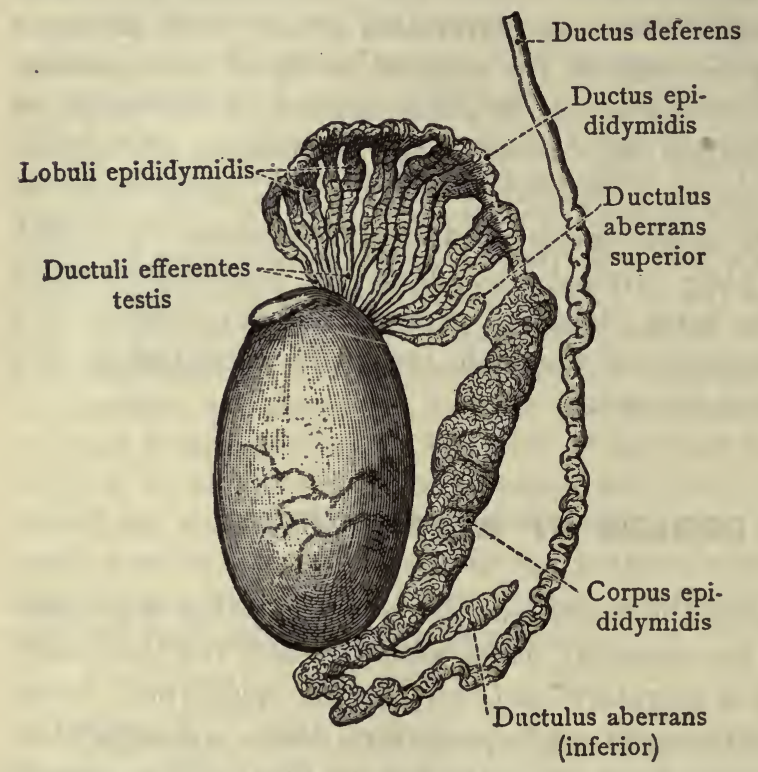

Fig. 425.-The Testicle with Its System of Efferent PASSAGES.

Natural size. (After Toldt.) divide it into a $\mathrm{number}$ of compartments or lobules, pyramidal in shape, the apices converging to an anterodorsal mass of dense connective tissue, the mediastinum testis (corpus Highmori), corresponding to a hilum. The lobules contain each several,frequently two, extensively convoluted tubules, the seminiferous tubules (tubuli contorti). When uncoiled they measure from one to two f e et. The entire testis contains several hundred lobules; the number has been variously estimated at from one hundred to four hundred.

Bremer (Amer. Jour. Anat., 11, 4, 1911) describes the tubuli contorti of man as tubules that may be single, ending blindly, may foranch or may anastomose. Huber and Curtis (Anat. Rec., 7, 6, 1913), on the contrary, state that in the adult rabbit the seminiferous tubules "present no blind ends, diverticula or nodular enlargements." Their simplest form is that of an arch beginning and ending in a tubulus rectus. The two limbs may lie in adjacent lobules. Complex tubules are also described, resulting from the linkage of from three to twelve 
simple arched tubules. According to Curtis (1915) branches and anastomoses of seminiferous tubules are infrequent in the mouse testis, more frequent in dog, and most frequent in rabbit.

The testis is lodged in the scrotum. The wall of the scrotum is essentially like the general integument, except that it may be more

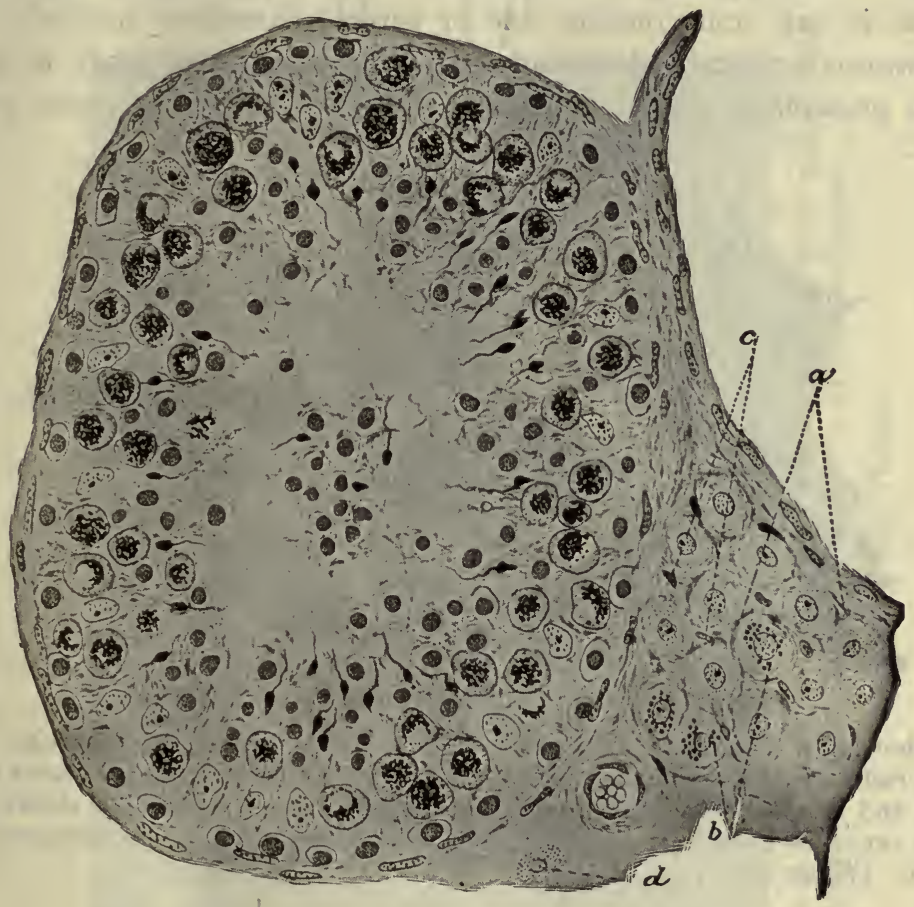

Fig. 426.-Seminal Tubule of a Man in Transection.

$a$ and $b$, interstitial cells, the latter containing coarse granules; $c$, connective tissue cells; $d$, a mast-cell of the connective tissue. Within the tubule several phases of spermatogenesis are well shown. Highly magnified. (After Spangaro.)

highly pigmented. Its subcutaneous layer, however, is looser in texture and contains more elastic tissue and smooth muscle. It is known as the dartos.

The wall of the seminiferous tubules consists of fibro-elastic tissue. They are lined with a several layered epithelium, the cells representing the several stages of spermatogenesis. In addition to the germ or sex cells, the epithelium contains sustentacular cells (Sertoli cells), to which the spermatids bejcome attached during process of metamorphosis into ripe spermatozoa (spermia). It seems probable that the spermatids also 
draw nourishment from the sustentacular cells for the work of metamorphosis, hence also known as 'trophocytes.' The sustentacular cell is roughly of tall columnar shape, tapering somewhat irregularly toward the distal pole. The proximal pole contains the nucleus and frequently flares somewhat giving the entire cell a tall pyramidal shape. The nucleus is pale and contains one or several chromatic nucleoli. The spermatozoa are embedded head first, four to eight or more to a cell, in the protoplasm of the trophocyte. Such a composite group consti-

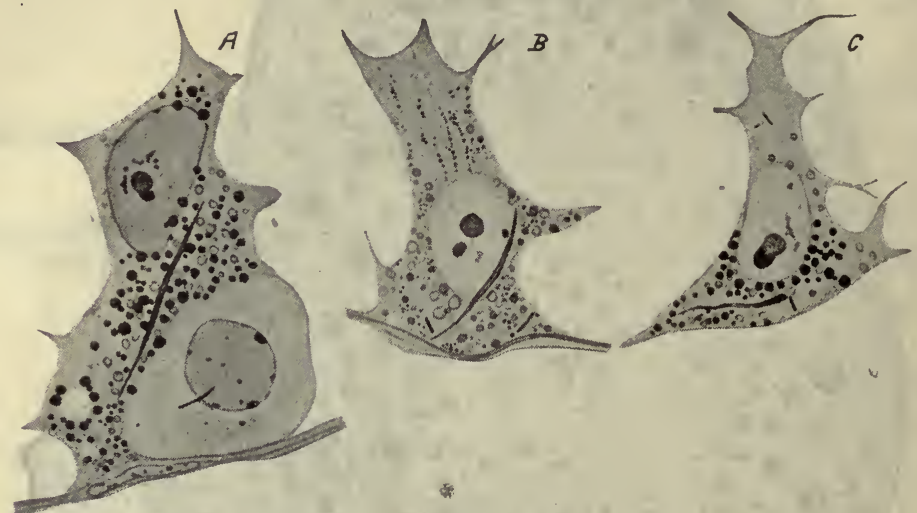

Fig. 427.-Sertoli Cells of the Human Testis.

$A$, showing a crystalloid (of Charcot) and lipoid granules and spherules; below to the right a spermatogonium with a crystalloid (of Lubarsch). $B$ shows a crystalloid and two accessory rods, and lipoid granules and droplets. $C$ shows at the base a crystalloid and one accessory rod, and at the summit two accessory rods. $\times 600$. (Winiwarter.)

tutes a so-called spermatoblast of von Ebner. The sustentacular cell contains one or several crystalloids. Their origin and function is unknown. They were regarded by Montgomery as probably sustentacular cell determinants, having been traced by him from the common mothercells of both trophocyte and spermatogonium into the former, the latter being said to lack these elements. However, Winiwarter (Fig. 427) reports similar crystalloids in both trophocytes and spermatogonia. Trophocytes more probably have an origin distinct from that of the germ-cells, according to certain investigators arising from the Bowman's capsule of the Wolffian tubules of the mesonephros.

Every section of a tubule of an active testis contains several, sometimes all the stages of spermatogenesis. Since the spermatogenetic process generally travels in waves, a longitudinal section is most favor- 


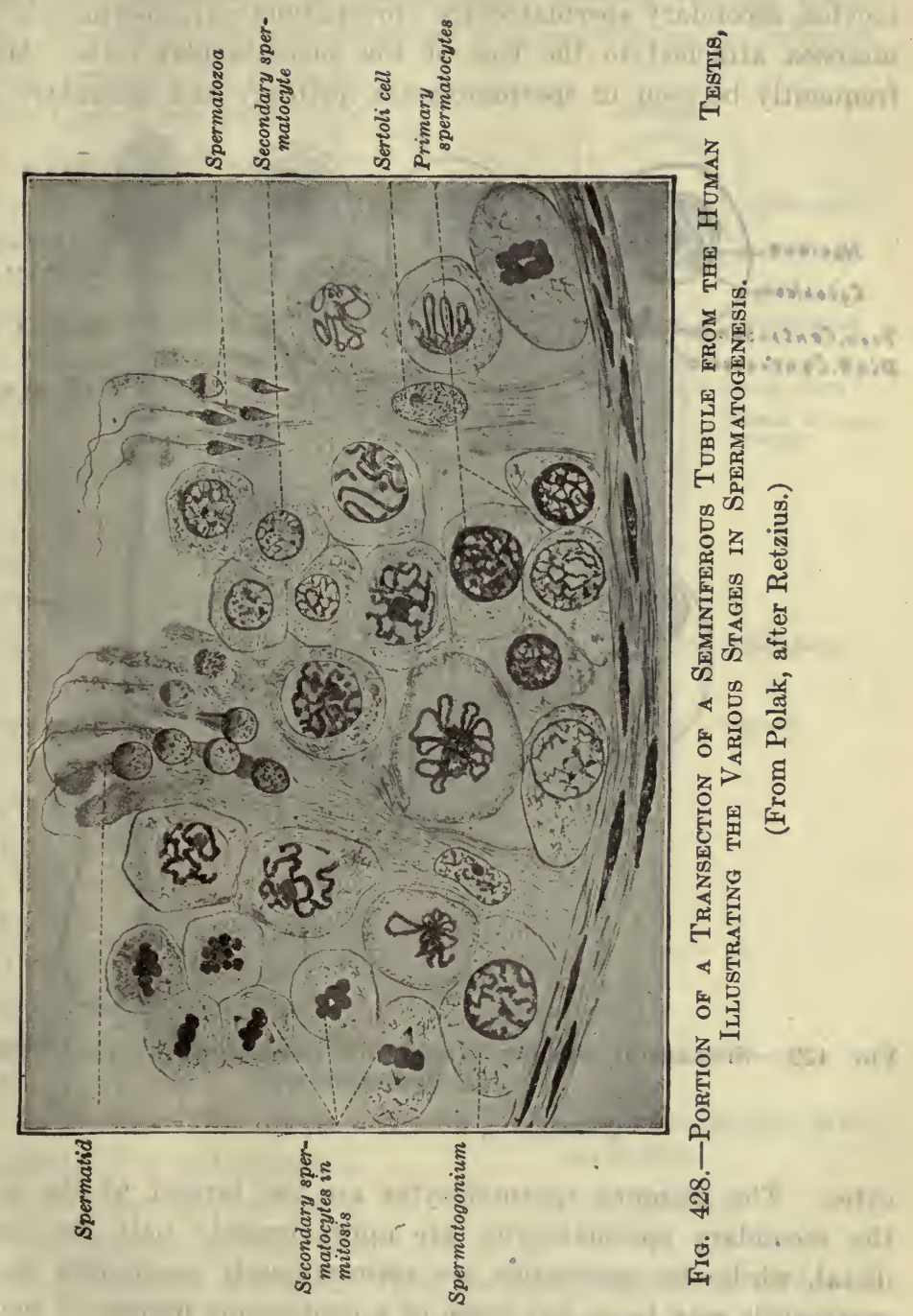

cthiตn 
able for a study of the continuous process. From wall to lumen of tubules can be seen, at successive levels, spermatogonia, primary spermatocytes, secondary spermatocytes (prespermatids), spermatids, and spermatozoa attached to the tips of the sustentacular cells. Mitoses can frequently be seen in spermatogonia, primary and secondary spermato-
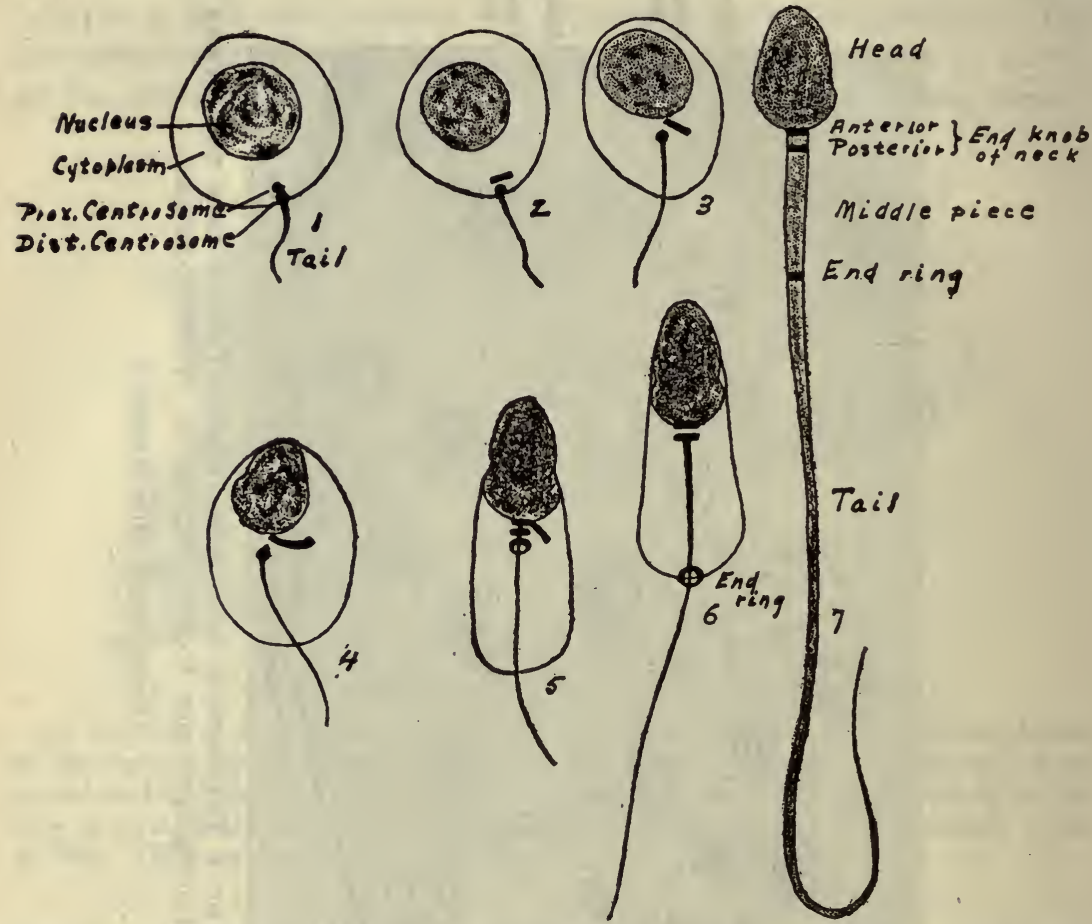

Fig. 429.-Successive Stages in the Metamorphosis of the Spermatid into THE SPERMatozoöN.

(Schematic, from Bonnet, after Meves.)

cytes. The primary spermatocytes are the largest of the germ cells; the secondary spermatocytes are approximately half the size and lie distal, while the spermatids are approximately one-fourth the size; the spermatids may be at any stage of a continuous process of metamorphosis involving nuclear, cytoplasmic, and general form changes. The spermatogonia are of various sizes, frequently approximating that of the primary spermatocytes, but always lie peripherally, on the basement membrane between sustentacular cells. The above named marks constitute the grosser criteria for identifying the several generations of 
cells involved in spermatogenesis. As concerns details, the criteria above given for the grasshopper testis, can be applied.

The Spermatozoön.-Spermiohistogenesis, however, differs somewhat. The spermatid nucleus becomes progressively more compact, chromatic and smaller. At the same time it moves toward one pole of the cell, the cytoplasm flowing backward, except for a thin envelope (galea capitis) which terminally forms a covering for the perforatorium which represents a portion (acrosome) of the original idiosome. The idiosome is the germ-cell representative of the archoplasmic complex. Concurrently with the changes outlined for the nucleus it liberates a centrosome, and centrosome and acrosome pass to opposite poles of the nucleus. The centrosome divides into two moieties, a proximal, which becomes attached to the nuclear membrane, and a distal, which moves peripherally at the same time sprouting a flagellum, the central filament. Meanwhile a peripheral portion separates from the more central portion of the distal centrosome and moves backward over the central filament enclosing an enveloping column of cytoplasm, the middle piece, in which mitochondria have aggregated. The mitochondria fuse to form a filament, the spiral

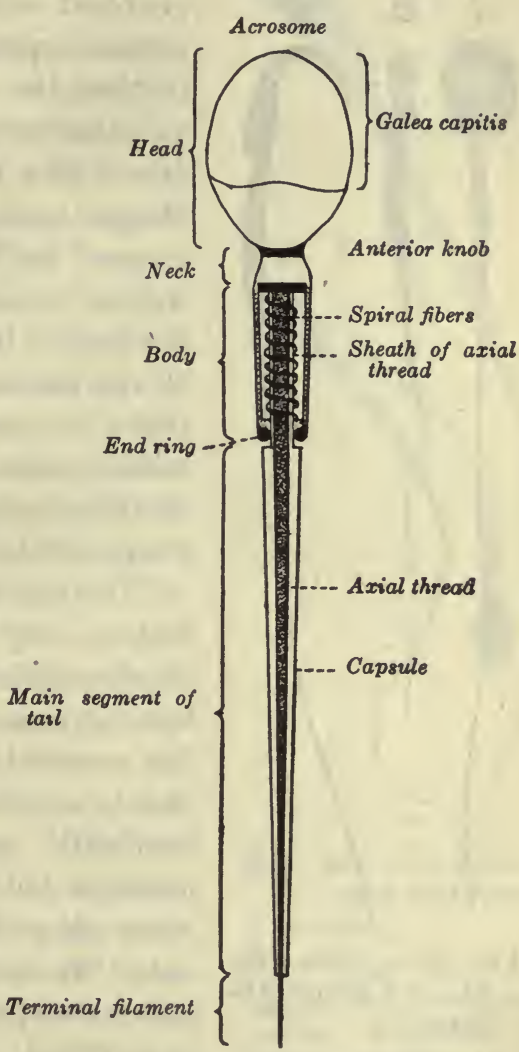

Fig. 430.-Diagram of Human SperMATOZOÖN.

(After Bonnet.) filament, which becomes spirally arranged about the central filament. The central filament continues to grow distally beyond the final locus of the ring centrosome, and together with an envelope of cytoplasm constitutes the tail of the spermatozoön. A short terminal naked portion of the central filament is known as the terminal filament.

Summarizing the above: The nucleus becomes the head of the 
spermatozoön. This is tipped with an acrosome, a derivative of the idiozome, and covered by the galea capitis, the two comprising the perforatorium. The other constituents of the idiozome contribute the

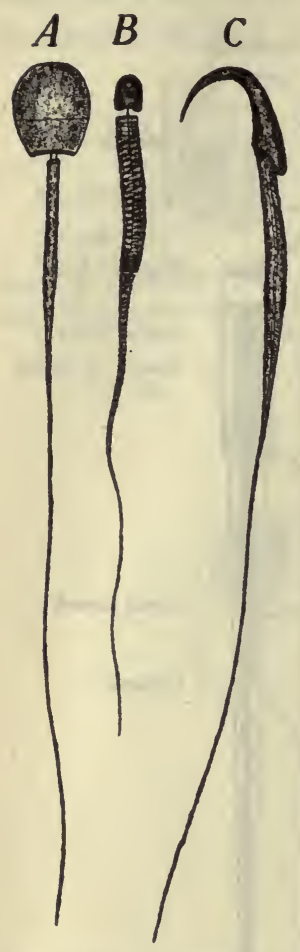

Fig. 431.-SpermatozOA OF VARIOUS ANIMALS.

$A$, from the badger; $B$, from the bat. Redrawn after Ballowitz. $\times 1200$. $C$, from the rat. Hematein and eosin. $\times 1200$. central filament. The extent between distal and proximal centrosomes constitutes the neck; the portion between distal centrosome and ring centrosome the connecting or middle piece (body), with its spiral filament derived from mitochondria. The tail or flagellum consists of central filament enveloped by cytoplasm. In many forms a spiral fin develops on the body. Also, there is endless variation with reference to the shape of the head. But the development and morphology of spermatozoa of all vertebrate forms is essentially the same. At fertilization only head and middle piece-nucleus and centrosome and mitochondria-enter into the egg, the tail being generally left behind.

The human spermatozoön has a length of from fifty to sixty microns. Of the total the head constitutes about $5 \mu$, the middle piece about $5 \mu$ and the end-piece about $10 \mu$. Seen en face the head has an ovoid shape; in profile it appears pyriform, due to a thinning along its terminal margin. Occasionally atypical or monstrous, double-headed, multiple-tailed, and giant spermatozoa appear; but these are probably non-functional. Though flagellate, the spermatozoa are non-motile until they reach the epididymis, where they become bathed in a secretion. Their motility, however, is not pronounced until they reach the place in the excretory passage where the secretion of the seminal vesicles, prostate and bulbo-urethral glands are present. The mixture of these secretions with the sperm forms the semen.

The spermatozoa can withstand considerable variations ${ }^{\text {in }}$ temperature, but they are very susceptible to acid solutions; they survive best in slightly alkaline media. Under favorable conditions spermatozoa may be kept alive for as long as eight days; it is probable that they remain alive for even a longer time in the female genital tract; but 
it is uncertain whether they can maintain their functional virility for this length of time. The number of spermatozoa per cubic millimeter has been estimated at 60,876 ; and the total in an average ejaculation at 200,000,000 (Lode, 1896).

The tubuli convoluti pass into short tubuli recti and the latter into the rete testis of the mediastinum. The rete testis is the beginning of the excretory duct, and consist of a meshwork of irregular tubules lined with a single layered cuboid non-ciliated epithelium. They connect with a group of greatly coiled tubules, the ductuli efferentes, from ten to fifteen in number, each terminally winding so as to assume a conical shape, hence coni vasculosi-which tubules empty into the distal portion of a common duct - the ductus epididymis. This portion of the duct, together with the efferent ductuli, constitute the globus major of the epididymis. Consideration of the duct system will follow below.

Interstitial Cells of Leydig.-Here should be considered the intertubular connective tissue of the testis. Fibro-elastic connective tissue acts as a supporting stroma for the testicular parenchyma of the seminiferous tubules. Scattered throughout this stroma, between the adjacent tubules, are peculiar large polygonal cells, interstitial cells of Leydig, either scattered or arranged in masses. The condition of the nucleus varies from pale vesicular to dense deeply chromatic. Division figures are exceedingly rare

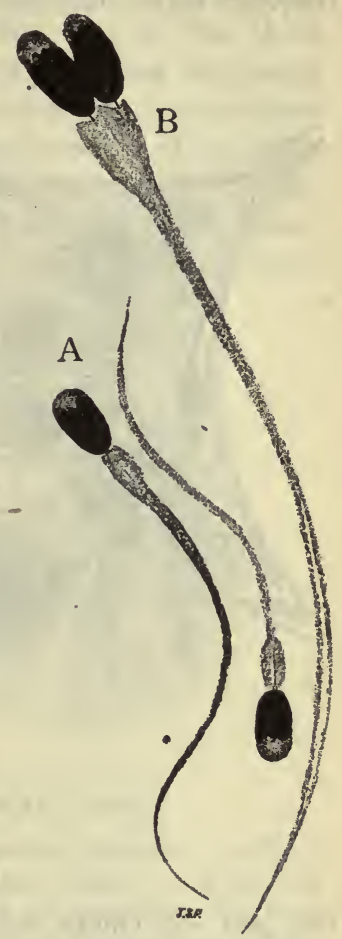

Fig. 432. - SPERMATOZOA FROM THE SEMEN OF MAN.

$A$, usual type; $B$, 'giant' double spermatozoön. Hematein and eosin. $\times 1200$. among them, though an occasional cell may, in young testes, be seen in mitosis. In older testes an occasional nucleus may be seen in what appears to be amitotic division. A number of the cells also are bi- and multinucleate. However, multiplication of specific interstitial cells is evidently exceptional. These cells arise directly through modification in shape, and growth from the fusiform and irregular connective tissue cells of the stroma. Moreover, this process seems to be reversible. They thus represent a transient phase in the life of certain cells of the intertubular connective tissue. Their cytoplasm contains crystalloids (mito- 
chondria) and fatty granules. The nature of the lipoid granules is discussed by Whitehead (Amer. Jour. Anat., 14, 1, 1912). These cells increase in number during senile atrophy of the testis, but later entirely

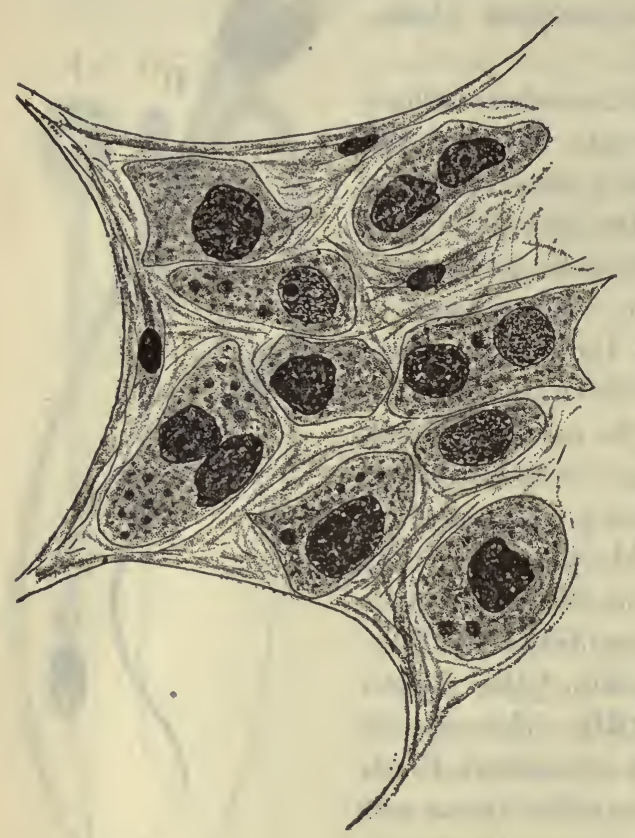

Fig. 433.-A Group of Interstitial Cells from the Testis of a Thirty-five Year Old Negro.

The outlines of three adjacent seminiferous tubules are shown. Among the interstitial cells are three nuclei of ordinary connective tissue. The interstitial cells are filled with lipoid spherules and granules: Formalin fixation, ironhematoxylin stain. $\times 1500$. disappear. They are relatively larger and more abundant also in the testes of tuberculous patients where there is considerable degeneration and a general infantile condition of the seminal epithelium. Various functions have been ascribed to them-that is, those obviously dependent upon the internal secretion of the testes-prominently, origin and maintenance of secondary sexual characters, and basis of sexual instinct. Obviously other cells of the testis might conceivably subserve these functions, namely, the sustentacular cells and the sexcells. However, observations on testes of mules (infertile) and cryptorchid horses, and the findings of Whitehead (Anat. Rec., 2, $5,1908)$ in a third abdominal testicle of a horse where only interstitial cells in great abundance persisted in normal condition, would seem to relegate these functions to the interstitial cells.

Kingsbury's careful studies (Amer. Jour. Anat., 16, 1914) of the interstitial cells of the ovary of the cat (elements presumably homologous with the interstitial cells of the testis) strongly support the interpretation of these cells in terms of absorbers of degenerating and disintegrating materials. In the ovary all of the earliest and many of the later primary oöcytes disintegrate. In the vicinity of such atretic follicles interstitial 
cells laden with lipoid débris are abundant. Their presence, however, is transient, and their abundance reciprocal to the abundance of degenerating follicles. This observation, reasoning by analogy, would explain their abundance in the testes of cryptorchid horses, mules, and in the third abdominal testicle; for here also degenerative processes are going on among the sex cells. Testicles of cryptorchid horses and mules are characterized by an unusual abundance of interstitial cells, and degeneration of sex cells. These animals experience heat, but are infertile; this was true also of the stallion from whom two testicles had been removed and in whom subsequently the third abdominal testicle was discovered. These observations
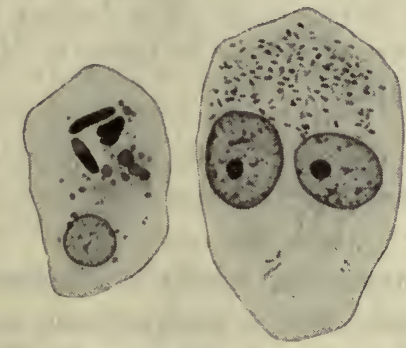

$B$

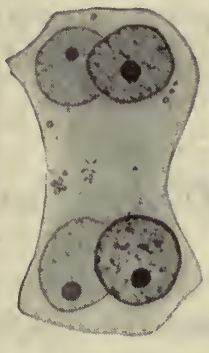

$C$

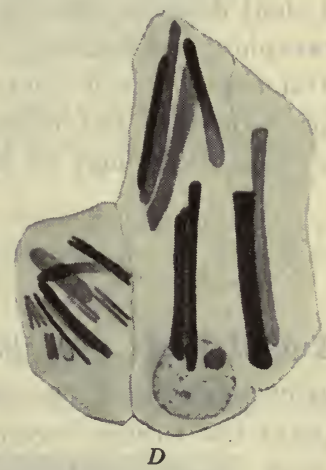

Fig. 434.-Interstitial Cells from the Human Testis.

$A, B$, and $C$, from a twenty-five year old man; $A$ shows the idiosome, bacillary centrosomes, lipoid granules and crystalloids; $B$, with two nuclei; $C$, with four nuclei (probably the result of amitotic division) and eight centrosomes. $D$, from a 41-year-old man, showing large and small crystalloids. Highly magnified. (Winiwarter, Anat. Anz., 41, 11, 1912.)

would seem to indicate that the sexual instinct depends upon an internal secretion on the part of the interstitial cells. The two results can be harmonized on this basis: The internal secretion may actually be formed by the cells of the seminiferous tubules; when these degenerate the products are removed by the interstitial cells; since even in healthy testes there is some degeneration of sex cells, all testes contain a few interstitial cells; these are more abundant at puberty; the secretory products of degenerating sex cells are included among the débris removed by the interstitial cells from which they may be passed into the capillaries of the testicular stroma.

The suggestion that the interstitial cells are in some way connected with secondary sex characters seems disproved by various castration experiments. For example, the spayed hen takes on male secondary sexual characters; if these were dependent exclusively upon the interstitial cells of the testis, they could not appear in the absence of both ovaries and testes. 
However, the experiments of Steinach (Zentralblatt f. Physiol., 27, 14, 1913) cannot be ignored in this connection. Steinach made reciprocal transplantations of testes and ovaries in young male and female rats and in guinea pigs, and claims to have succeeded in changing a potential female, from the view point of secondary sex characters (both physical and temperamental), into a male, and vice versa. A male is said to have developed functional mammary glands, and even to have suckled young. Steinach interprets his results to mean that not only do the secondary sex characters of the male and female depend upon the presence of the 'pubertal gland' (interstitial cells), but also the fact whether the undifferentiated gonad shall develop into a testis or an ovary. Microscopic examination of the transplant showed that everything suffered degeneration except the interstitial tissue, which underwent extensive hyperplasia. For further information on this and related subjects reference should be made to Marshall's "The Physiology of Reproduction," 1910.

\section{The Duct System}

Tubuli Recti.-At the apex of the testicular lobule the tortuous seminiferous tubules pass into the rete testis of the mediastinum. At this point the tubule becomes straight and is abruptly narrowed. Thus the short straight tubules, tubuli recti, are formed. In the straight tubules the stratified epithelium of the tortuous portions is abruptly exchanged for a very low columnar or flattened type of epithelium with which the Sertoli cells of the tortuous tubules seem to be continuous. The straight tubules are very short and are soon transformed into the irregular anastomosing canals of the rete testis.

Rete Testis.-The connective tissue of the mediastinum is permeated by a network of irregular channels of varying diameter which present frequent dilatations and often have the appearance of broad cleft-like spaces. These are the canals of the rete testis which form a dense network of anastomosing channels. On the one hand they receive the straight tubules, and on the other they pass into the ductuli efferentes, which convey the secretıon onward to the globus major of the epididymis.

The canals of the rete testis are lined by cuboidal or flattened epithelium, which rests upon a delicate basement membrane. This in turn is supported by the connective tissue of the mediastinum. The broad but irregular lumen of the canals is occupied by the secretion from the seminiferous tubules and contains many spermatozoa.

Ductuli Efferentes,-As the tubules of the rete testis leave the 
mediastinum they are abruptly transformed into peculiar efferent ducts, 10 to 15 in number, which pass into the globus major of the epididymis and by means of spiral windings form conical masses, coni vasculosi, whose apex projects into the globus major. The epithelium of these tubules is peculiar in that it contains two varieties of cells, and in that it is thrown into many prominent longitudinal folds or rugæ.

In the lining epithelium there are short columnar cells which rest upon the basement membrane and carry upon their free ends a tuft of short cilia. These cells have an ovoid nucleus and a

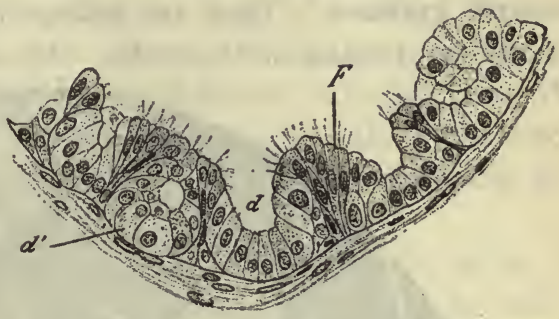

Fig. 435.-A Small Portion of the Wall of an Efferent Ductule of the TesTICLE.

$d$, 'glands' in longitudinal section; $d$ ', the same in oblique section; $F$, ciliated epithelium. $\times 140$. (After Kölliker.) very finely granular, eosinophil cytoplasm. Between and among the ciliated cells are many broad columnar or polyhedral cells, having remarkably clear cytoplasm, which

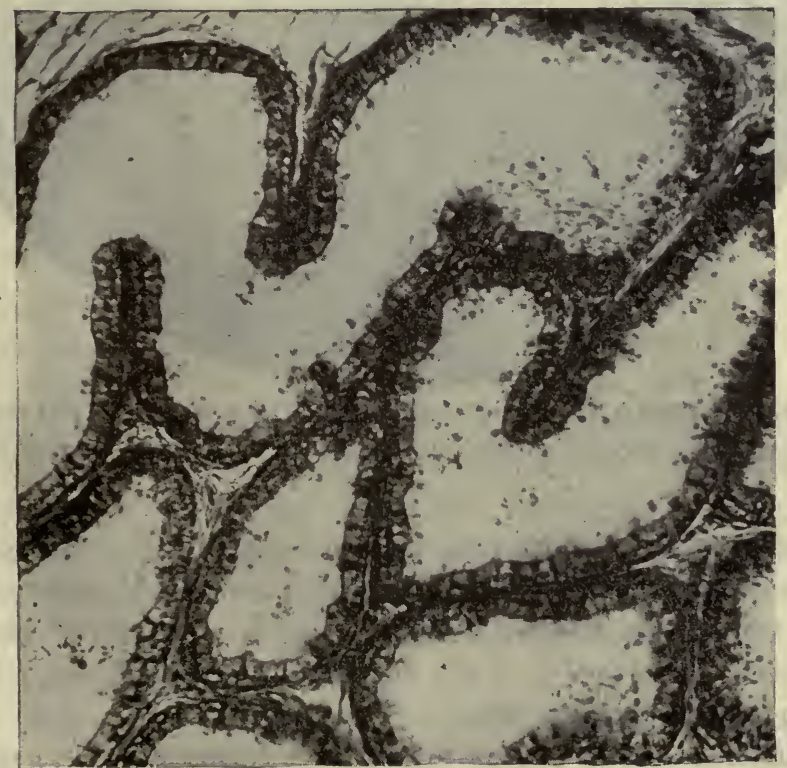

Fig. 436.-Efferent Ductules of the RabBit's Epididymis, Hematein and eosin. Photo. $\times 250$. 
chiefly occur between and at the base of the rugæ, and are frequently arranged in small groups simulating minute secreting glands. These clear cells have spheroidal nuclei and their cytoplasm is filled with large coarse granules. They are quite characteristic of this portion of the excretory tubules of the testis. The coni vasculosi form a considerable

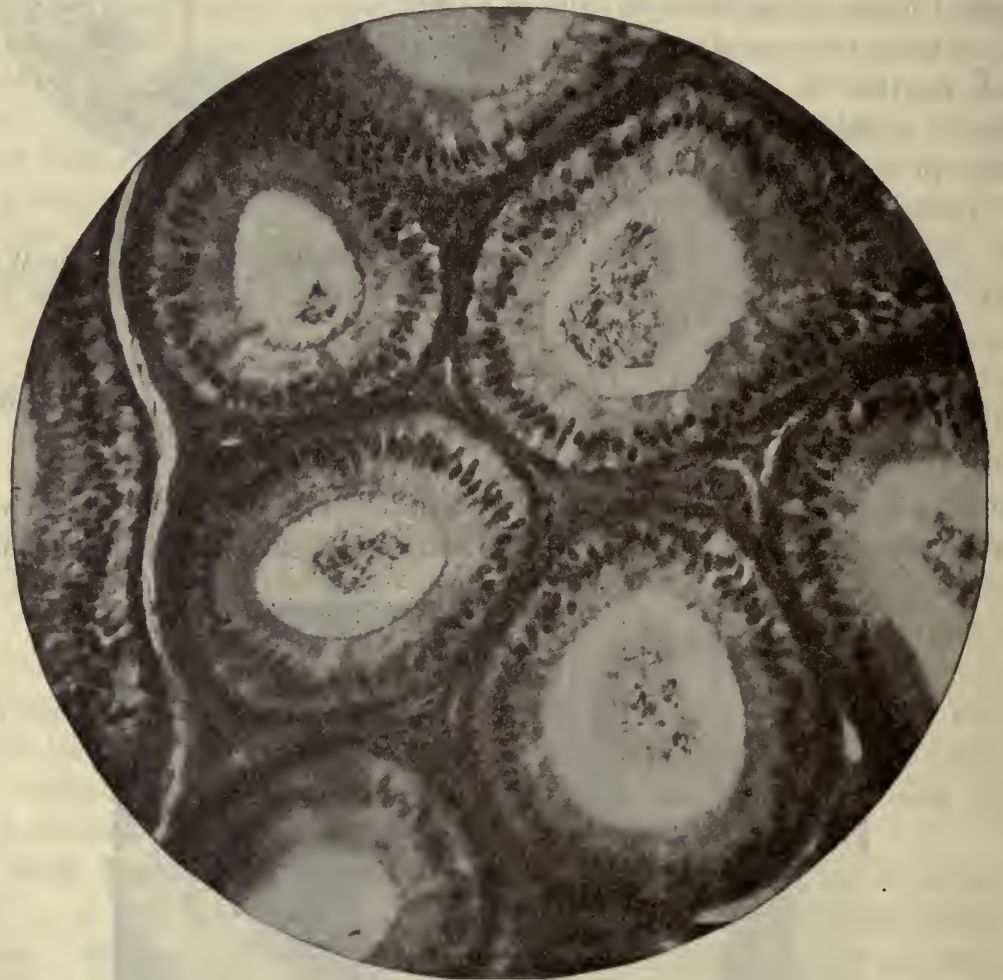

Fig. 437.-Several Coils of the Rabbit's Epididymis in Transection.

The lumen of the tubules contains groups of spermatozoa. Hematein and eosin. Photo. $\times 178$.

portion of the globus major of the epididymis. The epithelium rests upon a well-developed basement membrane, outside of which is a robust layer of circularly disposed, smooth muscle cells with intermingled elastic fibers. The nuclei of the lining cells can frequently be seen in some stage of amitotic division.

Epididymis.-The ductus epididymis forms a long coiled tubule whose convolutions, by their regular cylindrical form and their tall ciliated epithelium, are sharply distinguished from those of the ductuli 
efferentes, which have much thinner walls. The lining epithelium of the epididymis is of the tall, ciliated, simple columnar type with elongated ovoid nuclei, a finely granular cytoplasm, and a group of nonmotile cilia which often adhere together to form a peculiar tuft or cluster ('brush border'). At the base of the ciliated cells is an incomplete layer of basal epithelium, whose flattened cuboidal elements are wedged between the bases of the tall columnar cells. 'The twolayered epithelium is thus of the pseudo-stratified type. The cells apparently multiply largely by amitosis.

The epithelium rests upon a cellular basement membrane, which is supported by a connective tissue tunic of varying thickness. In addition to many elastic fibers, this coat contains a few smooth muscle cells. The coils of the epididymis are firmly united into a solid mass by means of the dense intervening connective tissue. They form the whole of the globus minor and a considerable portion of the globus major. When unraveled the duct of the epididymis measures about twenty feet in length.

The Ductus Deferens (Vas Deferens).-This duct is a continuation of the epididymis, whose course now becomes relatively straight. It measures about eighteen inches in length. In this portion of the excretory duct of the testis the lining epithelium soon loses its cilia, and the basal cells become more prominent. Hence in the greater portion of its course the ductus deferens is lined by tall, columnar, non-ciliated epithelium, with low basal cells between the attached ends of the columnar cells.

The epithelium rests upon a fibro-cellular basement membrane, which is supported by a fibrous tunica propria. This, in turn, passes almost insensibly into the muscular coat which consists of two layers, an inner circular and an outer longitudinal, both of which are highly developed. In the lower portions of the ductus deferens, a thin internal layer of longitudinal muscle fibers is also found. The fibers of the internal and middle circular layers are frequently less regularly arranged, in which ease their oblique bundles interlace with one another in a most intricate manner. The very thick, smooth muscular coat and the relatively narrow lumen give this portion of the duct a firm consistence and a cord-like feel.

In its ampulla-the dilated portion near its prostatic terminationthe mucous membrane of the ductus deferens is more loosely attached and the folds or rugæ, which elsewhere are few in number, are here very pronounced. The lumen of the ampulla is broad, but elsewhere 
the lumen of the duct is narrow, as compared with its exceptionally thick muscular wall. As elsewhere in the excretory canal of the testicle, the lumen of the ductus deferens contains many spermatozoa.

The Spermatic Cord.-The spermatic cord in its scrotal portion,

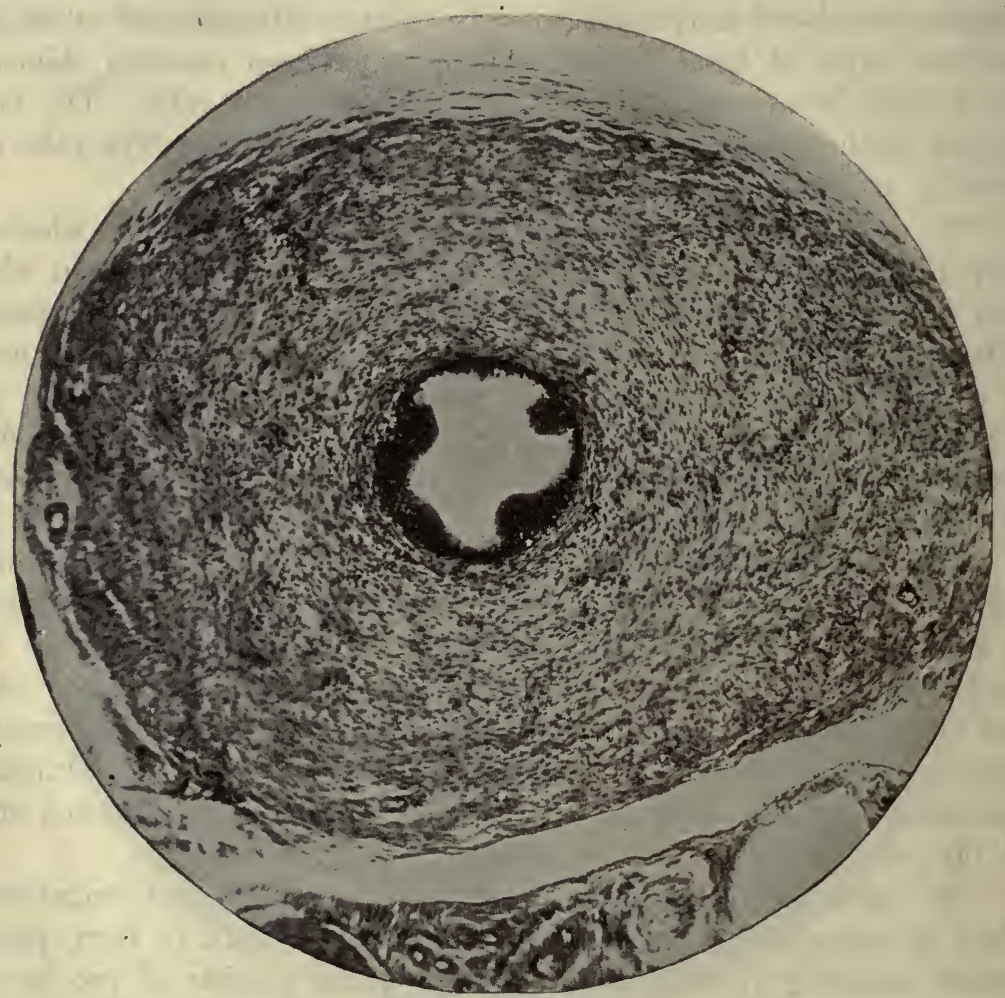

Fig. 438.-Transection of the Ductus Deferens of a Dog:

Hematein and eosin. Photo. $\times 104$.

in addition to the ductus deferens, contains a mass of connective tissue in which are embedded the smooth muscle fibers of the internal cremaster muscle, the spermatic artery, veins, and nerve plexus, and the vessels of the pampiniform plexus. Closely-associated with these constituents is also the striated cremaster muscle proper. The whole is invested by a reflection of the tunica vaginalis:

The pampiniform plexus is a considerable group of venous spaces, usually completely collapsed after death, which are characterized by 
very thick, firm, fibromuscular walls. The vessels are embedded in dense connective tissue, and the whole plexus in general appearance somewhat resembles the erectile tissues.

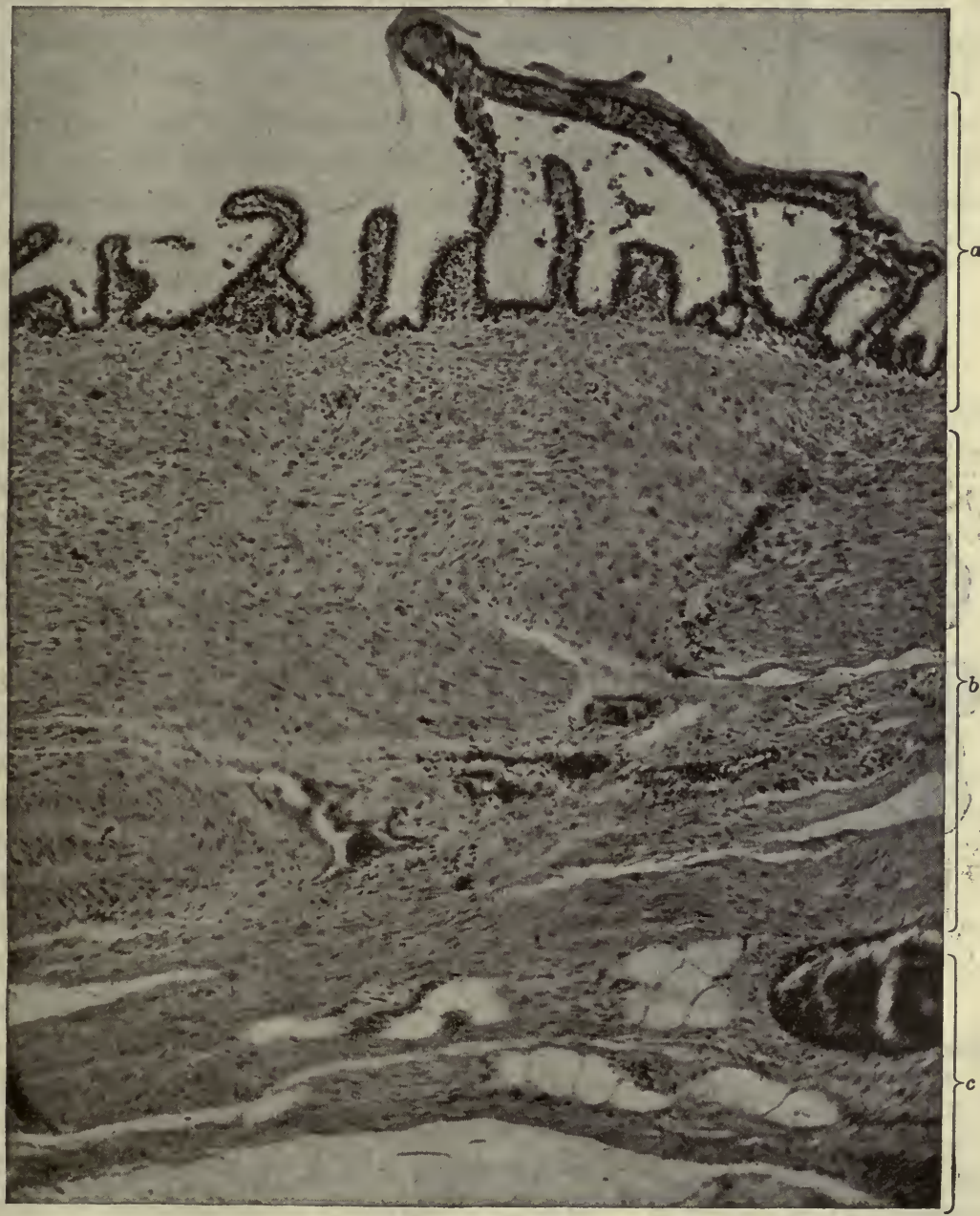

Fig. 439.-From a Section through the Wall of a Seminal Vesicle of Man. $a$, mucosa; $b$, muscular coat; $c$, fibrous coat. Hematein and eosin. Photo. $\times 185$.

The Seminal Vesicles. - The walls of the seminal vesicles consist of a thin outermost coat of connective tissue in which are many small ganglia, a muscular coat similar to that of the ductus deferens but 
much thinner, and a characteristic mucosa. The tunica propria of the mucous membrane is a thin layer of delicate cellular connective tissue

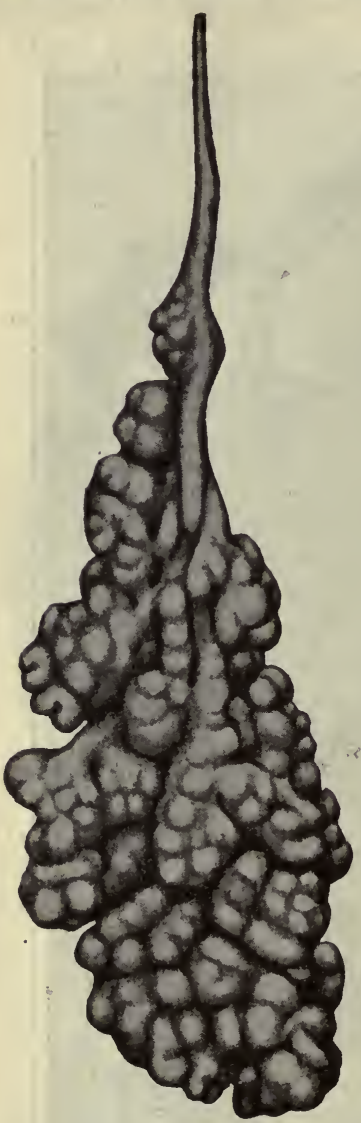

Fig. 440.-MODEL OF A Reconstructed Prostate Gland of Man.

The figure includes one lobule. The narrow duct expands and terminates in a large number of alveoli of very varied size and form. $\times$ 40. (After Maziarski.) which loosely attaches the lining epithelium to the muscular coat. The surface of the mucosa presents numerous folds which not only form longitudinal rugæ but also possess an intricate network of secondary ridges which are both longitudinal and transverse in direction. This peculiar arrangement results in the appearance of diverticula of various forms and sizes which, except that their epithelium does not differ from that of the surface, might often be interpreted, when seen in section, as representing secondary secreting glands within the mucosa. Slender processes of the corium extend into all the folds of the mucous membrane.

The lining epithelium of the seminal vesicles is of the columnar type and usually contains but a single layer of cells. Occasionally basal cells are also found in the deeper part of the epithelial layer; in such case the epithelium may be said to be of the pseudo-stratified type. This variation may possibly be partly dependent upon the distention or relaxation of the vesicles. The cells of the epithelium contain peculiar granules of yellowish pigment which are present in considerable numbers and are quite characteristic of the organ. The superficial cells appear to be readily desquamated, and together with coarse granules of secretion form the chief contents of the lumen. Occasional small concretions of irregular form, and homogeneous or lamellar structure, similar to those of the prostate gland, are also found. The seminal vesicles usually contain but few spermatozoa. Occasionally these are present in large numbers; at other times they may be entirely absent. Their chief function is now thought to be secretory. 
The Ejaculatory Ducts.-These ducts are formed by the union of the ampulla of a ductus deferens and the duct of a seminal vesicle and are similar in structure to the ampullæ of which they are the continuation. The ejaculatory ducts, however, possess a thinner wall and their mucosa presents the same folded condition as in the seminal vesicles, but to a lesser degree. In its prostatic portion the musculature of the ductus deferens blends with the muscular stroma of the prostate, so that in the ejaculatory duct the smooth muscle no longer forms a distinctly lamellated coat. On approaching the urethra, the epithelium of the ejaculatory ducts presents a gradual transition to the stratified epithelium of the urethral canal.

\section{Associated Glands}

The Prostate Gland.-This is a compound tubulo-alveolar gland consisting of from 30 to 50 lobules investing the urethra and the ejaculatory ducts. It pours its serous secretion, which has a characteristic odor, into the neighboring portion of the urethra by means of two large and many (15 to 30 ) small ducts. These open either directly into the urethral canal or indirectly through the utriculus prostaticus (sinus pocularis). The secreting alveoli are embedded in a very dense fibromuscular stroma which, at the surface of the organ, forms an unusually thick capsule in which interlacing bundles of smooth muscle are most prominent. This portion of the stroma also contains intrinsic striated muscle fibers in limited numbers. Broad bands of fibromuscular tissue pass inward from the capsule and form a network of thick septa in the meshes of which are the glandular alveoli. These septa converge toward the urethra, which penetrates the ventral portion of the organ, their muscular fibers finally blending with the sphincter fibers of the prostatic portion of this canal.

The stroma consists of smooth muscle and connective tissue; their fibers are intimately blended. The muscle and connective tissue constitute each about one-fourth of the organ. The muscle cells form either groups or bundles of variable size, or are frequently isolated within the meshes of the connective tissue. Their extreme abundance -in some parts exceeding the connective tissue in volume-is characteristic of the prostatic stroma. The connective tissue, which is sparingly supplied with elastic fibers, is rich in cells. Near the secreting alveoli the muscle fibers are absent and the cellular connective tissue becomes more prominent.

The lining cells are of the tall columnar type, sometimes forming 
a single, sometimes a multiple cell layer. They possess spherical or ovoid nuclei which lie in their deepest third. Their cytoplasm is finely granular and often contains small yellowish granules. The epithelium rests upon a prominent membrana propria, composed of peculiar coarse collagenous fibers, 'B-collagenous' fibers (Ferguson, Anat. Rec., 5, 12, 1911) somewhat resembling, but not identical with, reticulum. This

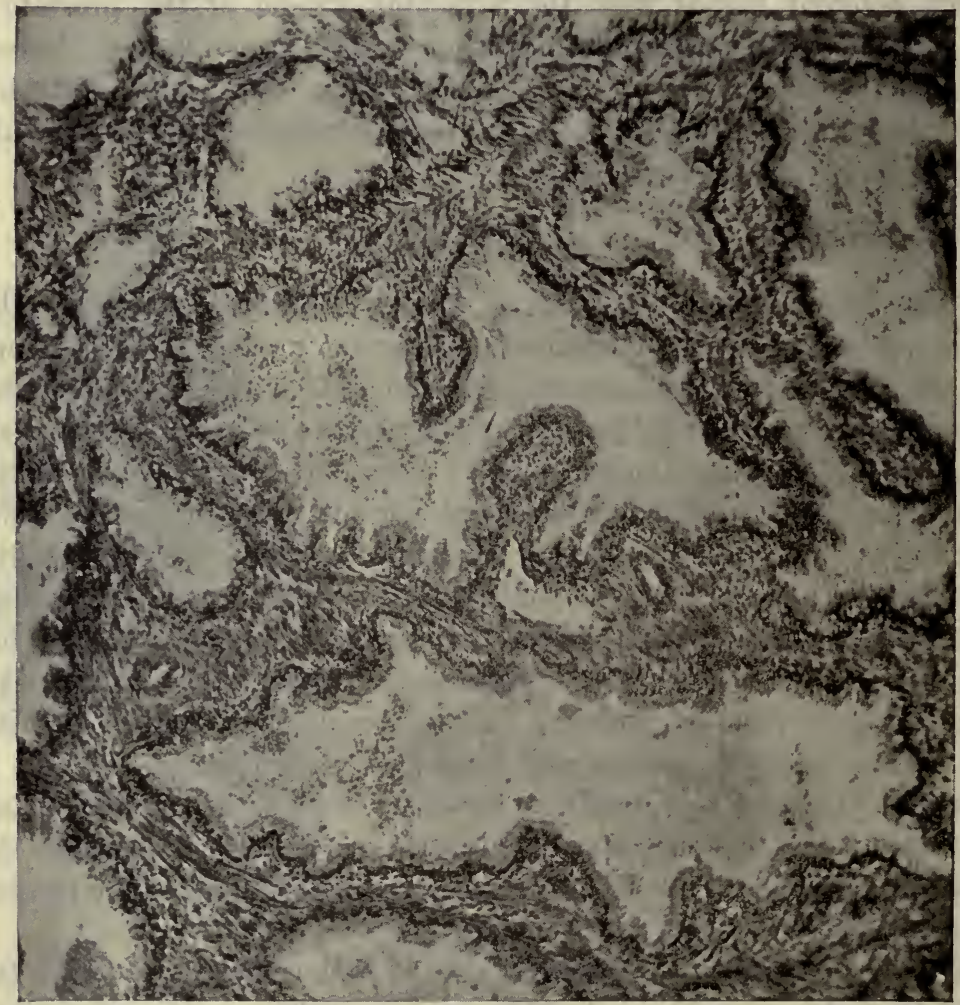

Fig. 441.-Several Alveoli of the Human Prostate Gland, Seen in Section. Hematein and eosin. Photo. $\times 160$.

type of fiber is present also throughout the stroma and distinguishable by the Bielschowsky technic from the usual collagenous ('A-collagenous') fibers.

The epithelium is remarkably folded upon itself, the narrow interval between the two layers of the epithelial folds being always occupied by delicate extensions of the connective tissue stroma. The prominence of the folds varies greatly in different tubules, some showing scarcely 
any such, the lumen of others being subdivided by deep rugæ into numerous anastomosing compartments. The amount of the folding also varies in different species, being more highly developed in some of the lower mammals, e.g., the dog, than in man.

The lumen of the prostatic tubules is broad, and is beset with numerous alveolar dilatations and shallow diverticula. It is usually broader

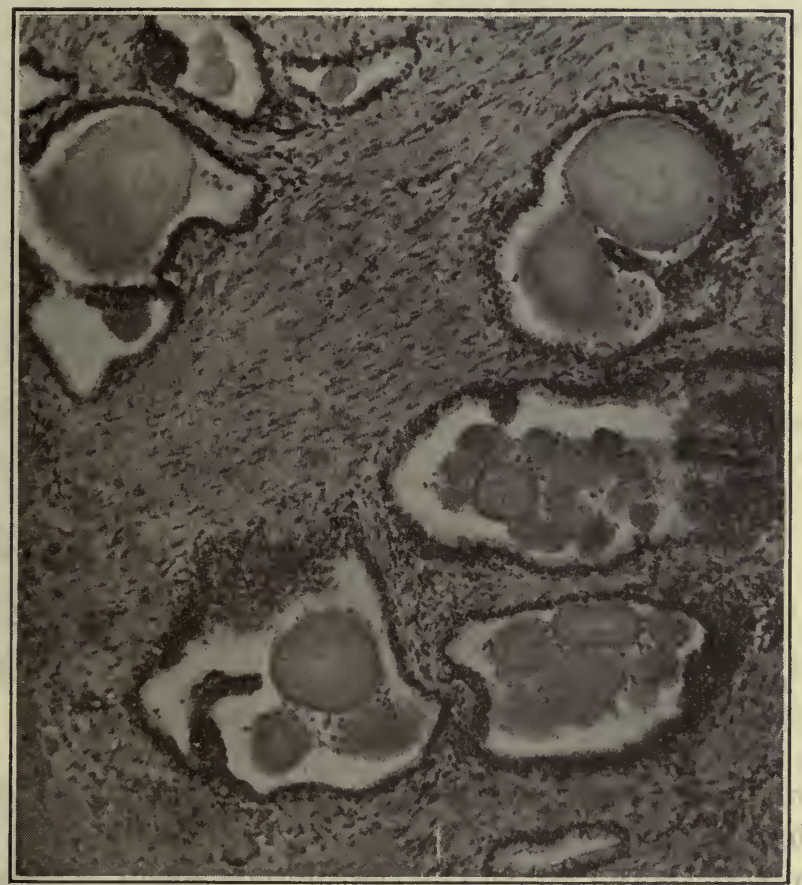

Fig. 442.-Portion of Prostate Gland of an Old Man, Showing the Prostatic Concrietions.

In the upper right hand concretion the concentric lamellæ are clearly discernible.

near the blind extremity and diminishes somewhat in diameter toward the duct. The caliber of the lumen also varies greatly, in different tubules and is possibly dependent in part upon the state of secretory activity. The contents of the lumen include the granular albuminous secretion, desquamated epithelial cells, and, as age advances, many socalled prostatic concretions ('corpora amylacea'; 'prostatic calculi'). The concretions vary greatly in size $(10 \mu$ to $1 \mathrm{~mm}$. in diameter $)$, and may be homogeneous, but more frequently present a distinctly 
lamellated appearance. Prostatic concretions may occur at all ages but increase both in number and size in later life. Occasionally they attain a large size and may become encysted.

The prostatic ducts are lined by either a single or a pseudo-stratified layer of columnar epithelium, and, except for their narrower caliber. and more regular contour, they closely resemble the secreting tubules. As the ducts approach their termination their epithelium increases the number of its cell layers. The larger ducts, just prior to their

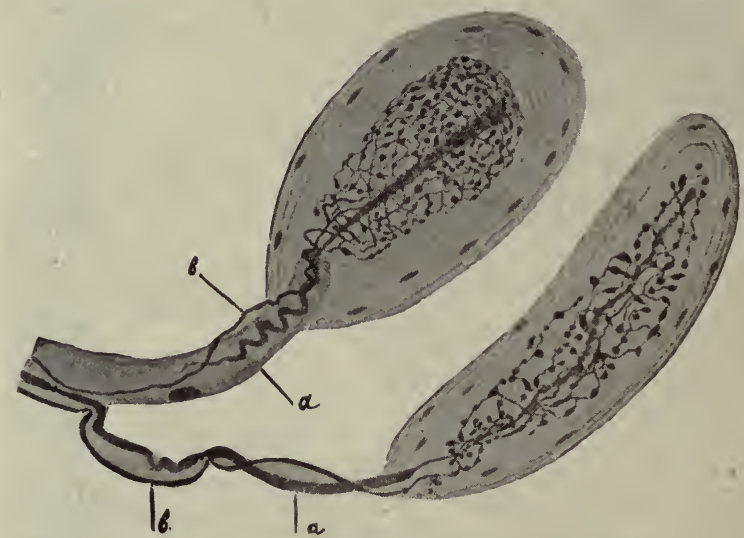

Fig. 443.-Prostatic Genital Corpuscles.

$a$, axial nerve fiber; $b$, peri-axial nerve fiber. . Methylene blue. Moderately magnified. (After Timofejew.)

termination, are lined by transitional epithelium similar to that of the urethra, into which they open.

Blood ANd LympH Supply.-The prostate gland possesses a rich blood supply. Its larger vessels are found in the capsule, whence they send branches into all portions of the fibromuscular stroma, and form a rich capillary plexus in the connective tissue layer about the epithelium of the secreting alveoli, and a second plexus in the substance of the stroma itself. The prostate is abundantly supplied, also, with lymphatic vessels, which are connected with the deep pelvic lymph nodes.

The capsule of the prostate, as also the neighboring connective tissue, both in relation with this organ and with the adjacent seminal vesicles, contains many nerve trunks, chiefly sympathetic, and small ganglia. The latter are especially numerous. In this region a peculiar variety of special nerve ending is found. It was formerly regarded as a Pa- 
cinian corpuscle, but differs somewhat from these bodies. It perhaps more nearly resembles the genital corpuscles. These bodies-are distinctly lamellated and possess a broad axial nerve fiber which somewhat resembles that of the end bulbs of Krause. This nerve fiber is,

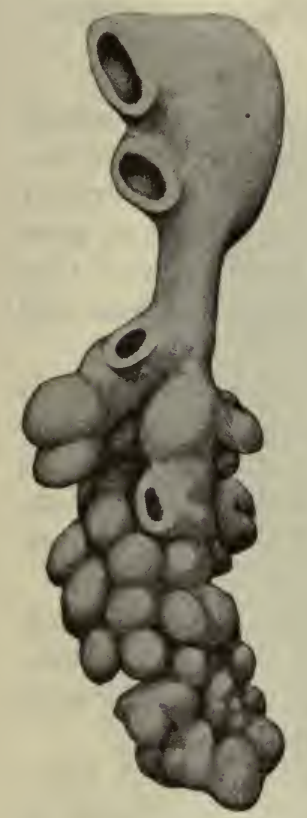

Fig. 444. - RECONSTRUCTION OF A BULBO-URETHRAL (COWPER's) GLAND OF MAN.

The tubular ducts are closely surrounded by the expanded alveoli. $\times 100$, (After Maziarski.)

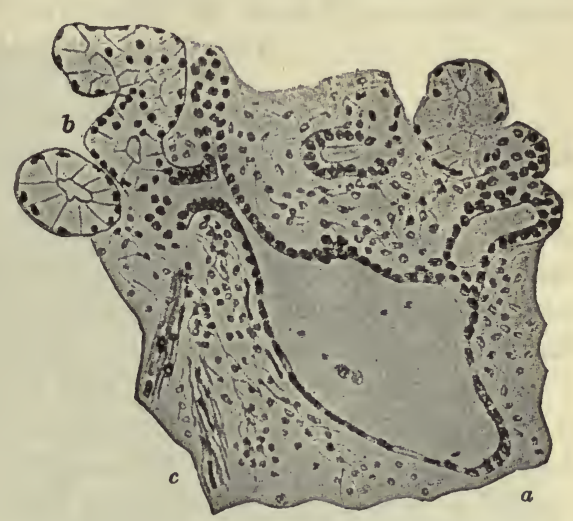

Fig. 445.-From a Section of the Bulbo-Urethral (Cowper's) Gland of MaN.

$a$, duct; $b$, tubules; $c$, stroma. $\times 130$. (After Braus.)

however, accompanied by another and finer fiber which, as Timofejew (Anat. Anz., 1896) has shown, breaks into a close network of fine fibrils surrounding the axial nerve fiber in a peculiar basket-like manner.

\section{The Bulbo-urethral Glands (Cowper's} Glands).-These are two small tubulo-acinar mucus-secreting glands which are divisible into numerous small lobules. The lobules are separated by connective tissue septa containing both smooth and striated muscle fibers. The latter are continuous with the adjacent compressor urethræ muscle. The secreting acini are lined by columnar cells, some of which are finely granular and stain with eosin and acid dyes, while others are apparently filled with mucous secretion and react to the specific dyes for mucin. Certain other tubular alveoli are lined by low cuboidal or flattened epithelium. The epithelium rests upon a distinct cellular basement membrane. 
The interlobular and the smaller intralobular ducts are also lined by a single layer of columnar cells. Their wall is supplied with smooth muscle, most of whose fibers have a longitudinal direction. The two ducts of Cowper's glands open into the bulbous portion of the urethra.

\section{Associated Vestigial Structures}

The vestigial structures associated with the male reproductive system include the appendices testis and epididymis, the superior and inferior ductuli aberrantes, the paradidymis and the sinus pocularis. Of these it may be said in general that they are more or less variable with respect of gross and microscopic structure and even with respect of presence; that they resemble histologically the structures with which they are homologous, and that they tend to become cystic.

The APPENDIX TESTIS is the least variable of the vestigial associates. It is present in about ninety per cent. of cases. It is a small spherical, frequently pedunculated, sac attached to the superior pole of the testis; it is covered with tunica vaginalis and lined with simple columnar epithelium, sometimes ciliated, and represents the end of the degenerated fetal Müllerian duct.

The APPENDIX EPIDIDYMIS is a very similar pedunculated structure, much less frequently present. It is situated on the globus major of the epididymis. It is supposed to represent a degenerated Wolffian tubule. By some it is regarded as the atrophic end of the Wolffian duct.

The DUCTULI ABERRANTES are blind tubules, the remnants of mesonephric tubules which failed of inclusion among the ductuli efferentes of the globus major. The superior ductule opens into the epididymis below the globus major; the inferior opens at the globus minor; both lie between the testis and the epididymis. They are lined with a single layer of columnar epithelium, sometimes ciliated. The inferior ductule is the more generally present, and has a length of about five centimeters.

The PARADIDYMIs (organ of Giraldès) lies within the spermatic cord between the head of the epididymis and the pampiniform plexus. It consists of a variable number of irregular branching tubules, blind at both ends, and lined with a single layer of columnar, ciliated epithelium. These tubules also represent persisting rudimentary mesonephric tubules.

The sINUS POCULARIS (sinus prostaticus) represents the remnant of the proximally fused degenerated fetal Müllerian ducts. It is the homologue of the vagina in the female. It is a shallow, blind pocket opening into the floor of the prostatic portion of the urethra; it may be bifid distally, varies in length from six to twelve millimeters, and is lined with a columnar epithelium which may be locally ciliated. 


\section{External Genital Organ}

The Penis.-The penis consists of three masses of erectile tissue, the two corpora cavernosa penis and the corpus spongiosum or corpus cavernosum urethra, which are firmly united by dense fibrous and areolar

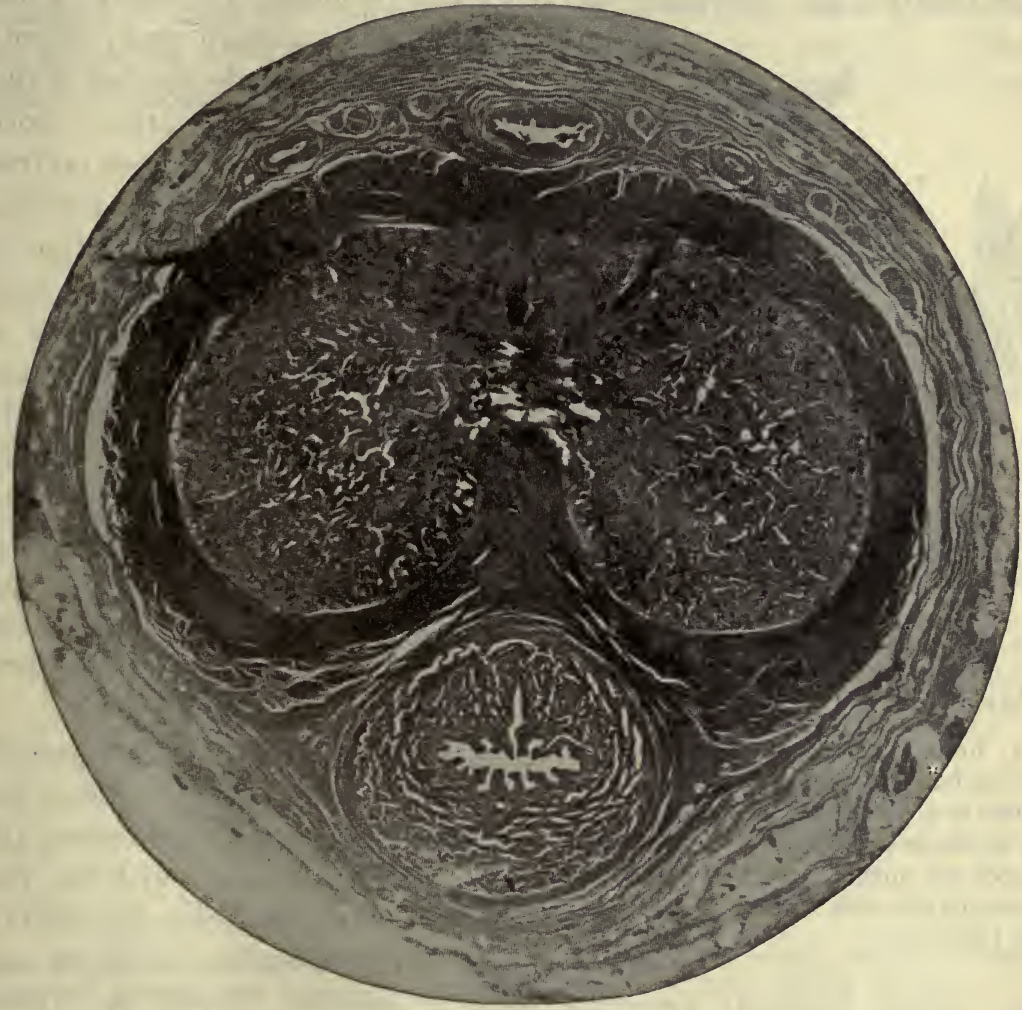

Fig. 446.-Transection of a Child's Penis, Just Back of the Glans.

The two corpora cavernosa (fused in the median line) and the corpus spongiosum, the latter containing the urethra, are well shown. The cutaneous surface is not included. Hematein and eosin. Photo. $\times 8$.

connective tissue. The outer cutaneous surface is loosely attached over the body of the organ; its structure does not differ materially from that of the skin of other parts. The subcutaneous tissue is remarkable for the extreme looseness of its areolæ and the absence of fat. In the glans penis the epithelial covering, which is continuous with the prepucial epidermis, is firmly united to the underlying erectile tissue. 
Each corpus cavernosum is invested with a thick sheath of very dense fibro-elastic tissue, the tunica albuginea, divisible into an inner circular and an outer longitudinal layer of fibers, and imperfect between the two corpora cavernosa penis where it forms the pectiniform septum. From the inner surface of this fibrous coat connective tissue septa, the trabeculæ, pass in all directions and form a reticular framework whose

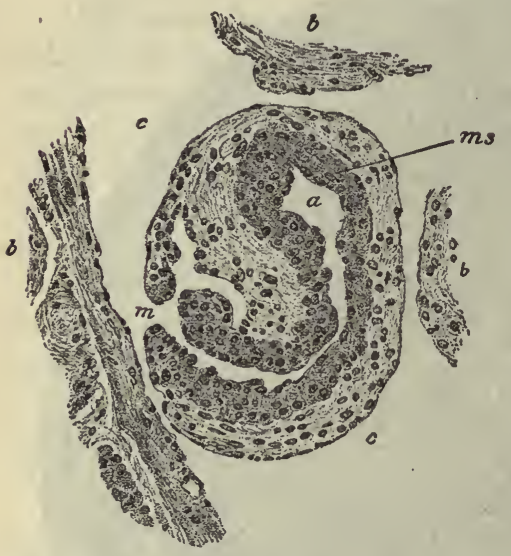

Fig. 447.-Helicine Artery in Section, From the URETHRAL BULB oF MAN.

$a$, lumen of a helicine artery; $b$, fibrous bands of the erectile tissue; $c, c$, lumen of a venous blood space; $m$, opening of the helicine artery into the blood space; $m s$, muscular coat of the artery. Hematoxylin and eosin. $\times 180$. (After Kölliker.)

fibrous bands contain many smooth muscle fibers. In the meshes of this framework are characteristic broad venous sinuses which possess no true wall other than their endothelial lining. In the flaccid condition of the organ the blood sinuses are completely collapsed, their walls are in contact, and their lumina almost obliterated, which gives them the appearance of mere slits in the - dense connective tissue of the cavernous body. When distended by injection, or, in the erectile condition of the organ, by blood, these spaces become widely dilated and form true blood sinuses of broad caliber.

The blood supply of the erectile tissue is peculiar. The arteries terminate either (1) in capillaries, (2) by direct anastomosis with the venules, or (3) by opening directly into the venous sinuses, in which case the minute terminal arterioles have a peculiar looped appearance and were described by J. Müller (1835) as helicine arteries. The capillaries form a superficial plexus beneath the tunica albuginea, which opens into a deeper plexus of broader vessels from which the venules take origin. Blood following this course through the capillaries and into the venules may not enter the venous sinuses-a direction which is assumed by the greater portion of the blood in the flaccid condition of the organ. The deeper venous plexus communicates freely with the venous sinuses so that the least obstruction to the usual venous outflow diverts the circulation through these channels.

The helicine arteries are confined to the corpora cavernosa penis, 
where they are most abundant near the root of the organ. The arterioles from which they are derived end by arborization in the connective tissue framework, their terminal twigs entering fibrous processes which project into the venous sinuses and are frequently bound down by delicate fibrous bands which unite their extremity to the wall of the sinus and produce the characteristic looped condition when the villuslike projection is distended by the injection of its arteriole. When partially injected the helicine arteries appear to end blindly, but when

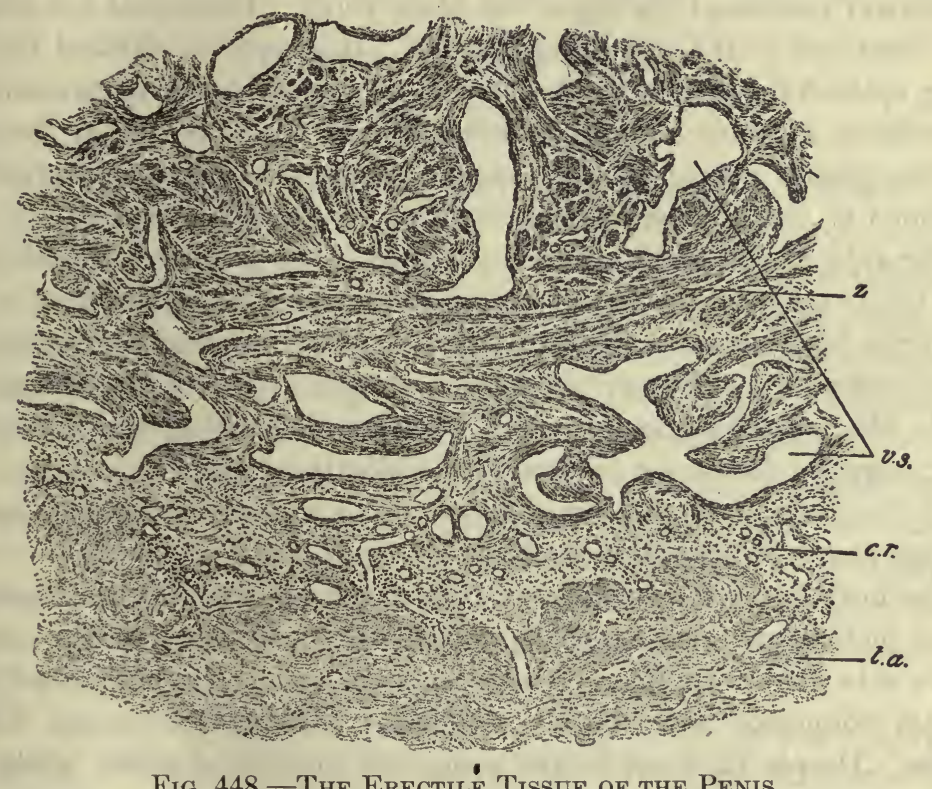

Fig. 448.-The Erectilé Tissue of the Penis.

$c r$, peripheral capillary plexus; $t a$, tunica albuginea; $v s$, venous spaces; $z$, bands of smooth muscle and vascular connective tissue. $\times 30$. (After Kölliker.)

completely distended they pour their contents into the venous sinuses.

The venous spaces at the periphery of the erectile body are relatively narrow and the intervening trabeculæ are thick; toward the axis of the body the sinuses become broader and occupy a relatively greater portion of the tissue. Here, also, their long axis, except in the corpus spongiosum, frequently lies in the transverse axis of the penis. Both the arteries and the veins of the erectile tissue possess very thick muscular walls, and in both, the intima becomes locally thickened by accumulations of longitudinal musclés and elastic tissue bulging into the lumen; these modifications are less pronounced in the veins than in the arteries. 
The tunica albuginea and trabeculæ of the corpus spongiosum are formed by less dense connective tissue than is found in the corpora cavernosa penis, and their venous spaces are not so broad. The broad anterior end of the corpus spongiosum forms almost the entire body of the glans penis, being only indented beneath the corona by the conical anterior ends of the corpora cavernosa penis which in this part are blended together to form a single median mass. The urethral canal occupies the axis of the corpus spongiosum from its bulb forward to the urinary meatus at the tip of the glans penis. This canal has already been described in the preceding chapter. It should be recalled that its lining epithelium differs in the several segments, being transitional in its prostatic and membranous portions, and stratified columnar throughout the greater length of the spongy portion, changing to stratified squamous in the dilated fossa navicularis of the glans penis.

The skin of the glans penis is peculiar in the relatively moist character of its epidermis and the consequent imperfect development of its superficial horny layer. Its dermal papillæ are conspicuously developed. In the region of the corona the derma contains a ring of large sebaceous glands, the preputial glands, which open on the free epithelial surface. Their secretion forms the smegma, a peculiarly odoriferous sebum. The so-called glands of Tyson are shallow, non-glandular epithelial pockets opening near the frenulum preputii (Lewis).

The medullated sensory nerves (dorsal nerves of the penis; branches of the pudic) are abundantly supplied with special nerve end-organs. In the skin they form free varicose endings among the epithelial cells, and are connected with tactile corpuscles of Meissner in the dermal papillæ. Deeper in the skin are many end bulbs of Krause, while still deeper are the peculiar genital corpuscles. Naked fibrils pass to the mucosa of the urethra. Pacinian corpuscles are also found in the loose connective tissue and in the tunica albuginea of the corpora cavernosa. Sympathetic nerve fibers are abundantly supplied to the walls of the blood-vessels and to the smooth muscle of the erectile tissue. Branches from the third and fourth sacral nerves also enter the penis as the nervi erigentes, supposed to convey the impulse to erection as vasodilator fibers.

The lymphatics of the penis form an abundant superficial set in the subcutaneous tissue; these follow the larger blood-vessels and empty into the inguinal lymph glands. A less abundant deep set of lymphatics in the erectile tissue, also, accompanies the blood-vessels of these parts, but is distributed to the pelvic lymph glands. 


\section{THE FEMALE REPRODUCTIVE ORGANS}

This system includes the ovaries, oviducts, uterus, vagina, and external genitals. All of these organs are concerned in the reproductive function, the ovary producing the germ cell or ovum, and the oviduct providing a suitable site for its maturation and fertilization and the uterus for the later development of the resulting embryo.

\section{Internal Genital Organs}

\section{The Ovary}

The ovary also is properly regarded as a gland with a double function, namely cytogenic and endocrin. The specific cells involved in the production of the internal secretion (exclusive of the lutein substance) are in doubt. As in the testis, the possibilities include the germ cells, the general connective tissue and the interstitial cells, homologues of the interstitial cells of Leydig of the testis. The.genetic and functional relationship between the several types of cells are similar to those described for the testis. The ovary, however, apparently does not contain a homologue of the Sertoli cells. As in the testis the internal secretory activity of the ovary sustains a reciprocal relationship to other internally secreting glands and in some manner, directly or indirectly, underlies normal development and the sexual instinct. The ovary, moreover, periodically elaborates still another internal secretion.

The organ involved is a transient structure, the corpus luteum of pregnancy. This will be described below. Its function pertains to an inhibition of ovulation during pregnancy (Loeb; Pearl), and a stimulation to secretory activity of the mammary glands (Bouin et Ancil; Ott and Scott), and apparently in part also to the preparation of the uterine mucosa for proper implantation and normal development of the fertilized ovum. As shown by extirpation experiments the internal secretions of both ovary and testis influence also the nervous system, and seem essential to normal nervous function.

The ovary is a solid ovoid body, about one and one-half inches long, three-quarters of an inch wide and one-half inch thick. It is attached to the margin of the broad ligament posteriorly by a short, thick connective tissue pedicle, the mesovarium, which transmits the blood-vessels with which the ovary is richly supplied. At its ovarian attachment the mesovarium becomes continuous with the connective 
tissue stroma of the ovary. The indentation which is thus produced is known as the hilum.

The substance of the ovary is divisible into a central medulla which reaches the surface only at the hilum; and a peripheral cortex which invests all other portions of the medulla and is in turn clothed by a layer of germinal epithelium, a continuation of 'the peritoneal epithelium, whose cells in this area are peculiar in that they possess a typically cuboidal shape, and are thus sharply distinguished from the flattened mesothelial cells of the surrounding portions of the peritoneum.

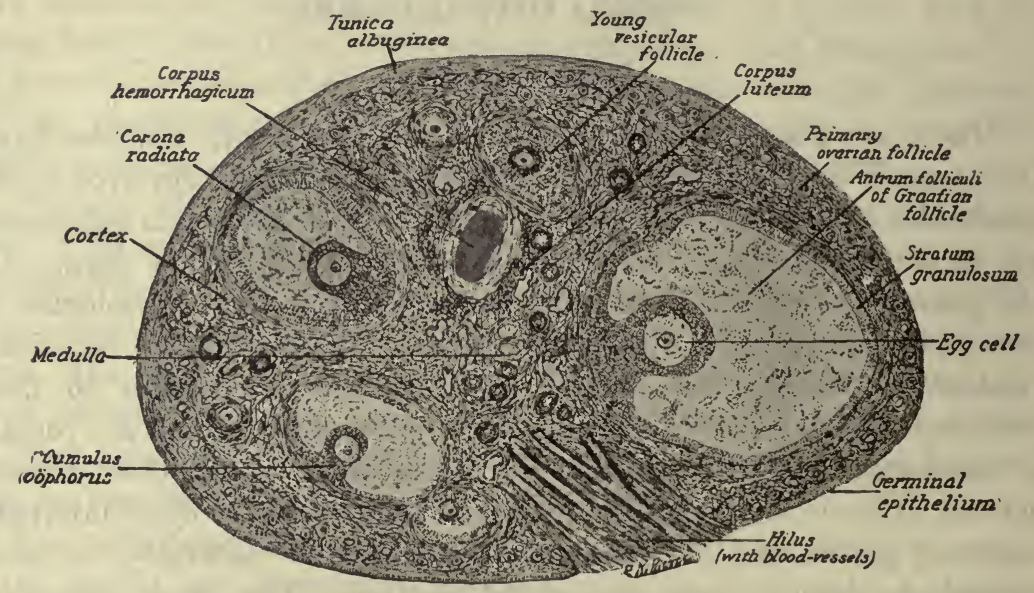

Fig. 449.-Section of Ovary of Adult Cat, Through Hilus, Showing Five Vesicular (Graaftan) Foldicles, with the Cumuldo Oöphorts and the ENCLOSED OVUM.

The antrum folliculi is filled with a granular material, a coagulum of the liquor folliculi. Note that the ovarian follicles are in the cortical portion, the medulla containing no follicles. $\times 7$.

The Medulla. - The medulla of the ovary consists of a fibromuscular stroma and large numbers of blood-vessels. Its arteries are characterized by their spirally tortuous course and thick muscular walls; its veins are numerous and large, and their endothelium rests almost directly upon the fibromuscular stroma. This portion of the ovarian stroma consists of fibrous connective tissue in which are elastic fibers and considerable numbers of smooth muscle cells. The connective tissue is richly supplied with cellular elements, most of which are ovoid or fusiform in shape.

The Cortex.-The cortex of the ovary likewise contains a vascular stroma and also large numbers of ova which are in all stages of develop- 
ment, from the genetic cells of the germinal epithelium up to the more mature germ cells, contained within epithelial sacs and known as ovarian follicles. During the menstrual epoch the ovaries also contain peculiar yellowish bodies, corpora lutea, resulting from the rupture of the largest

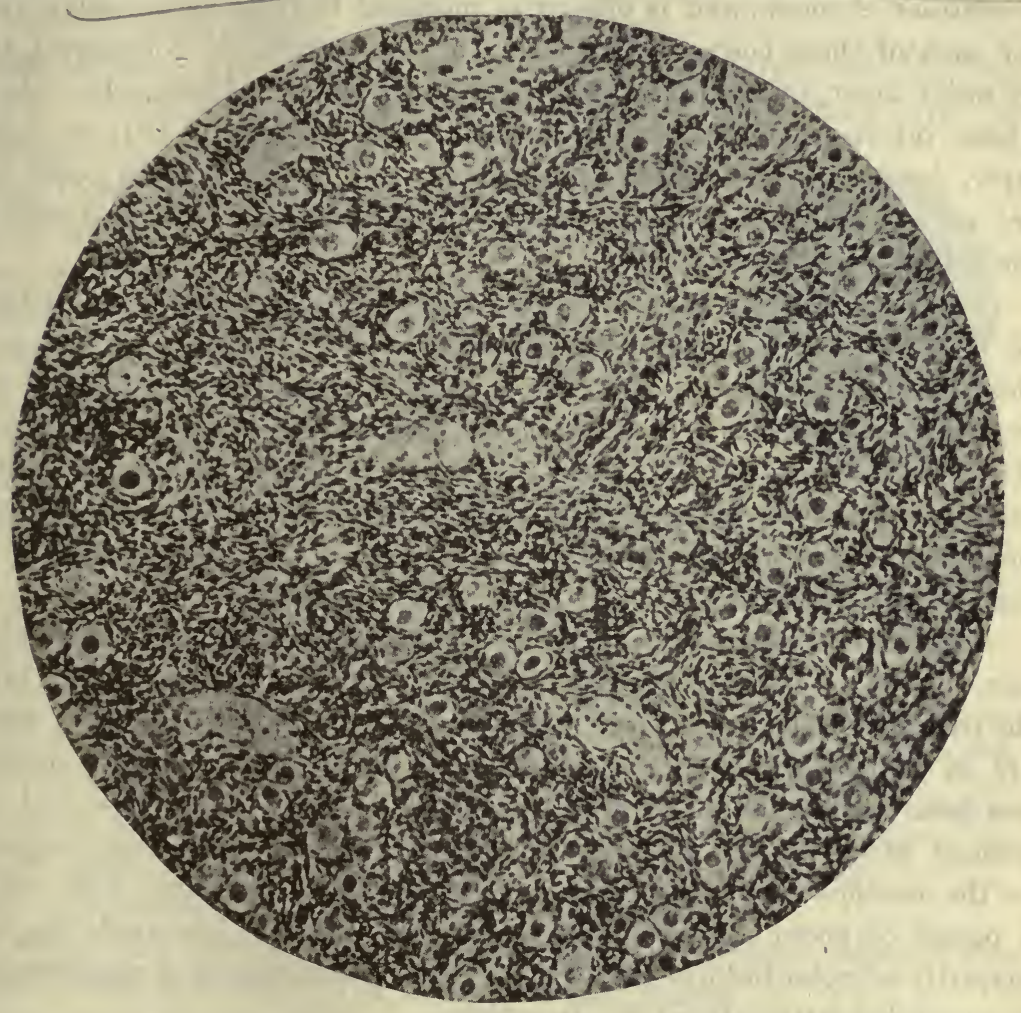

Fig. 450.-From the Ovarian Cortex of an Infant, Showing Manx Ova in the Primary Follicular Stage.

The portion above and to the right is near the free surface; that below and to the left adjoins the medulla. Hematein and eosin. Photo. $\times 200$.

follicles, a phenomenon which marks the climax of the process of ovulation.

The stroma of the ovarian cortex is a connective tissue structure which contains relatively few elastic fibers and, except near the medulla, very little if any smooth muscle. It is, however, abundantly supplied with connective tissue cells of large size, most of which are ovoid, fusiform, or even considerably elongated in shape. Many of these cells 
closely simulate smooth muscle on superficial examination, but are readily distinguished by careful study, especially if specimens are prepared by the various differential staining methods.

In the vicinity of the follicles the stroma is specially rich in cellular elements and is otherwise modified to form a concentric coat for each of these bodies. This coat, the theca folliculi, consists of $(a)$ an outer layer, or tunica externa, composed chiefly of connective tissue whose interlacing bundles are concentrically disposed, (b) an inner layer, tunica interna, which is peculiarly rich in large ovoid cells, and (c) an innermost memb'rana propria, upon which the epithelial cells of the follicle directly rest.

At the surface of the ovary the cortical stroma forms a dense layer of fine connective tissue fibers whose delicate bundles interlace in a close-meshed network. This layer, which immediately underlies the germinal epithelium at the surface of the ovarian cortex, is known as the tunica albuginea. It differs greatly in thickness in different mammalian species, in different individuals of the same species, and even in different portions of the same ovary. Its deeper surface blends insensibly with the underlying stroma of the cortex.

The general appearance of the ovary yaries according to the number, size, and stage of development of its ova and their follicles. At birth the cortex is packed with large numbers of newly formed ova, all of which are in approximately the same stage of development. Their number has been estimated at between 30,000 and $\% 0,000$. No new ova are formed after birth. Since in the normal sexual cycle from puberty to the menopause (from about the thirteenth to the forty-fifth year), a period of about 32 years, only about 400 eggs are liberated, the vast majority of potential ova must degenerate. This process of degeneration is especially active after the climacteric.

During childhood the formation of larger follicles goes forward at an unequal rate, some ova rapidly approaching maturity, others apparently remaining almost stationary, and still others undergoing retrograde development, so that at the age of puberty the ovary contains germ cells and follicles in all stages of development. After puberty the ripe follicles successively rupture and result in the formation of many corpora lutea which promptly degenerate, and are finally replaced by dense connective tissue in the form of small scar-like masses known as the corpora albicantia. Hence throughout the menstrual epoch the ovarian cortex contains many corpora lutea and corpora albicantia in addition to ova and follicles in various earlier stages of development. After the 
climacteric the remaining follicles degenerate and the process of ovulation gradually ceases.

We shall now discuss the structure of the ovum or female germ cell and shall then successively trace its development and maturation, the formation of its vesicular (Graafian) follicle, the rupture of the follicle, and the subsequent history of the corpus luteum.

The Ovum. - The ovum is a spherical cell of large size (200 to $300 \mu)$. When fully developed it is surrounded by a thick layer of exoplasm, the zona pellucida, which is probably derived from the cytoplasm of the follicular epithelium by which the ovum is closely invested. The ovum itself consists of a mass of cytoplasm, the vitellus, and a large vesicular nucleus or germinal vesicle, within which is frequently a single prominent nucleolus or germinal spot. The cytoplasm of the-mature ovum is inclosed by a very delicate cell membrane, known as the vitelline membrane, which is not demonstrable in the primitive ova of the younger follicles.

The CYTOPLASM of the ovum at first appears finely reticular, but as its development adyances a fatty material is deposited within its meshes, usually in the form of minute irregular spheroids, by the accumulation of which the reticular cytoplasm is in great part replaced by a granulofatty mass of faint yellowish color known as deutoplasm. Frequently this metamorphosis is not

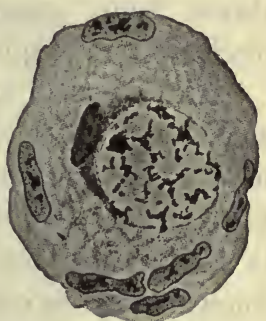

Fig. 451.-Ovum, ConTAINING A YoLK NuCleus (DotTerKERN) AT THE LEFT AND ABove THE NUCleUS.

The peripheral nuclei are derived from the adjacent stroma. Ironhematoxylin. Highly magnified. (After von Skrobansky.) quite complete, a remnant of the original cytoplasm persists beneath the vitelline membrane and in the vicinity of the nucleus.

Numerous cytoplasmic structures have been described in these cells, chief among which are the accessory nucleus (Nebenkern), and the yolk nucleus (Dotterkern). The accessory nuclei, occasionally chromatinic and therefore basophil, more frequently stain with cytoplasmic dyes and are at times attached, at other times separate from the true nucleus. They are more probably remnants of mitotic spindles. The yolk nuclei of mammalian ova most frequently take the form of crescentic masses of lightly staining chromatin ('chromidia') which partially surround the nucleus, forming a so-called nuclear cap. They are often found in various stages of disintegration, and the fragments may be transported to the peripheral portions of the cytoplasm, or may be irregularly scat- 
tered as small round chromatic granules, which occur throughout the cytoplasm. The physiological interpretation of these bodies is uncertain.

The NUCLEus of the ovum is a large spheroidal vesicle, the volume and distribution of whose chromatin is subject to great variation. Chromatin is present in greatest amount during the period of most active cell growth, in which the cytoplasm of the ovum is enormously increased in volume. At this time the nucleus often appears as a solid mass of chromatin. Later the chromatin is diminished in volume, portions of its substance being possibly extruded into the surrounding cytoplasm; the nucleus then acquires a characteristic vesicular appearance.

The nuclear membrane is sharply defined and is at most times prominent, except, as in other cells, during mitosis, a process which marks the final maturation of the germ cell. The nuclear matrix or nuclear sap abounds in the vesicular type of nucleus and the chromatin is scattered in small particles which adhere to the inner surface of the nuclear wall or to the delicate achromatic linin threads.

Each ovum as a rule contains a single nucleus (germinal vesicle), though occasionally two nuclei occur. The latter condition is presumed to arise either by the fusion of two ova within a single follicle or from incomplete cell division during development.

Each nucleus, during its vesicular stage, usually contains a single nucleolus (germinal spot), which forms a spherical mass of chromatin, situated, like the nucleus itself, eccentrically rather than centrally. The staining properties of the nucleoli vary remarkably. Usually they take the basic (nuclear) dyes to a greater or less depth; occasionally they exhibit an affinity for the acid (cytoplasmic) dyes; still other nuclei take a metachromatic or irregular tint with the ordinary nuclear stains. Many nuclei even in the absence of mitosis contain no nucleolus.

In the development of the ovum from the germinal epithelium, whose cells from their homology with the spermatogonia have been termed oögonia, there occur several mitoses which result in so-called oöcytes; these later develop into the mature ovum. At about the time of its extrusion from the ripe follicle a final series of mitoses occur, which distinguish the maturation of the ovum. In this process there is a series of two mitoses which result in the appearance of the polar bodies and produce a reduction in the number of chromosomes to one-half the number which is characteristic of the somatic cells. By the first mitosis the cell produces what may be termed a daughter ovum, together with the first polar body, a minute cell of insignificant/size. A second mitosis ensues giving origin to the mature ovum or oötid and the second polar 
body. 'The egg now contains only one-half the specific number of chromosomes; the full specific number is restored at fertilization by the addition of the male reduced complement carried by the sperm.

Development of the Ovarian Follicle.-The development of the ovarian follicle goes hand in hand with that of the ovum and can be readily followed in ovaries from individuals of different ages, children

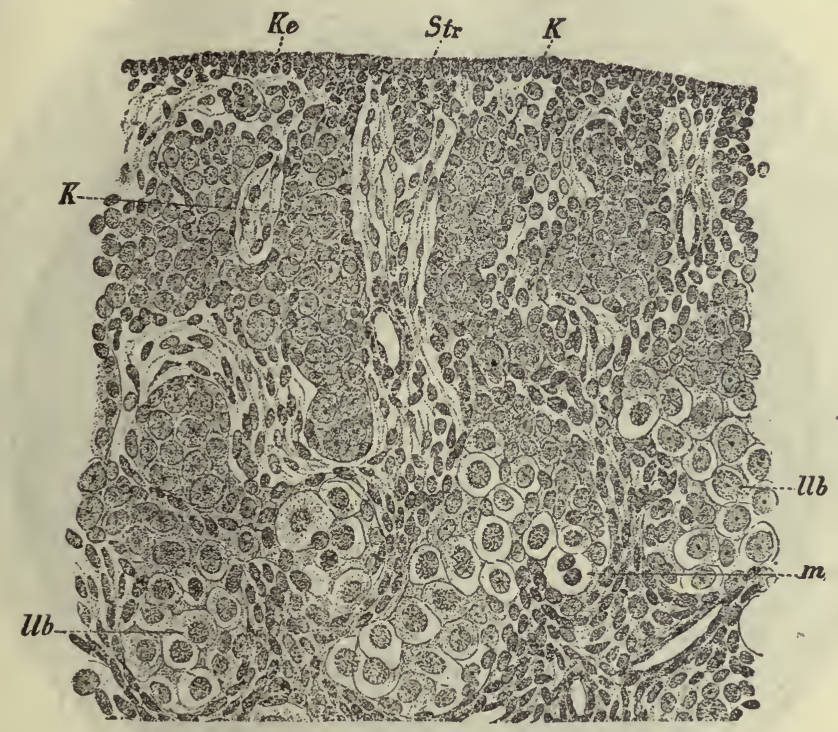

Fig. 452.-From a Section of the Ovarian Cortex of a New-born Kitten.

$K$, Pflüger's tubes; $K e$, germinal epithelium; $m$, mitosis; Str, ovarian stroma; $U b$, primitive follicles. Moderately magnified. (After Kölliker.)

and adults, the ripe follicles and corpora lutea appearing only after puberty. The process begins in the germinal epithelium in which certain cells so increase in size that they may be readily distinguished as future ova. As noted above, it is still an open question whether the primordial germ cells are genetic derivatives of the germinal epithelium or specific sex cells which have wandered into and become mingled with the cells of the peritoneal epithelium. More frequently, however, the earliest step in the process consists in the growth of solid cell columns from the layer of germinal epithelium into the cortical stroma of the ovary. These cell columns are known as Pfliger's tubes, though, except occasionally at the extreme surface of the organ, they lack a true tubular form and possess no vestige of a lumen. Felix, however, concludes that Pflüger's cords do not occur in the human female. 
Certain cells in these columns, by their increased size and prominent nucleus, become very early distinguishable as the primitive ova; their differentiation is rapidly followed by the constriction of the columns, through the activity of the surrounding tissue of the stroma, in such manner that one, rarely two or more ova, and several undifferentiated epithelioid cells are included in each portion whose connection with the

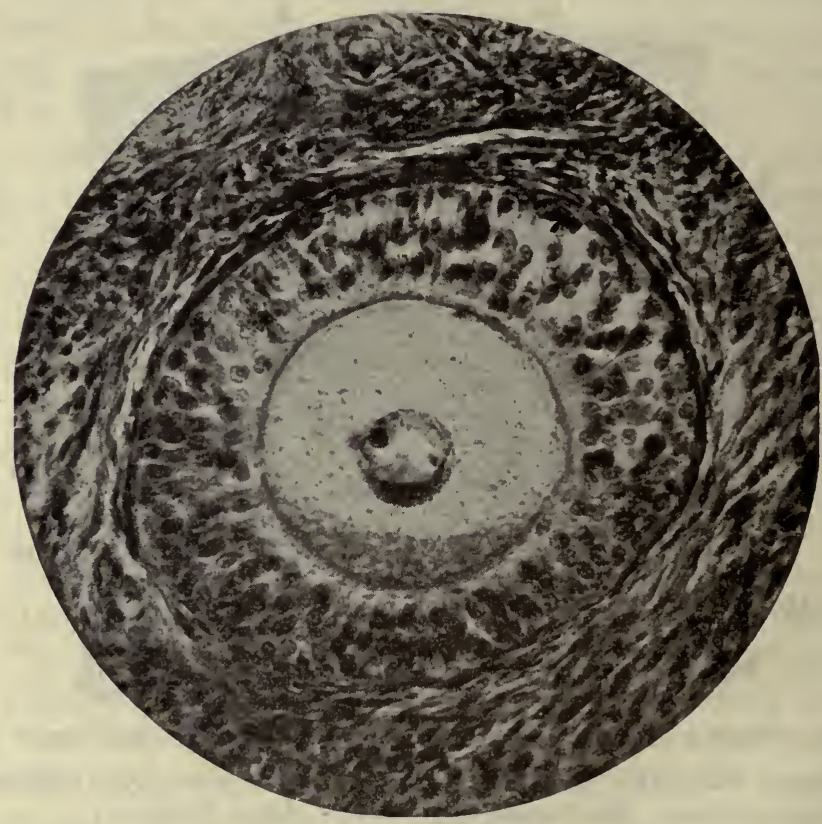

Fig. 453.-A Primary Ovarian Follicle of the Human Ovary.

From within outward are seen the germinal spot, germinal vesicle, vitellus, vitelline membrane, zona pellucida, granule cell layer, membrana propria, and theca folliculi. The ovarian stroma forms the border of the figure. Hematein and eosin. Photo. $\times 575$.

layer of germinal epithelium is thus severed. In this way the primitive follicles ('egg nests') are formed. In the ovary of the new-born hundreds of such primary follicles occur in all portions of the cortex (Fig. 450 ). They are also found persistent in large numbers in the ovary of the adult.

Many follicles never go beyond this primary stage of development, but after a time undergo retrograde metamorphosis either by gradual atrophy or by a process, known as atresia of the follicle, in which the 
chromatolysis in the ovum and its surrounding follicular cells is followed by growth and organization of the theca folliculi, the connective tissue which is thus formed finally replacing the atretic follicle.

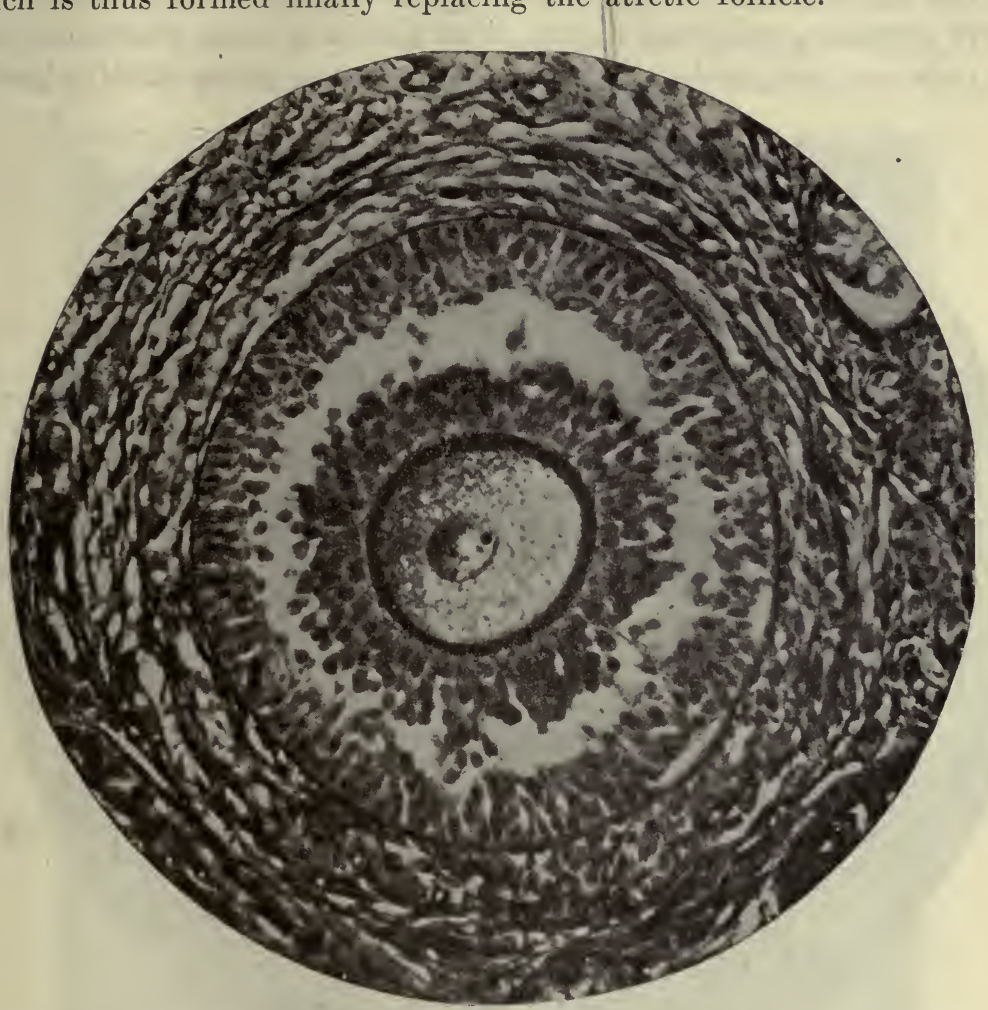

Fig. 454.-A Vesicular (Graafian) Follicle of the Human Ovary, Somewhat More Advanced than the Preceding.

The accumulation of liquor folliculi has separated the granular cells into a discus proligerus and a membrana granulosa. The membrana propria is very sharp, and the theca folliculi is almost divisible into an inner cellular and an outer fibrous layer. Hematein and eosin. Photo. $\times 460$.

After remaining stationary for a long period, often for years, certain of the primitive follicles enter upon a period of rapid growth. This process first affects the ovum and results in the appearance of the deutoplasm, zona pellucida, and other accessory structures, as already described. Cell multiplication now occurs in the surrounding epithelial cells, so that, instead of the single row of epithelium which surrounds the ovum of the primitive follicle, the ripening follicle soon acquires 
a layer of follicular epithelium several cells deep. This may be designated the mantle-layer.

The rapid multiplication of the epithelial cells is soon followed by astive secretion, resulting in the formation of a clear fluid by which the cells are more and more separated, and the cytoplasm of adjacent

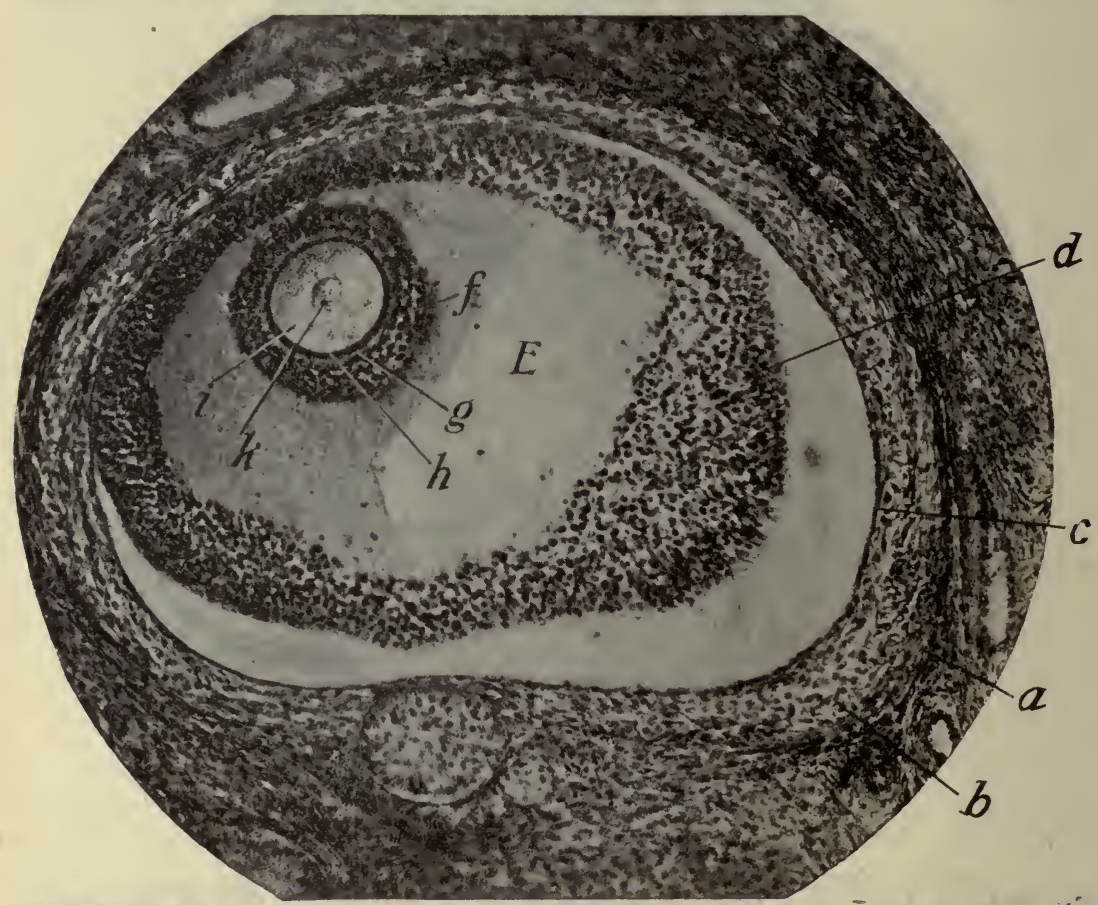

Fig. 455.-A Nearly Ripe Graafian Follicle from tile Ovary of a Dog.

$a$, fibrous, and $b$, cellular layer of the theca folliculi; $c$, membrana propria; $d$, membrana granulosa, which, as a result of contraction during hardening, has retracted from the membrana propria, leaving a broad artificial space; $E$, liquor folliculi; $f$, discus proligerus; $g$, zona pellucida; $h$, vitelline membrane; $i$, vitellus; $k$, the germinal spot, lying within the germinal vesicle. Hematein and eosin. Photo. $\times 150$.

cells is then readily seen to be firmly joined together by numerous delieate processes which may be regarded as intercellular bridges. Similar processes unite the neighboring cells to the-zona pellucida which has already formed about the ovum.

The accumulation of the fluid liquor folliculi within the follicle soon appears to tear apart certain of the epithelial cells, and a fluid-filled space, 
the antrum folliculi, is thus formed. Such a follicle is known as a vesicular or Graafian follicle. Follicles intermediate between the primary follicles with a single or double layer of mantle cells and vesicular follicles may for convenience be called intermediate or growing follicles. The epithelial cells are separated by the antrum into two layers: the one,

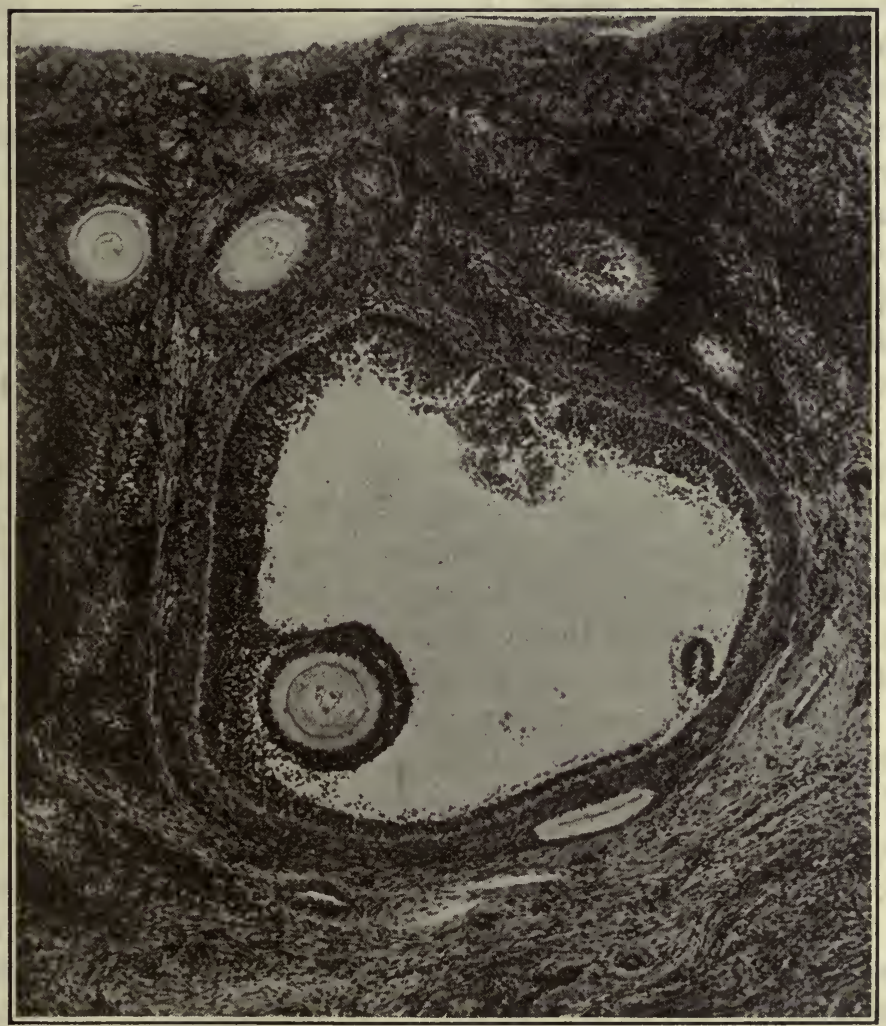

Fig. 456.-Photomicrograph of a Section of Cat's Ovary, Showing Two Primary Follicles and One Vesicular Follicle.

adherent to the membrana propria of the follicle, is known as the membrana or stratum granulosum; the other, adherent to the zona pellucida of the ovum, is designated the discus proligerus. The two layers remain in contact at one point, and as the liquor folliculi increases in volume, the attached discus proligerus with its contained ovum comes to occupy a more and more eccentric position, and the cells of the stratum granulosum, where the two layers are in contact, appear to pile up about the 
ovum in the form of a hillock, the so-called cumulus oöphorus. The latter term is now generally used exclusively in a sense to include and displace the term discus proligerus.

The cells of the cumulus, which adjoin the zona pellucida, become somewhat elongated and in this way they form a radiate investment consisting of one or two rows of columnar cells which surround the zona pellucida of the ovum and are known as the corona radiata. With the increase of the liquor folliculi the cumulus with its contained ovum is soon separated from its attachment to the stratum granulosum and the development of the folliculi is complete.

During this period of rapid growth and development the follicle has increased in size from a diameter which scarcely exceeded that of its ovum (about $300 \boldsymbol{\mu}$ ) to such a size that it occupies the entire breadth of the ovarian cortex. It is now ready for the final steps in the maturation of its ovum and for the rupture of the follicle coincident with the approach of the menstrual period.

The forces which lead to the rupture of the follicle are not fully determined. They are undoubtedly varied, and, in addition to the gradual attentuation of the layer of cortical stroma which covers the free surface of the follicle and is known as the stigma, they include the gradual accumulation of liquor folliculi under increasing tension, the marked congestion of the ovary at the approach of the menstrual period, which is accompanied by the determination of an undue proportion of blood to the theca of the ripe follicle (Clark), and possibly the contraction of the smooth muscle contained in the stroma of the deeper part of the cortex and adjacent portions of the medulla. In any event, as a result of the independent or combined action of these, or other unknown forces, the follicle ruptures in the direction of least resistance, viz., at the attenuated stigma, and the liquor folliculi gushes forth, carrying with it the detached ovum invested with its cumulus. The ovum is now free to enter the oviduct and prepare itself for fertilization and the development of the future embryo.

The ovum finds its way from the abdominal cavity into the oviduct by aid of currents established in the direction of the orifice by the cilia of the cells covering the fimbriæ; perhaps assisted by a direct grasping activity on the part of the fimbriæ. Since spermatozoa are free to wander out of the oviduct into the abdominal cavity, and since an egg may occasionally fail to reach the opening of the oviduct, the possibilities are offered for an abdominal pregnancy, a result occasionally consummated. Likewise an egg may fail to become expelled from its follicle and may 
then become fertilized within the ovary; or an egg fertilized in the abdominal cavity may perhaps subsequently become implanted in an empty follicle. Development proceeding under such conditions results in an ovarian pregnancy, at least seven well authenticated cases being now on record (Bryce, Kerr and Teacher, "An Early Ovarian Pregnancy," 1908). Ovarian pregnancies cannot proceed normally, and may early call for surgical intervention. Occasionally a follicle may contain more than one ovum, bi- and triovular follicles being common. Such follicles apparently present the possibilities for ordinary twinning and multiple births. Arnold (Anat. Rec., 6, 11, 1912) records follicles in the ovary of a negress also with from four to ten oöcytes, and one each with eleven and thirteen oöcytes.

The following table is offered for the benefit of the student as a résumé of the several structural layers of the ripe Graafian follicle. The structures are enumerated in order from without inward:

1. Theca folliculi $\left\{\begin{array}{l}\text { tunica externa } \\ \text { tunica interna } \\ \text { membrana propria. }\end{array}\right.$

2. Stratum (seu membrana) granulosum.

3. Liquor folliculi-occupying the antrum folliculi.

4. Discus proligerus-cumulus oöphorus.

5. Corona radiata.

6. Zona pellucida (seu striata).

\%. Perivitelline space (possibly an artifact).

8. Vitelline membrane.

9. Vitellus-egg cytoplasm.

10. Nucleus or germinal vesicle.

11. Nucleolus or germinal spot.

The Corpus Luteum. - The rupture of the follicle is accompanied by sudden relief of the intrafollicular tension and consequent hemorrhage from the thin-walled capillaries of the theca folliculi. Thus the cavity of the follicle is filled with blood; the ruptured follicle is then known as a corpus hemorrhagicum. This is the first stage in the formation of the corpus luteum.

Promptly succeeding the formation of the corpus hemorrhagicum, lutein cells appear at the periphery of the body. They are large, ovoid or polyhedral cells having a clear finely granular cytoplasm and a peculiar yellow color due to the presence of a pigment known as lutein. Moreover, the cytoplasm of the lutein cells becomes very rapidly infiltrated with droplets of fat, likewise deeply colored by the lutein pigment which 
is apparently held in solution. The origin of these cells is still a matter of controversy. By certain observers they have been thought to result from the growth and multiplication of those cells of the stratum granulosum which remain after the rupture of the follicle (Bischoff, Pflüger, Sobotta); by others they are derived from the connective tissue cells in the tunica interna of the theca folliculi (Kölliker, His, Palladino). Teacher (1908) interprets his preparations to 'indicate quite clearly that,

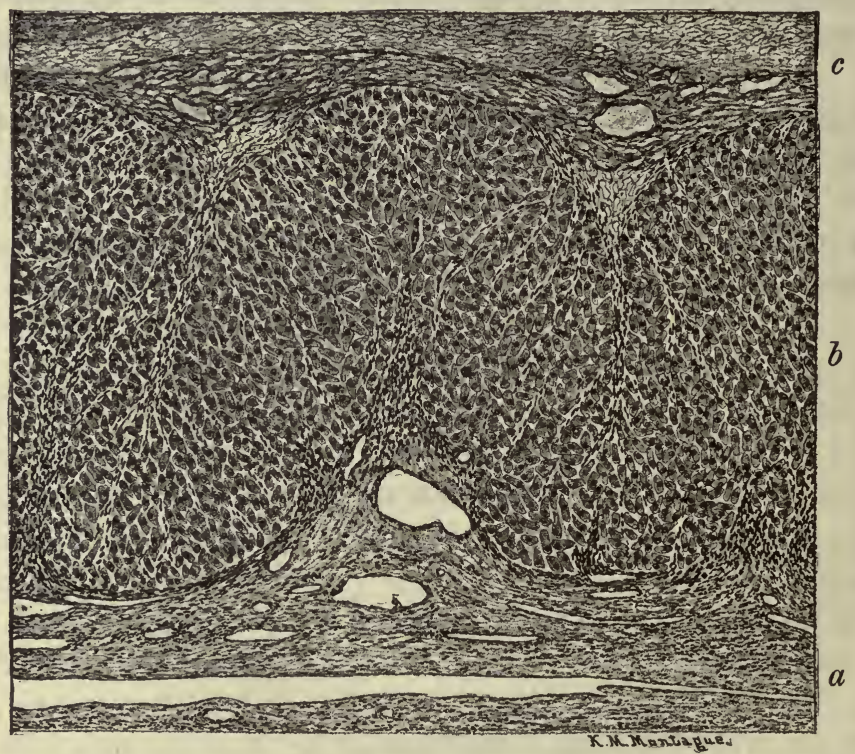

Fig. 457.-Section through the Peripheral Portion of a Corpus Luteum, Showing Lutein Cells.

$a$, the fibrous coat of the corpus luteum; $b$, lutein cells with bands of newly formed connective tissue; $c$, central blood clot, partially organized. Moderately magnified. (After Williams.)

whatever the source of the cells may be in lower animals, they do not in man arise from the membrana granulosa.' Lutein cells may multiply by mitosis.

The lutein cells increase rapidly both in number and in size, and gradually encroach upon the margin of the blood clot whose progressive absorption precedes the advance of the lutein cells. But not only docs the lutein mass grow centralward, it also, and especially in the event of fertilization of the discharged ovum with the consequently increased 
vascularity of the reproductive organs, grows at the periphery and in this way greatly increases the diameter of the corpus luteum.

Minute vascular sprouts of embryonic connective tissue now penetrate the lutein mass from the adjacent stroma of the theca folliculi, and growing centralward in septa-like processes, finally penetrate as far as the central blood clot. Hence the corpus luteum at this stage presents a more or less radiate structure. The central ends of the embryonic connective tissue septa frequently unite to inclose the remnant of the central blood clot, or by further proliferation they may entirely. replace the clot by a mass of newly formed gelatinous connective tissue.

The absorption of the blood clot usually proceeds slowly. Remnants of the disintegrating blood in the form of a central stellate mass, which often contains hematoidin crystals, frequently persist until the corpus luteum has become well organized with connective tissue.

The formation of new connective tissue is followed by its contraction. That this process occurs very early in the connective tissue first formed at the periphery of the body, may possibly be held to ac-

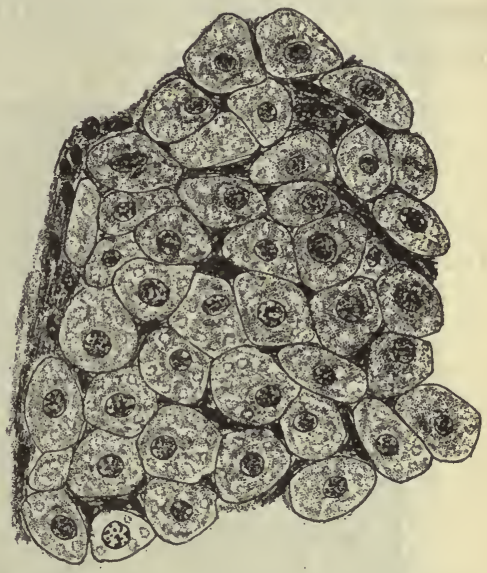

Fig. 458.-Portion of Corpus LuteUM OF RABBIT.

The lutein cells contain peripherally many fat vacuoles. The stroma consists of fibrous connective tissue. $\times 500$. count for the fatty infiltration and final degeneration of the lutein cells, because of the consequent interference with their vascular supply.

By continued development the entire mass of lutein cells is gradually replaced by connective tissue, which, by further contraction, finally produces a dense white fibrous scar, no longer containing lutein pigment, known as a corpus albicans. This body persists for a long period, but undergoes progressive contraction until only a minute scar of almost microscopical size remains to mark the site of the ruptured corpuscle and the highly developed corpus luteum. Such scars persist for years in the stroma of the ovarian cortex.

Finally it must be stated that there are no recognizable histological differences, other than those of size and duration, between the corpora lutea vera of pregnancy and the corpora lutea spuria whose formation 
accompanies the extrusion of the unfertilized ovum. The true corpora lutea are of relatively large size and persist for many months, the spurious are somewhat smaller and persist for little more than one month; yet both pass through the same histological process of development and degeneration and both leave their scars in the substances of the ovarian stroma.

Ovarian scars also arise through atresia of the larger follicles, the degeneration of whose epithelium is followed by an ingrowth of tissue derived from the theca folliculi, and the gradual development, organiza-

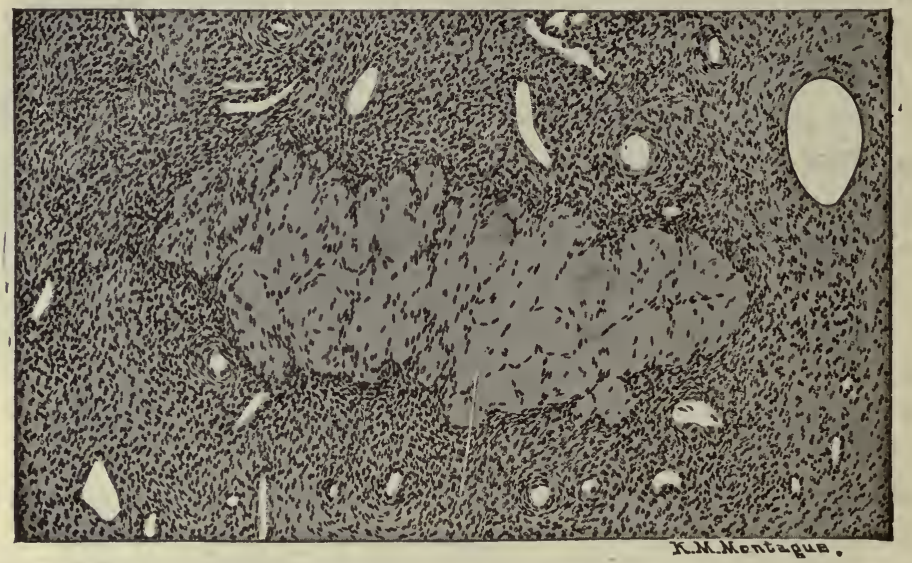

Fig. 459.-A Corpus Albicans, from a Section of the Human Ovary.

$\times 75$. (After Williams.)

tion, and final contraction of the connective tissue, forming, as it were, a minute but imperfect corpus albicans, in the center of which is often the shrunken degenerating remains of the ovum. Certain small acidophilic homogeneous bodies, the so-called Call-Exner bodies, of uncertain significance are also occasionally present in the stratum granulosa of the ovarian follicles.

Blood Supply.-The blood-vessels of the ovary are derived from the branches of the ovarian and uterine arteries. These vessels enter the ovary through the mesovarium and divide into numerous branches which pursue a peculiar spiral or corkscrew course through the stroma of the medulla, and finally enter the cortex. They possess thick muscular walls containing bundles of longitudinal smooth muscle fibers. In the cortex they supply capillaries to the stroma, and in the theca folliculi of the Graafian follicles they form rich plexuses of broad capillaries and 
thin-walled venules. As the follicle approaches maturity these plexuses become enormously developed and apparently bear an important relation to the rupture of the follicle and the rapid development of the corpus luteum. The veins, which take origin from the venules of these capillary plexuses, converge toward $t h \mathrm{e}$ medulla, where they form a plexus of la rge thin-walled vessels, the plexus venosus ovarii or pampiniform plexus, which is embedded in $\mathrm{the}$ connective tissue of the medulla, the mesovarium, and the adjacent portions of the broad ligament.

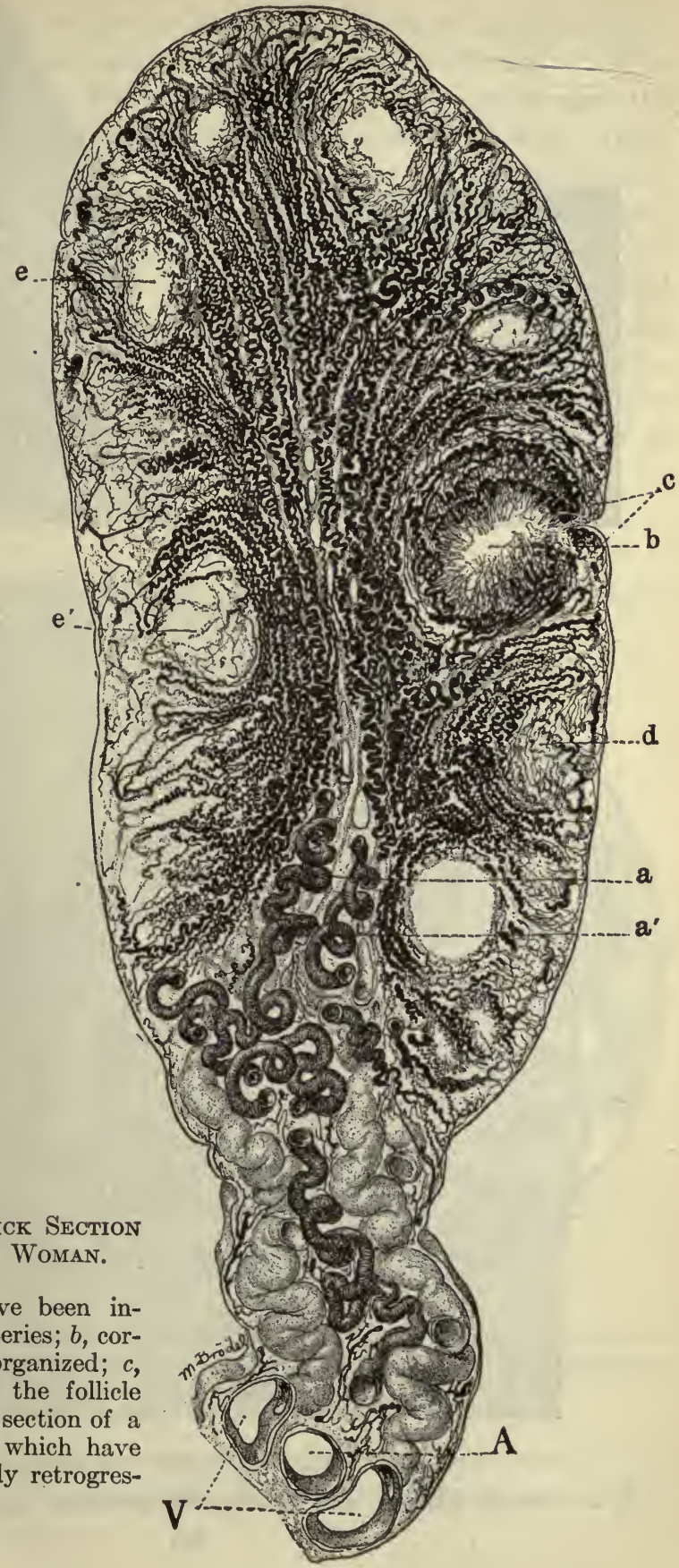

Fig. 460.-From a Thick Section OF THE OVARY OF A WOMAN.

The blood-vessels have been injected. $A, a$, and $a^{\prime}$, arteries; $b$, corpus luteum, partially organized; $c$, point where rupture of the follicle occurred; $d$, tangential section of a follicle; $e$, corpora lutea which have organized and are already retrogressive. (After Clark.) 

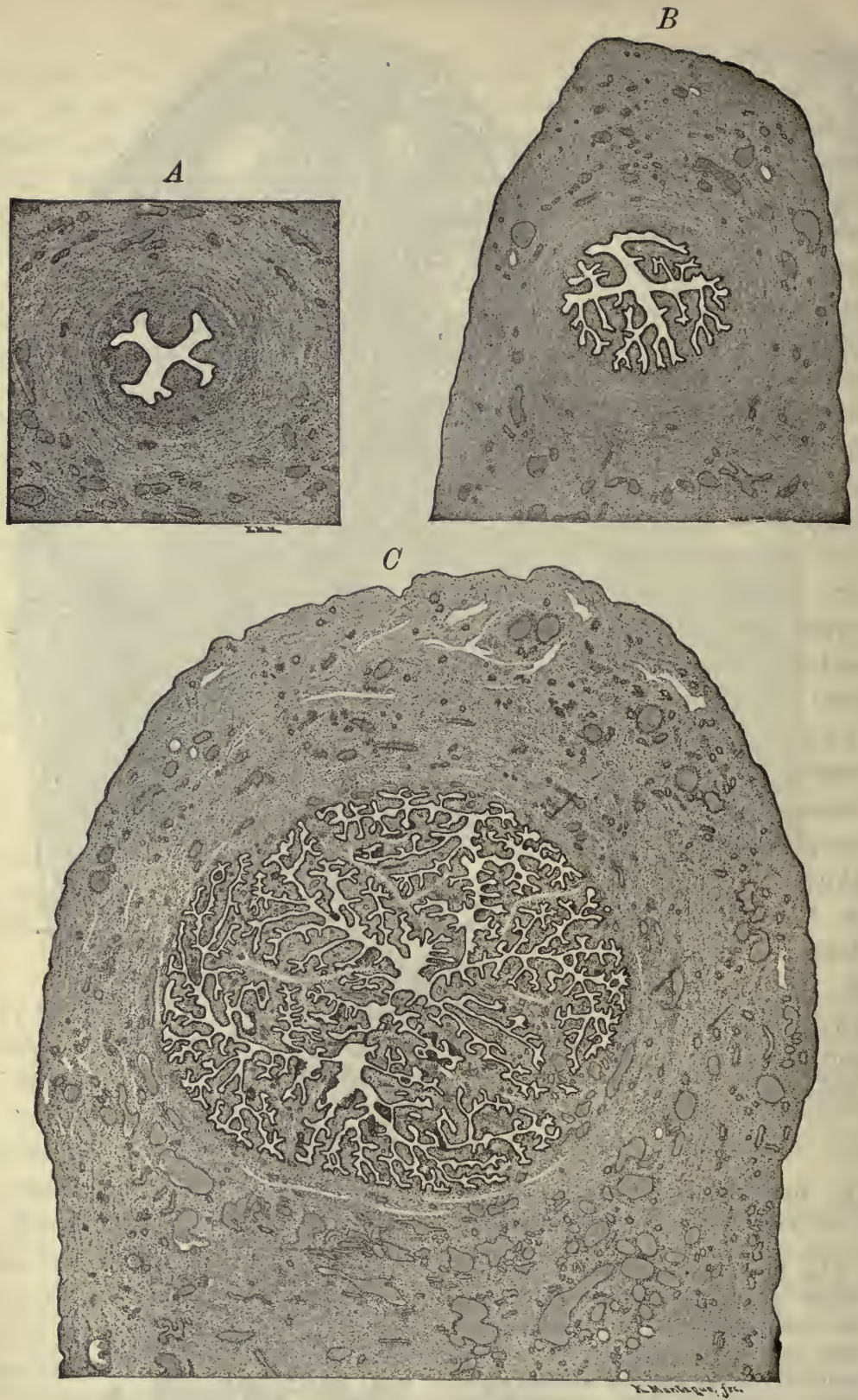

Fig. 461.-Transections of the Human Oviduct.

$A$, uterine; $B$, isthmic; and $C$, ampullar portions. $\times 15$. (After Williams.) 
The lymphatics arise in the cortical stroma by anastomosing canals and capillaries of irregular caliber, which are especially abundant in the walls of the Graafian follicles. These vessels converge toward the medulla, where they enter lymphatics which are supplied with valves, and find their way to the lymph nodes of the pelvic and lumbar regions.

The nerves are chiefly derived from the ovarian sympathetic plexus. They enter the hilum and are distributed to the walls of the bloodvessels, and to the stroma of the ovary; here they form a rich terminal plexus in the walls of the follicles. Whether or not the naked fibrils are distributed to the epithelial cells within the follicle has not been satisfactorily determined. The small ganglia of the medulla include also pheochrome cells (Winiwarter); and certain sensory fibers are said to end in lamellar corpuscles.

\section{The Oviduct}

The oviduct, uterine tube, or Fallopian tube is a narrow duct about $4 \frac{1}{2}$ inches $l \circ \mathrm{ng}$, leading from the ovary to the cavity of the uterus. It consists of a broad, funnel - s h a p e d, fringed or fimbriated extremity (or infundibulum), a constricted neck, an intermediate ampulla of considerable diameter, and a slender isthmus by which the oviduct communicates with the uterine cavity.

Throughout the entire tube its wall, which becomes gradually thinner

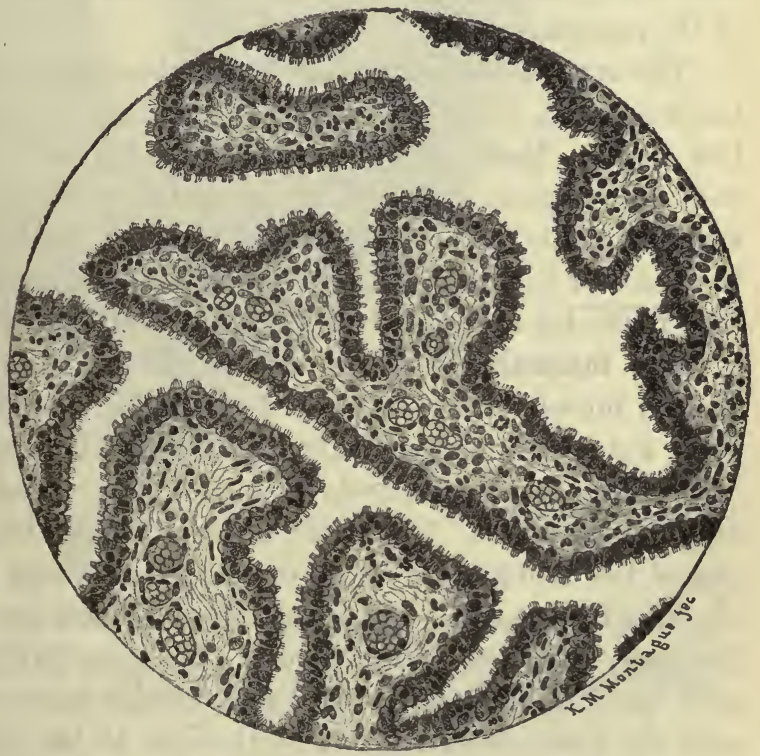

Fig. 462.-From a Transection of the Ampulla of the Oviduct, Showing the Structure of the Mucosa. $\times 280$. (After Williams.)

from isthmus to infundibulum, consists of three coats-mucous, muscular and serous-but the character of its mucous membrane differs 
somewhat in its several portions. In the isthmus it is relatively smooth and usually presents four longitudinal ridges which have few secondary or accessory folds; in the ampulla the mucosa is greatly folded, the primary rugæ possessing small secondary folds which extend in all directions, and by their very complexity nearly obliterate the otherwise broad lumen. In the fimbriated portion the folds of the mucosa are continued into the fimbriæ, at the margin of which the columnar ciliated epithelium of the oviduct becomes directly continuous with the serous mesothelium of the peritoneum investing the outer. surface of the tube.

The mucosa is lined by columnar epithelium, arranged either in a simple or pseudo-stratified manner, the greater portion of whose cells are provided with cilia. The ciliary motion is directed toward the uterus. The epithelial layer covers all the folds of the mucosa and, extending deeply into the crevices, forms invaginations which, in transections of the tube, simulate glandular structures. There are, however, no true secreting glands in the oviduct. Here and there groups of non-ciliated cells with clear cytoplasm occur among the more numerous ciliated cells of the mucosa.

The epithelium rests upon a thin homogeneous basement membrane beneath which is a tunica propria consisting of a cellular type of connective tissue. Many of the connective tissue cells are of fusiform shape, and, unless specially stained or carefully examined, they closely resemble smooth muscle cells. The mucosa, however, contains no muscle except at the bases of the largest folds, into which occasional fibers from the adjacent muscular coat penetrate.

The muscular wall of the oviduct is formed by two layers of smooth muscle - a broad inner circular layer, and an outer longitudinal coat, which is very unequally developed at different portions of the circumference, but is relatively thin in all parts, and is entirely wanting at frequent intervals. The outer layer is usually broadest at the free margin of the oviduct and at its opposite side where the tube is attached to the broad ligament. The inner circular fibers are more or less obliquely disposed, and, toward the mucosa, the muscular bundles fuse insensibly with the cellular connective tissue of the mucous membrane. In general the inner circular layer is thickest at the isthmus and thinnest at the infundibulum, while the longitudinal layer is thickest toward the fimbriated end.

The serous coat of the oviduct is continuous with the peritoneum. It consists of an outermost layer of mesothelium which rests upon a subepithelial layer of connective tissue, by which it is firmly united to the 
muscular wall. This portion of the serous coat contains the larger vessels and nerves, which are distributed to the inner coats.

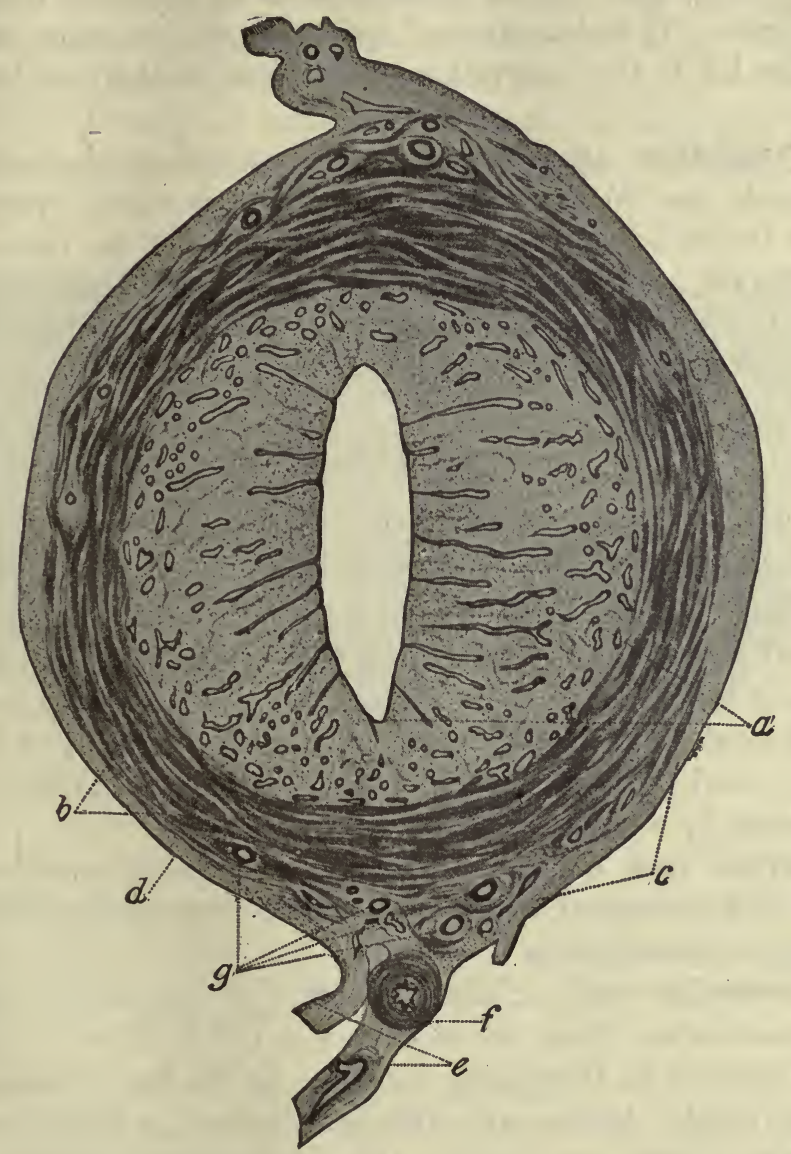

Fig. 463.-Transection of the Uterus of an Ape.

$a$, mucosa; $b$, circular muscle; $c$, longitudinal muscle; $d$, serous coat; $e$, lateral ligament; $f$, Wolffian tube; $g$, blood-vessels. $\times 4$. (After Sobotta.)

Blood Supply.-The arteries of the oviduct are derived from the uterine and ovarian vessels. The larger divisions find their way through the connective tissue of the serosa whence they: send smaller branches inward to form a plexus between the layers of the muscular wall and among the bundles of circular muscle fibers. From this plexus capillaries 
are distributed to the muscular coat, and to the mucous membrane in which they form a rich subepithelial capillary plexus. The veins follow a similar course, and like the arteries, form an extensive plexus in the muscular coat. The abundance of vessels in the muscular wall of the oviduct has led to the description of this coat as the vascular layer of the organ.

The lymphatics arise by anastomosing plexuses in the mucosa, from which vessels pass to the serous coat and enter valved lymphatics by which the lymph is conveyed to the lymph nodes of the lumbar region.

The nerves, which are chiefly sympathetic, are distributed from a plexus in the serous coat, to the muscular wall, and to the mucosa, in which they form a terminal subepithelial plexus.

\section{The Uterus}

The uterus is a hollow pear-shaped organ, divisible into a deepest or fundus portion, the body, and the cervix, about 3 inches in depth, $11 / 2$ in width and 1 in thickness. The cervix opens into the body through the internal os, into the vagina through the external os. Its wall consists of a mucous membrane, a muscular coat, and an outermost serous coat which is derived from the peritoneum and invests the body of the organ. The cervix uteri projects into the vaginal canal and the serous coat is there replaced by a reflection of the vaginal mucosa.

The serous coat, or perimetrium, of the uterus consists of mesothelium which rests upon a thin subepithelial layer of connective tissue. It presents no peculiarities.

The muscular coat, or myometrium, of the uterus consists of smooth muscle whose fibers are of large size (40 to $60 \mu$ in length) and which are disposed in interlacing bundles. In the lower mammals these form quite regular layers-an outer longitudinal, a thick inner layer, most of whose fibers are circular, and an innermost, but less distinct, submucous portion containing oblique and longitudinal fibers. The outer longitudinal and circular layers are separated by a fibromuscular stratum containing a rich plexus of large blood-vessels.

In the human uterus the arrangement of muscle fibers is much less regular, but follows a similar plan, though there is no distinct subdivision into layers. Nevertheless, careful examination reveals three indistinct strata which are intimately blended with one another. The outermost of these indistinct layers consists of irregularly disposed longitudinal fibers, the stratum supravasculare. This layer is in most parts very 
thin, and is best developed opposite the margin of the lateral ligament and in the cervix uteri. Within this is a broad layer of interlacing bundles of more or less circular fibers, which, from the slight obliquity of their course, frequently cross each other at acute angles. Intermingled with these circular bundles are many large blood-vessels, from which both the mucous and muscular coats are supplied. This broad middle layer is therefore known as the stratum vasculare. The inner

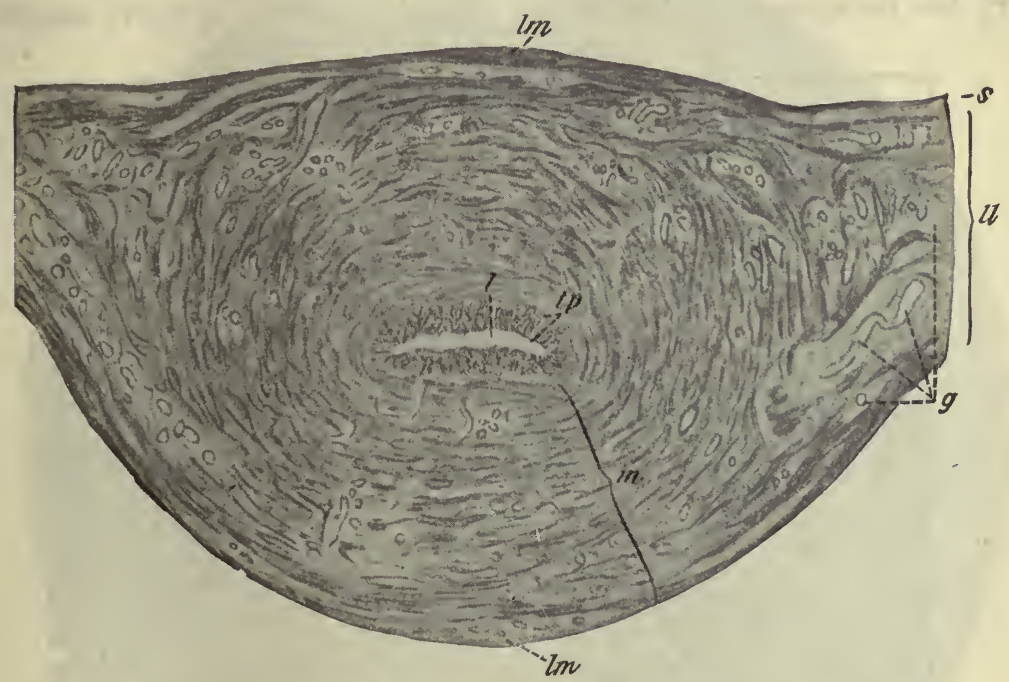

Fig. 464.-Transection through the Body of the Human Uterus.

$g$, blood-vessels; $l$, lumen; $l l$, broad ligament; $l m$, longitudinal muscle; $m$, circular muscle (the fibers are mostly oblique); $s$, serous coat; $t p$, mucosa. Hematoxylin and eosin. $\times 2$. (After Sobotta.)

portion of this second layer passes insensibly into a thin innermost stratum submucosum, which again contains many longitudinal fibers, and upon which the mucosa directly rests.

The uterine mucosa, or endometrium, is of considerable thickness ( 1 to $3 \mathrm{~mm}$.). It is clothed with epithelium, and its tunica propria contains numerous tubular glands.

The epithelium is of the ciliated columnar type, and consists of a single row of cells. Apparently not all of its cells are provided with cilia, areas of ciliated alternating with groups of non-ciliated epithelium. The epithelial layer is continuous with the epithelium of the uterine glands; in the region of the external os uteri it is replaced by the stratified squamous epithelium of the vaginal mucosa. Ofttimes, and espe- 
cially in multiparæ, the stratified squamous epithelium of the vagina is continued for some little distance within the canal of the cervix uteri; it never clothes more than the lower one-half to two-thirds of the cervical canal. The current resulting from the vibration of the intra-uterine cilia is directed toward the vagina.

The tunica propria of the mucosa consists of a peculiar embryonal type of connective tissue, similar to that of the oviducts, which contains very few collagenous and no elastic fibers, but which is richly supplied,

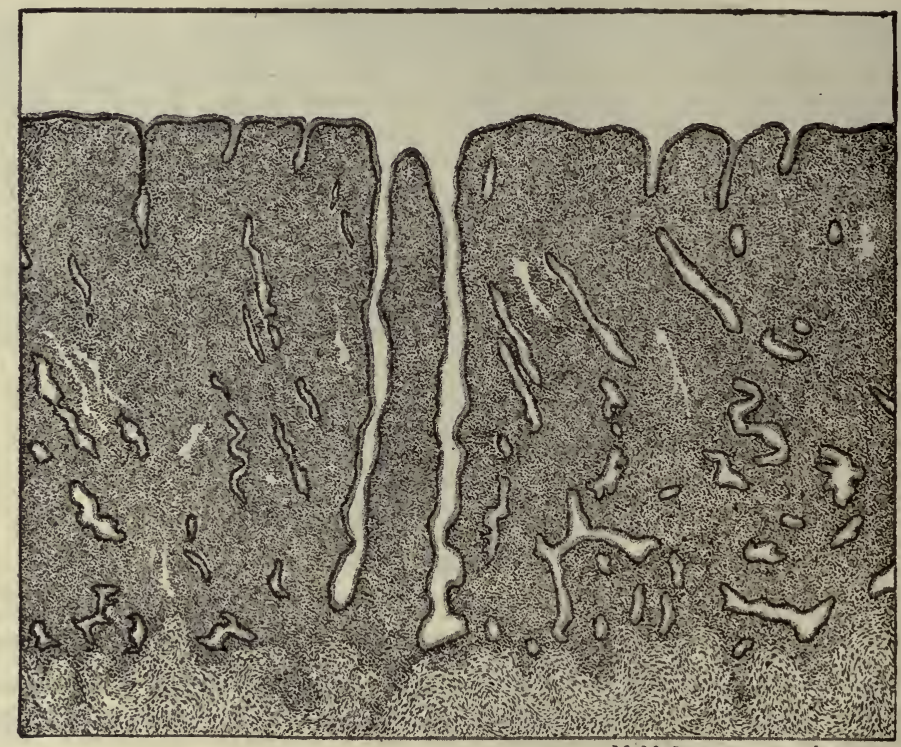

K.M.Mortigue,fer.

Fig. 465.-From a Transection of the Uterine Mucosa.

$\times 16$. (After Williams.)

in fact is literally packed, with cellular elements. These cells are ovoid or fusiform in shape, and many of them are branched; their nuclei, also, are ovoid and somewhat vesicular. Many lymphocytes are found in the tunica propria, but these mostly occur in the vicinity of the lymphatics and smaller blood-vessels with which the uterine mucosa is abundantly supplied. In the mucosa of the cervix uteri the development of the connective tissue appears to be more advanced, the cellular elements being relatively fewer; it also contains many fine fibers which appear to form a delicate network. At the external os uteri the tunica propria is con- 
tinuous with the similar, though still more fibrous, layer of the vaginal mucosa.

The uterine glands are divisible into two types-those of the body of the organ, and those of its cervix. The former are, perhaps, to be regarded as tubular invaginations of the lining epithelium, whose function

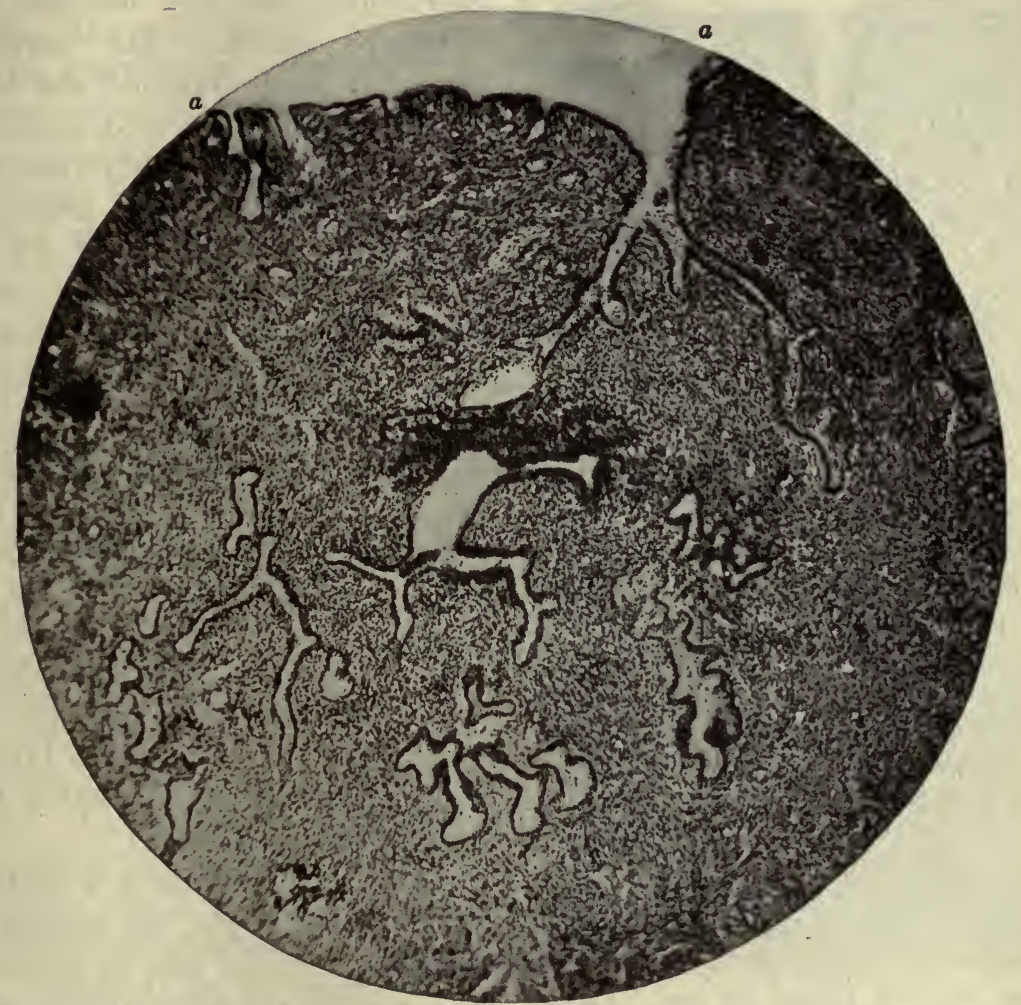

Fig. 466.-From the Cervix Uteri of a Girl of Sixteen Years, Showing the Cervical Glands in Section.

$a-a$, lining epithelium. Hematein and eosin. Photo. $\times 102$.

is one of epithelial regeneration rather than of glandular secretion. The tubules of the cervix uteri are true mucus-secreting glands.

The uterine glands proper, those of the body of the organ, are slightly branched or forked tubules which traverse the entire breadth of the mucosa, presenting a characteristic spiral or corkscrew course; their blind extremities are often bent or turned to one side, apparently from the proximity of the adjacent muscular coat. The glandular epithelium 
is of the columnar type and, like that of the free surface, is frequently provided with cilia, especially near the mouth of the gland. The epithelium rests directly upon the connective tissue of the tunica propria.

The cervical glands (glandulio uterince cervicales) resemble those of the body of the organ in their tubular form and the columnar shape of

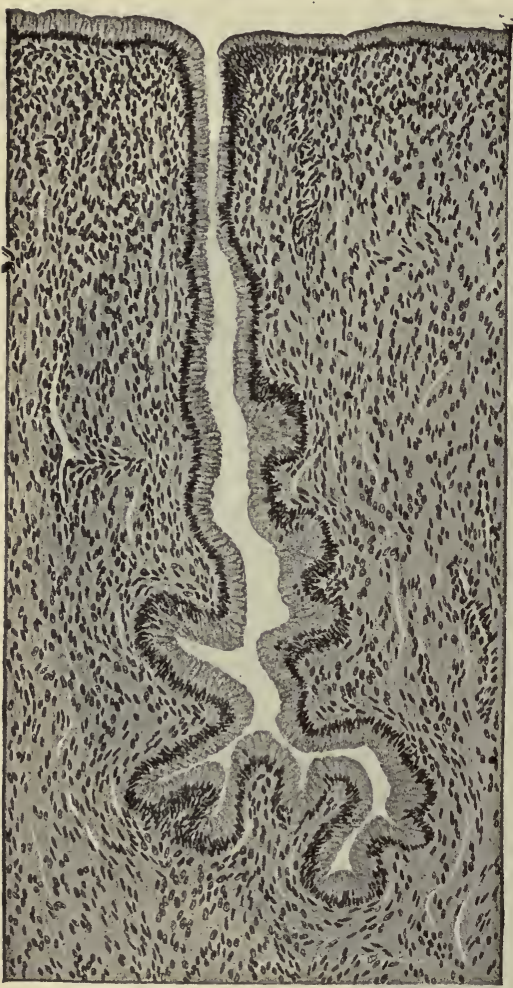

Fig. 467.-A Gland of the Human Cervix Uteri in Longitudinal SeCtion.

$\times 90$. (After Williams.) their epithelium, but here the resemblance ceases. The cervical glands are much branched, and their tubules present frequent dilatations, some of which, apparently from occlusion of their outlet, attain a macroscopic size, and are then known as Nabothian follicles (ovula Nabothii); they are filled with a tenacious mucous secretion. The glandular epithelium near the cryptlike ducts is usually ciliated, like that of the surface, but in the secreting portions it consists of tall, clear, columnar cells which are in varions stages of secretory activity, their product being a viscid glairy mucus, strings and granules of which are found within the lumen of the glands, as well as within the canal of the cervix uteri.

The uterine cavity is a relative term. In the virgin, the mucosa is considerably folded and its surfaces are almost in apposition, being only separated by a very limited amount of desquamated epithelium and cellular débris, to which, in the canal of the cervix uteri, the mucous secretion is added. During pregnancy, the development of the fetus within the uterine cavity distends its walls and so dilates the canal that it at last forms a sac of sufficient size to contain the fetus, which floats within the amniotic fluid inclosed by its membranes.

The blood-vessels of the uterus enter through the folds of the lateral ligament and find their way, through the subepithelial connective tissue 
of the serous coat and the muscular wall, to all portions of the organ. In the vascular layer of the muscular coat they form an extensive plexus from which branches are distributed to the musculature and to the mucosa, the branches to the latter penetrating nearly to the surface, where they form rich, subepithelial, capillary and venous plexuses. The uterine arteries, like those of the ovary, possess a peculiar, spirally tortuous course. The veins accompany the arteries, but are less tortuous.

The lymphatics of the uterus arise by anastomosing channels in the mucous and muscular coats. They form a vascular plexus in the serous coat and lead outward, through the lateral ligaments and pelvic connective tissue, to the lower lymph nodes of the lumbar region.

The nerves of the uterus are very numerous. They include both spinal and sympathetic fibers. They enter the serous coat from the ganglionic pelvic plexus, and are distributed to the vascular layer of the muscular coat. They there form a rich plexus, from which sympathetic motor fibers are distributed to the musculature and to the walls of the blood-vessels.

The distribution of nerves within the mucosa has not yet been thoroughly worked out. According to von Gawronsky (Arch.f. Gynäkol., 1894) and Köstlin (Fortschr. d. Med., 1894) sensory nerve fibrils penetrate nearly to the surface and form a scanty subepithelial plexus, whence are derived fibrils which terminate between the epithelial cells.

Since the uterus is subject to extensive structural variations dependent upon its functional phase and condition, it becomes important to recognize the differential marks of the menstruating and of the pregnant uterus. Besides general histologic alterations in the wall, especially in the mucous and muscular layers, additional structures involved in the pregnant uterus are the decidual cells and chorionic villi; these are diagnostic of pregnancy. Only the histology of these structures will be here described; for a consideration of their embryologic significance and relationship reference must be made to a text-book of Embryology.

\section{The Menstruating Uterus}

The appearance of the phenomena of menstruation is accompanied by decided alterations in the structure of the uterine mucosa. In spite of the difficulty of obtaining sufficiently fresh and well preserved material, certain changes which characterize the menstruating uterus are now definitely known. These chiefly consist in increased vascularity, hypertrophy of the elementary tissues of the mucosa, epithelial desquamation, and rupture of the blood-vessels, with consequent hemorrhages. These changes are fol- 
lowed by a process of regression and later of regeneration, by which the uterine mucosa rapidly returns to its former condition.

The first or hypertrophic stage involves the epithelium, whose cells are elongated, and the tunica propria, in which many of the connective tissue cells undergo multiplication and enlargement. Thus the mucous membrane becomes greatly thickened; its glands, also, are increased in both length and breadth, becoming at the same time even more tortuous than before. The glandular hypertrophy involves both the uterine and the cervical glands; the secretion of the latter is much increased.

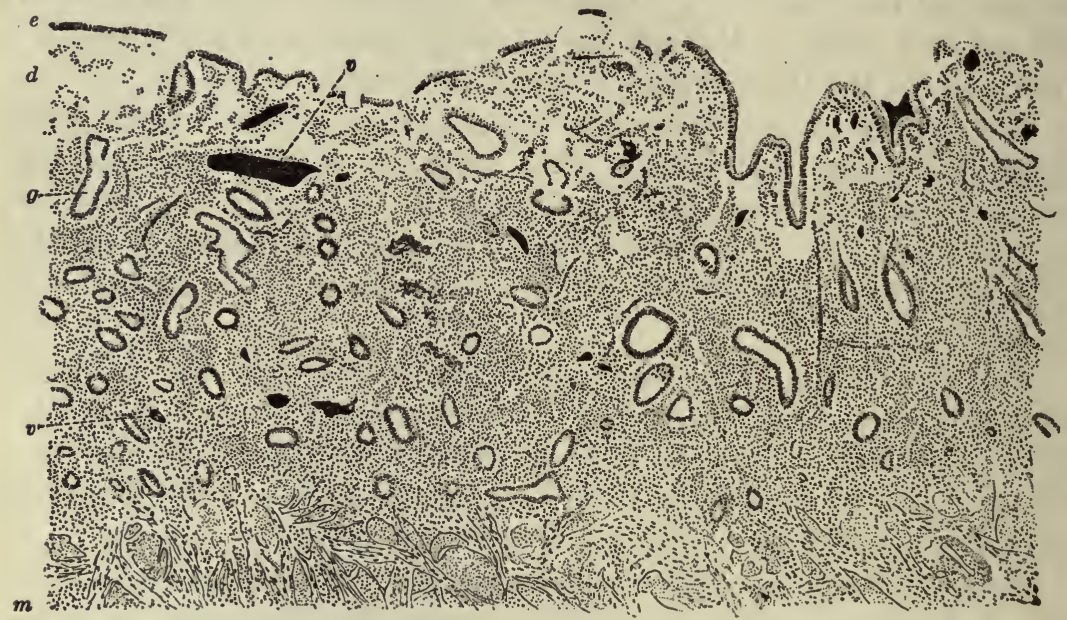

Fig. 468.-From a Section of the Human Uterine Mucosa at the First Day of Menstruation.

$e$, epithelium; $d$, disintegrating layer; $g$, gland; $v$, blood-vessel; $m$, muscular coat. $\times$ 44. (After Minot.)

At the same time, the blood-ressels become widely dilated, especially those near the surface, and broad thin-walled sinuses are formed beneath the epithelium. Finally these vessels rupture and hemorrhages occur into the substance of the mucosa as well as into the cavity of the organ; desquamation and disintegration of the superficial portions of the mucosa result. The menses which are thus formed contain blood, epithelium, connective tissue cells, and many leukocytes, which wander out from the bloodvessels of the mucosa in large numbers. The greatly thickened and hemorrhagic mucosa is known as the decidua menstrualis.

Regression and regeneration follow rapidly upon one another, the mucosa gradually regaining its former condition. During this process fat droplets appear in many of the connective tissue cells. The epithelium is rapidly regenerated, the new cells arising from the epithelial remnants at 
the mouths of the uterine glands. In the course of a few days the mucosa regains its former quiescent condition. The complete cycle includes 28 days, of which the menstrual process occupies about seven.

\section{The Gravid Uterus}

In the event of conception the uterine changes are more pronounced than during menstruation. These alterations include the same processes of hypertrophy and thickening as occur in the decidua menstrualis; they involve the musculature as well as the mucosa but are not followed by regressive changes,--hemorrhage, desquamation, etc.-until parturition occurs.

The muscular wall undergoes an enormous increase both in the number and size of its fibers. The relavively short (30 to $60 \mu$ ) smooth muscle fibers of the uterine wall gradually increase in size to as much as eleven times their former length and two to five times their breadth (Kölliker). The connective tissue of the muscular coat also increases in volume and becomes more distinctly fibrous. After parturition, fat droplets appear within the muscle cells, and the muscular wall by gradual atrophy returns to its former condition.

In the mucosa the formation of a decidual membrane goes forward in a manner similar to the development of the decidua menstrualis, but the process is exaggerated. The tunica propria soon becomes divisible into two distinct, though not sharply

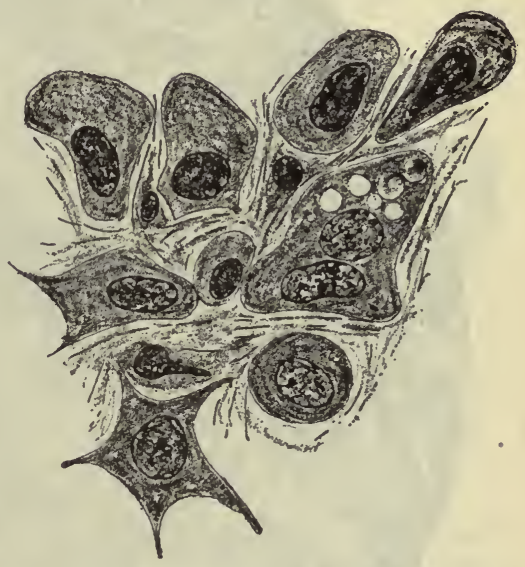

Fig. 469.-A Grodp of Deciddal Cells from the Human Uterus During the Early Stages of PregNANCY.

One of the cells contains two nuclei and a number of fat vacuoles. Three nuclei of the connective tissue stroma are also shown. $\times 750$. defined, layers, a deeper cavernous portion which is permeated by broad vascular channels together with the atrophied remains of the uterine glands, and a superficial compact layer in which the vascular channels, except for the thin-walled venous spaces, are smaller and the connective tissue cells more closely packed.

Many of the connective tissue cells attain a large size and their nuclei are frequently multiple, or they may acquire an irregular polymorphonuclear form. Giant cells are thus produced in the compact layer of the mucosa of the gravid uterus; they are highly characteristic of this tissue and are known as decidual cells. Though it is frequently asserted that 
similar cells occur in the decidua menstrualis, this is denied by Minot (1903), who states that in a considerable number of menstrual decidua examined, no such cells were ever found.

The superficial epithelium is soon desquamated and the tunica propria comes into contact with the fetal chorion. The glandular epithelium is also partially degenerated, often becoming flattened and of irregular shape. It is frequently desquamated into the glandular lumen; this lumen is thus

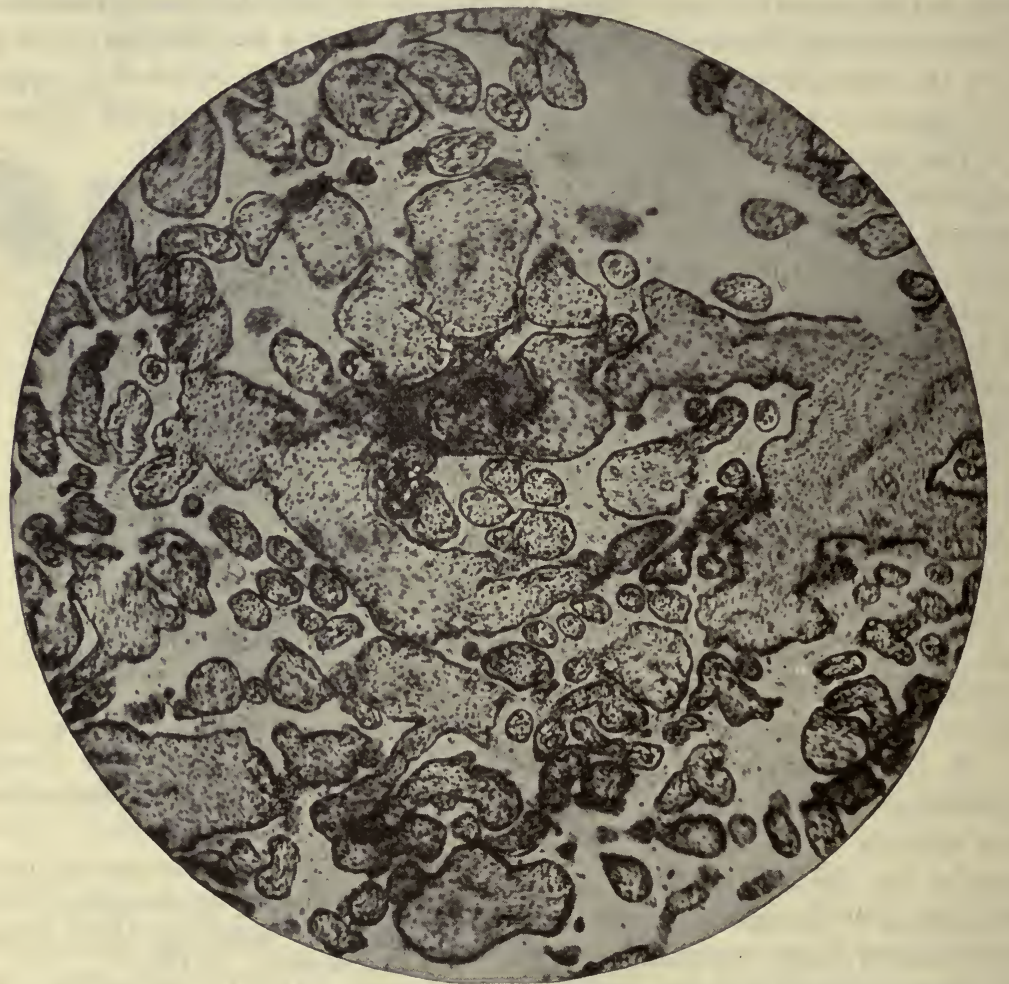

Fig. 470.-Chorionic Villi from the Human Placenta at Full Term. Hematein and eosin. Photo. $\times 114$.

reduced to a narrow crevice, which is so elongated during the dilatation of the uterine wall that the axis of the glandular remnant becomes nearly parallel to the surface of the decidua.

The decidual membrane which is thus formed is divisible into three portions, according to its relation to the tissues of the embryo: 1, that portion upon which the developing ovum directly rests, which is known as the decidua serotina or decidua basalis but later forms the placenta uterina or maternal portion of the placenta; 2 , at the margins of the 
implanted ovum the decidual tissues close up over the ovum which is thus surrounded by the so-called decidua reflexa or decidua capstlaris, which, after the early months of pregnancy, is gradually obliterated by the increasing growth of the fetus, and is finally replaced, its functions being progressively usurped by the newly formed placental tissues; 3 , all the remaining portions of the decidual mucosa, those which line the greater part of the uterine cavity, collectively form the decidua vera, with whose surface, in the later months of pregnancy, the fetal chorion is intimate in relation.

The mucosa of the cervix uteri meanwhile becomes greatly hyper-

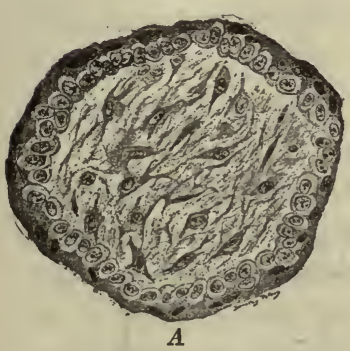

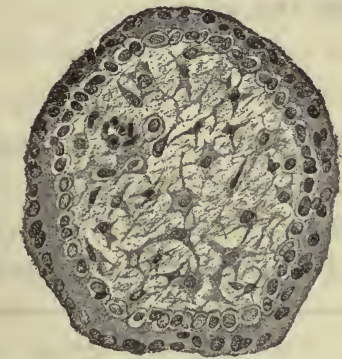

$B$

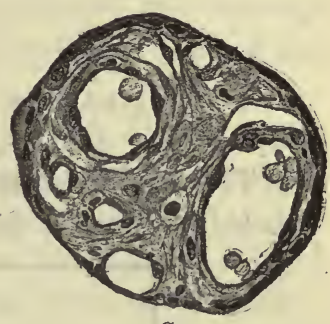

$C$

Fig. 471.-Chorionic Villus at Various Stages ó Development.

$A$, chorionic villus at third week; $B$, at fourth month; $C$, at term. (After Williams.) $\times 225$.

trophied and its glands much enlarged. This portion of the uterine mucosa does not, however, enter into the formation of the decidua vera; the changes occurring in its tissues, though similar, are much less pronounced.

The Chorionic Villi--These innumerable processes form the greater portion of the placental tissues. They vary in size from the broad main stems to the very slender terminal branches of the floating villi. They consist of a core of mesoderm covered with a variable layer of ectoderm. In the early condition of the placenta (fourth or fifth month of pregnancy) the villi are clothed with a double epithelial layer, of which the superficial takes the form of a syncytium (plasmoditrophoblast), while the deeper consists of a cellular layer, the cells of Langhans (cytotrophoblast). At later periods (seventh month to full term) the syncytium is found to have undergone a peculiar alteration, having become much thinner, and having even completely disappeared from considerable portions of the villi, it being replaced by canalized fibrin; at other points the syncytial cytoplasm is much thickened and the nuclei appear to be bunched or grouped within the thickened portions; these areas are known as cell-knots or proliferation islands. Here and there the degenerated cell-knots have been replaced by 
canalized fibrin. Wherever the main stems are inserted into the decidua the epithelium which formerly covered their tips appears to have also degenerated into a peculiar hyalin border zone. Towards the end of pregnancy the cytotrophoblast becomes converted into plasmoditrophoblast.

Within its syncytium the substance of the villus consists of the superficial cells of Langhans with their large ovoid nuclei, and a core of connective tissue of a delicate embryonic type, in which are the fetal bloodvessels. Even the smallest villi contain capillary loops of broad caliber, which are supplied by fetal arteries, derived from the umbilical arteries, which distribute their branches throughout the chorionic connective tissue. The fetal veins accompany the arteries.

\section{The Vagina}

The vagina is a fibromuscular sheath whose wall is divisible into three coats-mucous, muscular, and fibrous.

The mucous membrane is clothed by a layer of stratified squamous epithelium, and is thrown into numerous folds or rugæ. The epithelium

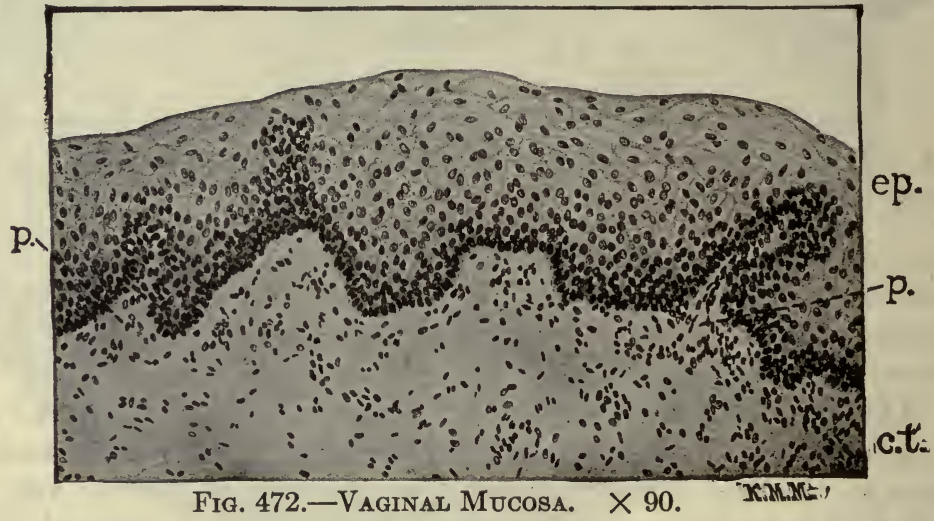

ep., epithelium; p., papilla; c.t., connective tissue. (After Williams.)

rests upon a fibrous basement membrane. The tunica propria is formed by a close-meshed areolar tissue which, in its deeper and looser portion, is permeated by vascular channels of considerable size. This deep vascular layer is frequently described as a submucosa; it rests directly upon the muscular wall. The surface of the mucosa presents numerous conical papillæ which project well into the epithelial layer.

The musculature of the vagina contains smooth or involuntary fibers, and is divisible into an inner circular and an outer longitudinal layer. The muscle fibers are long and slender. Considerable connective 
tissue is distributed among the muscle bundles. The latter are arranged in more or less parallel layers which are united by the delicate bands of connective tissue.

The outer fibrous coat consists of dense areolar tissue which is well supplied with elastic fibers. It loosely unites the vaginal wall to the surrounding tissues. In this coat is a plexus of blood-vessels and lymphatics, from which branches pass to the muscular coat, and to the mucosa, in which they form an abundant plexus. An extensive nerve plexus, including spinal and sympathetic fibers, among which are many small ganglia, is also found in the fibrous coat; it distributes motor branches to the muscular wall and to the blood-vessels, and sensory fibers to the mucosa, in which they end in relation with the cells of the lining epithelium.

The vaginal mucosa is reflected upon the outer wall of the cervix uteri, and at or near the external os it is continuous with the mucosa of the uterine cavity. Though occasional glands have been found in the vaginal mucous membrane, lined either by mucus-secreting or by ciliated cells, these glands would seem to be properly considered as anomalies, since they are usually absent, the mucoid secretions of the vaginal canal being chiefly provided by the abundant supply of mucus from the cervical glands of the uterus. The vaginal mucosa is continuous below with that of the vestibule.

\section{The Vestigial Structures}

The vestigial structures associated with the female reproductive system include the vesicular appendage (hydatid of Morgagni), the epoöphoron, and the paroöphoron. The same general statements made concerning the male vestigial structures hold likewise for those of the female.

The VESICULAR APPENDAGE is attached to the fimbriated end of the oviduct, its stalk being continuous with the collecting duct of the epoöphoron. It is a globular pedunculated structure of small size (three to six millimeters diameter); it is lined with cuboidal epithelium, and may contain fluid. It represents the atrophic end of the degenerated Wolffian duct. There are besides a variable number of smaller accessory vesicular appendages attached to the broad ligament.

The EPOöPHORON (parovarium; organ of Rosenmüller) lies between the layers of the broad ligament in the triangular area between the ovary and the ampulla of the oviduct. It consists of a variable number of tubules (eighteen to twenty), the homologues of the ductuli efferentes of the male. These tubules may be blind at only one or at both ends; they may be lined with ciliated columnar epithelium, or their lumina may become obliterated. 
Those blind only at one end connect with a longitudinal duct, a variable remnant of the Wolffian duct.

The PAROÖPHORON-homologue of the male paradidymis-is a more mesial collection of similar tubules of like structure and genetic significance. It is said to be present only in infants. The further extension mesially of the longitudinal duct, either in continuation with the collecting duct of the epoöphoron or paroöphoron, or as an isolated blind duct, usually in the wall of the uterus and vagina, is known as the canal of Gärtner. It is the vestige of the lower portion of the fetal Wolffian duct.

\section{The External Genitals}

The vestibule is supplied with a mucosa which offers a gradual transition from the vagina, on the one hand, to the skin on the other. Its

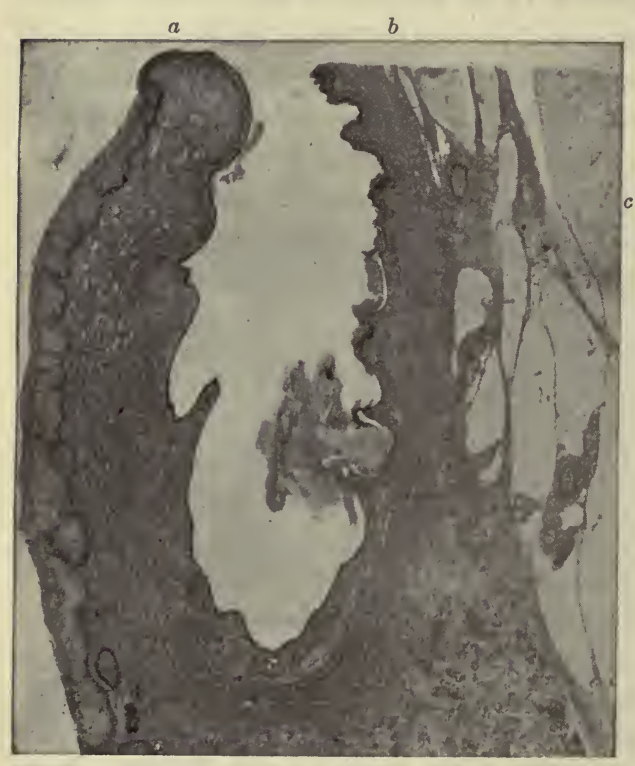

Fig. 473.-Transection of a Labium Minus of AN INFANT.

$a$, labium minus; $b$, border of the labium majus; $c$, adipose tissue of the latter. Hematein and eosin. Photo. $\times 12$. stratified squamous epithelium becomes in this way gradually more and more like that of the skin, eleidin granules first, and keratin later appearing on the outer surface of the labia minora. The epithelium of the labia. majora is identical with that of the skin.

The labia minora or nymphæ form the lateral walls of the vestibule and consist of a fold of the mucosa which is provided with exceptionally tall papillæ. Small sebaceous glands open directly upon the surface of the stratified squamous epithelium. There are no hair follicles in relation with these glands, and the labia minora centain no adipose tissue. They are richly supplied with blood-vessels, and with sensory nerve endings.

The labia majora are formed by similar folds whose inner surface resembles the adjacent portion of the labia minora, but whose outer 
surface is cutaneous and is supplied with sebaceous and sudoriparous glands and with numerous hair follicles. The subepithelial areolar tissue is very dense and its deeper portion contains much fat.

The clitoris consists of a mass of erectile tissue, homologous with the corpora cavernosa and glans penis of the male; it is covered by a fold of the mucosa. It is well supplied with nerves, which terminate in tactile corpuscles, end bulbs, and genital corpuscles. In this vicinity also, as well as in the region of the labia, Pacinian corpuscles are occasionally found.

The hymen is formed by a reduplication of the vestibular mucosa. Its inner surface is similar to that of the labia minora and vagina; its outer is like that of the cutaneous surface, except that it contains no hair follicles.

The glandulæ vestibulares minores are a group of small mucussecreting glands, similar in structure to the urethral glands of Littré in the male, which occur in the vestibular mucosa in the vicinity of the meatus urethræ.

The glandulæ vestibulares majores (glands of Bartholin) form a paired tubulo-alveolar mucus-secreting gland which opens by a narrow duct into the groove between the hymen and labium minus. The tubular alveoli are lined by columnar mucus-secreting cells; the ducts are clothed with columnar epithelium, which, as they approach their termination, becomes double-rowed, and finally changes to a stratified squamous epithelium similar to that of the surface upon which they open. These ducts frequently present saccular dilatations.

\section{The Mammary Glands}

From a strictly histogenic standpoint the mammary glands should be considered as appendages of the skin, and as such should more properly have been considered in the chapter devoted to that subject. Yet these glands are so closely related to the reproductive functions, attaining their full development only in the lactating female, that it seems equally proper to consider them at this time as accessory reproductive organs.

The mammary glands may be regarded as modified sweat glands. Though producing a fatty secretion they show no resemblance to sebaceous glands. The mammæ undergo the same slight but progressive development in both sexes until the time of puberty when they suffer regressive changes in the male, persisting thereafter only in rudimentary condition. In the female they continue to grow, but become functionally 
active only in the event of pregnancy. Upon the hemispherical corpus mamma can be distinguished the central raised nipple or mammilla, and the surrounding roughened and pigmented circular area, the areola.

Each mammary gland consists of fifteen to twenty lobes, each of which is of itself a branched saccular gland whose lactiferous duct opens

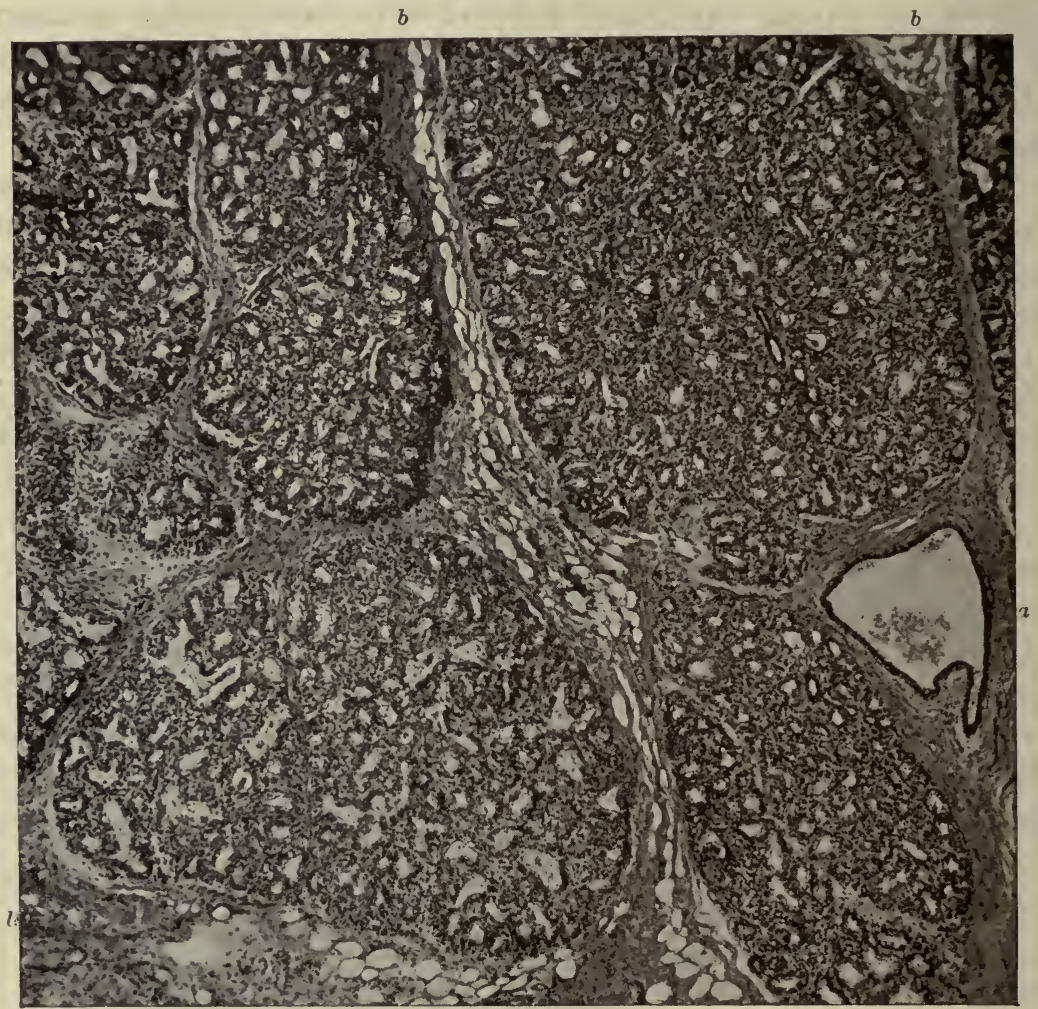

Fig. 474.-From the Actively Secreting Mammary Gland of a Woman.

Several lobules are included. $a$, interlobular duct; $b$, interlobular connective tissue. Hematein and eosin. Photo. $\times 52$.

on the surface of the nipple near its apex. The main lactiferous or lobar ducts subdivide in an arborescent manner into many interlobular ducts, about which are clustered the groups of secreting alveoli, each group forming one of the many lobules included in a lobe of the gland. The structure of the lobule, as well as the general appearance of microscopical sections of the gland, varies according to the stage of development and the condition of activity of the organ. 
The Active Gland.-During lactation the glandular alveoli are so numerous as to form by far the most prominent portion of the gland. Each lobule consists of a cluster of saccular alveoli which open by short alveolar ducts into the interlobular ducts of the connective tissue which invests the lobules of the gland. The alveoli are closely packed within the lobule.

The actively secreting alveoli are lined by cuboidal or low columnar cells which vary much in height even within the same alveolus, and are often considerably flattened. Fat droplets accumulate within the distal portion of their cytoplasm. The droplets increase, in size as well as in number, until they finally occupy the greater part of the distal end of the cell and are separated from each other by only a narrow interval of albuminous cytoplasm. Finally the fat droplets are discharged into the broad lumen of the alveolus, where they apparently still retain a thin albuminous envelope which prevents their cohesion and consequent fusion, and thus permits their suspension in the albuminous, fluid portion of the milk. The milk may include also cytoplasmic and nuclear débris.

The spherical nuclei of the secreting cells during this process are crowd-

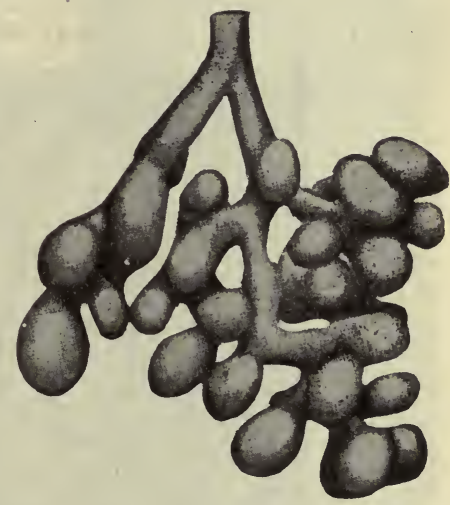

Fig. 475.-MODEL OF A ReCONSTRUCTION OF AN INTRALOBULAR DUCT AND ITS ACINI From - THe ACtive Mammary GLAND of a Woman.

$\times 200$. (After Maziarski.) ed to the base of the cell, and after the discharge of the secretion the shrunken but nucleated cell remnants remain in situ; after a period of rest the cells apparently resume their secretory function. It appears probable that each cell in its life history may repeatedly pass through the cycle of secretory changes, though the exact number of such cycles which an individual cell may present obviously does not admit of demonstration.

As a rule, the active epithelium consists of a single row of cells, though here and there they appear as if piled upon one another to form a double layer. During pregnancy many of these cells may be seen in mitosis. The actively secreting cells contain basal (ergastoplasmic) filaments. According to Hoven (Anat. Anz., 39, 1911) these filaments break up into granules from which minute fat spherules develop. The 
epithelium rests upon a reticular or homogeneous basement membrane, within which are occasional basket cells. 'These have been interpreted as smooth muscle cells, similar to those of the secretory portions of the sweat glands. The alveoli of the active gland are so closely packed that a connective tissue tunica propria is no more than scarcely demonstrable. The thin tunica propria is, however, richly supplied with blood capillaries, lymphatic vessels, and nerve fibers.

The ducts of the mammary gland are lined by either a single or double row of low columnar cells. They possess a relatively broad lumen. Their membrana propria is supporțed by a thin connective tissue wall,

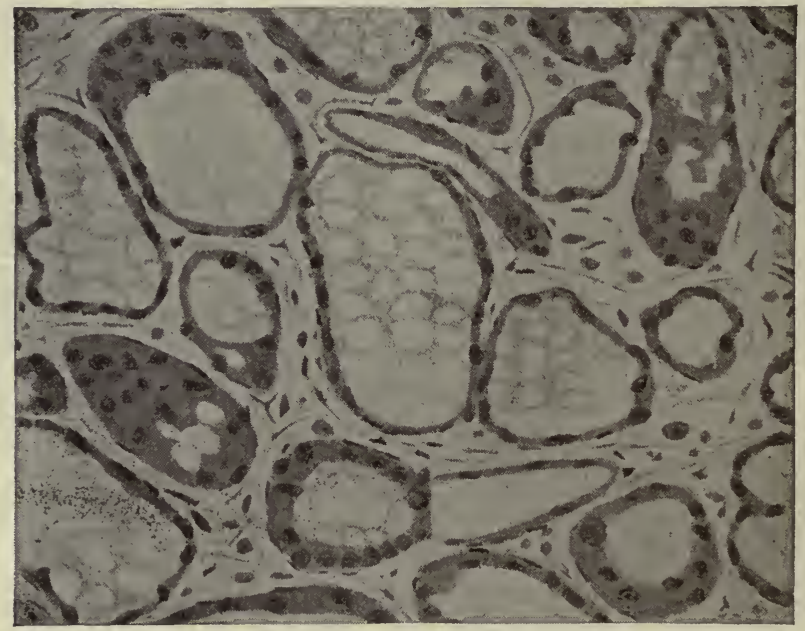

Fig. 476.-Active Mammary Gland of Rabirt (22 Days After Fecundation.) The alveoli are filled with milk containing fat droplets. $\times 416$. (After L. Schil.)

The alveoli are filled with a stainable milk coagulum in which are many fat spherules.

containing both circular and longitudinal elastic fibers but no muscle. The elastic fibers of the smaller ducts are poorly developed, but in suitable specimens the longitudinal fibers are readily seen even in very small branches. Beyond the lactiferous sinus the duct epithelium changes to a stratified squamous variety which is continuous with that of the cutaneous surface of the nipple.

The glandular lobules are firmly united by strong septa derived from the dense areolar tissue in which they are embedded. In the deeper parts of the gland occasional lobules of fat are found in this tissue. Within the nipple and beneath the adjacent portions of the areola, smooth 
muscle fibers are also found. These are arranged in circular bundles at the base of the nipple, with longitudinal fibers within its substance which, at the base of the mammilla, diverge in radiating bundles into the subcutaneous tissue of the areolar zone. Contraction of these fibers elevates and hardens the nipple, thus simulating the action of the erectile tissues.

According to Liperovsky (Anat. Anz., $45,20,1914)$ elastic fibers are more abundant than was formerly recognized in the walls of the alveoli and in the interalveolar connective tissue, frequently in intimate association with smooth muscle cells. Such 'elastico-muscular apparatus' is well developed in the peripheral portion of the glands, in the subpapillary layer of the skin, and especially in the vicinity of the nipple. The elastic fibers, which are superficially distributed, appear embedded at their deeper ends in the muscle fibers. This peculiar arrangement of elastic fibers in relation to smooth muscle probably aids in the expulsion of the secretion.

Embedded in the subcutaneous tissue of the areola are also a number of small accessory lactiferous glands known as the Glands of Montgomery (Areolar Glands of Duval). The nipple and areola contain also abundant sebaceous glands; and sweat glands are present in the periphery of the areola.

The Resting Gland.-With the cessation of lactation the glandular alveoli undergo a rapid atrophy, and are replaced by connective tissue derived from the interlobular stroma. 'The ducts contract and the epithelium piles up to form a two-

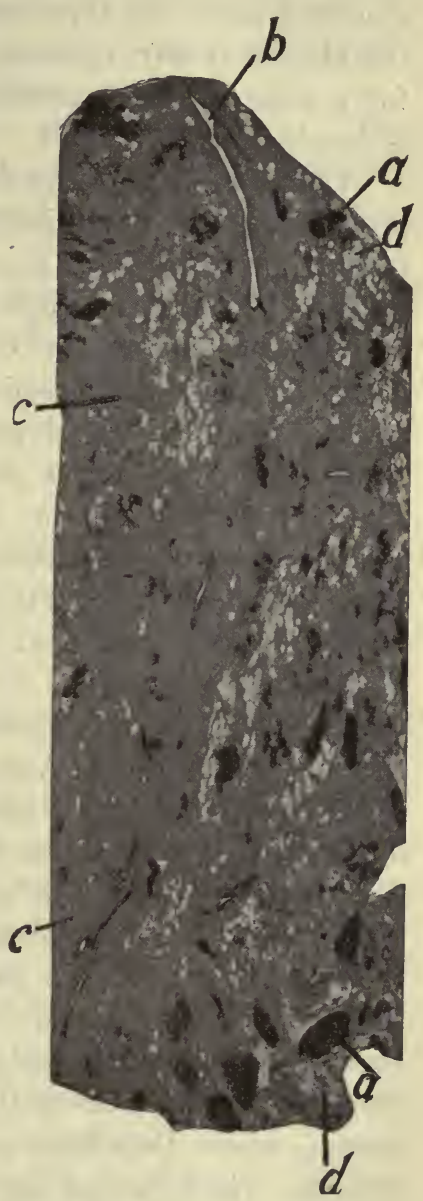

Fig. 477.-From a Section of THE HUMAN MamMary Gland in the Resting Condition.

$a$, remnants of the glandular alveoli; $b$, duct; $c$, connective tissue; $d$, adipose tissue. Hematein and eosin. Photo. $\times 10$. rowed, or even thicker, layer. The alveoli are reduced to mere buds from the terminal ducts, and their lumen is almost obliterated; their 
epithelium is similarly massed into a double layer of small cells. The lobules are reduced in size and consist only of a few shrunken alveoli clustered about the termination of an interlobular duct. The lumen of the alveoli, if any, contains no secretion, and that of the ducts, except for a little granular albuminous material and an occasional leukocyte, is empty.

The connective tissue stroma is much increased in volume, and in places shows a marked infiltration with fat. The alveolar tissue of the mammary gland at all times contains wandering leukocytes, and many granule cells, both acidophil and basophil in character.

With the appearance of pregnancy the gland promptly reënters a state of activity; its alveoli multiply; its connective tissue becomes relatively diminished in volume; its lobules are reformed and their alveoli finally begin secretion, a process which is heralded by the formation of a granulo-fatty colostrum, a rather serous fluid in which are suspended large numbers of colostrum corpuscles, large spheroidal cells, resembling leukocytes in their general form and in the character of their nuclei, but which possess a broad rim of cytoplasm often containing numbers of fat globules of varying size. Their cytoplasm has also been shown to contain neutrophil granules of Ehrlich similar to those of the polymorphonuclear leukocytes (Michaelis, Arch. mikr. Anat., 1898).

Colostrum discharge precedes and follows the period of lactation for a few days; it appears also in both sexes for several days after birth when it is commonly known as 'witch's milk.'

The origin of the colostrum corpuscles is still somewhat in doubt; though modern technic has gradually discredited the theory of their origin from desquamated remnants of the alveolar epithelium, and shows them to be more probably enlarged leukocytes which have wandered through the alveolar wall and have thus found their way into the lumen, where they take on a phagocytic activity and continue their growth. The following facts may be mentioned in support of this theory: $a$, leukocytes can be readily found between the cells of the alveolar epithelium as well as in the lumina of the saccules; $b$, the colostrum corpuscles examined in a fresh condition on a warmed slide have been repeatedly shown to possess the property of ameboid motion; $c$, the colostrum corpuscles, when stained, present the same granular and nongranular varieties as do the leukocytes of the blood; $d$, finally, the colostrum corpuscles have been shown to undergo mitotic cell division (Bizzozero and Ottolenghi), a phenomenon which we should hardly expect to find in degenerated and desquamated epithelial cell remnants. 
The blood-vessels of the mammary gland are specially abundant. They form rich capillary plexuses about the walls of the active alveoli. Many of the venules coming from these plexuses converge toward the areola, where they form an incomplete venous circle (circulus venosus of Haller) from which the efferent veins take their origin.

The lymphatics of the mammary gland are also numerous. 'They take origin from broad channels among the alveoli and enter a rich plexus about the interlobular ducts. From here several vessels pass to the lymph nodes of the axilla.

The nerves of the mammary gland include both spinal (sensory) and sympathetic fibers. The latter are distributed to the vascular walls, to the smooth muscle of the areola and nipple and to the alveolar epithelium. The sensory fibers supply the connective tissue of the nipple and areola where they occasionally terminate in tactile and Pacinian corpuscles.

Among the secreting alveoli the nerve fibers form an epilemmal plexus beneath the membrana propria, from which fibrils penetrate between the epithelial cells, upon which they end in minute granular varicosities (Arnstein, Anat. Anz., 1895).

Milk.-Milk, secreted by the active mammary gland, consists of an emulsion, in which fat droplets, varying in size from two to twenty microns or more, are suspended in a watery albuminous fluid. Water constitutes about eighty-six per cent. of the secretion; the protein constituent (three per cent.), which is largely nuclein, is derived in part from degenerating nuclei. Milk contains also a small amount of sugar (five per cent.) and a trace of salt. Each fat droplet is presumably invested with a thin coat of casein, derived from the cytoplasm of the secreting epithelium. Occasionally leukocytes occur in the milk, but never in large numbers, and like the similar colostrum corpuscles, they are mostly confined to the earlier periods of lactation. 


\section{CHAPTER XVI}

\section{THE DUCTLESS GLANDS-ENDOCRIN GLANDS}

(Organs of Internal Secretion)

Under this heading it will be convenient to consider the suprarenal, thyroid, parathyroid, thymus, carotid, and coccygeal glands, the hypophysis cerebri, the epiphysis cerebri and the paraganglia. This group of organs properly includes also the pancreatic islets, the interstitial cells of the testis and the ovary, and the corpora lutea already described.

\section{THE SUPRARENAL GLANDS}

The suprarenaij glands (bodies or capsules; also called adrenals) are two flattened irregular glandular masses situated close to the cranial extremity of each kidney, but without organic or genetic relationship with the renal system. On section the adrenal is seen to be readily divisible into a bright yellow or brownish-yellow cortex and a more vascular, and hence darker and somewhat reddish, medulla, whose central portion transmits several large veins which make their exit from an indentation in the anterior surface of the organ, known as the hilum.

Development and Function.-The suprarenal gland is a composite structure formed by the intimate association of two embryologically distinct anlages: one, the cortical component, of mesodermal, the other or medullary component, of ectodermal origin. The cortical component arises as a series of buds from the celomic epithelium covering the medial upper surface of the cephalic third of the Wolffian body; the medulla develops from cells which have migrated from the abdominal sympathetic plexus (celiac plexus), and which elaborate peculiar granules having an affinity for chromium, hence called chromaffin (or pheochrome) cells. The ontogenetic process recapitulates almost precisely the phylogenetic history of the suprarenals: In fishes the homologues of the two mammalian components remain separate, and the cortical representative includes a series of bodies, the interrenal bodies or organs; the medullary representative, 
also comprising a series of structures, is known throughout, due to its close topographical relationship to the kidney, as the adrenal. The cortex of the mammalian suprarenal gland thus represents the product of fusion of the ichthyoid interrenals. In the groups between fishes and mammals, the association of interrenals (cortical component) and adrenals (medullary component) becomes progressively more intimate.

The suprarenal is absolutely essential to life; removal promptly results in death. According to Crile (1914)/the brain is intimately dependent upon the suprarenals; when both glands are excised in the rabbit death follows in eighteen hours, the brain cells meanwhile exhibiting loss of chromophilic substance. The two portions are believed to have, in part at least, a different function, both inhering however in an internal secretion. The cortex is generally believed to elaborate an antitoxic secretion for neutralization of harmful products of destructive metabolism. The function of the medulla is dependent upon the adrenalin (adrenin; epinephrin) of the pheochrome granules, probably having to do with maintaining the proper tonus of the muscle of the heart and blood-vessels, thus underlying blood pressure. Minute amounts of epinephrin in the blood effect a sensitization of the vasoconstrictor nerve endings so that the efferent impulses discharged cause the muscular coats of arterioles to contract vigorously, the result being an increase in blood pressure. The most conspicuous diseases of the suprarenals involve hypersecretion, perhaps inducing to arteriosclerosis; and hyposecretion, frequently the result of tuberculous lesions, producing a clinical complex known as Addison's disease. (Reference should be made to Vincent's "Internal Secretions and the Ductless Glands," Longmans, 1912.)

The organ is enclosed by a connective tissue capsule of considerable thickness. 'From the inner surface of the capsule delicate fibrous trabeculæ pass inward and subdivide the epithelial parenchyma of the organ into cell groups and columns, which vary in their appearance according to the distribution of the connective tissue trabeculæ. The suprarenal parenchyma is exceptionally prone to post mortem changes. In the medulla the connective tissue presents an irregular areolar arrangement; the more regular, though varying form of the areolæ in the cortex, subdivides this portion of the organ into three more or less distinct layers, which were first described by Arnold (Arch. f. path. Anat., 1866) as the zona glomerulosa, zona fasciculata, and zona reticularis.

In the zona glomerulosa the connective tissue trabeculæ subdivide the epithelium into spheroidal groups of cells, many of which are continuous with the cell columns of the adjacent-zona-fasciculata. The glomerulate layer is relatively thin and lies close beneath the capsule. 
The stroma of the zona fasciculata is continued inward from the glomerulosa, but is so drawn out as to form elongated areolæ, inclosing

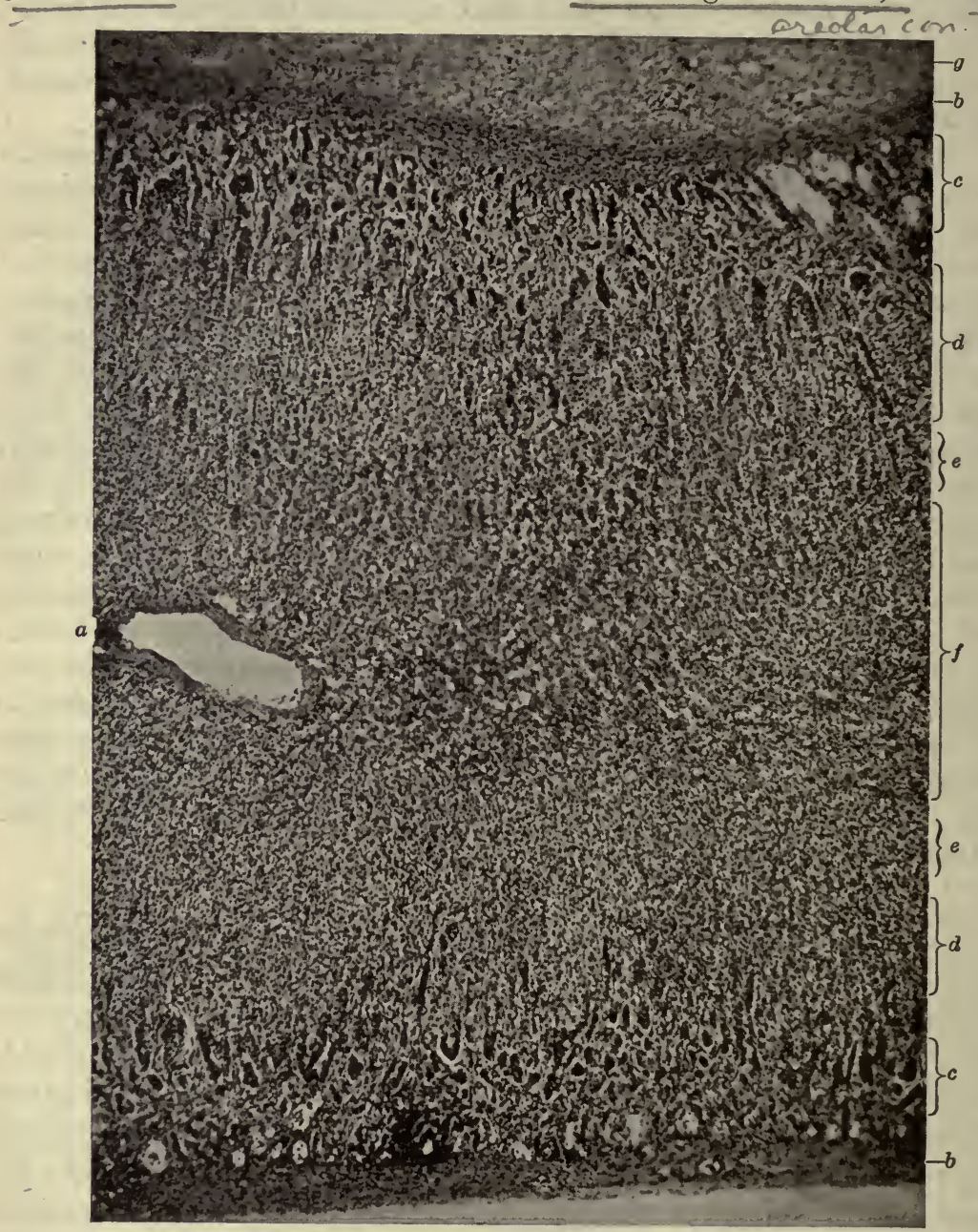

Fig. 478.-From a Section through the Human Adrenal.

$a$, central vein; $b$, capsule; $c$, zona glomerulosa; $d$, zona fasciculata; $e$, zona reticularis; $f$, medulla; $g$, peri-adrenal adipose and areolar tissue. Hematein and eosin. Photo. $\times 45$.

cell columns of considerable length, which are disposed in a radial manner. This is the broadest of the three cortical zones and is interposed between the glomerulosa and reticularis. 
At the inner border of the zona fasciculata the connective tissue bundles pass insensibly from the regular columnar arrangement of this layer into a reticular maze. The resulting cell groups are of very irregular form and compose the innermost cortical layer, the zone reticularis. This layer is the thinnest and least distinct of the three zones of the cortex. It can often be more readily distinguished by the highly pigmented condition of its cells, than by the mere form of its cell columns. In man it passes almost insensibly into the medulla; in many animalse.g., the dog, cat, and pig-there is a sharp demarcation between the zona reticularis and the medulla, produced by a thin membranous layer of connective tíssue which apparently results from the fusion of the central ends of the fibrous bands in the

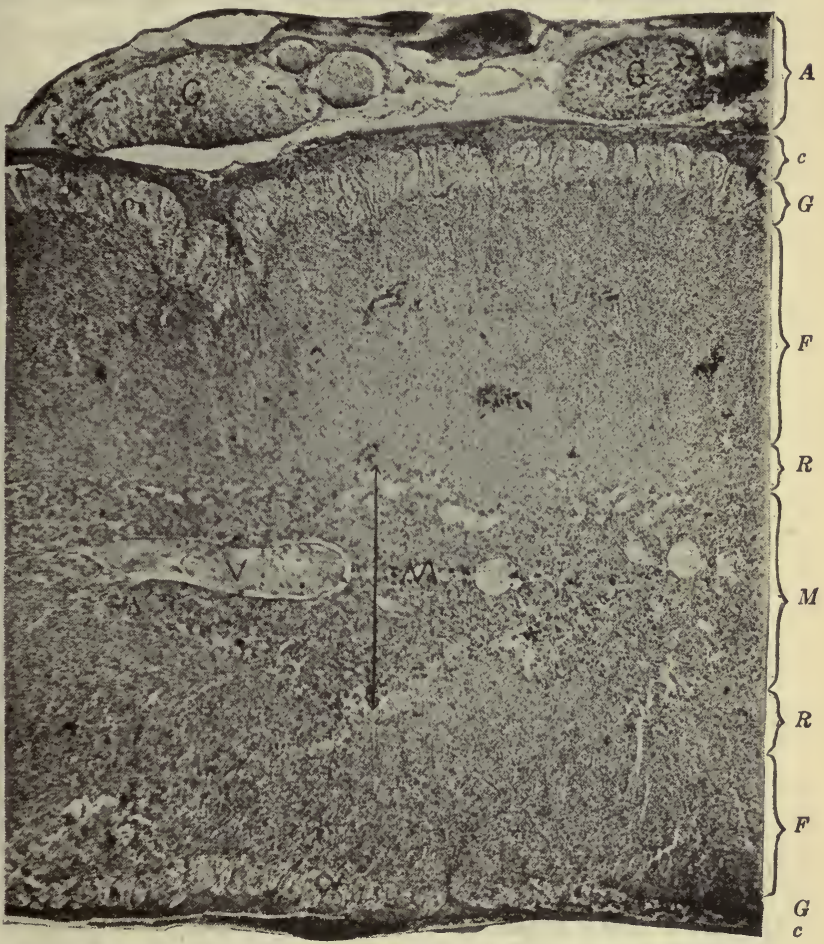

Fig. 479.-Photomicrograph of Suprarenal Gland of Dog. Magnification $\times 30$.

$A$, loose areolar connective tissue of outer portion of capsule containing two large $(G)$ and several small ganglia; $c$, capsule proper; $g$, zona glomerulosa; $F$, zona fasciculata; $R$, zona reticularis of cortex; $M$, medulla, showing the large central vein $(V)$.

cortical stroma. Such a membranous septum is usually wanting in the human adrenal.

The connective tissue stroma of the adrenal consists of a delicate vascular network, which in the cortex contains very few if any elastic fibers. Flint (1900) has shown that this connective tissue is, in large part, at least, a reticular tissue. The capsule consists of dense bundles 
of white fibrous tissue among which are many elastic fibers. The stroma of the medulla is also richly-supplied with elastie tissue.

The epithelium of the zona glomerulosa is arranged in spheroidal groups or in hooked or slightly coiled columns which are continuous with the straight columns of the fascicular zone. The cells of the zona glomerulosa are closely packed within the connective tissue meshes and

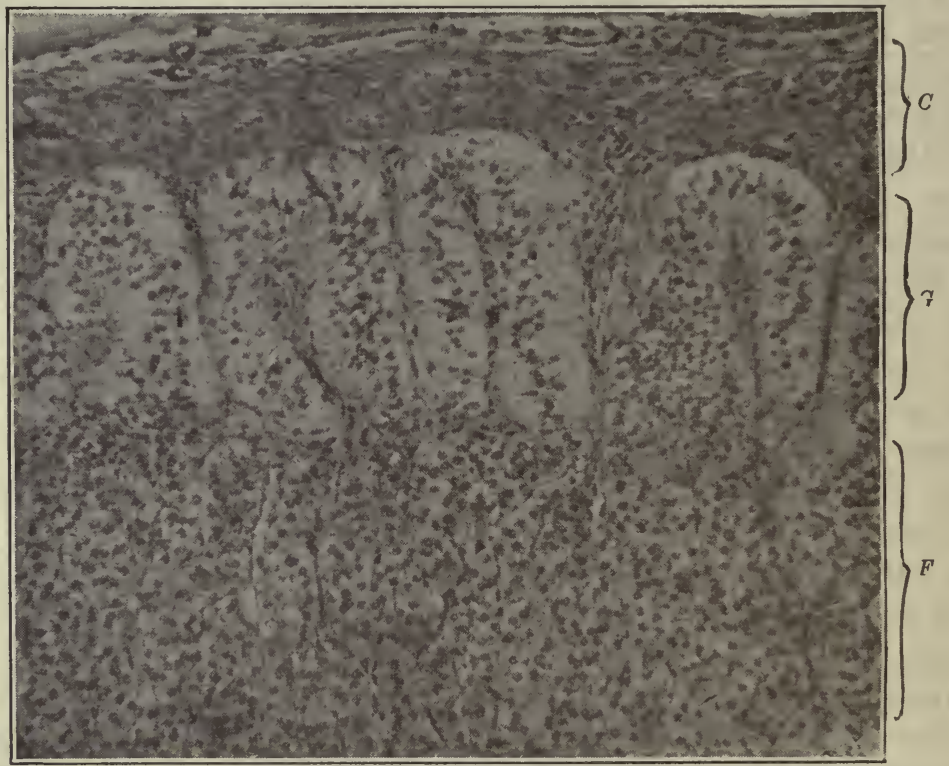

Fig. 480.-More Highly Magnified Region of the Preceding Section, to Show the Capsule $(C)$, Zona Glomerulosa $(G)$, and a Portion of the Zona Fasciculata $(F)$.

the cell outlines are very indistinct. Wherever their outlines can be readily distinguished the cells are seen to be of columnar shape and are arranged in slender columns whose cells are often grouped about an indistinct central lumen. The cytoplasm of the cells of this zone is finely granular and stains readily with acid dyes. Occasional minute fat droplets appear in the innermost cells of the group, but these are never so abundant as in the more internal portions of the cortex. The nuclei in this zone are spheroidal in shape and rich in chromatin; they present frequent mitoses (Canalis, 187\%), but these are more abundant in early life than in the adult.

The cells of the zona fasciculata are highly characteristic. They 
are arranged in long straight columns which extend from the zona glomerulosa inward to the zona reticularis. The cells are columnar or polyhedral in shape; many of them contain minute fatty droplets in great abundance. This fat is readily blackened by osmic acid. Arnold (1902), by extraction with ether, obtained crystals of palmatin and stearin from the suprarenal gland. Plechik (1902), however, considers that the adrenal fat differs in its ultinate chemical properties from the other fat of the body. Each columnar group consists of cells which are, as a rule, in approximately the same stage of fatty metamorphosis, and the cell columns of this zone may be divided into those which are distinctly acidophil and those which are distinctly fatty, though between these extremes there are many intermediate stages.

The acidophil cells are ovoid or polyhedral elements which possess one or two highly chromatic spheroidal nuclei and a finely granular cytoplasm. On careful examination with high magnification, extremely minute fat droplets may often be demonstrated even in the most characteristic of these cells; with loper magnification these are frequently invisible.

The fatty cells possess a spheroidal nucleus which is usually vesicular in character; occasionally it is highly chromatic. Frequently the apparent chromatolysis seems to progress in exact ratio to the accumulation of fat; those cells in which the fatty metamorphosis is more advanced present the more typically vesicular nucleus. With the progress of the fatty metamorphosis the cell outlines are again lost and the granular acidophil cytoplasm gradually replaced. The presence of fat in the broad zona fasciculata is partially responsible for the bright yellow color of the cortex of the organ.

The cells of the zona reticularis are similar to those of the zona fasciculata, though the fatty metamorphosis is less pronounced. In one particular, however, the cells of this layer are remarkable. They contain an abundance of a peculiar brownish-yellow pigment which occurs both in the form of coarse granules and as a diffuse coloration of the cytoplasm. The spherical nuclei, highly chromatic or only slightly vesicular in character, are not invaded by the pigmentation. The volume of pigment varies greatly in different individuals; it is usually absent in young persons, but is, as a rule, present after the twentieth year of life (Maass, 1889). In the suprarenal of the mouse many of these cells can be seen ip process of amitotic division.

The epithelial cells of the medulla are avoid elements with one or two spherical nuclei, which in many cases possess a vesicular char- 
acter; in other cells they consist of a dense, almost solid, mass of chromatin. The shape of the cell groups in the medulla varies greatly; usually they form small spheroidal masses or short columns. The cells are frequently arranged in a more or less tubular form but without a distinct Jumen. Frequently they surround a minute capillary vessel. The medullary cells presumably pour their secretion into the bloodvessels, whose broad sinusoidal capillaries permeate the delicate connective tissue bands which inclose the cell groups. Felicine (Arch. f. mikr. Anat., 1904) claims to have demonstrated the presence of minute intraand intercellular secretory canaliculi which open directly or indirectly through broader lacunce, into the blood-vessels.

The cell groups of the medulla, like those of the cortex, are divisible into the acidophil and the fatty types; the former are the more abundant, but the fatty metamorphosis is scarcely ever so advanced as in the cortex. There is, however, great variation in the size of the medullary cells. The larger ovoid elements form the typical groups; between these groups are narrow cell columns consisting of much smaller and less highly acidophil cells, which are arranged in slender columns and scattered irregular masses.

The striking feature of the medullary cells is their granular content. These chromaffin granules have a special affinity for chromic acid and its salts, and stain a light brown of yellow. Their staining capacity in chromium solutions is due to the presence of adrenalin (Kingsbury, Anat. Rec., 5, 1, 1911). The granules are very readily soluble in acids.

In the vicinity of the central veins, small nerve trunks are found, and occasional minute ganglia or isolated nerve cells occur along their course. These are not to be confused with the large ovoid epithelial cells of the medulla.

Blood Supply.-The arteries which supply the suprarenal glands form a plexus of vessels in the capsule of the organ and in the neighboring connective tissue. Some of the smaller branches of this plexus, the capsular arteries, supply the capsule itself, others enter the organ and are distributed to the cortex and to the medulla. The blood supplied to the capsular arteries, after traversing the capillaries, enters small venules which are tributary to the lumbar and phrenic veins. The course of the cortical and medullary vessels has been exhaustively studied by Flint (Proc. Bost. Soc. of Nat. Hist., 1900).

The cortical arteries enter the zona glomerulosa where they abruptly break up to form a capillary plexus which occupies the connective tissue between the cell columns. Capillary vessels are continued from this 
plexus through the intercellular reticulum of the zona faseiculata, where they are in intimate relation with the epithelial cells, and reach the zona reticularis. Here the capillaries are collected into thin-walled venules or sinusoids. These vessels, after some anastomoses, form venous stems which are continued, without further anastomosis, through the medulla to the central veins. The venules of the cortex possess no walls other than their endothelium.

The medullary arteries are also derived from the capsular plexus. They penetrate the cortex, and at the border of the medulla abruptly

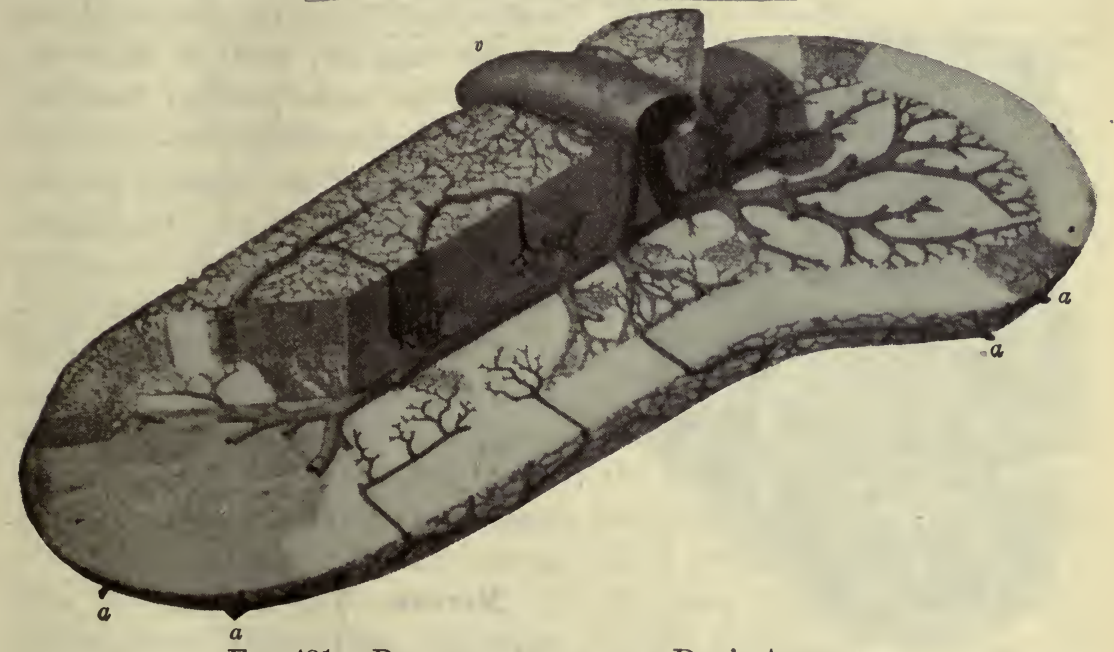

Fig. 481.-Reconstruction of a Dog's Adrenal.

$a$, arteries; $v$, vein. $\times 25$. (After Flint.)

terminate in a plexus of capillary vessels which lie in the connective tissue stroma and come into intimate relation with the medullary cells. These vessels possess extremely thin walls, their endothelium often being in direct contact with the adjacent epithelium, whose cells frequently impinge upon the lumen of the capillary vessel (see Fig. 481). The capillary plexus pervades the entire medulla, its vessels being here and there collected into small venules which unite to form the central veins. These form two, or sometimes four, main stems (Flint) which make their exit at the hilum and enter the lumbar or renal vein, or, on the right side, enter the inferior vena cava.

All of the efferent veins of the adrenal are characterized by a peculiar distribution of their smooth muscle fibers, which occur in considerable abundance, but are nearly all disposed in the axis of the vessel; the 
circular muscle fibers are confined to a very thin coat beneath the endothelium, or are often entirely absent. Frequently, and especially in the central veins of the adrenal, the coarse bundles of longitudinal muscle fibers project into the lumen of the vessel in a somewhat rugose manner. Whenever two veins unite to form a larger vessel, and at the junction of a central vein with any of its branches, these protuberant. muscular bundles are especially prominent. Moreover, Ferguson (Amer. Jour. Anat., 5, 1, 1905) describes anomalous vessels of a venous nature which

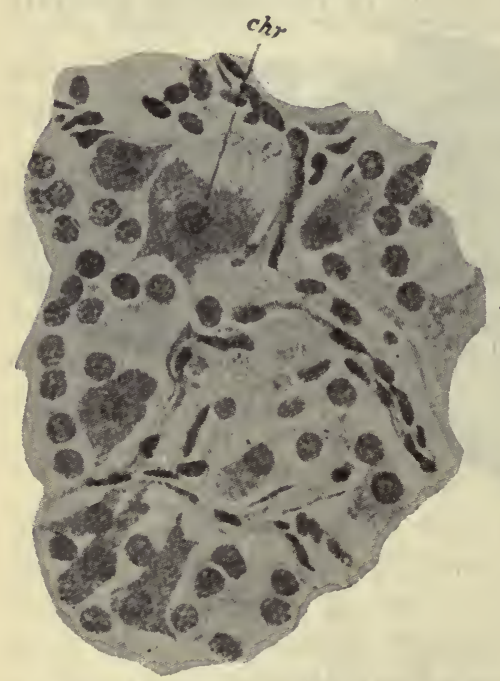

Fig. 482.-Section of Part of an AcCESSORY SUPRARENAL (ChromoPHill BOdY), NEW-BORN CHILD.

chr, a chromophil cell. (Schäfer, "Quain's Anatomy.") arise in the medulla, penetrate the cortex, and enter the venous plexus of the capsule; and in these instances the same peculiar distribution of the muscle has been observed in the veins of the capsular plexus.

Lymphatics.-The lymphatics of the suprarenal gland, according to Stilling (1887), form rich plexuses in the zona glomerulosa and in the medulla; elsewhere they are less abundant. They follow the course of the blood-vessels and are especially well developed in the vicinity of the central veins.

Nerves.-The adrenal is well supplied with small sympathetic nerve trunks from the solar plexus. They form a plexus in the capsule from which branches are distributed to the cortex and to the medulla. In the cortex they invest the bloodvessels with a delicate plexus, but have not been found within the epithelial cell columns. In the medulla they are also distributed to the bloodvessels and are supplied with occasional small ganglia. Passing from the plexus of sympathetic nerve fibers which invests the groups of medullary epithelium, Dogiel (1894) demonstrated delicate fibrils, supplied with minute varicosities, which penetrate between the epithelial cells and terminate in a manner very similar to that which is characteristic of the epithelial parenchyma of other secreting glands.

Accessory Suprarenals.-These include bodies of three types of structure: (1) bodies consisting exclusively of cortical substance; (2) 
broken into minute spherules. In general, the ratio of colloid content within the follicle, roughly stated, is in proportion to the age of the individual. The follicles at the periphery of the lobes of the gland are less fully distended than those in the interior.

Embedded in the colloid mass within the follicle, even in the apparently normal thyroid, red blood corpuscles and desquamated follicular epithelium are frequently found, but never in large quantity. Leukocytes are of less frequent occurrence and are more rarely found in the human thyroid than in that of the lower mammals.

The FOLLICULAR EPITHELIUM is typically cuboidal in shape; in young individuals it is somewhat taller than broad. In those follicles which are distended with colloid secretion the epithelium is relatively short; in those which are empty it is taller. Each cell contains a single spheroidal nucleus which lies in the center of the cell, or somewhat toward its basal extremity. This orderly disposition causes the nuclei, when seen in sections of the follicle, to appear as a continuous row in the wall of the alveolus, a disposition which is noticeable for its exceptional regularity.

The cytoplasm of the epithelium is finely granular and decidedly acidophilic. It usually contains some coarse granules and very small fatty droplets, which generally occupy the extremities of the cells. Minute spheroidal granules which give the color reactions of colloid are also found in the cytoplasm of the epithelial cells. Hürthle (Arch. f. d. ges. Physiol., 1894), by staining with the Biondi-Ehrlich mixture, succeeded in differentiating two types of cell, one lightly staining, the 'chief cells,' the other a darker colloid-containing type which he designated as 'colloid cells.' These variations probably only represent different stages of secretion in the same epithelial cell type. Minute intercellular canaliculi occur at the angles between adjacent cells.

In the thyroid of the opossum Bensley (Anat. Rec., 8, 9, 1914) also describes two types of cells, namely, the usual epithelial cells and ovoid cells. The latter hold a parietal position in the follicle; they are filled with fine eosinophilic granules which give to these cells a character strikingly similar to that of the acidophil cells of the anterior lobe of the hypophysis cerebri. He describes also large needle-shaped crystalloid bodies in the epithelial cells similar to those in the interstitial and Sertoli cells of the testis.

The epithelium rests upon a very delicate reticular basement membrane and is in close relation with the capillaries and lymphatic vessels 
of the interfollicular stroma. Colloid material, similar to that within the follicles, has been repeatedly found within the lymphatic vessels (Baber, Langendorf, Hürthle) and may be readily demonstrated in most sections

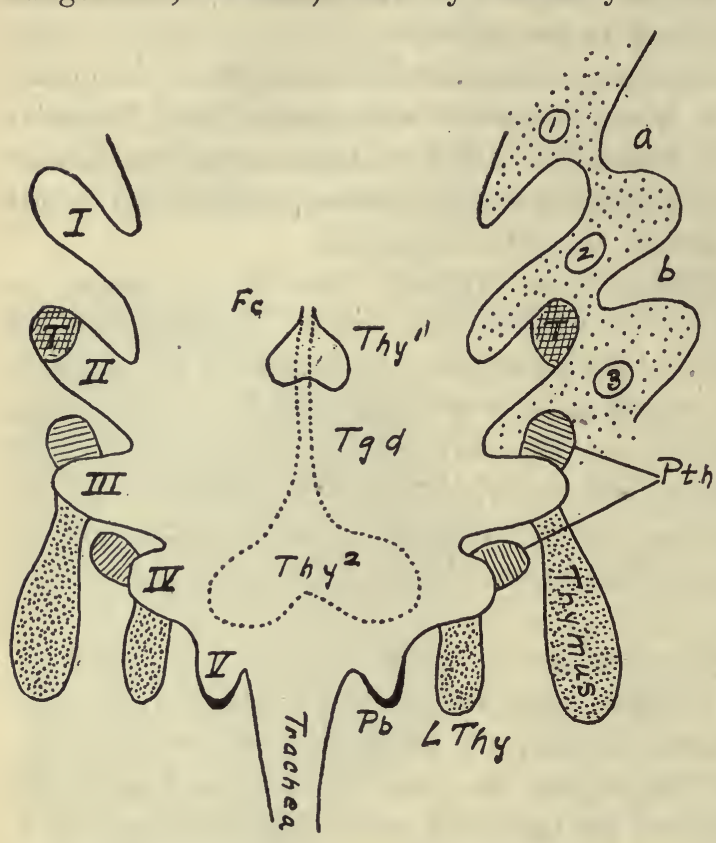

Fig. 484.-Diagram of Pharynx of Human Embryo, Showing the Origins of the Anlages of the Thymus, Thyroid and Parathyroids (Epithelial Bodies).

Thy ${ }^{1}$, earliest position of thyroid anlage; Thy ${ }^{2}$, secondary position of thyroid anlage; $T g d$, thyroglossal duct; Fc, foramen cecum; LThy, lateral thyroid anlages; Pthy, parathyroid bodies; $p b$, postbranchial body; $T$, tonsil; $I, I I, I I I, I V, V$, pharyngeal pouches; $a, b$, first and second branchial grooves ('gill clefts'); $1,2,3$, first, second and third aortic arches in the cores of the branchial arches. The complete wall of the pharynx is only shown at the right, above. (Adapted from Kohn.)

dantly supplied with lymphatic vessels. These form a plexus of very broad lacunar capillaries in the interfollicular connective tissue, where they stand in intimate relation with the follicular epithelium. From this plexus vessels pass to the interlobular connective tissue, in which they form a second plexus, whence lymphatic vessels pass out of

of the hyroid. Undoubtedly this d o e s not, however, represent the entire 'internal secudtion' of the gland.

\section{Blood Supply.}

The arteries form a rich plexus in and about the capsule of the thyroid, f r o m $y$ which $\mathrm{numerous}$ branches penetrate the organ, Tying in the connective tissue trabeculæ between the lobules; they are distributed to all parts of the gland. They supply a rich capillary plexus in the walls of the follicles. The veins retrace the course of the arteries. The wa $11 \mathrm{~s}$ of the smaller venules consist only of endothelium, with a very thin coat of fibro-elastic connective tissue.

Lymphatics.-The thyroid is very abun- 
the thyroid in company with the blood-vessels and enter the deep cervical lymph nodes.

Nerves.-The nerves of the thyroid are derived from the sympathetic system and are mostly non-medullated. They accompany the arteries and form a delicate terminal plexus in the walls of the follicles. The finer fibrils of this plexus end in contact with the epithelium. Berkeley (1895) describes also occasional fibrils which apparently penetrated between the epithelial cells.

Development.-The thyroid develops from three anlages, a median and a pair of lateral outgrowths from the primitive pharynx. The median anlage sprouts from the floor of the pharynx at the level of the first pharyngeal pouches. Its site of origin is marked in the adult by the foramen cecum of the tongue. It forms the isthmus and pyramidal lobe (process) of the definitive thyroid. The lateral anlages grow down from the ventral border of the fourth pharyngeal pouches; they form the lateral lobes of the gland. The pyramidal lobe represents the remnant of the embryonic thyroglossal duct; t varies greatly in length in different individuals; it may even retain a partial lumen which may be filled with colloid, but it does not open upon the surface. The primary anlages consist of solid cords of cells; the cords subsequently acquire a lumen, and become broken up into lobules and follicles through the invasion of connective tissue. According to Grosser the definitive thyroid arises exclusively from the median anlage.

Function.-Removal of the thyroid is followed by serious symptoms and frequently fatal results, particularly in the case of the carnivora. Its internal secretion is apparently necessary for normal metabolism and development. It governs the conditions favoring tissue oxidation (Crile). The essential secretion is probably something apart from the colloid and iodin content of the gland. According to Crile the active constituent, thyroiodin, is iodin in a special proteid combination. The belief that the thyroid has also an antitoxic rôle is based chiefly on the observation that thyroidectomized animals are extremely liable to certain infections. Gudernatsch's experiments with frog tadpoles show that a thyroid diet accelerates differentiation but inhibits growth: the tadpoles metamorphosed prematurely into diminutive frogs-a result the opposite of that obtained when thymus is fed. An enlarged thyroid is commonly known as a 'goitre.' Enlargement may be due to increase in the amount of the connective tissue or of the colloid parenchyma; one results in atrophy of the parenchyma and a condition of hyposecretion, associated with myxedema and in extreme cases with cretinism-an apparently hereditary defect; and the other in hypersecretion, associated with exophthalmic goitre (Grave's disease). In man and animals the thyroid shows a seasonal enlargement related to the sexual cycle. 


\section{- Accessory or Aberrant Thyroids}

These bodies, first described by Zuckerkandl (1879), are widely distributed through the connective tissue of the cervical region. They are most frequently found in the course of the embryonic thyroglossal

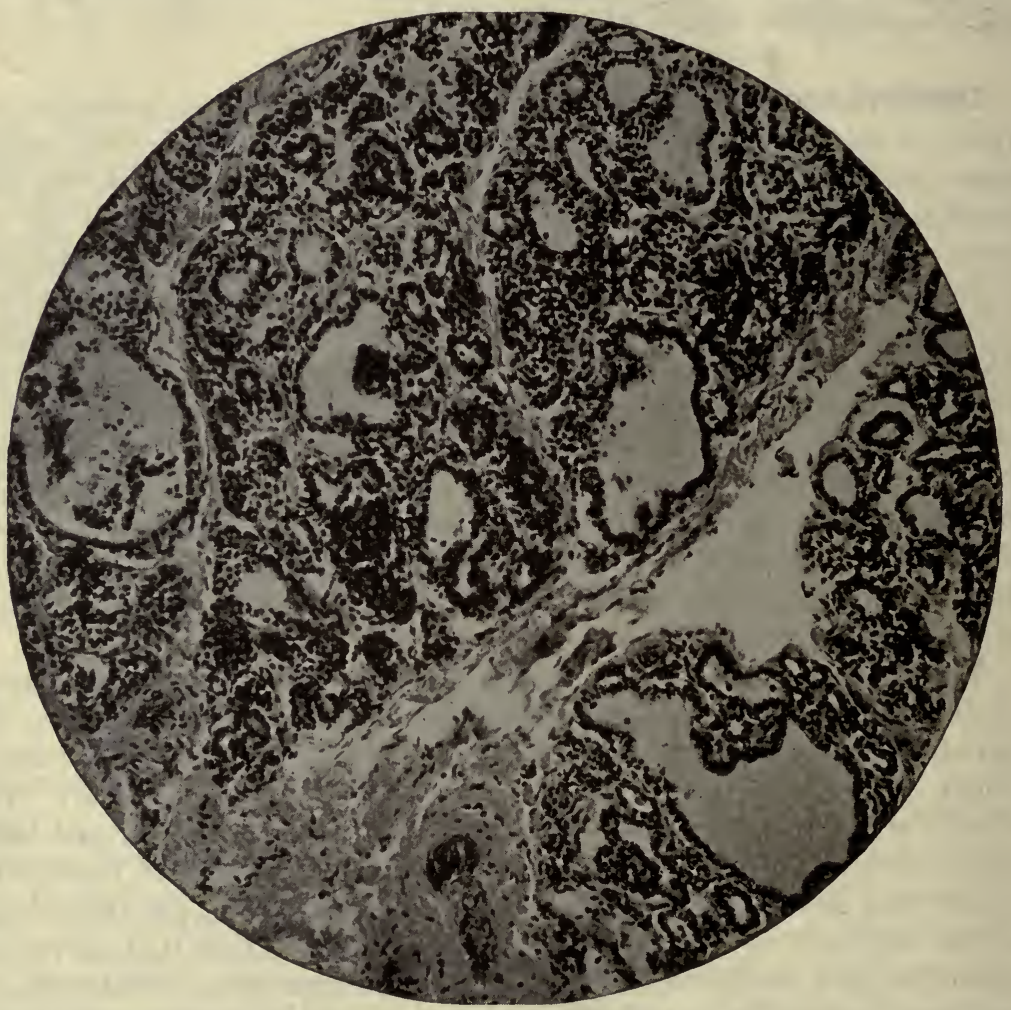

Fig. 485.-From the Border of a Mass of Aberrant Thyroid Tissue of Man, Occurring in the Region of the Parathyroid Glands.

Hematein and eosin. Photo. $\times 204$.

duct and in the immediate vicinity of the lateral lobes of the thyroid. They present the appearance of embryonal remnants of thyroid tissue, but are found in nearly all individuals.

The colloid follicles of the aberrant thyroids are usually small, though, in the larger specimens of these bodies, they may attain as great 
a size as those of the thyroid itself. The cell columns without colloid are more numerous than in the thyroid gland, giving to the aberrant bodies a decidedly cellular appearance. Each aberrant mass is usually inclosed by a very thin connective tissue capsule which sends delicate processes between the cell groups. The epithelial cells retain all the characteristics of those of the thyroid gland, and can be readily distinguished from the epithelium of the parathyroid glands with which the accessory thyroid bodies have been frequently confused. They are also much less vascular than the parathyroids.

\section{THE PARATHYROID GLANDS}

The PARATHYRoIDs, or epithelial bodies, are small glandular bodies of irregular distribution, usually found in relation with the posterior margin of the lateral lobes of the thyroid gland. They arise as buds from the dorsal pockets of the third and fourth pharyngeal pouches. Typically four parathyroids are present, the superior pair situated near the lateral, the inferior near the median margin of the lateral lobes of the thyroid. The inferior pair in the adult develops from the anterior pair of anlages, and frequently becomes embedded in the thyroid tissue. They may occur also in relation with the tracheal or laryngeal wall and may be found as high as the hyoid bone or as low as the border of the thymus. They also vary greatly in size and shape, but usually are of ovoid form and about three to five millimeters in diameter.

Each parathyroid is invested by a thin capsule of dense connective tissue and consists of a mass of epithelial cells supported by a delicate fibrous reticulum. The epithelial cells are of two chief types, designated by Welsh (Jour. Anat. and Physiol., 1898) as the 'principal' and the 'oxyphil' or acidophil cells.

The principal cells are the more abundant. They are ovoid or spheroidal elements, with a clear vesicular cytoplasm, a distinct cell membrane, and a large spherical nucleus, whose chromatin is irregularly distributed and often gives the nucleus a somewhat vesicular character.

The acidophil cells are of similar shape but are provided with a small spherical nucleus, which is very rich in chromatin, and a granular acidophilic cytoplasm. The acidophil are less numerous than the principal cells.

The distribution of the epithelial cells is subject to considerable variation. Most frequently they form an almost solid epithelial mass, in 
which capillary vessels are here and there found, the larger blood-vessels occupying the coarser bands of the fibrous stroma. In such glands the two cell varieties are either intermingled irregularly, or the acidophil cells may occur in scattered groups which are interspersed among the more numerous principal cells.

In certain instances the epithelial cells are arranged in small alveo-

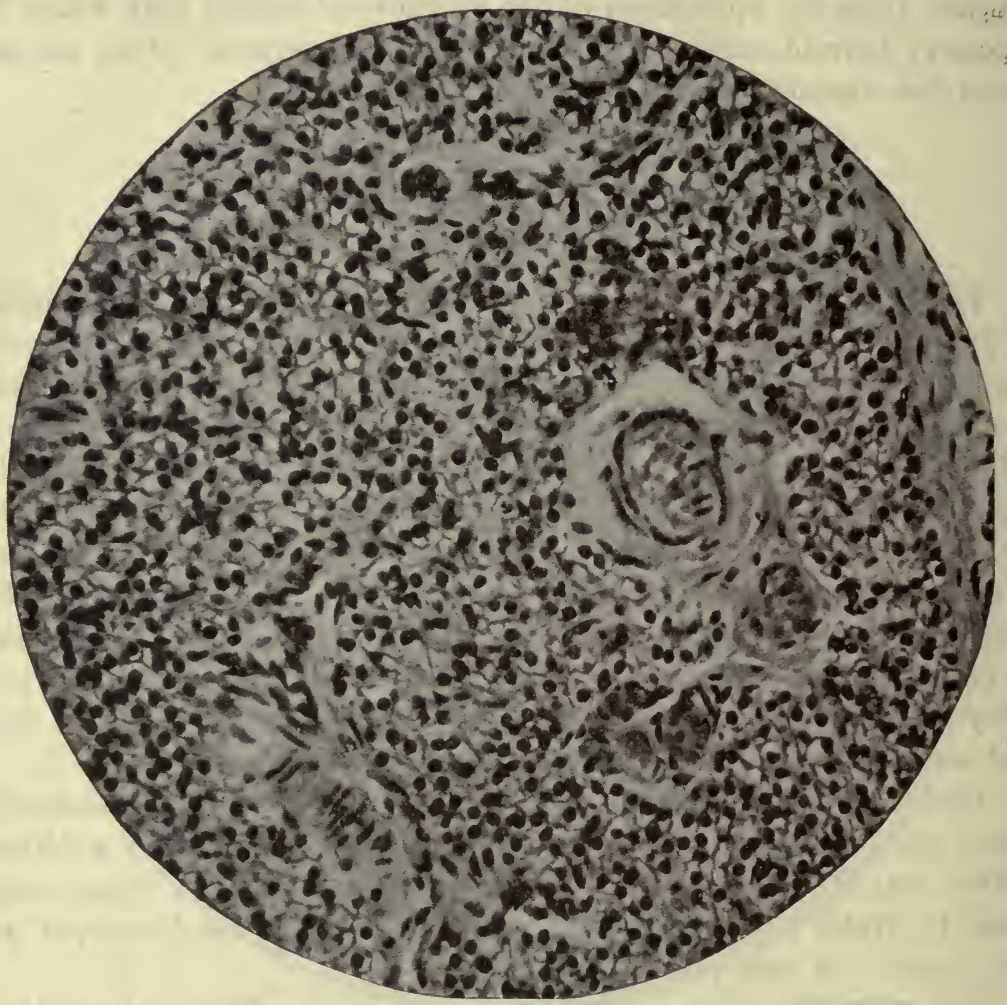

Fig. 486.-Human Parathyroid Tissue, Moderately Magnified.

Several blood-vessels are included. Capillary vessels can scarcely be recognized with this magnification. Hematein and eosin. Photo. $\times 300$.

lar groups which are surrounded by a network of capillary vessels. This arrangement appears to be more frequent in young individuals. The cell groups in this type of gland frequently form branching columns.

Occasionally, epithelial cells surround a central lumen, in which are small masses of an acidophil substance which resembles colloid in its reactions. This colloidal material is less abundant in the human para- 
thyroid than in that of the lower mammals. Likewise the cystic ducts, lined by columnar or ciliated columnar epithelium, which have been described by Kohn (189\%), though of frequent occurrence in the lower mammals are rarely, if ever, found in the human parathyroid.

The parathyroids and the thyroid are frequently in anatomical continuity; this area is characterized by transitional histologic conditions. Moreover, after thyroidectomy the parathyroids may alter their structure so as to resemble very closely thyroid tissue. The parathyroids are generally regarded as having a function absolutely essential to life. However, the experimental evidence touching this point is conflicting. But in certain forms, e.g., cat and dog, extirpation produces tetany which results in death. Vincent interprets the experimental data as indicating that the thyroid and the parathyroids constitute parts of the same apparatus, to which the pars intermedia of the hypophysis cerebri may have to be added as a third constituent.

The connective tissue of the gland is of variable quantity. It forms a thin but dense capsule; occasionally trabeculæ extend inward and partially outline indistinct lobules. In many instances a hilum transmits the larger blood-vessels by means of vascular trabeculæ which radiate to all portions of the organ. A delicate fibrous or reticular stroma invests the individual cells, or the cell groups, when these are present. Occasionally the cells are so closely packed that the stroma is scarcely demonstrable.

The blood supply of the parathyroid is exceedingly rich. Arteries enter from the capsule, or at the hilum, and rapidly break up into a plexus of broad capillary or sinusoidal vessels which follow the fibrous bands of the stroma and are in intimate relation with the epithelium. They are collected into thin-walled venules which retrace the course of the arteries.

\section{THE THYMUS}

The THYMUs was formerly regarded as an organ of fetal and infantile life, and was believed to attain its maximum development during the second year of childhood. After this time it was thought to become gradually replaced by adipose tissue, its retrograde metamorphosis reaching completion at about the age of puberty. However, Waldeyer (1890) showed that the thymus may persist even in advanced age as a body of considerable size, and apparently functional. The more recent 
studies of Hammar (1906) and others indicate that the thymus normally continues its development until about the time of puberty. Subsequently it loses slowly in weight, but a true atrophy of the parenchyma with cessation of function, does apparently not occur until about the age of fifty.

It is still uncertain whether the thymus should be classified with

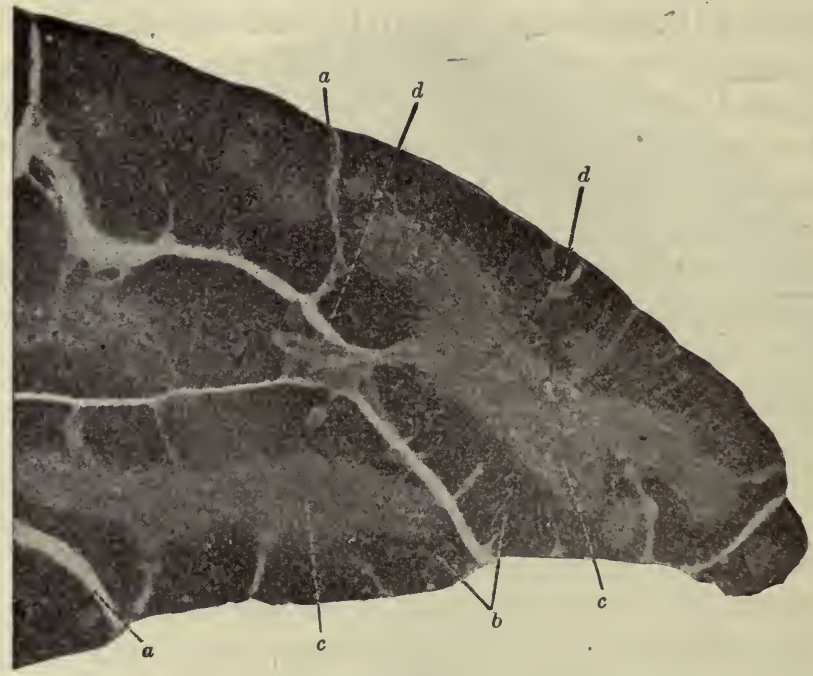

Fig. 487.-A Section through Several Lobules of the Thymus of an Infant. $a$, loose fibrous septum between the lobules; $b$, cortex, and $c$, medulla of the lobule; $d$, blood-vessels in the connective tissue. Hematein and eosin. Photo. $\times 60$.

lymphoid organs or with endocrin glands. Recent investigations increasingly indicate that it is essentially of lymphoid character but functions, in part at least, as an organ of internal secretion, related to normal growth and sexual development.

At its maximum the thymus forms a large lymphoid mass, embedded in areolar connective tissue, the trabeculx of which divide the organ into several lobes and innumerable minute lobules. Each lobule is surrounded by a thin fibrous capsule, by which it is loosely united to its neighbors.

The lobule consists of a mass of lymphoid tissue, which is dense at the periphery but looser in the central portion. It is thus divisible into a dense cortex and a loose medulla, both composed essentially of lymphoid tissue, but between which, because of the difference in density, there is a sharp line of demarcation. Frequently, at some point on 
its circumference, the medulla reaches the surface of the lobule, and at such locations a narrow column of medullary lymphoid tissue connects it with the adjoining lobule.

A close-meshed reticulum, within the narrow meshes of which are closely packed lymphoid cells, composes the lymphoid tissue of the lobule. That of the cortex and the medulla is alike, except for the fact that the meshes of the reticulum in the cortex are much more crowded with 'lymphocytes' than are those of the medulla. The medulla of each lobule is also characterized by the presence of several groups of concentrically arranged epithelioid cells, the thymic corpuscles (concentric corpuscles of Hassall).

Each thymic corpuscle consists of a large central cell or group of cells, which is surrounded by two to five layers of concentrically arranged flat epithelioid cells. These groups pr cell nests are strongly acidophil in their staining reactions, and therefore stand out in

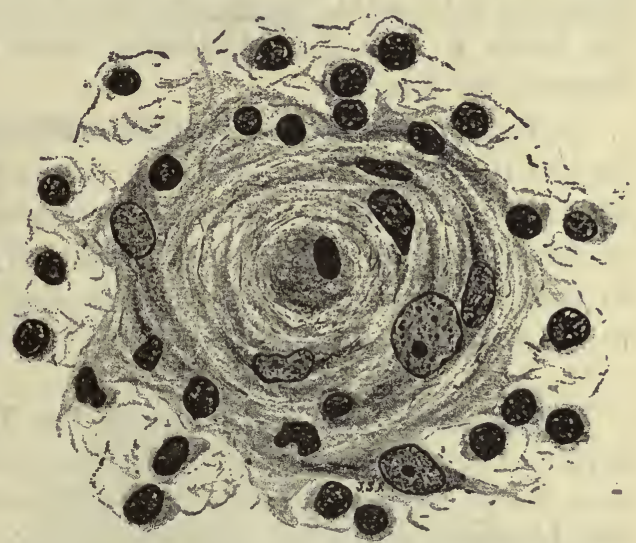

Fig. 488.-A Thymic Corpuscle from the ThYMUS OF AN INFANT.

Hematein and eosin. $\times 665$. marked contrast to the basophilic nuclei of the surrounding lymphoid tissue. A substance simulating the colloid of the thyroid may be present among the cells. Since no similar structure occurs elsewhere in the body, the concentric corpuscles are absolutely characteristic of the thymus lobule.

The nature of the thymic corpuscles is not satisfactorily understood. According to one hypothesis they represent blood-vessels whose lumen has been obliterated by proliferation of its endothelial cells. Another theory regards them as remains of the epithelial columns from which the organ arose in the embryo. They are also interpreted as masses of hypertrophied cells of the reticulum.

The types of lymphoid corpuscle which are found in the thymus are similar to those of the lymph nodes, though polymorphonuclear leukocytes are rather more frequent here, and giant cells, mononuclear or multinuclear in form, may be readily found in the medulla of this 
organ. Pappenheimer regards the specific cells of the thymus as epithelial in character, simulating, but not identical with, lymphocytes.

Blood Supply.-The larger arteries of the thymus are distributed within the interlobular connective tissue. They supply branches to the lobule which penetrate to the medulla, where they form a plexus of sinusoidal capillaries with elongated meshes, and also distribute radiating capillaries to the cortical portion of the lobule. These sinusoidal vessels are highly characteristic of the medulta of the thymus lobule. They unite to form venous radicals of considerable caliber, which leave the lobule to join the interlobular veins in the loose connective tissue between the lobules.

Lymph vessels are of frequent occurrence in the interlobular connective tissue, and their branches occasionally penetrate to the medulla of the lobule, but there is nothing corresponding to the lymph sinuses of lymph nodes. Small non-medullated nerve trunks are also found in the interlobular connective tissue, but seem to be chiefly distributed to the walls of the larger blood-vessels.

Development and Function of the Thymus.-The thymus arises as a pair of tubular outgrowths from the ventral aspect of the third pharyngeal pouch, which subsequently become solid and fuse to form a median, flat, bi-lobed organ lying in the root of the neck and the upper portion of the thorax. According to certain investigators (Stohr, et al.) the lymphoid cells of the definitive organ are derived from the entodermal cells of the initial anlages. The recent work of Maximow (Arch. mikr. Anat., 74, 3, 1909), however, seems to demonstrate that these cells are true lymphocytes and that they have their origin in the surrounding mesenchyme, from the cells of which they differentiate, and from which location they migrate into the thymus anlages-a conclusion confirmed by the still more recent work of Badertscher (Amer. Jour. Anat., 17, 4, 1915). The original lymphocytes are said to be of the large variety; these give rise through proliferation within the thymus to the smaller types. Among the invading leukocytes are also a small number of polymorphs. The original epithelial anlages continue to grow for a time, and gradually become differentiated into the definitive reticulum of the thymus. Hassall's corpuscles are believed to represent nonreticular remnants of the original entodermal anlages. According to Hammar, and Bell, these concentric corpuscles are derived from hypertrophic reticular cells. They first appear early in fetal life, and continue to form and increase in size during infancy. They are interpreted by some as thymic elements of internal secretion. The thymus is commonly regarded as a hemopoietic organ, but its activity is limited to the formation of lymphocytes and possibly a small number of granulocytes. Beard (1900) views it 
as the original source of leukocytes. The proliferative foci are the cortical portions of the lobules; the medulla docs not correspond to the germ centers of lymph nodules, but is probably an area of leukocyte dissolution. The thymus does not seem to be an organ essential to life. Extirpation in dogs and guinea pigs is not followed by death. However, the experimental evidence indicates a reciprocal functional relationship between the thymus and certain of the organs of internal secretion, especially the sex-glands. This suggests a secretory rôle; a conclusion supported by the results of the recent experiments of Gudernatsch (Amer. Jour. Anat., 15, 4, 1914) in which thymus fed to frog tadpoles, accelerated growth, but suppressed differentiation.

\section{THE CAROTID GLAND}

This body was first carefully described by Luschka (1862) and, from its intimate relation to the blood-vessels and nerves, is also known as the glomus caroticum or ganglion intercaroticum. It is about the size of a rice grain. It c onsists of scattered masses of epithelioid cells, usually grouped in small spheroidal clumps or 'cell balls,' embedded in the connective tissue at the point of bifurcation of the common carotid a r t e r y. Kohn (Arch. f. Mik. Anat., 1900) has described four types of the gland according to the density of its parenchyma - the type found in man consists of scattered cell

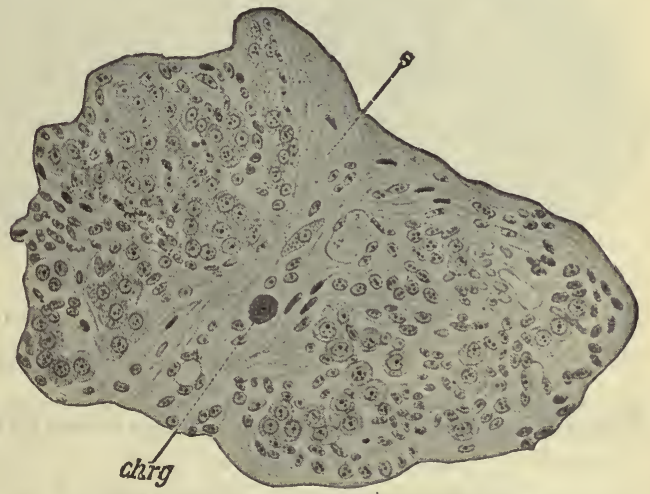

Fig. 489.-Carotid Gland of an Ape.

Chrg, a 'chromaffin cell'; $s$, connective tissue septum. Portions of two adjacent lobules are included in the figure. $\times 200$. (After Kohn.) groups; in the rabbit they

are even more diffuse. The carotid gland of a cat consists of a single cell mass, while that of the ape is intermediate between that of the cat and man.

The glandular elements are derived from embryonal sympathetic ganglion cells (Kohn). They are ovoid cells with finely granular cytoplasm and a spheroidal, somewhat vesicular nucleus. Many of them contain chromaffin granules. Because of its genetic relationship and 
histologic similarity to a sympathetic ganglion, Kohn proposes the name, paraganglion caroticum. Its function is obviously similar to that of the other organs of the chromaffin system, and probably chiefly dependent upon the presence of adrenalin.

The carotid gland is richly supplied with capillary blood-vessels and small non-medullated nerve trunks. The capillaries are in intimate relation with the glandular epithelium.

\section{THE COCCYGEAL GLAND}

This small body-2.5 $\mathrm{mm}$. in diameter (Eberth)-was discovered by Luschka in 1860 . 'Its structure closely resembles that of the carotid

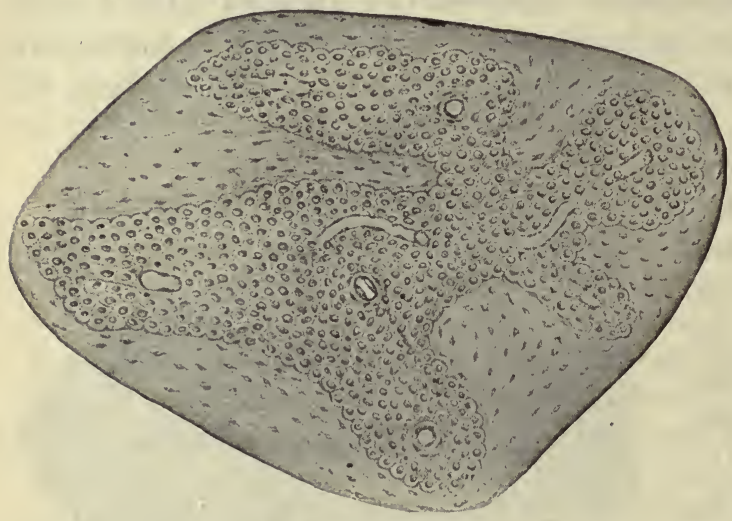

Fig. 490.-From a Section of the Coccygeal Gland of Man.

Highly magnified. (After Sertoli.) gland. It usually consists of several minute groups of epithelioid cells which are in relation with the terminal branches of the middle sacral artery. It is richly supplied with broad capillaries or sinusoids and hence is also known as the glomus coccygeum.

The parenchymal cells of the organ are ovoid elements which are closely $\mathrm{packed}$ about the walls of the blood-vessels in groups or short columns inclosed by delicate sheaths of connective tissue. The origin and function of these cells are unknown. No chromaffin cells have yet been certainly demonstrated. The organ is embedded in the dense connective tissue at the tip of the coccyx. Its general structure only suggests an internal secretory function.

According to Stoerck (Arch. mikr. Anat., Bd. 69, 1906), the cells of the coccygeal body do not give the pheochrome reaction at any period of life. They are believed to bear no genetic relation to the sympathetic nervous system. Schumacher regards the epithelioid cells as a transformation of the smooth muscle cells of the arterial vessels; 
others interpret them as modified endothelial cells of the blood-vessels. Vincent suggests that this body may function as a 'safety-valve' in the course of the peripheral circulation; he regards the balance of evidence as against any function involving an internal secretion.

\section{PARAGANGLIA}

The so-called chromaffin system includes besides the medulla of the suprarenal and the carotid gland, other larger and smaller groups of chromaffin cells ('pheochrome organs') derived from embryonal sympathetic cells and scattered along the entire sympathetic nervous system, the PARAGANGLia. They are therefore present in many organs which receive a considerable synipathetic innervation, e.g., kidney, liver, uterus, testis, etc. Chromaffin tissue composed of widely scattered cells is present also in the sympathetic trunks, particularly the various plexuses, among the definitive neurons of this system.

The largest paraganglia (organs of Zuckerkandl) occur in close relation to the inferior mesenteric artery, one on either side. Other smaller groups are found in relation to the abdominal a orta. Busachi has recently

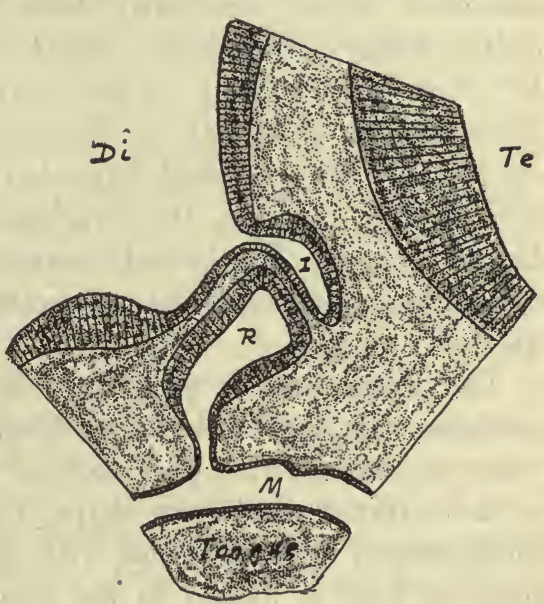

Fig. 491.-Median Section through the Anlages of the Hypophysis Cerebri of a 10 mm. Cat Embryo. (Bonnet.)

$D i$, diencephalon; Te, telencephalon; $M$, mouth; $R$, Rathke's pouch (pituitary anlage); $I$, infundibulum. $\times 50$. (1912) described chromaffin tissue in the human heart; and Thulin (Anat. Anz., 46, 22, 1914) reports a paraganglion in the striped muscle of the upper portion of the esophagus of man. In typical paraganglia the polygonal cells are arranged in irregular cords which form a fine-meshed network, the areolæ of which are occupied by sinusoidal blood-vessels, upon which the cells rest directly without the intervention of a basement membrane. The spherical nucleus is poor in chromatin; the reticular cytoplasm contains the pheochrome granules. The internal secretion of the paraganglia dẹ- 
pends upon the adrenalin content of the granules. Adrenalin produces a contraction of involuntary muscle. Its function appears to be to maintain the proper tonus of the blood-vessels.

\section{HYPOPHYSIS CEREBRI}

\section{(Pituitary Body)}

The HYPOPHYSIs is a gland of the internally secreting (endocrin) or ductless type. The secretion passes directly from the specific cells into capillaries. This gland is a composite structure, arising from the association of two originally distinct anlages; one taking origin as a median dorsal diverticulum (Rathke's pouch) from the primitive mouth, the other as a medial ventral evagination from the second cerebral vesicle (third ventricle) or diencephalon. Both components are thus ectodermal. The cerebral flask-shaped element invaginates the buccal element and modifies the original bulbar shape of the latter into that of a cup. The buccal connection disappears, while the cerebral connection persists as the infundibular stalk of the definitive structure.

The hypophysis is present as an essentially similar organ in all craniates. In man it measures about $12 \mathrm{~mm}$. in the transverse, about $7 \mathrm{~mm}$. in the sagittal, and about $5 \mathrm{~mm}$. in the vertical diameter. It has an inverted mushroom shape, the stalk or infundibulum, a hollow funnel-shaped structure lined with ependyma, attaching it to the brain, while its head is lodged in the sella turcica of the skull. The hypophysis was known to the early anatomists as the pituitary gland (Vesal, 1553), and was supposed to function as an excretory organ in the elimination of mucus (pituita) from the brain by way of the nose. The term pituitary gland is now generally applied only to the larger anterior component or epithelial lobe; the posterior component or neural. lobe is designated the infundibular process; the term hypophysis is applied to the associated elements. But hypophysis and pituitary body are still often used synonymously. The neural and glandular tissues are said to be connected with each other by means of nerve fibers, probably sympathetic, connective tissue, and blood-vessels. Certain investigators have described accessory pituitary bodies. Thus Haberfeld (1909) finds in man at all ages a 'pharyngeal pituitary,' a solid cord of neutrophilic cells about $5 \mathrm{~mm}$. in length immediately behind the vomer. This is frequently the initial seat of tumors. 
Function.-Very little is definitely known concerning the function of the hypophysis, notwithstanding extensive investigation. Much of the recorded clinical and experimental data is contradictory. However, the presence of the gland would seem to be essential to health, perhaps indispensable to the maintenance of life. It appears also that its two fundamental components (anterior and posterior lobes) subserve different functions, and that pathological alterations in the gland induce marked changes in some or all of the other ductless glands, which glands appear to sustain reciprocal functional relationships to each other. Absence of the hypophysis (apituitarism) in mammals is associated with grave nutritive disturbances usually leading to death. Disordered function (dyspituitarism) is accompanied by inordinate enlargement of the extremities (acromegaly) and gigantism, mental derangements, disturbances in the sexual organs and improper metabolism of sugar. Reduced secretion ('hypopituitarism') of the posterior lobe leads to an acquired high tolerance for sugars with the resultant accumulation of fat (Cushing; "The Pituitary Body and Its Disorders," 1910). Increased secretion may be responsible for some types of emaciation and for certain mental disturbances. Deficient secretion of the anterior lobe appears to inhibit complete development of the skeleton, resulting in dwarfism and infantilism; conditions of superabundant secretory activity (hyperpituitarism) are accompanied by acromegaly and gigantism (Cushing). Injection of extract of the posterior lobe (pituitrin) into the blood-vessels produces a rise of blood pressure accompanied by diuresis, believed to be due to a stimulation of the smooth musculature of the blood-vessels. In its effect upon smooth muscle, particularly of the uterus, which it stimulates to sharp and prolonged contraction, it resembles very closely the secretion of the suprarenal gland. Pituitrin is now extensively used in practical medicine and especially in obstetrics.

The anterior lobe is believed by some to be physiologically inactive, except in cases of defective secretion of the gland, while others hold that the removal of the posterior lobe gives rise to no symptoms, but that excision of the anterior lobe produces all the ill effects of total pituitary extirpation. The contradictory results of experimental work may be due to failure or inability to completely separate the several portions; moreover, certain elements of the supposed symptom complex accompanying hypophysectomy may be due to mechanical injury to associated parts of the brain.

Histologic Structure.-The hypophysis is usually described as consisting of three portions: (1) the anterior lobe or glandular portion; (2) the intermediate portion or boundary zone; and (3) the posterior lobe, or nervous portion. The recent more critical investigation of 
Tilney (Internationale Monatschrift f. Anat. u. Phys., Bd. 30, 1911), including careful reconstructions of a number of mammalian hypophyses, shows that this description is incomplete. The form, extent, relation and genetic significance of the pars intermedia had apparently hitherto not been fully appreciated, though Herring (1908) had already called attention to the fact that it comprised two histologically different areas. Tilney divides the hypophysis into: (I) pars neuralis (pars nervosa);

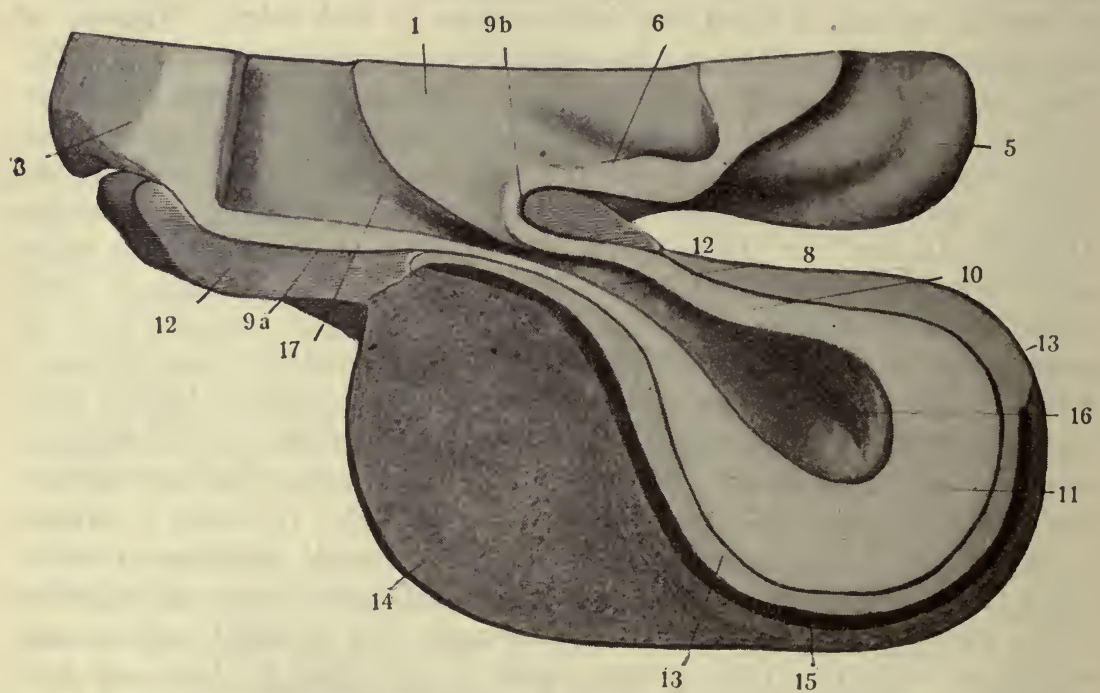

Fig. 492.-Sagittal View of a Wax Reconstruction of the Hypophysis CereBRI of the Adult Cat.

1 , third ventricle; $9 a$ and $b$, eminentia saccularis; 6 , area premammillaris; 5 , corpora mammillaria; 12 ; pars tuberalis; 8 , recessus infundibuli; 10 , infundibulum; 13, pars infundibularis; 16, recessus processi infundibuli; 11, processus infundibuli; 15, lumen residuale; 14, pars distalis; 17. recessus tuberis; 3 , optic chiasm. (Tilney, Internat. Monatschr., Bd. 30, 1913.)

and (II) pars buccalis. The pars neuralis consists of three distinct elements: (a) the eminentia saccularis of the tuber cinereum; (b) the infundibulum; and (c) the infundibular process. The pars buccalis consists of two elements: (a) the pars juxta-neuralis in close relation with and investing the neural portion; and (b) the pars distalis. A cleft, the residual lumen, remnant of the original cavity in the buccal diverticulum, appears between the two. Further analysis of the juxtaneural portion of the gland reveals two histologically different parts: (1) the pars infundibularis completely investing the infundibulum 
and the infundibular process; and (2) the pars tuberalis, which surrounds the eminentia saccularis.

The following outline presents this analysis in résumé:

\section{HYPOPHYSIS CEREBRI}

I. Pars Neuralis: (Posterior lobe).

(1) Eminentia Saccularis.

(2) Infundibulum.

(3) Processus infundibuli.

II. Pars Buccalis seu Glandularis:

(1) Pars juxtaneuralis. (Intermediate portion.)

(a) Pars tuberalis.

(b) Pars infundibularis.

(2) Pars distalis. (Anterior lobe.)

"The pars infundibularis makes its appearance almost immediately after the anlage of the buccal portion of the hypophysis is formed. 'The pars tuberalis arises as a relatively late structure. It has its origin in two secondary diverticula or sprouts from the body of the pituitary sac (buccal evagination). These sprouts, the lateral processes, ultimately fuse with each other across the median line, displace the body of the pituitary sac ventrad and thus secondarily assume their juxtaneural position. The juxtaneural portion of the hypophysis is intimately connected with the neural portion by means of nerve fibers, blood-vessels and connective tissue processes. Attempted separation consequently necessarily includes laceration of neural elements" (Tilney). Accordingly complete removal of the pars buccalis would seem to be impossible without the attendant removal of portions of the pars neuralis. This must be taken into account in interpreting symptoms following removal of the anterior lobe of the hypophysis.

I. Pars Neuralis : Infundibulum.-This consists of fusiform and multipolar neuroglia cells and fibers. A substance resembling colloid is also occasionally present. Small nerve cells have been reported, but their presence seems doubtful. The glia cells generally have a single nucleus, but two or more may appear. The protoplasm is finely granular, and occasionally contains pigment granules, which seem to be more abundant in aged specimens. Delicate and coarse glia fibers extend through the protoplasmic portions of the cells, frequently terminating the cell processes (Fig. 496).

II. Pars Buccalis Seu Glandularis: (1) Pars juxtaneuralis.- 
(a) Pars tuberalis.-The cells of this portion are arranged in large tubular acini; the lumina may contain colloidal material. The cells are of the cuboidal type, sometimes ciliated, frequently two layers deep.

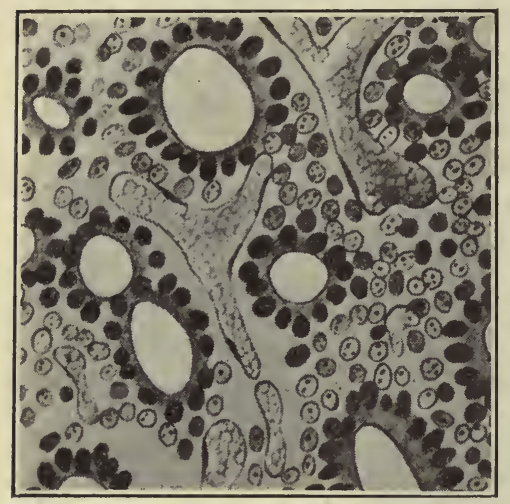

Fig. 493.-Pars Tuberalis, HypoPHYSIS OF Cat (Tilney.)

The cytoplasm of the cells is sparse, homogeneous and deeply basophilic. The nuclei are large and vesicular. Blood-vessels (sinusoidal capillaries) are numerous, and are said occasionally to contain colloid.

(b) Pars infundibularis.-Here the cells are arranged in a dense stratum several layers deep. Occasional acini (cysts) occur, but they are smaller than in the pars tuberalis and are lined with only a single layer of low cuboidal cells. These cells contain large vesicular centric nuclei surrounded by a considerable layer of faintly basophilic granular cytoplasm. Blood-vessels are very meager and thin-walled.

(2) Pars distalis. - This portion is surrounded by a robust fibrous capsule continuous posteriorly with a thinner investment for the neural lobe. Delicate trabeculæ traverse the parenchyma and support the very abundant plexus of arteriovenous sinusoidal blood spaces. The peripheral cells of the pars distalis constitute a narrow zone of almost exclusively basophitic cells. The cells of the main central portion are arranged in dense convoluted anastomosing cords. The cords comprise axial and parietal cells. The parietal cells are the larger, of polygonal shape, and with a granular cytoplasm deeply acidophilic. These are the 'eosinophilic' or acid-

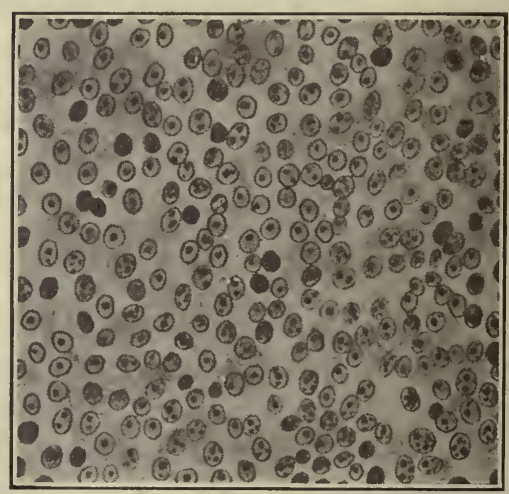

Fig. 494.-Pars Infundibularis, HyPOPHYSIS OF CAT. (Tilney.) ophitic ('chromophil') cells. The axial cells are smaller; their cytoplasm contains 'neutrophilic' granules. These are the 'chief,' or 'chromophobe' cells. The so-called 'chromophils' and 'chromophobes' of the 
earlier terminology (Flesch, 1880), which was based simply upon a difference in the degree of tingibility, probably represent merely different functional phases of the same cell type. The classification of the cells as acidophils and basophils, a distinction based upon a more precise microchemical difference, accords with a topographical segregation and more probably designates an actual functional division. True chromophobes, cells which stain only faintly and with difficulty in either acid or basic dyes-and probably functional phases of acidophil and basophil cellsappear first only in the higher sauropsids (Tilney). The nuclei of the several cell types are large and deeply staining. The cells in great part rest directly upon the endothelial walls of the capillaries. The blood-vessels are said to contain colloid.

Comparative histologic studies indicate that the pituitary gland has a twofold activity, one part dependent upon the basophils, the other upon the acidophils. The residual lumen is lined only with basophils, and occasionally contains

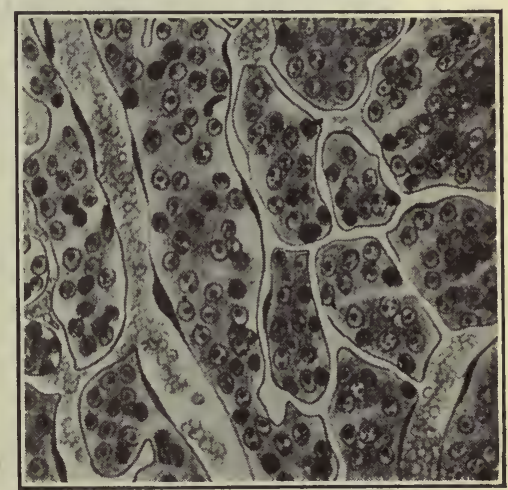

Fig. 495.-Pars Distalis, HypophySis of Cat. (Tilney.) colloid. Comparative morphology indicates that the neural portion may represent an original special sense organ. It has been suggested by Tilney (Mem. Wistar Inst., Biol. and Anat., No. 2, 1911) that the basophils contribute their secretion by way of the residual lumen and the infundibular process to the cerebrospinal fluid, and the acidophils directly through the blood spaces.

According to Dandy and Goetsch (Amer. Jour. Anat., 11, 2, 1911), the pars distalis receives from eighteen to twenty small arteries which converge to it from the circle of Willis. Within the lobe no true arteries or veins occur. The blood channels are sinusoidal in nature; they drain into a venous circle overlying the circle of Willis.

The above description of the hypophysis is based largely upon dog and cat material. Our knowledge of the human hypophysis is less complete; but as far as is known, the structural elements are essentially closely similar.

The nerve supply to the hypophysis in the dog is, according to Dandy (Amer. Jour. Anat., 15, 3, 1913), derived from the carotid plexus of the 
sympathetic system, which connects with the oculomotor and optic nerves. The innervation of the anterior lobe is described as abundant, that of the

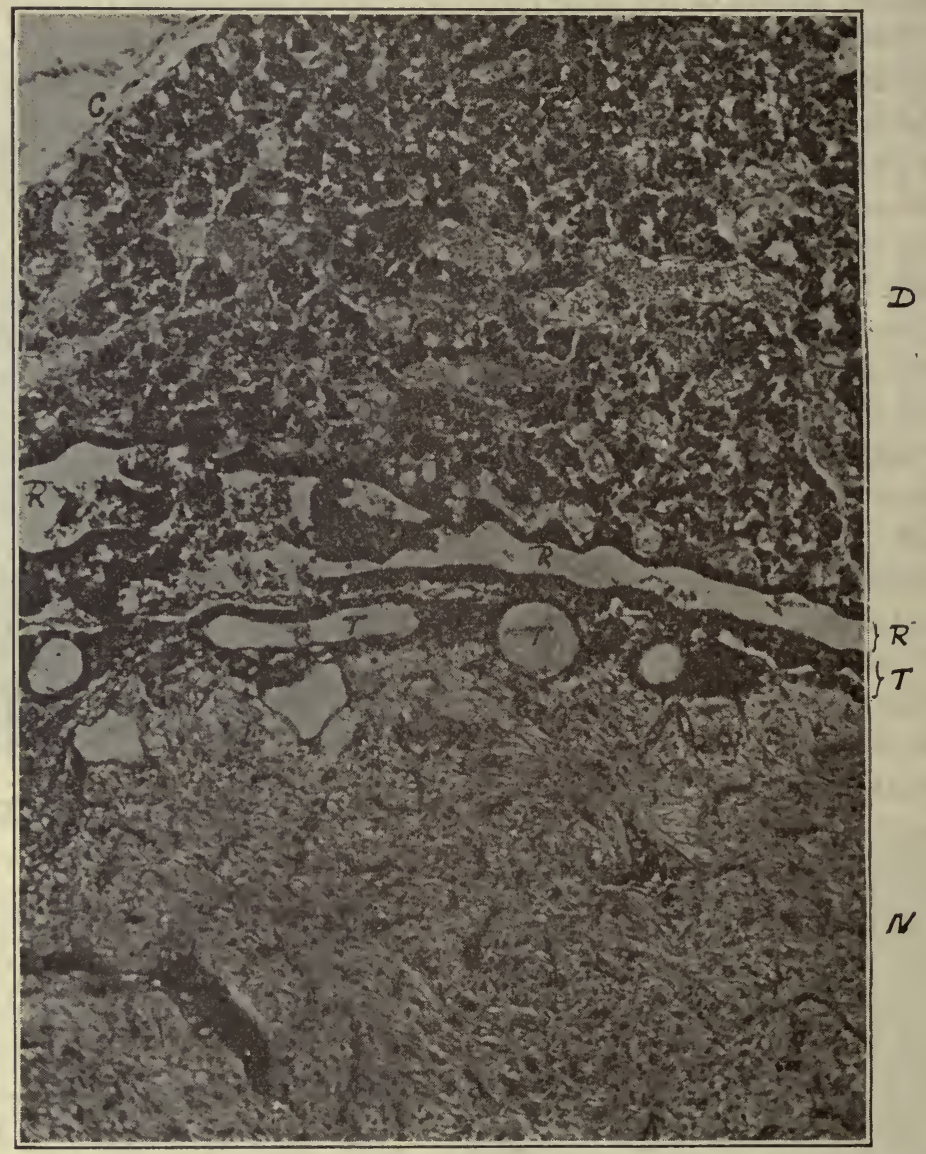

Fig. 496.-Section of Hypophysis Cerebri of Dog, Showing Portions of Pars Distalis $(D)$ of Anterior or Buccal Lobe, Residual lumen $(R)$, Pars Tuberalis (Pars Intermedia) ( $T$ ), Pars Neuralis $(N)$, and $C$, Capsule.

Note the abundant large blood sinuses in the glandular or buccal portion, and the tubules in the pars tuberalis (referring to the portion next the tuber cinereum). The pars neuralis is largely of neurogliar composition.

posterior lobe as very scanty. The fibers are thought to be secretory in character.

Dandy (ibid.) described also a small spherical body which he names parahypophysis in connection with the under central surface of the hypo- 
physis, resting in a depression in the floor of the sella turcica. This was found in over eighty per cent. of dogs examined. It is interpreted as a remnant of the embryonic Rathke's pouch. It is said to vary greatly in size and histological character, occasionally extending to the 'pars intermedia,' and to have an individual blood supply and possibly also a nerve supply. A parahypophysis has not yet been reported for man.

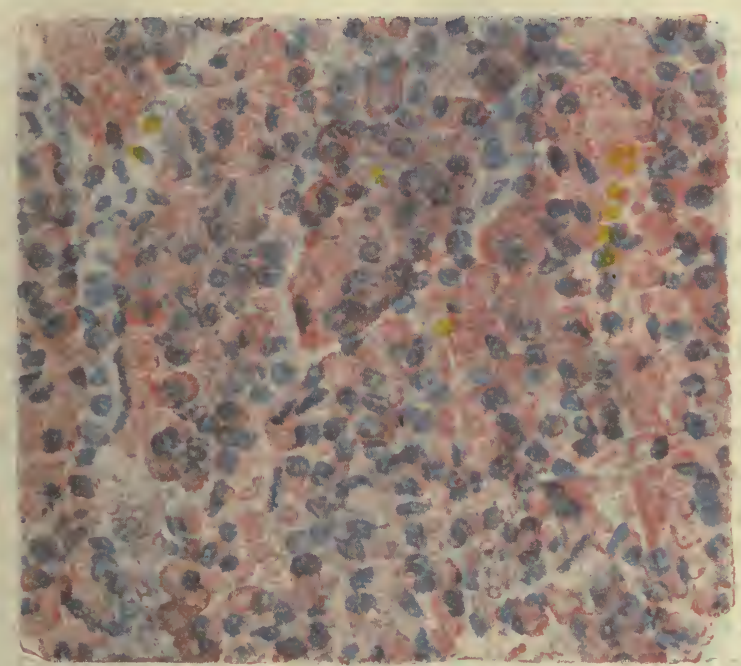

Fig. 497.-Field from the Center of a Normal Canine (Puppy) Pars ANTERIor (Distalis).

Note the excess of eosinophils lining the sinuses. No basophils in the field, as they are more in evidence in the glandular periphery. The central elements in the cell columns are neutrophilic. (Cushing.)

In the hypophysis of the ox, Wulzen (Anat. Rec., 8, 8, 1914) has described a cone-shaped structure in connection with the 'pars intermedia' resembling in cellular constitution the pars glandularis but differing in having finer connective tissue septa and smaller acini.

\section{EPIPHYSIS CEREBRI}

\section{(Pineal body)}

The EPIPHYsis (pineal body, pineal gland or conarium) may be provisionally regarded as an endocrin gland. Its glandular function may be of importance only during late fetal and infantile (or prepuberal) life, when it may control normal growth. The mammalian 
epiphysis has been known to anatomists as a probable gland since the time of Galenus (200). It has excited interest and been the subject of much speculation for centuries. Descartes (1649) regarded it as the seat of the 'soul.' Notwithstanding much study, it is still but imperfectly understood, and even its histological makeup is to some extent in dispute.

Development.-The epiphysis cerebri arises as an ependymal diverticulum from the roof of the diencephalon. The apical cells proliferate and undergo differentiation into neuroglia and interneuroglia cells. The latter may perhaps assume secretory activities, but no convincing cytological evidence appears indicative of such function apart from numerous melanic granules,-which, however, are present in all of the cells in early stages of development-and certain lipoid granules and spherules. In the sheep numerous cysts, lined by tall columnar (ependymal) cells appear at half term (21 cm. stage). The cysts contain a coagulum. The cysts progressively disappear, apparently through pressure from proliferating cells without, only a few persisting to birth and occasionally after. In the sheep the cysts arise through accumulations of fluid, compelling a cellular arrangement simulating acini, not as tubular outgrowths from the original lumen, as is apparently the case in birds. Both melanic granules and alveoli (cysts) are more probably to be interpreted as of ancestral significance. As far as is known an epiphysis is present in all vertebrates, with the exception of Myxinoides and Crocodilia. No trace of an epiphysis is said to appear even in embryos of Crocodilia. The pineal eye of certain reptiles is commonly regarded as the homologue of the pineal body of mammals. However, in the lower groups of animals the true homologue is generally a double structure, the pineal eye developing terminally on a secondary anterior evagination from the base of the primary one. This secondary process is not developed in mammals. In Hatteria (a New Zealand 'lizard') the pineal eye comes to the surface in the middle of the head, and consists of an optic cup with a lens covered by transparent epidermal scales, forming a cornea. It is believed to function only as a light or warmth perceptive organ. An additional structure, the paraphysis, found in certain lower forms and in the marsupialia, anterior to the pineal body, and arising similarly as an ependymal evagination, is commonly regarded as a choroid plexus, evaginated instead of being invaginated, as is usually the case in mammals. In sheep the pineal undergoes its greatest development during the first year of life, approximately fivefold.

The pineal body is attached to the posterior portion of the roof of the third ventricle by means of the pineal stalk. This contains the pineal recess, a cavity continuous with the ventricle. The anterior wall 
of the stalk is limited basally by the habenular conmissure, the posterior wall by the posterior commissure. The pineal proper thus lies underneath the posterior end of the corpus callosum and rests on the anterior pair of the corpora quadrigemina. It is a solid cone-shaped structure enveloped by a capsule of pia mater, and more or less distinctly lobed (Fig. 498). It measures in man $y$ mm. in length by 5 $\mathrm{mm}$. in transverse diameter. The mammalian pineal is perhaps rather a metamorphosed than a rudimentary organ. Its size bears no relation to the size of the brain or the size of the body; it is no

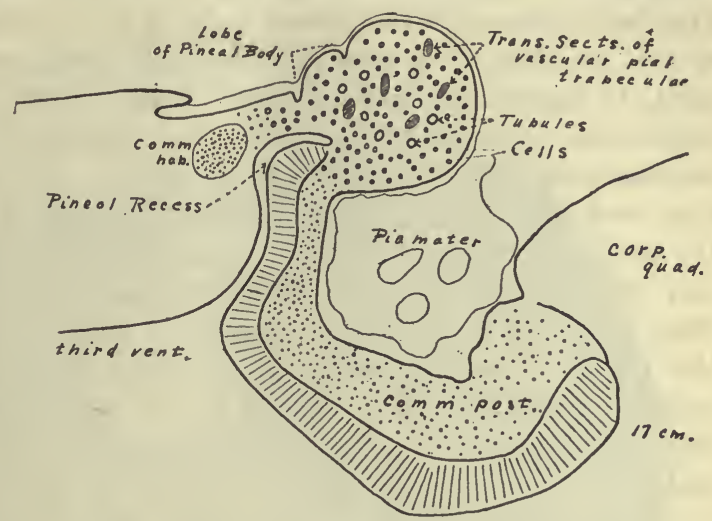

Fig. 498.-Semidiagrammatid Representation of a Median Longitudinal Section through the EpIphysis of a 17 cm. Sheep Fetus. larger in large than in small dogs, for example, and no larger in horse than in dog; that of sheep is about the size of that of man.

Function.-Injection of pineal extract of sheep into the blood of certain mammals produces only very slight effects. These, however, are fairly uniform, but very transient. The most conspicuous effect is a slight fall of blood pressure. There is also a slight improvement in the beat of the isolated cat's heart, a transient diuretic effect (in rabbit) and a slight irregular respiratory effect (in dog, eat and sheep-Jordan and Eyster, Amer. Jour. Physiol., 1911). These results are, to some extent, the reverse of those obtained with extracts of the hypophysis, and suggest a compensatory or antagonistic regulatory functional relationship. Extirpation experiments have not yet yielded perfectly satisfactory results. The evidence, however, indicates a functional rôle which is negligible or nil. Clinical evidence combined with autopsy findings indicate symptom complexes associated with pineal tumors or other structural alterations in general like, but of reverse relationship, to those present in case of hypophyseal alteration; i.e., the symptoms of hypopinealism are in general those associated with hyperpituitarism, and those of hyperpinealism like those accompanying hypopituitarism. The symptoms associated with morbid conditions of the pineal may possibly be the inherent result of pressure (or undue relaxa- 
tion) upon the hypophysis transmitted through the third ventricle with which both pineal and hypophysis are connected. Certain symptoms may also be due to a blocking of the aqueduct, and to pressure upon the corpora quadrigemina. A conservative estimate of all the evidence indicates very meager, if any functional activity, probably never essential to life. The practical absence of the pineal body in the opossum adds further support to this conclusion; as also its occasional disappearance, except for a mere shell, in apparently normal cats. Biedl (1910), however, arrives at the conclusion that the pineal body is an organ of internal secretion with metabolic significance limited to the young. Its general histological structure, and profuse vascularity, certainly suggest a glandular function. On the basis of feeding experiments with bullocks' pineals on certain laboratory animals and mentally defective children, Dana and Berkeley (Med. Rec., May, 1913) venture to suggest a relationship of pineal function in the young to bodily nutrition, including the development of the genital organs, the deposit of subcutaneous fat, general growth and mental progress. The pineal is a common seat of cysts and tumors, frequently gliomata.

Histologic Structure.-The epiphysis cerebri is divided imper-

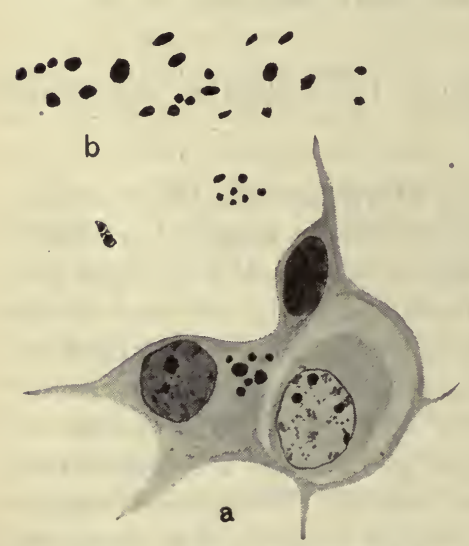

Fig. 499.-Cells from the PiNEAL Body of a $11 \mathrm{~cm}$. Sheep Fetus.

$a$, two neuroglia and one interr.euroglia cell; $b$, various forms and sizes of intracellular melanic granules. $\times 1500$. deeply staining nuclei, and glia fibers in the cytoplasm. Transition fectly into lobes by trabeculæ of fibroelastic connective tissue provided by the pia mater capsule. These support the larger blood-vessels. The finer trabeculæ shade into the fundamental reticular tissue which supports the parenchyma and the capillaries. The larger vessels are surrounded by considerable spaces, perhaps lymphatics. The parenchyma is aggregated into uncertain follicular masses and consists of two distinct types of cells: neuroglia and interneuroglia (secretory cells?). The abundant neuroglia fibers appear to blend intimately with the connective tissue framework of the structure. The interneuroglia cells are oval and polygonal, with a vesicular nucleus, and apparently lacking a cell membrane. The neuroglia cells are fusiform or stellate, with denser, more 
forms are abundant. In young sheep both types of cells are characterized by pigment granules; in aged specimens the pigment is for the most part present in the shape of extracellular clumps. Also in these stages the connective tissue is relatively much more abundant,

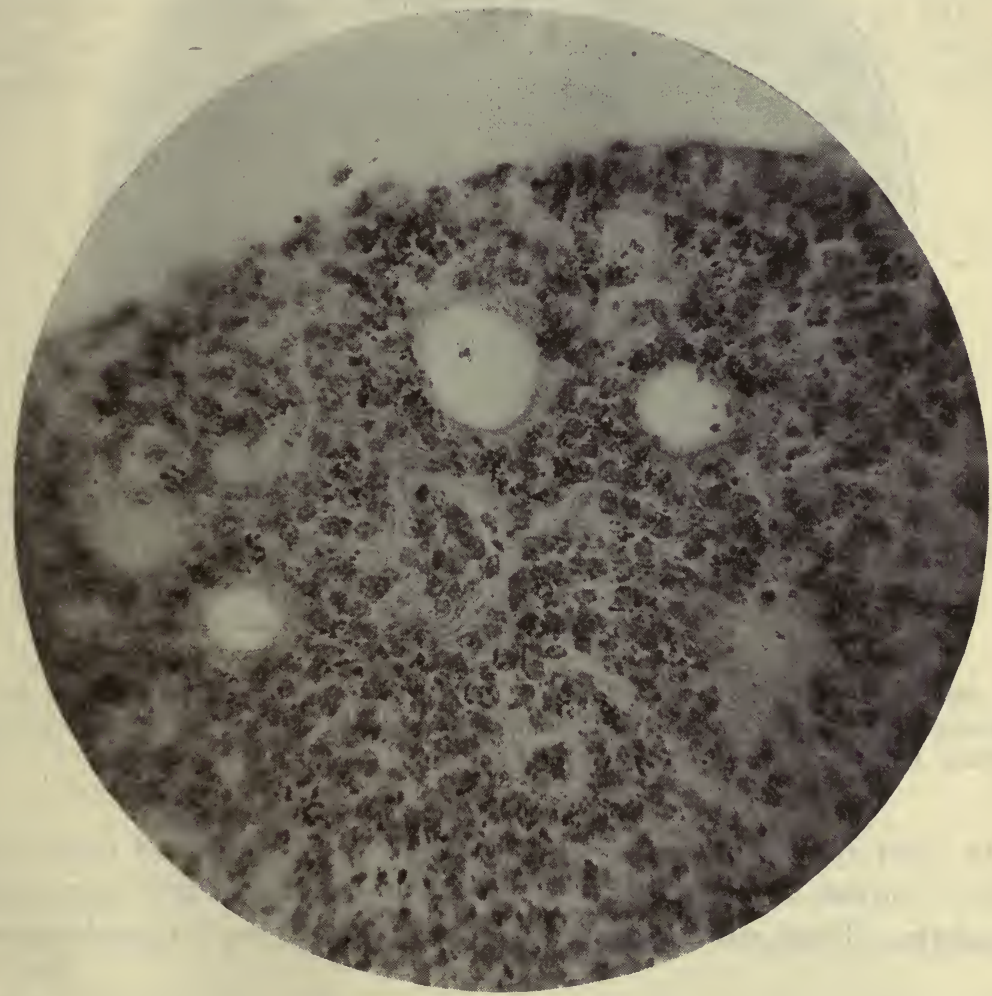

Fig. 500.-Photomicrograph of a Peripheral Portion of the Pineal Body of a 21 cm. Sheep Fetus, Showing Several Cysts and Vascular Trabecule, and an Enormous Number of Intracellular Melanic Granules. $\times 250$.

and the interneuroglia cells rare. The interneuroglia cells are probably less highly differentiated neuroglia cells, both types originally arising from ependyma. The interneuroglia cells also contain granules and vacuoles of a lipoid nature, perhaps degenerative in significance; no pheochrome granules are demonstrable. Involution begins about the age of 7 years in man and ends about the age of puberty, though regressive histological changes proceed throughout life. Adult corpora pinealia present very variable histologic pictures. 


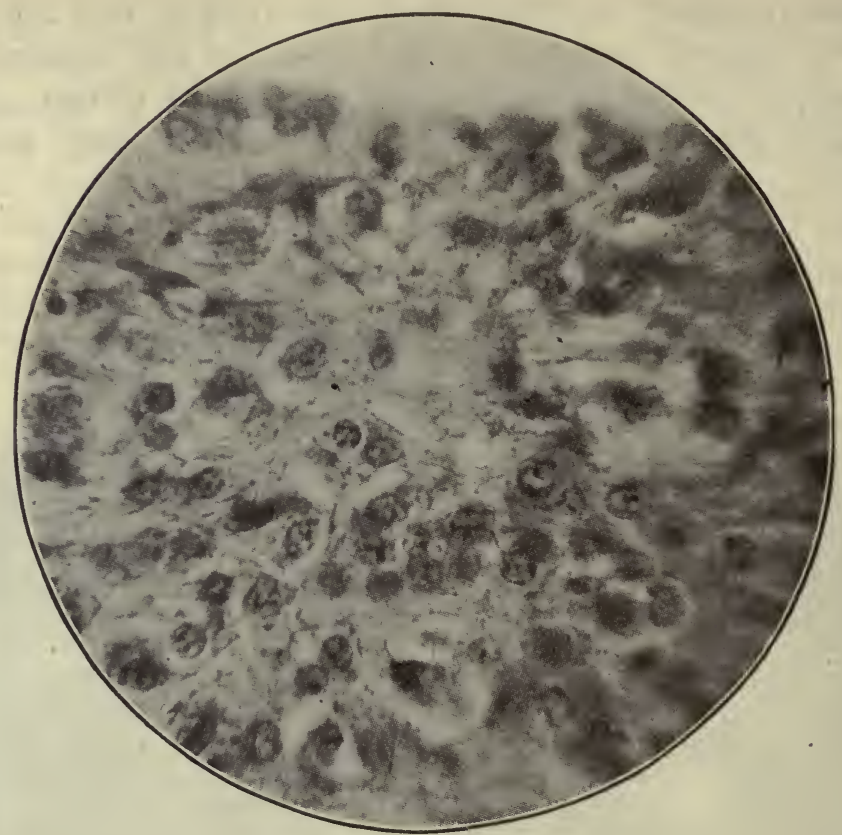

Fig. 501.-Photomicrograph of Peripheral Region of Pineal Body of a Yearling Sheep, to Show the Character of the Parenchyma, the Nedroglia Cells and Fibers, and the Interneuroglia Cells.

In older specimens large lamellated masses of brain sand (acervulus cerebri) appear, consisting chiefly of calcium carbonate and magnesium phosphate. These specimens are also characterized by large edematous

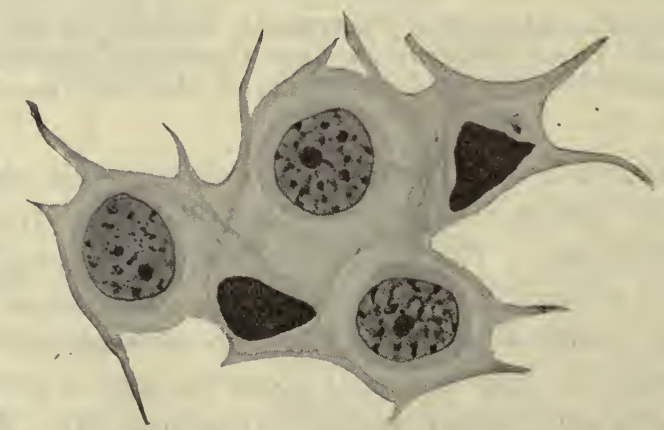

Fig. 502.-Two Neuroglia and Three Interneuroglia Cells from the Pineal Body OF A LAMB. $\times 1500$. 
areas. The interneuroglia cells have been variously designated as 'pineal cells,' 'epithelial cells,' 'lymphoid cells' and 'secretory cells.'- Certain workers claim to have distinguished, in certain mammals, two types of cells, basophils and acidophils. Occasionally smooth and striped muscle fibers have also been described in a few forms (e.g., ox). No nerve cells are demonstrable. Sympathetic nerves accompany the bloodvessels; and a few of the medullated type are found near the attached

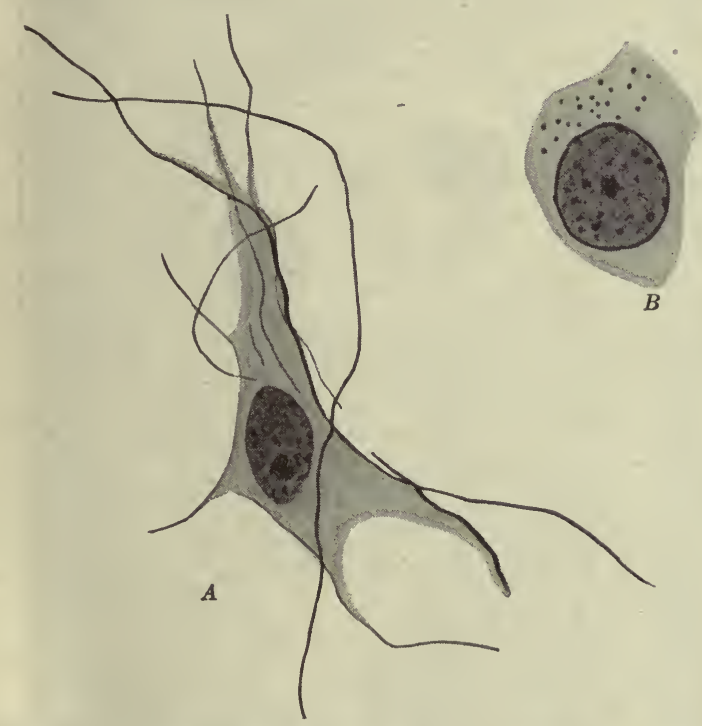

Fig. 503.-Cellls from Pineal of Yearling Sheep.

$A$, neuroglia cell and fibers; $B$, interneuroglia cell with melanic pigment granules. $\times 1500$.

portion, probably taking origin from the habenular and posterior commissures. The blood supply is very profuse. It takes origin from the pial vessels which are in union with the blood-vessels of the tela choroidea anteriorly. The vessels follow the ramifications of the pial septa. In the sheep the capillaries frequently terminate in the form of tangled loops or 'glomeruli' within spaces surrounded by more compact parenchyma ('follicles'). Cross sections of the coarser trabeculæ show a pair of vascular comites.

The dependence of normal liver function upon the integrity of the ductless glands is indicated by derangements following their removal (Whipple and Christian, Jour. Exp. Med., 29, 3, 1914). Many other 
organs (e.g., brain, and digestive system) no doubt hold a similar dependence upon the secretions (hormones) of the ductless glands. Moreover, the ductless glands themselves seem to sustain interdependent relationships among themselves and with the genital glands.

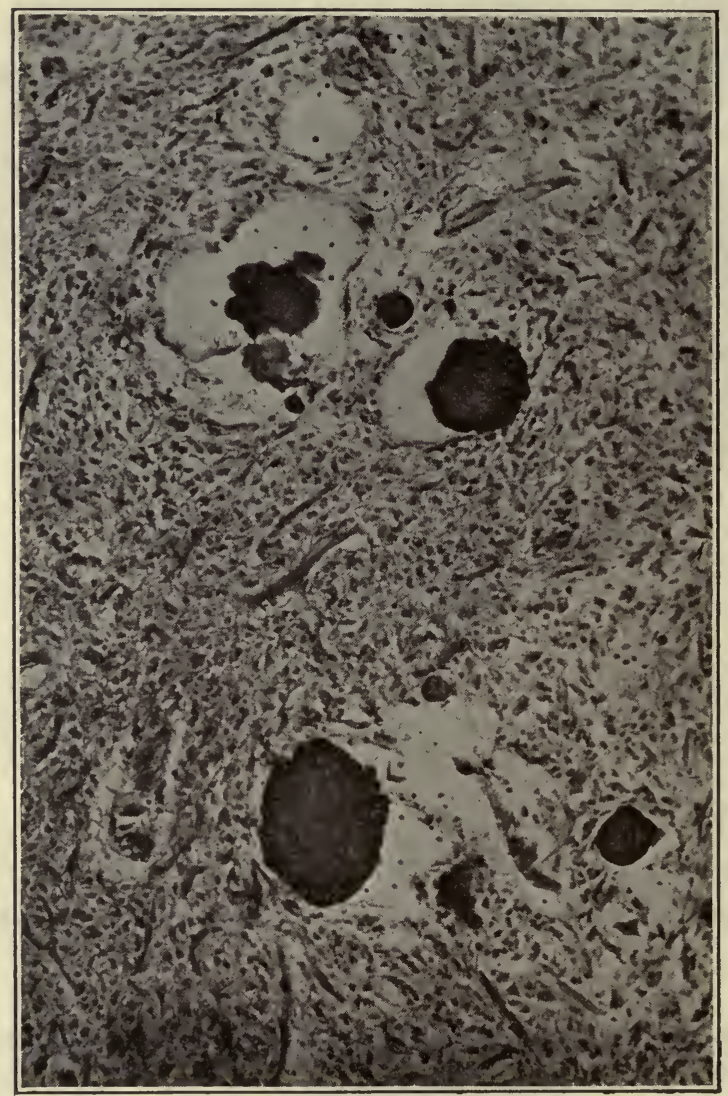

Fig. 504.- Section of Pineal Body of an Old Sheep, Showing 'Brain Sand' (Acervulus) in the Parenchyma.

(For recent reviews of literature on the epiphysis cerebri see Kidd, "The Pineal Body: a Review," Re. Neur. Psyc., Jan. and Feb., 1913; and Jordan, "Results of Recent Studies of the Mammalian Epiphysis Cerebri," Trans. Amer. Micr. Soc., 31, 4, 1912.) 


\section{CHAPTER XVII}

\section{THE NERVOUS SYSTEM}

\section{THE DEVELOPMENT OF THE NERVOUS SYSTEM}

The first external evidence of the beginning of the nervous system is the appearance of a median longitudinal furrow in the dorsal (neural) ectoderm of the very young embryo (about the fifteenth day in man). This is known as the neural or medullary groove. Still e a rlier stages, as revealed in sections, include first an increase in height of the ectodermal cells to form an axial plate of cuboidal cells, the neural plate; and secondly the conversion of this simple layered plate into a structure of several strata of cuboidal cells, which structure meanwhile forms the floor and lateral walls of the developing neural groove. The neural plate apparently grows more rapidly laterally causing thus a progressive elevation of its borders, the neural folds, and producing in consequence a gradually deepening neural groove (Fig. 506). According to Glaser (Anat. Rec., 8, 12, 1914), the inequality in growth. between neural plate and the adjacent ectoderm which causes folding is due to greater water absorption on the part of the plate. By continuation of this process of unequal growth medially

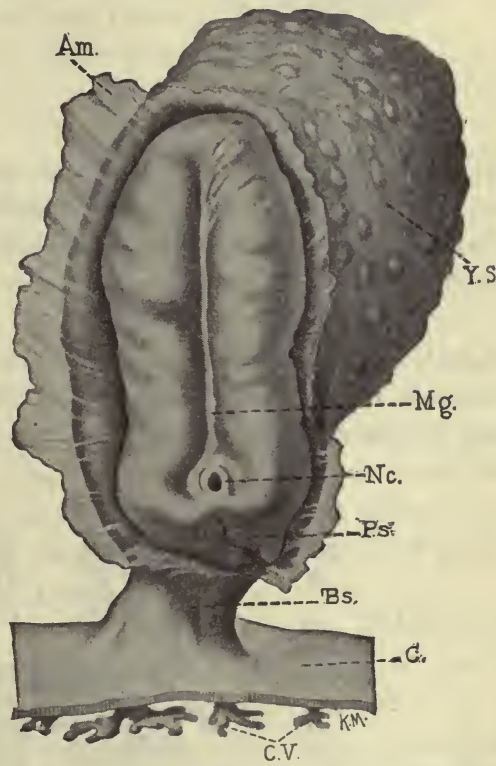

Fig. 505.-Human Embryo 2 MiluiMEters Long. (Graf Spee.) $\times 30$.

$A m$, amnion; $C$, chorion; C.V., chorionic villi; $B s$, body stalk (embryophore); $M g$, medullary groove; $N c$, neurenteric canal; $P s$, primitive streak; $Y s$, yolk-sac. (From Williams.) and laterally, the folds finally meet in the dorsal mid-line and fuse to form the neural tube. On both sides of the line of fusion a group of ectodermal cells appears, the neural or ganglionic crest, from which arise the neurons of the dorsal and sympathetic 
ganglia, and the neurolemma (sheath) cells of both the afferent and efferent fibers of the dorsal and ventral roots respectively. The neural tube (canal) is originally of approximately uniform caliber throughout; but shortly the

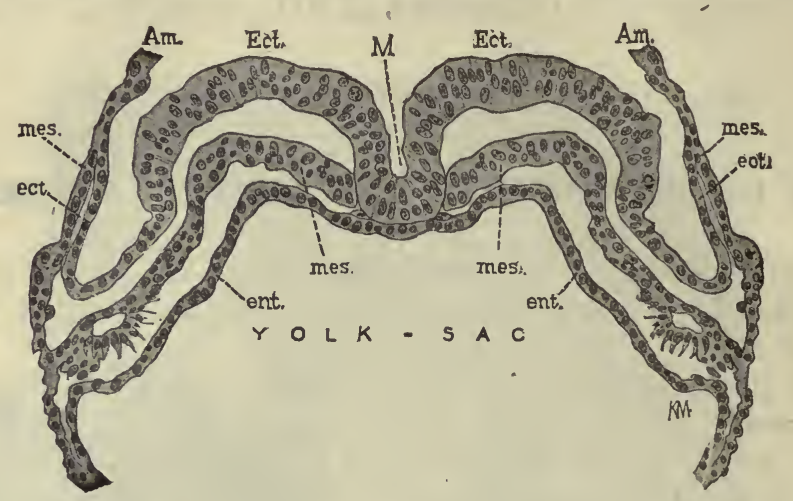

Fig. 506.-Transection throdgh the Graf Spee Embryo Shown in the Preceding Figure.

$A m$, amnion; ect, ectoderm; mes, mesoderm; ent, entoderm; $M$, medullary groove. (From Williams.)

cephalic end, by a series of processes involving dilatations, constrictions, foldings and fusions, develops into the brain; while the caudal portion gives rise to the spinal cord.

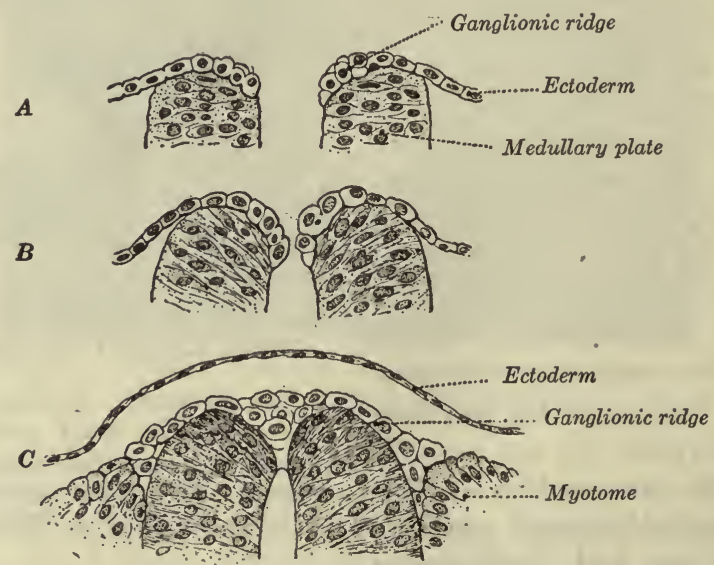

Fig. 507.-Three Successive Stages in the Process of Closure of the Medullary (Neural) Groove to Form the Medullary (Neural) Canal and Neural (Ganglionic) Crests.

(From Barker, after M. von Lenhossek.) 
These gross developmental changes-more appropriately considered in detail in works of Embryology-involve histogenetic processes in the walls of the canal. The earlier of these processes are essentially alike in both cerebral and spinal portions of the canal. Originally the ectodermal layer, which subsequently forms the wall of the tube, consists of a single layer of cuboidal cells similarly undifferentiated. Shortly spherical and oval germinal cells appear among the primitive ependymal cells. These indifferent cells migrate peripherally so that two layers can now be distinguished in the wall; an inner nuclear and an outer non-nuclear or marginal layer. Cell boundaries have meanwhile become obliterated and the entire wall is now essentially a dense syncytium. Still later the syncytium assumes a looser texture, forming the myelospongium, and the indifferent germinal cells give rise to two types of cells: the neuroblasts, from which differentiate the neurons; and the spongioblasts, from which the neuroglia cells and fibers develop.

Shortly after the time when neuroblasts and spongioblasts first become distinguishable the wall of the neural tube can be conveniently divided into three zones: (1) the ependymal layer, forming internally also a limiting membrane; (2) the inter-

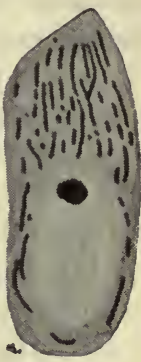

Fig. 508.-CELI LiNING THE NEÛRAL Canal of the NewLY-HATCHED RAINBow Trout, ShowING Mitochondria IN AN EMBRYONIC Nerve Cell.

Meves' technic. $X$ 2000. mediate, middle, nuclear or mantle layer, containing neuroblasts, spongioblasts and indifferent cells; and (3) the outer non-nuclear, or marginal layer, bounded externally by an outer limiting membrane. A glia frame-

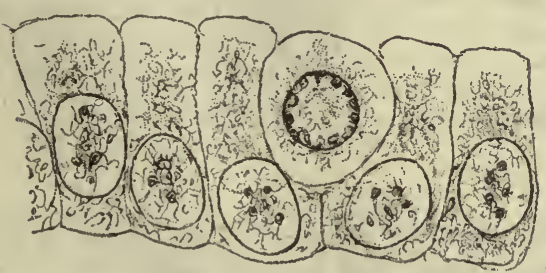

Fig. 509.-Section through Medullary Plate of a Rabbit Embryo.

The cells include a large round germinal cell. (After His.) work pervades the entire width of the wall.

Neuroglia cells differentiate from spongioblasts by a process involving chiefly the formation of glia fibers in their exoplasm. These fibers apparently arise from spongioplasmic fibrils of the protoplasm. Many glia fibers subsequently become disposed extracellularly. The primitive ependymal cells become greatly modified into stout, slightly branching fibers extending through the width of the walls of the neural tube. The main body, of columnar shape, with its nucleus and a distal tuft of cilia, retains its central position and forms an ependymal lining for the spinal canal and the ventricles of the brain. The several neuroglia constituents (astrocytes and 
glia fibers) form a loose-meshed syncytium, the myelospongium, for the support of the developing neuroblasts. Peripherally it is of denser texture and relatively free of nuclei, and is designated the marginal velum. Coincident with this process of neuroglia histogenesis, the neuroblasts enlarge, proliferate extensively and differentiate into neurons, their processes-axon and dendrons-arising as sprouts from the originally spherical neuroblasts.

The motor neurons remain located in the ventral horn of the spinal cord (Fig. 515), their axons (efferent fibers) passing out through the ventral root to unite with the fibers of the dorsal root

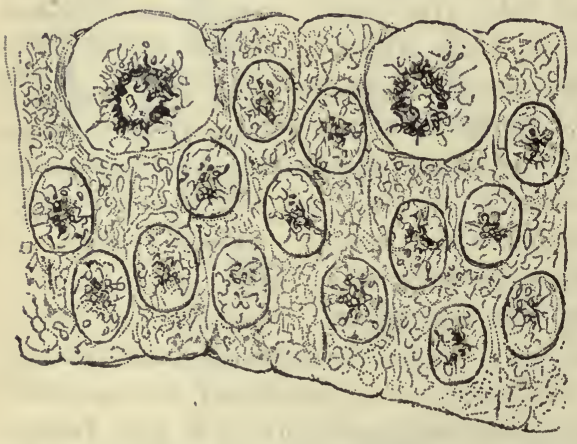

Fig. 510.-Section throdgh Medullary Plate of Closing Neural Groove of Rabbit Embryo. His.)

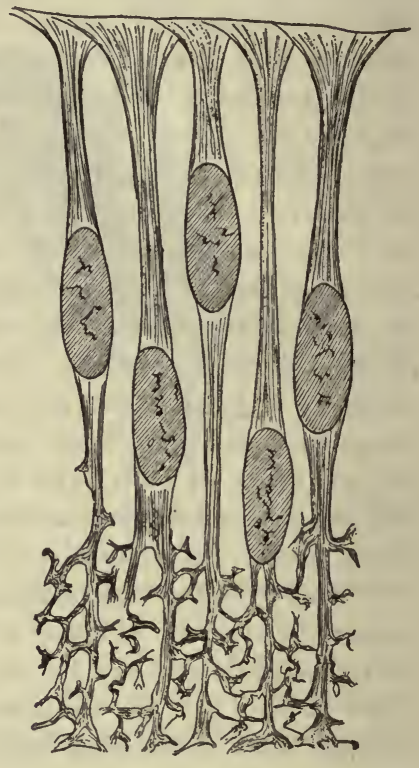

Fig. 511.-SECTION THROU'AH Wall of Later - Neural TUBE of RabBit EMbryo, Showing a Stage in the Differentiation of the Ependyma Celis AND THE Formation of A MYeLOSPONGIUM.

(After His.)

to form a spinal nerve. The sensory neurons arise external to the cord from neuroblasts of the linear neural crests, and migrate some distance ventrolaterally, where they aggregate into metameric oval masses which differentiate into the spinal ganglia. The differentiation process includes both capsule and neuron formation. The sensory neuron located in the dorsal (spinal) ganglia is also originally spherical or oval; subsequently it becomes bi-polar; and finally, by a process involving the retraction of the cell body (cyton) from its two processes and the fusion of these processes proximally, it; becomes a unipolar cell. The definitive axon thus divides in the manner of a $\mathrm{T}$ or $\mathrm{Y}$. Its central process, or axon proper, passes into the dorsolateral portion of the cord as an afferent fiber and here makes 
contact through a telodendrion either directly or indirectly (through association neurons) with the dendrons of motor neurons. Its peripheral process is to be regarded as the dendron, modified structurally so as to be essentially identical with the axon.

The sympathetic may be regarded, as a cenogenetic addition to the cerebrospinal nervous system. Ontogenetically it appears in man at the

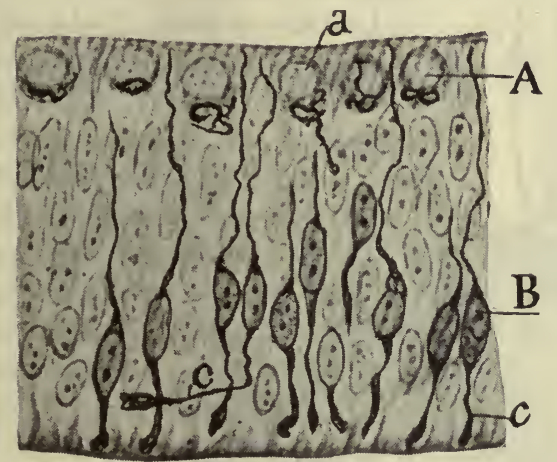

Fig. 512.-Section of Wall of Forebrain of Four-day Chick Embryo.

$A$, apolar neuroblast; $B$, bipolar neuroblast; $a$, the beginning of the sprouting of the axon; $c$, axons, showing the terminally expanding growth area ('cone of growth'). Cajal's silver technic. (Heidenhain, after Cajal.)

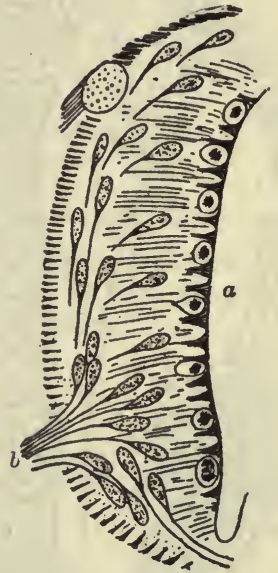

Fig. 513.-Diagram of a Transection of the Spinal Cord of aN Early Embryo, Showing the MigraTION OF NeUROBLASTS Toward the MargiNaL Veil, AND THE Ventral Nerve Rंоот.

$a$, neural canal; $b$, ventral root. (After His.)

beginning of the fifth week (7 mm. embryo). Phylogenetically, it first appears in cyclostomes. Kuntz (Jour. Comp. Neur. and Psyc., 29, 3, 1910) traces the neurons of the sympathetic trunks and the prevertebral plexuses to neuroblasts in both the neural crest and the medullary wall, from whence they migrate by both the dorsal and ventral nerve roots. The neurolemma cells, as also the capsular elements of the sympathetic neurons, arise from 'indifferent' cells which migrate from these same locations and proliferate and differentiate along their course. The cardiac plexus and the sympathetic plexuses in the walls of the visceral organs (terminal plexuses; 'vagal sympathetic' plexuses-Kuntz) have their origin, according to Kuntz, in nervous elements which migrate from the hind-brain and the vagus gan- 
glia along the fibers of the vagus nerves. Kuntz believes that indirect embryological and anatomical evidence warrants the conclusion that the sympathetic excitatory neurons arise from cells which migrate from the neural tube along fibers of the motor nerve roots, while the sympathetic sensory neurons, wherever such neurons exist, arise from cells which

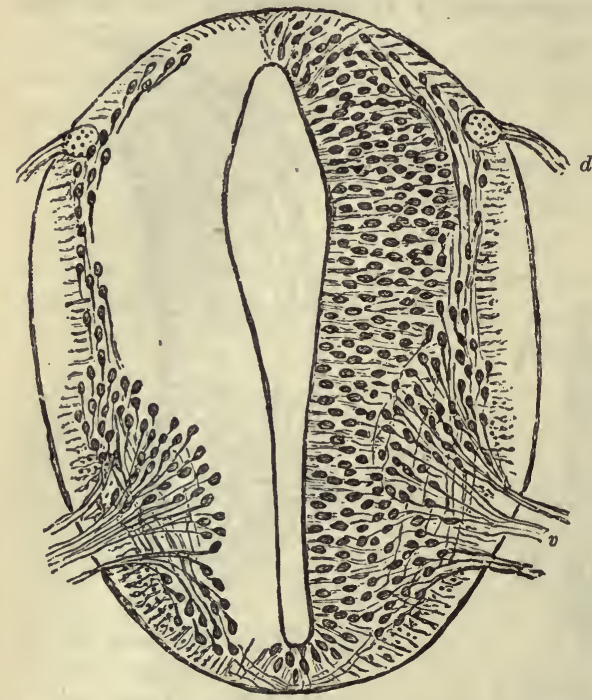

Fig. 514.-Transection of the Spinal Cord of a Human Embryo of Four WEEKS.

The central canal is immediately surrounded by ependyma cells. The peripheral nerve cells are shown on the left of the figure. The ventral nerve roots are already pushing outward from the primitive cord. $d$, dorsal; $v$, ventral nerve roots. (After His.) migrate from the cerebrospinal ganglia.' However, according to Carpenter (1914) the autonomic neurons show no distinct differences from the standpoint of their chromophilic content. Since such differences are conspicuous between the sensory and motor neurons of the cerebrospinal system (Malone), this observation arouses skepticism regarding the presence of sensory sympathetic ganglion cells.

The primary cerebral divisions (Fig. 516) and their major adult derivatives are enu- . merated in the appended outline. The microscopic anatomy of their definitive condition lies outside the scope of this book. These matters are more appropriately treated in special text-books of Neurology (e.g., Quain's "Anatomy," Vol. III, 1908; and Villiger's "Brain and Spinal Cord," translation by Piersol, 1912) and constitute the work of a separate course.

Likewise the developmental history is more advantageously considered in a special course in Embryology.

This work will include only the histology of sections of the spinal cord; and that of the cerebellar and cerebral cortex. 


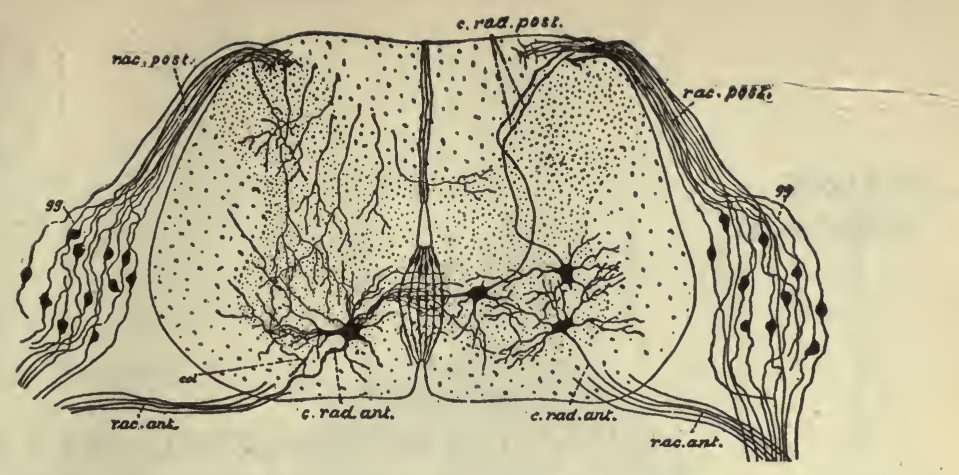

Fig. 515. - Transection of the Spinal Cord of an Embryo Chick.

c. rad. ant., axons to the ventral roots; c. rad. post., axons to the dorsal roots; col, collateral from an axon back to the gray matter; $g g$, dorsal root ganglion; rac. cint., ventral root; rac. post., dorsal root. (After van Gehuchten.)

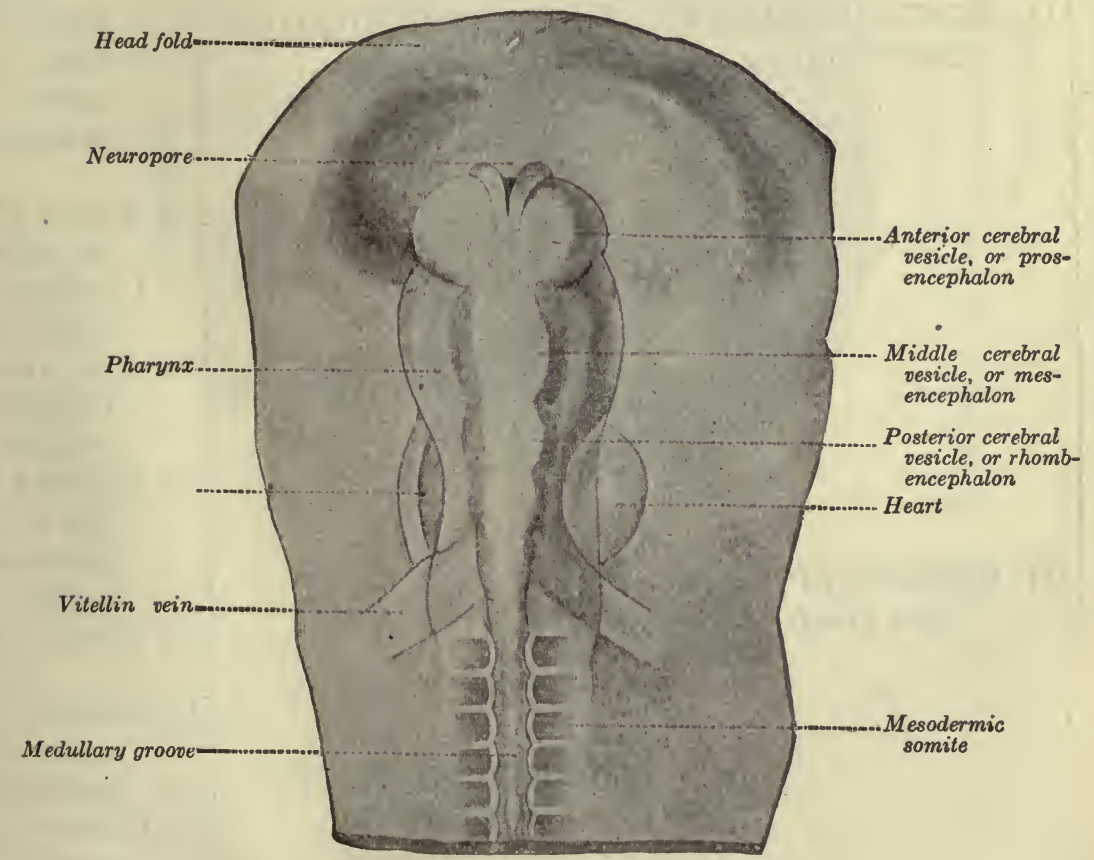

Fig. 516.-Reconstruction of the Anterior Portion of the Body of a Youn Chick Embryo, Seen from the Dorsal Surface. (After Kollmann.) 


\section{A. MYELON}

(spinal cord)
1. Sacral region

2. Lumbar region

3. Thoracic (dorsal) region

4. Cervical region
I. RHOMBENCEPHALON (rhomboid brain)

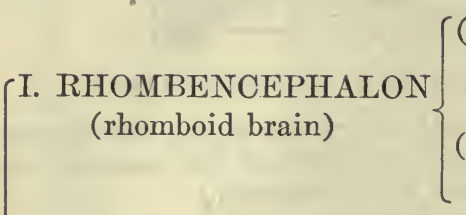

II. MESENCEPHALON (mid brain)

(c) MESENCEPHALON (mid brain)

(b) METENCEPHALON (hind brain)

(a) MYELENCEPHALON $\{$ 5. Medulla (after brain) Oblongata
III. PROSENCEPHALON (fore brain)

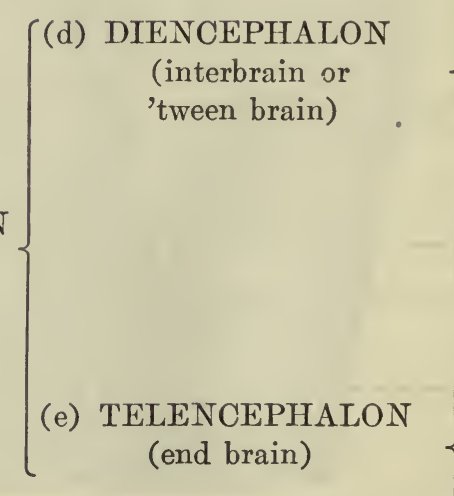

8. Region of the cruræ cerebri (brain stem); corpora quadrigemina

9. Region of the optic thalami (basal nuclei)

10. Optic tract (primitive optic evaginations)

11. Posterior lobe of hypophysis cerebri

12. Pineal body

13. Cerebral cortex (pallium)

14. Corpora striata 15. Olfactory lobes 


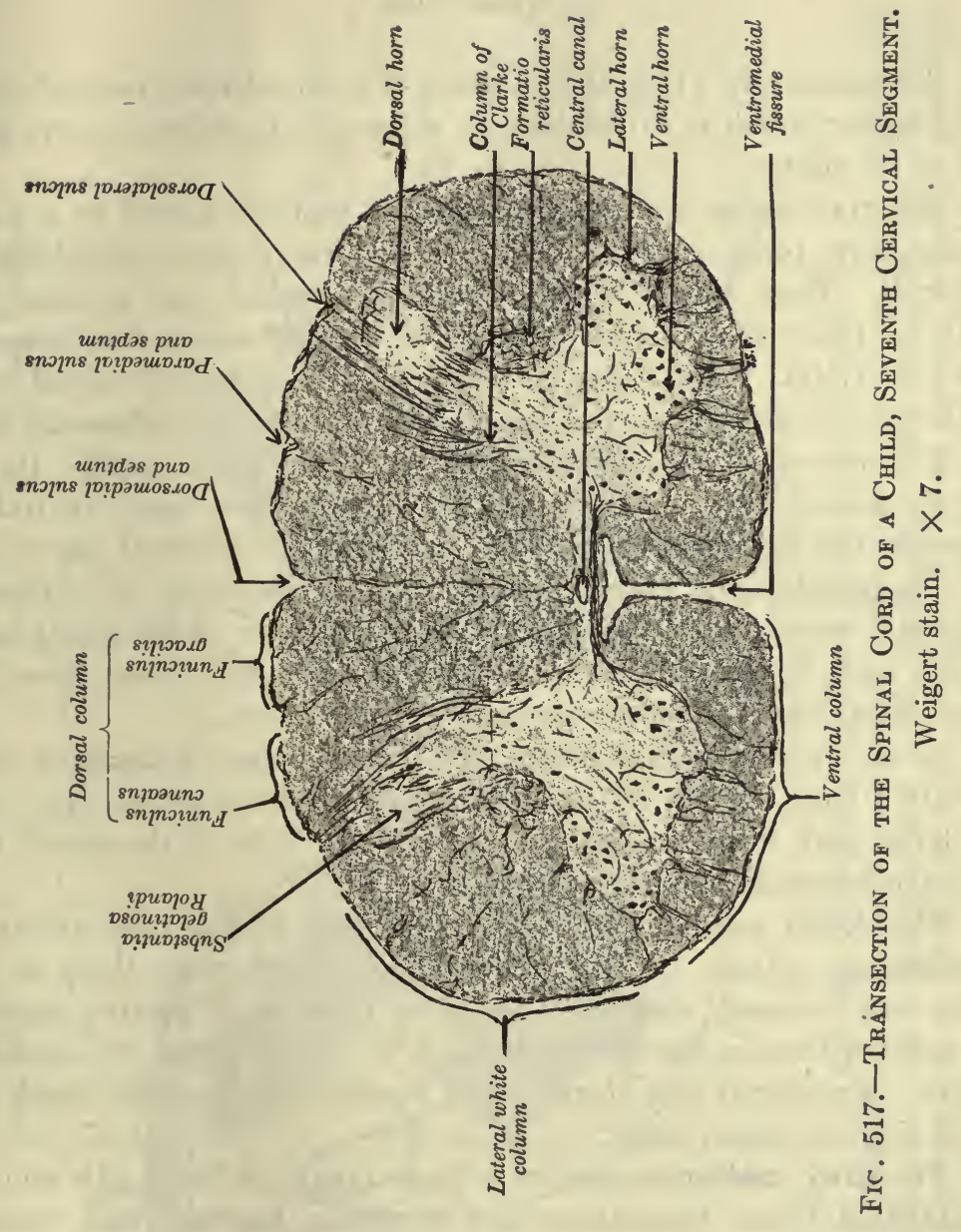




\section{THE SPINAL CORD}

\section{STRUCTURE}

The spinal cord (Fig. 51\%) consists of a considerable mass of central gray matter which is surrounded by a layer of medullated nerve fibers, the white matter.

The gray matter comprises two lateral portions united by a central commissure (gray commissure) in transverse section resembling the letter H. Each lateral portion includes a ventral and a dorsal horn with an intervening deeper portion, the central mass or 'intermediate zone' of Golgi. Each horn or cornu ends in a head or caput united with the intermediate portion or basis cornu by a neck or cervix cornu.

The ventral is somewhat broader than the dorsal horn. Its cells supply axons, which, after uniting into bundles, pass ventralward through the white matter to form the ventral (anterior) nerve roots.

The spinal cord may be considered as consisting of ontogenetic segments whose number corresponds to the number of the spinal nerves. Hence each segment contains the rentral horn cells whose axons form the ventral root of the corresponding spinal nerve.

In an entirely similar manner the dorsal horns of the gray matter receive a large portion of the incoming fibers of the dorsal roots, which in large part form end brushes around the cells of the dorsal horns and the intermediate zone (Fig. 518).

The dorsal roots enter through a distinct longitudinal groove, the dorsolateral sulcus. At the exit of the ventral roots there is, however, only a broad, shallow indentation, these roots making their exit in isolated bundles distributed through a vertical plane of considerable width. The dorsal root fibers of each segment, on the other hand, enter in a single compact mass.

The gray matter consists of a dense tangle of nerve cells and nonmedullated fibers, both axons and dendrons, together with neuroglia and blood-vessels. The fibers of a given area are derived not only from nerve cells in their immediate vicinity, but also include many processes which come from very distant regions. The gray reticulum is thus supplied from fibers of the ventral and dorsal nerve roots, together with innumerable collaterals, not only from the root fibers, but more especially from those fibers which collectively form the many large tracts passing up and down the spinal cord and placing each seg- 


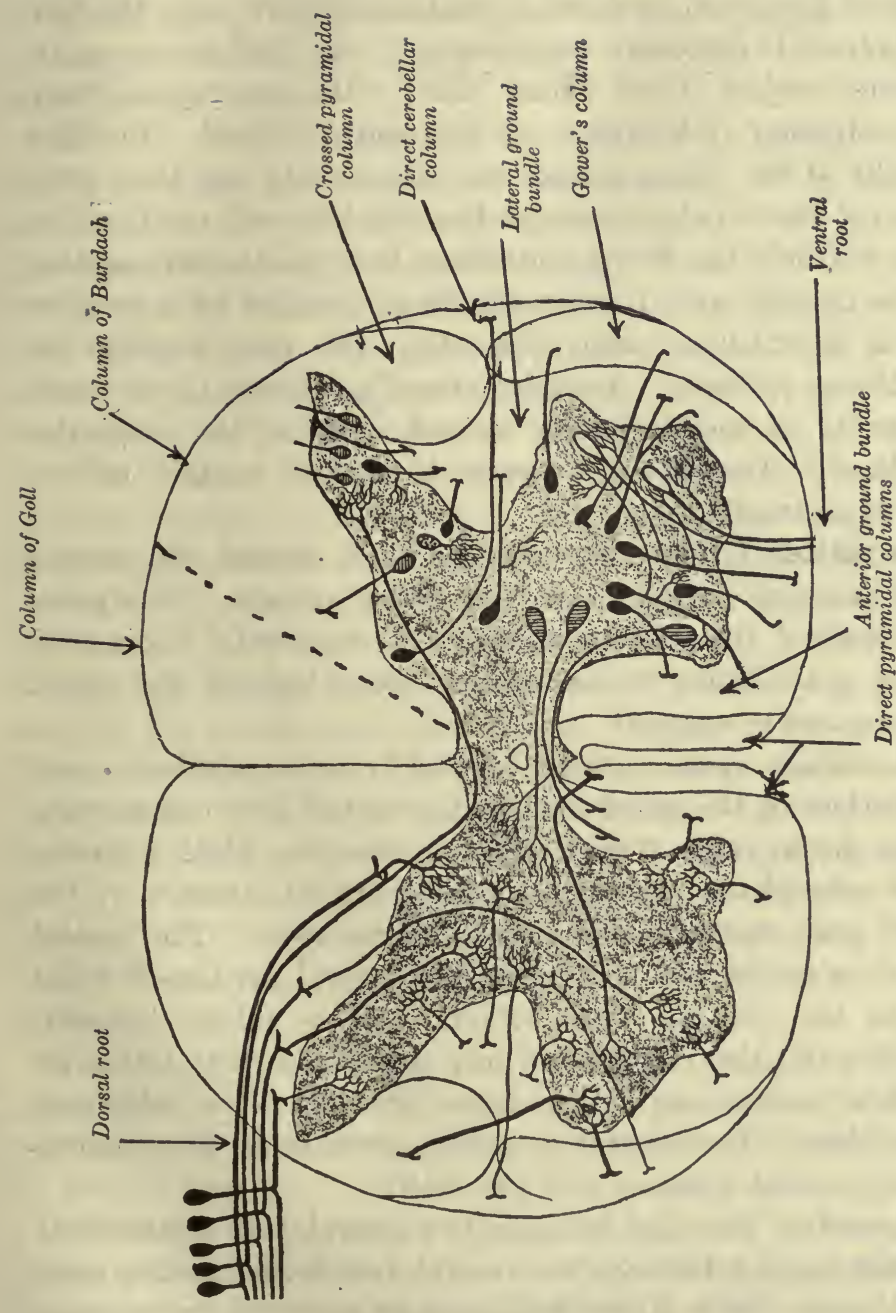

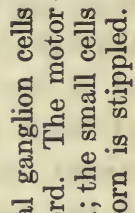

- 등 ठ워

ณี कृ 년 둥 क

누 0

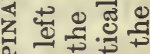

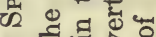

시구 $>$

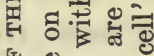

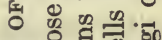

국엉엉

की สె

의 귝.

요 ⿷匚⿱

田

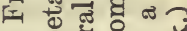

도 응 ठ…

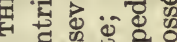

4

०. चี है

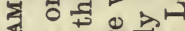

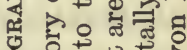

\& 눈

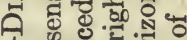

1. 넌 0 을

$\infty$ 경

드

ऽ

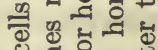

규휴

टै बै 그르 สํํㅇ 잉 영

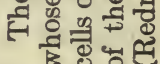


ment in communication with many other levels of both the spinal cord and brain.

The center of the gray commissure contains the central canal which lies in the axis of the spinal cord and is continuous above with the ventricles of the brain. It represents the remains of the fetal neural canal; and in the young subject is still patent, filled with cerebrospinal fluid, and lined by columnar cells which are frequently ciliated. In older subjects the cells of the lining epithelium have usually lost their cilia, and the lumen of the canal is more or less filled by cell proliferation which involves not only the lining epithelium but also the surrounding glia cells. The central canal is immediately surrounded by a peculiar gelatinous tissue in which are many glia cells. This mass is called the substantia gelatinosa centralis. A similar area of gelatinous tissue occurs near the extremity of the dorsal horns, and is called the substantia gelatinosa Rolandi. The latter, however, is said to contain only a scanty supply of neuroglia.

The white matter forms a covering or shell around the central gray mass. It increases in thickness from below upward. This pectliarity is the result of the progressive influx of centripetal fibers, and a corresponding contribution of centrifugal fibers, through the spinal nerves of each successive segment.

The dorsal median septum extends inward from the shallow sulcus on the dorsal surface of the spinal cord to the central gray commissure, and divides the dorsal mass of white matter into two white columns, lying on either side of the median line, and bounded laterally by the dorsal horns of gray matter and the dorsal nerve roots. The ventral median sulcus in a similar manner, splits the ventral portion of white matter into the two ventral white columns. This sulcus, however, does not penetrate all the way to the gray commissure but leaves an interval of white matter containing many transverse and obliquely disposed nerve fibers. The ventral or white commissure thus formed connects the two ventral columns of white matter.

The spinal cord is thus divided into two lateral and symmetrical halves by a plane passing through the ventral and dorsal median sulci and the central canal. Each lateral half includes a central curved mass of gray matter completely surrounded, except at the gray commissure, by the white matter. The latter is subdivided into a ventral, lateral, and dorsal column, each of which extends the entire length of the spinal cord and is apparently (to the naked eye only) continuous above with a similar column in the medulla oblongata. 
The ventral white column is included between the ventral median sulcus and the ventral gray horns and nerve roots; the lateral columns extend from the ventral roots in front, around the lateral surface of the spinal cord, to the dorsal roots; the dorsal columns are included between the dorsal horns of gray matter and dorsal nerve roots, and the dorsal median septum.

Each of these columns of white matter is again subdivided by neurogliar septa of variable size and number, which extend inward from the pia mater for a considerable distance. Such septa may even penetrate all the way to the central gray matter. One of these septa, the paramedian septum, more constant than the others, subdivides the dorsal column into two portions, a dorsomedial and a dorsolateral column, or the funiculus gracilis (column of Goll), and the funiculus cuneatus (column of Burdach) of the upper portions of the cord.

In the ventral white column two chief fiber tracts are recognized: a narrow median, the anterior or direct pyramidal tract; and a more lateral larger anterior ground bundle. In the lateral column four main tracts are recognized: three lateral, including an upper crossed pyramidal tract, a middle direct cerebellar tract, and a lower or Gower's column; and a large medial lateral ground bundle (Fig. 518). These tracts do not possess sharp boundaries. Moreover, the ground bundles at least are composite. The several elementary tracts contain fibers passing either from the periphery to the brain along the cord (ascending fibers) or from the brain through the cord to the periphery (descending fibers), or fibers connecting brain and cord or different segments of the cord. For a detailed description of the constitution of these fiber tracts reference must be made to the systematic text-books on the nervous system.

The larger blood-vessels are distributed along the fibrous septa, taking their origin from the vessels of the pia mater; the most of them are distributed to the white matter, but to some extent they also supply the gray matter.

The entire surface of the spinal cord presents, just beneath the pia mater, a thin superficial layer or marginal veil of glia tissue. In the brain this layer is somewhat exaggerated in thickness.

\section{The Regions of the Spinal Cord}

The varying number of fibers which are given off at different levels of the spinal cord results in considerable differences in size in its several portions. By means of these peculiarities, as well as by the spinal 
nerve roots to which they give origin, we distinguish a sacral, lumbar, thoracic, and cervical region. Each of these regions presents certain

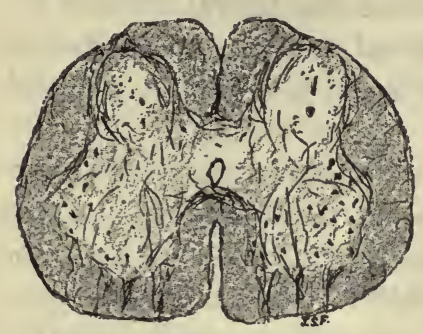

Fig. 519.-Transection of the Spinal Cord of a Child, Third Sacral Segment.

Weigert stain. $\times 7$. more or less important morphological characteristics.

In the sacral region the investment of white matter is very thin, the gray matter-though actually less in amount than in the more cephalad regions-appearing large by comparison. Both the ventral and dorsal horns of gray matter are short and thick. The substantia gelatinosa Rolandi is of considerable volume. The cell groups in the ventral horns of this region are a ventromedial and a dorsolateral.

The cord as a whole is small and its transection nearly circular in outline. The five segments of this region contain the neuron centers for the urinary bladder, the anus, some of the musculature of the lower limbs, and the sensory reflexes of the perineum and genitourinary organs.

Below the sacral region the spinal cord tapers rapidly (conus medullaris) and is continued downward for a considerable distance as the filum terminale. The surrounding leash of lumbar and sacral nerve roots forms the cauda equina. The fibrous membranes which surround the cord continue even farther downward in the medullary canal to form the central liga-

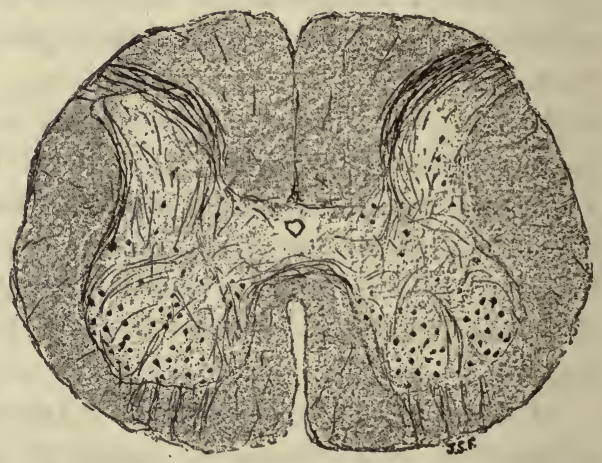

Fig. 520.-Transection of the Spinal Cord of a Child, Fifth Lumbar SegMENT.

Weigert stain. $\times 7$. ment, which is finally attached to the sacrum or coccyx.

In the lumbar region there is a distinct enlargement, chiefly involving the gray substance, which here includes the immense number of cells of the ventral horns whose 'motor' fibers enter the large lumbar nerve trunks for the supply of the lower limbs. These nerve trunks also supply to the cord a great number of centripetal or sensory fibers 
which enter the dorsal and, later (through secondary neurons), the lateral columns; thus both of these columns are of large size in and above the lumbar region. The dorsomedial column in this region attains an appreciable size, and a distinct pial septum marks its lateral boundary.

The spinal cord is now nearly circular in transection, its ventrodorsal being perhaps slightly less than its transverse diameter (10 $\mathrm{mm}$.). The gray commissure lies very near the middle of the spinal cord, and the ventral median fissure is, therefore, quite as deep as the dorsal median septum.

Both the ventral and dorsal gray horns are long and thick. Each dorsal horn contains a large apical area of gelatinous substance, is somewhat longer on its lateral than on its medial side, and reaches nearly to the dorsal surface of the spinal cord,

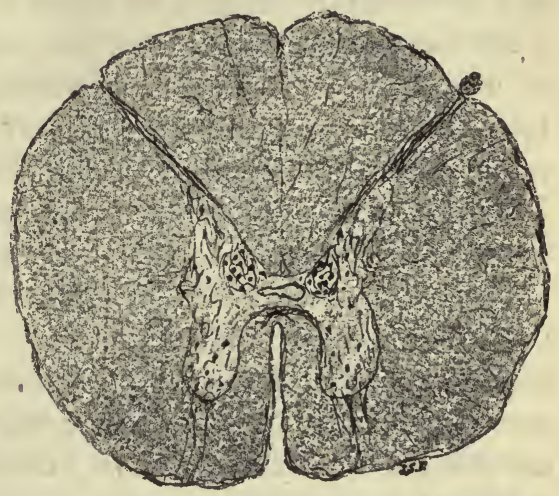

Fig. 521.-Transection of the Spinal Cord of a Child, Eighth Thoracic Segment.

Weigert stain. $\times 7$. opposite the dorsolateral sulcus.

The dorsal nerve roots entering at this level are apparently directed toward the middle of the tips of the dorsal horns of gray matter; once within the spinal cord they pass around to the mesial side of the dorsal horns.

The ventral horns, somewhat larger than the dorsal, present two short and broad protuberances, the one at the ventromesial, and the other and more prominent at the ventrolateral angle. A similar though less prominent protuberance is seen at the base of the ventral horn, on its lateral aspect. Each of these projections contains a more or less well-defined group of motor nerve cells. The cell groups of the ventral horns in the lumbar region are therefore a ventromedial, ventrolateral, and dorsolateral, together with an ill-defined central group occupying the deeper 'intermediate zone' of gray matter.

The nerve centers contained in the lumbar region control the reflexes and musculature of the lower limbs and the lower part of the abdominal wall.

A transection of the spinal cord in the thoracic region is of small 
diameter (eight millimeters), and is very nearly circular in outline. The white matter, since it contains the many nerve fibers going to and coming from the lumbar enlargement, is much more voluminous than the gray matter. The latter is reduced to a comparatively small central mass.

The dorsomedial column attains a considerable size in this region, and is distinctly marked off from the adjacent dorsolateral column by a fibrous septum derived in part from the pia mater, in part being of neuroglia composition. The dorsal and the lateral columns, having been much augmented by the influx of fibers from the large dorsal roots of the lumbar nerves, form the larger part of the white matter. The gray matter consequently appears to be pushed forward, its gray commissure lies considerably ventrad of the center of the spinal cord, the ventral median fissure is shorter than the dorsal median septum, and the tips of the dorsal gray horns are far removed from the surface, being only connected with the dorsolateral sulcus by the slender dorsal nerve roots. In fact, the dorsal horns of gray matter in this region are reduced to a minimum size; they are short and slender and contain comparatively few nerve cells.

At the base of each dorsal horn, on its mesial side, there is a distinctly outlined cell group whose transection is of oval or circular outline. Indeed, this cell group, the cell column of Clarke, begins in the second or third lumbar segment, and is continued upward to the second or third thoracic-at times even into the lowermost cervical segments - at which level it has dwindled to a relatively insignificant group. In the lower lumbar region an ill-defined group of cells occupying a similar position and having the same function is known as the nucleus of Stilling.

The ventral gray horns are very short and narrow, and their cells cannot be subdivided into groups as in the other regions of the spinal cord. In the upper part of the thoracic region a distinct protuberance makes its appearance at the base of the ventral horn, on its lateral aspect. This is the precursor of the larger lateral horn of the cervical region. In the upper thoracic region it contains a small cell group, the dorsolateral.

The nerve centers of the thoracic segments control the upper abdominal region, the thorax, and the viscera.

In the lower half of the cervical region the spinal cord presents a distinct enlargement, within the gray matter of which are the nuclei for the upper limbs. The spinal cord in this region is somewhat flat- 
tened, its transverse diameter $(14 \mathrm{~mm}$.) considerably exceeding its anteroposterior. The major portion of the white matter is still contained within its dorsal rather than its ventral portion, the gray commissure appearing to lie somewhat ventrad of the center. The dorsal median septum dips inward for a much greater distance than does the ventral median fissure.

The dorsal columns are decidedly larger than the ventral, and a distinct groove, the paramedial sulcus, from which a fibrous septum is continued inward, separates the dorsomedial from the dorsolateral. column.

The dorsal gray horns are long, relatively slender, and more divergent than in the lower levels. They do not reach the surface of the spinal cord, but are connected therewith by the long, slender dorsal nerve roots. The gray matter of the dorsal horns in this region is more or less invaded by bundles of nerve fibers derived from the lateral and dorsal columns; the tips of the dorsal horns are thus almost cut ofi from the deeper portions of gray matter.

The ventral horns are both long and broad. They present three noticeable promontories or processes-a medial (ventromedial), a ventral (ventrolateral), and a lateral. The lateral, because of its special prominence, is frequently called the lateral horn; it is one of the noticeable characteristics of the cervical region.

Each of these processes contains a corresponding cell group; hence we distinguish in the cervical enlargement a mesial, a ventral, and a lateral group, together with a small intermediolateral, which is partially or completely detached from the dorsal portion of the lateral group. There is also a small disseminated central group of nerve cells occupying the deeper portion of the ventral horn.

The nuclei of the segments included in the cervical enlargement contain the centers for the musculature and sensory reflexes of the upper limbs. The partial control of the pupillary movements in the eye is also located in the lowermost segments of this region.

In the upper half of the cervical region a transection of the spinal cord, except for its larger size, resembles very closely that of the thoracic region. The larger size is due to an increase in the white matter of the dorsal and lateral columns, consequent upon the acquisition of new fibers which enter the subjacent segments from the nerves supplying the upper extremities, together with an increased number of centrifugal fibers from the cerebrum which are distributed to the gray matter of this region. 
The ventral columns are also much increased in size by the addition of many fibers coming down from the medulla and cerebellum, which place the nerve centers of the spinal cord in close relation with those of the cranial nerves and with the association centers of the cerebellum.

In addition to the large size of its white columns, a noticeable characteristic of the upper cervical region is the prominence of its lateral

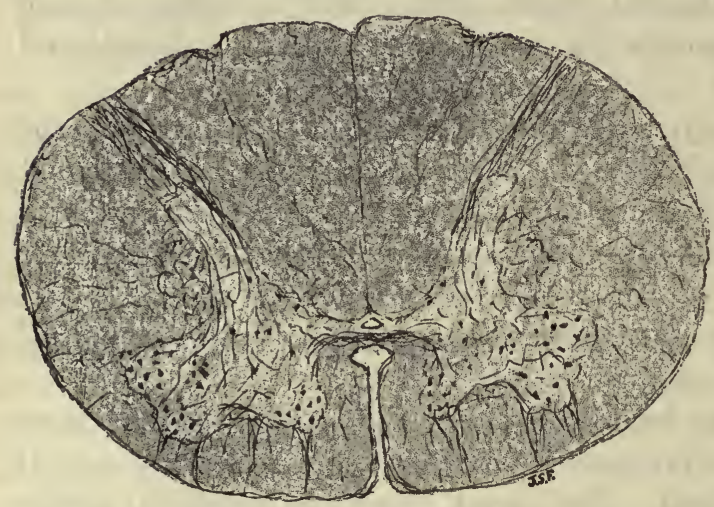

Fig. 522.-Transection of the Spinal Cord of a Child, Fourth Cervical Segment.

Weigert stain. $\times 7$. horns of gray matter. Just dorsal to the lateral horns is also a peculiar reticular formation which results from an invasion of the adjacent portions of the lateral white columns by bands of gray matter. The gray matter thus forms a coarse network whose meshes inclose isolated bundles of longitudinal nerve fibers.

The ventral horn cells of this region are scarcely divisible into groups, but a large and distinct cell group, the intermediolateral cell column, occupies the so-called lateral horn.

The nuclei of the upper cervical region innervate the skin and muscles of the neck and shoulder, they also supply the diaphragm. The nerve cells of this region not only supply the cervical spinal nerves, but they also send root bundles to the spinal accessory or eleventh cerebral nerve.

\section{THE CEREBELLUM}

The cerebellum consists of an irregular core of white substance, the medulla, and a thick mantle of gray matter, the cortex. It comprises two hemispheres or lobes connected by a third lobe, the vermis. Each lobe is a combination of lobules which include a variable number of transverse convolutions or folia. Each folium contains a medullary 


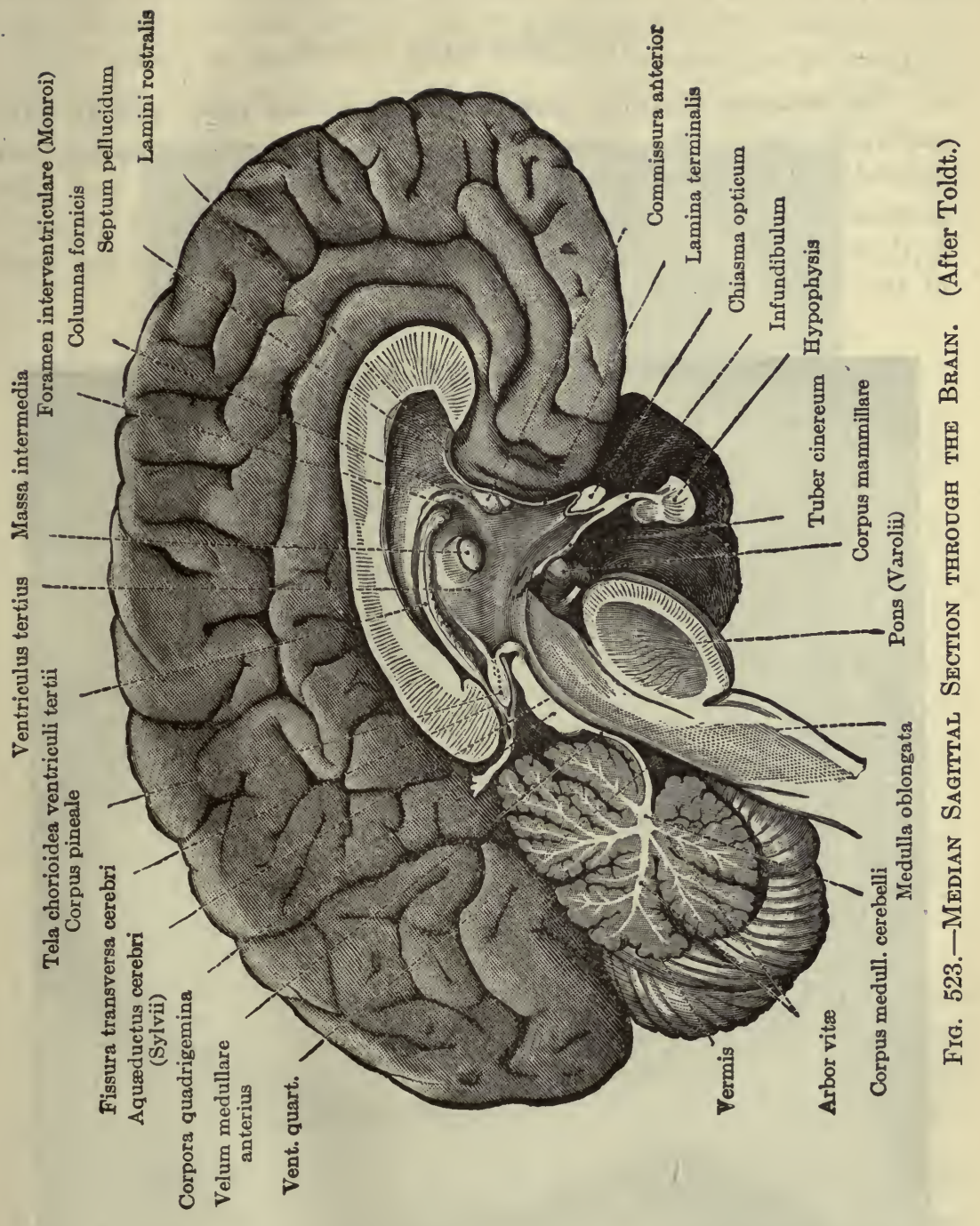


core and is covered by a cortical layer. The central medulla contains four paired masses of gray substance, the internal nuclei.

\section{The Cerebellar Cortex}

The cortex is divisible into an inner granular layer, and an outer molecular layer. The granular layer in a fresh section appears rustcolored; in a stained preparation it appears much darker and more granular than the molecular layer due to the abundance of nuclei; it is thickest over the summit of the folium, and thinnest in the depth of the sulcus between adjacent folia.

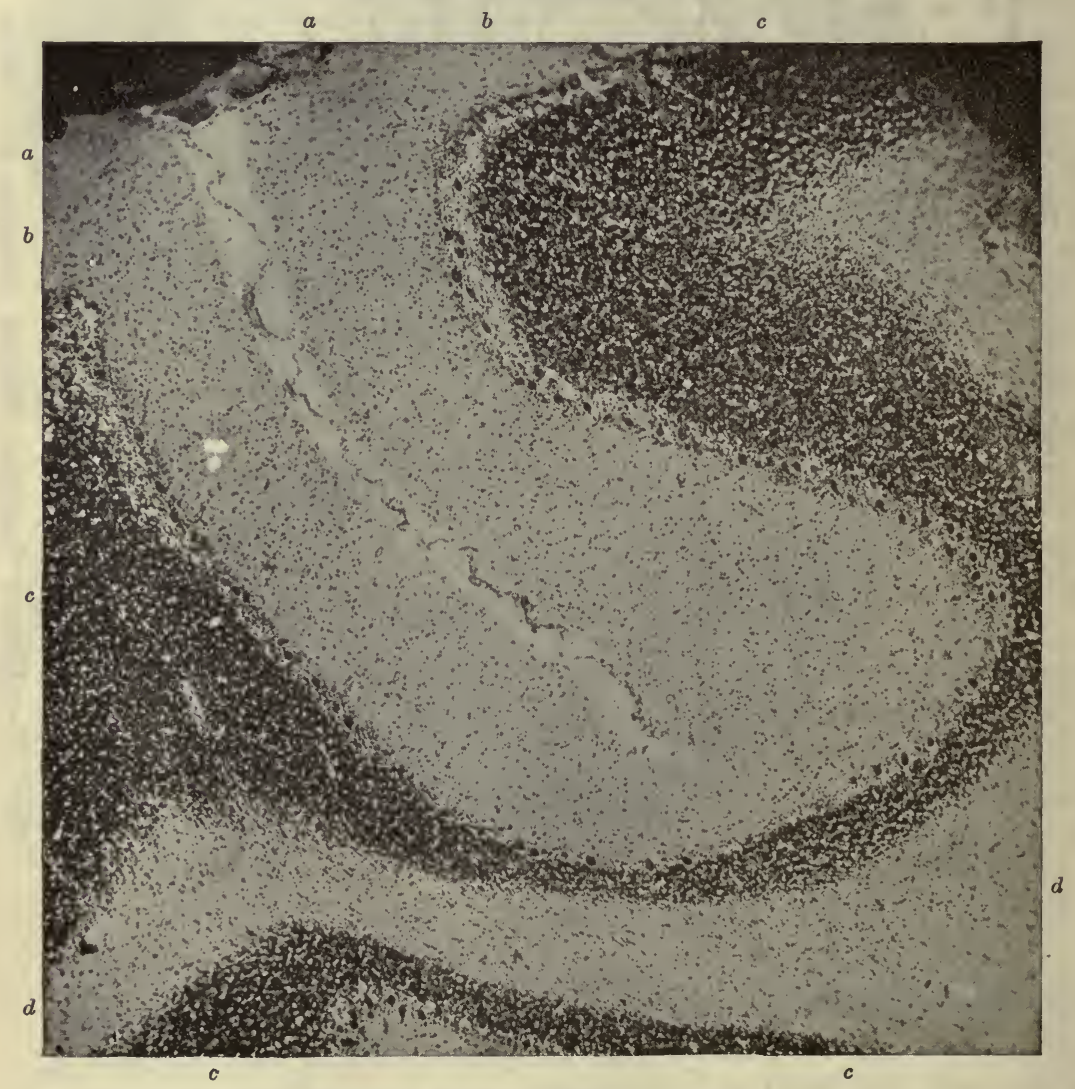

Fig. 524.-From a Section of the Cerebellar Cortex of Man.

$a-a$, pia mater; $b-b$, molecular layer; $c-c$, granular layer; $d-d$, white matter of the medulla. Nissl's stain. Photo. $\times 38$. 
The most conspicuous and distinctive elements of the cerebellar cortex are the Purkinje cells, situated along a line marking the division between molecular and granular layers. They are regarded as belonging to the molecular layer. The remaining cellular elements of the cortex cannot be studied in detail in ordinary histologic preparations. They must be identified mainly by their relative size and position. Our

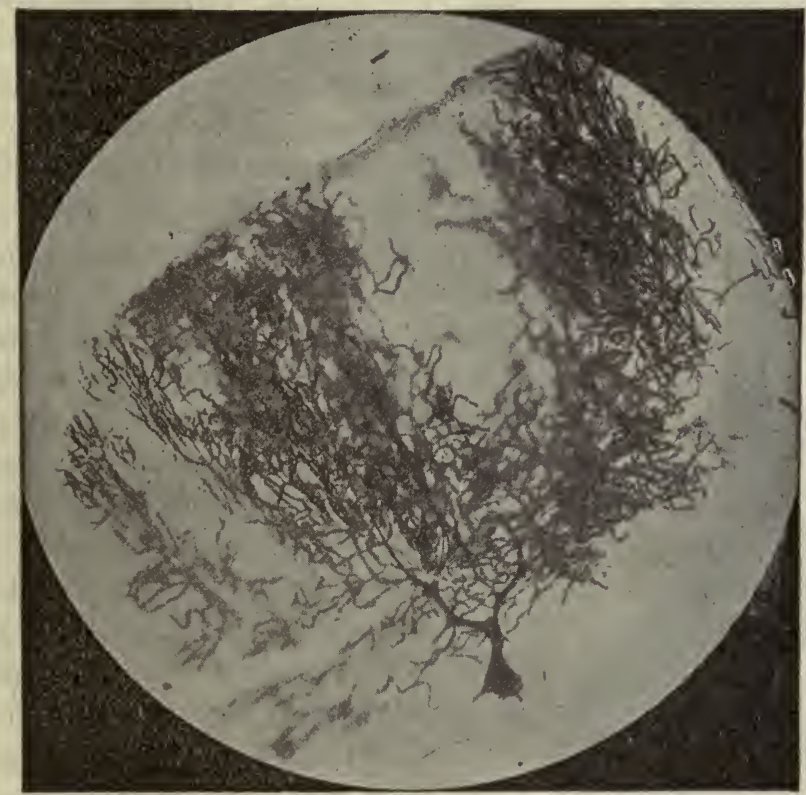

Fig. 525.-A Purkinje Cell from the Human Cerebellar Cortex.

Moderately magnified. Photo. (After Berkley.)

knowledge of their finer structure and their interrelationships has been gained by use of special staining technics, chiefly the Golgi process. By use of this technic two other types of cells can be distinguished in the molecular layer:'the small cortical cells, and the large cortical or basket-cells.

The Purkinje cells are large flask-shaped elements, with typically a robust dendron and a delicate axon at opposite poles. The axon passes centrally to contribute to the white matter 'of the medulla. It gives off numerous collaterals, some of which turn back into the molecular layer and terminate in relation with neighboring cells of Purkinje. The dendron passes toward the surface dividing almost immediately 
into two coarse branches, which each divides dichotomously into a succession of increasingly more delicate branches forming peripherally a dendronic field of extreme profusion of non-anastomosing fibrils. The

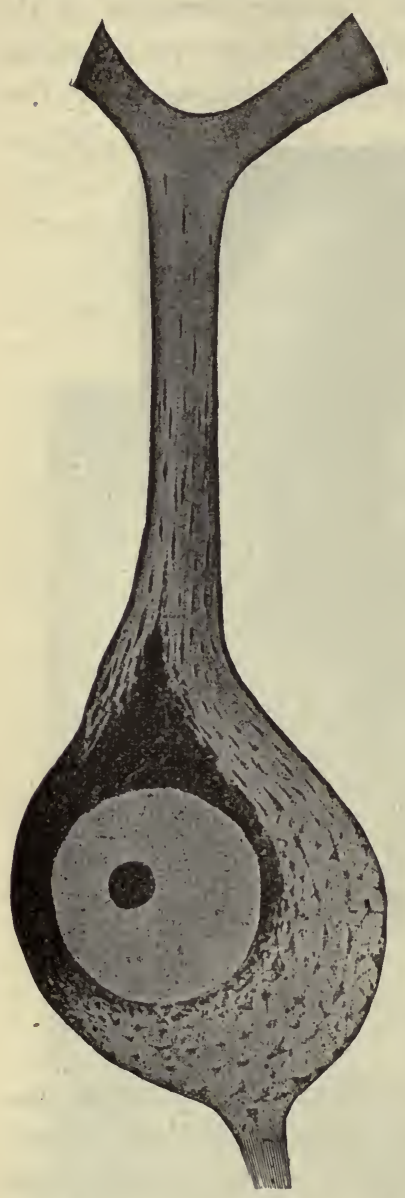

Fig. 526.-A Purkinje Celi From tHe CEREBellar Cortex of the Rabbit.

Highly magnified. (After Nissl.) looped. The axon of some of these cells has numerous collaterals.

The nuclear or granular layer also contains three distinct types dendron viewed as a whole constitutes a fanshaped structure. Its expansion is in a plane at right angles to the long axis of the convolution. In sections parallel with the long axis of the convolution the dendronic field is very narrow, and never wider than the diameter of the cell-body.

The basket-cells, or large cortical cells, are multipolar elements with relatively short robust branching dendrons, and a long axon which passes horizontally in the same plane in which the dendronic expansion of the Purkinje cells are placed. Along its course it gives off five or six collaterals which, as also the post-collateral portion of the axon itself, pass centrally toward the Purkinje cells where each breaks up into a profuse terminal arborization which invests the cell-body in the manner of a 'basket.' The basket cells occupy the middle and outer portions of the molecular layer; they are apparently of the nature of association neurons, perhaps coördinating the function of a number of Purkinje cells.

The small cortical cells are distributed throughout the molecular layer but are more abundant in the outer half. They are multipolar and vary considerably in size, some being almost as large as the basket cells. They possess from two to five delicate dendrons distributed for the most part in the same plane as those of the Purkinje cells. Their short slender axon, which is horizontally placed, is frequently characteristically

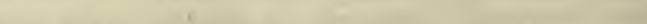


of cells: the granule cells, the large stellate cells and the so-called solitary cells. The latter are small fusiform elements, but of their significance and relationships little is definitely known.

The GRANULE CELLS have a general distribution throughout the granular layer. They are of relatively small size, multipolar, and possess short dendrons (frequently four) which end in claw-like processes. These latter are in close relationship with the so-called eosin bodies, small spheroidal finely granular masses having a special affinity for eosin. The eosin bodies are believed by some to represent synapses between the dendrites of the granular cells and the telodendria of the centripetal mossy fibers contributed by the medulla. The axon of the granule cell is unique among the cerebellar cortical neurons in that it passes toward the surface; here it divides in $\mathrm{T}$-fashion, the branches passing parallel to the long axis of the convolutions thus coursing at right angles to the dendronic expansion of the Purkinje cells, in relation with which they probably end.

The LARGE STELLATE CELLS have a limited distribution in the granular layer; they are more or less closely confined to the boundary zone between the molecular and granular layers. They are large multipolar cells, with a profuse dendronic contribution to the molecular layer, and an almost equally profuse short axonic and collateral contribution to the granular layer where the telodendria end in relation to the granule cells. These are typical Golgi cells of the second type.

\section{The Medulla}

The medulla next the cortex contain three important types of medullated fibers: the climbing fibers, the mossy fibers, and the axons of the Purkinje cells. The latter are centrifugal fibers passing to the internal nuclei. The climbing fibers are so designated by reason of their ending in profuse telodendria which are closely associated with the dendrons of the Purkinje cells, apparently 'climbing' over them. The mossy fibers owe their designation to the nodular mossy character of their terminal branches within the granular layer. Their end-arborizations are intimately related to those of the dendrons of the granule cells probably contributing to the formation of the eosin bodies.

Both cortex and medulla contain an abundant neurogliar supporting substance. The astrocytes of the cortex are largely of the short-rayed (mossy) type; those of the medulla are exclusively of the long-rayed 


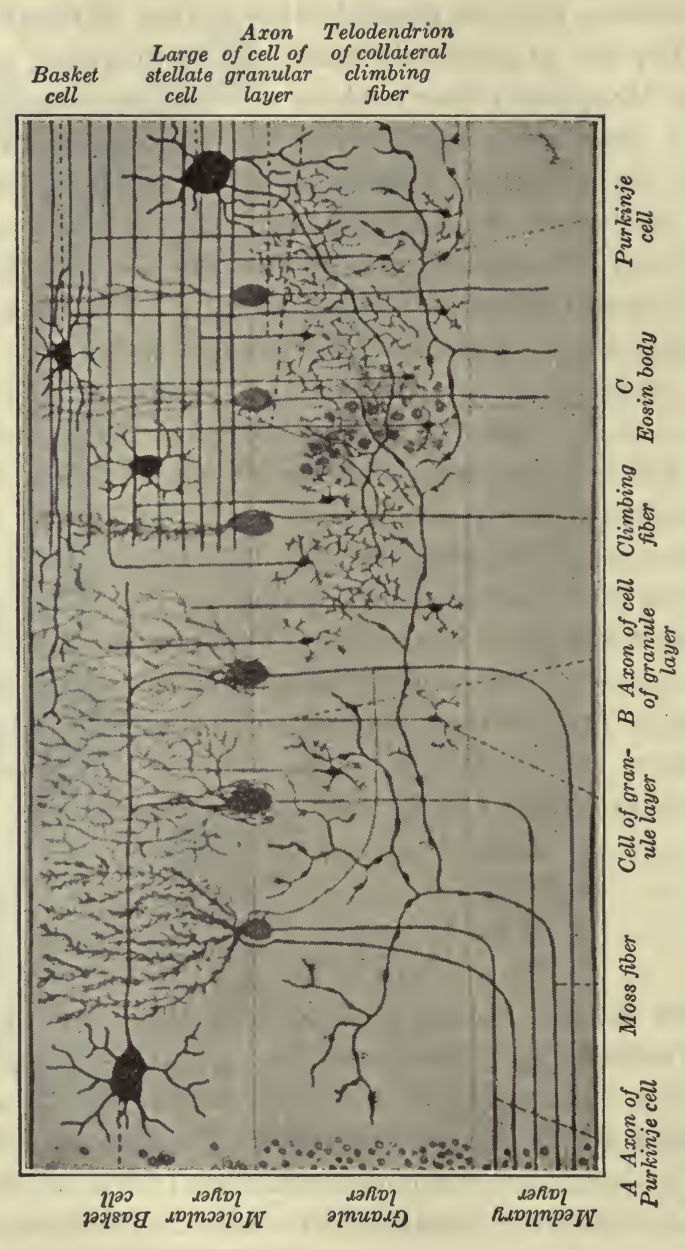

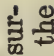

효요

o

ฮี డี

행

$>$...

ํ.?

홀

: 8

웡

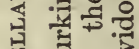

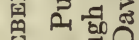

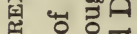

落

国

파 0.0

क

$50 \%$

承矛

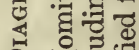

ค

1. .00 등

서 ․ㅡㅇㅡ 이

กิ 露语

อี

क षี ซี

를

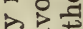

ฮี :

:

호 डेक्ष

४ 
type. Next the surface of the cortex the neuroglia tissue is condensed, forming a thick peripheral velum.

The foregoing is summarized in the following outline:

Cerebellum $\left\{\begin{array}{l}\text { Lobes } \\ \text { Lobules } \\ \text { Folia }\end{array} \quad\left\{\begin{array}{l}\text { Hemispheres } \\ \text { Vermis }\end{array}\right.\right.$

A. Molecular layer.

1. small cortical cells.

2. basket cells-small stellate cells-large cortical cells.

3. Purkinje cells.

B. Nuclear or Granular layer.

1. granule cells, with dendritic arborizations ending in close association with eosin bodies.

2. large stellate cells.

3. solitary cells.

II. Medulla-medullated nerve fibers.

(a) axon of Purkinje cells.

(b) mossy fibers, ending in eosin bodies of granular layer.

(c) climbing fibers.

\section{THE CEREBRAL CORTEX}

The cerebrum consists of two large symmetrically paired lobes or hemispheres connected by a bridge of white matter, the corpus callosum. Each hemisphere includes essentially a central mass of white substance or medulla, containing the internal nuclei or basal ganglia-paired masses of gray matter-and a covering of gray substance, the cortex or pallium. The cortex is greatly folded, thus becoming marked by convolutions or gyri with intervening fissures or sulci. The surface of each hemisphere may be divided into four principal lobes: (1) the frontal lobe, bounded posteriorly by the fissures of Rolando and Sylvius; (2) the parietal lobe, extending from the fissure of Rolando in front to the parieto-occipital fissure behind and the Sylvian fissure below; (3) the occipital lobe, bounded anteriorly by the parieto-occipital sulcus; and 
(4) the temporal lobe, lying below the fissure of Sylvius. The cortical portion folded under the lips of the Sylvian fissure is known as the insula (lobulus insula). The average thickness of the cortex is about three millimeters, but in the motor area of the frontal lobe, it may attain a depth of five millimeters, while in the occipital lobe it may become reduced to almost two millimeters.

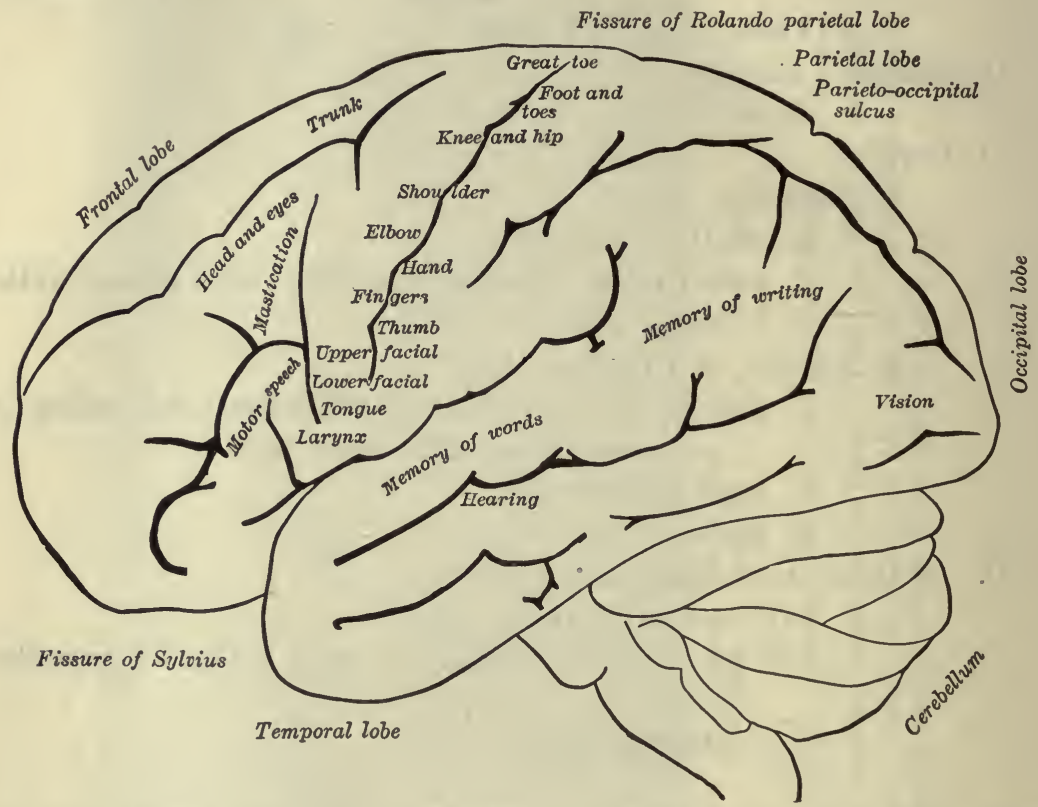

Fig. 528.-Left Lateral Surface View of Cerebral Cortex in Man, Showing the Lobes, Main Sulci, and the Larger Functional Areas.

Later researches have shown that the motor area is located entirely in the preRolandic region. (After Oppenheim.)

The nerve cells which enter into the formation of the gray matter of the cerebral cortex present a remarkable tendency to arrange themselves in more or less well-defined layers parallel to the surface of the cerebral convolutions. The number and arrangement of these layers in the various lobes varies, however, with the peculiar function of each of these areas. Thus, in the motor area there is a five layer type, in the parietal lobe a seven layer type, in the occipital lobe a six or eight layer type. The histologically different areas shade into each other by insensible gradations.

In general, it may be assumed that the nerve cells of all of these layers are included in one or two physiologically distinct groups or 
types: those whose axons enter the projection paths, and those whose axons enter the association paths; also that while these cells intermingle with each other in all portions of the cortex, yet certain areas are characterized by an undue proportion of one or the other type, and may accordingly be considered as either projection centers or association centers.

The larger cells belong, as a rule, to the projection centers, and the peculiar type of large cell contained in a given center may often be considered as characteristic of that particular area. Thus the motor area contains giant pyramidal cells (Betz cells), and the visual area the giant 'solitary cells' of Meynert.

The larger cells, being of Golgi's Type I, are assumed to * be connected with the projection fibers. On the other hand, the smaller cells-granule cells, polymorphous cells, etc.-which more frequently belong to Golgi's Type II, are thought to supply the axons of association paths. Those large areas-

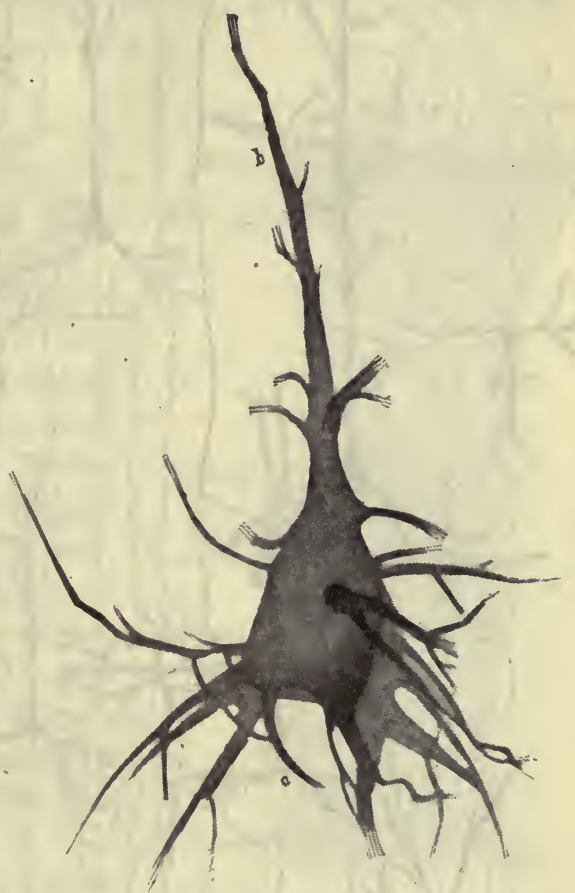

Fig. 529.-Large Pyramidal Cell of the Cortex. parietal lobe, frontal lobe, lobulus insulæ-which consist

(After W. von Bechterew.) "The Nervous System.")

(Barke:, in so large a part of the smaller type of cells, may therefore be supposed to contain the larger association centers.

The cells in any given portion of the cortex are not only arranged in layers parallel to the surface, of the cerebral convolutions, but the passage of fibers of the medulla to or from their terminations within the pallium, also separates the cells- of the cortex into irregular rows or striations, whose axis is perpendicular to the surface of the convolutions. 


\section{THE MOTOR AREA}

In the motor area, including the pre-Rolandic or pre-central gyrus and a posterior portion of the frontal lobe, the cortical cells form five tangential layers, as follows:

1. Molecular layer.

2. Outer polymorphous cell layer.

3. Small pyramidal cell layer.

4. Large pyramidal cell layer.

5. Inner polymorphous cell layer.

The MOLECULAR LAYER (stratum zonale) consists of a net work of fine dendronic fibers, derived from the deeper layers, which are disposed in tangential meshes beneath the pia mater. Occasionally small cells, apparently displaced from the deeper cell layers, are scattered among these fibers; they are of polymorphous form, and their processes are confined to the molecular layer. The surface of the molecular layer is covered by a marginal velum of neuroglia homologous with that beneath the pia mater of the spinal cord.

The SECOND, or OUTER POLYMORPHOUS, CELL LAYER, is a thin stratum. Its cells are frequently clumped, thus forming groups of various size. This grouping is, however, more distinct in some other regions, e.g., the olfactory area, than in the motor area itself.

The THIRD LAYER, SMALL PYRAMIDAL CELLS, is somewhat thicker than the above. It consists of numerous small cells-triangular, pyramidal, or pyriform in shape-whose pointed apices are directed toward the surface. Three sets

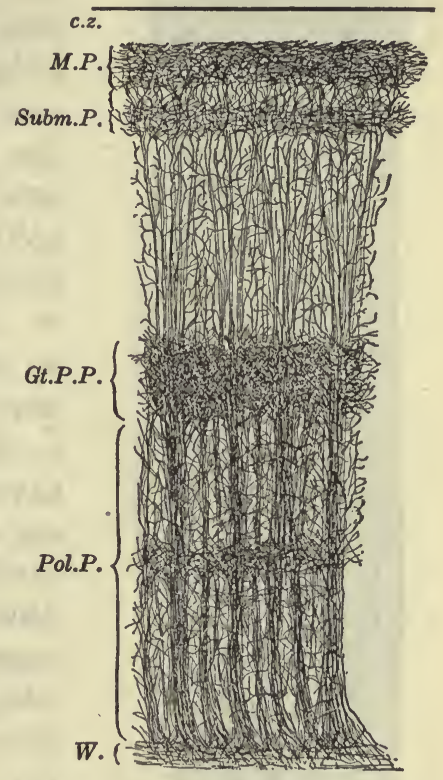

Fig. 531.-Disposition OF THE Nerve Fibers in the Cerebral Cortex of Man.

Between the vertical bands of radial fibers are the areas of interradial feltwork. The supraradial feltwork includes the tangential fiber layer or stratum zonale ( $M$. $P$.$) , and the stripe of Bechterew$ (Subm.P.). c.z., subpial neurogliar layer; Gt.P.P., outer stripe of Baillarger; Pol.P., inner stripe of Baillarger; $W$., white matter. (From . Barker, after L. Andriezen.) of dendrons are given off by these cells, an apical process which passes outward to ramify in the outer molecular layer, and from either side 
of the base of the cell a second set, whose processes are distributed in a plane nearly corresponding to that in which their cell bodies lie. The

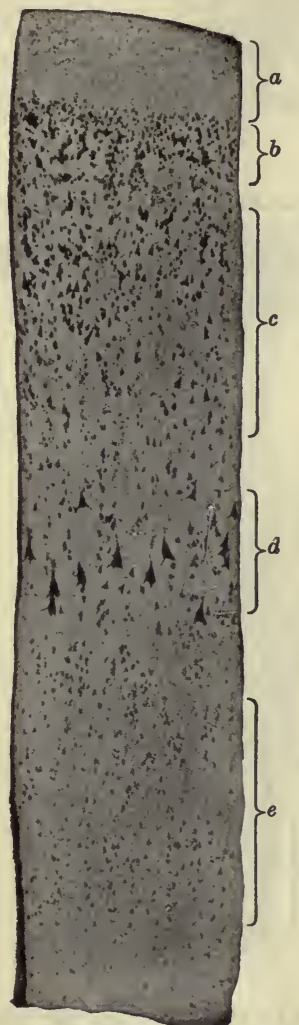

Fig. 532.-Human Cortex Cerebri, Motor Area.

$a$, tangential fiber layer; $b$, outer polymorphous cells; $c$, small pyramidal cells; $d$, large pyramidal cells; $e$, inner polymorphous cells. Nissl's stain. Moderately magnified. (After Schlapp.) axon is usually given off from the basal surface of the cell, and passes from this point directly inward to the white matter of the cerebral medulla.

The FOURTH LAYER, that of the LARGE PYRAMIDAL CELLS, is also a thick layer. Its cells are of the same shape, and distribute their processes after the same manner as those of the small pyramidal cell layer. The motor area is specially characterized by the large size of the cells of this layer. The largest of these, the Cells of Betz, are about six times as large as the small pyramidal cells, which have a fairly uniform size of about ten microns.

The FIFTH, Or INNER POLYMORPHOUS CELL, LAYER, is thicker than the preceding. Its cells are of very varied form-pyramidal, stellate, fusiform, and granule cells-and are less densely packed than is the case in the more superficial layers. They are intermediate in size, between the cells of the second and the third layers. The axons of the inner polymorphic cells, in large part, pass to the white matter of the medulla, though some of them are distributed laterally to neighboring convolutions. Their dendrons are partially distributed within the layer in which they arise, but by far the larger portion pass to the more superficial pyramidal cell layers. Many of the nerve cells of this layer, e.g., the granule cells, are very small, their nucleus being covered with an extremely narrow shell of cytoplasm.

It is noticeable that, as a rule, the dendronic processes from the cells of all five layers are distributed either in the same plane as their cell bodies, or they pass toward the surface, where many of them enter the superficial molecular layer. The axons, on the other hand, are di- 
rected inward toward the white matter of the cerebral medulla, in which they pass, either as association or as projection fibers, to many very distant parts. Notable exceptions to this latter rule, however, are the so-called cells of Martinotti, which occur to some extent in all layers, but which, though found in the pyramidal layers, are especially numerous among the polymorphous and granule cells. They are small polymorphic cells, which send their axons to the superficial molecular layer, giving off collaterals on their way.

The cell types in other portions of the cortex correspond very closely to those of the motor area. There are, however, slight but characteristic variations which are worthy of notice.

The cortex of the parietal lobe (also of the frontal, lemporal, convex surface of the occipital lobes, and the insula)-sensory area - presents a seven layer type, the additional layers resulting from an aggregation of the granule cells into one plane, which thus divides the large pyramidal cell layer. This type, therefore, presents the following layers:

1. Molecular or tangential fiber layer.

2. Outer polymorphous cell layer.

3. Small pyramidal cell layer.

4. Outer large pyramidal cell layer.

5. Granule cell layer.

6. Inner large pyramidal cell layer.

7. Inner polymorphous cell layer.

The distribution of this cortical type is suggestive of a close relation to the great association centers. Moreover, its most noticeable characteristics are the abundance of its granule cells and the relative paucity of pyramidal cells, especially those of the giant pyknomorphic

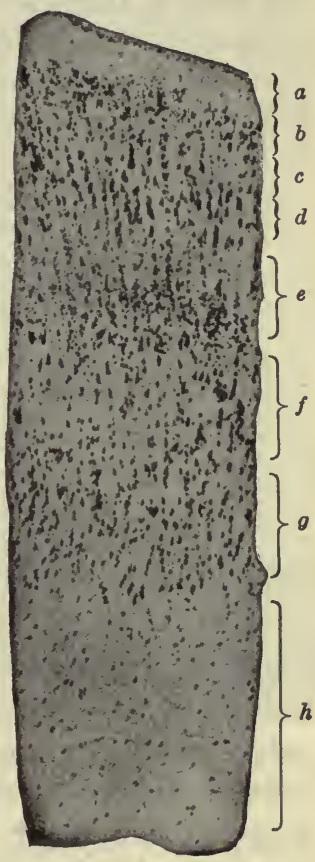

Fig. 533.-Human CoRtex Cerebri, ParieTAL LOBE.

$a$, tangential fiber layer; $b$, outer polymorphous cells; $c$, small pyramidal cells; $d$, outer large pyramidal cells; $e$, granule cells; $f$, inner large pyramidal cells; $g$, inner polymorphous cells; $h$, white matter of the medulla. Nissl's stain. Moderately magnified. (AfterSchlapp.) variety.

In the visual area-median surface of the occipital lobe-the formation is described as either a six or an eight layer type. The pyramidal cell layers are reduced to extreme thinness, the giant pyramids 
being noticeably deficient. The stripes of Baillarger, thin layers of tangential fibers on the deeper portions of the cortex, are especially distinct. So many granule cells are scattered among those of the pyramidal type that it becomes scarcely possible to distinguish from one another the second, third, and fourth layers. When these three layers are individually considered, the type presents eight layers; if, however, they are collectively considered as one stratum, the type presents six layers. With this reservation, the following layers may be distinguished:

1. Molecular or tangential fiber layer.

2. Outer polymorphous cell layer.

3. Small pyramidal cell layer.

4. The layer of granule and large pyramidal cells.

5. The outer stripe of Baillarger (great pyramidal plexus).

6. The granule cell layer.

\%. The inner stripe of Baillarger (polymorphous plexus).

8. Inner polymorphous cell layer.

The special characteristics of the visual area are the abundance of tangential fibers, as evidenced by the prominent stripes of Baillarger, the thick fiber layer in the deeper part of the molecular stratum, the abundance of granule cells, the paucity and irregular form of the pyramidal cells, and finally the presence in the inner stripe of Baillarger and in the outer portion of the deep polymorphous cell layer of numerous large isolated multipolar cells, the giant 'solitary cells' of Meynert. The outer stripe of Baillarger is especially prominent in the visual area (area striata) and is here known as the stripe of Gennari.

In the auditory area-temporal lobe-the seven layer type is found. The structure in this area is apparently identical with that previously described for the seven layer type in the parietal lobe.

In the olfactory area - hippocampal gyrus-the cells of the outer polymorphous layer arrange themselves in groups, and the pyramidal cells become largely transformed into polymorphic and fusiform cells; these lie between the characteristic outer layer and the inner layer of polymorphic cells, giving to the cortex of this region a three layered structure of indistinct outlines.

Fiber Tracts.-The corticifugal axons of the cells of the several layers are collected below the upper level of the large pyramidal cell layer into vertical columns which pass to the medulla as the bands of radial fibers. In these same columns course also the corticipetal axons. The cortex thus becomes divided vertically into cell rays and fiber 
rays, as was mentioned above. Between the fiber columns the dendronic network is known as the interradial feltwork; peripheral to where the fiber rays begin, this dendronic network forms the supraradial feltwork. At about the middle of the large pyramidal cell layer, abundant horizontal dendrons produce a distinct broad band, the outer stripe of Baillarger. Between the large pyramidal cell layer and the outer polymorphic cell layer, a similar but narrower band is known as the inner stripe of Baillarger. These stripes are most pronounced in the visual areas as was said above. Another band of similar nature at about the outer limit of the small pyramidal cell layer forms the stripe of Bechterew. Beneath the peripheral neurogliar marginal velum the dendrons of the pyramidal cells branch and form thus a band of horizontal fibers, the tangential fiber layer or molecular fiber layer. These fiber bands are conspicuous only in specimens prepared with the Weigert technic. Both cortex and medulla contain abundant neuroglia cells. Those contributing almost exclusively to the marginal velum are fusiform elements with lateral tufts of short horizontal fibers, and an expanding tuft of delicate fibers passing to the inner border of the small pyramidal cell layer. The neurogliar elements of the medulla are mostly of the long-rayed type.

\section{THE MENINGES AND BLOOD SUPPLY}

The brain and spinal cord are enveloped by the meninges, which include three fairly distinct membranes, the dura mater, arachnoid, and pia mater, and two cavities filled with lymph or a lymph-like fluid; by this arrangement the cerebrospinal axis is, as it were, sispended in fluid, and is everywhere surrounded by a watery cushion. 
The dura mater is the outermost of the three coats. Within the cranial cavity it is firmly attached to the bony walls, and serves as a periosteum for the internal surface of the bones which form the cranial cavity. Within the vertebral cavity the dura mater is distinct from the periosteum of the vertebræ, with which it is connected by loose fibrous tissue and masses of fat, which inclose large lymph spaces or chambers, lined by endothelium and collectively forming the epidural space.

The dura mater is composed of interlacing bundles of fibrous tissue containing few elastic fibers. The disposition of its fiber bundles varies somewhat in its different portions. In its spinal portion, most of the bundles are longitudinally disposed, comparatively few passing circularly around the circumference of the vertebral canal; within the cranial vault the bundles cross at acute angles; in the falces and in the tentorium cerebelli they are radially disposed.

The cranial dura consists of two distinct layers, an outer, which is very vascular and serves as the bony periosteum, and an inner, which is but "slightly vascular and may be considered as the dura proper. It is the inner layer only which is prolonged inward to form the falx cerebri and the falx and tentorium cerebelli. The venous dural sinuses of the cranium occupy clefts in the dura along the lines of attachment.

Although the dura mater is but poorly supplied with blood-vessels, it is relatively rich in lymphatics, which open into the subdural and epidural spaces and are continuous with the perivascular and perineural lymphatics which leave the cerebrospinal cavities in company with the cerebral and spinal nerves and the larger blood-vessels. In this way the lymphatics of the dura mater and its adjacent spaces are in communication with the lymphatic vessels of the eye, nose, ear, and cervical lymph nodes. These communications are of special importance as indicating the path followed by certain pathological processes which involve the meninges.

Where the outer surface of the dura is not attached to the surrounding bone or connective tissue, it is covered by a thin endothelioid coat, the lining endothelium (mesenchymal epithelium) of the epidural spaces. Its inner surface is lined by somewhat thicker endothelial cells, forming the wall of the subdural space. The dura contains sympathetic fibers for its blood-vessels, and also naked spinal and cerebral sensory fibers.

The arachnoid is a thin membranous sheet which is suspended between the dura and the pia mater. It is composed of a delicate areolar 
tissue which contains relatively few elastic fibers but is said to contain neither blood-vessels, lymphatics nor nerves. This thin fibrous membrane is covered on either side by a layer of endothelium; that upon its outer surface consists of cells of considerable thickness, which are derived from the lining membrane of the inner wall of the subdural space; the cells upon its inner surface are thinner and are derived from the walls of the $s u b$ arachnoid space.

Delicate septa-like bands pass from the inner surface of the arachnoid to the adjacent portions of the pia mater (Fig. 535). These processes are likewise invested by the endothelial lining of the subarachnoid space. A similar investment clothes the processes of the ligamentum denticulatum of the spinal cord which attaches the pia mater spinalis on either side to the

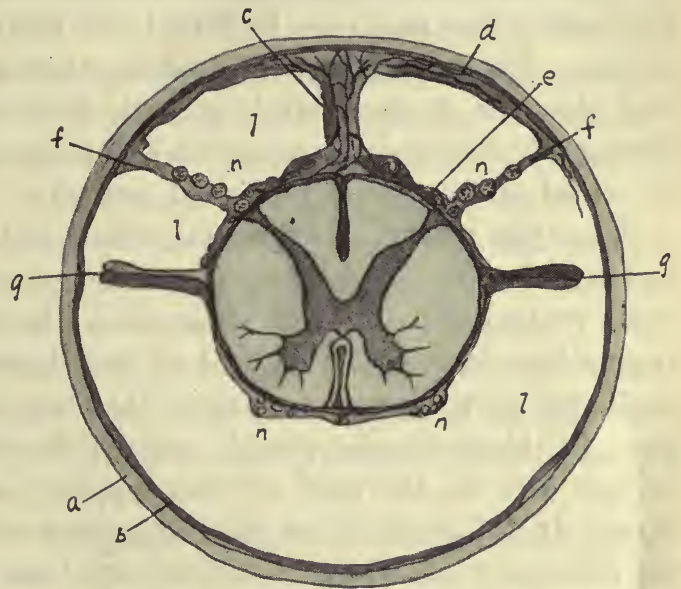

Fig. 535.-Section of the Spinal Cord and Its Membranes, From the UpPer Thoracic ReGION.

$a$, dura mater; $b$, arachnoid; $c$, septum posticum; $d, e, f$, subarachnoid trabeculæ; $n$, nerve fiber bundles; $g$, ligamentum denticulatum; $l$, subarachnoid space. (From Schäfer, after Key and Retzius.) adjacent portions of the dura mater. Subarachnoid trabeculæ support the nerve fibers of the dorsal roots.

A fibrous septum passing from the arachnoid to the pia mater, along a line opposite the dorsal median septum of the spinal cord, forms a fairly definite partition, the septum posticum. In the cervical region this is an interrupted septum, but in the thoracic and lumbar regions it becomes more or less complete.

The cranial arachnoid, in the vicinity of the cranial sinuses, notably the superior longitudinal sinus, sends outward many villus-like projections or arachnoid villi (Pacchionian bodies; granulationes arachnoidales) which protrude into the venous sinuses to such an extent as often to produce corresponding depressions in the inner surface of the bones of the cranial vault, into which they push, carrying before them a much 
attenuated portion of the dura mater. These villi are similar in structure to the membranous portion of the arachnoid. They are said to be absent at birth, small and inconspicuous in childhood, and to increase in size and number as age advances.

Fluid injected into the arachnoid or into the neighboring portions of the subarachnoid space passes readily into the lymph spaces of the dura mater, and may even be forced into the venous cavity of the cranial sinuses. While fluid thus injected may follow artificial rather than natural channels, it seems quite possible that the cerebrospinal fluid may 'during life find its way along such channels into the venous sinuses to the relief of excessive intracranial pressure.

The pia mater is intimately adherent to the surface of the brain and spinal cord. It follows all the irregularities of their surfaces and sends prolongations into all their sulci. In the larger fissures these invaginations form a double fold of pial tissue; in the smaller, the invaginated portions fuse to form a thin septum-like prolongation of the pia. In this particular the pia mater differs from the arachnoid, which bridges over all the sulci without dipping into any but the largest fissures. It differs also from the dura mater which, with the exception of the falces and tentorium, is not prolonged into any of the fissures or sulci of either the brain or the spinal cord.

The pia mater is a connective tissue membrane and is divisible into an inner and an outer layer. The outer layer is composed of coarse fibrous bundles the most of which in the pia mater of the spinal cord run longitudinally, while the finer fibers of the thin inner layer are circularly arranged.

Between the two layers are many blood-vessels and lymphatics, the pia mater being typically a vascular membrane. The larger bloodvessels are loosely embedded in the outer surface of the pia, some of them projecting into or even lying entirely within the subarachnoid space. The outer surface of the pia mater, as also the sheaths of the vessels which are loosely attached to its surface, is covered with a layer of very thin endothelial cells derived from the lining membrane of the subarachnoid space.

The inner surface of the pia is everywhere.firmly adherent to the surface of the brain and spinal cord. The slender trabeculæ and septalike processes which extend into the superficial portions of these organs, consist of connective tissues whose fibrous bands are continuous with those of the membranous pia mater. In the spinal cord many of these fibrous bundles extend inward as far as the gray matter, meanwhile be- 
coming intimately associated with neuroglia. In both the spinal cord and the brain the pial septa serve for the support of numerous-błoodvessels and perivascular lymphatics which are distributed through this connective tissue to all portions of the brain and spinal cord.

Within the cranium, reduplications of the pia mater, carrying between their folds a layer of arachnoidal tissue and an extensive plexus of small blood-vessels, push their way into the cerebral ventricles to form the superior and inferior tela choroidece. These choroid plexuses are separated from the ventricular cavities by an investment of cuboidal cells, which in fetal and infantile life are ciliated, and which are derived from and are continuous with the ependyma cells lining the walls of the ventricles. Thus the blood-vessels of the telæ choroideæ, in the strictest anatomical sense, lie without and not within the cavity of the cerebral ventricles, for they are everywhere separated from those cavities by the ependyma cells, which, ontogenetically at least, form a portion of the wall of these vesicles. The cerebrospinal fluid is supposed to originate largely by process of filtration from the blood-vessels of these choroid plexuses.

The pia mater contains mostly sympathetic fibers, but probably also a few sensory cerebrospinal fibers.

The peculiar arrangement of the three constituent membranes of the meninges leaves three distinct spaces or connected groups of spaces which are filled with fluid. These are the epidural, subdural, and subarachnoidal spaces.

The epidural space comprises a connected series of lymph cavities, which is of limited extent within the cranium, but of broad extent within the spinal canal. These spaces are lined by endothelium which is at many points continuous with the perivascular and perineural lymphatics and through them with the lymphatic vessels of the general systemic circulation. Obviously the epidural spaces serve as large lymphatic vessels and their cavities are consequently filled with lymph.

The subdural space has a complete lining of rather thick endothelial cells. The walls of this cavity are formed by the dura on the outer, and the arachnoid on the inner side. Its cavity is occupied by lymph and is continuous with the lymphatic vessels of the dura, and through them with the epidural spaces and systemic lymphatics.

This space is penetrated by the outgoing cranial and spinal nerves, which receive an investment from all three of the meningeal coats. The three layers composing this investment soon lose their distinctive charac- 
teristics, fuse together, and blend with the epineurium of the nerve trunks.

Fluid injected into the subdural space may be readily forced into the lymphatics of these epi- and perineural sheaths and may thus travel to parts quite remote from the central nervous system.

The subarachnoid space within the cranium is of limited breadth, but within the spinal canal it is much broader and contains not only the larger blood-vessels which are loosely attached to the surface of the pia, but also the many spinal nerve roots pass downward through this space toward their foramina of exit.

The subarachnoid space is lined by a thin endothelial layer, its outer wall being formed by the arachnoid, its inner by the outer surface of the pia mater; its cavity is filled with cerebrospinal fluid, which closely resembles, yet differs somewhat in chemical composition from the lymph. It contains a few lymphocytes, estimated at five per cubic millimeter of fluid. This space is in communication through the foramen of Majendie, an opening in the roof of the fourth ventricle, with the central canal of the spinal cord and the ventricular cavities of the brain. It is also thought to communicate with the cerebral ventricles at several other points.

The spinal portion of the subarachnoid space is crossed by a posterior median septum, the septum posticum, laterally by the ligamentum denticulatum, and by several irregular but incomplete septa which, like the ligamentum posticum, connect the pia mater with the arachnoid.

The ligamentum denticulatum is a dense mass of fibrous tissue containing a few elastic fibers, which, beginning at the lateral surface of the pia as a complete septum, passes, by about twenty-eight serrations, across the subarachnoid space, and pushing the arachnoid before it, is attached to the inner surface of the dura mater. The serrations of the dentate ligament do not penetrate the subdural space, for around the point of their attachment the surface of the arachnoid is firmly adherent to the dura mater. Each serration is invested by an endothelial coat continuous with the lining of the subarachnoid space.

Blood Supply.-The blood supply of the central nervous system is derived from vessels which lie within the folds of the pia mater, branches of the internal carotid and the vertebral arteries. The larger arteries form an anterior longitudinal group represented in the spinal cord by the anterior spinal artery and its branches, and in the brain by the vessels of the circle of Willis and their immediate branches.

Two sets of vessels may be said to be distributed from these sources 
-one of which is distributed through the pia mater to the adjacent white matter of the spinal cord and to the gray pallium of the brain; the other penetrates the spinal cord through the anterior median fissure by a series of small fissural arteries to be distributed to the central gray matter, and in the brain is represented by the branches of the middle cerebral arteries which penetrate directly to the ganglionic gray matter in the interior of the cerebrum.

In the spinal cord the vessels of the former set are mostly distributed to the white cortex, the larger branches, howęver, penetrate the white matter and aid in the formation of the capillary network of the gray medulla. In the brain their distribution is similar, the smaller pial vessels, the cortical arteries, being distributed to the cortex, which in this case is formed by the gray matter; the larger, the medullary arteries, penetrating to the white medulla in which they break up into capillary vessels.

The veins trend in the opposite direction and in the pia mater collect into large vessels, which in the brain open into the sinuses of the dura mater, and which in the spinal cord form the ventral and dorsal median veins.

All the larger vessels receive thin fibrous investments from the pia mater; the smaller vessels and capillaries are surrounded by neuroglia.

There are frequent anastomoses between the larger veins; the arteries, however, are all terminal arteries according to Cohnheim's classification, possessing no anastomoses with the capillary areas of other vessels.

Neither brain nor cord possess true lymphatics. The sole lymphatic representatives within the central nervous system are pericellular and perivascular spaces communicating with subpial spaces and ultimately through uncertain clefts and channels with the subarachnoid spaces.

The bulk of the cerebro-spinal fluid is secreted by the cells of the choroid plexuses directly into the cerebral ventricles. It escapes into the subarachnoid spaces through the roof of the fourth ventricle. From here it is absorbed into the venous sinuses by way of the arachnoid villi. A small quantity of cerebro-spinal fluid is contributed also by the blood capillaries of the central nervous system. The lymphlike fluid passes from these capillaries directly into the pericapillary spaces and thence to each nerve cell or outward through the perivascular channels to the subarachnoid spaces. Besides the fluid which escapes through the venous sinuses of the dura a small amount drains also by way of the perineural spaces indirectly into the lymphatic system. (See L. H. Weed; Anat. Rec., Vol. 12, 191\%.) 


\title{
CHAPTER XVIII
}

\author{
THE EYE
}

\section{GENERAL CONSIDERATIONS}

The eye may be said to consist of the visual organ, or globe, and its appendages - the eyelids, conjunctiva, and lacrimal apparatus-whose function is chiefly protective.

The globe of the eye, or eye proper, is contained within the cavity

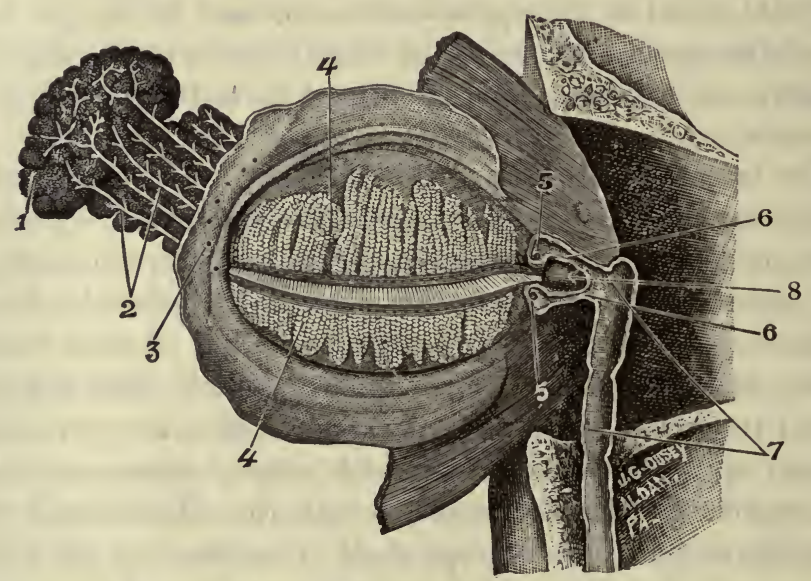

Fig. 536.-Dissection of Eyelids and Lacrimal Apparatus.

1, upper lacrimal gland; 2 , lower lacrimal gland and excretory ducts; 3 , mouths of excretory ducts; 4, tarsal (Meibomian) glands; 5, puncta lacrimalia; 6, lacrimal canaliculi; 7, lacrimal sac and nasal duct; 8, caruncula. (After Fox.)

of the orbit, its posterior two-thirds being embedded in a mass of intraorbital fat whose inner surface is covered by a thin fibrous membrane or fascia which is clothed with mesenchymal epithelium. The epithelium is reflected from this fascia to the surface of the ocular globe, along a line just posterior to the border of the conjunctiva, whence it passes over the surface of the globe as far posteriorly as the optic nerve, on 
the surface of which it again becomes continuous with the mesenchymal epithelium of the fascia. Thus a serous sac or lymphatic space is formed by the parietal layer of this sac, which lines the orbital cavity, in conjunction with the visceral layer which covers the posterior two-thirds of the globe of the eye; this sac is the capsule of Tenon.

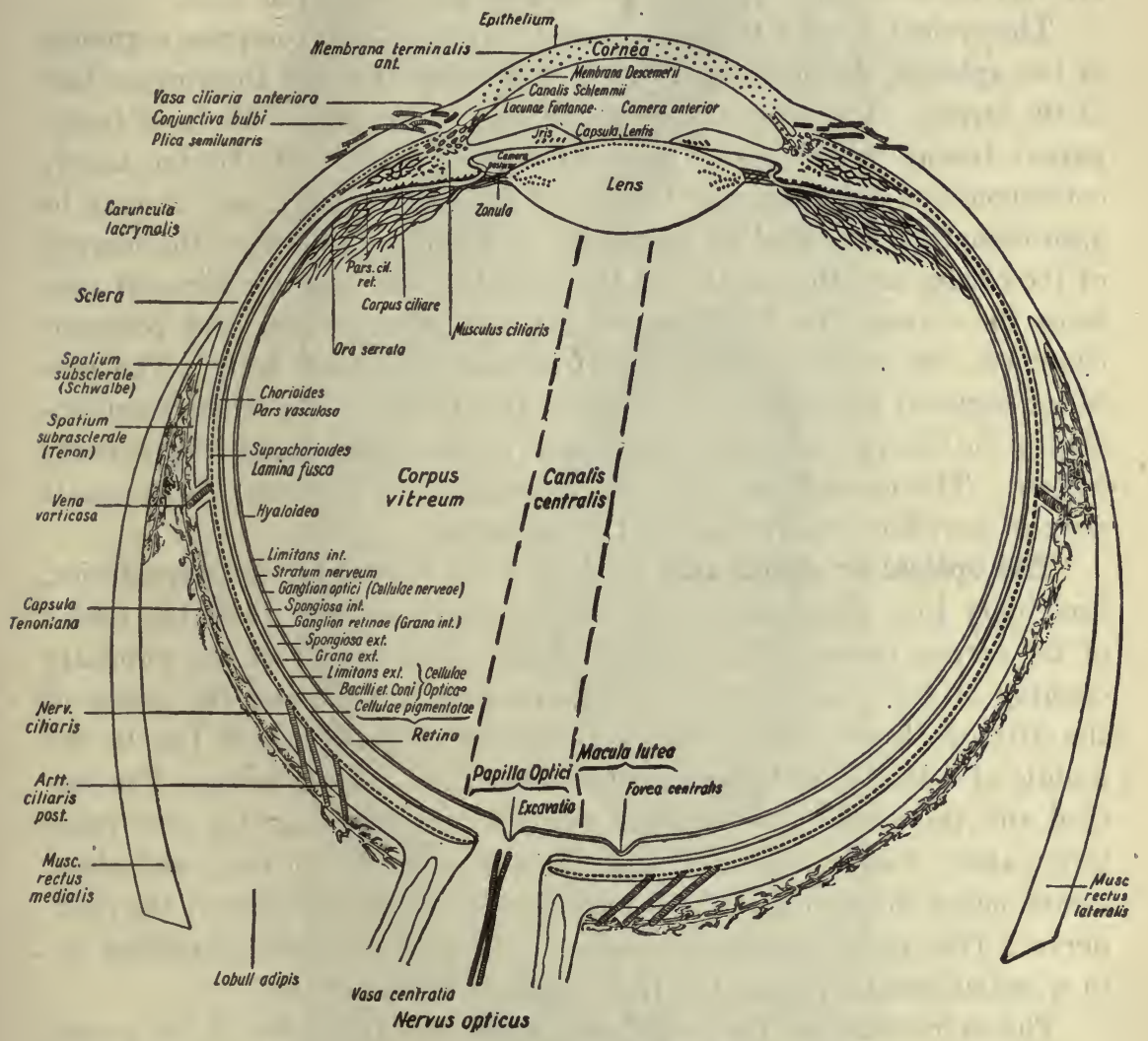

Fig. 537.-Horizontal Section of the Right Eyeball.

(From Fox, after Magnus.)

The anterior third of the globe is covered by a reflection, at the fornix conjunctivæ, of the conjunctival layer which clothes the inner surface of the palpebræ or eyelids. The conjunctiva is a continuation of the integument of the lid, modified so as to simulate a mucous membrane. The portion associated with the eyelid is known as the palpebral conjunctiva, that covering the eye as the ocular, bulbar or scleral conjunc- 
tiva. Where the sclera passes into the cornea, the conjunctival epithelium becomes continuous with the anterior corneal epithelium, the tunica propria blending with the corneal stroma.

The globe of the eye or eyeball is a spheroidal body whose surface consists of three coats, an outer, middle, and inner, and whose contents are the vitreous and aqueous humors and the crystalline lens.

The eyeball is not a true sphere, but may be said to comprise segments of two spheres, the smaller of which is inserted into the anterior surface of the larger. The anterior or smaller segment consists chiefly of transparent tissues which permit the entrance of light. Its border nearly corresponds to the posterior margin of the ciliary body, and it may be approximately indicated by a parallel circle midway between the margin of the cornea and the equator of the eyeball. The anterior segment contains the cornea, the sclerocorneal junction, the anterior and posterior chambers, the aqueous humor, the iris, and the ciliary body. The posterior segment comprises the posterior two-thirds of the eyeball and includes the sclera, choroid, retina, and, within these coats, the vitreous humor. The crystalline lens with its suspensory ligament forms, as it were, a partition separating the two segments.

The optical or visual axis of the eye is a horizontal, anteroposterior, imaginary line, about an inch in length, which extends from the center of the cornea through the anterior chamber, the center of the pupillary opening of the iris, the center of the crystalline lens, and the center of the vitreous humor, and reaches the fovea centralis which lies in the middle of a thickened portion of the retina, the macula lútea. The vertical and transverse axes measure about $1 \mathrm{~mm}$. less than the anteroposterior axis. Toward the inner side, at a distance of $3.5 \mathrm{~mm}$., and about $1 \mathrm{~mm}$. below the center of the fovea centralis, is the entrance of the optis nerve. This nerve pierces the coats of the eye, its fibers spreading ou in a radial manner, upon the inner surface of the retina.

The extremities of the visual axis mark the two poles of the ocula i globe; the anterior extremity, lying in the center of the cornea, is in the anterior or smaller spheroidal segment, the posterior extremity, in the fovea centralis, lies in the posterior segment of the eye.

\section{THE EXTERNAL COAT-THE FIBROUS TUNIC}

The outer tunic of the eyeball includes the cornea, the sclera, and the sclerocorneal junction. 


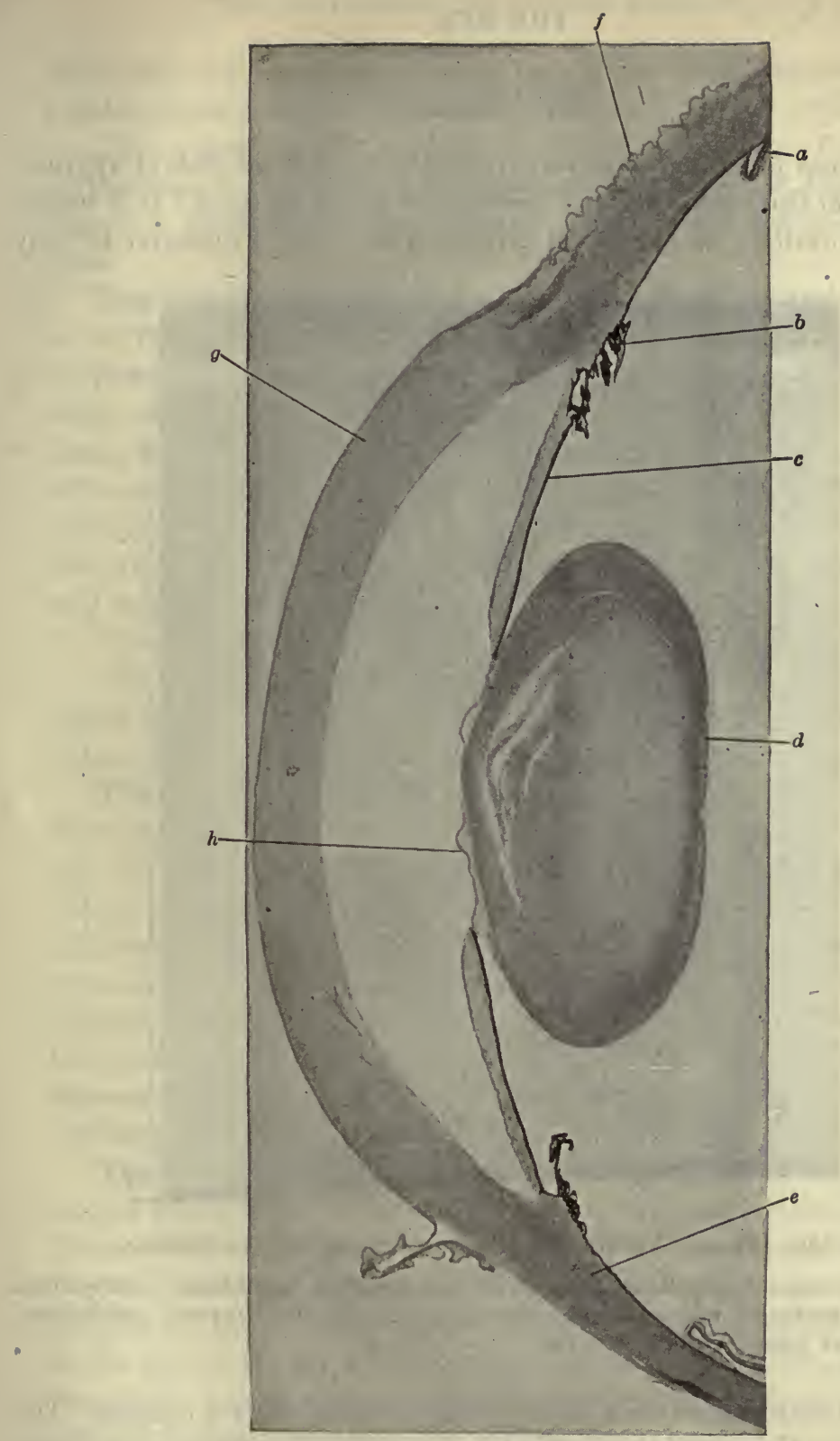

Fig. 538.-The Anterior Segment of a Child's Eye; Meridional Section.

$a$, ora serrata $; b$, ciliary processes; $c$, iris; $d$, crystalline lens; $e$, ciliary muscle; $f$, ocular conjunctiva; $g$, cornea; $h$, the capsule of the lens, partially detached. Hematein and eosin. Photo. $\times 10$. 


\section{The Cornea}

The cornea is a concavoconvex, transparent, colorless disk of approximately equal thickness $(1 \mathrm{~mm}$.) throughout all its portions. It is nearly circular in outline, its horizontal exceeding its vertical diameter by only

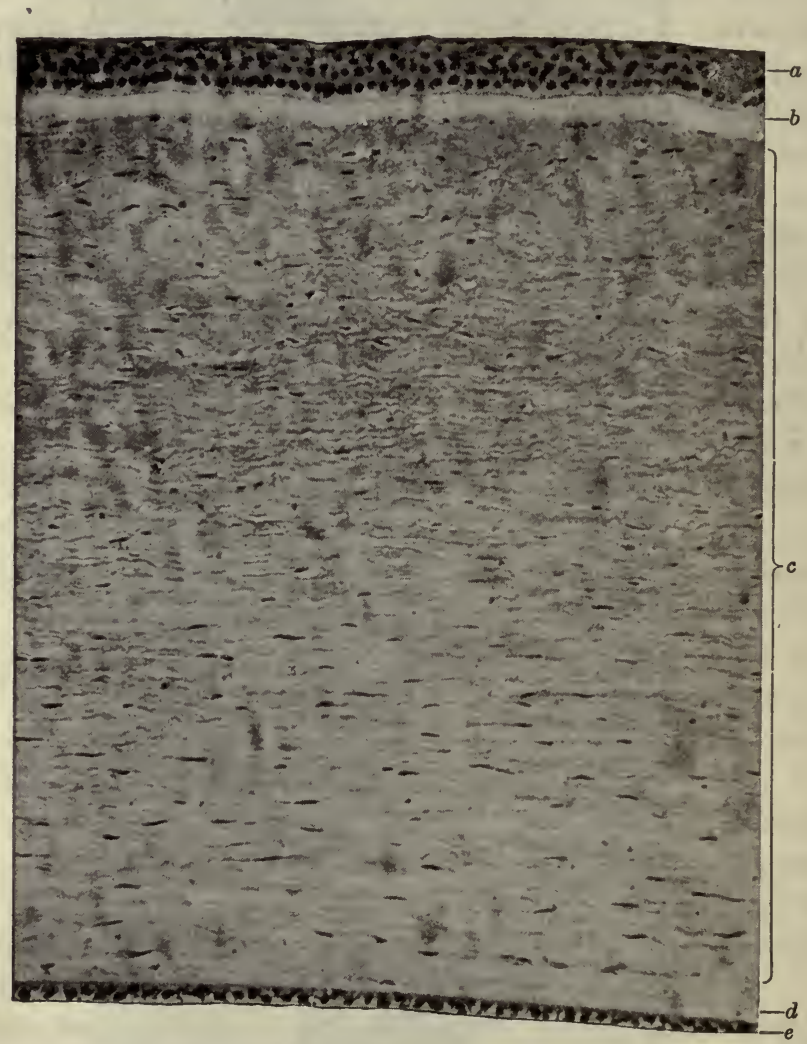

Fig. 539.-From a Meridional Section of the Human Cornea.

$a$, anterior corneal epithelium; $b$, anterior homogeneous membrane; $c$, substantia propria; $d$, posterior homogeneous membrane; $e$, posterior corneal epithelium. Hematein and eosin. Photo. $\times 180$.

$0.5 \mathrm{~mm}$.; its external surface is convex, its internal surface concave. The cornea forms the anterior one-fourth of the tunica externa, and represents a spheroidal segment whose radius is somewhat shorter than that of the posterior segment of the eyeball. It is inserted into the anterior margin of the sclera much after the manner in which a watch-glass is 
set in its rim; hence the inner surface of the cornea possesses a siightly greater diameter than the outer.

The cornea may be said to consist of five layers: 1 , the anterior epithelium; 2, the anterior homogeneous membrane; 3 , the corneal substance; 4 , the posterior homogeneous membrane; 5 , the posterior epithelium.

The anterior epithelium (corneal epithelium, corneal conjunctiva) at the margin of the cornea is continuous with the scleral portion of the conjunctiva. It consists of a relatively thin layer-six to eight cells deep-of stratified squamous epithelium, the deepest cells of which are elongated or columnar, the middle cells polyhedral, and the superficial cells somewhat flattened. The cells at all levels are nucleated and, like the other corneal tissues, perfectly transparent. The columnar cells are often slender and much elongated, their pointed apices extending well toward the surface of the epithelial layer.

The epithelium rests directly upon the anterior homogeneous lamella.

The deeper cells of the epithelium present distinct intercellular lymphatic spaces and intercellular bridges. Between the cells are the terminal ramifications of nerve fibrils from the plexus in the corneal substance.

The anterior homogeneous membrane (anterior basal lamella, elastic membrane of Bowman) was formerly thought to consist of elastic tissue, but this supposition is disproved by its ready solubility on boiling (His), as well as by the fact that it does not react typically to the specific stains for this tissue. Bowman's membrane is apparently a homogeneous or structureless coat except that it is slightly fibrillar at its extreme margin where it becomes continuous with the fibrous tissue of the sclera. It resembles elastic tissue in that it is highly refractive and possesses a shining glassy appearance. It does not stain readily with the ordinary dyes.

The corneal substance (substantia propria) forms the greater portion of the cornea. It consists of a lamellated connective tissue, which forms about sixty fibrous layers, parallel to the corneal surface. The fibrous bundles of these lamellæ, being arranged in meridional curves parallel to the surface, appear to cross one another at right angles in the central portion of the circular cornea. Other fibers, arcuate fibers, pass from one layer to another; so firmly uniting them that it is impossible to tease the cornea into its component lamellæ.

The intervals between the fibrous layers are occupied by interlamellar cement, or ground substance, in which lymphatic channels and large flattened cells, the corneal corpuscles, can be demonstrated. The cor- 
neal 'corpuscles' are branched lamellar connective tissue cells, which occupy the large lymphatic spaces or lacunæ of the interlamellar ground
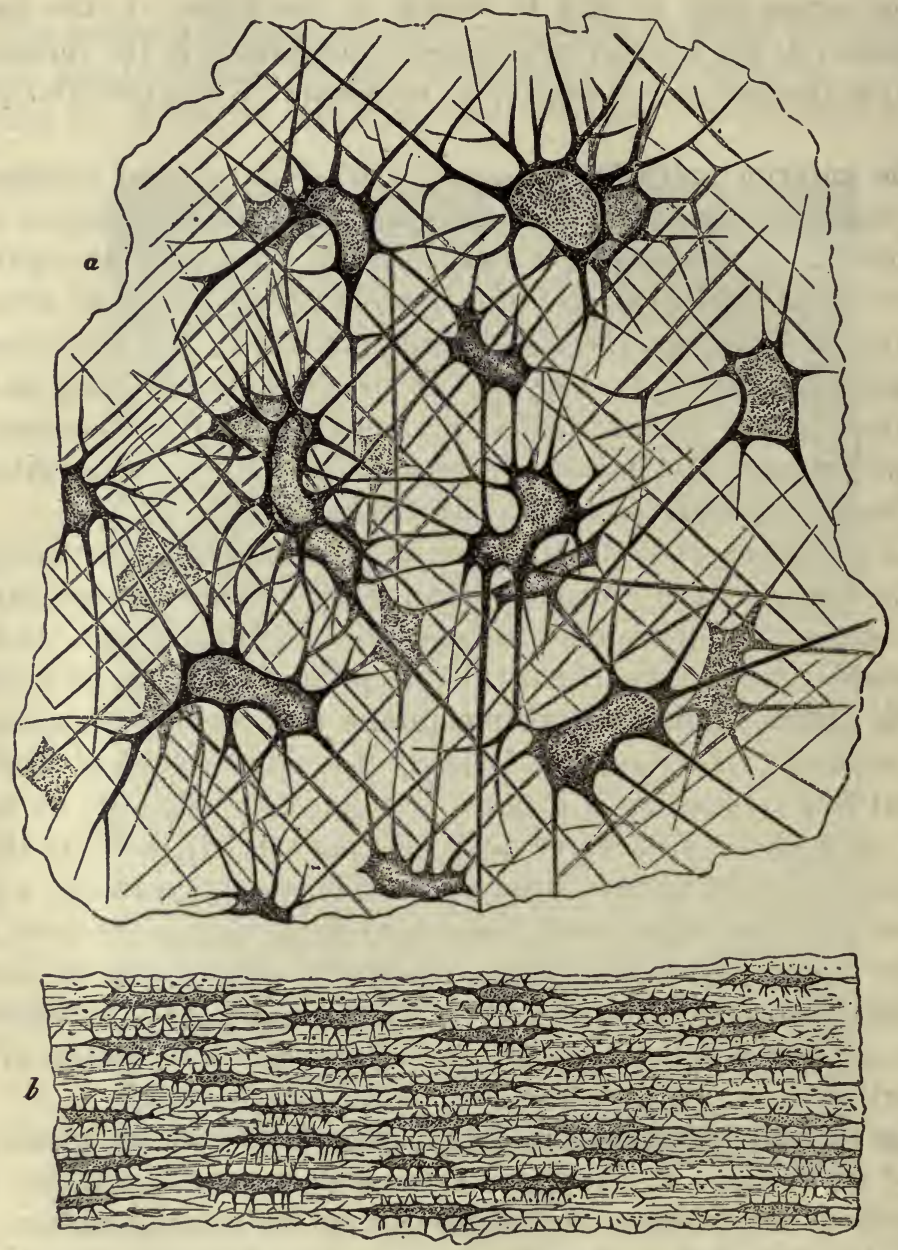

Fig. 540.-Corneal Corpuscles of the Frog.

$a$, as seen in tangential section; $b$, as seen in transection of the cornea. Chlorid of gold. Highly magnified. (After Rollett.)

substance, and which send fiber-like processes into the interlacing lymphatic channels.

The posterior homogeneous membrane, or membrane of Descemet (posterior basal lamella), is similar in structure to the anterior. Like 
the latter, though formerly considered an elastic membrane it does not give the specific reactions of elastic tissue. It is somewhat thicker than Bowman's membrane. At its margin the membrane is continuous with fibrous bundles which are directed outward into the ligamentum pectinatum, and, at least in some animals, through this ligament into the ciliary margin of the iris. The membrane of Descemet can be readily detached from the corneal substance by teasing. It prevents filtration of fluid from the anterior chamber into the corneal stroma.

The posterior epithelial layer (corneal endothelium) is a mesenchymal epithelium consisting of clear, cuboidal or flattened cells, placed edge to edge; and bound together by intercellular bridges. At the margin of the cornea it is reflected over the lateral wall of the anterior chamber to the anterior surface of the iris. Its cells rest upon the posterior homogeneous membrane.

All the tissues of the cornea, during life, are absolutely trans-

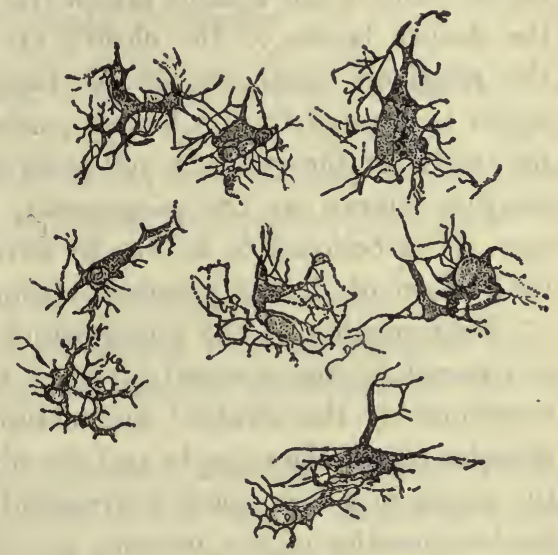

Fig. 541.-Corneal Cells, Isolated. Highly magnified. (After Waldeyer.) parent. The elements of which they consist are of almost identical refractive indices, and about that of water, so that in fresh, or in living tissue, it is almost impossible for the microscope to discover any of the structure of the cornea. After death the cornea becomes opaque and its elements are then easily distinguished.

Vascular and Nerve Supply.-The cornea itself is an absolutely non-vascular tissue, having neither blood nor true lymphatic vessels. It is, however, well supplied with nerve fibers, derived from the ciliary nerves, which form an annular plexus in the sclera about the margin of the cornea, from which point bundles of naked axis-cylinders pass into the corneal substance to form a basal plexus, near the anterior homogeneous membrane. From this latter plexus, fibers are distributed to the corneal substance and to a subepithelial plexus, anterior to Bowman's membrane, whence the terminal sensory fibrils penetrate the anterior epithelium. For a distance of several millimeters within the margin of the cornea special nerve endings (bulbous corpuscles) may also occur. 


\section{The Sclera}

The sclera (sclerotic coat) is a firm opaque connective tissue membrane which forms the outermost layer of the posterior segment of the eyeball. It consists of two layers, the thick, firm, substantia propria, and the very thin, innermost, delicate, lamina fusca.

By reflected light the sclera of the adult is of a lustrous white color. In the child it has a faint bluish tint, due to the presence of pigment in the deeper layers of the child's eye which shows indistinctly through the relatively clear superficial tissues. The anterior portion of the sclera is covered by the bulbar conjunctiva and is familiarly known as the 'white of the eye.' A yellowish patch in the vicinity of the corneal margin, known as the pinguecula, may be present, especially in old age. It is believed to be due to irritation from dust, leading to colloid infiltration of the conjunctival stroma.

That portion of the sclera which is posterior to the ocular equator is covered by the visceral layer of the capsule of Tenon except at the insertions of the straight and oblique muscles. The tendons of these muscles pierce the capsule and are obliquely inserted into the surface of the sclera in a line nearly corresponding to the equator of the eye. The tendon bundles of the muscles are directly continuous with the fibrous bundles which compose the sclera.

The Substantia Propria.-The collagenous fibrous tissue of the sclera is disposed in bundles which are arranged along meridional and equatorial lines; they interlace with one another to form a dense network. A few elastic fibers are interspersed among the bundles of this network. Stellate connective tissue cells, the scleral corpuscles, lie in the interfascicular clefts. Occasional pigmented cells are also sometimes present.

The Lamina Fusca.-The inner surface of the sclera' presents a fine gauzy membrane which can be readily detached by teasing. This is the lamina fusca scleræ. It consists of delicate interlacing fibrous bundles and numerous pigmented connective tissue cells. The lamina fusca near the posterior pole is firmly adherent to the scleral substance.

At the posterior pole of the eye the sclera is pierced by the optic nerve, whose numerous bundles penetrate the coats of the eyeball and give to this portion of the sclera a cribrose appearance. This area of the sclerotic coat is known as the lamina cribrosa sclerce. It is a circular zone whose border is outlined by the entrance of the posterior ciliary arteries and the ciliary nerves. $\checkmark$ This is the thickest portion of the sclera, 
the coat becoming progressively thinner toward the equator of the eye; near its anterior margin it is again thickened by the tendinous insertions of the extrinsic muscles.

The sclera is chiefly supplied by branches from the posterior ciliary arteries, which form a wide-meshed plexus in its substance, its vessels anastomosing freely with those of the choroid coat.

\section{The Sclerocorneat Junction}

The sclerocorneal junction (Fig. 543) is a narrow circular zone at the margin of the cornea, where it is inserted into the sclera. Across this narrow zone the fibrous bundles of the opaque sclera are continued directly into the similar, though perfectly transparent, bundles of the corneal substance.

The anterior or outer surface of this zone is covered by the ocular portion of the conjunctiva. Its epithelium is of the stratified squamous variety and is continuous with the anterior epithelium of the cornea.

From the inner surface of this junctional zone the anterior extremities of the muscle fibers composing the ciliary muscle take their origin. The fibers of this muscle intermingle with the marginal fibers of the posterior homogeneous layer of the cornea to form the ligamentum pectinatum, which connects the sclerocorneal junction with the base of the iris. This pectinate ligament is very much more highly developed in certain animals, e.g., cow and horse, than in man. .

Toward the inner side of the scleral margin and near the border of the cornea is the canal of Schlemm (sinus venosus sclerce). This is an annular venous channel (or network of channels), draining into the anterior ciliary veins. Though venous in character it serves also as a drainage channel for the lymphatic spaces of Fontana, which lie in the lateral wall of the anterior chamber and between the fiber bundles of the ligamentum pectinatum. The spaces of Fontana are true lymphatic spaces and are in communication with the anterior chamber of the eye.

Through the canal of Schlemm, the aqueous humor of the anterior chamber is put into communication with the veins of the sclera, and a system is thus formed by which the intra-ocular pressure is maintained at normal. A blocking of the canal of Schlemm interferes with the drainage of the anterior chamber, and is believed to produce the serious pathologic condition of the eyeball, known as glaucoma, due to progres- 
sive increase of intra-ocular tension which causes atrophy of the optic nerve and the retina.

Blood Supply. - The sclerocorneal junction is abundantly supplied with blood from the anterior ciliary vessels, which, with the posterior conjunctival vessels, form loops at the margin of the cornea and anastomose freely with the vessels of the ciliary body. The sclera contains no true lymphatic vessels.

\section{THE MIDDLE COAT-THE VASCULAR TUNIC}

The middle tunic (uvea, uveal tract) includes the choroid coat, ciliary body, and iris. The latter is perforated centrally by an approximately circular aperture, the pupil.

The iris divides the cavity of the anterior segment of the eye into an anterior chamber, included between it and the posterior or inner surface of the cornea, and a posterior chamber, which is bounded by the iris in front and the crystalline lens and its suspensory ligament behind. The free or pupillary margin of the iris is in light contact with the anterior surface of the lens. The posterior chamber is therefore an annular compartment.

\section{The Choroid Coat}

The choroid coat (tunica choroidea) consists of three layers: 1 , the lamina suprachoroidea; 2 , the lamina vascularis; 3 , the lamina capillaris. The function of the very vascular choroid is to supply nutrition to the outer portions of the retina.

The lamina suprachoroidea (suprachoroid layer) is a very delicate membrane which contains many pigmented cells and is similar in structure to the lamina fusca of the sclera.

The flattened pigmented cells are brownish-black in color from the many coarse granules which they contain, and are irregularly disposed, either separately or in groups. Lymphatic spaces occur between this layer and the sclera and communicate through the interfascicular lymphatic clefts of the sclera with the capsule of Tenon.

The fibers of this layer are not only distributed in its own plane but pass obliquely to the lamina fusca, thus loosely attaching the suprachoroid layer to the sclera. Similar, obliquely disposed fibers pass to the deeper portions of the choroid, with the fibers of which they blend.

The lamina vasculosa (vascular layer, choroid proper), so called 
because it contains the ramifications of the ciliary arteries and veins, is by far the thickest of the three layers of the choroid. It may be arbitrarily separated into an outer stratum, consisting chiefly of dense interlacing bundles of connective tissue fibers which inclose only the larger blood-vessels, and an inner stratum of similar structure, but everywhere permeated by a close network of small vascular twigs. So dense is this network near the posterior pole of the eye, as to give the layer the appearance of an almost continuous sheath of small blood-vessels.

The Lamina Capillaris.-Within the vascular layer is the capillary membrane (lamina capillaris, lamina choriocapillaris, tunica Ruyschi-

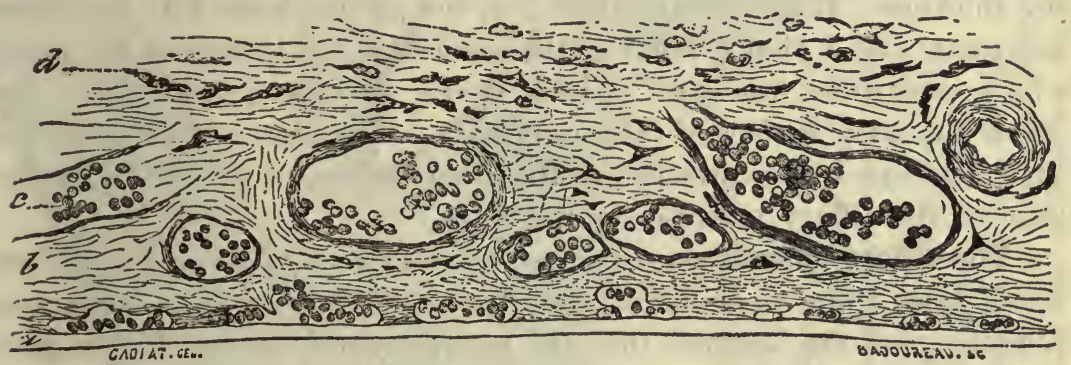

Fig. 542.-From a Meridional Section of the Choroid Coat.

$a$, membrane of Bruch; $b$, the inner margin of the vascular layer. Between $a$ and $b$ is the capillary layer or choriocapillaris; $c$, venule containing blood corpuscles; $d$, fibrous layer of the choroid or lamina suprachoroidea. Highly magnified. (After Cadiat.)

ana), which contains an exceedingly close-meshed capillary network. This network is specially dense near the macula lutea at the posterior pole of the eyeball. Its inner surface forms a very thin homogeneous membrane, the lamina basalis, lamina vitrea or membrane of Brich, which increases somewhat in thickness as age advances. The inner surface of the lamina basalis is indented by the bases of the adjacent pigment cells of the retina. Anteriorly the vessels of the choriocapillaris, like those of the vascular layer, become continuous with the vessels of the ciliary body and iris.

Between the pars vascularis and the choriocapillaris may be distinguished a narrow dense fibro-elastic boundary zone, free of pigment. In ruminants this layer becomes pronounced, due to the presence of robust connective tissue fibers, and is known as the tapetum fibrosum. This layer gives to the eyes of ruminants their characteristic metallic luster. In the eyes of carnivora and certain fishes the tapetum is composed of rectangular epithelioid cells, filled with peculiar glistening 
crystals giving to these eyes an iridescent sheen, and is known as the tapetum cellulosum.

\section{The Ciliary Body}

The ciliary body (corpus ciliare) represents the thickened anterior border of the choroid coat. It is, therefore, of annular shape and occupies a zone ${ }^{\circ}$ whose posterior border blends with the choroid at a point opposite the ora serrata of the retina, and whose anterior margin is continued into the iris opposite the sclerocorneal junction. It may be said to consist of three structures arranged in layers of varying thickness: 1 , the ciliary muscle; 2 , the fibrous layer with its ciliary processes; and 3 , that portion of the pigmented epithelium of the retina which constitutes the pars ciliaris retince or ciliary epithelium, and covers the inner surface of the ciliary body. The suspensory ligament of the crystalline lens is attached to the inner surface of the retinal epithelium of the ciliary processes and grooves.

The ciliary muscle consists of an annular mass of non-striated fibers which arise from the inner surface of the sclera near the sclerocorneal junction, and are inserted into the entire breadth of the fibrous mass of the ciliary body as far back as the anterior margin of the choroid. The muscle fibers are divisible into three sets, according to the direction of their long axis; these are the meridional, the radial, and the circular.

The meridional fibers form the outer and greater portion of the muscle. They begin just posterior to the corneal margin, taking their origin from the inner surface of the sclera, and radiate backward in a meridional direction for a variable distance, to be finally inserted into the fibrous bundles of the posterior half of the ciliary body (ciliary ring), the longest fiber bands passing as far back as the choriociliary junction, where they are attached to the anterior margin of the choroid.

The radial fibers simulate the meridional fibers in that they radiate from the corneal margin. They pursue, however, a shorter course. From their origin they pass backward with a sharp inward curve to assume a direction which approaches that of the radii of the ocular globe (hence their name) ; they are inserted into the anterior half of the fibrous layer of the ciliary body. Their radial disposition becomes progressively more apparent toward the axial margin of the ciliary body. These fibers are far less numerous than the meridional.

The circular fibers comprise numerous small non-striated muscle bundles which are interspersed among the bundles of radial fibers. 


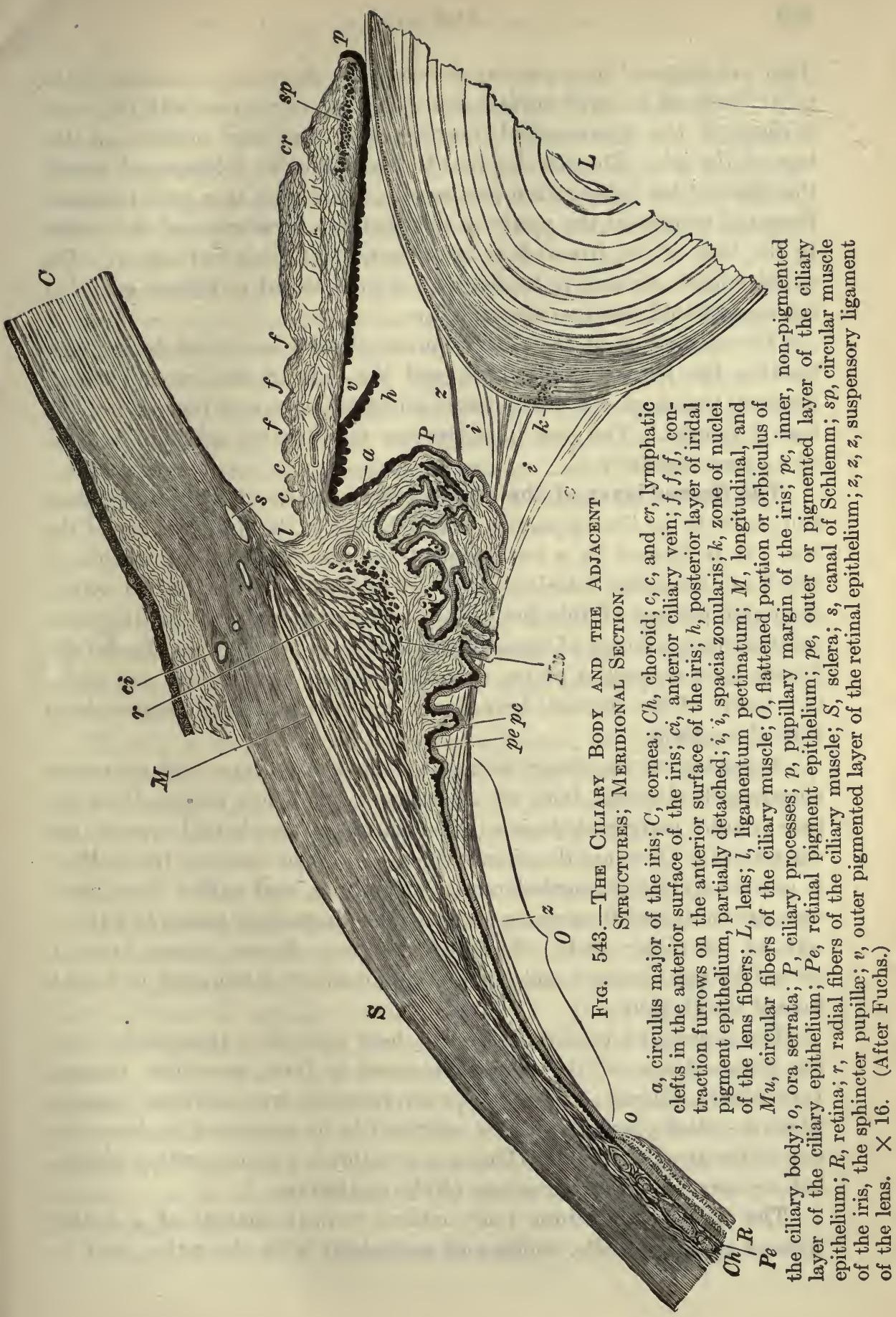


They are disposed in a circular direction about the axial margin of the ciliary body on its outer surface, and hence are in relation with the inner surface of the sclerocorneal junction and the outer margin of the base of the iris. The circular muscle fibers are also interspersed among the fibers of the ligamentum pectinatum, which pass in a radial manner from the margin of the posterior homogeneous membrane of the cornea to the base of the iris and anterior margin of the ciliary body. The circular fibers are said to be deficient or even absent in myopic eyes, but are exaggerated in hypermetropic eyes.

The disposition of the ciliary muscle fibers is such that during contraction the fibrous ciliary body and the base of the iris are drawn forward, the choroid is made tense, and the suspensory ligament of the lens is relaxed. The lens then becomes more nearly spherical because of its own elasticity.

The fibrous layer of the ciliary body consists of connective tissue, and connects the fibrous portion of the choroid to the similar tissue of the iris. It is formed by a reticulum of the fine fibers in the meshes of which are numerous lamellar and a few pigmented cells. Buried within the outer portion of this fibrous mass and intermingling with its fibers are the fiber bundles of the ciliary muscle. Into the inner portion of the fibrous layer a vascular plexus is continued from the vascular and capillary layers of the choroid; branches of the ciliary arteries communicate with this plexus.

Appended to the inner surface of the fibrous layer are numerous meridionally disposed folds of connective tissue which radiate from the base or outer margin of the iris to the margin of the choroid opposite the ora serrata. These are the ciliary processes. Their inner or free surface is covered by the pigmented retinal epithelium, and within these processes are contained the greater portion of the pigmented connective tissue cells of the ciliary body. Each fold is much deeper (about $1 \mathrm{~mm}$.) toward its axial margin and becomes progressively diminished in height toward the choroid.

The pigmented epithelial layer is here and there invaginated into the fibrous tissue of the ciliary processes to form ampullate recesses (the ciliary glands), which somewhat resemble true secreting glands. These so-called glands have been supposed to be concerned in the secretion of the aqueous humor. They are probably not true secreting glands, but represent mere invaginations of the epithelium.

The ciliary epithelium (pars ciliaris retince) consists of a double layer of epithelial cells, continuous posteriorly with the retina, and in 
front with the pars iridica retinæ. The superficial (innermost) cells present a clear or slightly granular cytoplasm with a centrally situated nucleus. Their cytoplasm is but slightly pigmented, and ofttimes is indistinctly rodded or fibrillated. In shape, these cells are of the low columnar type, but they become progressively flattened toward the iris, where they are continuous with the pars iridica retinæ. They represent a continuation of the sustentacular cells of the retina.

The cells of the deeper (outer or anterior) layer vary in height from a low columnar at the ora serrata to a somewhat flattened cell near the iridal margin, and are continuous with the pigmented cell layer of the retina. This cell layer is deeply pigmented, the entire cytoplasm being filled with the dark brown pigment granules. The nucleus, however, as in the pigmented cells of the choroid, contains no pigment, and therefore, in unstained preparations, appears under the microscope as a clear opening in the dark background of pigmented cytoplasm.

\section{THe IRIS}

The iris (Figs. 538 and 543) forms an annular curtain which projects from the anterior margin of the ciliary body toward the axis of the eye. It presents a central circular opening, the pupil, which lies in the visual axis.

The iris is suspended in the aqueous humor, its pupillary margin resting gently upon the anterior surface of the lens, its base or ciliary margin being separated from the lens by an interval, the posterior chamber, which is also filled by the aqueous humor.

The iris may be said to consist of three layers: 1 , the external epithelium; 2, the fibrous stroma; 3 , the internal epithelium.

The external epithelium (endothelium of the iris) is. a mesenchymal epithelium continuous at the margin of the anterior chamber with the posterior epithelial layer of the cornea, which appears to be reflected upon the anterior surface of the iris. At the pupillary border it is also continuous with the internal epithelium of the iris (pars iridica retinæ). The cells of the anterior or external epithelium are very much flattened and almost endothelioid in appearance; at occasional intervals the epithelium is incomplete. These intervals occur either near the pupillary or the ciliary margin, and correspond to recesses which open directly into the fibrous stroma of the iris and become continuous with its lymphatic interstices.

To the naked eye the anterior surface of the iris presents an uneven 
appearance, which is apparently due to the presence of slight meridional ridges, with shallow intervals, which extend from the pupillary margin of the iris to its outer border." The lighter radial and circular markings are due to the blood-vessels.

The fibrous stroma of the iris (pars choroidalis iridis, pars uvealis iridis) consists of a loose spongy connective tissue of an almost embryonal type. Its fibers are scanty and are gathered into small bundles, which interlace somewhat, but which are for the most part disposed in a meridional direction. This disposition is especially noticeable near the ciliary margin.

The fibrous stroma is very rich in connective tissue cells, which are mostly stellate and branch and interlace freely. They contain more or less brownish pigment, which is most abundant near the posterior (inner) surface. The color of the iris, when viewed with the naked eye, is dependent upon the depth of pigmentation in these connective tissue cells, as well as in the cells of the internal epithelial layer, and to the relative transparency of the stroma. In dark blue and black eyes the stroma pigment is scanty, and the very dark epithelial pigment shows through the more anterior clear layers of the iris. In the brown eye the stroma pigment is dense and opaque. A gray color is produced by a scanty stroma pigment clouded by a rather dense fibrous stroma. In the eyes of albinos the iris lacks pigment altogether; the pink color of the iris is due to the blood in the numerous vessels of the stroma.

Embedded in the fibrous stroma, near its pupillary margin, is a small bundle of non-striated muscle fibers, which are circularly disposed, to form the so-called sphincter muscle of the iris. Its fibers are distributed in a plane parallel to the surface of the iris, and within the inner (posterior) part of its fibrous stroma. They are most abundant near the pupillary margin, and become progressively thinner toward the base of the iris. Internally to the sphincter muscle, and in contact with the basement membrane of the internal epithelium, is an incomplete layer, more distinct toward the ciliary margin of the iris, which contains radially disposed smooth muscle fibers, the dilator muscle of the iris, apparently of ectodermal origin. Mydriatics (e.g., atropin) produce an enlargement of the pupil, presumably through inhibition of the constrictor and stimulation of the dilator muscles; miotics (e.g., morphin) produce a contrary effect.

The stroma of the iris is exceedingly vascular, the arteries and veins being meridionally disposed, the capillaries forming an irregular plexus. Near the pupillary margin the vessels form a rich capillary anastomosis, 
the circulus minor. The entering arteries likewise form a circulus major by anastomoses at the ciliary margin of the iris.

The internal epithelium (posterior epilhelium, pars iridica retina) resembles that of the ciliary body or pars ciliaris retinæ, with which it is continuous. The innermost (superficial) layer of epithelial cells, in the iridal epithelium, is deeply pigmented and somewhat flatter than in the ciliary body. The pigmentation is so deep that in the adult iris it is scarcely possible to distinguish the two epithelial layers. These can, however, be readily seen in the fetal eye, and even in that of the child,

\section{THE ANTERIOR CHAMBER}

The anterior chamber is bounded in front by the posterior (internal) surface of the cornea, and behind by the anterior surface of the crystalline lens and the anterior (external) aspect of the iris; it contains the aqueous humor presumably largely a filtration product from the numerous blood-vessels of this region, in part a secretion product of the ciliary epithelium. Its anterior boundary is convex, its posterior concave, and its circular margin is limited by an area which is known as the iridocorneal angle.

At this angle the mesenchymal epithelium is reflected from the posterior surface of the cornea upon the anterior surface of the iris. The latter portion of the epithelial layer is incomplete, since it presents numerous openings which communicate with the lymphatic spaces between the fibers of the ligamentum pectinatum and ciliary muscle. These lymphatic recesses are the spaces of Fontana.

The ligamentum pectinatum consists of fibers which arise from the margin of Descemet's membrane, and pass backward and inward, in a radial direction, to the fibrous stroma of the iris and ciliary body. Viewed from the cavity of the anterior chamber the fibers of this ligament, with the intervening spaces of Fontana, present a toothed appearance; the ligament derives its name from this peculiarity.

\section{THE POSTERIOR CHAMBER}

The posterior chamber is an annular cavity, somewhat triangular or trapezoidal in transection, whose lumen, like that of the anterior chamber, is occupied by aqueous humor, suspended in which are the fibers of the suspensory ligament of the crystalline lens. 
It is limited anteriorly by the internal surface of the iris, and antero-externally by the ciliary processes. Its postero-internal boundary is formed by the marginal portion of the lens, together with the adjacent portion of the hyaloid membrane, which incloses the vitreous humor.

\section{THE INTERNAL COAT-THE NERVOUS TUNIC}

The internal coat of the eyeball is divisible into three portions: 1 , the pars optica retinæ or retina proper; 2 , the pars ciliaris retinæ, and 3 , the pars iridica retinæ.

The last two portions, though morphologically continuous with the pars optica retinæ, differ therefrom in their physiological function; they respectively form the innermost layer of the ciliary body and iris. As such they have already been described.

\section{General Consideration of the Retina}

The retina (pars optica retince) may be said to be formed by the radial expansion of the fibers of the optic nerve which enter the eye at the inner side of its posterior pole, piercing the sclera and choroid and spreading out over the inner surface of the eyeball.

These nerve fibers arise from groups of nerve cells which are disposed in layers to form the optic and retinal ganglia (ganglion nervi optici and ganglion retince). The association of nerve cells and fibers with their supporting tissues forms the inner, cerebral, or neural portion of the retina. The dendritic arborizations of many of these nerve cells lie within the outer half, or neuro-epithelial portion of the retina.

The retina may be said to extend forward from the entrance of the optic nerve (optic disk) as far as the posterior margin of the ciliary body, where it apparently ends abruptly with an indented border, the ora serrata. From this border the retina is continued farther forward, but only as the dark pigmented layers of the ciliary processes and iris. In the usual preparations these layers contrast intensely with the opaque white color of the true retina. Like all the other tissues which are placed in the optical axis of the eye, the retina, during life, with the exception of its pigment layer, is perfectly transparent, but becomes opaque immediately after death or local injury.

The retina presents on its inner surface a slightly elevated yellow spot, the macula lutea, which lies exactly at the posterior pole of the 
visual axis. The fovea centralis is the slight depression in the center of the macula lutea, and is the result of an apparent thinning of the retinal layers at this point.

The papilla optica, or entrance of the optic nerve, also forming a slight elevation with a central depression, the physiologic excavation, is placed 3.5 to $4 \mathrm{~mm}$. to the nasal side of the masula lutea, and at a slightly lower horizontal plane.

\section{Development of the Eye}

A word as to the development of the organ will make clearer the description of the several layers of the retina. The retina is developed as an evagination of the first cerebral vesicle, and is, therefore, to be regarded as a detached lobe of the cerebrum itself. The evagination or optic vesicle grows forward in the embryo, and soon forms a flask-shaped process whose expanded extremity is early infolded in a cuplike manner forming the optic cup. The peripheral layer of the cup becomes the pigmented layer, the lining layer differentiates into the neural layers, of the retina. The inferior surface of this optic cup and the connecting optic stalk at first presents a slit-like deficiency, the choroidal fissure, into which grows the mesoblastic tissue which ultimately forms the vitreous humor and conveys the central artery of the optic nerve. The

$A$

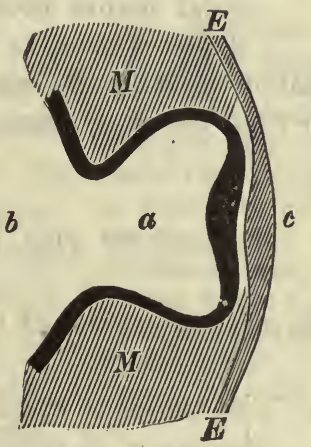

Fig. 544.-The Developing Eye in Meridional Section; Diagrammatic.

$A$, early; $B$, later stage. $E, E$, ectoderm; $L$, lens; $M, M$, mesoblast; $a$, optic vesicle, protruding from, $b$, the first cerebral vesicle; $c$, a thickening of the ectoderm, anlage of the lens; $o$, constricted pedicle of the optic cup; $p$, outer coat of the optic vesicle, anlage of the retinal epithelium; $r$, inner wall of the vesicle, anlage of the neural portion of the retina. (After Fuchs.)

indented extremity of the optic cup is soon occupied by the developing lens, which arises, under the influence of the optic cup itself (Lewis, Amer. Jour. of Anat., 1904), but is formed from the overlying area of the epidermal ectoderm. 
According to certain investigators (Szily, 1908, et al.) the definitive vitreous humor is preceded by a primitive structure in the form of a loosemeshed reticulum of protoplasmic fibers (cytodesmata) derived from, and

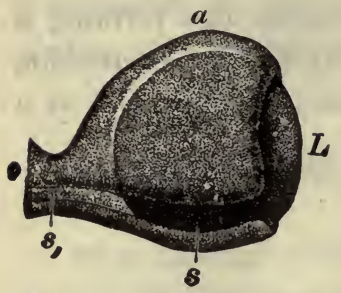

Fig. 545.-SChematic ReCONSTRUCTION OF THE Developing Eye.

$a$, optic cup; $s$, choroidal fissure; $s_{1}$, optic nerve; $L$, developing lens. (After Fuchs.) in continuity with, both the lens and the retina, hence ectodermal in origin. This primitive vitreous body is subsequently invaded by vascular mesenchyma, the two tissues uniting to produce the definitive vitreous. 'The hyaloid membrane, surrounding the vitreous body, and the fibers of the suspensory ligament of the lens are thought by some to represent the persistent peripheral remains of the original ectodermal stroma. Others, among them most recently Baldwin (Arch. mikr. Anat., vol. 80, 1912), describe the origin of the suspensory ligament from mesenchyme.

Failure of closure on the part of the choroidal fissure gives rise to various degrees of a defect known as coloboma. This condition may appear simply as a shallow cleft in the iris, or as a more or less extensive fissure in the retina or even the optic nerve, which seriously interferes with vision.

\section{LAYERS OF THE Retina}

The retina may be said to consist of ten layers, which from without inward are:

1. The pigment epithelium.

2. The layer of rods and cones.

3. The external limiting membrane.

4. The outer nuclear layer.

5. The fiber layer of Henle.

6 . The outer reticular layer.

7. The inner nuclear layer.

8. The inner reticular layer.

9. The ganglion cell layer.

10. The nerve fiber layer.

To these several layers an additional one, the internal limiting membrane, is frequently added. The first five of these layers are contained within the neuro-epithelial portion of the retina, the last five form its cerebral or neural portion.

1. The pigment epithelium (layer of pigmented cells) consists of 
a single layer of columnar epithelial cells whose bases, of generally hexagonal outline, rest upon and are firmly adherent to the inner strface of the choroid coat, and from whose free borders irregular processes extend inward between the elements of the rod and cone layer. These epithelial cells have a finely ${ }^{b}$ granular cytoplasm. Their nucleus is oval, somewhat flattened, and placed near the base of the cell; it is, however, obscured or even entirely hidden by the mass of dark pigment granules (fuscin) by which the cytoplasm of the cell is more or less completely filled.

The disposition of the pigment within the epithelial cell apparently corresponds to, and is dependent upon, the effect of light upon the retina. In an eye exposed to the action of light at the instant of death, the pigment granules accumulate in the irregular processes of the cells which surround the rods and cones, the outer or basal portion of the cell being relatively free from pig-

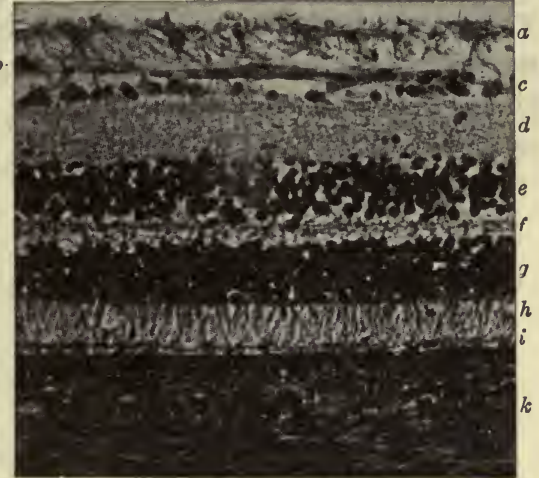

Fig. 546.-The Retina of a Child's Eye; Meridional Section.

$a$, nerve fiber layer, the broad bases of Müller's fiber cells show distinctly; $b$, blood-vessel; $c$, large ganglion cell layer; $d$, inner reticular layer; $e$, inner nuclear layer; $f$, outer reticular layer, with a prominent fiber layer of Henle; $g$, outer nuclear layer; $h$, layer of rods and cones; $i$, layer of pigmented epithelium; $k$, choroid coat. Hematein and eosin. Photo. $\times 225$. ment. In an eye which is shaded from the light, or in one removed in comparative darkness, the pigment has apparently retracted until it lies entirely with the body of the cell. Even under these conditions the extreme base of the cell frequently presents a narrow zone which is relatively free from pigment. Similar changes in the disposition of the pigment undoubtedly occur in the living eye under the influence of exposure to varying degrees of light.

The function of this pigment and of the peFig. 547.-Pigmented

EPITHELIUM OF THE Retina, Viewed in Transection.

$\times$ 500. (After Fuchs.) culiar changes in its disposition is still somewhat speculative, but it may, without doubt, be safely asserted that these phenomena are concerned with the renewal of the visual purple of the outer segments of the rods after the same has 
been bleached by exposure to light. Possibly these changes possess a stimulant action upon the neuro-epithelial elements.

2. The rod and cone layer (bacillary layer) consists of a series of columnar elements which are disposed in a palisade-like manner, and whose narrow extremities are embedded in the surface of the layer of pigment epithelium. The rod and cone layer contains elements of two distinct types, the rods and the cones, specialized receptors of the rod and cone visual cells, and very similar to each other in their structure. Each

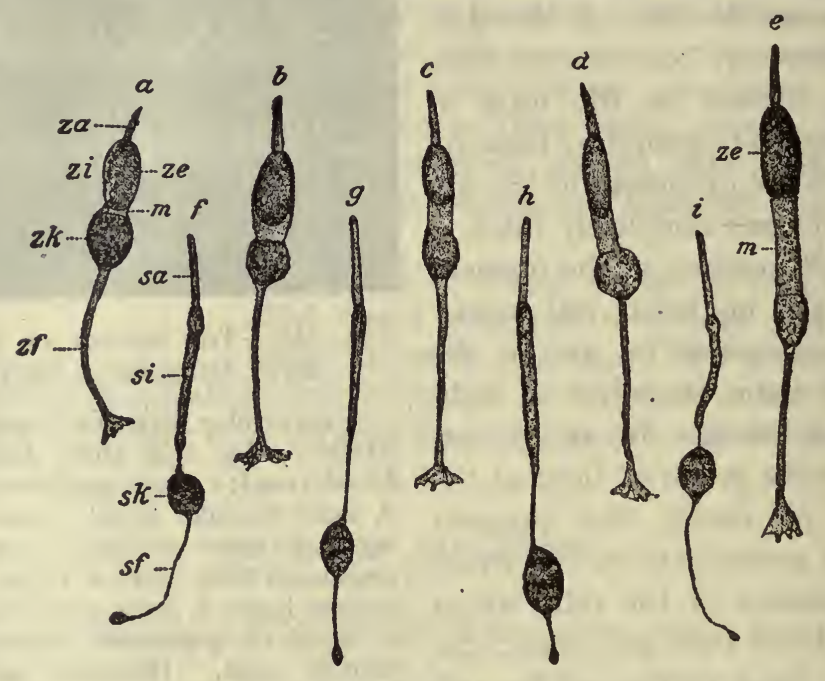

Fig. 548.-Isolated Rod and Cone Visual Celis of the Pig.

$a-e$, cones; $f-i$, rods; $z a$, outer segment; $z i$, inner segment of the cone, the latter consisting of an ellipsoid, $z e$, and a more or less elongated neck, $m$; $z k$, cone nucleus; $z f$, cone fiber; $s a$, outer, and $s i$ inner segment of the rod; $s k$, rod nucleus; $s f$, rod fiber. (After Kölliker.)

rod and each cone visual cell consists of two distinct portions, the outer of which, alone, lies in the bacillary layer; the inner portion is included in the outer nuclear layer of the retina. The outer portion is cytoplasmic, and its broad base rests upon the external limiting membrane; the inner portion is narrow, nucleated near its center, and extends entirely through the outer nuclear layer.

The Rods.-The outer, cytoplasmic, or bacillary portion of each rod visual cell consists of a somewhat thickened spheroidal base, the inner segment, and an outer filamentous extremity, the outer segment. These two segments are quite as distinct in fresh unstained tissue as in fixed 
and stained preparations, the distinction being due to the fact that the inner segment of each rod, while finely granular and easily stained, is also singly refractive; the outer homogeneous segment, on the other hand, not only stains with difficulty but is doubly refractive or anisotropic. The outer, therefore, under all conditions appears bright and lustrous as compared with the isotropic inner segment. The outer segment is said to be covered by a delicate sheath of neurokeratin, and to consist of a substance chemically very similar to that of the myelin of medullated nerve fibers.

The outer segment contains the visual purple or rhodopsin which, during life, is rapidly bleached by exposure to light, and is as rapidly renewed through the agency of the pigment epithelium.

Both segments, but especially the inner, under favorable conditions, present slight longitudinal striations. These striations, when present, are most distinct in the outer half of the inner rod segment where they form the so-called ellipsoid; they are generally interpreted as due to linear surface grooves in the outer segments, and to fibrils in the ellipsoid. The outer filamentous segment of each rod sometimes exhibits transverse markings, possibly indicating a minute structure which is comparable to a series of superposed disks.

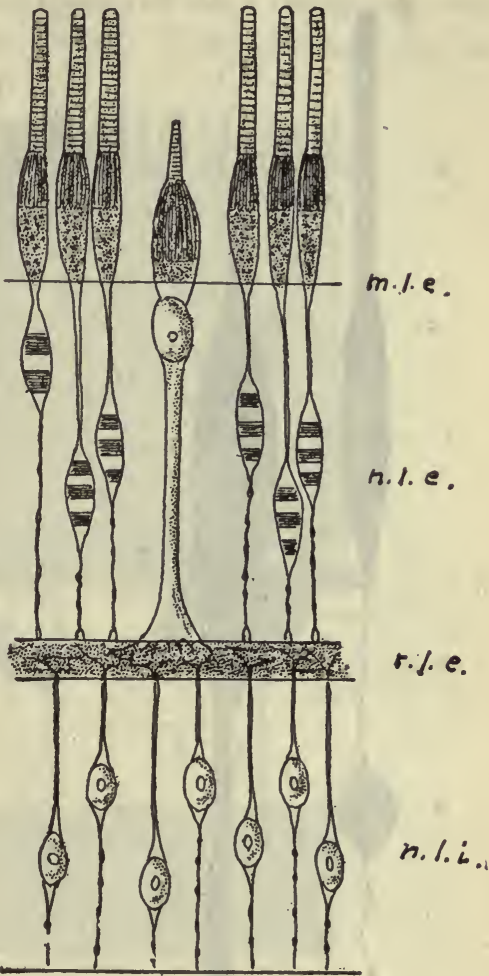

Fig. 549.-Diagram of the Rod and Cone Visual Cells, and Their Respective Bipolar NeuRoNs. (Schwalbe.)

m.l.e., external limiting membrane; n.l.e., external nuclear layer; r.l.e., external reticular (molecular) layer; n.l.i., internal nuclear layer (layer of rod and cone bipolars).

The rods have an average length of 60 microns, and an average diameter of 2 microns; of the entire extent, the outer cylindric segment and the inner spheroidal segment contribute approximately equal portions.

The inner or nucleated portion of each rod, the rod fiber, is found 
in the outer nuclear layer and is continued as a fine filament, which, having penetrated the external limiting membrane, extends as far as the border line between the outer nuclear and outer reticular layers, at which

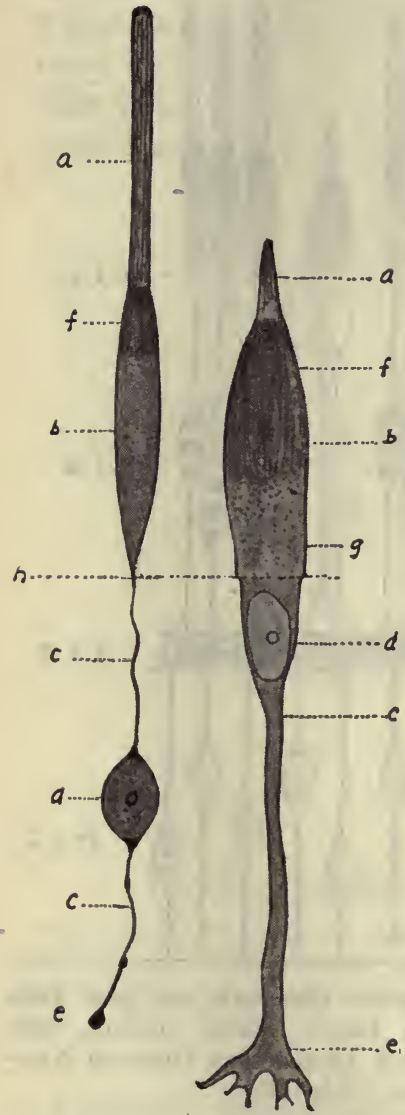

Fig. 550.-A Rod and a Cone Visual Cell from the Fundus of the Human Retina, Outside the Macula LUTEA.

$a$, outer segment; $b$, inner segment; $c$, rod- or cone-fiber; $d$, nucleus; $e$, rod- or cone-foot; $f$, ellipsoid; $g$, myoid (of cone); $h$, external limiting membrane. (Schäfer, Greeff.) $\times$ 1000.

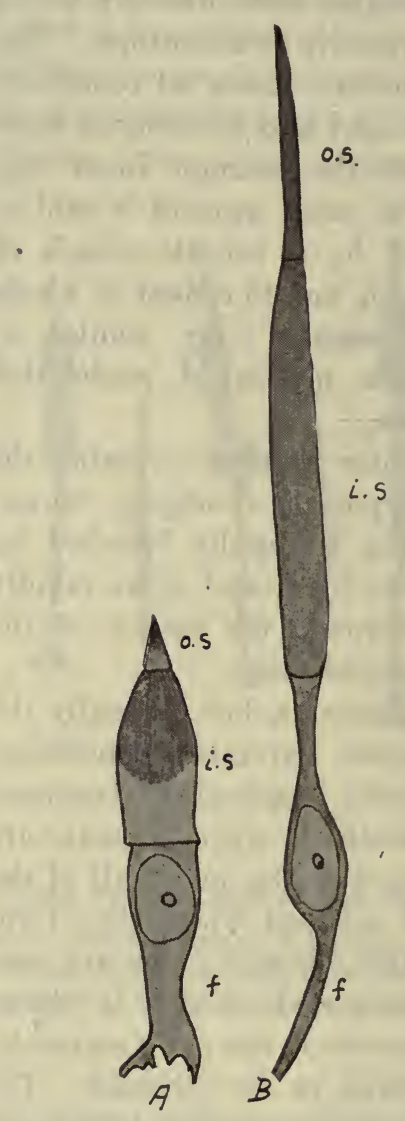

Fig. 551.-Two Cones from the Human Retina.

$A$, from close to the ora serrata; $B$, from near the margin of the forea centralis. The fiber (f) is cut short beyond the nucleus. Between the ora and the fovea cones of intermediate lengths are found, forming a series from $A$ to $B$. In the central part of the fovea, the cones are still longer than $B$, the increase being due mainly to an elongation of the outer segments (os) to about twice the length in $B$. (Greeff.) $\times 1000$. 
level the rod filament ends in a knob-like expansion. Similar knobs may appear along its course. At some point in its course through the nuelear layer the rod fiber presents a nucleated enlargement, which, under some conditions, shows one to three alternate light and dark transverse striations, the optical expression of the distribution of the chromatin in the

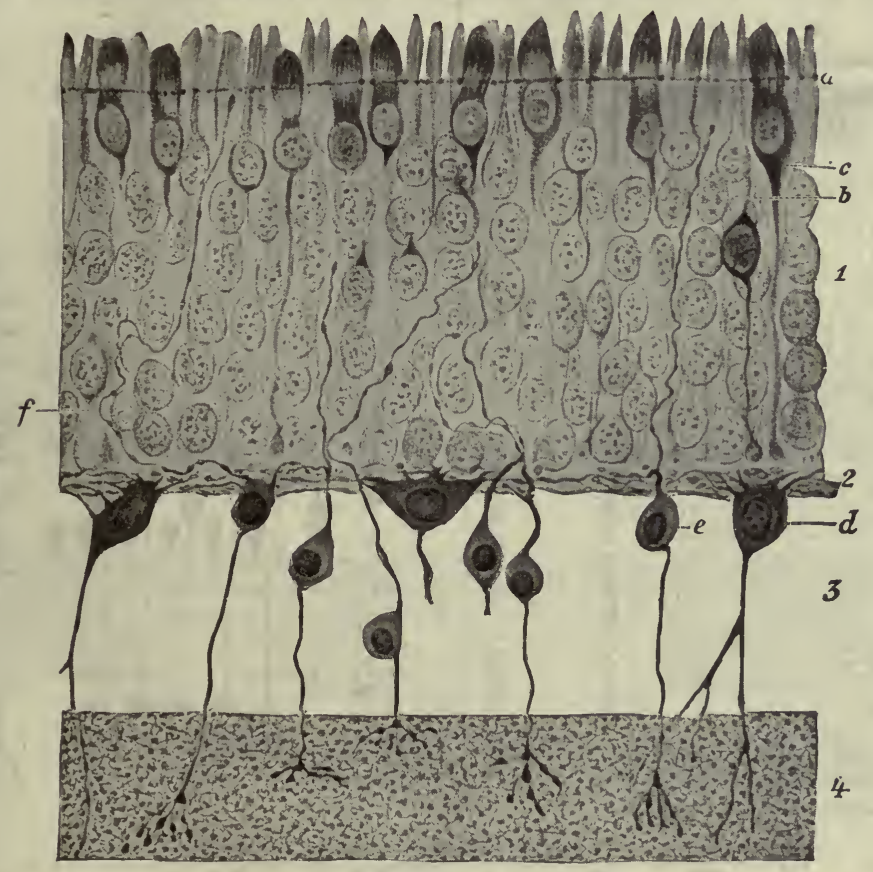

Fig. 552.-From the Human Retina.

1 , outer nuclear layer; 2 , outer reticular layer; 3 , inner nuclear layer; 4 , inner reticular layer; $a$, external limiting membrane; $b$, rod cell nuclei; $c$, cone cell nuclei; $d$, cone bipolars; $e$, rod bipolars; $f$, an exceptionally long process of a rod bipolar. Methylene blue. Highly magnified. (After Dogiel.)

form of bands. The nuclei of the rod fibers are placed at various levels in the nuclear layer, and collectively occupy nearly its entire thickness. Its outer border, however, contains relatively few rod nuclei.

The Cones.-The cones resemble the rods in structure, but their cytoplasmic portion is shorter, the inner segment of which is several times as broad ( $35 \mu$ by $7 \mu$ ). The outer anisotropic segment is especially short and does not contain visual purple, while the isotropic basal segment, whose fibrillated ellipsoid occupies a somewhat greater propor- 
tion of its length than is the case with the homologous rod segment, rests directly upon, and may even project through the external limiting membrane. The inner or nucleated portion, therefore, begins as a broad nucleated mass, equal in diameter and continuous with the bacillary portion of the cone element, to which it is ofttimes united by a slightly constricted neck. In this inner portion, just within the external limiting
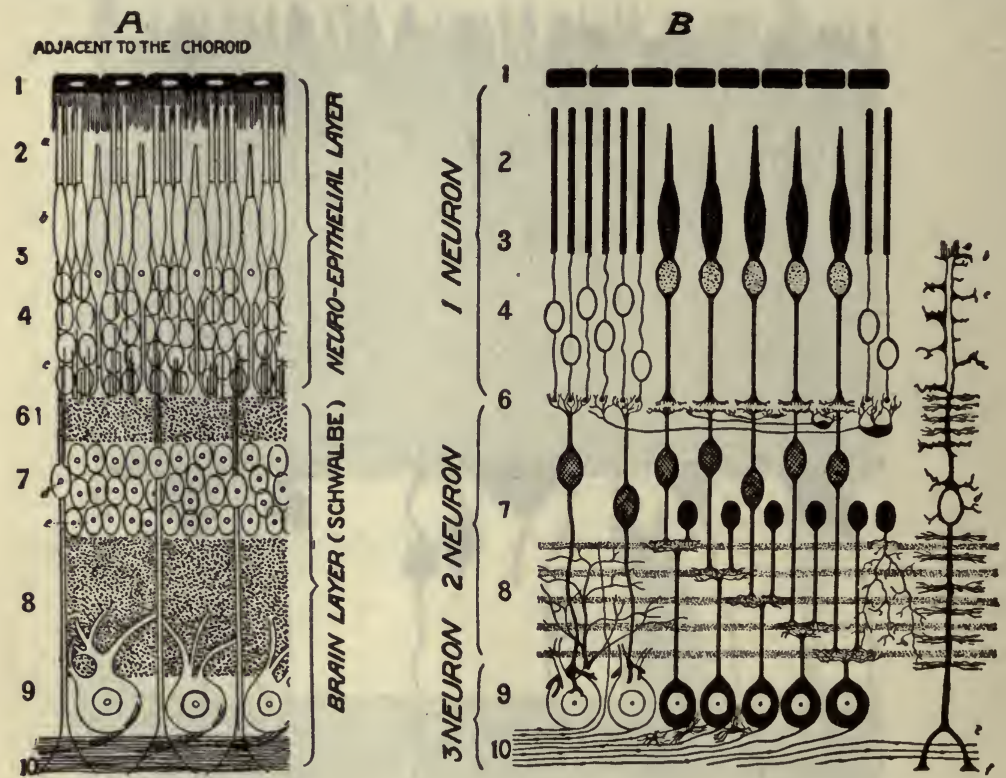

ADSACENT TO THE VITEOUS

Fig. 553.-Diagrams of the Human Retina, Showing the Relationships to Each Other of the Retinal Neurons, and Their Disposition in the DifFERENT LAYERS.

(From Fox's "Ophthalmology.")

membrane, is the cone nucleus; it differs from the rod nucleus in that it stains less deeply, presents no transverse striations, and frequently incloses a distinct nucleolus. From its nucleated portion the cone fiber is continued as a rather broad cytoplasmic filament straight inward to the border of the nuclear layer, where it terminates in an expanded portion or cone foot, from the flattened inner surface of which the fine filaments penetrate the margin of the outer reticular layer.

The outer segments of both rods and cones are embedded in the cells of the pigment layer, whose delicate filamentous processes project between the rods and cones, frequently extending almost to the external limiting 
membrane. Both rods and cones are hexagonal or nearly circular in transection. The slight intervals between the neighboring elements and the processes of the pigment epithelium are occupied by a homogeneous fluid, probably a somewhat modified lymph. The rods far outnumber

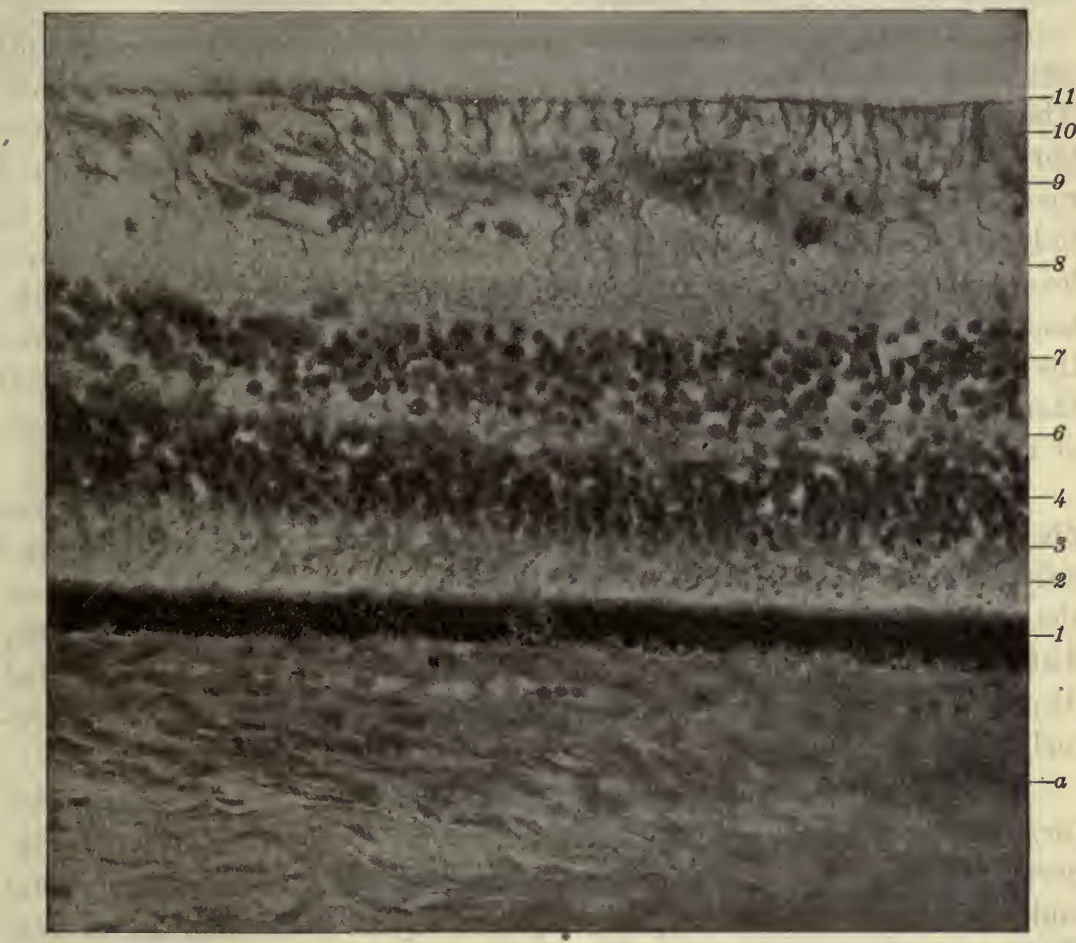

Fig. 554.-From a Meridional Section of a Child's Eye, Showing the Layers of the Retina at a Point Midway Between the Macula Lutea and the Ora Serrata.

1, pigment layer; 2, bacillary layer; 3 , external limiting membrane (indistinct); 4, outer nuclear layer; 6 , outer reticular layer; 7 , inner nuclear layer; 8 , inner reticular layer; 9 , ganglion cell layer; 10 , nerve fiber layer; 11 , internal limiting membrane; $a$, choroid coat. Hematein and eosin. Photo. $\times 400$.

the cones; three to four rods generally appear between two successive cones. The total number of cones in the human retina has been estimated at $70,000,000$; that of rods at $130,000,000$.

3. The external limiting membrane (membrana limitans externa) consists of the flattened and amalgamated extremities of the sustentacular cells (Müller's fibers), which form the chief supporting tissue, the neu- 
roglia element, of the retina, and which extend from the extreme inner surface outward to the external limiting membrane. It will therefore be more convenient to defer further description of this membrane until the remaining layers have been described, and the Müllerian fibers can be considered in their entirety.

4. The outer nuclear layer (outer granular layer) consists, for the most part, of the nucleated portions of the rod and cone elements. The outermost zone of this layer contains only cone nuclei; the inner portion, comprising about three-fourths of its thickness, contains only rod nuclei. The former, with occasional exceptions (Stöhr), are situated in only one relatively narrow plane; the latter are distributed at various levels, though they occur more abundantly in the mid-region of the nuclear layer. In addition to portions of Müller's fibers which serve for the support of the nucleated elements, this layer contains the terminal filaments of the distal processes of some of the small bipolar nerve cells of the inner nuclear layer.

5. The fiber layer of Henle is formed by that portion of the cone fibers which is internal to the layer of rod nuclei. It is a thin layer and only acquires importance in the neighborhood of the macula lutea, where the cones are most abundant. In this portion of the retina it is easily distinguished from the outer reticular layer by the somewhat radial disposition of its fibers, the fibers of the reticular layer having an irregularly meridional direction.

6. The outer reticular layer (outer molecular layer; outer plexiform layer) presents a dense tangle of neural tissues consisting of supporting neuroglia fibers and interlacing processes from the horizontal and bipolar nerve cells of the inner nuclear layer. Terminal fibrils from this network intermingle in the fiber layer of Henle with terminal fibrils from the cone feet; more externally they are in intimate relation with the end knobs of the rod fibers. This arrangement permits the transmission of stimuli from the neuro-epithelium to the retinal ganglion.

7. The inner nuclear layer (inner granular layer, ganglion retince, outer ganglionic layer) contains a mass of nerve cells, together with the nucleated portion of the sustentacular fiber cells of Müller. The nerve cells may be described as corresponding to one of three types, which, from the plane in which they are distributed may be termed the outer, middle, and inner.

The Outer Nerve Cells.-The outer nerve cells (horizontal cells, basal cells) possess pyramidal, stellate, or flattened cell bodies whose dendrons are distributed to the horizontal plexus of the outer reticular 
layer. These cells vary in size; the dendritic or distal processes of the smaller cells on reaching Henle's layer are in relation with the terminal fibrils of the cone feet; those from the larger nerve cells are in relation with the terminal knobs of the rod fibers. The axis cylinders or central processes from all these cells after traveling horizontally-viz., in a plane parallel to the layers of the retina-for a greater or less distance, turn inward and pass to the inner reticular layer, where they come into

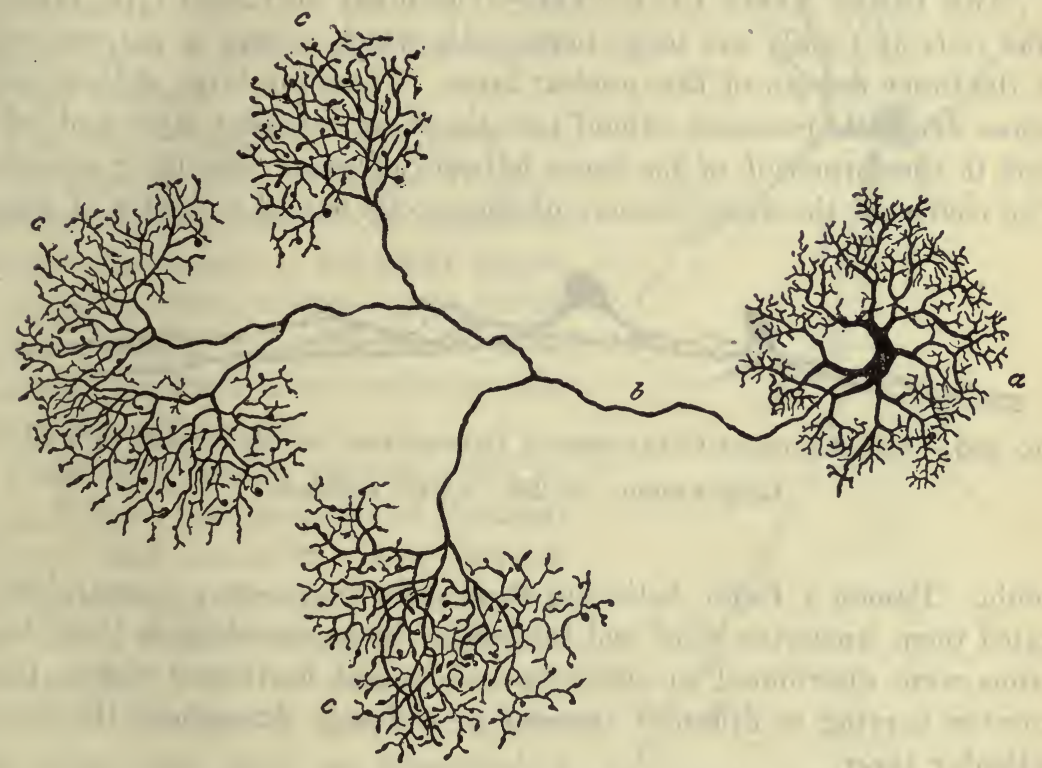

Fig. 555. - Horizontal Cell from the Retina of a Calf.

$a$, cell body; $b$, axon; $c$, terminal arborizations of the axon. Golgi's stain. $\times 150$. (After Marenghi.)

relation with the dendrons of the large nerve cells of the ganglion cell layer. Other processes, mostly from the smaller cells, terminate in the outer reticular layer, probably serving the purpose of association neurons.

The Middle Type.-The nerve cells of the middle type are usually of bipolar form, and are the most abundant elements of the inner nuclear layer. The one set of their processes is directed outward (peripheralward); they pass to the outer reticular layer where they eventually come into relation with either the rod fibers or the cone fibers. Hence those cells which are in relation with the visual rods are classified as 
rod bipolars, those in relation with the visual cones as cone bipolars (Fig. 553). The terminal fibrils of the cone bipolars are horizontally, those of the rod bipolars radially, disposed.

The central processes, axons, of the bipolar cells are directed inward (centralward), and on entering the inner reticular layer terminate in an end brush which is in relation with the dendritic processes from the large ganglion cell layer.

The Inner Nerve Cell Type.-The inner nerve cell type (amacrine cells of Cajal) are large nerve cells which occupy a narrow zone at the inner margin of this nuclear layer. These are large stellate cells whose dendritic processes extend into the inner reticular layer and take part in the formation of the dense feltwork of which that layer consists. The course of the axis-cylinders of these cells is still a matter of some

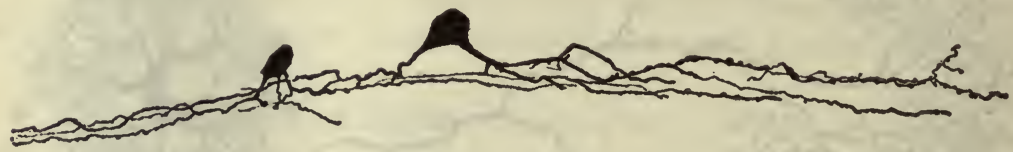

Fig. 556.-Two Amacrine Cells from a Transection of the Retina of a Calf. Golgi's stain. $\times 260$. (After Kölliker.)

doubt. Ramón y Cajal, believing these cells to possess no axon, designated them 'amacrine cells' and subdivided them according as their dendrons were distributed in either one of several horizontal planes (the number varying in different species) or diffusely throughout the inner reticular layer.

Some of the amacrine cells, however, send an axon in a horizontal direction to the inner reticular layer, and are also in relation with the terminal arborizations of centrifugal nerve fibers which enter from the nerve fiber layer. These have been regarded by some observers as 'dislocated nerve cells' of the ganglion cell layer; Cajal named them 'association amacrins.'

8. The inner reticular layer (inner molecular layer, inner plexiform layer) is a densely tangled network of nerve-cell processes, a neurospongium. To these are added a much branched portion of Müller's fibers, which form the chief supporting tissue of this layer. The cell processes entering into this formation are derived from the cells of the inner nuclear and ganglion cell layers, and it is here that the processes of these cells interlace so closely as to permit the transmission of impulses from the one neuron to the other. Their terminal arborizations are, for the 
most part, disposed in horizontal planes, though a few spread throughout the entire thickness of the reticular layer.

9. The ganglion cell layer (ganglion nervi optici, inner ganglionic layer, layer of large nerve cells) is of variable thickness. Its greatest depth is in the region of the macula lutea, where it consists of five or six superposed ganglion cells. Toward the equator of the eye it becomes progressively thinner, until near the ora serrata its single layer of cells only forms an incomplete stratum.

The cells comprising this layer are mostly large, stellate, pyriform, or spheroidal nerve cells, from whose peripheral border dendrons pass to the inner reticular layer, and from whose central border an axon passes to the nerve fiber layer to eventually become the axis cylinder of a fiber of the optic nerve.

These cells, though varying much in size and shape, possess a body of the usual structure with neurofibrils and chromophilic granules, and a pale vesicular nucleus with a distinct chromatic nucleolus. Intermingled with the nerve cells are many fine branches of the sustentacular cells which here form an open-meshed network within whose spaces the nerve cells are inclosed.

10. The nerve fiber layer, in intimate relation with the preceding, forms the innermost of the retinal zones. It consists of naked axis-cylinders passing from their origin in the ganglionic layers

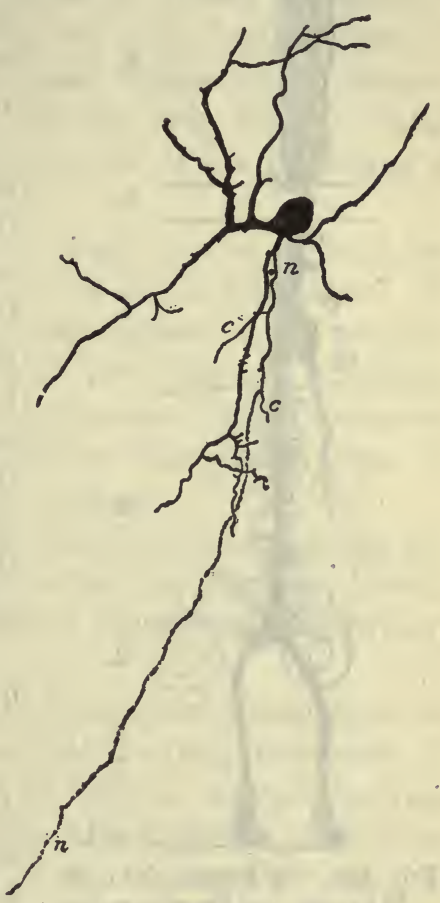

Fig. 557.-A Nerve Cell of the large Ganglion Cell LAYER; FROM the RETINa OF A CAT.

$n, n$, axon; $c, c$, collaterals. Golgi's stain. $\times 325$. (After Kölliker.) to their immediate destination, the optic nerve. They are, therefore, mostly if not wholly centripetal fibers. A few centrifugal fibers have been demonstrated in this layer, but they would appear to be probably vasomotor in function, a few possibly ending in relation to the amacrine cells.

The nerve fibers of this layer converge from all portions of the retina, follow a meridional course through the open meshes of the network of branching sustentacular cells, and converge toward the optic papilla, the 
entrance, or rather the point of exit, of the optic nerve. Hence the nerve fiber layer, being augmented by the constant acquisition of new

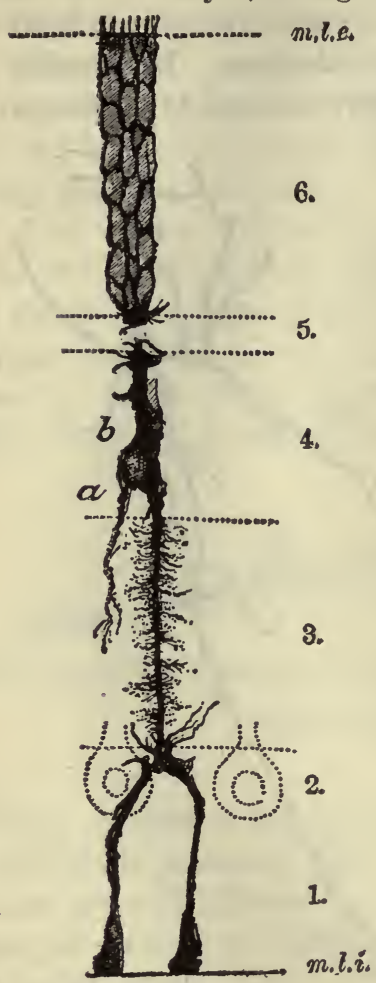

Fig. 558.-A Fiber Cell of

MÜller, or SustentacutaR CELL, FROM the DoG'S RETiNa.

1 , nerve fiber layer; 2 , ganglion cell layer; 3 , inner reticular layer; 4, inner nuclear layer; 5 , outer reticular layer; 6 , outer nuclear layer; $a$, a process extending into the inner reticular layer; $b$, nucleus of the cell; m.l.e., external limiting membrane; m.l.i., internal limiting membrane. Golgi's stain. Highly magnified. (After Cajal.) axons from the ganglion cells, becomes progressively thicker toward the posterior pole of the eye, and is thickest at the margin of the optic papilla, where it is so highly developed as to almost exclude the other retinal layers.

The course of these non-medullated nerve fibers is not straight; on the contrary, they interlace to form a delicate fibrillar network. At the margin of the papilla optica the nerve fibers bend outward with a sharp curve almost at right angles to their former course. At this point also they gradually acquire a medullary sheath and, uniting into many bundles, penetrate the numerous openings of the lamina cribrosa of the sclerotic and choroid coats to form the optic nerve.

\section{The Supporting Tissues of the Retina}

These consist of a gliaform reticulum distributed throughout the cerebral portion of the retina, and of a special supporting tissue, Müller's fibers, which may also be regarded as glia tissue, though they are common to both the neural and epithelial portions.

The fibers of Müller (radial fibers; sustentacular cells) comprise numerous large glia cells whose processes begin with an expanded base at the inner surface of the nerve fiber layer, and can be traced all the way through the retina to the membrana limitans externa, which is likewise formed by the terminal expansions of these cells. The nucleus of the fiber cell lies in the mid-region of the inner nuclear layer. 
The expanded and flattened bases or inner extremities of these glia cells are so closely approximated to one another as to form a complete investment for the inner surface of the retina, which is known as the internal limiting membrane (membrana limitans interna) and is frequently classed as the innermost layer of the retina. Under low magnification it appears as a continuous membrane, but under higher powers it is readily resolved into the broad, conical, basal expansions of which it consists. From these initial expansions the glia cells may be traced outward through the nerve fiber and ganglion cell layers by means of the numerous coarse processes or glia fibers.

The glia fibers then pass in a fairly straight course through the inner reticular layer. In this portion numerous short, fine, lateral offshoots from the main stem support the neurospongium of the reticular layer. Continuing through the inner nuclear layer the glia substance is somewhat thickened; it sends off fewer but coarser lateral processes, and in the mid-region of this layer presents an enlargement which is almost entirely occupied by the large ovoid nucleus.

The fiber cell, somewhat narrowed, may then be traced through the outer reticular to the outer nuclear layer, where its processes form a dense network about the nucleated segments of the rod and cone visual cells.

The terminal processes of the fiber cells become again flattened, somewhat after the manner in which the internal limiting membrane is formed, and are so closely approximated as to form an external limiting membrane, a distinctly membranous structure which derives a reticular appearance from being pierced by each of the innumerable rod and cone elements.

From the outer surface of the expanded ends of the Müllerian fiber cells which form the external limiting membrane, minute fibrils are continued between the bases of the non-nucleated portions of the rod and cone cells to form shallow sockets, the rod and cone sockets, into which the bacillary portions of these elements are fixed. The neuroglia supporting tissue includes also the ordinary long- and short-rayed astrocytes, limited, however, to the cerebral portion of the retina.

\section{The Macula Lutea}

\section{(Yellow Spot)}

The macula lutea being apparently the most highly developed portion of the retina, deserves some special consideration. The macula is a cir- 


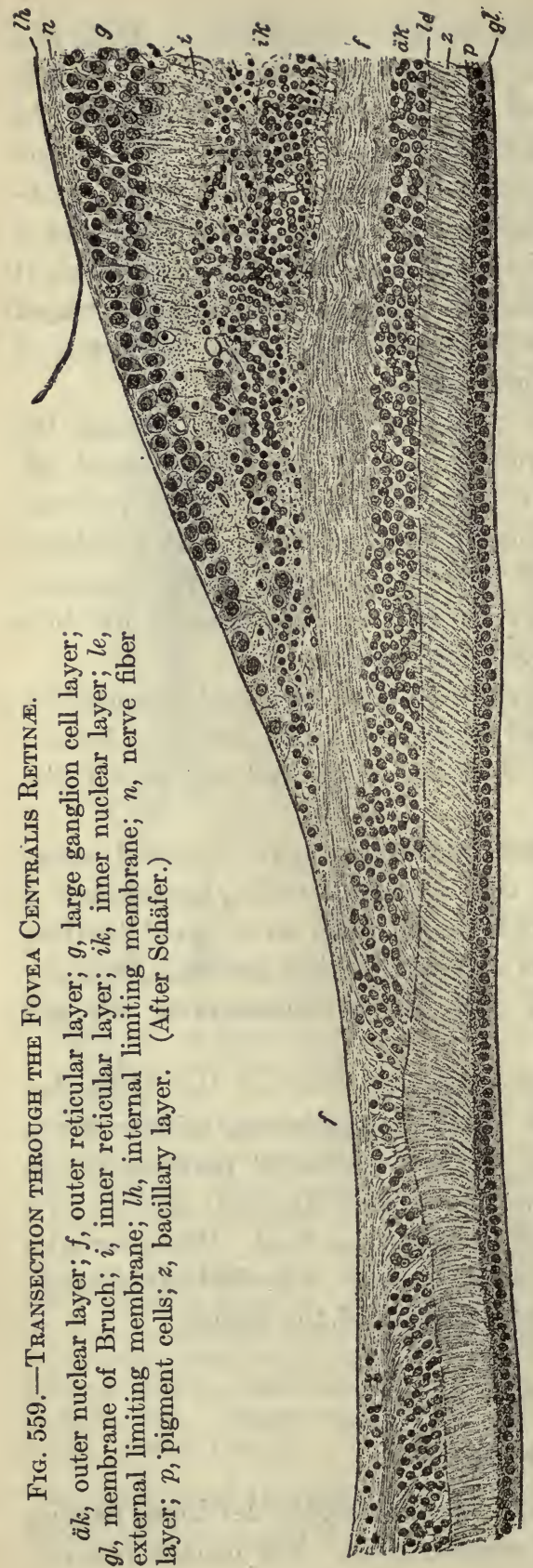

cular elevation about $2 \mathrm{~mm}$. in diameter, in the center of which is a marked depression, the foved centralis. It is yellow in color, due to the presence of a pigment. The elevation results from an increased thickness of all the retinal layers, but especially of the ganglion cell layer, which in this portion of the retina is five or six cells deep. The reticular layers are also much thickened in this area. In the bacillary layer, within the area of the macula, the cones are far more numerous than elsewhere, especially when considered in relation to the rods, which are greatly diminished peripherally and absent centrally. The cones of the macula are almost twice as long as those of the equatorial region of the retina, the increased length being due to elongation both of the intermal and external segments, but mainly the latter; they are also somewhat more slender, and their nuclei may be placed some distance beyond the external limiting membrane.

Toward the fovea centralis the inner layers of the retina become very much thinned, until at its center the nerve tissues are merely represented by scattered cells of the inner nuclear and ganglion cell layers. Rod elements are not found in this area; the bacillary layer consists entirely of elongated, slender cones. 
The much elongated nuclear portion of the cones deviates in a slanting direction toward the margin of the macula, and the cone nuclei are further removed from the external limiting membrane than elsewhere in the retina.

The pigment of the epithelial layer is much diminished and may even be absent at the fovea. Because of the diminution in the number of ganglion cells in this area the nerve fiber layer is greatly diminished in thickness on approaching the margin of the fovea, and toward its center entirely disappears.

The fovea centralis lies at the posterior pole of the anteroposterior axis. The light stimulus here meets least obstruction in passing to the neuro-epithelial elements; this is the point of acutest vision.

Since the fovea centralis contains no rods, it lacks the visual purple; and since vision is sharpest at this point, the visual purple would seem unessential to sight. The visual purple is said to be absent in the eyes of the pigeon, the hen, some reptiles and some bats. It is supposed to enhance the irritability of the rods in dim lights.

In the eyes of birds and reptiles the cones outnumber the rods; and in certain reptiles, e.g., lizards, rods are entirely lacking. In sharks and rays, most nocturnal animals, and the owl, cones are either absent or few in number or rudimentary in structure. According to the view of Kreis (1895) the cones function in the

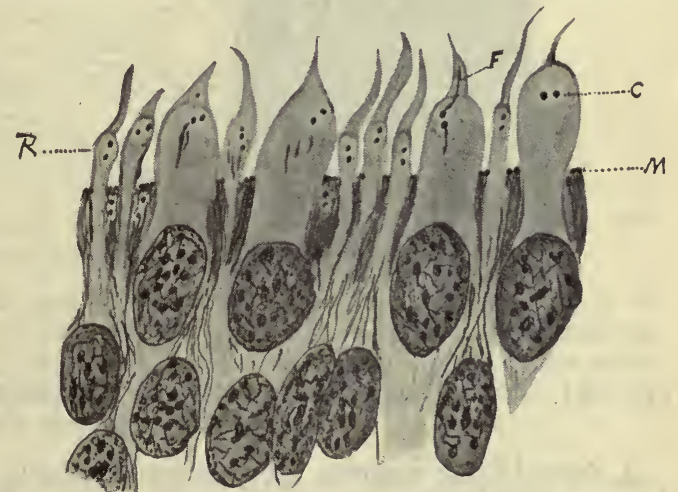

Fig. 560.-Developing Rod and Cone Visual Cells, from the Retina of a 345 ma. (6 mos.) Human Fetus.

$M$, diplosome in a Müller's fiber at the level of the external limiting membrane; $C$, diplosome in a cone cell; $F$, fiber growing out from the cone diplosome; $R$, diplosome in a rod visual cell. (Seefelder.) $\times 1000$.

perception of color, the rods are sensitive only to light and darkness. In color blindness the cones are defective; in night-blindness the rods are affected.

Development of Rods and Cones.-The rods and cones arise and by an essentially identical mode of differentiation from apparently similar embryonic cells. A protoplasmic bud is pushed beyond the external limiting membrane towards the layer of pigmented cells. The cone bud is from 
four to five times as stout as that of the rod cell. These processes carry apically a diplosome, surrounded by a lighter cytoplasmic halo. From each member of the diplosome a fiber-process grows out, the one passing towards the elongating distal, the other towards the proximal, pole of the cell. The distal process thus becomes enveloped by an extension of the cell cytoplasm, the two constituents, fiber and investing cytoplasm, uniting in the forma-
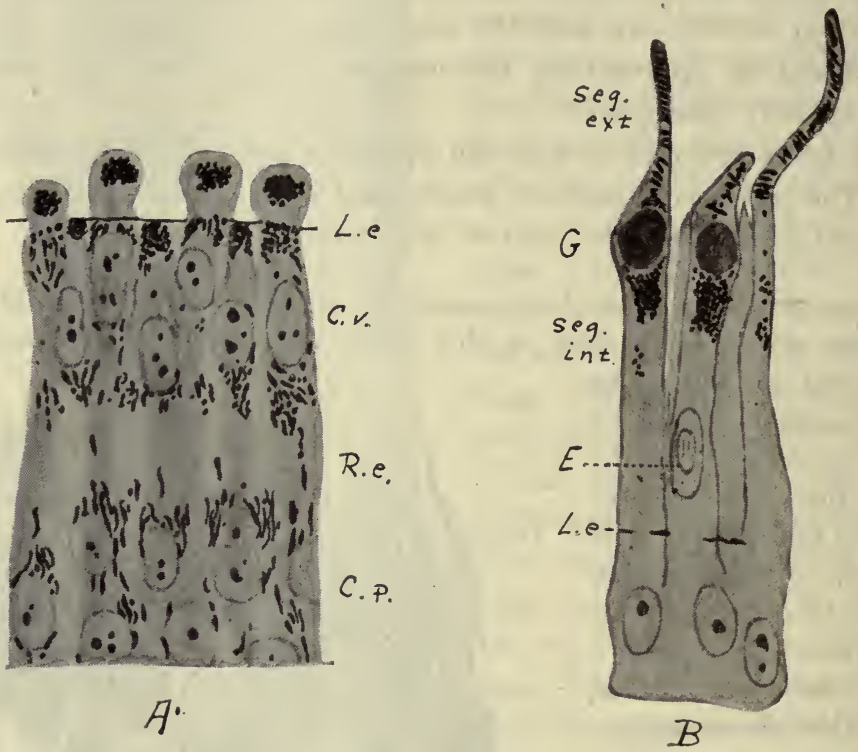

Fig. 561.-Two Early Stages in the Development of the Rod and Cone Visual Cells in the Chick.

$A$, from 12-day embryo, showing the visual cell buds containing mitochondria; L.e., external limiting membrane; C.V., visual cell; R.e., external reticular layer; $C . p$., bipolar cells; $B$, from 1-day-old chick, showing two complete rods and one cone, both elements containing a large lipoid spherule $(G)$ and mitochondria. The cone contains an ellipsoid $(E)$; seg. int., internal segment; seg. ext., external segment. (Leplat.) $\times$ about 2000.

tion of the outer segment of the rods and cones. Seefelder ("Atlas zum Entwickslungsgeschichte des Menschlichen Auges," Leipzig, 1914) has recently confirmed the essential points in the earlier descriptions of rod and cone differentiation by Leboucq (1909) and by Magiott (1910). Leplat (Anat. Anz., 45, 8, 1913) has investigated the rôle of the mitochondria in connection with this process. He describes the migration of the. 'plastosomes' into the external segment where they become chemically altered and disappear as such in their contribution to the homogeneous cytoplasmic sheath of the centrosomal filament of the external segment. Leplat inclines to regard the transverse striation of this segment and its cleavage into 
dises by maceration as the expression of its mode of construction from mitochondria. It remains uncertain whether the longitudinal fibrillation of the internal segments is likewise the probable result of an arrangement of mitochondria in the developing cell; the complete history of these mitochondria has not yet been traced.

The Inversion of the Retina. - The receptive cells of the vertebrate retina exhibit, in contrast to all other neuro-epithelial cells, a reversed polarity with respect to the source of their special stimulus, the ether waves; the transmitting end of the cells is nearer the source of the light than the percipient end. The human retina is appropriately described as

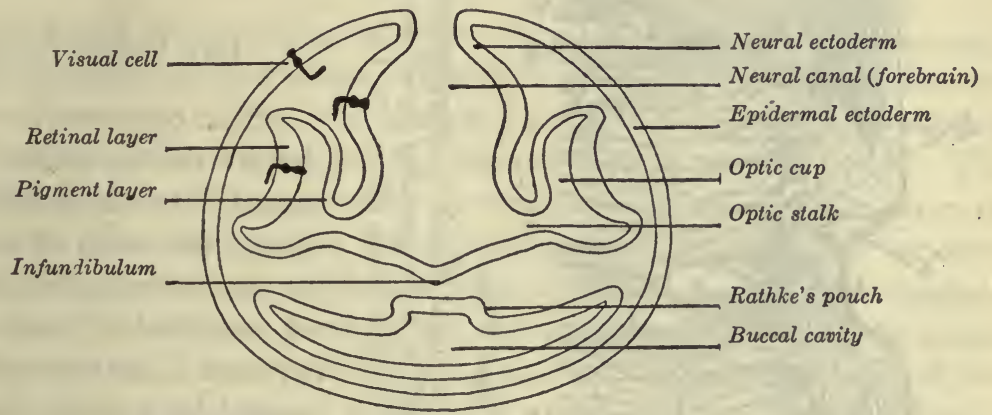

Fig. 562.-Diagram Illustrating Balfour's Theory to Account for the Inversion of the Visual Cells of the Vertebrate Retina.

Transverse section through the head of a hypothetical vertebrate embryo, to show the morphological relations of the surfaces of the ectoderm of the integument, the neural tube, and the forming retina. In each of these situations a single sense cell is indicated. (After Parker, Amer. Nat., 42, 501, 1908.)

an inverted sense-organ. The most plausible theory yet proposed in explanation of this inversion of the visual cells seems to be the one outlined by Balfour (1881), expressed in terms of the ancestral history' of the vertebrate eye. (Among the invertebrates an inverted retina is known only in certain mollusca, e.g., Pecten, and in certain spiders and the scorpion.) 'According to this view the vertebrate retina originated on the outer surface of the ancestral vertebrate in much the way that the eyes of many invertebrates have been produced. The primitive retinas thus formed were implanted in that portion of the surface of the animal from which the central nervous system was destined to develop, and when this was infolded these retinas were carried in with it and came thus to be involved in the central organ. If the morphological position of a sensory cell, such as may have existed in the primitive external retina, is supposed to have been thus retained as this organ was carried from its superficial location into the central nervous system and out again almost to the external sur- 
face, the resulting retina would be composed of inverted elements (Fig. 562). Thus this theory at once offers an explanation for the two most striking features of the vertebrate retina, namely, its formation as an apparent outgrowth from the central nervous system and the inverted condition of its receptive cells' (Parker). The difficulties of this theory are

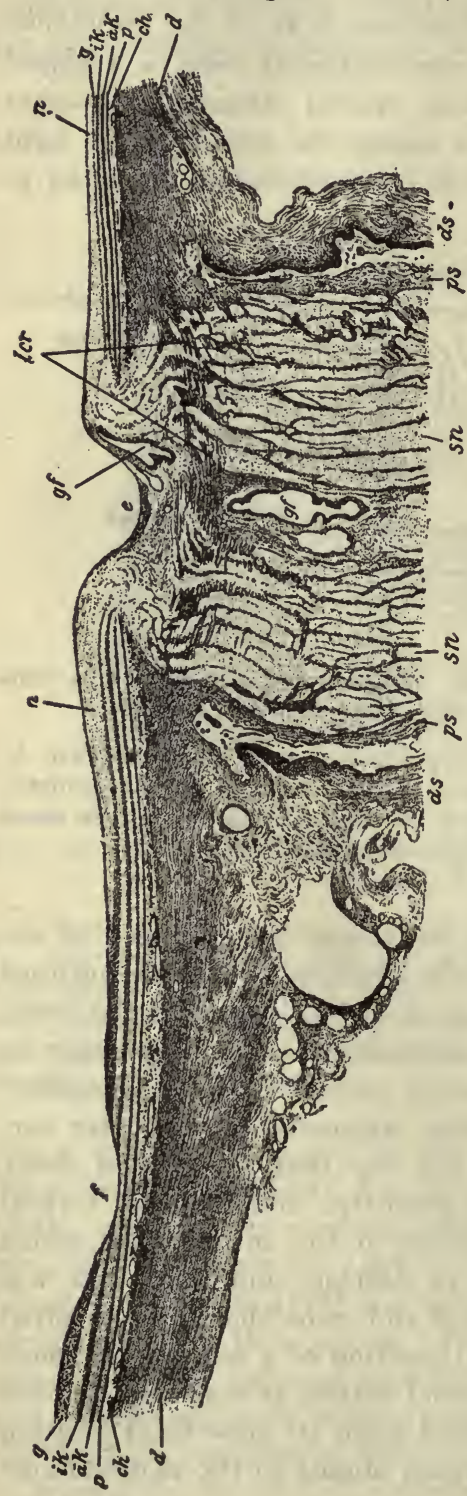

范渠 discussed, and an alternative theory, based upon the direction eyes of Amphioxus, is presented by G. H. Parker (Amer. Nat., 42, 501, 1908).

\section{The Optic Nerve}

The optic nerve is a large nerve trunk, composed, like the white matter of the brain of which it is an ontogenetic portion, of medullated nerve fibers without a neurolemma, supported by a neurogliar network containing long-rayed astrocytes. It receives an investing sheath from each of the cerebral membranes, septa from the pia mater enveloping the several funiculi. These sheaths are continued as far forward as the eyeball, at $\mathrm{wh} \mathrm{i} \mathrm{ch}$ point they become continuous with the sclera. Though the choroid corresponds to the pia mater, the two are not apparently in direct continuity.

Lying in the axis of the nerve, the arteria centralis retince with its accompanying vein enters the eye and appears on the inner sur- 
face of the retina at the porus opticus (physiologic excavation; optic disk) in the center of the optic papilla. Here it divides, its two branches at first pursuing a meridional course between the hyaloid membrane and the retinal surface; soon they pierce the latter to supply the cerebral portion of the retina. No vessels penetrate the neuro-epithelial portion of the retinal layers; these are nourished by the choroid. 'The vena centralis retince pursues a course exactly similar to that of the artery.

\section{The Ora Serrata}

At the ora serrata (Fig. 538) the typical layers of the retina, already much thinned, abruptly cease. The first elements to disappear are the rods and cones; the cones, which become much shorter, practically lacking the outer segment, extend farther toward the ora than the rods. The remaining layers are continued forward only as the double layer of epithelial cells belonging to the pars ciliaris retinæ, the inner stratum of which appears to be analogous to and continuous with the cerebral portion of the retina, apparently the sustentacular cells; while the outer, deeply pigmented layer apparently represents the pigmented layer of the retina. For some distance toward the ora serrata, the retina becomes modified by the presence of large vacuoles, probably lymph spaces.

\section{THE OCULAR CONTENTS}

Within the ocular globe, whose walls are formed by the three coats of the eye, are certain structures which may be collectively considered as its contents. They are:

1. The aqueous humor.

2. The crystalline lens.

3 . The vitreous humor.

4. The hyaloid membrane.

5. The suspensory ligament.

\section{The Aqueous Humor}

The aqueous humor is a fluid, closely allied to lymph, which occupies the anterior and posterior chambers of the eye. Microscopically it is structureless. Occasional leukocytes, migrants from adjacent lymph channels, may be encountered. 


\section{The Crystalline Lens}

The crystalline lens with its suspensory ligament forms a sort of diaphragm which separates the ocular cavity into two compartments, of which the anterior is occupied by the aqueous humor, the posterior by the vitreous humor.

The lens is a biconvex transparent body having a somewhat greater convexity on its posterior than on its anterior surface; its curvature is greater at its margin than toward its center. It has a transverse diameter of from $8 \mathrm{~mm}$. to $9 \mathrm{~mm}$., and an anteroposterior diameter of from $3.5 \mathrm{~mm}$. to $4 \mathrm{~mm}$. according to the degree of accommodation. It consists of a capsule, epithelium, and a substantia lentis.

The capsule of the lens is a homogeneous membrane which covers its entire surface and receives the attachment of the suspensory ligament. It presents faint meridional striations and may sometimes be separated into several lamellæ (Berger, 1893); this lamellation may be purely artificial, but appears to be somewhat dependent upon the attachment of the fibers of the suspensory ligament to the surface of the lenticular capsule.

The capsule is about twice as thick over the anterior as over the posterior surface of the lens. On the former surface it is in relation with the lenticular epithelium, but on the posterior surface the capsule rests directly upon the substantia lentis. The anterior surface of the capsule is in gentle contact with the free margin of the iris.

The lenticular epithelium consists of a single layer of cells which covers the entire anterior convexity of the lens, extending as far back as its equator. The height of these cells varies with the age of the individual. In fetal life they are distinctly columnar, in youth short columnar or cuboidal, in adult life low cuboidal or flattened. Toward the margin of the lens the epithelial cells become progressively lengthened, and at its equator are transformed directly into the fibers of the lenticular substance. This definitive structure of the lens recalls its manner of development from the original lens vesicle; the hollow vesicle becomes solid by the elongation of the cells of the posterior wall.

The substantia lentis is, therefore, the product of the epithelium of the lens, whose cells become greatly elongated to form slender hexagonal prisms, known as the lens fibers. When it is first formed each prism exhibits a nucleus which persists for some tirne, but gradually disappears as in the process of growth the older fibers become farther and farther removed from their source of nutrition, the lymph and the 
aqueous humor in which the surface of the lens is bathed. This change is accompanied by a hardening or cornification and slight shrinkage of the lens fibers, so that those prisms which come to occupy the center of the lens form a dense, hard mass of non-nucleated fibrous cells with faintly serrated margins; the peripheral fibers retain their smooth edges and their nuclei, and form a protoplasmic mass of much softer consistency. The hardened central mass is the so-called nucleus of the lens. Any opacity of the lens or its capsule is known as a cataract.

The nuclei of the lens fibers remain in the neighborhood of the equator, where they are first formed, and are thus contained within a narrow, superficial, equatorial zone, the nuclear zone.

Each lens fiber is disposed along a meridian of the lens, and extends from its anterior to its posterior hemisphere; the fibers are so-arranged that they abut upon one another, end to end, along Vshaped lines which radiate from either pole. This. union is often quite firm, and thus are formed long fibrous bands which can be traced from the anterior to the posterior hemispheres of the lens. These bands are distributed in a peculiar manner. Near each pole along the line of abutment, the band may be said to bend upon itself with a sharp curve-making

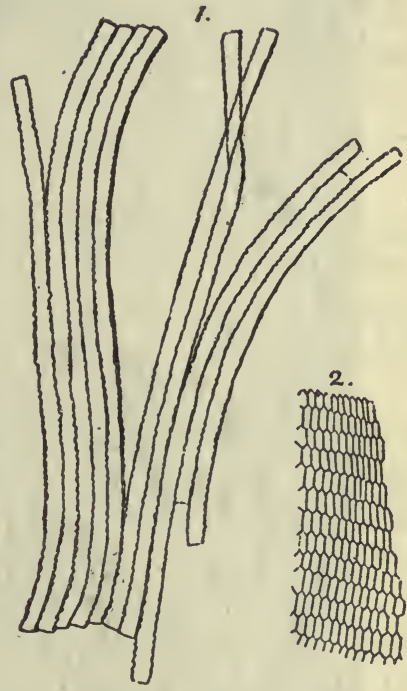

Fig. 564.-Lens Fibers.

1 , in profile, from the crystalline lens of the ox's eye; 2 , in transection, from thehuman crystalline lens. $\times 350$. (After Kölliker.) an angle of about 60 degrees-whose convexity is directed toward the pole, the parallel fibers being so arranged as to form a sector whose apex is also directed toward the pole. The corresponding sectors of opposite poles overlap one another so that the fibrous bands are continued from one side of one polar mass to the reverse side of the overlapping sector and so back, on the farther side, to the adjacent sector of the former hemisphere. By teasing, fibrous bands can sometimes be traced successively through all of the polar sectors and thus back to a sector beneath that from which the start was made. Obviously no individual lens fiber is of sufficient length to extend from pole to pole of the lens. 
The polar figures formed by the lines of terminal union of the lens fibers are known as lens starts; these are at first three-rayed, but later become six- and even nine-rayed.

During the earlier stages of the development the lens is invested

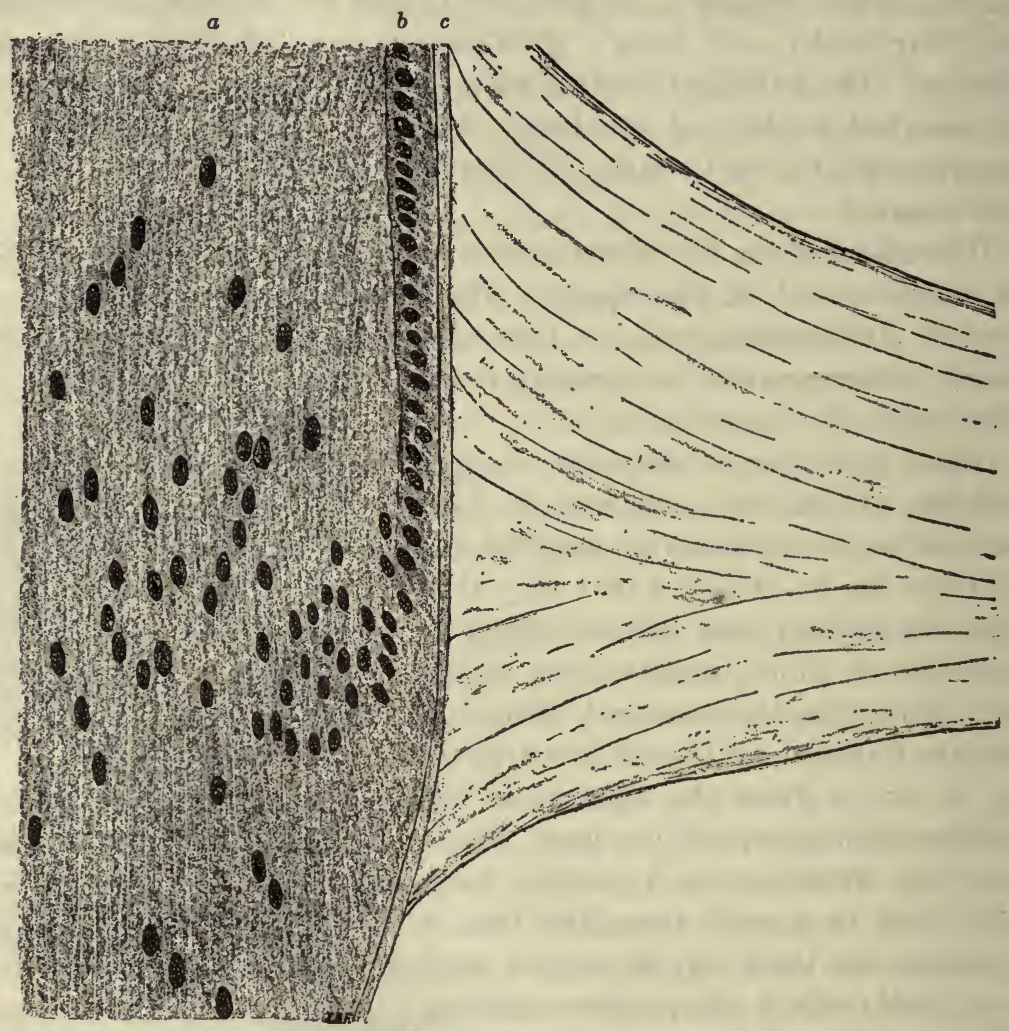

Fig. 565.-The Nuclear Zone at the Margin of the Crystalline lens of a Child's Eye, Showing the Transition of the Lens Epithelium to the Lens Fibers and the Attachment of the Suspensory Ligament.

$a$, lens fibers; $b$, lenticular epithelium; $c$, capsule of the lens; $d$, suspensory ligament. Hematein and eosin. $\times 273$.

by a delicate vascular membrane, the tunica vasculosa lentis (designated pupillary membrane in front), supplied by the hyaloid artery continued from the central artery of the retina. This tunic normally disappears before birth; its atypical persistence in whole or in part seriously interferes with vision. 


\section{The Vitreous Humor}

The vitreous humor (vitreous body) is a soft jelly-like mass which fills the entire cavity of the eye behind the line of the ora serrata and crystalline lens. About 98 per cent. of its composition is water. It is completely invested by the hyaloid membrane. Its anterior excavation which holds the posterior convexity of the lens is known as the hyaloid or patellar fossa. The vitreous humor appears to be a peculiarly delicate form of very loose gelatinous connective tissue whose scanty fibers present a somewhat concentrically lamellated arrangement and are so very delicate as to be recognized under ordinary conditions only with the greatest difficulty.

Occasionally stellate and fusiform cells, remarkable for their large vacuoles and varicose processes, have been demonstrated in small numbers within the vitreous body. Small rounded cells somewhat resembling leukocytes are also found, but for the most part they are flattened against the hyaloid membrane; they occur in very limited numbers.

These various cells, as well as occasional filamentous remnants of the original mesenchymal constituents of the vitreous humor, may cast shadows upon the retina within the visual field. Such shadows, called musca volitantes, on account of their 'flitting' motion when the eyes are moved, are seen when looking at a bright light, or frequently in looking through the microscope. In advanced age crystals may form in the vitreous which are observed to settle to the bottom of the eye when the eyes are held still.

\section{The Hyaloid Membrane}

The hyaloid membrane is a very thin structure which surrounds the vitreous humor and unites it to the inner surface of the retina and the crystalline lens. It consists of delicate glassy fibers so disposed as to form an extremely thin reticular membrane. It passes forward over the inner surface of the retina, to which it is loosely united, until at the ora serrata its fibers leave the retinal surface and pass inward to the margin of the lens to become inserted into the lenticular capsule.

\section{The Suspensory Ligament}

\section{(Zonula Ciliaris)}

Certain fibers from the hyaloid membrane pass forward from the ora serrata and are firmly adherent to the ciliary processes, or become 
attached in the grooves between the processes. From the sides of these processes fibers diverge at frequent intervals and pass to the margin of the lens, where they are attached on either side of the equator, spreading over a zone which is somewhat narrower posteriorly than anteriorly. These fibers form the suspensory ligament of the crystalline lens (Figs. 543 and 565). They occupy an annular zone which is included between the ciliary processes and the margin of the lens, and which is known as the zonula ciliaris (of Zinn).

The glassy fibers of this ligament take origin from the sides of the ciliary processes along which they are firmly attached, becoming free only near the apices of these processes. They pass thence to the margin of the lens and spread out upon the surface of the capsule to which they are intimately adherent. The fibers arising more posteriorly are said to be attached to the lens anteriorly to the equator, those arising from the more anterior portions of the ciliary processes becoming attached posteriorly to the equator of the lens.

The most anterior of these fibers form a somewhat plicated but incomplete membrane which serves as the anterior boundary of an annular series of connecting lymphatic spaces collectively forming the spatia zonularis (canals of Petit). This irregularly sacculated, annular canal is bounded posteriorly by the hyaloid membrane, anteriorly by the incomplete membranous wall of the posterior chamber through which the aqueous humor readily diffuses, internally by the margin of the crystalline lens, and antero-externally by the ciliary processes. Besides supporting the lens, the suspensory ligament assists in the accommodation of the lens to far and near vision, a process involving a change of convexity, and dependent upon the activity of the ciliary muscle.

\section{BLOOD-VESSELS OF THE EYE}

The circulation of blood in the globe of the eye is maintained through four sets of vessels:

1. The arteria and vena centralis retinæ.

2. The short ciliary arteries and venæ vorticosæ.

3. The long ciliary arteries.

4. The anterior ciliary arteries and veins.

1. The arteria centralis, destined for the supply of the retina, enters the optic nerve about midway between the optic commissure and the ocular globe, and arriving at the center of the nerve runs in its axis 
to the papilla optica, at which point it divides into two branches, an inferior and a superior branch, which, by rapid dichotomous division, radiate from the optic papilla to all parts of the retinal surface, thereby $\mathrm{f}$ or $\mathrm{m}$ ing a plexus of small arteries within the nerve fiber and gangìion cell layers. From this plexus capillaries are distributed to all the cerebral layers of the retina. No blood-vessels are found within the neuro-epithelial layers. The retinal arteries, like those of the brain, do not anastomose with one another; they are terminal arteries.

The retinal veins follow a course exactly similar to that of

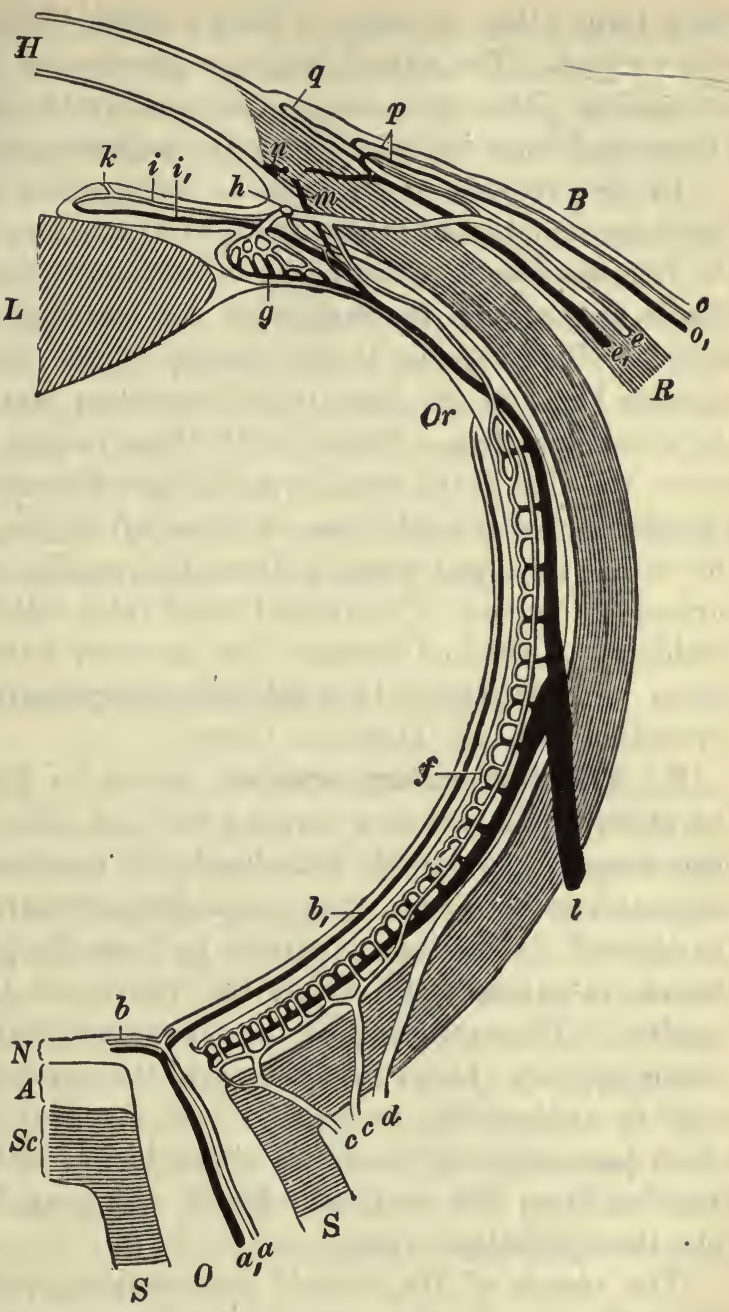

Fig. 566.-Schematic Representation of the Intrinsic Blood-Vessels of THE EYE.

Arteries in outline, veins in solid black. $A$, choroid; $a$, central artery, and $a_{1}$, vein of the retina; $B$, conjunctiva; $b$, retinal arteries; $b_{1}$, retinal veins; $c, c$, short ciliary arteries; $d$, long ciliary artery; $e, e_{1}$, anterior ciliary arteries and veins; $f$, choriocapillaris; $g$, capillaries of the ciliary body; $H$, cornea; $h$, circulus major of the iridal arteries; $i$, arteries, and $i_{1}$, veins of the iris; $k$, circulus minor of the iridal arteries; $L$, crystalline lens; $l$, venæ vorticosæ; $m$, anastomosis of ciliary and anterior ciliary veins; $N$, retina; $n$, canal of Schlemm; $O$, optic nerve; $o$, posterior conjunctival artery, and $o_{1}$, vein; $p$, anterior conjunctival vessels; $q$, vascular loops at the margin of the cornea; $R$, internal rectus muscle; $S$, sheath of the optic nerve; Sc, sclera. (After Leber.) 
the arteries; they converge to form a single efferent vessel, the vena centralis retinæ. The retinal veins are peculiar in that their walls contain no muscle. The optic nerve is supplied with small branches from the arteria and vena centralis retinæ in their passage through its substance.

In the fetus a small branch, the hyaloid artery, apparently the direct continuation of the arteria centralis retinæ, passes forward through the vitreous humor to the posterior surface of the lens, whence capillary vessels pass around the margin of the lens and are connected with the anterior ciliary vessels at the margin of the iris. The hyaloid artery supplies blood to the fetal tunica vasculosa lentis for the nutrition of the developing lens. Before birth these vessels disappear; the hyaloid artery remains for a time as a delicate fibrous strand, occupying the persistent canalis hyaloideus or canal of Stilling, which lies almost in the visual axis and extends from the papilla optica to the posterior surface of the lens. The hyaloid canal (also called the canal of Cloquet) establishes a channel between the aqueous humor and the lymphatic spaces of the retina. In adult life both the vitreous humor and the crystalline lens are bloodless tissues.

2. The short ciliary arteries, twelve to fifteen in number, enter the globe of the eye in a circle (circle of Zinn) which surrounds the optic nerve. They supply branches to the meningeal sheaths of the optic nerve and to the sclera, their main stems penetrating this coat to enter the choroid. Here they subdivide to form the plexus of arteries in the lamina vasculosa from which the vessels of the choriocapillaris are supplied. The capillaries of the last-named layer unite to form small venous radicals which converge toward the equator of the eye, where they unite in a whorl-like manner to form the four or five vence vorticosa, which pass obliquely backward through the sclera, receiving. additional branches from this coat, and finally emerging from the eye to empty into the ophthalmic vein.

The vessels of the choroid communicate posteriorly with those of the optic nerve, and anteriorly, by a free anastomosis, with those of the ciliary processes.

3. The long ciliary arteries, two in number, enter peripherally at the circle of Zinn on either side of the optic nerve, and pass horizontally forward upon the outer surface of the choroid to the ciliary muscle. Near the base of the iris they divide, and by anastomosis with each other and with the anterior ciliary arteries form a vascular circle, the circulus major, about the base of the iris.

From this circle recurrent branches supply the ciliary body and 
anastomose with the vessels of the choroid; other branches pass into the iris and, converging toward the visual axis form, just outside the pupillary margin, a second circle of anastomosis, the circulus minor.

The veins of the iris and ciliary body follow closely the distribution of the arteries, the greater portion of their blood returning through the veins of the choroid and the venæ vorticosæ. Some, however, is returned by means of anastomoses with the anterior ciliary veins.

4. The anterior ciliary arteries, derived from the muscular and lacrimal branches of the ophthalmic, distribute branches to the conjunctiva and sclera, and within the latter membrane, about $2 \mathrm{~mm}$. outside of the corneal margin, pass to the circulus iridis major and partially supply the iris and ciliary body as already described.

The anterior ciliary veins follow the course of the corresponding arteries. They empty into the vessels of the ocular conjunctiva.

\section{THE LYMPHATIC SYSTEMS OF THE EYE}

The lymphatic systems of the eye include very few true lymphatic vessels, but consist rather of a series of channels which may be arbitrarily considered as an anterior and a posterior set of intercommunicating spaces. The former set includes the lymphatic spaces of the cornea, the spaces of Fontana, the anterior and posterior chambers, the lymphatic clefts of the ciliary muscle and iris, and the zonular spaces or canals of Petit. The posterior set includes the subdural and subarachnoid spaces in the sheath of the optic nerve, the capsule of Tenon, the lymphatic spaces of the lamina suprachoroidea, the perivascular spaces of the choroid and retina, the irregular clefts between the pigmentary and bacillary layers of the retina, the similar clefts of the ganglion cell layer, the lymphatic spaces of the hyaloid membrane, the hyaloid canal, and the interstices of the vitreous humor.

These two sets of lymphatic channels communicate with each other by means of the perivascular spaces of the two outer tunics, as well as through that portion of the hyaloid membrane which forms the posterior. wall of the spatia zonularis, through the clefts of which and the hyaloid canal the lymph of the vitreous body communicates freely with the aqueous humor of the spatia zonularis and posterior chamber. Consequently, if the cornea be penetrated either accidentally or otherwise, and the anterior and posterior chambers be emptied, their aqueous humor is rapidly replaced, not only from the adjacent spaces of the 
anterior set of lymphatic vessels, but from the vitreous humor and posterior set as well.

It is also important to note that the posterior set of lymphatic spaces is directly connected through the meningeal sheaths of the optic nerve with the subdural and subarachnoid spaces of the cerebral meninges.

\section{THE NERVES OF THE EYE}

The nerves of the eye, in addition to the optic, are the long and short ciliary branches of the ophthalmic nerve. The former, two or three in number, and the latter, six to ten, after supplying a vasomotor branch to the arteria centralis retinæ, pierce the sclera in company with the corresponding ciliary arteries and pass meridionally forward on the inner surface of the sclera, supplying branches to this tunic and to the vessels of the choroid, and finally reaching the ciliary muscle, where their branches form an annular plexus containing a few ganglion cells.

From this plexus fibrils are supplied to the blood-vessels and muscular tissues of the ciliary body and iris, and to the cornea. The corneal branches pass to the annular plexus at the sclerocorneal junction, whence they are distributed to the corneal tissues, as already described (page $633)$.

\section{APPENDAGES OF THE EYE}

The appendages of the eye include the eyelids, conjunctiva, and lacrimal glands.

\section{The EyeLids}

The eyelids are developed in the embryo through an invagination of the skin, which, leaving a slit-like aperture (the palpebral fissure) between its involuted margins, covers the inner surface of the lid to form the palpebral conjunctiva, and is reflected over the globe of the eye as the ocular conjunctiva and anterior corneal epithelium.

The lids, therefore, may be said to consist of two membranous portions, the cutaneous (outer or anterior) and the conjunctival (inner or posterior). Between these two portions the orbicularis palpebrarum forms a septum of striated muscle fibers.

The cutaneous portion of the eyelid differs from other portions 
of the skin only in that its subcutaneous tissue contains no fat. The derma is loosely connected with the muscle by a wide-meshed areolar tissue. Fine hairs are distributed over the cutaneous surface, their follicles extending well down through the derma. Small sebaceous

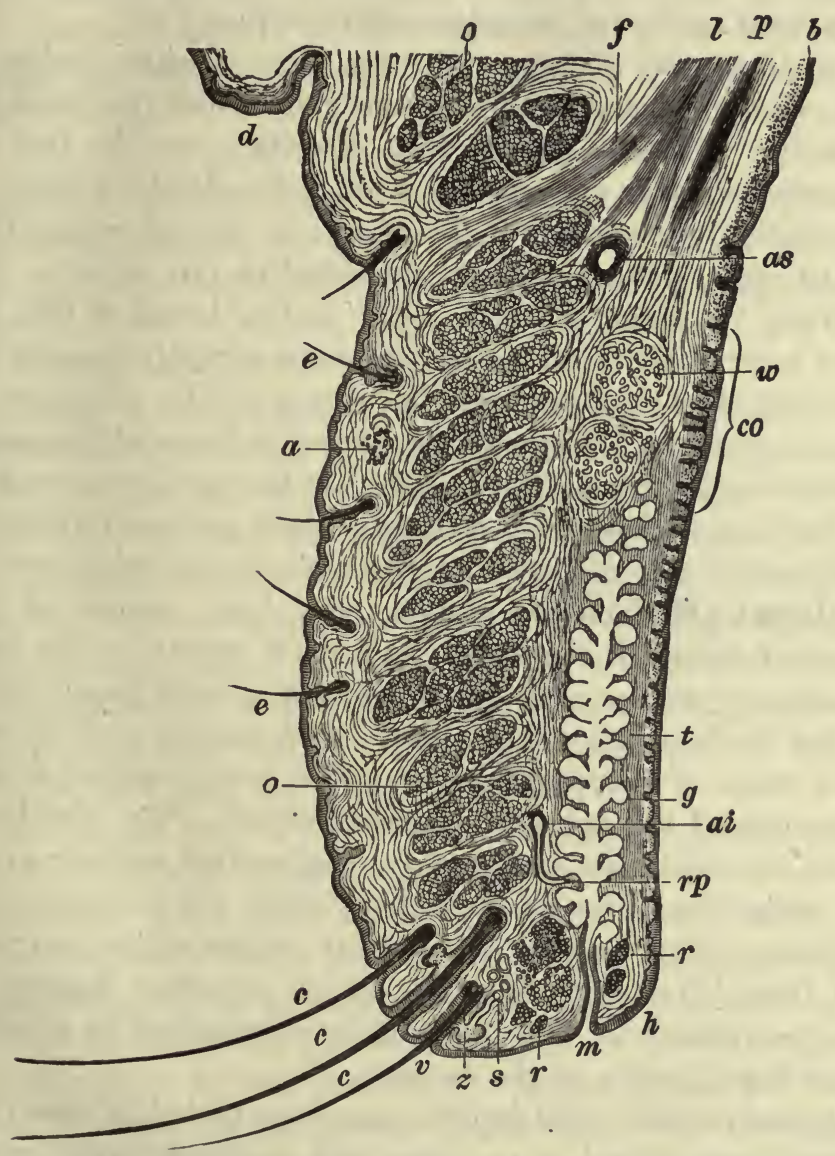

Fig. 567.-Vertical Section throdgh the Upper Eyelid.

$a$, sweat glands; $a i$, primary tarsal arch; $a s$, secondary tarsal arch; $b$, conjunctival epithelium; $c$, eyelashes; co, rugated portion of the conjunctiva; $d$, epidermis; $e$, fine hairs; $f$, process of the levator palpebræ superioris which is inserted into the skin; $g$, Meibomian gland; $h$, internal angle of the margin of the lid; $l$, levator palpebræ superioris; $m$, duct of a Meibomian gland; $o$, orbicularis palpebrarum muscle; $p$, superior palpebral muscle of Müller; $r$, ciliary muscle of Riolan; $s$, glands of Moll; $t$, fibrous tissue of the tarsus; $v$, external angle of the margin of the lid; $w$, posterior tarsal glands; $z$, sebaceous glands. (After Fuchs.) 
glands open into the hair follicles and occasional sudoriparous glands pour their secretion upon the epidermal surface.

At the margin of the lid its cutaneous portion is reflected inward, and at its inner angle becomes directly continuous with the palpebral conjunctiva. The free margin of the lid presents, therefore, an outer angle, an inner angle, and an intermediate surface.

Two or three rows of large stiff hairs, the eyelashes or cilia, project from the outer angle, and large sebaceous glands open into their follicles. Other smaller sebaceous glands. open directly upon the free surface. These sebaceous glands are sometimes called the glands of Zeiss.

The intermediate surface of the margin of the lid retains the character of the skin, though no hairs are found in this portion. Peculiar sweat glands, the glands of Moll, occur in the derma of this part.

At the inner angle of the lid the epidermis abruptly changes its character to that of the conjunctiva, the derma of the cutaneous surface being continuous with the submucous connective tissue of this membrane. At the inner angle also, are the openings of the peculiar large sebaceous glands, the tarsal glands (of Meibom), their orifices forming a continuous punctate row of pores barely visible to the naked eye.

The tarsal (Meibomian) glands are long compound saccular glands, about thirty in the upper, and about twenty in the lower lid, whose secreting saccules open into a common, axially placed duct which extends the whole length of the gland. Each saccule is filled with cells in various stages of fatty degeneration and is exactly similar in structure to the saccules of the ordinary sebaceous glands. The glands are embedded in the connective tissue of the conjunctiva and are so large as to form projecting ridges on its surface, which are disposed in vertical lines radiating from the row of glandular orifices at the margin of the lid. At their blind extremities the glands are often slightly bent or curved upon themselves, and this portion is embedded in a dense mass of fibrous tissue known as the tarsus.

The tarsus in each eyelid forms a very dense plate-like mass of areolar connective tissue which is so dense and resistant as to erroneously suggest a cartilaginous structure. It is inserted between the conjunctiva and the orbicularis muscle. It is thickest toward the free margin of the lid, but becomes progressively thinner in the opposite direction, until, as a mere fibrous membrane, the palpebral fascia, it is continued to the margin of the orbit.

The conjunctival portion of the lids, the palpebral conjunctiva, consists of a peculiar stratified epithelium and a thin connective tissue 
corium. Its epithelium comprises four or five layers of cells, the deeper of which are small and spheroidal, and the superficial elongated or conical, their blunt ends forming the free surface of the conjunctiva, their pointed extremities buried between the cells of the deeper layers. The bases of these elongated cells become somewhat expanded and broader from the increased tension of the conjunctiva when the lids are closed; they retract and become narrower when the lids are separated and the conjunctiva relaxed.

The cells of the superficial layer are often so distinctly elongated as to possess a columnar form. They may, however, be spheroidal or even somewhat flattened, in which case they very closely resemble the ordinary type of stratified squamous epithelium. The epithelial layer rests almost directly upon the connective tissue corium, the basement membrane being imperfectly developed.

The corium of the conjunctiva is thin. With the aid of a thin layer of submucous areolar tissue it unites the epithelium to the tarsus and to the fibers of the orbicularis muscle; near the margin of the lid its submucous tissue incloses the Meibomian glands. Opposite the plane at which the blind ends of the Meibomian glands are embedded in the free margin of the tarsus, the conjunctival surface is thrown into eight to twelve horizontal folds, beneath which, in the connective tissue, are a few minute tubulo-alveolar glands, the posterior tarsal glands (glands of Waldeyer; glands of Henle). Their ducts open upon the free surface of the conjunctiva near the fornix conjunctivæ.

At the attached base of the lid a narrow band of smooth muscle extends from the levator palpebræ and inferior oblique muscles into the body of the lid. These fibers have been described by H. Müller (1858) as the superior and inferior palpebral muscle of the upper and lower lid, respectively, and have come to be known as the muscle of Müller.

The fold by which the palpebral conjunctiva is reflected upon the globe of the eye to become continuous with the ocular portion of the membrane is known as the fornix conjunctive. The extremely loose attachment of the conjunctiva of the fornix to the underlying connective tissue and intra-orbital fat permits the great freedom of motion which is characteristic of the ocular globe. The small accessory lacrimal glands (glands of Krause) open into the margin of the fornix conjunctivæ. The superior fornix contains from eight to twenty, the inferior from two to five. In this region, also, occasional goblet cells occur in the superficial layers of the epithelium.

The ocular conjunctiva is likewise very loosely attached to the sclera. 
The scleral portion of the conjunctiva is nearly identical in structure with the palpebral portion already described. Near the margin of the cornea the superficial cells of the epithelium become at first spheroidal and then, as the cornea is approached, they are progressively flattened, so that, just outside of the corneal margin, the conjunctival epithelium conforms to the stratified squamous type which forms the anterior epithelium of the cornea.

The blood supply of the eyelids is derived from the internal palpebral artery which furnishes a branch to each lid; these two branches

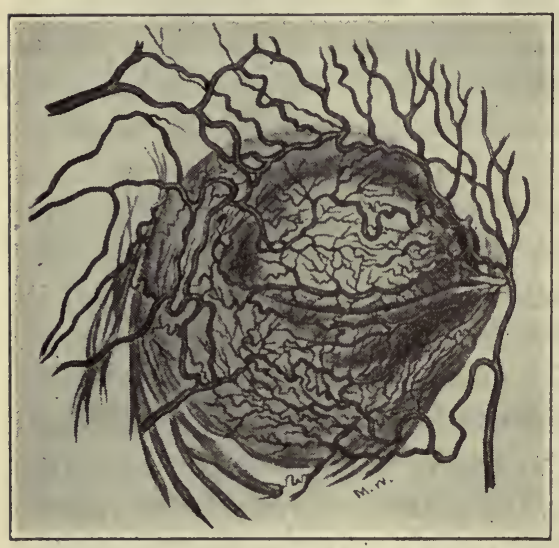

Fig. 568.-Arterial Supply of the EyeLID.

(After Fox.) anastomose at the external angle with the lacrimal branch of the ophthalmic, through the external palpebral artery. These transverSe ARTERIAL VESSELS (primary tarsal arches) lie near the free margin of the lids between the tarsus and the orbicularis palpebrarum muscle. Each tarsal arch sends pre-tarsal twigs forward to supply the muscle and integument, and post-tarsal twigs backward to supply the tarsal fascia, the tarsal glands and the conjunctiva. At the basal end of the tarsus, just back of the levator palpebræ muscle, a second arch (the (secondary tarsal arch) may be present, more commonly in the upper lid; this second arch represents a larger branch of the palpebral artery, and it anastomoses freely with the primary arch. The veIns follow an essentially similar course. The LYмPнатісs likewise include a pre- and post-tarsal set, which unite to form larger tarsal tributaries which drain along the facial vein toward the submaxillary lymph nodes.

The nerve supply includes sensory, motor and sympathetic fibers. The main trunks lie between the tarsus and the orbicularis palpebrarum muscle. The sympathetic fibers supply the blood-vessels, the smooth muscle of Müller, and the glands. Motor fibers contributed by the facial nerve, supply the annular sheet of striped muscle; the oculomotor nerve contributes motor fibers to the levator palpebræ muscle. The sensory fibers, which are derived from the trigeminal nerve, end in naked fibrils among the cells of the external integument and the internal con- 
junctiva, and in the subcutaneous connective tissue and the tarsus. Numerous sensory fibrils end also in special end-bulbs, especially along the inner margin of the lids; similar endings occur in the palpebral and bulbar conjunctiva.

\section{The Lacrimal Lake}

In the $\supset$-shaped area of the internal canthus, the lacus lacrimalis, lies a roughened irregular reddish mass of delicate modified skin, the caruncle (caruncula lacrimalis). It is embedded in fat and contains a number of very delicate hairs, large sebaceous glands and a few sweat glands. Where the borders of the lake pass into the palpebral margins there appears a slightly raised papilla lacrimalis, each of which contains an apical opening, the puncta lacrimalia, leading into the two canaliculi lacrimales, which conduct the excess of lacrimal secretion or tears into the nasolacrimal duct. To the outer side of the lacrimal lake there appears a vertical crescentic fold of delicate skin, the plica semilunaris, which is the homologue of a functional third eyelid (nictitating membrane) of birds and reptiles.

\section{The Lacrimal GLand}

The lacrimal glands are two flattened, lobulated, glandular masses situated at the upper and outer angle of the orbit, one in relation with each eye. They secrete a clear watery fluid, the tears. These glands are somewhat molded to conform to the shape of the orbit and the globe of the eye, between which they are inserted.

Each lacrimal gland is a secreting gland of the compound tubular type (Marziarski) (Fig. 25\%, page 258), and consists of eight to twelve small lobules which open into the fornix conjunctivæ by about as many minute ducts. The lobules are aggregated into two fairly distinct lobes, separated by a denser fascia, the superior lobe or orbital portion and the inferior lobe or palpebral portion, and are united by thin fibrous fasciæ which contain the larger ducts.

Each lobule of the gland contains many serous-secreting acini and numerous small intralobular ducts. The secreting acini are lined by tall, columnar cells, resting upon a thin basement membrane, which is supplied with 'basket cells' and is invested with a delicate fibrous tunica propria. The appearance of the secreting epithelium differs some- 
what according to its state of activity. After a period of rest, and in the ordinary condition of relative inactivity, the epithelium becomes distended with secretion and is either clear in appearance or at most is only very finely granular, the nuclei are crowded to the base of the swollen cells, and the lumen of the acinus is very small. After a period of excessive activity the secreting cells become shrunken and more dis-

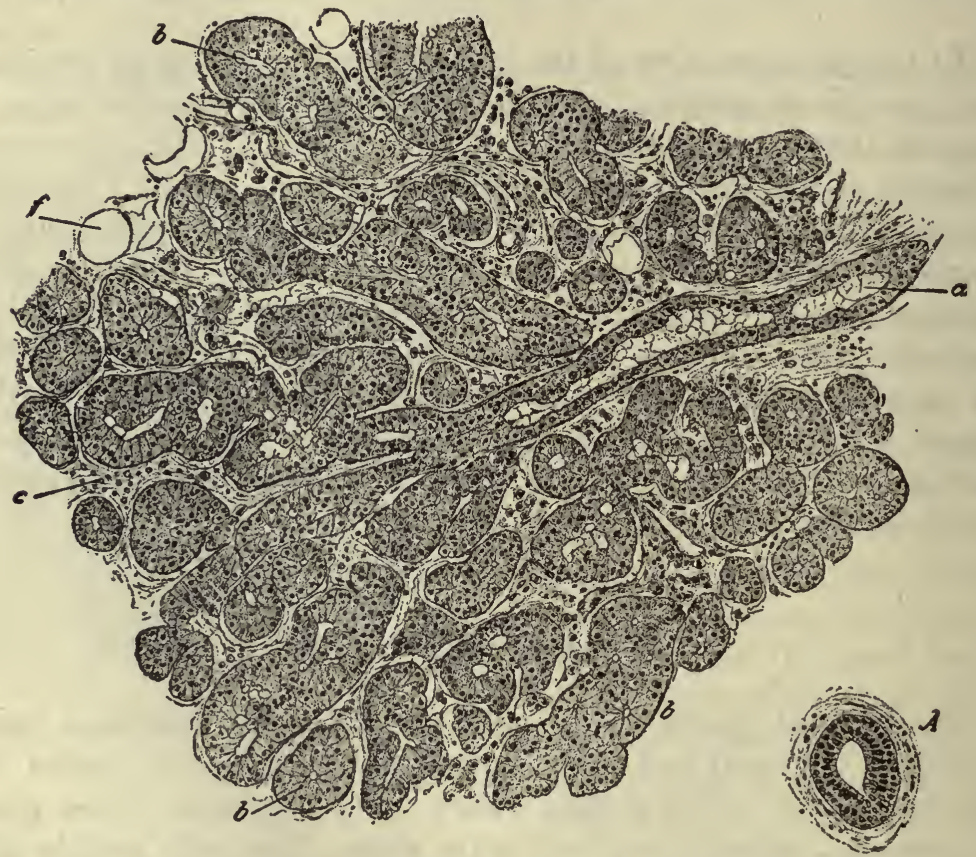

Fig. 569.-Section throdgh a Lobule of the Lacrimal Gland of Man.

$a$, small duct branching within the lobule; $b$, intercalary ducts; $c$, connective tissue; $f$, fat cells. $A$, transection of an interlobular duct. Hematoxylin and eosin. $\times$ 112. (After Kölliker.)

tinctly granular, and the lumen of the acinus appears much dilated.

The secreting acini empty into narrow intercalated ducts which lie within the lobule, have a considerable lumen, and are lined by tall columnar cells resting upon a second incomplete layer of small, somewhat flattened basket cells. .

These intralobular ducts unite at the margin of the lobule to form the larger interlobular ducts, which are contained in the interlobular connective tissue. Here the duct is lined by low columnar or even somewhat flattened cells, at first disposed in a single, but later in a 
double layer. As the duct approaches the conjunctival surface the number of cell layers increases until their lining epithelium finally comes to resemble the stratified epithelium of the conjunctiva with which it is continuous.

Minute collections of diffuse lymphoid $\mathrm{t}$ is s u e and even $\mathrm{s}$ mall lymph nodules are occasionally found $\mathrm{just}$ beneath the epithelium of $\mathrm{the}$ conjunctiva in the neighborhood of the lacrimal glands of the fornix; occasionally the lymphoid tissue is quite abundant.

In animals possessing a membrana nictitans (" $t$ h i r d lid') a small, mucus-secreting gland occurs at the inner angle of the orbit; this is known as the gland of Harder. It is well developed also in some mammals, e.g., rabbit, but in man and other primates it is

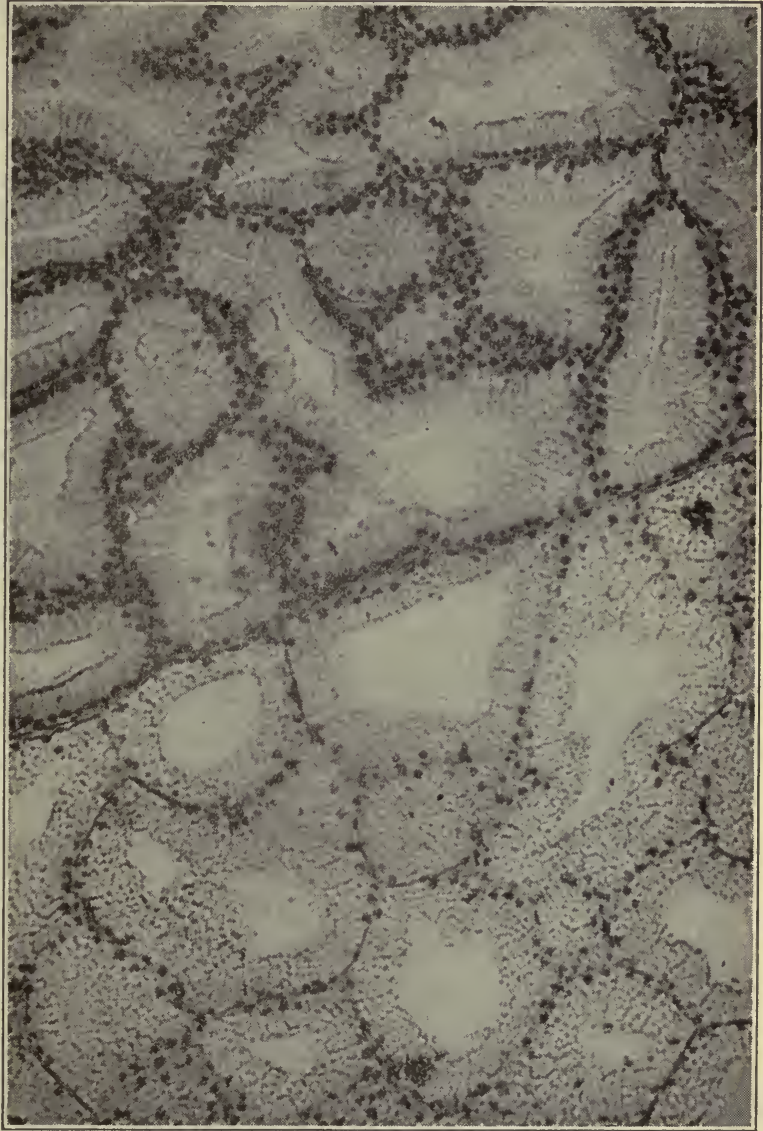

Fig. 570.-Portions of Two Adjacent Lobules of the Lacrimal Gland of the Rabbit, Showing Two Stages in Secretory Activity of the Tubules. Magnification $\times 100$.

In the upper lobule the cells are filled with minute, in the lower lobule with large, secretion spherules. usually absent, though in an extremely vestigial condition it may occasionally be found within the basal portion of the plica semilunaris. 


\section{CHAPTER XIX}

\section{THE EAR}

This organ may be subdivided for description into the external, the middle, and the internal ear. The first two portions serve for the collection and transmission of sound waves, the last for the transformation of the sound waves into nerve stimuli which are then transmitted through the path of the acoustic nerve to the cerebrum.

\section{THE EXTERNAL EAR}

The external ear includes an auricular or free portion and an external acoustic (auditory) meatus.

\section{The Auricle}

The auricle, or pinna, contains a thin cartilaginous plate of peculiar form which is covered on both sides by the skin. The cartilage is of the elastic variety, but differs from the similar cartilages of other parts in the abundance of its large cartilage cells; in occasional areas the elastic reticulum is deficient. This reticulum is closely connected with the fibrous perichondrium, beneath which it forms a complete layer. The extrinsic muscles of the ear are inserted into the perichondrium and the fibrous tissue by which it is surrounded.

The skin of the external ear does not essentially differ from that of other parts. It is supplied with fine hairs and with many large sebaceous glands; sweat glands also occur on the outer surface. The derma is united to the underlying cartilage by connective tissue; on the concave surface this union is very firm and permits but little motion. The subcutaneous tissue, except in the lobule, contains but little fat. The lobule does not contain cartilage. 


\section{The External Acoustic Meatus}

The external acoustic Meatus (or auditory canal) is divisible into an outer cartilaginous and an inner bony portion; the walls of the two

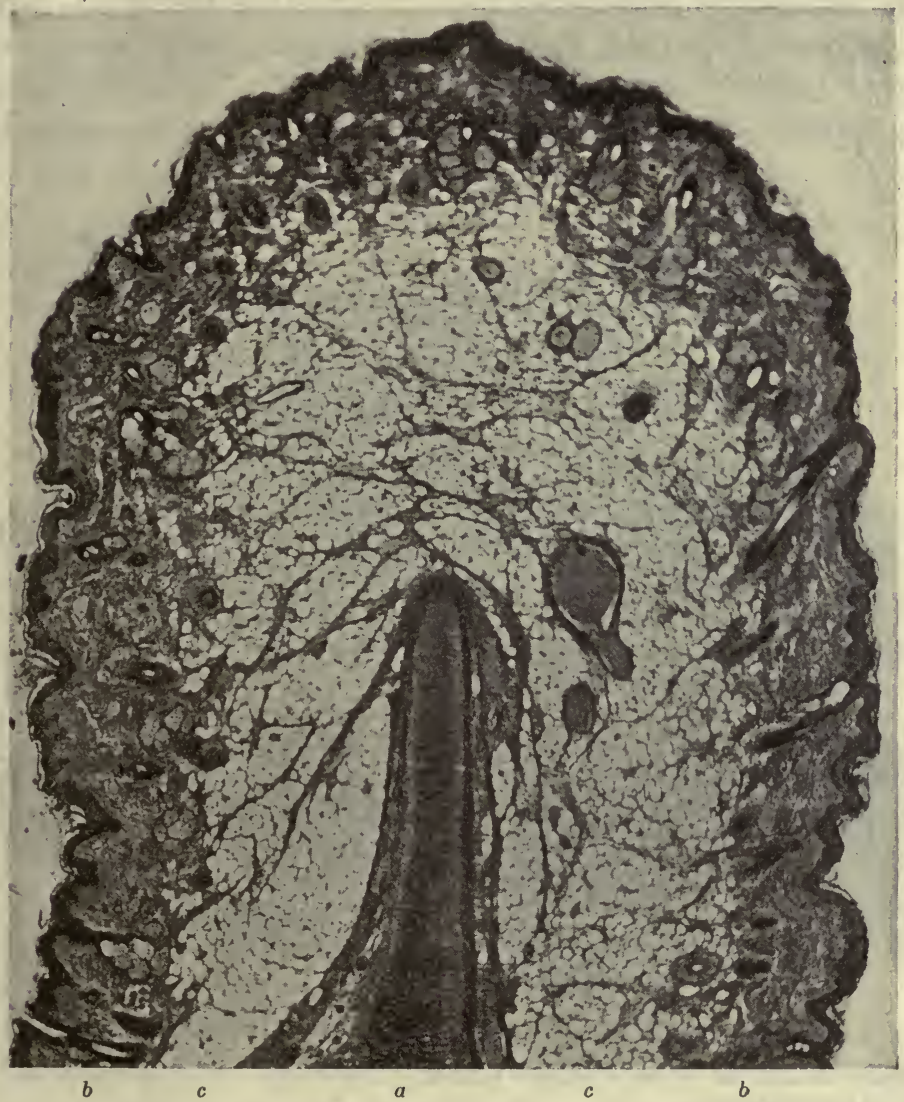

Fig. 571.-Transection of the Lobule of the External Ear of an Infant.

$a$, cartilage; $b$, skin; $c$, adipose connective tissue. Hematein and eosin. Photo. $\times 20$.

portions, except for this difference, are quite similar in structure. The cartilage is continuous with that of the auricle, and is of the cellular elastic variety. The skin of this portion contains large stiff hairs and both sebaceous and ceruminous glands. The former, as in the auricle, 
open either upon the free surface of the skin or into the adjacent portion of the hair follicles.

\section{The Ceruminous Glands}

The ceruminous glands resemble in structure the sweat glands of other portions. They are coiled tubular glands which open upon the surface of the skin by means of a narrow duct. The coils of their secreting portion are lined by columnar cells with spheroidal, basally

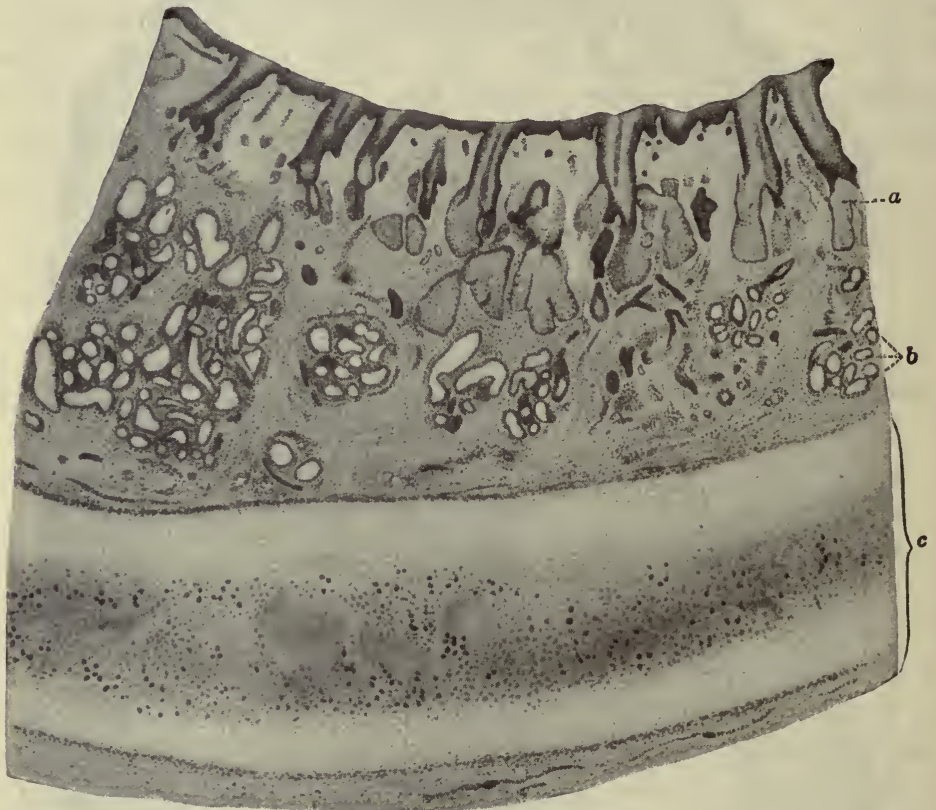

Fig. 572.-From the External Acoustic Meatus of Man.

$a$, sebaceous gland; $b$, ceruminous gland; $c$, cartilage. Hematoxylin and eosin. $\times 15$. (After Sobotta.)

situated nuclei and a clear cytoplasm containing many small brownish granules of pigment and a few fatty particles. The cytoplasm is often diffusely colored by the brownish pigment. Between the lining cells and the basement membrane appear slender fusiform elements resembling similarly placed elements in the sweat glands, and likewise interpreted as muscle cells. The secretion of these glands, the cerumen, in addition to the pigmented and fatty secretion of the glands, contains 
sebum, desquamated epithelial cells and occasional fine hairs, together with foreign particles of a very varied sort.

In the bony portion of the meatus the corium or derma is firmly adherent to the periosteum of the bone, and all the layers of the skin are much reduced in thickness. The scanty hairs are very fine, and, with the glands, are continued inward to the tympanic membrane only in the superior portion of the wall of the canal. Papillæ are present as far as the margin of the tympanic membrane. Upon the surface of this membrane, which closes the inner end of the external acoustic meatus and separates it from the cavity of the middle ear, the skin is reduced to an extremely thin cutaneous coat, devoid of hairs, glands, and papillæ.

\section{THE MIDDLE EAR}

\section{General Considerations}

The middle ear or tympanum ('ear-drum') is an irregular cavity, broad above and behind, narrow below and in front, which lies just within the external acoustic meatus. Its outer wall is largely formed by the tympanic or drum membrane, its inner by the osseous wall of the internal ear.

The contour of the tympanum is very irregular, its cavity being encroached upon by numerous bony elevations which are most pronounced on its internal wall. Externally the tympanic membrane is attached to a bony and fibrocartilaginous ring, the annulus tympanicus, which projects somewhat into the tympanic cavity. In front, the orifice of the auditory (Eustachian) tube is marked by a slight cartilaginous projection near the floor of the cavity.

Above and behind, the tympanic cavity is prolonged into a deep recess, the epitympanic cavity, in the upper part of whose posterior wall are the orifices of the mastoid cells. The upper portion of the cavity contains the rounded heads of the malleus and incus, the two largest of the auditory ossicles. The internal wall of the tympanum presents anteriorly a bulging prominence which is known as the promontory, and which indicates the position of the first or broadest turn of the spiral canal of the cochlea. Beneath this prominence is a recess leading to a bony 'window,' the fenestra cochlea (or fenestra rotunda), which, in life, is closed by a delicate membrane, the secondary tympanic membrane. Behind the promontory and at a slightly higher level a 
deep recess, the pelvis ovalis, leads inward to the fenestra vestibuli (or fenestra ovalis), which is closed by the base of the stapes; the body of this ossicle is contained entirely within the pelvic recess, and near its mouth the stapes articulates with the orbicular extremity of the long process of the incus. The superior portion of this deep recess is encroached upon by the projecting wall of the aqueductus Fallopii which transmits the facial nerve, and posteriorly, near the point where it merges with the general tympanic cavity, a low, conical, bony projection known as the pyramid transmits the stapedius muscle. The canal of the tensor tympani muscle contained within a still more prominent, conical, bony projection, the processus cochleariformis, is found near the anterointernal angle of the tympanic cavity just above and parallel to the auditory tube. The narrowest portion of the tympanum is, perhaps, almost its very center, and is included between the promontory on the inner and the tympanic membrane on the outer side. Extending from this narrowed central portion upward, backward, and inward, are expanded recesses which are partially occupied by the three auditory ossicles; the remaining portions of the tympanum are filled by air which gains access to the cavity through the auditory tube.

\section{The Tympanic Mucosa}

The tympanic mucosa consists of a thin but dense tunica propria which is firmly attached to the underlying periosteum and softer parts by loose connective tissue, and is clothed with a layer of flattened entodermal epithelium, which, in the vicinity of the origin of the auditory tube, is of low columnar form and is provided with cilia, but in most other portions of the tympanum is squamous in character and of the tessellated type, closely resembling endothelium. The floor of the tympanum and the lower portions of its anterior, internal, and posterior walls also possess a partial clothing of low ciliated cells (Kessel). Occasional gland-like folds of the mucosa occur near the orifice of the auditory tube, though the true glandular character of these folds is very questionable.

\section{The Mastoid Cells}

The mastoid cells (cellula mastoidece seu pneumatica) are numerous small spaces situated within the mastoid process of the temporal bone; they are lined by a continuation of the tympanic mucosa, which is everywhere clothed by flattened epithelium. The corium is closely attached to the periosteum of the bony wall, the periosteum also serving 
as a vascular layer of the mucosa in the mastoid cells, as well as in the general tympanic cavity.

\section{The Trmpanic Membrane}

The tympanic membrane is a thin delicate partition which is formed by a reflection of the cutaneous layer of the external acoustic meatus on the one hand, the tympanic mucosa on the other, and between these two membranes a layer of dense fibrous tissue whose tendinous bands are disposed in radial and circular directions. The margin of the tympanic membrane is inserted into a fibrocartilaginous ring which rests upon a. bony elevation, the annulus tympanicus.

The slender manubrium or handle of the malleus projects from the superior margin of the ring and is inserted between the folds of the tympanic membrane, extending downward to about the center of the membrane, at which point is the deepest part of its concavity, its umbo. The bony handle of the malleus, lying between the cutaneous and mucous layers of the tympanic membrane, is covered by a thin cartilaginous layer, and receives the insertions of the tendinous fibers. These fibers are divisible into an outer radial layer which extends from the fibrocartilaginous ring at the

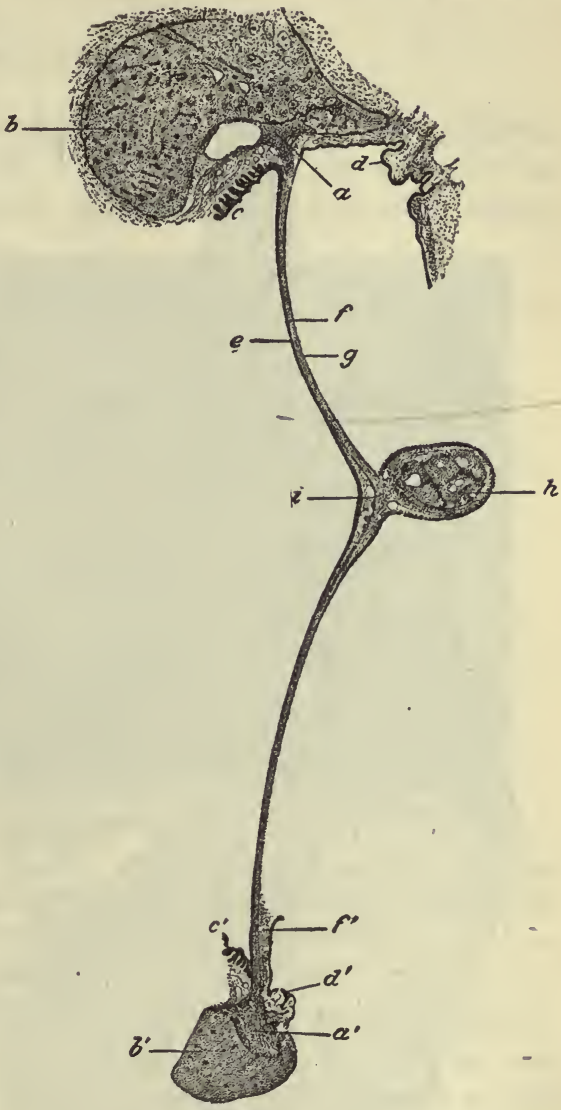

Fig. 573.-Transection of the Tympanic Membrane of a Child.

$a, a^{\prime}$, fibrocartilaginous ring; $b, b^{\prime}$, bone; $c, c^{\prime}$, skin of the external acoustic meatus; $d, d^{\prime}$, tympanic mucosa; $e$, cutaneous layer of the tympanic membrane; $f$, fibrous layer, obliquely cut at $f^{\prime} ; g$, layer derived from the tympanic mucosa; $h$, handle of the malleus; $i$, blood-vessels. Hematoxylin and eosin. $\times 11$. (After Kölliker.) periphery inward to the manubrium mallei, and an inner circular lay" whose thickest portions are found close to the manubrium and near tine 
periphery of the membrane; between these points the circular layer of fibers is partially or entirely deficient. Just within the fibrocartilagi-

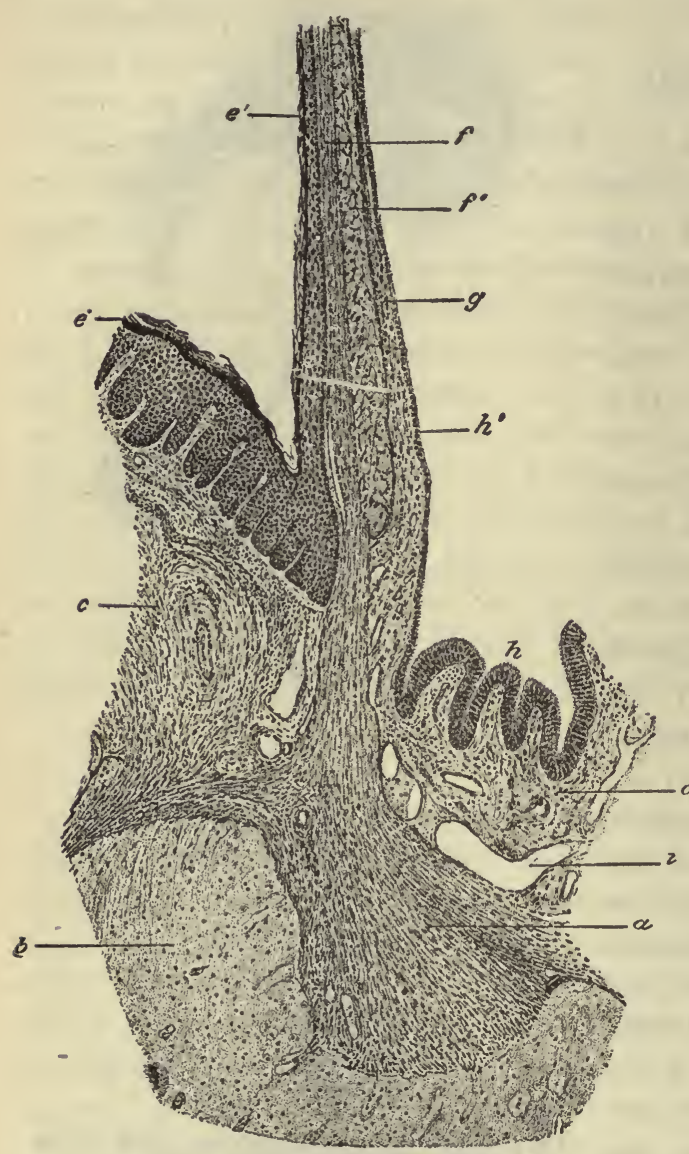

Fig. 574.-Section throdgh the Margin of the Trmpanic Membrane of a Child.

$a$, fibrocartilaginous ring; $b$, bone; $c$ derma of the external auditory canal; $d$, tympanic mucosa; $e, e^{\prime}$, epidermis; $f$, radial fibers, and $f^{\prime}$, circular fibers of the tympanic membrane; $g$, mucosa of the membrane; $h$, epithelium of the tympanum; $i$, blood-vessels. $\times 55$. (After Kölliker.) nous ring at the periphery of the membrane the circular layer of fibers abruptly ends.

The cutaneous layer of the tympanic membrane forms a very thin coat, its epidermis consisting of a germinal layer one' or two cells deep, which is covered by several flattened non-nucleated cells of the horny portion. The derma or corium is very thin, contains no papillæ, and is intimately adherent to the fibrous layers of the membrane; it contains neither glands nor hairs.

The mucous layer of the tympanic membrane is even thinner than the cutaneous. It consists of a flattened entodermal epithelium which rests almost directly upon the layer of circular fibers. A few connective tissue fibers p a s s irregularly from the mucous, through the fibrous, to the cutaneous layer, thus firmly uniting the several layers into a compact membrane.

In the upper quadrant of the tympanic membrane, above the attachment of the malleus, the fibrous layers are wanting; the mucous and cutaneous layers are therefore in contact, and the membrane presents 
a flaccid appearance in comparison with the tense condition of its other parts. This portion is known as the membrana flaccida or Shrapnell's membrane.

\section{The Auditory Ossicles}

These are three in number, the malleus, the incus, and the stapes; they form a continuous bony chain, extending from the insertion of the manubrium mallei in the tympanic membrane to the fenestra vestib-

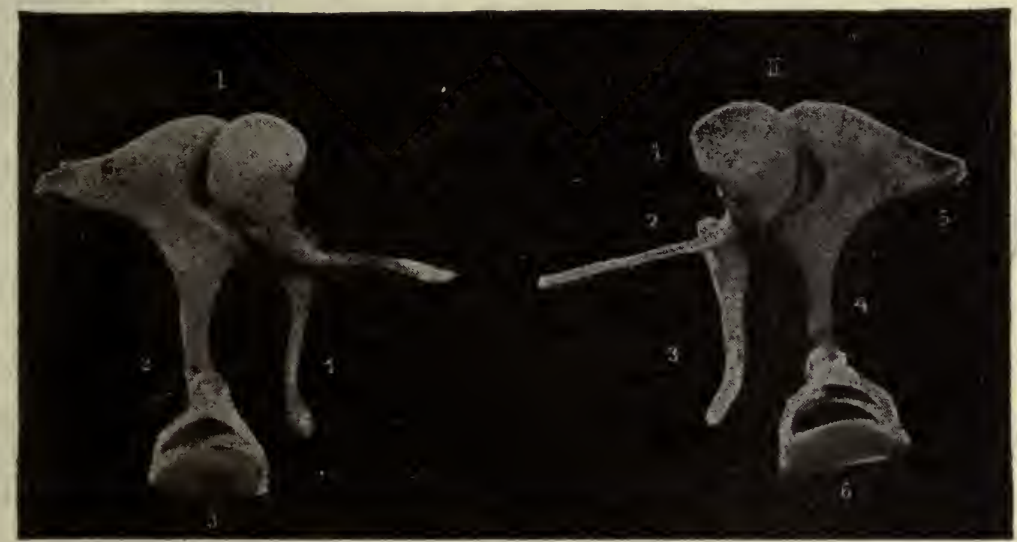

Fig. 575.-The Auditory Ossicles.

$I$, ossicular chain of the left ear; 1 , malleus; 2 , incus; 3 , stapes. II, ossicular chain of the right ear; 1 , malleus; 2 , processus gracilis; 3 , manubrium; 4 , long process of the incus; 5 , short process of the incus; 6, stapes. (After Rüdinger.)

uli, with whose margin the foot of the stapes articulates. The ossicles consist of compact bony tissue containing loosely packed Haversian systems; they are united with each other by firm fibrocartilaginous articulations. With the exception of the stapes, none of the ossicles contain a marrow cavity.

The manubrium of the malleus is firmly fixed in the tympanic membrane, as already described, the head of the bone articulating with the head of the incus in the epitympanic recess. The long process of the incus, circular in transection, extends downward along the tympanic wall in a course nearly parallel to that of the manubrium mallei, being, in a portion of its course, contained within a recess in the osseous wall of the tympanum. Finally, at the level of the stapes it makes a sharp bend, almost at right angles with its former course, to articulate, by means of a rounded end or orbicular process, with the head of the 
stapes. This latter bone is deeply placed within the recess of the pelvis ovalis, and continues the bony chain to the fenestra vestibuli, where the foot plate of the stapes is in relation, by its inner surface, with the vestibular perilymphatic space of the internal ear.

The course of the chain of ossicles is such that they form a lever; the long process of the incus being shorter than the manubrium mallei, the vibrations of the tympanic membrane in response to sound waves are transmitted to the internal ear diminished in amplitude but ex-

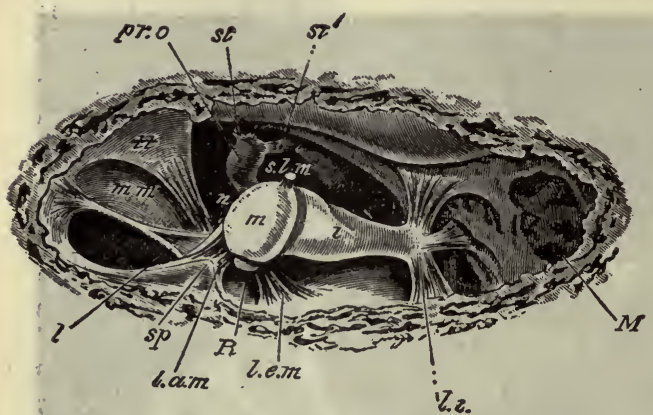

Fig. 576.-The Cavity of the Tympanum, Viewed From ABOVE.

$i$, the body of the incus; $l$, ligamentous fold of the mucosa; l.a.m., anterior ligament of the malleus; l.e.m., external ligament of the malleus; l.i., posterior ligament of the incus; $M$, mastoid cell; $m$, head of the malleus; m.m., mucous membrane; $n$, chorda tympani nerve; $p r . o$. , orbicular process of the incus articulating with the stapes in the depth of the cavity; $R$, beneath this space is the flaccid portion of the tympanic membrane; s.l.m., cut end of the superior ligament of the malleus; $s p$, spina tympanica anterior; $s t, s t^{\prime}$, tendon of the stapedius muscle; $t t$, tendon of the tensor tympani muscle. $\times$ 4. (After Schäfer.) aggerated in intensity. The combined activity of the tympanic membrane and the auditory ossicles is said to reduce the amplitude as much as seventy-six times, and to increase the force of the vibrations thirty times.

Two muscles and several ligaments are connected with the ossicles.

\section{The tensor tympani} muscle is mostly contained within a canal which is parallel to and lies just above the auditory tube, and from its bony wall the muscular fibers arise. The wall of the canal forms a conical projection known as the processus cochleariformis, which projects well into the cavity of the tympanum, being directed toward the neck of the malleus. Leaving its canal at the apex of this conical process the tendon of the muscle bends sharply over the margin of the processus cochleariformis and passes directly to its insertion into the neck and the adjoining part of the manubrium of the malleus. Hence the naked tendon of the muscle lies within the tympanic cavity.

The stapedius muscle is similarly, contained within the cavity of the pyramid, from whose bony wall its fibers take origin. Passing for- 
ward, the muscle makes its exit at the apex of the pyramid, and is directly inserted into the neck of the stapes close to the articttation of the orbicular process of the incus.

The ligaments of the malleus are the anterior, the external, and the superior. The anterior ligament firmly attaches the head of the malleus to the margin of the Glaserian fissure in the anterior wall of the tympanium. The processus gracilis of the malleus is inclosed by the fibers of this ligament. It is also in close relation with the chorda tympani nerve, which, being clothed by the tympanic mucosa, traverses this portion of the tympanic cavity and enters the iter chordæ anterius.

The external ligament connects the neck of the malleus with the upper portion of the external wall of the tympanum. It is somewhat fan-shaped. The space lying between the external ligament of the malleus and the membrana flacida is known as Prussak's space. The superior ligament is a looser fibrous band which passes from the head of the malleus to the superior wall of the tympanum.

The ligament of the incus is decidedly fan-shaped, its straight, coarse, fibrous bands. radiating from the short process of the ossicle to the adjacent portion of the posterior wall of the tympanum.

The articulation of the malleus with the incus, as also that of the latter bone with the stapes, is supplied with a delicate capsular ligament.

The annular ligament of the stapes connects the margin of the foot plate of this bone with the adjacent portions of the cartilaginous and bony wall of the vestibule at the margin of the fenestra vestibuli. The articulation which is thus inclosed is directly formed by an annular plate of cartilage investing the margin of the oval foot of the stapes, and a similar annular plate of hyaline cartilage which lines the borders of the foramen vestibuli. The fibers of the annular ligament are continuous with those of the perichondrium and adjacent periosteum.

\section{The Auditory (Eustachian) Tube}

The auditory or Eustachian tube connects the cavity of the tympanum with that of the nasopharynx. Its first portion, including about one-third of its entire length, is surrounded by a bony wall; beyond this it is supplied with a cartilaginous plate; its pharyngeal ostium s: entirely membranous.

The mucosa consists of an entodermal epithelium, which is of the 
columnar ciliated variety, continuous with, and similar to the respiratory epithelium of the nasopharynx, together with a fibrous membrana propria which is loosely connected with the surrounding bony, cartilaginous, and muscular walls. The lower portions of the tube are richly supplied with mucus-secreting, tubulo-acinar glands, and toward

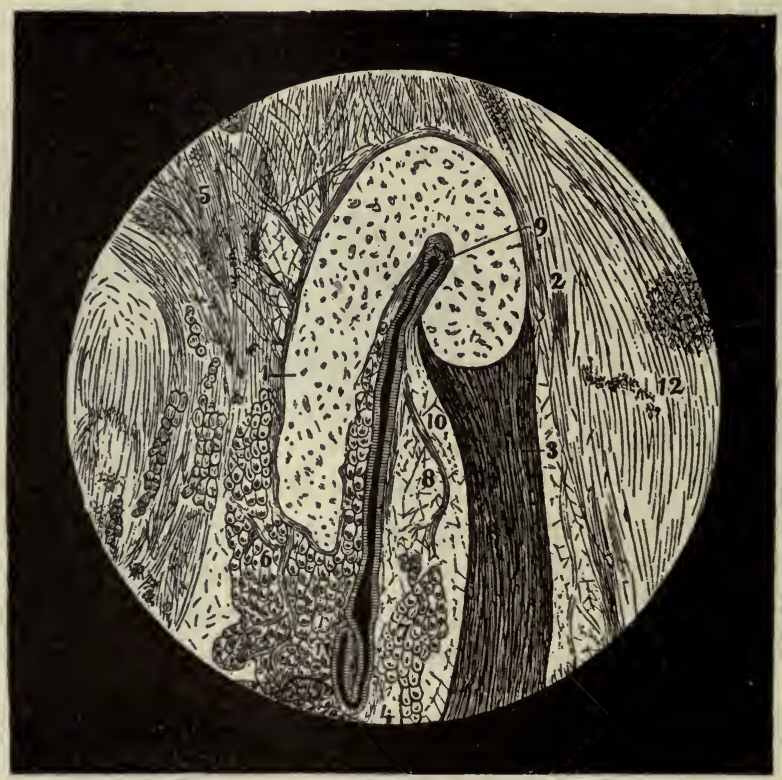

Fig. 577.-Transection of the Eustachian Tube; Diagrammatic.

1, cartilaginous plate; 2 , lateral or hooked end of the cartilage; 3 , 'dilator tubæ (tensor palati); 4 , levator palati; 5 , fibrocartilage at the base of the skull; 6 and 7 , mucous glands; 8 , adipose tissue; 9,11 , lumen of the tube; 10,12 , connective tissue. Low magnification. (After Rüdinger.)

its pharyngeal end the mucosa is much infiltrated with lymphoid tissue, thus forming the tubal tonsil of Gerlach.

The cartilage of the auditory tube is firmly adherent to the bony wall. At the point of attachment it has a hyaline structure, the fibers of the perichondrium penetrating only the surface of the cartilaginous plate. Lower down the cartilage becomes infiltrated with fibers and conforms to the typical elastic variety. Like the cartilage of the auricle it is rich in cellular elements. Its transection presents a peculiar hooklike form, by means of which the posterior surface, the superior margin, and the upper portion of the anterior surface are invested by cartilage, 
while the remaining portions of the anterior surface and the whole of the inferior margin are entirely membranous. Closely associated with the auditory tube are the tensor and levator palati muscles, lying laterally and ventrally to the tube. These muscles serve also as dilators for the tube.

\section{Vascular Supply}

The Blood-vessels. - The mucosa of the middle ear is richly supplied with blood-vessels, the larger of which lie in the deeper part of the membrane and supply capillary vessels to the tunica propria. The bloodvessels of the auditory tube are especially numerous.

In the tympanic membrane the arteries and veins form an annular plexus at the margin; and a group of similar vessels surrounds the manubrium mallei, lying in the deeper layers of the cutaneous portion of the membrane.

The mucosa of the tympanum is peculiar in the relative deficiency of capillary vessels (Prussak, 1869); the veins are numerous. The veins of the auditory tube empty into the internal jugular; they also communicate with the cavernous sinus by a trunk of considerable size (Dench, 1895).

The lymphatics of the middle ear form plexuses in the connective tissue of the mucosa and in a general way follow the course of the smaller veins. They lead in part to the lymphatic nodes behind the ear, and in part to the parotid group (Kölliker). They also communicate with the perilymphatic spaces of the internal ear.

\section{THE INTERNAL EAR}

\section{General Considerations}

The internal ear includes a series of membranous structures together with the terminal fibers of the acoustic nerve; these are contained within a series of connected cavities hollowed out of the petrous portion of the temporal bone, and in relation with the mesial wall of the tympanum. The central portion of this bony cavity, an ovoid space, is known as the vestibule; its outer wall presents the orifice of the fenestra vestibuli which leads to the tympanum, but during life is closed by the base of the stapes. Opening from the vestibule, at one end, are the bony cavities occupied by the three semicircular canals which, in a general way, 
project from the posterodorsal aspect of the vestibule; at the other end the bony cochlea containing its series of spiral canals projects anteriorly from the vestibule. Collectively these spaces, with several diverticula, form the bony labyrinth, and within them in life are contained a number of membranous sacs whose general form corresponds more or less

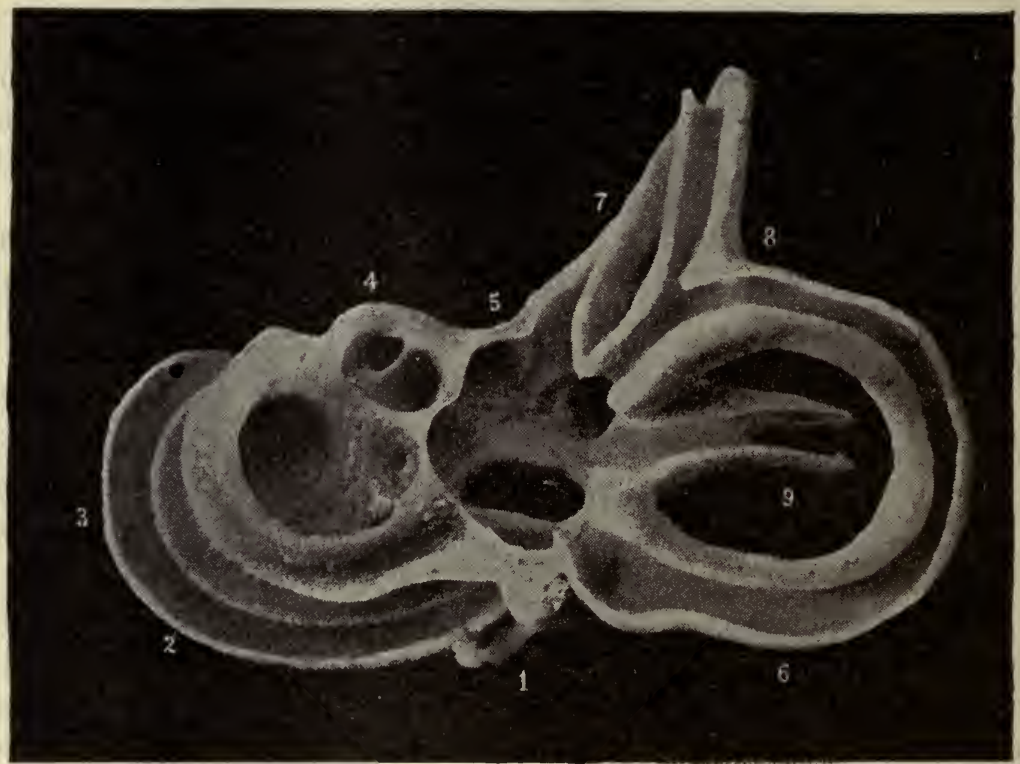

Fig. 578. - The Bony Labyrinth.

1 , round window; 2 , osseous lamina spiralis; 3 , osseous cochlear canal; 4 , floor of internal acoustic meatus; 5 , vestibule; $6,7,8,9$, semicircular canals. The figures are placed at that portion of the margin which is nearest the structure indicated. (After Rüdinger.)

closely to that of the bony cavity; these sacs collectively form the membranous labyrinth:

The vestibule contains two of these membranous sacs, the sacculus and the utriculus, which are connected by means of the slender utriculosaccular canal, from which a much prolonged diverticulum enters the aqueductus vestibuli to penetrate to the posterior surface of the petrous bone where it comes into relation with the cerebral meninges; this diverticulum is known as the ductus endolymphaticus. Its dilated terminal portion constitutes the endolymphatic sac, which lies upon the dura and probably opens into the subdural space. The utricle and saccule, as also all other portions of the membranous labyrinth, contain a watery fluid, 
the endolymph; they do not entirely fill the bony cavity of the labyrinth in which they lie, the intervening space being occupied by a retiform connective tissue with broad interstices which are permeated by an aqueous fluid, the perilymph.

\section{The Saccule}

The saccule is a rounded membranous cavity which is connected, on the one hand, by means of the slender canalis reuniens, with the cochlear duct or scala media, and on the other hand with the ductus endolymphaticus and utricle, as already stated. Its wall consists of an

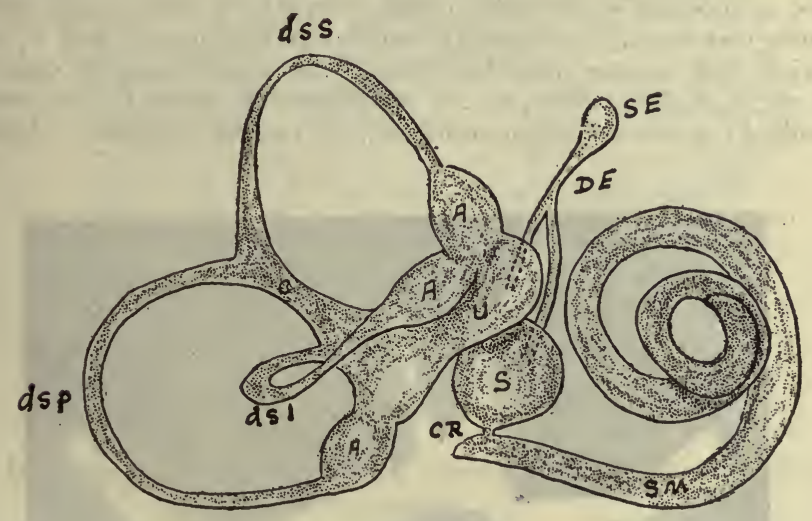

Fig. 579.-Diagrain of the Membranous Labyrinth in Lateral View.

$A$, ampullæ; $U$, utricle; $S$, saccule; $S M$, scala media or cochlear duct; $C R$, canalis reuniens; $C$, crus, or common canal; $S E$, saccus endolymphaticus; $D E$, ductus endolymphaticus; $d s s, d s p, d s l$, superior, posterior and lateral (external) semicircular ducts. The utricle and saccule are connected by the utriculosaccular duct. The cochlear duct terminates in the ceca vestibulare and cupulare. (After Gray.)

ectodermal epithelium, a membrana propria and a fibrous coat. The epithelium consists of flattened squamous cells; it completely lines the cavity. The epithelial surface is somewhat irregular from the papillary elevations of the fibrous coat. On the antero-inferior surface of the saccule the epithelium is peculiarly altered so as to form a layer of columnar cells, many of which are provided with stiff cilia. This neuroepithelium is distributed over an oval area ( $3 \mathrm{~mm}$. by $2 \mathrm{~mm}$. in extent) beneath which the fibrous coat is much thickened by the entrance of many fibers derived from the vestibular portion of the acoustic nerve. 


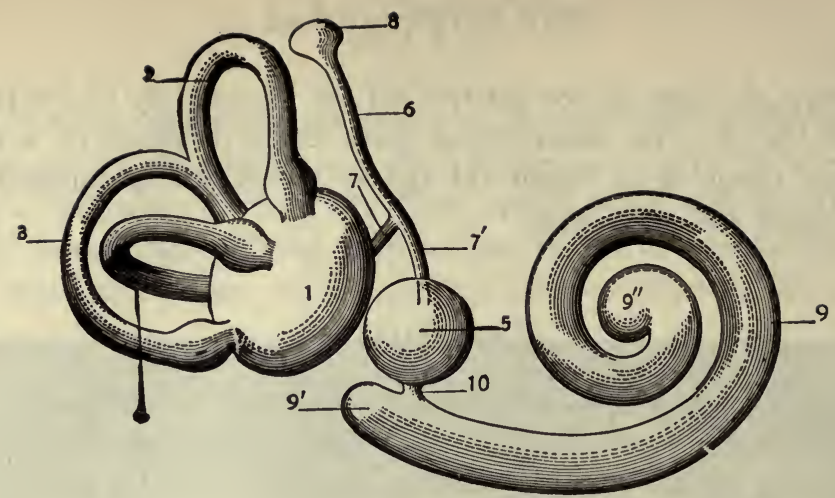

Fig. 580.-Diagram of the Right Membranous Labyrinth.

1, utricle; 2 , superior semicircular canal; 3 , posterior semicircular canal; 4 , external semicircular canal; 5 , saccule; 6 , endolymphatic duct; 7 and $7^{\prime}$, canals connecting utricle and saccule respectively with the endolymphatic duct; 8 , endolymphatic sac; 9 , cochlear duct; $9^{\prime}$, its vestibular cul-de-sac (cecum vestibulare); $9^{\prime \prime}$, its terminal cul-de-sac (cecum cupulare); 10, canalis reuniens. (After Testut.)

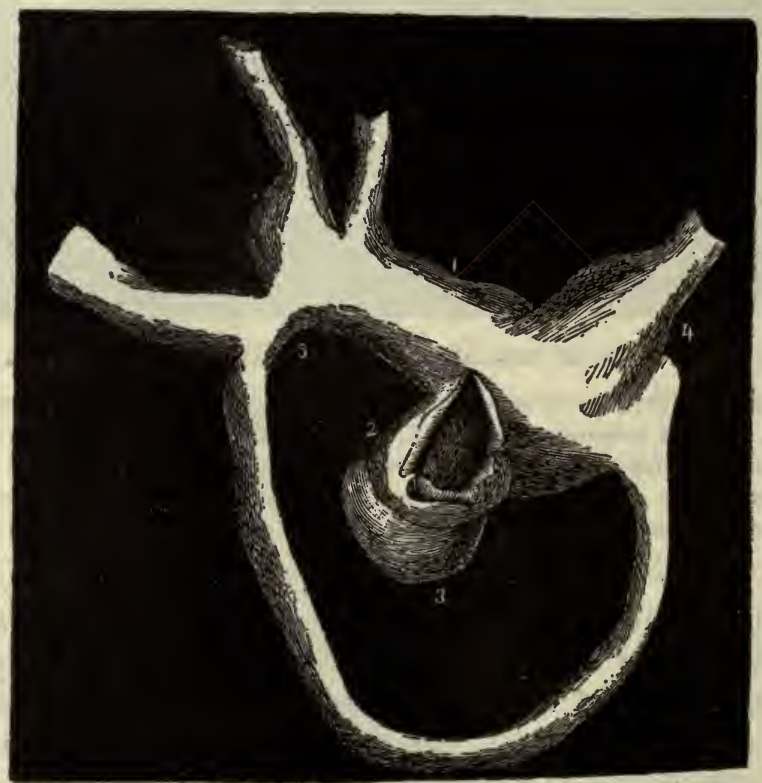

Fig. 581.-The Isolated Membranods Labyrinth.

1 , utricle; 2 , saccule (opened); 3 , location of the macula acustica sacculi ; 4 , ampulla of a semicircular canal; 5, canalis communis. Low magnification. (After Rüdinger.) 
This elevation with its neuro-epithelial covering is known as the macula acustica sacculi.

The neuro-epithelium contains two varieties of cells, the sustentacular and the hair cells. The former, fiber cells of Retzius, form a layer, two or three cells deep, which rests upon the basement membrane, and whose broad basal portion contains a spheroidal nucleus. Beyond the nucleated portion the cytoplasm of the sustentacular cell is con-

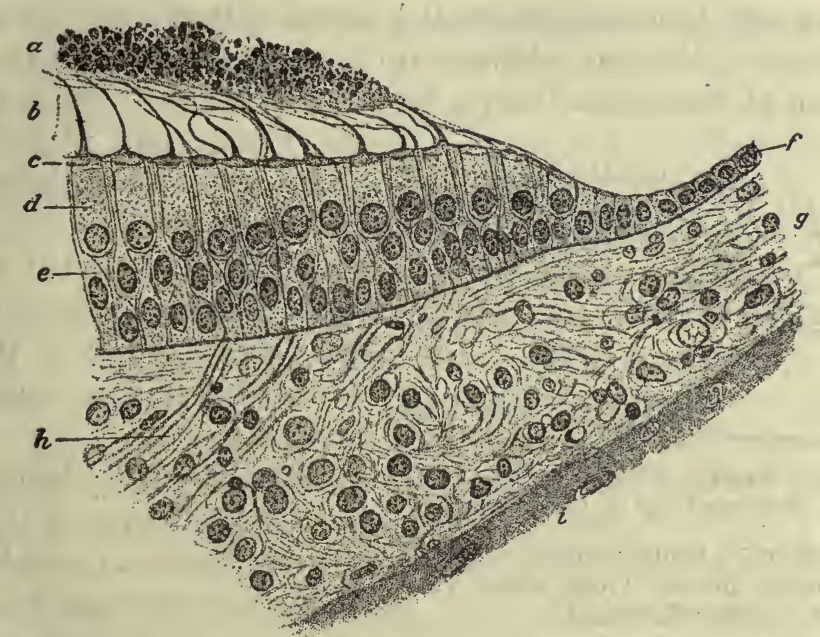

Fig. 582.-Transection of the Margin of the Macula Acustica Sacculi of a Guinea-Pig.

$a$, otolithic membrane; $b$, hairs; $c$, cuticular membrane; $d$, hair cells; $e$, sustentacular cells; $f$, epithelium of the saccule; $g$, tunica propria; $h$, nerve fibers; $i$, bone. Hematoxylin and eosin. $\times 325$. (After Kölliker.)

tinued inward between the bodies of the hair cells to the surface of the epithelial layer, this portion of the cell being relatively slender.

The hair cells occupy the superficial part of the epithelial layer by their broad nucleated portions, which carry upon their free extremity a single tuft of long stiff cilia, having the appearance of a delicate hairlike process which projects into the endolymphatic cavity. That portion of the endolymph which immediately overlies the macula, and into which the hair-like processes project, though not essentially different in microscopic appearance in fresh tissues, appears to possess a somewhat gelatinous consistence, and in it are suspended various forms of minute crystals of calcium carbonate which are known as otoconia or 'otoliths.' The free surface of the neuro-epithelium is also provided 
with a reticulated cuticular membrane which presumably is formed by the amalgamation of the free ends of the sustentacular cells. Through the openings in this reticular membrane the ciliary tufts of the hair cells project.

The central ends of the hair cells, beneath the nucleated enlargement which is found near the middle of the epithelial layer, are prolonged outward between the nucleated portions of the sustentacular cells and frequently terminate in small knobbed extremities. This portion of the cells is in intimate relation with the terminal fibrils of the vestibular portion of the acoustic nerve, which, coming from a nerve plexus in

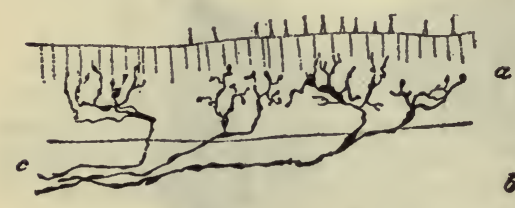

Fig. 583.-Nerve Endings in the Macula Acustica of a Guinea-pig.

$a$, epithelium; $b$, tunica propria; $c$, three terminal nerve fibers. Golgi stain. $\times$ about 200. (After Retzius.) the fibrous wall of the saccule, forms an intra-epithelial plexus of delicate varicose fibrils. Frequently the epithelial coat contains coarse granules of a brownish pigment which, at times, also produces a diffuse coloration of the cells.

The lining epithelium of the saccule rests upon a thin homogeneous basement membrane and is further supported by a delicate fibrous coat or tunica propria. The connective tissue of this coat forms interlacing bundles the most of which are distributed in a circular manner about the wall of the ovoid sacculus. At the macula this coat is much thickened by the entrance of the fibers from the vestibular nerve. It also contains the minute blood-vessels which supply the organ.

As is the case with the other divisions of the membranous labyrinth, the fibrous wall of the saccule is in contact on one aspect of its surface with the periosteum which lines the osseous labyrinth; elsewhere it is separated from the periosteum by the perilymphatic cavity.

\section{The Utricle}

The utricle is somewhat larger than the saccule. It lies behind and somewhat above the saccule, is of a very irregular oblong form, and receives the insertions of the semicircular canals. Its anterior portion is provided with a macula and the structure of its wall differs in no wise from that of the saccule; both of these mem- 
branous sacs are contained within the irregular cavity of the vestibule. The structure of the utriculus, therefore, requires no further description.

\section{The Semicircular Canals}

The semicircular canals or ducts are three in number, the posterior, superior, and lateral. The last is also horizontal in its position; the first two are vertical, but are so placed as to form a right angle with one another. The posterior lies in the long axis of the petrous bone and its plane is therefore more nearly sagittal, while that of the superior canal is more nearly coronal. Each canal forms something more than half a circle, its two ends opening separately into the cavity of the vestibule, with the exception of the posterior and superior canals whose internal ends open by a common orifice, the canalis communis. The unjoined orifices of the posterior and superior canals, as also the outer extremity of the lateral canal, present a marked dilatation at their termination in the vestibule. These dilatations are known as the ampulla. They lodge the neuro-epithelial patches, the cristæ acusticæ. The osseous and membranous canals are of similar shape; the latter is, of course, contained within the former.

The membranous semicircular canals open into the utricle. They do not entirely fill their bony canal, but, like the utricle and saccule, lie in contact with the periosteum at one surface only; this surface being that of the outer wall or periphery of the semicircle, while in the remaining portion of the circumference of the cylindrical bony duct, the membranous canal is loosely united to the periosteum of the osseous wall by a retiform connective tissue whose loose meshes are filled with perilymph and lined with mesenchymal epithelium.

The wall of the membranous canal is similar in structure to that of the saccule and utricle and consists of an ectodermal epithelium, a membrana propria, and a fibrous tunic. Each of the three ampullæ presents a marked differentiation of the epithelial lining, which is there raised in the form of a prominent crescentic fold, inappropriately termed by the older anatomists the crista acustica, from its supposed connection with the auditory function. Like the maculæ of the saccule and utricle, the cristæ are supplied by the vestibular nerve and are concerned with the function of equilibration.

The utricle and saccule represent the original anlage of the earthe otocyst-from which the canals and cochlea arise as evaginations; they correspond most closely also to the 'ear' of certain invertebrates, 
e.g., crustacea, which is simply an equilibrating organ. They are believed to function as static organs of equilibration, giving information as to position at rest or during progressive movements; the semicircular canals on the other hand are commonly conceived of as dynamic organs of equilibration and are thought to furnish information regarding the direction and extent of rotatory movements.

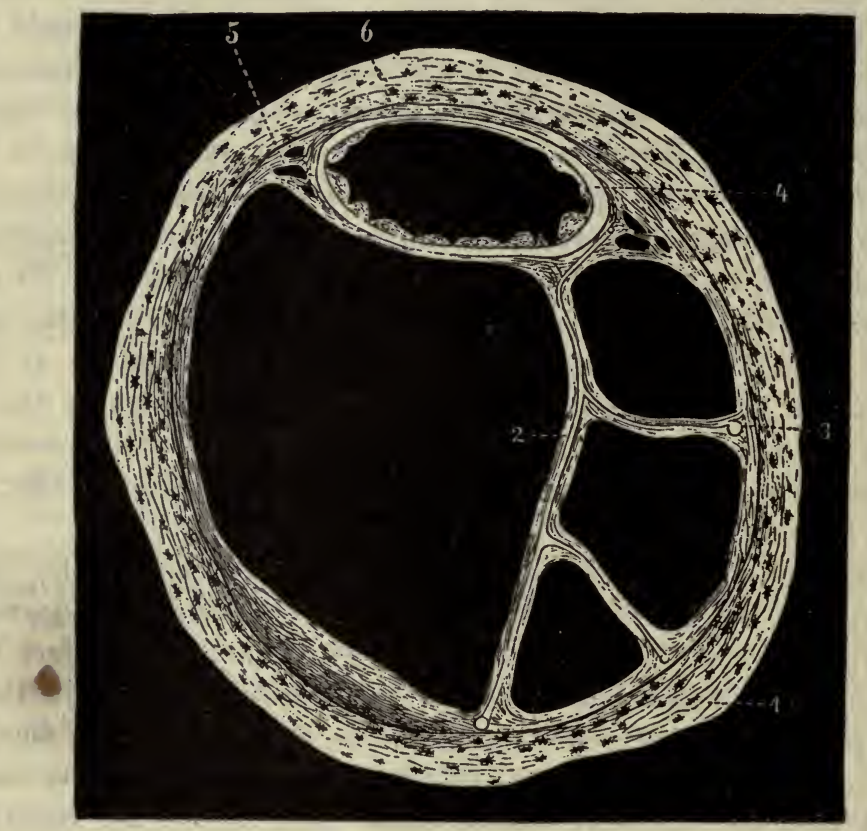

Fig. 584.-Transection of a Human Semicircular Canal.

1 , bone; 2 , retiform connective tissue membranes; 3 , at this point a band of connective tissue joins the periosteum; 4 , membranous semicircular canal; 5 , ligamentous attachment of the canal; 6 , at this point the membranous and osseous canals are in contact. Moderately magnified. (After Rüdinger.)

The cristæ are clothed with tall columnar cells which, though somewhat taller, are otherwise similar in structure to those of the maculæ, and are similarly divisible into sustentacular cells and hair cells. They are also covered by a gelatinous cuticular formation, containing otoliths, which is here known as the cupola. The vibratory stimulus is transmitted from the endolymph to the hair cells through the medium of the otolithic membranes. 


\section{The Cochlea}

The cochlea, like the vestibular portion of the internal ear, consists of a bony case which incloses a membranous organ.

Structure.-The bony cochlea possesses a peculiar flat pyramidal shape. The base of the pyramid is in contact with the anterior aspect of the vestibule; its apex or cupola is directed forward, outward, and slightly downward. The pyramid is hollow and contains in its axis a conical bony support, the modiolus, which tapers from a broad base to a pointed apex beneath the broader, blunt, and rounded cupola of the outer bony wall. The modiolus contains a broad canal which receives the cochlear division of the acoustic nerve as it enters from the internal meatus.

The outer surface of the modiolus supports a bony shelf, the lamina spiralis ossea, which winds in a spiral manner from its base to its apex, and ends in a hook-like process, the hamulus. This shelf only partially spans the interval between the modiolus and the outer wall of the cochlea. In life the remaining

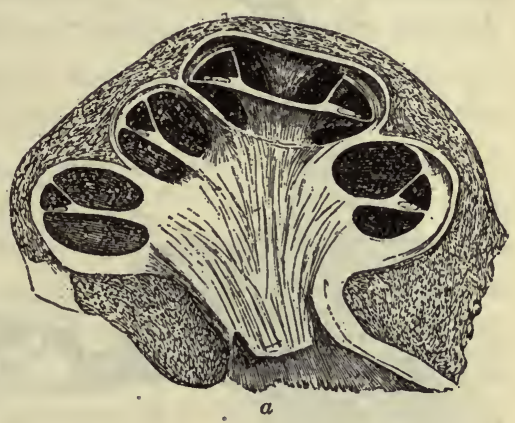

Fig. 585.-Axial Section through the Cochlea of a Fetal Calf.

$a$, internal acoustic meatus in which is the cut end of the cochlear nerve as it enters the modiolus. $\times 6$. (After Kölliker.) interval is completed by a firm fibrous membrane, the basilar membrane (lamina spiralis membranacea). Thus the cylindrical canal of the cochlea, which is wound spirally around the modiolus making two and one-half turns from the base to the apex, is subdivided into two parallel longitudinal divisions, which are respectively known as the scala vestibuli and the scala tympani. They are so disposed that in a given turn of the canal the former is always nearer the apex, the latter nearer the base of the cochlea. According to Wiedersheim (1893) the human cochlea has nearly three turns, the pig four, the cat three, the rabbit two and one-half, the ox three and one-half, and cetacea one and one-half turns.

The osseous lamina spiralis presents a grooved margin or sulcus, from the basal or tympanic lip of which the lamina basilaris is continued to the opposing surface of the bony wall. The lamina spiralis ossea is hollowed out in a diploic manner for the transmission of the 
branches of the cochlear nerve, which are continuously given off all the way from the base to the apex of the osseous spiral lamina, and which pass outward through the foramina nervosa upon the basilar membrane

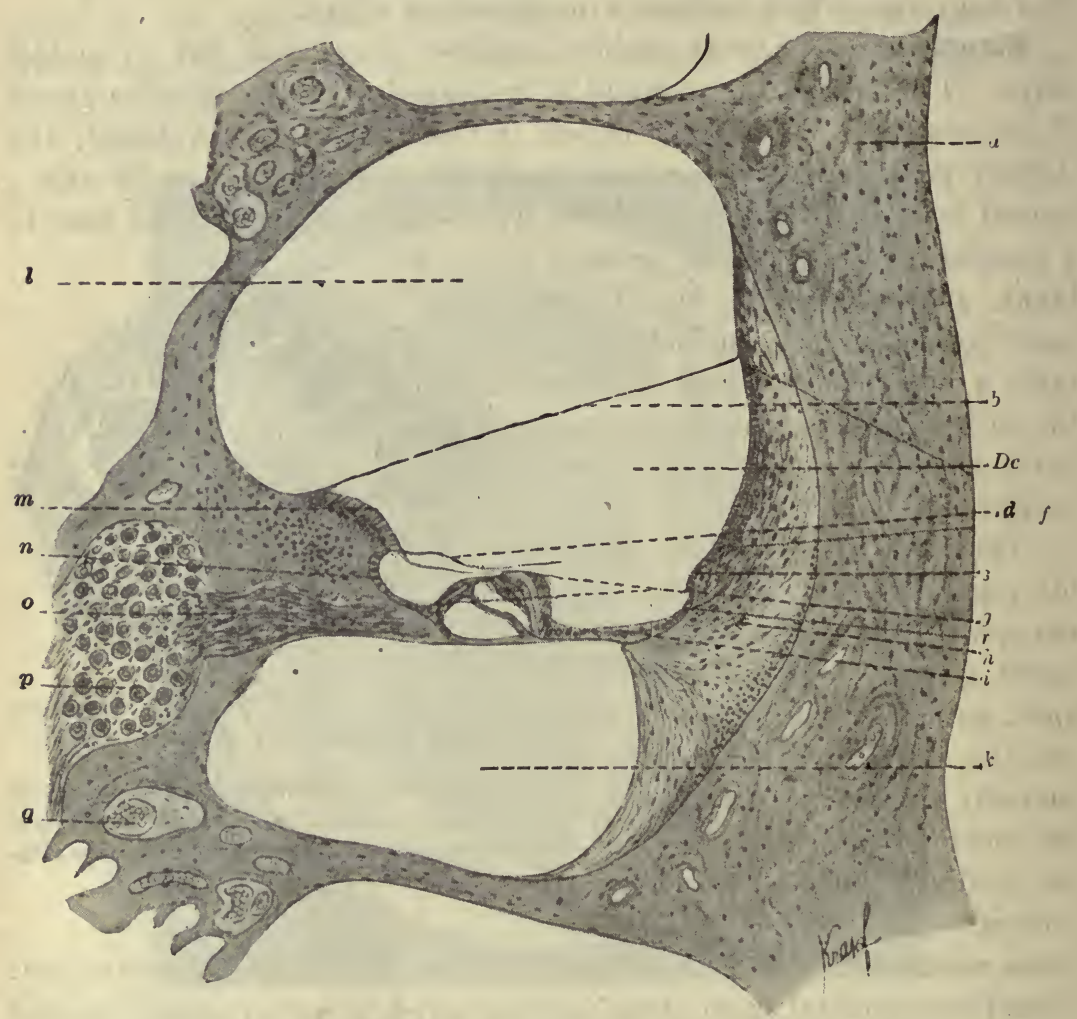

Fig. 586.-Axial Section through a Turn of the Cochlea of a Guinea-pig.

$a$, bone of the outer wall of the cochlea; $b$, membrane of Reissner; $d$, membrana tectoria; $D c$, cochlear duct or scala media; $f$, stria vascularis; $g$, organ of Corti; $h$, spiral ligament; $i$, cells of Claudius; $k$, scala tympani; $l$, scala vestibuli; $m$, vestibular lip of the limbus spiralis; $n$, internal spiral sulcus; $o$, nerve fibers of the cochlear nerve, contained within one of the radiating canals within the osseous spinal lamina; $p$, nerve cells of the spiral ganglion; $q$, blood-vessel; $r$, external spiral sulcus, upon which open Shambaugh's glands; $s$, prominentia spiralis, containing the vas prominens. $\times 90$. (After Böhm and von Davidoff.)

to be distributed to the epithelium of the spiral organ (of Corti). This organ is a peculiar spiral group of neuro-epithelial cells which extends the whole length of the basilar membrane from the base to the cupola of the cochlea. 
The margin of the osseous spiral lamina is much thickened by the fibrous and epithelial tissues by which it is invested, so that a membranous sulcus of considerable depth is formed between the two lips (vestibular and tympanic lips) of the bony sulcus spiralis internus. This is further thickened by a marked elevation of fibrous tissue covered by columnar cells, from the outer margin of which a delicate membrane, the membrana tectoria, extends outward and overhangs the epithelium of Corti's organ. From the inner margin of the elevation of fibrous tissue, the limbus spiralis, which is supported by the vestibular lip of the bony lamina, a delicate membrane, the vestibular membrane (of Reissner), passes obliquely outward to the bony wall of the cochlea, and in transections appears to cut off a corner of the scala vestibuli, thus marking off a triangular space whose base is formed by the outer wall of the cochlea, its sides by the membrane of Reissner and the basilar membrane upon which rests the organ of Corti; its blunt apex is found at the sulcus spiralis internus. Since these membranes extend the entire length of the bony spiral canal of the cochlea, the space which is thus apparently cut off from the scala vestibuli must form a spiral canal, included between the scala tympani on the one side and the scala vestibuli on the other; this canal is the scala media or cochlear duct.

The scala media is an endolymphatic canal. At the apex of the cochlea it ends in a blind extremity which is known as the lagena or cecum cupulare; its opposite end forms a blind pouch between the fenestra cochleæ and the fenestra vestibuli, at the base of the cochlea, which is termed the cecum vestibulare. The scala media is connected with the saccule and utricle by means of the canalis reuniens, as described above.

The scala tympani and scala vestibuli, on either side of the scala media, extend spirally from the base to the apex of the cochlea. At the apex they are united by the helicotrema, a continuation of these canals which curves around the hamulus. At the base of the cochlea the two canals diverge, the scala tympani ending abruptly at the fenestra cochleæ, which is closed by a fibrous membrane, clothed on its tympanic surface by the flattened epithelium of the tympanic mucosa, and on its cochlear surface by the epithelium of the scala tympani. This secondary tympanic membrane serves for the relief. of tension in the cochlea when the perilymph is set into motion by the stapes. The scala vestibuli, on the other hand, is continued backward into the vestibule, where it is in relation with the external surface of the saccule and utricle, and, 
since it is in contact with the outer wall of the bony vestibule, this portion of the scala vestibuli receives the opening of the fenestra vestibuli, which is closed by the foot plate of the stapes. Corresponding to the relative positions of the fenestra vestibuli and fenestra cochleæ, the scala vestibuli in the first turn of the cochlea lies above the scala tympani, and being somewhat the longer it also extends farther backward.

Having traced the general form and relations of the several portions of the cochlea, we are now in a position to study more carefully the finer structure of its more important parts.

The membranous wall of the scala tympani and scala vestibuli is clothed by a mesenchymal epithelium of flattened endothelioid cells, which rest upon a double layer of fibrous tissue. Thus the tunica propria also serves as a periosteum for the inner surface of the bony wall of the cochlea, and conveys the blood and lymphatic vessels. The scalæ are perilymphatic canals. They communicate with the subdural space through the aqueductus vestibuli and the aqueductus cochleæ. The latter opens from the scala tympani near its beginning at the fenestra cochleæ, and passes below the pyramid to the dura transmitting a small vein.

The vestibular membrane (of Reissner) is an extremely delicate structure which consists of a thin central substantia propria, covered on either surface by epithelium, that on the one surface being continuous with the mesenchymal epithelium of the scala vestibuli, that on the other with the ectodermal epithelium of the scala media. It is non-vascular in the adult.

The outer wall of the scala media is lined by a continuation of the epithelium in that portion which adjoins the membrane of Reissner, and this rests upon a fibrous membrane similar to that which forms the walls of the other scalæ. Toward the attachment of the membrana basilaris, however, the tissue of the outer fibrous wall of the scala media is much thickened, and forms a dense ligamentous structure, triangular in shape as seen in a longitudinal section of the cochlea, which receives the insertion of the membrana basilaris at its apex, and being, like the basilar membrane, continued from the base to the apex of the cochlea, is known as the spiral ligament. Its dense fibrous bands radiate from the attachment of the basilar membrane to all portions of the ligament, and are firmly attached to the bony wall of the cochlea, with whose periosteum the deeper fibers of the spiral ligament are blended.

The surface of the spiral ligament, which forms the outer wall of the scala media, slopes gradually away from the attachment of the basilar 
membrane; that which impinges upon the scala tympani slopes more abruptly. The greater portion of the spiral ligament, therefore, is contained within the scala media. Here it is lined by low columnar or cuboidal epithelium whose cells blend, without demarcation, with the underlying vascular connective tissue, so that the minute blood-vessels frequently appear as if lying within the epithelial layer, although they probably are always contained within the connective tissue processes which project into the attached surface of the epithelial layer.

This very vascular subepithelial portion of the spiral ligament is known as the stria vascularis. A short distance above the point of attachment of the basilar membrane to the spiral ligament appears a prominent spiral ridge, the prominentia spiralis, the intervening groove constituting the sulcus spiralis externus. The larger blood-vessel (venous) within the prominence is the vas prominens. From the external sulcus there extend into the subjacent ligamentous tissue numerous large clear clumps of epithelioid cells. These have been variously interpreted as neuro-epithelial elements and as smooth muscle cells. But Shambaugh (Archives of Otology, 37, 6, 1908) has shown that they contain tubules which open into the sulcus, and that they are in reality branched tubular glands. He ascribes to them the function of producing at least a portion of the endolymph of the scala media; the stria vascularis is probably also an important source of endolymph.

The tympanic wall or floor of the scala media presents for examination several structures, which, from within outward (viz., from the modiolus to the ligamentum spirale), are the limbus spiralis, membrana tectoria, sulcus spiralis internus, basilar membrane, and the organ of Corti which rests upon the basilar membrane (Fig. 587).

The vestibular lip of the limbus spiralis presents a distinct elevation, which is formed by a peculiar cellular variety of connective tissue, and is covered by columnar epithelium, whose cells are not sharply defined from those of the underlying connective tissue. The surface of the epithelium presents a distinct cuticular formation of considerable thickness, which seems to be prolonged outward from the margin of the vestibular lip, and forms the membrana tectoria.

The surface of the limbus spiralis, when viewed from the scala media, presents slight elevations which, at the margin of the vestibular lip, are prolonged into prominent ridges whose indented borders overhang the sulcus and are known as the auditory teeth (of Huschke).

The Membrana Tectoria (Membrane of Corti).-This is an exoplasmic or cuticular tissue, formed by the epithelium of the inner or 
limbus portion of the embryonic cochlear duct. It has a gelatinous fibrillar structure, but lacks nuclei; and, unlike the otherwise very similar otolithic membranes of the maculæ and cristæ, it contains no calcareous products. Its free margin overhangs, or rests lightly upon, the hair cells of Corti's organ.

The tectorial membrane has been very carefully studied by Hardesty (1908) in the pig, from whose cochlea he has been able to remove it entire. It is said to measure about $30 \mathrm{~mm}$. in length, to occupy the four turns of the cochlea, to be about five times as wide and five times as thick in the apical turn as at the basal end, and to have a section area in the apical turn approximately twenty-one times and a volume ninety-five times the area and volume of its basal end. It is described as consisting 'of a hyaline matrix, probably keratin, in gelatinous form, in which are embedded the very numerous fine fibers or threads of uniform size' (Anat. Rec., 8, 2, 1914). The membrane has a slight amount of elasticity, is of a semi-solid character and possesses 'marked adhesiveness'; its specific gravity is said to be but little greater than that of the endolymph. None of the fibers extend the entire width of the membrane, none are attached at both ends, and the greater number are attached at neither end (Hardesty, Amer. Jour. Anat., 8, 2, 1908). Hardesty describes a stripe (Hensen's stripe) on the under surface of the tectorial membrane opposite the row of inner hair cells, which he explains 'as a line of intererossing ends of fibers of the under surface resulting from the process by which the growth of the membrane terminates.' He describes also a thin, exceedingly delicate, 'accessory tectorial membrane,' along the under surface of the outer portion of the chief membrane; only its outer edge is attached to the latter, and it is bounded internally by Hensen's stripe, thus covering only the outer hair cells.

The Sulcus Spiralis Internus.-This is a deep groove included between the vestibular lip of the limbus and the basilar membrane which is attached to the tympanic lip. The sulcus is lined by flattened epithelial cells, which are apparently continuous with those of the vestibular lip, and like them are not readily distinguished from the underlying connective tissue. The epithelium is continued outward upon the basilar membrane to the margin of Corti's organ, with the innermost cells of which it is continuous.

The Basilar Membrane (Membrana basilaris).-This is a thin but resistant membranous structure, upon which rests the epithelium of Corti's organ. Hardesty (1908) describes it as a 'flat tendon . . . whose purpose is merely to strengthen the floor of the ductus cochlearis and 

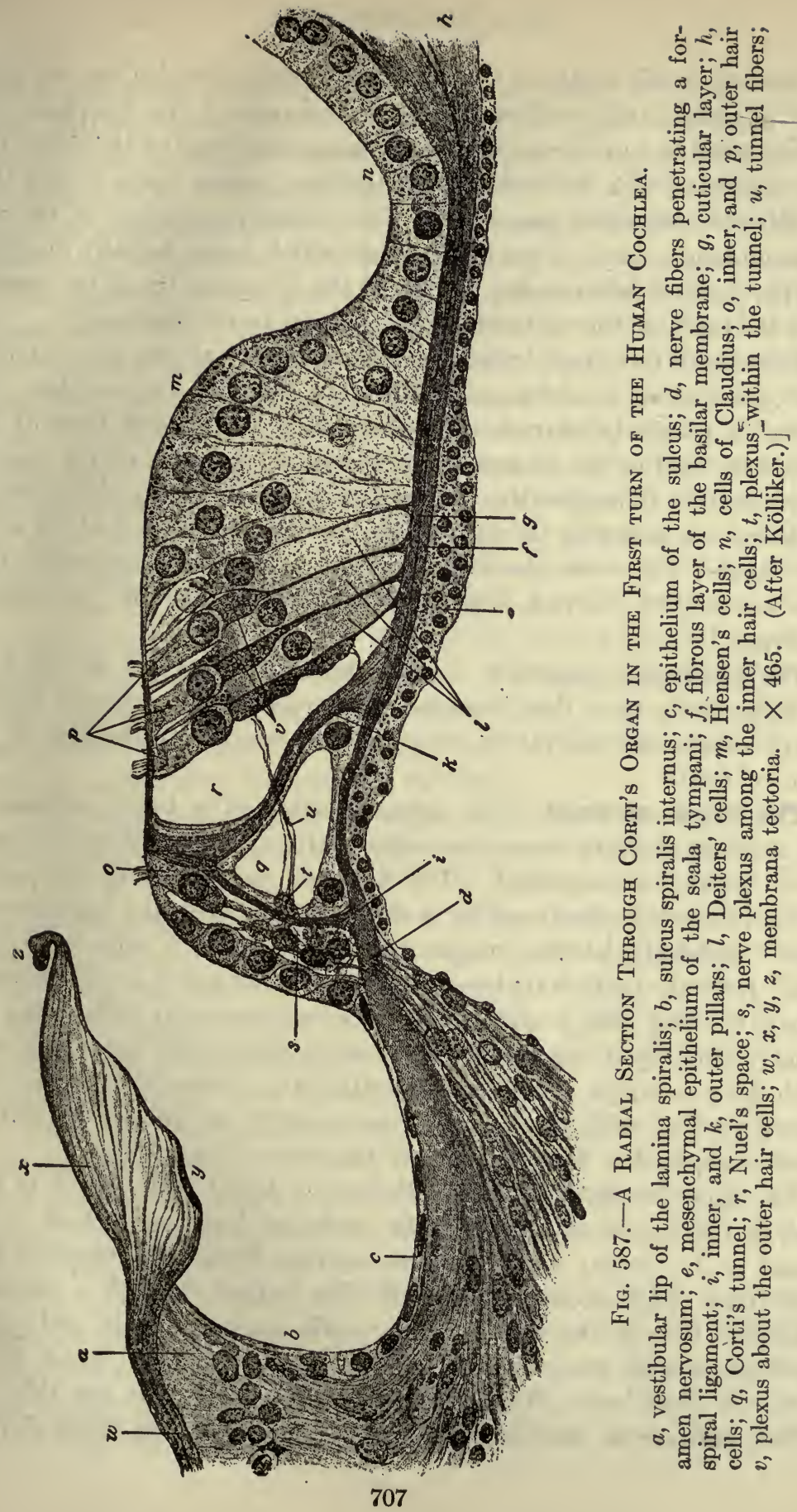
the position of the organ of Corti, and the fibers of which are too rigid and firmly associated to allow of resonant vibration.' Its tympanic surface is clothed by a continuation of the lining membrane of the scala tympani, consisting of a mesenchymal epithelium, resting upon a very thin and delicate connective tissue layer. The substantia propria of the basilar membrane consists of tendinous bands which, being radially disposed, span the interval between the margin of the tympanic lip of the osseous spiral lamina and the opposed margin of the spiral ligament.

Because of the great breadth of the modiolus at the base, and its rapid diminution in thickness toward the apex of the cochlea, this interval is relatively narrow at the beginning of the first turn of the spiral scala media, but progressively widens as the apex of the cochlea is approached. Consequently, the shortest tendinous fibers of the basilar membrane are found at the base of the cochlea, the longest at its apex. The shortest fibers are also the coarsest. It has been estimated that there are 24,000 distinct fibers or 'auditory strings' in the basilar membrane.

The substantia propria is covered upon that surface which faces the scala media by a thin homogeneous membrane, a cuticular formation or exoplasmic derivative, upon which rests the epithelium of the organ of Corti.

The Organ of Corti.-This organ consists of a highly differentiated neuro-epithelium whose specialized cells are disposed according to a very regular arrangement. The flattened epithelium of the sulcus spiralis internus is continued for a short distance upon the basilar membrane. Suddenly, at the margin of Corti's organ, it alters its character. Here the epithelium becomes abruptly changed to a tall columnar variety, the first cells, known as the inner sustentacular cells, being apparently piled upon one another and resting against the inner hair cells, which form a single row of neuro-epithelium; these, like all the succeeding rows of cells, can be traced as a continuous line in the spirally wound scala media, from the base to the apex of the cochlea.

The inner auditory or hair cells have a broad body which is confined to the superficial third of the epithelial layer and which is nucleated at its deeper enr. Its free surface forms an expanded oval plate from which about twenty stiff cilia project through a cuticular membrane toward the cavity of the scala media. These end plates interdigitate with the phalanges of the inner pillar cells, which are to be shortly described. The bases of the inner hair cells are thin and slender, and are in relation with a nerve plexus of fine fibrils derived 
from the terminal processes of the cochlear nerve. These nerve fibrils make their exit in small bundles from the bony spiral lamina, through the foramina nervosa and passing outward upon the basilar membrane are distributed in a plexus beneath the epithelium, some of their naked processes almost immediately penetrating the epithelial layer to end between the bases of the inner hair cells.

The inner hair cells rest against the inner pillar cells, or rods, of Corti's arch. This arch is formed by two rows of highly specialized cells, the inner and the outer pillars, which are widely separated where their bases are attached to the basilar membrane, but are in contact at their free ends; in fact, the free extremity of the inner pillar is prolonged into a broad flattened plate-like process whose inner margin interdigitates with the head plate of the inner hair cells, as stated above, and whose outer margin is so prolonged as to almost, though not completely, cover the rounded head of the outer pillar. The head of the outer pillar, being similarly flattened, expanded, and prolonged outward. beyond the margin of the head plate of the inner pillar cell, comes into contact with the phalanges of Deiters' cells and with the cilia of the outer hair cells which lie next without; they leave a space between the outer pillars and the outer hair cells which is known as Nuel's space, filled by a semi-fluid intercellular substance.

The inner pillar cells are rather more numerous than the outer -in the entire length of the scala media, according to Retzius, there are 5,600 of the former to 3,850 of the latter-so that about three of the expanded head plates of the inner pillars overlap two of the rounded heads of the outer pillar cells. The arch formed by the opposed pillar cells, being succeeded by similar arches of successive pillars, forms a continuous tunnel, triangular in transection, which extends the whole length of the scala media, and is known as the canal of Corti. This canal is also filled with a semi-fluid substance.

Each pillar cell is differentiated into two portions, the pillar proper and the basilar cell, the latter containing the nucleus. The pillar presents a fibrillar appearance, the fibrils being disposed in the long axis of its body. This portion of the cell reaches from the basilar membrane to the free surface of the neuro-epithelium.

The basal part of the cell, the basilar cell, probably represents the undifferentiated portion of the primordial pillar cell. It consists of a clear, finely granular cytoplasm and contains the spheroidal nucleus. It lies on that side of the pillar which faces the canal of Corti, the bases of the opposed cells being expanded until they meet, thus forming a 
cuticular floor for the tunnel. This undifferentiated basilar portion occupies only the deeper half of the pillar cell.

The outer hair cells form three to five rows of ciliated cells which are similar in structure to the inner hair cells, and which are supported by the sustentacular cells of Deiters. Their cylindrical cell bodies occupy the superficial third of the epithelial layer and at the deeper extremity present a nucleated enlargement, beyond which they are continued only as an extremely slender basal process. The free ends of the outer hair cells present an expanded oval surface from which the hairs project. The outer hair cells are about five times as numerous as the inner, that is, there are about 3,600 of the inner to 18,000 of the outer (Waldeyer). According to certain authorities the hair cells lack the delicate basal process in the adult condition.

The outer sustentacular cells (Deiters' cells) are cylindrical cells whose expanded bases rest upon the basal membrane and whose distal portions extend toward the surface between the outer hair cells. The superficial portion of these cells, being encroached upon by the broad outer hair cells, is very slender; the broader basal portion occupies the deeper two-thirds of the neuro-epithelium, the spheroidal nuclei being found at the level of the middle third. Each sustentacular cell contains a cuticular filament (fiber of Retzius) which begins in contact with the cuticle of the basal membrane, and extends through the axis of the cell to its free border, where it expands to form a broad flattened plate of peculiar shape, known as the phalangeal process. These cuticular processes surround and overlie the margins of the head plates of the hair cells, thus forming a reticular layer through the openings of which the cilia of the hair cells project.

The cells of Deiters are succeeded by the sustentacular cells of Hensen. These are tall columnar cells about eight rows broad, the innermost of which equal in height the tall cells of the preceding type, but which at their outer border become abruptly shortened. Here they pass into the cuboidal cells of Claudius, and are thus continued outward to the spiral ligament.

The nuclei of the cells of Hensen are found in their superficial third, those of the cells of Claudius in the center of the cell. Beneath Hensen's cells other small nucleated elements are occasionally found; they give to this layer somewhat the appearance of a two-rowed epithelium and are known as the cells of Böttcher.

Both the cells of Hensen and those of Claudius are provided with a cuticular margin which, with the similar cuticle of the cells of Deiters, 
forms a continuous, membranous, cuticular layer known as the lamina reticularis. The inner portion of this cuticular membrane is pierced by the cilia of the three to five rows of outer hair cells, as already described.

A fibrillar axial core is a common feature of all of the sustentacular elements of Corti's organ. This fiber of Retzius becomes progressively

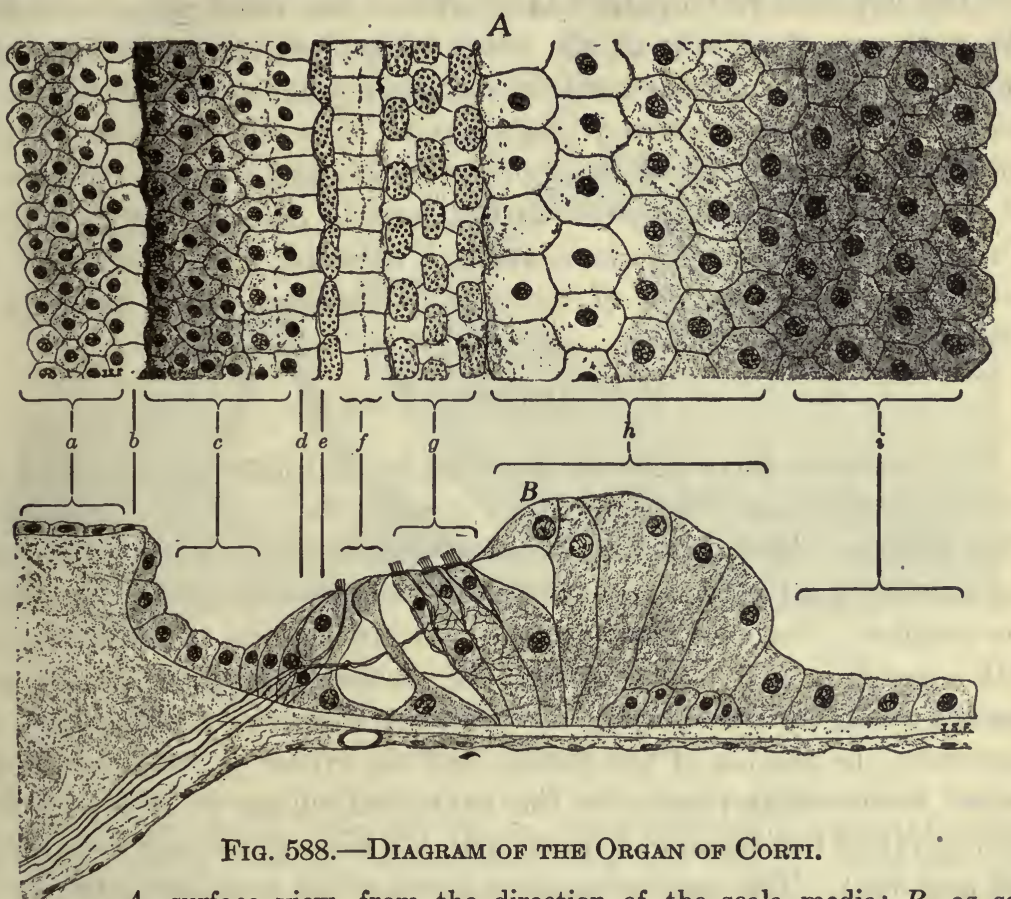

$A$, surface view, from the direction of the scala media; $B$, as seen in section, profile view. $a$, the vestibular lip of the lamina spiralis; $b$, margin of same; $c$, sulcus spiralis internus; $d$, inner sustentacular cells; $e$, inner hair cells; $f$, pillar cells; $g$, outer hair cells and phalanges of Deiters' cells; $h$, cells of Hensen; $i$, cells of Claudius. Very highly magnified.

less pronounced in passing from the pillar cells, where it is very highly developed, to the cells of Claudius, where it is barely discernible.

In the above description we have directed attention to the appearance of transections of the organ of Corti. In the study of this organ in the fresh condition, and occasionally in fixed and stained preparations, it is possible to obtain a surface view of this organ from the direction of the scala media. In such preparations the polygonal outlines of the columnar cells of the limbus spiralis, beneath which are the auditory teeth, are seen on the outer side of the attachment of Reissner's mem- 
brane. Beneath the overhanging vestibular lip of the limbus the mosaic of large polygonal epithelial cells of the internal sulcus comes into view. At the margin of the organ of Corti these are exchanged for the broader cell ends of the inner sustentacular cells and the adjacent single row of inner hair cells.

The flattened rectangular head plates of the inner pillar cells form the next row, the heads. of the outer pillars projecting from beneath, and extending beyond the heads of the inner pillar cells. These are followed by the interdigitating phalanges of the cells of Deiters, which enter into the formation of the reticular membrane, through the fenestra of which the cilia of the three to five rows of outer hair cells project. This cuticular membrane is continued outward, and beneath it are successively seen the ends of the cells of Hensen, and of the cells of Claudius.

\section{The Acoustic Nerve}

The acoustic nerve presents two distinct divisions both of which are sensory, but which differ greatly as regards their central termination. They likewise differ in their peripheral distribution. Within the internal acoustic meatus the nerve divides, each branch consisting of numerous bundles. The vestibular (superior or anterior) division is supplied with a ganglion of considerable size, the vestibular ganglion (of Scarpa), beyond which the nerve separates into three branches which supply, respectively, the macula of the utricle, and the cristæ of the superior and lateral semicircular canals, in the neuro-epithelium of each of which their terminal fibrils end in relation with the bases of the hair cells (Figs. 582 and 583). The remaining nerve fibers which are distributed to the vestibule are derived from a branch of the cochlear (inferior or posterior) division, and they supply in a similar manner the macula of the saccule and the crista of the posterior semicircular canal. According to Streeter (Amer. Jour. Anat., 1907), the vestibular nerve contributes also the innervation to the posterior canal and to the saccule, the cochlear nerve supplying only the cochlea.

The cochlear branch proper, cochlear nerve, enters the modiolus, where it becomes abruptly narrowed by giving off numerous fine branches which pass outward between the layers of the bony spiral lamina. Here they form a continuous spiral succession of small nerve trunks, supplied with many bipolar ganglion cells, which collectively form the spiral ganglion (Fig. 586). They penetrate the margin of the bony sulcus through the foramina nervosa, a succession of perforations, in the tym- 
panic lip of the sulcus. Here the nerve fibers lose their medullary sheath and come almost at once into relation with the inner-hair cells. From this point the path of the non-medullated fibers varies, most of them passing for some distance along a spiral course through the organ of Corti. One such spiral bundle is found on the inner, and another on the outer side of the inner pillars, the latter lying within the canal of Corti. Still other fibers, the tunnel fibers, cross the canal of Corti to form a spiral plexus beneath the outer hair cells and the cells

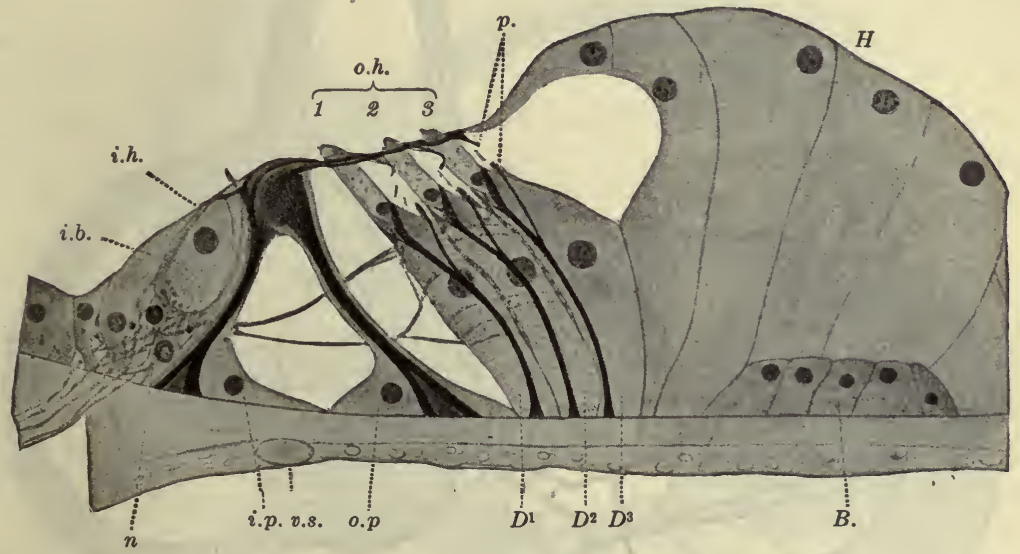

Fig. 589.-Axial Section through Corti's Organ of the Guinea-pig, Showing the Terminal Nerve Fibrils.

$B$, cells of Böttcher; $D^{1}, D^{2}, D^{3}$, three rows of Deiters' cells; $H$, cells of Hensen; $i . b$., inner border cell; i.h., inner hair cell; $i . p$., inner pillar cell; $n$, terminal branch of the cochlear nerve; o.h. $-1,2,3$, three rows of outer hair cells; o.p., outer pillar cell; $p$., phalangeal process of the outer sustentacular process. Very highly magnified. (After Held.)

of Deiters. Terminal fibrils from these spiral plexuses end in relation with both the inner and the outer hair cells.

The relation of the nerve cells of the spiral ganglion and the vestibular ganglion to the termination of the nerve fibrils about the hair cells of the organ of Corti, the maculæ, and the cristæ, is essentially the same. The ganglia contain the cell bodies of the peripheral sensory neurons of the eighth cerebral or acoustic nerve. These are bipolar cells, of which the central process or axon enters a medullated nerve fiber of the acoustic nerve, while the peripheral process is distributed to the hair cells of the several areas of specialized neuro-epithelium, as above described. 


\section{The Vascular Supply}

Blood Supply. - The internal ear is supplied by the internal auditory artery, a branch of the basilar artery, which enters the labyrinth along with the acoustic nerve, and at once divides into two main stems, the vestibular and the cochlear (arteria cochlearis communis, Sieben-

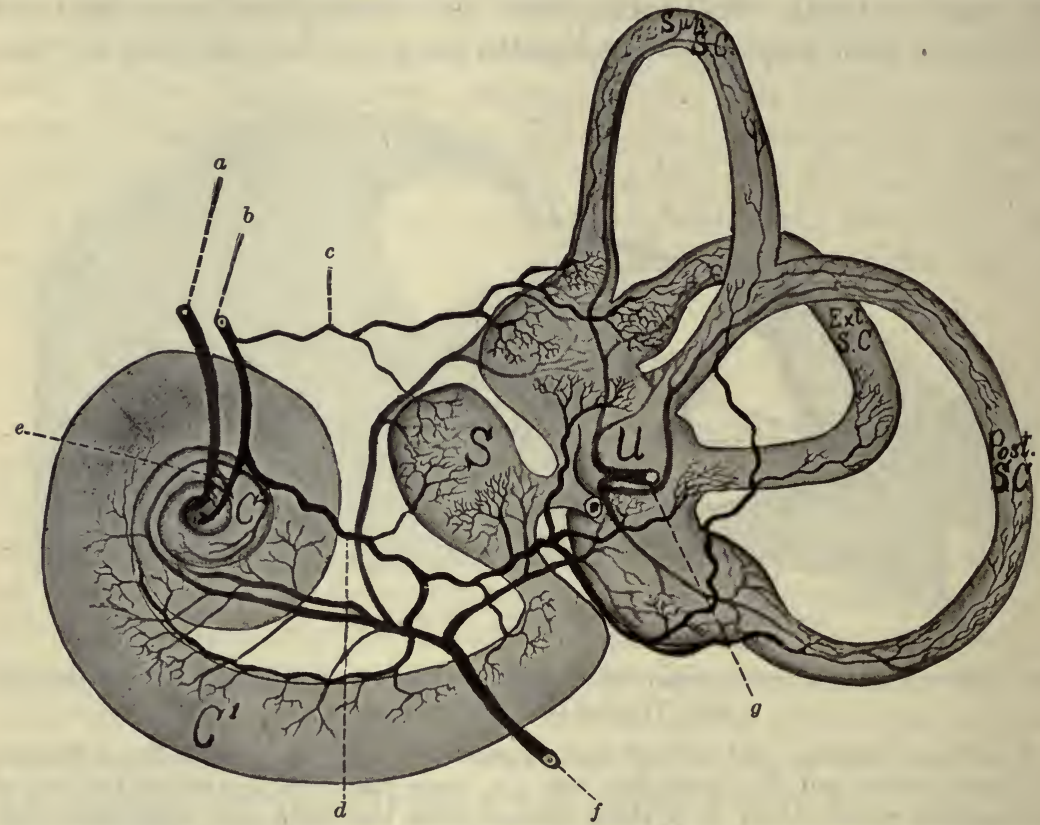

Fig. 590.-Scheme of the Vascular Supply of the Internal Ear.

$C^{1}$, first turn of the cochlea; S, saccule; Sup.S.C., Ext.S.C., and Post.S.C., superior, external, and posterior semicircular canals; $U$, utricle. The arteries are in heavy black, the veins somewhat lighter; $a$, central vein, and $b$, central artery of the cochlea; $c$, vestibular artery; $d$, vestibulocochlear artery; $e$, arteria propriæ cochleæ; $f$, vena aqueductus cochleæ; $g$, vena aqueductus vestibuli.

mann). The vestibular artery accompanies the branches of the vestibular nerve to the saccule, utricle, and semicircular canals, supplying these structures in the posterior portion of the vestibule, and forming a rich plexus in the connective tissue of the maculæ and cristæ, and a more scanty network in the remaining portions of the membranous labyrinth.

The cochlear division of the internal auditory artery, according to Siebenmann, promptly subdivides into the cochlear artery proper, which appears as the continuation of the vessel, and the vestibulocochlear 
artery, which supplies the macula sacculi, the posterior ampulla, and the adjacent portions of the utricle and posterior semicircular canal. This vessel also supplies the early portion of the first turn of the spiral cochlea.

The true cochlear artery enters the modiolus and supplies a branch to the remaining portion of the first cochlear turn, and a terminal branch which passes as far as the apex of the cochlea, distributing its

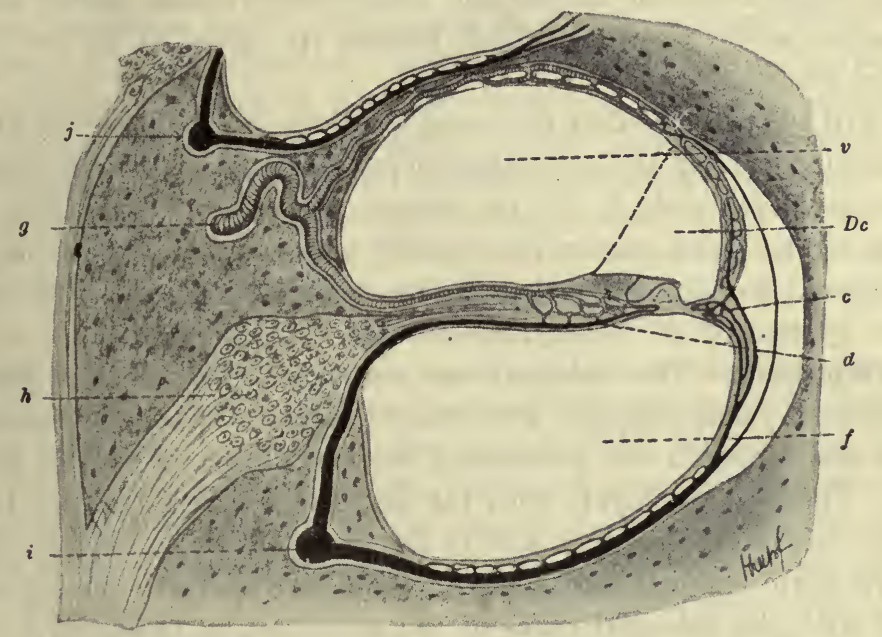

Fig. 591.-Scheme of the Vascular Terminations in the Wall of the Cochlear Canals.

$c$, capillary vessels in the spiral ligament; $D c$, cochlear duct or scala media; $d$, capillaries in the limbus spiralis; $f$, scala tympani; $g$, arteriole; $h$, spiral ganglion; $i$, vena spiralis inferior; $v$, scala vestibuli; $j$, vena spiralis superior. (After Böhm and von Davidoff.)

branches to the last two turns. All of these vessels are characterized by their peculiarly tortuous course. They distribute terminal branches to the limbus spiralis and to the connective tissue of the membranous scala vestibuli, extending as far around this canal as the spiral ligament. No vessels cross in the basilar membrane.

The veins collect the blood from the limbus spiralis and the wall of the scala tympani and form venous trunks within the modiolus, which correspond more or less closely with the arteries. Two of the cochlear radicals of the venous tributaries are important by reason of their position and relative size: the vas prominens of the prominentia spiralis of the stria vascularis, and the vas spiralis beneath the organ of Corti. 
Those veins coming from the wall of the scala tympani unite to form superior and inferior spiral veins in the inner wall of the scala tympani. These vessels chiefly empty into the vena aqueductus cochlea which finds its way through the aqueduct to the internal jugular vein. Other branches from the interior of the cochlea unite to form the central vein of the cochlea, which becomes the chief radical of the internal auditory vein, and thus enters either the transverse or inferior petrosal sinus.

The veins from the utricle and semicircular canals mostly enter the vena aqueductus vestibuli, which follows its aqueduct to the superior petrosal sinus.

It will be perceived that the blood has three chief avenues of exit from the labyrinth: 1 , by the vena aqueductus vestibuli; 2 , by the vena aqueductus cochleæ; and, 3, by the internal auditory vein. The greater portion of the blood pursues the second course and thus finds its way to the internal jugular vein, the smaller remainder entering the petrosal sinuses by one of the other two avenues.

Lymphatics.-The internal ear contains relatively few lymphatic vessels but is richly supplied with broad lymphatic spaces. Anastomosing vessels are found in the periosteum and membranous wall of the labyrinth. These communicate with the perilymph spaces between the periosteum and the membranous wall in the vestibule, and with the vestibular and tympanic scalæ in the cochlea. The perilymphatic spaces are connected with the subdural space of the meninges by means of lymphatic channels in the aqueductus cochleæ. The perilymph of the vestibule also communicates with the subdural space through vessels which follow the sheaths of the nerves.

The endolymph cavities of the several divisions of the membranous labyrinth communicate freely with one another; by means of the ductus endolymphaticus a connection is also established though the aqueductus vestibuli with the subdural space, the blind terminal saccule of this canal, the saccus endolymphaticus lying upon the posterior surface of the petrous bone and in contact with the dura mater.

\section{FUNCTION OF THE COCHLEA}

The cochlea is the essential organ of hearing. The fundamental structure concerned in audition is the spiral organ of Corti. The physiology of sound perception involves proximally the stimulation of the hair cells of Corti's organ by the tectorial membrane. This membrane is thrown 
into synchronous vibrations by the undulations in the endolymph of the cochlear duct transmitted through the vestibular membrane from the perilymph. The latter receives the sound waves through the foot-plate of the stapes.

The Helmholtz (1896) theory of tone perception, until recently widely accepted, postulated sympathetic vibrations, in resonance with atmospheric waves, on the part of the fibers of the basilar membrane. This view has been shown to be untenable, notably by Shambaugh (Archives of Otology, 37, 6, 1908) and by Hardesty (Amer. Jour. Anat., 8, 2, 1908). The basilar membrane contains only a little more than half as many fibers as the maximum number of vibrations (40,000 double vibrations per second) commonly audible; moreover, it does not possess the physical and histologic properties demanded by the 'resonance theory' of tone perception. Hardesty has shown that the tectorial membrane on the contrary does answer the requirements of an alternative theory, a modification of the earlier 'telephone theory' of Rutherford (1886); and he has succeeded in constructing an apparatus which simulates the cochlea, and imitates its presumed functional activity at least in the lower ranges of the tone scale.

Hardesty suggests 'that notes up to a certain pitch throw the entire natural tectorial membrane into vibrations of corresponding frequencies and that sensations of pitch are determined by the frequency of impingement of the membrane upon the auditory hairs, intensity being determined by the amplitude and quality by the quality of the wave motion imparted. Further, that the highest notes within the range of the auditory apparatus throw, according to their frequency, only varying extents of the smaller, basal end of the tectorial membrane into vibration, being so damped out in passing toward the apex of the cochlea, overcoming friction, the inertia of the endolymph and that of the membrane itself, as not to produce vibrations in the heavier, apical portions.' (Anat. Rec., 8, 2, 1914).

In essence, the tectorial membrane is conceived to respond in its several parts in the manner of a physical resonator to tones of different pitch (Shambaugh). According to this conception, tone analysis is accomplished peripherally, the specific stimulus being carried to the brain by the special sets of cochlear nerve fibers.

\section{DEVELOPMENT OF THE EAR}

The external ear develops in connection with the first branchial furrow: the meatus from the deepened groove, the auricle through the fusion of definite tubercles on the adjacent branchial arches.

The middle ear and auditory tube arise from the corresponding pharyngeal pouch, the process involving, a ventral elongation of the groove to form 


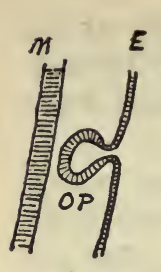

$\boldsymbol{A}$

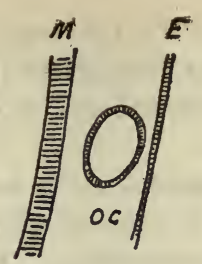

$B$
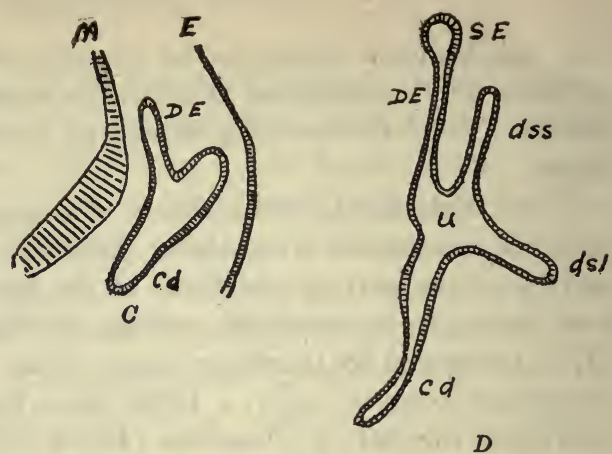

Fig. 592.-Semidiagrammatic Illugtrations of Successive Stages in the Development of the Internal Ear of the Chick.

$A, 45$ hour embryo; $B, 60$ hour embryo; $C, 5$ day embryo; $D, 7$ day embryo; $M$, wall of neural canal at level of metencephalon; $E$, epidermal ectoderm; $o p$, otic pit; $o c$, otocyst (otic vesicle); $D E$, ductus endolymphaticus; $S E$, saccus endolymphaticus; $c d$, cochlear duct; $u$, utriculus; $d s s$, superior semicircular duct; $d s l$, lateral semicircular duct.

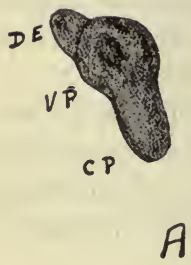

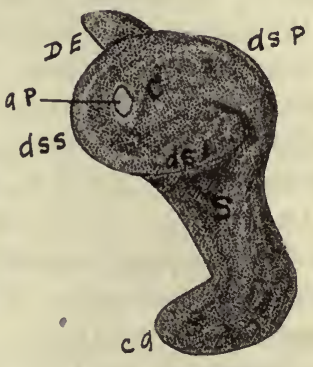

B

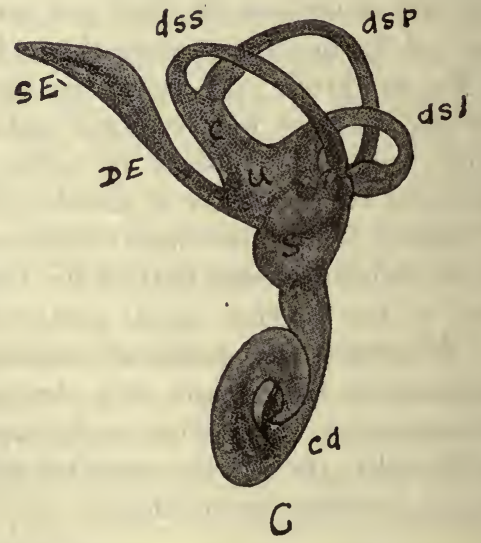

Fig. 593.-Wáx Reconstructions of Three Early Stages in the Development of the Internal Ear (Membranous Labyrinth) of Man.

$A$, lateral view, from a $6.6 \mathrm{~mm}$. embryo; $B$, lateral view from an $11 \mathrm{~mm}$. embryo; $C$, front view from a $20 \mathrm{~mm}$. embryo ( $A$ and $B$ correspond approximately to stages $C$ and $D$ of the preceding figure). $D E$, ductus endolymphaticus; $S E$, saccus endolymphaticus; $V P$, vestibular pouch; $c p$, cochlear pouch; $a p$, absorption focus; $c$, crus; $s$, sacculus; $u$, utriculus; $c d$, cochlear duct; $d s s, d s p$, and $d s l$, superior, posterior, and lateral semicircular ducts. (After Streeter.) 
the tube and a subsequent dorsal dilatation, expanding as the tympanum to include the auditory ossicles which have meanwhile taken form in the adjacent mesenchyma. The mastoid cells are formed by a late erosion and invasion of the bone by the mucous membrane of the tympanum.

The internal ear develops from a thickening in the epidermal ectoderm at the level of the third primary cerebral vesicle. This auditory anlage becomes invaginated to form an otic pit, the aperture of which subsequently closes and thus separates an auditory vesicle or otocyst from the overlying parent ectoderm. At the point of closure medially a dorsal evagination arises to form the endolymphatic duct. At the upper pole of the vesicle appear the semicircular ducts through a process involving the elevation of three circular folds, the lateral walls of which fuse proximally, and subsequently disappear leaving a peripheral duct dilated at one end to form an ampulla. The cochlea arises at the opposite pole as a tubular evagination which becomes spirally disposed. The original vesicle persists as the utricle; on its anteromedial border is formed an alveolar evagination, the saccule, which remains connected by a constricted duct, the utriculosaccular canal, to which is attached the ductus endolymphaticus.

Each ampulla differentiates an elongated patch of neuro-epithelium, the crista; in both utricle and saccule a similar oval patch appears, the macula; the spiral organ of the cochlea develops in like manner through a specialization of the ectoderm along the floor of the membranous duct.

The bony labyrinth develops from the mesenchyma originally enveloping the membranous labyrinth. The mesenchyma immediately surrounding the membranous labyrinth becomes converted into a mucoid tissue which eventually disappears, leaving the perilymphatic spaces; these are bounded by periosteum, the innermost layer of which becomes modified into a mesenchymal epithelium.

The scala tympani and scala vestibuli of the cochlea are formed by the coalescence and subsequent dilatation of small mesenchymal tissue spaces in two distinct regions: one between the saccule and the oval window, the other between the saccule and the round window. From these two areas the two great scalæ of the cochlea proceed in a definite and constant direction to their definite position and condition (Streeter, Proc. Amer. Assoc. Anat., 1916). 


\section{CHAPTER XX}

\section{HISTOLOGIC TECHNIC}

The satisfactory examination of the tissues with the aid of the modern microscope requires certain preparatory steps which are in certain cases very simple, in others very complicated. The present chapter deals briefly with the more important and simpler methods, and the general principles upon which they are based.

\section{THE EXAMINATION OF FRESH TISSUES}

Certain tissues may be examined immediately after they have been removed from the body. This method is applicable to blood, lymph, scrapings from the spleen, liver, uterus, and similar organs, small fragments of muscle, connective tissue, etc.

A small drop of blood may be collected upon the under surface of a cover glass, which is then quickly dropped upon a glass slide and examined at once. The glass must be thoroughly cleaned, otherwise a thin preparation can not be obtained. Slides and covers should be washed in very dilute hydrochloric acid (about 10 per cent.), then washed in running water for several hours, and finally rinsed in 95 per cent. alcohol. Ordinarily slides and cover glasses can be sufficiently cleaned by simply dipping in alcohol and drying with a linen cloth.

Scrapings from the epithelium of the mouth, or from similar mucous membranes, may be prepared in the same manner as blood, and examined while still suspended in their own fluids. Most tissues, however, are not sufficiently well moistened for examination after this manner; the preparation must then be diluted with some inert fluid. Normal saline solution may be used for this purpose; the following formula is recommended :

Sodium chlorid ..............75 to $0.9 \mathrm{grm}$.

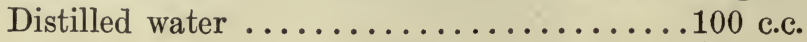


Some histologists regard a 0.6 per cent. solution preferable. Normal salt solution has become quite generally displaced by Ringer's -Solution, which corresponds more nearly to blood plasma and is less likely to produce distortion. This is prepared, adjusted to tissues of warmblooded animals, according to the following formula:

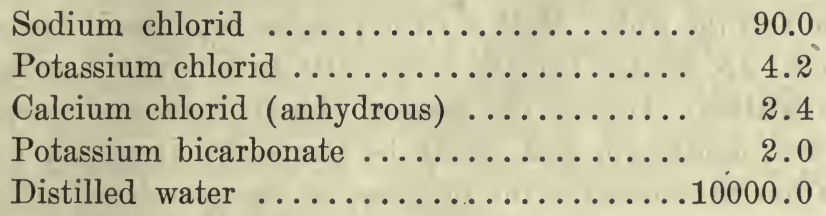

In order to render the nuclei more conspicuous a drop of a 5 per cent. aqueous solution of acetic acid may be added. This, however, will dissolve the collagenous fibers and albuminous granules. The nuclei may be stained by the addition of a drop of a 1 per cent. aqueous solution of methylene blue.

A 40 per cent. solution of glycerin in distilled water also is useful as an examining medium; better still, the tissues may be suspended in a mixture of equal parts of 95 per cent. alcohol, glycerin, and distilled water. This mixture is especially useful, for in it tissues may be kept for a long period without deterioration.

Amniotic fluid obtained from pig fetuses is also valuable as a medium for the examination of fresh tissues.

Hogan (Jour. Amer. Med. Assoc., 64, 9, 1915) has devised a normalsalt-gelatin mixture with a colloidal constitution like that of blood serum, which serves as a favorable indifferent medium for the examination of fresh blood and other delicate tissues. For the method of preparation reference should be made to the original article.

\section{THE DISSOCIATION OF TISSUES}

It is frequently desirable to dissociate tissues to a certain extent into their component elements prior to microscopical examination. This is accomplished by teasing, or by the solvent action of relatively strong acids or alkalies. Isolation by the latter method is known as maceration.

For teasing, minute fragments of tissue are separated by the aid of needles or scissors and placed on a clean slide, where they are to be kept always moistened with normal. saline solution or other isotonic 
fluid. For their further manipulation a dissecting microscope is useful, though not essential. Two sharply pointed needles, mounted in wooden handles, are to be used.

The fragment of tissue is pinioned with the needle held in the left hand, and with that in the right the tissue is gently torn by a rhythmic combing motion, being very careful to avoid squeezing the tissue between the needle and the slide. With a little practice bundles of fibers, groups of cells, etc., are readily isolated sufficiently to be studied under moderate magnification. During the teasing, the fragments of tissue should be kept well moistened, and are to be frequently inspected under low magnification to determine the progress of the operation. When satisfactorily prepared, a cover glass may be applied, and the preparation examined under higher magnification.

In applying a cover glass care should be taken to permit one edge of the cover to first touch the slide while being held at an angle of 30 degrees to 40 degrees. If the cover is then gently lowered into place, the air is forced out before the advance of the fluid, and the many air bubbles which would otherwise be included are not found in the preparation.

The method of teasing is particularly applicable to the study of the connective, and peripheral nervous tissues. Collagenous fibers, elastic fibers, fat cells, and nerve fibers are readily isolated in this way. If desired, they may be stained by the addition of a drop of a solution of methyl green, picrocarmin, etc.

Chemical Dissociation:-It is necessary to dissociate many tissues by chemical means, either because of the firm union of the elements composing the tissue or because they may be too delicate and fragile to withstand the mechanical teasing. Epithelial cells, nerve cells, and muscle fibers are readily macerated in this way.

For the dissociation of muscle fibers small cubes ( 0.25 to 0.5 c.c.) are placed for ten to thirty minutes in the following solution:

Strong nitric acid ...................

Potassium chlorate, sufficient to saturate.

The fragments of tissue should be handled with glass rods. After some minutes they begin to disintegrate at the surface, and should then be transferred to running water, where they are left to wash from three to twelve hours. The pieces of tissue are then transferred to a mixture of equal parts of alcohol, glycerin, and water, and thoroughly shaken. 
Muscle fibers isolated in this way may be kept for months or even years.

Caustic potash (potassium hydroxid) is also a valuable dissociator for muscle tissue. The solution commonly used is made by dissolving 40 grams of the potash in 60 c.c. of water. Cardiac muscle is sufficiently macerated in twenty minutes. The tissue must then be thoroughly washed, and may be preserved in the alcohol-glycerin mixture.

Epithelium may be dissociated by teasing or by the action of a 40 per cent. solution of potassium hydroxid, or by means of a 10 or 20 per cent. aqueous solution of 'Tysol,' and preserved, if desired, in the mixture of alcohol, glycerin, and water.

Nerve cells may be isolated from the anterior horns of the spinal cord or other gray matter of the central nervous system by teasing. They may also be isolated by immersion in a 0.2 per cent. aqueous solution of formalin in normal salt solution for two to twenty-four hours, or in a 0.2 per cent. aqueous solution of potassium bicarbonate, two to five days. Afterward they are transferred to a normal saline solution or to a mixture of alcohol, glycerin, and water, and isolated. by shaking, assisted, if necessary, by gentle teasing.

Similar preparations may also be made by placing. small fragments of tissue in 30 per cent. alcohol (Ranvier's alcohol; 2 parts water, 1 part 95 per cent. alcohol) for two days or more; then shake thoroughly, allow the débris to settle, remove a drop of the fluid with a pipette, and examine.

For the isolation of the elements of the epidermis, hair and nails, stronger solutions are required. Concentrated sulphuric acid may be employed. After maceration is complete, the cells must be thoroughly washed in water and may then be preserved in the alcohol-glycerin mixture. A caustic potash solution, acting for two to three hours, is also serviceable with these tissues.

Any of the above preparations may be stained by the addition of a small drop of a solution of eosin, picrocarmin, or methyl green, to the fluid in which they are examined.

\section{FIXATION}

For the preservation of tissue, and as a preparation for further manipulation, most tissues require to be 'fixed.' Innumerable formulas have been advocated for this purpose, many of them having as their 
object the demonstration of certain structural features by the after application of special staining methods.

The action of the fixing fluids is in most cases dependent upon the combination of the reagent with the chemical elements of which the tissue consists; very elaborate compounds are thus formed.

The distinction between a physical and a chemical combination of a dye with particular tissue elements must be emphasized.

The following reagents are recommended for general use. The choice of a fixative is in great measure determined by the staining method which is to be afterward applied.

Alcohol.-This is especially useful for the fixation of the glandular organs. Tissue may be placed directly in 95 per cent., or in absolute alcohol. The fluid is to be changed in twenty-four hours, and again in five to seven days. This method of fixation is desirable for after-staining of the nervous tissues with methylene blue and for the demonstration of glycogen in the hepatic cells, cartilage, etc. Alcohol causes considerable distortion of the internal architecture of the cell by its rapid and forceful diffusion from the surface toward the center of the tissue, the cytoplasmic granules often being in this way forced to one side of the cell. This result may be partially avoided by the use of 'graded alcohol,' viz., 67 per cent. alcohol for three to twelve hours, 82 per cent. for twenty-four hours, and finally 95 per cent. alcohol, which should be once changed after a few days. Glycogen, however, is partially dissolved by the action of the dilute alcohols.

The graded alcohols may be derived from the 95 per cent. stock supply by the following dilutions:

95 per cent. alcohol, 1 part; distilled water, 1 part $=48$ per cent.

95 per cent. alcohol, 3 parts; distilled water, 1 part $=67$ per cent.

95 per cent. alcohol, 5 parts; distilled water, 1 part $=82$ per cent.

For practical purposes these grades may be regarded as 50, 70 and 80 per cent. respectively; they may be derived also by the use of the following formula:

$$
\begin{aligned}
& 265 \text { c.c. } 95 \% \text { alc. }+235 \text { c.c. aq. dist. }=50 \% \text { alc. } \\
& 370 \text { c.c. } 95 \% \text { alc. }+130 \text { c.c. aq. dist. }=70 \% \text { alc. } \\
& 425 \text { c.c. } 95 \% \text { alc. }+75 \text { c.c. aq. dist. }=80 \% \text { alc. }
\end{aligned}
$$

The distortion from the use of strong alcohol, as well as the dissociation which follows the use of the weaker strengths, may also be par- 
tially avoided by the addition of a little iodin to the stronger alcohol, or by combination with acetic acid, thus:

Glacial acetic acid ............. 5 c.c.

95 per cent. alcohol ...........6.6 60 c.c.

Distilled water ................... 35 c.c.

After fixation for three to twenty-four hours the tissues are washed, and hardened by immersion for twenty-four hours in each strength of graded alcohol (50, 70, 80 and 95 per cent.), and may be kept indefinitely in 80 per cent. alcohol. Tissues should always be stored in 80 per cent. alcohol. Lower grades tend to macerate, higher grades to harden unduly.

Carnoy recommends also a mixture of glacial acetic acid and absolute alcohol in the proportion of 1 to 3 ; this gives excellent results with muscular tissues.

Tissues for fixation by these or any subsequent method are preferably cut into small cubes; a size not exceeding 0.5 to $1 \mathrm{~cm}$. is most desirable. If larger pieces of tissue are necessarily used, the reagents will each require increased time to insure complete penetration.

Mercuric Chlorid (Corrosive Sublimate).-This salt is to be used in saturated aqueous solution. As it dissolves with difficulty in cold water, the use of a hot, normal saline solution hastens the operation.

Mercuric chlorid is an excellent fixative for cytoplasm, but gives still better results when combined with a nuclear fixative such as acetic acid. The following is an excellent method for general use:

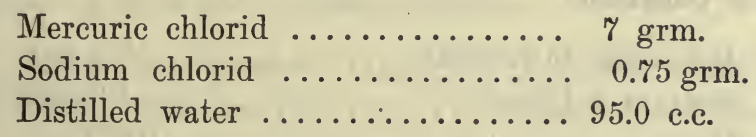

Just prior to use add 5 c.c. of acetic acid.

Small pieces of tissue remain in this corrosive acetic mixture for two to twenty-four hours, and are then transferred to graded alcohol, beginning with 70 per cent. and remaining twenty-four hours in each strength. Most dyes will act perfectly on tissue fixed in this way. If, however, the presence of mercury interferes with the action of a dye, this salt can be readily removed by the addition of a few crystals of iodin to the higher grades of the graded alcohols, renewing the iodin if necessary until it is no longer decolorized.

Formalin.-Tissues may be fixed in formalin (formol), a 40 per 
cent. solution of formaldehyd gas, which is to be used in strengths varying from 5 to 20 per cent. Small pieces should be left in the weaker solutions (5 to 10 per cent.) from six hours to two days, not longer. In the stronger solutions (10 to 20 per cent.) tissues should remain for only two to six hours. More prolonged immersion in the fixative causes considerable swelling. As a rule, the stronger solutions are preferable; this is especially true for the fixation of the cells of lymphoid tissue. The 10 per cent. formalin solution is valuable for the fixation of human embryos. For the hardening of nervous tissues, also, the 10 per cent. solution is very serviceable.

After fixation, the tissues are transferred directly to 80 per cent. alcohol, with one or several changes. This method gives excellent results with lymphoid and epithelial tissues, but does not bring out the finer details of cytoplasmic structure.

Potassium Bichromate (Dichromate).-This salt has been used in all sorts of combinations; those which follow may be specially recommended. Müller's solution is employed for the fixation of the tissues of the central nervous system, and must be used when fixation is to be followed by any of the Weigert hematoxylin staining methods. Applied to the fixation of other organs, Müller's fluid is apt to produce some maceration and better results are usually obtained with Müller-formol or with Tellyesniczky's solution.

For the special fixation of cytoplasmic granules, and also for afterstaining with Mallory's connective tissue stains, Zenker's solution yields better results.

\section{Müller's Solution:}

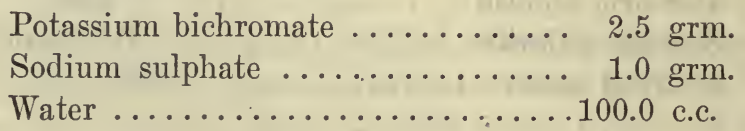

Pieces of tissue are left in the fluid for one to six weeks; large pieces of the spinal cord or brain require four to six weeks. If left too long the tissues will become brittle. After fixation, the tissue is washed thoroughly in running water for twelve to twenty-four hours, and hardened in graded alcohol.

A relatively large volume of the fixing fluid should be used, and it should be frequently changed. It should not be allowed to become turbid nor to deposit crystals: this is avoided by frequent changes and by keeping the jars in the dark, or at least in such a position that they are not exposed to a bright light. 


\section{Tellyesniczky's Fluid:}

Potassium bichromate 3 grm.

Water ........................... 100 c.c.

Glacial acetic acid (added just before use) .... 5 c.c.

Pieces of tissue are placed in a considerable volume of the fixing fluid and left for three to seven days. They are then washed in running water for twelve.to twenty-four hours, and hardened in graded alcohol. (Where tissues are first washed in water, the graded alcohols must begin with the 50 per cent. strength.) This fluid yields excellent results with muscular and glandular tissues, and is particularly serviceable where pieces of considerable size must be used, e.g., whole embryos.

Orth's Fluid (Müller-formol) :

Müller's fluid .................. 95 to 90 c.c.

Pure formalin ............. 5 to 10 c.c.

This is an excellent fixative for general use, for by it most tissues are well preserved. Small pieces of tissue are left in a considerable volume of the fluid for one to five days, washed thoroughly in running water for twelve to twenty-four hours, and hardened in graded alcohol. The washing should be so thorough as to remove all excess of the chromium compounds, otherwise difficulty will be experienced in obtaining satisfactorily stained preparations.

Chrom-Acetic-Formalin Mixture.-This makes an excellent fixing fluid for general embryologic work.

Mix and keep in stock the following solution:

1 per cent. chromic acid ..........16 volumes

Glacial acetic acid .............. 1 volume

At the time of using add to 2 volumes of the stock mixture, 1 volume of formalin.

Wash in water from twenty minutes to two hours according to size.

\section{Zenker's Solution:}

Potassium bichromate ............ $2.5 \mathrm{grm}$.

Sodium sulphate $\ldots \ldots \ldots \ldots \ldots \ldots \ldots \ldots \ldots \quad 1.0 \mathrm{grm}$.

Mercuric chlorid .............. 5.0 grm.

Distilled water ................. 100.0 c.c.

Just prior to use add 1 c.c. of glacial acetic acid to each 20 c.c. of the fluid. 
Small pieces of tissue, only, should be used. They remain in a considerable volume of the solution for three to twenty-four hours, after which they are thoroughly washed in running water for twelve to twenty-four hours, and hardened in graded alcohol. The corrosive sublimate deposits crystals in the tissue. These may be removed by the addition of a crystal of iodin (or tincture of iodin) to the stronger alcohols until decolorization no longer occurs. If the mercury is not thus removed it will be difficult to obtain well stained specimens, and the presence of the crystals may cause much confusion. In obstinate cases Lugol's solution is recommended in place of tincture of iodin (alcoholic solution of iodin), to be added until the alcohol has a 'port wine' color. (See page 758.)

This is probably the best fixing fluid for general routine histologic work. The presence of the acetic acid, however, dissolves the more delicate albuminous granules. To avoid this, formalin may be substituted in the same proportion, and added in the same way, for the acetic acid. This modified Zenker's fluid (HELLY's FLUID) is particularly valuable for the preservation of tissues where it is desired to investigate the granular cytoplasmic contents, e.g., blood cells in embryos, etc.

Flemming's Fluid (strong solution) :

Chromic acid, 1 per cent. aqueous solution.... 15 parts Osmic acid, 2 per cent. aqueous solution...... 4 parts Glacial acetic acid ............... 1 part

The mixture should be newly made, from stock solutions of the ingredients, immediately before using. The stock mixture undergoes a deteriorating chemical transformation.

Pieces of tissue should not be more than 2 to $3 \mathrm{~mm}$. in thickness and should be left in the solution and kept in the dark for one to twentyfour hours, according to the results desired. For mere fixation a short immersion is sufficient; for blackening fat and the myelin of medullated nerve fibers the longer period is necessary. After fixation the tissues are to be washed in running water for three to twenty-four hours, and hardened in graded alcohol.

This fluid gives splendid results for the fixation of the finer cytological elements of glandular epithelium; and for the demonstration of nuclear constitution, chromosomes, and mitotic figures, it is unexcelled. It serves also to demonstrate the presence of fat and myelin, which are blackened by the osmium tetroxid. When used for the demonstration of fat in sections, chloroform should be substituted for 
xylol in the embedding process. It does not penetrate the tissues very readily, and the surfaces of the piece are usually destroyed by overfixation.

\section{Kleinenberg's Fluid:}

Saturated aqueous solution of picric acid..... 99 c.c. Sulphuric acid $\ldots \ldots \ldots \ldots \ldots \ldots \ldots \ldots \ldots \ldots$ c.c.

Shake well, filter and dilute the filtrate with 200 c.c. of distilled water.

Small pieces of tissue should be left in the fluid for about three hours and then transferred to 70 per cent. alcohol, which is changed two or three times during the first day. Hardening is continued in 80 and 95 per cent. alcohols, which are to be frequently changed. The picric acid will be slowly dissolved by the alcohol, but will not be entirely removed even after a considerable time; a trace does no harm.

This fluid gives excellent results with small pieces of embryonic tissue, and possesses the additional advantage of removing the calcareous salts from partially calcified bone; it is not, however, a strong decalcifying reagent.

When a fixative is employed which contains picric acid, the tissue must never be washed in water, but is transferred directly to $\% 0$ per cent. alcohol.

\section{Bouin's Fluid:}

Picric acid, saturated aqueous solution........75 c.c.

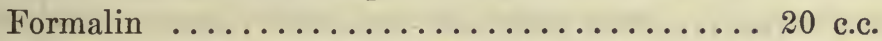

Glacial acetic acid............... 5 c.c.

Small pieces of tissue are fixed from three to twelve hours, transferred to 70 per cent. alcohol, and then passed through the higher grades. This is an excellent fluid for the fixation of embryonic material. For chromosome studies it is probably surpassed only by Flemming's fluid.

Van Gehuchten's Fluid (Carnoy's fluid, No. I) :

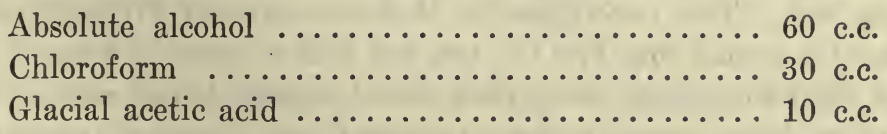

Very small pieces of tissue should be used. They should be left in the fluid three to twelve hours, and the vessel tightly closed to prevent evaporation of the volatile fluid. The tissues are then transferred to 
several changes of absolute alcohol to remove the fixing fluid and complete the dehydration.

This fluid is especially valuable for the fixation of lung tissue. It can be advantageously applied only to perfectly fresh, viz., living tissues.

Gilson discovered that this fluid could be advantageously modified for cytologic purposes by saturation with mercury bichlorid. About 20 grams should be added to the above mixture. As thus modified the solution is commonly known as Carnoy's fluid, No. II. Its penetrating properties are very high and the degree of fixation delicate. Tissues should not be left in the fluid for more than an hour. The aftertreatment is the same as with the simple solution, except that tincture of iodin must be used with the alcohols.

Gilson's Fluid (Mercuro-nitric mixture):

Bichlorid of mercury ............. 5 grm.

Nitric acid (approximately 80 per cent.) ..... 4 c.c.

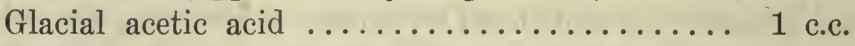

Alcohol (70 per cent.).............25 c.c.

Distilled water ......................

Fix small pieces of tissue from three to twelve hours, transfer directly to 70 per cent. alcohol, and pass through the higher grades, with the addition of tincture of iodin. This fluid has a high degree of penetration, and produces faithful and delicate fixation.

Heat.-This is a useful agent for the fixation of blood, marrow cells, and scrapings from glandular and other organs, which are not to be afterward stained with methylene blue or its compounds. For this purpose smears made upon glass slides or cover glasses are quickly dried in the air, heated to 110 degrees C. for twenty to thirty minutes, and are then ready for immediate staining.

The smears are made in the following manner: Slides or cover glasses should be thoroughly cleaned with a final rinsing in equal parts of absolute alcohol and ether. A small drop of blood or other fluid is collected by quickly touching the center of a cover glass to a drop of ordinary size. This cover glass is then immediately dropped upon the surface of a second one, and the two are drawn apart by a rapid sliding motion, the two surfaces being maintained parallel to one another during the motion. The success of the maneuver depends upon its rapidity, and to obtain very thin preparations some little dexterity is required.

Fairly good smears are more easily made with slides. A drop of blood is collected upon the end of one glass slide whose edge must have been 
ground. The end of the slide with the drop of blood is then touched to the middle of a second slide, the drop spreads out between the two, and the first slide is rapidly drawn over the surface of the second, while being held at an angle of about 45 degrees. A broad smear is thus left upon the surface of the second slide, some portions of which are sufficiently thin, other portions too thick for use. Like the former maneuver the success of this depends upon rapidity, cleanliness, and the use of a sufficiently small drop of fluid.

Fixation by Vapors.-Smears of fluids or very thin pieces of tissue may be fixed by a very brief exposure to the vapor of osmium tetroxid, formalin, etc. This method is only useful in occasional instances. Osmium tetroxid vapor has been shown to preserve faithfully the mitochondria in young cells from tissue cultures (M. R. and W. H. Lewis, Amer. Jour. Anat., 17, 3, 1915).

In all methods of fixation where pieces of tissue are immersed in the fixing and hardening fluids, it is desirable to prevent the distortion of the object from the pressure of contact with the glass container. This is accomplished by suspending the object by means of a thread, or by resting the tissue upon a thin layer of cotton placed in the bottom of the jar.

\section{DECALCIFICATION}

Tissues containing bone or other calcareous material require decalcification before they can be sectioned for examination. If the calcareous deposit is limited in amount, as in early fetal tissues, this can be accomplished and the tissue fixed at the same time by the use of Kleinenberg's fluid, a saturated aqueous solution of picric acid, or a 5 to 10 per cent. aqueous solution of sulphurous acid, the tissues being permitted to remain in the decalcifying fluid until a needle or slender scalpel can be readily pushed to the most central portions without producing great resistance or any grating sensation.

For well developed and mature bones the above methods are insufficient, and stronger acids must be relied upon. Nitric acid is the one most generally used for this purpose. The tissue should have been previously fixed, Müller-formol, Zenker's fluid, or mercuric chlorid being the preferable fixatives. The fixed and washed tissues are placed in 2 to 5 per cent. nitric acid, and the fluid changed daily until decalcification is complete. They are then thoroughly washed in running water for twelve to twenty-four hours and hardened in graded alcohol. 


\section{INJECTION}

Injection is used either for the rapid dissemination of fixing fluids through whole organs, embryos, etc., or for the demonstration of blood or lymphatic vessels. For the former purpose mercuric chlorid is the most useful fixative, since it may be immediately followed by the injection of a hardening fluid, alcohol, by which the remaining mercury is dissolved out of the tissue before overfixation occurs. For the latter purpose a colored fluid, either aqueous or gelatinous, is forced into the blood or lymphatic vessels. Berlin blue, carmin, vermilion, and lampblack are the coloring matters most frequently used. The last two merely require suspension in a gelatinous or an aqueous menstruum; the preparation of Berlin blue and carmin is somewhat more complicated.

\section{Berlin Blue Gelatin Mass:}

Saturated aqueous solution ( 1 to 20) of

Berlin blue (Grübler's)........... 100 c.c.

Pure French gelatin (in sheets)...... 5 to $10 \mathrm{grm}$.

The gelatin should be quickly washed to remove dust, etc., and then placed for several hours in a very little distilled water until it becomes swollen and soft. The superfluous water is then poured off, and the gelatin melted over a water bath. The warmed solution of Berlin blue may now be added, a little at a time, and continuously stirred. Finally, the mixture is filtered through cotton flannel (or flannel) which has been previously wrung out of hot water. If the mass is not to be used at once, a few crystals of thymol may be added as a preservative, or, after cooling, a little methylic alcohol may be floated upon the surface of the solidified mass. It is better to use it at once.

\section{Carmin Gelatin Mass:}

Carmin (Grübler's) ........................... 3 grm.

Ammonium hydrate, strong...........6. 6 c.c.

Pure French gelatin.............. 7 grm.

Distilled water....................... 80 c..

The gelatin is prepared and melted as above, 50 c.c. of the water being used, and the evaporation replaced. The carmin is rubbed up in a mortar with the remaining 30 c.c. of the water, and the ammonia is added to render the carmin soluble. The mixture is now permitted to 
stand for two hours, after which it is neutralized by the gradual addition of 4 to 6 c.c. of glacial acetic acid, the mixture being constantly stirred, and the latter portions of the acid diluted with four volumes of distilled water, and added drop by drop. The acid soon changes the color of the mixture from a purplish carmin to a bright crimson. Care should be taken not to add too much acid. When properly prepared, the sense of smell should detect both ammonia and acetic acid, and the fluid should have a dark crimson color (the addition of too much acid produces a brighter crimson). Should the mixture be slightly overacidified a few drops of diluted ammonia will restore the proper condition. The carmin solution is now added to the gelatin mass, a little at a time and with constant stirring, and the whole is filtered through cotton flannel wrung out of hot water.

The gelatin mass may be kept for a short time by being covered with methylic alcohol, but is better used at once.

The pressure required for injection may be obtained by the gentle use of a hand syringe; by the displacement of the confined air in a large bottle or carboy by tap water; or much better by the use of a water blast, of which the small glass type is relatively inexpensive and will furnish a pressure for injection about equal to $180 \mathrm{~mm}$. of mercury. The air outflow of the water blast is connected by rubber tubing with a glass canula of proper size to fit the vessel injected, a Wolff bottle containing the warm injection mass being interposed. If a manometer is connected, by means of a $\mathbf{T}$-canula, on the proximal side of the Wolff bottle, a relatively even and accurately measured pressure is assured. The amount of pressure should be at first low (20 to 40 $\mathrm{mm}$. of mercury), and should be gradually increased up to, but not much beyond, the normal blood pressure in the vessel injected.

The injected organ is cooled rapidly in a refrigerator, or by being packed in ice or immersed in ice water. After solidification small pieces are immediately placed in 95 per cent. alcohol for fixation, dehydration, and hardening.

\section{HARDENING}

After proper fixation nearly all tissues require to be further hardened before satisfactory sections can be cut. This is accomplished by immersion in alcohol until dehydration is complete. The process requires from a day to a week, according to the size of the tissue and the volume and strength of the fluid. Various strengths of alcohol are advised. For 
general use the procedure recommended by Gage. ("The Microscope") is found to be very satisfactory. The tissues after fixation are successively placed for one or two days in each of the following strengths of alcohol-70, 80 and 95 per cent. They are then returned to 80 per cent. alcohol, where they may remain indefinitely, but it is generally safer to embed for sectioning without great delay; this is especially true of tissue which has been fixed with Zenker's solution. Most fixing fluids also harden; the terms 'fixing' and 'hardening' are consequently often used synonymously.

\section{EMBEDDING}

Thick sections may be obtained from the firmer tissues by freehand sectioning with a razor, but for the satisfactory preparation of thin sections a microtome is a necessity and the tissues must have been previously embedded to render them sufficiently firm. This is accomplished by infiltrating the tissue with celloidin or paraffin, either of which yields a firm, waxy consistence.

Embedding in Celloidin.-Make a saturated solution of a little celloidin (Schering's) in a mixture of equal parts of alcohol and ether. The alcohol should contain no trace of copper sulphate. This solution is for convenience known as number III and should have a very thick, syrupy consistence.

A small portion of number III is mixed with three to five times its volume of the alcohol and ether mixture, to obtain number II, which should have a somewhat viscid consistence.

A second small portion of number III is diluted with ten to fifteen times its volume of the alcohol and ether, to produce celloidin number I, which should have a thin, watery consistence.

Small pieces of tissue which have been thoroughly hardened in 95 per cent. alcohol are treated as follows:

1. Dehydrate in absolute alcohol, six to twenty-four hours.

2. Place in the absolute alcohol and ether mixture, twelve to twentyfour hours.

3. Place in celloidin number $I$, twelve to twenty-four hours.

4. Place in celloidin number II, twelve to twenty-four hours.

5. Place in celloidin number III, twenty-four to forty-eight hours, or longer.

Pieces of tissue of considerable size may be satisfactorily embedded 
in celloidin, but should be passed through the suecessive solutions in a much more leisurely manner. Thus an eye requires two-to-three weeks, a large piece of the central nervous system three to four weeks for proper embedding. The tissue should now be fastened to a wooden block and the celloidin hardened. Ordinary wood yields its resins to the alcohol in which the blocks are to be kept; the white pine blocks which are commercially known as 'deck plugs' contain very little resin and are admirably adapted for the purpose. Vulcanized fiber blocks are still better, and glass blocks are also serviceable. The piece of tissue should be so oriented upon the block that the future sections may be cut nearly parallel to the block surface. Thick celloidin is then poured over the tissue; a few moments' exposure to the air cements it firmly to the block. After the celloidin has become firm by partial drying in air, the block may be stored indefinitely in 70 per cent. alcohol (stronger alcohol is apt to soften the celloidin), where the hardening is completed. If it is desired to harden the celloidin rapidly for early cutting, the block may be floated in a jar of chloroform, tissue down, for two or three hours.

The final step in the embedding process may be accomplished advantageously by allowing the tissue to remain in the slowly hardening thick celloidin in a shallow dish. Care must be taken to begin this step with sufficient celloidin to keep the tissue covered until the hardening is completed. The tissue may then be cut from the firm celloidin and blocked.

Embedding in Paraffin.-If sections thinner than $15 \mu$ are desired, paraffin embedding must be used; it is impossible to cut celloidin sections with any great degree of certainty thinner than $10 \mu$ to $15 \mu$. Paraffin sections are readily cut at 5 , and may be cut as thin as $2 \mu$. The paraffin method is also to be selected for the rapid preparation of tissues for sectioning, but it is only applicable to small pieces of tissue. For large pieces better results will be obtained with celloidin. Many methods for the employment of paraffin have been extolled; the following can be recommended. The tissue, after fixation, should have been hardened in alcohol.

1. Dehydrate in absolute alcohol twenty-four to forty-eight hours; very small pieces of tissue ( 1 to $2 \mathrm{~mm}$.) may be completely dehydrated in three to six hours.

2. Place in equal parts of absolute alcohol and xylol (xylene), one to three hours.

3. Place in pure xylol until clear and translucent, one-half to two 
hours. (For relatively large pieces of tissue cedar-wood oil or pure anilin oil may be substituted for the xylol).

4. Place in melted paraffin containing a little xylol (or cedar oil); that which has been previously used for embedding does very well.

5. Transfer to pure melted paraffin.

6. Transfer to a second dish of pure melted paraffin (with melting point of about $54^{\circ}$ ). The object of these changes is to replace the xylol (or oil) with pure paraffin. If the xylol is not completely removed the tissue will contain bubbles and satisfactory sections cannot be made.

7. Embed in a paper box or a watch glass. If glass is used the surface should be smeared with the least trace of glycerin to prevent adhesion. The box should be filled with pure melted paraffin, the tissue handled with warmed forceps, and placed with proper orientation so that it is completely covered with the melted paraffin. The paraffin is now rapidly cooled by immersion in cold water; in summer months ice water must be used. If a paper box is used it can be left to float on the water until the paraffin is thoroughly congealed. The manner of preparing these boxes is shown in Fig. 594.

Considerable depends upon the choice of a proper grade of paraffin. That which melts at $58^{\circ}$ to $65^{\circ}$ is most desirable for use in temperate climates during the warmer months; during the winter months paraffin of $54^{\circ}$ to $56^{\circ}$ is preferable. If too hard, the paraffin cracks; if too soft, it fails to retain its form during sectioning. The former condition may be improved, if necessary, by the proximity of a small flame during the sectioning process, or by breathing upon the knife blade and tissue block; the latter fault may be remedied by placing the tissue for a short time in the refrigerator, just prior to cutting.

\section{SECTIONING}

The cutting of free-hand sections is so simple an operation as to scarcely require description. A small, inexpensive hand microtome and a sharp razor whose surfaces are ground flat, not concave, are all that is necessary.

For more precise sectioning a stationary microtome is a necessity. Many types of these instruments are on the market. The Thoma type of instrument is specially adapted for celloidin work, but may also be used for paraffin sections. The Schanze instrument is very useful for 
celloidin sections and may also be used for small paraffin sections. The Minot and the new Spencer rotary microtomes are specially adapted for the production of serial sections in paraffin. These instruments of themselves suggest the manner in which they are to be used, and the technic is easily acquired. Like all delicate instruments, they must be kept well cleaned and properly oiled, to do good service.

Much depends upon the choice and care of the knife. The micro-
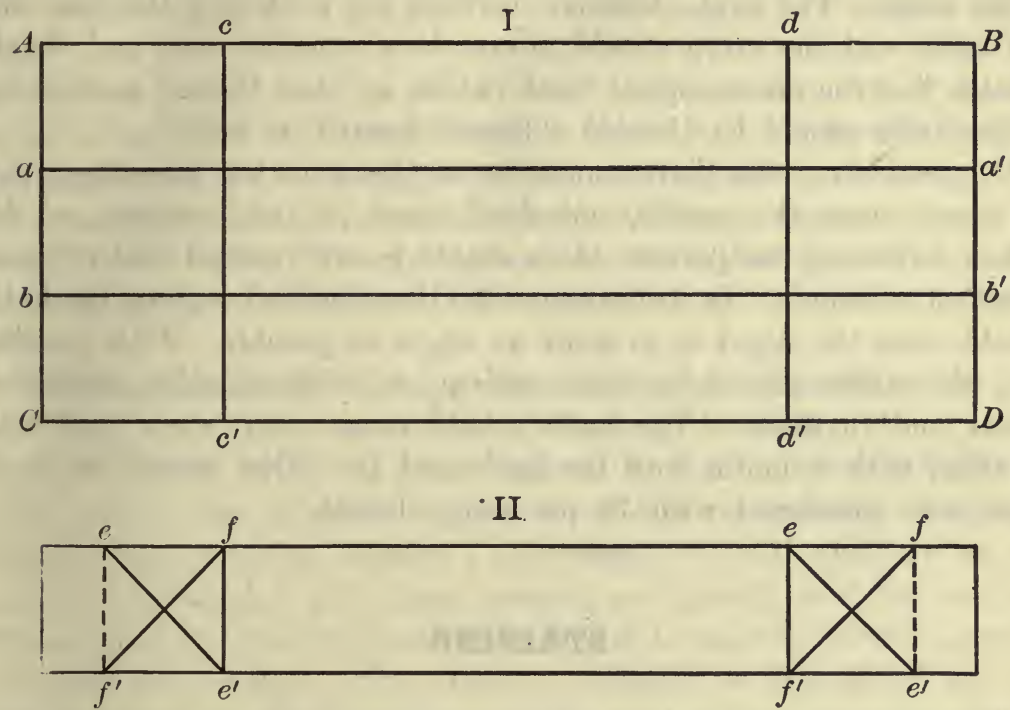

Fig. 594.-A Method of Preparing a Paper Box for Paraffin Embedding.

I. A slip of paper, $A, B, D, C$, is folded, both ways, on the lines $a-a^{\prime}, b-b^{\prime}, c-c^{\prime}$, and $d-d^{\prime}$. Then being folded into the form shown in $I I$, it is laid flat, the section $a, a^{\prime}, b^{\prime}, b$, shown in $I$, being uppermost, and the paper is creased on the lines $e-e^{\prime}$ and $f-f^{\prime}$. It is then opened, folded in the shape of a box, $f, e, f^{\prime}, e^{\prime}$, forming the bottom, and is secured by folding down the ends after creasing the paper on the lines $e-f^{\prime}$ and $f-e^{\prime}$.

tome knives of Jung are of excellent quality and should be kept in good condition by the frequent use of the hone and strop.

The hone should be of fine Belgian stone. It should be well moistened with water, the addition of a little fine soap being a distinct advantage. The edge of the knife, carefully applied to the hone, should first be drawn obliquely from heel to toe and toward the operator, being held at a constant angle and drawn the whole length of the stone. The knife is then turned over and the motion is reversed, the knife being held obliquely at an angle equal to the previous one, the edge directed 
away from the operator, and the knife pushed from heel to toe, the whole length of the stone. The motion being repeated, a sharp edge is gradually acquired, which can be finished by the use of the strop.

In the use of the strop the motions are the reverse of those with the hone, the back of the knife in this case preceding its edge as it is drawn along the leather, and the draw should be from the toe to heel of the knife. The angle, however, between the knife and the hone and the knife and the strop should always be a constant one, and should be such that the microscopical 'teeth' which are thus formed on the edge of the knife should be directed obliquely toward its heel.

In sectioning, the knife should be so placed in the microtome that its edge crosses the paraffin-embedded object at right angles, and for ribbon sectioning the paraffin block should be so trimmed that it forms a perfect rectangle. In sectioning celloidin-embedded objects, the knife should cross the object at as acute an angle as possible. With paraffin, also, the stroke should be sharp and quick; with celloidin, somewhat slower and rhythmic. The knife should remain dry when used with paraffin; with celloidin both the knife and the object should be at all times well moistened with 70 per cent. alcohol.

\section{STAINING}

The sections, having been cut, are at once ready for staining, provided they were embedded in celloidin. If paraffin was used for embedding, the sections have first to be fastened to the slide. This is accomplished in the following manner.

The paraffin sections are properly arranged upon the surface of a clean slide, a few drops of water from a pipette are allowed to flow between the slide and the sections, so that the latter float upon the surface of the water; and the slide is gently heated over a small flame. Thus the paraffin sections are straightened; care should be used not to melt them. The excess of water is now carefully drained off and the slide placed in an oven and heated to about $40^{\circ} \mathrm{C}$. for several hours, until thoroughly dried. Most tissues will now adhere firmly to the slide. If, however, the tissue was fixed with solutions containing bichromate of potassium the sections are liable to come off the slide, a misfortune which may be avoided by the use of a celloidin adhesive, with or without the previous use of Mayer's albumin. As a precautionary 
measure against the loss of sections, the routine use of albumin adhesive is recommended.

\section{Mayer's Albumin:}

White of egg, chopped with scalpel or scissors and filtered.................... 10 c.c.

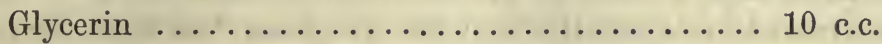

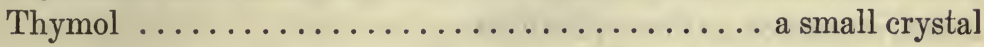

A drop of this fluid is rubbed evenly upon the surface of a clean slide. Distilled water is added with a pipette and the paraffin sections arranged upon the slide by the method detailed above. In the process of drying the albumin is coagulated and the tissue becomes firmly attached to the slide. The drying may be accomplished without special heating, but requires at least twelve hours at ordinary room temperatures.

Celloidin Adhesive.-The sections having been fastened to the slide and dried, the paraffin is removed by dipping the slide into one or two changes of pure xylol, the xylol removed by washing with absolute alcohol, and a few drops of very thin solution of celloidin (made by diluting thin celloidin, number I (see page 734) with eight or ten volumes of the alcohol and ether solvent) are poured over the sections. The solution of celloidin should be so thin as to scarcely leave an appreciable film on the slide. The excess of celloidin is drained off and the film hardened by first flooding the slide with 70 per cent. alcohol, and after a few minutes transferring it to water. The sections will not now be removed from the slide except by mechanical violence. In handling serial sections of embryos, time may be saved, and the same end accomplished, by simply spreading by means of a soft brush a thin film of the very thin celloidin solution over the dried paraffin sections. The sections may then be manipulated for staining in the usual way.

Celloidin sections do not need to be fastened to the siide before being stained; they are sufficiently firm to be gently handled with a needle.

Staining in Bulk.-It is occasionally desirable to stain tissue in bulk so that sections once cut can be immediately mounted. This is best accomplished by the use of a single stain applied to small blocks of tissue immediately after fixation and hardening, and is usually done in aqueous media. Borax carmin is the most useful dye for the purpose, and is used as described below, except that it will require two or three 
days to penetrate the tissue. Delafield's hematoxylin also gives brilliant results in bulk staining.

Regressive Staining.--Staining in bulk is necessarily a regressive process, viz., the tissue is first overstained and then partially decolorized. With borax carmin the decolorization is accomplished by acid alcohol (hydrochloric acid 1 c.c., 70 per cent. alcohol 100 c.c.). Since the stain is removed more rapidly from the cytoplasm than from the nucleus, a differentiation is thus produced.

Progressive Staining.-In progressive staining the dye, having been once taken up by the tissue, is not removed, the differentiation of nucleus and cytoplasm being accomplished by the selective affinity of the dye. Thus, certain dyes are nuclear (basic), others are cytoplasmic (acid). The former possess a special affinity for the nucleus, the latter stain both nucleus and cytoplasm.

Certain dyes may be used either progressively or regressively; in the former case care must be exercised that the section be not overstained; in the latter case overstaining is impossible, but decolorization must be watched with care.

Classification of Dyes.-Dyes may be classified according to their affinity for certain granules or other portions of the cytoplasmic structure. A classification of this kind was advanced by Ehrlich through his pupil, G. Schwartze (Inaug. Dissert., 1880), and has been greatly elaborated by Pappenheim (Grundriss der Farbechemie, 1901). Such a classification is very incomplete and unsatisfactory, but in a very general way serves a useful purpose. The following is sufficient for our present needs :

1. Basic dyes, those which color the chromatin of the nucleus (largely nucleic acid) and the so-called basophil granules. Hematein, methylene blue, methyl green, safranin, and basic fuchsin are examples.

2. Acid dyes, those which are usually cytoplasmic dyes, and have an affinity for the acidophil (i.e., basic) granules. Such are eosin, Congo red, orange $\mathrm{G}$, methyl blue, and acid fuchsin.

3. Neutral dyes, which result from a due admixture of acid and basic colors, and which gives a specific tint to the so-called neutrophil or azure granules. Such dyes are Ehrlich's triacid mixture, eosinated methylene blue, etc.

4. Specific dyes, which result from the due admixture of dyes with certain reagents, other dyes or chemicals, and which have a selective affinity for particular tissues. This is an indefinite class which includes Weigert's elastic tissue stain, Mallory's connective tissue stain, Sudan III 
for fat, the intra vitam staining of nerve tissues with methylene blue, etc.

Mordants.-The successful application of a dye requires that the tissue shall have a chemical affinity for the stain. This affinity may be either natural or artificial, e.g., eosin will color nearly all tissues under any ordinary conditions without the aid of any other reagent; the coloration is, however, largely a physical phenomenon, due to inbibition of the dye; hematoxylin, on the other hand, stains ordinary tissue but slightly, but its action is much enhanced by first acting upon the tissue with alum or a similar reagent. The alum, in this case, serves as a mordant.

A mordant should have a strong affinity for both the stain and the tissue. Hence it is that, after a tissue has been once stained by the aid of a mordant, it may be decolorized, partially or completely, by the second application of the same or another mordant of equal strength.

\section{Single Stains with Nuclear Dyes}

Hematein.-Hematein is the active principle of the dye, hematoxylin, obtained by extracting logwood. Hematein is derived from the solutions of hematoxylin by oxidation, either by chemical reagents or by prolonged exposure to the air. As a dye it must be combined with a mordant, which, most frequently, is some form of alum. The following formulas are recommended:

Alum Hematein (Mayer) :

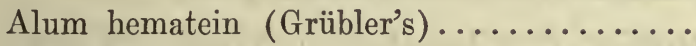

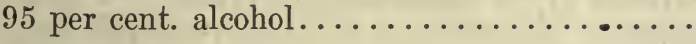

0.2 grm.

Saturated aqueous solution of ammonium alum

5 c.c.

100 c.c.

About 10 grm. of alum are required for this solution. The hematein should be first dissolved in the alcohol, with gentle heat if necessary, and afterward added to the warm solution of alum. The fluid is ready for use in two or three days, but will increase in strength for several weeks; it then requires dilution.

\section{Böhmer's Hematoxylin:}

Hematoxylin 0.5 grm.

Absolute alcohol.................. 5 c.c.

Saturated aqueous solution of potassium alum 100 c.c. 
The ingredients should be mixed as above and allowed to stand for eight to ten days in an open bottle and exposed to sunlight. Filter. Ripening will continue for some weeks, and the dye will require dilution from time to time with a saturated solution of potassium alum.

\section{Delafield's Hematoxylin:}

Hematoxylin

4 grm.

95 per cent. alcohol................. 25 c.c.

Saturated aqueous solution of ammonium alum 400 c.c.

Mix as above and permit the fluid to stand in an open vessel exposed to the air and sunlight for three or four days. Add:

Glycerin .................. 100 c.c.

Methylic alcohol ................. 100 c.c.

Expose to light in a cotton-plugged bottle. After two days filter. After several days filter again, and keep in tightly stoppered bottle. The fluid will ripen for several weeks and may require dilution with a saturated aqueous solution of alum, containing glycerin and methylic alcohol in the above proportions.

This is a very deep nuclear stain, in fact it is so deep that, while the nuclei are sharply differentiated, the intranuclear structure is nearly obliterated. The stain is somewhat improved in this particular by the slight regressive action of very weak acids-e.g., hydrochloric, picric, or dilute acetic acid. This is a very useful dye for the toto staining of small embryos.

Mann's Acid Hematein (Ehrlich's Hematoxylin) :

Hematein (Grübler's) or hematoxylin crystals $2 \mathrm{grm}$. Absolute alcohol ................... 100 c.c.

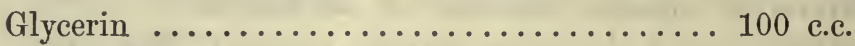

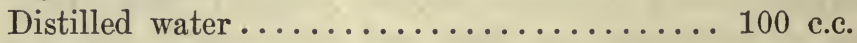

Potassium alum ............... 10 grm.

Glacial acetic acid............... 10 c.c.

The hematein (or hematoxylin) is dissolved in the acetic acid, with 25 c.c. of the alcohol; the glycerin and the remainder of the alcohol are then added. The alum is dissolved in water by the aid of heat, and the warm solution is poured slowly while stirring into the solution of hematein. The fluid keeps indefinitely and is an excellent hematein stain for general use. Hematoxylin solutions may be ripened immediately by the addition of small amounts of hydrogen peroxide (about 1 c.c. to 200 c.c. of stain, neutralized by a few grains of sodium chlorid). 
Application of the Hematein Stains.-All of the above solutions are used in a similar manner. Sections, either free or attached to the slide, are taken from water and immersed in the dye for three to five minutes; they are then thoroughly washed in water. The stained sections are at first of a reddish-purple color, but soon become a deep blue from the slight alkalinity of the tap water used for washing. If necessary this alkalinity may be increased by the addition of one or two ảrops of ammonia to 500 c.c. of the water used for washing.

Hematoxylin solutions require air and sunlight for ripening. After ripening is complete the solution should be kept tightly stoppered. The solutions should be filtered before using. Ehrlich's hematoxylin is probably the best nuclear dye for general routine work. Better differentiation is usually obtained if the sections are over-stained ( 5 to 10 minutes) and then, after thorough washing, partially decolorized in a 1 per cent. solution of hydrochloric acid in 70 per cent. alcohol (a few seconds is usually sufficient), wasked, and the color restored in an ammoniacal solution ( 1 per cent. aqueous solution of ammonium hydroxid). The sections must again be thoroughly washed, and the best differentiation is secured if the sections are now placed in very dilute watery eosin for ten to twelve hours.

Methylene Blue.-This dye is a derivative of thionin, and may be similarly used. For staining fresh tissues the dye is used in 2 per cent. aqueous solution. Its preparations are not very permanent. The chief uses of this dye are in combination with eosin as a stain for blood; as a stain for nerve cells according to the method of Nissl; and as applied to living organs as a specific stain for nerve tissues after the method of Ehrlich. These methods will be described below.

Methyl Green.-This dye is preferable to methylene blue as a stain for fresh tissues. It is used in 2 per cent. aqueous solution and applied as a progressive stain. It also enters into the composition of Ehrlich's triacid mixture. It is strongly basic.

Carmin.-This valuable dye is derived from the cochineal bug, and is used either as a progressive or a regressive stain. For the former, picro-carmin or alum carmin are recommended; for the latter, borax carmin is preferable.

\section{Borax Carmin:}

Borax................ 4 grm.

Distilled water (boiling) ...... 100 c.c.; cool, filter, and add

Carmin................ 3 grm.; when dissolved, add

ro per cent. alcohol......... 100 c.c. 
Mix the ingredients in the above order, and after twenty-four hours filter. It may be necessary to use a drop or two of ammonia to complete the solution of the carmin. This is again removed by evaporation.

Tissues are to be overstained in the carmin solution, and differentiated in acid alcohol ( 70 per cent. alcohol containing 0.5 to 1 per cent. of hydrochloric acid) until the red color is no longer removed in clouds. The sections are then well washed with several changes of 95 per cent. alcohol, cleared and mounted. This is a most excellent dye for staining embryos in toto.

\section{Alum Carmin:}

Potassium alum .............. 5 grm.

Distilled water (hot)................. 100 c.c.

Carmin ..................... 1 grm.

Mix in the order given, boil for twenty minutes, and when cold filter. Picro-Carmin :

Ammonium hydrate....... 5 c.c.

Distilled water.......... 50 c.c.

Carmin............... 1 grm.; when dissolved, add

Saturated aqueous solution of picric acid............ 50 c.c.

Expose to light and air for two days; filter.

Picro-carmin is used as a progressive stain. Since the picric acid is soluble in alcohol, dehydration should be rapid, or a crystal of picric acid should be added to the alchohol used for dehydration.

Aceto-Carmin.-This is an invaluable fluid for certain purposes, especially where it is desired to study nuclear structure, chromosomes and spindles in fresh and uncut (teased) tissue. The tissue is simultaneously fixed and stained in this mixture, and remains preserved in the fiuid in a condition favorable for study for a considerable time. The stained tissue, e.g., insect testes, etc., is teased out on a slide in the fluid, covered with cover glass, and may then be studied under an oil immersion lens. The mitotic figures are almost as clear as in sectioned material. The solution is made by dissolving to saturation carmin in boiling acetic acid of 45 per cent. strength, and filtering.

Safranin.-This dye is a coal-tar derivative; it is an excellent nuclear stain. Like carmin, safranin yields a deep red color.

Safranin 0 (Grübler's) ............. 1 grm.

Distilled wäter ................... 100 c.c. 
1. Tissues taken from water are stained five minutes.

2. Wash in water.

3. Dehydrate rapidly in absolute alcohol. The alcohol removes some of the safranin, giving a regressive effect.

\section{Single Stains with Cytoplasmic Dyes}

Eosin.-This dye is a coal-tar derivative. There are no less than seventeen varieties of the dye on the market, of which five are in general use. These are: (1) yellowish alcoholic; (2) bluish alcoholic; (3) yellowish watery; (4) bluish watery; (5) pure French eosin. The most reliable of these dyes are manufactured by Grübler. The first and fifth varieties are to be recommended as blood stains, the first and fourth are the best for general use. Two distinct methods are based upon this choice of dyes.

Method I:

Yellowish alcoholic eosin............. 1 grm.

70 per cent. alcohol................. 100 c.c.

This stock solution is usually diluted with four to ten volumes of 70 to 95 per cent. alcohol just before using. The stain is preferably preceded by the use of a nuclear dye, after which the sections should be dehydrated in 95 per cent. alcohol.

1. Stain in the diluted eosin, one to five minutes, or until the sections become a bright red color.

2. Wash quickly in absolute alcohol, clear, and mount. The color is dissolved out during this process, producing some differentiation by regression.

Method II:

Bluish watery eosin............. 1 grm.

Distilled water .................... 100 c.c.

The stock solution should be diluted with one to four volumes of distilled water before using.

1. Tissues are taken from water and placed in the dilute eosin, one to five minutes.

2. Wash quickly in water to remove the excess of the dye.

3. Pass radially through graded alcohol, clear, and mount.

Slight differentiation may be obtained by prolonging the washing, in water, otherwise the stain is progressive. Much greater differenti- 
ation is possible with either eosin method by making the stain very dilute and staining for twenty-four to forty-eight hours.

Congo Red:

Congo red ................. 1 grm.

Distilled water .................... 100 c.c.

A few drops of dilute acetic acid should usually be added to the above; the bright red color is then exchanged for a dull bluish red, and in this neutralized condition the stain usually gives the highest differentiation. The dye should be used in the same manner as watery eosin (see Method II, above). Congo red gives especially good results when applied to fetal and young tissues.

Orange G.-There are many varieties of orange. The orange G and the aurantia of Grübler will be found satisfactory. As a cytoplasmic stain the former is preferable. It should be used in the same manner as alcoholic eosin (see Method $I$, above).

Fuchsin.-Two distinct dyes, the one of acid, the other of basic, properties, pass under this name. Acid fuchsin is a cytoplasmic dye, but when used in acid solution has a slight selective affinity for the nuclei. Basic fuchsin is chiefly useful in bacteriology. It is also used in preparing Weigert's elastic tissue stain.

It is recommended that euparal be used as a mounting medium with this group of stains. The tissue may be mounted directly from 90 per cent. alcohol.

\section{Double Staining}

\section{Hematein and Eosin:}

1. Stain with one of the hematein solutions, preferably Mann's or Ehrlich's for general use, five minutes.

2. Wash well in water.

3. Stain in watery eosin, one to ten minutes. Oralcohol.

3. Dehydrate in 95 per cent.

4. Wash quickly in water.

5. Dehydrate in absolute alcohol.

6. Clear and mount.

4. Stain in alcoholic eosin, one to five minutes.

5. Dehydrate quickly (one to five minutes) in absolute alcohol.

6. Clear and mount. 
Methyl Blue and Safranin. - Methyl blue is a very different dye from methylene blue but is practically identical with water blue (Wasser blau). It is an acid or cytoplasmic stain.

1. Stain with a 2 per cent. aqueous solution of methyl blue, three minutes.

2. Rinse in water.

3. Stain in a 1 per cent. aqueous solution of safranin, five minutes.

4. Wash in water.

5. Differentiate and dehydrate quickly in absolute alcohol, till the sections become again blue.

6. Clear and mount.

This method gives a permanent stain which yields excellent results with certain tissues-e.g., the skin.

Other nuclear and cytoplasmic dyes may be combined in a similar manner to the above methods.

\section{Spectal Staining Methods}

\section{Iron Hematoxylin (Heidenhain) :}

I. Mordant:

Ferric alum (iron ammonium sulphate-violet

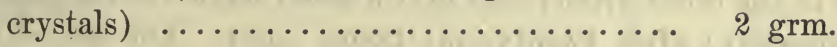

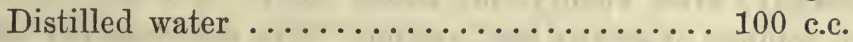

II. Stain :

Hematoxylin $\ldots \ldots \ldots \ldots \ldots \ldots \ldots \ldots \ldots .1$ grm.

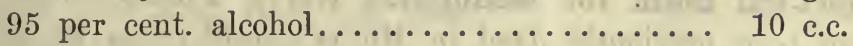

Distilled water $\ldots \ldots \ldots \ldots \ldots \ldots \ldots \ldots$ c.c.

1. Mordant the sections four to twelve hours in I.

2. Rinse in water.

3. Stain twelve to twenty hours in II.

4. Wash well in water. The sections should become very black; a drop or two of ammonia to one-half liter of water often improves the color.

5. Decolorize in the mordant, watching each section under the microscope, and stopping the decolorization at the proper time by-

6. Wash thoroughly in slowly running water, or in several changes of still water.

7. Counter-stain if desired, dehydrate, clear, and mount. 
This method gives an excellent stain for the finer nuclear structure, mitosis, etc.

\section{Muchematein (Mayer) :}

Hematein (Grübler's)............. 0.2 grm.

Glycerin .................. 40 c.c.

Aluminium chlorid ............. $0.1 \mathrm{grm}$.

Distilled water ...............6. 60 c.c.

Mix the ingredients in the order given, rubbing the hematein with the glycerin in a mortar. One or two drops of nitric acid added to the final mixture will sharpen its properties as a nuclear stain.

This dye is used as a specific stain for mucinous tissues. It is used in the same manner as hematein, and stains rapidly (three to ten minutes).

Mucicarmin (Mayer) :

Carmin ...................... 1 grm.

Aluminium chlorid $\ldots \ldots \ldots \ldots \ldots \ldots \ldots .0 .5 \mathrm{grm}$.

Distilled water ............... 2 c.c.

50 per cent. alcohol.............. 100 c.c.

Mix in the order given; heat over a small flame till the fluid darkens (two minutes); after twenty-four hours, filter. For use, dilute with five to ten volumes of 50 per cent. alcohol. Like muchematein, mucicarmin is a specific stain for mucus-containing cells. It also stains rapidly.

Weigert-Pal Stain for Medullated Nerve Fibers.-The tissues must have been previously fixed in Müller's fluid or in 10 per cent. formalin, washed in water, hardened in alcohol, and sectioned.

I. Stain:

Hematoxylin ................. 1 grm.

Absolute alcohol .................. 10 c.c.

Distilled water ....................... 90 c.c.

Saturated aqueous solution of lithium carbonate

$(1$ to 80$) \ldots \ldots \ldots \ldots \ldots \ldots \ldots \ldots \ldots \ldots \ldots \ldots \ldots \ldots$ c.c.

II. Differentiating Solution:

Potassium permanganate ........... 0.25 grm.

Distilled water ............... 100 c.c. 


\section{Decolorizing Solution:}

1 per cent. aqueous solution of oxalic acid...... 50 c.c.

1 per cent. aqueous solution of potassium sulphate 50 c.c.

This last solution should be freshly prepared by mixing the two stock solutions just prior to use.

1. Stain sections (six to twenty-four hours) until black.

2. Wash well in water. A few drops of lithium carbonate solution added to the water may improve the color, which should become a deep blue-black.

3. Differentiate until the gray matter becomes brown (one-quarter to two minutes).

4. Rinse in water.

5. Decolorize until the white matter becomes a steel blue, the gray matter a light brown (one-quarter to one minute), watching each section with care.

6. Wash thoroughly in several changes of water, or in running water.

7. If desired, counter-stain with alum carmin, and wash in water.

8. Dehydrate, clear, and mount.

Methylene Blue, for non-medullated nerve fibers (Intravitam Method):

I. Stain:

Methylene blue (Grübler's 'rectif. nach Ehrlich') $\ldots \ldots \ldots \ldots \ldots \ldots \ldots \ldots \ldots \ldots \ldots \ldots \ldots \ldots \ldots \ldots \ldots \ldots \ldots$

Distilled water ............. 100 c.c.

Dissolve with heat, cool, and filter.

II. Fixing Solution (Bethe's) :

Ammonium molybdate .............. 1 grm.

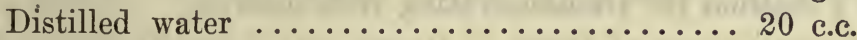

Hydrochloric acid, C. P............... 1 drop.

The solution should be freshly made and kept at or near $0^{\circ} \mathrm{C}$.

1. The method is only applicable to living tissues, by injecting the blood-vessels with the stain, or by partially immersing in the staining fluid small pieces of tissue, freshly removed from the living animal.

2. After ten to thirty minutes, rinse in normal saline solution, and place in the cold fixing solution for two to six hours, according to the size of the pieces. The tissue should be kept cold.

3. Wash well in distilled water. 
4. Dehydrate quickly in 95 per cent. and absolute alcohol, kept at or near $0^{\circ} \mathrm{C}$.

5. Embed in paraffin. At a convenient time, cut and mount.

The stain is rather unstable, but may be kept fairly well if mounted in glycerin or in neutral balsam.

This technic is invaluable for the demonstration of the terminal nerve supply of tissues.

Methylene Blue, for chromophilic (tigroid) granules in cyton and dendron (Nissl's Method):

I. Stain:

Methylene blue (Grübler's 'B pat.')..... 3.75 grm.

Venetian soap (white Castile)........ 1.75 grm.

Distilled water ................ 1000 c.c.

II. Differentiating Solution:

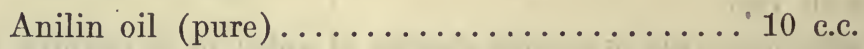

95 per cent. alcohol............... 90 c.c.

This method is only applicable to tissue which has been fixed in 95 per cent., or in absolute alcohol. Thionin may be substituted for the methylene blue in the stain.

1. Warm the stain till steam begins to rise; then immerse the sections for four to six minutes. They acquire a deep blue color.

2. Rinse in distilled water.

3. Differentiate in the anilin alcohol till the sections become a light blue, carefully observing each section (twenty to sixty seconds).

4. Wash in 95 per cent. alcohol.

5. Clear in equal parts of origanum and cajuput oils, and mount in neutral balsam or in colophonium dissolved in xylol.

\section{Cajal's Method for Demonstrating Neurofibrils:}

1. Fix small pieces of tissue in 10 per cent. formalin for six hours.

2. Wash in water for four hours.

3. Transfer to 40 per cent. alcohol for six hours.

4. Place in 50 c.c. of 40 per cent. alcohol, adding 5 drops of ammonia, for twenty-four hours.

5. Transfer to $\mathbf{1 . 5}$ per cent. silver nitrate solution at incubator temperature $\left(38^{\circ}\right.$ C. $)$ for five days.

6. Rinse, and place in a solution of 1 gram pyrogallic acid or hydrochinon, 100 c.c. water, and 15 c.c. formalin for twenty-four hours.

7. Pass through graded alcohols, and embed in paraffin or celloidin. 
For other more complicated Cajal technics for demonstrating neurofibrils, also for the Bielschowsky, Bethe and Apáthy methods for the same purpose, reference must be made to the special works on technic.

Golgi's Stain for Nerve Cells (cyton, axis cylinder, and dendrons, and neuroglia):

I. Mordant:

1 per cent. aqueous solution of osmium tetroxid 10 c.c.

$3 \frac{1}{2}$ per cent. aqueous solution of potassium bichromate ................... 40 c.c.

\section{Silver Solution:}

Silver nitrate (crystals) ........... 0.75 grm.

Distilled water ............... 100 c.c.

This method is only applicable to fresh tissues, and the best results are obtained when the tissue is taken from a fetus or from an animal not over three days old. Thin slices or small fragments of tissue must be used.

1. Fix in the mordant for ten days, frequently changing the fluid, which should not become turbid, nor should its odor of osmium tetroxid entirely disappear.

2. Rinse quickly in water.

3. Place tissues in the silver solution, diluted with two volumes of distilled water, for fifteen minutes.

4. Place in the undiluted silver solution twenty-four to fortyeight hours. If several pieces of tissue are prepared they should be removed at intervals, as the duration of the impregnation by silver is always an experiment.

5. Dehydrate in absolute alcohol, one hour. hour.

6. Transfer to equal parts of absolute alcohol and ether, half an

7. Thin celloidin (number 1), thirty minutes.

8. Thick celloidin (number 3), thirty to forty-five minutes.

9. Transfer to a wooden block and fasten with celloidin.

10. Harden the celloidin block in chloroform, one-half to one hour.

11. Cut at once, the sections being $50 \mu$ to $100 \mu$ thick. While cutting, the knife should be well moistened with bergamot oil, not alcohol, and the sections, if not mounted at once, may be preserved for a short time in the same oil. Oil of lavender, cajuput, or origanum may be used in a similar manner. 
Nitrate of Silver for Cement Substances.-This reagent is used to outline epithelial cells by blackening the intercellular substance, the silver, after impregnation, being reduced or blackened by exposure to light.

1. The fresh tissue is immersed in a 0.25 per cent. to 0.5 per cent. aqueous solution of silver nitrate (crystals), and left in the dark for ten to twenty minutes. Protargol may be substituted for silver nitrate crystals.

2. Wash in distilled water, and while still in water, expose to direct sunlight until the object becomes a dark reddish-brown color (ten to thirty minutes).

3. Transfer to 70 per cent. alcohol, three to twelve hours.

4. Preserve in 95 per cent. alcohol. The tissue may be mounted in the usual way.

Since nitrate of silver will attack metal instruments, the tissues while in this solution should be handled with glass rods. In silvering serous membranes, it is well to slightly stretch the object by tying it over a cork with a thread tightly fastened around the edge.

Gold Chlorid for Nerve Plexuses and Nerve Endings (Ranvier's Method).-1. Wash the fresh tissues in normal saline solution, and place them in pure lemon-juice until they appear clear (five to ten minutes).

2. Wash quickly in distilled water.

3. Place in the dark in a 1 per cent. aqueous solution of chlorid of gold for ten to forty-five minutes, according to the permeability of the tissue.

4. Wash in distilled water.

5. Place in a 25 per cent. aqueous solution of formic acid, and keep in the dark for twenty-four to forty-eight hours.

6. Wash thoroughly in water.

\%. The tissue is now properly teased and mounted in glycerin, or sections may be dehydrated, cleared, and mounted in balsam.

The gold method is used for the demonstration of nerve plexuses and nerve terminations. It is applicable also for the demonstration of certain connective tissue cells, e.g., corneal cells, and tendon cells.

Picro-Fuchsin (Van Gieson).-This method is used as a specific stain for connective tissue; it colors the collagenous fibers a bright red, all other tissues appearing yellow. Picric-acid-fuchsin may be used as an after stain with nuclear dyes, e.g., hematoxylin, though the tissue 
must be greatly overstained with the nuclear dye, since the picric acid will decolorize hematein.

Saturated aqueous solution of picric acid.... 100 c.c.

1 per cent. aqueous solution of acid fuchsin... 5 c.c.

The acid fuchsin solution must be boiled well and filtered before adding to the picric acid solution, otherwise a precipitate will occur and the mixture be spoiled.

1. Stain with Delafield's or Böhmer's hematoxylin, fifteen to thirty minutes.

2. Wash well with water. The sections should be almost black.

3. Stain with picro-fuchsin, three to five minutes.

4. Rinse quickly in water (water removes the fuchsin).

5. Dehydrate in absolute alcohol, clear, and mount.

Weigert's Elastic Tissue Stain.-This method gives a specific stain for elastic fibers; it may be used alone, or in combination with hematein and picro-fuchsin.

1 per cent. aqueous solution of basic fuchsin.. 100 c.c.

2 per cent. aqueous solution of resorcin...... 100 c.c.

Boil the mixture in a porcelain dish, and while hot, add liquor ferri sesquichlorati (Pharm. Ger., III), 25 c.c.

Heat and stir for five minutes; a heavy precipitate is formed. Cool and filter. Dry the precipitate in a porcelain dish over a water bath or sand bath. Dissolve the dried precipitate in 200 c.c. of 95 per cent. alcohol, filter and replace the alcohol lost by evaporation. Add 4 c.c. of pure hydrochloric acid.

Tissues should be stained twenty to sixty minutes, then thoroughly washed in water, dehydrated, cleared, and mounted.

The following method gives very beautiful results:

1. Stain in Delafield's or Böhmer's hematoxylin, twenty to thirty minutes.

2. Wash well with water.

3. Stain in Weigert's elastic tissue stain, twenty minutes.

4. Wash in water.

5. Stain in picro-fuchsin, three to five minutes.

6. Rinse quickly in water.

\%. Dehydrate in absolute alcohol, clear, and mount.

Orcein may also be employed as a specific stain for elastic fibers.

Mallory's Connective Tissue Stain.-This method is applicable 
only to tissues which have been fixed in Zenker's solution and dehydrated with alcohol. Somewhat inferior results are obtained after fixation with mercuric chlorid.

It stains collagenous fibers and reticulum, blue; nuclei, neuroglia and cytoplasm, red; and hemoglobin (red blood corpuscles), yellow. Elastic fibers remain unstained.

I. Stain:

Acid fuchsin .................... $0.1 \mathrm{grm}$.

Distilled water .................. 100 c.c.

II. Fixative:

Phosphomolybdic acid ............ 1 grm.

Distilled water ................. 100 c.c.

III. Counter-Stain:

Anilin blue (soluble in water)........ $0.5 \mathrm{grm}$.

Orange G (Grübler's).............. 2 grm.

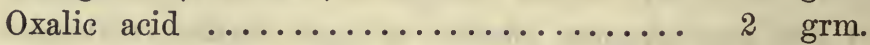

Distilled water .................. 100 c.c.

1. Stain in the fuchsin solution, three to twenty minutes. The sections should become a bright red.

2. Wash in water.

3. Fix in the phosphomolybdic acid solution, one minute. This 'fixes' the fuchsin and prevents decolorization.

4. Wash well in water.

5. Counter-stain in the anilin blue solution (No. III), five to twenty minutes. The section should become decidedly blue.

6. Wash in water.

7. Dehydrate, clear, and mount.

Eosinate of Methylene Blue (Hasting's Method for Blood).-For the somewhat complicated method of preparing the stain the reader is. referred to the original article, Johns Hop. Hosp. Bull., 1904, vol. xv, p. 122. The stain is applicable to smears of blood, marrow, splenic cells, etc. When used with smears which contain traces of fat, a preparatory treatment with a 2 per cent. aqueous solution of sodium metaphosphate, which probably serves as a mordant, improves the staining properties. Otherwise the stain is applied without previous fixation.

1. Stain for one minute.

2. Dilute the stain with several volumes of distilled water, and 
continue the stain for five minutes, or until satisfactorily differentiated.

3. Wash with distilled water.

4. Dry and mount.

The Giemsa and the Wright blood stains (other modifications of the original Romanowsky stain) may be used for the same purposes, and are applied in a similar manner.

\section{Eosin and Methyl Blue Mixture (Mann):}

1 per cent. aqueous solution of methyl blue..... 35 c.c.

1 per cent. aqueous solution of bluish watery eosin 45 c.c.

Distilled water .................. 100 c.c.

1. Mordant the sections in water, leaving them till all alcohol has been replaced (five to thirty minutes).

2. Stain in the above mixture, five to ten minutes.

3. Wash well and differentiate in water; the effect may be varied by the duration of the washing (ten to forty minutes).

4. Dehydrate in alcohol, clear, and mount.

When a sharper nuclear dye is desired the stain may be used as a counter-stain after hematein. In this case the methyl blue solution should be allowed to act only three to five minutes. The methyl blue used in this method is a cytoplasmic dye and should not be confounded with methylene blue. This is a valuable stain for differentiating cytoplasmic granules.

\section{Triacid Stain (Ehrlich):}

Saturated aqueous solution of orange G..... 13 c.c.

Saturated aqueous solution of acid fuchsin.... 7 c.c.

Distilled water ................ 15 c.c.

95 per cent. alcohol............... 15 c.c.

Saturated aqueous solution of methyl green.... 12.5 c.c.

95 per cent. alcohol . ............... 10 c.c.

Glycerin ................... 10 c.c.

Be certain that the solutions of the dyes are saturated, and mix in the order given.

The following formula by Mayer may be substituted:

Distilled water ................ 45 c.c.

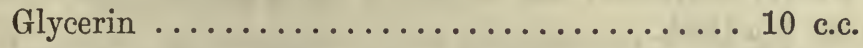

95 per cent. alcohol............... 25 c.c.

Acid fuchsin ................... 3 grm. 
Orange $G \ldots \ldots \ldots \ldots \ldots \ldots \ldots \ldots \ldots \ldots \ldots \ldots \ldots \ldots \ldots \ldots$ grm.

Methyl green .................. 1 grm.

Mix the fluids and dissolve the dyes in the order given.

When used for staining sections, either of these formulas should be diluted with five to ten volumes of the following mixture:

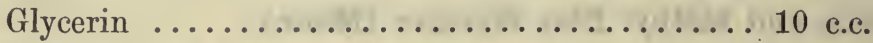

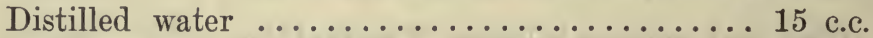

95 per cent. alcohol . ................ 25 c.c.

1. Stain five to ten minutes in the diluted solution. (Use full strength for blood smears.)

2. Rinse in water.

3. Dehydrate in absolute alcohol. (Smears are dried in the air.)

4. Clear and mount.

The best results are obtained with tissues fixed in a sublimate mixture. It is a delicate cytologic stain, and is serviceable in differentiating between chromatin (chromosomes) and plastin (plasmosomes and linin); also in differentiating cytoplasmic granules. Sections should not be over $3 \mu$ in thickness. The stain is more or less capricious.

Auerbach's Fuchsin-Methyl-Green Stain.-This stain does well only after a sublimate fixative. It is serviceable in cytologic work for differentiating between 'active' and 'resting' chromatin. When successfully manipulated it stains the chromosomes green and the plasmosomes and linin red.

Keep in separate bottles the following stock solutions:

I. Acid fuchsin 1 part, distilled water 1,000 parts.

II. Methyl green 1 part, distilled water 1,000 parts.

Mix when ready to use in the following proportions:

2 parts of I, acidulating every 50 c.c. with 1 drop of a 10 per cent. solution of glacial acetic acid.

3 or 4 parts of II.

Fix tissues (e.g., insect testes, etc.) in the sublimate acetic mixture, transfer direct to 80 per cent. alcohol (removing the mercury with tincture of iodin), dehydrate, and embed in paraffin. Sections should not be cut over $3 \mu$ thick.

Stain for fifteen minutes, and pass directly to 95 per cent. alcohol. When the green stain no longer leaves the sections in clouds, pass 
rapidly through absolute alcohol and xylol, and mount in balsam. The sections may also be mounted directly from 95 per cent. alcohol in euparal.

Mitochondrial Technics.-Mitochondria (chondriosomes, plastosomes) are readily destroyed by acids, hence these must be eliminated from fixing solutions, or greatly reduced.

I. MEVes' MeTHoD.-This is the simplest method for demonstrating mitochondria, and in many instances it is quite satisfactory.

Very small pieces of tissue are fixed for eight days in Flemming's strong solution in which the acetic acid has been reduced to 3 or 4 drops in the simplest formula (see page 728). The tissue is then embedded in paraffin in the usual way, and the sections cut at 3 to $6 \mu$ are stained by the iron-hematoxylin method. The nuclei and cytoplasm are a uniform pale gray; the mitochondria appear as deep black granules, rods or filaments.

II. Benda's Metrod.-This is a more complicated technic, but gives excellent results, and is claimed to be specific.

1. Fixation in strong Flemming's fluid (acetic acid reduced to 3 drops) for eight days.

2. Wash in water one hour.

3. Place in a mixture of equal parts of pyroligneous acid and $\mathbf{1}$ per cent. chromic acid for twenty-four hours.

4. Transfer to a 2 per cent. solution of potassium bichromate for twenty-four hours.

5. Wash, dehydrate and embed in paraffin and fix on slide in the usual way.

6. Mordant sections on slide in 4 per cent. solution of ferric alum for twenty-four hours.

7. Rinse with water, and place in an amber-yellow aqueous solution of Kahlbaum's suphalizarinate of soda (prepared by dropping 1 c.c. saturated alcoholic solution in 100 c.c. of water) for twenty-four hours.

8. Rinse with water, flood slides with a solution of crystal violet ( 1 vol. sat. sol. of dye in 70 per cent. alc.; 1 vol. 1 per cent. $\mathrm{HCl}$ in 70 per cent. alc.; 2 vols. anilin water) and warm until vapor is given off.

9. Rinse, differentiate one or two minutes in 30 per cent. acetic acid solution.

10. Wash in running water five to ten minutes. 
11. Dry with blotting paper, drop in absolute alcohol, pass through bergamot oil into xylol and mount in balsam.

According to Wildman (Jour. Morph., 24; 3, 1913) the slides may be passed from the alizarin solution after rinsing, 1 , into a 3 per cent. solution of crystal violet ( 3 c.c. anilin stain in 100 c.c. distilled water) for ten minutes; 2 , rinse and place in 80 per cent. alcohol for five seconds; 3, pass through 95 per cent. and 100 per cent. alcohol, where the differentiation is watched; 4 , clear and mount in the usual way.

III. Regaud's Metion (Arch. d'Anat. Micr., t. 11, 1910, p. 296). -This constitutes a very satisfactory routine technic.

1. Fix small piece of tissue for twenty-four hours in a mixture of Potassium bichromate, 3 per cent. aqueous solution'. . 80 parts

Formalin ...................... 20 parts

2. Transfer to a 3 per cent. aqueous solution of potassium bichromate. This acts as a mordant. The tissue should be left in this solution for from 1 to 4 weeks, with several changes of fluid.

3. Wash, harden, embed in paraffin, and cut sections of from 3 to 5 microns.

4. Stain with iron-hematoxylin.

Mallory's Phosphotungstic Acid Hematoxylin for Neuroglia and may be used to advantage with the connective tissues) (Jour. Exp. Med., Vol. 19, No. 5, 1900). - Tissues should be fixed in Zenker's fluid or in Helly's fluid. It stains neuroglia, myoglia and fibroglia fibrils and fibrin and nuclei blue; collagen fibrils are stained reddish-brown; other intercellular substances pink.

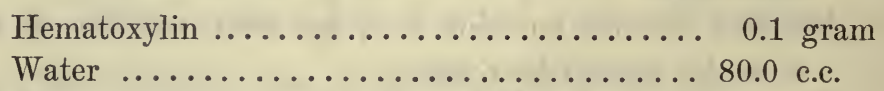

Phosphotungstic acid (Merck), 10 per cent. aqueous solution $\ldots \ldots \ldots \ldots \ldots \ldots \ldots \ldots$. 20.0 c.c.

Dissolve the hematoxylin by heating in a small amount of water; when cool add to rest of solution. Allow to ripen several weeks. The solution can be ripened for immediate use by addition of 10 c.c. of $1 / 4$ per cent. solution of potassium permanganate, or by the addition of 0.2 gram peroxid of hydrogen.

1. Pass sections through graded alcohols to water.

2. Place in $1 / 4$ per cent. aqueous solution of potassium permanganate for five to ten minutes. 
3. Wash in water.

4. Place in a 5 per cent. aqueous solution of oxalic acid for-five to ten minutes.

5. Wash thoroughly in water.

6. Place in phosphotungstic acid hematoxylin solution for twelve to twenty-four hours.

7. Dip for a few seconds in 95 per. cent. alcohol.

8. Clear in carbol-xylol and xylol and mount in xylol-damar.

Vance's Method for Staining Bile Canaliculi (Anat. Anz., Vol. 44, No. 1\%, pp. 412-413).

1. Fix tissues in Helly's fluid; or in a mixture of equal parts of 10 per cent. formalin and 5 per cent. mercuric chlorid.

2. Embed in colloidin.

3. Place sections in a dilute solution of iodin in 95 per cent. alcohol for five to fifteen minutes.

4. Remove excess iodin by washing in several changes of 95 per cent. alcohol.

5. Stain in Mallory's phosphotungstic acid hematoxylin for twentyfour houl's.

6. Transfer directly to 95 per cent. alcohol.

7. Clear in carbol-xylol or in oil of origanum, and mount in balsam.

Jenner's Blood Stain.-This solution acts as a fixative as well as a stain. Blood smears are immersed in the stain for two to five minutes, washed in water, dried and mounted in balsam.

Water soluble eosin (Griibler's), 1 per cent. aqueous solution ..................... 100 c.c.

Methylene blue (Grübler's), 1 per cent. aqueous solution $\ldots \ldots \ldots \ldots \ldots \ldots \ldots \ldots \ldots \ldots \ldots \ldots \ldots \ldots$ c.c.

Mix the above solutions; after twenty-four hours, filter. Dry the filtrate at $65^{\circ} \mathrm{C}$., wash the dry filtrate, and again dry and powder. Dissolve 0.5 gram of the powder in 100 c.c. pure methyl alcohol. The result constitutes the stock solution.

\section{Zimmermann's Technic, for demonstrating the intercalated disks} of cardiac muscle.

1. Small pieces of tissue are fixed for twenty-four hours in a solution of 90 parts absolute alcohol and 10 parts 25 per cent. nitric acid.

2. Wash in several changes of 95 per cent. alcohol, or until it remains neutral to litmus paper. 
3. Pass through graded alcohols to distilled water.

4. 'Transfer to solution of 1 gram hemalum (Grübler's) to 10 c.c. water, where it remains for eight to ten days.

5. Wash in distilled water, embed in paraffin, and section.

The intercalated disks are conspicuously stained a dark blue, which contrasts sharply with the light-blue background.

'These disks can be well demonstrated also by fixation in Carnoy's fluid, followed by iron-hematoxylin stain. For the same purpose Heidenhain employs vanadium hematoxylin after sublimate fixation (see Lee's Vade Mecum).

Gage's Method, for Demonstrating Glycogen.-Glycogen granules are readily soluble in aqueous media. Tissue which is to be tested for this substance should be fixed in 95 per cent. alcohol.

1. Embed in paraffin in the usual way.

2. Flatten sections on slide by use of the following iodin solution (Lugol's solution), which is subsequently used for staining:

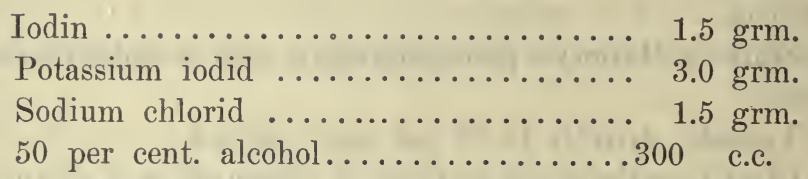

3. Stain, dehydrate, dissolve paraffin with xylol, mounted in melted vaselin, and seal cover glass with shellac. The glycogen grains are stained mahogany red.

Osmic Acid Technic for Fat.-Free fats and lipoids are soluble in ether, chloroform, absolute alcohol, xylol, benzene, and essential oils. Since these must be employed in both paraffin and celloidin embedding, fat cannot be very satisfactorily demonstrated by these technics. Frozen sections (made with a freezing microtome) of fresh or formalin-fixed tissues are therefore preferable (in some instances indispensable); these may be satisfactorily stained with Sudan III (red), Sharlach R (red), Nile blue (blue); they must be mounted in glycerin jelly.

Osmic acid, however, renders certain fats resistant to solution in oils, and tissue so fixed may accordingly be treated by the paraffin technic; but thick cedar oil must be substituted for xylol or chloroform, and absolute alcohol should be avoided:

1. Fix small pieces of fatty tissue in Flemming's fluid for two days.

2. (a) Embed in paraffin (use cedar oil), section, and mount in euparal; or 
(b) Embed in celloidin, the different solutions of which should be made up in 95 per cent. alcohol (see Kingsbury, Anat. Rec., $5,6,1911)$.

Technic for Demonstrating Chromaffin Granules.-Chromaffin material ('pheochrome' granules; adrenin granules) is highly susceptible to solution by acids. When preserved the chrornaffin granules react to chromium (acid and solutions of salts) and stain light brown. The best method for their preservation is Helly's fluid (see page 728), which coincidently stains the granules. Paraffin or celloidin sections may be stained with varions nuclear dyes for the clear differentiation of the nuclei.

Celloidin-paraffin Method (Apáthy; Ztschr. wiss. Mikr., Bd. XXIX, 1912, S. 449-515).- This method obviates the difficulties encountered in sectioning hard and brittle objects, e.g., chitin, and eggs with yolk.

1. Place thoroughly dehydrated tissues in ether-alcohol for at least five hours.

2. Thin celloidin ( 2 per cent. solution) for twenty-four hours.

3. Thick celloidin (4 per cent. solution) for twenty-four hours.

4. Pour into a paper embedding box or a glass dish, and harden in chloroform vapor for twelve hours.

5. Trim the block quickly, leaving a few millimeters of celloidin on each side of object, and place in test tube of chloroform for twelve hours.

6. Transfer to tube of following oil mixture until the block becomes clear and sinks:

Chloroform, by weight $\ldots \ldots \ldots \ldots \ldots \ldots \ldots 4$ parts

Oil of origanum, by weight ........... 2 parts

Oil of cedar wood, by weight $\ldots \ldots \ldots \ldots \ldots \ldots 4$ parts

Absolute alcohol, by weight $\ldots \ldots \ldots \ldots \ldots \ldots 1$ part

Carbolic acid crystals, by weight ......... 1 part

(Put small amount of dried sodium sulphate into bottom of test tube to take up the water brought into the mixture by the celloidin.)

\%. Wash cleared block in three or more changes of benzol; this removes the oils and alcohol, and prepares for paraffin filtration.

8. Embed in paraffin, section and mount as in ordinary paraffin technic. (See also Kornhauser, Science, July 14, 1916, 5\%-58.) 


\section{MOUNTING}

After staining, the sections are opaque; they must be rendered transparent for microscopic examination. This is accomplished by permeating the sections with oil; but since oil and water are not miscible, the tissue must first be thoroughly dehydrated with alcohol. Immersing thin sections in 95 per cent. alcohol for three to five minutes is usually sufficient for this purpose unless xylol is to be used as the clarifying oil or unless the stain is injured by so prolonged an immersion. In either of these cases absolute alcohol is to be used for dehydration, because of its more rapid and thorough action.

Clarification.-Sections, either free or fastened to the slide, are immersed in oil until clear. Free sections will at first float on the oil, but when fully permeated will sink. Attached sections should lose all traces of 'milky' appearance. The following oils are commonly used for clarification: Bergamot, origanum cretici, cajuput, clove, carbol-xylol (pure carbolic acid, melted, 25 to 33 c.c.; xylol, 75 to 67 c.c.), and xylol. Xylol (xylene) is the most desirable in that it is perfectly miscible with the balsam in which the section is usually mounted, and is finally lost by evaporation. It will not act in the presence of the least trace of water. Carbol-xylol has the advantage of a slight affinity for water; this is also true of the heavier oils. Complete dehydration is therefore not required. Bergamot is desirable for celloidin sections, but has the disadvantage of rapid deterioration, after which it dissolves the celloidin. Either origanum or cajuput oil, or a mixture of the two, serves well for celloidin sections, but leaves them somewhat stiffer than does bergamot oil. The latter is therefore preferable for elastic tissues. On the whole, origanum serves best for routine work with celloidin sections, xylol or carbol-xylol for paraffin.

After clarification celloidin sections must be transferred to a slide. This is accomplished by means of a metal lifter or by a strip of rice paper (ordinary cigarette paper does nicely). The section, lying on the paper, is inverted upon the surface of the slide, to which it remains adherent after the paper is gently lifted. The excess of oil is then removed with blotting paper or by gentle pressure with a folded towel, a drop of xylol-balsam applied, and the cover glass dropped into position. The preparation is permanent.

Xylol-balsam is prepared by adding to Canada balsam sufficient xylol so that the mixture will have a thick, syrupy consistence, but will drop from a glass rod without stringing.

Sections may also be permanently mounted in glycerin without pre- 
vious dehydration, the edge of the cover glass being, after some hours, covered with a ring of King's cement.

Glycerin jelly is also serviceable, and does not require cementing of cover glass.

Neutral Balsam.-Sections may frequently be rendered more permanent by the use of neutral balsam, prepared as follows:

Dilute Canada balsam with xylol until it acquires a very thin watery consistence. Add sodium bicarbonate in excess. Shake thoroughly, and allow to stand in a stoppered bottle for twelve hours or more. Filter; this is readily, though slowly, accomplished if the dilution is sufficient. Permit the solution to stand in an open vessel, protected from dust, until it evaporates to the proper consistence for use.

Gum-Damar.-This material, also dissolved in xylol, is in some respects even a better mounting medium than balsam; it does not turn yellow with age.

Euparal.-This new mounting medium is for most purposes the best. Sections are mounted direct from 95 per cent. alcohol. Delicate tissues may thus be spared the passage through absolute alcohol and oil. This curtailment of the technic is also a saving of expense. Euparal has, moreover, a lower index of refraction than balsam or gum-damar, and is thus more favorable for the demonstration of cytologic details (see Lee, "Vade Mecum," 8th ed., p. 247).

For more detailed and extensive information on histologic technic, and microchemic methods, the following books should be consulted:

1. Lee: "The Microscopist's Vade Mecum" (8th ed., 1913). Blakiston, Phila.

2. Mann: "Physiological Histology-Methods and Theory." Clarendon Press, Oxford, 1902.

3. Guyer: "Animal Micrology." Univ. of Chicago Press, 1906.

4. Hardesty: "Neurological Technique." Univ. of Chicago Press, 1902.

5. Kingsbury: "Laboratory Directions in Histology-Histological T'echnique." Ithaca, N. Y., 1910.

6. Mallory and Wright: "Pathological Technique." Saunders, Phila.

7. "Enzyklopædie der mikroskopischen Technik" (2d ed., 1910). Urban und Schwarzenberg, Berlin.

8. Gage: "The Microscope" (11th ed., 1911). Comstock Pub. Co., Ithaca, N. Y. 



\section{DIRECTIONS FOR LABORATORY WORK}

\section{INTRODUCTORY EXERCISE}

This exercise is designed to acquaint the student with the diagnostic features of certain more common laboratory materials which may become incorporated with histologic preparations; and to serve as a preliminary test of the student's facility in the use of the microscope and of his acuity of observation.

(1) Mount under cover glass in a drop of water a human hair, a strand of wool, and a few rabbit hairs. Examine with the low power (l.p.) of the microscope. Sketch (1) a short segment of each as seen with the high power (h.p.).

(Use only medium hard pencil. Make no mark that does not correspond with some structural feature of specimen under observation.)

In what features do the three hairs resemble each other?

In what features do they differ?

Compare surface features with structure of axis by changing the level of focus.

(2) Mount on separate slides in water:

(a) a few cotton fibers

(b) a strand of silk

(c) some finely separated threads of linen.

Examine with l.p. Note differential features. Sketch $(2,3,4)$ short segments under h.p.

(Label all drawings. Print labels. Use broken line leaders, ending with arrow at point or structure to be indicated.)

(3) Make water mount of a small mass of a common mould. Notice the long branching filaments (hyphæ); and the spheroidal spores, from which hyphæ may occasionally be seen sprouting. Sketch (5) portion of a hypha. Are the hyphæ segmented?

(No attempt should be made to execute a drawing of a preparation until after a precise conception has been acquired of the structure under examination as the result of an intelligent study with both the low and high powers of the microscope.) 


\section{THE CELL}

\section{(A) THE PLANT CELL.}

(1) Mount in water a small piece of the membrane separating the inner layers (leaves) of an onion. Note shape and size of cells, manner in which the cells are grouped, location and relative size of nucleus, nucleoli, cell wall, and character and conter.ts of the cytoplasm. Identify the rod-shaped mitochondria.

Make l.p. sketch (6) of a small group of cells; and h.p. sketch (7) of a single cell. Compare with section of cork. Difference? Propriety of name 'cell'?

(2) Make similar preparation, study and sketches $(8,9)$ of a thin slice of the onion leaf. Identify an air globule.

(3) Mount in water a thin slice of the potato. Sketch (10) under h.p. a cell, noting carefully the nucleus and the starch grains. Make careful sketch (11) of a large starch grain. Add a drop of tincture of iodine, and note the color change of the starch grains; and observe again the shape, size and location of the nucleus.

(4) Examine small portion of the leaf of a common higher moss (e.g., Bryum), and sketch h.p. (12) a cell, noting especially its robust CELL WALL, and its content of chlorophyll bodies (chloroplasts).

\section{(B) THE ANIMAL CELL.}

(1) Scrape gently with finger inner surface of cheek and mount the débris. Note the greatly flattened plate-like cells (squamous cells). Make 1.p. sketch (13) of a small group; h.p. sketch (14) of a typical isolated cell.

(2) Ovum (egg cell). Make a careful drawing (15) (from demonstration slide of starfish ovary) of a large egg. Note endoplasm, exoplasm, cell membrane, and nuclear membrane. In the cytoplasm observe spongioplasm, hyaloplasm, microsomes; in the nucleus, chromatin, nucleolus, linin, chromioles, and karyosomes. Character of cytoplasm?

(3) Sketch $(16, a)$ cell with mitochondria (demonstration slide); $(16, b)$ cell with ergastoplasmic fibrils (e.g., acinar cell of pancreas, or lining entodermal cell of yolk-sac of $10 \mathrm{~mm}$. pig embryo).

(4) Sketch (17) astral system (demonstration slide of maturing or segmenting egg of some invertebrate, e.g., starfish, clam). Note centrosome, centriole, centrosphere, astrosphere, and achromatic spindle. 
(5) Sketch (18) section of full grown ovum of blood-starfish (Henricia sanguineolenta); noting especially the multinucleolated condition of the nucleus, and the granulo-alveolar character of the cytoplasm. The eggs of many amphibians during later growth stages also have a multinucleolated nucleus.

Sketch (19) a small area of this cytoplasm demonstrated with an oil immersion lens.

(6) Living Protoplasm. Mount a drop of a hay infusion culture of amẹæ and paramecia.

Examine first a paramecium. Observe ciliary movement. When the protozoon comes to rest, note exoplasm, endoplasm, metaplasm, nuclei (macronucleus and micronucleus), contractile vacuoles and the finely granulo-alveolar character of the cytoplasm. Sketch (20). Observe the cytoplasmic changes occurring during the death of the animal.

In an ameba, observe manner of progression (ameboid movement), the nucleus, food vacuoles, water and contractile vacuoles; and note the homogeneous or very finely granular character of the protoplasm. Sketch (21). Observe the protoplasmic changes during death.

(Unless otherwise specified all drawings are in future to be made with the high power of the microscope.)

\section{CELL DIVISION}

\section{(A) AMITOSIS (Akaryokinesis, Direct division).}

(1) Sketch (22) several stages in the amitotic division of the nucleus (from demonstration preparation, e.g., ductuli efferentes of epididymis of mouse; mesenchyma of young embryo-10 da. turtle embryo). Study direct division or 'budding' of yeast cells.

(2) Sketch (23) a multinucleated ('giant') cell from the yolk sac of a 10 to $15 \mathrm{~mm}$. pig embryo (demonstration preparation). These cells arise from mononucleated cells through amitotic division of the nucleus.

\section{(B) MITOSIS (Karyokinesis, Indirect division).}

(1) Sketch (24) successive steps in the mitotic division of cells from the root tip of some vigorously growing young plant (e.g., dog-tooth violet, onion, hyacinth, spiderwort (tradescantia), etc.):

(a) cell with nucleus in the resting condition.

(b) nucleus with close spireme.

(c) with loose spireme. 
(d) segmented spireme (Prophase stages).

(e) chromosomes in equatorial plate (monaster figure); Metaphase stage.

(f) some stage of the migration of the daughter chromosomes (derived from a longitudinal splitting of the prophase mother chromosomes) towards the poles (Anaphase). (The double group of chromosomes of the anaphase stage is designated the disaster figure.) ('The achromatic spindle holding the chromosomes is called the amphiaster.)

$(g, h, i)$ three stages in the reconstitution of the nuclei of the two daughter cells (Telophase); these are the same as the prophase stages, but follow in inverse order. Note presence of mid-body or cell-plate at anaphase.

How do the two daughter cells resulting from a division differ in shape, and in the disposition of their major axis, from the mother cell?

Note changes in nuclear membrane; formation of achromatic spindle (centrosomes are lacking in flowering plants); different position of chromosomes on spindle at metaphase and anaphase.

(2) Make a similar series of drawings (25) of stages of mitosis in the epithelial cells from sections of the skin of the tail of an amphibian larva (e.g., salamander, frog). (Demonstration slide.)

\section{HISTOGENESIS}

(1) Sketch (26) several phases in the segmentation of the egg of the starfish, including the two and four cell (blastomere) stages.

(2) Sketch (27) a transverse section of a vertebrate embryo (e.g., frog or salamander larva), showing an early stage in the formation of the germ layers. Label ectoderm, mesoderm, entoderm, notochord, neural groove (canal), somite, celom, and the primitive intestine.

\section{EPITHELIUM}

\section{Simple (Non-stratified).}

(A) POLYHEDRAL (e.g., column of liver cells).

The polyhedral represents the least modified type of cell, from the standpoint of shape. The original or embryonic shape of all cells is spheroidal. All the morphologic types of cells may be interpreted in terms of a mechanically modified spheroidal cell. 
(1) Sketch (28) a few isolated cells from a dissociated (macerated) preparation of the liver. Note the number and position of the nuclei, general shape of the cell, character of cytoplasm, cytoplasmic granules (glycogen) and droplets (fat), and cell membrane (?). (Dissociated tissues are best preserved for study in a solution of equal parts of absolute alcohol, glycerin and water).

To a teased fresh preparation of liver in physiologic salt solution add a drop of a 5 per cent. aqueous solution of acetic acid. This will cause the nucleus to appear more distinctly. It dissolves also all albuminous granules, but leaves unaltered fat and lipoids. (This method of treatment may be profitably employed with all fresh dissociated (teased) preparations. In the case of the connective tissues, the acetic acid will cause the collagen fibers to swell and finally disintegrate, but will have no effect upon the elastic fibers.)

Subsequently add to the same preparation a drop of a 1 per cent. solution of methylene blue. This will stain the nucleus and thus cause it to contrast more sharply with the cytoplasm. (Other equally good staining solutions for isolated tissues are: (a) a 1 per cent. solution of methyl green in 20 per cent. alcohol; (b) 1 per cent. aqueous solution of borax carmin; (c) dilute solution of any of the hematoxylin stains; (d) or a 1 per cent. aqueouis solution of eosin may be employed to stain the cytoplasm, producing in this way also a heightened contrast between nucleus and cytoplasm.)

(The best way to add any fluid to a macerated preparation under a cover-glass is to place a few drops of the solution at one edge of the cover-glass, and cause it to flow under the glass by withdrawing fluid from the opposite edge by means of blotting paper.) Distinguish between physical and chemical coloration.

(2) Sketch (29) a small area of a stained section of the liver.

(B) SQUAMOUS (Pavement epithelium).

(1) Mesothelium. Sketch (30) small area of toto mount of piece of mesentery of cat, treated with silver nitrate solution to outline the intereellular cement and lightly stained with Delafield's hematoxylin. Note the character of the cell borders, shape of cell, position and shape of nucleus, stomata (?), guard cells (?), and stigmata. Study a stained section of the amnion.

(2) Endothelium. Sketch (31) small area of lining of capillary or small vein (e.g., from toto mount of mesentery, or pia mater of brain). Note sinuous character of cell outline. What is the relation of 
the long axis of the endothelial cell to the long axis of the blood vessel? How does it differ in shape from the mesothelial cell? Sketch (32) also a few endothelial cells (profile view) from a stained section of a small blood vessel.

(3) Mesenchymal Epithelium (ranges from the cubic to the squamous type of cell). Sketch (33) a group of the epithelial cells from a stained section of the synovial membrane. (This is more generally of the cubic type of epithelium.) Note membrana propria and tunica propria.

(4) Sketch (34) a few of the squamous cells (flattened entodermal cells) lining the pulmonary alveoli. from a stained section of the lung; also (35) a small area of the superficial cells of the skin or of the mucosa of the esophagus.

(C) COLUMNAR.

\section{(a) Plain.}

(1) Sketch (36) a few isolated columnar cells from a macerated preparation of the mucosa of the stomach, or of the small intestine. Note striated border, and position of nucleus.

(2) Sketch (37 a) a small area of the epithelium from a stained section of the stomach or small intestine. Note proximal (basal, attached) and distal (free) ends of cell, striated border and terminal bars. Note terminal bars in those portions of the sections where the columnar epithelium is cut in a tangential plane near the distal border. Sketch $(3 \%, b)$.

(b) Modified.

(1) Goblet cell. Sketch (38) several isolated goblet cells from a macerated preparation of colon.

(2) Sketch (39) small area of epithelial lining of colon, from stained section. Functional tenure of a goblet cell?

(3) Pyramidal (glandular) cell. Sketch (40) a transverse section of fundus of a tubular or tubo-acinar gland (e.g., gastric, salivary), showing the pyramidal shape of the glandular epithelial cell of the secretory fundus or acinus. Note the lumen of the gland, and the character of the cytoplasm at the distal and proximal poles of the cells.

(4) Ciliated cell. Sketch (41) several isolated ciliated cells from a macerated preparation of the trachea. (These columnar ciliated cells formed part of a pseudostratified columnar ciliated epithelium. See below.)

(5) Sketch (42) a portion of the epithelium of the uterus or oviduct, from a stained section. 
(6) Study in physiologic salt solution ciliated cells scraped from the roof of the mouth of a pithed frog. Observe the activity of the long cilia. (An equally instructive preparation can be obtained from the gills of a living oyster or clam.) Sketch (43).

(7) Pigmented cell. Sketch (44) a few pigmented cells (cubic) from the pigment layer of the retina or the iris, from a stained section of the eye.

(8) Flagellate cell. Sketch (45) a spermatozoön from a stained section of a mammalian testis; or from a preserved specimen of human semen.

(9) Neuroepithelium. Sketch (46) a few cells from the bacillary (rod and cone cell) layer of the retina, in a stained section of the eye.

(D) $C U B I C$.

Sketch (47) a small extent of cubic epithelium in the loop of the uriniferous tubule, from a stained section of the kidney.

\section{Complex (Stratified) Epithelium.}

(1) Squamous. Sketch (48) portion of the mucosa of the esophagus, from a stained section. (A stained section of cornea or of thin skin is almost equally favorable.) Note the intercellular bridges between cells of the middle layers, especially prominent in skin.

(2) Sketch (49) the several types of cells included in a stratified squamous epithelium, from a macerated preparation of the esophageal mucosa.

(3) Pseudostratified columnar. (This type is almost invariably ciliated-exceptions, larger ducts of glands, portion of ductus deferens.) Sketch (50) small extent of mucosa of trachea or bronchus, from a stained section. (This type of epithelium is sometimes erroneously referred to as 'stratified columnar'. True stratified columnar epithelium is of meagre and variable occurrence, sometimes as patches among the pseudostratified epithelium. Stratified columnar epithelium occurs in the mucosa of the intermediate portion of the male urethra.)

(4) Transitional. Study carefully and draw (51) a small area of the lining epithelium of the bladder or ureter (preferably in the slightly distended condition) from a stained section.

(5) Sketch (52) the several characteristic types of cells which constitute a transitional epithelium from a dissociated preparation of the bladder mucosa. 


\section{CONNECTIVE TISSUE}

(A) FIBROUS VARIETIES (or connective tissue proper). These develop from the middle germ layer (mesoderm).

(1) Embryonal. Study the subcutaneous tissue in a stained transverse section of a 10 to $20 \mathrm{~mm}$. pig embryo. Note differences in appearance of deeper and more superficial regions, representing earlier and later stages in the histogenesis. Draw (53 a and b) small area from each of the two regions. Note the shape of the cells, and the character and quantity of the intercellular substance. Is embryonic connective tissue (mesenchyma) composed of discrete cells or does it correspond to a syncytium? Does the matrix contain fibrils?

(2) Mucous (Gelatinous). This type is found typically only in the umbilical cord, where it is known as the jelly of Wharton. The vitreous humor of the eye is also comparable to mucous connective tissue. In a stained section of the umbilical cord study a region, some distance removed from the large central blood vessels, and note the various forms of cells; and the character and quantity of the intercellular substance (matrix), including the fibrils. Draw (54) a small area. Is mucous connective tissue syncytial in character? Is it vascularized? Define 'fibroglia.'

(3) Reticular (Retiform). This forms the supporting framework for the cells (lymphocytes) in lymphoid tissue. Select for study a thinner region of stained section of some lymphatic organ (e.g., hemal node, lymph node, spleen) where the lymphocytes are sparse. Draw (55) a small area, noting the character of the fibril bundles, and their relation to the lymphocytes and to certain stellate connective tissue cells. Do the bundles anastomose? (A demonstration preparation of the reticulum of a lymph node or of the spleen, from which the lymphocytes have been removed by 'digestion', or by shaking, or by washing in warm,water after gelatin injection and freezing-Mall's technic-may be substituted to advantage for this exercise.)

(4) Areolar (Loose fibro-elastic). This type includes fibro-elastic structures of greatly varying densities. Spread out taut on slide a thin film of fresh subcutaneous or intermuscular tissue of some mammal (e.g., guinea pig, rabbit, rat). Allow the ends to dry fast to the slide, keeping the center moist with physiologic salt solution. Mount under cover-glass and examine the several types of fibers. Note the dull, white, wavy bundles of collagen fibrils, and the clear, shiny, stouter elas- 
tic fibrils. The latter may be arranged in a wide-meshed network. Sketch (56) a bundle of white fibers. Do the bundles anastumose? Sketch (57) also several elastic fibers. Do they branch? Explain the curled character of some of the fibers. Add several drops of a 5 per cent. aqueous solution of acetic acid. Note that the collagen fibrils swell, some of the bundles become beaded, and finally disintegrate. What is the significance of the beaded condition of the bundles of collagen fibrils? Note difference in structural and chemical characteristics of the collagen (white) and elastic (yellow) fibers. Add a drop of a nuclear dye (e.g., methylene blue solution) and examine and sketch (58) the several types of cells: (a) spindle-shaped cell; (b) plasma cell; (c) lamellar cell; (d) clasmatocyte; (e) granular leukocyte. Wash the preparation in distilled water and add several drops of a 1 per cent. solution of magenta (basic fuchsin) in 70 per cent. alcohol. This will stain the elastic fibers (red). Examine the preparation for final study of the elastic fibers.

Sketch (59) a small area of subcutaneous tissue from a section of skin stained with Weigert's elastic tissue stain (resorcin-fuchsin), Delafield's hematoxylin and Van Gieson's stain (picric-acid-fuchsin). Note cells, fibers and intercellular spaces ('tissue spaces', filled with 'tissue fluid').

(5) Dense White Fibrous Tissue (e.g., tendon, sclera of eye). Study a longitudinal section of tendon. Sketch (60) a small area under the l.p. noting the fasciculi. Sketch (61) h.p. a small area from a transverse section of tendon. Note shape of cells and nuclei, their arrangement with respect to each other and the bundles (fasciculi) of white fibrils. Tease a fragment of tendon (from tail of rat or leg muscle of rabbit) in salt solution; add a few drops of a 5 per cent. aqueous solution of acetic acid to destroy some of the collagen fibrils; add a few drops of methylene blue solution; sketch (62) several isolated cells. (A stained macerated preparation of tendon is an equally favorable material for study of tendon cells.)

(6) Dense Elastic Tissue. Study a teased or macerated preparation of ligamentum nuchæ of ox, and make drawing (63) of a few fibers. Note transverse markings in portions of some fibers. Sketch (64) small areas of stained (Van Gieson's dye) sections, (a) transverse and (b) longitudinal, of ligamentum nuchæ.

(B) MODIFIED.

(1) Adipose (Fat) Tissue. This is modified areolar tissue. Study a cover-glass mount of fresh fat, from the subcutaneous tissue of 
a mammal (e.g., mouse, rabbit). As the fat cools note the formation of margaris crystals. Add a drop of methylene blue and sketch (65) several cells. Note shape and size of cell, position of nucleus, and character of cytoplasm. Sketch (66) several cells from a teased specimen of fresh fat preserved in osmic acid. What is the color of the fat globule? Add eosin stain and note cytoplasm, fat globule and nucleus. Sketch (67) small area of fat in subcutaneous tissue from stained section of skin or capsule of some gland, e.g., pancreas, kidney. Note position of nucleus, and distribution of cytoplasm. What effect has alcohol and ether preservation on fat? Distinguish between nuclei of connective tissue and fat cells.

Sketch (68) developing adipose tissue from stained section of subcutaneous tissue of young animal. (Developmental stages are commonly to be found also in the areolar tissue enveloping the pancreas, kidney and other viscera.)

(2) Lymphoid (Adenoid) Tissue. (Modified reticular tissue.) Study a stained section of a small lymph node. Note cortex and medulla. Sketch (69) small area of medulla, including the denser tissue of the medullary cords and the enveloping looser tissue of the sinuses. In the sinuses the reticular tissue is especially prominent.

(3) Pigmented Connective Tissue. (Modified areolar tissue.) Study section of derma and subcutaneous tissue of negro skin; or the choroid coat (middle tunic) of the eye; (or toto mount of skin of young salamander or lizard). Sketch (70) several pigment cells. Note various irregular shapes of cells, and form and distribution of the pigment granules (melanin granules). Does the nucleus contain pigment? What is the distinction between a 'pigment cell' and a 'pigmented cell'?

(4) Notochordal Tissue. This is of entodermal origin (ectodermal in the guinea pig; G. Carl Huber), and resembles connective tissue only in its, grosser features. It is represented in the adult only in the nuclei pulposi of the intervertebral discs. Sketch (71) small section of notochord from stained transverse section of a 10 to 20 mm. pig embryo.

(5) Neuroglia. This is the connective tissue of the central nervous system (brain and spinal cord). It is of ectodermal origin. It will be considered in connection with nervous tissues.

(C) SUSTENTATIVE.

(a) Cartilage (Gristle).

(1) Hyaline. Make l.p. study of cartilage plate and enveloping 
perichondrium (articular, costal, tracheal or bronchial). Sketch (72) narrow segment of entire plate. Make h.p. drawing (73) of (a) perichondrium and (b) central portion of plate. Note carefully transition from perichondrium to cartilage; cell groups, lacuna, capsule and hyaline matrix. Are blood vessels, lymph vessels or nerves discernible in the matrix? The fetal precursor of the majority of bones is also hyaline cartilage. What is the fundamental structure of the hyaline matrix?

(2) Elastic Cartilage (Yellow fibro-cartilage). Study cartilage plate of the epiglottis or the external ear (e.g., ox) in stained section. Note elastic fibers in the hyaline matrix. (Some specific elastic tissue stain should have been employed with the section.) Sketch (74) a segment from perichondrium to center inclusive.

(3) Fibro-Cartilage (White fibro-cartilage). Study a stained section of an intervertebral disc (e.g., ox) or the semilunar cartilage of the knee. Is a perichondrium present? Note the collagen fibers in the matrix (a specific collagen fiber stain should be employed, e.g., Mallory's or Van Gieson's). How does fibro-cartilage differ from hyaline cartilage and from tendon? Sketch (75) a small area of central portion of cartilage disc. Structural differences between intervertebral disc and semilunar cartilage of knee?

(4) Precartilage. Sketch (76) a small area of the condensed mesenchyma in the region of a future bone in a transverse stained section of some mammalian embryo, e.g., pig of 10 to $15 \mathrm{~mm}$.

(5) Vesicular Supporting Tissue. A variety of connective tissue found in the sesamoid bone of the tendon of Achilles of the frog, characterized by abundant large clear cells scattered among bundles of collagen fibrils, is somewhat similar to mammalian fibro-cartilage, and represents a transition from dense connective tissues to cartilage. Study a section of the tendon of Achilles of the frog. Note differences and similarities between this tissue and mammalian tendon and fibro-cartilage. Sketch small area.

(b) Bone (Osseous tissue).

(1) Gross Study. Study macroscopically and with hand lens a longitudinally cut long bone (e.g., femur). Note: central marrow cavity, peripheral compact bone of diaphysis, and terminal cancellous bone of epiphyses. Note also jagged border of marrow cavity terminally.

(2) Macerated Compact Bone (ground section of bone). Study l.p. a transverse section through the diaphysis. Note: central marrow cavity, outer circumferential (periosteal) lamellæ; inner circumferential (endosteal) lamellæ; interstitial lamellæ; Haversian systems 
(canal and lamellæ); lacunæ, and canaliculi. Sketch (7\%) a complete Haversian system. What are the contents of the Haversian canal? What is the structure of the osseous lamellæ? Content of lacunæ? Significance of canaliculi? Study a fragment of a ground longitudinal sẹction of the shaft (diaphysis) of a long bone. Sketch (78) a Haversian canal at the point of branching, with the enveloping lamellæ. Note: Volkman's canals of the circumferential and interstitial lamellæ. Are the latter connected with the Haversian systems? Do the Haversian systems connect with the periphery and the central marrow cavity? What are the nutrient foramina of a bone? How many to the shaft of a long bone? to an epiphysis?

(3) Decalcified Coмpact Bone: Study stained longitudinal section of end of long bone including epiphysis and part of diaphysis. Note: periosteum, muscle attachments, fibers of Sharpey, Haversian systems, bone cells, endosteum, marrow. Sketch (79) typical areas from epiphysis (cancellous bone) and diaphysis (compact bone), and periosteum.

(4) Bone Development.

(a) Enchondral Ossification (Substitution Bone). Study a longitudinal stained section of a decalcified small fetal long bone (e.g., phalanx of finger, or metacarpal; or some small bone of fetal pig). Sketch (80) l.p. a narrow strip of section through center of ossification and including terminal articular cartilage and central marrow cavity. This drawing should illustrate the successive stages in the process of enchondral ossification. Sketch (81) h.p. a strip through area of ossification. Note: calcified cartilage remnants and adjacent rows of disintegrating cartilage cells; primary marrow spaces; periosteal bone; spicules of primary bone; and epiphyseal line of cartilage? Sketch (82): (a) periosteum; (b) strip of periosteal bone, including a periosteal bud of osteogenic tissue within a Haversian space (the precursor of a Haversian system); (c) plate of enchondral bone with central calcified cartilage core and surface layer of primordial bone, bone cells, bone lacunæ, and peripheral osteoblasts and asteoclast (in a Howship's lacuna) ; (d) primordinal marrow areola with marrow cells, including blood cells, osteoblasts and giant cells (megakaryocytes and polykayocytes-osteoclasts).

(b) Intramembraneous Ossification. Study stained section of jaw of some mammalian fetus (e.g., of pig fetus of about $35 \mathrm{~mm}$.) for developing membrane bone (cancellous bone). Sketch (83 a) small area of core of plate. Sketch $(83 \mathrm{~b})$ an osteoclast, noting the content of resorbed bone globules. 


\section{MUSCULAR TISSUE}

(A) SMOOTH (Unstriped, Plain, Non-striated, Involuntary Smooth).

(1) Sketch (84) several isolated cells from a macerated preparation of smooth muscle from the wall of the bladder, stomach or intestines of the cat, or from a teased fresh preparation of bladder of frog. Note shape of cell, position of nucleus, fibrillar and granular content of cytoplasm, perinuclear coarsely granular sarcoplasm, and cell membrane (sarcolemma).

(2) Study smooth muscle in a stained section of the stomach or intestines. Make l.p. sketch (85) of area including fibers cut transversely and longitudinally. Sketch (86) h.p. a small area of cross-cut fibers. Note variations in shape of cross sections and the position of the nucleus. Why do not all of the cross sections of the fibers contain a nucleus? Sketch (87) h.p. small area of longitudinally cut fibers. Note the manner in which the fibers are joined into membranes. Why do many of these cells appear much shorter than the isolated cells? Study wall of one of the blood vessels of the umbilical cord. Sketch (88) a few cells. Is an intercellular cement present? intercellular bridges? Study also smooth muscle of bladder, and of a pregnant uterus. Where in the body are the longest smooth muscle cells found? Study the nerve supply of the smooth muscle of the intestine in a demonstration preparation.

\section{(B) CARDIAC (Involuntary striped).}

(1) Sketch (89) a fragment of the cardiac syncytium (myocardium) from a macerated preparation of the heart; or from a teased fresh specimen. Note the branching of the muscle trabeculæ, position of nuclei, fibrils, sarcoplasm, cross striations, and sarcolemma.

(2) Make sketch (90) of small area of a stained section including both transversely and longitudinally cut trabeculæ ('fibers'). Note position of the nucleus, myofibrils, cross striations (ground membranes, Z lines, or telophragmata) and intercalated discs. What is the relationship between the telophragmata and the sarcolemma? between the telophragmata and the nuclear membrane? Are other striations present besides the Z-stripes?

(3) Sketch (91) small area of a specially prepared demonstration slide (Zimmermann's technic) to show the several types of intercalated discs. Tease in glycerin a fragrnent from a hemalum-stained block of myocardium. Determine the complete form of the intercalated discs. 
(4) Sketch (92) portion of developing myocardium from a stained transverse section of some mammalian embryo (e.g., pig embryo of from 10 to $20 \mathrm{~mm}$. length). Note the shape, and the myofibrillar cytoplasmic content, of the constituent myoblasts. Do the myoblasts anastomose? Is heart muscle originally syncytial in character?

\section{(C) STRIPED (Striated; Voluntary striped).}

(1) Tease a fragment of fresh frog's or other vertebrate's muscle in normal salt solution, or in Ringer's solution. Mount under cover-glass and study carefully, noting position of nuclei, longitudinal myofibrils, and cross striations ( $\mathrm{J}$ or isotropic dises; $\mathrm{Q}$ or anisotropic dises, and ground membranes or membranes of Krause). By exerting pressure on cover-glass, crush some of the fibers. Look for the sarcolemma spanning a break in the sarcoplasm. (This is especially readily demonstrated in the frog's muscle.) Add drop of dilute acetic acid and note effect. Then add a drop of the methylene blue stain, observe the shape and position of the nuclei, and sketch (93) a fiber, including the sarcolemma at the level of fracture.

(2) Study fibers from a macerated preparation, preserved in the alcohol-glycerin mixture. Note the terminal cleavage of some of the fibers into discoid structures, the sarcomeres; and the splitting of others into the constituent myofibrillæ (sarcostyles). Sketch (94) a fiber showing cleavage into sarcomeres and (95) one showing splitting into sarcostyles.

(3) Study a stained transverse section of striped muscle (e.g., tongue; skeletal muscle). Draw (96) a number of adjacent fibers, indicating nuclei, myofibrils, Cohnheim's areas (Kölliker's columns), and sarcolemma. Sketch (97) a fiber in longitudinal section, showing the several cross striations. How do the cross striations in striped voluntary muscle differ from those in cardiac muscle?

(4) Make drawing (98) of a portion of a longitudinal section of some arthropod striped muscle fiber (e.g., insect leg or wing muscle) from a demonstration preparation, showing several phases in the contraction process. Note the several striations: isotropic and aniostropic discs, $\mathrm{Z}$ and $\mathrm{M}$ membranes (telophragmata and mesophragmata); and the additional stripes of: (a) the accessory disc (of von Rollet and Englemann) and (b) the resulting terminal disc (of Merkel). Explain different arrangement of the striations in contracted and extended fibers. Difference between a contraction 'band' and a contraction 'wave'?

(5) Sketch (99) l.p. a portion of a transverse section of a small 
muscle, showing epimysium, muscle fasciculi and enveloping perimysium, individual fibers and enveloping endomysium.

(6) Study an injected specimen of skeletal muscle in a stained longitudinal section. Draw (100) a few fibers showing the abundant distribution and intimate disposition of capillaries and arterioles.

\section{(D) TENDON.}

(1) Study a stained transverse section of a tendon. Note epitendineum, peritendineum enveloping the tendon fasciculi, and the endotendineum. Make a sketch of same under the low power (101). Sketch (102) h.p. small area of a transversely cut tendon bundle. Note the shape of the tendon cells and their relation to the smallest subdivisions (primary bundles) of the fasciculi. Sketch (103) two adjacent tendon fasciculi, from a stained longitudinal section. Note peritendineum; the disposition of the cells with respect to the tendon bundles, and to each other; and the disposition of the nuclei in successive cells. Genetic relationship between tendon and muscle?

(2) Study the area of junction between muscle and tendon, from a stained longitudinal section (e.g., leg muscle of frog). Sketch (104). What is the relation of the tendon fibrils to the muscle fibrils? to the sarcolemma? Note the increased abundance of nuclei at this level. Significance? Two chief modes of muscle-tendon connections?

(3) Sketch from specially prepared demonstration slides: neuromuscular (sensory) end organ, or muscle spindle (105); (d) neurotendinous (sensory) end organ, or tendon spindle (106); (c) motor end plate (10\%).

\section{NERVOUS TISSUE}

\section{(A) THE NEURON (Nerve cell).}

(1) Study under cover-glass an isolated nerve cell (neurocyte) from a macerated preparation, or a teased fresh specimen, of the gray matter of the spinal cord of the ox. Add a drop of stain.' Sketch (108) a complete cell, noting axon, dendrons, axon hillock (implantation cone), nucleus, nucleolus, and granular and fibrillar cytoplasmic contents. What are the marks of differentiation between an axon and the dendrons?

(2) Sketch (109) a similar cell from a stained transverse section of the spinal cord. 
(3) Sketch (110) a large nerve cell (e.g., large pyramidal cell of cerebral cortex) in a demonstration slide prepared by the Beilschowsky technic to show neurofibrils.

(4) Sketch (111) a similar cell from a demonstration preparation stained by Nissl's method to show the chromophilic substance (Nissl granules, chromophilic granules, tigroid substance).

(5) Sketch also (112) from Golgi preparation (a) a large pyramidal cell of the cerebral cortex; and (113) (b) a Purkinje cell of the cerebellar cortex.

(B) THE AXON ('Nerve fiber'; Axis cylinder process; Neurite).

(a) Medullated.-(1) Tease in Ringer's solution a fragment of a fresh medullated (myelinated) nerve (e.g., great sciatic; peroneal) from a mammal or the frog. Mount under a cover-glass and study a wellpreserved fiber, noting the axial axis cylinder and neuroplasm, the enveloping medullary (myelin) sheath, the peripheral nucleated sheath of Schwann (neurolemma), and the nodes of Ranvier. Add a drop of some nuclear stain, and sketch (114). Are all of the fibers myelinated? Are all of the same girth?

(2) Tease a fragment of a medullated nerve preserved in osmic acid. Mount and study an individual fiber, noting, besides the abovespecified constituents, also the Schmidt-Lantermann lines or incisures. What is the significance of the Lantermann segments? Sketch (115).

(3) Sketch (116) a small area from a stained cross section of the white matter of the spinal cord. Note the central axis cylinder and the enveloping myelin sheath, with its neurokeratin framework. Is a neurolemma present? Note also the neurogliar connective tissue filling the spaces between the fibers.

(4) Study under l.p. a cross section of a large medullated nerve (e.g., tibial; peroneal) in a stained preparation. Sketch (117) showing epineurium, perineurium, funiculus, endoneurium, Henle's sheath, and nerve fibers. Sketch h.p. (118) a small area of a funiculus. Are all the fibers of the same girth? Sketch (119) also several fibers from a stained transverse section of a medullated nerve preserved in osmic acid. Chemical character of myelin?

(5) Study under l.p. a longitudinal stained section of a medullated nerve. Sketch (120) a small extent of two adjacent funiculi. Sketch under h.p. (121) several adjacent fibers showing at least one node of Ranvier. What are the criteria for distinguishing between longitudinal 
section of a medullated nerve, a tendon or compact white fibrous tissue and smooth muscle?

(b) Non-Medullated Axon (Remak's fibers; Unmyelinated fibers). -(1) Search for sympathetic (autonomic) nerves, both transversely and longitudinally cut, in a stained section of some portion of the alimentary tract, in the connective tissue between the two muscle layers. Note also an occasional sympathetic ganglion cell. Sketch: (a) a bundle of fibers cross cut (122); (b) longitudinally cut (123) ; and (c) several ganglion cells (124). Study also a cross-section of the trunk of the vagus nerve; here non-medullated fibers are mixed with medullated fibers of all sizes. Sketch (125). Tease a fragment of the vagus nerve in Ringer's solution. Add a drop of stain. Differentiate between the myelinated and unmyelinated fibers and sketch (126) a few of the latter.

(C) GANGLIA.

(a) Spinal (Dorsal, Sensory):- (1) Study a stained section of a spinal ganglion. Sketch (12\%) several cells. Note nucleated capsule. How is this capsule related to the dendrons and to the neurolemma of the axon? Compare these cells with those of the sympathetic ganglia.

(2) Sketch (128) the several types of atypical cells in a spinal or cerebral ganglion (e.g., Gasserian) in a specially prepared section (Ranson-Cajal's pyridin-silver method). Note: (a) 'fenestrated' cells; (b) multipolar cells; (c) cells with collaterals ending in 'end bulbs'; and (d) the usual unipolar type. Explain the origin of the unipolar type. Which of the two divisions of its single process corresponds to a dendron?

(b) Sympathetic (Autonomic).-(1) Sketch (129) small area of a sympathetic ganglion (e.g., ciliary; otic; ganglia of myenteric plexus). Are the cells of the sympathetic ganglia enveloped by a nucleated capsule?

(D) NEUROGLIA.

Study a section of the cerebral cortex or spinal cord treated according to the Golgi technic for neuroglia. Sketch: (a) a larger long-rayed astrocyte (130); (b) a smaller short-rayed astrocyte or mossy cell (131). Study a second section treated according to the Benda or Weigert neuroglia technic. Sketch (132) a few cells and fibers. What structure in ganglia is the homologue of the neuroglia of the brain and spinal cord? What is the analogue? 


\section{(E) PERIPHERAL NERVE TERMINATIONS (END-ORGANS).}

(a) Nerve Endings in Epithelium.-(1) Sketch (133) a bundle of naked nerve end-fibrils in a demonstration slide of thick skin or cornea, stained by the intravitain methylene-blue method. In the same preparation search for tactile cells of Merkel. Note tactile meniscus at end of nerve fibril and the associated tactile cell. Sketch (134).

(2) Sketch (135) a taste bud from a circumvallate papilla of the human tongue or a foliate papilla of the tongue of the rabbit, in stained section. Note the gustatory cells, the sustentacular cells and the basal cells; and in specially stained preparation note also the nerve fibrils terminating in relation to the cells of the taste bud.

(b) Nerve Endings in Connective Tissue.-(1) Study a stained section of skin of finger tip. Distinguish between vascular and tactile papillæ of the derma. Sketch (136) a tactile corpuscle (of Meissner) from a tactile papilla.

(2) Sketch (13\%) a lamellar (Pacinian) corpuscle from a stained toto mount of a fragment of the cat's mesentery. Sketch (138) a similar corpuscle in section in the interlobular connective tissue, from a stained section of the pancreas. Sketch: (a) a corpuscle of Herbst (139); (b) a corpuscle of Grandry (140), from a stained section of duck's bill.

(c) Nerve Endings in Striped Muscle and in Tendon.-(1) sensory; muscle spindles and tendon spindles.

(2) motor; motor end-plates in muscle.

Study and sketch (141) from special demonstration slides. What is the character of the sole plate? relation to 'intermediate' or 'receptor' substance of myoneural junction? function of the different nerve endorgans? (Consult following scheme of classification, arranged in accordance with the terminology. proposed by Sherrington.) 


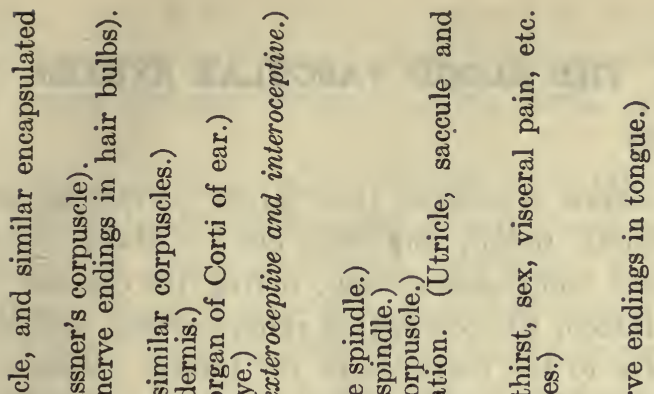

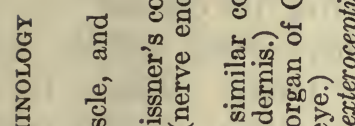

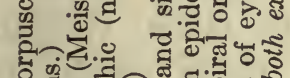

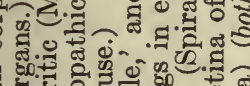

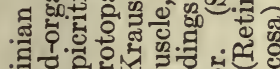

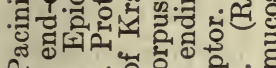
ब.

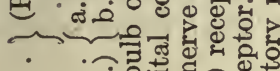

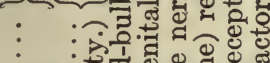

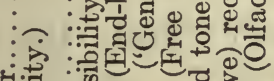

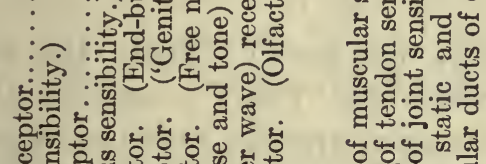

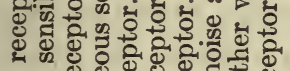

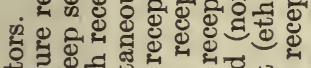

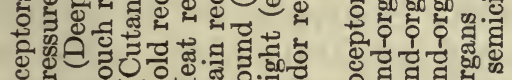

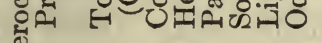

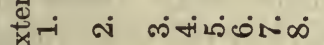
畕

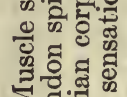
द्र. 투유. 㱐

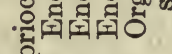

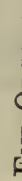
4
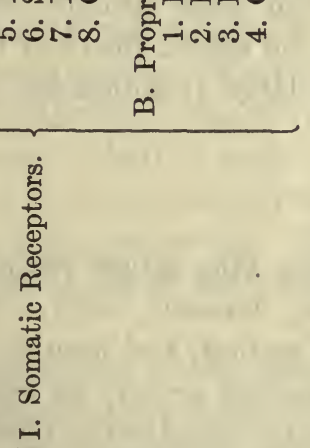

政高

空

ฮ્ఠ

फั

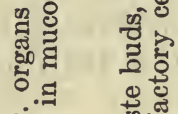

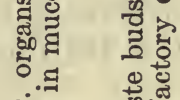

क्ष

कू

융ㅎㅀ

ठ․ㅇ

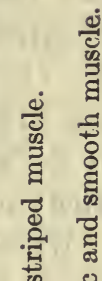

我

สై .

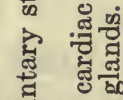

킁 ․․

$\rightarrow \quad 3050$

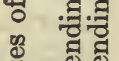

苛 00

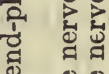

ठ্․

ठ की ठे छा

$\dot{4}$

(2)

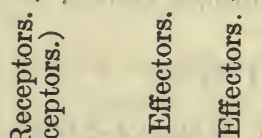

舅

ศि

동

'

乘造

¿ं

通
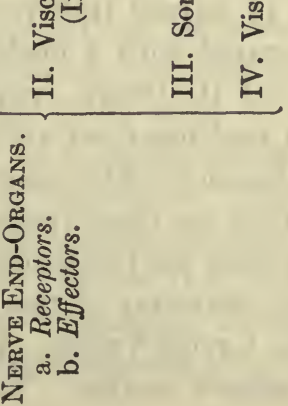


\section{THE BLOOD VASCULAR SYSTEM}

(A) ARTERIES.

(1) Medium-Sized Arteries (the larger peripheral arteries, e.g., the carotids, femoral, radial, popliteal, etc.). Study the vessel as a whole, in a stained transverse section, noting its circular outline, the generally empty lumen, the corrugated inner surface, and the thickness of the wall relative to the diameter of the lumen. Make a l.p. sketch (142) of entire vessel, indicating the three tunics: (a) tunica interna (intima); (b) tunica media, and (c) tunica externa (adventitia). Sketch h.p. (143) a narrow segment of the wall, noting the fundamental tissues of the several layers. Identify the internal and external elastic (fenestrated) membranes, and the vasa and nervi vasorum of the tunica externa. Which is the thickest tunic and what tissue elements does it include?

(2) Large Arteries (e.g., the aorta).-Study the wall of the aorta in stained section. Sketch h.p. (144) a segment of the wall. Compare with wall of medium sized artery. Note the relatively wider tunica interna, the much narrower tunica externa, the apparent absence of the internal and external elastic membranes, and the great abundance of elastic tissue among the muscle cells of the tunica media. Study also a similar section stained with some specific elastic tissue stain (e.g., resorcin-fuchsin), and note the elastic fibers in the three tunics. Compare aorta with some other large (conducting) artery, e.g., subclavian and common iliac.

\section{(B) VEINS.}

(1) Medium-Sized Veins (the larger peripheral veins, e.g., external jugular, basilic, median, femoral, etc.). Study the vessel as a whole, in stained transverse section, and note its relatively thinner wall as compared with a medium-sized artery, its generally collapsed condition, and its lumen frequently filled with blood. Compare in detail with a medium-sized artery, noting differences in composition and relative thickness of the several tunics. Sketch l.p. (145) the entire vessel, indicating the three tunics. Sketch h.p. (146) a narrow segment of the wall, noting the fundamental component tissues in each tunic. Note the delicate character of the internal and external elastic membranes.

(2) Larae Veins. (a) The Inferior Vena Cava.-Study the wall in a stained section. Compare with aorta. Note great thickness of 
tunica externa. Sketch h.p. (147) a segment of the wall, noting the fundamental tissues in each tunic. Note the longitudinal musele-bundles in the tunica externa. Compare with portal vein; with hepatic vein.

(b) The Superior Vena Cava. Sketch h.p. (148) a segment of a section stained with a specific elastic tissue stain noting the distribution of elastic fibers. Compare with pulmonary vein.

(3) VAlves. Study a longitudinal stained section of a large peripheral vein of the lower extremities (e.g., long saphenous vein). Identify the valves, and note their distribution, position with respect to tributaries, and their gross and minute structure. Sketch (149) a segment of the wall, including a valve. How many cusps are generally included in a valve? Why are valves less numerous in the veins of the upper than lower extremities, and why are they entirely lacking in the abdominal and thoracic veins, and in veins of smaller calibre than 2 $\mathrm{mm}$.? What specific structural differences obtain among the following veins: venæ cavæ, mesenteric, external jugular, pulmonary, umbilical and cranial? What is the functional significance of these differences?

\section{(C) SMALL VASCULAR COMITES.}

(1) Small Arteries and Veins. Many sections will contain pairs of smaller vessels. Study a larger pair in a stained section, and make detailed comparison with respect of relative gross size and form, condition of lumen, thickness of wall relative to diameter of bore, and relative thickness of the several tunics. What can you determine regarding the presence of elastic membranes? Make (a) l.p. sketch of pair (150); (b) h.p. sketch of segment of wall of each (151).

(2) Arterioles and Venules.-(a) Find a pair of vascular comites of this size in almost any stained section. Make h.p. sketch (152), noting especially relative thickness of the several tunics in the vessels of the pair.

(b) In a stained toto mount of the mesentery or the pia mater find a typical pair of comites and trace from arteriole and venules through precapillary twigs into the capillaries. Sketch (153) such a system, noting the differential marks between arteriole and venule, precapillary: artery and vein; and between the foregoing and capillaries. What are sinusoids? Retia mirabilia? How do they differ from capillaries?

(c) In stained sections identify precapillary arteries and veins and capillaries. Sketch (154). What is the chief differential feature between a precapillary artery and vein, and between these and a capillary? 
(d) In a toto mount of mesentery, treated with silver nitrate, sketch (155) the endothelial lining of one of the smaller vessels.

(e) Sketch (156) in longitudinal or oblique section a capillary, and a precapillary vein in the bone marrow of a developing membrane bone, e.g., jaw of pig fetus of $35 \mathrm{~mm}$.

(At this stage the student should review his slides, noting in each section: (a) the type of epithelium present; (b) the type of connective tissue; (c) muscle; (d) nerves; and (e) types of blood vessel. Several laboratory periods may be spent to advantage on this exercise.)

\section{(D) HEART (Cor).}

(1) Make gross study of heart (human, hog, dog or beef) and note in intact organ: (a) ventricles, (b) atria, (c) auricular appendages, (d) pericardium (visceral and parietal portions), and (e) the roots of the several large arteries and veins (aorta, pulmonary artery, inferior and superior vena cava, and the pulmonary veins). Open the several chambers and note in the ventricles: (a) columnæ carneæ, (b) papillary muscles, (c) chordæ tendineæ, attached to the valves guarding the atrioventricular orifices, (d) the moderator band of the right ventricle, and (e) the semilunar valves guarding the aortic and pulmonary orifices. In the atria note the (a) pectinate muscles of the auricular appendages and (b) the crista terminalis. Note also the interatrial and the interventricular septa, and expose the atrio-ventricular bundle (bundle of His) in the anterior septal portion of the heart.

(2) Study a stained transverse section of the heart of a mouse (or other small mammal) cut through the ventricles. Note (a) epicardium, (b) myocardium, (c) endocardium, (d) papillary muscles, (e) columnæ carneæ. Sketch under l.p. (15\%).

(3) Study a stained section through the ventricular wall of the human heart. Note the several layers, and compare with the three tunics of a large blood vessel. Make l.p. sketch (158).

(4) From stained sections make h.p. sketches of (a) epicardium (159), (b) endocardium (160), (c) Purkinje fibers, in transverse section (most conspicuous in rabbits and sheep's heart) (161), and a cusp of an atrio-ventricular valve, including base (annulus fibrosus) and apex, noting in each case the component tissue elements (162).

(5) Make drawings from demonstration preparation of the node of the sino-ventricular bundle, showing the constituent muscular and connective tissue elements (163), and the nerve and blood supply (164). Structure of sino-ventricular bundle of His? of the moderator band? 
(6) Sketch small area of myocardium from injected specimen of cat's heart to show vascular supply (165).

(7) Sketch from a demonstration slide (stained with methylene blue) a small area of the myocardium, showing the manner of the nerve supply (166).

\section{BLOOD}

\section{(A) THE RED BLOOD CORPUSCLES (Erythroplastids, 'Erythro- cytes').}

(1) Sterilize tip of finger or lobe of ear with an alcohol-ether solution, and allow to dry thoroughly. Sterilize a needle in the flame of a Bunsen burner. Prick finger tip or lobe of ear, and mount a considerable drop of blood under a cover-glass. Examine quickly. Compare color as viewed macroscopically and under the microscope. Note shape and form of red corpuscles, as seen en face and in profile. Note the formation of rouleaux. Sketch (167). After a time crenated corpuscles appear near the edges due to evaporation of the blood plasma which in consequence has become hypertonic. Sketch (168). Note also the white glistening leucocytes, and the clumps of platelets.

(2) Place on a slide a small drop of Ringer's solution (an isotonic solution). Prick finger tip and touch a small drop of blood to the Ringer's solution. Mount the mixture under a cover-glass supported by a hair (or prepare the mixture on the cover-glass and mount as a hanging drop over a hollow ground slide, sealing the cover-glass with vaselin). Follow a red corpuscle as it floats and turns in a current, noting carefully its form. What is the shape of the red blood corpuscle? Is it nucleated? Sketch (169). What is the significance of the cupshaped corpuscles? Sketch (1\%0). Do rouleaux appear in the circulating blood? Note also the white glistening round and irregular leucocytes, and the granular masses of platelets.

(3) Make successive mounts of blood in Ringer's solution and add to (1) a drop of a one per cent solution of acetic acid; to (2) a drop of a one per cent solution of tannic acid; to (3) a drop of a solution of bile. Note the results and explain. How is the third result related to the morbid condition known as jaundice or icterus?

(4) Mount a drop of blood in distilled water (a hypotonic solution). Follow the successive changes undergone by the red corpuscles. Sketch (171). Explain. Recognize the final steps when blood shadows and blood dust (hemokonia) appear. 


\section{(B) THE WHITE BLOOD CELLS (Leucocytes; Amebocytes).}

(1) Mount a drop of blood in Toison's solution under cover-glass. Note the several types of white blood cells: (a) small mononuclear; (b) large mononuclear; (c) polymorphonuclear; and (d) note also a group of blood platelets (plaques). Are the latter elements capable of ameboid motility? What is their derivation? Are they true cells? What is their most probable function?

(2) Study carefully with oil immersion lens a blood smear stained with Wright's (or Hasting's, Ehrlich's triacid, or Jenner's) stain. Identify and sketch (172) the various types of leucocytes: (a) lymphocytes (large and small); (b) large mononuclear and 'transitional' nongranular leucocytes; (c) polymorphonuclear neutrophil leucocyte; (d) eosinophil leucocyte; (e) basophil leucocyte (mast cell) ; and (f) blood platelets. Note relative size, shape of nuclei, granular cytoplasmic content, and relative abundance of each type. Make similar study of rabbit's blood. (Here the neutrophils are replaced by similar cells with fine eosinophilic, 'special', granules. In amphibia and reptiles the neutrophils are replaced by eosinophils with ellipsoidal granules.)

(3) Study the contents of a vein in a stained section. Note the various shapes of the red corpuscles, and identify the white blood cells present.

(4) Make mount in Toison's solution of frog's blood. Note the ellipsoidal nucleated red corpuscles, the white blood cells (a, small, large and 'transitional' non-granular leucocytes; $b$, polymorph neutrophils; c, eosinophils; and d, basophils) and the small spindle-shaped thrombocytes. Sketch (173). Watch a large leucocyte in ameboid progression. Sketch several steps (174).

(5) Make similar mount and study of bird's or turtle's blood. How does it differ from frog's blood? How do sauropsid bloods differ from mammalian? rabbit's blood from human blood?

\section{(C) FIBRIN.}

Place a small drop of blood on, slide and spread out thin, allow to coagulate slowly. Breathe on the coagulum at intervals through a period of about a quarter of an hour. Add a drop of methylene blue, and allow it to act for several minutes. Rinse in water; dry thoroughly and mount under a cover-glass in balsam. Examine the thinner portions of the coagulum for the fibrin net. Sketch (175). Study and sketch (176) also a small area of the fibrin net from a stained section of a blood clot. Difference between plasma and serum? 
(D) HEMOGLOBIN CRYSTALS.

Place large drop of blood on slide in a small drop of distilled water. Allow to dry slowly. Mount when dry in balsam under cover-glass. Examine h.p. for crystals. Note shape, size and color. Sketch (177).

\section{(E) HEMIN CRYSTALS.}

Place a drop of blocd on slide. Add 2 or 3 grains of sodium chloride and a drop of glacial acetic acid. Heat slowly over flame of a Bunsen burner until bubbles begin to appear. Mount in balsam. Examine h.p. for crystals. Note shape, size and color. Sketch (178) several isolated crystals and several groups.

\section{(F) BLOOD DEVELOPMENT (Hemopoiesis).}

(1) IN Embryo. Sketch (179) a few blood cells from a young mammalian embryo, noting the several types of embryonic erythrocytes: (a) hemoblasts, (b) erythroblasts, (e) normoblasts.

(2) In Adult Bone Marrow. Study with oil immersion lens smear preparation or a section of red bone marrow (e.g., of femur of rabbit or guinea pig), stained in Wright's solution. Identify and sketch (180) the several types of myelocytes: (a) myeloblast, (b) leucoblast, (c) erythroblast; also (d) myeloplaxes (giant cells), (e) erythrocytes, (f) the several types of granular' myelocytes: eosinophil, basophil and 'special' (neutrophil of higher mammals), and $(\mathrm{g})$ origin of platelets from pseudopods of megakaryocytes. Note cells in process of division, both mitotic and amitotic. Note also the framework of reticular connective tissue (reticulum); and the process of extrusion of the nucleus of a normoblast in the formation of an erythroplastid. Compare with section of red marrow of femur of frog. Differences? Make similar study of fragment of marrow of femur of rabbit or guinea pig, preserved in aceto-carmin, and teased in glycerin.

\section{THE LYMPHATIC SYSTEM}

\section{(A) $L Y M P H$.}

Study a smear preparation of lymph stained with Wright's stain from the thoracic duct of a dog. Sketch (181) the several types of leucocytes. Which type predominates? Do blood platelets occur in lymph? Red blood corpuscles? Origin of lymph? Consider the coagulation of lymph. 


\section{(B) LYMPH VESSELS (Lymphatics).}

(1) Lympi Capillaries. Identify a lymph capillary in a stained section (e.g., lymph node, subcutaneous tissue, submucosa of trachea or large intestine) and sketch (182). How does it differ from a blood capillary? a venule? a sinusoid?

(2) Lacteal. Sketch (183) the terminal lymph capillary in a villus of a stained section of the small intestine.

(3) Thoracic Duct. Sketch (184) a segment of the wall of a stained section of the thoracic duct (Weigert's elastic tissue stain, and picric-acid-fuchsin). Note the several layers, and the fundamental tissues of each. How can you distinguish between a large lymphatic and a vein of the same calibre? Do lymphatics contain valves?

\section{(C) SEROUS MEMBRANES (Peritoneum, Pleura, and Pericar- dium).}

Sketch (185) h.p. a small area of a stained toto mount of a fragment of the peritoneum treated with silver nitrate.' Note the character of cells and intercellular stomata. Sketch (186) also a short segment of a stained section of same. Note shape and character of cells; intercellular bridges, striated border, basement membrane, and the submesothelial fibro-elastic corium.

\section{(D) LYMPH NODULES (Lymph follicles).}

Sketch (18\%) l.p. a small lymph nodule from the submucosa of a stained section of large intestine, stomach, or vermiform appendix.

\section{(E) LYMPH NODE (Lymph gland).}

Study a stained section of a mesenteric lymph node. Sketch (188) 1.p. Sketch (189) h.p. small area including a medullary cord and the adjacent lymph sinus. What is the predominant cell type? Note dividing cells in germinal center of cortical nodules. Examine in Ringer's solution scrapings from a lymph node of cat, after addition of drop of methylene blue. Examine also a glycerin mount of aceto-carmin preserved material.

\section{(F) LYMPHOID ORGANS.}

(1) Hemolymph Node. (Hemal Node). Study a stained section of a hemolymph node of sheep. Sketch (190) h.p. a small area including the denser and looser parenchyma. Differential characteris- 
tics of lymph, hemal and hemolymph nodes? Blood and lymph supply of each? Giant cells?

(2) Tonsils (Faucial tonsil). Study a stained section of the faucial tonsil. Make h.p. sketch (191) of the infiltrated stratified squamous epithelium lining a crypt. Function of tonsils?

(3) Spleen (Lien).-(a) Study a stained section of spleen. Note (1) robust fibromuscular capsule; (2) similar trabeculæ; and (3) splenic (Malpighian) corpuscles with central or subcentral arteriole. Sketch segment under l.p. (192). Study under h.p. the spleen pulp, noting difference between pulp cords and intercordal pulp (venous sinuses). Sketch (193) a small area of this portion of the parenchyma, noting types of cells and character of the terminal arterioles. Study injected specimen of spleen, noting vascular terminals within the lobule.

(b) From a smear preparation of spleen, stained with Wright's blood stain, sketch (194) the several types of parenchymal cells: (1) lymphocytes; (2) polymorphonuclear neutrophil leucocytes; (3) large mononuclear leucocytes ('splenic cells') ; (4) eosinophil leucocytes; (5) basophil leucocytes; (6) erythrophages; (7) erythroblast; (8) giant cells. Make similar study of an aceto-carmin preparation mounted in glycerin. What are the chief criteria for distinguishing the several lymphoid organs from lymph glands, and from each other? Function of spleen?

\section{SKIN AND APPENDAGES}

\section{(A) THE SKIN (Integument).}

(1) Study under l.p. a vertical stained section of thick skin (from palm of hand or sole of foot). Note epidermis (cuticle), dermis (corium, cutis), and the tela subcutanea. Distinguish between the stratum corneum and the stratum germinativum; between the pars papillare and the pars reticulare. Note the spiral epidermal portion of the ducts of the sweat glands. Note also the panniculus adiposus of the subcutaneous tissue. Sketch a narrow segment through entire thickness (195). Make h.p. sketch (196) of several adjacent cells from each of the six distinct layers of the epidermis. What are the several factors (physical, chemical and mechanical) which operate upon a cell of the cylindrical cell layer during its metamorphosis into an element of the scaly cell layer?

(2) Study under l.p. a vertical stained section of thin skin (e.g., from abdomen). Sketch (19\%). What are the chief structural differ- 
ences between thick and thin skin? Between thin skin and the mucous membrane lining the mouth? Note the delicate hairs.

(3) Make h.p. sketches $(198, a, b, c)$ from different portions of a section through the head of a $20-\mathrm{mm}$. pig fetus, illustrating three earlier stages in the development of skin: one consisting of a single layer of cells (with superjacent periderm or epitrichium), one of two or three layers, and one of a larger number of layers.

(4) Compare sections of thin skin of: (a) dark negro; (b) light mulatto; (c) brunette; (d) blond. Note quantity and distribution of the pigment (melanin) granules in the several specimens of skin.

\section{(B) SWEAT GLANDS (Sudoriparous glands).}

(1) In a stained vertical section of skin identify and study a sweat gland (complete if possible). Note: (a) its duct (excretory) including the spiral 'mouth' within the epidermis; (b) its coiled fundus (secretory). Under h.p. draw a cross-section of the dermal (or subcutaneous) duct and fundus (199). Note the longitudinally disposed smooth muscle cells between the secretory cells of the fundus and the membrana propria.

\section{(C) THE NAILS (Unguis; Onyx).}

(1) Examine a finger-nail and note: (a) body; (b) root; (c) lunula; (d) nail sulcus; (e) nail fold or vallum; (f) eponychium; (g) hyponychium. Sketch (200). Explain the opacity and color of the lunula.

(2) Study l.p., a stained transverse section of the finger tip including the nail. Note the homologous layers of nail and adjacent skin. What differences obtain? What layer of the epidermis does the nail body represent? How does the pars papillare differ in the two regions? Sketch (201).

(3) Make l.p. sketch (202) of a stained vertical longitudinal section of the unguinal phalanx. Note nail matrix and nail bed. What is the epidermal homologue of the nail matrix? The relation of the matrix to the lunula? Sketch (203) h.p. a small area through the body of the nail including its corium.

(D) THE HAIR (Pilus; Thrix).

(1) Mount in water under cover-glass a freshly extracted hair. Note: (a) hair bulb; (b) root (radix); (c) shaft (scapus); (d) medulla; (e) cortex; (f) pigment granules; and (g) cuticle. Explain 
differences in color of hair. Sketch under h.p. a portion of the shaft (204).

(2) In a stained vertical section of the scalp examine, under the low power, a hair follicle with its included hair in longitudinal section. Note: (a) hair papilla; (b) hair bulb (pigmented?); (c) hair root; (d) epidermal root sheath; (e) arrector pili muscle; (f) sebaceous gland. Sketch (205). Make h.p. sketch (206) of the hair and its follicle, cut longitudinally, at the level of the sebaceous gland (about the middle third of the root), noting the several layers of the inner and outer root sheaths (the epidermal sheath), and of the dermal sheath. What is the function of the arrectores pilorum muscles? Make h.p. sketch (20\%) of the region of transition from root and follicle to the hair bulb.

(3) Sketch h.p. (208) a transverse section of a hair follicle at or near the level of the middle third, showing the several layers of the follicle as above. What layers are homologous in hair follicle and skin? Explain the homologies in terms of development. Explain the cause of the curling of certain types of hair.

(E) SEBACEOUS GLAND.

(1) Study a sebaceous gland in a stained vertical section of the scalp. Note its relation to the hair follicle. Note also its duct and saccules. Sketch l.p. (209). Make h.p. sketches (210 a, b, c) of cells of the sebaceous gland at three successive stages of their metamorphosis into the sebum 'secretion'. How many glands to a hair follicle?

(F) THE BLOOD SUPPLY.

(1) Study in stained section injected specimens of skin and scalp. Sketch (211). Note the precise location of the several vascular plexuses of the derma and the tela subcutanea; and the blood supply of the sweat glands, hair follicles, sebaceous glands, and the fat lobules of the tela.

\section{THE RESPIRATORY SYSTEM}

\section{(A) THE NASAL CAVITY.}

(1) Study in a stained section the lining mucous membrane of the vestibule. Note transition from external skin to internal mucous membrane; note, also the vibrissæ and the associated sebaceous glands. Make h.p. sketch of a narrow segment (212).

(2) Study mucosa of respiratory portion (Schneiderian membrane). 
Note the pseudo-stratified ciliated columnar epithelium, goblet cells, the extensive vascular corium, and the sero-mucous glands. Sketch h.p. a narrow segment (213).

(3) Make h.p. sketch (214) of olfactory mucous membrane, noting: a, sustentacular cells; b, olfactory cells; c, basal cells; and d, serous glands of Bowman. In what respect is the olfactory cell unique?

(4) In a section of the cat's nose, note the vomero-nasal organ of Jacobson in the median septum, one on either side of the cartilaginous plate. Sketch a narrow segment.

(B) THE LARYNX.

(1) Study a stained vertical section through lateral wall of the larynx. Note vocal cords (true and false) and ventricle. Sketch (215), indicating the types of epithelium and the contents of the corium at different levels. What are the fundamental tissues comprised in the true vocal cords?

(C) THE TRACHEA.

(1) Study a stained transverse section of the trachea. Note: a, character of the lining epithelium; b, goblet cells; c, elastic layer in tunica propria; d, muscularis mucosæ? e, tela submucosa, with its mucous glands with demilunes; f, fibro-cartilaginous tunic. Trace the duct of a mucus-secreting gland to the surface. Note its apulla in the corium. What is the character of the cartilage of the adventitia? Explain the trachealis muscle. Sketch h.p. (216) a narrow segment of the complete width of the wall.

\section{(D) THE BRONCHI.}

(1) Study a stained transverse section of a primary bronchus. Compare with trachea. In what points do they differ?

\section{(E) THE LUNG (Pulmo).}

(1) Make h.p. sketch (21\%) of narrow segment of wall of secondary or tertiary bronchus. Note: a, muscularis mucosæ; b, lymphoid tissue (solitary nodules?); c, mucous glands; $d$, character of lining epithelium. How are the pulmonary artery and vein, and the bronchial artery and vein, related to these bronchi?

(2) Sketch h.p. (218) a bronchiole. What are the chief structural differences between a bronchiole and a tertiary bronchus? Relation of pulmonary artery and vein to bronchiole? Structure of terminal (respiratory) bronchiole? 
(3) Make l.p. sketch (219) of a primary lobule (histologic and functional unit), including: a, transition from respiratory (terminal) bronchiole to b, alveolar duct; c, atria; d, pulmonary sacs; e, pulmonary alveoli. Study the several portions of the primary pulmonary lobule under the high power and note the structural differences. At what point in the lobule does the smooth muscle end? Where do the lymphatics end? Relative abundance of the elastic fibers? Relation of pulmonary artery and vein to the primary lobule? Define a secondary pulmonary lobule (anatomic unit).

(4) Make h.p. sketch (220) of wall of pulmonary alveolus (air cell) cut tangentially. Note the flat respiratory cells and the expansive nonnucleated respiratory plates.

(5) Sketch (221) several adjacent alveoli from a section of an injected specimen of the cat's lung.

(6) Make h.p. sketch (222) of small extent of the visceral pleura.

(7) Consider the distribution of the lymphatics, the lymph nodes, lymph nodules and the more diffuse lymphoid tissue in the lung. Function of lymphoid tissue in the lung? Relation to inhaled dust? to infecting bacteria? to tuberculosis? Consider also the distribution and relative abundance of smooth muscle and of elastic fibers. Significance? Relation to asthma? to anaphylaxis, e.g., in guinea pig? Explain absence of bronchial veins beyond the bronchioles.

\section{THE ALIMENTARY TRACT}

\section{(Digestive Canal)}

\section{(A) THE LIP.}

(1) Study l.p. stained section (vertical) of lip. Distinguish cutaneous and mucous surfaces. Note: a, bundles of striped muscle fibers of orbicularis oris muscle in transverse section; b, mucous glands; $c$, hair follicles; d, sweat glands; e, sebaceous glands; f, coronary (labial) artery. Sketch (223):

\section{(B) THE TEETH.}

(1) Study a stained axial section of a decalcified molar tooth within its alveolar socket. Note: a, crown; b, neck; c, root; d, fangs; e, pulp cavity; f, root canal; g, foramen apicis dentis. Sketch h.p. (224) small area of a, enamel; b, dentin; c, cementum; d, dental pulp reticulum; e, granular layer of Tomes; $f$, an odontoblast with its Tomes' fibril within 
a dentinal tubule. Sketch l.p. (225) a portion of the area of contact between root of tooth and alveolar process. Note: a, alveolar bone; b, periosteum and pericementum; c, circular dental ligament; $d$, epithelial remnants of dental lamina; e, fibers of Sharpey; $f$, cementum cells.

(2) Study l.p. an axial ground section of tooth. Note in the enamel, the contour lines of Retzius and the prism stripes of Schreger; in the dentin, the dentinal tubules, the contour lines of Owen, and the incremental lines of Schreger. Make h.p. sketches (226) of small areas of the enamel showing the prism bundles cut transversely and longitudinally; also (22\%) of small areas of the dentin showing the tubules cut transversely and longitudinally. Sketch also (228) a small area of the cementum; and (229) of the granular layer of Tomes in the region of the neck of the tooth. Describe the complete form, structure, content and significance of the dentinal tubules.

(3) Sketch (230) several successive early stages in the development of the deciduous teeth in a stained vertical section of the jaw of some mammalian fetus (e.g., of $25 \mathrm{~mm}$. pig embryo). Note: a, labiodental strand and groove; b, labial lamina ; $c$, dental lamina ; $d$, enamel organ ; e, dental papilla.

(4) Study a stained axial section of an infant's tooth some time before eruption. Note: a, enamel organ (enamel, inner enamel epithelium, outer enamel epithelium, and enamel pulp) ; b, dental pulp, odontoblasts and the strata of older and younger dentin; c, the dental sac; $d$, anlage of the permanent tooth; e, remnant of the dental lamina. Make l.p. sketch (231). Sketch h.p. (232) a small area near the apex of the crown, showing from without inward: $a$, enamel pulp; $b$, layer of ameloblasts; c, Tomes' processes ; d, enamel ; e, membrana performativa ; $\mathrm{f}$, stratum of older basophilic dentin; g, stratum of younger acidophilic (only slightly calcified) dentin with Tomes' fibrils; h, layer of odontoblasts; i, dental pulp.

\section{(C) THE TONGUE (Lingua).}

(1) Make diagram l.p. (233) of transverse section of a cat's tongue to show the disposition of the striped muscle. Note the bilaterally symmetrical structure of the tongue; the extent of the medial septum linguæ; the difference between the mucous covering on the dorsum and ventrum of the tongue. Note also the superficial longitudinal muscles, the genio-glossus ventrally, and the deep transverse and vertical fibers. Note further the glands imbedded in the muscle.

(2) Sketch from stained sections of the human tongue the several 
types of lingual papillæ: filiform (conical) (234); fungiform (235) ; circumvallate (vallate) (236); and foliate (237). Note the taste-buds in the latter two types of papillæ.

(3) Study an injected specimen of cat's tongue and note the blood supply of the muscle, and of the several papillæ.

(4) Study a transverse section of the foliate papillæ of the rabbit's tongue. Note the numerous taste buds.

\section{(D) THE PALATE (Palatinum).}

(1) Study a stained longitudinal section of the palate (including the uvula, the soft palate, and a portion of the hard palate), noting the character of the epithelium on the nasal and oral surfaces. Sketch (238).

\section{(E) THE PHARYNX.}

(1) Study l.p. a stained section of the oropharynx. Note: a, type of epithelium of the mucosa; b, character of the tunica propria; c, lymphoid tissue; $d$, boundary of fibro-elastic tissue, the representative of both the tela submucosa and the muscularis mucosa of other portions of the digestive tube (the elastic fibers are mostly longitudinally disposed) ; and e, the outermost layer of obliquely disposed striped muscle fibers, among the connective tissue of which are embedded many tuboacinar mucous glands. Sketch (239).

(F) THE ESOPHAGUS (Gullet).

(1) Study l.p. a stained transverse section through the lower third of the esophagus. Note the four tunics from within outward: a, tunica mucosa; b, tela submucosa; $c$, tunica muscularis; and d, tunica adventitia (fibrosa). In the tunica mucosa, note the type of the epithelium, the character of the corium (lamina propria mucosæ), and the lamina muscularis mucosæ. (Near the cardiac orifice the muscularis mucosæ consists of an incomplete inner layer of circularly disposed smooth muscle cells, and a more extensive outer stratum of longitudinally arranged cells; elsewhere in the esophagus generally the latter stratum only occurs; below the esophagus both layers occur.) In the tela submucosa note the character of the tissue and the mucous glands. Do the glandular alveoli contain demilunes? (Mucus-secreting glands are absent in the esophagus of the cat, very abundant in that of the dog, and very variable in man. They are generally more abundant towards the oral end.) In the tunica muscularis, note the two strata of smooth muscle cells, the outer longitudinal and the inner circular (at the cardiac orifice 
an additional innermost oblique layer may appear). Is a mesothelial layer present in the tunica adventitia? Sketch l.p. (240) a narrow segment through the complete wall.

(2) Compare the above section with sections through the middle and upper third of the esophagus. What differences obtain at these levels in the several tunics?

(3) Compare a section through the upper third of the esophagus with one through the pharynx. (A longitudinal section through the area of transition from pharynx to esophagus is preferable.) What takes the place in the pharynx of the muscularis mucosæ of the esophagus? Note that in the upper end of the esophagus a third innermost stratum of oblique or longitudinal striped muscle fibers may occur in the tunica muscularis.

(4) Study sections through the upper cardiac glands (superficial esophageal glands). Sketch (241).

(5) Sketch small portions of the myenteric (242), and the submucous nerve plexuses (243).

\section{(G) THE STOMACH (Ventriculus; Gaster).}

(1) Study l.p. a stained longitudinal section through the area of transition from the esophagus to the cardiac portion (pars cardiaca) of the stomach. Note the tunica serosa of the cardia. Make sketch (244) including the four tunics. Sketch h.p. (245) a cardiac gland.

(2) Study a section through the pars fundica of the stomach (fundus ventriculi). Sketch l.p. (246). Examine carefully under h.p. a gastric gland of this region and note: a, mouth; b, foveola (crypt, pit); c, neck (cervix); d, body (corpus); and e, fundus. Note the different types of epithelium occurring in the several regions. Sketch h.p. (247) a complete gland, noting especially the chief and parietal cells. Sketch h.p. (248) a transverse section through the body of a gastric gland. Look for a 'lenticular gland' (solitary lymph nodule) in the tunica mucosa.

(3) From a demonstration slide prepared by the Golgi technic sketch h.p. (249) a portion of a longitudinal section of the secretory region of the fundic gland to show the main lumen and the system of inter- and intracellular secretory canaliculi.

(4) Study a section of the pars pylorica of the stomach. Sketch l.p. (250). Sketch h.p. (251) a gastric gland of this region. In what features do the pyloric and fundic glands differ? How do they differ functionally as indicated by these features? Enumerate four salient 
differential characteristics between the pars fundica and the pars pylorica of the stomach.

(5) Study an injected specimen of cat's stomach showing the blood supply of the several tunics. Sketch (252).

(6) Study demonstration preparations (methylene blue technic) of cat's stomach showing the nerve supply. Note especially the myenteric and the submucous plexuses. Sketch (253).

\section{(H) THE SMALL INTESTINE (Intestinum tenue).}

(1) Study l.p. a longitudinal section through the area of transition from the pylorus to the duodenum. Compare part for part. In the duodenum note: $a$, villi; $b$, intestinal glands (crypts of Lieberkúhn); $c$, duodenal (Brunner's) mucous glands; and $d$, the plicæ circulares (valvulæ conniventes). Sketch (254). Enumerate the chief criteria for distinguishing between the duodenum (small intestine) and the pyloric portion of the stomach.

(2) Sketch h.p.: a, a villus in transverse and longitudinal sections (255) ; b, intestinal gland (256). Identify a cell of Paneth.

(3) Study l.p. a stained transverse section through the jejunum or the ileum. Sketch (257) at the level of a Peyers patch (agminated nodule). Difference in shape of villi in the three segments of the small intestine? Distribution of lymphoid tissue? Presence of glands in the tela submucosa?

\section{(I) THE LARGE INTESTINE (Intestinum crassum).}

(1) Draw l.p. (258) a portion of a stained section of the colon, including the four tunics. How does it differ in grosser features from the small intestine? In the relative abundance of goblet cells? of solitary lymph nodules? of granule cells of Paneth? Note the teniæ coli of the tunica muscularis.

\section{(J) THE VERMIFORM APPENDIX (Processus vermiformis).}

(1) Study a transverse section of a normal human appendix. Note the relative abundance of the crypts of Lieberkühn and the solitary lymph nodules; and the differences in the lamina muscularis mucosæ and the tunica muscularis, as compared with the colon. Sketch (259).

(K) CAECUM.

Study a longitudinal section through the area of transition (ccecum) from the ileum to the ascending colon. Note the iliocolic valve. Note 
also in the colon the haustra, the plicæ semilunares and the appendices epiploicæ. Sketch l.p. (260).

\section{(L) RECTUM.}

Sketch l.p. (261) the area of transition from the rectum to the anus to show the structural differences in the several tunics. Compare the rectum with the colon. Enumerate the grosser differential marks among colon,' appendix and rectum. Note rectal valves, rectal columns, and anal valves.

Study an injected specimen of the small intestine of the cat. Sketch the blood supply of a villus and of a crypt of Lieberkühn (262).

\section{THE LARGE GLANDS OF THE DIGESTIVE CANAL}

\section{(A) THE LARGER SALIVARY GLANDS.}

(1) The Parotid Gland. Study under the low power of the microscope a stained section of the parotid gland. Sketch (263), showing the division into lobes and lobules, and the location of the interlobular and intralobular excretory ducts. Note the distinction between the parenchyma and the stroma or interstitial tissue.

Sketch h.p. (264) an alveolus (acinus) in connection with its intercalary duct. Note the shape, manner of distribution within the alveolus, stage of secretion as indicated by the cytology, staining reaction and relation to the 'basket cells' of the constituent secretory cells of the acinus. Sketch h.p. (265) also transverse sections of the secretory (salivary) and the excretory portions of the intralobular duct.

(2) The Sublingual Gland. Study a stained section of the chief sublingual gland (of man, dog, cat or rabbit). Sketch h.p. (266) an alveolus with a large demilune ('crescent' of Gianuzzi). Explain the functional significance of the difference in staining reaction between the cells lining the alveolus and those of the demilune. Probable function of the demilune cells? Chief difference in duct systems of parotid and sublingual glands?

(3) The Submaxillary Gland. Compare the submaxillary with the parotid and sublingual glands. Enumerate the chief differential characteristics among these three glands. Sketch h.p. (267) two adjacent alveoli, one of the mucous the other of the serous type. From an injected specimen of cat's submaxillary gland sketch (268) the intra- 
lobular blood supply. Nerve supply of the salivary glands? Note differences between the resting and active (or stimulated) gland.

(B) THE PANCREAS.

(1) Study a stained section of the pancreas. Make 1.p. sketch (269) to show the arrangement into lobes and lobules, and the location of the ducts. Sketch h.p. (2\%0) an acinus with its intercalary duct (intermediate duct), and a centro-acinal cell group. Note the polar differentiation of the zymogenous acinar cells; and the different staining reaction of the zymogenous and the centro-acinal cells. Significance? What is the relationship between the distal granular zone of the zymogenous cells and their basal filar zone? the mitochondria? the 'nebenkern'? How do these structures vary according to the phase of functional activity?

(2) Sketch h.p. (2\%1) a pancreatic islet (island of Langerhans). From a specially prepared demonstration slide of the pancreas sketch (272) cells of the A and B types. Number, form, distribution, staining reaction, and significance of the islets?

(3) Sketch h.p. (273) a cross-section of a large interlobular duct.

From an injected specimen of a cat's pancreas sketch (274) the blood supply of the zymogenous parenchyma and of the islets. Significanie of the relatively great vascularity of the islet? Relation of the capllaries to the cords of islet cells? Enumerate the differential marks between the pancreas and the parotid gland.

\section{(C) THE LIVER (Hepar).}

(1) Study l.p. a stained section of the liver: a, of pig or camel; b, turtle or frog; c, cat or human. Enumerate differences. From sections a and c sketch l.p. (275) a hepatic lobule including the capsule of Glisson. From section b, sketch h.p. (276) a small extent of the liver tubule, noting the definite structural polarity of the constituent cells. Functional significance of the latter phenomenon? Similarity between embryonic mammalian liver and the adult reptilian and amphibian livers? Significance? What is the shape of the liver lobules as revealed by reconstructions? Difference between a hepatic lobule and a portal lobule?

(2) Study h.p. a stained section of the human or cat's liver. Sketch h.p. (27\%) a small area, noting the hepatic cell-cords (trabeculæ), the interstitial reticulum, the capilliform sinusoids (intralobular capillaries), the relation of the latter to the cell cords, and of the stellate cells (of von Kupfer) to the endothelial lining cells and to the liver 
cells proper. From a demonstration preparation according to the Golgi technic or the Vance technic, draw h.p. (278) a cell cord with its interand intracellular secretory canaliculi. In a section prepared according to Mallory's technic for connective tissue, study the character of the inter- and intralobular connective tissue.

(3) Sketch h.p. (279) several types of liver cells: a, granular (with zymogenic and glycogenic granules); b, fatty; c, pigmented; $d$, binucleated. Consider the questions regarding the presence, character, significance and relation to the bile capillary (ductule) and to the capillary blood supply (in health and in disease, e.g., in jaundice) of the intracellular bile canaliculi.

(4) Sketch h.p. (280) a portal canal (interlobular vein, artery, lymphatic and bile duct); a central (intralobular) vein (281); a sublobular vein (282). Sketch h.p. (283) the area of transition from an intralobular to the interlobular bile duct.

(5) From an injected specimen of the cat's liver sketch (284) a small area of the parenchyma. Trace the path of the portal and the hepatic blood through the liver. Diagram (285).

(D) THE GALL BLADDER (Vesica fellea).

(1) Make h.p. sketch (286) through the wall of gall-bladder, noting the constituents of the several tunics.

\section{THE URINARY SYSTEM}

\section{(A) THE KIDNEY (Ren).}

(1) From a gross specimen of an adult human kidney divided in the median longitudinal plane, make a sketch (287) to show the general topography. Note: capsule (tunica fibrosa, tunica adiposa); cortex (pars radiata, pars convoluta); medulla (renal pyramids, renal columns, renal papillæ); renal sinus (in hilus), containing the renal pelvis (with infundibula or major calyces and the minor calyces). Note also the ureter, and the distribution of the branches of the renal artery and vein in relation to this duct.

(2) Study l.p. a stained longitudinal section of the kidney of the mouse or rat (or other small mammal). Sketch (288). In capsule note: mesothelium; tunica albuginea; smooth muscle? Define a renal lobule (renculus). How does the adult human kidney differ from that of the fetus and the infant? from that of reptiles? from that of mouse and cat? from that of horse? from that of pig? 
(3) Study a stained longitudinal (sagittal) or radial section of the mammalian kidney. Make l.p. sketch (289) (diagrammatic) to show the finer topographic relationship of the several divisions and their larger constituents. Note cortex corticis, pars radiata, pars convoluta, renal corpuscle, renal column, and boundary and papillary zones of the medulla.

Sketch h.p. (290) a renal corpuscle. Note: glomerulus; capsule (visceral and parietal layers); arterial pole (with afferēnt and efferent arterioles); uriniferous pole (neck).

Sketch h.p. (291) portions of the several segments of a renal (uriniferous) tubule: proximal convoluted tubule; descending limb of Henle's loop; ascending limb of Henle's loop; distal convoluted tubule; arched collecting tubule; straight collecting tubule; papillary duct.

Note the difference in staining reaction of the two portions of the tubule, divided at the point where the distal convoluted joins the arched collecting tubule. Significance with respect of function? of origin?

(4) From a demonstration slide prepared according to Huber's technic for isolating the renal tubules (Anat. Rec., vol. 5, 1911), sketch a complete tubule (292).

(5) Study a stained coronal (tangential) section of the kidney through cortex. Identify the several constituents of the pars radiata and the pars convoluta. Make a similar study of a similar section through the medulla.

(6) Study a longitudinal section of the kidney stained with Mallory's connective tissue stain. Note the types and distribution of the connective tissue.

(7) From a demoustration slide of the kidney prepared by Meve's mitochondrial technic, sketch (293) a portion of the proximal convoluted tubule. Shape of the mitochondria? Function?

(8) Study a radial section of an injected cat's kidney. Sketch (294) the blood supply. Note interlobar arteries and veins; arciform (arcuate) vessels; interlobular vessels; afferent and efferent glomerular arterioles; the glomerular rete mirabile; the capillary supply of the convoluted tubules; the stellate veins; the arteriolæ and venulæ rectæ (veræ and spuriæ) of the medulla.

\section{(B) THE URINARY BLADDER (Vesica urinaria).}

Study l.p. a stained section of the urinary bladder. Make l.p. sketch (295) of segment of complete wall; h.p. sketch (296) of tunica mucosa. How does the mucosa vary with the degree of distension? How does the 
tunica muscularis differ from that of the digestive tube? Character, origin and relationship of the trigonum vesicæ?

\section{(C) THE URETER.}

Make similar study and sketches $(29 \%, 298)$ of ureter cut transversely.

(D) THE URETHRA.

Make similar study and sketches $(299,300)$ of female urethra. (The male urethra should be studied in connection with the penis.)

\section{THE MALE ORGANS OF REPRODUCTION}

\section{(Male Genital Apparatus)}

\section{(A) THE TESTIS (Orchis).}

(1) Study a gross fresh specimen of a mammalian testis, with its associated duct system. Identify: a, globus major (caput), globus minor (cauda) and corpus of epididymis; b, ductuli efferentes (and coni vasculosi); c, ductuli abberentes (superior and inferior); d, ductus deferens; e, paradidymis; and $f$, the appendices testis and epididymis. Sketch (301).

(2) Study with hand lens a median longitudinal section of a mammalian testis. Identify: a, capsule; b, hilus; c, mediastinum (corpus Highmori) with rete testis; $d$, lobular compartments (lobules of testis); e, semniferous tubules (tubuli contorti); and f, tubuli recti. Sketch (302).

(3) Study under h.p. a stained section of an active mammalian testis (e.g., mouse, guinea-pig, human). Sketch (303) a segment (or segments) of the wall showing besides the several phases of Spermogenesis (viz.: a, spermogonium; b, primary and c, secondary spermocytes-both resting and in mitosis - $d$, spermatids in several stages of metamorphosis into e, spermium or spermozoön), a Sertoli cell (sustentacular cell or trophocyte) with attached spermia, forming a 'spermoblast'. Note also: $a$, tunica vaginalis; $b$, tunica albuginea; $c$, tunica vasculosa; and $d$, interlobular septa. Sketch h.p. (304) a portion of wall of: a, a tubulus rectus; and $b,(305)$ the rete testis. What is the form of a seminiferous tubule? Its relation to the lobule of the testis? How may the testis be classified among glands?

(4) Study h.p. a section of the active testis of a grasshopper. Identify the successive stages in spermogenesis. Count the number of 
chromosomes in the metaphase plates of: a, the dividing spermogonium; $b$, the primary spermocyte; $c$, the secondary spermocyte. Note the accessory or sex chromosome. What is its position and behavior in: a, the resting primary spermocyte; $b$, the dividing primary spermocyte; $c$, the resting and dividing secondary spermocytes; $d$, the spermatid? What is its probable significance with respect of sex determination? sex control? How do the maturation mitoses differ from ordinary somatic cell division? Significance of this difference from the standpoint of inheritance? Probable significance of the chromosomes?

(5) The Spermatozoön (spermozoön; spermium; sperm). Sketch (306) from a stained section under the oil immersion lens a human spermatozoön. Compare with a cover-glass preparation of preserved sperm. Identify: a, head (with perforatorium, consisting of acrosome and galea capitis); b, neck; $c$, middle piece or body (with distal centrosome, end ring, central filament and spiral filament); $d$, tail; and e, terminal filament.

Compare with spermium of dog and mouse, and some other mammal (e.g., opossum).

From what constituents of the spermatid are the several aboveenumerated portions of the fully developed spermium derived?

Origin, function and fate (in fertilization) of: $a$, the head; $b$, the middle piece (with its centrosome and mitochondrial spiral filament); and $\mathrm{c}$, the tail?

(6) The Interstitial Cells (of Leydig). Identify among the intertubular connective tissue (testicular stroma) the interstitial cells. Sketch (30\%) several adjacent cells. Origin, structure, probable function and fate? Condition of the interstitial cells, and of the seminal epithelium, in cryptorchism?

\section{(B) THE DUCT SYSTEM OF THE TESTIS.}

(1) Tubuli Recti. Sketch (308) h.p. a segment of the wall of a tubulus rectus at the point of transition to the tubulus contortus. With what cells of the latter tubule are the lining cells of the tubulus rectus homologous?

(2) Rete Testis. Study l.p. the mediastinum of the testis with its included rete tubules. Sketch (309) a small area. Make h.p. sketch (310) of the lining epithelium.

(3) Ductuli Efferentes. Study 1.p. a stained section through the efferent ductules of the epididymis. Sketch (311) a small area. Relation of the ductuli efferentes to the coni vasculosi and to the 
globus major (caput epididymis). Make h.p. sketch (312) of a segment of the wall, showing the character of the epithelium (with its groups of ciliated and non-ciliated columnar cells), the basement membrane, and the muscular tunica propria.

(4) The Epididymis. Make l.p. sketch (313) of a stained section of this extremely convoluted duct; and a h.p. sketch (314) of a segment of the wall, noting the character of the epithelium, the basement membrane, and the muscular tunica propria. How can the duct of the corpus epididymis be distinguished in section from the ductuli efferentes of the caput epididymis?

(5) The Ductus Deferens (Vas deferens). Study 1.p. a stained section of the ductus deferens. Note the tunica mucosa, the lamina propria mucosæ and the tunica muscularis. Sketch (315). Make h.p. sketch (316) through the mucosa. How does the epithelial lining differ in the upper, middle and lower portions? How does the tunica muscularis of the lower portion differ from that of the remainder of the duct? Difference between tunica mucosa and tunica muscularis of the ureter and the ductus deferens?

(6) The Seminal Vesicles. Sketch l.p. (317) a segment of the entire wall; and h.p. (318) a small extent of the mucosa.

(7) The Ejaculatory Ducts. Sketch 1.p. (319) a segment of the complete wall; and h.p. (320) a portion of the mucosa.

\section{(C) THE SPERMATIC CORD.}

Study l.p. a stained transverse section of the spermatic cord. Note the following constituents: a, fibrous stroma; b, ductus deferens; $c$, external cremaster (striped) muscle; d, internal cremaster (smooth) muscle; e, spermatic artery and veins, including $f$, the pampiniform plexus. Sketch (321).

\section{(D) THE SCROTUM.}

Study l.p. a stained section of the wall of the scrotum. Note relatively abundant pigmented cells in stratum germinativum of epidermis; loose corium (derma) with a considerable number of elastic fibers and smooth muscle cells (constituting the dartos). In what respects does the skin of the scrotum differ from ordinary integument? Sketch (322).

(E) THE GLANDS ASSOCIATED WITH THE MALE GENITAL SY STEM.

(1) The Prostate Gland. Study l.p. a stained section of the human prostate. Sketch (323) a small area showing several alveoli 
and the abundant fibro-muscular stroma. Contents of the alveoli? Make h.p. sketch (324) of a small extent of the mucosa. Significance of the alveolar content?

(2) The Bulbo-Urethral Glands (Cowper's glands). Make h.p. sketch (325) of several adjacent alveoli and included fibro-muscular stroma. Character of the muscle content? Sketch h.p. (326) a portion of the alveolar lining.

\section{(F) THE PENIS (Phallus).}

(1) Study a stained transverse section of the human penis, through its middle portion. Note: a, corpus spongiosum (corpus cavernosum urethræ) with the urethra; b, urethral glands (glands of Littré) ; $c$, the two corpora cavernosa (penis) ; $d$, the enveloping tunica albuginea, and c, the peripheral cutaneous envelope. Sketch (327). Sketch h.p.: a, the lining of the urethra including a urethral gland (328); b, a small area of erectile tissue from one of the corpora (329); and e, a helicine artery (330). How does the epithelium of the urethra differ in its several segments, namely the prostatic, the membraneous, the penile, and the glans (fossa navicularis) portions?

Sketch 1.p. (331) portion of a preputial gland from a section through the corona. Location and significance of Tyson's glands? Note character of the skin of the glans penis.

Trace the course of the blood through the penis in the flaccid, and in the erect condition. Nerve supply and nerve end-organs?

\section{THE FEMALE ORGANS OF REPRODUCTION}

\section{(Female Genital System)}

\section{(A) THE OVARY (Ovarium).}

(1) Study l.p. and h.p. a stained section of the ovary of a full-term fetus or young infant. Note: a, the peripheral 'germinal epithelium', with its larger spherical primordial germ cells (ova); b, egg tubes of Pflueger: c, 'egg nests'; and d, a typical primitive ovarian follicle with its central ovum (primary oöcyte) and its enveloping mantle layer. Sketch each under the h.p. (332 a, b, c and d). Derivation of the ovarian germ cells?

(2) Study l.p. a stained section of the ovary of an adult. Note: a, the enveloping peritoneal layer ('germinal epithelium'); b, the peripheral cortex with its ovarian follicles and the superficial tunica albuginea; 
and c, the medulla, coming to the surface at the hilus. Sketch (333). In the cortex note also the corpora lutea and the corpora albicantia. What is their significance? Make h.p. sketches $(334,335)$ of portions of each. Make h.p. sketch (336) of a typical area of the medulla.

Sketch h.p. also: a, a primary ovarian follicle (33\%); b, a follicle at an intermediate stage of growth (338); and c, an older vesicular (Graafian) follicle (339), noting all the constituent elements. Manner of derivation of the secondary oöcyte from the primary oöcyte of the vesicular follicle? oötid from secondary oöcyte?

(3) Study an injected specimen of a cat's ovary. What is remarkable about the arteries? Sketch (340) an area of the medulla, and an adjacent vesicular follicle.

(4) Oögenesis. Sketch the several salient stages of oögenesis from a series of demonstration slides of eggs of mouse or starfish: a, unripe (small) ovarian egg (primary oöcyte) (314); b, ripe (full-grown) primary oöcyte (342); c, first maturation spindle (343); d, secondary oöcyte with first polocyte (344); e, second maturation spindle (345); f, oötid or mature ovum with second polocyte (346).

\section{(B) THE UTERINE TUBE (Oviduct; Fallopian tube; Salpinx).}

Make a comparative study of transverse sections of the uterine tube at: $a$, the isthmus; $b$, the ampulla; $c$, the infundibulum, noting differences in the size of the lumen, and the character and constituents of the three tunics (tunicæ mucosa, muscularis and serosa). Diagram (34\%). Sketch h.p. (348) a segment of the mucosa from the isthmic region. Differential features among uterine tube, ductus deferens and the ureter?

\section{(C) TH்E UTERUS (Metra; Hystera).}

Compare vertical sections through the body and cervix of the uterus. Sketch l.p. (349) a narrow segment of the entire wall from both regions. Enumerate the chief differences. Sketch h.p.: a, the mucosa of the body (350) ; b, a cervical gland (351). Significance of non-ciliated areas? How many strata in the tunica muscularis? Which is the stratum vasculare? How does the resting uterus differ from the menstruating uterus? from the pregnant uterus? How does the human uterus differ in gross form and with respect of the muscular tunic from that of certain common mammals, e.g., dog, cat, etc.?

(D) THE $V A G I N A$.

Study l.p. a section from the middle portion of the vagina. Note: $a$, the tunica mucosa; b, tunica muscularis; c, tunica fibrosa. Sketch 
(352). Type of lining epithelium? Are glands present? What is the striking common characteristic of the tunica propria of the entire duct system of the female genital apparatus?

(E) THE EXTERNAL GENITALS.

These include: $a$, the vestibule; $b$, the labia minora; $c$, the labia majora; $d$, the clitoris; e, the hymen; $f$, the minor vestibular glands, and $\mathrm{g}$, the major vestibular glands. Enumerate the histologic characteristics of each. What are the male homologues for $d, f$, and $g$ ? The major vestibular glands (glands of Bartholin) open into the groove between the hymen and the labium minus. Sketch h.p. several adjacent alveoli of this gland (353).

(F) THE MAMMARY GLAND (Mamma).

(1) Study l.p. a section of the active (lactating) gland. Note the arrangement into lobes and lobules. Sketch (354), showing the interlobar lactiferous duct and the inter- and intralobular ducts. Make h.p. sketch (355) of an alveolus, noting especially the cytoplasmic contents of the lining cells. Significance of the basket cells? of the basal striations of the secretory cells?

(2) Make l.p. study of a resting mammary gland. Sketch (356).

(3) Examine a cover-glass preparation of fresh milk stained with methylene blue. Note especially the leucocytes and fat droplets. Sketch (35\%). Derivation of the leucocytes?

(4) Enumerate the histologic features of: $a$, the mammilla; $b$, the areola; c, the glands of Montgomery, and $d$, the integument of the mammæ. Character and significance of 'witches milk'?

\section{DUCTLESS GLANDS}

(Endocrine Glands; Organs of Internal Secretion; Cryptorhetic Glands)

(A) THE SUPRARENAL GLANDS (Adrenals).

(1) Examine a divided fresh suprarenal gland of some mammal. Note the yellowish coloration of the cortex, and the red color of the medulla; note also the capsule and the hilus. Cause of difference in color between cortex and medulla?

(2) Study l.p. stained vertical sections of the human and the dog's adrenal. Chief differences? Sketch (358) a narrow segment from capsule to center of medulla. Make h.p. sketches (359) of groups of cells 
from the zonæ glomerulosa, fasciculata and reticularis. Enumerate the chief differences in gross arrangement of the cells and in their cytoplasmic contents. Sketch also (360) a group of cells from the medulla. What is the chief granular content of these cells? Significance? Sketch also (361) a segment of the central vein. What is remarkable about this vessel?

(3) Study a stained horizontal section through the zona glomerulosa. Arrangement of the glomerular cells? Function of the adrenal? Reptilian and ichthyoid homologues?

(4) Study an injected specimen of cat's adrenal. Diagram the arterial blood system (362). Relation of the cells to the venous capillaries? Significance of the separate blood supply of the cortex and the medulla?

\section{(B) THE THYROID GLAND.}

Study l.p. a stained section of the human thyroid gland. Note: capsule, lobules, follicles and colloid content of latter. Sketch (363) a small area including the capsule and the interfollicular stroma. Sketch h.p. (364) a folicle, noting especially the two types of lining cells: a, chief cells; $b$, colloid.cells. What are the striking characteristics of the colloid content of the follicle? Cellular inclusions in the colloid?

From an injected specimen of the cat's thyroid diagram the blood supply of a lobule (365).

What morbid condition is associated with atrophy of the thyroid? with hypertrophy (hypersecretion)? with arrested development? Normal function?

How does aberrant or accessory thyroid tissue differ from that of the thyroid proper?

What are the salient differential characteristics of sections of the thyroid, active mammary gland and prostate gland?

\section{(C) THE PARATHYROID GLANDS.}

Make h.p. sketch (366) of a small area of a stained section of a parathyroid (epithelial body). Note the 'acidophil' and 'principal' types of cells. Does colloid material occur? Spatial and functional relationship between thyroid and parathyroids. Function of the parathyroids?

\section{(D) THE THYMUS GLAND.}

Study l.p. a stained section of the human thymus. Note: capsule, lobules (with cortex and medulla) and interlobular connective tissue 
septa. Sketch (367) several adjacent lobules. Note continuity of medullary substance between adjacent lobules. Make h.p. sketch of a lobule (368); also of a thymic corpuscle (concentric corpuscle of Hassall) (369). Where are the latter located? Origin and function of the thymic corpuscles? Is the thymus a lymphoid organ or an endocrin (cryptorhetic) gland? Ontogenetic history of the thymus? From an injected specimen of cat's thymus, sketch (370) the blood supply of a lobule.

THE CAROTID GLAND.

Location, origin and probable function of this gland? Sketch h.p. (371) a small area. Compare with the Coccygeal Gland and the Paraganglia. Origin and function of the latter?

\section{THE HYPOPHYSIS CEREBRI (Pituitary Gland).}

In a stained sagittal section of the human hypophysis identify: a, pars neuralis (posterior lobe) ; b, pars juxtaneuralis (intermediate portion); c, residual lumen; d, pars distalis (anterior lobe). Make h.p. sketches (372) of a small area from each of these portions. In the pars distalis note the cords of central neutrophilic and parietal eosinophilic cells. Significance of these two types? Over the periphery of the pars distalis and lining the residual lumen note the basophilic cells. Significance? What are the 'chromophobe' cells?

Origin and probable function (normal and morbid) of this gland? Blood and nerve supply?

\section{(G) THE EPIPHYSIS CEREBRI (Pineal Body).}

Study l.p. a stained sagittal section of the adult sheep's pineal body. Note: pineal stalk with its pineal recess; capsule, 'lobes', trabeculæ, and acervulus (brain sand). Compare with the human pineal body. Slretch h.p. (373) a small area near the periphery. Note the two chief types of cells : neuroglia and 'interneurogliar'. Significance of the two types? Note also the intercellular clumps of pigment, and the lamellated and conglomerate types of acervulus, and the larger edematous areas. Compare with a similar section of this gland from a young sheep. In the latter note the intracellular pigment granules and the intercellular cysts. Origin and probable function (normal and morbid) of this gland? Blood and nerve supply? Significance of the melanic pigment? of the cysts? of the brain sand? 


\section{THE EYE}

(Bulbus Oculi, and Appendages: Palpebra and Lacrimal Glands)

\section{(A) MACROSCOPIC STUDY.}

(1) With the aid of a mirror, sketch your eye (374). Note: $a_{\text {s }}$ supercilium (eye-brow); b, superior and inferior palpebræ (eye-lids), with their cilia (eye-lashes) at the base of which internally may be seen' a row of whitish pits, the orifices of the tarsal (Meibomian) glands; c, the medial and lateral anguli.oculi and the canthi (commissures); $d$, in the depth of the medial angle (the lacus lacrimalis) a reddish roughened area, the caruncula lacrimalis; e, at the base of this angle the plica semilunaris (homologue of nictitating membrane or third eye-lid of birds, reptiles, etc.), and $f$, at either basal angle of the internal canthus an elevated papilla lacrimalis, with its punctum lacrimalis leading to the canaliculus lacrimalis and the naso-lacrimal duct. Note also the fine hairs on the caruncula. Retract the lower lid and note the conjunctive palpebræ; its continuity with the conjunctiva oculi across the fornix conjunctivæ can readily be observed.

Within the rima palpebrarum, the opening between the lids, note: $a$, the pupil; b, the iris; and c, the overlying transparent cornea, continuous peripherally with the opaque sclera. What is the pinguecula?

(2) In a formalin-preserved specimen of the eye-ball of sheep or ox, dissect out carefully the six extrinsic eye-muscles. Note the point and manner of insertion of the muscles and the relation of the superior and inferior obliques to the superior and inferior recti muscles respectively. The levator palpebræ superioris muscle may also have remained intact; also a muscle attached by a stout tendon to the well-developed cartilaginous plica semilunaris, the musculus plicæ. In the eyes of ruminants: an additional muscle occurs, not present in the human eye, the retractor: bulbi; this large stout muscle completely envelops the optic nerve. Separate the retractor bulbi muscle from the optic nerve down to the point: of exit of the nerve from the bulb. By noting the position of the point: of exit with respect to the posterior pole of the bulb, and the relation of this point to the plica semilunaris and levator palpebræ muscle, determine which eye you have been dissecting. Sketch anterior and posterior poles (375).

(3) Bisect the eye-ball in the horizontal meridian. (This should be done with a sharp razor, proceeding from the posterior pole, first divid- 
ing the optic nerve.) Examine under water. Note: cornea, iris, crystalline lens, suspensory ligament (zonula ciliaris of Zinn) of tens, anterior and posterior chambers with their content of aqueous humor; the ciliary body with the ciliary folds and processes; the ora serrata; the vitreous humor (body); the retina; the tunica choroidea; the sclera; the lamina fusca and the lamina suprochoroidea between the separated portions of the choroid and sclerotic coats; the macula lutea with the fovea centralis; the optic papilla (disc) with the central depression (physiologic excavation); hyaloid canal; and the hyaloid membrane. Sketch (376). Significance of the hyaloid canal (canal of Cloquet; canal of Stilling)?

Remove the lens. Note the difference in curvature of its anterior and posterior surfaces. Remove the anterior lens epithelium, and the lens capsule. Remove the more superficial layer of lens fibers and free the central non-nucleated lens 'nucleus'. Explain the arrangement of the lens fibers, with relation to each other, the 'nucleus', and the poles of the lens (lens 'stars'). Sketch (37\%).

How is the lens held in place? Describe the action of the lens. What is a cataract of the eye? Difference between the anatomic axis of the bulb and the visual axis? Embryonic origin of the several tunies and humors of the bulbus oculi, and of the lens? What is the capsule of Tenon?

\section{(B) MICROSCOPIC STUDY.}

(1) Study l.p. a stained horizontal section of the human eyeball (or that of cat, dog or monkey). (The bulb should have been preserved in Perenyi's fluid,* which softens the lens). Sketch (378). Note the difference in curvature between the cornea and the posterior scleral segment of the bulbus oculi; also between the anterior and posterior surfaces of the lens. Identify the several chambers, angles and tunics of the bulb, and the several laminæ of each tunic. Note the disposition of the nuclei in the lens. Enumerate and identify the intrinsic muscles of the eye. Explain their nerve supply. Explain the action of the ciliary body. Function of the aqueous chambers and the canal of Schlemm (sinus venosus scleræ)? Relation to glaucoma? Define astigmatism.

(2) Study h.p. the sclero-corneal junction. Identify all of its con-

* Perenyi's fluid: 4 parts 10 per cent nitric acid

3 parts alcohol $(95 \%)$

3 parts 0.5 per cent chromic acid.

Fix 5 to 10 hours, and pass directly into $70 \%$ alcohol. 
stituent elements, and note its relationship to adjacent structures. Sketch (379). Function of ligamentum pectinatum and the included spaces of Fontana?

(3) Sketch h.p: (380) the cornea. Identify its five layers. Innervation of the corneal conjunctiva? Function of the membrane of Des cemet? Changes in cornea after death or fixation?

(4) Sketch h.p. (381) the sclera. Location and character of the lamina fusca?

(5) Sketch h.p. (382) the tunica choroidea. Note the several laminæ: suprachoroidea, vasculosa, tapetum fibrosum (cellulosum), choriocapillaris, and lamina basalis (vitrea). Function of the choroid?

(6) Sketch h.p. (383) the equatorial margin of the crystalline lens, including the zonula ciliaris of Zinn (suspensory ligament) with its spacia zonularis (canals of Petit). Note the lens capsule, lenticular epithelium, and the substantia lentis composed of nucleated lens fibers, with serrated margins.

(7) Sketch h.p. (384) a segment of the iris. Note: a, its external layer of mesenchymal epithelium ('endothelium') ; b, its fibrous stroma, with its pigmented cells; $\mathrm{c}$, the internal epithelial layer (pars iridica retinæ) ; d, the stromal recesses; e, the dilator and sphincter muscles; and $f$, the circuli major and minor. Origin and function of the iridal muscles? Relation of pigmented stroma to 'color of eye'? Effect of certain drugs upon the size of the pupil?

(8) Sketch h.p. (385) the pars optica retinæ, or the retina proper, at a point about midway between the macula lutea and the ora serrata. Relation to pars ciliaris retinæ and the pars iridica retinæ? Identify the eleven different layers. Compare this portion of the retina with that near the fovea centralis and that near the ora serrata. Probable function of : $a$, the pigment (fuscin) of the pigmented layer; $b$, the visual purple (rhodopsin) of the rod visual cells; and c, the myoid element of the rods and cones? Explain the inversion of the retina. Explain coloboma and scotoma.

(9) In a Golgi preparation of the retina identify the different neurons of the several layers and determine their interrelations (synapses). Construct a diagram (386) showing the interrelationships of the elements of the different layers, including the neuroglia supporting cells of Müller.

(10) (a) Study an injected specimen of cat's eye. Note the distribution of the branches of the central artery of the retina, the short ciliary arteries, the long ciliary arteries, and the anterior ciliary arteries, 
especially with respect to the uvea or complete middle tunic. Do these several arterial systems anastomose? Note also the radicles of the rena vorticosæ. Sketch (387) lateral half of bulbus oculi.

(b) Trace out also the lymphatic systems of the eye.

(C) THE OCULAR APPENDAGES.

(1) The Palpebre (eye-lids). Study 1.p. a stained vertical section of the human superior eye-lid. Note: cutaneous surface, rimal margin and the conjunctival surface; the orbicularis palpebrarum muscle, insertion of the musculus levator palpebræ superioris, the superior palpebral muscle of Müller, the ciliary muscle of Riolan, and the tarsal fascia (tarsus); the cilia (eye-lashes), sebaceous glands of Zeiss, glands of Moll, tarsal glands of Meibon, and the glands of Waldeyer (posterior tarsal glands); the primary and secondary tarsal (arterial) arches. Sketch (388).

Make h.p. sketch (389) of the palpebral conjunctiva near the level of the fornix conjunctivæ.

(2) The Lacrimal Gland. Study a stained section of the lacrimal gland. Sketch l.p. (390) a lobule, showing the ducts and the secretory tubules. Make h.p. sketches (391, a, b, etc.) of sections of tubules at several stages of secretory activity. Where are the accessory lacrimal glands of Krause? Location and significance of the gland of Harder?

\section{THE GAR}

\section{(A) THE EXTERNAL EAR.}

(1) Auricle (Pinna). Study l.p. a stained section of the lobule of the ear, including adjacent portion of the pinna. Note the absence of cartilage in the lobule proper, and the abundant adipose tissue. Sketch (392). Make h.p. sketch (393) of portion of the elastic cartilage of the pinna. Note the relatively large number of cells.

(2) Study l.p. a stained section of the cartilaginous portion of the External Acoustic Meatus. Note the abundant large stiff hairs, sebaceous glands, and ceruminous glands. Sketch (394). Sketch h.p. (395) a transverse section of the secretory portion of the coiled, simple tubular, ceruminous gland. Enumerate differences and resemblances between the ceruminous glands, the sudoriparous glands, and the mammary glands.

\section{(B) THE MIDDLE EAR (Tympanum).}

(1) Study 1.p. a stained radial section of the tympanic membrane. Note: annulus tympanicus; umbo, with attached manubrium of the 
malleus; membrana flaccida (Shrapnell's membrane)? and cutaneous layer, mucous layer, and intermediate radial and circular collagen fibers. Sketch (396). Sketch (397) h.p. a small segment of the tympanic membrane near the umbo. Function of tympanic membrane? Relation to the auditory ossicles? Structure of the ossicles? Relation of tympanim to the mastoid 'cells'? to the auditory tube?

(2) The Aúditory Tube (Éustachian Tube). Study a stained transverse section of the cartilaginous portion of the tuba auditiva. Note character of the lining epithelium, and the fibro-cartilaginous wall. Relation of cartilage plate to medial and lateral surfaces, and to the tensor and levator palati muscles. Sketch (398). Function of the auditory tube? Position and significance of the tubal tonsil? Function of the musculus tensor palati in relation to the auditory tube?

\section{(C) THE INTERNAL EAR}

(1) Vestibule (Utriculus and Sacculus). Study a stained section of the wall of the utricle or saccule through the macula. Note character of the lining epithelium. Sketch h.p. (399) a segment of the macula. Function of the otolithic membrane?

(2) Semicircular Ducts (Canals). Study h.p. a stained transverse section of a membraneous semicircular duct. Compare with a section through the ampulla. Sketch segments of the lining epithelium (400), and of the crista ampullæ acustica including the overlying cupola (401).

(3) Cochlea. Study l.p. a stained axial section of the cochlea of some mammal (cat; rabbit). Sketch a complete vertical section of a turn of the cochlea (402). Identify and label all the included parts. Function of the vestibular (Reissner's) membrane? tectorial membrane? basilar membrane? Shambaugh's glands? the stria vascularis? vas prominens?

Make h.p. sketch (403) of the spiral organ (of Corti), identifying the various cells involved.

Study an injected specimen of the cat's cochlea. Diagram (404) the blood supply of a turn of the cochlea.

Describe the nerve supply of the membraneous labyrinth of the internal ear. Where is the internal acoustic meatus? Function of the utriculus? sacculus? semicircular canals? cochlea? 


\section{THE NERVOUS SYSTEM}

\section{(A) THE SPINAL CORD (Medulla spinalis).}

(1) Study l.p. a stained (preferably by the Weigert-Pal technic) transverse section of the spinal cord in the lower cervical region, including a spinal ganglion. Compare with the upper cervical and thoracic levels. Note the enveloping membranes (spinal meninges) : dura mater, arachnoidea, and the pia mater; and their continuity with the connective tissue envelopes of the spinal nerve. 'Sketch (405) a segment through these membranes with the included lymph spaces. Note the greater vascularity of the pia mater spinalis. Note further the compressed oval shape of the lower cervical section, and its division into symmetrical halves by the dorsal longitudinal septum and the ventral longitudinal fissure; also the central canal; the central gray matter arranged in the form of an $\mathrm{H}$ with its dorsal, ventral, and lateral horns and their constituent cell groups; the peripheral white substance, divisible into dorsal, lateral, and ventral columns; the gray and white commissures; the substantia gelatinosa centralis, and the gelatinous substance of Rolandi; the formatio reticularis; the cell column of Clarke; nucleus of Stilling; the connective tissue and neuroglia septa of the white substance; and the dorsal and ventral roots of the spinal nerves. Sketch (406). Describe the structural differences between gray, white, gelatinous, and reticular substances. Compare the lower cervical with the lumbar and sacral sections. Differences? Development of the spinal medulla? Define tract (fasciculus), column (funiculus), and nucleus (of origin and termination) of the spinal cord. Define: conus medullaris, filum terminale, and the cauda equina. Describe the relations to each other of the sensory, motor and association neurons in the spinal cord.

(2) Study l.p. an injected specimen of the spinal cord of the cat. Note the five longitudinal arterial trunks in the pia mater spinalis: the larger antero-median artery, and the more slender postero-lateral longitudinal arterioles (arranged anteriorly and posteriorly to the line of entrance of the posterior nerve roots). Note their further penetration and distribution within the gray and white substances. (Sketch (407).

Location, structure and function of the septum posticum, the ligamenta denticulata and the subarachnoid spaces? origin of cerebrospinal fluid? Trace the course of the lymph circulation through the central nervous system. 


\section{(B) THE CEREBELLUM.}

(1) Study l.p. a stained parasagittal section of a hemisphere of the human cerebellum. Note the arrangement into lobules composed of folia. Note also that each folium comprises a central medulla and a peripheral cortex. In the cortex identify the superficial molecular layer, the deeper nuclear or granular layer, and between the two the intermediate layer of Purkinje cells (commonly classified as the innermost stratum of the molecular layer). Sketch (408). Make h.p. sketch (409) of a Purkinje cell.

, (2) From a specially prepared demonstration slide of the cerebellum (Golgi technic) make a diagram (410) showing the location and interrelations of the constituent cells and fibers: In the molecular layer, the large ('basket') and small cortical cells; the Purkinje cells; and in the nuclear layer, the granule cells, eosin bodies, large stelate cells, and the solitary cells. In the medulla note the axons of the Purkinje cells, the mossy fibers and the climbing fibers. What is the significance of the eosin bodies? Relation between granule cells and mossy fibers? How does a sagittal section differ in appearance from a frontal section, with respect to the Purkinje cells and the large cortical (basket) cells? Note also the types and disposition of the neuroglia cells.

\section{(C) THE CEREBRAL CORTEX (Pallium).}

(1) Study l.p. a stained (hematoxylin and eosin) vertical section of the human cerebral cortex in the motor area (precentral gyrus). Note the arrangement of the cells into five tangential strata: molecular layer, outer polymorphous cell layer, small pyramidal cell layer, large pyramidal cell layer, and inner polymorphous cell layer. Sketch (411). Compare with similar sections from the parietal lobe (a sensory area), the visual area of the occipital lobe, the auditory area of the temporal lobe, and the olfactory area of the hippocampal gyrus. Note also the cerebral meninges (dura mater, arachnoidea, and pia mater) and the enclosed lymph spaces. Describe the granulationes arachnoidales (Pacchionian bodies; arachnoidal villi).

(2) From a Golgi preparation of the motor cortex, sketch (412) a segment showing the character and arrangement of the neurons and the neuroglia elements. Identify a cell of Betz; a cell of Martinotti. Significance of these cells? Distribution of the 'solitary cells' of Meynert?

(3) From a Weigert-Pal preparation of the motor cortex sketch (413) the arrangement of the nerve fibers. Note the bands of radial 
fibers alternating with the interradial feltwork; the supraradial feltwork; and the tangential fiber strata: the stratum zonale, the stripe of Bechterew, and the outer and inner stripes of Baillarger. Relation of the tangential stripes to the cell strata? Compare with a similar preparation of the visual cortex. Define the stripe of Gennari. 



\section{INDEX}

Abiogenesis, 17

Absorption, bone, 83

Accessory suprarenal, 556

Accessory thyroid, 562

Acervulus, 584

Aceto-carmin stain for tissues, 744

Acid dyes, 740

Acidophil cells, of small intestine, 371 of pituitary body, 576

Acoustic meatus, external, 683

Acoustic nerve, 712

cochlear branch of, 712

spiral ganglion of, 712

vestibular branch of, 712

vestibular ganglion (of Searpa), 712

Acromegaly, 573

Adenoid tissue, 84

Adipose tissue, 61 .

Adrenal (suprarenal) gland, blood supply of, 554-555

cells of medulla, 553

chromaffin granules in, 554

lymphatics and nerves of, 556

zona fasciculata of, 552

zona glomerulosa of, 552

zona reticularis of, 553

Adrenalin (epinephrin), 549-554

Adrenin, 549

Agminate nodules of small intestine (Peyer's patches), 366

Alcohol for fixation of tissues, 724

Alimentary canal, fibroserous coat of, 345

glands of, (crypts of Lieberkühn), 366

ileocecal valve of, 379

intestinal villi in, 366

intestine, large, 377

appendices epiploicæ of, 377
Alimentary canal, intestine, large, lymphoid tissue and lymph nodes

$$
\text { of, } 378
$$

mucous membrane of, 377

plicæ semilunares of, 377

rectum of, 379

sacculations or haustra of, 377

teniæ (lineæ) coli, 377

vascular and nerve supply of, 379

vermiform appendix of, 378

intestine, small, 375

absorption from, 375

agminate nodules of (Peyer's patches), 366

blood supply of, 373

corium of, 366

duodenal glands of (Brunner's glands), 365-372

granules of Paneth in, 370

intestinal glands of, 370

intestinal villi in, 368

lacteals of, $369-374$

lining epithelium of, 369

lymphatics of, 374

mucous membrane of, 366

nerve supply of, 374

Peyer's patches in, 366

solitary nodules of, 366

structure of, 363

submucosa of, 364

valvulæ conniventes of, 364

mucous membrane of, 347

muscular coat of, 346

submucous coat of, 346

stomach, blood supply of, 360

cardiac glands of, 360

fundus glands of, 354

lenticular glands of, 360

lymphatics of, 362 
Alimentary canal, stomach, mucous coat of, 353

muscular coat of, 353

nerve supply of, 362

oxyntic or delomorphous cells of, 356

peptic or aủelomorphous cells of, 355

parietal cells of, 356

pyloric glands of, 357

secretion of, 357

serous coat of, 352

submucous coat of, 353

Alimentary tract, tabular statement of characteristics, 381

Alum carmin for staining tissues, 744

Alum hematein (Mayer) for staining tissues, 741

Ameboid mobility, 16

Ameloblasts (adamantoblasts), 333

Amitosis, 19

Anaphase, 25

Anatomy, microscopic, 1

Angioblast, 215

Annulus tympanicus of ear, 685

Anterior chamber of eye, 636

Appendix epididymis, 502

Appendix testis, 502

Appendix vermiformis, 378

Aqueductus Fallopii, relations of, to ear, 686

Aqueous humor of eye, 665

Arachnoid membrane, 620

granulations, 621

villi, 621

Arciform veins of kidney, 443

Areas of Cohnheim in muscle, 112

Areolar connective tissue, 56

Arterial wall, general characteristics of, 180

Arteries, 176

anterior ciliary, of eye, 673

anterior spinal, 624

arciform, of kidney, 440

arteria centralis of retina, 664, 670

arteriæ propriæ renales, 440

arteriæ rectæ of kidney, 441
Arteries, atypical, structure of, 182 auditory, 714

bronchial, 317

central, of retina, 664,670

circle of Willis, of brain, 624

circle of Zinn, of eye, 672

cortical, of cerebrum, 625

external elastic membrane of, 179

fissural, of brain, 625

helicine, of corpus cavernosum penis; 504

hyaloid, of eye, 672

interlobular, of kidney, 441

of liver, 415

internal elastic membrane of, 178

large, structure of, 181

large and small compared, 184

long ciliary, of eye, 672

medium-sized, coats of, 177

medullary, of cerebrum, 625

middle cerebral, 625

pulmonary, 314

structure of, 183

precapillary, structure of, 182

short-ciliary, of eye, 672

small, structure of, 181

tunica adventitia of, 177-178

tunica intima of, 177

tunica media of, $177-178$

umbilical, structure of, 182

vasa vasorum of, 180

Arterioles, structure of, 181

Atrioyentricular bundle of His, 102

Auerbach's fuchsin methyl green stain, 756

Auditory area of cerebral cortex, cell layers of, 618

Auditory canal, 683

Auditory ossicles, 689

Auditory tube of ear, 691

Auerbach's stain for chromatin, 756

Auricle or pinna of external ear, 682

Axis cylinder, the, 132

Axon, the, 128

Balsam (Canada) for mounting sections, 760 
Bartholin's glands of the vulva, 541

Basic dyes, 740

Basket cells of cerebellum, 608

Basophilic cells of pituitary body, 576

Bellini's ducts of kidney, 438

Benda's technic for demonstrating mitochondria, 757

Berlin blue gelatin mass for injection of tissues and organs, 732

Betz cells of cerebral cortex, 613

Bibliography of histologic technics, 761

Bioplasm, 1

Bladder, urinary, 448

Blastomeres, 27

Blood, 203

angioblast, 215

coagulation of, 214

development of, 24

fibrin of, 203

hematin of, 212

hematoidin, 214

hemin, 212

hemoglobin of, 203-211

hemokonia of, 206

hemolysis of, 206

hemosiderin, 213

hypertonic solution for, 206

hypotonic solution for, 206

isotonic solution for, 206

leukoblasts in, 218

mononuclear leukocytes in, 218

oxyhemoglobin, 211

prothrombin, 203'

Teichmann's crystals in, 212

thrombin, 203

Blood cells, basophils, 210

erenation of, 206

dyes for, 210

eosinophils, 210

erythroblasts, 217

erythrocytes, 203-217

erythroplastids, 203-217

giant, 209-218

granulocytes, 209

hyaloplasm of, 206

ichthyoid stage, 217

leukocytes, 203
Blood cells, lymphocytes, 207

megaloblasts, 217

megalocytes, 209

neutrophils, 209

non-granular leukocytes, 208

normoblasts, 217

plastids, 203

plates (plaques), 209

polykaryocytes, 209

primitive, derivatives from, 219

red, 203

rouleaus, 206

sauroid stage, 217

spindle cells, 209

spongioplasm of, 206

thrombocytes, 209

transitional leukocytes, 208

white, 207

Blood clot, 203

Blood corpuseles, 203

crenated corpuscles, 206

Blood crystals, 212

Blood plasma, 203

Blood platelets, 203-209

Blood serum, 203

Blood shadows, 206

Blood stain, eosinate of methylene blue, Hastings' method, 754

Blood vascular system, 176

Blood vessels, circulus major of iris, 672

circulus minor of iris, 673

development of, 192

essential structure of, 194

fetal, 538

of brain and spinal cord, 619-624. 625

of bronchi, 317

of eye, 636-643-664-670-673

of eyelids, 678

of heart, 201

of internal ear, 714

of kidney, 440-444

of large intestine, 379

of liver, 415-417

of lungs, 314

of lymph nodes, 242 
Blood vessels, of mammary gland, 547 of middle ear, 693

of olfactory mucosa, 298

of ovary, 522

of oviduct, 527

of pancreas, 403

of parathyroid glands, 565

of penis, 503-504

of prostate gland, 500

of renal pelvis and ureter, 448

of salivary glands, 392

of skin, 290

of small intestine, 373

of spleen, 246

of stomach, 360

of suprarenal gland, 554

of thymus gland, 568

of thyroid gland, 560

of tongue, 343

of urinary bladder, 452

of uterus, 532

Böhmer's hematoxylin for staining tissues, 741

Bone, 72

absorption of, 83

cancellous, 85

eanaliculi of, 75

circumferential lamellæ of, 76

compact, 74

development of, 79

diploe of, 88

endochondrial ossification, 85

epiphyseal line, 85

epiphyseal ossification, 85

external circumferential lamellæ of, 84

general considerations of, 72

Haversian canals of, 85

Haversian lamellæ of, 75

Haversian spaces of, 84

Haversian systems of, 75-84

internal circumferential lamellæ of, 85

interstitial lamellæ of, 76-85

intramembranous ossification, 86

lacunæ of, 75

lacunæ of Howship, of, 84
Bone, lymphatics of, 78

nerves of, 78

osteoblasts of, 83

osteoclasts of, 77

periosteum of, 74

primary, 83

red marrow of, 77

tables of, 88

Volkmann's canals of, 76

Bone marrow, 77-220

Bone marrow cells, basophil granulocytes, 223

eosinophil granulocytes, 222

erythrocytes, 224

giant cells, 223

hemoblasts, 221

large mononuclear leukocytes, 222

lymphocytes, 221-222

mast cells, 223

mesameboid cells, 221

myeloblasts, 221

myelocytes, 220

myeloplaxes, 223

polymorphonuclear neutrophil gran. ulocytes, 222

premyelocytes, 221

primitive blood cells, 221

Bony labyrinth of the internal ear, 694

Borax carmin stain for tissue, 743

Bouin's fluid for fixation of tissues, 729

Bowman's elastic membrane of cornea, 631

Box for embedding in paraffin, 737

Brain sand (acervulus), 584

Bronchi, 304

structures of, 304

Bronchial arteries, 317

Bronchioles, 307

Brownian movement, 17

Bursæ, 117-233

Cajal's method for demonstrating neurofibrils, 750

Calcified cartilage, 80

Canal of Stilling (canalis hyaloideus), 672 
Capillary plexuses, 187

Capillary wall, stigmata of, 186

Capillaries, structure of, 184

Cardiac glands of the stomach, 360

Cardiac muscle, 94, 99

Cardiac muscle, intercalated dises of, Zimmerman's method of staining, 758

Cardiac Purkinje fibers, 102

Cardiac plexus, origin of, 591

Carmin gelatin mass for injection of tissues and organs, 732

Carmin stain for tissues, 743

Carnoy's fluid No. 1 for fixation of lung tissue, 729-730

Carotid gland, the, 569

Cartilage, 67

blastema of, 69

calcified, 80

cells, enlargement of, 80

elastic, 71

fibro-, 71

hyaline, 68

matrix of, 69

origin of, 70

primordial marrow cavities in, 82

Cell, definition of, 1

Cell layers of motor area of cerebral cortex, 615,616

Cell plate (midbody), 25

Celloidin, adhesive for sections, 739 embedding of tissues in, 734

Cells, acidophil, of parathyroid, 564 acidophilic, of pituitary body, 576

acidophil, of small intestine, 371 amitosis of, 19

anaphase of, division of, 25

archoplasm of, 7

astral rays of, 7

astral system of, 7 .

basophilic, of pituitary body, 576

basket, of salivary glands, 385

basophil granules of, 52

Betz, of cerebral cortex, 613

cartilage, enlargement of, 80

cementum, of teeth, 337
Cells, of cerebral cortex, Golgi's, types 1 and 2, 613

centro-acinal, of Langerhans, 396

centriole of, 7

centrosome of, 7

centroplasm of, 7

centrosphere of, 7

chondrioconts of, 8

chondriomites of, 8

chromaffin of small intestine, 371

chromaffin, of suprarenal gland, 548

chromidia of, 8

chromophobe, of pituitary body, 576

chromosomes of, 24

cilia of, 16

close spireme, 24

connective tissue, 51

cytology of, 1

cytoplasm of, 7 .

decidual, of gravid uterus, 535

deutoplasm of, 8

diplosome of, 7-24

division of, 18

eosinophil granulocytes, 52

ependyma, of nervous system, 589

general statements in regard to, 5

hepatic, 412

pigment in, 414

goblet, of small intestine, 370

granular, of cerebellum, 609

granule of Paneth in small intestine, 370

kinoplasm of, 7

lamellar, 52

of Langhans, of chorionic villi of gravid uterus, 537

large stellate, of cerebellum, 609

loose spireme of, 24

lutein, of ovary, 519

of Martinotti of cerebral cortex, 617

mastoid, of ear, 686

metaphase, of division, 25

metaplasm of, 8

microsome of, 8

mitochondria of, 8

mitosis of, 22

nucleus of, 6 
Cells, neuroglia, of nervous system, 589

osteoblasts, 77

osteoclasts, 78

parietal (oxyntic or delomorphous), of stomach, 356

peptic or adelomorphous, of stomach, 355

pheochrome, of suprarenal gland, 548

pigment, 52

plasma of, 51

plastosomes of, 8

prickle, 44

pseudochromosomes of, 8

Purkinje, of cerebellum, 607

pyramidal, of cerebral cortex, 613

reproduction of, 17

resting nucleus of, 24

segmented spireme of, 24

Sertoli, of seminiferous tubules, 481

small cortical, of cerebellum, 608

solitary, of Meynert of cerebral cortex, 613-618

spindle, 51

telophase of, division of, 25

tendon, 117

interstitial, of Leydig of testis, 487

trophospongium of, 8

varieties of, 32

vital properties of, 14

Cement substance, intercellular, 30

nitrate of silver stain for, 752

terminal bars of, 34

Cementoblasts, 337

Cementum, development of, 336

Centers of ossification, 79

Central artery of retina, 664

Central nervous system, blood supply of, 624

veins of, 625

Central spindle, 25

Cerebellum, 603

basket cells of, 608

climbing fibers of medulla of, 609

cortex of, 606

granule cells of, 609
Cerebellum, large stellate cells of, 609 medulla of, 609

Purkinje cells of, 607

résumé of structures of, 611

small cortical cells of, 608

Cerebral cortex, 611

auditory area, cell layers of, 618

cell layers of motor area, 615, 616

cells (solitary) of Meynert, 613-618

fiber tracts of, 618

Golgi's types of cells of, 613

motor area of, 615

motor area, cells of Martinotti of, 617

nerve cells of, 612

olfactory area, cell layers of, 618

stripes of Baillarger, 619

stripe of Bechterew, 619

visual area, cell layers of, 617

Cerebrum, cortical arteries of, 625

lobes of, 611

medullary arteries of, 625

meninges and blood supply of, 619 620-621

middle cerebral artery of, 625

Cervical glands of uterus, 532

Ceruminous glands of ear, 684

Chondrioconts, 50

Chondroclasts, 82

Chordæ tendineæ of heart, 197

Chorionic villi, 537

Choroid coat of eye, 636

lamina basalis of, 637

lamina capillaris of, 636

membrane of Bruch, 637

suprachoroid layer of, 636

tapetum cellulosum of, 638

tapetum fibrosum of, 637

tunica Ruyschiana of, 637

vascular layer of, 636

Choroidal fissure of the eye, 645

Chromaffin cells of small intestine, 371

of suprarenal gland, 548

Chromaffin granules, technic for demonstrating, 759

Chromaffin system, 571

Chromatin, Auerbach's fuchsin methylgreen stain for, 756 
Chromidia, in spermatogenesis, 474

Chromo-acetic formalin mixture for fixation of tissues, 727

Chromophobe cells of pituitary body, 576

Chromosomes, 24-476

sex, in spermatogenesis, 475

in spermatogenesis, 464

Chyle, 226

in small intestine, 374

Cilia, 16

Ciliary body of eye, 638

fibrous layer of, 640

Ciliary epithelium of eye, 640

Ciliary glands of eye, 640

Ciliary motility, 16

Ciliary processes of eye, 638

(iiliary muscle of eye, 638

Circle of Willis, 604

Circle of Zinn, 672

Circulus venosus of Haller, 547

Uiliated epithelium, 40

Clarification of sections, 759

Classification of dyes, 740

Clitoris, 541

Coagulation of blood, 214

Coceygeal gland, 570

Cochlea of internal ear, accessory tectorial membrane of, 706

basilar membrane of, 706

cochlear artery of, 714

function of, 716

hamulus of, 701

Hensen's stripe of tectorial membrane in, 706

limbus spiralis of, 705

membrana tectoria (membrane of Corti), 705

membranous wall of scala tympani and scala vestibuli, 704

organ of Corti, 708

varieties of cells of, 708-709

prominentia spiralis of, $\overline{705}$

spiral ligament of, 704

stria vascularis of, 705

structure of, 701

sulcus spiralis externus of, 705
Cochlea of internal ear, sulcus spiralis internus of, 706

vestibular membrane of Reissner, 704

Collagen, 57

Colloid in thyroid gland, 557

Coloboma, eye, 646

Colostrum, 546

Columnæ carnæ, 198

Comparison of artery with vein of corresponding size, 79

Concentric corpuscles (Hassall) of thymus, 567

Congo red for tissue stain, 746

Conjunctiva, ocular, 674

palpebral, 674

Conarium (pineal body), 579

Connective tissue, Mallory's stain for,

- 753

Conus medullaris, 600

Corium of skin, 268

Cornea of eye, 630

anterior homogeneous membrane in, 631

Bowman's elastic membrane of, 631

endothelium of, 633

epithelium of, 631-633-674

layers of, 631

membrane of Descemet, 632

posterior epithelial layer of, 633

posterior homogeneous membrane of, 632

vascular and nerve supply of, 633

Corneal corpuscles, 631

Corneal epithelium, 631-674

Corneal substance, 631

Corpora albicantia, of ovary, 510

Corpora lutea spuria, 521

Corpora lutea vera of pregnancy, 521

Corpus cavernosum penis, 503

helicine arteries of, 504

pectiniform septum of, 504

tunica albuginea of, 504

Corpus hemorrhagicum of ovary, 519

Corpus Highmori testis, 480

Corpus luteum of ovary, 519

Corpus spongiosum penis, 503-506 
Corpuscles, Pacinian, 174

Cover glass, how to apply, 722

Cowper's glands of urethra, 501

Cretinism, 561

Crusta petrosa of teeth, 329

Crystalline lens of eye, 666

capsule of, 666

lenticular epithelium of, 666

nuclear zone of, 667

nucleus of, 667

substantia lentis, 666

suspensory ligament of, 669

Cutaneous appendages, 271

Cutis vera, 268

Cytogenesis, 1

Cytologic stain, Ehrlich triacid, 755

Cytology, 1

Cytolymph, 8

Cytomorphosis, 29

Cytoplasm, 7

Cytoplasmic dyes, $\mathbf{7 4 5}$

Cytoplasmic granules, eosin and methyl blue mixture (Mann), stain for, 755

Cytoreticulum, 8

Dartos of scrotum, 481

Decalcification of tissues, 731

Decidua reflexa of the gravid uterus, 537

Decidua vera of the gravid uterus, 537

Delafield's hematoxylin for staining tissues, 742

Dendrons (dendrites), 128

Dental cement, 329

Dental euticular membrane,

Dental enamel, layers of, 335

Dental groove, 330

Dental lamina, 330

Dental papilla, 330-336

Dental pulp, 323

odontoblasts in, 324

Dentin, 325

contour lines of Owen in, 327

globules in, 326

granular layer of Tomes in, 327

incremental lines of Schreger in, 327
Dentin, interglobular spaces of, 326

sheaths of Neumann in, 327

tubules in, 326

Derma, 268

Descemet's membrane of cornea, 632

Determiners, chromosomal in sex heredity, 477

Digestive system, 320

alimentary canal, character of wall in, 344

fibroserous coat of, 345

mucous membrane of, 347

muscular coat of, 346

submucous coat of, 346

esophagus, coats of, 348

glands of, 351

mucous coat of, 350

muscular coat of, 350

ileocecal (colic) valve, 379

intestinal glands (crypts of Lieberkühn), 366

intestine, large, 377

appendices epiploicæ of, 377

lymphoid tissue and lymph norles of, 378

mucous membrane of, 377

plicæ semilunares of, 377

rectum, 379

sacculations or haustra of, 377

teniæ (lineæ) coli, 377

vascular and nerve supply of, 379

vermiform appendix, lymphoid tissues in, 378

intestine, small, absorption from, 375

agminate nodules of (Peyer's patches), 366

blood supply of, 373

corium of, 366

duodenal glands (Brunner's), 365372

granule cells of Paneth in, 370371

intestinal glands of, 370

intestinal villi in, 368

lacteals of, $369-374$ 
Digestive system, intestine, small, lining epithelium of, 369

lymphaties of, 374

mucous membrane of, 366

nerve supply of, 374

Peyer's patches, 366

solitary nodules of, 366

structure of, 363

submucosa of, 364

valvulæ conniventes of, 364

liver, bile capillaries of, 411

blood supply of, 417

capsule of Glisson of, 408

cells of, 412

course of blood through, 419

gall-bladder, 420

hepatic connective tissue in, 408

hepatic lobule, 409

interlobular arteries and veins of, 415

interlobular bile ducts, 415

lymphatics of, 419

nerves. of, 420

pigment cells in, 414

portal canals, 408-415

structure of, 405

mouth, 320

lymphoid tissues in mucous membrane of, 321

mucous membrane of, 320

secreting glands in mucous membrane of, 321

pancreas, 394

acini of, 396

blood supply of, 403

nerve supply of, 404

résumé, 404

pharynx, 347

portal vein, 417

salivary glands, 382

blood supply of, 392

general considerations of, 382

mucous acini of, 386

nerve supply of, 393

parotid gland, 390

serous acini, 385

sublingual gland, 392
Digestive system, salivary glands, submaxillary gland, 391

stomach, 360

blood supply of, 360

cardiac glands of, 360

fundus glands of, 354

lenticular glands of, 360

lymphatics of, 362

mucous coat of, 353

muscular coat of, 353

nerve supply of, 362

peptic or adelomorphous cells of, 355

parietal cells (oxyntic or delomorphous cells) of, 356

pyloric glands of, 357

serous coat of, 352

secretion of, 357

submucous coat of, 353

teeth, 337

ameloblasts (adamantoblasts) of; 333

cementoblasts of, 337

cementum, 329

development of, 336

deciduous, 331

papilla of, 336

pulp of, 323

dentin of, 325

development of, 330

enamel of, 328

enamel germ of, 330-331

enamel layers of, 335

enamel organ of, 330

layers of, 331

Tome's processes in, 333

odontoblasts, 324

odontoclasts, 337

permanent, 331

structure of, 323

tongue, 337

blood vessels of, 343

foramen cecum of, 343

lymphatics of, 344

mucous membrane of, 337

muscle fibers of, 337

nerve supply of, 344 
Digestive system, tongue, papillæ of, $339,340,341$

taste buds of, 341

tonsil of, 343

Diploe, of bone, 88

Diploid number of chromosomes, 363

Diplosome, 24

Double staining of tissues, 746

Ductless glands, 260-548 hormones of, 586

Ductuli aberrentes, mesonephric, 502

Duodenal glands (Brunner's), 365372

Dura mater, 620

Dyes, 740

acid, 740

basic, 740

classification of, 740

cytoplasmic, 745

mordants for, 741

neutral, 740

specific, 740

Dyspituitarism, 573

\section{Ear, 717}

development of, 717-719

external, 682

acoustic meatus of, 683

auricle of, 682

ceruminous glands of auditory canal, 684

pinna of, 682

internal, 712

acoustic nerve, 712

cochlear branch of, 712

vestibular ganglion of Scarpa, 712

aqueductus vestibuli of, 694

auditory artery, 714

blood supply of, 714

bony labyrinth of, 694

eanalis communis of semicircular canals, 699

cochlea, 706

accessory tectorial membrane of, 706

basilar membrane of, 706
Ear, internal, cochlea, cecum vestibulare of scala media, 703

cochlear artery, 714

cochlear nerve, spiral ganglion of, 712

cupola of, 701

development of, 719

foramina nervosa of, 702

function of, 716

hamulus of, 701

helicotrema of, 703

Hensen's stripe of tectorial membrane in, 706

lagena or cecum cupulare of scala media in, 703

limbus spiralis of, 705

membrana tectoria, membrane of Corti, 703-705

membranous wall of scala tympani and scala vestibuli, 704 organ of Corti, 708, 709-710

organ of Corti, cells of, 708-709

prominencia spiralis of, 705

scala media, or cochlear duct, 703

scala tympani, 701

secondary tympanic membrane of, 703

scala vestibuli of, 701

spiral ligament of, 704

spiral ligament, stria vascularis of, 705

spiral organ of Corti, 702

structure of, 701

sulcus spiralis externus, 705

sulcus spiralis internus, 703-706

vestibular membrane of Reissner, 703-704

vestibular artery, 714

cupola of semicircular canals in, 700

ductus endolymphaticus, 694

endolymph of, 695

endolymphatic sac, 694

fenestra vestibuli, 693

general considerations of, 693

internal auditory vein, 716 
Ear, internal, cochlea, lymphatics of, 716

macula acustica sacculi, 697 macula acustica utriculi, 698 membranous labyrinth, 694 neuro-epithelium of saccule, 695 neuro-epithelium, varieties of, 697 organ of equilibration, 700 otoliths in, 697

otocyst, 699

perilymph of, 695

saccule of, 694

- sacculus of vestibule in, 694

semicircular canals of, 699

ampullæ of, 699

utricle of, 698

utriculosaccular canal of vestibule, 694

utriculus of vestibule, 694

veins of, 715-716

vestibule of, 693

middle, 686

aqueductus Fallopii, relations of, 686

annulus tympanicus, 685-687

auditory canal, 683

auditory ossicles of, 689

auditory tube (Eustachian tube), 691

blood vessels of, 693

epitympanic cavity of, 685

Eustachian tube, 691

fenestra ovalis of tympanum, 686

fenestra rotunda of tympanum, 685

general considerations of, 685

incus, 689

orbicular process of, 689

lymphatics of, 693

malleus, 689

ligaments of, ' 691

mastoid cells, 686

membrana flaccida of, 689

Prussak's space, 691

Shrapnell's membrane, 689

stapedius muscles of, 690

stapes, 689

tensor tympani muscle, 690
Ear, middle, tympanic membrane, 687

tympanic mucosa, 686

tympanum, 685

pelvis ovalis of, 686

processus cochleariformis of, 690 promontory of, 685

tubal tonsil of, 692

umbo of tympanic membrane in, 687 .

Ectoderm, 28

Ehrlich's triacid stain, 755

Ejaculatory ducts, 497

Elastic fibers, 57

Elastic tissue, Weigert's stain for, 753

Elastin, 58

Eleidin, 45

Embedding, in paraffin, box for, 737

of tissues, 734

in celloidin, 734

in paraffin, 735

Embryonic connective tissue, 50-53

Enamel, 328

contour lines of Retzius in, 329

prism, 328

radial lines or prism stripe of

Schreger in, 329

Enchylema, 8

Endocardium, 195-196

Endocrin glands, 260-548

hermones of, 586

Endomysium, 195

Endothelium, 37

Enlargement of cartilage cells, 80

Entoderm, 28

Eosin, as a tissue stain, 745

and methyl blue mixture (Mann), stain for cytoplasmic granules, 755

Eosinate of methylene blue stain (Hasting's method) for blood, 754

Ependyma cells, 141-589

Epicardum, 195

Epicritic sensibility, 164

Epidermis, 262

Epididymis, 492 
Epidural space, 620-623

Epinephrin (adrenin), 549

Epiphysis cerebri, 579

blood supply of, 584

development of, 580

function of, 581

habenular commissure of, 581

histologic structure of, 582

nerves of, 584

posterior commissure of, 581

Epithelia, classification of, 34

Epithelial tissues, 30-33

Epithelium, 40

ciliated, 40

endothelial, 37

glandular, 40

goblet cell, 41

mesothelial, 37

modified columnar, 40

neuro-, 42

non-stratified, 37

pavement, 37

plain, 39

pseudo-stratified columnar, 46

simple columnar, 39

simple squamous, 37

stratified, 43

stratified squamous, 43

transitional, 47

varieties of, 33

Epitympanic eavity of ear, 685

Epoöphoron (parovarium), of female genitalia, 539

Equatorial plate, 25

Eruptive tissue, of developing bone, 82

Esophagus, 348

coats of, 348

glands of, 351

mucous coat of, 350

muscular coat of, 348

submucous coat of, 348

Euparal, for mounting sections, 74

Eustachian tube, 691

Exophthalmic goitre, 561

External ear, 682

External genitals, female, 540

clitoris, 541
External genitals, female, glandulæ vestibulares $\mathrm{m}$ a jo $\mathrm{r}$ e $\mathrm{s}$ (Glands of Bartholin), 541 glandulæ vestibulares minores, 541 hymen, 541

labia majora, 540

labia minora, 540

vestibule, 540

External genitals, male, 503

penis, 503

corpora cavernosa of, 503-504

corpus spongiosum urethræ of, 506

helicine arteries of, 504

lymphatics of, 506

nerves of, 506

nervi erigentes of, 506

pectiniform septum of, 504

preputial glands of, 506

tunica albuginea of corpus cavernosum, 504

Tyson's glands of, 506

Eye, anterior chamber of, 636-643

appendages of, 674

aqueous humor of, 665

blood supply of sclerocorneal junction, 636

blood vessels of, 670

canalis hyaloideus, or canal of Stilling, 672

canal of Schlemm (sinus venosus scleræ), 635

capsule of Tenon, 627

choroid coat of, 636

lamina basalis of, 637

lamina capillaris of, 636

membrane of Bruch, 637

tapetum cellulosum of, 638

tapetum fibrosum of, 637

tunica Ruyschiana of, 637

vascular layer of, 636

ciliary arteries, anterior, 673

long, 672

short, 672

ciliary body of, 638

fibrous layer of, 640

ciliary epithelium, 640

ciliary glands, 640 
Eye, ciliary muscle, 638

ciliary processes, 640
circle of Zinn, 672
coats of, 628
conjunctiva, 674
ocular, 674
palpebral, 674
contents of, 665
cornea, 630
anterior epithelium of, 674
anterior homogeneous membrane
$\quad$ of, 631

Bowman's elastic membrane of, 631 corpuscles of, 631

endothelium of, 633

epithelium of, 631

layers of, 631

membrane of Descemet, 632

posterior epithelial layer of, 633

posterior homogeneous membrane of, 632

substance of (substantia propria), 631

vascular and nerve supply of, 633

erystalline lens of, 666

capsule of, 666

lenticular epithelium of, 666

nuclear zone of, 667

nucleus of, 667

substantia lentis, 666

suspensory ligament of, 669

development of, 645

choroidal fissure, 645

optic cup, 645

optic stalk, 645

optic vesicle, 645

external or fibrous tunic of, 629

general considerations of, 626

gland of Harder, 681

glaucoma, 635

hyaloid artery of, 672

hyaloid membrane of, 669

iridocorneal angle, 643

iris, 641

circulus major of, 672

circulus minor of, 673

coloboma of, 646
Eye, iris, eolor of, 642

dilator muscle of, 642

endothelium of, 641

fibrous stroma of, 642

internal epithelium of, 643

layers of, 641

sphincter muscle of, 642

lacrimal gland, 679

lacrimal lake, 679

ligamentum pectinatum of, 635-643

lymphatic systems of, 673

middle coat of, vascular tunic, 636

muscæ volitantes, 669

nerves of, 674

optic nerve, 664

optical or visual axis, 628

ora serrata, 665

pineal, 580

posterior chamber of, 636-643

retina of, 644

cell types of inner nuclear layer of, 654-655-656

central artery of, 664-670

cones of, 651-652-653

external limiting membrane of, 653

fibers of Müller of, 658

fiber layer of Henle, 654

fovea centralis of, 660

ganglion cell layer of, 657

general considerations of, 644

inner nuclear layer of, 654

inner reticular layer of, 656

inversion of, 663

layers of, 646

macula lutea of, 659

nerve fiber layer of, 657

optic papilla of, 665

optic disk of, 665

outer nuclear layer of, 654

outer reticular layer of, 654

pigment epithelium of, 646

porus opticus of, 665

rods of, 648-649-650

rods and cones, development of, 661-663

layer of, 647

supporting tissues of, 658 
Eye, retina of, visual purple of, 647649

yellow spot of, 659

sclerocorneal junction of, 635

sclerotic coat of, 634

corpuscles of, 634

lamina fusca of, 634

lamina cribrosa of, 634

pinguecula of, 634

substantia propria of, 634

spaces of Fontana of, 635-643

spatia zonularis of (canals of $\mathrm{Pe}$ tit), 670

suprachoroid layer of, 636

uvea of, 636

uveal tract of, 636

vascular tunic of, 636

venæ vorticosæ of, 672

vitreous humor of, 669

zonula ciliaris of Zinn, of, 670

Eyelid, 674

accessory lacrimal glands of Krause of, 677

blood supply of, 678

cutaneous portion of, 674

conjunctival portion of, 676

glands of Moll of, 676

glands of Zeiss of, 676

lymphatic and nerve supply of, 678

palpebral muscle of Müller, 677

posterior tarsal glands of Waldeyer, 677

tarsal (Meibomian) glands, 676

tarsus of, 676

Fallopian tube, 525

blood supply of, 527

mucosa of, 526

lymphatics and nerves of, 528

muscular wall of, 526

serous coat of, 526

structure of, 525

Female reproductive organs, 507. See also Reproductive system.

Fat, osmic acid test for, 758 stains for, 62, 759

Fat tissue, 61
Fibers, collagenous, 49

elastic, 49-57

white, 49

Fiber tracts of cerebral cortex, 618.

See also Nervous system.

Fibrocartillage, 71

Filar mass, 8

Filum terminale of spinal cord, 600 . See Nervous system.

Flagellate motion, 16

Flagellum, 16

Flemming's fluid for fixation of tissues, 728

Fontana, spaces of, 635. See also Eye.

Formalin, for fixation of tissues, 725

Fovea centralis of retina, 660. See also Eye.

Fresh tissues, examination of, 720

Fuchsin for tissue stain, 746

Gage's method for demonstrating glycogen, 750

Gall-bladder, 420. See also Digestive system.

Gametogenesis, 460. See also Reproductive system.

Ganglia, 148. See also Nervous system.

Gastric glands, 354. See also Digestive system.

Gigantism, 573

Gilson's fluid for fixation of tissues, 730

Glands, 253

accessory lacrimal, of Krause, 677

adrenal, 548

lymphatics and nerves of, 556

anterior lingual (of Nuhn), 342

branched saccular, 260

branched tubular, 258

bulbo-urethral, 501

cardiac, of stomach, 360

carotid, 569

ceruminous, of ear, 684

ciliary, of eye, 640

cervical, of uterus, 632

classification of, 253 
Glands, coceygeal, 570

compound saccular, 260

compound tubular, 258

compound tubulo-alveolar, 258

convoluted tubular, 257.

Cowper's, of urethra, 501

ductless, 260-548

hormones of, 586

duodenal (Brunner's), 365-372

endocrin, 260-548

hormones of, 586

histologic types of, 254

internal secretion of, 261

intestinal (crypts of Lieberkühn), 366

of small intestine, 370

lacrimal, 679

lenticular, of stomach, 360

mammary, 541

active, 543-545

blood vessels of, 547

milk, 547

of Montgomery (areolar glands of Duval), 545

Marchand's (Marchand's adrenal), 557

Meibomian (tarsal), of eyelid, 676

mucous, 255

mucus of, 256

of Bartholin (Bartholin's glands of vulva), 541

of esophagus, 351

of eyelid (glands of Moll), 676

of Harder, 681

of tongue, 342

of Tyson, of penis, 506

of von Ebner, 343

of Zeiss, of eyelid, 676

parathyroid, acidophil cells of, 564

blood supply of, 565

structure of, 562

parótid, 390

physiologic types of, 254

pineal, 579

posterior tarsal, of Waldeyer, 677

preputial, 506

prostate, 497
Glands, pyloric, 357

racemose, 258

secreting, of mouth, 321

suprarenal, 548

chromaffin granules in, 554-555

development and function of, 548

lymphaties and nerves of, 556

medulla, cells of, 553

zona glomerulosa of, 552

zona reticularis of, 553

zona fasciculata of, 552

salivary, 382

serum-secreting cells of, 256

simple saccular, 259

simple tubular, 257

submaxillary, 391

thymus, 565-567

thyroid, blood supply of, 560

colloid in, 557

development of, 561

follicles of, 557

follicular epithelium of, 559

function of, 561

lymphaties of, 560

nerves of, 561

structure of, 557

urethral (Littre's), 454

uterine, 531

Glandulæ vestibularis, majores (glands of Bartholin), 541

minores of vulva, 541

Glandular epithelium, 40

Glaucoma, 635

Glia cells, 141

Glomus caroticum, 569

Glomus coccygeum, 570 .

Glycerin jelly, for mounting sections, 760

Glycogen, Gage's method for demon. strating, 758

Goblet cell epithelium, 41

Goitre, 561

exophthalmic, 561

Gold chlorid for demonstrating nerve plexuses and nerve endings (Ranvier's method), 752 
Golgi's cells of cerebral cortex, types 1 and 2,613

Golgi's stain for nerve cells, 751

Graafian follicle, 517

Graafian follicle, Résumé of structures, 519

Graves' disease, 561

Guard cells, 38

Gum-damar, for mounting sections, 760

Hair, 277

development of, 277

regeneration of, 286

root of, 281

root sheaths of, 283

shaft of, 281

structure of, 277

Haploid group of chromosomes in spermatogenesis, 464

Hardening of tissues, 733

Hasting's method for blood (eosinate of methylene blue stain), 754

Haversian spaces in bone, 84

Haversian systems of bone, 75-84

Heart, 195

annulus fibrosus of, 197

atrioventricular bundle of, 199

blood vessels of, 201

chordæ tendineæ of, 197

columnæ carnæ of, 198

development of, 201

endocardium of, 196

endomysium of, 195

epicardium of, 195

moderator bands of, 199

myocardium of, 195

nerve supply of, 202

valves of, 196

venæ minimæ of, 201

Heat, fixation of tissues by, 730

Helly's fluid for fixation of tissues, 728

Hemal nodes, 239

Hematein, for staining tissues, 741 stains, application of, 743 and eosin stains for tissues, 746

Hemoglobin, 211

Hemolymph nodes, 239
Hemopoiesis, 214

Heterozygote, 478

Histogenesis, 1-27

Histologic technic, 720

bibliography of, 761

Histology, definition of, 1

historical development of, 2

relations of, to other biological sciences, 2

Homozygote, 478

Hone for microtome knife, 737

Howship, lacunæ of, 84

Hyaline cartilage, 68

Hyaloid artery, of eye, 672

Hyaloid membrane, of eye, 669

Hyaloplasm, 8

Hydatid of Morgagni, 539

Hymen, 541

Hypopituitarism, 573

Hypophysis cerebri, 572-573-574-575

function of, 573

histologic structure of, 573-574-575

pars buccalis seu glandularis, 575

pars neuralis; infundibulum, 575

parts of, 575

Rathke's pouch, 572

Ileocecal (colic) valve, 379

Incus, of ear, 689

Injection of tissues, 732

pressure required for, how obtained, 733

with Berlin blue gelatin mass, 732

with earmin gelatin mass, 732

Intercalated dises of cardiac muscle, 99

Internal ear, acoustic nerve, 702

aqueductus vestibuli of, 694

auditory artery of, 714

blood supply of, 714

bony labyrinth of, 694

canalis communis of, 699

cecum vestibulare of scala media, 703

cochlea, accessory tectorial membrane of, 706

basilar membrane of, 706

function of, 716

foramina nervosa of, 702 
Internal ear, cochlea, hamulus of, 701

helicotrema of, 703

Hensen's stripe of tectorial membrane, 706

limbus spiralis of, 705

membrana tectoria (membrane of Corti) of, 703-705

membranous wall of scala tympani and scala vestibuli, 704

organ of Corti, cells of, 708-709

prominentia spiralis of, 705

spiral ligament of, 704

structure of, 701

sulcus spiralis externus of, 705

sulcus spiralis internus of, 703

vestibular membrane (of Reissner), 704

cochlear artery, 714 .

cochlear nerve, spiral ganglion of, 712

ductus endolymphaticus of, 694

endolymph of, 695

endolymphatic sac of, 694

fenestra vestibuli of, 693

general considerations of, 693

helicotrema, 703

internal auditory vein, 716

lagena or cecum cupulare of scala media, 703

lymphatics of, 716

macula acustica sacculi, 697

macula acustica utriculi, 698

membranous labyrinth of, 694

neuro-epithelium, of saccule, 695

varieties of, 697-708-709

organ of equilibration, 700

otocyst, 699

otoliths in, 697

perilymph of, 695

saccule of, 695

sacculus of vestibule, 694

scala media or cochlear duct of, 703

scala tympani of cochlea, 701

scala vestibuli of cochlea, 701

secondary tympanic membrane of cochlea, 703
Internal ear, semicircular canals of, 699

ampullæ of, 699

spiral organ of Corti, 702

utricle of, 698

utriculosaccular canal of vestibule, 694

utriculus of vestibule, 694

veins of, 715-716

vestibular artery, 714

vestibular ganglion (of Scarpa), 712

vestibular membrane (of Reissner), 702

vestibule of, 693

Internal genital organs, female, 507. See also Reproductive system.

Interrenal bodies, 548

Interstitial granules of Kölliker, 99'

Interfilar mass, 8

Intestine, large, 377

appendices epiploicæ of, 377

lymphoid tissue and lymph nodes of, 378

mucous membrane of, 377

plicæ semilunares of, 377

rectum, 379

sacculations or haustra of, 377

tæniæ coli (lineæ coli), 377

vascular and nerve supply of, 379

vermiform appendix of, 378

Intestine, small, absorption from, 375

acidophil cells of, 371

agminate nodules of (Peyer's patches), 366

blood supply of, 373

chromaffin cells of, 371

chyle in, 374

corium of, 366

duodenal glands (Brunner's), 365372

glands of, 370

glands (crypts of Lieberkühn) of, 366

granule cells of Paneth, 370-371

lacteals of, 369 
Intestine, small, lining epithelium of, 369

lymphatics of, 374

mucous membrane of, 366

myenteric ganglionic plexus (Auerbach's), 375

nerve supply of, 374

Peyer's patches of, 366

solitary nodules of, 366

structure of, 363

submucosa of, 364

submucous plexus (Meissner's), 375

valvulæ conniventes of, 364

villi of, $366-368$

Intracartilaginous ossification, 79

Intra vitam method of staining nonmedullated nerve fibers with methylene blue, 749

Iridocorneal angle, 643

Iris, 641

circulus major of, 672

circulus minor of, 673

color of, 642

dilator muscle of, 642

endothelium of, 641

fibrous stroma of, 642

internal epithelium of, 643

layers of, 641

sphincter muscle of, 642

Iron hematoxylin, for staining tissues, 747

\section{Joints, 88}

adaptation cartilages of, 89

diarthroses, 88

intra-articular menisci, 88

labra glenoidalia, 89

sutura, 88

synarthroses, 88

synchondroses, 88

syndesmoses, 88

synovia, 89

synovial membrane, 89

Karyon, 18

Keratin, 45

Kidney, 423
Kidney, arched collecting tubule of (junctional tubule), 437

arciform arteries of, 440

arciform veins of, 443

arteries, interlobular, of, 441

arteriolæ rectæ of, 441

ascending limb of Henle's loop, 435

Bellini's duct of, 438

blood vessels, lymphatics and nerves of, 440

capsule of Bowman, 429

corpuscles in, 425

cortex of, 424

descending limb of Henle's tubule, 434

distal convoluted portion of tubule in, 436

divisions of the renal tubule, 429

glomerulus of, 429

loop of Henle, 435

lymphatics and nerves of, 444

medulla of, 424

neck of tubule of, 431

papillary ducts of, 438

proximal convoluted portion of tubule of, 431

renal connective tissue, 426

renal corpuscles (Malpighian body), 425-429

renal lobule, 426

renal pelvis and ureter, 445

blood supply, lymphatics and nerves of, 448

mucosa of, 445

muscular coat of, 448

tunica propria of, 447

straight collecting tubules of, 437

table of divisions of renal tubule, 439

table showing the course of the renal circulation, 443

topography of, 423

tubules, peculiarities of, 440

uriniferous, or renal tubules of, 427

venæ propriæ renales, 443

veins, stellate of, 443

Kleinenburg's fluid, for fixation of tissues, 729 
Knife, microtome, hone for, 737

Kölliker's muscle columns, 112 venulæ of, 443

Labia majora, of pudendum, 540

Labia minora, of pudendum, 540

Lacrimal gland, 679

Lacrimal lake, 679

Lacteals, of small intestine, 369-374

Lactiferous duct, of mammary gland, 542

Lamellæ, external circumferential, of bone, 84

internal circumferential, of bone, 85

interstitial, of bone, 85

Larynx, 300

structures of, 300

vocal cords of, 301

Lenticular glands, of stomach, 360

Ligamentum denticulatum, of spinal cord, 621-624

Ligamentum pectinatum, of eye, 643

Ligaments, 117

circular dental, 329

Lingual glands, anterior, 342

Lingual tonsil, 244-343

Littre's glands, urethral, 454

Liver, 405

bile capillaries of, 411

blood supply of, 417

capsule of Glisson, 408

cells of, 412

connective tissue of, 408

course of blood through, 419

interlobular arteries and veins in, 415

interlobular bile ducts of, 415

lobule of, 409

lymphatics of, 419

nerves of, 420

pigment in, 414

portal canals of, $408-415$

portal vein of, 417

structure of, 405

Loose spireme, of cell nucleus, 24

Lumbar region, of spinal cord, 600
Lung, 304

atria of, 310

blood supply of, 314

lobule of, 314

lymphaties of, 318

nerve supply of, 319

pulmonary alveoli of, 311

tissue fixation of, 730

Lutein cells, of ovary, 519

Lymphatic system, 225

amygdalæ (faucial tonsils), 242

bursæ, 233

cisterna chyli, 230

chyle, 226

hemolymph, or hemal nodes, 239

lymph, 225

lymph cells, 235-238

lymph corpuscles, 225

lymph follicles, 233

lymph glands, 235

lymph nodes, 235

blood vessels of, 239

development of, 242

structure of, 236

lymph nodules, 233

lymph sinus, 236

lymph vessels, development of, 230

lymphatic capillaries, 227

lymphatic vessels, 226

lymphatics, of adrenal gland, 556

of bone, 78

of eye, 673

of eyelid, 678

of internal ear, 716

of kidney, 444

of large intestine, 378

of liver, 419

of lung, 318

of mammary gland, 547

of middle ear, 693

of penis, 506

of salivary glands, 393

of small intestine, 374

of stomach, 362

of thyroid gland, 560

of tongue, 344

lymphoid tissue, 64 
Lymphatic system, marrowlymph spleen, 245

blood vessels of, 246

development of, 250

differentiation of, from lymph node, 249

functions of, 249

pulp veins of, 248

splenic cells, 249

splenic nodule (Malpighian corpuscle), 247

splenolymph glands, 240

thoracic duct, 230

tonsils, faucial, 242

lingual, 244

palatine, 242

pharyngeal, 244

salivary corpuscles of, 243

tubal tonsil (of Gerlach), 692

Malleus, of ear, 689

ligaments of, 691

Mallory's connective tissue stain, 753

Mammary glands, 541

active gland, 543

blood vessels of, 547

circulus venosus of Haller, of, 547

colostrum, 546

lactiferous duct of, 542

lymphatics and nerves of, 547

milk, 547

resting, gland, 545

Mann's acid hematein (Ehrlich's Hematoxylin) stain, for tissues, 742

Marchand's glands (Marchand's adrenal), 557

Marginal velum, 590

Marrow, of bone, 77

blood supply of, 78

cavities, primordial, in, 82

primary osteogenic, 82

red, 77

Mastoid cells, of ear, 686

Maturation, of germ cells, 460
Mayer's albumin, for fastening sections to slide, 739

Medullary sheath, 134

Meibomian glands, of eyelid, 676

Meissner's submucous plexus, 347

Membrana propria, 34

Membrane, mucous, of large intestine, 377

mucous, of small intestine, 366

mucous, of mouth, 320

synovial, 89

Membranous labyrinth, of internal ear, 694

Meninges and blood supply of cerebrum, 619-620-621

Mendelism, 477

Mercuric chlorid, for fixation of tissues, 725

Mereuro-nitric mixture, for fixation of tissues, 730

Mesenchyma, 50

Mesoderm, 28

Mesothelium, 37

Mesovarium, 507

Metabolism, 14

Metaphase, of mitotic cell division, 25

Methyl blue and safranin stain, for tissues, 747

Methyl green stain, for tissues, 743

Methylene blue, for chromophilic (tigroid) granules in cyton and dendrons, 750

stain for non-medullated nerve fibers (intra vitam method), 749

tissue stain, 743

Meves' method for staining mitochondria, 757

Microtome, for sectioning tissues, 736

Microtome knife, for sectioning tissues, 737

hone for, 737

use of strop for, 738

Middle ear, 685

blood vessels of, 693

general considerations of, 685

lymphatics of, 693

Milk, 547 
Mitochondria, 8-50

Mitochondrial technics, Meves' method, 757

Benda's method, 757

Mitome, 8

Mitosis, 22

Montgomery's glands, of mammæ, 545 Molecular motility, 17

Monaster stage of mitotic cell division, 25

Mordants, for dyes, 741

Morphogenesis, 29

Motor area, of cerebral cortex, 615

Mounting sections, 759

euparal for, 761

glycerin jelly for, 760

gum-damar for, 760

neutral balsam for, 760

xylo-balsam for, 760

Mouth, 320

lymphoid tissue of, 321

mucous membrane of, 320

secreting glands of, 321

Muchematin stain, for tissues, 748

Mucicarmin stain, for tissues, 748

Mucous membranes, 251

of alimentary canal, 347

of mouth, 320

structure of, 251

Mucous tissue, 54

Mueus, 256

formation of, 41

Müller's fibers, of retina, 658

Müller's solution, for fixation of tissues, 726

Müllerian duets, 456

Museæ volitantes, 669

Muscle, striped, 112

areas of Cohnheim, 112

as a whole, 112

blood supply of, 114

cardiac (involuntary striped), 90-94,99

atrio-ventricular bundle of His, 102

interealated aiises in, 99, 104

interealated dises (Zimmermann's technic for demonstrating), 758
Muscle, eardiac, nerve endings in, 175

Purkinje fibers' of, 102 contraction bands of, 93 endomysium of, 112 epimysium of, 112 fasciculus of, 112 histogenesis and structure of, 91 inokommata of, 98-104 interstitial granules of Kölliker, 99 Kölliker's columns, 112 mesophragma of, 98 myoblasts, 90 myofibrils, 90 myochondria of, 92 myofibrils of, 104 myoglia of, 93 myotomes, 106. nerve endings in, 114-115, 171, 175 of heart, 94-99 perimysium of, 112 red, 110

sarcolemma of, 92 sarcomeres of, 98-104 sareoplasm of, 90 sarcosomes, of Retzius, 99 sarcostyle of, 104 skeletal, 90 smooth (involuntary; unstriped), 90, 91

histogenesis, and structure of, 91 myofibrils, 93 nerve endings in, 175

situation of, 94

structure of border fibrils (myoglia) in, 93

telopragma of, 98

types of, 90

unstriped, 90

voluntary striped, 104

(skeletal), 90

Muscle spindles, 172

Muscular contraction, 111

Myelospongium, of developing spinal cord, 509

Myenteric plexus, and ganglion, 346

Myoblasts, 90 
Myocardium, 195

Myofibrils, 90

Myxedema, 561

Nabothian follicles, of uterus, 532

Nails, 274

structure of, 275

Nasal eavity, 293

blood vesséls of, 298

neuro-epithelium of, 297

olfactory portion of, 296

respiratory portion of, 294

vestibule of, 293

vomero-nasal organ of Jacobson, 295

Nasopharynx, 299

Nerve cell, 121

apyknomorphous condition of, 124

association neurons, 131

axis cylinder process of, 128

axon, 125

axon hillock, 129

axoplasm, 129

cellulifugal process of, 130

chromophilic substance of, 123

collaterals of, 129

commissural neurons, 131

cytochromatin of, 123

cytoplasm of, 121

dendrons (dendrites) of, 127-128

end arborization of, 129

ganglia, 148

Golgi's cells, type 1 (Deiter's cells), 131

type 2, 131

implantation cone of, 129

mitochondria in, 125

neuraxis, 128

neuraxon, 128

neurite, 128

neuro-epithelium, 42

neurons, 125

Nissl's substance of, 123

nucleus of, 121

origin of, 131

processes of, 127

projection neurons, 131

protoplasmic processes of, 128
Nerve cell, pyknomorphous condition of, 123

size of, 131

spinal ganglia, 150

telodendrion of, 129

- tigroid substance of, 123

Nerve endings, 159

circumgemmal fibers in taste buds, 163

in connective tissue, 163

corpuseles of Grandry, 169

corpuseles of Herbst, 169

end bulbs of Krause, 174

end fibrils, 159

epicritic sensibility of, 164

epithelial cells in, 159

genital corpusele, 166

Golgi end organs, 174

Golgi-Mazzoni corpuseles, 169

gustatory cells, 161

gustatory organs, 160

intergemmal fibers, 163

intragemmal fibers, 163

Key-Retzius corpuseles, 169

lamellar (Pacinian) corpuscles, 167

Merkel's corpuscles, 169

motor end plates, 171

muscular, 171

muscle spindles, 172

neuro-epithelium, 160

neuromuscular end organs, 171

neuromuscular spindles, 172

neurotendinous end organs, 174

Pacinian corpuseles, 167-174

peripheral end organs, 159

protopathic sensibility of, 164

Ruffini's end organs, 165

sole plate, 172

sustentacular cells, 161

tactile cells, 159

tactile corpuseles, 163

tactile meniscus, 160

taste buds in, 160

tendon in, 171

tendon spindles, 174

terminal cylinders, 165

touch corpuscles of Meissner, 163 
Nèrve endings, trefoil plates, 159

Vater's corpuseles, 167

Vater-Pacinian corpuscles, 167

Nerve fibers, 132

axis eylinder of, 132

axolemma of Kühne, 134

axon fibrils, 134

axoplasm of, 134

endoneurium of, 132-147

fiber bundles, 139

funiculi of, 139

glia fibers, 144

incisures of Schmidt, 135

internodal segments of, 135

medullary segments of, 136

medullary sheath of, 132-134

medullated, 132

with a neurolemma, 132

without a neurolemma, 137

myelin of, 135

sheath of, 132-134

neuroglia, occurrence of, in, 145

neurolemma of, 136

neurokeratin of, 135

neuroplasm of, 134

nodes of Ranvier, 132

non-medullated, 132

with a neurolemma, 137

without a neurolemma, 137

nucleated sheath of Schwann of, 136

Remak's fibers, 137

Schmidt-Lantermann lines in, 135

sheath cells of, 137

sheath of Henle, 132-147

sympathetic nerve fibers, 137

telodendrions of, 140

terminal arborizations of, 140

tracts, 139

trophic center of neuron, 137

ultimate fibrillæ of, 133

Wallerian degeneration of, 140

white substance of Schwann, 132134

Nerve trunks, funieulus of, 146

lymphatic vessels of, 147

nervi nervorum, 148

nervi erigentes, of penis, 506
Nerve trunks, perineurium of, 146

structure of, 146

vascular supply of, 147

Nervous system, 587

blood supply of, 624

cerebellum, 603

basket cells of, 608

climbing fibers of medulla, 609

cortex of, 606

granule cells of, 609

large stellate cells of, 609

medulla of, 609

Purkinje cells of, 607

résumé of structures of, 611

small cortical cells of, 608

cerebral cortex, 611

arteries of, circle of Willis, 624

cortical, 625

fissural, 625

medullary, 625

middle cerebral, 625

terminal, 625

auditory area of, cells of, 618

cell layers of, 612

cells, Betz, of, 613-616

giant pyramidal, 613

Golgi's, types 1 and 2, 613

solitary, of Meynert, 613

frontal lobe of, 617

granule cell layer of, 617

inner large pyramidal cell layer of, 617

inner polymorphous cell layer of, 617

molecular layer of, 617

occipital lobe of, 617

outer large pyramidal cell layer of, 617

outer polymorphous cell layer of, 617

outer stripe of Baillarger, 619

parietal lobe of, 617

radial fibers of, 618

small pyramidal cell layer of, 617

temporal lobe of, 617

thickness of, 612

visual area of, 617 
Nervous system, cerebrum, 611

and spinal cord, meninges and blood supply of, 619

basal ganglia of, 611

corpus callosum, 611

gyri of, 611

hemispheres of, 611

insula of, 612,617

medulla of, 611

occipital lobe of, 611

pallium of, 611

parietal lobe of, 611

sulci of, 611

Sylvian fissure of, 612

temporal lobe of, 612

veins of, 625

development, 587-595

of cardic plexus, 591

of ependyma cells, 589

of marginal velum, 590

of myelospongium, 589

of neural groove (medullary groove), 587

of neural or ganglionic erest, 587

of neural plate, 587

of neural tube, 587

derivatives of, 594

of neuroblasts, 589

of neuroglia cells, 589

of neurons, 587

of spongioblasts, 589

of sympathetic plexus, 591

meninges, 620

arachnoid, 620

granulations of, 621

Pacchionian bodies of, 621

subarachnoid space, 621

villi of, 621

dura mater, 620

epidural space of, $620-623$

subdural space of, 620

pia mater, 622

choroid plexus of, 623

telæ choroideæ of, 623

motor area of cerebral cortex, 615

cells of Martinotti, 617
Nervous system, inner polymorphous cell layer of, 616

layers of, 615

marginal velum, 615

molecular layer, 615

outer polymorphous cell layer, 615

outer polymorphous cell layer of, 618

outer stripe of Baillarger of, 618

pyramidal cell layer, 616

small pyramidal cell layer, 615

small pyramidal cell layer of, 618

solitary eells of Meynert of, 618

stratum zonale, 615

stripe of Bechterew of, 619

supraradial felt work, 619

tangential fiber layer, 619

spinal cord, 596

anterior spinal artery of, 624

basis cornu, 596

caput cornu, 596

cell column of Clarke, 600

central eanal of, 598

central commissure, 596

cervical region, lower half, 602

upper half, 613

cervix cornu, 596

columns of, 598

conus medullaris, 600

filum terminale, 600

gray matter of, 596

intermediate zone of, 596

ligamentum denticulatum, 621

lumbar region of, 601

nerve roots of, 596 .

nucleus of Stilling, 602

regions of, 599

sacral region of, 600

segments of, 596

septum posticum, 621

structure of, 596

thoracic region of, 607

veins of, 625

white matter of, 598 
Nervous system, sympathetic division of nervous system, 153

cranial autonomic, 153

sacral autonomic, 153

sympathetic ganglia, 154

sympathetic proper, 153

tissues of nervous system, 119

axon, 119

cell body, 119-121

eyton, 121

dendrites, 119

dendrons, 119

epineurium of, 146

ganglion, 121

ganglion cell, 121

glomerulus of, 152

general considerations of, 119

nerve fiber, 119

neuroglia (glia tissue), 121

astrocytes (neuroglia cells), 141

long rayed or spider cells, 143

short rayed or mossy cells, 143

ependyma, 141

neuron, 119

neurone theory, 157

perikaryon, 121

stains of, 748

Cajal's method for demonstrating nerve fibrils, 750

gold chlorid, Ranvier's method, for nerve plexuses and nerve endings, 752

Golgi's, 751

methylene blue, for non-medullated nerve fibers (intra vitam method), 749

Nissl's method for staining chromophilic (tigroid) granules in cyton and dendron with methylene blue, 750

Weigert's, for medullated nerve fibers, 748

Neurone theory, 157

Neutral balsam, for mounting sections, 760

Neutral dyes, 740

Normal salt solution, 720
Nucleus, basic chromatin of, 6

chromatin, 6

chromatin nucleoli, 6

chromioles, 6

karyolymph, 6

karyoplasm, 6

karyosomes, 6

linin, 6

net knots in, 6

nucleoplasm, 6

nuclear sap, 6

oxychromatin of, 6

paralinin, 6

plasmosomes, 6

Ocular conjunctiva, 674

Ocular contents, 665

Odontoblasts, 324

Odontoclasts, 337

Olfactory area, of cerebral cortex, 618

Oögenesis, $460-478$

oögonia, in ovary, 512

oötid, or mature ovum, 479

primary oöcyte, 478

polar body, 479

secondary oöcyte, 479

Optic eup, 645

Optic nerve, 664

Optic stalk, 645

Optic vesicle, 645

Optical axis, of eye, 628

Ora serrata of eye, 665

Orange G. tissue stain, 746

Organ, of Corti, of ear, 708-709

of equilibration, 700

of hearing, 716

of Jacobson, 295

of Rosenmüller, 539

Orth's fiuid, for fixation of tissues, 727

Osmic acid stain, for fat, 758

Ossification, centers of, 79

endochondral, 85

epiphyseal, 85

intramembranous, 86

intracartilaginous, 79

perichondrial, 84

Osteoblasts, 77-83 
Osteoclasts, 78

Otoliths, 697

Ovarian follicle, 518

atresia of, 514

corona radiata of, 518

cumulus oöphorus, 518

development of, 513

discus proligerus, 517

liquor folliculi, 514

Pflüger's tubes, 513

résumé of structures, 519

stigma of, 518

stratum granulosum, 517

vesicular, or Graafian follicle, 517

Ovarian scars, 522

Ovary, 507

- $\quad$ blood supply of, 522

corpora albicantia, of, 510-521

corpora lutea of, 509

spuria, 521

vera, of pregnancy, 521

corpus hemorrhagicum, 519

corpus luteum, 507-519

cortex of, 508

hilum of, 508

lutein cells of, 519

lymphatics of, 525

medulla of, 508

nerves of, 525

ova, 510

ovarian follicles, 509

pampiniform plexus of, 523

plexus venosus of, 523

primitive follicles of (egg nests), 514

tunica albuginea of, 510

Oviduct, blood supply of, 527

lymphatics and nerves of, 528

mucosa of, 526

muscular wall of, 526

structure of, 525

Ovum, the, 511

accessory nucleus of, 511

eytoplasm of, 511

deutoplasm of, 511

nuclear cap of, 511

nucleus or germinal vesicle of, 511512
Ovum, the, oöcytes, 512

oötid, 512

vitelline membrane of, 511

vitellus, 511

yolk nucleus of, 511

zona pellucida of, 511

Oxyhemoglobin, 211

Pacchionian bodies, 621

Pacinian corpuscles, 174

Palpebral conjunctiva, 674

Palpebral muscle, of Müller, 677

Pampiniform plexus, of ovary, 523

of spermatic cord, 494

Pancreas, 394

centro-acinal cells, of Langerhans, of, 396

general considerations of, 394

islands of Langerhans, 399

nerve supply of, 404

pancreatic islets, 399

résumé of, 404

Papillæ, circumvallate, of tongue, 341

conical, of tongue, 340

filiform, 340

foliate, of tongue, 341

fungiform, of tongue, 340

lenticular, of tongue, 342

Paradidymis, organ of Giraldès, 502

Paraffin, embedding of tissues in, 735

Paraganglia, 571

Paraganglion caroticum, 570

Parahypophysis, 578

Paramitone, 8

Paraplasm, 8

Parasynapis, 463

Parathyroid gland, 562

acidophil cells of, 564

blood supply of, 565

structure of, 562

Parietal lobe, of brain, 617

Paroöphoron, 540

Parovarium, 539

Parotid gland, 390

Stenson's duct of, 390

Pectinate ligament, of eye, 635 
Penis, 503

corpus cavernosum, pectiniform septum of, 504

corpus spongiosum of, 503-506

glands of Tyson of, 506

helicine arteries of, 504

lymphatics of, 506

nerves of, 506

nervi erigentes of, 506

preputial glands of, 506

tunica albuginea of, 504

Peptic glands, 354

or adelomorphous glands, of stomach, 355

Pericementum, of teeth, 329

Perichondrial ossification, 84

Perichondrium, 72

Periodontium, 329

Periosteum, 74

Peyer's patches, 366

Pharyngeal tonsil, 244

Pharynx, 347

Pheochrome cells, of adrenal, 548

Pheocrome organs, 571

Pia mater, 622

Picrocarmin stain, for tissues, 744

Picro-fuchsin (Van Gieson's) stain, for

Pigment cells, 52 connective tissue, 752

Pineal body (conarium; epiphysis cerebri), 579

blood supply of, 584

development of, 580

function of, 581

habenular commissure of, 581

posterior commissure of, 584

structure of, 582

Pineal eye, 580

Pineal recess, 580

Pineal stalk, 580

Pinna, 682

Pituitrin, 573

Pituitary body, 572

pars buccalis of, 575

pars neuralis of, 575

Rathke's pouch, 572

structure of, 573-575
Placenta, 536

Pleura, 312

Pleural pores, 313

Polarity, of cells, 39

Portal vein, 417

Posterior chamber of eye, 636-643

Potassium bichromate, for fixation of tissues, 726

Preputial glands, 506

Prickle cells, 44

Prophase, 24

Prostate gland, 497

blood supply of, 500

concretions of, corpora amylacea, 499

structure of, 497

Protopathic sensibility, 164

Protoplasm, 1

alveolar, 13

ameboid motility of, 16

chemical constitution of, 2

ciliary motility, 16

circulatory movement of, 15

colloidal biogens of, 10

colloids, 4

contractility of, 15

erystalloids, 4

emulsoid of, 14

gel state of, 4

granular, 13

irritability of, 15

metabolism of, 14

molecular motility of, 17

muscular movement, 17

physical constitution of, 3

sol state of, 4

structure of, 10

Prussak's space, of ear, 15

Pseudopodium, 16

Pseudo-stratified columnar epithelium, 49

Pulmonary artery, 314

Pulmonary veins, 317

Pyloric glands, of stomach, 357

Pyramidal cells, of brain, 613

Racemose glands, 258

Ranvier's method of staining nerve plexuses and endings, 752 
Rathke's pouch, of hypophysis cerebri, 572

Rectum, 379

Renal pelvis and ureters, 445. See also Kidney.

Reproductive system, 479 development of, 456

female organs of generation, 507 external organs of, 540 clitoris, 541

hymen, 541

glands of Bartholin, 541

glandulæ vestibulares minores, 541

labia majora, 540

labia minora, 540

vestibule, 540

mammary gland, 541

active, 543

blood vessels of, 547

colostrum, 546

glands of Montgomery, 545

lactiferous duct of, 542

lymphatics of, 547

milk of, 547

resting, 545

internal organs of generation, 507

Fallopian tube, 525

- lymphatics of, 528

mucosa of, 526

muscular wall of, 526

serous coat of, 526

structure of, 525

Graafian follicle, résumé, of structures, 519

liquor folliculi of, 514

stratum granulosum of, 518

Pflüger's tubes, 513

primitive follicles (egg nests), 514

ovarian follicles, 509

atresia of, 514

corona radiata of, 518

cumulus oöphorus, 518

development of, 513

discus proligerus of, 517

Graafian follicle, 517

ovarian scars, 522
Reproductive system, female organs of generation, internal organs of generation, o v a r y, 507

blood supply of, 523

corpora lutea spuria, 521

corpora lutea vera, of pregnancy, 521

corpus albicans of, 510-521

corpus hemorrhagicum of, 519

corpus luteum of, 507-519

cortex of, 508

hilum of, 508

lutein cells of, 519

lymphatics of, 525

medulla of, 508

mesovarium, 507

nerves of, 525

ova, 510

pampiniform plexus of, 523

tunica albuginea of, 510

oviduct, 525

blood supply of, 527

lymphatics of, 528

mucosa of, 526

muscular coat of, 526

structure of, 525

ovum, 511

accessory nucleus of, 511

cytoplasm of, 511

deutoplasm of, 511

nucleus of (germinal spot, germinal vesicle), 511-512

nuclear eap, 511

oöcyte, stage of, 512

oögonial, stage of, 512

oötid, stage of, 479-512

vitelline membrane of, 511

vitellus, 511

yolk nucleus of, 511

zona pellucida of, 511

uterine cavity, 532

uterine glands, 531

- uterus, 528

blood vessels of, 532

cells of Langhans of chorionic villi, in, 537 
Reproductive system, female organs of generation, internal organs of generation, uterus, cervical glands of, 532

chorionic villi of pregnant uterus, 537

decidua menstrualis of, 534

decidua reflexa of, 537

decidua serotina of, 536

decidua vera of, 537

decidual cells of, 535

fetal blood vessels in, 538

gravid, the, 535

lymphatics and nerves of, 533

menstruating, 533

mucosa, or endometrium, of, 529

muscular coat of, or myometrium, 528

Nabothian follicles of, 532

placenta uterina, 536

serous coat of, or perimetrium, 528

structure of, 528

vagina, 538

mucosa of, 538

musculature, 538

outer fibrous coat of, 539

structure of, 538

vestigial structures, 539

epoöphoron (parovarium, organ of Rosenmüller), 539

hydatid of Morgagni (vesicular appendage), 539

paroöphoron, 540

fetal, derivatives of, 459

gametogenesis, 460

chromosomes in, 464

determination of sex, 475

chromosomes in, 476

diploid number of chromosomes, 463

gonad, 460

haploid chromosome group, 464

heterotypic division, 460

maturation, 460
Reproductive system, gametogenesis, Mendelian inheritance, 478

Mendelism, 477

determiners in, 477

homozygote, 478

heterozygote, 478

spermatocytes, primary, in, 463

synapsis phenomena, in, 461

synizesis in, 463

telosynapsis in, 463

tetrads, formation of, in, 460

unit characters in heredity, 474

zygote, 460

general considerations of, 455

gonads, or sex glands, 456

male organs of generation, 501

external organ, 503

penis, 503

corpora cavernosa of, 503

corpus spongiosum of, 503-506

erectile tissue of, 503-505.

glands of Tyson, 506

helicine arteries of, 504

pectiniform septum of, 504

lymphatics of, 506

nerves of, 506

nervi erigentes of, 506

preputial glands of, 506

tunica albuginea of, 504

internal organs, 479

Cowper's glands, 501

ejaculatory ducts, 497

prostate gland, 497

blood supply of, 500

structure of, 497

prostatic concretions, 499

scrotum, dartos of, 481

seminal vesicles, 495

testis, 479

corpus Highmori (mediastinum) of, 480

duct system of, 490

ductuli efferentes of, 490

epididymis of, 492

functions of, 479

histogenesis and structure of, 485 
Reproductive system, male organs of generation, internal organs, testis, interstitial cells of Leydig, of, 487

lipoid granules in, 488 pampiniform plexus of, 494

rete of, 490

semen, 486

spermatic cord, 494

spermatid, 484

spermatoblasts, 482

spermatozoön, human, 486

structure of, 479

trophocytes (Sertoli cells) of, 482.

tubules of, 487

tubuli recti of, 490

tunica albuginea of, 480

tunica vaginalis of, 480

tunica vasculosa of, 480

vas (ductus) deferens of, 493

vestigial structures, associated with, 502

appendix epididymis, 502

appendix testis, 502

ductuli aberrentes, 502

paradidymis (organ of Giraldès), 502

sinus pocularis (sinus prostaticus), 502

Müllerian ducts, 456

oögenesis, $460-478$

parasynapsis in, 463

polar body in, 479

primary oöcyte in, 478

secondary oöcyte in, 479

sex chromosome in, 475

spermatogenesis, 460

chromidia in, 474

idiozome in, 475

prespermatid in, 474

spermatogonia in, 463

spermatozoön in, 475

Wolffian body, or mesonephros, 456

Wolffian ducts, 456

Resting nucleus, 24
Respiratory system, 292

development of, 293

larynx, the, 300

structures of, 300

vocal cords of, 301

lung, the, 304

alveolar ducts of, 310

alveoli of, 311

atria of, 310

blood supply of, 314-319

bronchi of, 304

bronchial arteries, 317

bronchioles of, 307

lobule of, 314

lymphatics of, 318

pleura of, 312

pleural pores of, 313

pulmonary artery, 314

pulmonary veins, 317

nasal cavity, 293

blood vessels of, 298

neuroepithelium of, 297

olfactory portion of, 296

organ of Jacobson, of, 295

respiratory portion of, 294

vestibule of, 293

nasopharynx, 299

trachea of, 302

Retia mirabilia, of kidney, 187

Reticulum, 49-55

Retina, arteria centralis of, 664-670

cell types of inner nuclear layer of, 654

cones of, 651

external limiting membrane of, 653

fiber layer of, 654

fibers of Müller, 658

fovea centralis of, 660

ganglion cell layer of, 657

general considerations of, 644

inner nuclear layer of, 654

inner reticular layer of, 656

inversion of, 663

layers of, 646

macula lutea of, 659

nerve fiber layer of, 657

optic dise of, 665 
Retina, optic papilla of, 665 outer nuclear layer of, 654 outer reticular layer of, 654 pigment epithelium of, 646 porus opticus of, 665

rod and cone layer of, 647

rods of, 648-658

and cones, development of, 661 663

supporting tissues of, 658

visual purple of, 647-649

yellow spot of, 659

Ringer's solution, 720

Safranin stain, for tissues, 744

Salivary corpuscles, 243

Salivary glands, 390

blood supply of, 392

lymphaties of, 393

nerve supply of, 393

parotid gland, 390

sublingual gland, 392

submaxillary gland, 391

Santorini's duct, 394

Sarcoplasm, 90

Sarcosomes, of Retzius, 99

Scala media, of cochlea, 703

Scala tympani, 703

Scleral corpuscles, 634

Sclerotic coat of eye, 634

Scrotum, 481

Sectioning of tissues, 736 microtome for, 736

Sections, colloidin adhesive, for, 739 clarification of, 759

Mayer's albumin for, 739

mounting, of, 759

in euparal, 761

in glycerin jelly, 760

in gum damar, 760

in neutral balsam, 760

in xylol-balsam, 760

Segmentation process, 27

Segmented spireme, 24

Semen, 486

Semicircular canals, of ear, 699

Seminal vesicles, 495
Septum posticum, 621

Serous membranes, 232 mesothelia of, 232

stomata in, 232

tunica propria of, 232

Sertoli cells, 482

Shrapnell's membrane, of ear, 689

Sinus prostaticus, 502

Sinusoids, vascular, 187

Skin, 262

blood supply of, 290

capillary layer of, 268

eylindrical cell layer of, 264

derma, the, 268

eleidin layer of (stratum lucidum), 267

epidermis, the, 262

flattened cell layer and scaly layer (stratum corneum) of, 267

granular layer or stratum granulosum, of, 266

growth and development of, 270

hair, development of, 277

mature, 279

regeneration of, 286

root of, 281

shaft of, 281

sheaths of, 283

structure of, 277

keratin of, 267

layers of, 262

nails, 274

growth and development of, 276

structure of, 275

nerve supply of, 291

prickle cell layer (stratum spinosum) of, 265

reticular layer of, 269

sebaceous glands of, 286

stratum germinativum, 265

sudoriparous glands of, 271

Small intestine, 374. See Digestive system.

Smears, method of making, 730

Solution, Hogan's, 721

normal saline, 720

Ringer's, 721 
Spaces, of Fontana, 643

Specific dyes, 740

Spermatic cord, 494

Spermatid, and spermatozoön, 484

Spermatocytes, primary, 463

Spermatogenesis, 460

idiozome in, 475

prespermatid in, 470

Spermatogonia, 463

Spermatozoön, 475

histogenesis, and structure of, 485

human, 486

Spinal cord, 596

basis cornu of, 596

caput cornu of, 596

cell column, of Clarke, 602

central canal of, 598

central commissure of, 596

cervical region, lower half of, 602

cervical region, upper half of, 603

cervix cornu of, 596

columns of, 598

filum terminale of, 600

gray matter of, 596

intermediate zone of, 596

lumbar region of, 600

nerve roots of, 596

nucleus of Stilling, of, 602

regions of, 599

segments of, 596

sacral region of, 600

thoracic region of, 601

white matter of, 598

Spiral organ, of Corti, 702. See also Ear.

Spireme, loose, 24

Spleen, 245

blood vessels of, 246

development of, 250

differentiation of, from lymph nodes, 249

functions of, 249

pulp veins of, 248

Splenic cells, 249

Splenic nodules, 247

Spongioblasts, 589

Spongioplasm, 8
Staining methods, special, 747

Benda's mitrochondrial technic, 757

blood, Hasting's eosinate of methylene blue, for, 754

chromaffin granules, technic for, 759 chromophilic (tigroid) granules in cyton and dendron, with methylene blue, 750

connective tissue, Mallory's, 753

with picro-fuchsin (Van Gieson's), 752

cytoplastic granules, eosin and methylene blue mixture, Mann's, 755

Ehrlich's triacid, 755

elastic tissue, Weigert's, 753

fat, osmic acid for demonstrating, 758

glycogen, Gage's method for demonstrating, 758

medullate nerve fibers, Weigert-Pal method for demonstrating, 748

mitochondria, 757

mucinous tissues, specific, 748

nerve cells, Golgi 's, 751

nerve plexuses and endings, gold chlorid (Ranvier's) for, 752

neurofibrils, Cajal's, 750

nitrate of silver, for cement substances, 752

Staining of tissues, 738

double, 746

in bulk, 739

progressive, 740

regressive, 740

Stains, aceto-carmin, 744

alum carmin, 744

Auerbach's fuchsin-methyl-green, 756

borax carmin, 743

carmin, 743

Congo red, 746

Delafield's hematoxylin, 742

eosin, 745

fuchsin, 746

hematein, 743

and eosin, 746

iron hematoxylin, 747 
Stains, Mann's acid hematein (Ehrlich's hematoxylin), 742

methylene blue, 743

methyl blue and safranin, 747

methyl green, 743

muehematein, 748

mucicarmin, 748

orange $G, 746$

picro-carmin, 744

safranin, 744

single, with cytoplasmic dyes, 745

with nuclear dyes, 741

Stapedius muscle, 690

Stapes, 689

Stenson's duct, 390

Stigmata, 38

Stomach, 353

blood supply of, 360

cardiac glands of, 360

fundus glands of, 354

lenticular glands of, 360

lymphatics of, 362

mucous coat of, 353

muscular coat of, 353

nerve supply of, 362

parietal cells (oxyntic or delomorphous cells) of, 356

peptic, or adelomorphous cells, of, 355

pyloric glands, 357

secretion of, 357

serous coat of, 352

submucous coat of, 353

Stomata, 38

Stratified epithelium, 43

Stratified squamous epithelium, 43

Stripe of Bechterew, 619

Stripes of Baillarger, 619

Strop, use of, 738

Subarachnoid space, 621-624

Subcutaneous tissue, 269

Subdural space, $620-623$

Sublingual gland, 392

Submaxillary gland, 391

Submucous plexus (Meissner's), of small intestine, 375

Suprarenal glands, 548

accessory, 556
Suprarenal glands, chromaffin granules in, 554

blood supply of, 554-555

development and function of, 548

lymphatics and nerves of, 556

medulla, cells of, 553

zona fasciculata, 552

zona glomerulosa, 552

zona reticularis, 553

Sweat glands, 271

Sympathetic plexus, 461-463

Synapsis phenomena, 461-463

Syncytia, 31

Synizesis phase of gametogenesis, 463

Synovia, 89

Synovial membranes, 232

Tapetum cellulosum, of eye, 638

Tapetum fibrosum, of eye, 637

Tarsus of eyelid, 676

Technic, for demonstrating chromaffin

Teeth, 333 granules, 759

ameloblasts (adamantoblasts), 333

cementoblasts, 337

cementum, 329

development of, 336

deciduous, 331

dental enamel, layers of, 335

dental papilla, 336

dental pulp, 323

dentin of, 325

development of, 330

enamel germ of, 330-331

enamel organ, of, 330

layers of, 331

Tomes' processes, 333

odontoclasts, 337

permanent, 331

root membranes of, 329

root eanals of, 336

Sharpey's fibers in, 329

structure of, 323

Telæ choroideæ, 623

Tellyesniczky's fluid, for fixation of tissues, 726

Telophase of mitotic cell division, 25 
Telosynapsis, 463

Tendon, 115

nerve endings in, 171

peritenoneum of, 115

vagina fibrosa, 115

vagina mucosa, 115

Tendon cells, 117

Tendon fasciculi, 115

Tendon spindles, 174

Testis, $479-480$

corpus Highmori of, 480

duct system of, 490

ductuli efferentes of, 490

epididymis, 492

human, 486

interstitial cells, of Leydig, 487

lipoid granules in, 488

mediastinum of, 480

rete of, 490

semen, 486

spermatic cord of, 494

spermatid, and spermatozoön, 484

spermatoblasts, 482

spermatozoön, histogenesis and structure of, 485

trophocytes of, 482

tubules of, 487

tubuli recti of, 490

tunica albuginea of, 480

tunica vaginalis of, 480

tunica vasculosa of, 480

vas (ductus) deferens of, 493

Thoracic duct, 230

Thrombin, 203

Thymus gland, 565

blood supply of, 568

development and function of, 568

lymphoid corpuscles of, 567

Thyroid gland, 557

accessory, 562

blood supply of, 560

colloid in, 557

development of, 561

follicles of, 557

follicular epithelium of, 559

function of, 561

lymphatics of, 560
Thyroid gland, nerves of, 561 structure of, 557

Thyroidin, 561

Thyroglossal duct, 343

Tissue

adenoid, 64

adipose, 61

bone, 72

compact, 74

general consideration of, 72

bone marrow, 77

cartilage, 67

blastema of, 69

elastic, 71

fibro-, 71

hyaline, 68

matrix of, 69

origin of, 70

connective, acidophil granulocytes, 52

areolar, 56

basophil granule cells, in, 52

blood and nerve supply of, 67

cells of, 51

dense elastic, 60

dense fibrous, 59

elastic fibers of, 57

embryonal, 50-53

eosinophil granulocytes of, 52

fibrocartilage, 71

general statements, 49

lamellar cells of, 52

loose fibro-elastic, 56

marrow, blood supply, 78

mucous, 54

oxyphil granulocytes in, 52

perichondrium, 72

periositeum, 74

plasma cells of, 51

red marrow, 77

reticular, 55

spindle cells of, 51

types of, 53

yellow elastic, 61

ectoderm, 28

entoderm, 28

epithelia, classification of, 34 
Tissue, epithelial tissue, 30-3

epithelium, transitional, 47

varieties of, 33

eruptive, 82

fat, 61

stains for, 62

intercellular cement substance, in, 30

lymphatic corpuscles, 66

lymphoid, 31-64

mesoderm, 28

mucous, 54

muscular, 90

Tissue fixation, by, 723

alcohol, 724

Bouin's fluid, 729

Carnoy's fluid (lung), 729

chromo-acetic-formalin mixture, 727

Flemming's fluid, 728

formalin, 725

Gilson's fluid, 730

heat, 730

Helly's fluid, 728

Kleinenberg's fluid, 729

mercuric chlorid, 725

mercuro-nitric mixture, 730

Müller's solution, 726

Orth's fluid, 727

potassium bichromate, 726

Tellyesniczky's fluid, 726 .

vapors, 731

van Gehuchten's fluid, 729

Zenker's solution, 727

Tissue fixation, of human embryos, 726

lung tissue, 730

Tissue juice, 55

Tissue staining, in bulk, 739

progressive, 740

regressive, 740

Tissue stains, 744

aceto-carmin, 744

alum carmin, 744

alum hematein (Mayer), 741

Böhmer's hematoxylin, 741

borax carmin, 743

carmin, 743

double staining, 746
Tissue stains, Congo red, 746

Delafield 's hematoxylin, 742

eosin, 745

fuchsin, 746

hematin, 741-743

and eosin, 746

iron-hematoxylin, 747

Mallory's, for connective tissue, 753

Mann's hematein (Ehrilich's hematoxylin), 742

methyl green, 743

methyl blue, and safranin, 747

methylene blue, 743

muchematein, 748

mucicarmin, 748

mucinous, specific stain, 748

orange $\mathrm{G}, 746$

picro-carmin, 744

picro-fuchsin, for connective tissue (Van Gieson), 752

safranin, 744

single, with nuclear dyes, 741

Weigert's, for elastic tissue, 731

Tissues, 30

box for embedding for sectioning of, 737

decalcification of, 731

dissociation of, 721

chemical, 722

embedding of, 734

in celloidin, 734

in paraffin, 735

hardening of, 733

injection of, 732

maceration of, 721

sectioning of, 736

staining of, 738

teasing of, 721

Tongue, 337

blood vessels of, 343

foramen cecum of, 343

glands of, 342

lymphatics of, 344

mucous membrane of, 337

nerve supply of, 344

papillæ of, 339 
Tongue, serous glands of, 343

taste buds of, 341

Tonsil, faucial (palatine), 242

lingual, 244, 343

pharyngeal, 244

tubal, 692

Trachea, 302

1 structures of, 302

Tunica propria, corium of skin, 34

Tympanic membrane, 687

Tympanum of ear, 685. See also ear

Tyson's glands, in penis, 506

Umbo, of tympanic membrane, 687

Unit characters, in Mendelian inheritance, 477

Urinary system, 423

kidney, 423

arciform veins of, 443

arteries, arteriæ propriæ renales, 440

arciform, 440

arteriæ rectæ, 441

interlobular, 441

Bellini's ducts of, 438

capsule of Bowman, of, 429

corpuscles (renal) of, 425

cortex of, 424

glomerulus of, 429

lymphatics and nerves of, 444

medulla of, 424

papillary ducts of, 438

pelvis and ureter of, 445

blood supply of, 448

mucosa of, 445

muscular coat of, 448

tunica propria of, 447

renal circulation, table showing course of, 443

renal connective tissue, 426

renal corpuscles (Malpighian bodies), 425-429

renal lobule, 426

topography of, 423

tubules of, arched connecting (junctional tubule), 437
Urinary system, kidney, tubules of, ascending limb of Henle's loop, 435

descending limb of Henle's loop, 434

distal convoluted portion of, 435

divisions of renal tubule, 429

loop of Henle, 435

neck of, 431

peculiarities of, 440

proximal convoluted portion of, 431

straight collecting, 437

table of divisions of renal tubule, 439

uriniferous, or renal, 427

urethra, female, 452

structure of, 452

urethra, male, structure of, 454

urinary bladder, 448

mucous membrane of, 448

muscular coat of, 451

vascular and nerve supply of, 452

veins of, stellate, 443

venæ propriæ renales, 443

venulæ rectæe of, 443

Uterus, the, 532

blood vessels of, 532

cells of Langhans of chorionic villi of, 537

cervical glands of, 532

chorionic villi of pregnant uterus, 537

decidua menstrualis of, 534

decidua reflexa of, 537

decidua serotina of, 536

decidua vera of, 537

decidual cells of, 535

fetal blood vessels, 538

glands of, 531

gravid, 535

lymphatics and nerves of, 533

menstruating, 533

mucosa, or endometrium, of, 529

muscular coat, or myometrium, of, 528 
Uterus, Nabothian follicles of, 532 placenta uterina, 536 serous coat, or perimetrium, of, 528 structure of, 528

Utricle, of ear, 698

Vagina, mucosa of, 538 musculature of, 538 outer fibrous coat of, 539 structure of, 538

Valves, of heart, 196

Valvulæ conniventes, 364

Vas (ductus) deferens, 493

Vascular sinusoids, 187

Vascular tissue, 176

Veins, 187

arciform, of kidney, 443

atypical, 189

auditory, 716

circulus venosus, of Haller, 547

eranial, 190

larger, structure of, 188

of central nervous system, 625

of ear, 715

of kidney, 443

of liver, 415

of ovary, 523

of sclera, 635

of spermatic cord, 494

portal, 417

pulmonary, 190-317

small, 188

smaller and larger, comparison of, 190
Veins, valves in, 191

vena cava, 190

vena comes, 191

venæ vorticosæ, of eye, 672

Velum, marginal, 590

Venous spaces, 190

Venules, precapillary, 187

Vermiform appendix, 379

Vesicular appendages, 539

Vestibule, of pudendum, 540

Vestigial structures, associated with reproductive organs, 539 . 540

Visual area, 617

Visual axis, 628

Vitreous humor, 669

Vocal cords, 301

Weigert-Pal stain, for medullated nerve fibers, 748

Weigert's elastic tissue stain, 753

Wirsung's duct, 394

Wolffian body, or mesonephros, 456

Xylo-balsam, for mounting sections, 760

Zenker's solution, 727

Zimmermann's technic, for intercalated dises of cardiac muscle, 758 .

Zonula ciliaris, of eye, 670

Zygote, 27-460 







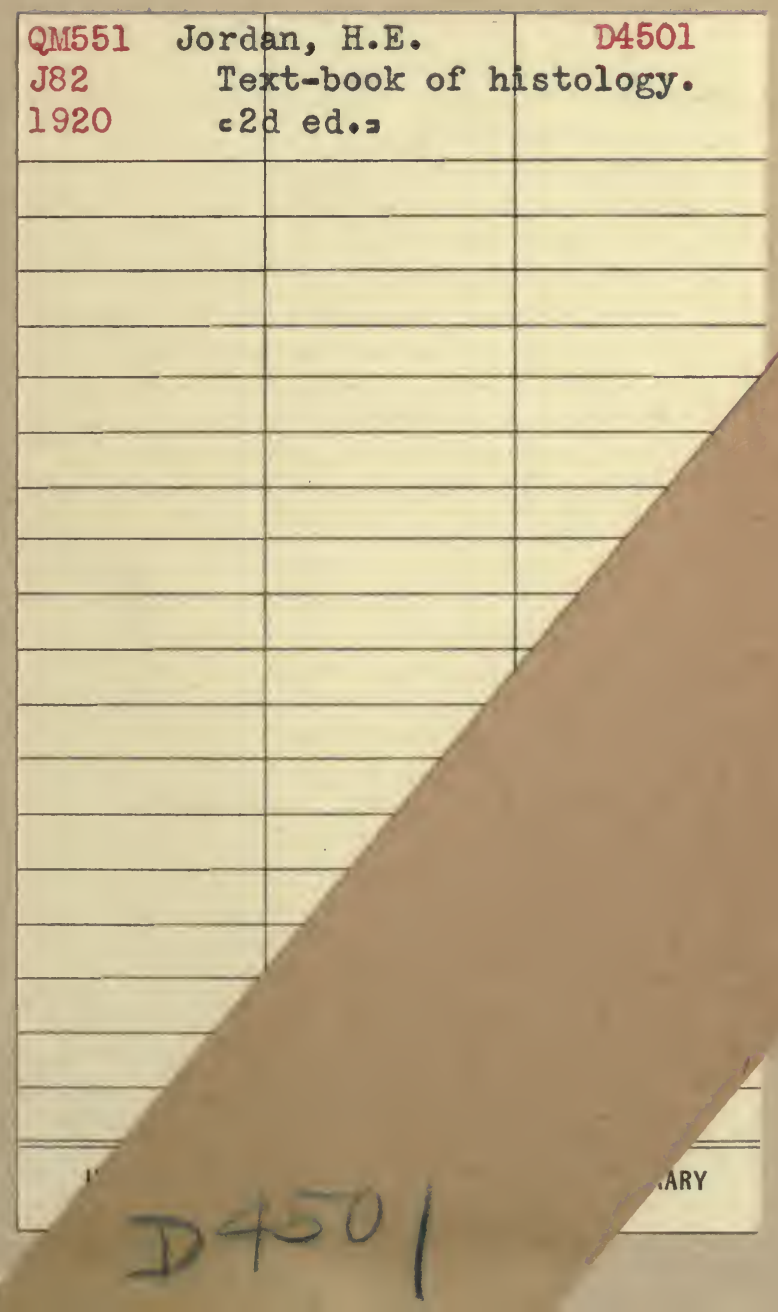


